This report was funded by the Bonneville Power Administration (BPA), U.S. Department of Energy, as part of BPA's program to protect, mitigate, and enhance fish and wildlife affected by the development and operation of hydroelectric facilities on the Columbia River and its tributaries. The views in this report are the author's and do not necessarily represent the views of BPA.

For additional copies of this report, write to:

Bonneville Power Administration

Public Information Center - CKPS-1

P.O. Box 3621

Portland, OR 97208

Please include title, author, and DOE/BP number from the back cover in the request. 


\title{
APPLICATION OF THE ECOSYSTEM DIAGNOSIS AND TREATMENT METHOD TO THE GRANDE RONDE MODEL WATERSHED PROJECT
}

\author{
Prepared by: \\ L. Mobrand \\ and \\ L. Lestelle \\ Mobrand Biometrics \\ Vashon Island, WA
}

Prepared for:

U. S. Department of Energy

Bonneville Power Administration

Environment, Fish and Wildlife

P.O. Box 3621

Portland, OR 97283-3621

Task Order Number 95AT6 1148

Contract Number 94AM33243

JANUARY 1997 


\section{CONTRIBUTORS}

This work product is truly the result of a collaborative effort of all the participants in this project.

The contributors in alphabetical order were:

Don Bryson, Nez Perce Tribe

Rich Carmichael, ODFW

Errol Claire, consultant to ODFW

Ron Costello, Mobrand Biometrics Inc.

Boyd Hadden, private consultant

Kim Jones, ODFW

Bill Knox, ODFW

Lyle Kuchenbecher, Grande Ronde Model Watershed

Larry Lestelle, Mobrand Biometrics Inc.

Kevin Martin, USFS

Glenn McDonald, private consultant

Lars Mobrand, Mobrand Biometrics Inc.

Bob Rose, USFS

Mark Shaw, BPA

Brad Smith, ODFW

Ken Witty, Cramer and Assoc.

Patty Perry and Board members of the Grande Ronde Model Watershed Project provided valued advice, direction and patient support during the course of this project. Several additional individuals contributed to the earlier phases of this project; they are included in the list of participants at the beginning of this report. 


\section{LIST OF TABLES}

Table 1. Definition of spring chinook salmon life stages within the Grande Ronde Basin and corresponding time periods..................................................... 17

Table 2. Descriptions of key habitat used by spring chinook salmon by life stage within the Grande Ronde Basin. ................................................................................ 18

Table 3. Description of environmental quality attributes affecting responses of salmonids within freshwater. This list was applied to spring chinook salmon in the Grande Ronde Basin; other attributes may be needed for other species or in different types of environments.

Table 4. List of reach ranges used in the analysis Sample table showing evaluation of a reach based on its importance to primary and secondary life history pathways.

Table 7. Sample table showing evaluation of a reach based on its importance to vacant life history pathways. 


\section{LIST OF FIGURES}

Figure 1. Technical and policy related components of Watershed Management Process. . . . . 3

Figure 2. Annual planning and decision making cycle. .........................................4

Figure 3. Map showing location of Grande Ronde / Imnaha Basins. .................................6

Figure 4. Sample key habitat landscape for a tributary (Catherine Cr.) .......................... 14

Figure 5. Sample productivity landscape for a tributary(Catherine $\mathrm{Cr}$.)...................... 15

Figure 6. $\quad$ Sample environmental attributes landscapes for a tributary (Catherine

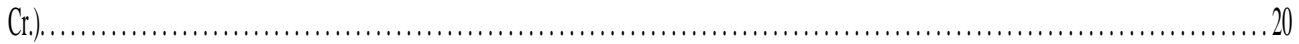

Figure 7. Sample key habitat landscape for basin (Catherine Cr./Grande Ronde R.)..........21

Figure 8. Sample productivity landscape for basin (Catherine Cr./Grande Ronde R.)........22

Figure 9. Sample environmental attributes landscapes for basin (Catherine Cr./Grande

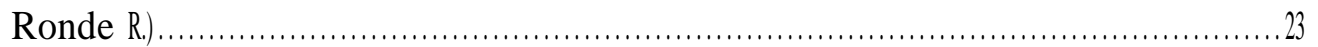

Figure 10. Sample graphic showing nine typical life history pathways used in the diagnosis (spawning reach in this example is the South Fork of Catherine Cr.). . . . . . . . . . . 29

Figure 11. Sample graphic showing productivity and capacity profiles for pathways with common spawning reach (in this example the spawning reach is the South Fork of

Catherine Cr.). ......................................................................................... 31

Figure 12. Comprehensive performance plot for the Grande Ronde Basin.......................33

Figure 13. Performance plots for pathways within the Grande Ronde Basin, grouped by subbasin where the spawning stage occurs.............................................34

Figure 14. Productivity frequency charts for pathways associated with spawning reaches within subbasins of the Grande Ronde Basin. Frequencies are weighted by spawning reach lengths. Shaded bars represent Template, and white bars

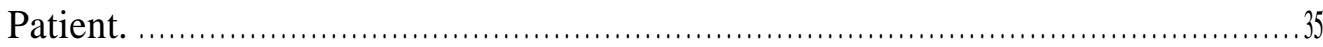

Figure 15. Comprehensive performance plot for the Imnaha Basin. ...............................36

Figure 16. Performance plots for pathways within the Imnaha Basin, grouped by subbasin

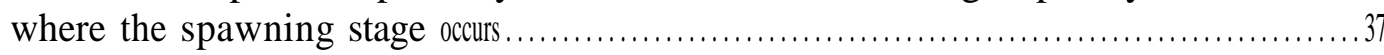

Figure 17. Productivity frequency charts for pathways associated with spawning reaches within subbasins of the Imnaha Basin. Frequencies are weighted by spawning reach lengths. Shaded bars represent Template, and white bars Patient ............ 38

Figure 18. Potential benefits to primary and secondary life histories from reach specific habitat quality improvements in the Grande Ronde and Imnaha basins. Each point in the graph represents one stream reach. The rectangle marked "A" contains the reaches that are most important to chinook salmon and have suffered the most severe loss of habitat quality. The benefit categories are defined in the text. . . ....5 1

Figure 19. Potential benefits to lost(vacant) life histories from restoration of spring chinook performance characteristics in specific reaches in the Grande Ronde and Imnaha basins. Each point in the graph represents one stream reach. The categories defined by the rectangles and marked with the letters $\mathrm{A}-F$ are discussed in the

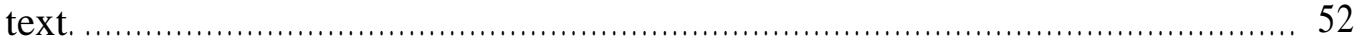




\section{LIST OF APPENDICES}

APPENDIX A: Productivity and Capacity Profiles for Life History Pathways in the Grande Ronde River and Tributaries

APPENDIX B: $\quad$ Productivity and Capacity Profiles for Life History Pathways in the Imnaha River and Tributaries

APPENDIX C: Patient Template Analysis. Landscapes showing Key Habitat, Productivity, and Environmental Attributes for each Range

APPENDIX D: $\quad$ Grande Ronde Stream Reach Summaries for Primary and Secondary Life History Pathways

APPENDIX E: $\quad$ Grande Ronde Stream Reach Summaries for Vacant Life History Pathways

APPENDIX F: Imnaha Stream Reach Summaries for Primary and Secondary Life History Pathways

APPENDIX G: Imnaha Stream Reach Summaries for Vacant Life History Pathways APPENDIX H: BIBLIOGRAPHY 


\section{LIST OF PARTICIPANTS}

Ralph Browning, USFS

Don Bryson, Nez Perce Tribe

Rich Carmichael, ODFW

Errol Claire, consultant to ODFW

Ron Costello, Mobrand Biometrics Inc.

Larry Gilbertson, Mobrand Biometrics Inc.

Jerry Gildemeister

Boyd Hadden, private consultant

Charles Huntington, consultant to Umatilla Tribe

Kim Jones, ODFW

Bill Knox, ODFW

Lyle Kuchenbecher, Grande Ronde Model Watershed

Mark Lacy

Larry Lestelle, Mobrand Biometrics Inc.

Kevin Martin, USFS

Glenn McDonald, private consultant

Lars Mobrand, Mobrand Biometrics Inc.

Bob Rose, USFS

Mark Shaw, BPA

Brad Smith, ODFW

Ken Witty, Cramer and Assoc.

Jeff Zakel, ODFW 


\section{PREFACE}

The results of the first phase of the GREDT project were reported in three documents: A "Recommendations" report, a "Template for Project Planning Status Report", and a Progress Report. This document replaces the Recommendations and Progress Reports. The recommendations put forth in the first report are included in Part I of this document without significant change. The Template Project Status Report, which shows how an annual planning report is structured, is a stand alone piece which is not incorporated here. The contents of the Progress Report have been updated and expanded into the methods (Part II) and results sections (Part III) of this report. The phase one Progress Report covered the upper portion of the Grande Ronde basin only, this report covers all of the Grande Ronde and Imnaha basins.

The data and information included in this analysis were obtained from numerous sources. Lengths and widths for individual reaches were obtained from the StreamNet database and checked against Oregon State Resource maps. Seasonal and historic variations in width were provided by Mr. Boyd Hadden, former water master in the basin. Studies and reports dating back as far as 1845 were consulted, a bibliography is included as Appendix H. Most of the information, however was assembled through a series of workshops where biologists familiar with the streams in the basin provided input from survey records, field notes, and personal experience. The specific sources of each data item used in this report are documented in the projects electronic database. This database, which also includes the "raw data" is housed on the computers at the offices of Mobrand Biometrics Inc. awaiting transfer to a more appropriate locally accessible data keeper. 


\section{PART I. AN OVERVIEW OF THE GREDT PROJECT}

In the spring of 1994 a technical planning support project was initiated by the Grande Ronde Model Watershed Board of Directors (Board) with funding from the Bonneville Power Administration. The project was motivated by a need for a science based method for prioritizing restoration actions in the basin that would promote effectiveness and accountability. In this section we recall the premises for the project. We also present a set of recommendations for implementing a watershed planning process that incorporates a science-based framework to help guide decision making. This process is intended to assist the Grande Ronde Model Watershed Board in its effort to plan and implement watershed improvement measures. The process would also assist the Board in coordinating its efforts with other entities in the region.

The planning process is based on an approach for developing an ecosystem management strategy referred to as the Ecosystem Diagnosis and Treatment (EDT) method (Lichatowich et al. 1995, Lestelle et al. 1996). The process consists of an on-going planning cycle. Included in this cycle is an assessment of the ability of the watershed to support and sustain natural resources and other economic and societal values. This step in the process, which we refer to as the diagnosis, helps guide the development of actions (also referred to as treatments) aimed at improving the conditions of the watershed to achieve long-term objectives.

The planning cycle calls for routinely reviewing and updating, as necessary, the basis for the diagnosis and other analyses used by the Board in adopting actions for implementation. The recommendations offered below address this critical need to habitually updated the information used in setting priorities for action.

\section{PREMISES}

This project and the recommendations that follow are based upon a set of premises drawn from the purpose of the model watershed program in general and the Grande Ronde Model Watershed in particular. Those premises are:

Ecosvstem strategv -- Watershed planning should employ a holistic approach that incorporates human economies and values, while being consistent with ecological principles that promote sustainability. The strategy should incorporate the broad range of values and objectives held by stakeholders in the Grande Ronde Basin. Although many of the initial actions may target spring chinook salmon, the scope of watershed planning is much broader. The salmon should be seen as an indicator, or diagnostic species, of the general condition of the watershed and its ability to sustain a diversity of values and objectives.

Sustainabilitv -- Communities generally desire that resource-based values and objectives associated with the water and land of a watershed be sustainable. These communities embrace basic land and water ethics that recognize the need for sustainability within the context of a watershed that has undergone major changes to accommodate human needs.

Grande Ronde Ecosystem Diagnosis and Treatment /January 1997 /Page I 
Therefore, land and water processes of a watershed should continue to function in a manner that can sustain both human and natural resources. If such processes are rapidly changed without regard to consequences, those resources may not be sustainable. Species like salmon that are dependent on the relative stability of those processes over a wide expanse of the watershed can therefore help us diagnose conditions for sustainability.

Scientific method -- Current understanding of how to achieve resource sustainability is limited and inadequate for meeting the challenges of growing communities and their associated needs for natural resources. Therefore, a necessary part of watershed management should be the use of the scientific method to improve understanding over time. Fundamental to this method of learning is the use of an explicit conceptual framework within which information about the system of interest is gathered, analyzed and organized. A logical linkage between actions and events within the watershed and their effect on values and objectives must be presumed and explicitly addressed. The scientific method promotes learning and assures accountability.

Adaptive Management -- Watershed management should be driven by a decision process that is based upon learning by doing, often referred to as adaptive management. This approach to decision making allows action in the face of scientific uncertainty. It serves two important functions: it provides assurance that watershed management is progressive-i.e. those actions that are effective are continued and those proven ineffective or damaging are discontinued: and it also provides the means for an open decision making process where the public has the opportunity to remain informed and participate effectively. Adaptive management requires a strong commitment to learning through research, monitoring and evaluation. Both scientific information and community values must be effectively integrated into the adaptive management decision process.

\section{PROCESS RECOMMENDATIONS}

In order to make the best use of the framework and information developed by the GREDT project, in the context of adaptive management, we offer the following four recommendations:

1. We recommend that the Board organize its planning process so that both policy and technical roles are clearly identified and supported.

The watershed management process consists of both policy and technical related components (Fig. 1). The Board is responsible for policy related elements, including such items as identification of objectives, selection of actions for implementation, scheduling, and review of program performance.

Technical support information is needed by the Board in fulfilling its functions (Fig. 1). This information needs to be organized and understood through a science-based framework. The information should be supplied to the Board by individuals qualified to perform necessary analyses and to communicate the results. 


\section{Watershed Management Process \\ (Adaptive Management)}
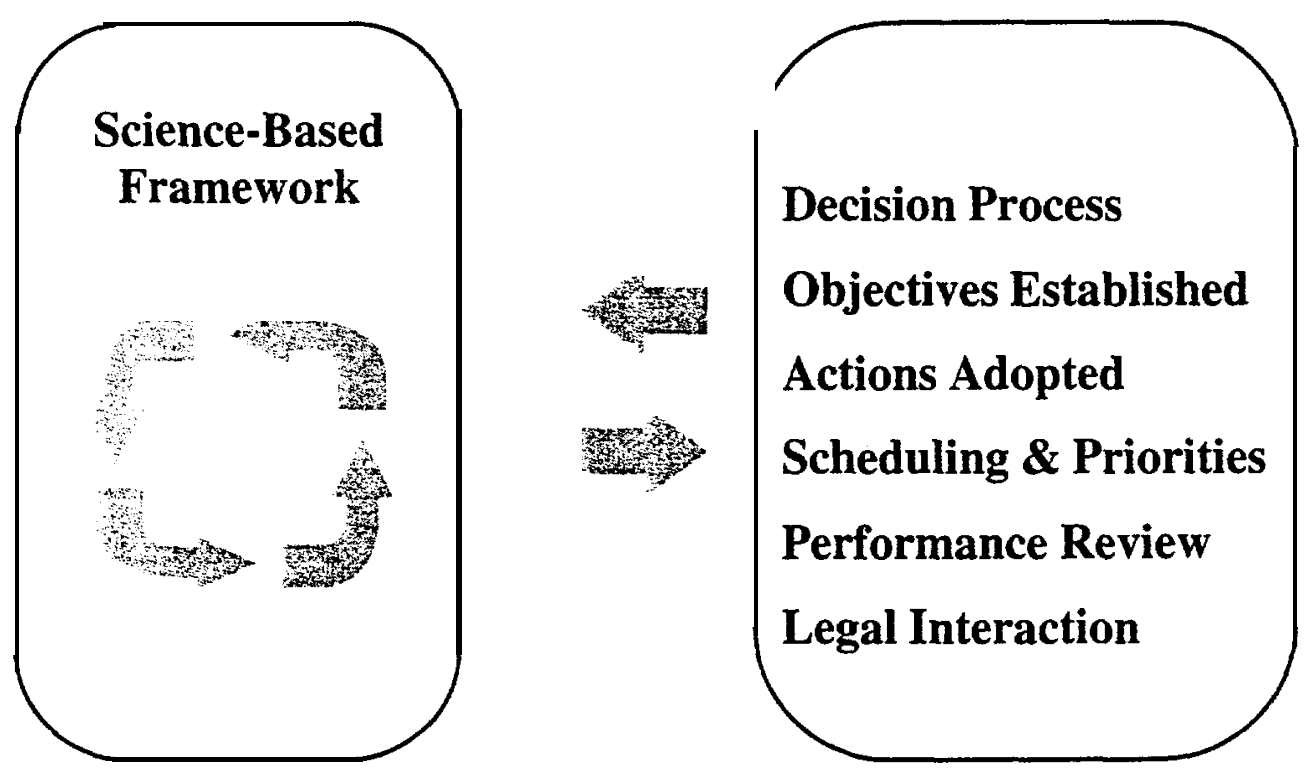

Figure 1. Technical and policy related components of Watershed Management Process.

We assume that the technical aspects of the program are to be handled by existing staff of the Grande Ronde Model Watershed Program at least in the short-term. In the future, the Board may want to consider other options for handling technical needs, such as drawing on the expertise of other governmental agencies in the region or hiring its own specialists as may be necessary.

2. We recommend that the Board formalize an annual planning cycle, through which the Board would review the progress of the program, consider proposed actions, and adopt appropriate actions for implementation.

Such an annual planning cycle would revolve around the Board's responsibilities for considering proposed actions and for adopting and implementing those that are selected (Fig. 2). Technical staff would supply information to the Board consistent with the annual cycle.

The planning cycle would set a schedule for accomplishing the following each year:

Produce an annual planning report (Planning Status Report or PSR)

Review a range of candidate actions for the upcoming year

Prioritize candidate actions on the basis of how consistent they are with program strategies and for their expected results

Adopt actions for implementation

Perform an annual review of the performance of the program

Grande Ronde Ecosystem Diagnosis and Treatment/January 1997/Page 3 


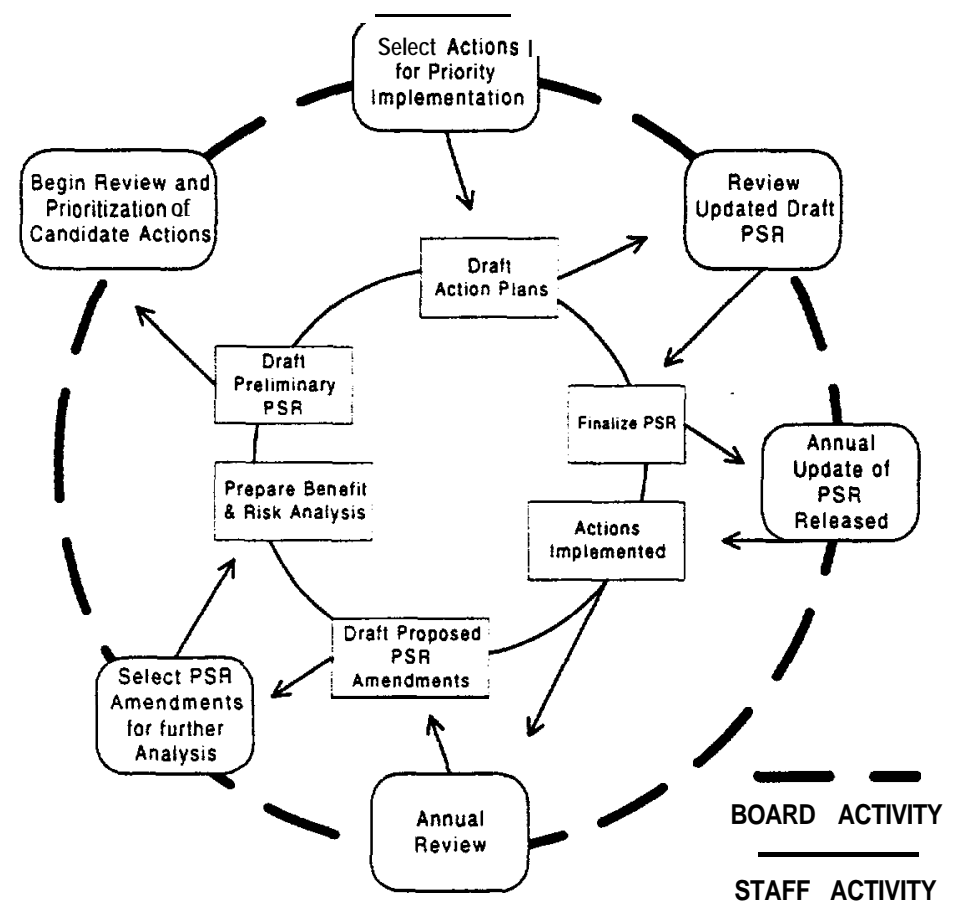

\section{Figure 2. Annual planning and decision making cycle.}

3. We recommend that the Board modify its existing technical support activities so that staff support would include the following, consistent with the annual cycle:

Prepare a set of proposed actions that are developed consistent with the watershed diagnosis

Prepare a technical analysis (including trade-offs) of all candidate actions (those based on the scientific diagnosis as well as any other proposed actions)

Prepare drafts of the annual Planning Status Report (see Fig. 2)

Coordinate the annual performance review

Update analytical tools as required

4. We recommend that a standard set of analytical tools be used in generating technical support information for the Board.

These tools are described in detail in a BPA report titled "Applied Ecosystem Analysis - A Primer" (Lestelle et al. 1996). The tools include the following items:

A database of information used in diagnosing watershed conditions

An analytical model for computing productivity and capacity indices of spring chinook in the basin

A series of graphical displays to assist in performing analyses and communicating results

$\mathrm{Cl}$ A watershed restoration strategy that incorporates the metapopulation concept to form guidelines to prioritize actions 


\section{PART II. APPROACH AND METHODOLOGY}

This part of the report covers five topics: an overview of the Grande Ronde watershed; chinook salmon as a diagnostic species: the EDT approach; the data analysis methods used; and principles for prioritization of actions.

\section{THE GRANDE RONDE WATERSHED - A Brief Orientation}

The Grande Ronde Basin is located in northeastern Oregon and southeastern Washington (Fig. 3). The basin drains an area of approximately 4,000 square miles and connects to the Snake River 169 miles upstream of its mouth. The confluence of the Snake and Grande Ronde rivers is located 493 miles upstream of the mouth of the Columbia River.

The basin is characterized by rugged mountains and two major river valleys-the Grande Ronde and Wallowa valleys. The Blue Mountains and the Wallowa Mountains rise to 7,700 and 10,000 feet respectively.

The basin has a semi-arid climate. Temperatures and precipitation vary with elevation. Average annual rainfall in the valleys ranges from 12 inches to 23 inches, while at higher elevation it can exceed 50 inches. The valleys tend to have warm, dry summers and cold, moist winters (NPPC 1990).

Approximately 45 percent of the basin is public land managed by the U.S. Forest Service (NPPC 1990). Privately owned land is located primarily at lower elevations along streams and on the valley floors, Primary land uses in the basin are forest, range, and cropland.

The Grande Ronde Basin contains aquatic resources of considerable importance to local, regional, and national interests. These include approximately 2,300 miles of salmonid streams (Huntington 1994). The majority of these miles support anadromous salmonids.

Alterations of the watershed started soon after settlement in the 1860s. Agricultural development, timber harvest and mining were among the land use practices that stimulated early development and supported the growth of local communities. These activities have gradually changed the natural landscape and altered the physical and biological processes that characterized the system.

Reviews of changes that have occurred to the basin's stream system are given in West and Zakel (1993), Smith and Knox (1993), and McIntosh (1992).

\section{CHINOOK SALMON - A Diagnostic Species}

Early in this project we concluded that chinook salmon are indeed a useful indicator of watershed health. The history of chinook salmon abundance and distribution is a reflection of the changes that its environment has undergone over the past century. 


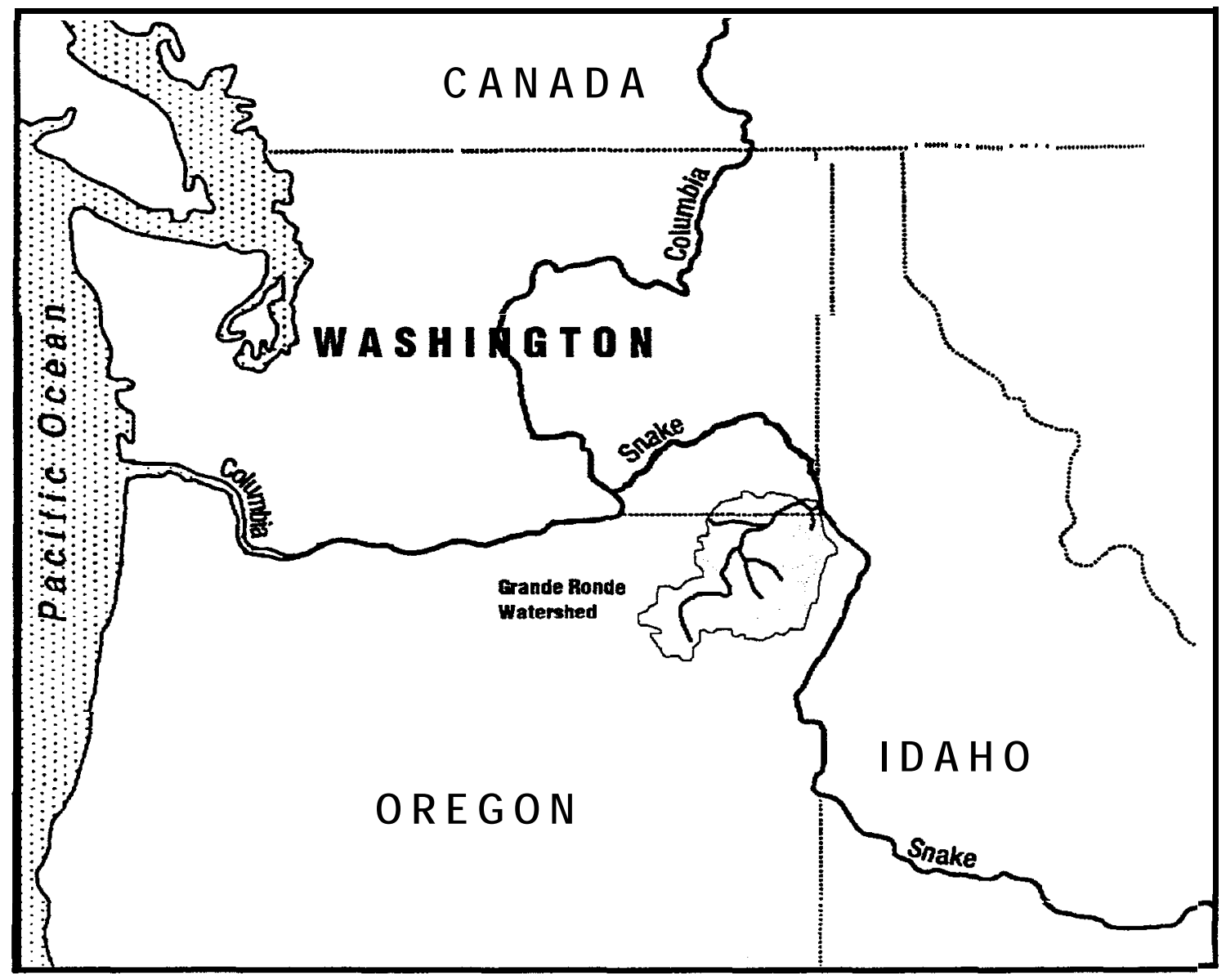

Figure 3. Map showing location of the Grande Ronde / Imnaha Basins. 
The Grande Ronde Basin historically produced large runs of spring chinook salmon. Fisheries managers estimated that 12,200 spring chinook entered the river in 1957 (NPPC 1990). Run sizes were estimated to be about 8,400 fish in the early 1970s (Smith 1975). Prior to all dam construction and alteration of the watershed, run sizes were likely much larger than these levels (Lichatowich and Mobrand 1995).

Spring chinook are widely distributed in the basin. Twenty-one streams historically supported spawning. The Wenaha River, Catherine Creek, Minam River, Lookingglass Creek, Lostine River, and the Upper Grande Ronde River were considered highly productive for these fish (NPPC 1990).

Redd counts made in the basin indicate that spawner abundance remained relatively high until the early 1970s. A sharp decline has occurred since then. Run sizes in some recent years have numbered several hundred fish.

Outplanting (supplementation) of spring chinook has occurred in the basin in recent years in an attempt to bolster the run. See NPPC (1990) for a more complete description.

The suitability of chinook salmon as a diagnostic species is further supported by arguments that:

- it is sensitive to water quality and quantity, which is important to the ability of the watershed to support many other objectives,

- it is a highly migratory species and therefore utilizes most of the watershed - from mouth to headwaters,

- it has many distinct life stages and a complex history, with specific and varying

environmental requirements,

- through its life history it connects the different environments and cycles nutrients between ocean and headwaters,

- and last but not least chinook salmon are an integral part of the Northwest heritage and represent an important component of its culture and quality of life.

\section{THE EDT APPROACH - An Overview}

The approach we are about to describe is referred to as the Ecosystem Diagnosis and Treatment (EDT) method (Lichatowich et al. 1995, Lestelle et al. 1996). It was devised to help diagnose the condition of resources like salmon and the environments upon which they depend. A diagnosis guides the development of rational actions and strategies. Actions may involve land use practices, water management, hatcheries or habitat restoration. The EDT method is presented in greater detail in a report titled "Applied Ecosystem Analysis - A Primer" (Lestelle et al. 1996), which we refer to in this report as the "Primer". We urge the reader who wishes a more complete description of the EDT approach to consult the "Primer".

The EDT method was developed with strong emphasis on the importance of a science-based approach. Fundamental to the scientific method is the use of an explicit conceptual framework 
within which information about the natural system is gathered, analyzed and organized. A logical linkage between actions and events within the watershed and their effect on values and objectives must be presumed and explicitly addressed. This process promotes learning and provides accountability.

While the ideas we present are based on concepts that are familiar, we believe the EDT method provides a new and useful way of thinking about resources like salmon and their ecosystems.

The Ecosystem Diagnosis and Treatment (EDT) method is a science-based approach to formulating and analyzing actions to maintain or improve the sustainability and production of natural resources. Its chief aim is to provide a theory, a set of tasks, and a collection of tools which can form the basis for technical input in watershed management processes.

The approach provides an ecosystem perspective to natural resource management. The analysis focuses on one or more species, whose dependence on the ecosystem is extensive both in space and time. The approach has been developed and tested using the life histories of migratory salmonids. Their broad migratory range and sensitivity to human activities make them well suited as indicators or diagnostic species. Species other than salmon could be analyzed using the same approach.

The EDT method is formulated around five premises that support the concept of watershed management: 1) Management actions, whether aimed at biotic (e.g., fish populations) or abiotic (e.g., water and land) elements, should be built on an ecosystem-directed strategy; 2) A primary goal for management is to ensure the sustainability of renewable natural resources and, where possible, to allow their sustainable use; 3) Certain species or populations that are dependent on the relative stability of ecological processes over a wide expanse of the watershed can serve to help diagnose conditions for sustainability; 4) Use of the scientific method in management will increase understanding of important ecological relationships; and 5) Information generated through using the EDT method will be of most benefit if it is incorporated into an on-going and iterative management process.

The EDT method is comprised of three components: Tasks, Theory, and Tools These components complement each other. The Tasks set forth a specific sequence of actions; they explain what needs to be done and in which order. The Theory is the conceptual framework needed to apply the scientific method, and the Tools are methods, procedures and aids designed for analysis and communication. Each component is described briefly below.

The Tasks are composed of a sequence of planning and implementation steps introduced in Lichatowich et al. (1995). The sequence forms an iterative cycle of planning, implementing, and learning: 1) Identification of objectives; 2) Diagnosis; 3) Formulation of strategies and actions; 4) Trade-off analysis; 5) Revision of objectives; and 6) Prioritization and sequencing and implementation of actions, including monitoring and evaluation. Several of these steps require policy direction-we confine our discussion to the technical elements of the process.

The Theory is the conceptual framework that gives the tasks purpose and meaning. Watershed management has a logical requirement-we must be able to see the whole and its parts in a 
consistent way. This requires conceptualizing the system in a manner that is useful and consistent with ecological theory. We call this conceptualization a framework or a theory. The architecture of the framework is prevailing ecological theory, and its building blocks are the assumptions which explain our observations and rationalize our actions. The EDT method incorporates an explicit conceptual framework for understanding how actions will affect the ecosystem. The EDT framework partitions the relationship between goals and actions into three components: (1) it relates goals to biological performance, (2) biological performance to environmental attributes, and (3) environmental attributes to actions.

The Tools are analytical procedures or conceptual devices used in performing analyses. There are four categories of tools: 1) diagnostic procedures and models; 2) procedures for strategy development; 3) procedures for trade-off analysis; and 4) monitoring and evaluation procedures. These tools include procedures for capturing data (a database system), for analyzing information (models and analytical routines), for displaying results (graphics and reports), and for prioritizing monitoring and evaluation.

\section{DATA AND ANALYSIS}

The data and information that is analyzed and presented in this report were obtained through a series of workshops conducted in La Grande and Enterprise over the past three years. The methods used to organize, analyze and display the data are described in detail in the Primer (Lestelle et al. 1996). We also refer to that report for a discussion of assumptions associated with the approach.

The workshops focused primarily on the second component of the framework - the relationship between biological performance and environmental attributes (see previous section). This relationship was examined for each stream reach from the perspective of seven chinook salmon life stages. Both the present and the historic (prior to Euro-American influences) conditions were evaluated as a part of a Patient-Template Analysis (PTA) (Lichatowich et al. 1995). The PTA formed the basis for the "diagnosis" of the watershed.

Part III of this document presents the results of our analysis of the Grande Ronde watershed from the perspective of chinook salmon.

\section{PRIORITIZATION PRINCIPLES}

The diagnosis tells us why resource objectives are not being met under current environmental conditions. We can now describe lost productivity and capacity in terms of location, time and life stage, and we can identify and describe the loss of life history diversity. Given the diagnosis and

the stated objectives, there still remains the question of which are the most important problems to address, what are the appropriate remedies and in what order should they be applied? 
The PTA and the diagnosis tell us the condition of the environment from the salmon's point of view. In particular it tells us the past and present conditions along a suite of potential life history pathways. It does not tell us which of the lost or damaged life history pathways are most important to rebuild or how to identify and prioritize potential remedial actions. This is done as a part of the "treatment selection" step in the EDT process.

The treatment selection must be consistent not only with the diagnosis of the environment but also with the present status of the chinook populations in the Grande Ronde and Imnaha basins. It also needs to be consistent with the mechanisms whereby chinook populations organize themselves within a changing environment. Those mechanisms include metapopulation organization and genetic diversity (ISG 1996). Analysis of the metapopulation structure of the historic Grande Ronde and Imnaha chinook populations was not attempted in this project (although the PTA analysis does shed light on this subject). Instead the assumption was made that the maintenance and enhancement of existing populations of spring chinook would be a high priority in the process of identifying and restoring core populations and their habitats.

Several hundred potential life history pathways for spring chinook within the study area were identified. Based upon present usage they were classified as primary, secondary, or vacant (perhaps the ghosts of pathways lost). Then the following strategic priorities were recommended:

1. Maintain the quality of habitat associated with primary life history pathways.

2. Improve the quality of habitat associated with primary life history pathways.

3. Maintain the quality of habitat associated with secondary life history pathways.

4. Improve the quality of habitat associated with secondary life history pathways.

5. Begin to improve habitat quality along lost life history pathways - with highest priority for potential core population pathways.

6. Reconnect the lost (and thus vacant) life history pathways to begin their repopulation.

In order to translate these strategic priorities into reach specific action priorities, we need to know (a) which reaches are most important for spring chinook, and (b) among those, which ones offer opportunities for improvement, To address question (a) we computed a "chinook usage score" for each reach based on the numbers of primary and secondary life histories it supported. Since all priority one life history pathways cannot be improved simultaneously, we need to further refine these strategic priorities. We suggest that the potential for biological performance improvement is an appropriate and useful criterion for such refinement. By biological performance we mean the combination of productivity, capacity and diversity. We argue that, under present conditions, productivity is perhaps the most important element of performance. The potential for improvement also depends upon the feasibility to enhance productivity -question (b) above. If we assume that historic performance represents an upper limit to future performance, then the difference between the present and the past is a measure of potential gain. In order to identify the priority of a specific stream reach we must take into consideration the potential cumulative gain in performance (primarily productivity) in that reach, which depends on how much time the fish spend there. These considerations were assimilated into a score for "habitat productivity change." 
Part III of this report includes tables and charts that enable decision makers to translate these strategic priorities into reach specific action priorities by referring to chinook usage scores, habitat productivity change scores, and other information. 


\section{PART III: RESULTS AND DISCUSSION}

This part of the report introduces the results of the EDT analyses performed to date. There are three subsections covering respectively: patient-template analysis (PTA), diagnosis, and treatment selection. The actual study results are contained in the Appendices at the end of the report. This section is a road map to the lookup charts and tables in the Appendices. 


\section{PATIENT-TEMPLATE ANALYSIS}

\section{Purpose and Terminology}

The Patient-Template Analysis (PTA) is an examination of watershed conditions from the perspective of spring chinook salmon, the diagnostic species. The PTA compares existing environmental conditions (the Patient) to historic reference conditions (the Template). The Template is a reference point that represents the spring chinook performance potential within the climatic, geologic, and biologic setting of the watershed.

Both the PTA and the subsequent diagnosis (next section) use the term "performance" to describe watershed conditions. Performance consists of three elements that together measure the suitability of the watershed to support spring chinook salmon. These elements are productivity', capacity', and diversity.' Roughly speaking, these terms represent the quality, the quantity, and the distribution and connectivity of conditions for spring chinook within the watershed.

Results of the analysis are presented as a series of charts and summary narratives. The narratives highlight the major differences between Template and Patient conditions seen in each chart. This information which is grouped by "stream reach ranges" (see definition below), is provided in Appendix $\mathrm{C}$ for both the Grande Ronde and Imnaha basins.

\section{Description of PTA Graphics}

The graphics are designed to facilitate comparison between Patient and Template. Each display is a visualization of spring chinook performance patterns over space and time within the watershed.

Two types of graphic layouts are used. The first displays either the quantity of key habitat -- a measure of habitat capacity (Fig. 4), or relative density-independent survival --a measure of productivity (Fig. 5). These graphics depict each of the two performance-related measures as a "landscape" across a grid that defines location and time. The landscapes are shown as threedimensional surfaces projected above a contour plot on the space-time plane.

\footnotetext{
1/ Productivity is the reproductive rate of a salmon life cycle: it can be disaggregated into a density-independent survival rate and reproductive potential (e.g., the salmon's fecundity) components. The survival component can be further disaggregated into life stage survivals. The cumulative value for an entire life history is referred to as cumulative productivity. The analysis presented here focuses on the survival component, which is affected by environmental quality.

2 / Capacity represents the upper limit on abundance that a given unit of habitat can support within some time period. Within a short period of time, it is largely determined by the quantity of habitat available (with a given quantity of food). Over longer periods of time, such as covering the full period of freshwater utilization by spring chinook, there is a cumulative capacity for production, which is affected by productivities and capacities at intermediate life stages. Capacity regulates the density-dependent survival component of total survival.

$3 /$ Life history diversity represents the range of pathways through space and time available to, and used by, a species in completing its life cycle.
} 


\section{Catherine Cr Key Habitat Quantity}

\section{Patient}

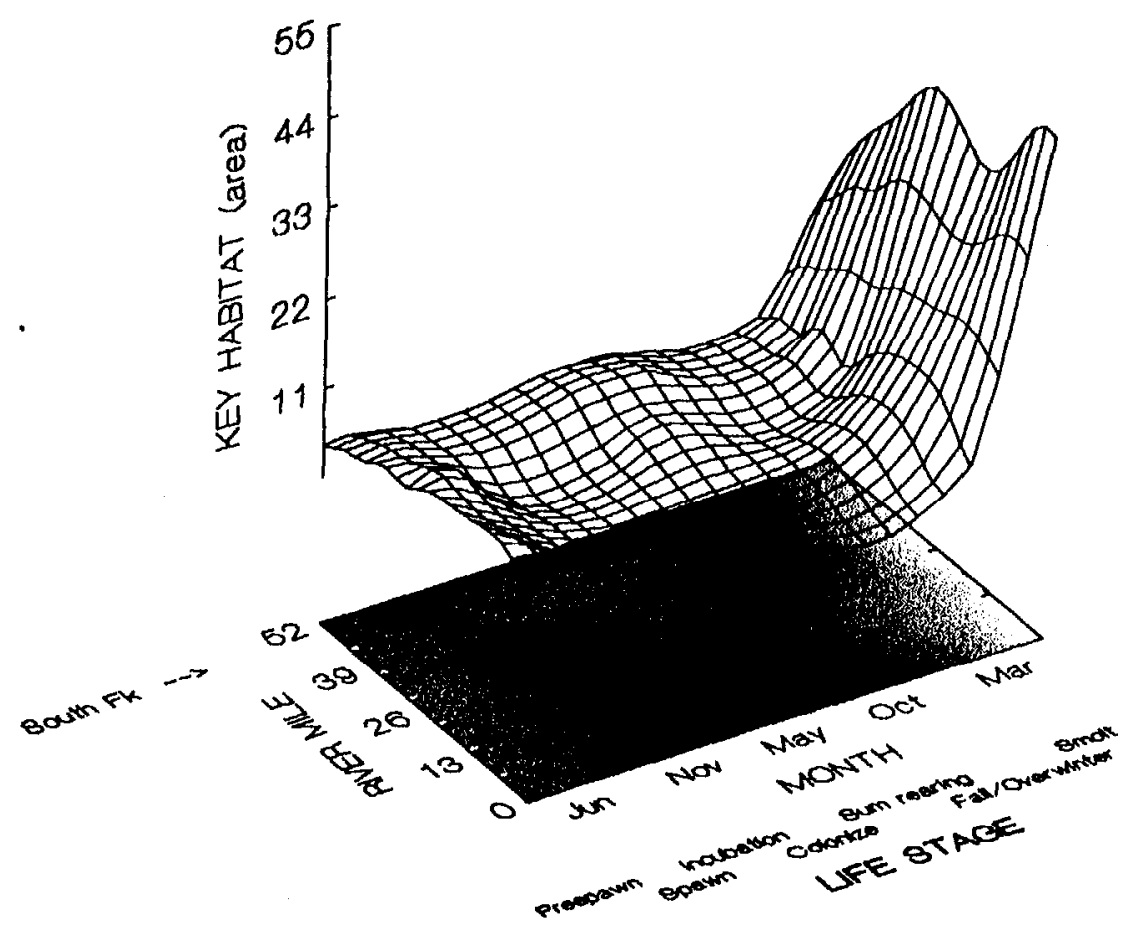

Template

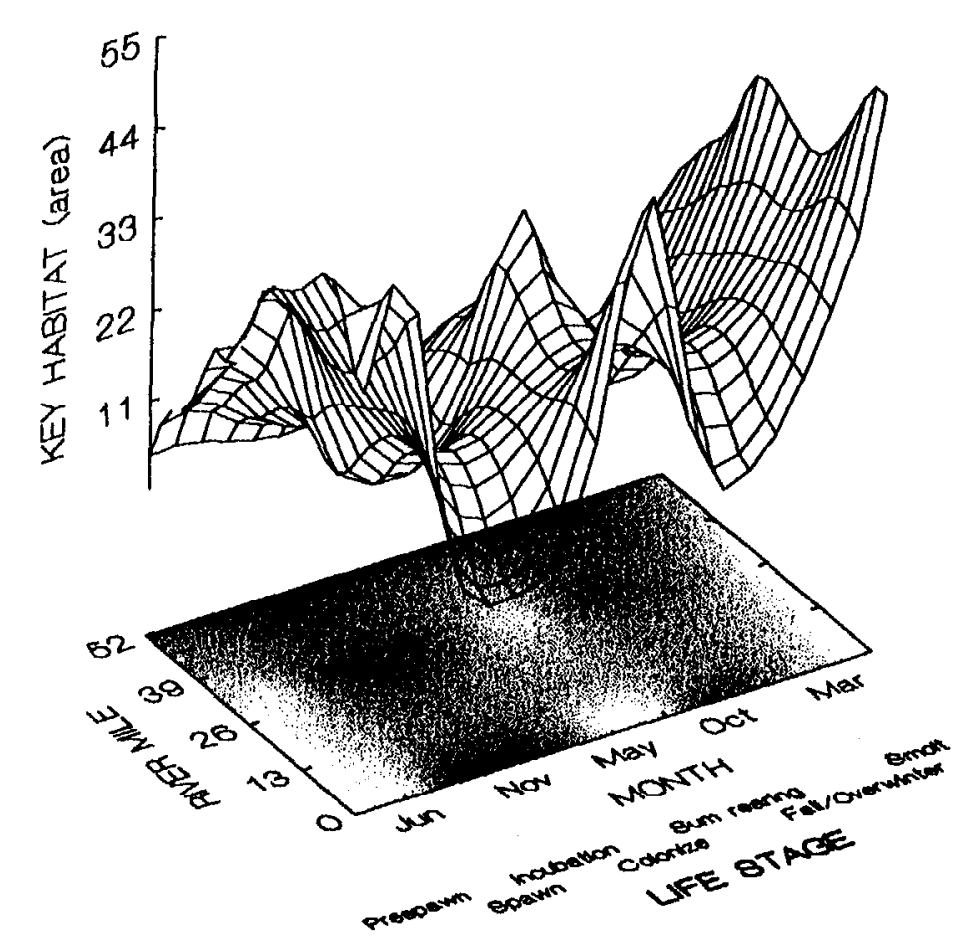

Figure 4. Sample key habitat landscape for a tributary (Catherine Cr.) 


\section{Catherine Cr Relative Survival}
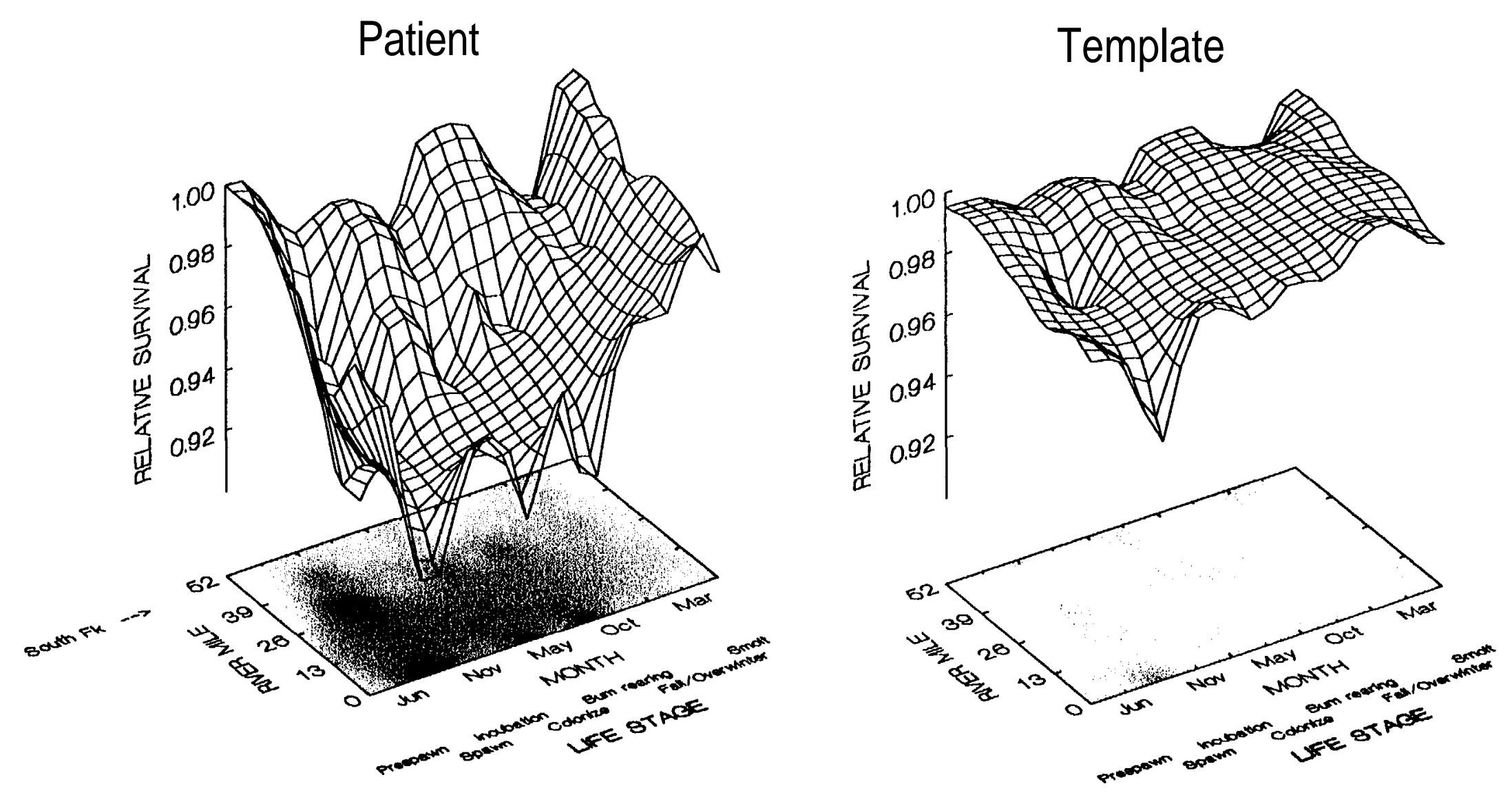

Figure 5. Sample productivity landscape for a tributary (Catherine Cr.) 
The space dimension of each graphic is shown as location (river mile) along a continuously connected stream channel, from its headwaters to its mouth. The time dimension is measured in months. Corresponding to time are the life stages (Table 1) of spring chinook salmon, shown on each chart to identify the approximate time period associated with each life stage.

The two measures (key habitat and relative survival) illustrated in these graphics are indirect measures of life stage capacity and life stage productivity. Key habitat is the portion of the total quantity of habitat available that is strongly preferred or required for a given life stage (Table 2); Fig. 4 depicts quantity as the amount of stream surface area within a defined stream reach comprising key habitat. Relative survival (Fig, 5) is an index of productivity, where a value of 1 indicates that survival conditions at a given location and time are optimal; values less than 1 indicate less than optimal conditions.

The second type of graphic is composed of 28 separate charts (Fig. 6), each with the same space and time dimensions that are used in the first type of graphic. The charts compare broad patterns of environmental quality for Patient and Template for each of 14 attributes that affect survival (Table 3), all displayed on one page to facilitate comparison. The display for each attribute is a 2D contour plot showing the effects of the attribute on productivity (i.e., relative survival, as illustrated in Fig. 5). These graphics are meant to show only broad patterns of attribute effects, highlighting differences between stream reaches and life stages-they do not show differences in effects within life stages at a particular location.

\section{Stream Reach Ranges}

The landscapes displayed in Figs. 4-6 use Catherine Creek as an example. Location (river miles) is displayed as distance from the mouth of Catherine Creek. Another way of viewing Catherine Creek is as an extension of the Grande Ronde River. Here location is shown along the river channel beginning at the Grande Ronde River's entry into the Snake River, extending upstream to the mouth of Catherine Creek, then continuing upstream the length of Catherine Creek (for example, Figs. 7-9). Hence location (distance in river miles from the Snake River) can always be viewed along a single, linear dimension in relation to the Snake River. The connected stream reaches that form the $y$-axis on the chart are referred to as a stream reach range.

A reach range begins at the main river's entry into the Snake River and terminates at or near the headwaters of a contiguous drainage. The locations where one stream enters the next larger channel are denoted along the reach range in the larger charts. Each reach range ends at or near the upstream margin of the habitat utilized by spring chinook in the drainage.

By displaying environmental information along the major reach ranges used by spring chinook salmon in a watershed, a series of landscapes can be presented that show the portions of the watershed that are most important, relevant to different spawning subpopulations. 
Table 1. Definition of spring chinook salmon life stages within the Grande Ronde Basin and corresponding time periods.

\begin{tabular}{|c|c|c|c|}
\hline Life Stage & Description & Months & $\begin{array}{c}\text { Statistical } \\
\text { weeks }\end{array}$ \\
\hline Pre-spawning adult & $\begin{array}{l}\text { Upstream adult migration and holding } \\
\text { prior to spawning. }\end{array}$ & April-August & $15-33$ \\
\hline Spawning & $\begin{array}{l}\text { Spawning period, including } \\
\text { establishment and defense of redd } \\
\text { sites. }\end{array}$ & $\begin{array}{l}\text { August- } \\
\text { September }\end{array}$ & $33-38$ \\
\hline Incubation & Egg deposition to fry emergence. & August-April & $33-70$ \\
\hline Fry colonization & $\begin{array}{l}\text { Fry emergence until establishment of } \\
\text { summer rearing locations. }\end{array}$ & April-June & $61-71$ \\
\hline Summer rearing & $\begin{array}{l}\text { After colonization ceases when fish } \\
\text { are largely stationary and activities are } \\
\text { mainly directed at feeding and growth } \\
\text { (large fish may outmigrate near end of } \\
\text { period). }\end{array}$ & June-September & $71-95$ \\
\hline $\begin{array}{l}\text { Fall redistribution } \\
\text { and } \\
\text { overwintering }\end{array}$ & $\begin{array}{l}\text { Beginning with drop in temperature in } \\
\text { early fall until the onset of yearling } \\
\text { smolt migration at the end of winter. }\end{array}$ & $\begin{array}{l}\text { September- } \\
\text { March }\end{array}$ & $96-114$ \\
\hline Smolt to smolt & $\begin{array}{l}\text { Onset of seaward migration to } \\
\text { departure from the Grande Ronde } \\
\text { Basin. }\end{array}$ & April-June & $114-125$ \\
\hline
\end{tabular}


Table 2. Descriptions of key habitat used by spring chinook salmon by life stage within the Grande Ronde Basin.

\begin{tabular}{|c|c|}
\hline Life stage & Key habitat \\
\hline Pre-spawning adult & $\begin{array}{l}\text { Large, deep pools with sufficient connecting flow for } \\
\text { adult migration. }\end{array}$ \\
\hline Spawning & $\begin{array}{l}\text { Riffles containing a mixture of gravel and cobble sizes } \\
\text { with flow of sufficient depth for spawning activity. }\end{array}$ \\
\hline Incubation & $\begin{array}{l}\text { Riffles as described for spawning with sufficient flow } \\
\text { for egg and alevin development. }\end{array}$ \\
\hline Fry colonization & $\begin{array}{l}\text { Shallow and relatively slow velocity areas within stream } \\
\text { channel, often associated with stream margins and in } \\
\text { relatively low gradient reaches. }\end{array}$ \\
\hline Summer rearing & $\begin{array}{l}\text { Pool type habitat associated with relatively low gradient } \\
\text { stream channel reaches (usually not in backwaters nor } \\
\text { slow eddies). }\end{array}$ \\
\hline Fall redistribution/overwintering & $\begin{array}{l}\text { Areas containing structural complexity (wood matrices, } \\
\text { brush, or large cobbles) within flowing channel, not } \\
\text { usually in swift or higher gradient reaches; off-channel } \\
\text { areas (ponds, oxbows, etc.). }\end{array}$ \\
\hline Smolt to smolt & $\begin{array}{l}\text { Sufficient flow for free movement of smolts } \\
\text { downstream. }\end{array}$ \\
\hline
\end{tabular}


Table 3. Description of environmental quality attributes affecting responses of salmonids within freshwater. This list was applied to spring chinook salmon in the Grande Ronde Basin; other attributes may be needed for other species or in different types of environments.

\begin{tabular}{|c|c|}
\hline Attribute & Describtion \\
\hline C.hannel stability & $\begin{array}{l}\text { Stability of the reach with respect to its streambed, banks, and its channel } \\
\text { shape and location. }\end{array}$ \\
\hline F'low & Pattern and extent of flow fluctuations within the stream reach. \\
\hline $\begin{array}{l}\text { Habitat type } \\
\text { diversity }\end{array}$ & $\begin{array}{l}\text { The extent of habitat complexity within a stream reach. Complexity is the } \\
\text { opposite of uniformity; greater complexity increases density-independent } \\
\text { survival; woody debris, brush, and other structure add complexity. }\end{array}$ \\
\hline Sediment load & $\begin{array}{l}\text { The amount of sediment present in, or passing through, the stream reach. } \\
\text { Sediment may be suspended (turbidity), moving along the substrate } \\
\text { (bedload), or within the substrate (percent fine particles). }\end{array}$ \\
\hline T'emperature & $\begin{array}{l}\text { Water temperature in the stream reach. Density-independent survival is } \\
\text { affected by rapid fluctuations, or by conditions near the extremes of } \\
\text { tolerance. }\end{array}$ \\
\hline $\begin{array}{l}\text { I?:iparian } \\
\text { condition }\end{array}$ & $\begin{array}{l}\text { The state of the vegetation component of the narrow strip of land } \\
\text { bordering the stream where vegetation species occur that are dependent on } \\
\text { the stream or its adjacent water table. }\end{array}$ \\
\hline P'redators & $\begin{array}{l}\text { The relative abundance of predators that feed upon the diagnostic species. } \\
\text { The effects of poaching and human harassment are included here." }\end{array}$ \\
\hline C.hemicals & Concentrations of toxic chemicals from point and non-point sources. \\
\hline C'ompetitors & $\begin{array}{l}\text { The relative abundance of other species in the stream reach that compete } \\
\text { with the diagnostic species for food or space. }\end{array}$ \\
\hline Cobstructions & $\begin{array}{l}\text { Physical structures that impede the use of a stream reach, such as dams, } \\
\text { weirs, or waterfalls. }\end{array}$ \\
\hline VVithdrawals & Water withdrawals from the stream reach. \\
\hline Nutrient load & $\begin{array}{l}\text { The concentration of dissolved nutrients due to natural or man-induced } \\
\text { causes. (Only nutrient loads that affect density-independent survival are } \\
\text { considered here; hence, food enrichment for the diagnostic species is not } \\
\text { addressed here.) }\end{array}$ \\
\hline Cxygen & Mean concentration of dissolved oxygen in the stream reach. \\
\hline P'athogens & $\begin{array}{l}\text { The abundance, concentration, or effect of pathogens in the stream reach. } \\
\text { For example, the presence of a fish hatchery or large numbers of livestock } \\
\text { along the reach could cause unusually high concentrations of pathogens. }\end{array}$ \\
\hline Other & Any other attribute unique to the stream reach that may affect survival. \\
\hline
\end{tabular}

'Subsistence, ceremonial, recreational and commercial fishing impacts are not included. 


\section{Catherine $\mathrm{Cr}$ Environmental Attributes}

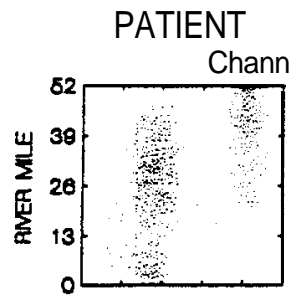

TEMPLATE

hannel stabilitv
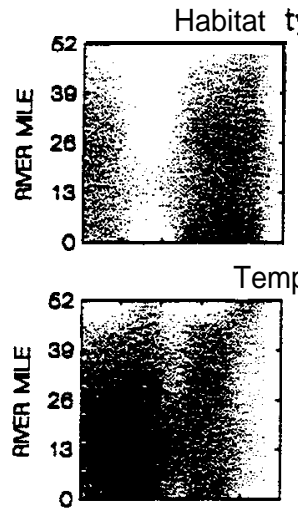

Temperature
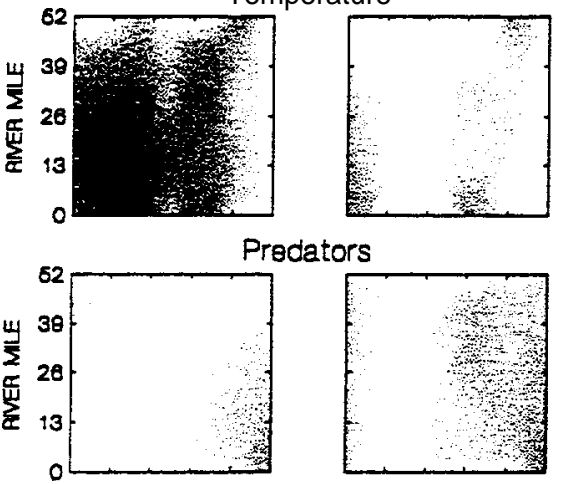

Predators

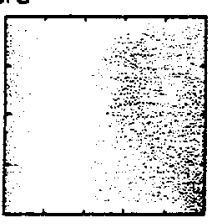

Competitors
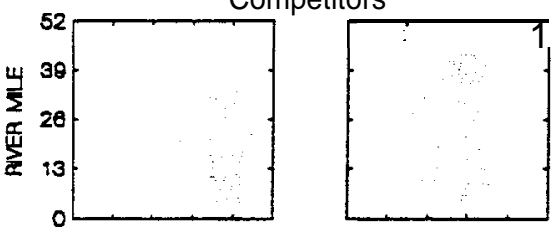

Withdrawals
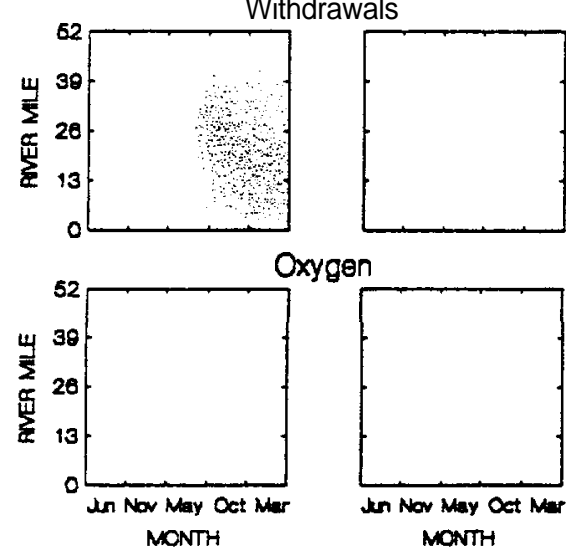

Oxygen

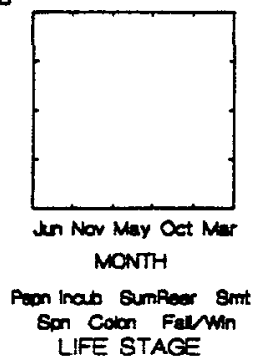

PATIENT

TEMPLATE Flow
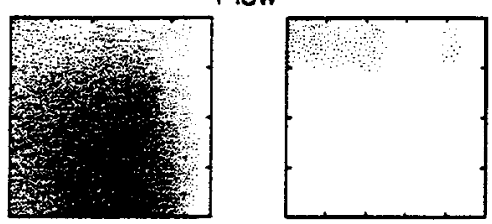

Sediment load

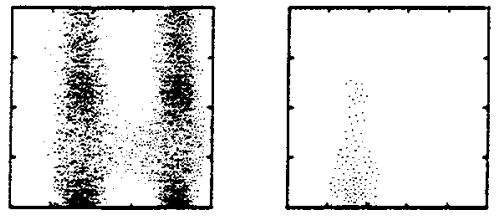

Riparian condition
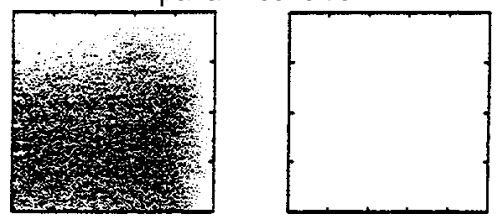

Chemicals

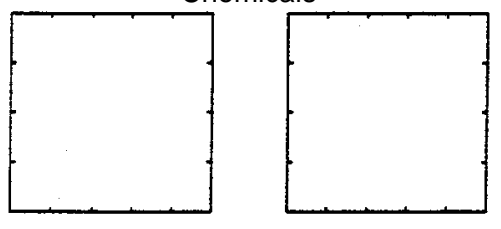

Obstructions

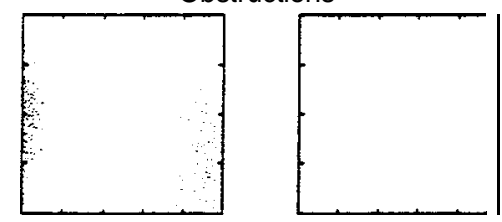

Nutrient load

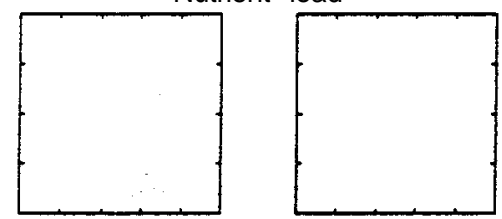

Pathogens

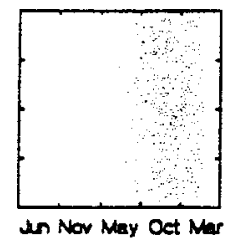
MONTH

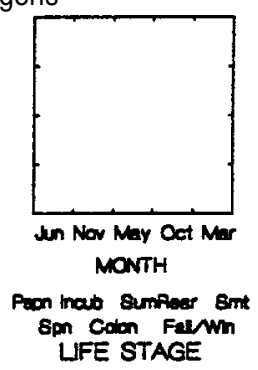

Level of

Effect

No effect

$\square$ Low

a Moderate

a High

- Lethal

Figure 6. Sample environmental attribute landscapes for a tributary (Catherine Cr.) 


\section{Catherine Cr/Grande Ronde R Key Habitat Quantity}

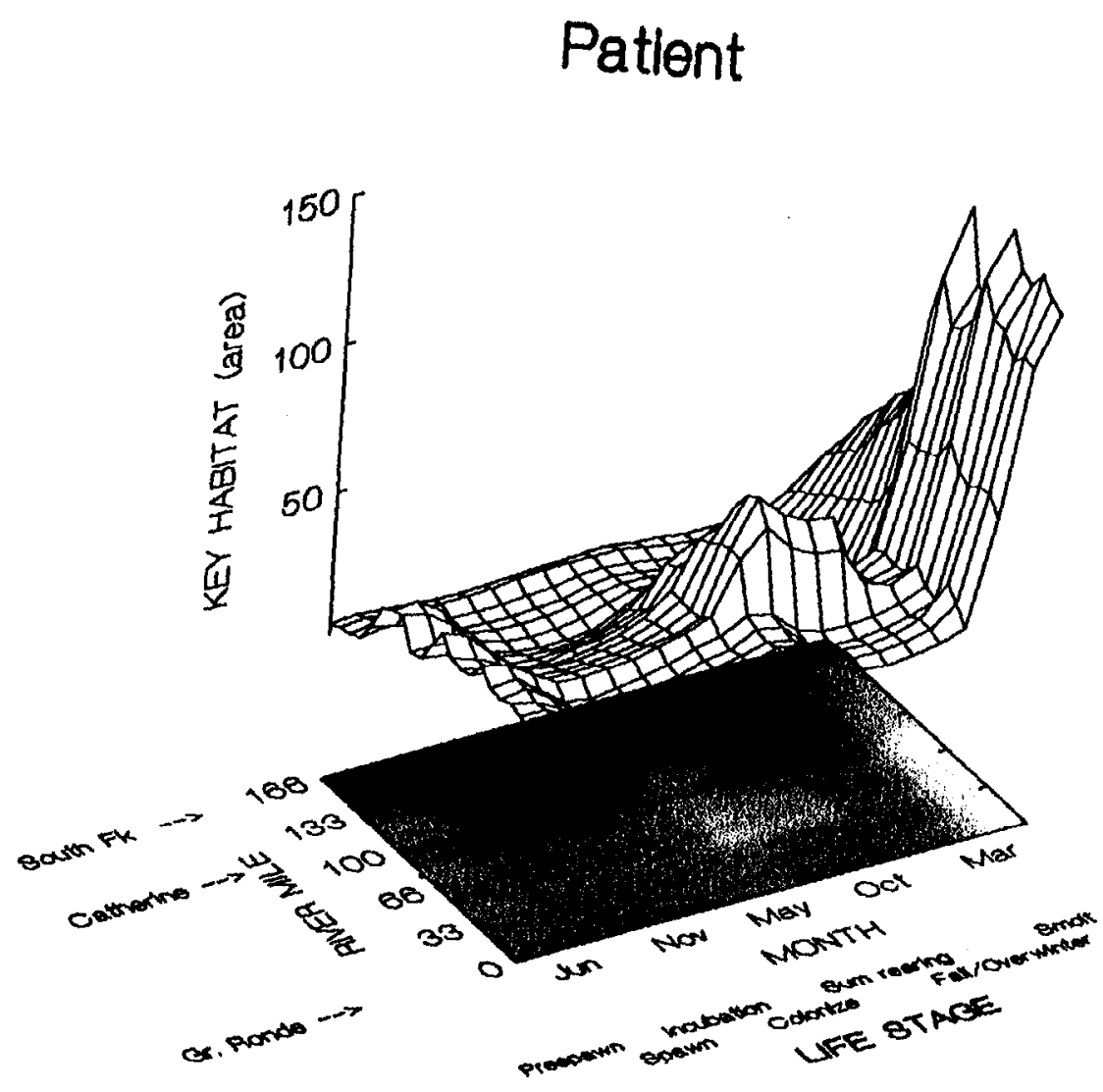

Template

Figure 7.

Sample key habitat landscape for basin (Catherine Cr./ Grande Ronde R.)

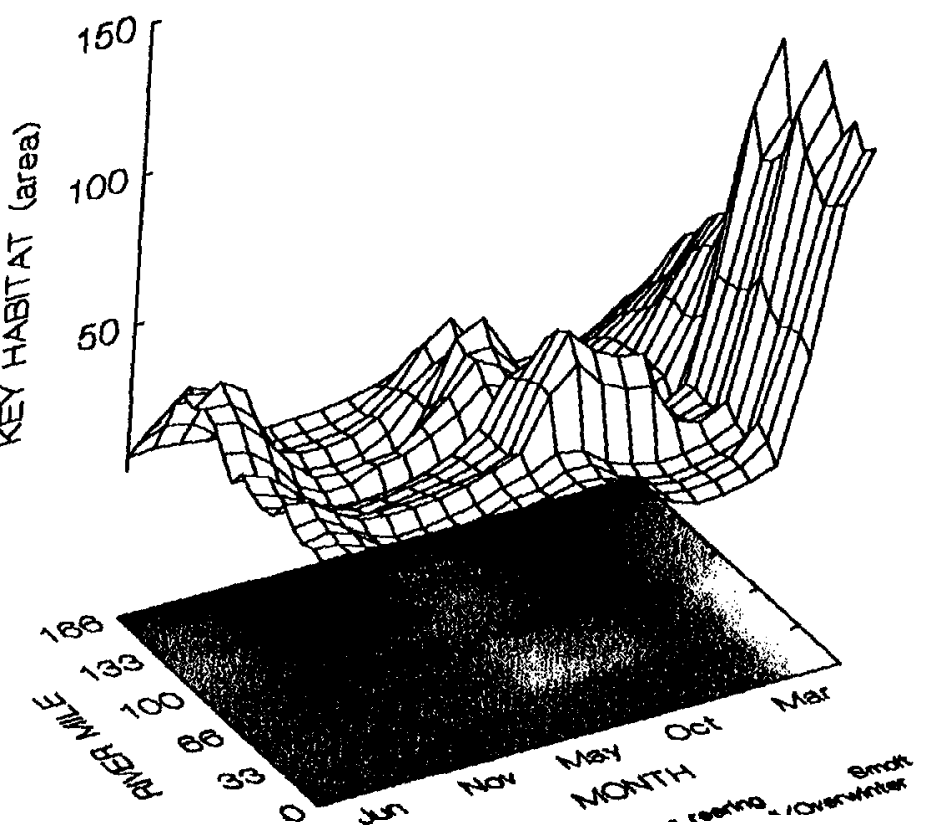

< 40 


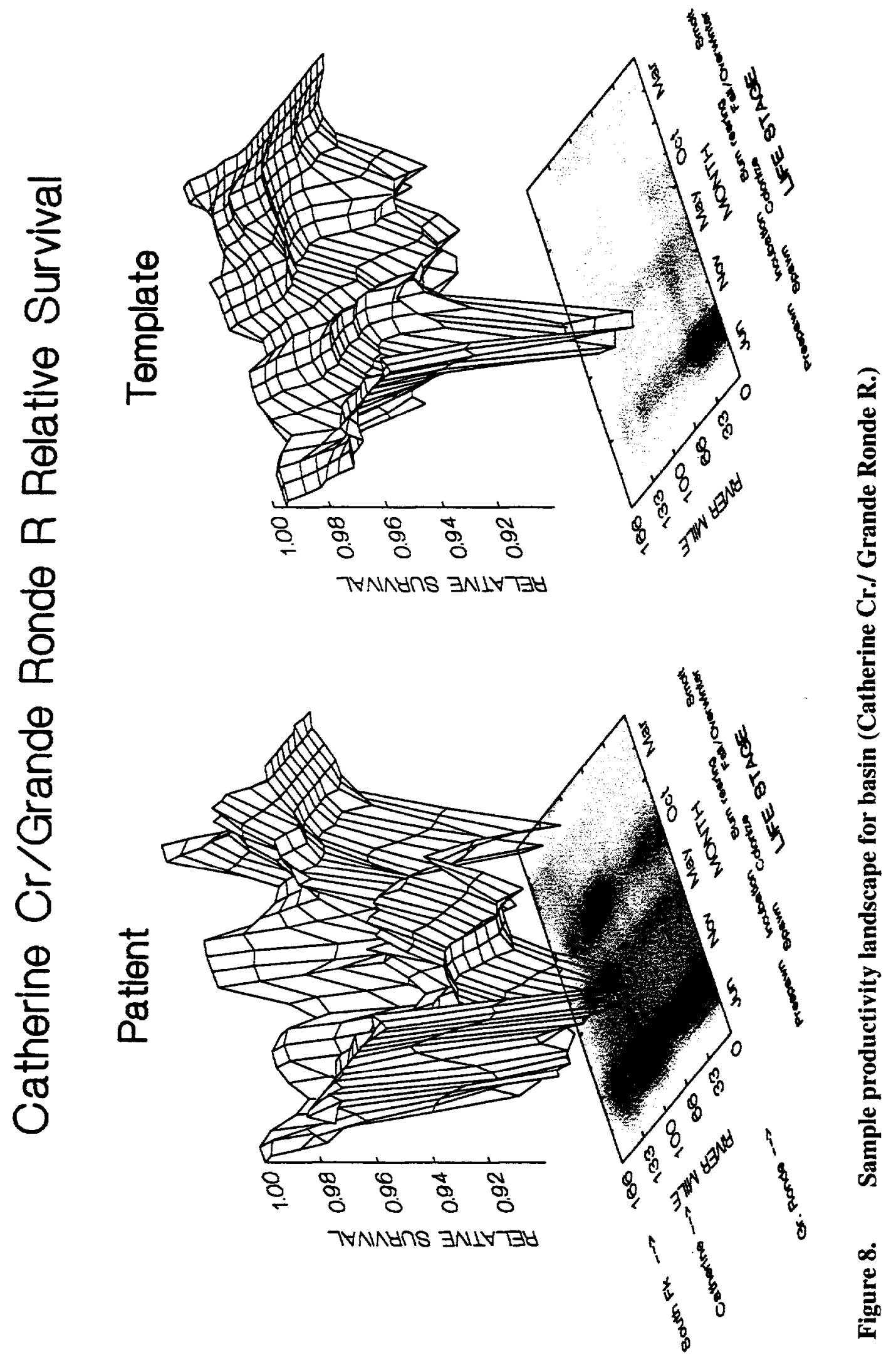




\section{Catherine $\mathrm{Cr}$ /Grande Ronde R Environmental Attributes}

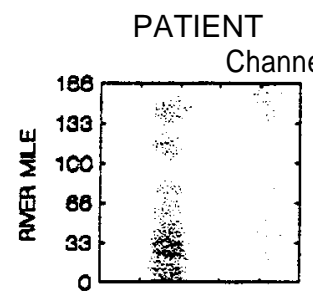

TEMPLATE

Channel. stability
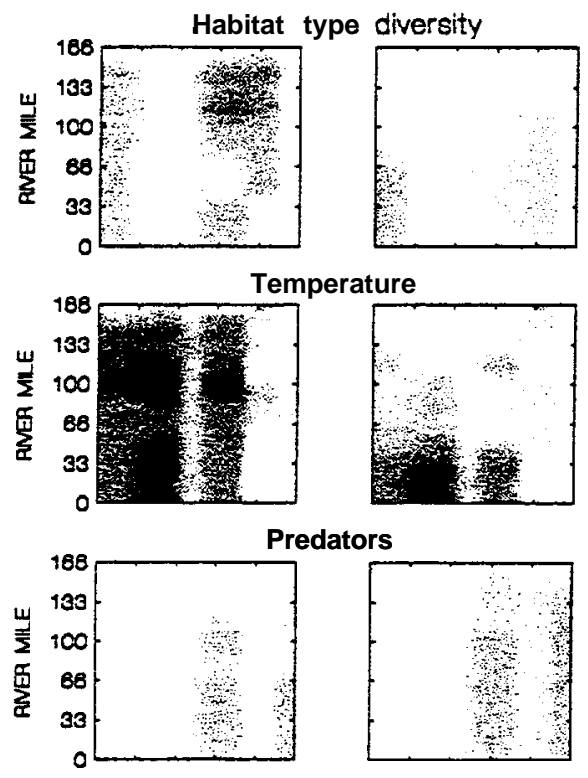

Predators

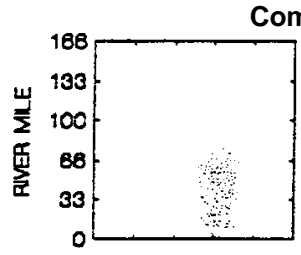

Competitors
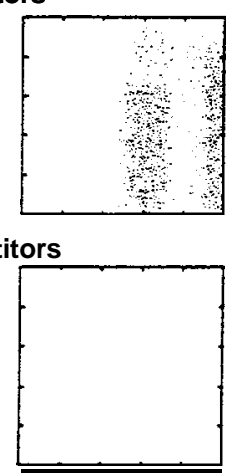

Withdrawals
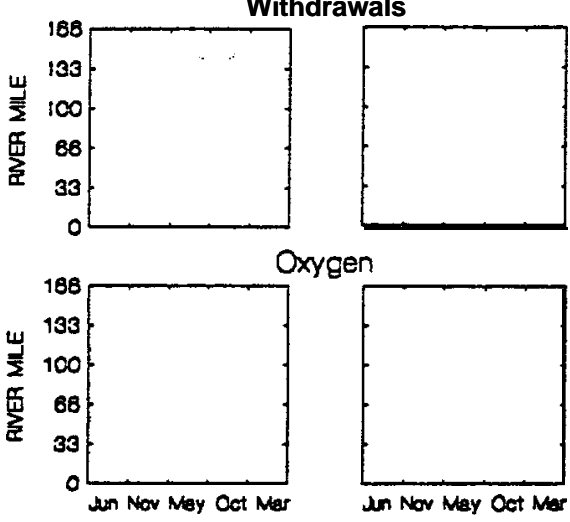

$$
\text { MONTH }
$$

Peon Inab Sumfeer smt
Son Colon Falwh Son Colon Faly

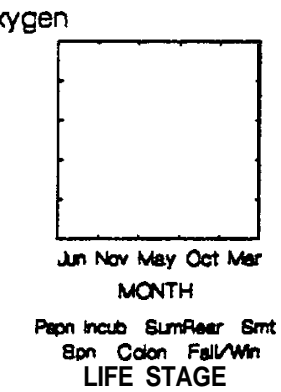

PATIENT

Flow

TEMPLATE

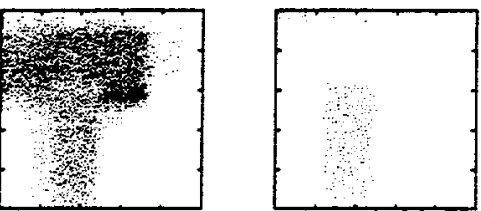

Sediment load

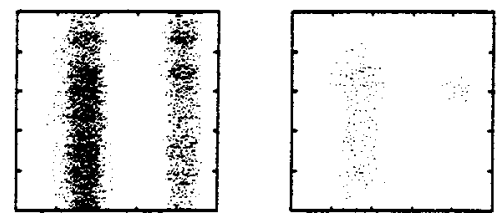

Riparian condition

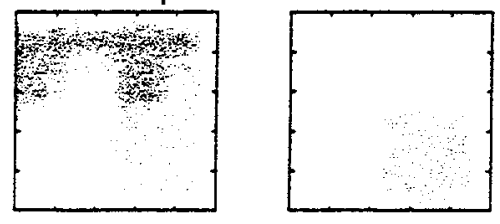

Chemicals

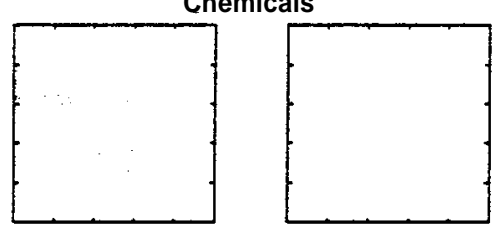

Obstructions

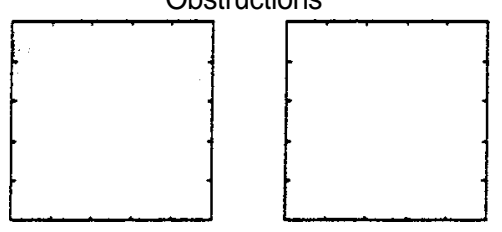

Nutrient load

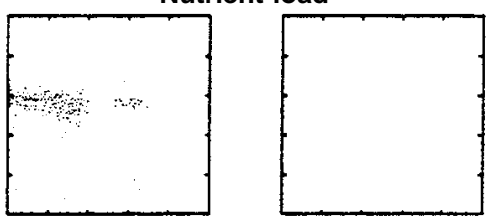

Level of

Effect

$\square$ No effect

$\square$ Low

- Moderate

High

Lethal

Figure 9. Sample environmental attribute landscape for basin (Catherine Cr./ Grande Ronde R.) 
Where applicable, a set of six landscape graphics are presented together, as in Figs. 4-9. Figs. 7-9 show the Catherine Creek/Grande Ronde River reach range, whereas Figs. 4-6 show a close-up view of just Catherine Creek for greater resolution within this smaller drainage.

A list of stream reach ranges used in the analysis is given in Table 4.

Table 4. List of reach ranges used in the analysis.

\begin{tabular}{|c|c|}
\hline \multirow{12}{*}{ Grande Ronde Basin } & Bear Cr. / Wallowa R. / Grande Ronde R. \\
\hline & Catherine Cr. / Grande Ronde R. $\stackrel{\underline{I}}{ }$ \\
\hline & Chesnimnus Cr / Joseph Cr. / Grande Ronde R. \\
\hline & Grande Ronde R. \\
\hline & Hurricane Cr. / Wallowa R. / Grande Ronde R. \\
\hline & Lookingglass Cr. / Grande Ronde R. \\
\hline & Lostine R. / Wallowa R. / Grande Ronde R. \\
\hline & Minam R. / Wallowa R. / Grande Ronde R. \\
\hline & OK Gulch Cr. / Prairie Cr. / Grande Ronde R. \\
\hline & Sheep Cr./ Grande Ronde R. \\
\hline & Wallowa R. / Grande Ronde R. \\
\hline & Wenaha R. / Grande Ronde R. $\stackrel{\text { II }}{ }$ \\
\hline \multirow{4}{*}{ Imnaha Basin } & Big Sheep Cr. / Imnaha R. \\
\hline & Imnaha R. \\
\hline & Lightning Cr. / Imnaha R. \\
\hline & Little Sheep Cr. / Big Sheep Cr. / Imnaha R \\
\hline
\end{tabular}

Example of PTA Summary

A narrative interpretation of the PTA, for the Catherine Creek/Grande Ronde River reach range is shown below as an example.

\section{Key Habitat Quantity}

The quantity of key habitat for spring chinook in Catherine Creek is greatly reduced compared to historic levels (Fig.4). These losses are due to land development, channel straightening and realignment, removal of large organic structure from the channel, and dewatering of the channel. Loss of key habitat has occurred for nearly every life stage, but it has been particularly severe for the prespawning, fry colonization, summer rearing, and overwintering life stages.

The quantity of key habitat within the Grande Ronde River downstream of Catherine Creek is substantially reduced compared to historic levels for a distance of approximately 30 miles (Fig. 5). These losses have been greatest for the spawning, egg incubation, fry colonization, and overwintering life stages. Further downstream, loss in key habitat has been comparatively minor. 


\section{Relative Productivity (Survival)}

The capability of Catherine Creek of supporting spring chinook productivity (survival) is sharply reduced compared to historic conditions for nearly every life stage (Fig. 6). This reduction has been most severe in the lower 40 miles of stream, with conditions improving in the upper stream reaches. Survival conditions approach historic levels for some life stages in the Catherine Creek forks. Survival conditions are especially low in the later phase of the prespawning stage and in the spawning, incubation, fry colonization, and summer rearing stages in the lower 30 miles of stream. Survival conditions have declined in these reaches due primarily to increased water temperature, increased sediment load, loss in habitat diversity, loss in flow, channel and bank destabilization, and alteration of the riparian corridor (Fig. 7).

The capability of the Grande Ronde River for approximately 70 miles downstream of Catherine Creek for supporting spring chinook productivity is sharply reduced compared to historic conditions for many life stages (Fig. 9). This loss has been most severe for a distance of approximately 40 miles downstream of Catherine Creek. Survival conditions in these reaches are especially poor in the prespawning, spawning, incubation, and summer rearing life stages. Survival conditions have declined in these reaches due primarily to increased water temperature during mid to late summer, increased sediment load, alteration of runoff patterns, and channel destabilization (Fig. 8).

\section{Results}

Graphic results and narrative interpretations of the Patient-Template Analysis are presented for all reach ranges in Appendix $\mathrm{C}$. 


\section{DIAGNOSIS}

\section{Purpose}

The diagnosis is a determination of the potential of the Grande Ronde and Imnaha watersheds to support persistence, abundance, and distribution goals for spring chinook salmon. It is performed by analyzing the information assembled in the PTA from a spring chinook life history perspective with these restoration goals in mind. The diagnosis synthesizes the information to guide the formulation and prioritization of actions that are consistent with watershed goals and that effectively target the impediments to spring chinook performance.

\section{Overview of Diagnostic Steps}

A major conclusion of the PTA for both the Grande Ronde and Imnaha basins is that environmental conditions affecting salmon survival differ greatly over time and space within these watersheds. Salmon performance can, therefore, vary dramatically, depending on how members of a salmon population utilize the river system by location and time of year. The diagnosis is designed to test the performance characteristics of the watershed along a large and diverse series of pathways that represent the range of possible life history patterns of spring chinook. A life history pathway, or trajectory, is the specific route that a single salmon and its offspring, might follow through a river system.

The diagnosis is made through four steps: 1) identify representative life history pathways (trajectories) which will serve as "probes" of the performance landscapes; 2) compute numerical indices of performance for each life history pathway and graph the separate performance measures along each pathway for both Patient and Template; 3) review the performance graphs to assess the changes in salmon performance potential of the reach range; and 4) summarize findings into a short narrative.

\section{Step 1: Definition of Life History Pathways}

A life history pathway begins at the point (time and location) where a maturing adult enters the river system, continues upstream to the point of reproduction, then proceeds through the locations where juvenile rearing and downstream migration occur. The pathway is a trajectory through time and space -- it serves as a "probe" of the environmental conditions a salmon might encounter within the watershed. Many such probes are needed to obtain a useful picture of the salmon performance potential of the entire watershed.

Life history pathways can be defined consistent with several general life history patterns that spring chinook salmon exhibit. We define a life history pathway as a unique route through a river system. A pattern, on the other hand, refers to a more general way in which salmon move through and use the system - it is a collection of pathways. In one pattern, for example, emergent fry take up residence within their natal stream reach and remain there until smolt migration one year later. In another pattern, emergent fry take up residence in their natal reach and remain there 
until fall, when they move downstream to overwintering habitat. In the latter case, the over-wintering site may be several miles downstream or scores of miles, but in either case, the general pattern is to redistribute downstream in the late summer/early fall. A large watershed, therefore, contains a myriad of possible pathways but comparatively few life history patterns. For purposes of this analysis, we identify five distinct life history patterns (Table 5).

Associated with each potential spawning stream, a reach range (as defined above) was identified. For each reach range nine different life history pathways were defined by - one or more for each of the five life history patterns. The nine distinct pathways were defined by systematically varying spawning location (within the reach range) and migration timing consistent with the given life history pattern

The nine pathways associated with a spawning reach are best explained with an example (Fig. 10). The spawning reach of the South Fork of Catherine Creek is located approximately 165 miles upstream of the mouth of the Grande Ronde River. Each of the nine charts in Fig. 10 traces a pathway in space and time along the Catherine Cr./Grande Ronde reach range. One pathway (\# 1) represents life history pattern 1 in Table 5. Three pathways (\#'s 2, 3, and 4) represent pattern 2 , each differing in the distance to the overwintering site. One pathway (\#5) represents pattern 3. Three pathways (\#'s 6, 7, and 8) represent pattern 4, each differing in the distance to the over-wintering site. The last pathway (\# 9) represents pattern 5 -- fish migrating to the Snake River as subyearlings in late summer.

All life history patterns were defined so that they enter the Grande Ronde (Imnaha) River between mid April and mid May as maturing adults. Patterns 1-8 leave the Grande Ronde (Imnaha) in May as smolts. Pattern 9 was defined to leave the Grande Ronde (Imnaha) River as subyearlings in October.

The analyses in this report by necessity assume that survival outside the Grande Ronde and Imnaha subbasins is the same for all life history pathways, even though observations suggest that this assumption probably is false. Studies have indicated that arrival times at Lower Granite of different life history patterns are not the same (Keefe et al. 1996) and consequently different survival conditions in the mainstem Snake and Columbia Rivers are most likely encountered. Our study is limited to the reaches within the subbasins and hence cannot account for the potentially important implications of diversity of life history pathways in the remainder of the life cycle.

A total of 71 and 28 spawning reaches are used to define unique pathways in the Grande Ronde and Imnaha basins respectively. Combined, a total of 881 pathways are used in analyzing salmon performance in the two basins. This large, diverse set of pathways enable us to systematically probe potential salmon performance over the entire watershed. 
Table 5. Five generalized life history patterns of spring chinook salmon in the Grande Ronde and Imnaha basins used in the diagnostic analysis. Note: This is not intended to be a complete list of all patterns within these river basins.

\begin{tabular}{|c|c|c|c|c|c|c|}
\hline Pattern & Adult entry timing & Spawning timing & $\begin{array}{l}\text { Emergent fry } \\
\text { colonization }\end{array}$ & Summer rearing & Overwintering & $\begin{array}{c}\text { Emigration to } \\
\text { Snake River }\end{array}$ \\
\hline I & April - mid June & August - September & $\begin{array}{l}\text { Residency established near } \\
\text { natal site }\end{array}$ & $\begin{array}{l}\text { Remain in same vicinity where } \\
\text { fry colonization occurs }\end{array}$ & $\begin{array}{l}\text { Remain in same vicinity as } \\
\text { summer residents }\end{array}$ & $\begin{array}{l}\text { April - May } \\
\text { as yearlings }\end{array}$ \\
\hline 2 & April - mid June & August - September & $\begin{array}{l}\text { Residency established near } \\
\text { natal site }\end{array}$ & $\begin{array}{l}\text { Remain in same vicinity where } \\
\text { fry colonization occurs }\end{array}$ & $\begin{array}{l}\text { Movement occurs in late } \\
\text { summer/early fall } \\
\text { downstream to suitable } \\
\text { hahilat }\end{array}$ & $\begin{array}{l}\text { April - May } \\
\text { as yearlings }\end{array}$ \\
\hline 3 & April - mid June & August - September & $\begin{array}{l}\text { Dispersal downstream to } \\
\text { suitable hahitat }\end{array}$ & $\begin{array}{l}\text { Remain in same vicinity where } \\
\text { fry colonization occurs }\end{array}$ & $\begin{array}{l}\text { Remain in same vicinity as } \\
\text { summer residents }\end{array}$ & $\begin{array}{l}\text { April - May } \\
\text { as yearlings }\end{array}$ \\
\hline 4 & April - mid June & August - September & $\begin{array}{l}\text { Dispersal downstream to } \\
\text { suitable habitat }\end{array}$ & $\begin{array}{l}\text { Remain in same vicinity where } \\
\text { fry colonization occurs }\end{array}$ & $\begin{array}{l}\text { Movement occurs in late } \\
\text { summer/early fall } \\
\text { downstream to suitable } \\
\text { habitat }\end{array}$ & $\begin{array}{l}\text { April - May } \\
\text { as yearlings }\end{array}$ \\
\hline 5 & April - mid June & August -September & $\begin{array}{l}\text { Dispersal downstream IO } \\
\text { suitable hahitat }\end{array}$ & $\begin{array}{l}\text { Remain in same vicinity where } \\
\text { fry colonization occurs }\end{array}$ & $\begin{array}{l}\text { Overwintering does not } \\
\text { occur within the basin }\end{array}$ & $\begin{array}{l}\text { September - } \\
\text { October as } \\
\text { Subyearlings }\end{array}$ \\
\hline
\end{tabular}




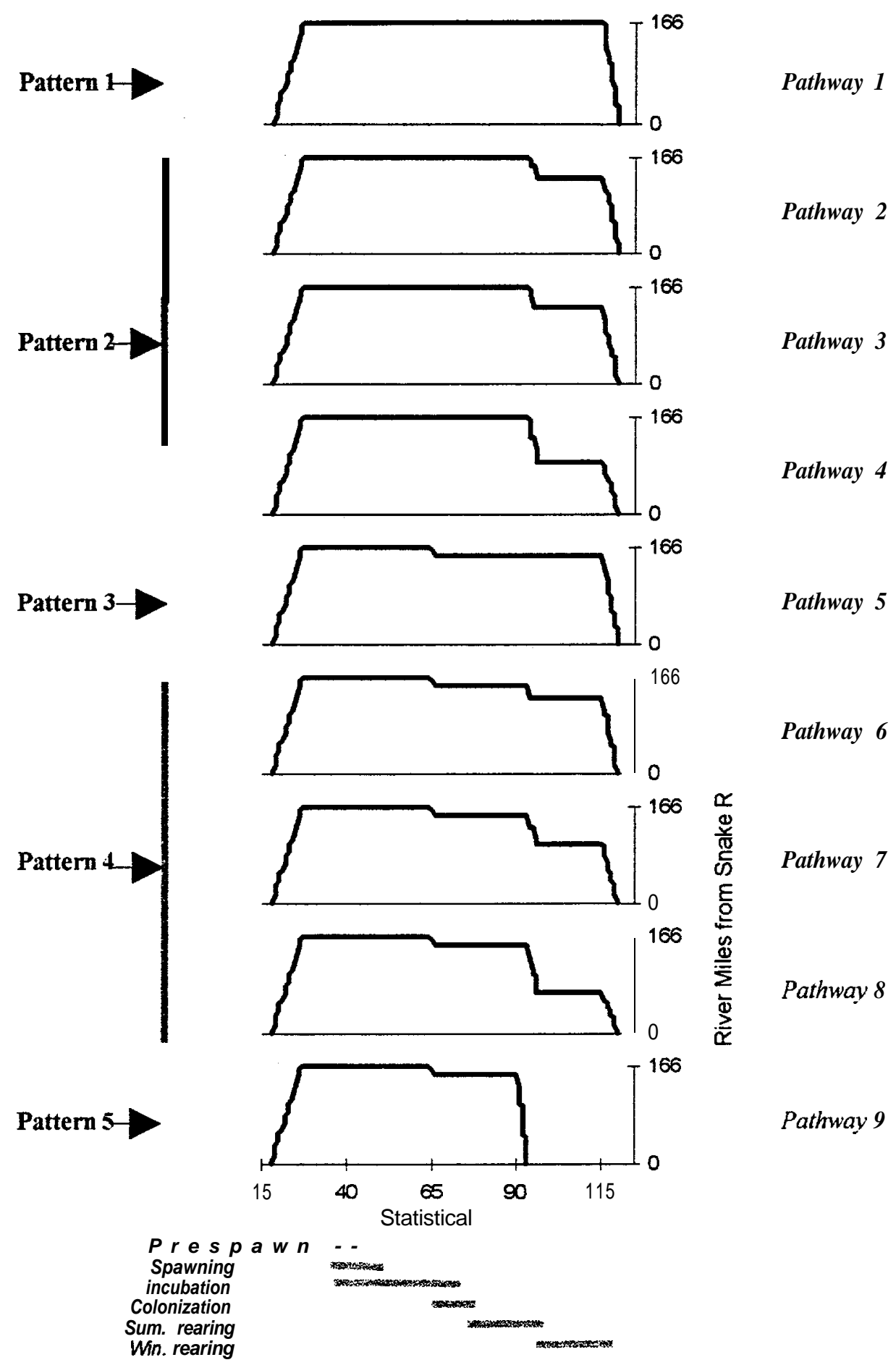

Figure 10 Sample graphic showing nine typical life history pathways used in the diagnosis (spawning reach in this example is the South Fork of Catherine $\mathrm{Cr}$ ). 


\section{Step 2: Computation of Salmon Performance Indices}

To understand the relative importance of various stream reaches to spring chinook salmon, performance needs to be analyzed in a way that synthesizes all conditions of all reaches encountered by individual salmon pathways. This is done by computing numerical indices of what we call cumulative productivity and cumulative capacity for each life history pathway (see EDT Primer, Lestelle et al. 1996, for details). These indices reflect the cumulative effects of all environmental conditions on a group of fish that would utilize the watershed along a specific pathway.

The cumulative productivity index compares the capability of pathways to sustain spring chinook salmon. As a rough guide, populations that must rely on pathways with index values less than about 150 could be considered at extreme risk given the survival conditions outside of the Grande Ronde and Imnaha basins. We also suggest that populations relying on pathways with values less than 300 be considered at high risk.

The cumulative capacity index reflects the maximum abundance of spring chinook salmon (computed as smolt equivalents) that could be produced along the different pathways, if spawning reaches were fully utilized.

Performance index values for all life history pathways analyzed are listed in Appendices A and B for the Grande Ronde and Imnaha basins respectively. Also given are profiles of productivity and quantity of key habitat associated with each pathway. Results are grouped by spawning reach; each page provides results for the nine pathways associated with the reach range defined by the given spawning reach.

An example is given in Fig. 11 to explain the graphics in Appendices A and B using the South Fork Catherine Creek spawning reach. The figure is composed of 18 individual profile charts, nine for relative survival (productivity) and nine for quantity of key habitat quantity. Each chart compares Patient and Template values of either relative survival or key habitat; also shown on each chart for reference is the pathway (trajectory). These profiles show values corresponding to the route that would be followed by specific pathways traced across the appropriate landscape (Appendix C). The profiles identify the specific places and times where productivity or quantity of key habitat is different between Template and Patient and the extent of change.

\section{Step 3: Review of Performance Plots}

Performance plots are pictures of how potential salmon performance has changed within the watershed. They, more comprehensively than the other graphics, illustrate the implications to salmon performance as a result of altered environmental conditions in the watershed. The plots combine the three elements of performance-productivity, capacity, and life history diversity-into one picture to facilitate diagnosis. 
Spawning reach: Catherine $\mathrm{Cr}$.

South Fork Catherine Cr (RM 164.4 - 166.8 from Snake R)

\section{Relative Productivity}
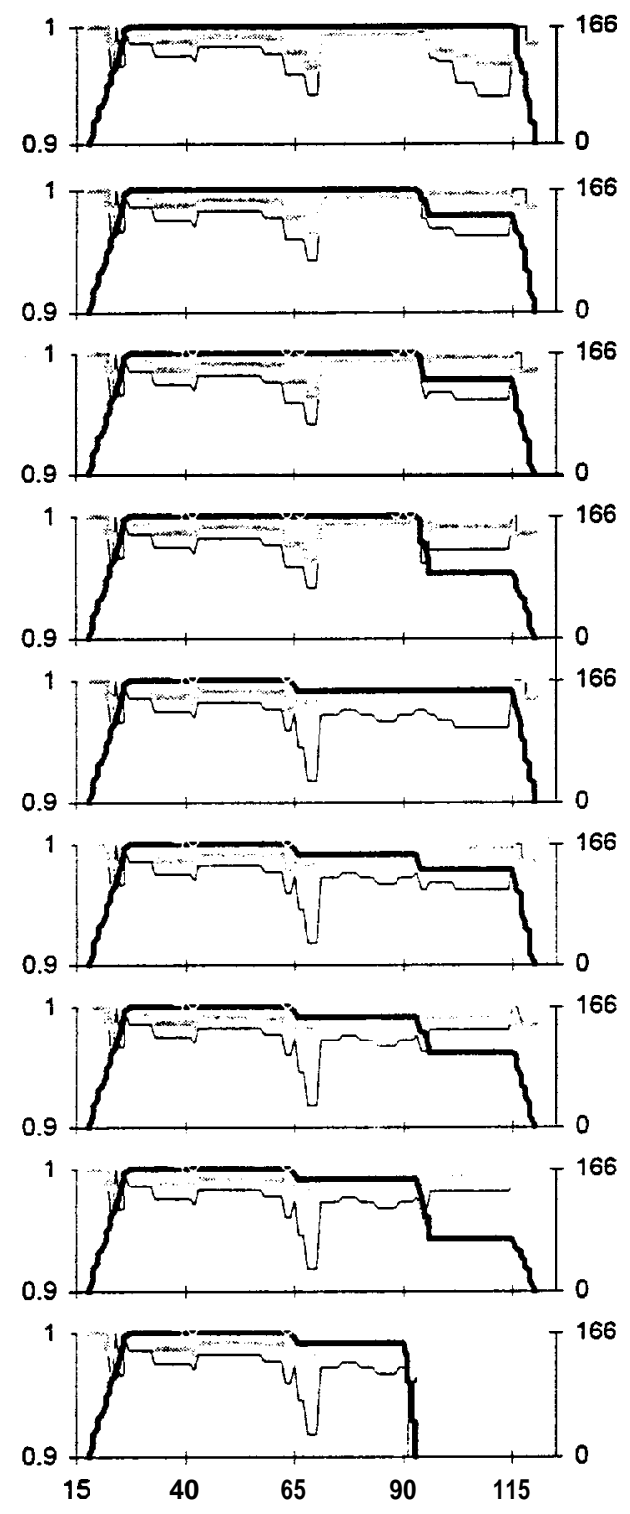

Statistical week

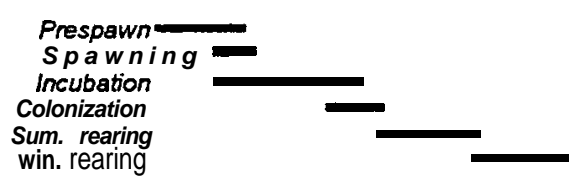

Relative Key Habitat Quantity
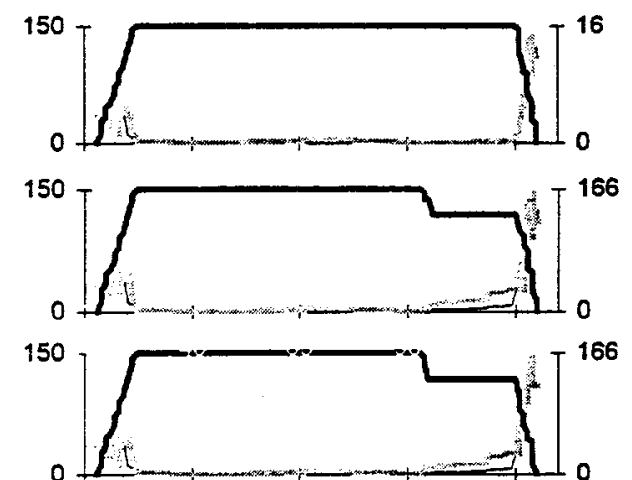

150
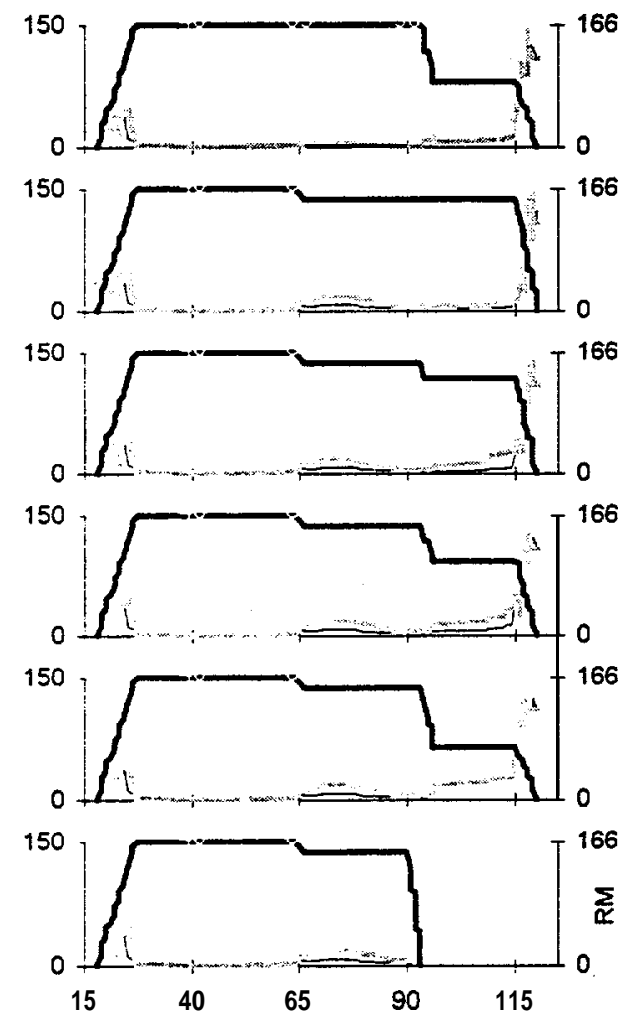

15

40

Statistical week

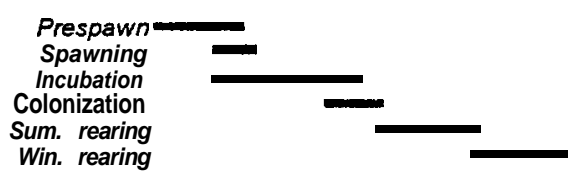

\begin{tabular}{|c|c|}
\hline \multicolumn{2}{|c|}{$\begin{array}{c}\text { Template } \\
\text { Patient }\end{array}$} \\
\hline Cum. & \\
\hline IProd. & Cap. \\
\hline \multicolumn{2}{|c|}{ Pathway \#1 } \\
\hline 225 & 0.13 \\
\hline 85 & 0.07 \\
\hline \multicolumn{2}{|c|}{ Pathway \#2 } \\
\hline 347 & 0.25 \\
\hline 102 & 0.09 \\
\hline \multicolumn{2}{|c|}{ Pathway \#3 } \\
\hline 345 & 0.25 \\
\hline 99 & 0.08 \\
\hline \multicolumn{2}{|c|}{ Pathway \#4 } \\
\hline 310 & 0.22 \\
\hline 117 & 0.09 \\
\hline \multicolumn{2}{|c|}{ Pathway \#5 } \\
\hline 347 & 0.32 \\
\hline 54 & 0.05 \\
\hline \multicolumn{2}{|c|}{ Pathway \#6 } \\
\hline 358 & 0.35 \\
\hline 52 & 0.05 \\
\hline \multicolumn{2}{|c|}{ Pathway \#7 } \\
\hline 322 & $\overline{0.32}$ \\
\hline 72 & 0.07 \\
\hline \multicolumn{2}{|c|}{ Pathway \#8 } \\
\hline 338 & 0.34 \\
\hline 69 & 0.07 \\
\hline \multicolumn{2}{|c|}{ Pathway \#9 } \\
\hline 577 & 0.62 \\
\hline 136 & 0.15 \\
\hline
\end{tabular}

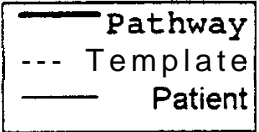

Figure 11. Sample graphic showing productivity and capacity profiles for pathways with common spawning reach (in this esample the spawning reach is the South Fork of Catherine Creek. 
Plots are provided at two scales for each basin. One plot compares all pathways in each basin together for both Patient and Template (Figs. 12 and 15). The same information is then shown plotted separately for the major subbasins in each basin (Figs. 13 and 16). Each plot is composed of data points for both Patient and Template, where a single data point depicts cumulative productivity and cumulative capacity for one pathway (as listed in Appendices A and B). The number of data points in the chart is an indicator of life history diversity - more abundant and productive pathways suggest greater diversity of life histories.

Accompanying the plots at the subbasin scale is another graphic that provides additional information on the potential of each subbasin for salmon production (Figs. 14 and 17). Because spawning reaches vary in length, the points in the performance plots do not necessarily represent the same number of fish. Figs. 14 and 17 attempt to correct for this by weighting the heights of the bars in the histogram by spawning reach length. Bar height in these histograms represents the relative number of potential pathways linked to spawning reaches in each subbasin when reach lengths are standardized among subbasins. The x-axes in these plots are identical to those in Figs. 13 and 16 to facilitate comparison between the graphs.

\section{Step 4: Diagnostic Summaries}

In the following, the diagnosis for each subbasin within the Grande Ronde and Imnaha basins are discussed. It is important to note that problems stated for life histories of a given subbasin are the result of habitat changes both inside and outside that subbasin. To understand the significance of changes to reaches within the subbasin we refer to Appendices D - H.

\section{Grande Ronde Basin}

The current potential of the Grande Ronde Basin to produce and sustain spring chinook salmon in relation to conditions within the basin has been severely reduced compared to historic potential (Fig. 12). The large majority of life history pathways (represented by data points in Fig. 12) that historically were capable of supporting spring chinook can no longer do so due to changes in the quality of habitat that have occurred over the past 150 years. ${ }^{4}$

In general, data points for the Patient in Fig. 12 show a dramatic shift to the lower left portion of the chart, indicating that fish following these life history pathways are less able to sustain themselves against additional mortality pressures than they could historically. Moreover, the capacity of these pathways for smolt abundance has declined sharply.

Life history pathways with productivity index values less than about 150 are probably at extremely high risk of extinction given the current conditions outside of the Grande Ronde basin. High risk of extinction may also exist for pathways with values as high as 300 . These life history

\footnotetext{
4 / Major losses in sustainable life history pathways can be attributed to changes within the Grande Ronde (and Imnaha) watershed, however the problem is exacerbated by a reduction in survival outside the basin. It is the cumulative effect of productivity losses both within and outside the basins that has caused the severe decline in the abundance and sustainability of salmon produced from the Grande Ronde and Imnaha basins.
}

Grande Ronde Ecosystem Diagnosis and Treatment /January 1997/ Page 32 


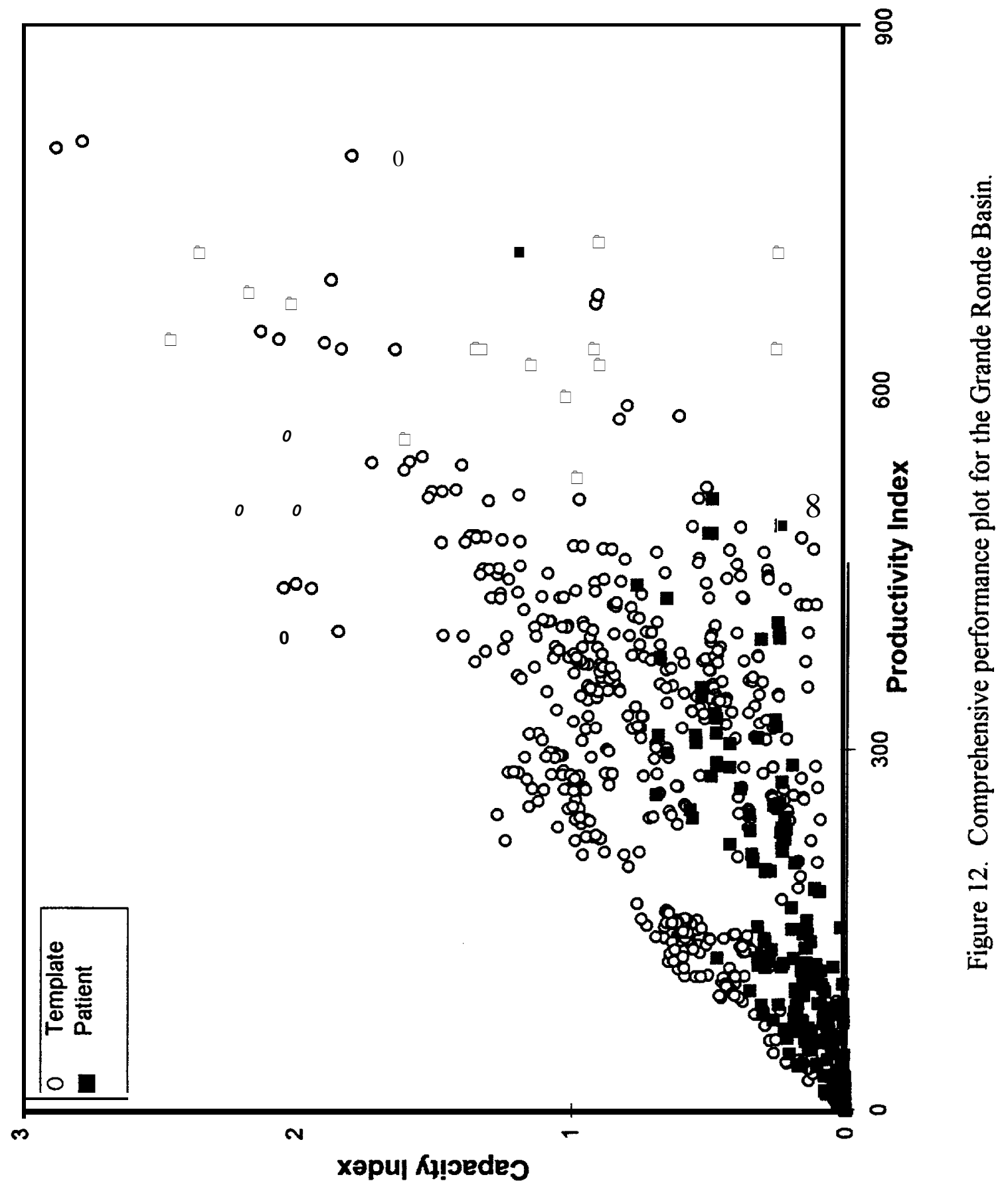



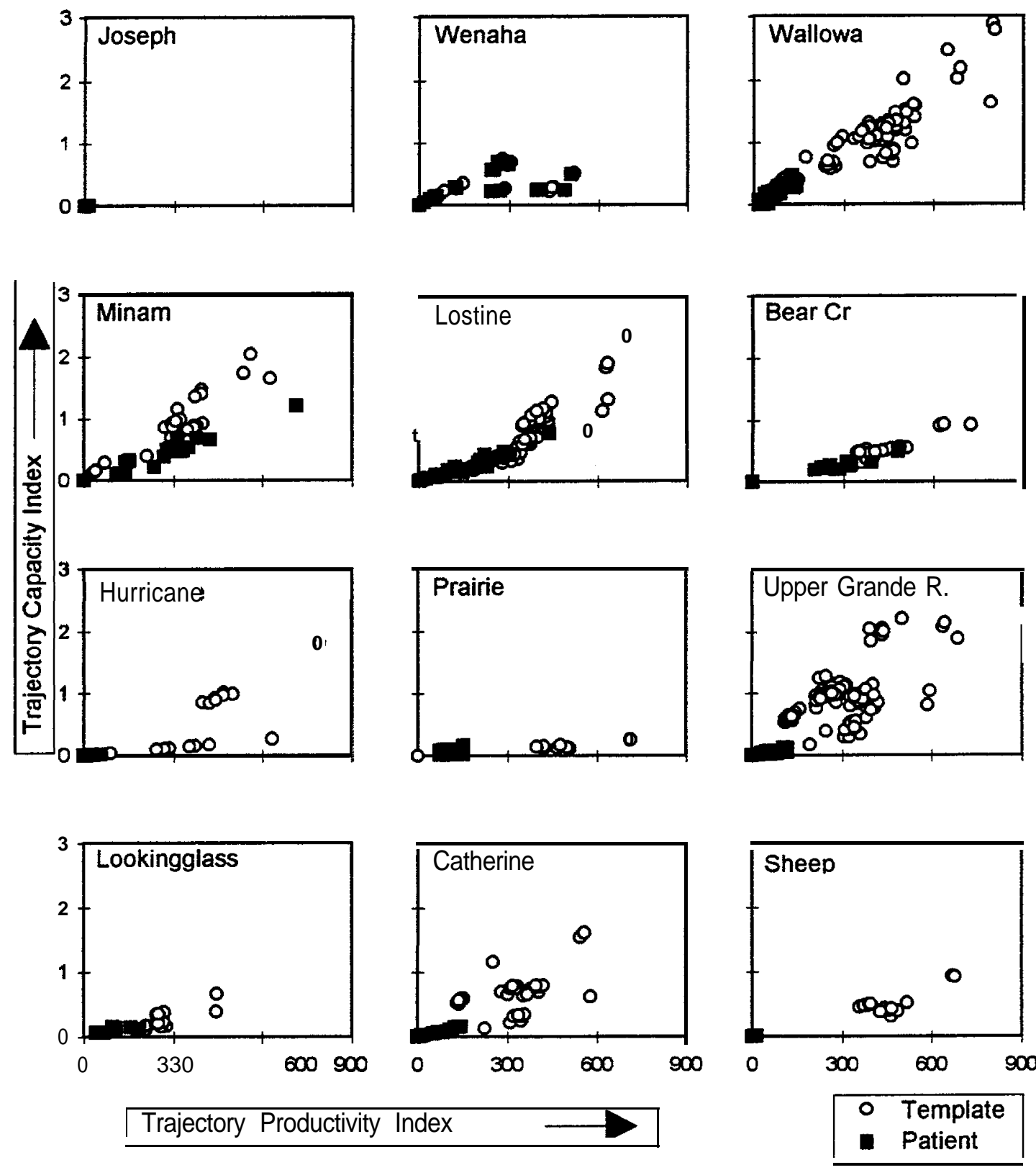

Figure 13. Performance plots for pathways within the Grande Ronde Basin, grouped by subbasin where the spawning stage occurs. 

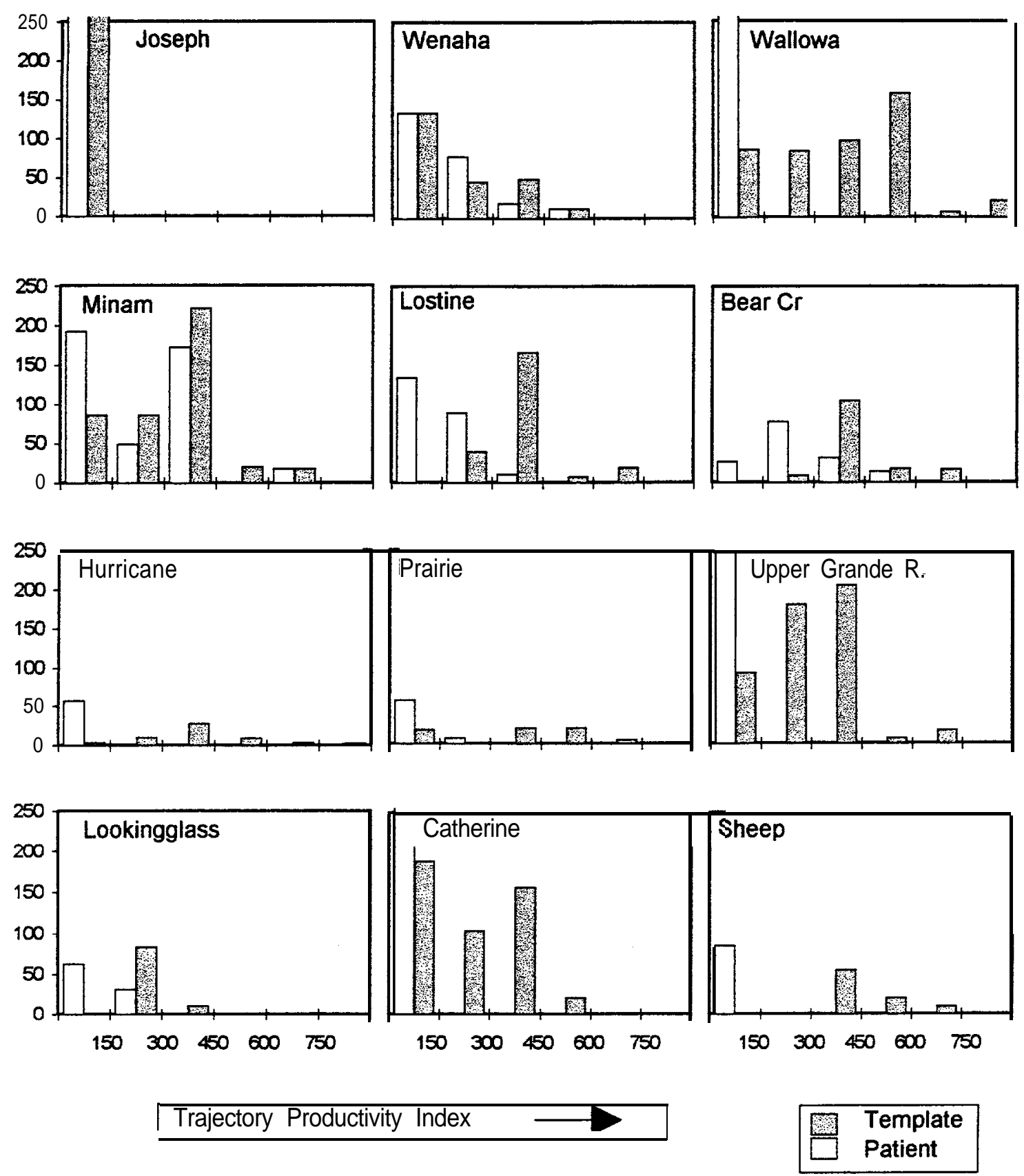

Figure 14. Productivity frequency charts for pathways associated with spawning reaches within subbasins of the Grande Ronde Basin. Frequencies are weighted by spawning reach lengths. Shaded bars represent Template, and white bars Patient. 


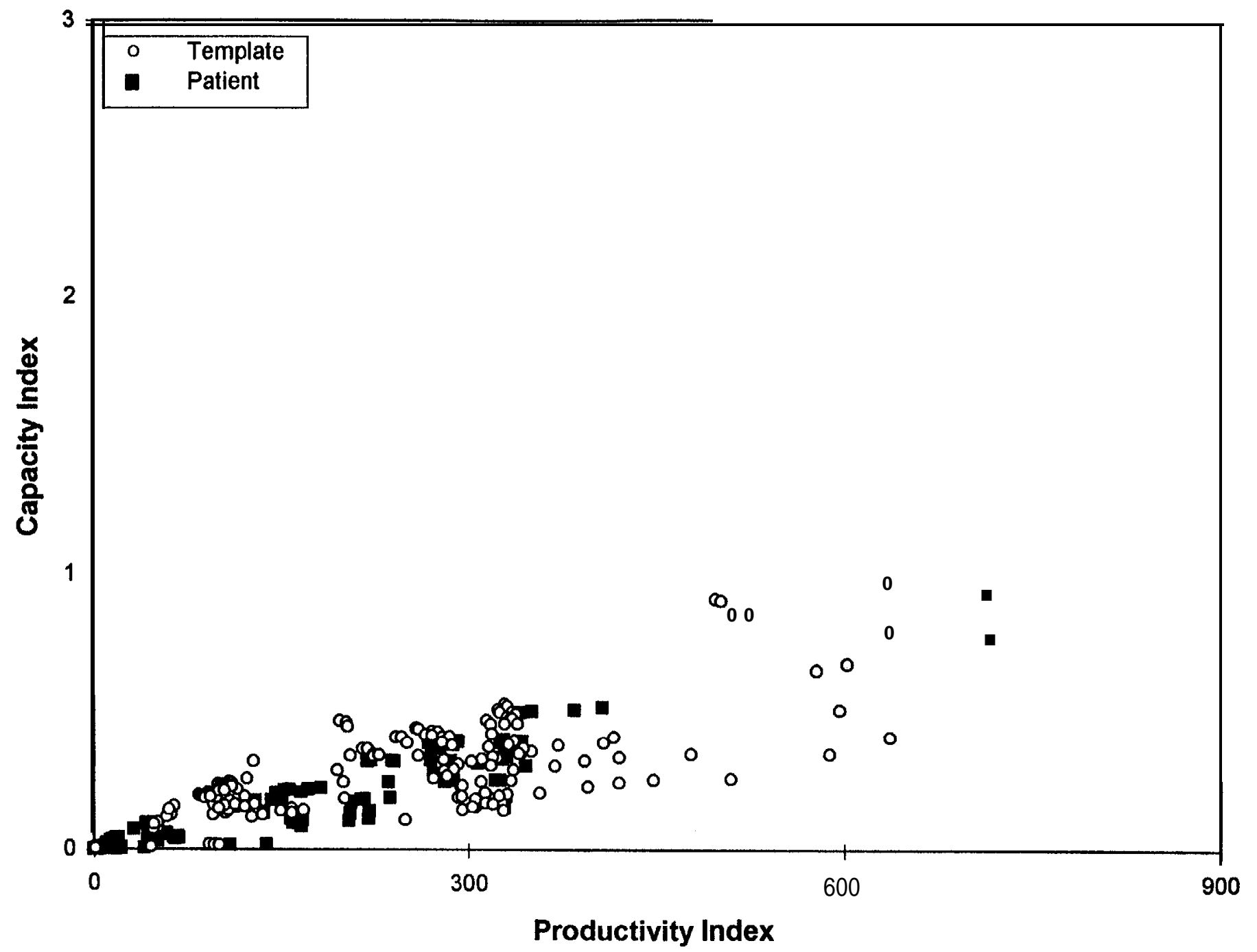

Figure 15. Comprehensive performance plot for the Imnaha Basin 


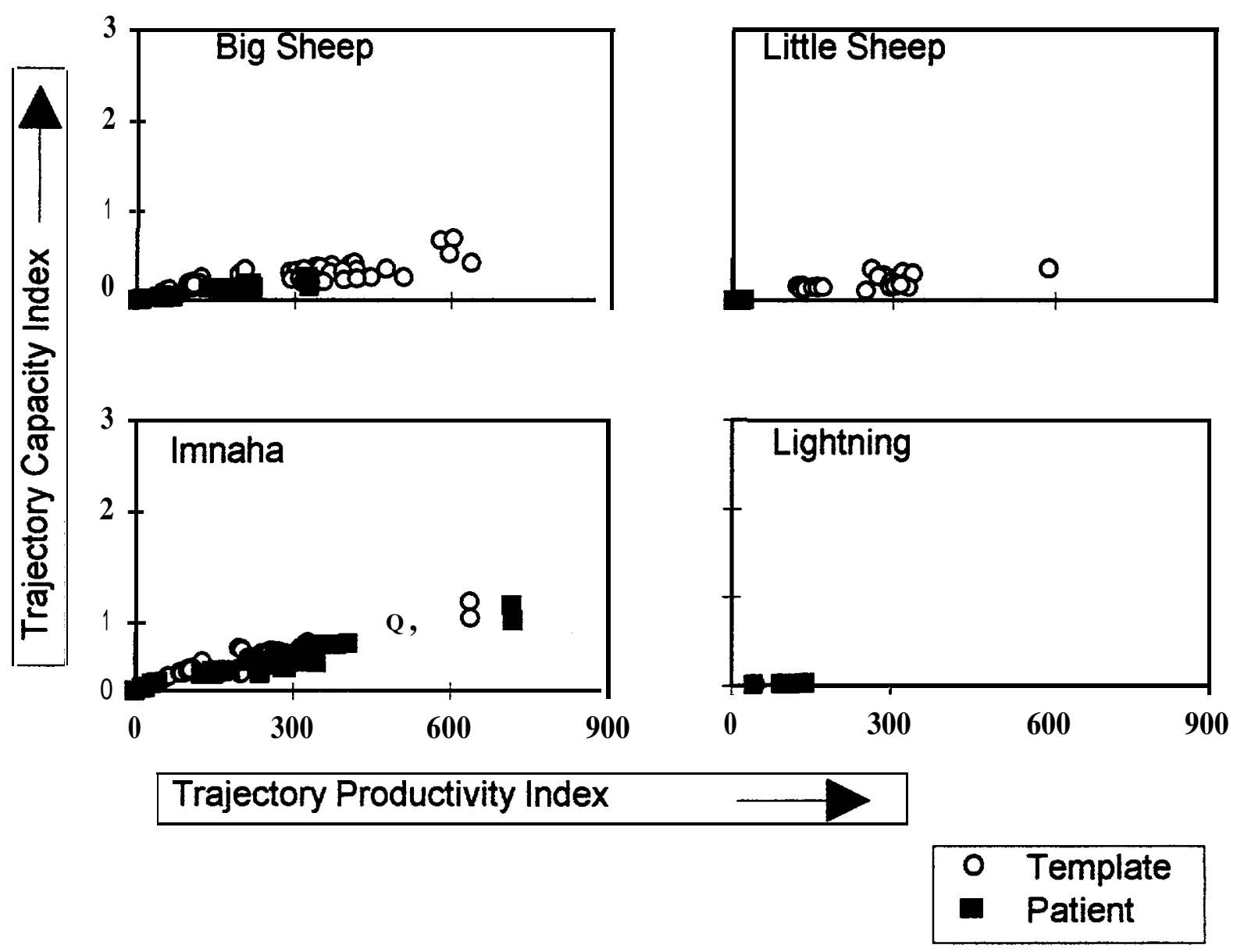

Figure 16. Performance plots for pathways within the Imnaha Basin, grouped by subbasin where the spawning stage occurs. 

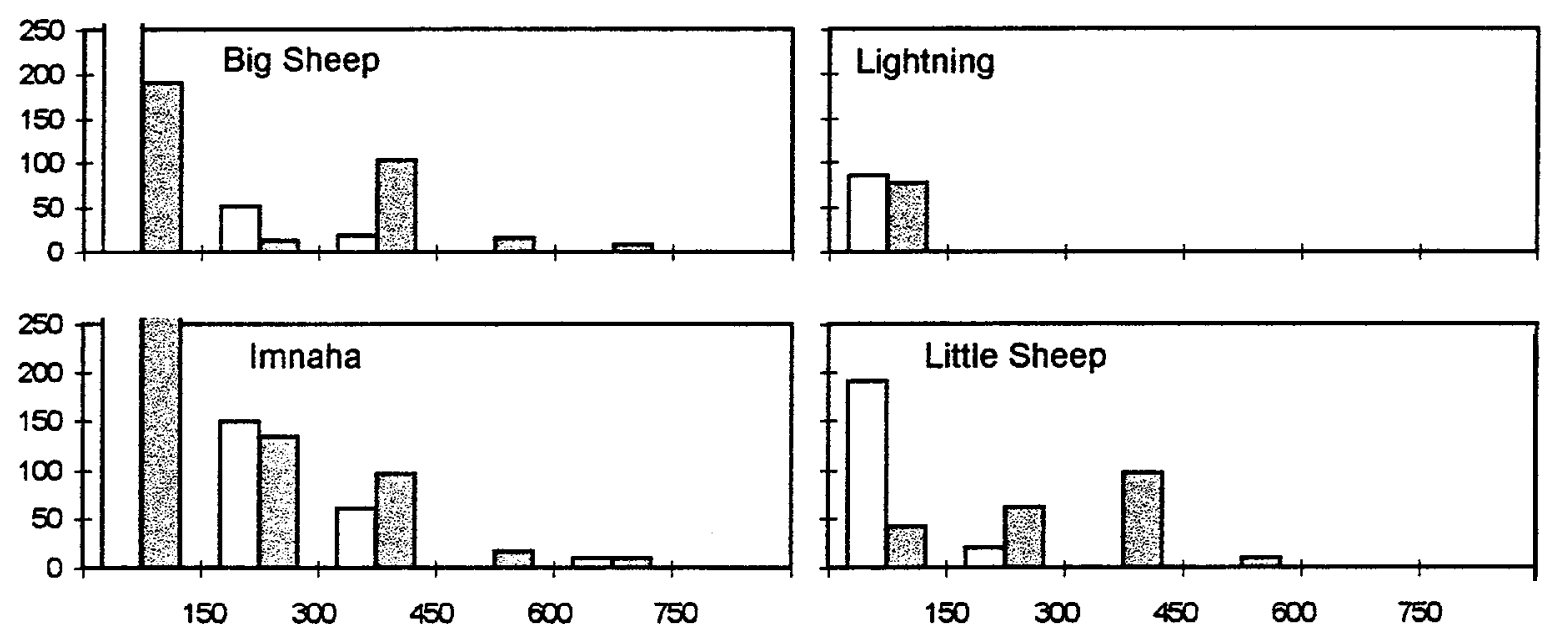

Trajectory Productivity Index

Figure 17. Productivity frequency charts for pathways associated with spawning reaches within subbasins of the Imnaha Basin. Frequencies are weighted by spawning reach lengths. Shaded bars represent Template, and white bars Patient. 
pathways continue into the mainstem Snake and Columbia rivers, where the quality of habitat for spring chinook has also deteriorated over the past century (Lichatowich and Mobrand 1995).

Conditions within the Grande Ronde Basin to support spring chinook salmon differ significantly between subbasins (Fig. 13). The degree of habitat change (degradation from the salmon perspective) over the past 150 years also varies among subbasins. These sharp differences between subbasins have created a situation today where the Grande Ronde spring chinook population contains no strong core population to support metapopulation function in the basin. Life history pathways that remain relatively strong are associated with a few geographic areas of the basin where spawning still occurs; these areas tend to be isolated from one another, hampering metapopulation function.

A diagnostic summary follows for each of the major subbasins.

Joseph Creek Subbasin: Over the past 150 years, this subbasin appears to have been unable to sustain any level of spring chinook production. Low performance potential under Template conditions (Fig. 13) indicates that the lack of spring chinook production in this subbasin is not due to man-induced changes that occurred in the past century, either within or outside this subbasin. This subbasin appears to offers no restoration potential for spring chinook salmon.

Productivity - This component of survival for Joseph Creek (including Chesnimnus) life history pathways was historically extremely low and remains so. Water temperature $\backslash$ and flow patterns appear to have always been natural impediments to survival along these pathways (Appendix C). salmon

Capacity - This subbasin has virtually no natural capacity to produce spring chinook

Diversity - This subbasin supports virtually no diversity of life history pathways capable of producing spring chinook.

Wenaha River Subbasin: This subbasin is largely unchanged from historic conditions (Fig. 13). It offers little or no additional restoration potential for spring chinook salmon.

Productivity - This component of survival for Wenaha River life history pathways is highly variable between pathways. Some life history pathways have among the highest productivities of any current day life history pathways for the Grande Ronde basin. Other pathways appear to have very low productivity; these are associated with the lower most spawning reach or with pathways that take juveniles into the mainstem Grande Ronde for summer rearing. This variability between pathways suggests that overall productivity for fish spawned in this subbasin may be highly variable between years.

Capacity - This subbasin supports life history pathways that have some of the highest capacities of any that are currently populated in the Grande Ronde basin. These capacity levels are relatively low, however, compared to the high historic capacities of pathways in other parts of 
the basin. Other life history pathways associated with the Wenaha subbasin have relatively low capacities.

Diversity - The potential for life history diversity associated with Wenaha River pathways has remained essentially unchanged compared to historic conditions. Only those life history pathways with relatively high productivity are sustainable, however, due to deterioration in habitat quality outside the basin. The subbasin has greater diversity of life history pathways than shown in Fig. 13 because the North Fork is not depicted on the chart; that reach supports life history pathways with productivities comparable to the higher values shown for the basin.

Wallowa River Subbasin (excluding tributaries to Wallowa R.): Life history pathways associated with spawning reaches in the mainstem Wallowa River show severe declines in potential salmon performance compared to historic levels (Fig. 13). Restoration potential is significant for this subbasin. The most unique feature of this subbasin compared to all others in the basin is the presence of Wallowa Lake, which historically enhanced the productivity and capacity of the subbasin for salmon. The results depicted in Fig. 13 suggest that the Wallowa River was historically the most productive subbasin within the Grande Ronde basin for spring chinook.

Productivity - This component of survival for Wallowa River life history pathways is currently low; groups of fish using these pathways appear to be at extremely high risk of extinction. This subbasin supported life history pathways under historic conditions that were the highest in the Grande Ronde basin. Major impediments to productivity (Appendix C) are sediment load, affecting both egg incubation and overwinter survival, flow patterns immediately downstream of Wallowa Lake; nutrient loading, and pathogens (whirling disease).

Capacity - The mainstem Wallowa River supports life history pathways that currently have low capacity for spring chinook. Historically, these same pathways had exceptionally high abundance, the highest of any pathway? in the Grande Ronde basin.

Diversity - The potential for life history diversity associated with Wallowa River pathways is significantly reduced compared to historic conditions. The resilience of the population to withstand additional environmental changes has been lessened accordingly. Life history pathways that may currently be used by spring chinook show relatively uniform productivity and capacity at low to extremely low levels. Improvements in productivity in these stream reaches could significantly improve overall capacity for salmon within the Grande Ronde Basin.

Minam River Subbasin: Life history pathways associated with spawning reaches in the mainstem Minam River show small to moderate changes in current potential salmon performance compared to historic levels (Fig. 13).

Productivity - This component of survival differs widely between Minam River pathways. In general, within basin productivity does not appear to have changed greatly compared to 
historic levels. The wide differences in productivity between pathways suggest that overall productivity for fish spawned in this subbasin may be highly variable between years.

Capacity - The capacity of Minam River life history pathways for smolt production has declined, on the average, by over half compared to historic levels. These changes are apparently due largely to cumulative effects of reductions in life history productivity.

Diversity - The current potential for life history diversity associated with Minam River pathways is reduced slightly compared to historic conditions. The resilience of the population to withstand additional environmental changes has been lessened accordingly. Life history pathways that may currently be used by spring chinook show a relatively wide range in productivity and capacity. Only those life history pathways with relatively high productivity are capable of being sustained, however, due to deterioration in habitat quality outside the basin.

Lostine River Subbasin: Life history pathways associated with spawning reaches in the Lostine River show moderate to severe declines in current potential salmon performance compared to historic levels (Fig. 13). Restoration potential is significant for pathways associated with this subbasin.

Productivity - This component of survival for Lostine River life history pathways has declined substantially compared to historic levels. The subbasin supported life history pathways under historic conditions that were among the highest in the Grande Ronde basin. Major impediments to productivity (Appendix C) are altered flow patterns, channel and substrate stability, and water temperature-all of these occur in the lower reaches of the Lostine River.

Capacity - The capacity of life history pathways associated with this subbasin for smolt production has declined, on the average, by well over half compared to historic levels. These changes are apparently due largely to cumulative effects of reductions in life history productivity.

Diversity - The current potential for life history diversity associated with Lostine River pathways is reduced substantially compared to historic conditions. The resilience of the population to withstand additional environmental changes has been lessened accordingly. Life history pathways that may currently be used by spring chinook show a fairly wide range in productivity and capacity, but many of the pathways have low productivity. Only those life history pathways with relatively high productivity are capable of being sustained, however, due to deterioration in habitat quality outside the basin.

Bear Creek Subbasin (tributary to Wallowa River): Life history pathways associated with spawning reaches in Bear Creek show moderate to severe losses in current potential salmon performance compared to historic levels (Fig. 13).

Productivity - This component of survival differs widely between Bear Creek pathways (Fig. 13). In general, within basin productivity has declined substantially compared to historic levels. The wide differences in productivity between pathways suggest that overall productivity for fish spawned in this subbasin may be highly variable between years. Major impediments to 
productivity (Appendix C) are altered flow patterns in the lower reach of the stream, loss of habitat diversity, and altered temperature regimes.

Capacity - The capacity of life history pathways associated with this subbasin for smolt production has declined, on the average, by over half compared to historic levels. These changes are apparently due largely to cumulative effects of reductions in life history productivity and loss of key habitat in the lower reach.

Diversity - The current potential for life history diversity associated with Bear Creek pathways is reduced substantially compared to historic conditions. The resilience of the population to withstand additional environmental changes has been lessened accordingly. Life history pathways that may currently be used by spring chinook show a fairly wide range in productivity and capacity. Only those life history pathways with relatively high productivity are capable of being sustained, however, due to deterioration in habitat quality outside the basin.

Hurricane Creek Subbasin: Life history pathways associated with spawning reaches in Hurricane Creek show extremely severe losses in current potential salmon performance compared to historic levels (Fig. 13). Historically, those life history pathways with the highest productivities and capacities made use of the Wallowa River for some or all of the post fry emergence stages. Restoration potential is significant for pathways associated with this subbasin.

Productivity - This component of survival for Hurricane Creek life history pathways has declined severely compared to historic levels; groups of fish attempting to use these pathways are at extremely high risk of extinction. Major impediments to productivity (Appendix C) are alterations in flow pattern, sediment load, channel and bed instability, and lack of habitat diversity.

Capacity - The current capacity of life history pathways associated with this subbasin for smolt production is a small fraction of what it was historically. These changes are due largely to cumulative effects of reductions in life history productivity.

Diversity - The current potential for life history diversity associated with Hurricane Creek pathways is only a very small fraction of what it was historically. None of the life histories examined appear capable of sustaining production.

Prairie Creek Subbasin: Life history pathways associated with spawning reaches in Prairie Creek show extremely severe losses in current potential salmon performance compared to historic levels (Fig. 13). This potential is reduced because of losses in productivity and not capacity.

Productivity - This component of survival for Prairie Creek life history pathways has declined severely compared to historic levels; groups of fish attempting to use these pathways are at extremely high risk of extinction. Major impediments to productivity (Appendix C) are sediment load and lack of habitat diversity. 
Capacity - The current capacity of life history pathways associated with this subbasin for smolt production is low due to the cumulative effects of reductions in life history productivity (including loss of productivity outside Prairie Creek). The amount of key habitat has been increased in this subbasin because of flow augmentation through the irrigation system, but low productivity prevents this habitat from being used to much extent.

Diversity - The current potential for life history diversity associated with Prairie Creek pathways is low. None of the life histories examined appear capable of sustaining production..

Upper Grande Ronde Subbasin (upstream of Wallowa River, excluding tributaries to Grande Ronde $\boldsymbol{R}$.): Life history pathways associated with spawning reaches in the upper Grande Ronde River show severe declines in potential salmon performance compared to historic levels (Fig. 13). Restoration potential is significant for this subbasin. The results depicted in Fig. 13 show that the upper Grande Ronde River was historically extremely productive for spring chinook.

Productivity - This component of survival for Grande Ronde River Iife history pathways is currently low; groups of fish using these pathways appear to be at extremely high risk of extinction. This subbasin supported life history pathways under historic conditions that were the highest in the entire Grande Ronde basin. Major impediments to productivity (Appendix C) are alterations in temperature regimes, sediment load, loss of habitat diversity, channel and bed instability, and alterations in flow patterns.

Capacity - The mainstem Grande Ronde River supports life history pathways that currently have very low capacity for spring chinook. Historically, these same pathways had exceptionally high abundance, among the highest in the Grande Ronde basin. These changes are due largely to cumulative effects of reductions in life history productivity, although the quantity of key habitat has also been reduced.

Diversity - The potential for life history diversity associated with Grande Ronde River pathways is severely reduced compared to historic conditions. The resilience of the population to withstand additional environmental changes has been lessened accordingly. Life history pathways that may currently be used by spring chinook show relatively uniform productivity and capacity at low to extremely low levels. All of the life histories examined appear to be at extremely high risk of extinction. Improvements in productivity associated with stream reaches supporting these life histories would significantly improve overall sustainability and capacity for salmon within the Grande Ronde Basin.

Lookingglass Creek Subbasin: Life history pathways associated with spawning reaches in the Lookingglass Creek show moderate declines in current potential salmon performance compared to historic levels (Fig. 13).

Productivity - This component of survival for Lookingglass Creek life history pathways has declined slightly to moderately compared to historic levels. Productivity levels for these pathways are uniformly low. All of these pathways have low productivity, in part, because of losses that occur in the mainstem Grande Ronde River. 
Capacity - The capacity of life history pathways associated with this subbasin for smolt production has declined, on the average, by nearly half compared to historic levels. These changes are apparently due largely to cumulative effects of reductions in life history productivity.

Diversity - The current potential for life history diversity associated with Lookingglass Creek pathways is reduced substantially compared to historic conditions. The resilience of the population to withstand additional environmental changes has been lessened accordingly. Life history pathways that may currently be used by spring chinook show a relatively narrow range in productivity and capacity. Few, if any, life history pathways appear to be currently capable of sustaining production.

Catherine Creek Subbasin: Life history pathways associated with spawning reaches in Catherine Creek show severe declines in potential salmon performance compared to historic levels (Fig. 13). Restoration potential is significant for this subbasin.

Productivity - This component of survival for Catherine Creek life history pathways is currently low; groups of fish using these pathways appear to be at extremely high risk of extinction. Major impediments to productivity (Appendix C) are alterations in temperature regimes, sediment load, alterations in flow patterns, and lack of habitat diversity.

Capacity - Catherine Creek supports life history pathways that currently have low capacity for spring chinook. Historically, these same pathways had high abundance-These changes are due largely to cumulative effects of reductions in life history productivity, although the quantity of key habitat has also been reduced.

Diversity - The potential for life history diversity associated with Catherine Creek pathways is significantly reduced compared to historic conditions. The resilience of the population to withstand additional environmental changes has been lessened accordingly. Life history pathways that may currently be used by spring chinook show relatively uniform productivity and capacity at low to extremely low levels. All of the life histories examined appear to be at extremely high risk of extinction. Improvements in productivity associated with stream reaches supporting these life histories would significantly improve overall sustainability and capacity for salmon within the Grande Ronde Basin.

Sheep Creek Subbasin: Life history pathways associated with spawning reaches in Sheep Creek show extremely severe losses in current potential salmon performance compared to historic levels (Fig. 13). Historically, those life history pathways with the highest productivities and capacities made use of the mainstem Grande Ronde River for some or all of the post fry emergence stages. This subbasin is currently not capable of sustaining spring chinook production. Restoration potential is significant for pathways associated with this subbasin.

Productivity - This component of survival for Sheep Creek life history pathways has declined severely compared to historic levels. Productivity is currently too low to sustain production. Major impediments to productivity (Appendix C) are alterations in temperature 
regimes, sediment load, loss of habitat diversity, channel and bed instability, and alterations in flow patterns.

Capacity - Life history pathways associated with this subbasin currently appear to have virturally no capacity for smolt production. Historically, capacity for these pathways was substantial.

Diversity - No potential for life history diversity associated with Sheep Creek pathways currently exists. None of the life histories examined appear capable of sustaining production. Restoration potential for the subbasin is significant.

\section{Imnaha Basin}

The current potential of the overall Imnaha basin to produce and sustain spring chinook salmon in relation to conditions within the basin has been severely reduced compared to historic potential (Fig. 14). The large majority of life history pathways (represented by data points in Fig. 14) that historically were capable of supporting spring chinook can no longer do so, due to changes in the quality of habitat that have occurred over the past 150 years.

In general, data points for the Patient in Fig. 14 show a shift to the lower left portion of the chart, indicating that fish following these life history pathways are less able to cope with additional mortality pressures than they were historically - they have lost much of their resilience. The capacities of these pathways are also reduced, though the extent of loss appears to be relatively small.

The patterns of data points in Fig. 14 indicate that the Imnaha basin was historically a much less productive environment for spring chinook salmon than the Grande Ronde basin; both productivity and capacity were substantially less in the Imnaha. However, the loss of potential salmon performance has been much less severe in the Imnaha basin.

Life history pathways with productivity index values less than about 150 are likely at extremely high risk of extinction, given the current conditions outside of the Imnaha basin. High risk of extinction may also exist for pathways with values as high as 300 . These life history pathways continue into the mainstem Snake and Columbia rivers, where the quality of habitat for spring chinook has also deteriorated over the past century (Lichatowich and Mobrand 1995).

Conditions within the Imnaha basin to support spring chinook salmon differ significantly between subbasins (Fig. 15). The degree of habitat change (degradation from the salmon perspective) over the past 150 years also varies among subbasins within the Imnaha.

A diagnostic summary follows for each of four subbasins. The subbasins examined are the mainstem Imnaha, Big Sheep, Little Sheep, and Lightning. Other relatively small tributaries exist, similar to Lightning, but were not analyzed. Results for those streams would resemble those for Lightning Creek. 
Imnaha River Subbasin (excluding tributaries): Life history pathways associated with spawning reaches in the mainstem Imnaha River and South Fork show small changes in current potential salmon performance compared to historic levels (Fig. 15).

Productivity - This component of survival differs widely between Imnaha River pathways. In general, within basin productivity does not appear to have changed greatly compared to historic levels. The wide differences in productivity between pathways suggest that overall productivity for fish spawned in this subbasin may be highly variable between years.

Capacity - The overall capacity of Imnaha River life history pathways for smolt production appears to have changed little compared to historic levels. Changes that have occurred are apparently due to small reductions in life history productivity.

Diversity - The current potential for life history diversity associated with mainstem Imnaha River pathways is reduced slightly compared to historic conditions. The resilience of the population to withstand additional environmental changes has been lessened accordingly. Life history pathways that may currently be used by spring chinook show a relatively wide range in productivity and capacity. Only those life history pathways with relatively high productivity are capable of being sustained, however, due to deterioration in habitat quality outside the basin. In general, productivities of many Imnaha life history pathways appear to fall within a range that places groups of fish following these pathways at moderate to high risk of extinction with current conditions outside the basin.

Big Sheep Creek Subbasin: Life history pathways associated with spawning reaches in Big Sheep Creek show moderate to severe declines in current potential salmon performance compared to historic levels (Fig. 15). Restoration potential is significant for pathways associated with this subbasin.

Productivity - This component of survival for Big Sheep Creek life history pathways has declined substantially compared to historic levels. The subbasin supported life history pathways under historic conditions that were among the highest in productivity in the Imnaha basin. Major impediments to productivity (Appendix C) are alterations in temperature regimes, sediment load, channel or streambed instability, and loss of habitat diversity.

Capacity - The capacity of life history pathways associated with this subbasin for smolt production has declined compared to historic levels. However, smolt capacity of these pathways appears to have always been low.

Diversity - The current potential for life history diversity associated with Imnaha River pathways is reduced substantially compared to historic conditions. The resilience of the population to withstand additional environmental changes has been lessened accordingly. Groups of fish using these life history pathways are at high to extremely high risk of extinction. 
Little Sheep Creek Subbasin: Life history pathways associated with spawning reaches in Little Sheep Creek show extremely severe losses in current potential salmon performance compared to historic levels (Fig. 15). This subbasin is currently not capable of sustaining spring chinook production. Restoration potential is significant for pathways associated with this subbasin.

Productivity - This component of survival for Little Sheep Creek life history pathways has declined severely compared to historic levels. Productivity is currently too low to sustain production. Major impediments to productivity (Appendix C) are alterations in flow regimes, channel or streambed instability, changes in temperature regimes, and loss of habitat diversity, and water withdrawals.

Capacity - Life history pathways associated with this subbasin currently appear to have virtually no capacity for smolt production. Historically, capacity for these pathways was relatively low.

Diversity - No potential for life history diversity associated with Little Sheep Creek pathways currently exists. None of the life histories examined appear capable of sustaining production.

Lightning Creek Subbasin: Life history pathways associated with spawning reaches in Lightning Creek show little or no loss in current potential salmon performance compared to historic levels (Fig. 15). However, potential salmon performance associated with this subbasin is low.

Productivity - This component of survival for Lightning Creek life history pathways has declined severely compared to historic levels. Productivity is currently too low to sustain production.

Capacity - Life history pathways associated with this subbasin have very little capacity for smolt production. However, smolt capacity of these pathways appears to have always been low.

Diversity - Little life history diversity associated with Lightning Creek pathways currently exists. None of the life histories examined appear capable of sustaining production. 


\section{TREATMENT SELECTION}

\section{Purpose}

The purpose of this step is to identify reach specific action priorities within the watersheds based on the diagnosis. These priorities are presented as guidelines to assist watershed planners---not as rigid rules. Under these guidelines, each reach is assigned to an action priority category. Results are given as four sets of reference tables (Appendices D-G).

\section{Application of Prioritization Principles}

A general set of principles for applying these guidelines is described under "Prioritization Principles" earlier in this document; we refer to the EDT Primer (Lestelle et al. 1996) for a more complete description. In simplest terms, these principles call for setting the following strategic priorities within each watershed: first, maintain the performance of existing life histories; second, improve the performance of existing life histories; and third, restore lost life histories.

These principles give the two highest priorities to maintaining and improving existing quality and quantity of habitat associated with the primary and secondary life histories of spring chinook in the basins. These life histories are still populated: therefore, we assume that these should be protected and enhanced as a starting point in watershed restoration.

Primary and secondary life histories are those that utilize pathways in the watershed still capable of supporting spring chinook production. Fish continue to use these pathways because their connecting habitats have been able, so far, to sustain spring chinook populations. These are also the pathways with the greatest potential to continue to support spring chinook populations in the immediate future. Pathways incapable of sustaining salmon are either unpopulated or act as survival "drains" or impediments to long term sustainability.

The third strategic priority calls for restoring life history pathways that have been lost to the population. These vacant pathways can generally not be repopulated (through supplementation or otherwise) without first restoring quality (and perhaps quantity) of habitat so that it can support and sustain fish production. Restoration of vacant life histories may be required in the long term, in order to meet model watershed objectives and to restore metapopulation function for the salmon in the basin.

These strategic priorities are intended to direct efforts where they are likely to derive the most benefit. Watershed improvement efforts should be guided by a long-term vision if salmon are to be restored. The priorities suggested here should be viewed as constructive advice on how to invest in salmon restoration, not as rigid rules. It is important to take advantage of special opportunities (that are consistent with the diagnosis) and also to periodically review the prioritization principles. 


\section{Overview of Treatment Identification Steps}

The procedure for identifying reach specific action priorities has four steps: 1) classify each life history pathway used in the diagnostic analyses (Appendices A and B) as primary, secondary, or vacant; 2) assign a weighting factor to each pathway, based on current usage (relative abundance of fish), or in the case of vacant pathways, based on potential production; 3) compute cumulative usage scores and habitat productivity loss scores for each stream reach within each basin; and 4) plot scores and assign priority to each reach based on its potential for improving spring chinook performance.

Summary results are tabulated in a set of reference tables (Appendices D-G). The purpose of these tables is to help identify a) the stream reaches where improvements are most likely to produce benefits to spring chinook salmon, and b) the habitat attributes within those reaches that need to be addressed.

\section{Step 1: Classification of Life History Pathways}

All life history pathways used in the diagnostic analyses (Appendices A and B) are classified as primary, secondary, or vacant on the basis of the average spawner abundance in recent years and on the basis of observed life history patterns in the basins.

First pathways with cumulative (Patient) productivities too small $(<50)$ to possibly sustain populations of salmon are eliminated. Information listed in Appendices A and B is used for this purpose. Based upon personal observations, biologists working in the basin determine which of the remaining pathways are still populated and classify them as either primary (those that support most of the remaining spring chinook) or secondary (those that are used to lesser degree, perhaps intermittently). All other pathways are classified as vacant.

\section{Step 2: Assignment of Weighting Factors for Computation of Relative Importance of Reaches}

Weighting factors are assigned to each populated life history pathway based on whether it is primary (weight $=2$ ) or secondary (weight $=1$ ) and on the relative spawner abundance. Using spawning survey information and personal observations, team members categorize life history pathways into four relative abundance levels. For example, a primary pathway with the lowest relative abundance are given a weight of 2.1 , while the same pathway having the most relative abundance are given a weight of 2.4. Hence primary and secondary life history pathways are organized into eight different weight categories: $1.1,1.2,1.3,1.4,2.1,2.2,2.3$, or 2.4.

Vacant life history pathways are categorized into two groups of relative potential abundance based on their cumulative capacity index values under template conditions (the breakpoint was an index value of 1 .O). Lower index values are given a weight of 0.1 , while greater values are assigned a weight of 0.15 . Since reach length is not incorporated in the cumulative capacity 
index, weights are also applied for the length of the spawning reach associated with each pathway.

\section{Step 3: Computation of Reach Scores}

To get a better sense for the relative importance of each stream reach in prioritizing habitat improvements two "scores" are computed: 1) a chinook usage score reflecting the combined usage by all life history pathways of the reach; and 2) a habitat productivity change score reflecting the percent change in productivity between Template and Patient conditions. These scores provide a means for comparing the potential benefit to spring chinook performance of improvements in different stream reaches in the basin.

The usage score is computed as the weighted sum of the lengths of time (in weeks) that the reach was used by each pathway, where pathways were weighted as described in step 2.

The habitat productivity change score is computed as the weighted sum of the percentage change in productivity between Template and Patient for the segment of each pathway passing through the reach, with each pathway weighted as described in step 2 .

\section{Step 4: Review of Reach Importance Plots}

The two types of scores for each stream reach are plotted for primary and secondary life history pathways (combined on one plot) and for vacant pathways.

The plots provide a convenient way of categorizing the relative benefits that would accrue to spring chinook abundance and sustainability by targeting actions in different reaches within a basin.

Fig. 18 compares chinook usage (Y-axis) and habitat change (X -axis) scores for primary and secondary life histories among the reaches in the Grande Ronde and Imnaha basins. Each data point represents one stream reach. The rectangle marked " $A$ " contains the reaches that have the highest utilization by life history pathways and also the greatest loss in habitat quality. Actions aimed at improving habitat quality in these reaches would most likely produce the greatest benefit to chinook abundance and sustainability. Rectangles marked "B", "C", "D", "E", and "F" categorize other reaches in declining order of potential benefits from habitat improvements.

Fig. 19 compares reaches in the Grande Ronde and Imnaha basins in terms of their potential to contribute to restoration of vacant life history pathways. Each data point represents one stream reach. Rectangles marked "A", "B", "C", "D", "E", and "F" categorize stream reaches in declining order of benefits (to vacant life histories) from habitat quality improvements.

In Appendices D - G, the categories "A" through " $F$ ' are referred to as "Restoration benefit categories". 


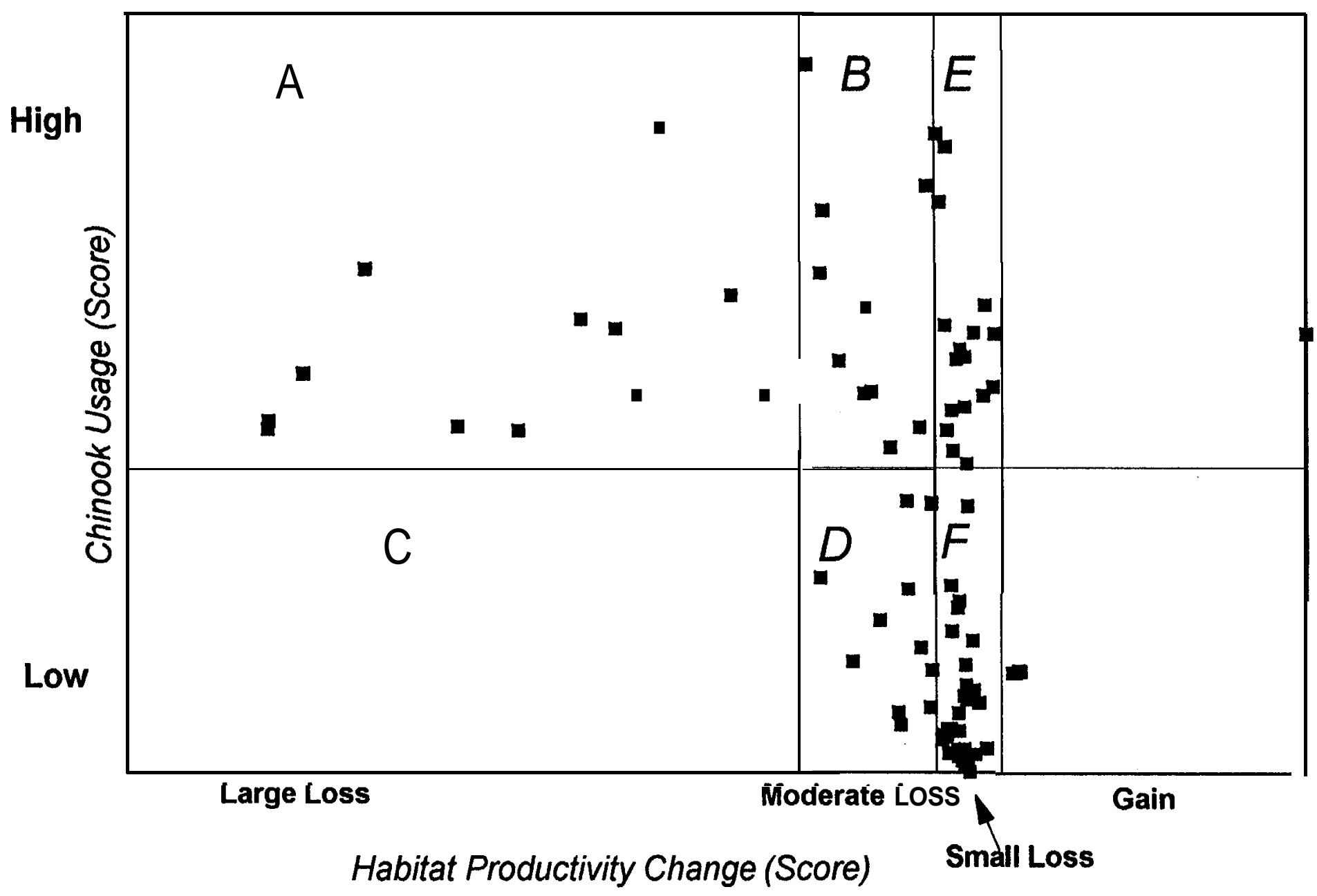

Figure 18. Potential benefits to primary and secondary life histories from reach specific habitat quality improvements in the Grande Ronde and Imnaha basins. Each point in the graph represent one stream reach. The rectangle marked "A" contains the reaches that are most important to chinook salmon and have suffered the most severe loss of habitat quality. The benefit categories are defined in the text. 

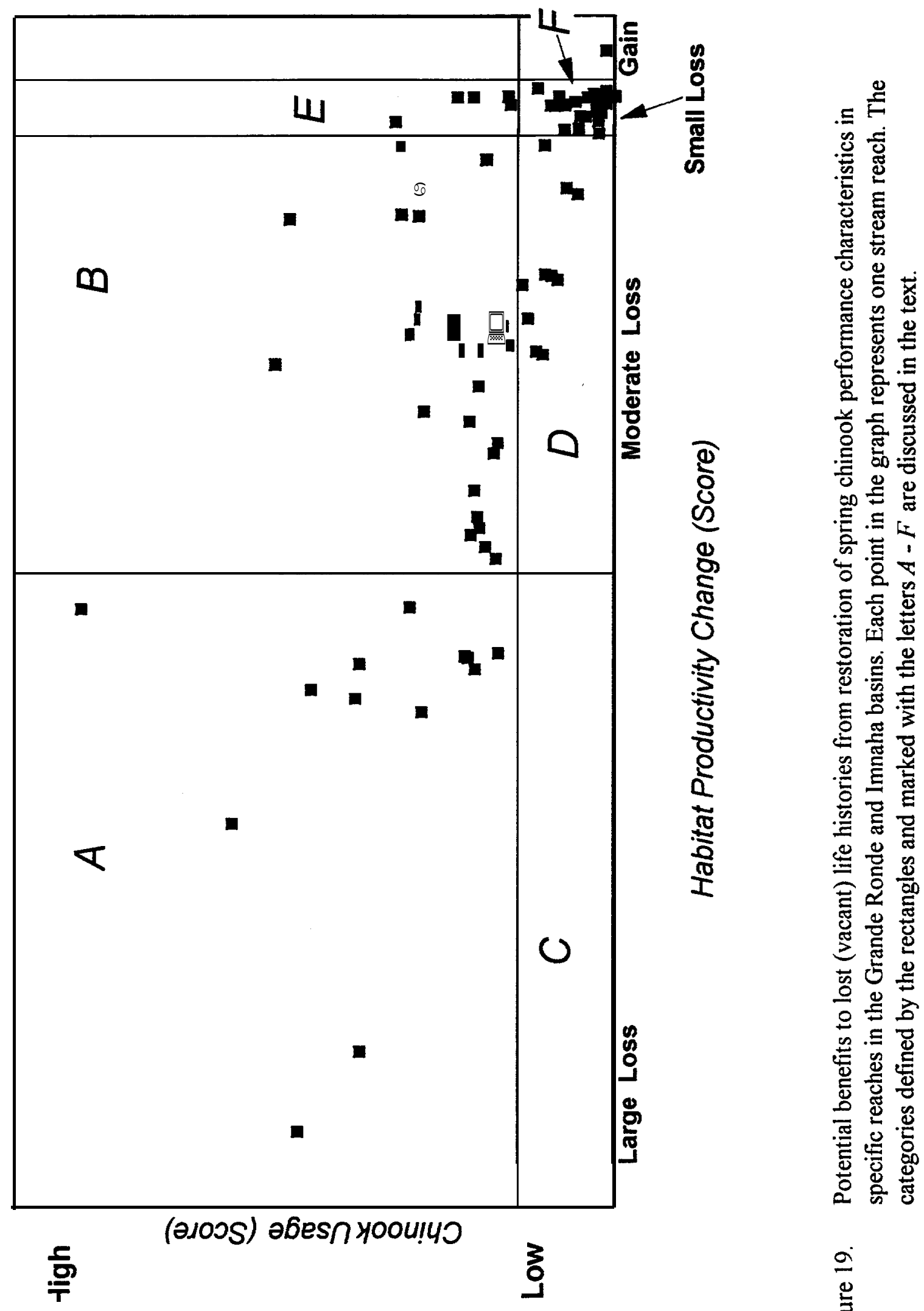

$\frac{9}{0}$ 


\section{Guide to Targeting Reach Specific Actions}

Strategic priorities for targeting reach specific actions are aimed at either maintaining the performance of existing life histories, improving the performance of existing life histories, or restoring lost life histories.

\section{Actions Intended to Maintain Performance of Existing Life Histories}

The GREDT work group suggests that maintenance of all existing life histories should be the highest priority. Appendices D (Grande Ronde) and F (Imnaha) identify the reaches that are used by existing life histories. The chinook usage index shown in these appendices also provide a sense of the relative importance of each reach to those life histories. The reaches with the highest chinook usage scores are most important to the maintenance of spring chinook performance.

\section{Actions Intended to Improve Performance of Existing Life Histories}

Appendices D (Grande Ronde basin) and F (Imnaha basin) summarize the importance of each reach to existing spring chinook life histories. One table for each stream reach is provided. Tables are arranged alphabetically by stream reach for easy reference. Included in each table is the restoration benefit category ("A" through " $F$ ") of the reach. Reaches in category "A" should be the highest priority for habitat quality improvement.

Each table also includes detailed information by life history stage and relevant time of year about the number of primary and secondary life history pathways that utilize the reach (by relative abundance), percent loss of key habitat, and habitat productivity loss. Finally, each table identifies the specific environmental attributes that have affected the loss in productivity.

Table 6 shows an example of one of the reference tables from Appendix D, the South Fork Catherine Creek. The table indicates that the reach is assigned to benefit category B, implying that it is of high importance to existing life histories and that it has experienced a moderate loss in spring chinook productivity (see Figure 18). Only reaches belonging to category A might offer greater potential for improvement, since here the productivity loss is greater and therefore perhaps also the opportunity for increasing habitat quality.

Actions aimed at improving the quality of egg incubation habitat would offer the greatest benefit to spring chinook (habitat productivity loss score of -166.8 ; relevant months would be October through March), followed by actions aimed at improving an earlier time period (August through October) that affect the pre-spawning, spawnirg, and incubation life stages, and so on.

If actions were to be taken to improve the incubation environment, the most important attribute to target would be sediment load, followed by flow patterns, water temperature, and channel stability. By far, the greatest impediment to productivity is considered to be sediment load. 
Table 6. Sample table showing evaluation of a reach based on its importance to primary and secondary life history pathways.

Stream: Catherine Cr.

Reach: South Fork Catherine Cr (RM 164.4 - 166.8 from Snake R)

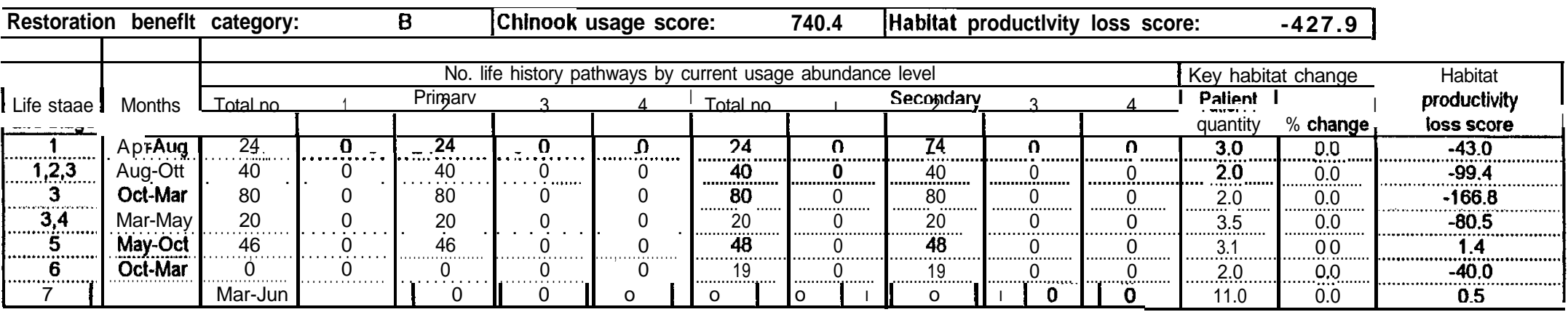

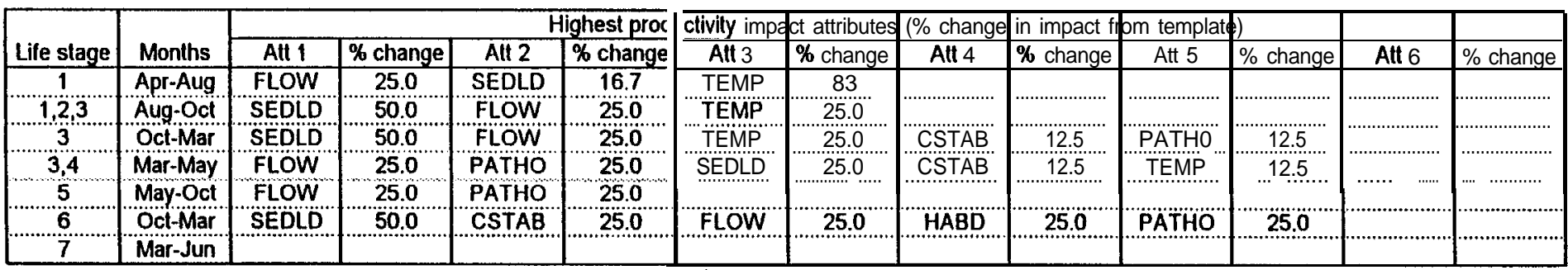

Abundance /eve/ refers lo the average abundance of spawners that spawn in the natal stream reaches that populate the life history pathways using the reach in this table: $\begin{array}{ll}\text { Level 1: } & \text { O-I } 0 \text { spawners } \\ \text { Level 2: } & \text { IO-30 spawners } \\ \text { Level 3: } & 30-60 \text { spawners } \\ \text { Level 4: } & \quad>60 \text { spawners }\end{array}$

Life stages: 1 - prespawner; 2 - spawner; 3 - incubation; 4 - fry colonization; 5 - summer rearing; 6 - overwintering; 7 - yearling presmolt/smolt

Attributes: CHEM - chemicals

CSTAB - channel stability

NUTLD - nutrient load

COMP - competition (wilh other species)

OBST - obstructions

OXYG - dissolved oxygen

PATHO - pathogens

RIPCN - riparian condition

FLOW - flow

PRED - predators (includes fishing-related losses)

SEDLD - sediment load

TEMP - water temperature

HABD - habitat diversity

WITH - water withdrawals

"NA" indicates that no life history pathway meeting the criteria used in defining these pathways was present.

Blank cells under attributes indicate no additional attributes with negative effects on productivity were evident compared to those present in the template. 


\section{Actions Intended to Restore Performance of Vacant Life Histories}

The information needed to set stream reach specific priorities for restoring spring chinook performance in vacant life history pathways are provided in Appendices E (Grande Ronde) and G (Imnaha). One table is provided for each stream reach. Tables are arranged alphabetically by stream reach for easy reference.

Each table lists the benefit category (based on Fig. 19), chinook usage score, and habitat productivity loss score for the associated reach. The same information is provided as that given in Appendices D and F.

Table 7 is an example of one of the reference tables in Appendix E, the South Fork Catherine Creek. This reach is assigned to benefit category $\mathrm{F}$, meaning that it is given relatively low priority for restoring vacant life histories. This low benefit category suggests that habitat quality improvements in this reach would be of little value to vacant life histories, as indicated above however existing ones would benefit significantly.

Actions aimed at improving the quality of egg incubation habitat would offer the greatest benefit to achieve a restoration objective (habitat productivity loss score of -20.9 ; relevant months would be October through March), followed by actions aimed at improving the earlier time period (August through October) that affect the prespawning, spawning, and incubation life stages, and so on.

If actions were to be taken to improve the incubation environment, the most important attribute to target would be sediment load, followed by flow patterns, water temperature, and channel stability. By far, the greatest impediment to improving productivity was considered to be sediment load. 
Table 7. Sample table showing evaluation of a reach based on its importance to vacant life history pathways.

Stream: Catherine $\mathrm{Cr}$

Reach: South Fork Catherine Cr (RM 164.4 - 166.8 from Snake R)

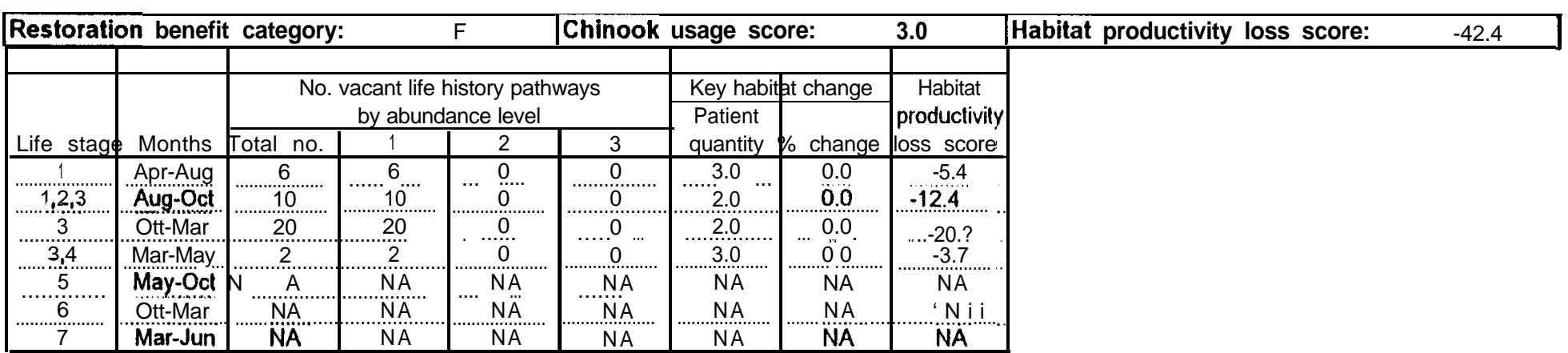

\begin{tabular}{|c|c|c|c|c|c|c|c|c|c|c|c|c|c|}
\hline \multirow[b]{2}{*}{ Life stage } & \multirow[b]{2}{*}{ Months } & & & \multicolumn{7}{|c|}{ 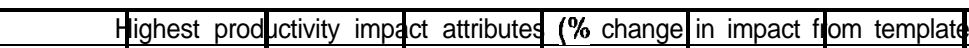 } & \multirow{2}{*}{\multicolumn{3}{|c|}{ 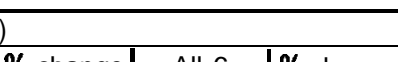 }} \\
\hline & & All 1 & $\%$ change & All 2 & $\%$ change & All 3 & $\%$ change & All 4 & $\%$ change & All 5 & & & \\
\hline 1 & Apr-Aug & FLOW & 25.0 & SEDLD & 16.7 & TEMP & 6.3 & & & & & & \\
\hline $1,2,3$ & Aug-Ott & SEDLD & 50.0 & FLOW & 25.0 & TEMP & 25.0 & & & & & & \\
\hline 3 & Ött-Mar & S̈EDL̈D & 50.0 & FLÖẄ & 25.0 & TEMMP & 25.0 & $\dot{C} \bar{S} A \ddot{B}$ & 12.5 & PATHOO & 12.5 & & \\
\hline 3,4 & Mar-May & FLOW & 25.0 & SEDLD & $25 \quad 10$ & CSTAB & 12.5 & PATHO & 12.5 & TEMP & 12.5 & & \\
\hline 5 & May-Ott & $\mathrm{NA}$ & NA & $\mathrm{NA}$ & N A & NA & NA & NA & NA & NA & NA & NA & NA \\
\hline 6 & Oct-Mar & 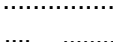 & $\mathrm{NA}$ & $\mathrm{NA}$ & NA & $\mathrm{NA}$ & $\mathrm{NA}$ & $N A$ & $\mathrm{NA}$ & $\mathrm{NA}$ & NA & $\mathrm{NA}$ & $\mathrm{NA}$ \\
\hline 7 & M̈ar-Jun & $\mathrm{NA}$ & $\mathrm{NA}$ & $N A$ & NA & $\mathrm{NA}$ & $\mathrm{NA}$ & $\mathrm{NA}$ & $\mathrm{NA}$ & & NA & $\mathrm{NA}$ & NA \\
\hline
\end{tabular}

Abundance level refers lo estimated relative abundance of spawners that spawned in the natal stream reaches that populated the life history pathways using the reach in this table: Level 1: Low lo average (relative lo other reaches) abundance

Level 2: $\quad$ High (relative lo other reaches) abundance

Level 3: Exceptionally high (relative lo other reaches) abundance

Life slages: 1 - prespawner; 2 - spawner; 3 - incubation; 4 - fry colonization; 5 - summer rearing; 6 - overwintering; 7 - yearling presmolt/smolt

Attributes: CHEM - chemicals CSTAB - channel stability

COMP - competition (with other species)

$\mathrm{FLOW}$ - flow

HABD - habitat diversity
NUTLD - nutrient load

OBST - obstructions

OXYG - dissolved oxygen

PATHO - pathogens

PRED - predators (includes fishing-related losses)
RIPCN - riparian condition

SEDLD * sediment load

TEMP - waler temperature

WITH * waler withdrawals

"NA" indicates that no life history pathway meeting the criteria used in defining these pathways was present.

Blank cells under attributes indicate no additional attributes with negative effects on productivity were evident compared to those present in the template. 
The Appendices that follow provide the information necessary to prioritize activities to improve habitat conditions in the Grande Ronde and Imnaha basins from the spring chinook perspective. This information takes into account the habitat conditions throughout these basins, the requirements of spring chinook at each life stage, and the connectivity of habitat segments. It contrasts the present condition (Patient) with a historic reference condition (Template). The information in this report greatly increases our ability to set biologically and economically sound priorities, simply because it is based upon a logical framework. We need to keep in mind however that there is still uncertainty associated with both the information and the framework, and in the spirit of adaptive management we must continue to question and improve our knowledge. For example the implications of a metapopulation structure on the prioritization of restoration of vacant life histories is not yet fully understood for spring chinook in the Grande Ronde/Imnaha basins. We hope and expect that this will be an area of further development. 


\section{LITERATURE CITED}

Huntington, C.W. 1994. Stream and riparian conditions in the Grande Ronde Basin 1993. Final report. Prepared for the Grande Ronde Model Watershed Board, La Grande, OR.

Independent Scientific Group, 1996. Return to the river: restoration of salmonid fishes in the Columbia River Ecosystem. 96-6, Prepublication Copy. Northwest Power Planning Council, Portland, Oregon.

Keefe, M., R.W. Carmichael, B.C. Jonasson, R.T. Messmer, and T.A. Whitesel 1996. Fish research project (Oregon) - Investigations into the life history of spring chinook salmon in the Grande Ronde River Basin. Annual Report 1994 to Bonneville Power Administration. Project number 92-026-01, Portland, Oregon.

Lestelle, L.C., L.E. Mobrand, J.A. Lichatowich, and T.S. Vogel, 1996. Applied ecosystem analysis - a primer, EDT: the ecosystem diagnosis and treatment method. Project number 9404600, Bonneville Power Administration, Portland, Oregon.

Lichatowich, J.A., and L.E. Mobrand. 1995. Analysis of chinook salmon in the Columbia River from an ecosystem perspective. Final Report, Contract No. DE-AM79-92BP25 105. Bonneville Power Administration, Portland, OR.

Lichatowich. James, L. Mobrand, L. Lestelle, and T. Vogel. 1995. An approach to the diagnosis and treatment of depleted Pacific salmon populations in freshwater ecosystems. Fisheries (Bethesda) 20(1): 10- 18.

McIntosh, B.A. 1992. Historical changes in anadromous fish habitat in the upper Grande Ronde River, Oregon, 1941-1990. Masters thesis. Oregon State University, Corvallis, OR.

Northwest Power Planning Council. 1990. Grande Ronde River Subbasin salmon and steelhead production plan. Columbia Basin system planning. Published by the Northwest Power Planning Council, and the Agencies and Indian Tribes of the Columbia Basin Fish and Wildlife Authority, Portland, OR.

Smith, A.K. 1975. Fish and wildlife resources of the Grande Ronde Basin, Oregon, and their water requirements. Federal Aid to Fish Restoration Project Completion Report. Oregon Department of Fish and Wildlife, Portland, OR.

Smith, B., and B.U. Knox. 1993. Wallowa District 1993 stock status review. Oregon Department of Fish and Wildlife, March, 1993.

West, D., and J. Zakel. 1993. La Grande Fish District annual report. Oregon Department of Fish and Wildlife, March 1993.

Grande Ronde Ecosystem Diagnosis and Treatment /January 1997 /Page 58 


\section{LIST OF APPENDICES}

APPENDIX A: $\quad$ Productivity and Capacity Profiles for Life History Pathways in the Grande Ronde River and Tributaries

APPENDIX B: $\quad$ Productivity and Capacity Profiles for Life History Pathways in the Imnaha River and Tributaries

APPENDIX C: $\quad$ Patient Template Analysis. Landscapes showing Key Habitat, Productivity, and Environmental Attributes for each Range

APPENDIX D: $\quad$ Grande Ronde Stream Reach Summaries for Primary and Secondary Life History Pathways

APPENDIX E: $\quad$ Grande Ronde Stream Reach Summaries for Vacant Life History Pathways

APPENDIX F: Imnaha Stream Reach Summaries for Primary and Secondary Life History Pathways

APPENDIX G: Imnaha Stream Reach Summaries for Vacant Life History Pathways APPENDIX H: Bibliography 


\section{APPENDIX A}

Productivity and Capacity Profiles for Life History Pathways in the Grande Ronde River and Tributaries 


\title{
Spawning reaches analyzed in the Grande Ronde, Iisted in the order they appear in Appendix A.
}

\author{
Stream \\ Reach \\ Bear Cr. \\ Bear Cr. \\ Bear Cr. \\ Catherine Cr. \\ Catherine $\mathrm{Cr}$. \\ Catherine $\mathrm{Cr}$. \\ Catherine $\mathrm{Cr}$. \\ Grande Ronde R. \\ Cirande Ronde R. \\ Grande Ronde R. \\ Grande Ronde R. \\ Grande Ronde R. \\ Grande Ronde R. \\ Grande Ronde R. \\ Grande Ronde R. \\ Grande Ronde R. \\ Grande Ronde R. \\ Grande Ronde R. \\ Grande Ronde R. \\ Grande Ronde R. \\ Grande Ronde R. \\ Grande Ronde R. \\ Grande Ronde R. \\ Grande Ronde R. \\ Grande Ronde R. \\ Grande Ronde R. \\ Grande Ronde R. \\ Grande Ronde R. \\ Grande Ronde R. \\ Grande Ronde R. \\ Grande Ronde R. \\ Grande Ronde R. \\ Grande Ronde R. \\ Grande Ronde R. \\ Grande Ronde R. \\ Grande Ronde R. \\ Hurricane Cr. \\ Hurricane Cr. \\ Joseph Cr. \\ Joseph Cr. \\ Chesnimnus Cr. \\ Lookingglass $\mathrm{Cr}$. \\ Lookingglass $\mathrm{Cr}$. \\ Lostine $\mathrm{R}$. \\ Lostine R. \\ Lostine R. \\ Lostine R. \\ Lostine R. \\ Mouth - Chamberlain Ditch (RM 101.8 - 104.7 from Snake R) \\ Chamberlain Ditch - Little Bear Cr (RM 104.7 - 109.3 from Snake R) \\ Little Bear Cr - headwaters (RM 109.3 - 1 18.3 from Snake R) \\ Mouth - 10th Street Bridge in Union (RM 114.5 - 146.0 from Snake R) \\ 10th Street Bridge in Union - Little Catherine Cr (RM 146.0 - 157.5 from Snake R) \\ Little Catherine Cr - forks (RM 157.5 - 164.4 from Snake R) \\ South Fork Catherine Cr (RM 164.4 - 166.8 from Snake R) \\ Joseph Cr - Buford Cr (RM 3.7 - 26.2 from Snake R) \\ Buford Cr - Bear Cr (RIM 26.2 - 28.2 from Snake R) \\ Bear Cr - Menatchee Cr (RM 28.2 - 34.8 from Snake R) \\ Menatchee Cr - Wenaha R (RM 34.8 - 45.1 from Snake R) \\ Wenaha R - Courtney Cr (RM 45. 1-46.2 from Snake R) \\ Courtney Cr - Mud Cr (RM 46.2 - 51.4 from Snake R) \\ Mud Cr - Wallupa/Wildcat Cr (RM 5 1.4 - 52.8 from Snake R) \\ Wallupa/Wildcat Cr - Grossman Cr (RM 52.8 - 6 I. 1 from Snake R) \\ Grossman Cr - Wallowa R (RM 6 I. 1- 80.0 from Snake R) \\ Wallowa R - Lookingglass Cr (RM 80.0 - 83.4 from Snake R) \\ Lookingplass $\mathrm{Cr}$ - Gordon Cr (RM 83.4 - 94.2 from Snake R) \\ Gordon Cr - Phillips Cr (RM 94.2 - 96.9 from Snake R) \\ Phillips Cr - Clark Cr (RM 96.9 - 97.3 from Snake R) \\ Clark Cr - Indian Cr (RM 97.3 - 99.3 from Snake R) \\ Indian Cr - Willow Cr (RM 99.3 - 103.5 from Snake R) \\ Willow Cr - downstream end of State Ditch (RM 103.5 - 1 14.5 from Snake R) \\ Downstream end of State Ditch - Upstream end of State Ditch (RM 1 14.5 - 1 18.9 from Snake R) \\ Upstream end of State Ditch - I-84 crossing below Devil's Slide (RM 118.9 - 128.4 from Snake R) \\ I-84 crossing below Devil's Slide - Five Points Cr (RM 128.4 - 133.4 from Snake R) \\ Five Points Cr - Rock Cr (RM 133.4 - 133.8 from Snake R) \\ Rock Cr - Whiskey Cr (RM 133.8 - 136.6 from Snake R) \\ Whiskey Cr - Jordan Cr (RM 136.6 - 139.1 from Snake R) \\ Jordan Cr - Beaver Cr (RIM 139. I - 145.8 from Snake R) \\ Beaver Cr - Meadow Cr (RM 145.8 - 147.0 from Snake R) \\ Meadow Cr - Fly Cr (RM 147.0 - 15 I ..3 from Snake R) \\ Fly $\mathrm{Cr}$ - head of canyon 1.3 miles downstream of Sheep Cr (RM15 1.3 - 159.4 from Snake R) \\ Head of canyon 1.3 miles downstream of Sheep Cr - LimberJim Cr (RM 159.4 - 164.2 from Snake R: \\ LimberJim Cr - Clear Cr (RM 164.2 - 166.9 from Snake R) \\ Clear Cr - headwaters (RM 166.9 - 170.1 from Snake R) \\ Mouth - Lower Alderslope Ditch (RM 12 I .9 - 124.9 from Snake R) \\ Lower Alderslope Ditch - Upper Alderslope Ditch Diversion (RM 124.9 - 128.2 from Snake R) \\ Cottonwood Cr - Swamp Cr (RM 8.2 - 37.7 from Snake R) \\ Swamp Cr - Chesnimnus Cr (RM 37.7 - 52.7 from Snake R) \\ Confluence with Crow Cr - South Fork (RM 52.7 - 77.7 from Snake R) \\ Mouth - Little Lookinpglass Cr (RM 83.4 - 87.6 from Snake R) \\ Little Lookingglass Cr - headwaters (RM 87.6 - 93.8 from Snake R) \\ Mouth - Clearwater Ditch (RM 105.8 - 108.3 from Snake R) \\ Clearwater Ditch - Cross Country Ditch (RM 108.3 - 110.8 from Snake R) \\ Cross Country Ditch - Sheep Ridge Dam (RM 110.8 - 115.0 from Snake R) \\ Sheep Ridge Dam - Silver Cr (RM 115.0 - 120.0 from Snake R) \\ Silver Cr - Lake Cr (RM 120.0 - 124.6 from Snake R) \\ Lake Cr - East Lostine R (RM 124.6 - 13 I .7 from Snake R)
}




\title{
Spawning reaches analyzed in the Grande Ronde, listed in the order they appear in Appendix A.
}

\author{
Stream \\ Minam R. \\ Minam R. \\ Minam R. \\ Minam R. \\ Prairie Cr. \\ Prairie Cr. \\ Sheep Cr. \\ Sheep Cr. \\ Wallowa R. \\ Wallowa R. \\ Wallowa $\mathrm{R}$. \\ Reach \\ Mouth - Cougar Cr (RM 89.8 - 99.4 from Snake R) \\ Cougar Cr - Little Minam R (RM 99.4 - 108.4 from Snake R) \\ Little Minam R - splash dam site (1 m above Garwood Cr; RM 108.4 - 119.7 from Snake R) \\ Splash dam site - falls (RM 119.7 - 138.0 from Snake R) \\ Mouth - OK Gulch confluence (RM 126.8 - 131.8 from Snake R) \\ OK Gulch fork (RM 131.8 - 133.8 from Snake R) \\ Mouth - Chicken Cr (RM 164.2 - 165.7 from Snake R) \\ Chicken Cr - headwaters (RM 165.7 - 173.7 from Snake R) \\ Mouth - Minam R (RM 80.0 - 89.8 from Snake R) \\ Minam R - Deer Cr (RM 89.8 - 90.8 from Snake R) \\ Deer Cr - Dry Cr (RM 90.8 - 98.3 from Snake R) \\ Dry Cr - Bear Cr (RM 98.3 - 101.8 from Snake R) \\ Bear Cr - Whiskey Cr (RM 101.8 - 103.9 from Snake R) \\ Whiskey Cr - Lostine R (RM 103.9 - 105.8 from Snake R) \\ Lostine R - Parsnip Cr (RM 105.8 - 109.0 from Snake R) \\ Parsnip Cr - Hurricane Cr (RM 109.0 - 12 I .9 from Snake R) \\ Hurricane Cr - Lower Alderslope Ditch (RM 121.9 - 126.8 from Snake R) \\ Lower Alderslope Ditch - Wallowa Lake (RM 126.8 - 130.8 from Snake R) \\ Mouth - Crooked Cr (RM 45.1 - 5 I .8 from Snake R) \\ Crooked Cr - Fait-view Cr (RM 5 1.8 - 55.3 from Snake R) \\ Fairview Cr - forks (RM 55.3 - 65.9 from Snake R) \\ South Fork Wenaha R (RM 65.9 - 72.4 from Snake R) \\ Wenaha R.
}


Spawning reach: Bear Cr.

Mouth - Chamberlain Ditch (RM 101.8 - 104.7 from Snake R)

Relative Productivity
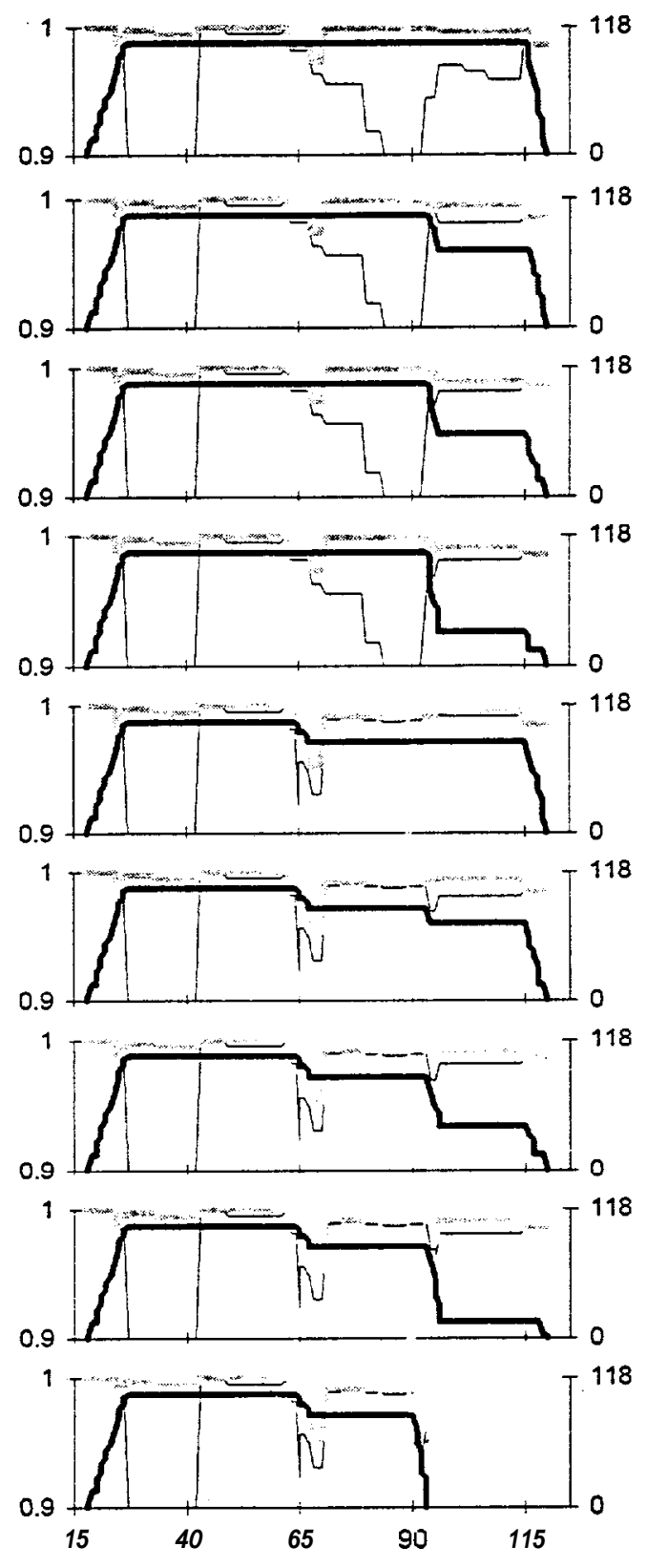

Statistical week
Relative Key Habitat Quantity

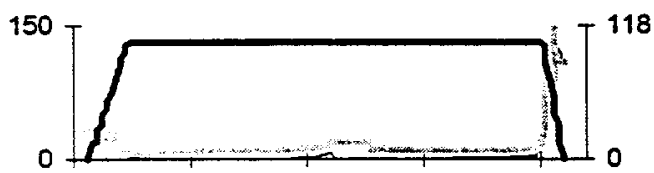

$150-$

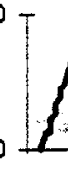

150

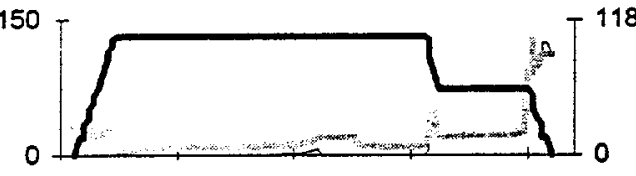

150
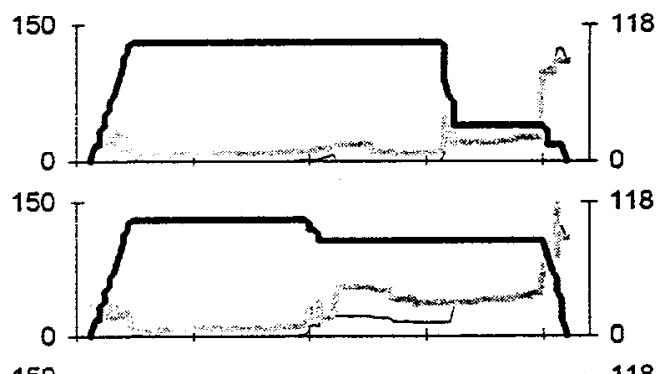

150 .

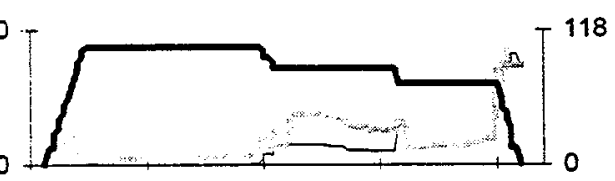

$150-$

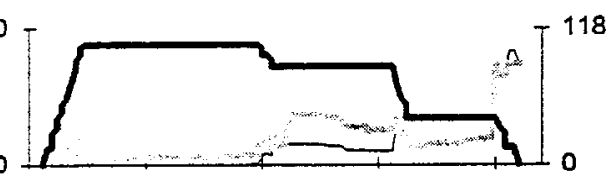

150

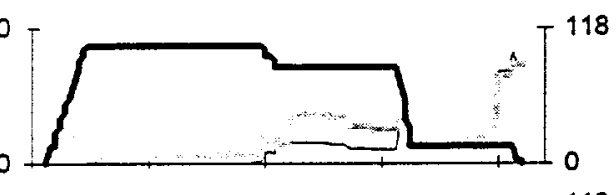

$150-$

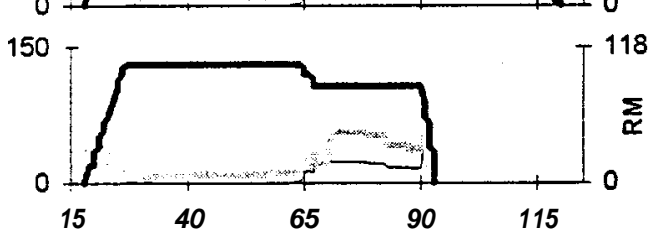

Statistical week

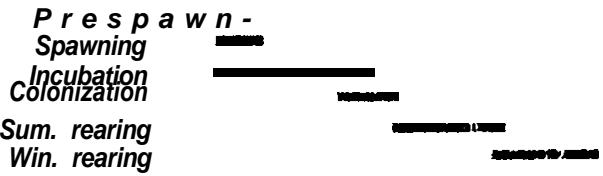

Prespawn -

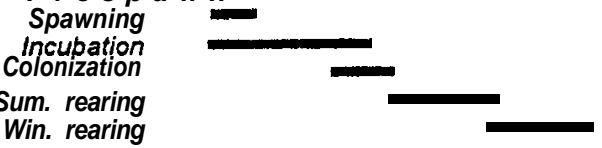

\section{Template \\ Patient}

Cum. Cum. Prod. Cap.

Traj. \#1

\begin{tabular}{l|l}
\hline 509 & 0.54 \\
\hline & 0.00
\end{tabular}

o) 0.00

Traj. \#2

$486 \quad 0.57$

이 0.00

Traj. \#3

\begin{tabular}{|l|l|}
439 & 0.51 \\
\hline & 0.00
\end{tabular}

0) 0.00

Traj. \#4

\begin{tabular}{l|l}
\hline 460 & 0.54 \\
\hline
\end{tabular}

0.00

Traj. \#5

\begin{tabular}{l|l}
375 & 0.53 \\
\hline
\end{tabular}

0.00

Traj. \#6

\begin{tabular}{l|l}
\hline 77 & 0.52 \\
\hline
\end{tabular}

0) 0.00

Traj. \#7

$355 \quad 0.49$

Traj. \#8

$353 \quad 0.49$

0) 0.00

Traj. \#9

\begin{tabular}{|r|r|}
\hline 635 & 0.93 \\
0 & 0.00
\end{tabular} 
Spawning reach: Bear $\mathrm{Cr}$.

Chamberlain Ditch - Little Bear Cr (RM 104.7 - 109.3 from Snake R)

Relative Productivity
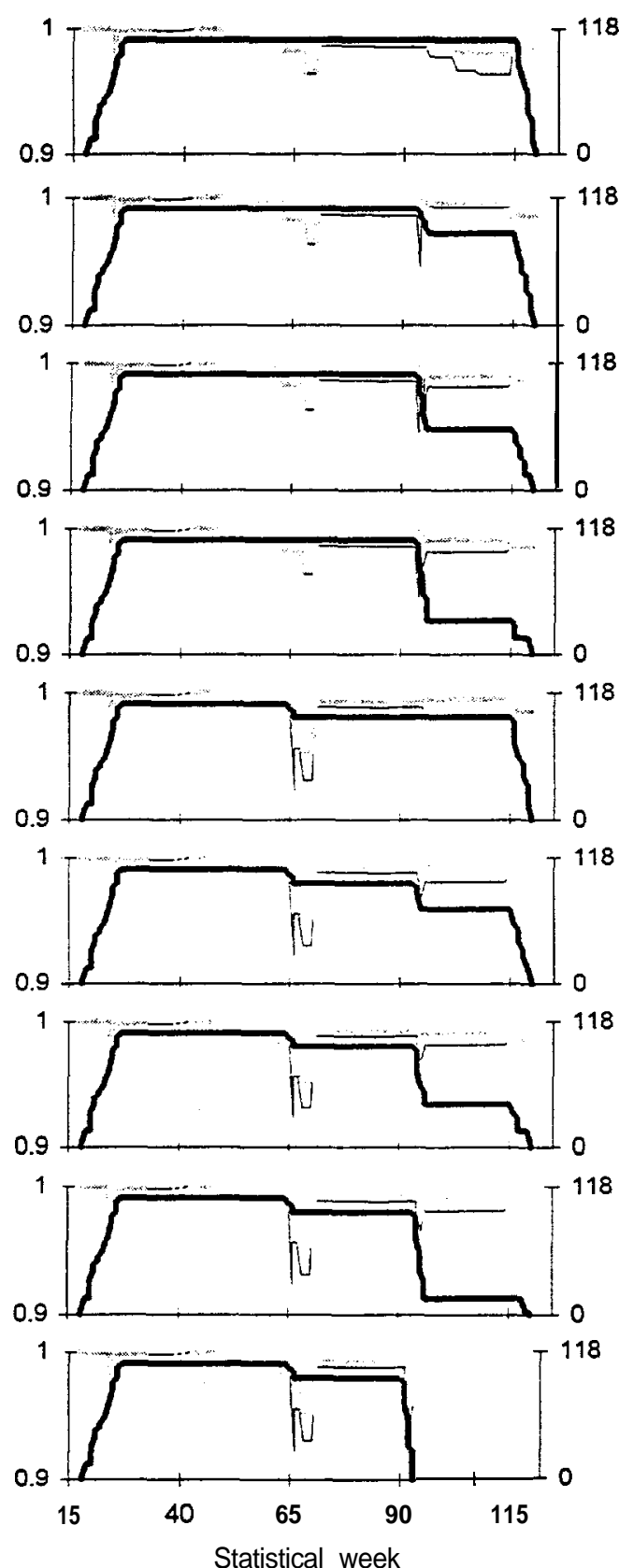

Relative Key Habitat Quantity
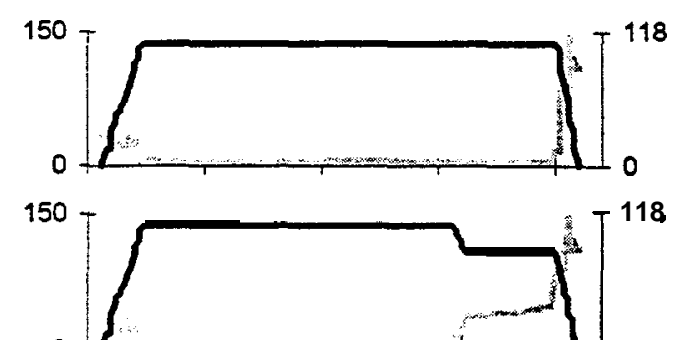

0

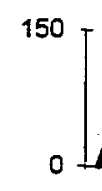

150

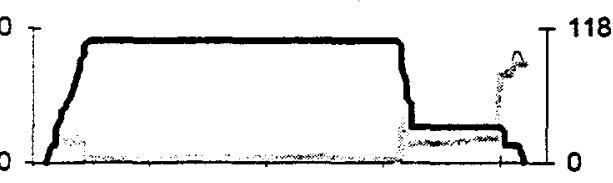

150

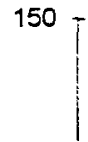

0

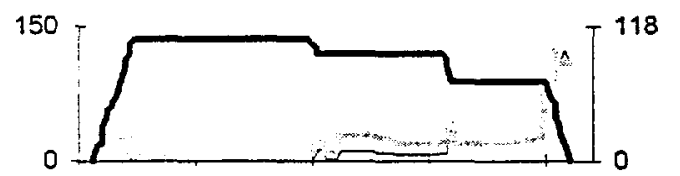

0

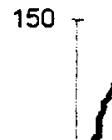

$\sqrt{1}$

0

$150+$
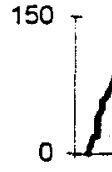

150
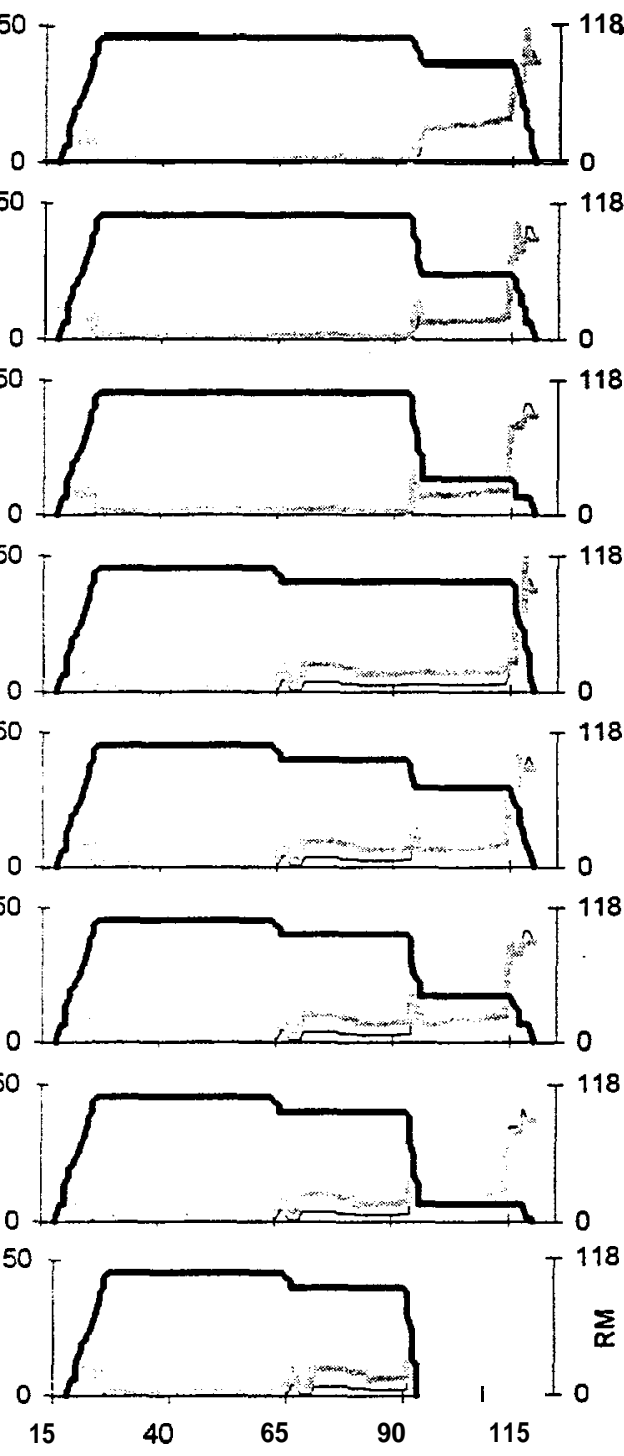

$T^{118}$
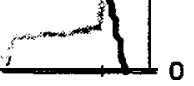

$T^{118}$

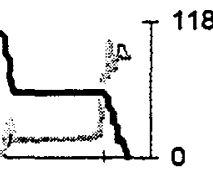

118
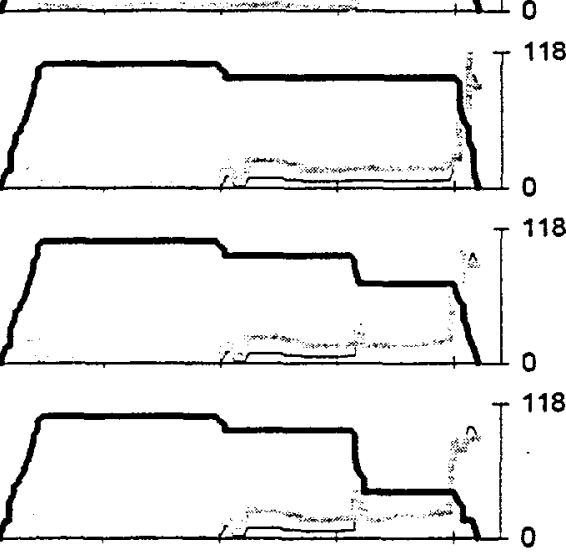

espawn -

Spawning

Incubation

Colonization

Sum. rearing
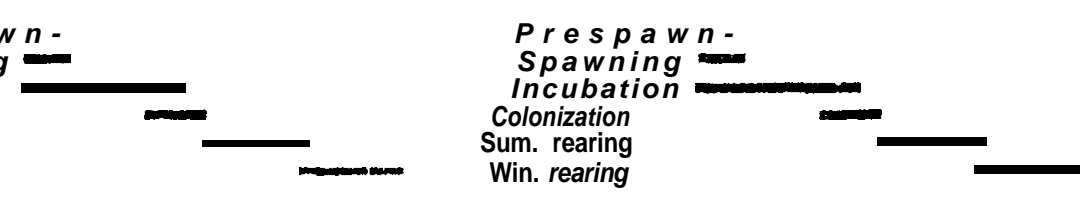

Trajectory

Template

Patient 
Spawning reach: Bear $\mathrm{Cr}$.

Little Bear Cr - headwaters (RM 109.3 - 118.3 from Snake R)

Relative Productivity
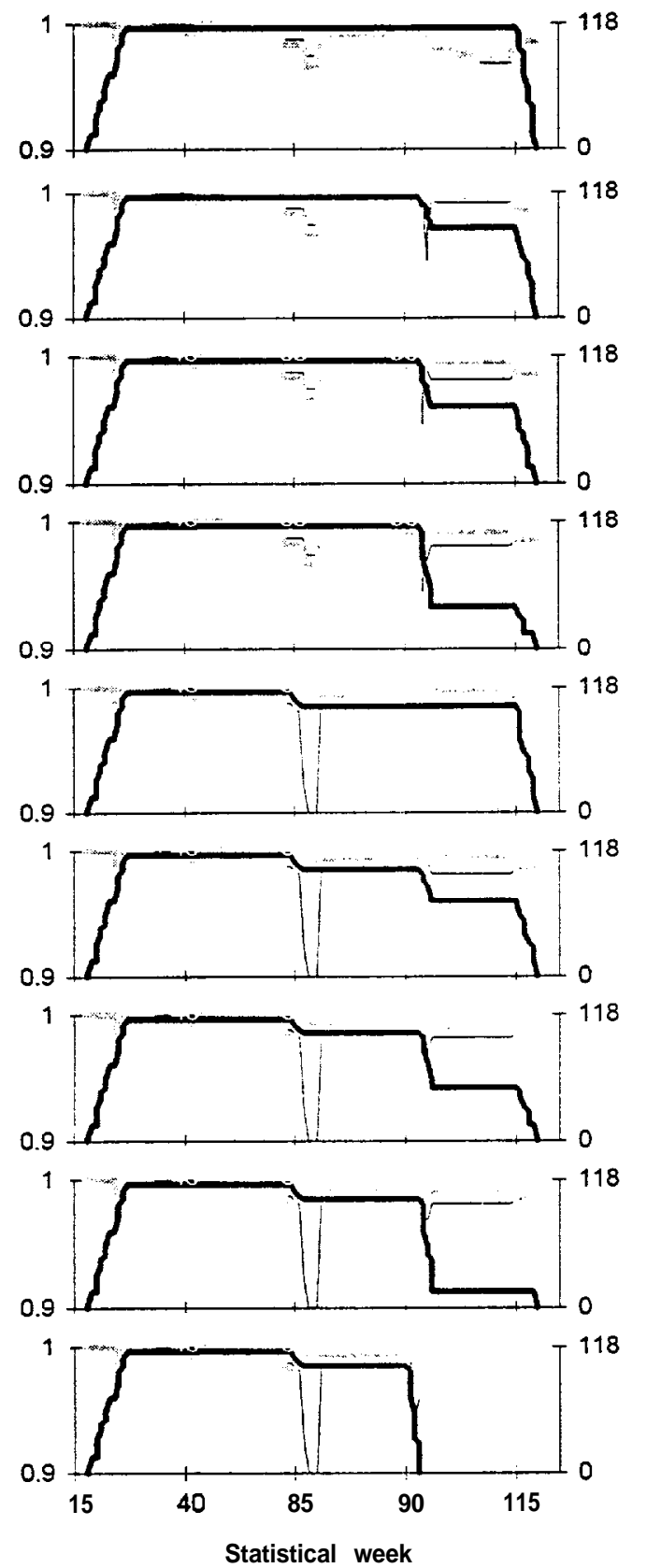

Prespawn -

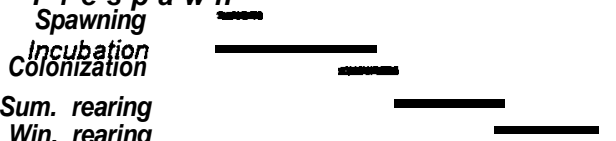

Win rearing

Win. rearing
Relative Key Habitat Quantity
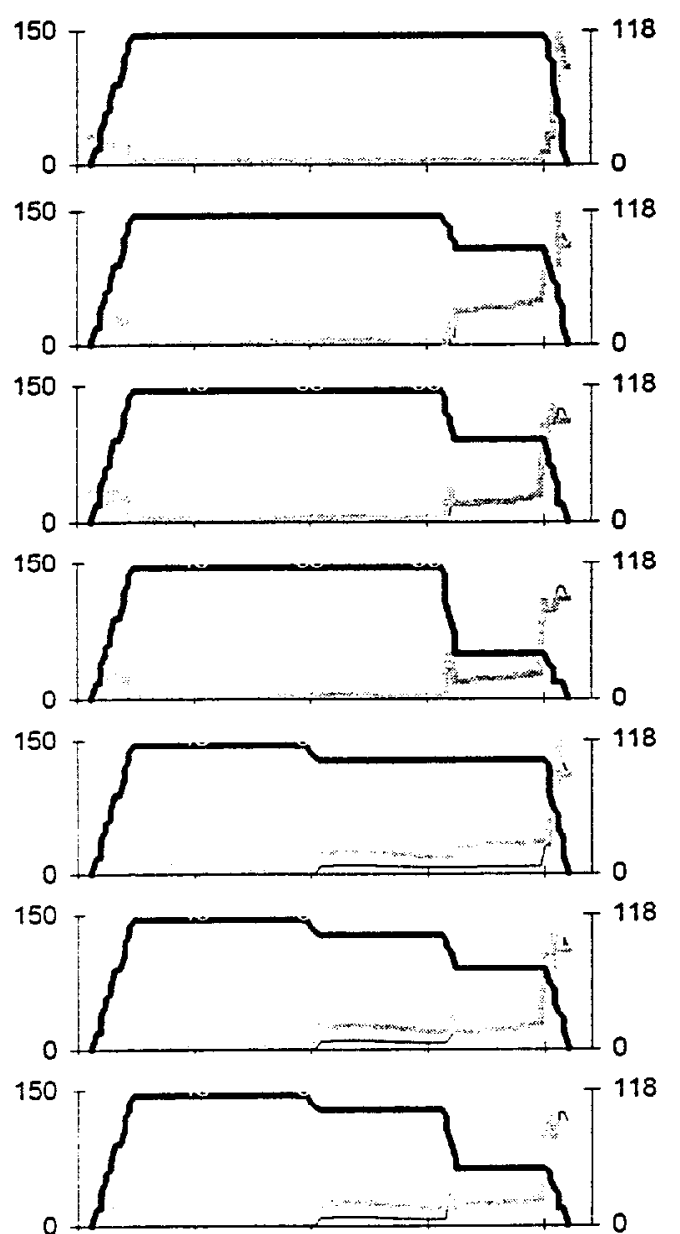

150
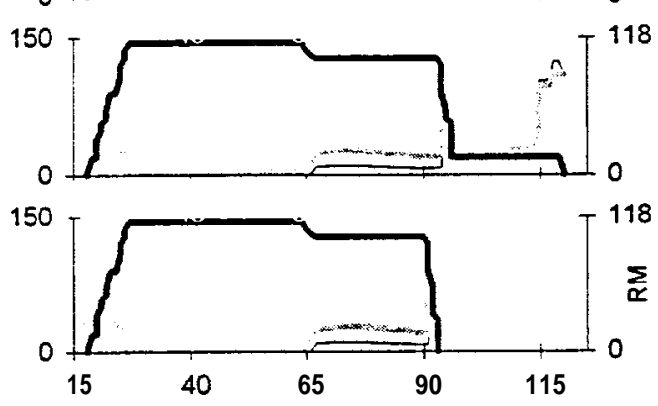

Statistical week
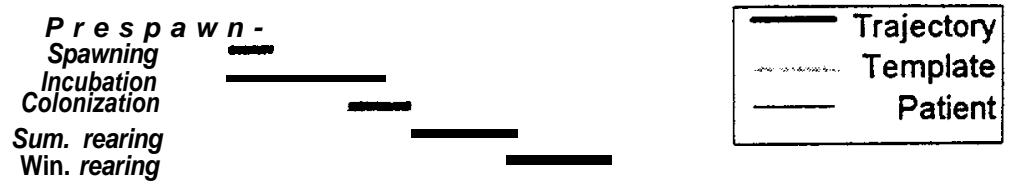
Spawning reach: Catherine $\mathrm{Cr}$.

Mouth - 10th Street Bridge in Union (RM 114.5 - 146.0 from Snake R)

Relative Productivity
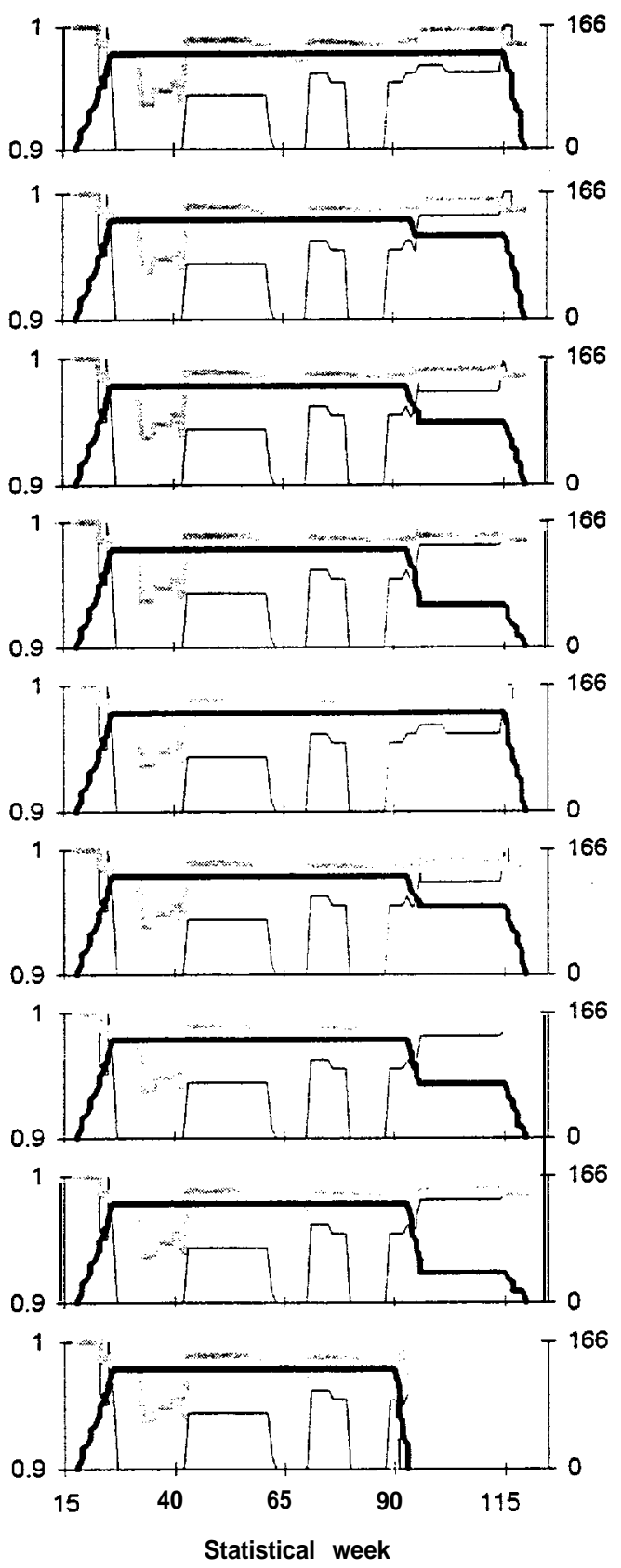

$P$ respaw $n$ -
Spawning

Spawni

Colonization

sum. rearing

Win. rearing

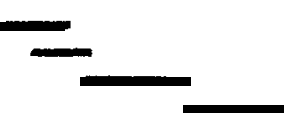

Relative Key Habitat Quantity

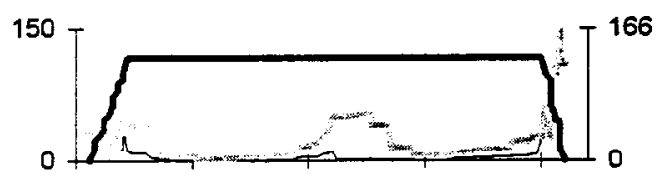

$150-$

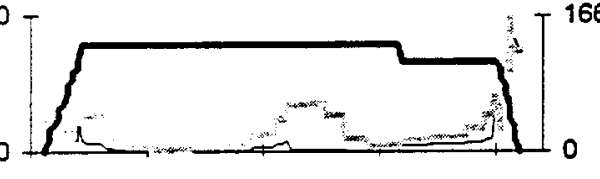

150

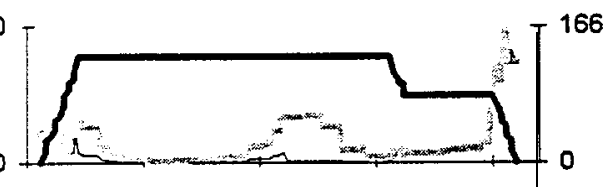

150 .

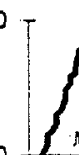

150 .
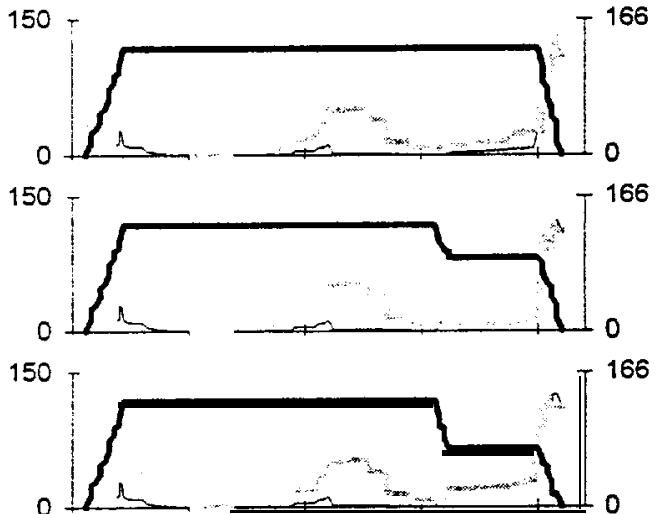

150 .
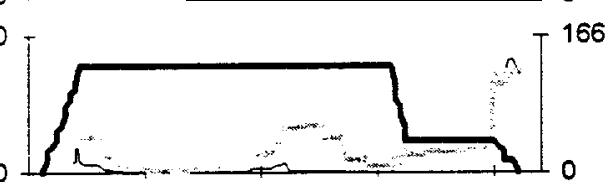

150
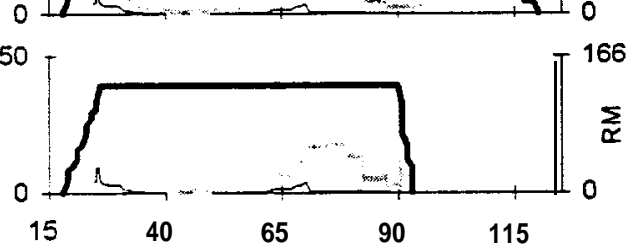

Statistical week

Prespawn-

Spawning

Incubation

Colonization

Sum. rearing

Win. rearing

\begin{tabular}{|r|c|}
\hline \multicolumn{2}{|c|}{ Template } \\
Patient \\
\hline Cum. & Cum. \\
Prod. & Cap. \\
Traj. \#1 \\
\hline $\mathbf{1 5 4}$ & 0.59 \\
0 & 0.00 \\
\hline
\end{tabular}

Traj. \#2

\begin{tabular}{l|l}
145 & 0.57
\end{tabular} 0 0.00

Traj. \#3

$138 \quad 0.54$ 0) 0.00

Traj. \#4

$132 \quad 0.53$

0.00

Traj. \#5

\begin{tabular}{|l|l|}
\hline 154 & 0.59 \\
\hline
\end{tabular}

0.00

Traj. \#6

$138 \quad 0.51$

0) 0.00

Traj. \#7

\begin{tabular}{l|l}
\hline 146 & 0.59 \\
\hline
\end{tabular}

0) 0.00

Traj. \#8

\begin{tabular}{l|l}
\hline 138 & 0.56 \\
\hline
\end{tabular}

0) 0.00

Traj . \#9

\begin{tabular}{l|l}
\hline 253 & 1.16 \\
\hline & 0.00
\end{tabular} 0) 0.00 
Spawning reach: Catherine $\mathrm{Cr}$.

10th Street Bridge in Union - Little Catherine Cr (RM 146.0 - 157.5 from Snake R)

Relative Productivity
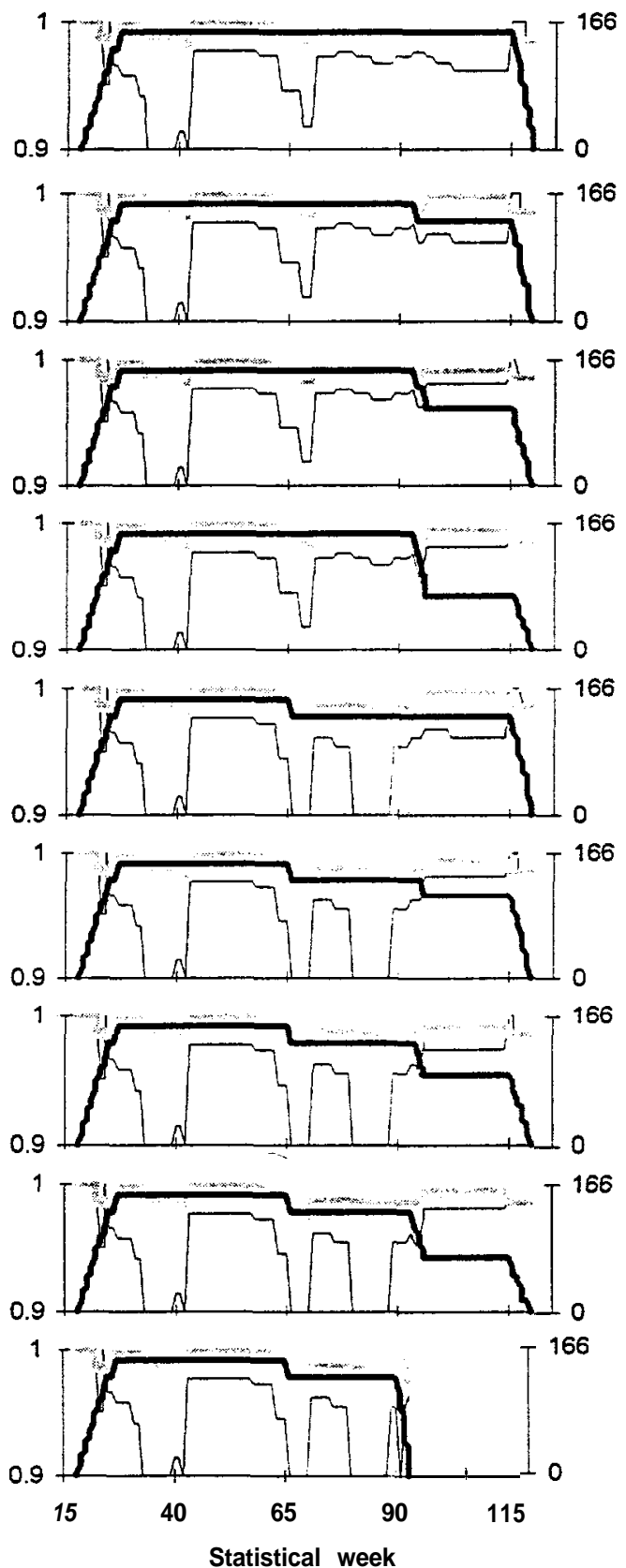

Prespawn -

Spawning

Incubation

Colonization

Sum. rearing

Win. rearing
Relative Key Habitat Quantity
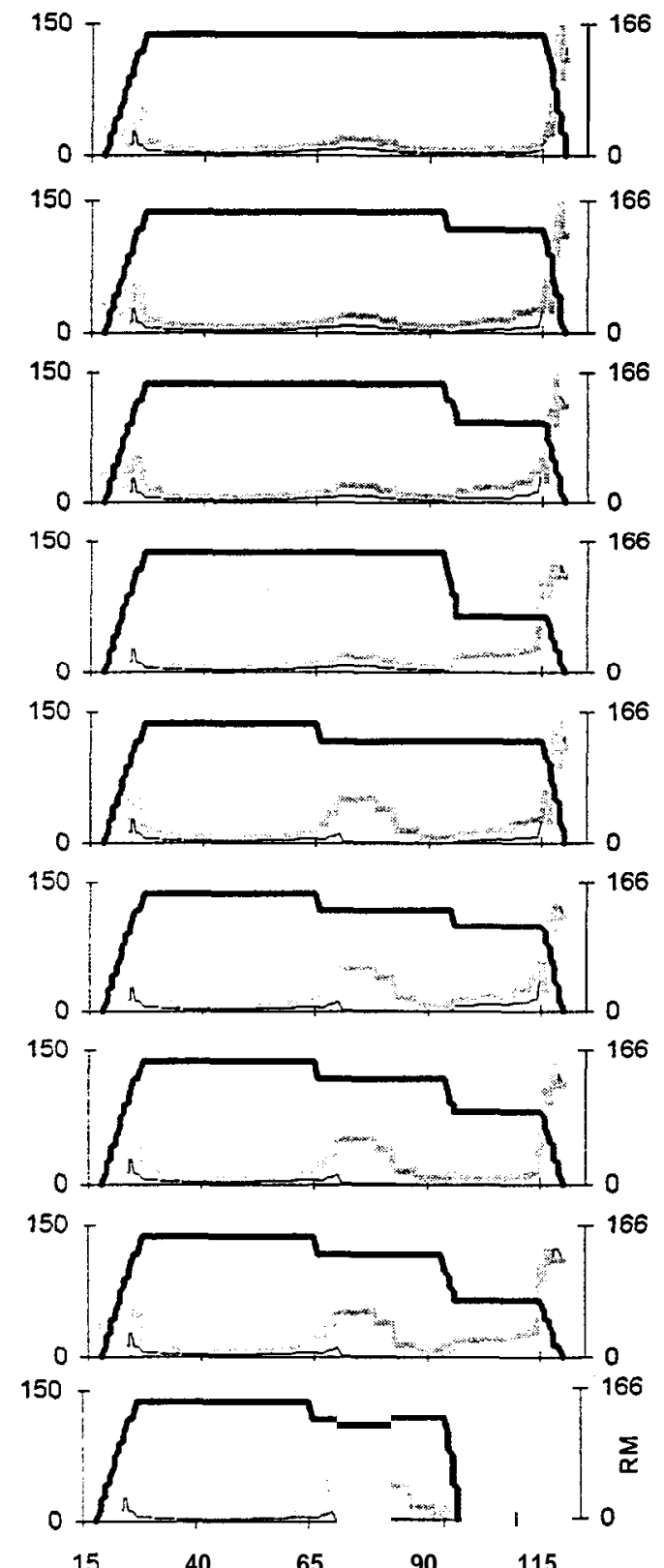

$\begin{array}{lllll}15 & 40 & 65 & 90 & 115\end{array}$

Statistical week

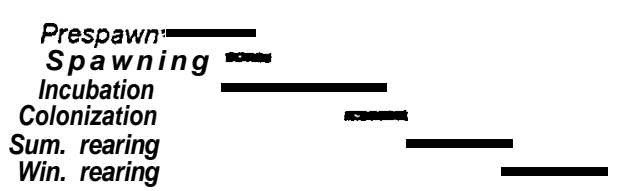

Template

Patient

Cum. Cum.

Prod. Cap.

Traj. \#1

\begin{tabular}{l|l|}
\hline 406 & 0.65 \\
\hline 14 & 0.02
\end{tabular}

14) 0.02

Traj. \#2

\begin{tabular}{l|l}
\hline 419 & 0.75 \\
13 & 0.02
\end{tabular}

13) 0.02

Traj. \#3

\begin{tabular}{l|l}
\hline 788 & 0.74 \\
\hline
\end{tabular}

$18 \quad 0.03$

Traj. \#4

\begin{tabular}{l|l}
\hline 396 & 0.75 \\
\hline
\end{tabular}

17 0.03

Traj. \#5

\begin{tabular}{l|l} 
“ & \multicolumn{1}{c}{ । } \\
\multicolumn{1}{c}{ Traj. \#6 } \\
\hline 319 & 0.75 \\
3 & 0.01
\end{tabular}

Traj. \#7

\begin{tabular}{l|l}
\hline 302 & 0.65
\end{tabular}

2) 0.00

Traj . \#8

\begin{tabular}{r|r}
\hline 318 & 0.78 \\
3 & 0.00
\end{tabular}

Traj. \#9

\begin{tabular}{r|l}
\hline 557 & 1.61 \\
5 & 0.01
\end{tabular} 
Spawning reach: Catherine $\mathrm{Cr}$.

Little Catherine Cr - forks (RM 157.5 - 164.4 from Snake R)

Relative Productivity
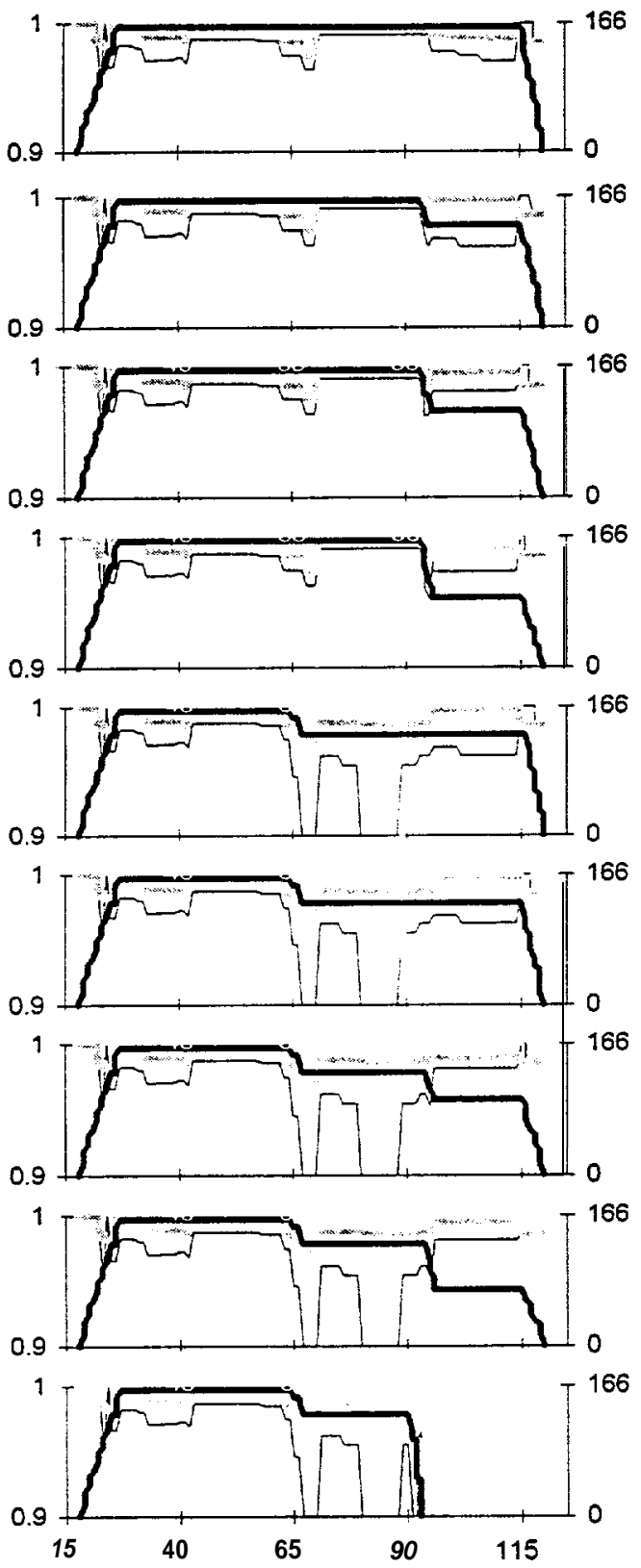

Statistical week

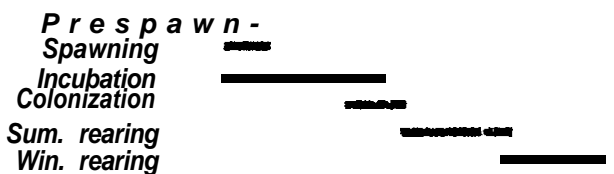

Relative Key Habitat Quantity

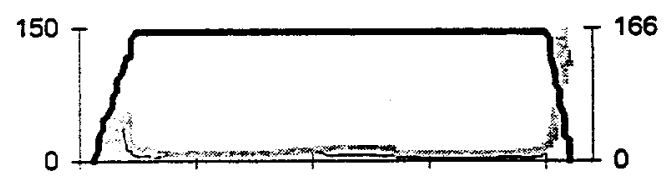

150

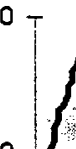

150 -

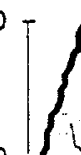

150

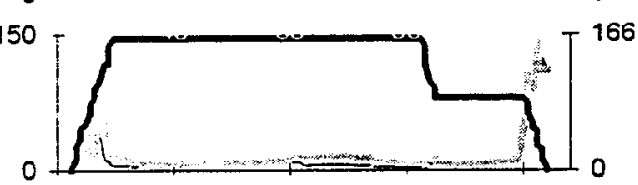

150

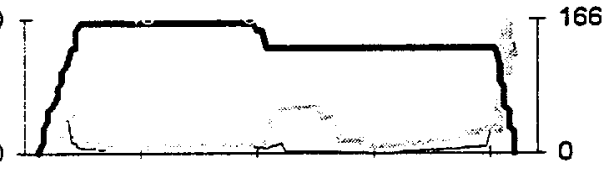

150

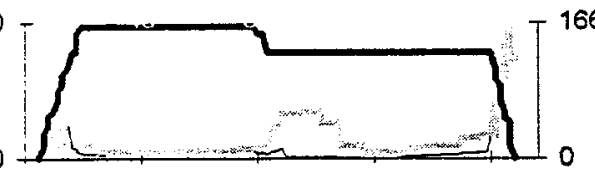

150
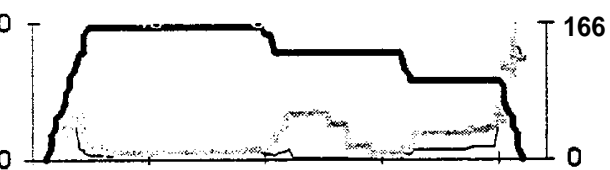

150

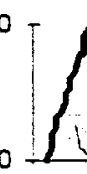

150

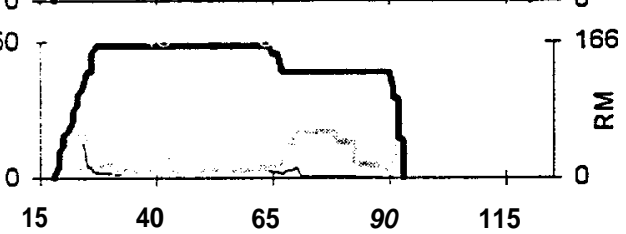

Statistical week

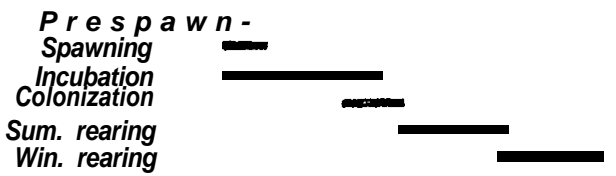

\section{Template \\ Patient}

Cum. Cum.

Prod. Cap.

Traj. \#1

$354 \quad 0.63$

136 0.15

Traj. \#2

\begin{tabular}{|l|l|}
\hline 411 & 0.78 \\
\hline
\end{tabular}

$108 \quad 0.11$

Traj. \#3

\begin{tabular}{l|l}
\hline 388 & 0.76
\end{tabular}

147 0.16

Traj. \#4

\begin{tabular}{|l|l|}
\hline 367 & 0.66 \\
\hline
\end{tabular}

\begin{tabular}{l|l}
124 & 0.15
\end{tabular}

Traj. \#5

$328 \quad 0.74$

\begin{tabular}{l|l}
9 & 0.01
\end{tabular}

Traj. \#6

$328 \quad 0.75$

\begin{tabular}{l|l}
9 & 0.01
\end{tabular}

Traj. \#7

\begin{tabular}{|l|l|}
\hline 280 & 0.69 \\
\hline
\end{tabular}

120.02

Traj. \#8

311 0.75

12 0.02

Traj. \#9

\begin{tabular}{r|r|}
\hline 543 & 1.55 \\
21 & 0.03 \\
\hline
\end{tabular} 
Spawning reach: Catherine $\mathrm{Cr}$.

South Fork Catherine Cr (RM 164.4 - 166.8 from Snake R)

Relative Productivity
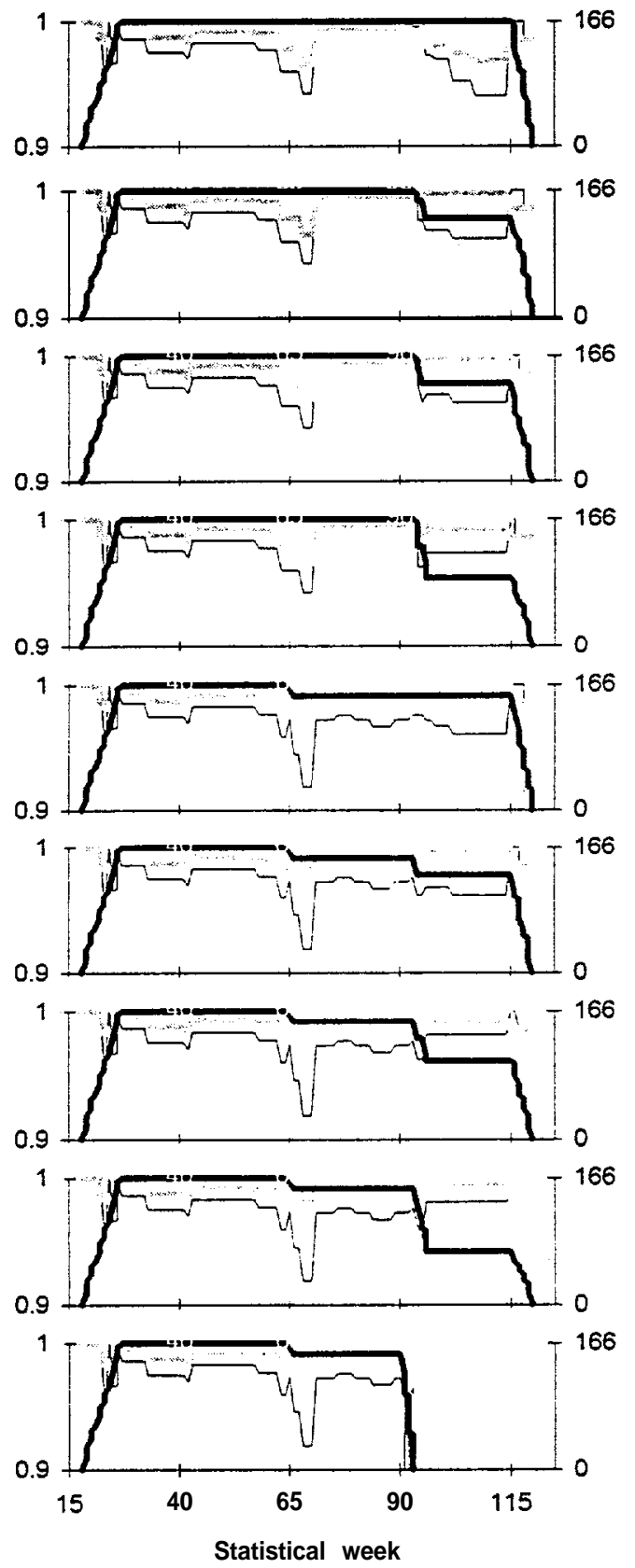

Prespawn -

Spawning

Incubation

Colonization

Sum. rearing

Win. rearing
Relative Key Habitat Quantity
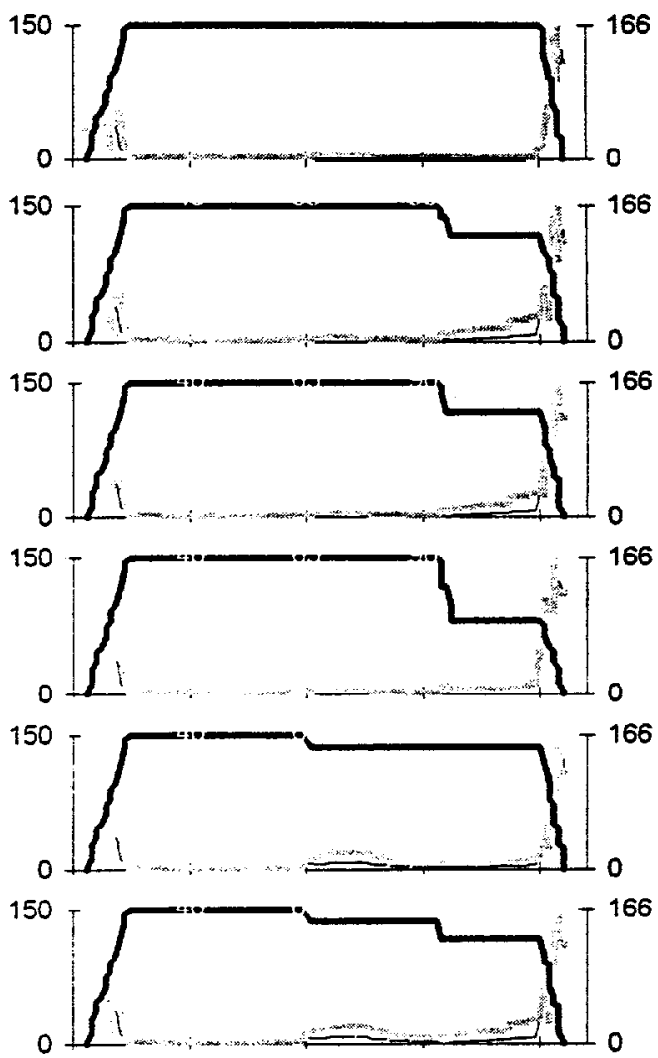

150
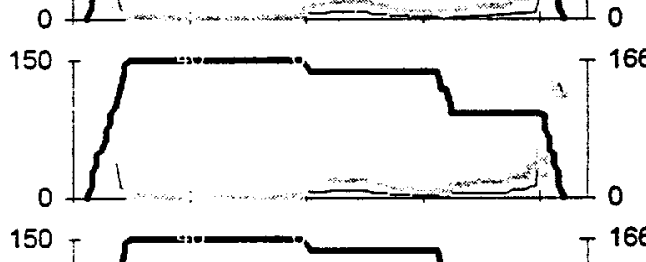

166
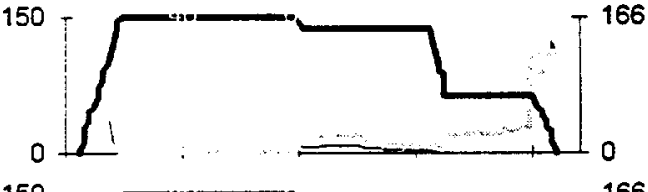

150 -

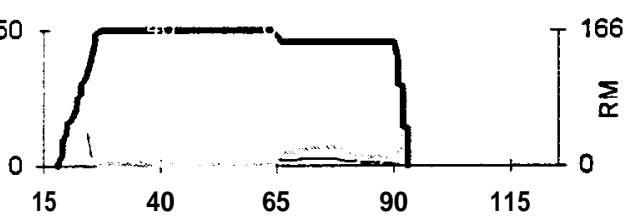

Statistical week

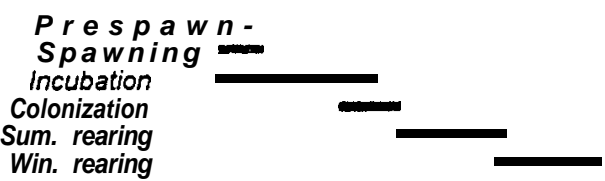

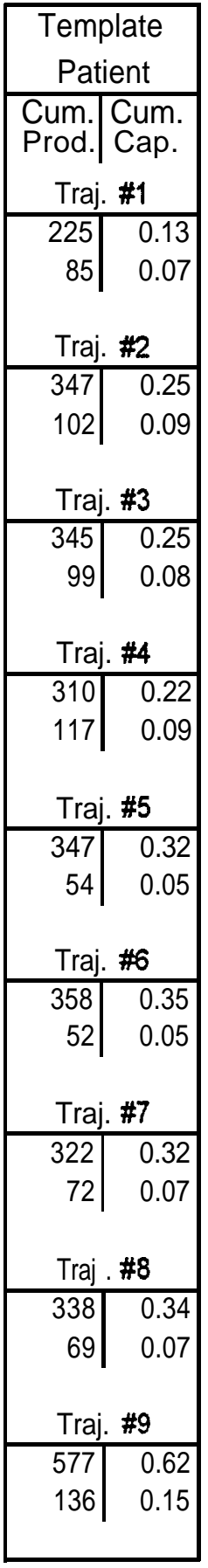


Spawning reach: Grande Ronde R. Joseph Cr - Buford Cr (RM 3.7- 26.2 from Snake R)

Relative Productivity
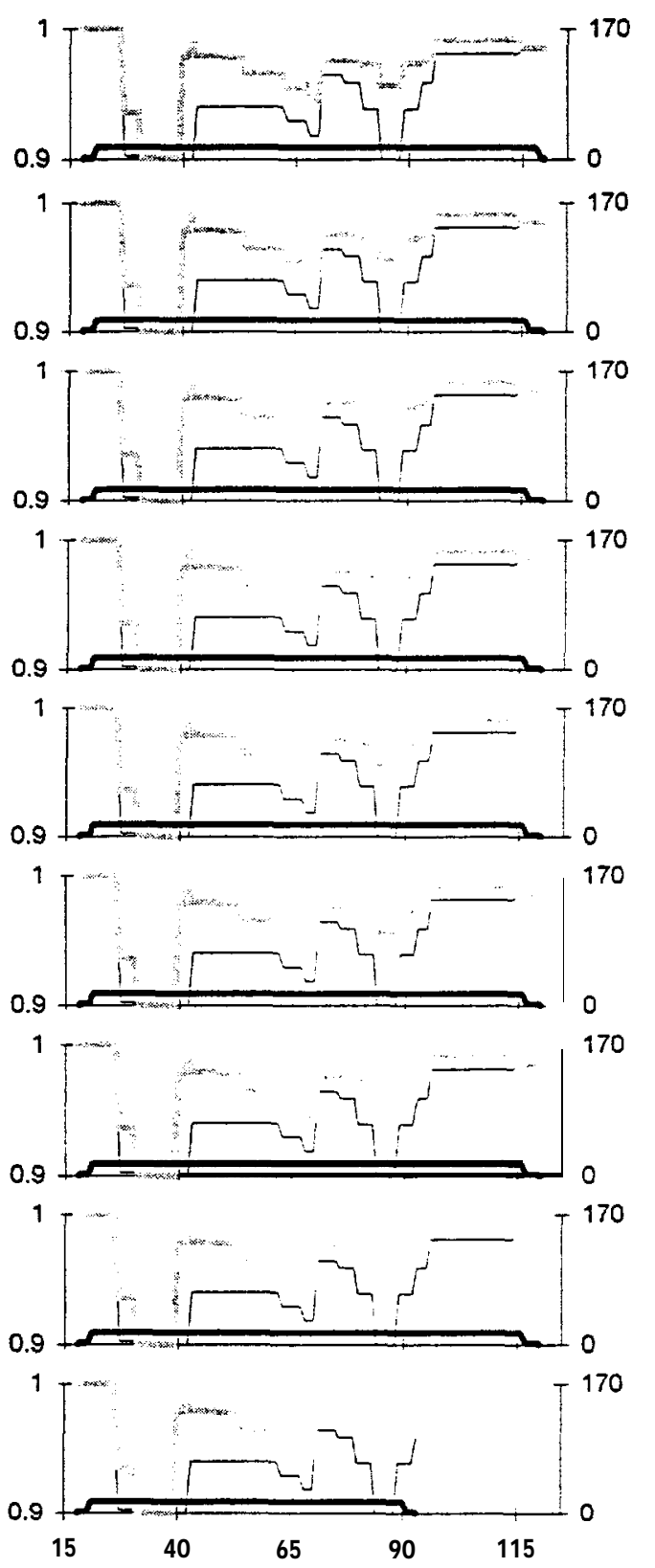

Statistical week

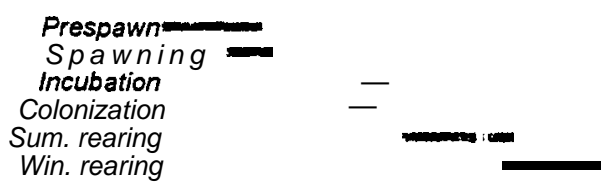

Relative Key Habitat Quantity
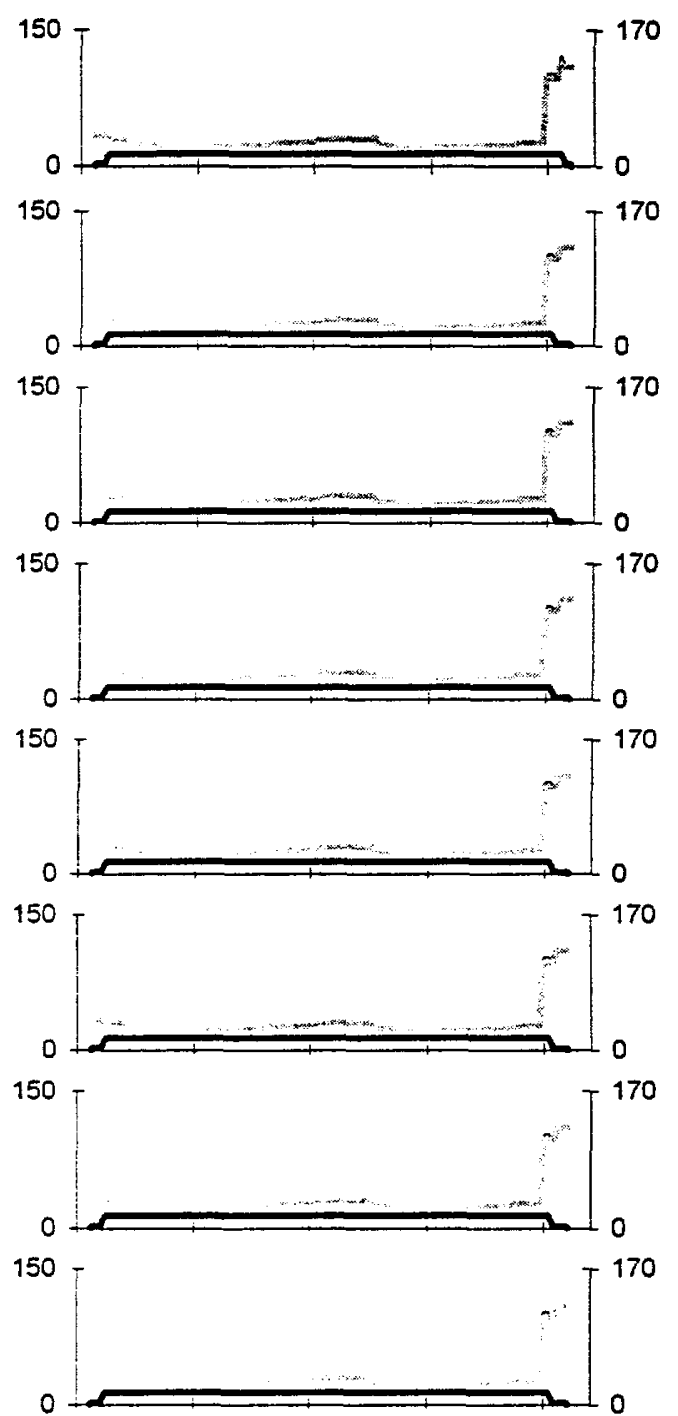

150

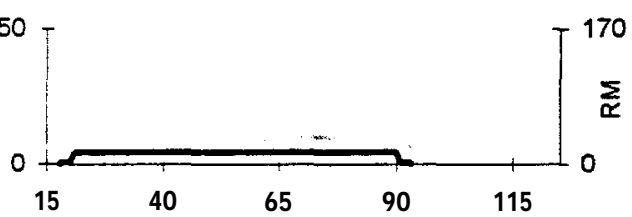

Statistical week

\begin{tabular}{|c|c|}
\hline \multicolumn{2}{|c|}{ Template } \\
Patient \\
\hline Cum. & Cum. \\
Prod. & Cap. \\
\hline
\end{tabular}

Traj.\#1

10.00

o) 0.00

Traj.\#2

\begin{tabular}{l|l|}
\hline 1 & 0.00 \\
0 & 0.00
\end{tabular}

Traj. \#3

\begin{tabular}{l|l}
1 & 0.00 \\
\hline & 0.00
\end{tabular}

0) 0.00

Traj.\#4

\begin{tabular}{l|l}
\hline & 0.00 \\
\hline & 0.00
\end{tabular}

0) 0.00

Traj. \#5

10.00

0 0.00

Traj.\#6

\begin{tabular}{l|l}
1 & 0.00 \\
\hline & 0.00
\end{tabular}

0.00

Traj.\#7

\begin{tabular}{l|l}
1 & 0.00 \\
0 & 0.00
\end{tabular}

0.00

Traj. \#8

\begin{tabular}{l|l}
1 & 0.00 \\
\hline
\end{tabular}

0) 0.00

Traj. \#9

\begin{tabular}{l|l}
2 & 0.01
\end{tabular}

0.00 
Spawning reach: Grande Ronde R.

Buford $\mathrm{Cr}$ - Bear Cr (RM 26.2 - 28.2 from Snake R)

Relative Productivity
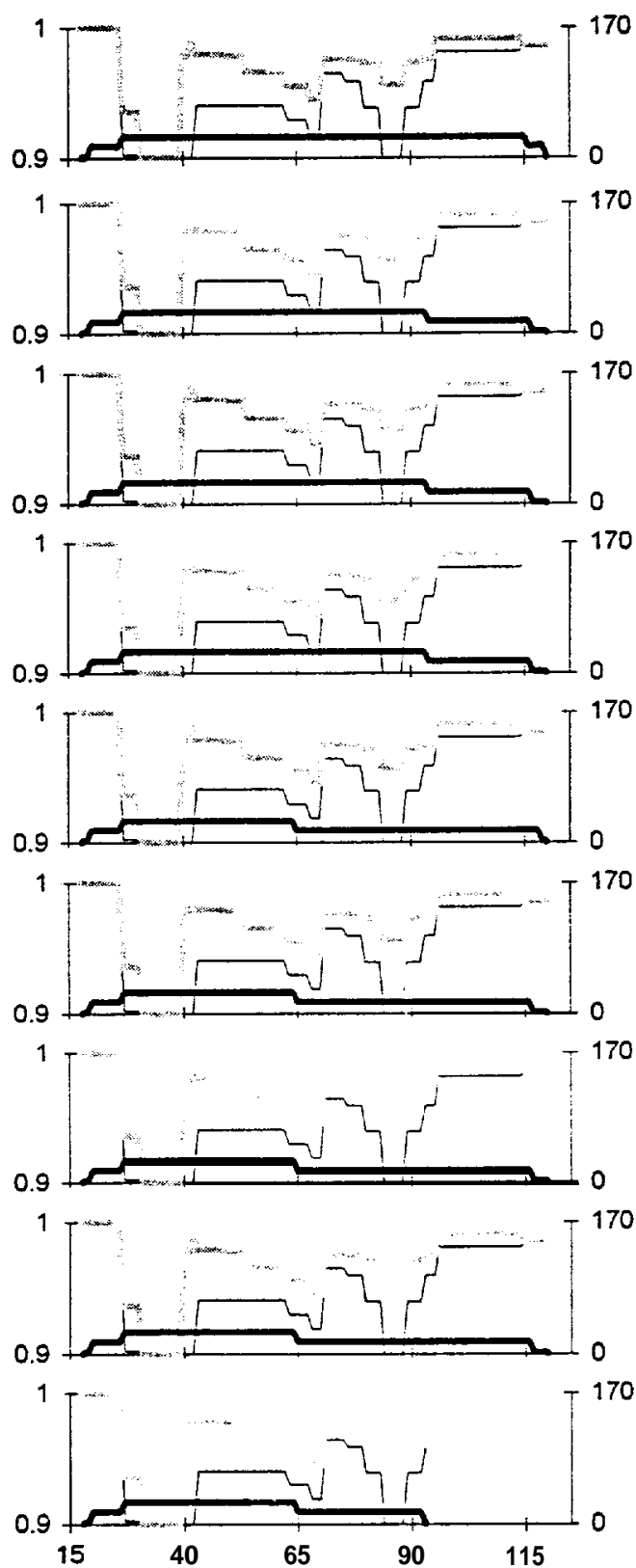

Statistical week

\section{Prespawn -}

Spawning
incubation

Sum. rearing

Win. rearing
Relative Key Habitat Quantity
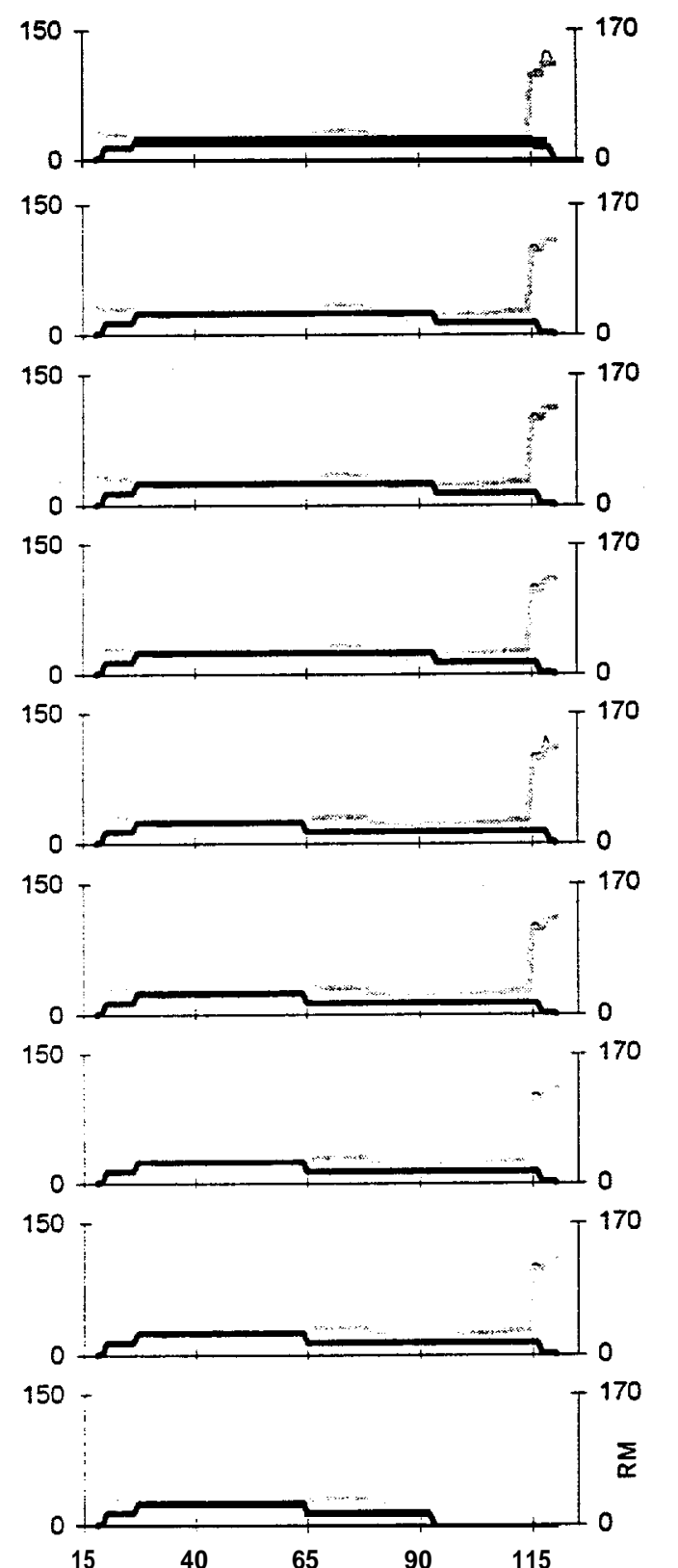

Statistical week \begin{tabular}{|c|c|}
\hline $\begin{array}{c}\text { Template } \\
\text { Patient }\end{array}$ \\
\hline $\begin{array}{c}\text { Cum. } \\
\text { Prod. }\end{array}$ & Cam. \\
\hline
\end{tabular}

Traj. \#1

\begin{tabular}{l|l}
1 & 0.01 \\
0 & 0.00
\end{tabular}

Traj. \#2

\begin{tabular}{l|l}
1 & 0.01 \\
0 & 0.00
\end{tabular}

Traj. \#3

\begin{tabular}{l|l}
1 & 0.01 \\
0 & 0.00
\end{tabular}

Traj. \#4

\begin{tabular}{l|l}
1 & 0.01 \\
0 & 0.00
\end{tabular}

Traj. \#5

\begin{tabular}{l|l|}
\hline 1 & 0.01 \\
0 & 0.00
\end{tabular}

Traj. \#6

\begin{tabular}{l|l}
\hline 1 & 0.01 \\
0 & 0.00
\end{tabular}

Traj. \#7

\begin{tabular}{|l|l|}
\hline 1 & 0.01 \\
\hline & 0.00 \\
\hline
\end{tabular}

o) 0.00

Traj. \#8

\begin{tabular}{l|l|}
\hline 1 & 0.01 \\
0 & 0.00
\end{tabular}

Traj. \#9

\begin{tabular}{l|l|}
2 & 0.01 \\
\hline
\end{tabular}

o) 0.00

Trajectory

Template

Patient 
Spawning reach: Grande Ronde R.

Bear $\mathrm{Cr}$ - Menatchee $\mathrm{Cr}$ (RM 28.2 - 34.8 from Snake R)

Relative Productivity
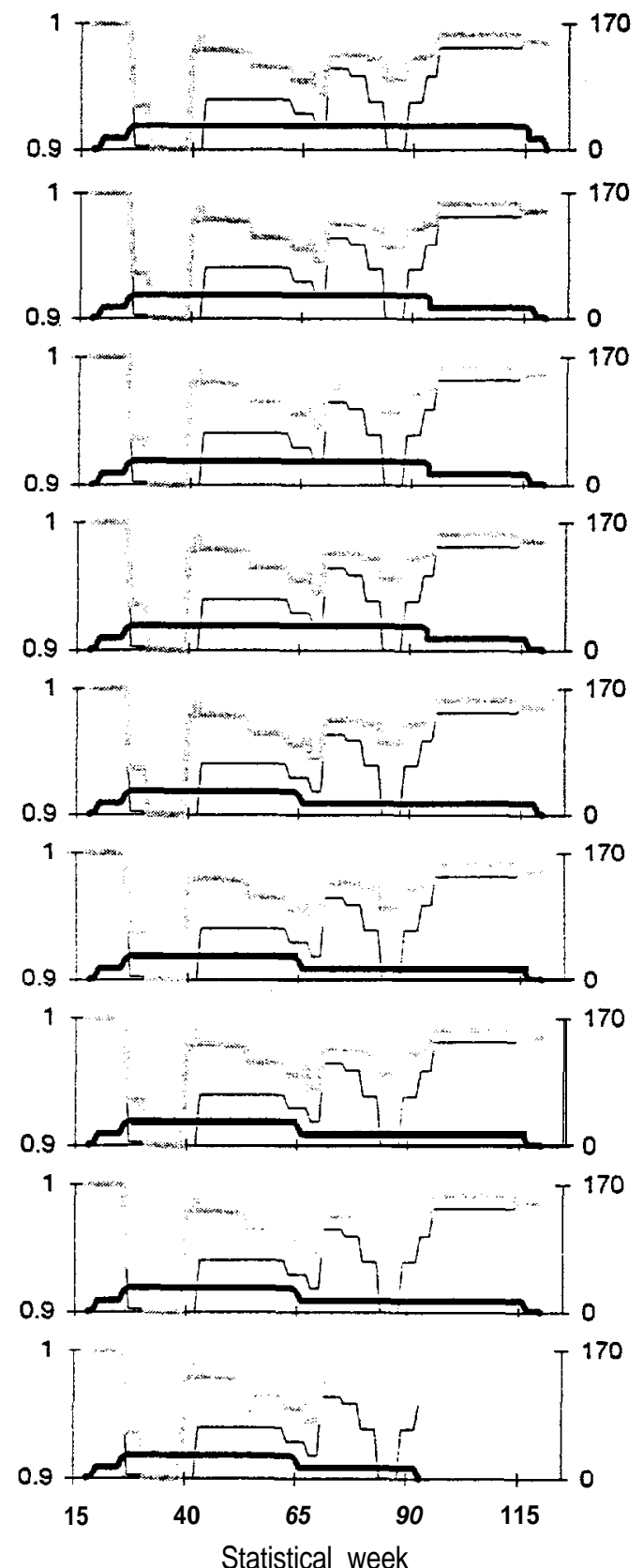

Statistical week

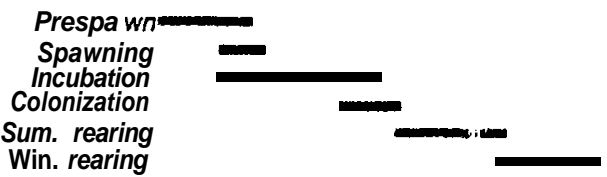

Relative Key Habitat Quantity
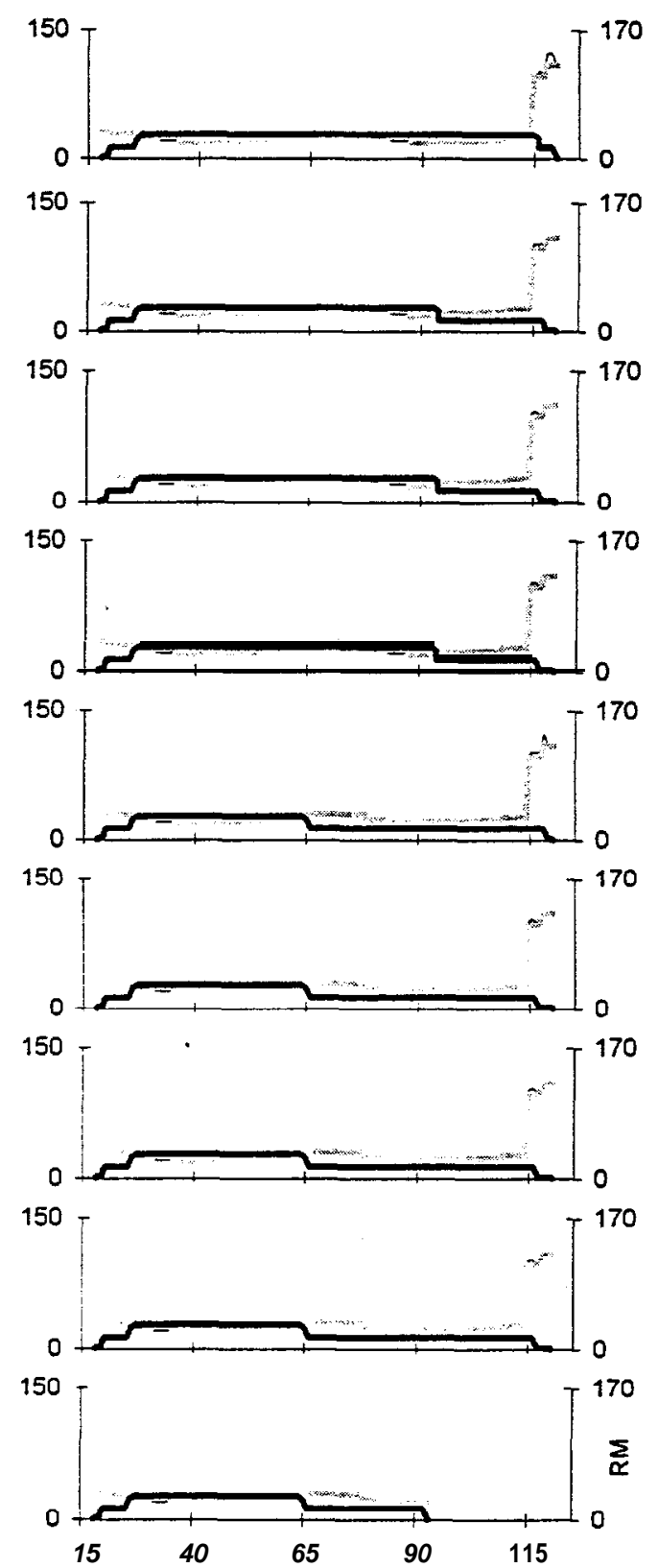

Statistical week \begin{tabular}{|c|c|}
\hline \multicolumn{2}{|c|}{ Template } \\
Patient \\
\hline Cum. & Cum. \\
Prod. & Cap.
\end{tabular}

Traj. \#1

\begin{tabular}{l|l|}
\hline 1 & 0.01 \\
0 & 0.00 \\
\hline
\end{tabular}

Traj. \#2

\begin{tabular}{l|l|}
1 & 0.01 \\
0 & 0.00
\end{tabular}

Traj. \#3

\begin{tabular}{l|l|}
\hline & 0.01 \\
0 & 0.00
\end{tabular}

Traj. \#4

\begin{tabular}{l|l|}
1 & 0.01 \\
0 & $0.0 \mathrm{C}$
\end{tabular}

Traj. \#5

\begin{tabular}{l|l}
\hline 1 & 0.01 \\
0 & 0.00
\end{tabular}

Traj. \#6

\begin{tabular}{l|l}
1 & 0.01 \\
0 & 0.00
\end{tabular}

Traj. \#7

10.01

0.00

Traj. \#8

$1 \longdiv { 0 . 0 1 }$

0) 0.00

Traj. \#9

\begin{tabular}{l|l|}
\hline 2 & 0.01 \\
0 & 0.00
\end{tabular} 
Spawning reach: Grande Ronde R.

Menatchee Cr - Wenaha R (RM 34.8 - 45.1 from Snake R)

Relative Productivity
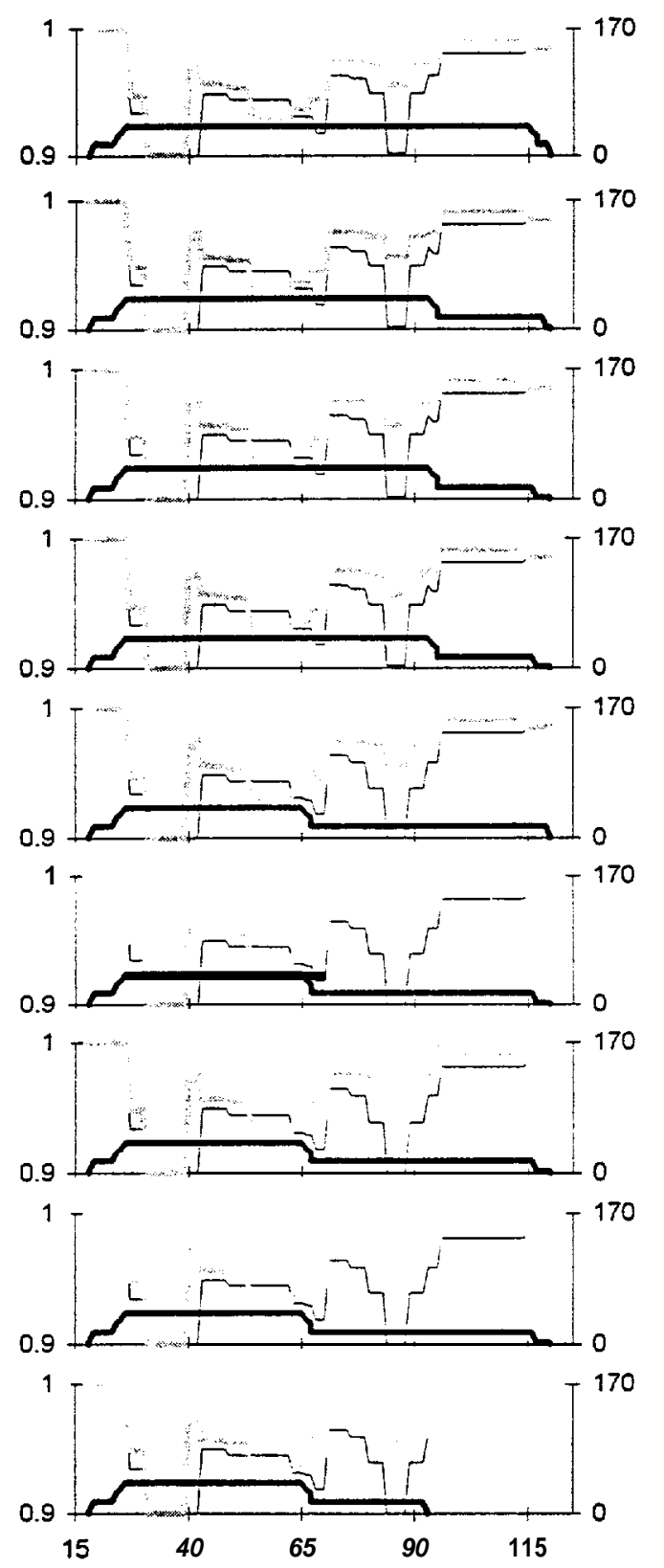

Statistical week

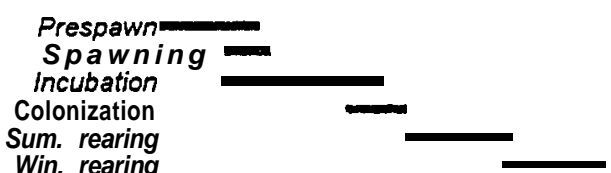

Relative Key Habitat Quantity

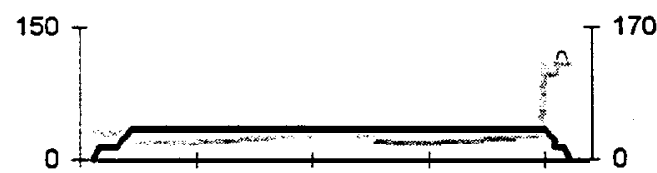

150
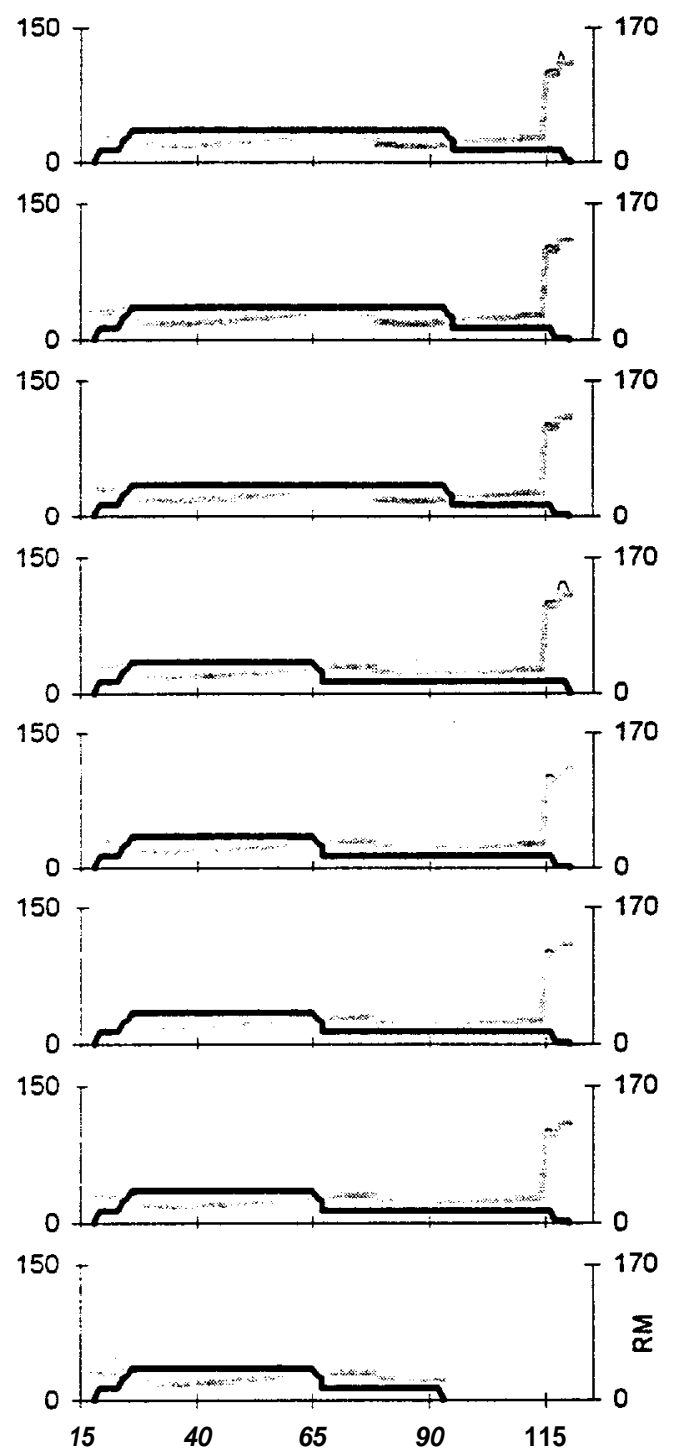

Statistical week

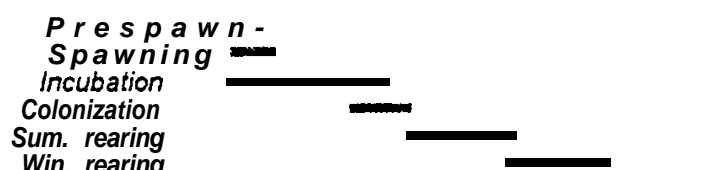

Trajectory -.- Template

Patient

Traj. \#5

\begin{tabular}{|l|l|}
\hline 5 & 0.02 \\
0 & 0.00
\end{tabular}

Traj. \#6

$5 \mid 0.02$

0.00

Traj. \#7

0.02

00.00

Traj. \#8

\begin{tabular}{l|l}
5 & 0.02 \\
0 & 0.00
\end{tabular}

0) 0.00

Traj. \#9

\begin{tabular}{l|l}
9 & 0.04 \\
0 & 0.00
\end{tabular}

0) 0.00 
Spawning reach: Grande Ronde R.

Wenaha R - Courtney Cr (RM 45.1 - 46.2 from Snake R)

Relative Productivity
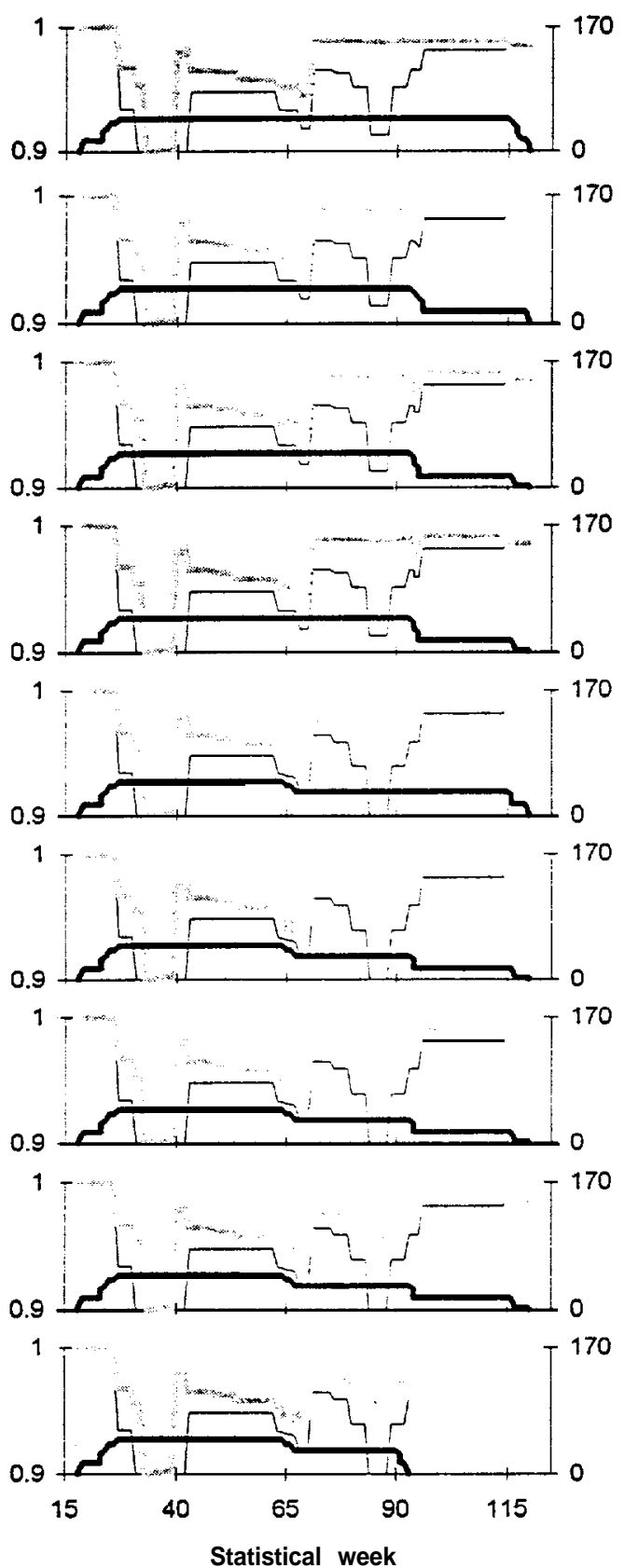

Prespawn -

Spawning

Incubation

Colonization

Sum. rearing

Win. rearing
Relative Key Habitat Quantity
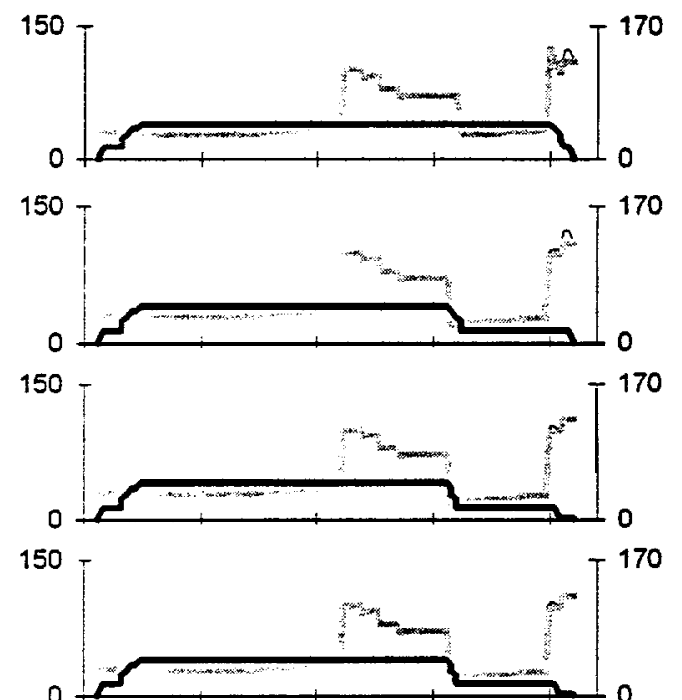

150

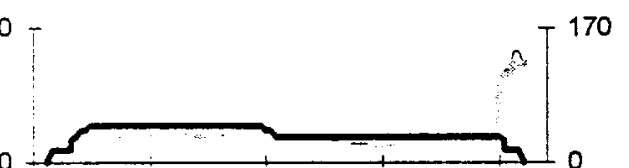

150
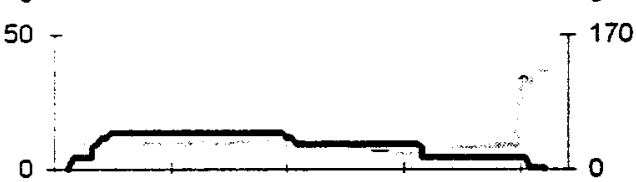

150
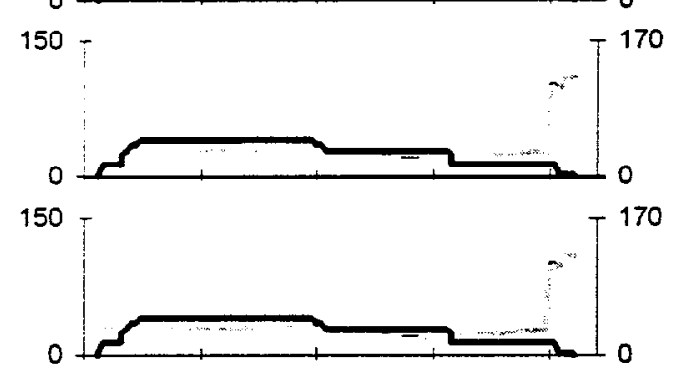

150

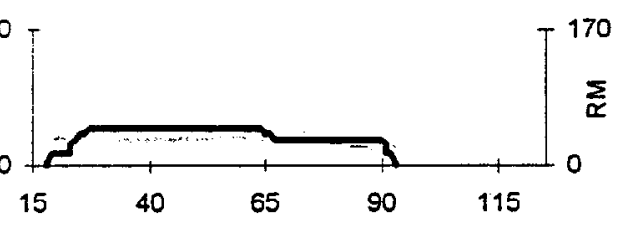

Statistical week

\section{Template \\ Patient \\ Cum. Cum. \\ Prod. Cap.}

Traj. \#1

$40 \mid 0.22$

0.00

Traj. \#2

\begin{tabular}{|r|r|}
\hline 40 & 0.22 \\
0 & 0.00
\end{tabular}

Traj. \#3

\begin{tabular}{r|r}
40 & 0.22 \\
0 & 0.00
\end{tabular}

Traj. \#4

400.22

0.00

Traj. \#5

25 0.14

0.00

Traj. \#6

250.14

0.00

Traj. \#7

\begin{tabular}{r|r|}
\hline 25 & 0.14 \\
0 & 0.00
\end{tabular}

Traj. \#8

\begin{tabular}{|r|r|}
\hline 25 & 0.14 \\
0 & 0.00
\end{tabular}

Traj. \#9

\begin{tabular}{r|r|}
48 & 0.26 \\
0 & 0.00
\end{tabular} 
Spawning reach: Grande Ronde R.

Courtney Cr - Mud Cr (RM 46.2 - 51.4 from Snake R)

Relative Productivity
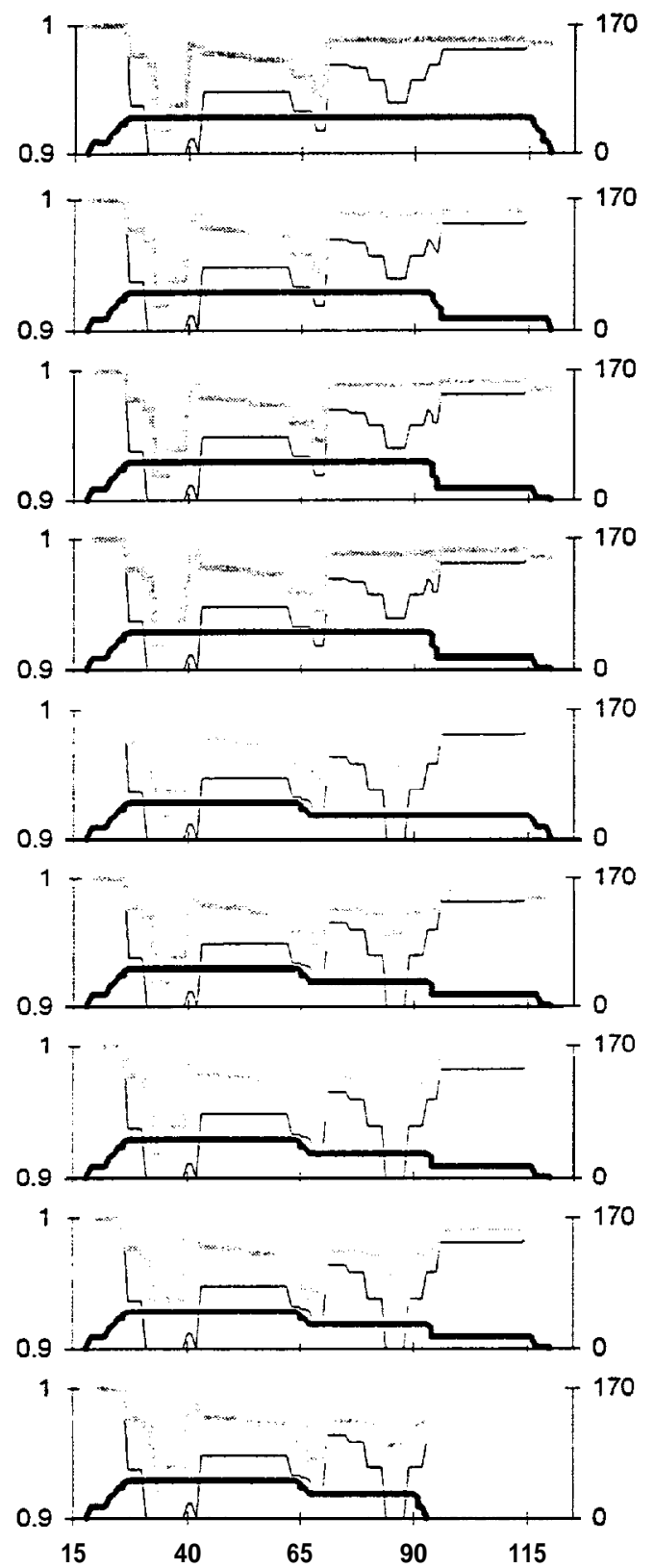

Statistical week

Prespawn

Spawning

Incubation

Colonization

Sum. rearing

Win. rearing
Relative Key Habitat Quantity

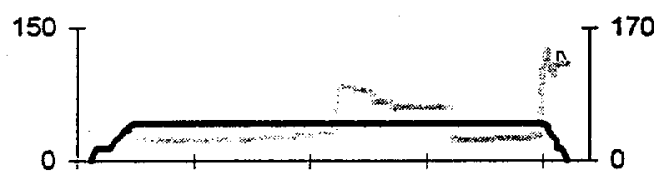

$150-$
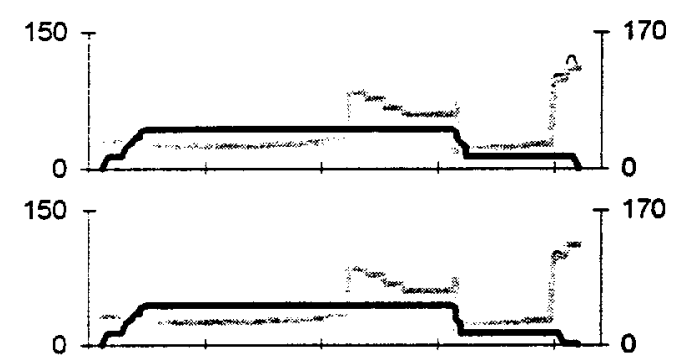

150
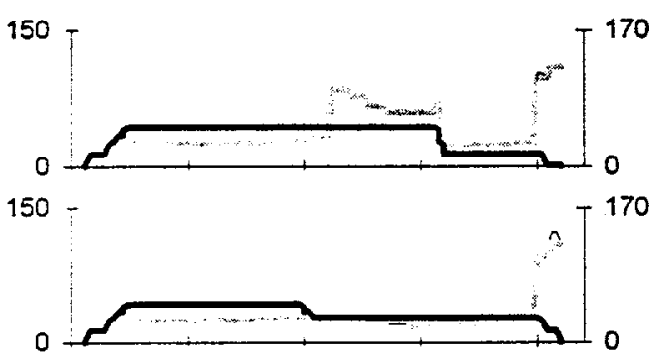

150 -
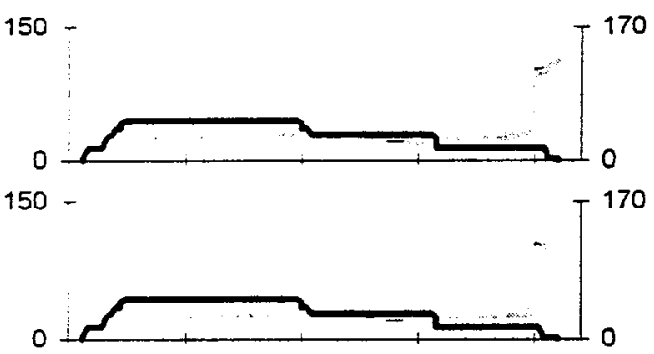

$150-$
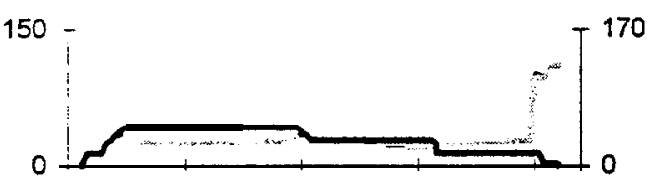

150

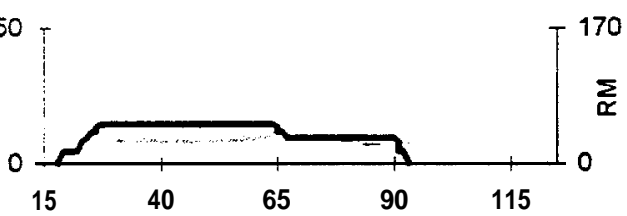

Statistical week \begin{tabular}{|c|c|}
\hline \multicolumn{2}{|c|}{ Template } \\
Patient \\
\hline Cum. & Cum. \\
Prod. & Cap. \\
\hline
\end{tabular}

Traj. \#1

\begin{tabular}{r|r|}
94 & 0.46 \\
0 & 0.00
\end{tabular}

Traj. \#2

\begin{tabular}{l|l|}
96 & 0.46 \\
\hline
\end{tabular}

이 0.00

Traj. \#3

\begin{tabular}{l|l|}
96 & 0.46
\end{tabular}

o) 0.00

Traj. \#4

\begin{tabular}{l|l}
96 & 0.46 \\
\hline
\end{tabular}

0) 0.00

Traj. \#5

590.28

o) 0.00

Traj. \#6

590.28

이 0.00

Traj. \#7

590.28

o) 0.00

Traj. \#8

\begin{tabular}{|l|l|}
\hline 59 & 0.28 \\
\hline
\end{tabular}

0 0.00

\begin{tabular}{|r|r|}
\hline \multicolumn{2}{|c|}{ Traj. \#9 } \\
\hline 112 & 0.55 \\
0 & 0.00
\end{tabular}

Trajectory

Template

Patient 
Spawning reach: Grande Ronde R.

Mud $\mathrm{Cr}$ - WallupaNildcat $\mathrm{Cr}$ (RM 51.4 - 52.8 from Snake R)

Relative Productivity
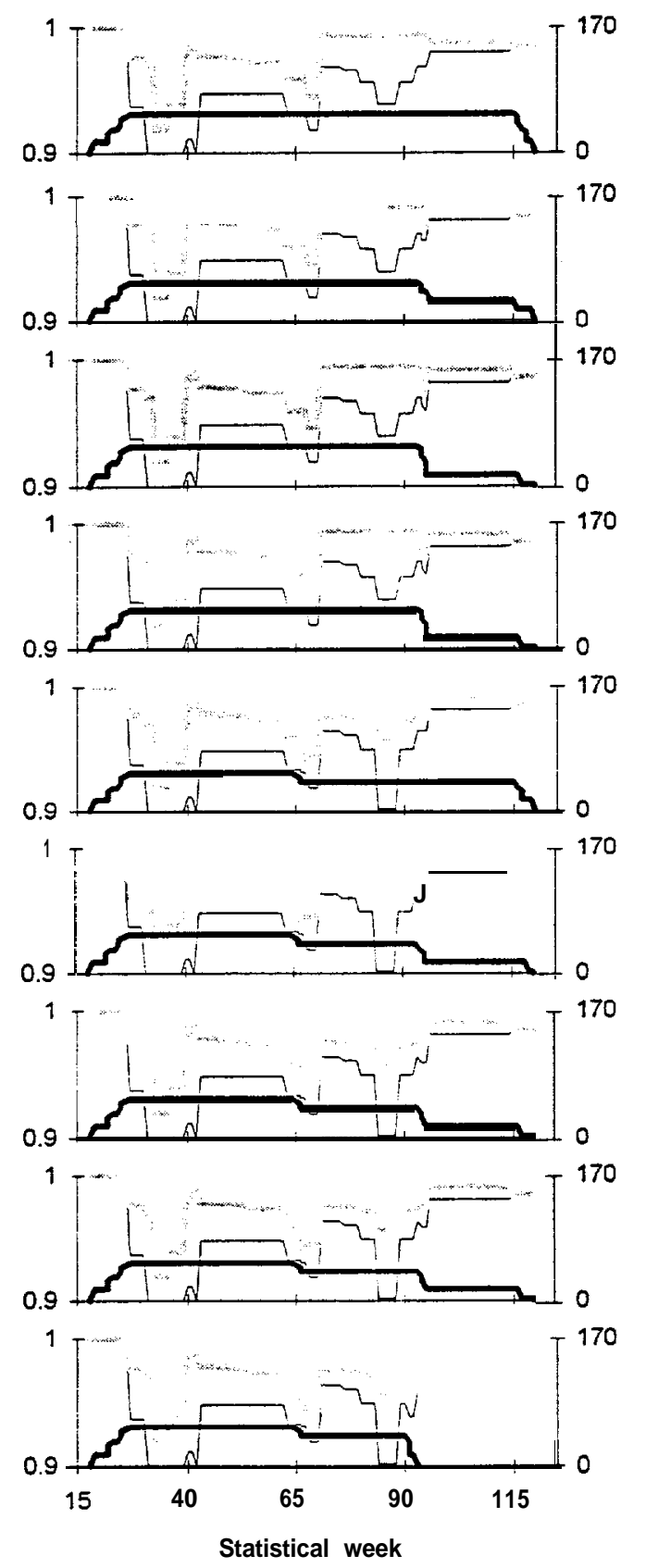

Prespawn -

Spawning

Incubation

Colonization

Sum. rearing

Win. rearing
Relative Key Habitat Quantity

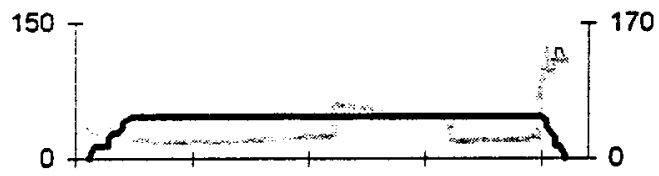

150

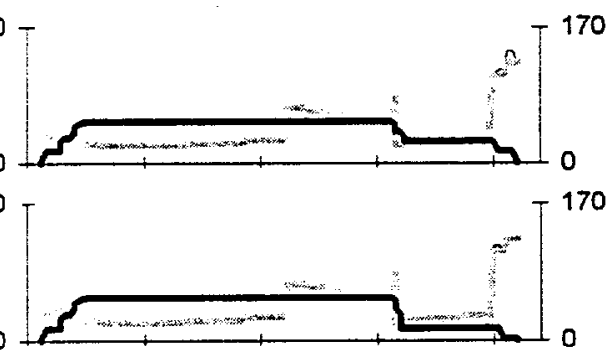

150
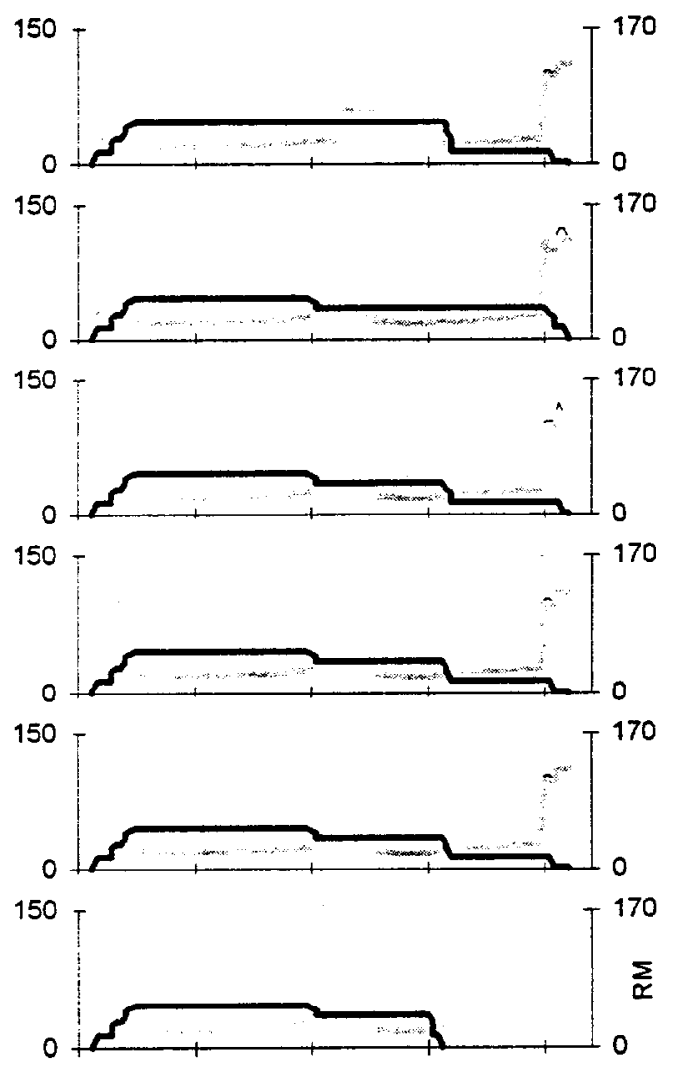

Statistical week

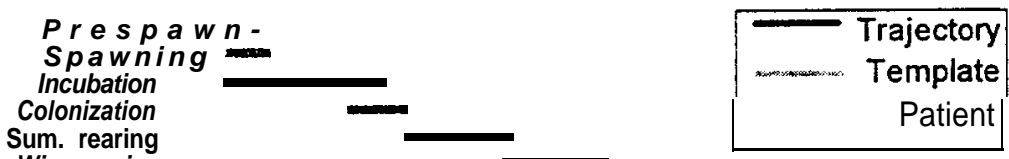


Spawning reach: Grande Ronde R.

WallupaMildcat Cr - Grossman Cr (RM 52.8 - 61 .1 from Snake R)

Relative Productivity
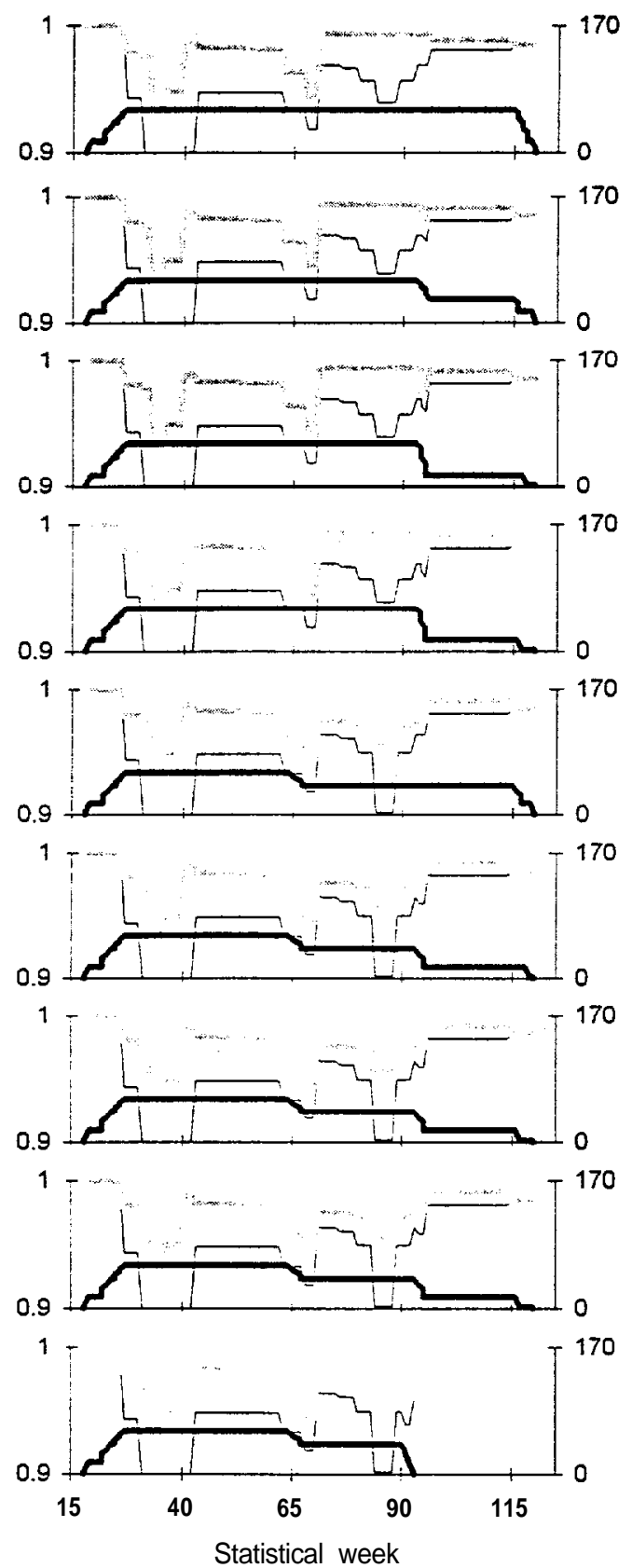

Prespawn -

\section{Spawning}

Incubation

Colonization

Sum. rearing

Win. rearing

Relative Key Habitat Quantity
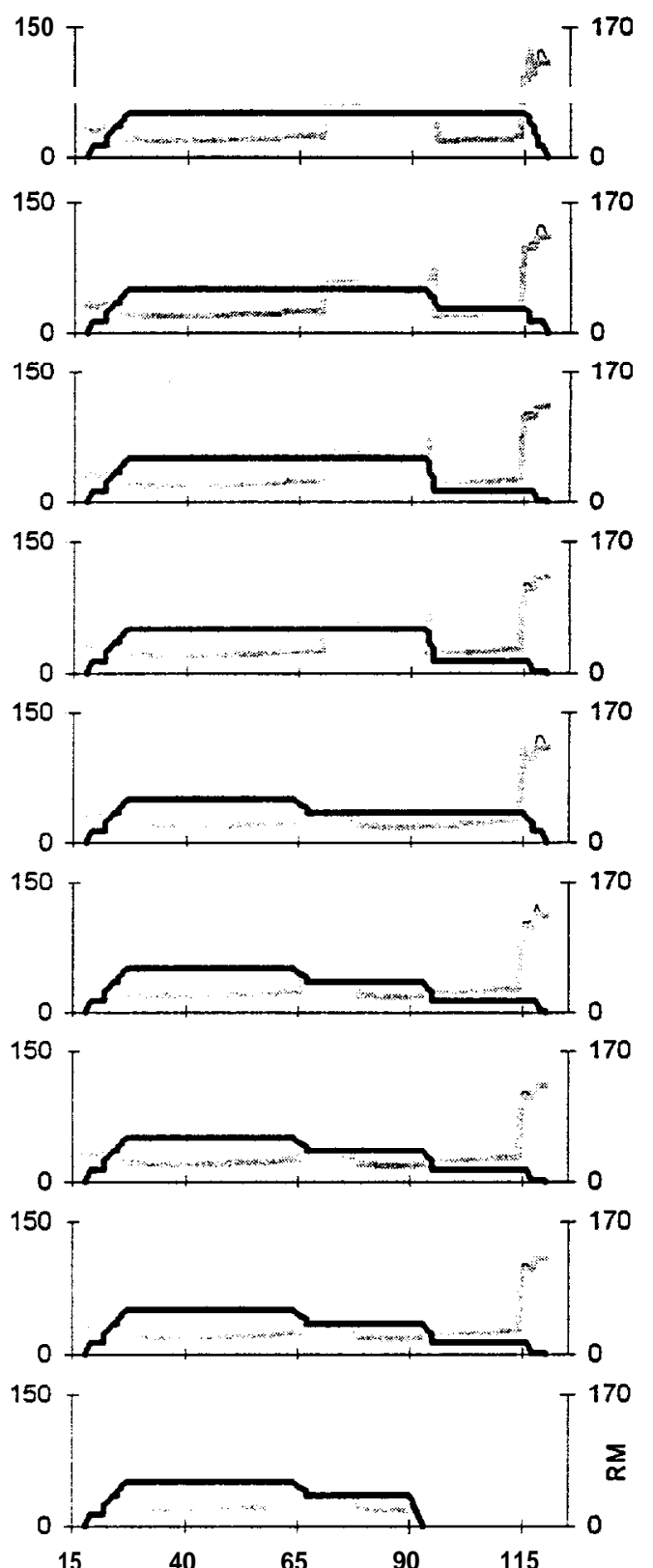

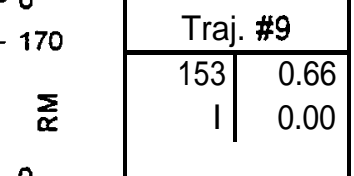

Statistical week

Prespawn -

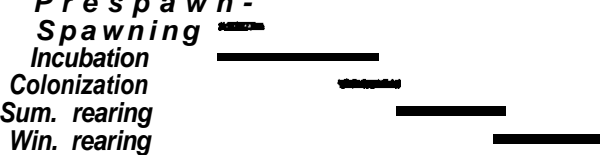

\begin{tabular}{|c|c|}
\hline \multicolumn{2}{|c|}{$\begin{array}{c}\text { Template } \\
\text { Patient }\end{array}$} \\
\hline Cum. & Cum. \\
Prod. & Cap.
\end{tabular}

Traj. \#1

\begin{tabular}{r|r|}
142 & 0.57 \\
1 & 0.00
\end{tabular}

Traj. \#2

1450.58

110.00

Traj. \#3

\begin{tabular}{r|r}
144 & 0.58 \\
1 & 0.00
\end{tabular}

Traj. \#4

$144 \quad 0.58$

10.00

Traj. \#5

$80 \mid 0.33$

0.00

Traj. \#6

800.33

0.00

Traj. \#7

\begin{tabular}{l|l|}
\hline 80 & 0.33 \\
\hline
\end{tabular}

0.00

Traj. \#8

80.0 .33

0) 0.00 
Spawning reach: Grande Ronde $\mathbf{R}$.

Grossman $\mathrm{Cr}$ - Wallowa R (RM 61 .1- 80.0 from Snake R)

Relative Productivity
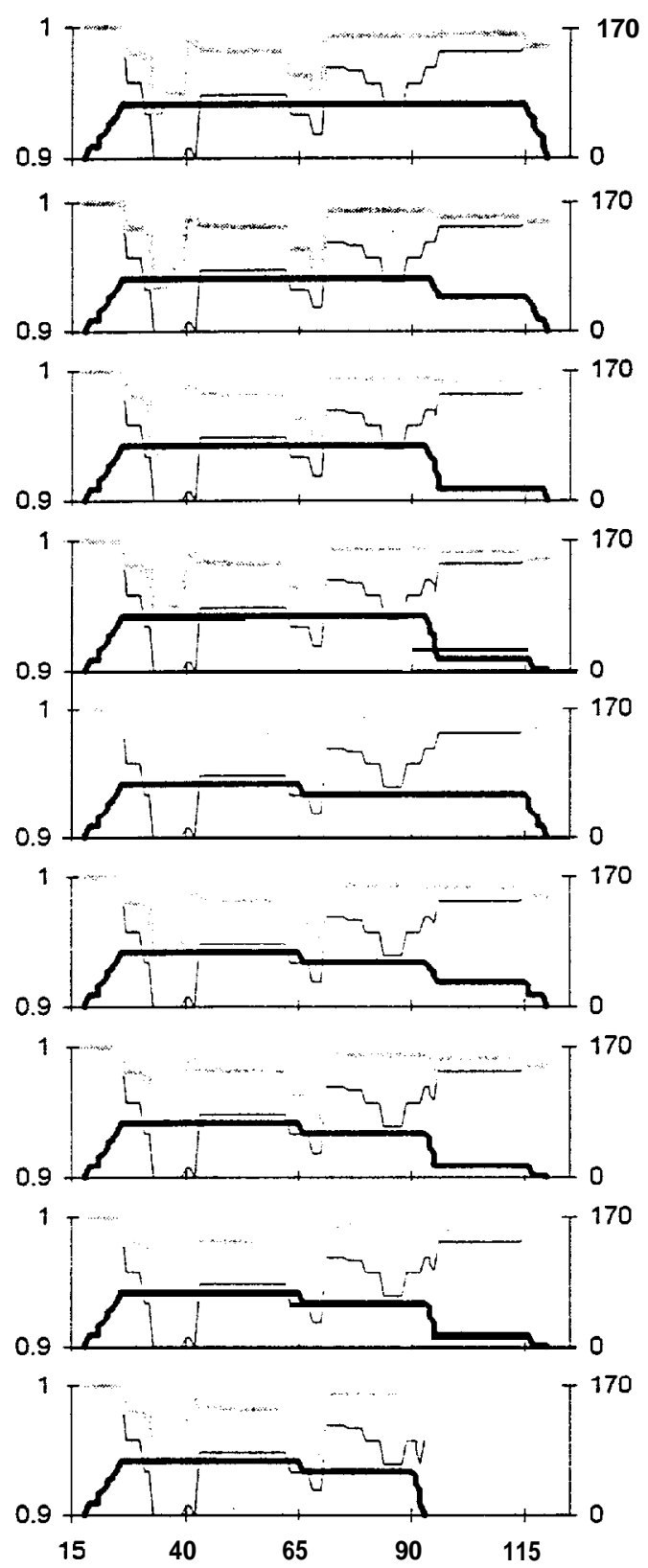

Statistical week

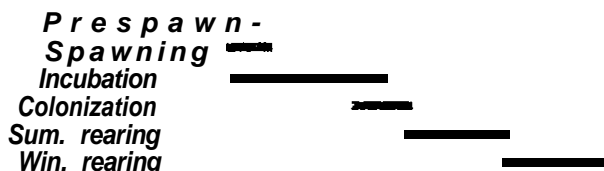

Relative Key Habitat Quantity

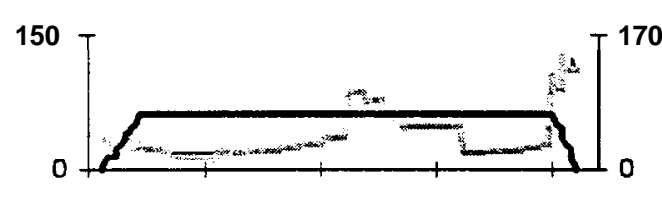

150

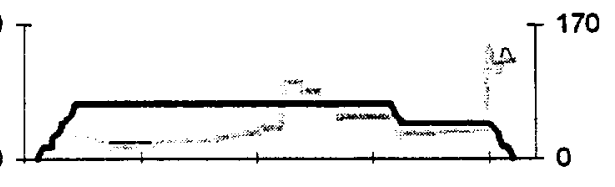

150

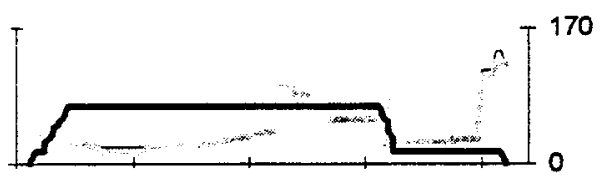

150
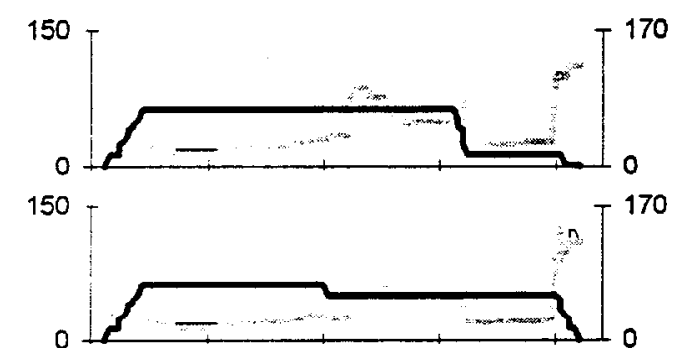

150

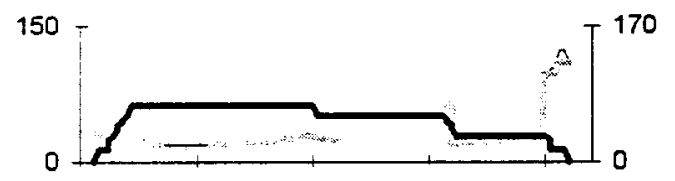

150

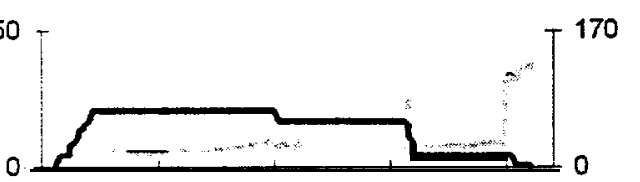

150

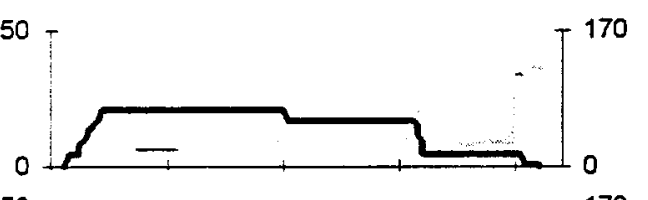

150

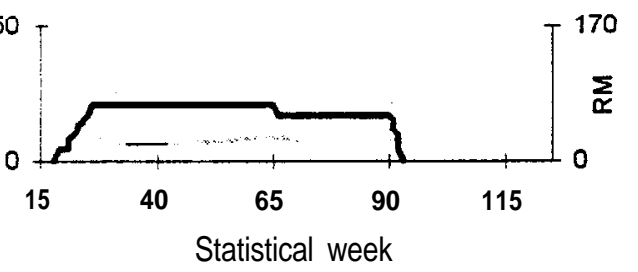

so

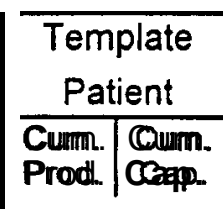

Traj. \#1

$157 \quad 0.65$

6 6) 0.03

Traj. \#2

\begin{tabular}{r|r|}
142 & 0.60 \\
6 & 0.03
\end{tabular}

Traj. \#3

$146 \quad 0.61$

6) 0.03

Traj. \#4

$145 \mid 0.61$

6) 0.03

Traj. \#5

\begin{tabular}{l|l}
142 & 0.57 \\
\hline
\end{tabular}

\begin{tabular}{l|l}
6 & 0.03
\end{tabular}

Traj. \#6

\begin{tabular}{|l|l|}
145 & 0.59 \\
\hline & 0.03
\end{tabular}

6) 0.03

Traj. \#7

\begin{tabular}{r|r|}
144 & 0.59 \\
6 & 0.03
\end{tabular}

Traj. \#8

\begin{tabular}{|l|l|}
\hline 144 & 0.59 \\
\hline
\end{tabular}

6) 0.03

Traj. \#9

\begin{tabular}{|r|r|}
\hline 258 & 1.12 \\
14 & 0.07 \\
\hline
\end{tabular}

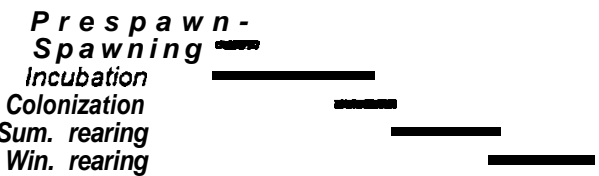


Spawning reach: Grande Ronde R.

Wallowa R - Lookingglass Cr (RM 80.0 - 83.4 from Snake R)

Relative Productivity
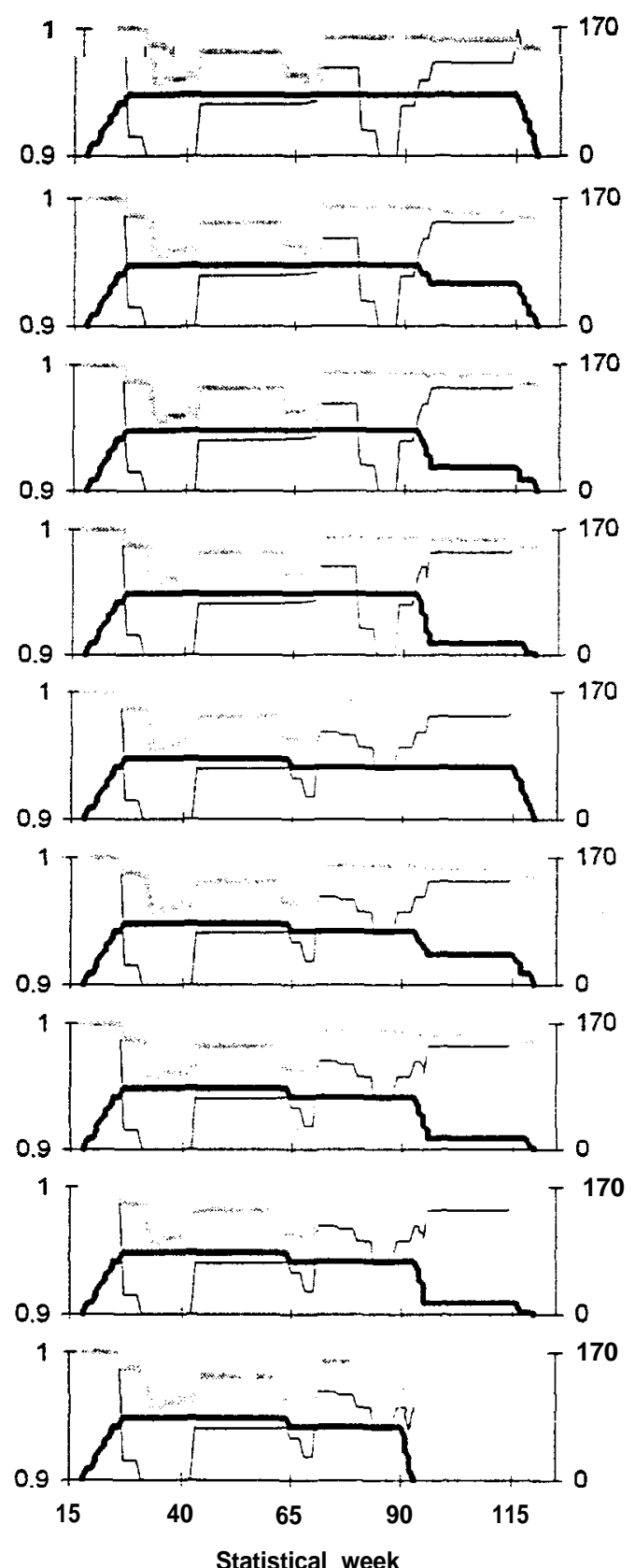

Prespawn -

Spawning

Incubation

Colonization

Sum. rearing

Win. rearing
Relative Key Habitat Quantity
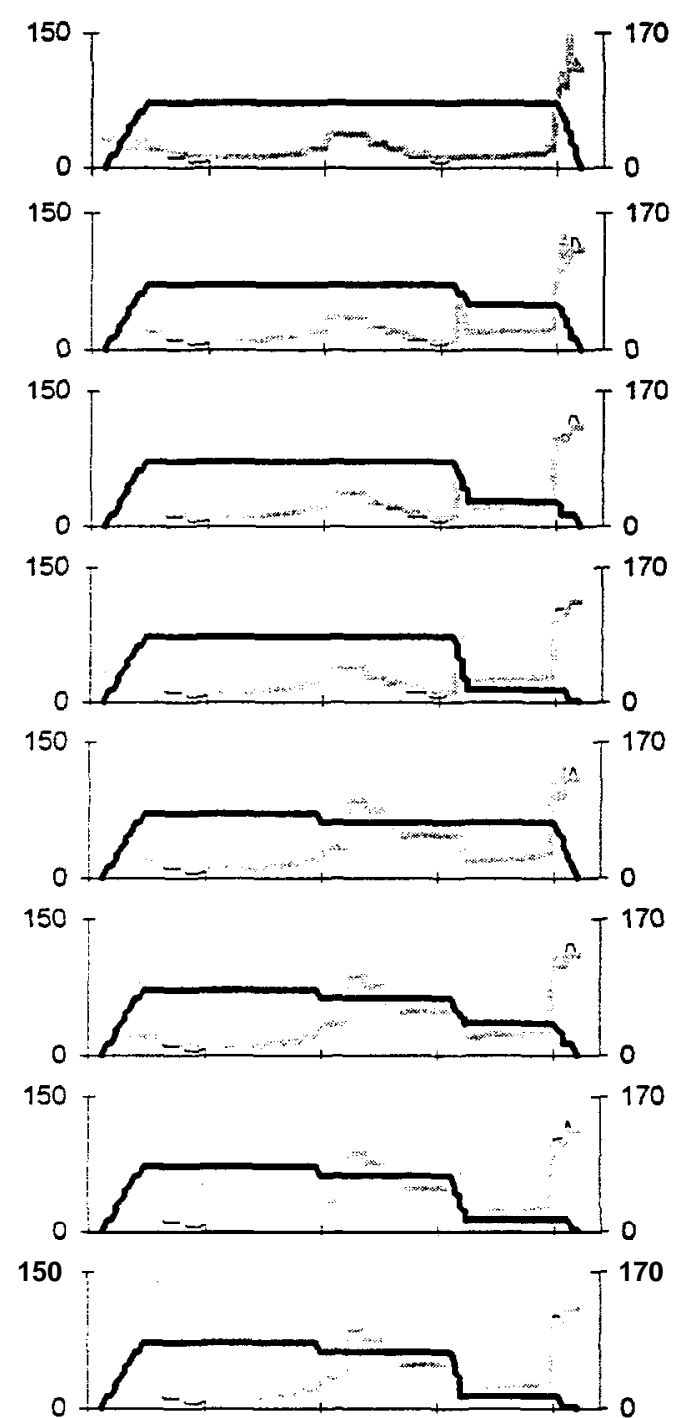

$150 T$

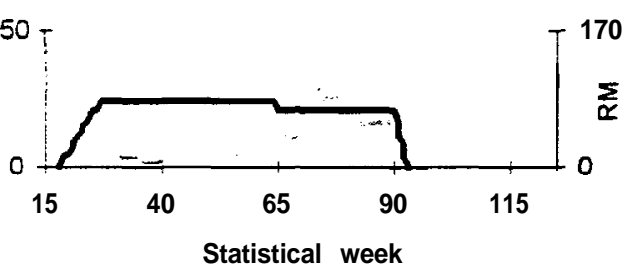

Prespawn -

Spawning Incubation Colonization Sum. rearing Win. rearing

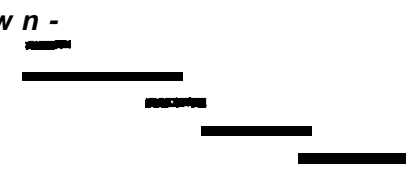


Spawning reach: Grande Ronde $\mathbf{R}$.

Lookingglass $\mathrm{Cr}$ - Gordon $\mathrm{Cr}$ (RM 83.4 - 94.2 from Snake R)

Relative Productivity
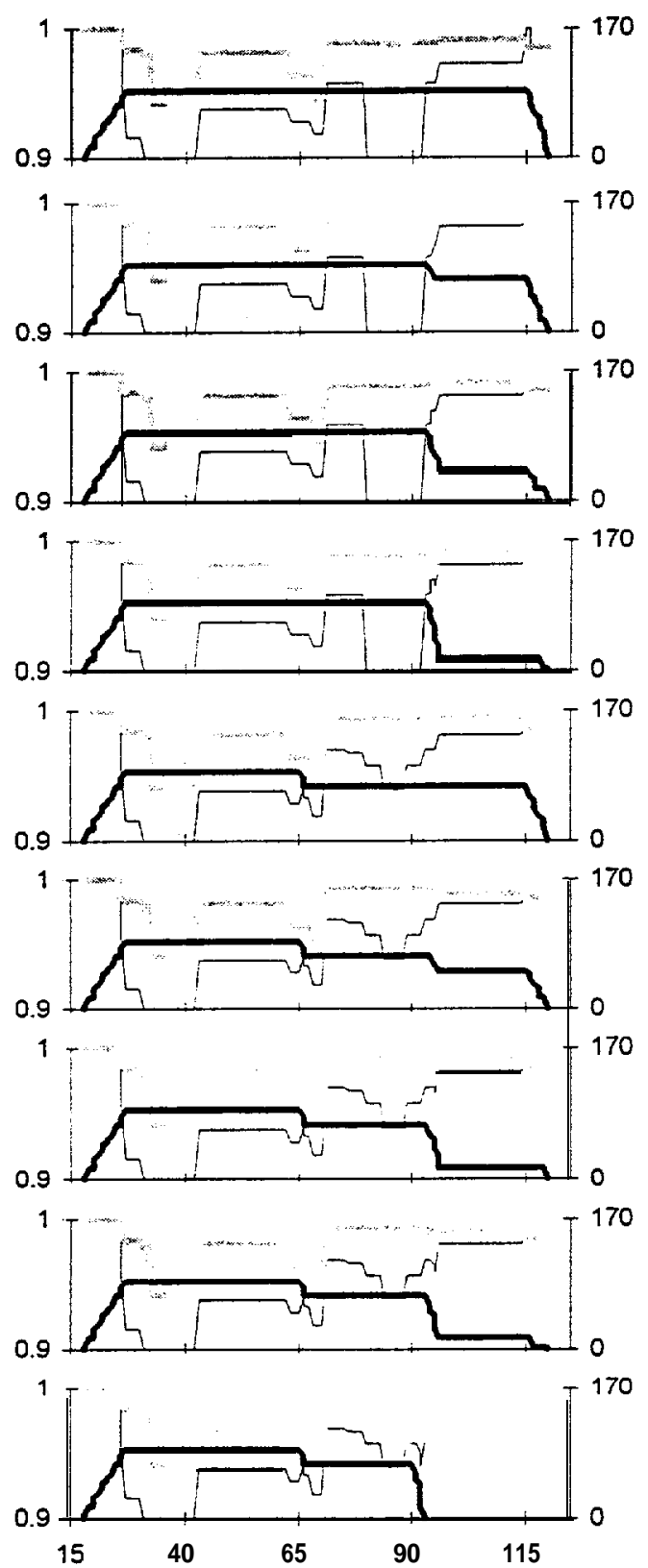

Statistical week

\section{Prespawn -}

Spawning

Incubation

Colonization

Sum. rearing

Win. rearing
Relative Key Habitat Quantity
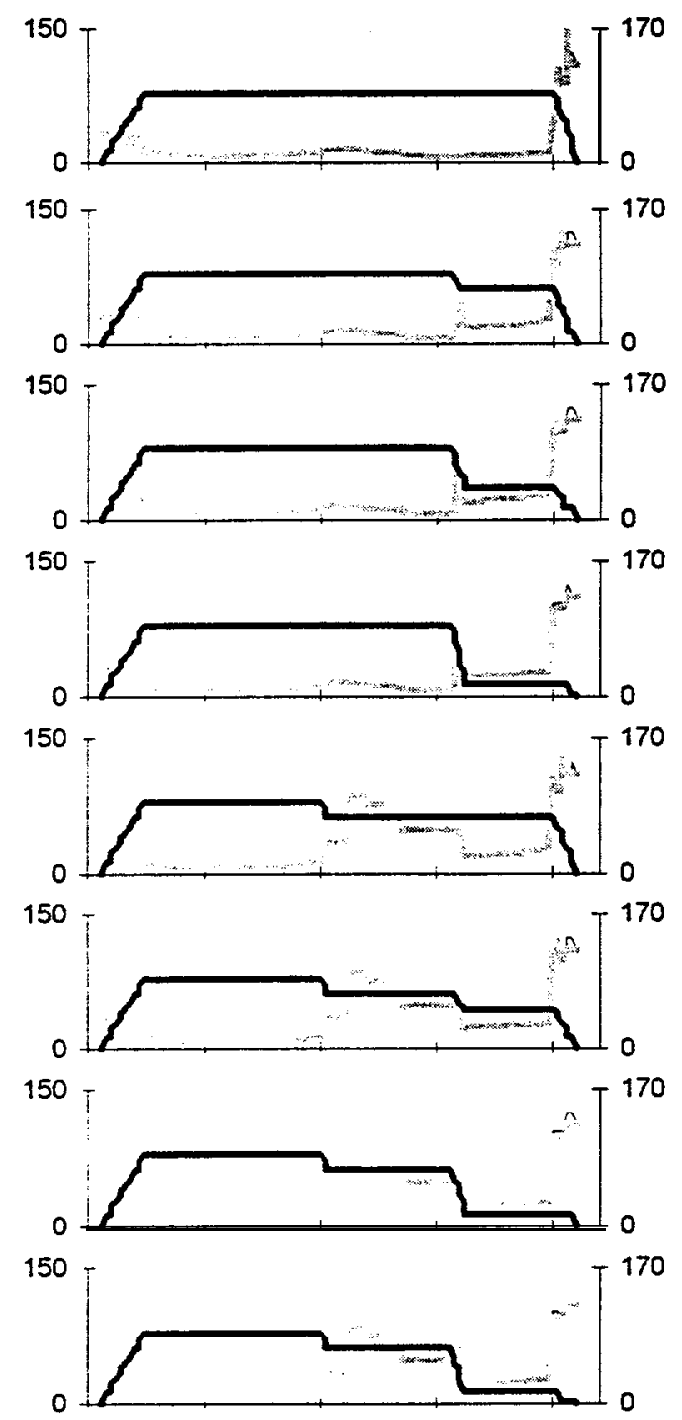

150

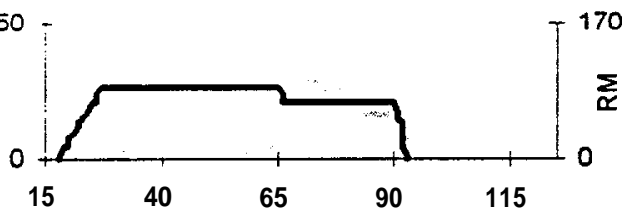

Statistical week

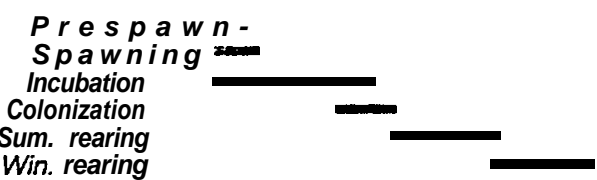

\section{Template \\ Patient}

Cum. Cum. Prod. Cap.

Traj. \#1

1240.29

0) 0.00

Traj. \#2

$132 \quad 0.32$

0) 0.00

Traj. \#3

$124 \mid 0.31$

0) 0.00

Traj. \#4

\begin{tabular}{r|r|}
123 & 0.30 \\
0 & 0.00
\end{tabular}

Traj. \#5

1470.41

o) 0.00

Traj. \#6

\begin{tabular}{|l|l|}
\hline 133 & 0.38 \\
\hline
\end{tabular}

$\begin{array}{ll}0 & 0.00\end{array}$

Traj. \#7

\begin{tabular}{|l|l|}
137 & 0.39
\end{tabular}

0) 0.00

Traj. \#8

$136 \quad 0.38$

이 0.00

Traj. \#9

\begin{tabular}{r|r|}
244 & 0.72 \\
0 & 0.00 \\
\hline
\end{tabular} 
Spawning reach: Grande Ronde R.

Gordon Cr - Phillips Cr (RM 94.2 - 96.9 from Snake R)

Relative Productivity
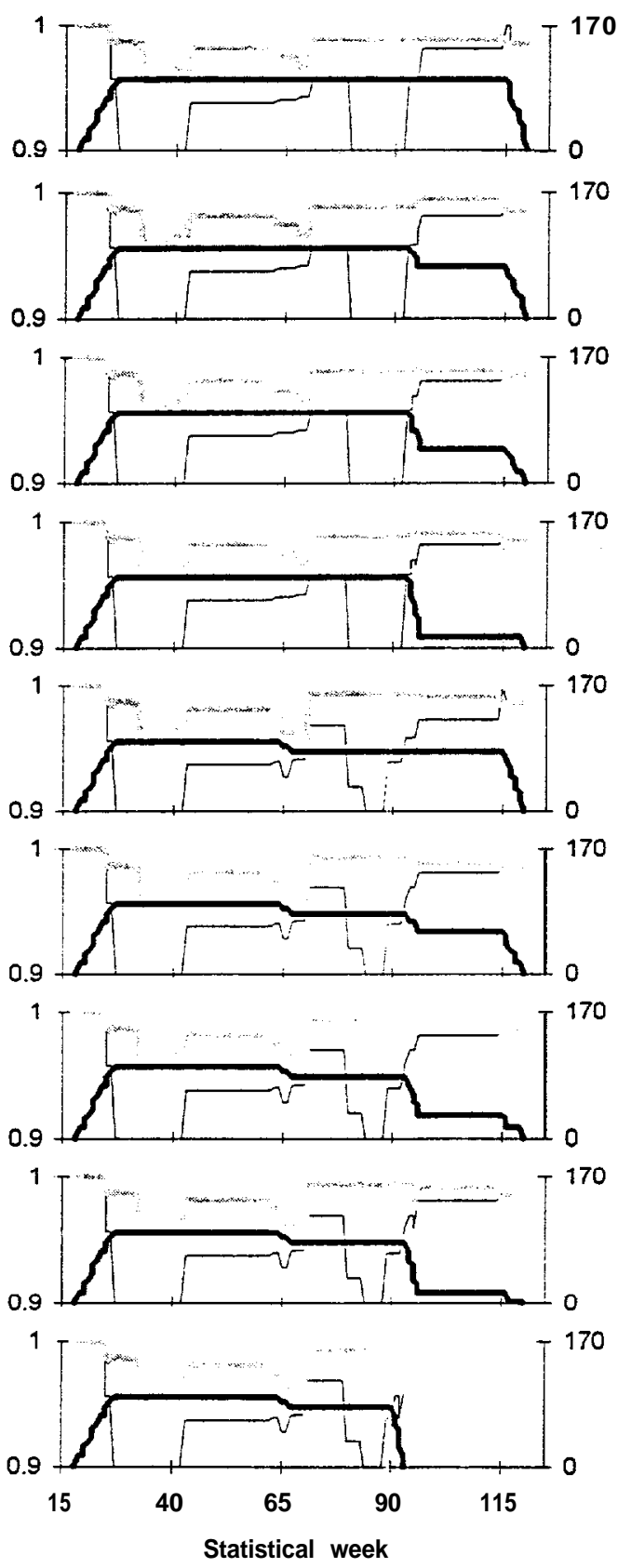

Prespawn -

Spawning

Incubation

Colonization

Sum. rearing

Win. rearing

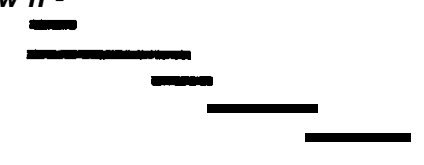

Relative Key Habitat Quantity

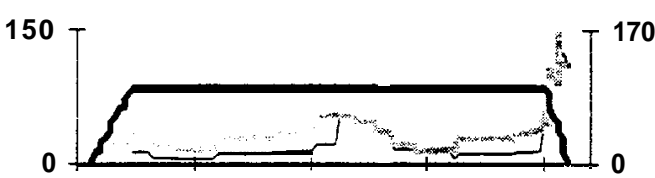

150

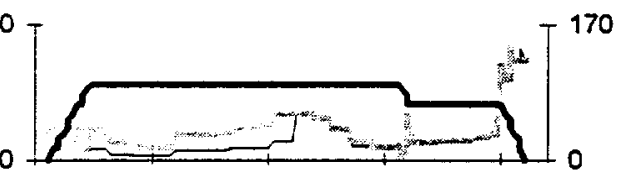

150
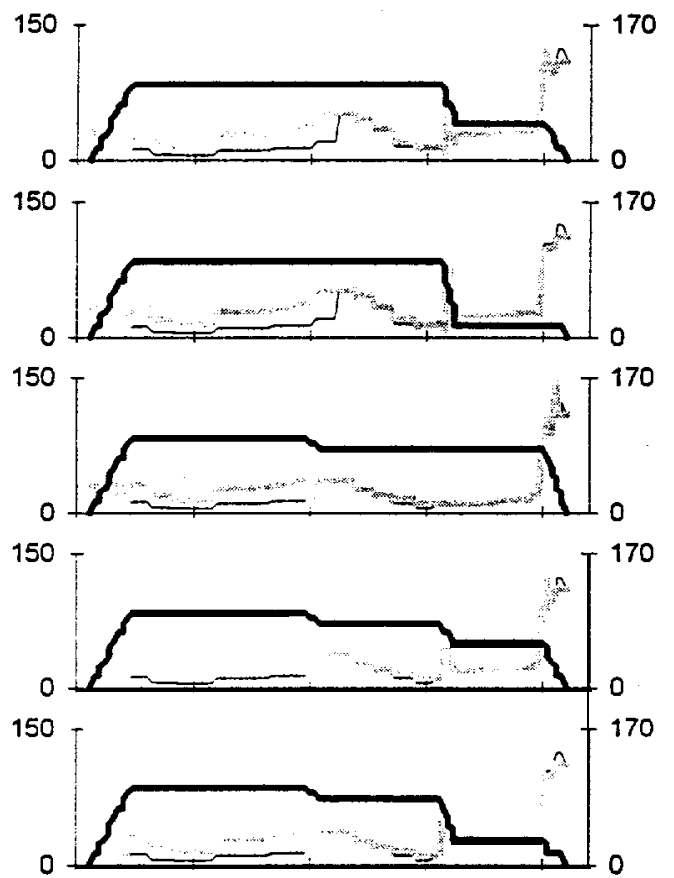

150 T

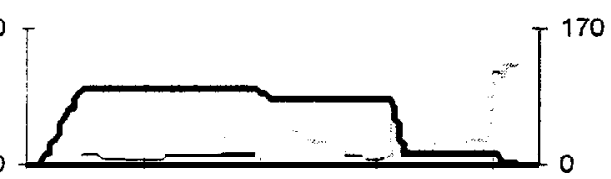

150 -

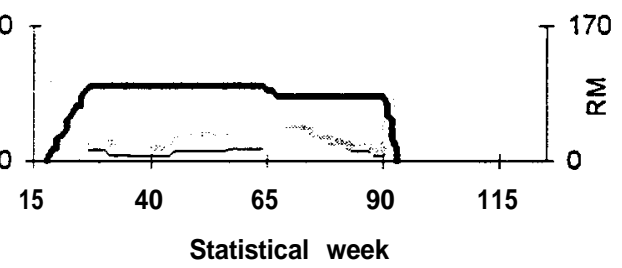

170

$\sum_{\alpha}^{5}$

Prespawn -

Spawning

Colonization

Sum. rearing

Win. rearing

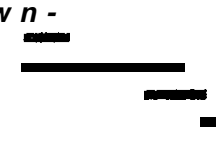

\section{Template}

Patient

Cum. Cum.

Prod. Cap.

Traj. \#1

1490.59

0) 0.00

Traj. \#2

$165 \mid 0.65$

0.00

Traj. \#3

$150 \quad 0.61$

0.00

Traj. \#4

156 0.62

o) 0.00

Traj. \#5

\begin{tabular}{l|l}
160 & 0.58
\end{tabular}

0) 0.00

Traj. \#6

1520.58

o) 0.00

Traj. \#7

$158 \quad 0.61$

0) 0.00

Traj. \#8

\begin{tabular}{l|l|}
\hline 157 & 0.60
\end{tabular}

이 0.00

Traj. \#9

\begin{tabular}{|r|r|}
\hline 281 & 1.19 \\
0 & 0.00
\end{tabular} 
Spawning reach: Grande Ronde R.

Phillips $\mathrm{Cr}$ - Clark Cr (RM 96.9 - 97.3 from Snake R)

Relative Productivity
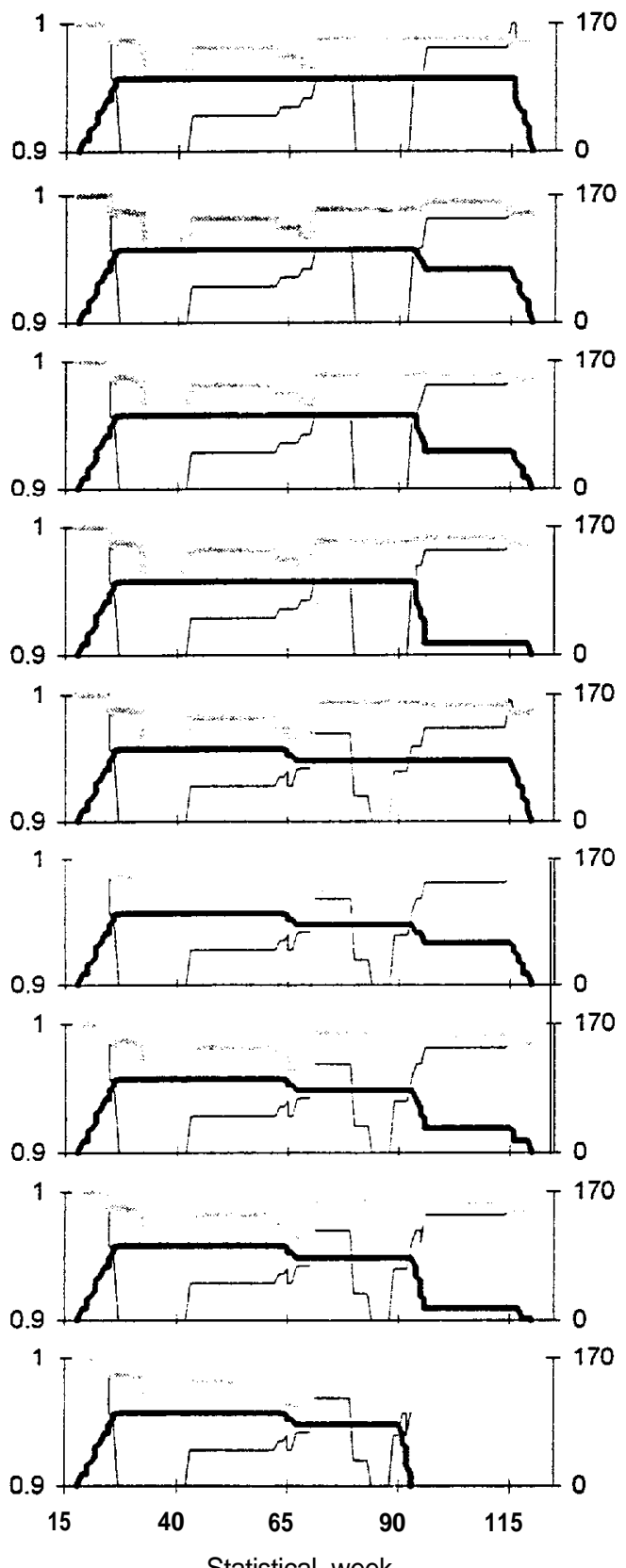

Statistical week

Prespawn Spawning Incubation Colonization Sum. rearing Win. rearing

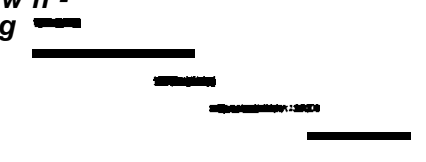

Relative Key Habitat Quantity
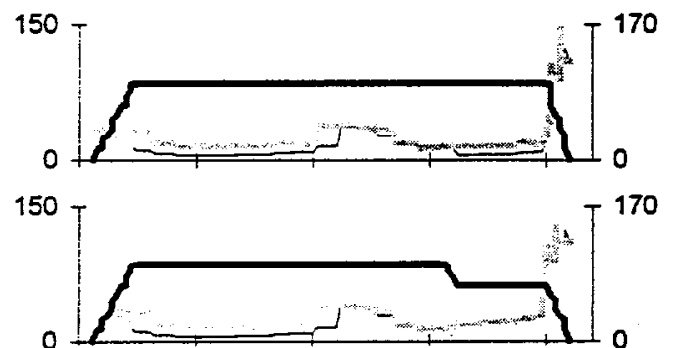

150

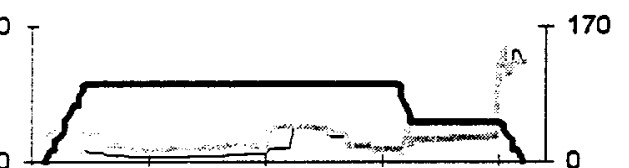

150

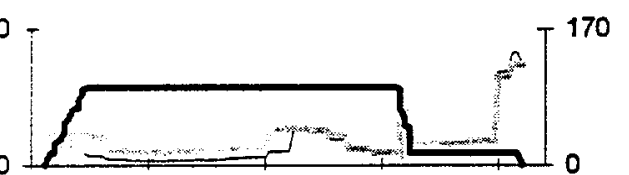

150
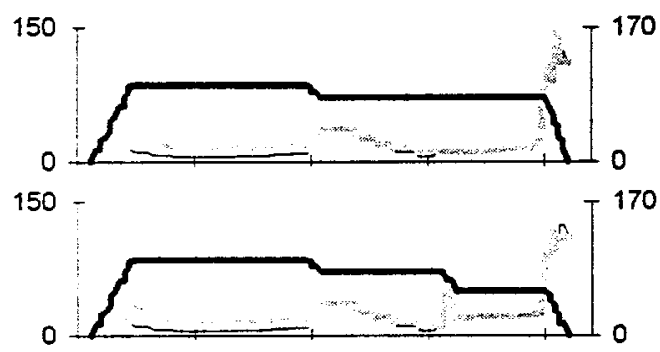

150 -

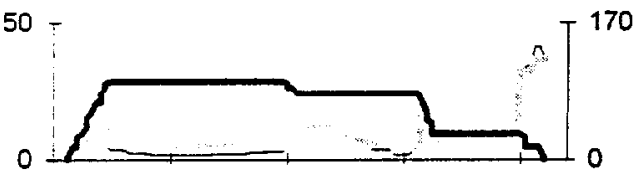

150

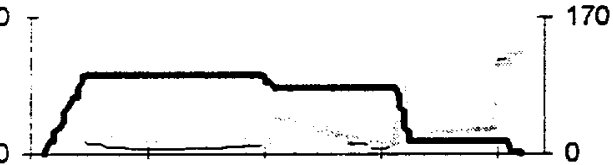

150

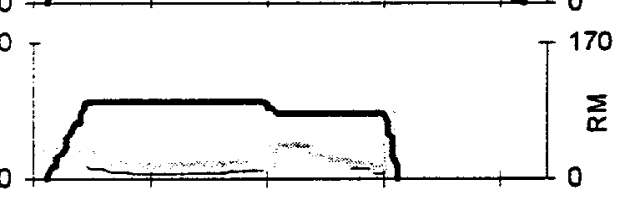

$\begin{array}{lllll}15 & 40 & 65 & 90 & 115\end{array}$

Statistical week

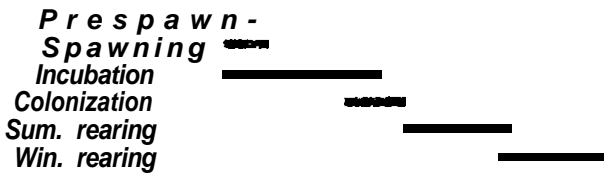

Template

Patient

Cum. Cum.

Prod. Cap.

Traj. \#1

\begin{tabular}{l|l|}
149 & 0.58 \\
\hline
\end{tabular}

$0 \quad 0.00$

Traj. \#2

16060066

0) $\quad 0.00$

Traj. \#3

\begin{tabular}{l|l|}
\hline 150 & 0.61 \\
\hline
\end{tabular}

0) 0.00

Traj. \#4

\begin{tabular}{|l|l|}
157 & 0.64 \\
\hline
\end{tabular}

Traj. \#5

\begin{tabular}{l|l|}
160 & 0.60
\end{tabular}

o) 0.00

Traj. \#6

$152 \quad 0.60$

0) 0.00

Traj \#7

$159 \quad 0.63$

\begin{tabular}{l|l}
0 & 0.00
\end{tabular}

Traj. \#8

$157 \quad 0.62$

0) 0.00

Traj. \#9

\begin{tabular}{r|r}
282 & 1.23 \\
0 & 0.00 \\
\hline
\end{tabular}

- Trajectory Template

Patient 
Spawning reach: Grande Ronde R.

Clark Cr - Indian Cr (RM 97.3 - 99.3 from Snake R)

Relative Productivity
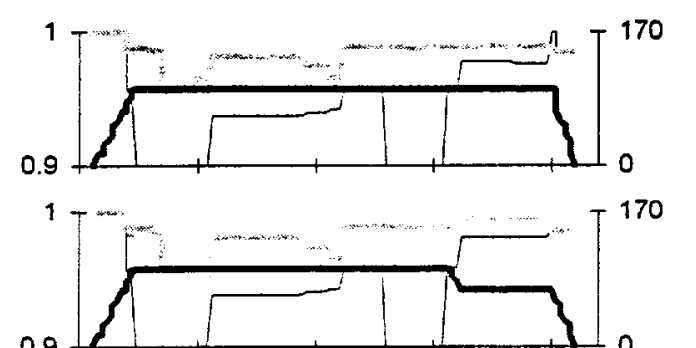

0.9
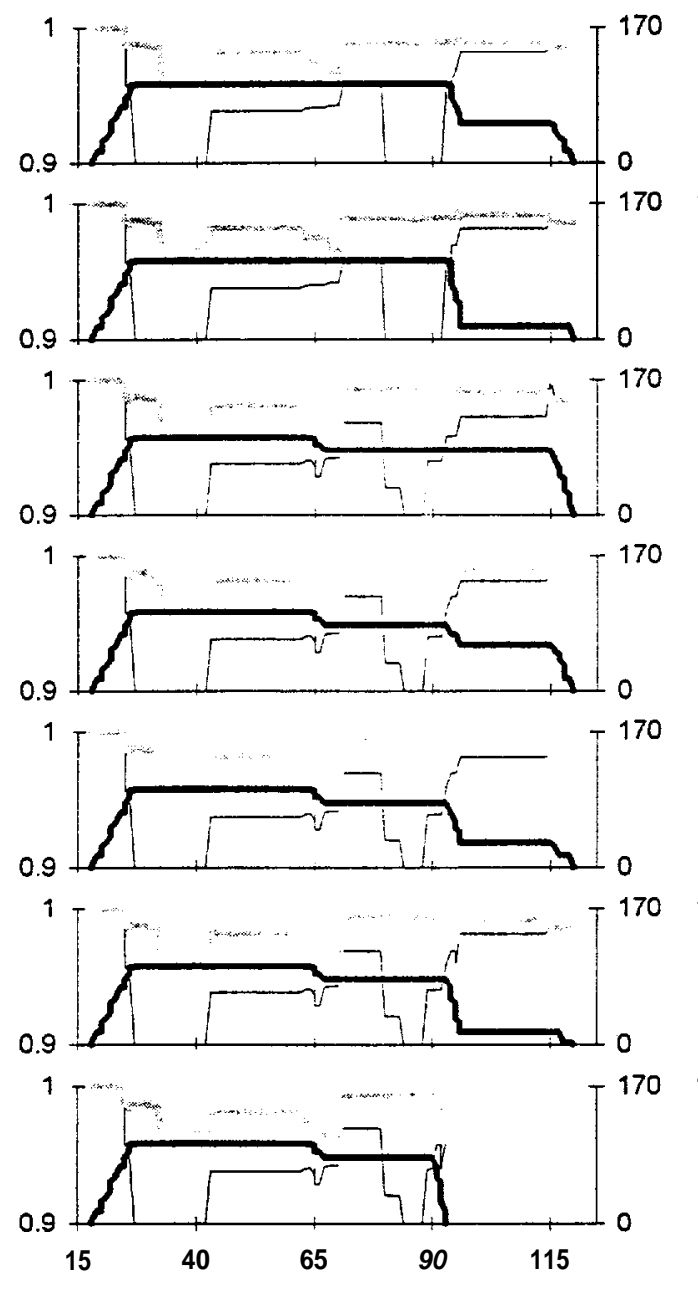

Statistical week
Relative Key Habitat Quantity

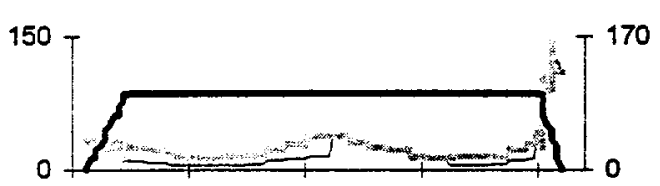

150

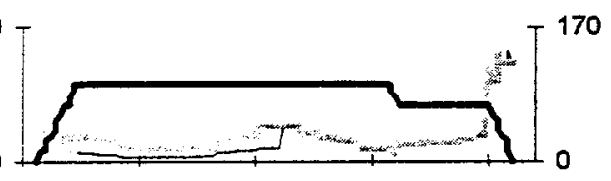

150

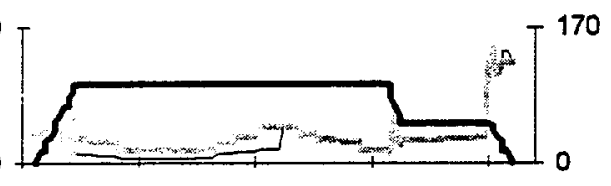

150

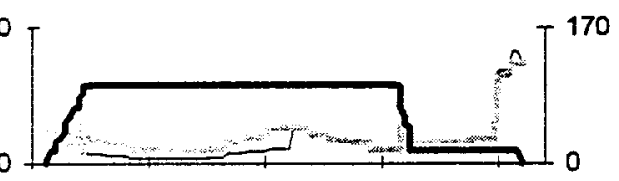

150

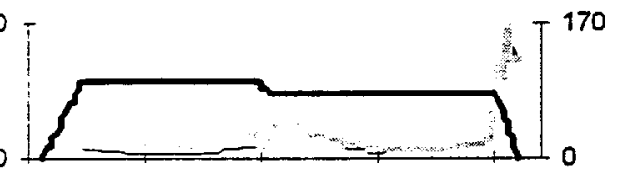

150

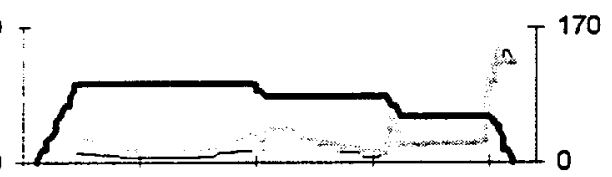

150

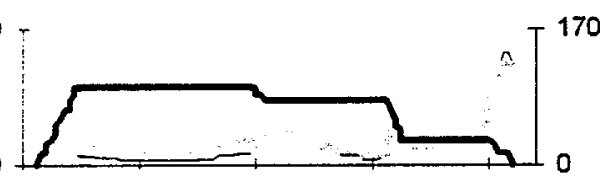

150 T
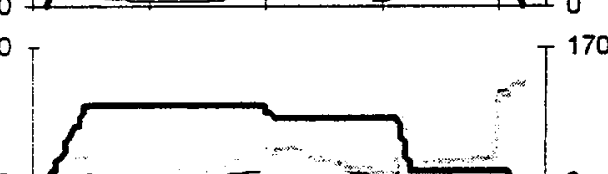

01

150 -

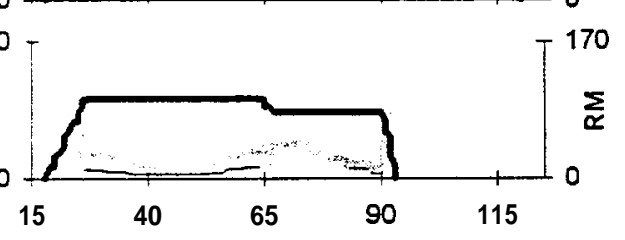

Template

Patient

Cum. Cum.

Prod. Cap.

Traj. \#1

1490.58

0) 0.00

Traj. \#2

\begin{tabular}{l|l}
\hline 166 & 0.65 \\
\hline
\end{tabular}

이 0.00

Traj. \#3

\begin{tabular}{l|l}
\hline 150 & 0.60 \\
\hline
\end{tabular}

o) 0.00

Traj. \#4

\begin{tabular}{l|l}
\hline 157 & 0.63 \\
\hline
\end{tabular}

0) 0.00

Traj. \#5

$160 \quad 0.59$

0) 0.00

Traj. \#6

\begin{tabular}{l|l}
152 & 0.59 \\
\hline
\end{tabular}

0) 0.00

Traj. \#7

\begin{tabular}{l|l}
\hline 159 & 0.62 \\
\hline
\end{tabular}

0) 0.00

Traj. \#8

$157 \quad 0.61$

0.00

Traj. \#9

\begin{tabular}{r|l|}
\hline 282 & 1.21 \\
0 & 0.00
\end{tabular}
Prespawn -

Spawning

incubation

Colonization

Sum. rearing

Win. rearing

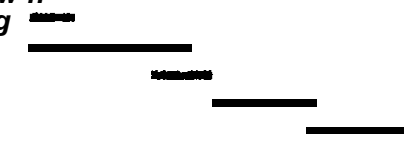

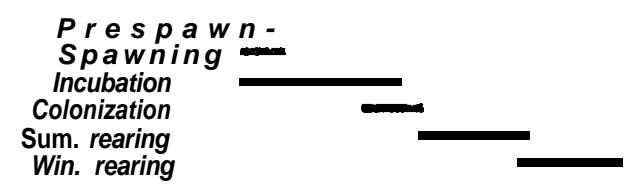

Trajectory

Template

Patient 
Spawning reach: Grande Ronde $\mathbf{R}$.

Indian $\mathrm{Cr}$ - Willow $\mathrm{Cr}$ (RM 99.3 - 103.5 from Snake R)
Relative Productivity
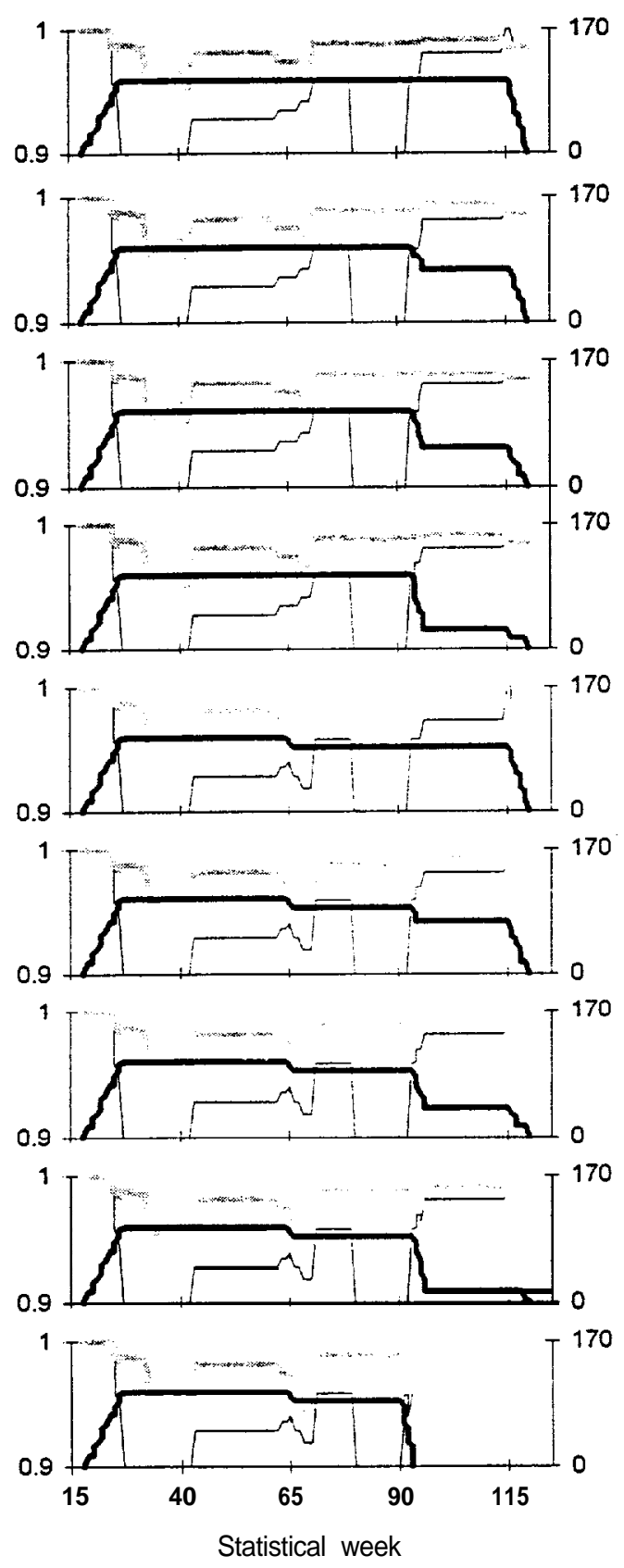

Relative Key Habitat Quantity
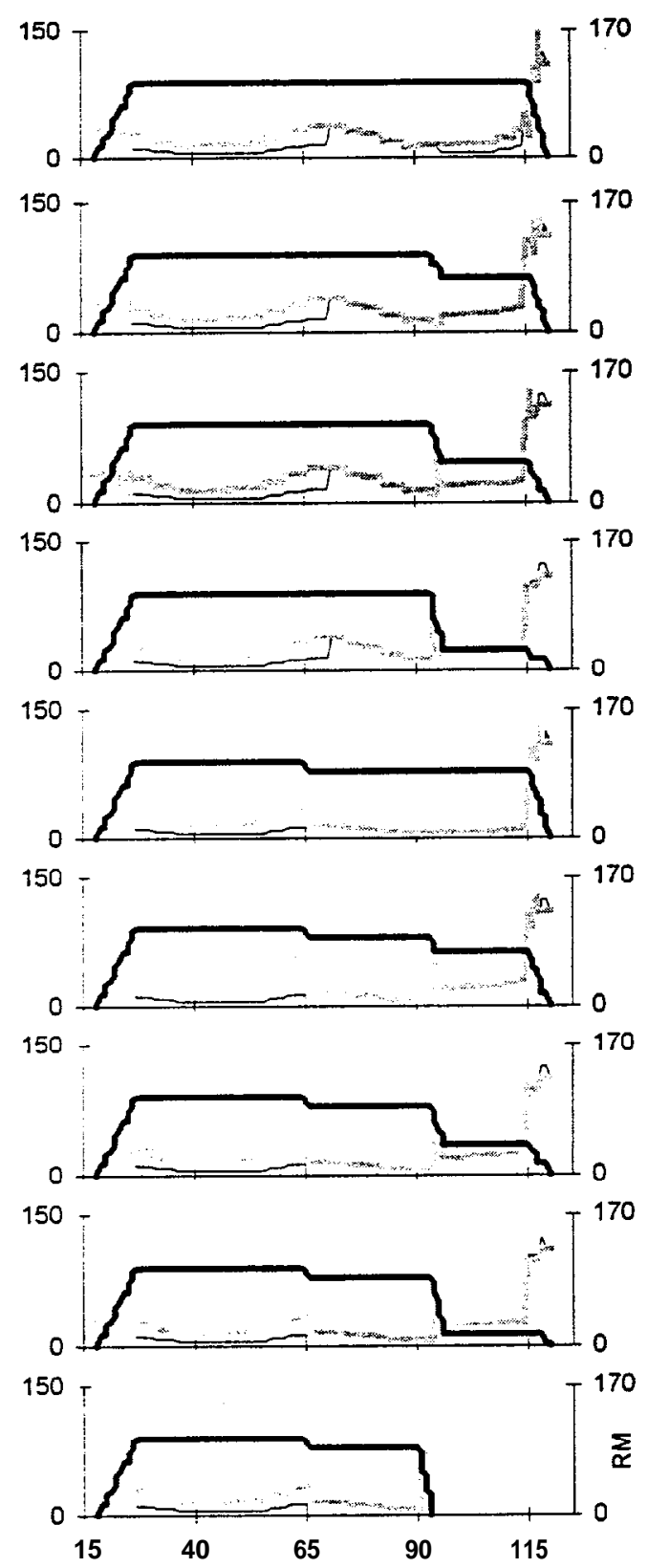

Statistical week

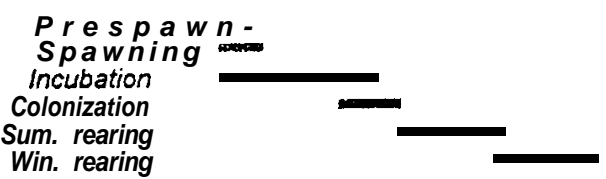

Win. rearing

\begin{tabular}{|c|c|}
\hline \multicolumn{2}{|c|}{$\begin{array}{c}\text { Template } \\
\text { Patient }\end{array}$} \\
\hline $\begin{array}{c}\text { Cum, Cum. } \\
\text { Prod. Cap. }\end{array}$
\end{tabular}

Traj. \#1

\begin{tabular}{l|l|}
\hline 157 & 0.61 \\
\hline & 0.00
\end{tabular} 0.00

Traj. \#2

$165 \mid 0.65$ \begin{tabular}{l|l}
0 & 0.00
\end{tabular}

Traj. \#3

1490.59

0.0 .00

Traj. \#4

$157 \quad 0.63$

0.0 .00

Traj. \#5

$144 \quad 0.44$

0.00

Traj. \#6

$153 \quad 0.53$

0.00

Traj. \#7

$144 \quad 0.50$

0.00

Traj. \#8

\begin{tabular}{|c|c|}
143 & 0.49 \\
\hline & 0.00
\end{tabular}

0) 0.00

Traj. \#9

2590.98

0) 0.00

Presp a w n -

Spawning

Colonization

Sum. rearing

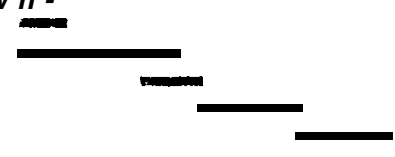

\begin{tabular}{|r|r|}
259 & 0.98 \\
0 & 0.00 \\
\hline
\end{tabular}


Spawning reach: Grande Ronde R.

Willow $\mathrm{Cr}$ - downstream end of State Ditch (RM 103.5 - 114.5 from Snake R)

Relative Productivity
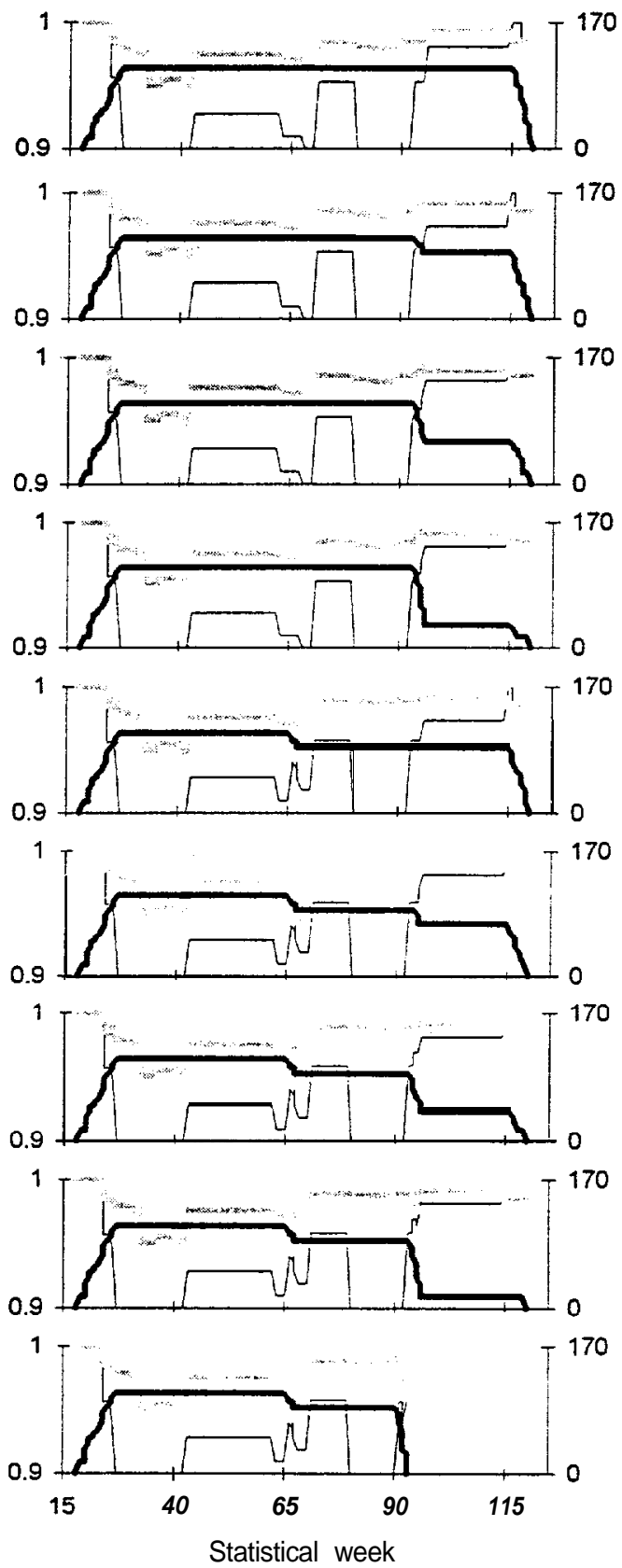

Prespawn -

Spawning

Incubation

Colonization

Sum. rearing

Win. rearing

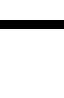

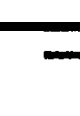

Relative Key Habitat Quantity

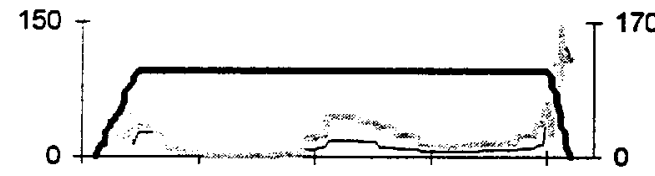

150
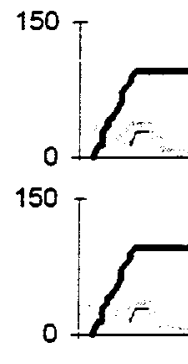

150
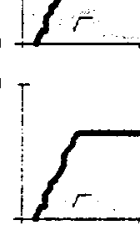

100
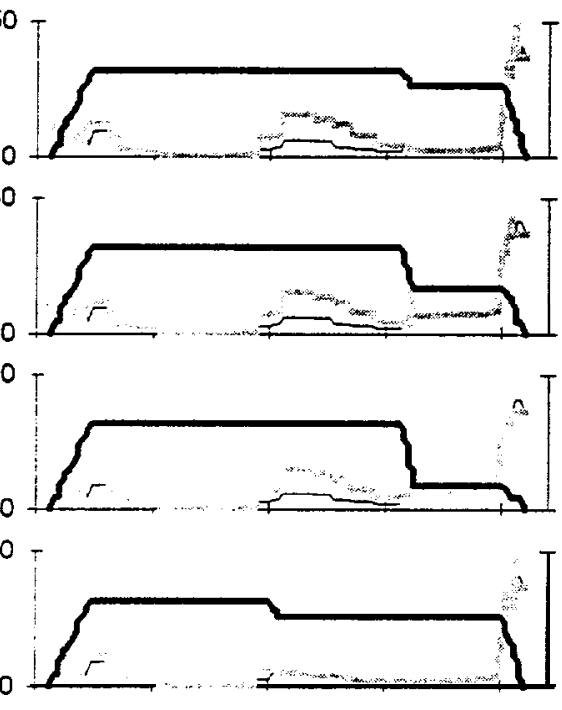

$T^{170}$

150

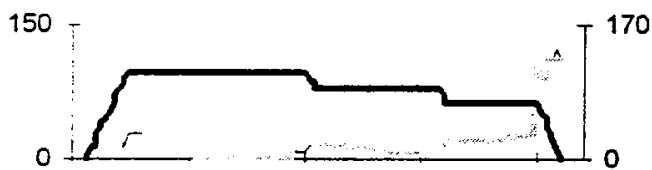

$150-$
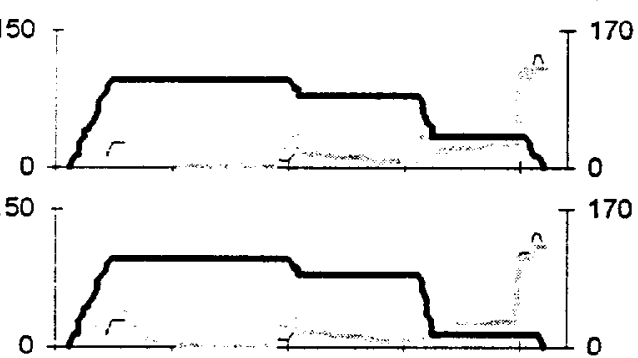

150

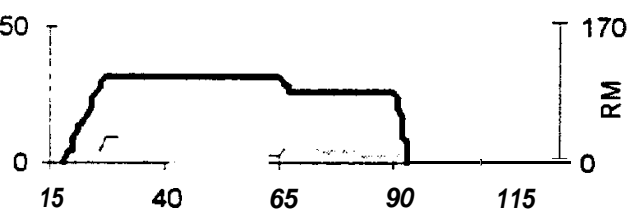

Statistical week
Template

Patient

Cum. Cum.

Prod. Cap.

Traj. \#1

\begin{tabular}{l|l}
111 & 0.45 \\
\hline
\end{tabular}

$\begin{array}{ll}0 & 0.00\end{array}$

Traj. \#2

$106 \quad 0.40$

0) 0.00

Traj. \#3

1010.42

0) 0.00

Traj. \#4

\begin{tabular}{r|r|}
106 & 0.44 \\
0 & 0.00
\end{tabular}

Traj. \#5

\begin{tabular}{r|r|}
113 & 0.37 \\
0 & 0.00
\end{tabular}

Traj. \#6

1190.42

0.00

Traj. \#7

1130.41

0) 0.00

Traj. \#8

\begin{tabular}{r|r|}
112 & 0.40 \\
0 & 0.00
\end{tabular}

Traj. \#9

\begin{tabular}{r|l}
203 & 0.79 \\
0 & 0.00
\end{tabular} 
Spawning reach: Grande Ronde R.

Downstream end of State Ditch - Upstream end of State Ditch (RM 114.5 - 118.9 from Snake R)

Relative Productivity
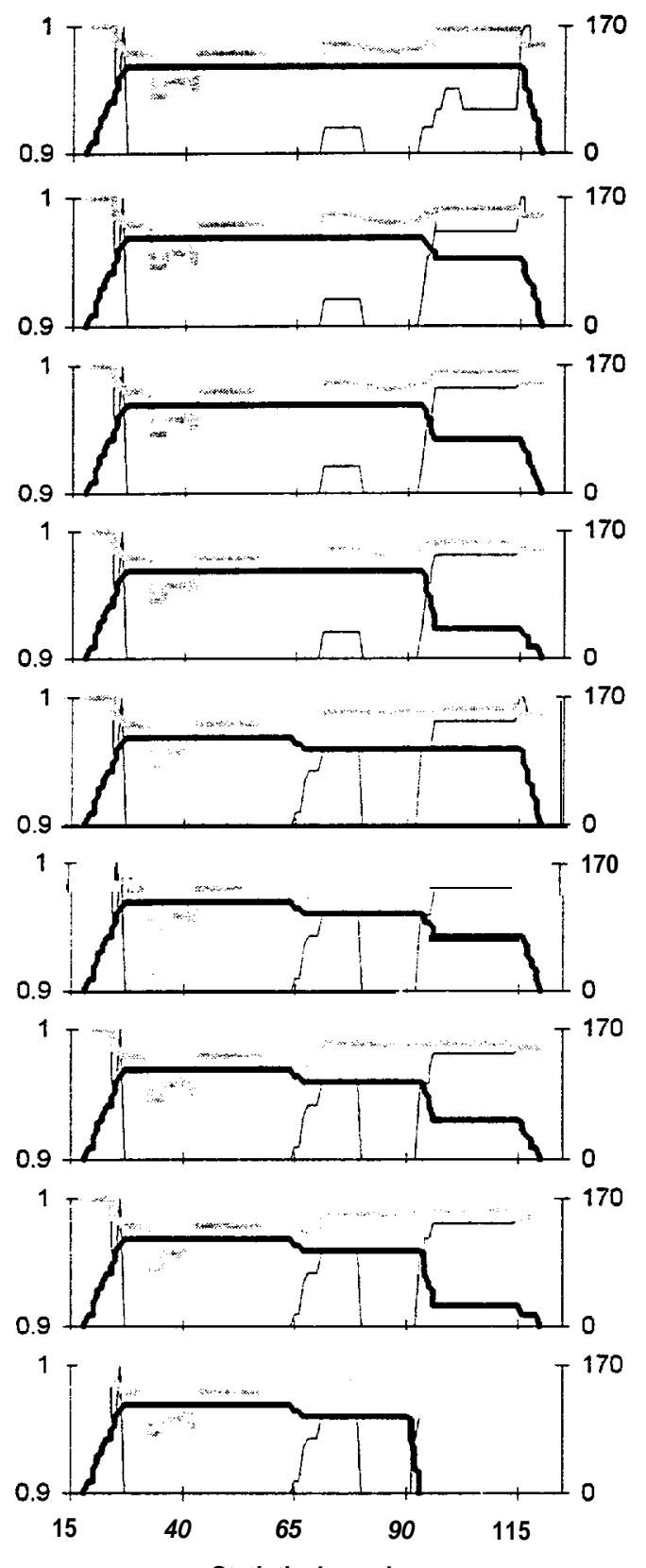

Statistical week

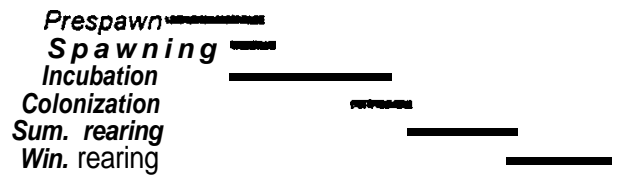

Relative Key Habitat Quantity

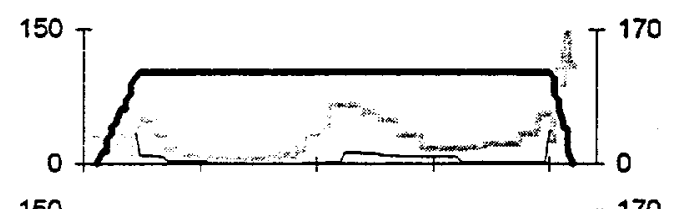

150

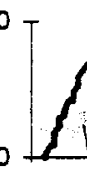

$150 \mathrm{~T}$

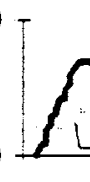

$150-$

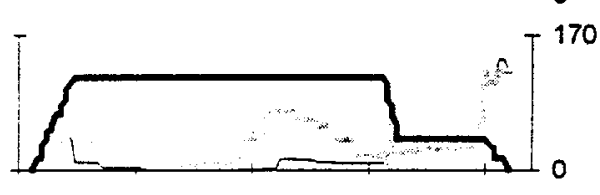

150

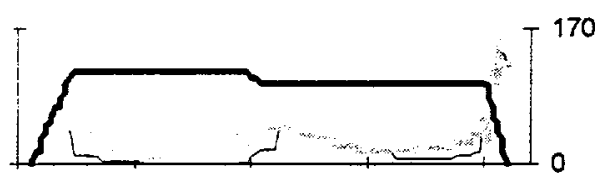

150

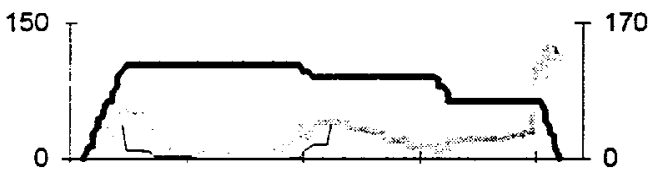

$150-$

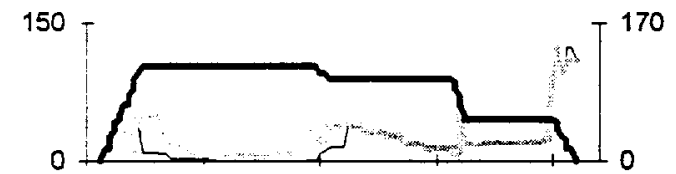

150 .

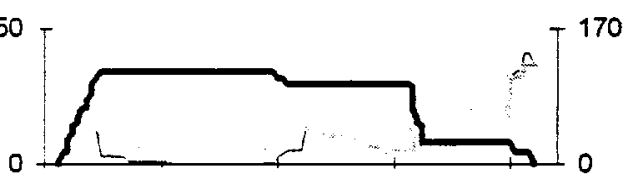

150

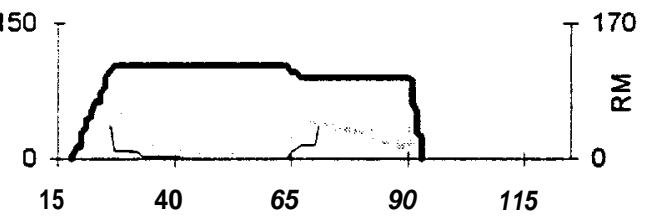

Statistical week

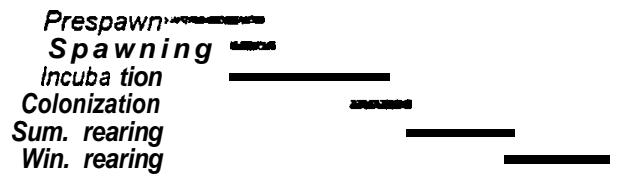

\begin{tabular}{c} 
Template \\
Patient \\
\hline Cum. Cum. \\
Prod. Cap.
\end{tabular}

Traj. \#1

\begin{tabular}{r|r}
124 & 0.65 \\
0 & 0.00
\end{tabular}

Traj. \#2

$112 \quad 0.53$

0) 0.00

Traj. \#3

\begin{tabular}{r|l|}
118 & 0.61 \\
0 & 0.00
\end{tabular}

Traj. \#4

$\begin{array}{lll}112 & 0.59\end{array}$

0) 0.00

Traj. \#5

\begin{tabular}{l|l}
125 & 0.61
\end{tabular}

0) 0.00

Traj. \#6

1310.64

0) 0.00

Traj. \#7

\begin{tabular}{|r|r|}
\hline 119 & 0.59 \\
0 & 0.00
\end{tabular}

Traj. \#8

\begin{tabular}{l|l}
\hline 125 & 0.63
\end{tabular}

o) 0.00

Traj. \#9

\begin{tabular}{|r|r|}
\hline 225 & 1.24 \\
0 & 0.00
\end{tabular} 
Spawning reach: Grande Ronde R.

Upstream end of State Ditch - I-84 crossing below Devil's Slide (RM 118.9 - 128.4 from Snake R)

Relative Productivity
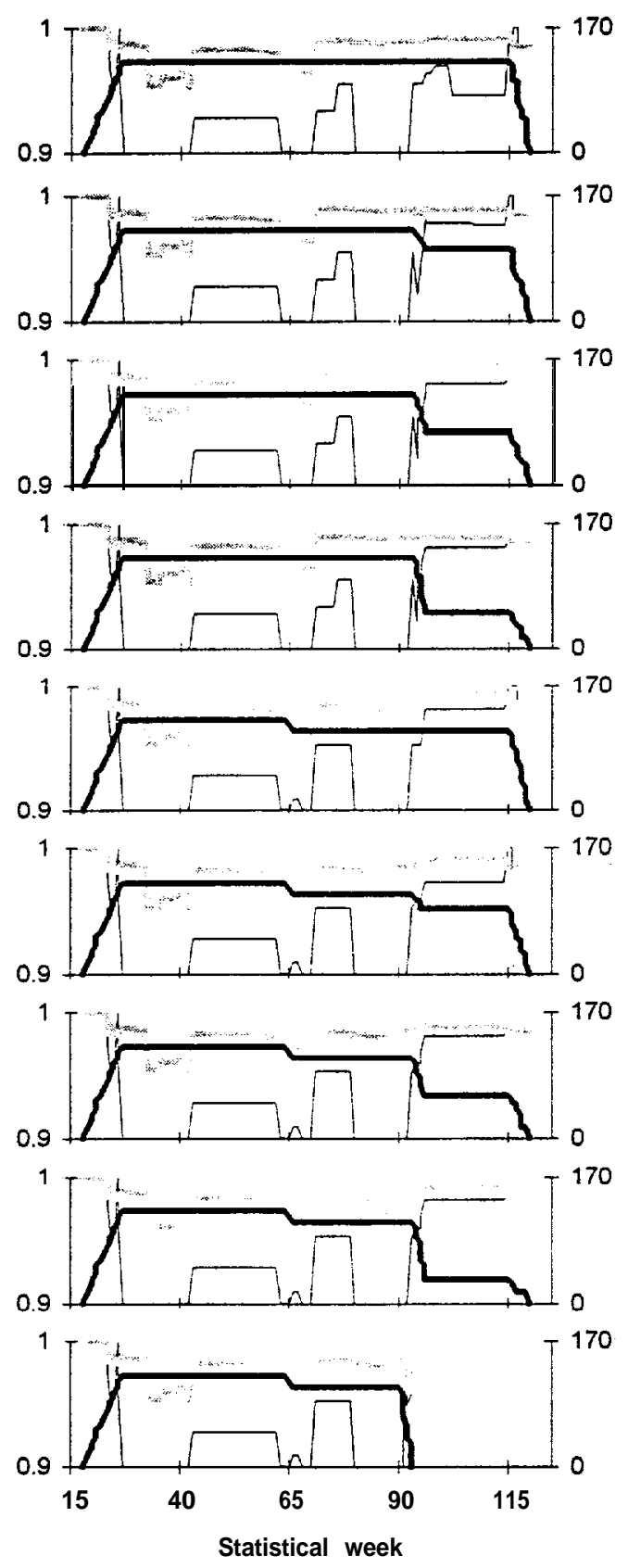

Prespawn -

Spawning Incubation
Colonization

Sum. rearing Win. rearing
Relative Key Habitat Quantity

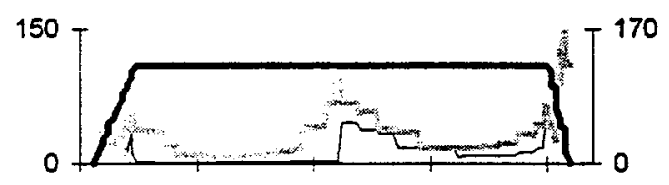

150

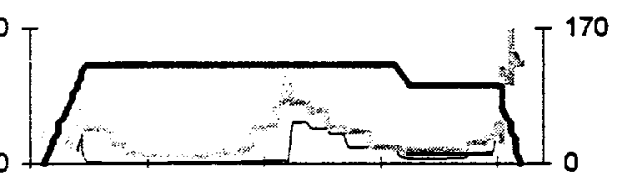

150
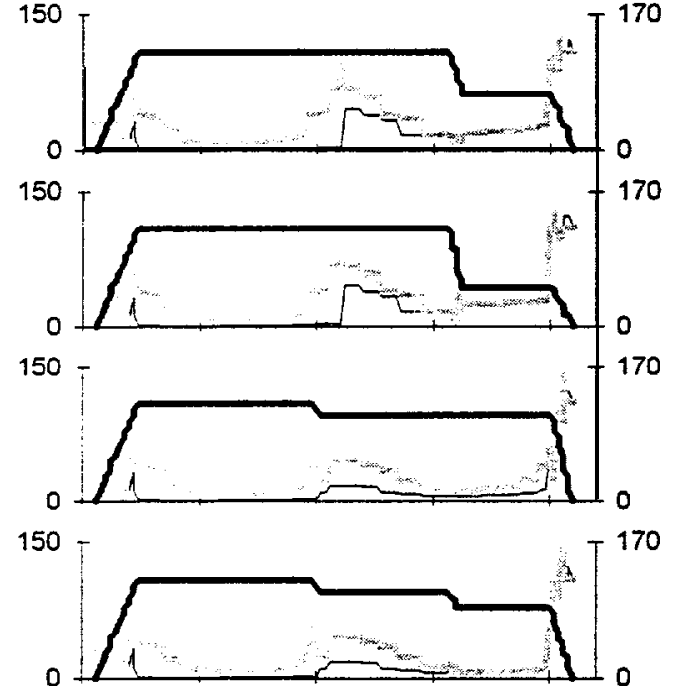

150

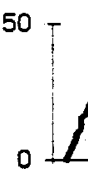

150

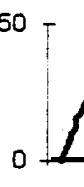

150

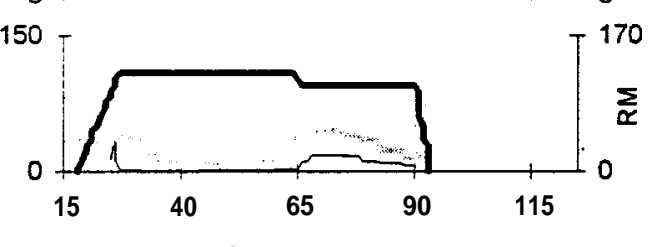

Statistical week

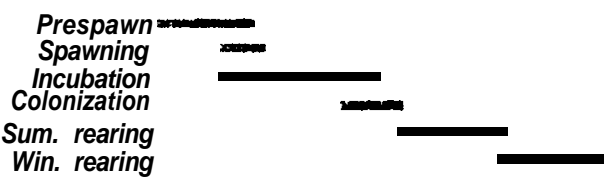

Template

Patient

Cum. Cum.

Prod. Cap.

Traj. \#1

\begin{tabular}{l|l}
\hline 155 & 0.73 \\
\hline
\end{tabular}

0) 0.00

Traj. \#2

\begin{tabular}{l|l}
\hline 144 & 0.66 \\
\hline
\end{tabular}

0) 0.00

Traj. \#3

\begin{tabular}{l|l}
\hline 160 & 0.75 \\
\hline
\end{tabular}

0 0.00

Traj. \#4

\begin{tabular}{l|l}
\hline 145 & 0.69 \\
\hline
\end{tabular}

00.00

Traj. \#5

1410.63 이 0.00

Traj. \#6

1350.56

0) 0.00

Traj. \#7

1290.59

0) 0.00

Traj. \#8

1340.62

0) 0.00

Traj. \#9

2471.27

0) 0.00 
Spawning reach: Grande Ronde R.

I-84 crossing below Devil's Slide - Five Points Cr (RM 128.4 - 133.4 from Snake R)

Relative Productivity
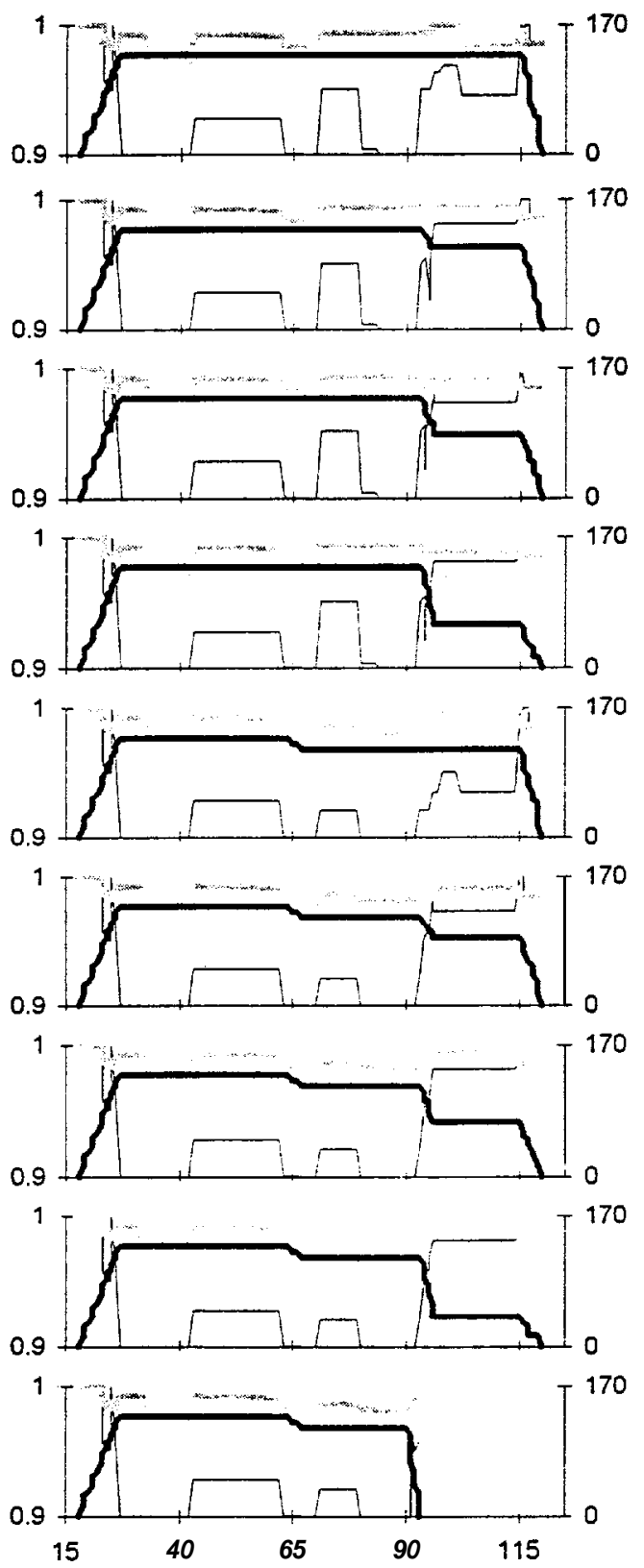

Statistical week

Prespawn.

Spawning

Incubation

Colonization

Sum. rearing

Win. rearing
Relative Key Habitat Quantity
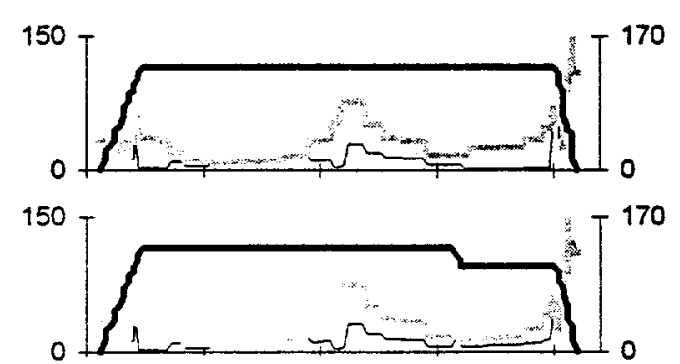

150

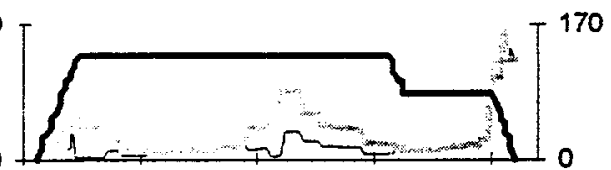

150
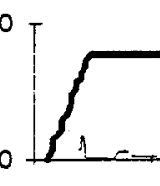

150
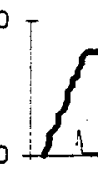

${ }^{150} \mathrm{~T}$
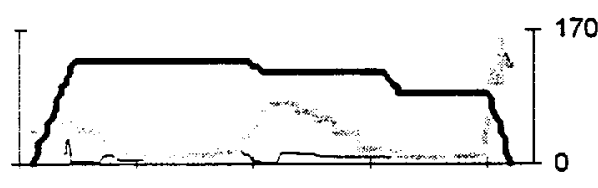

$150-$

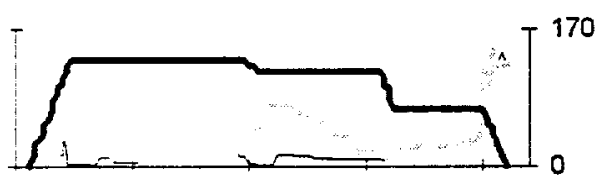

0

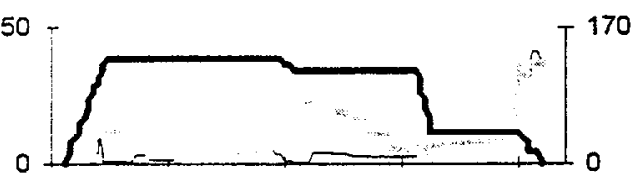

:

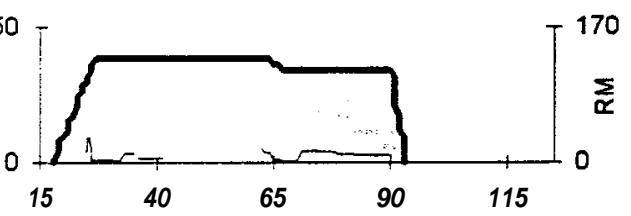

Statistical week

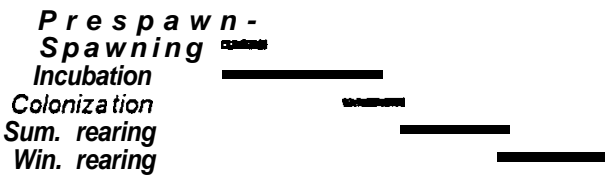

Template

Patient

Cum.|Cum.

Prod. Cap.

Traj. \#1

$268 \quad 1.14$

0) 0.00

Traj. \#2

\begin{tabular}{l|l}
294 & 1.17
\end{tabular}

$\begin{array}{ll}0 & 0.00\end{array}$

Traj. \#3

\begin{tabular}{l|l}
280 & 1.07 \\
\hline
\end{tabular}

0) 0.00

Traj. \#4

\begin{tabular}{l|l|}
\hline 267 & 1.10 \\
\hline
\end{tabular}

0.00

Traj. \#5

$237 \quad 1.05$

0) 0.00

Traj. \#6

213 0.81

0) 0.00

Traj. \#7

\begin{tabular}{r|r|}
225 & 0.99 \\
0 & 0.00
\end{tabular}

Traj. \#8

\begin{tabular}{l|l}
\hline 213 & 0.96
\end{tabular}

0.00

Traj. \#9

\begin{tabular}{r|r|}
394 & 2.04 \\
0 & 0.00 \\
\hline
\end{tabular} 
Spawning reach: Grande Ronde R.

Five Points $\mathrm{Cr}$ - Rock Cr (RM 133.4 - 133.8 from Snake R)

Relative Productivity
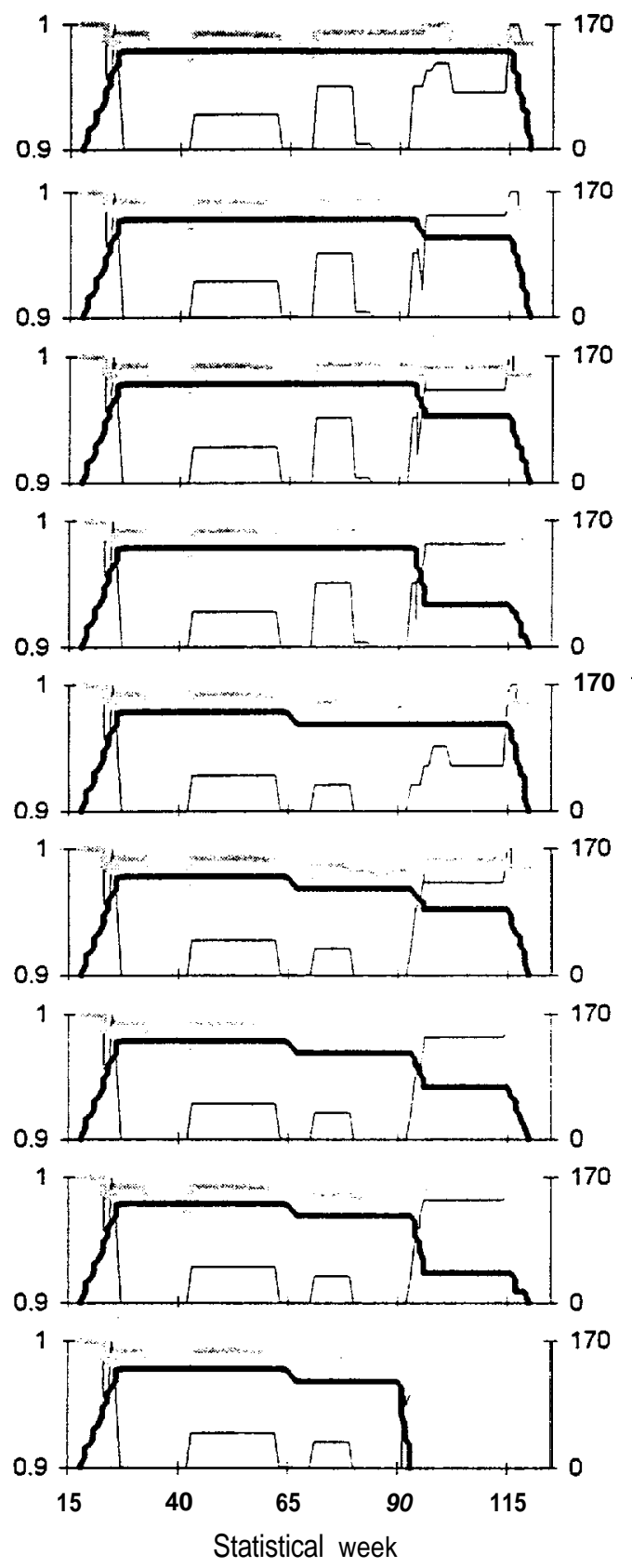

Prespawn -

Spawning

Incubation

Colonization

Sum. rearing

Win. rearing
Relative Key Habitat Quantity

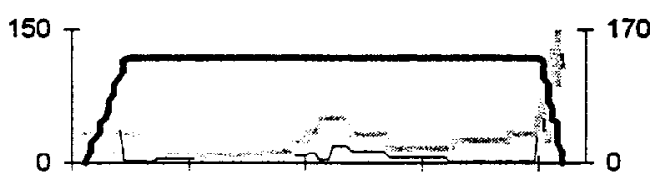

$150-$

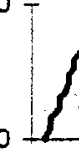

150
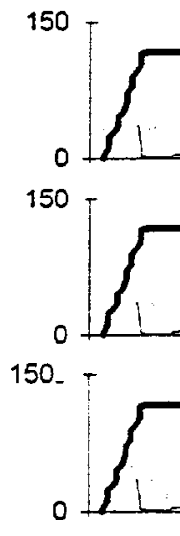

150 T

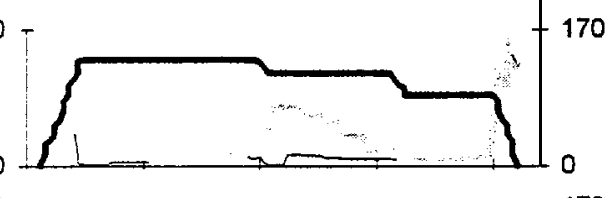

50
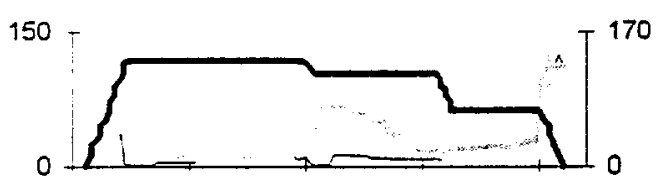

: 50
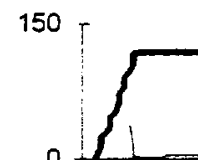

150

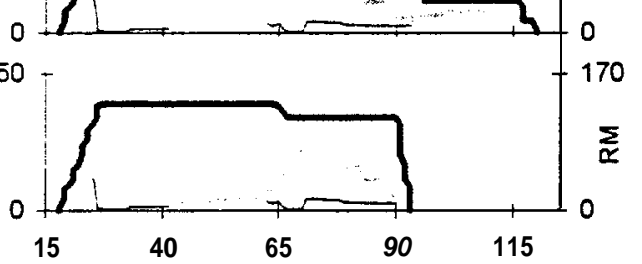

Statistical week

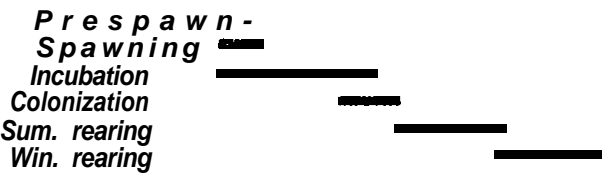

Template

Patient

Curm. Qum.

Prod. Caap.

Traj. \#1

26901.01

이 0.00

Traj. \#2

$295 \mid 1.05$

0) 0.00

Traj. \#3

2800.87

0) 0.00

Traj. \#4

2670.98

0.00

Traj. \#5

\begin{tabular}{l|l}
239 & 0.97 \\
\hline
\end{tabular}

\begin{tabular}{l|l}
0 & 0.00
\end{tabular}

Traj. \#6

\begin{tabular}{l|l}
\hline 216 & 0.75 \\
\hline
\end{tabular}

0.00

Traj. \#7

\begin{tabular}{l|l}
\hline 227 & 0.91
\end{tabular}

0) $\quad 0.00$

Traj. \#8

\begin{tabular}{|l|l|}
\hline 216 & 0.88 \\
\hline
\end{tabular}

0) 0.00

Traj. \#9

\begin{tabular}{l|l|}
\hline 398 & 1.85 \\
\hline
\end{tabular}

ㅇ 0.00 
Spawning reach: Grande Ronde R.

Rock $\mathrm{Cr}$ - Whiskey $\mathrm{Cr}$ (RM 133.8 - 136.6 from Snake R)

Relative Productivity
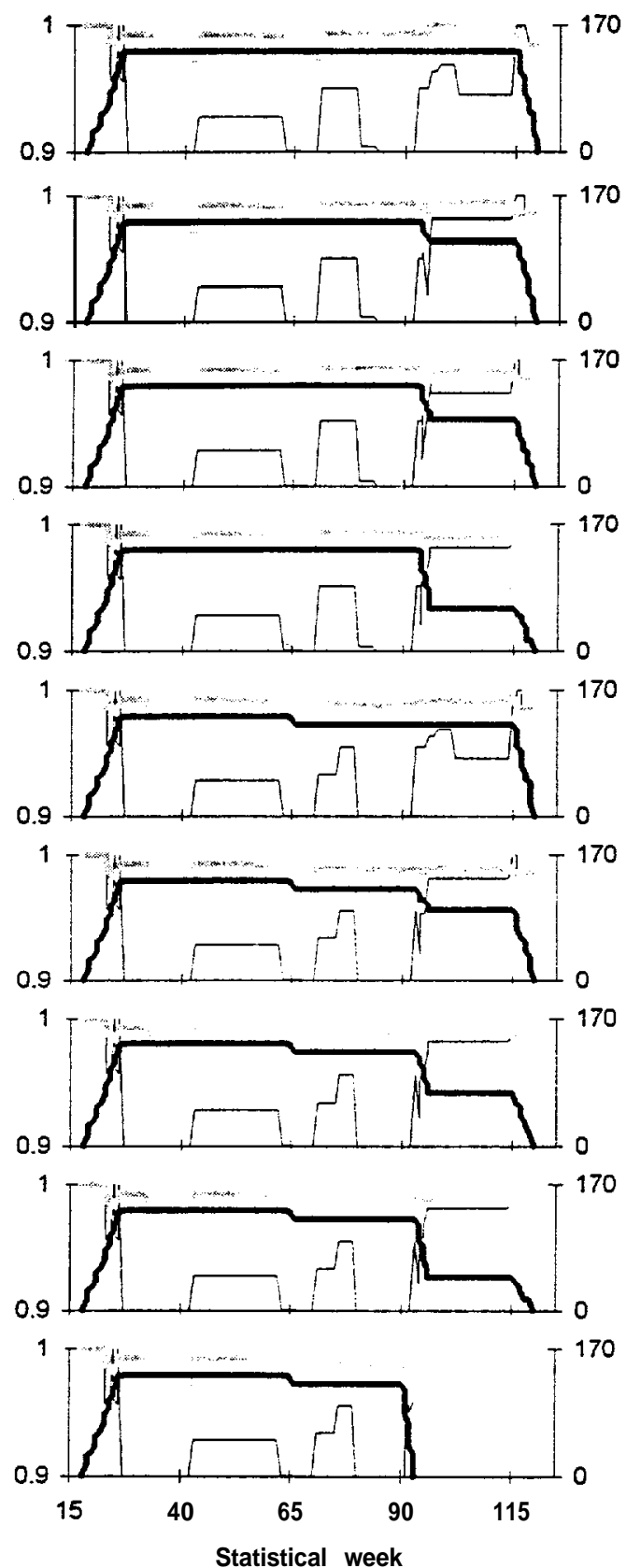

Prespawn -

Spawning

Incubation

Colonization

Sum. rearing

Win. rearing
Relative Key Habitat Quantity

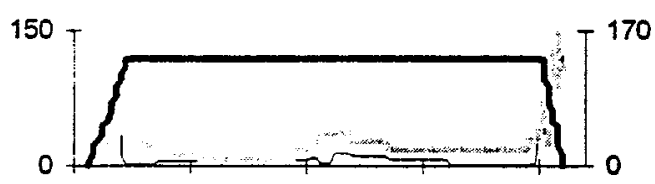

150

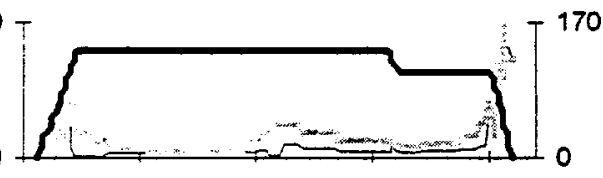

150

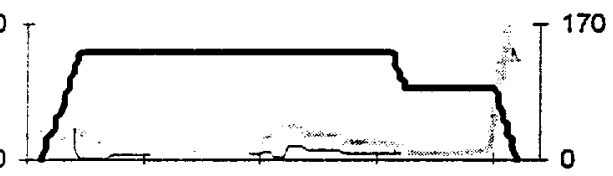

150 .

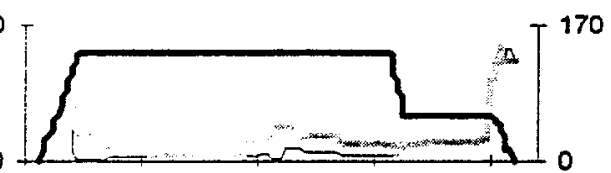

150 -

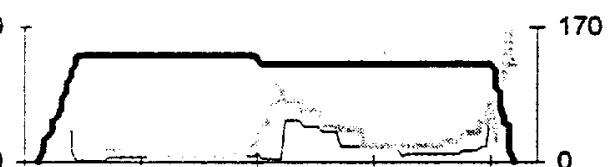

150

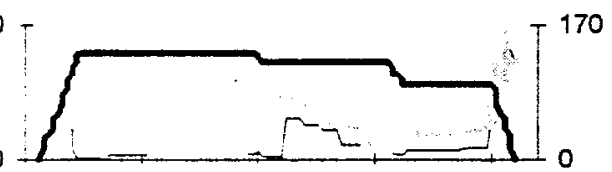

150
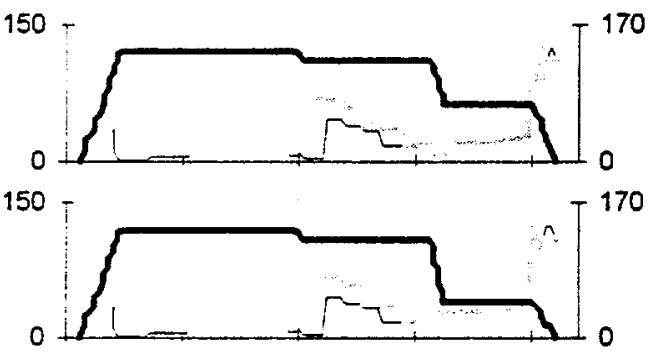

$150-$

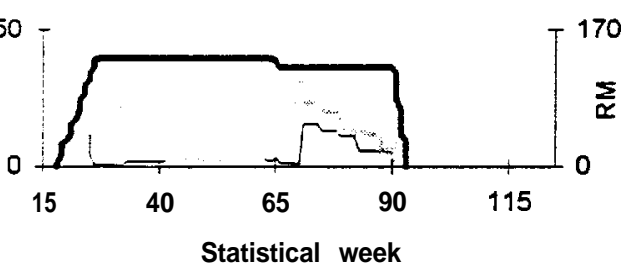

170

$\sum_{x}$

Prespawn -

Spawning

Incubation

Colonization

Sum. rearing

Win. rearing

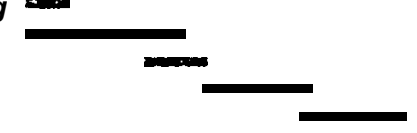

\begin{tabular}{|c|}
\hline $\begin{array}{c}\text { Template } \\
\text { Patient }\end{array}$ \\
\hline $\begin{array}{c}\text { Cum. } \\
\text { Prod. }\end{array}$ Cam. \\
\hline
\end{tabular}

Traj. \#1

2690.96

이 0.00

Traj. \#2

\begin{tabular}{l|l}
\hline 296 & 1.03 \\
\hline
\end{tabular}

0.00

Traj . \#3

$280 \quad 0.86$

$0 \quad 0.00$

Traj. \#4

$268 \quad 0.96$

0) 0.00

Traj. \#5

\begin{tabular}{l|l}
243 & 0.95 \\
\hline
\end{tabular}

0) 0.00

Traj. \#6

\begin{tabular}{l|l|}
226 & 0.90 \\
\hline
\end{tabular}

0.00

Traj . \#7

$251 \quad 0.97$

0) 0.00

Traj. \#8

\begin{tabular}{r|r}
228 & 0.92 \\
0 & 0.00
\end{tabular}

Traj. \#9

\begin{tabular}{l|l}
\hline 434 & 1.95 \\
\hline & 0.00
\end{tabular}

0) 0.00 
Spawning reach: Grande Ronde R.

Whiskey Cr - Jordan Cr (RM 136.6 - 139.1 from Snake R)

Relative Productivity
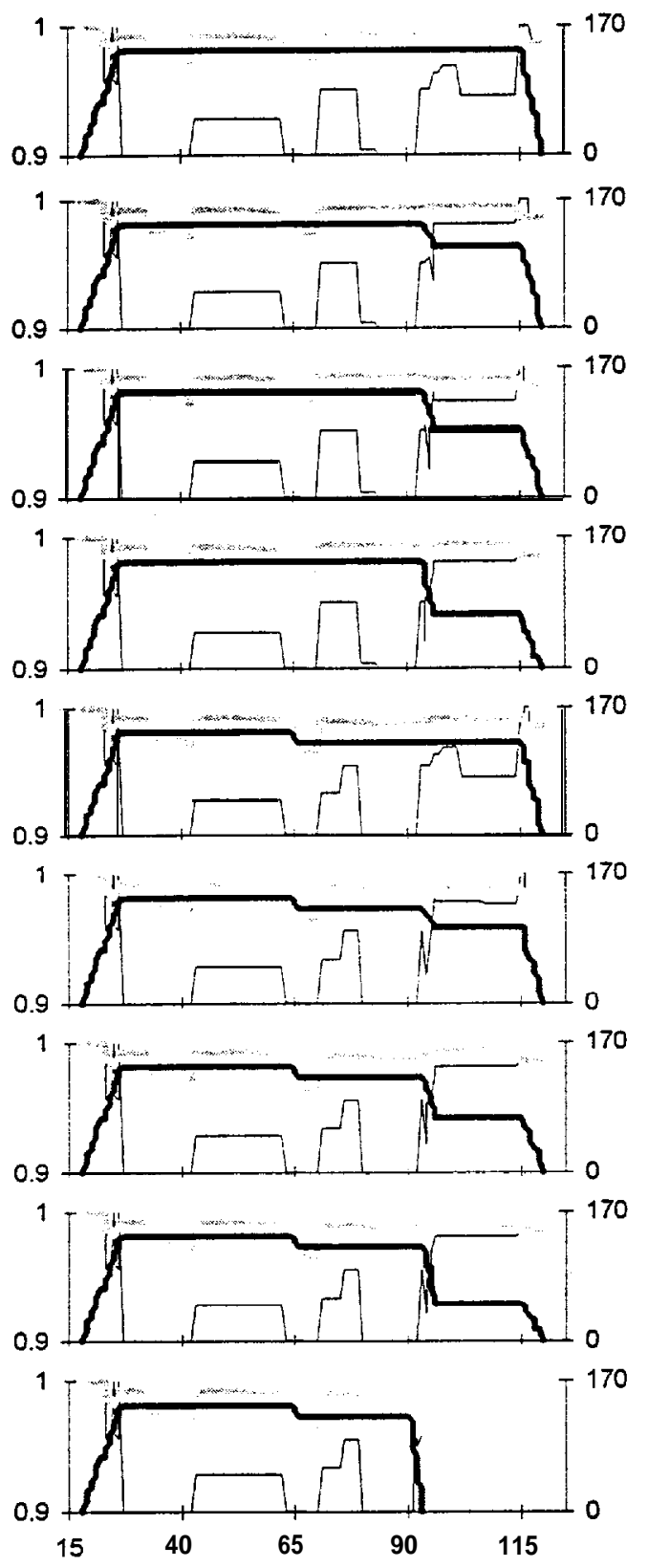

Statistical week

\section{Prespaw n -}

Spawn
Incubation

Colonization

Sum. rearing

Win. rearing
Relative Key Habitat Quantity
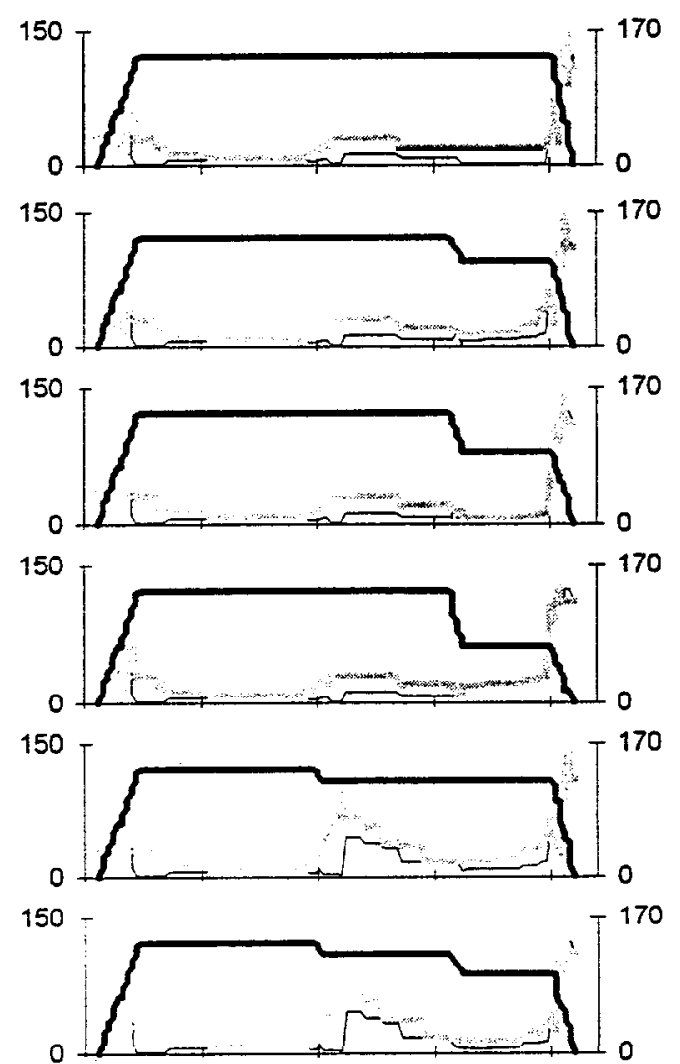

150

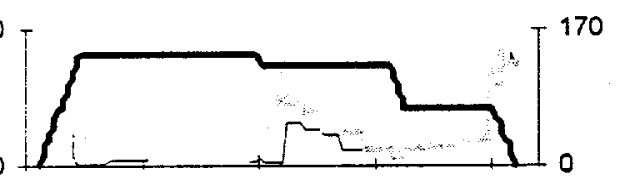

150

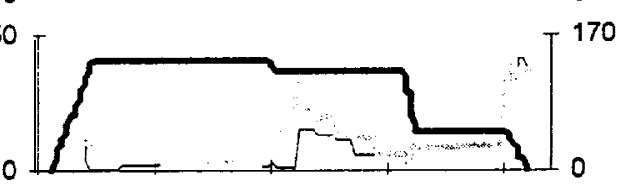

150

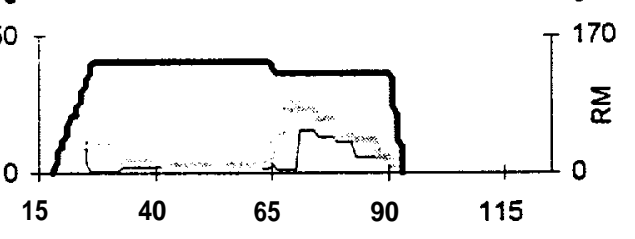

Statistical week \begin{tabular}{|c|c|}
\hline \multicolumn{2}{|c|}{ Template } \\
Patient \\
\hline Cum. & Cum. \\
Prod. & Cap.
\end{tabular}

Traj. \#1

$270 \quad 0.99$

0.00

Traj. \#2

\begin{tabular}{r|l|}
298 & 1.06 \\
0 & 0.00
\end{tabular}

Traj. \#3

2810.88

0) 0.00

Traj. \#4

\begin{tabular}{l|l|}
296 & 1.08 \\
\hline & 0.00
\end{tabular}

이 0.00

Traj. \#5

$243 \quad 0.99$

0) 0.00

Traj. \#6

\begin{tabular}{l|l|}
\hline 227 & 0.89 \\
\hline & 0.00
\end{tabular}

0) 0.00

Traj. \#7

252 1.01

0) 0.00

Traj. \#8

\begin{tabular}{r|r|}
\hline 228 & 0.95 \\
0 & 0.00
\end{tabular}

Traj. \#9

$434 \quad 2.05$

0) 0.00 
Spawning reach: Grande Ronde R.

Jordan $\mathrm{Cr}$ - Beaver Cr (RM 139.1 - 145.8 from Snake R)

Relative Productivity
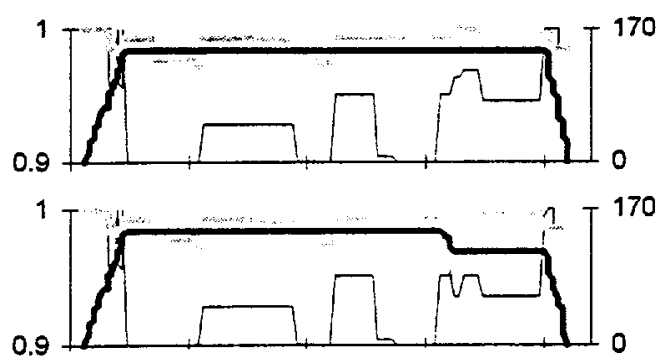

$1 T+\ldots \ldots$
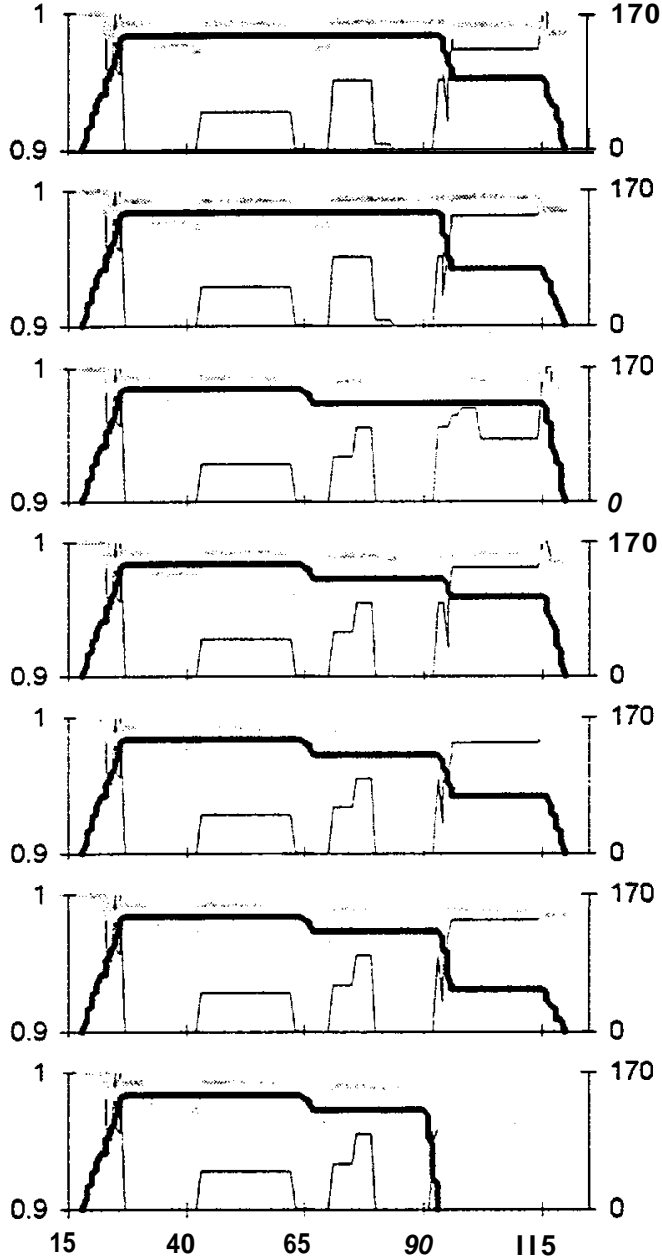

Statistical week

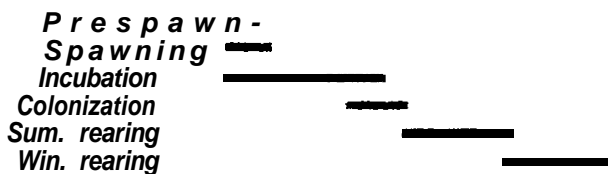

Relative Key Habitat Quantity

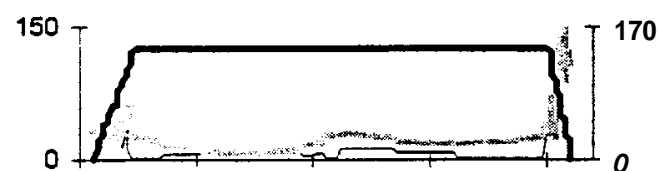

$150-$
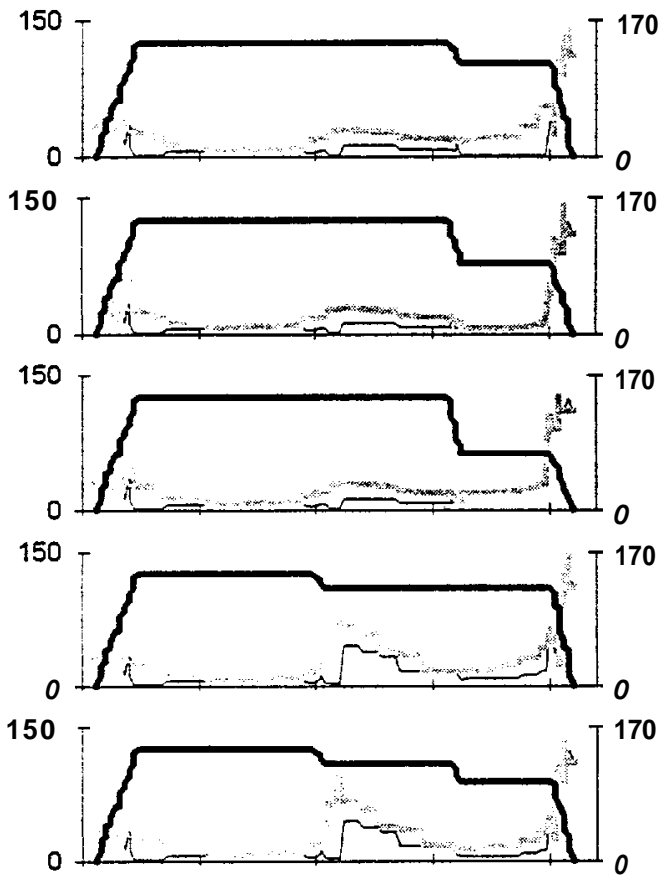

$150-$

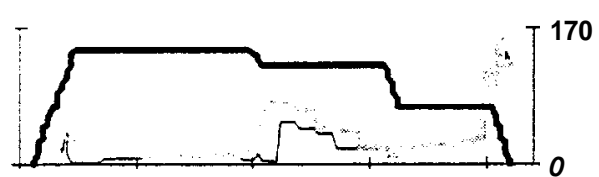

150 r

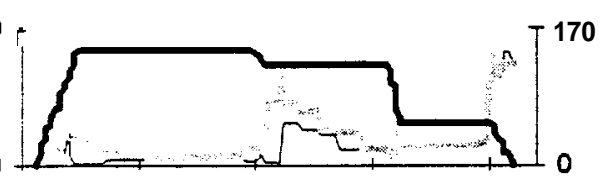

150

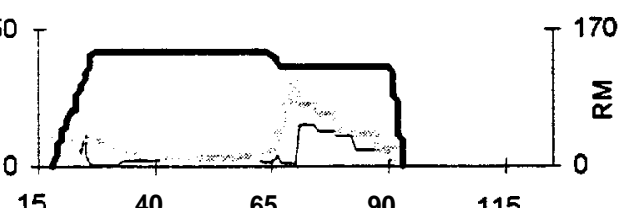

Statistical week

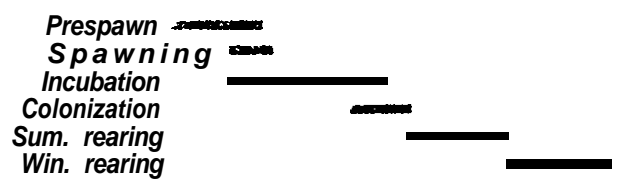

\begin{tabular}{|r|c|}
\hline \multicolumn{2}{|c|}{$\begin{array}{c}\text { Template } \\
\text { Patient }\end{array}$} \\
\hline Cum. & Cum. \\
Prod. & Cap. \\
Traj. \#1 \\
\hline 269 & 0.96 \\
0 & 0.00 \\
\hline
\end{tabular}

Traj. \#2

$314 \quad 1.12$

00.00

Traj. \#3

2800.85

0.00

Traj. \#4

295 1.05

0.00

Traj. \#5

\begin{tabular}{l|l|}
\hline 245 & 0.97 \\
\hline
\end{tabular}

0.00

Traj. \#6

\begin{tabular}{l|l|}
\hline 241 & 0.93 \\
\hline & 0.00
\end{tabular}

0.00

Traj. \#7

254 1.00

0) 0.00

Traj. \#8

\begin{tabular}{l|l|}
230 & 0.91 \\
\hline
\end{tabular}

0) 0.00

Traj. \#9

\begin{tabular}{|r|r|}
\hline 438 & 2.01 \\
0 & 0.00
\end{tabular} 
Spawning reach: Grande Ronde R.

Beaver Cr - Meadow Cr (RM 145.8 - 147.0 from Snake R)

Relative Productivity
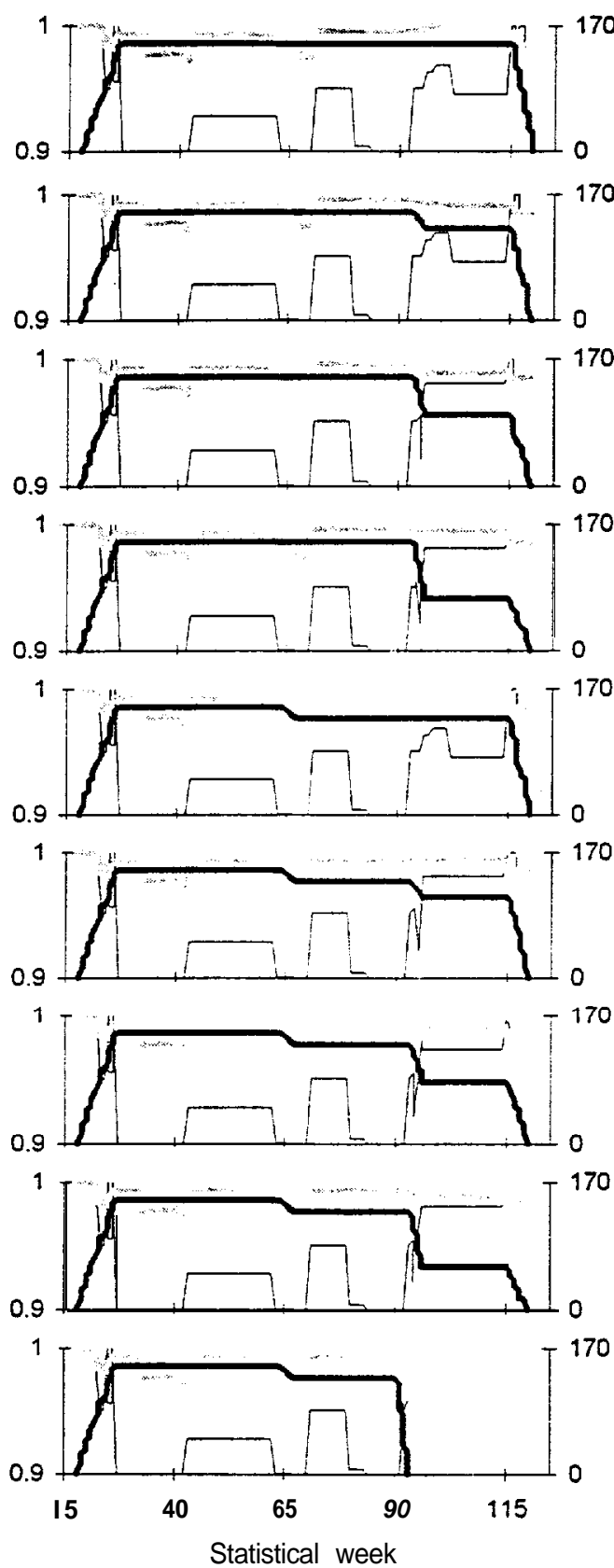

Prespawn -

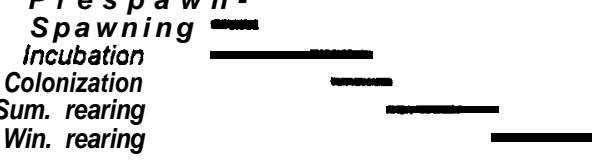

Relative Key Habitat Quantity

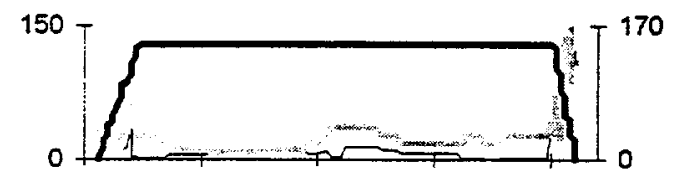

$150 T$
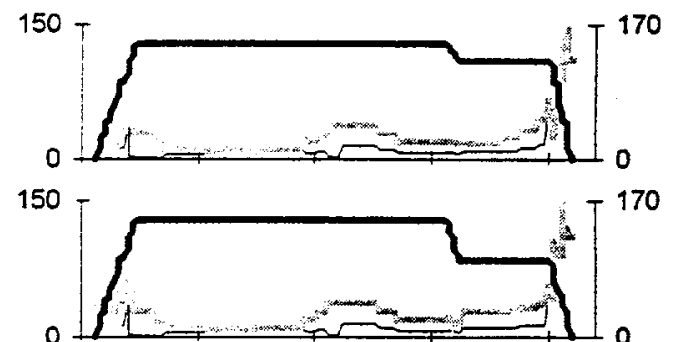

150

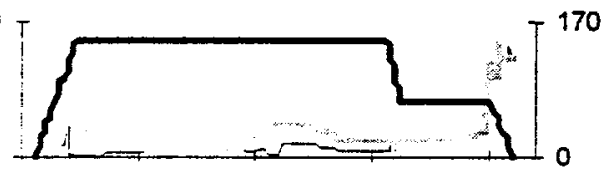

150 -

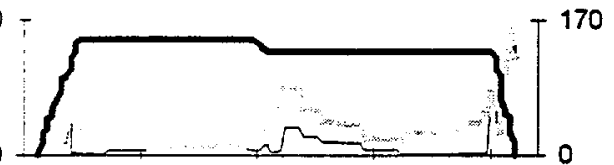

0
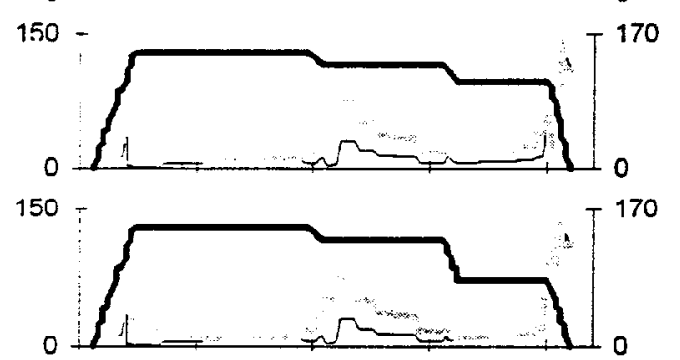

150

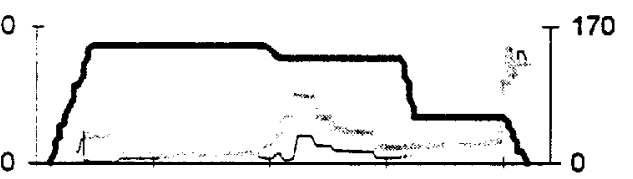

$150 \mathrm{~T}$

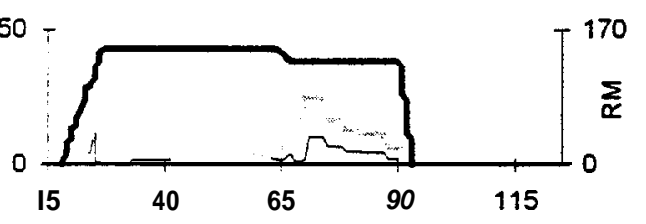

Statistical week

\begin{tabular}{|c|}
\hline $\begin{array}{c}\text { Template } \\
\text { Patient }\end{array}$ \\
\hline Cum. \\
Prod.| Cam. \\
\hline
\end{tabular}

\begin{tabular}{|r|r|}
\hline Traj . \#1 \\
\hline 283 & 1.03 \\
0 & 0.00
\end{tabular}

Traj. \#2

2991.08

0.00

Traj. \#3

\begin{tabular}{l|l|}
280 & 1.04 \\
\hline
\end{tabular}

0.0 .00

Traj. \#4

3090

0) 0.00

Traj. \#5

\begin{tabular}{l|l}
\hline 268 & 1.03
\end{tabular}

0.00

Traj. \#6

$294 \quad 1.06$

0) 0.00

Traj. \#7

\begin{tabular}{|l|l|}
\hline 279 & 0.97 \\
\hline
\end{tabular}

\begin{tabular}{l|l}
0 & 0.00
\end{tabular}

Traj. \#8

\begin{tabular}{r|r|}
266 & 0.99 \\
0 & 0.00
\end{tabular}

Traj. \#9

4992.21

0) 0.00

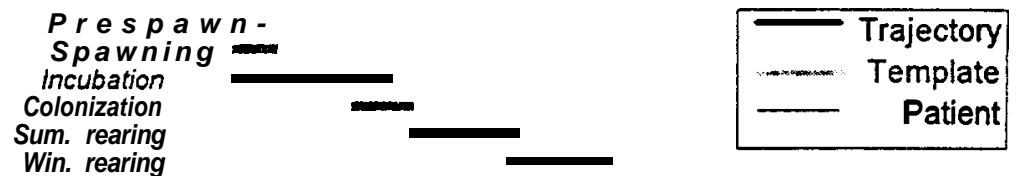


Spawning reach: Grande Ronde R.

Meadow Cr - Fly Cr (RM 147.0 - 151.3 from Snake R)

Relative Productivity
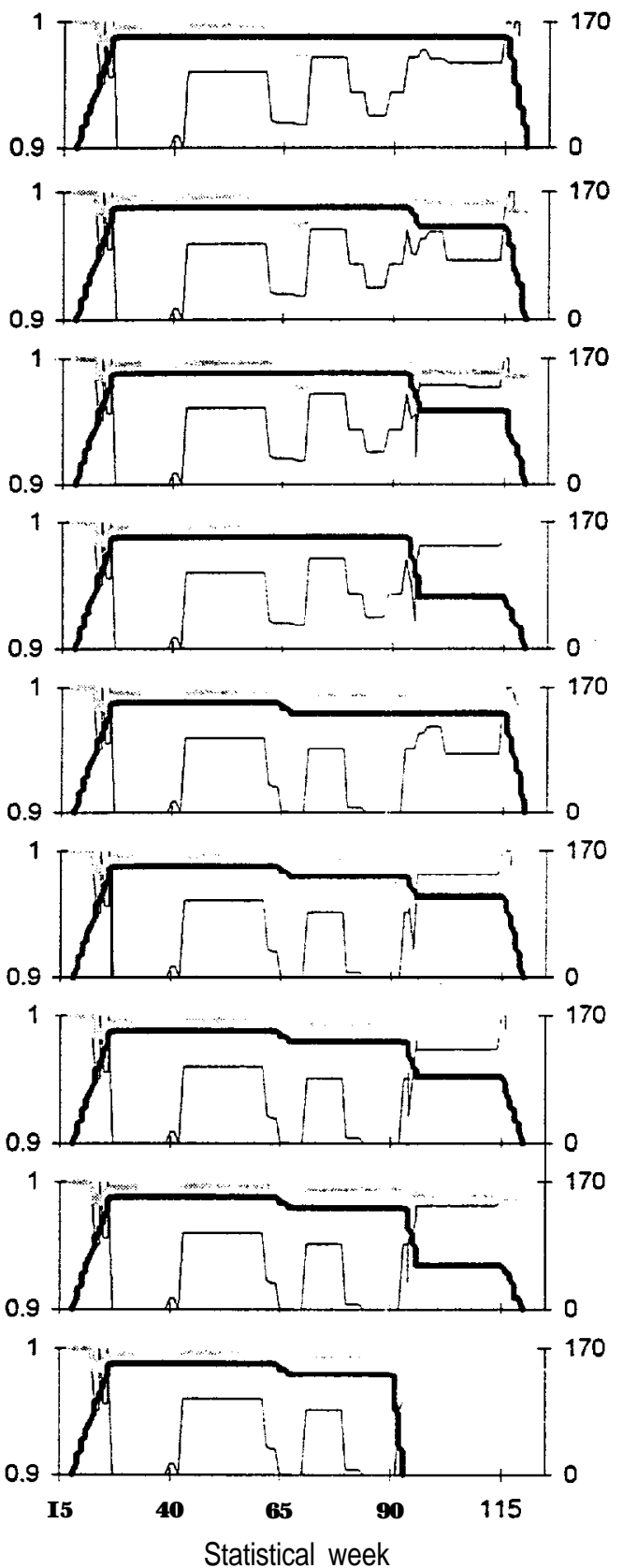

Relative Key Habitat Quantity
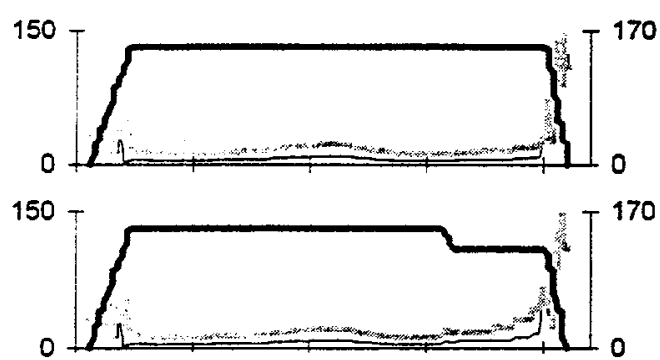

150 -

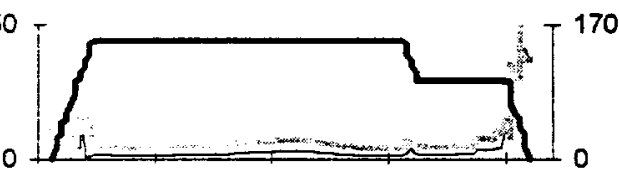

150

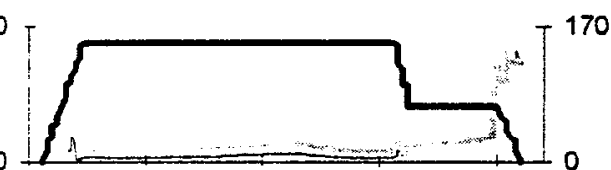

$150+$

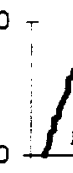

150 -

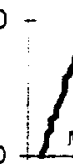

$150-$

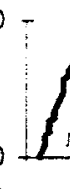

$150-$

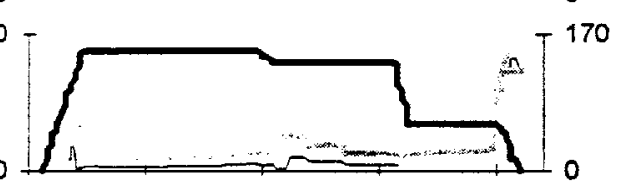

150 .
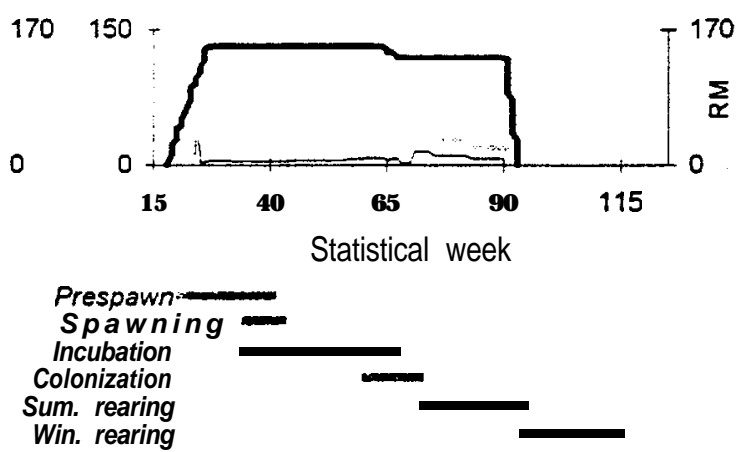

Win. rearing

\begin{tabular}{l} 
Template \\
Patient \\
\hline Cum. Coum. \\
Prodi. | Coap.
\end{tabular}

Traj. \#1

\begin{tabular}{l|l|}
\hline 352 & 0.83 \\
\hline
\end{tabular}

2) 0.00

Traj. \#2

3500 0087

1) 0.00

Traj. \#3

3290.80

2) 0.00

Traj. \#4

\begin{tabular}{l|l}
361 & 0.89 \\
\hline
\end{tabular}

2) 0.00

Traj. \#5

\begin{tabular}{r|r|}
\hline 344 & 0.94 \\
0 & 0.00
\end{tabular}

Traj. \#6

$377 \quad 1.01$

0) 0.00

Traj. \#7

\begin{tabular}{l|l|}
\hline 358 & 0.85 \\
\hline & 0.00
\end{tabular}

0) 0.00

\begin{tabular}{r|r} 
Traj. \#8 \\
\hline 342 & 0.94 \\
0 & 0.00
\end{tabular}

Traj. \#9

\begin{tabular}{l|l}
\hline 641 & 2.07 \\
\hline & 0.00
\end{tabular}

0) 0.00

Prespawn
Spawning

Incubation
Colonization

Sum. rearing
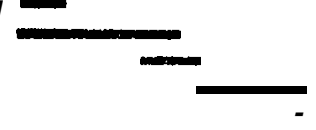
Spawning reach: Grande Ronde R.

Fly $\mathrm{Cr}$ - head of canyon 1.3 miles downstream of Sheep $\mathrm{Cr}$ (RM 151.3 - 159.4 from Snake R)

Relative Productivity
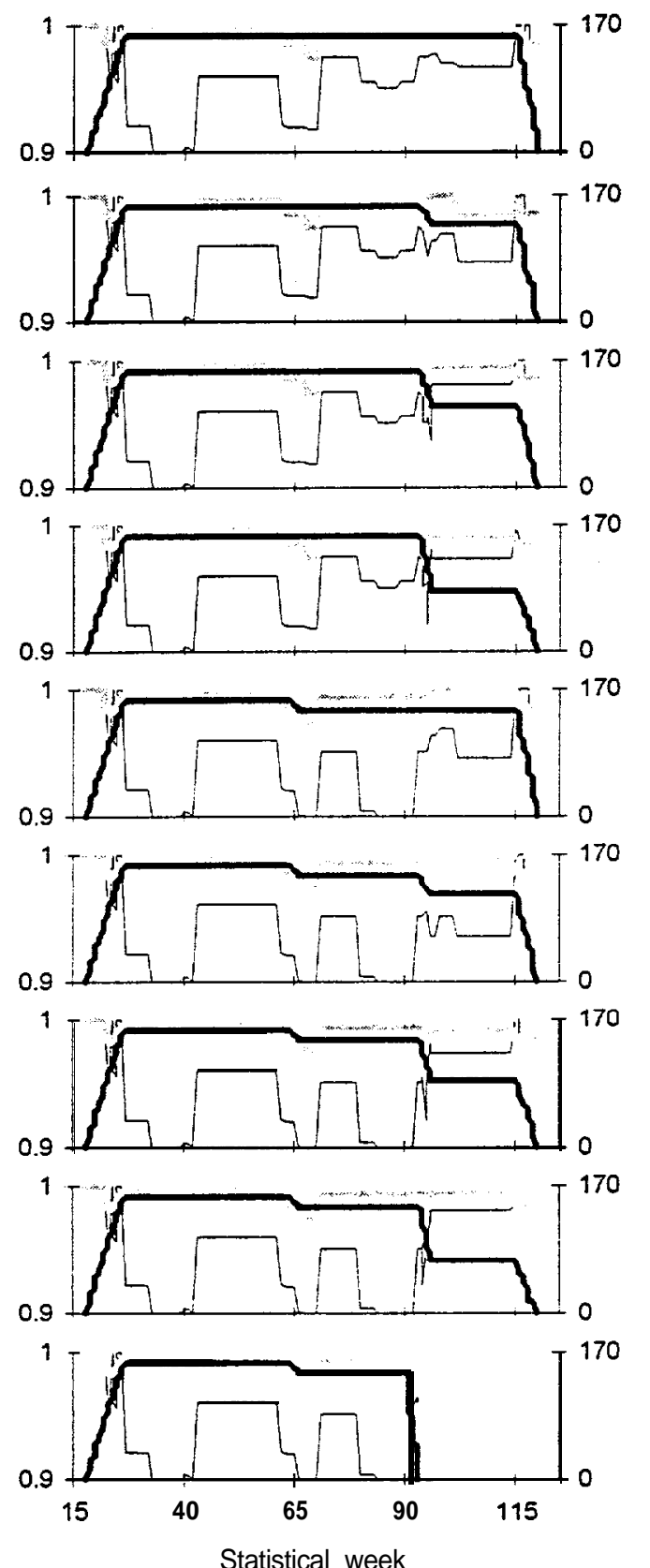

Relative Key Habitat Quantity
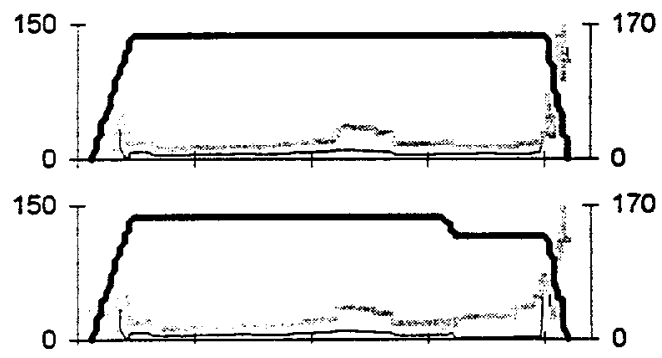

150

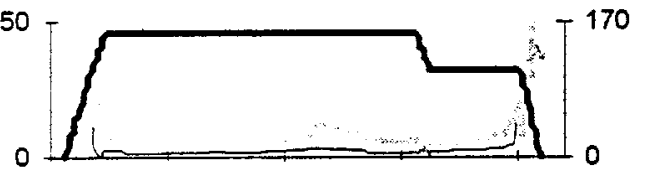

$150+$

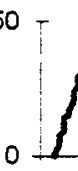

150 -

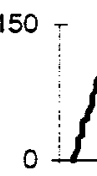

150 .

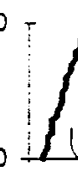

150 T
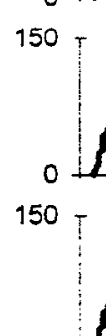

${ }^{150}$

150
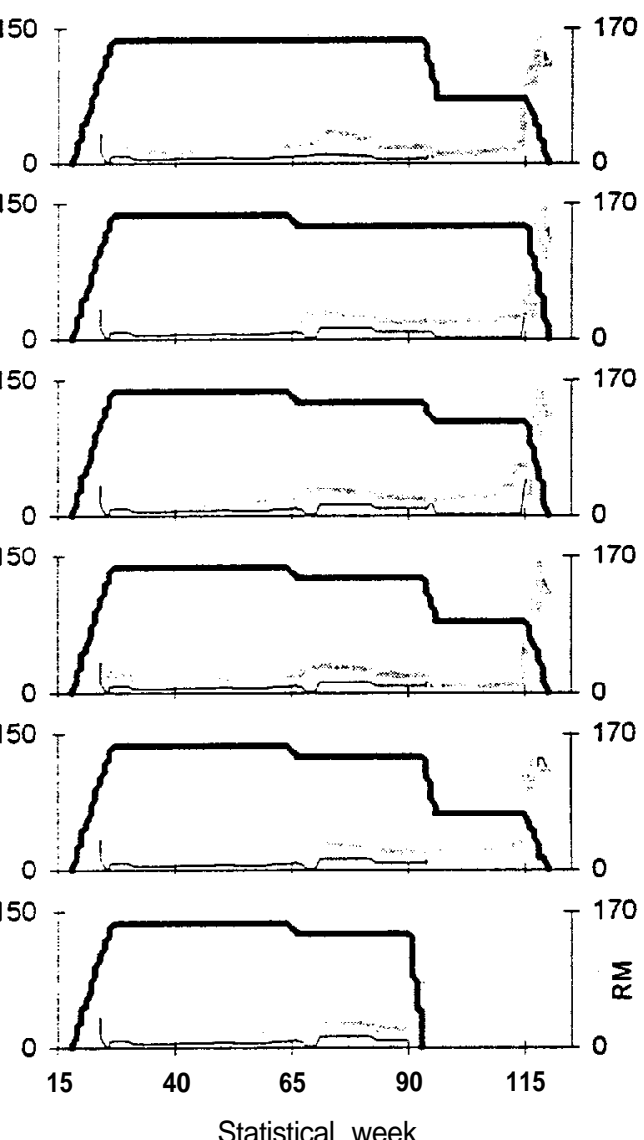

$-170$

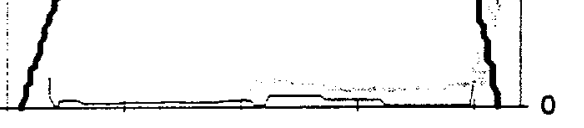

res p a w n -

Spawning

Incubation

Colonization

Sum. rearing
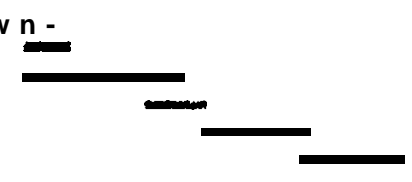

Win. rearing

Prespawn -

Spawning
Incubation
Colonization
Sum. rearing

Win. rearing

\begin{tabular}{|r|c|}
\hline \multicolumn{2}{|c|}{$\begin{array}{c}\text { Template } \\
\text { Patient }\end{array}$} \\
\hline Cum. & Cum. \\
Prod. & Cap. \\
Traj. \#1 \\
\hline 352 & 0.94 \\
4 & 0.01 \\
\hline
\end{tabular}

Traj. \#2

3280.94

3) 0.00

Traj. \#3

$364 \quad 0.99$

\begin{tabular}{l|l}
4 & 0.01
\end{tabular}

Traj. \#4

344 0.91

4) 0.01

Traj. \#5

345 0.97

0) 0.00

Traj. \#6

\begin{tabular}{r|l}
402 & 1.14 \\
0 & 0.00
\end{tabular}

Traj. \#7

$360 \quad 0.86$

0) 0.00

Traj. \#8

\begin{tabular}{|l|l|}
\hline 379 & 1.06 \\
\hline
\end{tabular}

0) 0.00

Traj. \#9

\begin{tabular}{l|l}
647 & 2.14 \\
\hline
\end{tabular}

\begin{tabular}{l|l}
0 & 0.00 \\
\hline
\end{tabular} 
Spawning reach: Grande Ronde R.

Head of canyon 1.3 miles downstream of Sheep Cr - LimberJim Cr (RM 159.4 - 164.2 from Snake F!

Relative Productivity
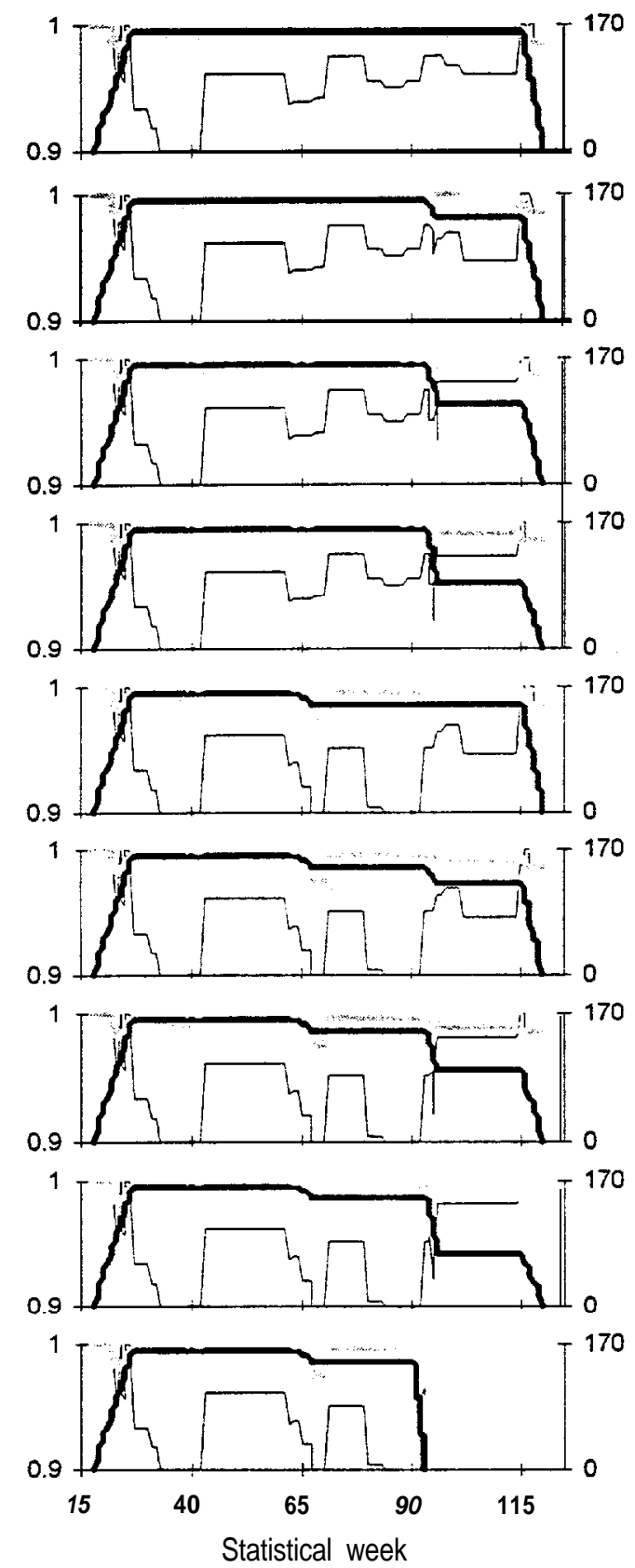

Prespawn -

Spawning

Incubation

Colonization

Sum. rearing

Win. rearing
Relative Key Habitat Quantity
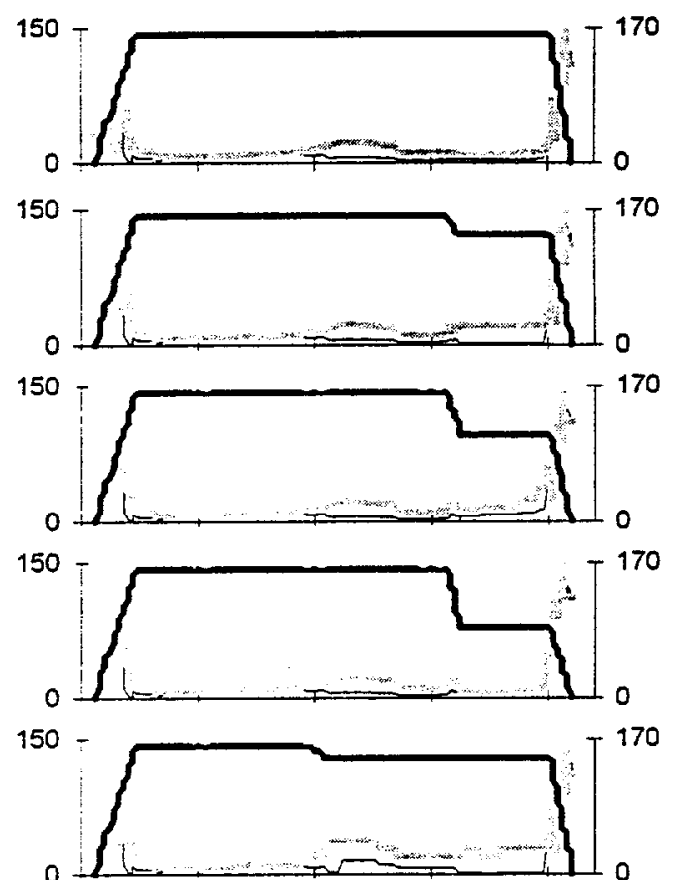

$150 T$

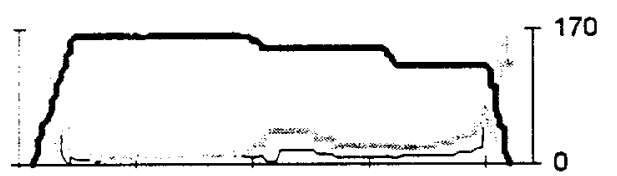

150

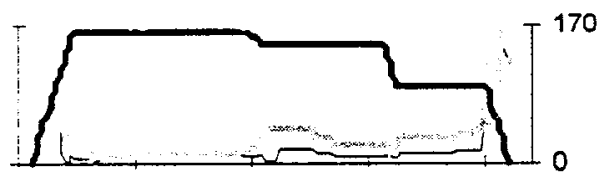

150 -
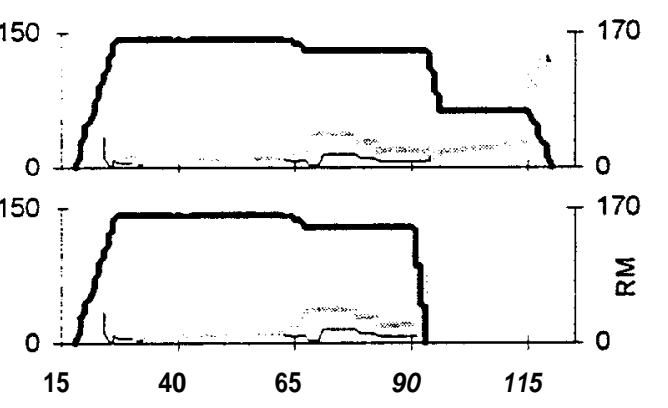

Statistical week

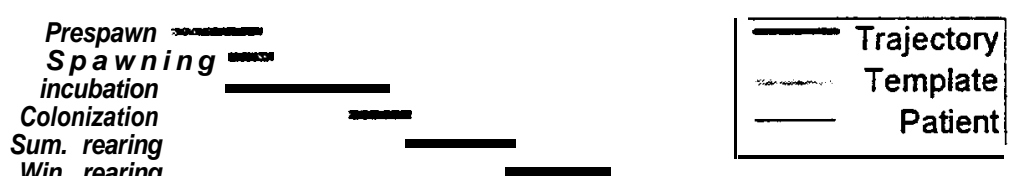


Spawning reach: Grande Ronde R.

LimberJim Cr - Clear Cr (RM 164.2 - 166.9 from Snake R)

Relative Productivity
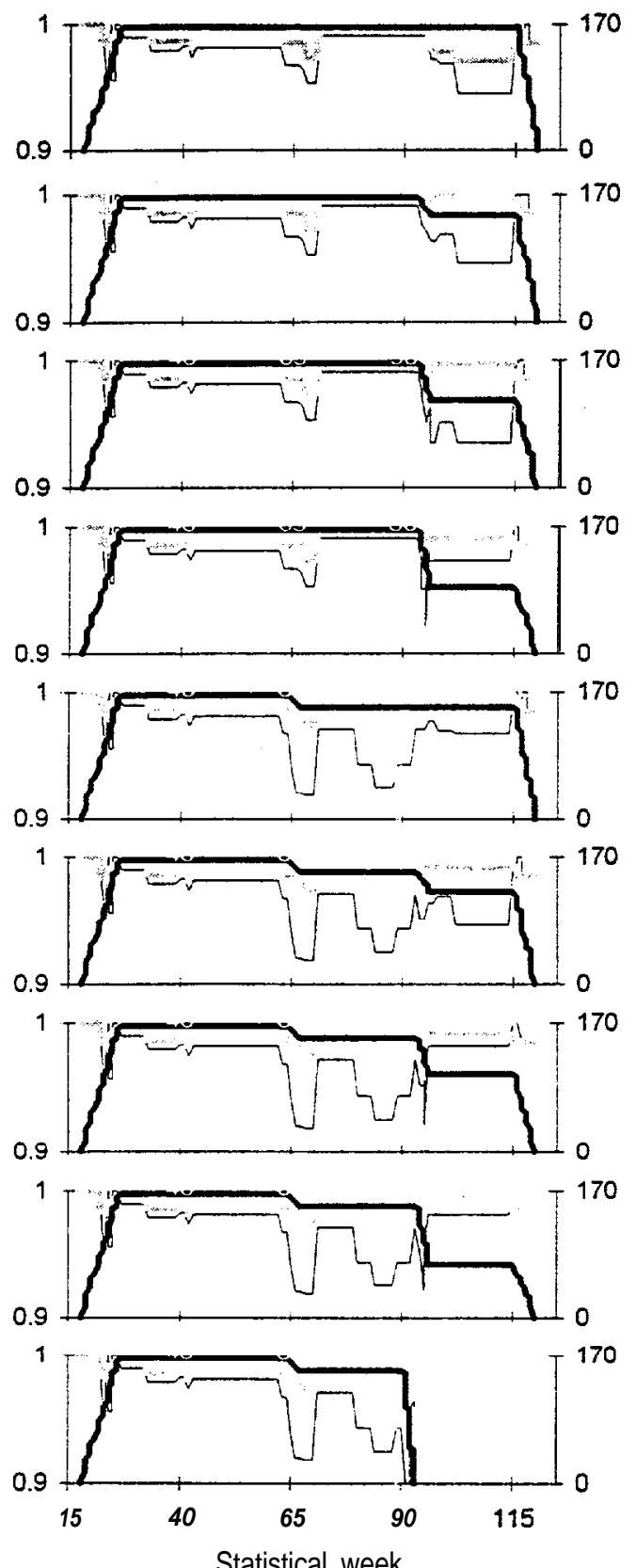

Prespawn -

Spawning

Incubation

Colonization

Sum. rearing

Win. rearing
Relative Key Habitat Quantity
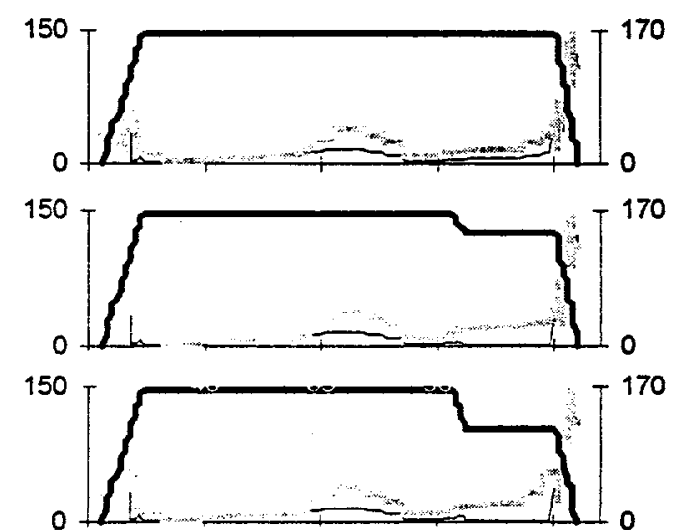

$150-$

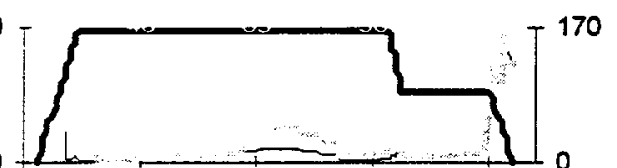

150

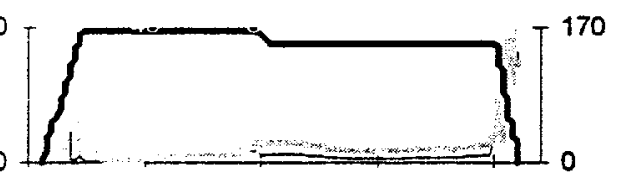

$0+12,110$

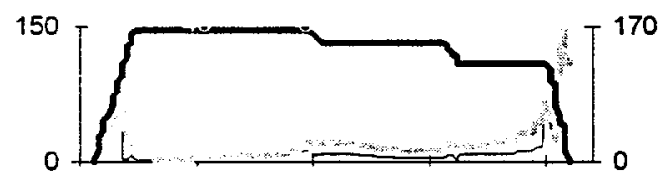

150
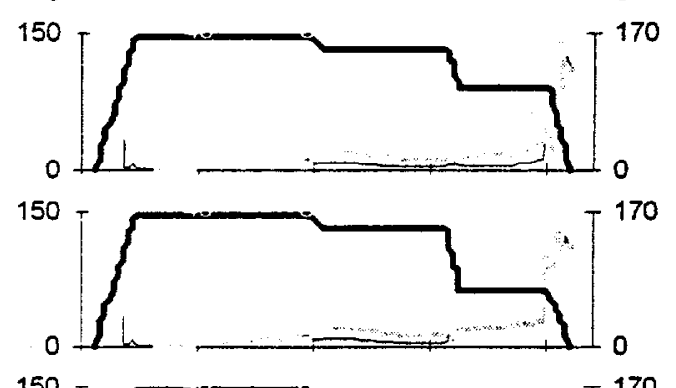

$\int_{15}^{150}$

Prespawn -

Spawning

Incubation

Colonization

Sum. rearing

Win. rearing

Statistical week

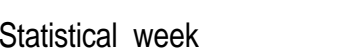

\begin{tabular}{|c|c|}
\hline \multicolumn{2}{|c|}{$\begin{array}{c}\text { Template } \\
\text { Patient }\end{array}$} \\
\hline Cum. & Cum. \\
Prod. & Cap. \\
\hline
\end{tabular}

Traj. \#1

\begin{tabular}{r|r|}
248 & 0.39 \\
88 & 0.03
\end{tabular}

Traj. \#2

$326 \quad 0.52$

$84 \quad 0.03$

Traj. \#3

3810.61

$62 \quad 0.02$

Traj. \#4

\begin{tabular}{l|l|}
\hline 344 & 0.50 \\
\hline
\end{tabular}

120 0.05

Traj. \#5

\begin{tabular}{|l|l|}
\hline 336 & 0.52 \\
\hline
\end{tabular}

\begin{tabular}{l|l}
36 & 0.01
\end{tabular}

Traj. \#6

\begin{tabular}{l|l}
336 & 0.54 \\
\hline
\end{tabular}

25 0.01

Traj. \#7

\begin{tabular}{l|l|}
\hline 331 & 0.52 \\
\hline
\end{tabular}

$\begin{array}{ll}43 & 0.02\end{array}$

Traj. \#8

\begin{tabular}{r|r|}
\hline 345 & 0.55 \\
41 & 0.02
\end{tabular}

Traj. \#9

594 1.03

$80 \mid 0.03$

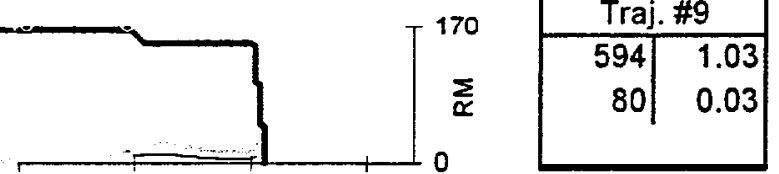

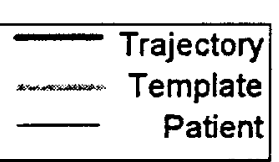


Spawning reach: Grande Ronde R.

Clear $\mathrm{Cr}$ - headwaters (RM 166.9 - 170.1 from Snake R)

Relative Productivity
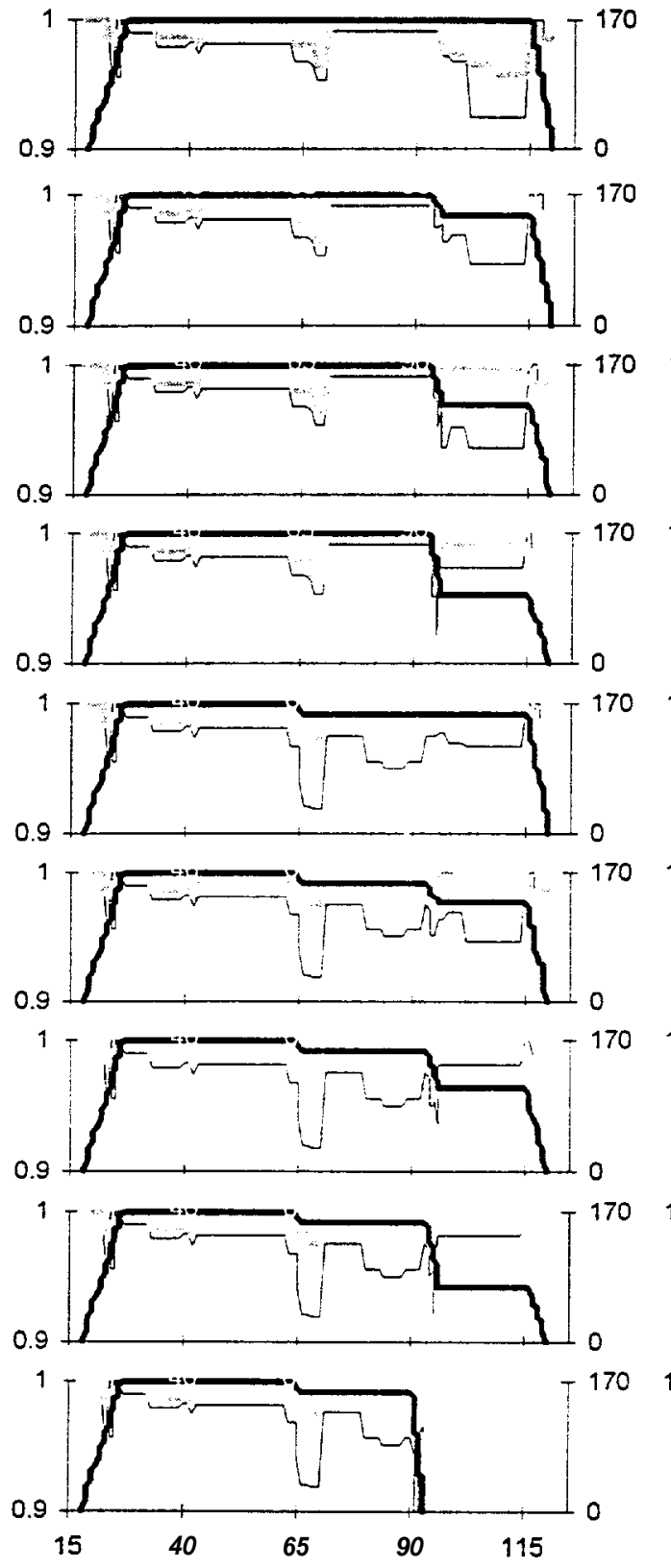

Statistical week

Prespawn -

Spawning

Incubation

Colonization

Sum. rearing

Win. rearing
Relative Key Habitat Quantity
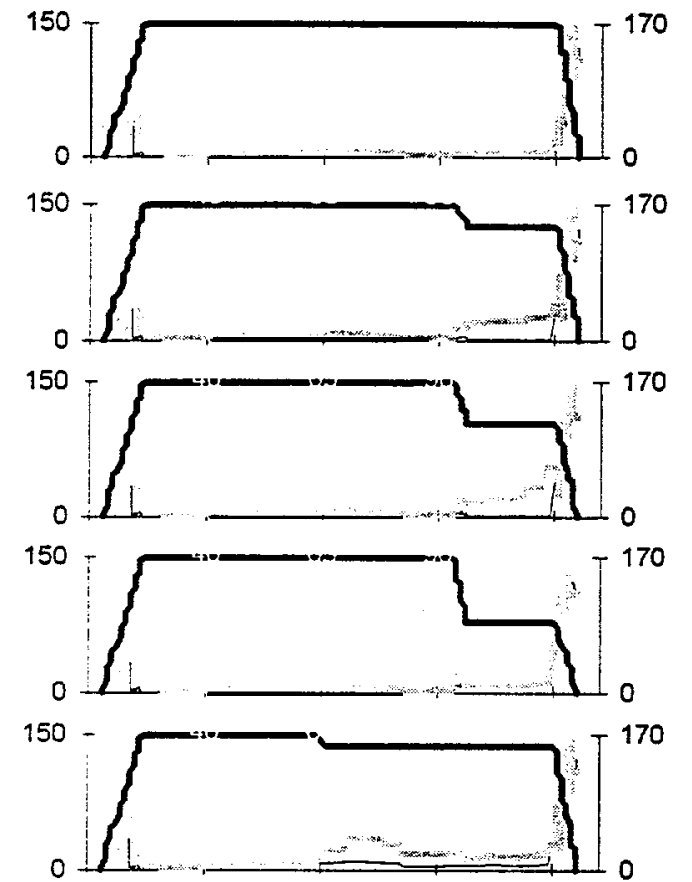

150
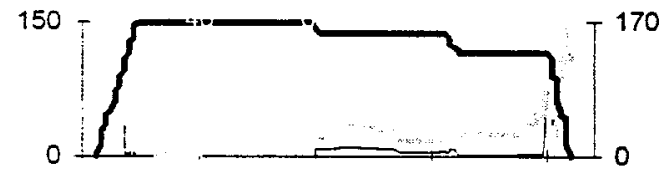

$150 \div-170$
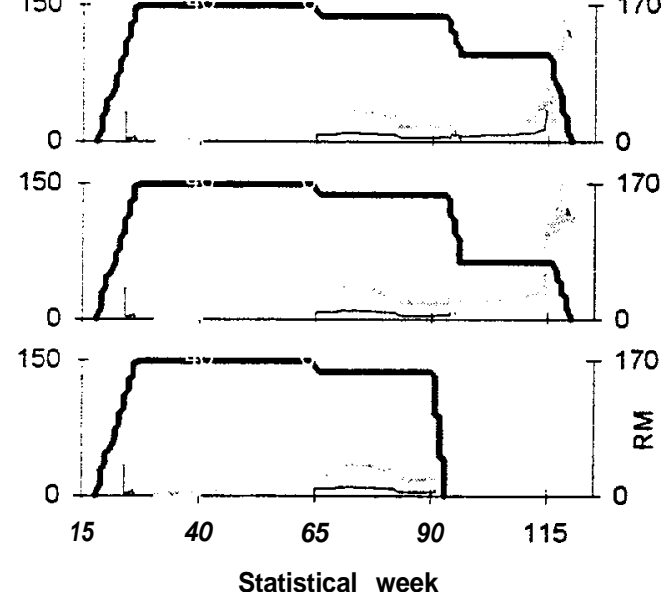

Prespawn -

Spawning

Incubation

Colonization

Sum. rearing

Win. rearing \begin{tabular}{|c|c|}
\hline \multicolumn{2}{|c|}{ Template } \\
Patient \\
\hline Cum. & Cum. \\
Prod. & Cap. \\
\hline
\end{tabular}

Traj. \#1

1950.17

$66 \quad 0.06$

Traj. \#2

3090.29

$86 \quad 0.07$

Traj. \#3

$361 \quad 0.34$

$63 \quad 0.05$

Traj. \#4

3250.29

$120 \quad 0.11$

Traj . \#5

$332 \quad 0.42$

48 0.05

Traj. \#6

3100.40

33 0.03

Traj. \#7

$344 \quad 0.44$

$57 \quad 0.06$

Traj. \#8

3410.44

56 0.06

Traj. \#9

586 0.81

106

0.12 
Spawning reach: Hurricane Cr.

Mouth - Lower Alderslope Ditch (RM 121.9 - 124.9 from Snake R)

Relative Productivity
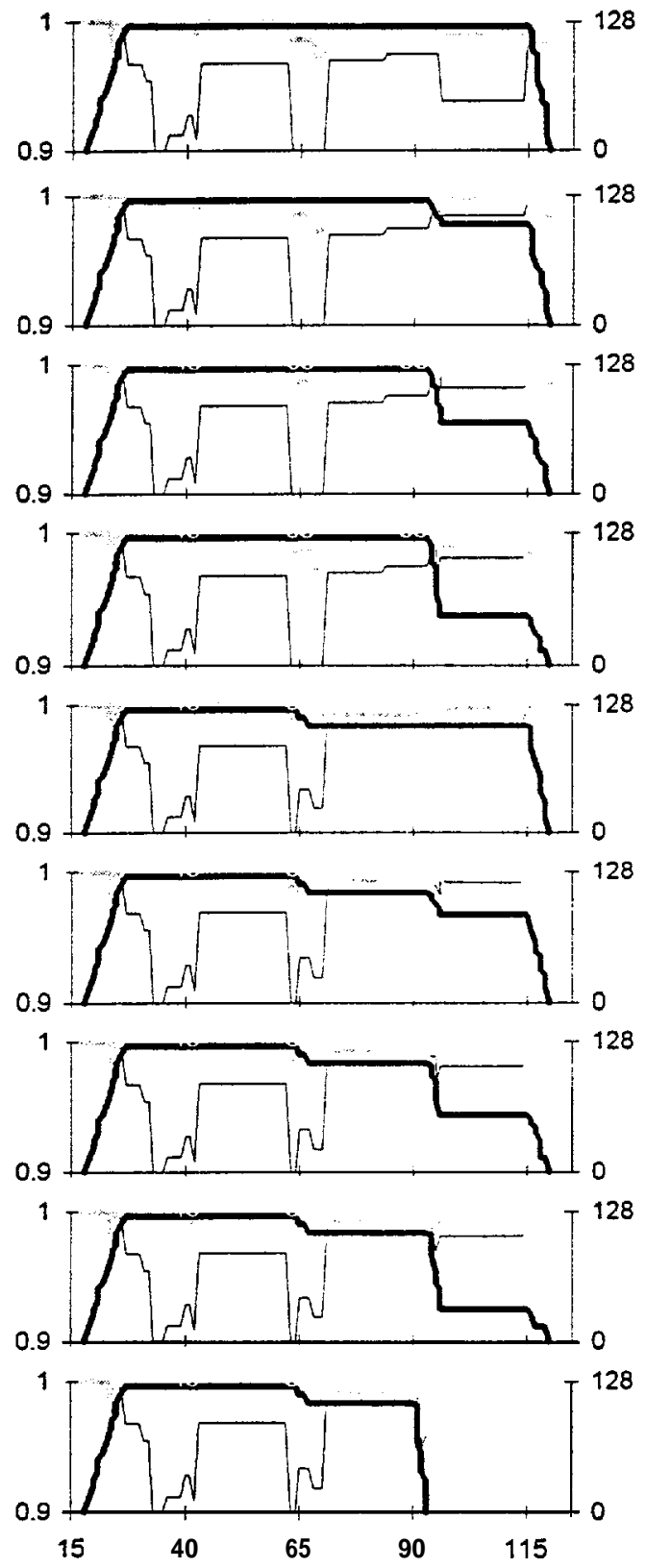

Statistical week

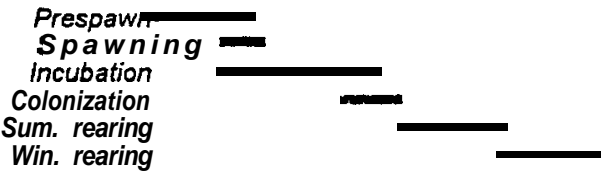

Relative Key Habitat Quantity
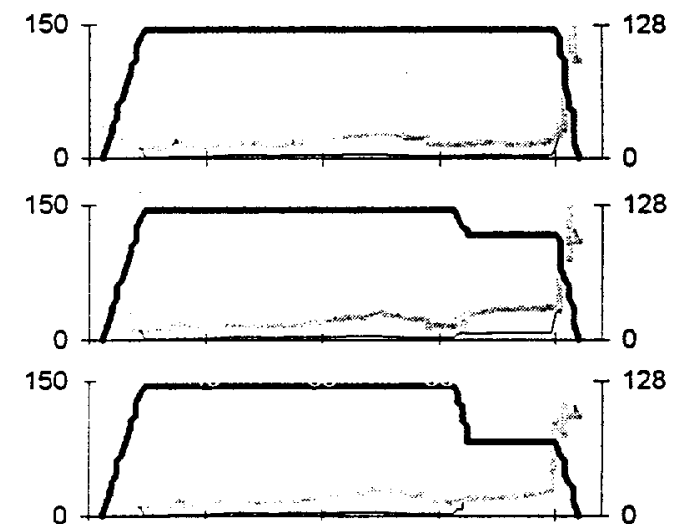

150

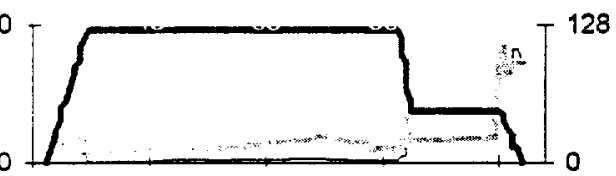

150

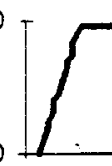

150
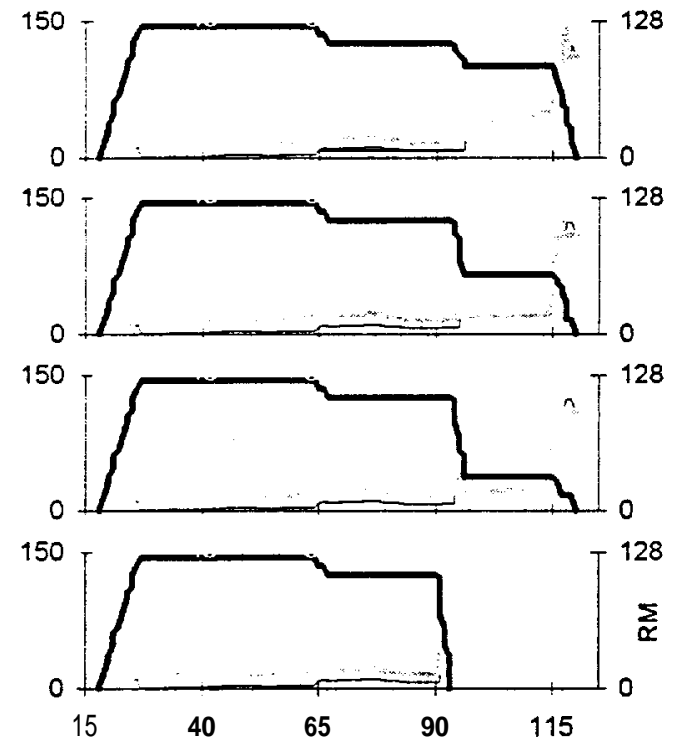

Statistical week

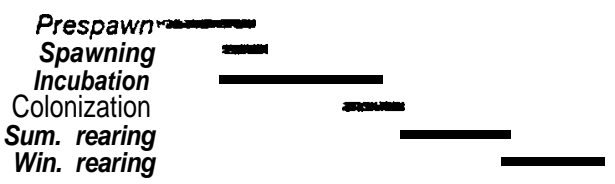

\begin{tabular}{|c|}
\hline $\begin{array}{c}\text { Template } \\
\text { Patient }\end{array}$ \\
\hline $\begin{array}{c}\text { Cum. } \\
\text { Prod. }\end{array}$ Cam. \\
Cap.
\end{tabular}

Traj. \#1

\begin{tabular}{l|l|}
\hline 420 & 0.84 \\
\hline & 0.00
\end{tabular} 40.00

Traj. \#2

\begin{tabular}{l|l|}
468 & 1.00 \\
\hline & 0.00
\end{tabular}

\begin{tabular}{l|l}
9 & 0.00
\end{tabular}

Traj . \#3

\begin{tabular}{l|l|}
439 & 0.92 \\
\hline & 0.00
\end{tabular}

9) 0.00

Traj. \#4

$397 \quad 0.85$

9 0.00

Traj. \#5

$501 \quad 0.98$

$32 \quad 0.01$

Traj. \#6

$468 \quad 0.97$

35 0.01

Traj. \#7

$423 \quad 0.84$

29 0.01

Traj . \#8

\begin{tabular}{l|l}
442 & 0.89 \\
\hline
\end{tabular}

28 0.01

Traj. \#9

\begin{tabular}{|l|l|}
792 & 1.81 \\
\hline
\end{tabular}

$60 \quad 0.02$ 
Spawning reach: Hurricane $\mathrm{Cr}$.

Lower Alderslope Ditch - Upper Alderslope Ditch Diversion (RM 124.9 - 128.2 from Snake R)

Relative Productivity
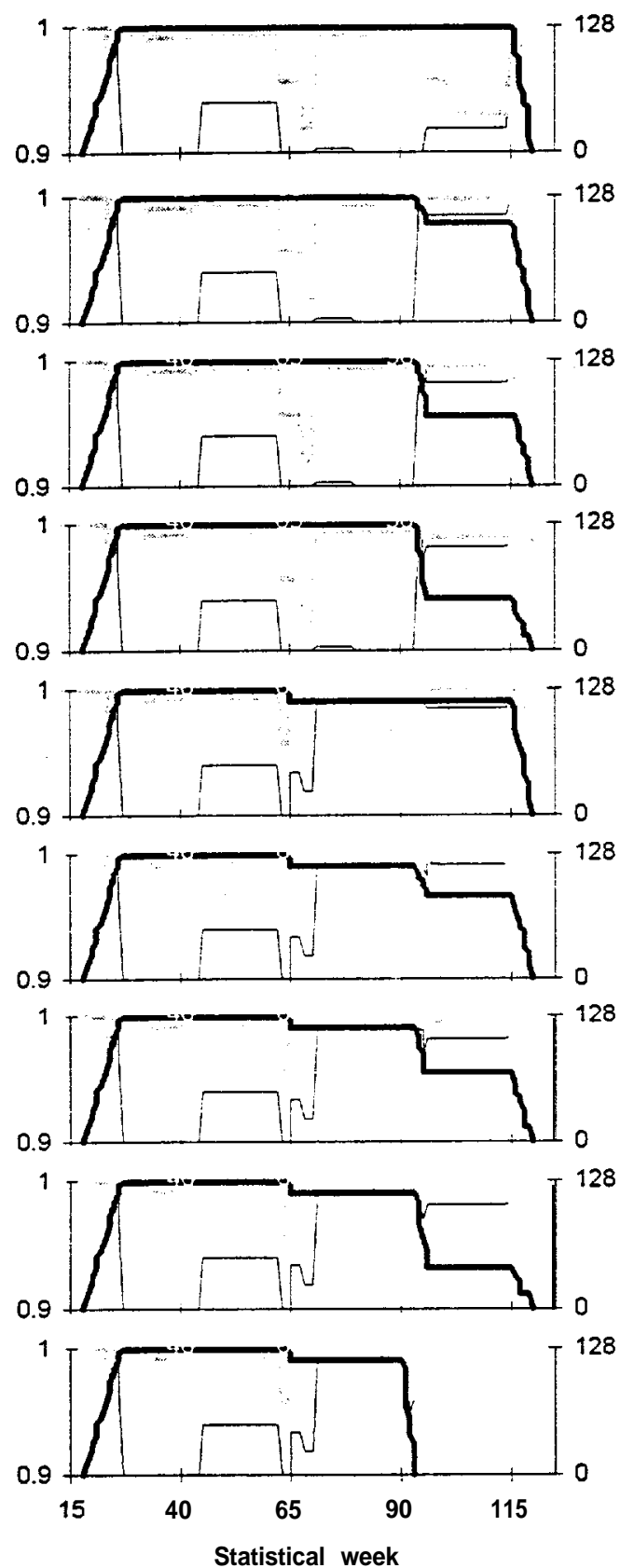

Prespawn -

Spawning

Incubation

Colonization

Sum. rearing

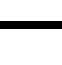

Relative Key Habitat Quantity
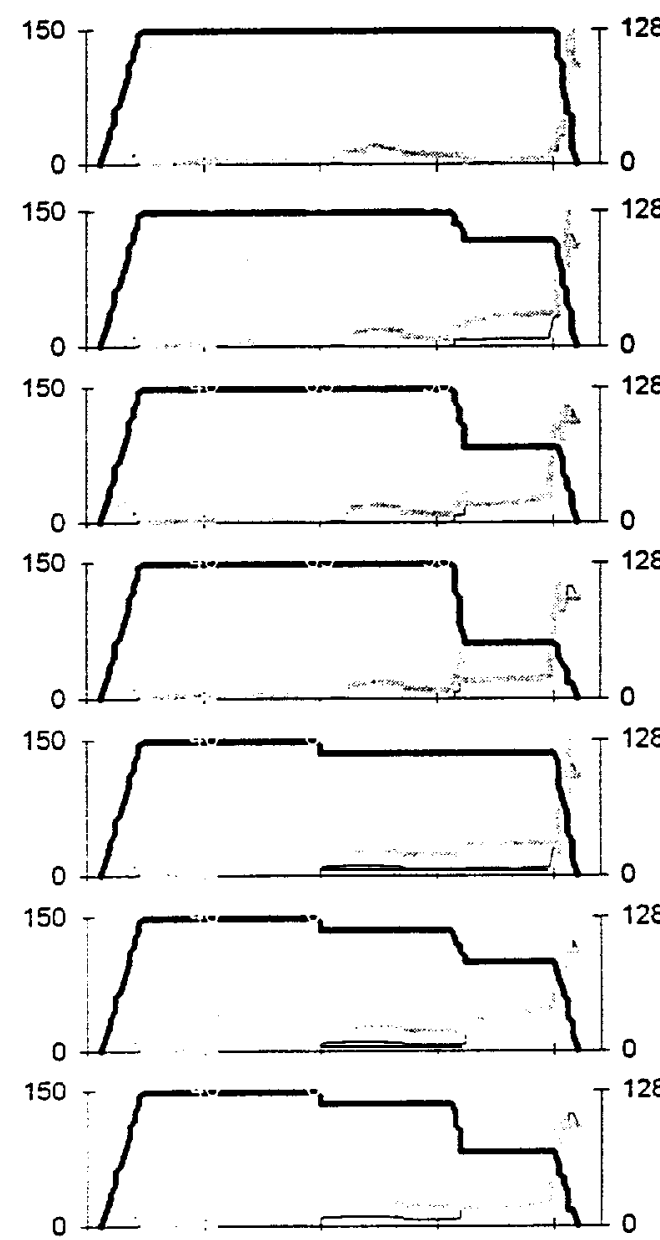

150 -
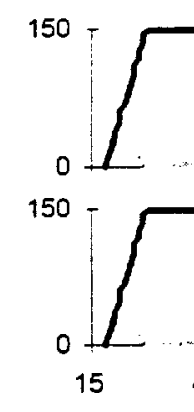

40
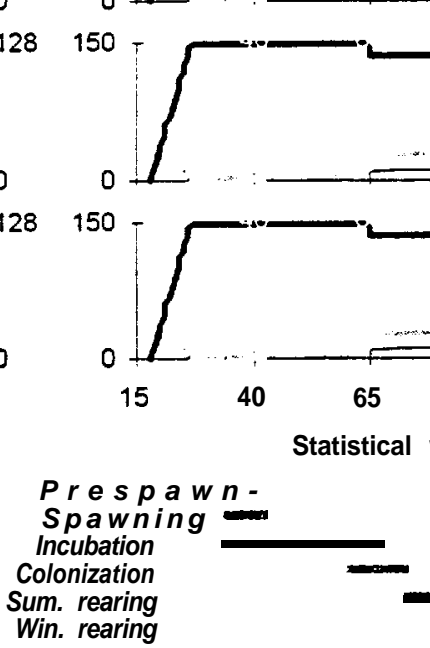

\begin{tabular}{|r|c|}
\hline \multicolumn{2}{|c|}{$\begin{array}{c}\text { Template } \\
\text { Patient }\end{array}$} \\
\hline Cum. & Cum. \\
Prod. & Cap. \\
Traj. \#1 \\
\hline 92 & 0.04 \\
0 & 0.00 \\
\hline
\end{tabular}

Traj. \#2

2870.11 0.00

Traj. \#3

2690.11 0) 0.00

Traj. \#4

$243 \quad 0.10$

0) 0.00

Traj. \#5

\begin{tabular}{l|l}
422 & 0.17 \\
\hline
\end{tabular}

0) 0.00

Trinj j\#6

374 00.115

0) $\quad 0.00$

Traj. \#7

$374 \quad 0.15$

0) $\quad 0.00$

Traj. \#8

\begin{tabular}{l|l}
353 & 0.14
\end{tabular}

0) 0.00

Traj. \#9

\begin{tabular}{r|r|}
633 & 0.26 \\
0 & 0.00 \\
\hline
\end{tabular}

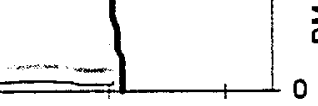

$65 \quad 90 \quad 115$

tatistical week 
Spawning reach: Joseph $\mathrm{Cr}$.

Cottonwood Cr - Swamp Cr (RM 8.2 - 37.7 from Snake R)

Relative Productivity

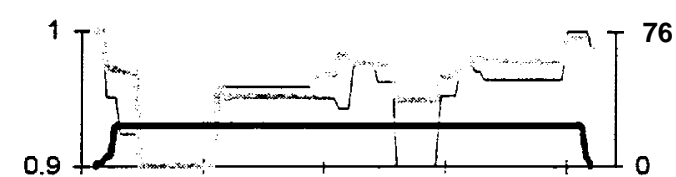

Relative Key Habitat Quantity
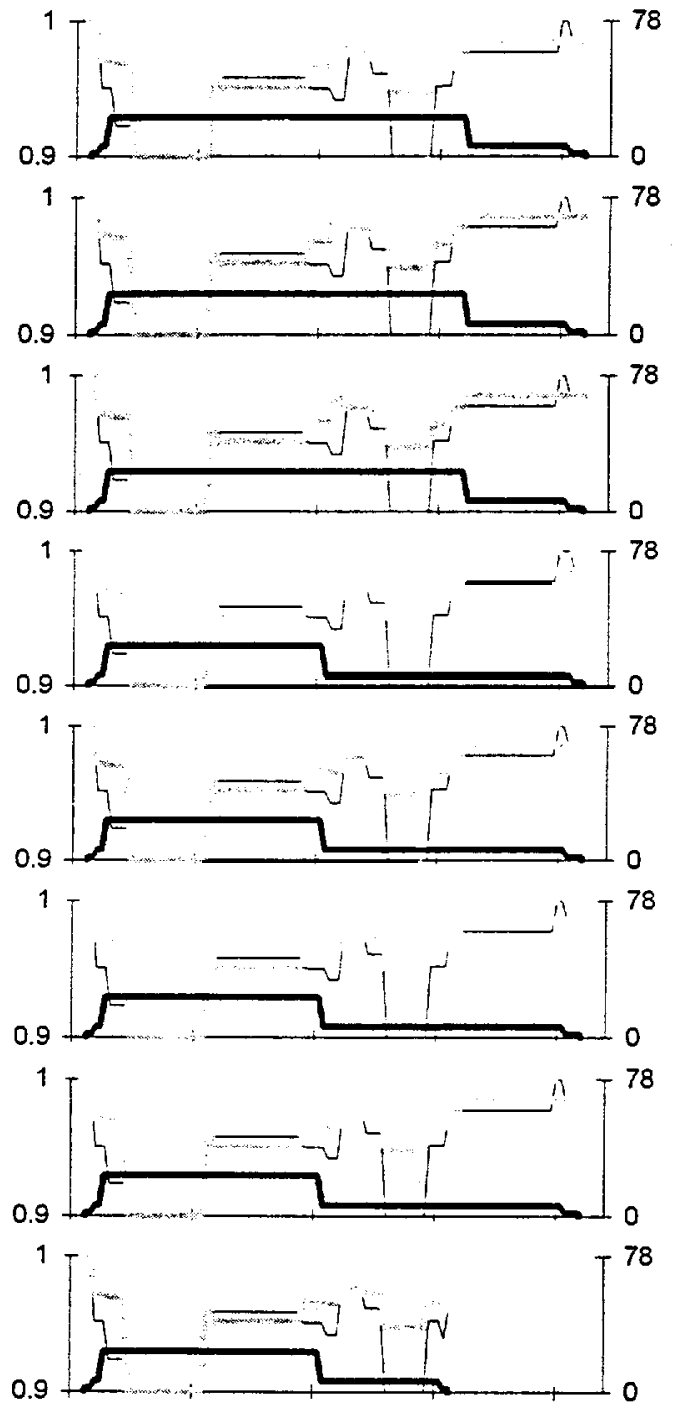

0.9

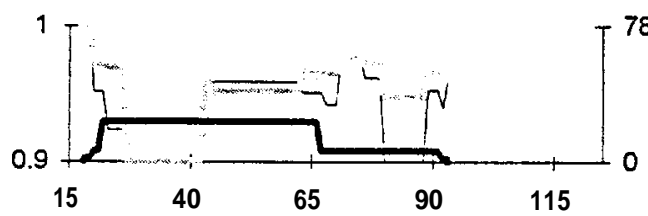

Statistical week

Prespaw $n$ -

Spawning

Incubation

Cobnization

Sum. rearing

Win. rearing
$6150-$

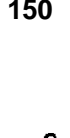

\section{Relative Key Habitat Quantily}
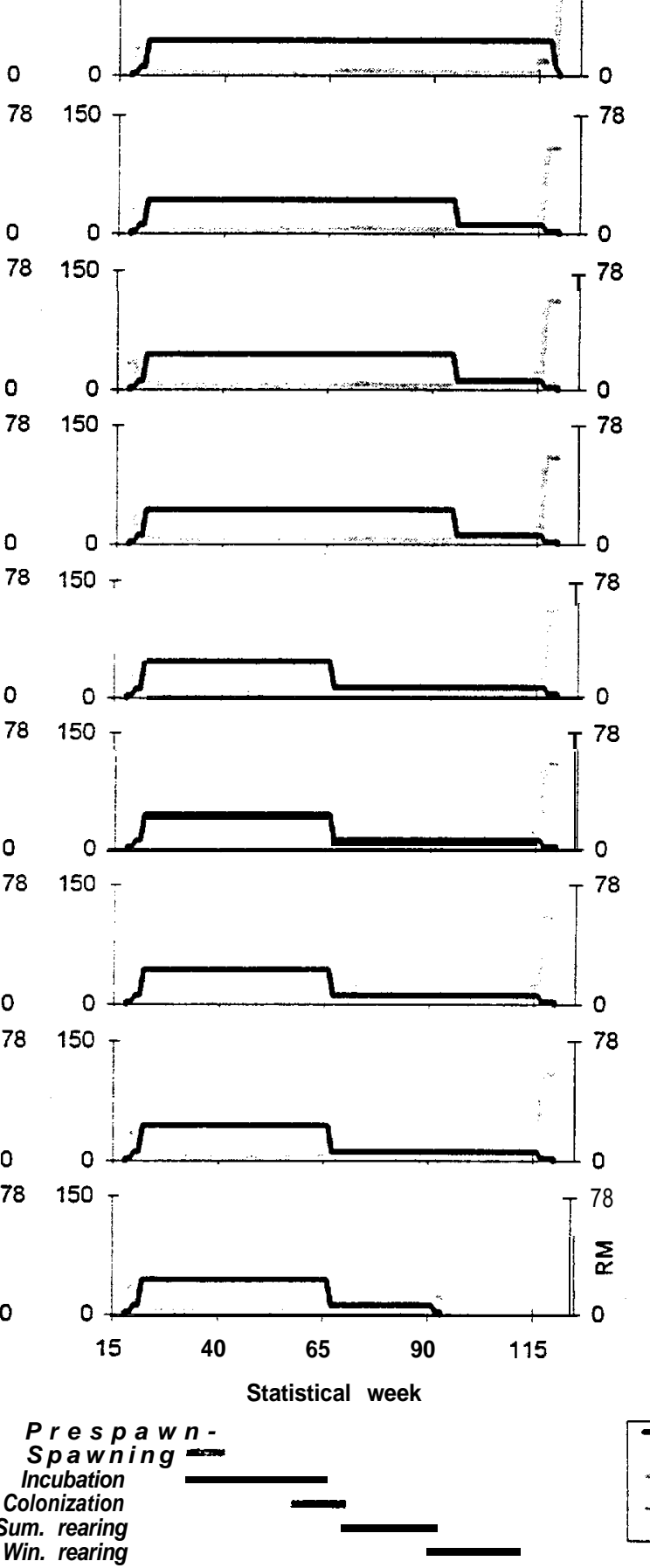

\begin{tabular}{||r|c|}
\hline \multicolumn{2}{||c|}{ Template } \\
Patient \\
\hline Cum. & Cum. \\
Prod. & Cap. \\
Traj. \#1 \\
\hline 0 & 0.00 \\
0 & 0.00 \\
\hline
\end{tabular}

Traj. \#2

00.00

o) 0.00

Traj. \#3

\begin{tabular}{l|l}
0 & $0.0 c$ \\
0 & $0.0 c$
\end{tabular}

Traj. \#4

\begin{tabular}{l|l}
0 & $0.0 C$ \\
0 & $0.0 C$
\end{tabular}

Traj. \#5

\begin{tabular}{l|l}
$0.0 \mathrm{C}$ \\
\hline
\end{tabular}

이 0.00

Traj. \#6

0) 0.00

Traj. \#7

0. 0.00

0) 0.00

Traj. \#8

\begin{tabular}{l|l}
0 & 0.00 \\
0 & 0.00
\end{tabular}

Traj. \#9

\begin{tabular}{l|l}
\hline 0 & 0.00 \\
0 & 0.00
\end{tabular}

0.00 
Spawning reach: Joseph Cr.

Swamp Cr - Chesnimnus Cr (RM 37.7 - 52.7 from Snake R)

Relative Productivity
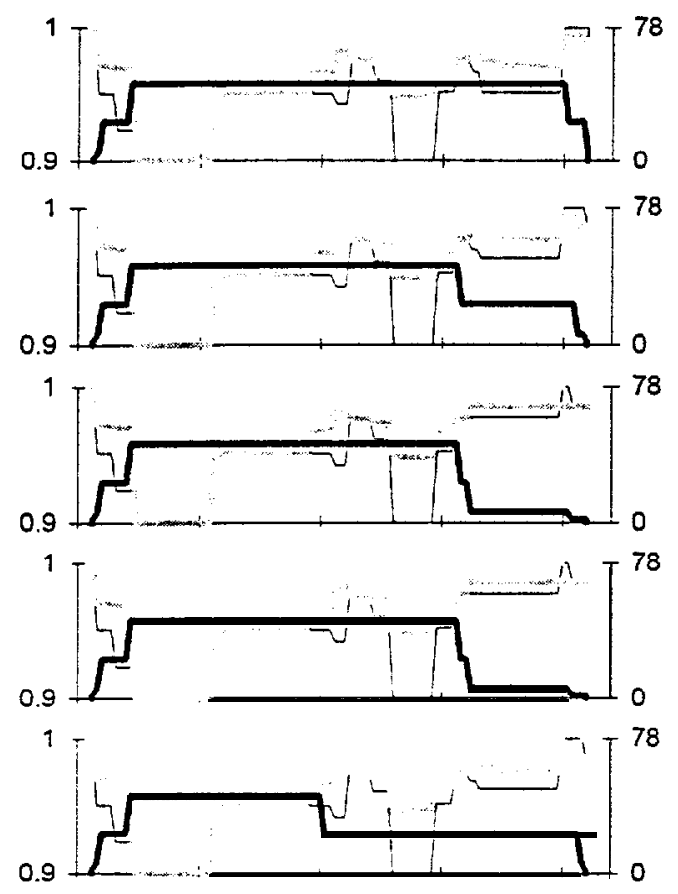

1
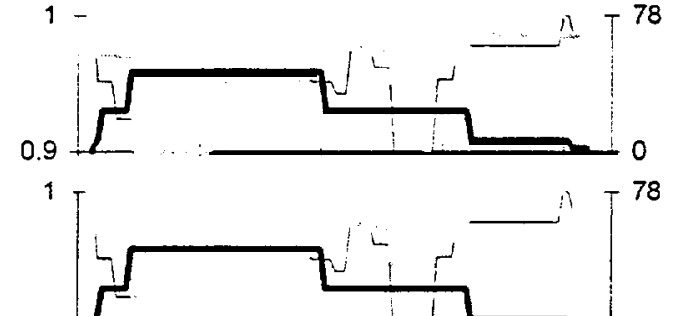

09.5
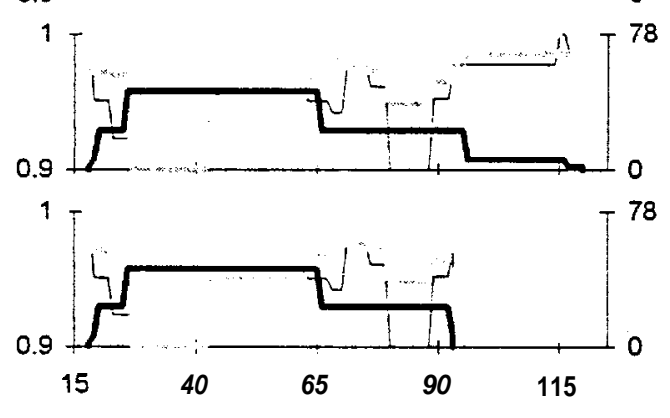

Statistical week
Relative Key Habitat Quantity

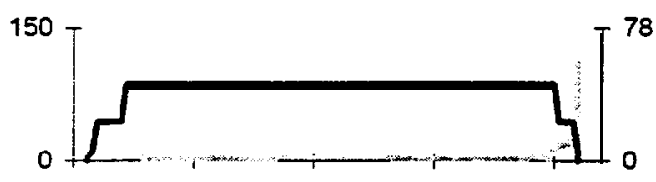

$150 \mathrm{~T}$
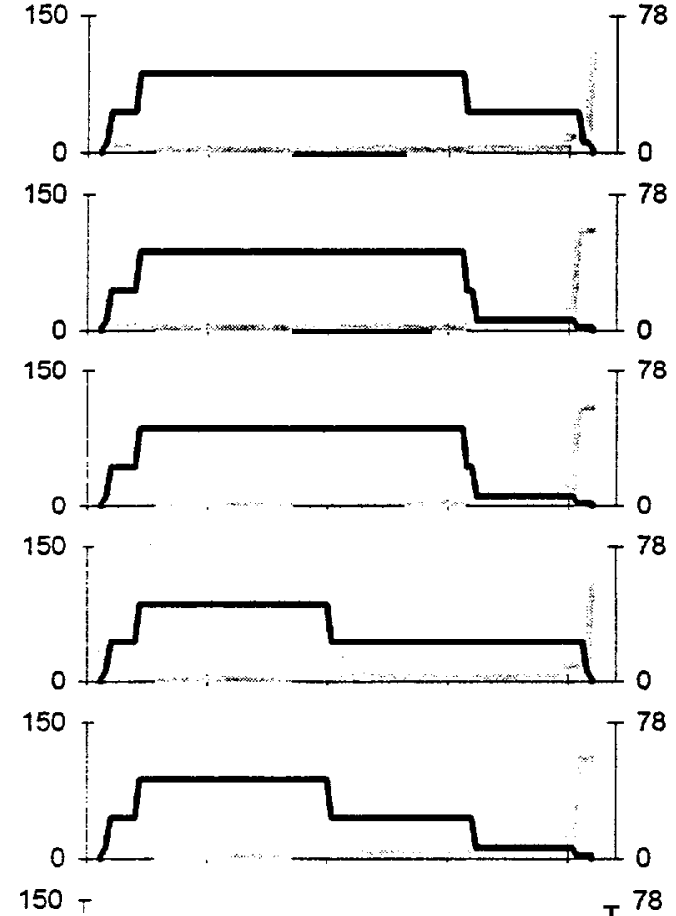

$150-$
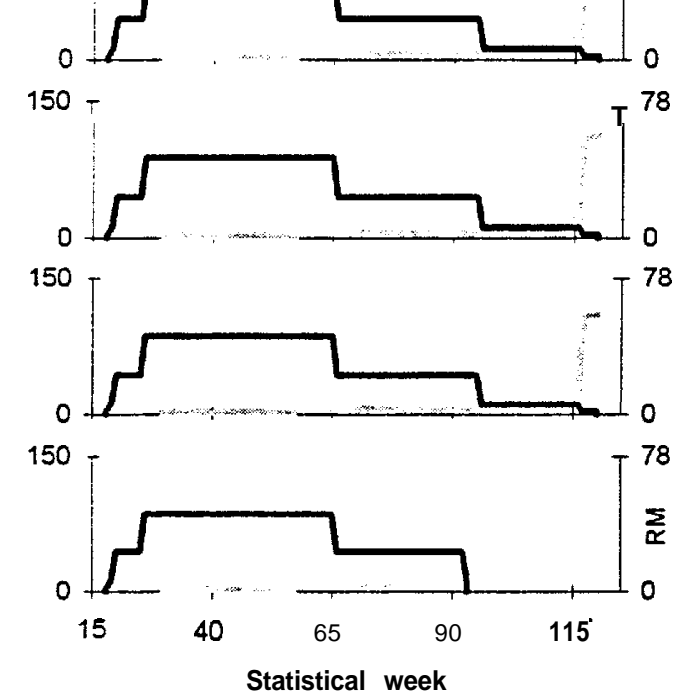

Prespawn -

Spawning

Incubation

Colonization

Sum. rearing

Win. rearing

Statistical week
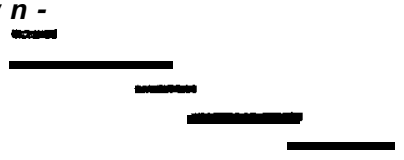

\section{Template}

Patient

Cum. Cum.

Prod. Cap.

Traj. \#1

\begin{tabular}{l|l|}
\hline 0 & 0.00 \\
0 & 0.00
\end{tabular}

Traj. \#2

00.00

00.00

Traj. \#3

\begin{tabular}{l|l|}
0 & 0.00 \\
0 & 0.00
\end{tabular}

Traj. \#4

00.00

0) 0.00

Traj. \#5

\begin{tabular}{l|l}
0 & 0.00 \\
0 & 0.00
\end{tabular}

0.00

Traj. \#6

\begin{tabular}{l|l}
0 & 0.00 \\
0 & 0.00
\end{tabular}

Traj. \#7

\begin{tabular}{l|l}
0 & 0.00 \\
0 & 0.00
\end{tabular}

Traj. \#8

\begin{tabular}{l|l}
0 & 0.00 \\
0 & 0.00
\end{tabular}

Traj. \#9

\begin{tabular}{l|l}
\hline 0 & 0.00 \\
0 & 0.00
\end{tabular}

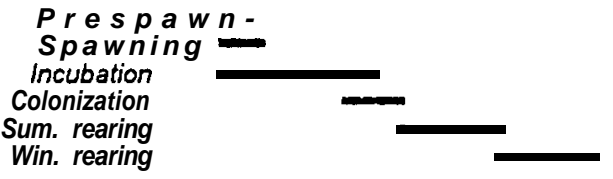


Spawning reach: Chesnimnus Cr.

Confluence with Crow $\mathrm{Cr}$ - South Fork (RM 52.7 - 77.7 from Snake R)

Relative Productivity
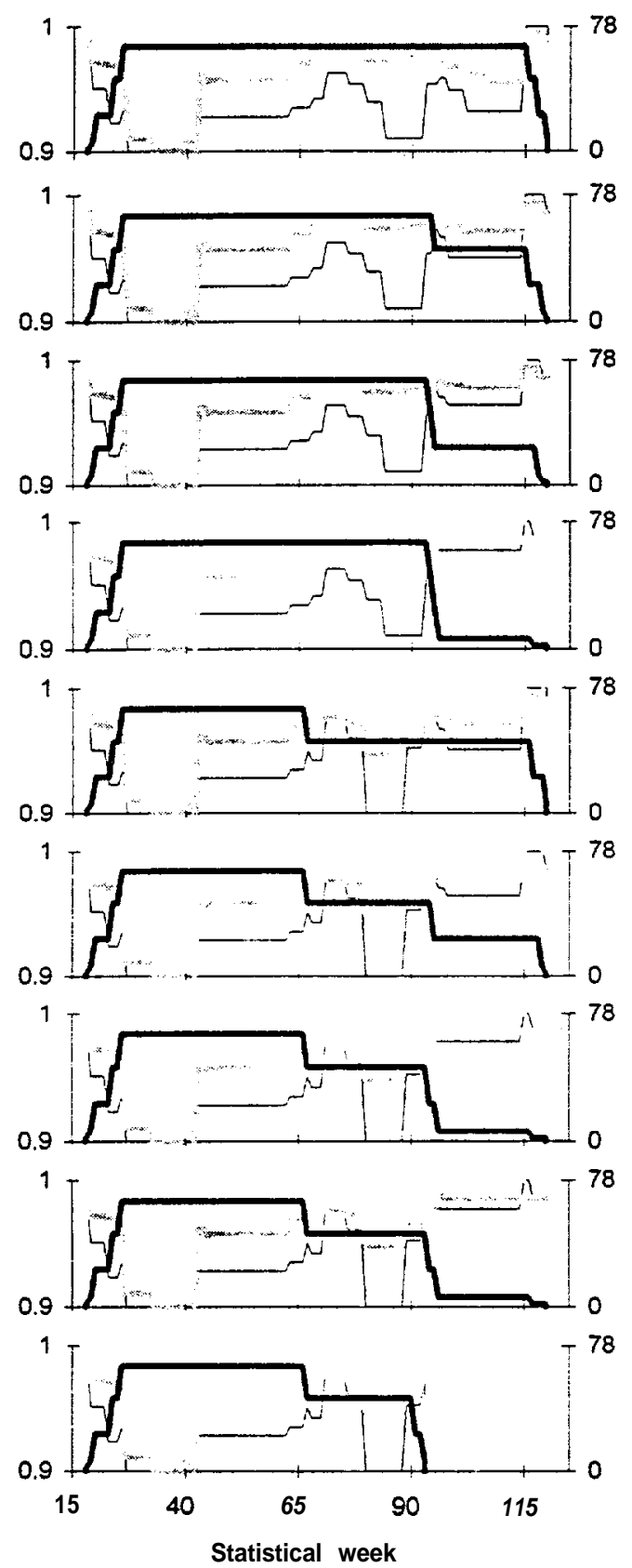

Prespawn -

Spawning

Incubation

Colonization

Sum. rearing

Win. rearing
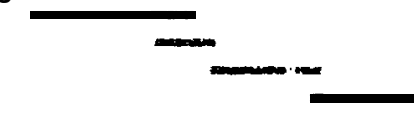

\section{Relative Key Habitat Quantity}

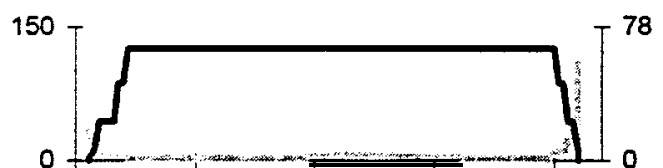

$150-$

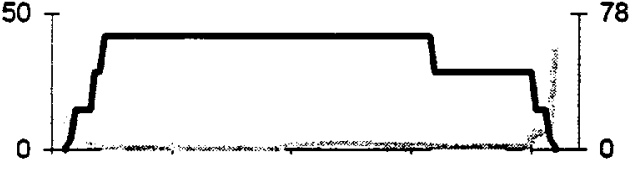

150 T

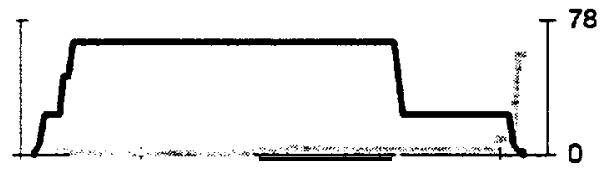

150
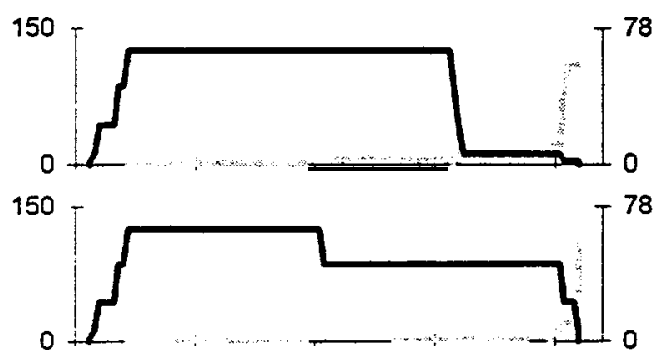

150
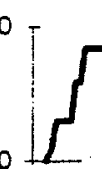

150

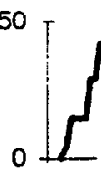

150
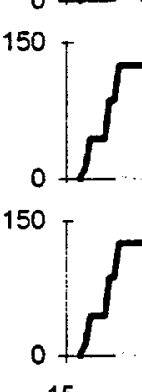

15
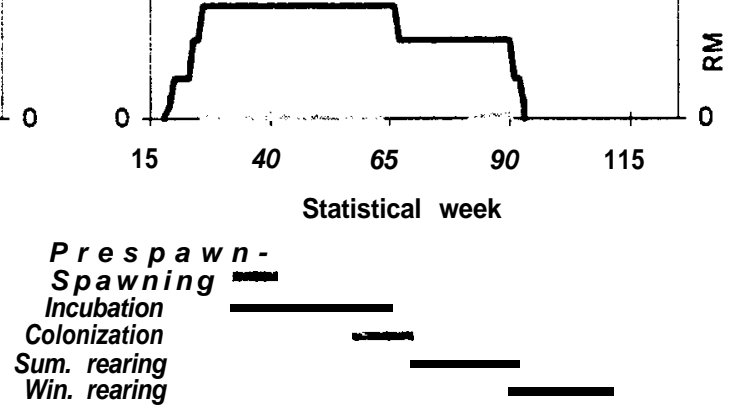

115

Statistical week

78

0

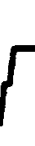

Template

Patient

Cum. Cum.

Prod. Cap.

Traj. \#1

60.01

o) 0.00

Traj. \#2

\begin{tabular}{l|l}
8 & 0.01
\end{tabular}

이 0.00

Traj . \#3

90.01

$0 \quad 0.00$

Traj. \#4

\begin{tabular}{|l|l|}
\hline 0 & 0.01 \\
\hline
\end{tabular}

00.00

Traj. \#5

\begin{tabular}{l|l|}
\hline 6 & 0.01 \\
\hline
\end{tabular}

$0 \quad 0.00$

Traj. \#6

6 0.01

$0 \quad 0.00$

Traj . \#7

70.01

0) 0.00

Traj. \#8

\begin{tabular}{l|l|}
7 & 0.01 \\
0 & 0.00
\end{tabular}

\begin{tabular}{l|r|r}
78 & \multicolumn{2}{|c}{ Traj . \#9 } \\
\cline { 2 - 3 }$\sum_{\propto}$ & 15 & 0.01 \\
& 0 & 0.00
\end{tabular}

\begin{tabular}{l|r|r}
78 & \multicolumn{2}{|c|}{ Traj . \#9 } \\
\cline { 2 - 3 }$\sum_{\propto}$ & 15 & 0.01 \\
& 0 & 0.00
\end{tabular} 
Spawning reach: Lookingglass $\mathrm{Cr}$.

Mouth - Little Lookingglass Cr (RM 83.4 - 87.6 from Snake R)

Relative Productivity
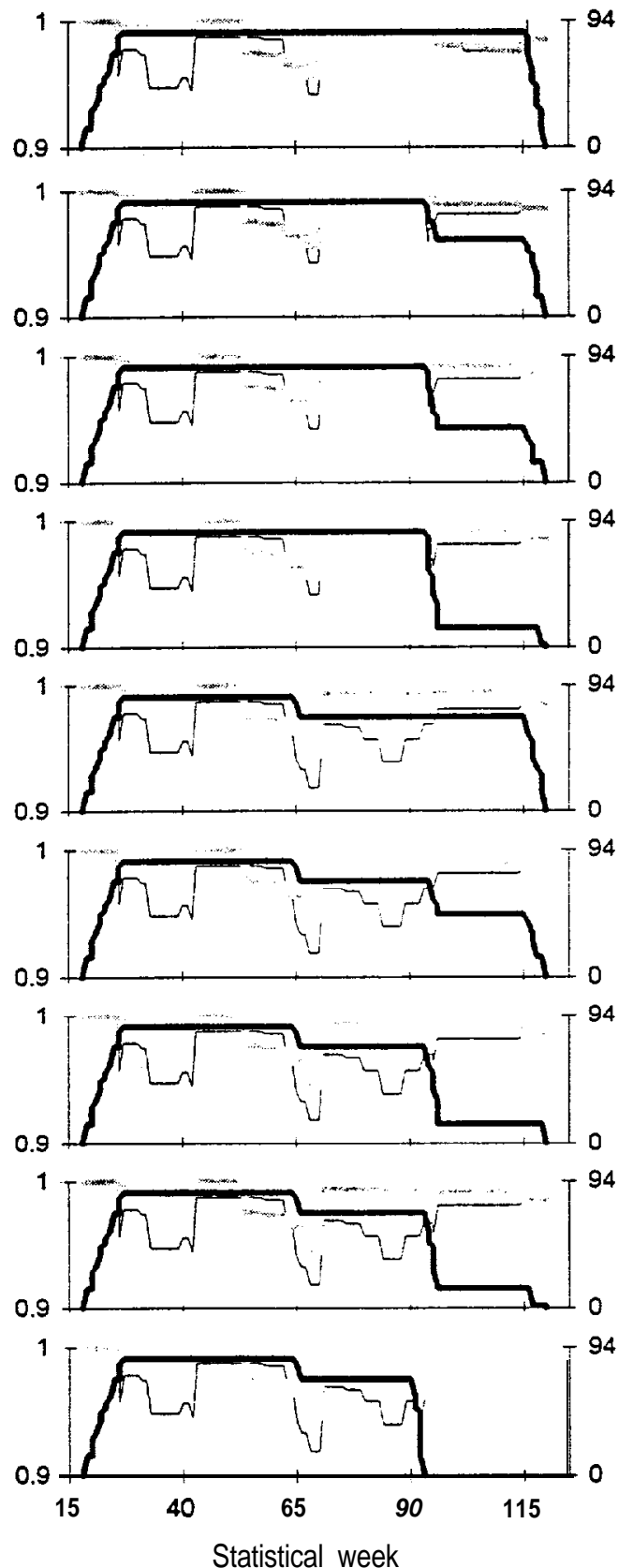

Prespawn -

Spawning

Incubation

Colonization

Sum. rearing

Win. rearing
Relative Key Habitat Quantity
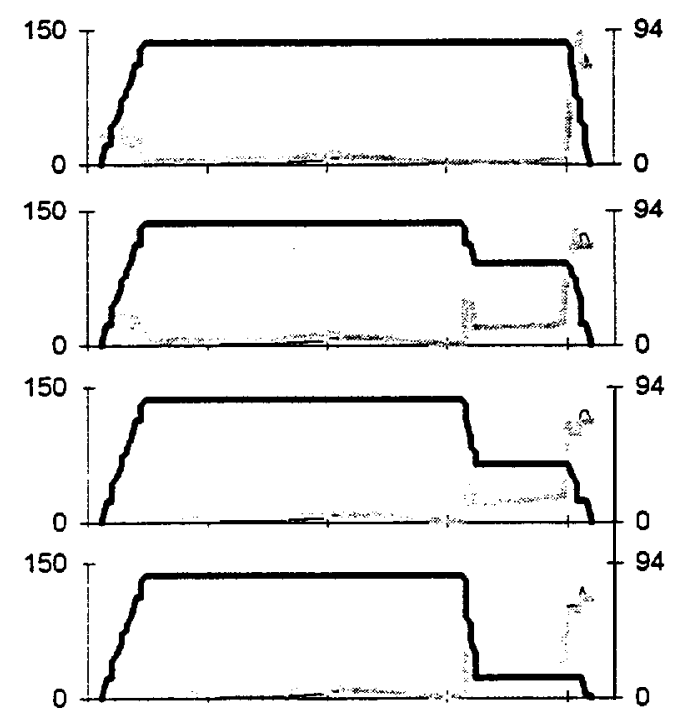

150
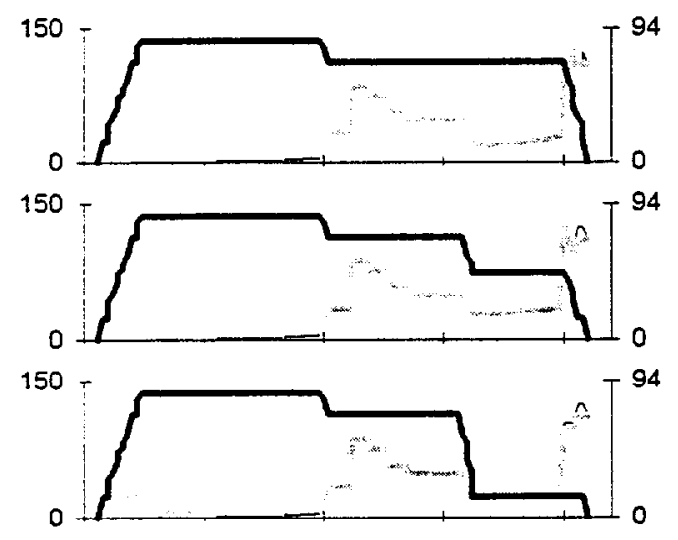

$150-$

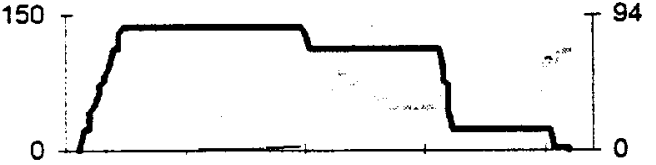

150

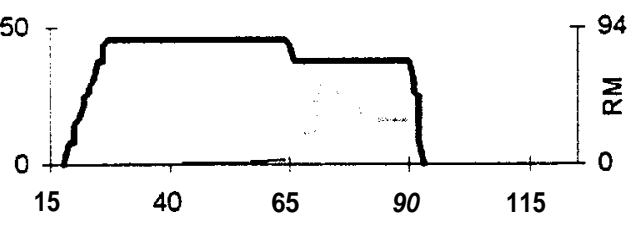

Statistical week

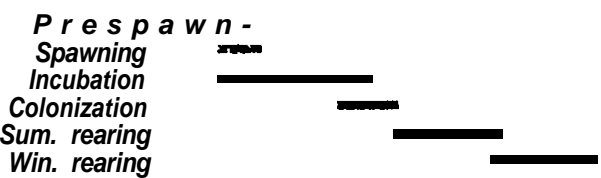

\begin{tabular}{|c|c|}
\hline \multicolumn{2}{|c|}{$\begin{array}{c}\text { Template } \\
\text { Patient }\end{array}$} \\
\hline \multicolumn{2}{|c|}{ Cum. Cum. } \\
Prod. Cap. \\
Traj. \#1 \\
\hline 208 & $\mathbf{0 . 1 8}$ \\
107 & $\mathbf{0 . 1 2}$
\end{tabular}

Traj. \#2

2510.26

$109 \quad 0.14$

Traj. \#3

\begin{tabular}{l|l}
\hline 263 & 0.27 \\
\hline
\end{tabular}

1090.14

Traj. \#4

\begin{tabular}{l|l}
\hline 260 & 0.27 \\
\hline
\end{tabular}

1090.14

Traj. \#5

\begin{tabular}{l|l}
\hline 269 & 0.38 \\
\hline 42 & 0.07
\end{tabular}

42 0.07

Traj. \#6

\begin{tabular}{l|l}
\hline 243 & 0.35 \\
\hline 42 & 0.07
\end{tabular}

42) 0.07

\begin{tabular}{c|c} 
Traj . \#7 \\
\hline 251 & 0.36
\end{tabular}

42) 0.07

\begin{tabular}{r|c}
\multicolumn{2}{c}{ Traj. \#8 } \\
\hline 249 & 0.36 \\
42 & 0.07
\end{tabular}

Traj. \#9

\begin{tabular}{l|l}
\hline 448 & $0.6 \varepsilon$ \\
\hline
\end{tabular}

96 0.15 
Spawning reach: Lookingglass $\mathrm{Cr}$.

Little Lookingglass $\mathrm{Cr}$ - headwaters (RM 87.6 - 93.8 from Snake R)

Relative Productivity
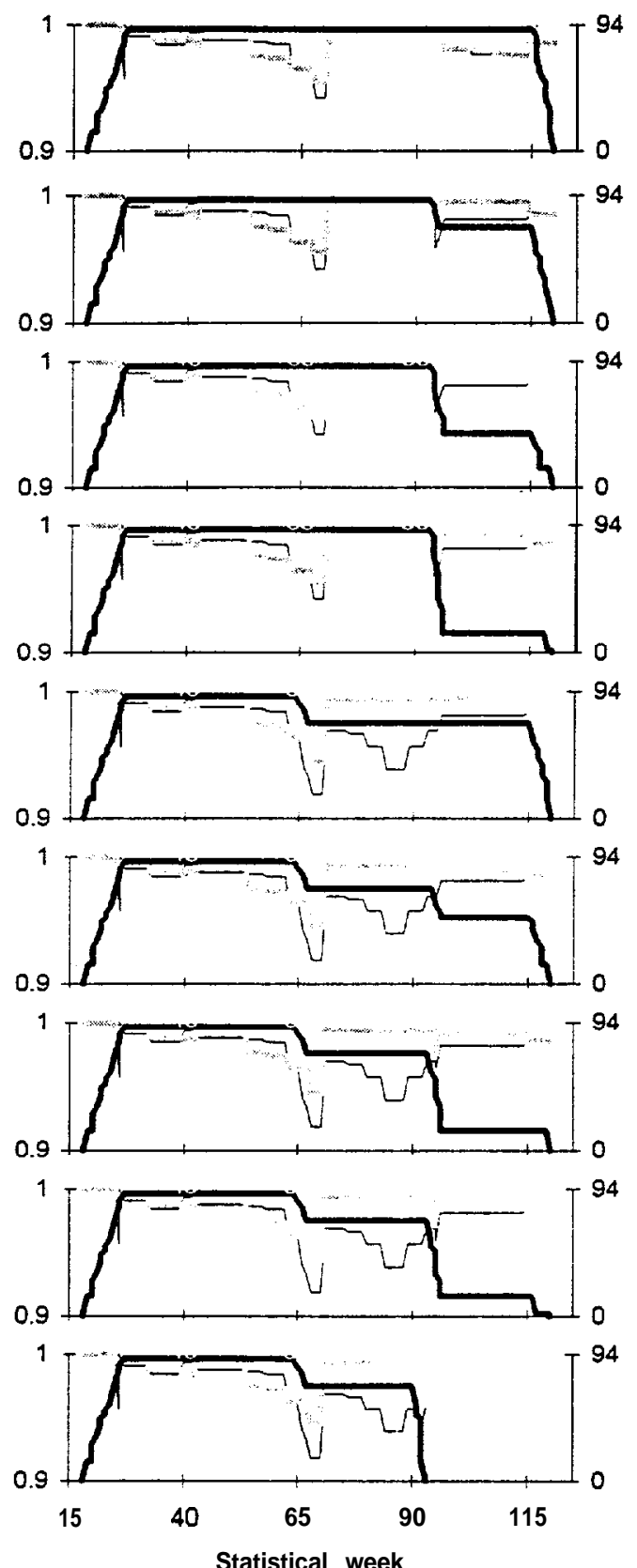

Prespawn -

Spawning

Incubation

Colonization

Sum. rearing

Win. rearing
Relative Key Habitat Quantity
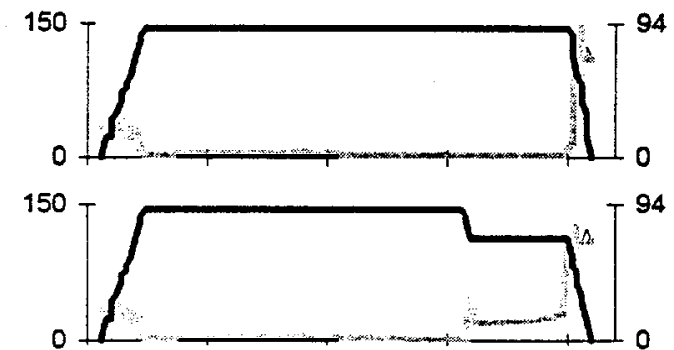

150
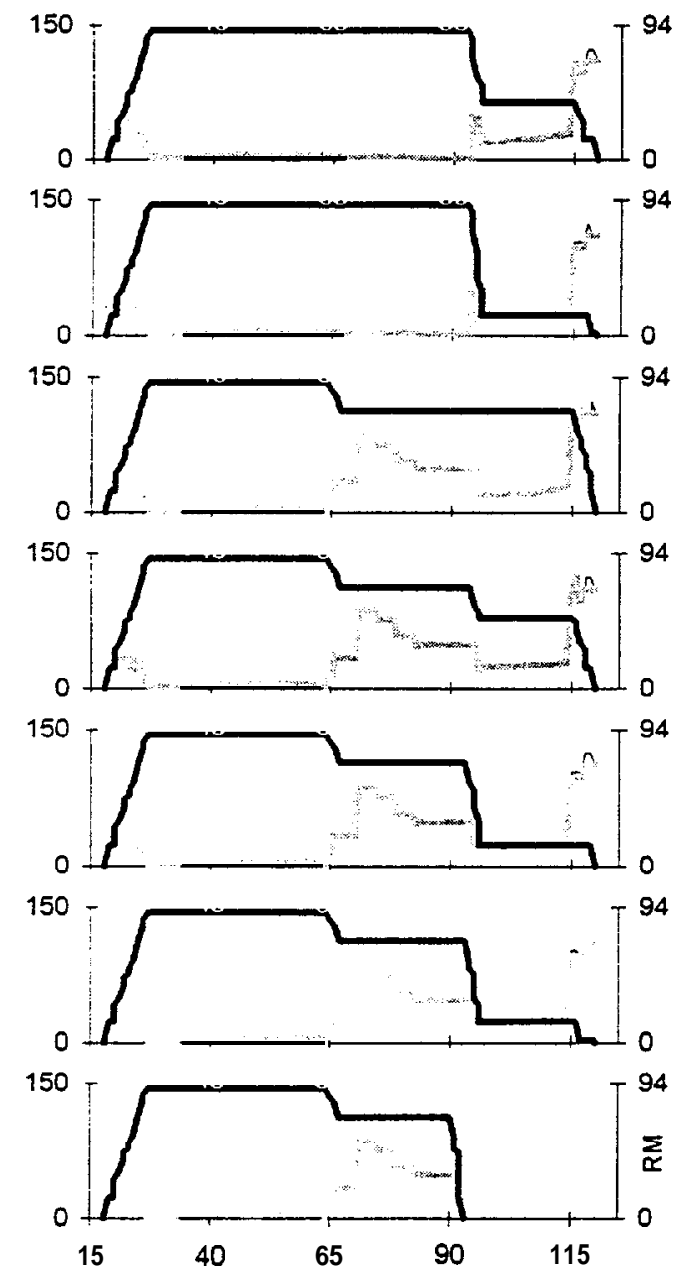

94
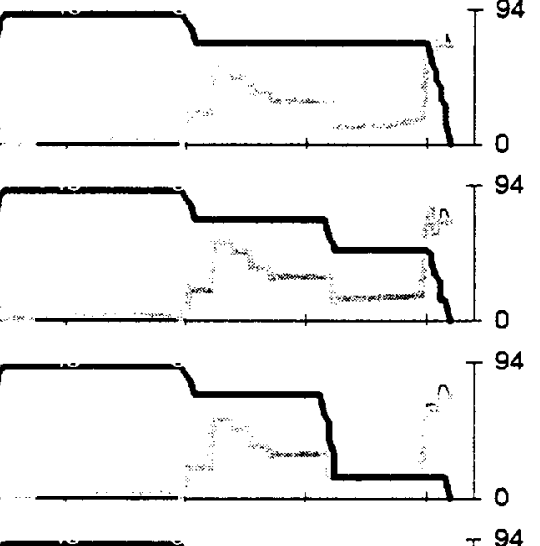

15
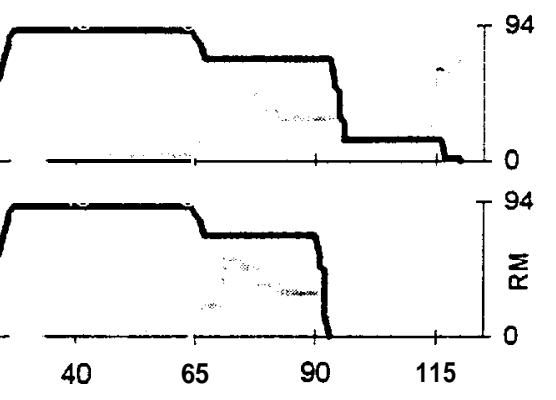

Statistical week

Prespawn -

Spawning

Incubation

Colonization

Sum. rearing

Win. rearing

\begin{tabular}{|c|c|}
\hline $\begin{array}{l}\text { Tem } \\
\text { Pat }\end{array}$ & \\
\hline $\begin{array}{l}\text { Cum. } \\
\text { Prod. }\end{array}$ & $\begin{array}{l}\text { Cum. } \\
\text { Cap. }\end{array}$ \\
\hline Traj & $\# 1$ \\
\hline 207 & 0.11 \\
\hline 182 & 0.10 \\
\hline
\end{tabular}

Traj. \#2

\begin{tabular}{l|l}
\hline 277 & 0.17 \\
\hline
\end{tabular}

186 0.12

Traj. \#3

: “, : :

Traj. \#4

\begin{tabular}{l|l|}
\hline 259 & 0.16 \\
\hline
\end{tabular}

184

0.12

Traj. \#5

\begin{tabular}{|c|c|}
\hline 268 & 0.23 \\
\hline
\end{tabular}

$70 \quad 0.06$

Traj. \#6

\begin{tabular}{|l|l|}
\hline 242 & 0.21 \\
\hline
\end{tabular}

$\begin{array}{ll}70 & 0.06\end{array}$

\begin{tabular}{r|r|}
\multicolumn{2}{|c|}{ Traj. \#7 } \\
\hline 250 & 0.21 \\
70 & 0.06
\end{tabular}

Traj. \#8

\begin{tabular}{|l|l|}
\hline 249 & 0.21 \\
\hline
\end{tabular}

690.06

Traj. \#9

\begin{tabular}{|l|l|}
\hline 445 & 0.39 \\
\hline
\end{tabular}

159 0.14

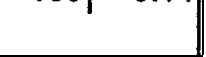


Spawning reach: Lostine $\mathbf{R}$.

Mouth - Cleatwater Ditch (RM 105.8 - 108.3 from Snake R)

\section{Relative Productivity}
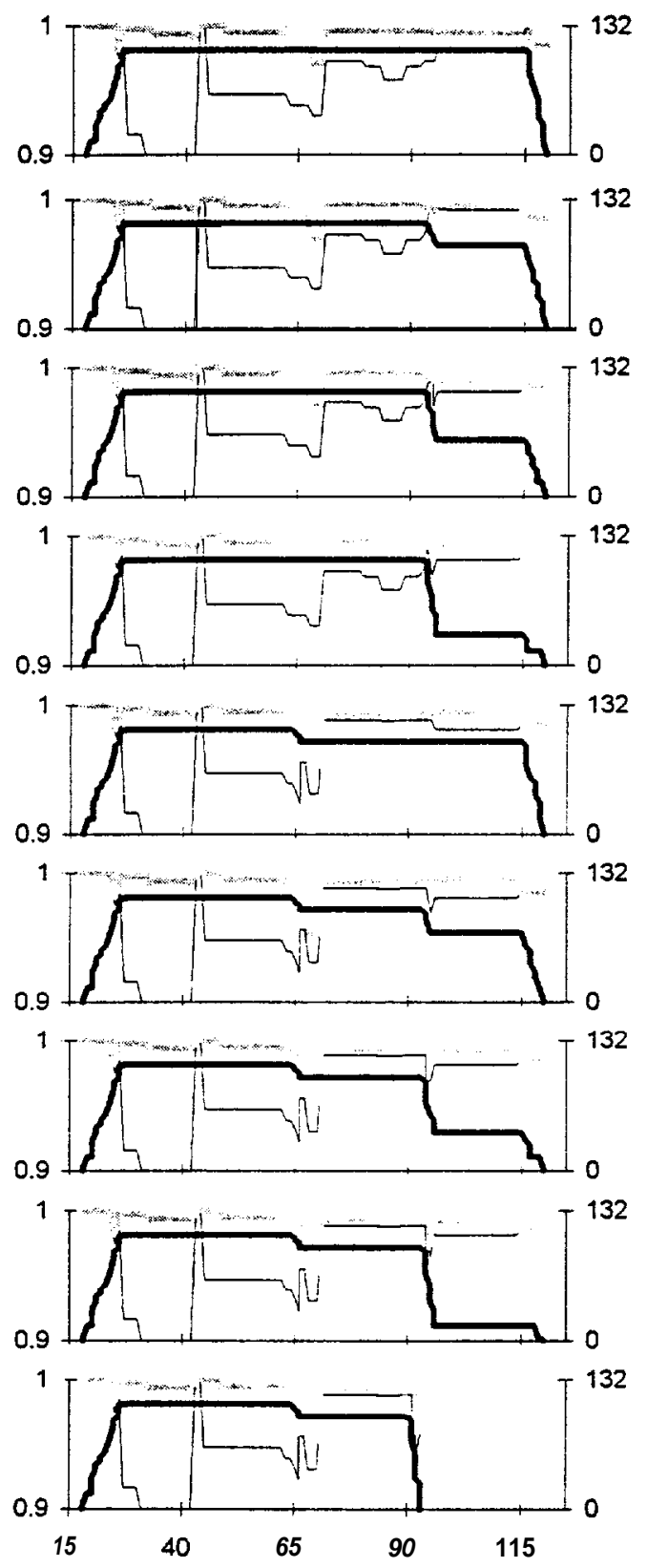

Statistical week

Prespaw -

\section{Spawning}

Incubation

Colonization

Sum. rearing

Win rearing
Relative Key Habitat Quantity
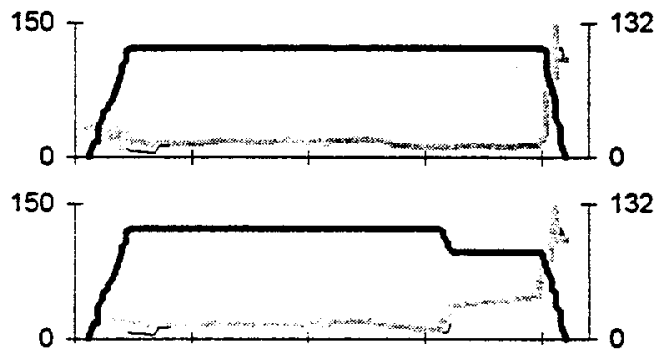

$150 r$

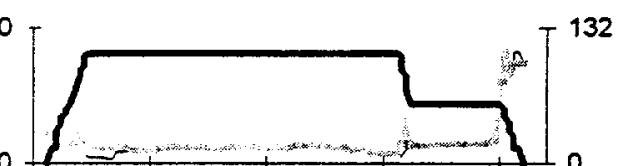

150

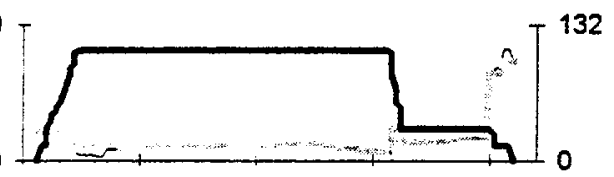

150

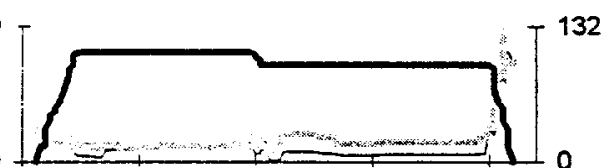

150

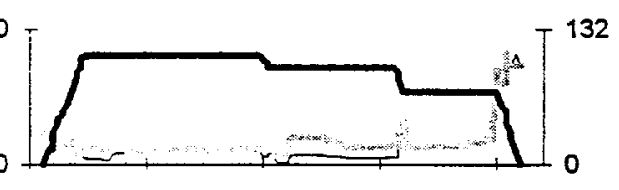

150

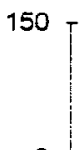

150
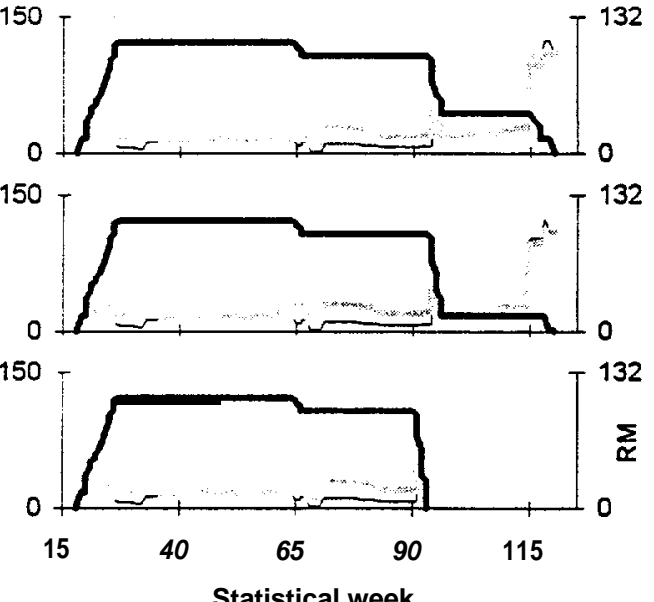

P res p a w n -

Spawning

Incubation

Colonization

Sum. rearing

Win. rearing \begin{tabular}{|r|r|}
\hline \multicolumn{2}{|c|}{ Template } \\
Patient \\
\hline Cum. & Cum. \\
Prod. & Cap. \\
Traj. \#1 \\
\hline 432 & 0.92 \\
2 & 0.01 \\
\\
Traj. \#2 \\
\hline 427 & 1.05 \\
3 & 0.01 \\
\hline
\end{tabular}

Traj . \#3

"

Traj. \#4

\begin{tabular}{l|l}
\hline 403 & 0.96 \\
\hline
\end{tabular}

2) 0.01

\begin{tabular}{r|r} 
Traj . \#5 \\
\hline 372 & 0.94 \\
4 & 0.01
\end{tabular}

Traj. \#6

\begin{tabular}{l|l}
\hline 372 & 0.96 \\
\hline & 0.01
\end{tabular}

4) 0.01

Traj. \#7

\begin{tabular}{l|l}
\hline 352 & 0.92 \\
\hline
\end{tabular}

4) 0.01

Traj. \#8

\begin{tabular}{|l|l|}
\hline 349 & 0.91 \\
\hline
\end{tabular}

40.01

Traj. \#9

\begin{tabular}{l|l}
\hline 628 & 1.85 \\
\hline & 0.02
\end{tabular}

\begin{tabular}{l|l}
8 & 0.02 \\
\hline
\end{tabular} 
Spawning reach: Lostine $\mathbf{R}$.

Cleatwater Ditch - Cross Country Ditch (RM 108.3 - 110.8 from Snake R)

Relative Productivity
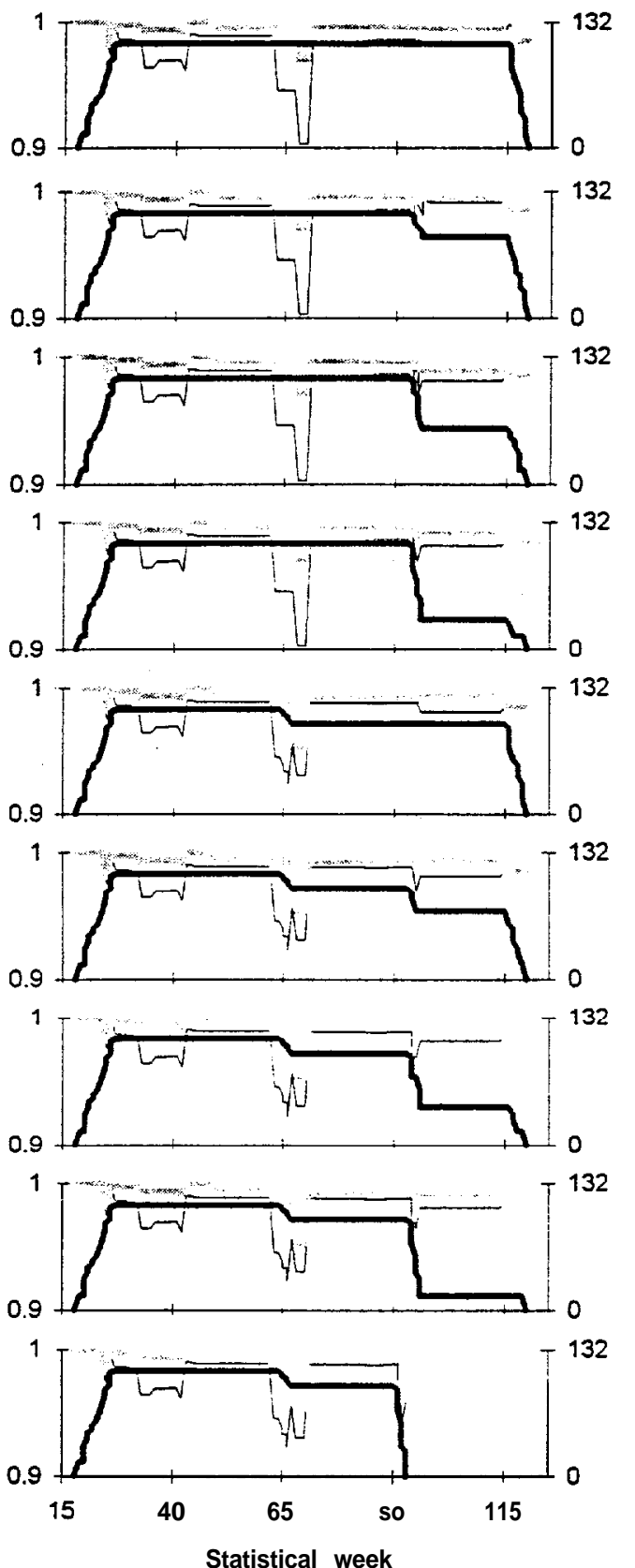

Prespawn -

Spawning

Incubation

Colonization

Sum. rearing

Win. rearing
Relative Key Habitat Quantity
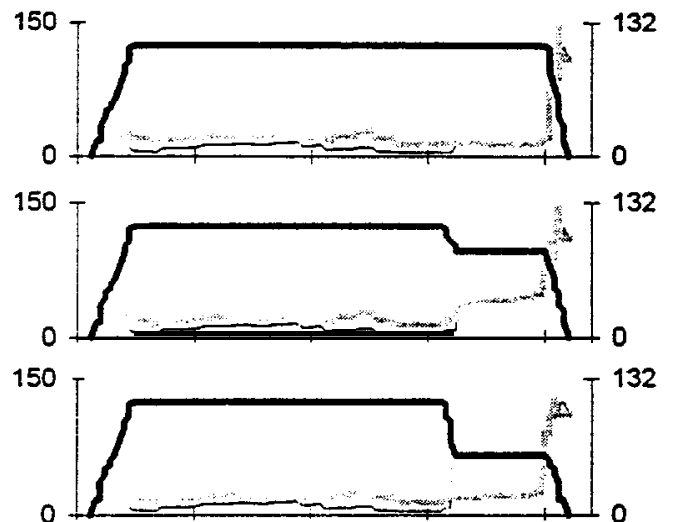

150 -

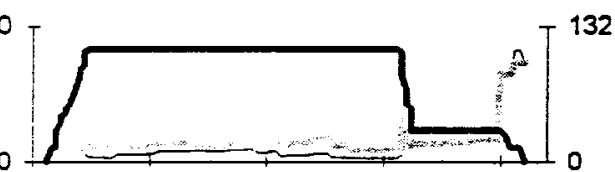

150

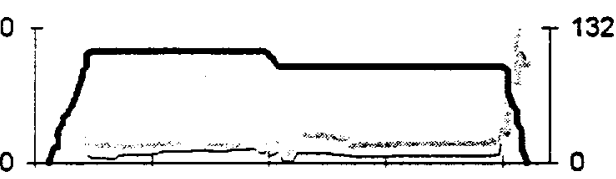

150

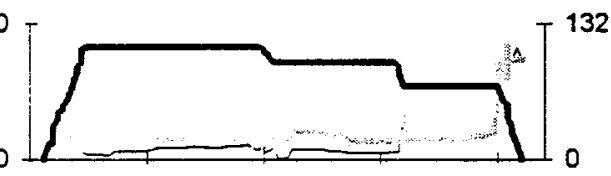

150

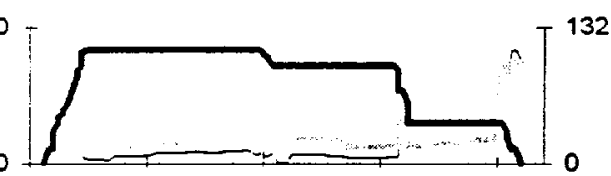

150

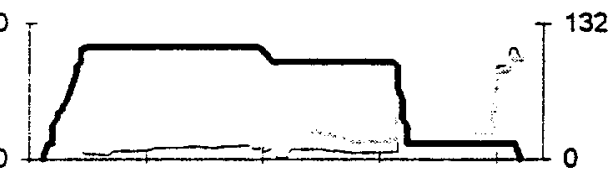

150

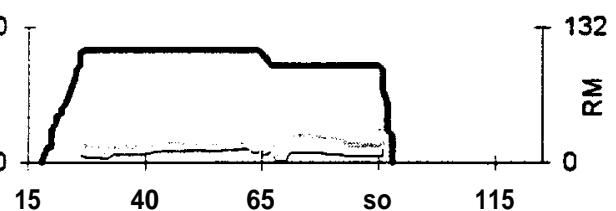

Statistical week

Prespawn -

Spawning

Incubation

Colonization

Sum. rearing

Win. rearing \begin{tabular}{|r|c|}
\hline \multicolumn{2}{|c|}{$\begin{array}{c}\text { Template } \\
\text { Patient }\end{array}$} \\
\hline $\begin{array}{r}\text { Cum. } \\
\text { Prod. }\end{array}$ & Cum. \\
Cap. \\
Traj. \#1 \\
\hline 433 & 0.99 \\
108 & 0.16 \\
\hline
\end{tabular}

Traj. \#2

\begin{tabular}{l|l|}
\hline 428 & 1.11 \\
\hline
\end{tabular}

127

0.19

Traj. \#3

386 0.96

$104 \quad 0.16$

Traj. \#4

\begin{tabular}{l|l|}
404 & 1.02 \\
\hline
\end{tabular}

103

0.15

Traj. \#5

3750.97

120 0.16

Traj. \#6

\begin{tabular}{|l|l|}
\hline 375 & 0.99 \\
\hline
\end{tabular}

$118 \quad 0.16$

Traj. \#7

\begin{tabular}{l|l}
\hline 354 & 0.94 \\
\hline
\end{tabular}

117

0.16

Traj. \#8

$352 \quad 0.93$

$116 \quad 0.16$

Traj. \#9

\begin{tabular}{l|l}
\hline 633 & 1.91 \\
\hline
\end{tabular}

247 0.35 
Cross Country Ditch - Sheep Ridge Dam (RM 110.8 - 115.0 from Snake R)

Relative Productivity
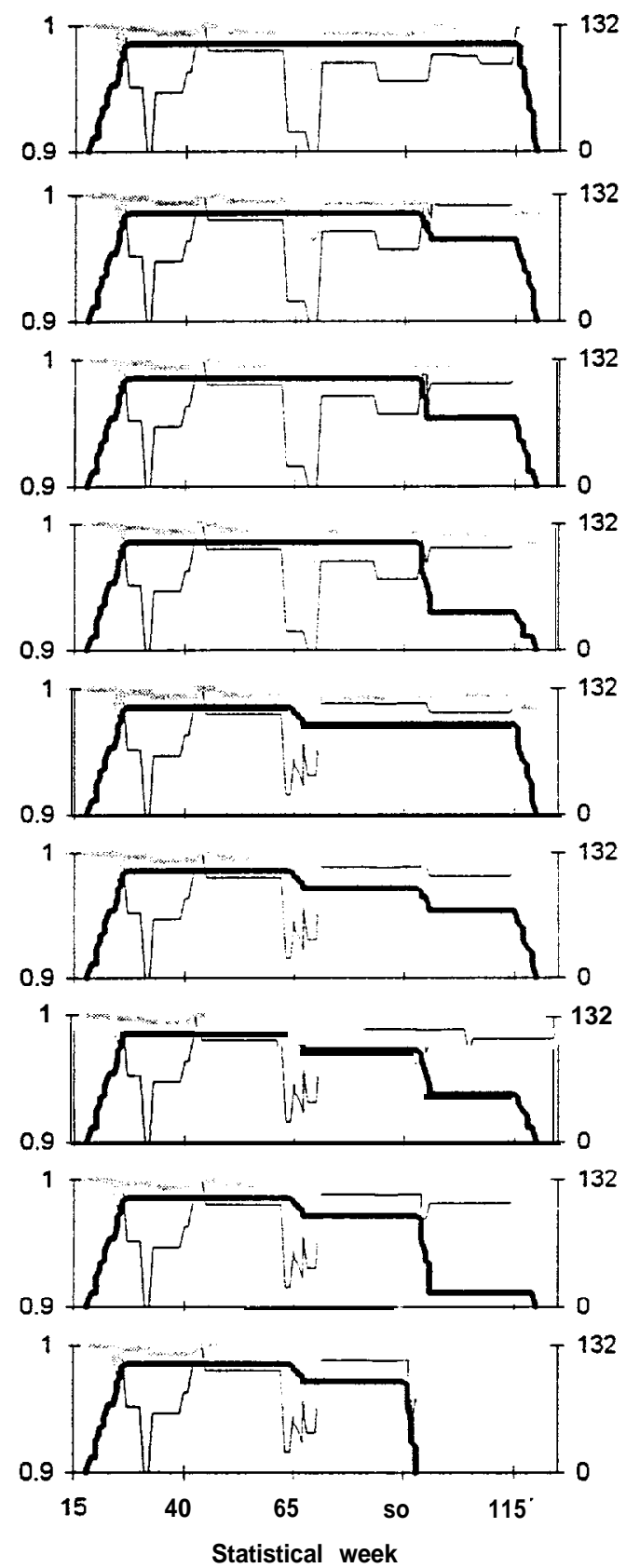

Prespawn -

Spawning -

incubation

Colonization

Sum. rearing

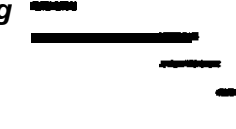

Spawning reach: Lostine $\mathbf{R}$.
Relative Key Habitat Quantity
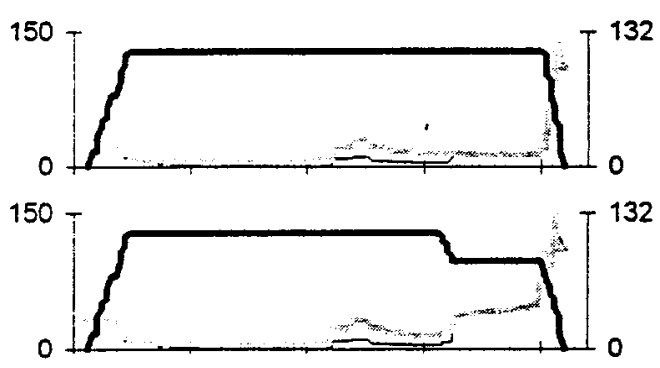

${ }_{150}^{150}$
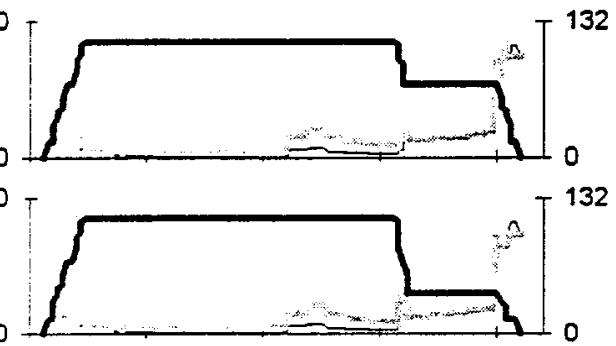

$150-$

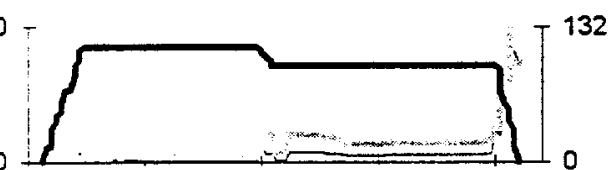

150 .

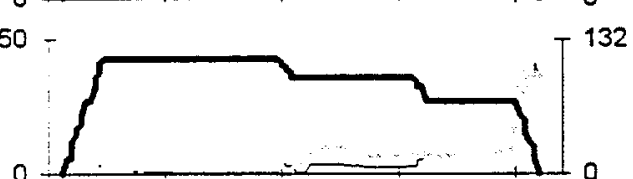

0
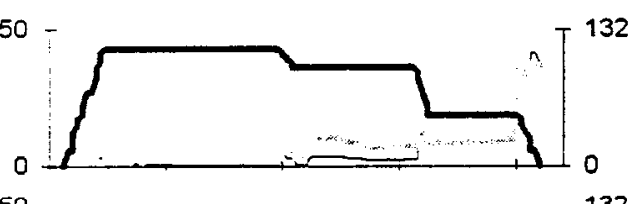

150 -

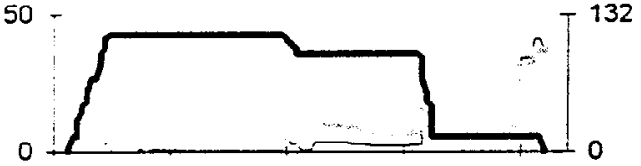

0

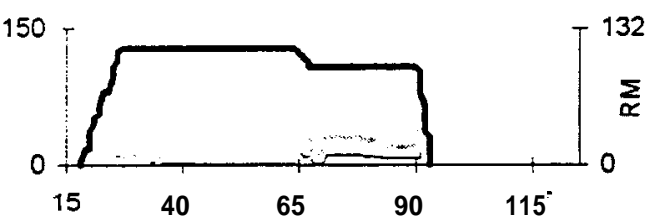

15

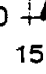

40

Statistical week

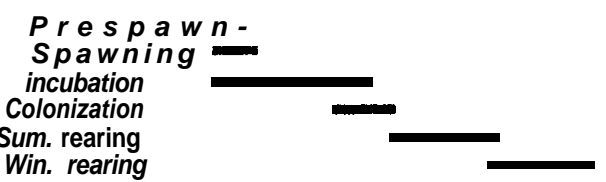

$15^{\circ}$

Win. rearing \begin{tabular}{|c|}
\hline \multicolumn{2}{|c|}{$\begin{array}{c}\text { Template } \\
\text { Patient }\end{array}$} \\
\hline Cum. Cum. \\
Prod. Cap.
\end{tabular}

Traj. \#1

333 (1) 0.57

$19 \quad 0.04$

Traj. \#2

\begin{tabular}{l|l|}
\hline 399 & 0.73 \\
\hline
\end{tabular}

28 0.06

Traj. \#3

\begin{tabular}{l|l}
398 & 0.71 \\
\hline
\end{tabular}

23) 0.05

Traj. \#4

\begin{tabular}{l|l}
375 & 0.68 \\
\hline
\end{tabular}

\begin{tabular}{l|l}
23 & 0.05 \\
\hline
\end{tabular}

Traj. \#5

\begin{tabular}{l|l}
376 & 0.71
\end{tabular}

\begin{tabular}{l|l}
59 & 0.11
\end{tabular}

Traj. \#6

$375 \quad 0.71$

$59 \quad 0.11$

Traj. \#7

\begin{tabular}{l|l}
\hline 40 & 0.66 \\
\hline
\end{tabular}

$58 \quad 0.11$

Traj. \#8

\begin{tabular}{|l|l}
\hline 355 & 0.68 \\
\hline
\end{tabular}

\begin{tabular}{l|l}
57 & 0.11
\end{tabular}

Traj. \#9

\begin{tabular}{l|l}
\hline 636 & 1.33 \\
\hline
\end{tabular}

120 0.24

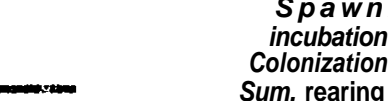


Spawning reach: Lostine $\mathbf{R}$.

Sheep Ridge Dam - Silver Cr (RM 115.0 - 120.0 from Snake R)

Relative Productivity
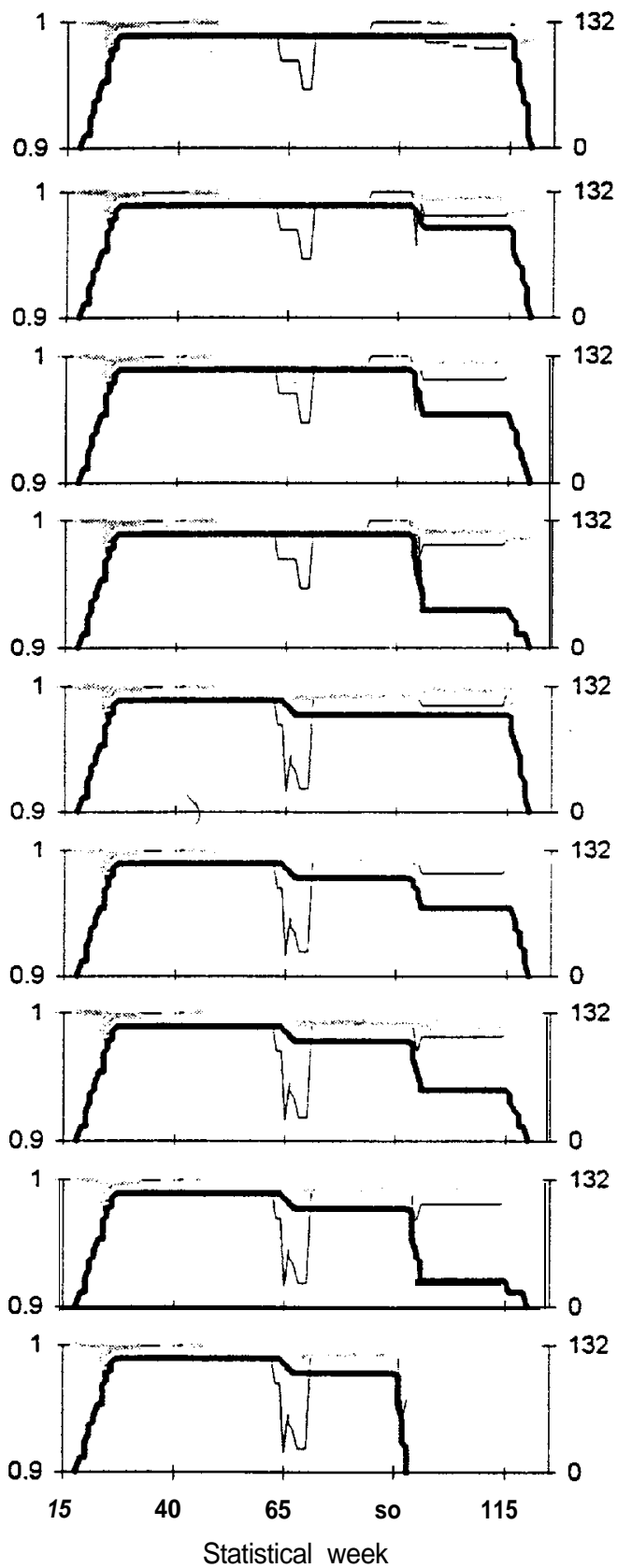

Prespa wn -

Spawning Incubation
Colonization Colonization
Sum. rearing Win. rearing

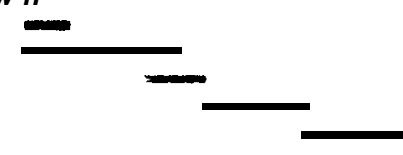

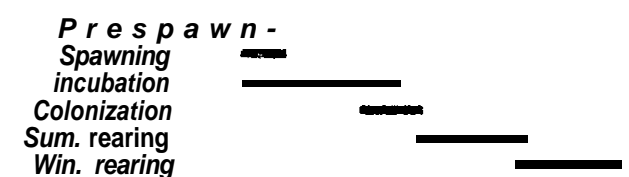

Relative Key Habitat Quantity
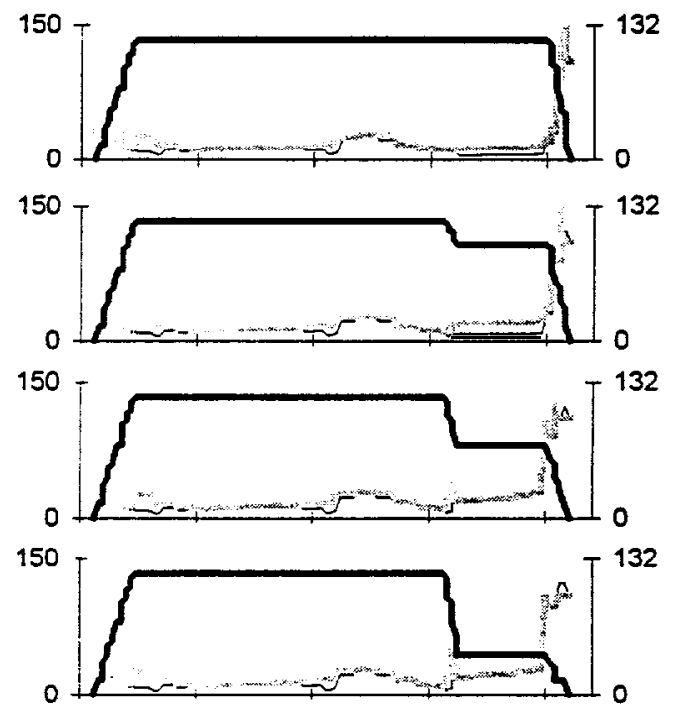

150

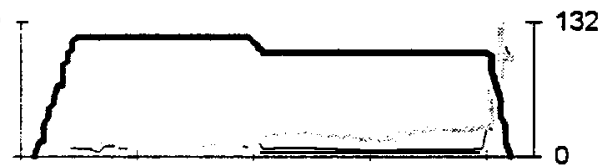

150

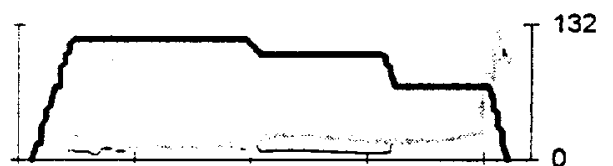

$150+$

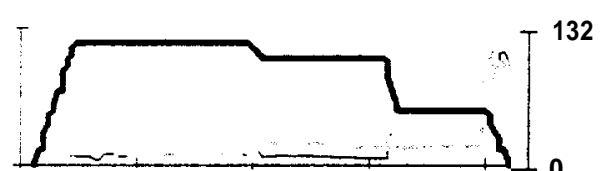

150

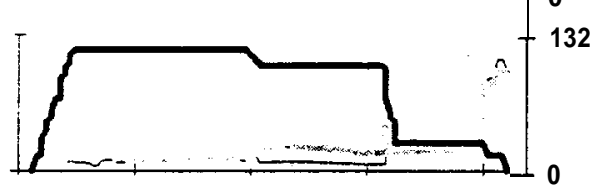

150

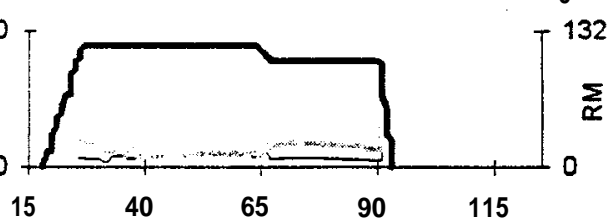

132

\begin{tabular}{|c|c|}
\hline \multicolumn{2}{|c|}{$\begin{array}{c}\text { Template } \\
\text { Patient }\end{array}$} \\
\hline $\begin{array}{c}\text { Cum. } \\
\text { Prod. }\end{array}$ & Cum. \\
\hline
\end{tabular}

Traj. \#1

$365 \mid 0.90$

$306 \quad 0.43$

Traj. \#2

\begin{tabular}{l|l|}
\hline 408 & 1.08 \\
\hline
\end{tabular}

286 0.43

Traj . \#3

\begin{tabular}{l|l}
\hline 407 & 1.09 \\
\hline
\end{tabular}

$290 \quad 0.47$

Traj. \#4

\begin{tabular}{l|l}
384 & 1.05 \\
\hline
\end{tabular}

287

0.47

Traj. \#5

\begin{tabular}{l|l}
\hline 446 & 1.27 \\
\hline
\end{tabular}

\begin{tabular}{l|l}
233 & 0.35
\end{tabular}

Traj. \#6

\begin{tabular}{l|l}
\hline 417 & 1.18 \\
\hline
\end{tabular}

214 0.35

Traj. \#7

\begin{tabular}{l|l}
\hline 377 & 1.07 \\
\hline
\end{tabular}

210 0.34

Traj . \#8

\begin{tabular}{l|l}
395 & 1.13 \\
\hline
\end{tabular}

208 0.34

Traj. \#9

\begin{tabular}{l|l}
\hline 705 & 2.36 \\
\hline
\end{tabular}

\begin{tabular}{ll|}
437 & 0.77
\end{tabular} 
Spawning reach: Lostine $\mathbf{R}$.

Silver Cr - Lake Cr (RM 120.0 - 124.6 from Snake R)

Relative Productivity
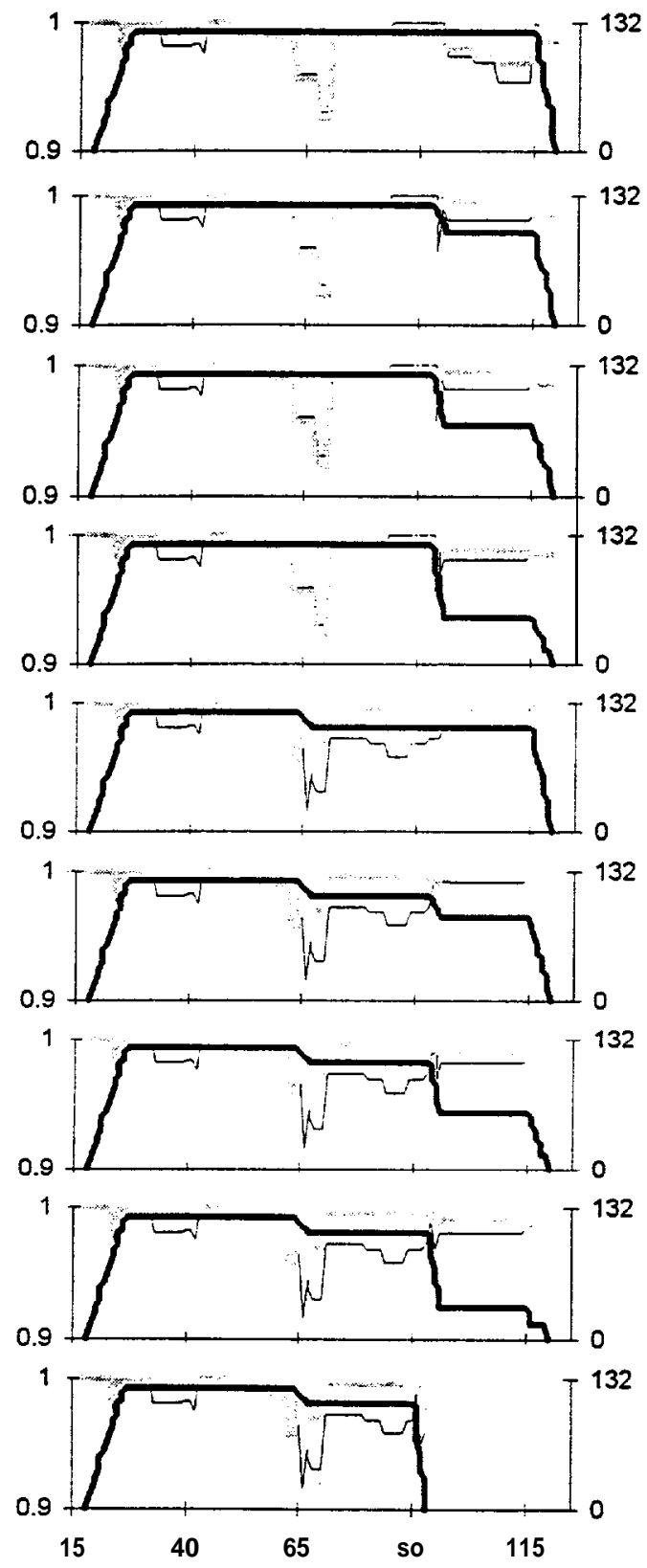

Statistical week

\section{Prespawn -}

Spawning Incubation Colonization Sum. rearing Win. rearing
Relative Key Habitat Quantity
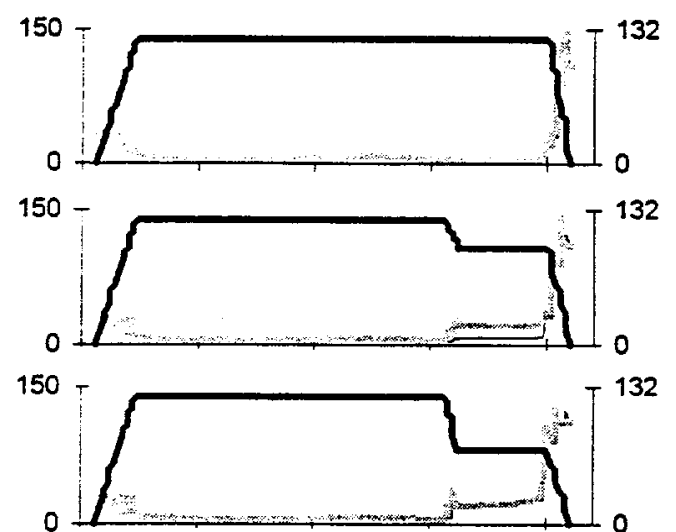

150

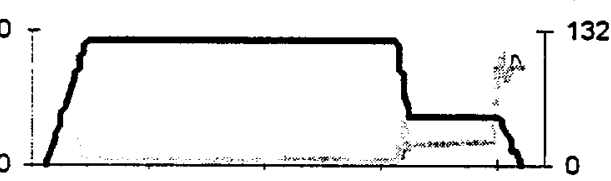

$150=$

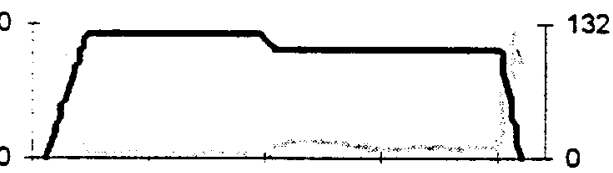

$150-$

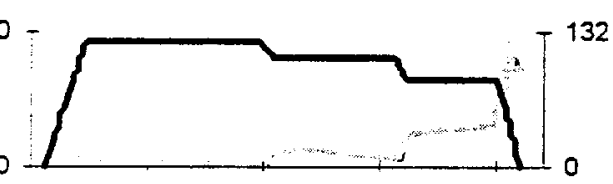

150 -
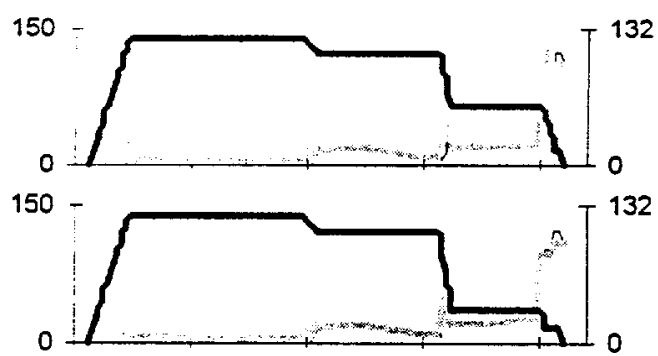

150 ;

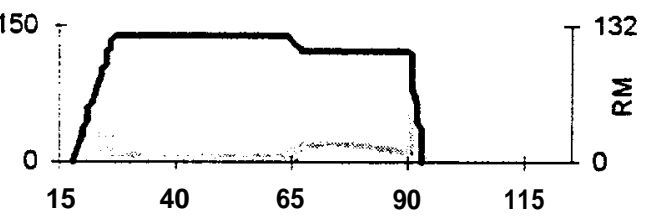

Statistical week

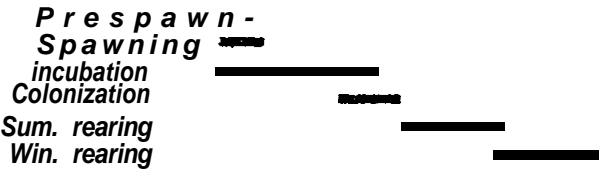

\begin{tabular}{|c|c|}
\hline \multicolumn{2}{|c|}{ Template } \\
Patient
\end{tabular}

Traj. \#1

\begin{tabular}{l|l}
176 & 0.23 \\
\hline
\end{tabular}

$151 \quad 0.20$

Traj. \#2

$262 \quad 0.39$

$2 0 0 \longdiv { 0 . 2 8 }$

Traj. \#3

\begin{tabular}{l|l}
\hline 261 & 0.39 \\
\hline
\end{tabular}

201.

0.29

Traj. \#4

\begin{tabular}{l|l|}
\hline 236 & 0.36 \\
\hline
\end{tabular}

1990.29

Traj. \#5

\begin{tabular}{l|l}
\hline 373 & $0.5 \mathrm{~s}$ \\
\hline
\end{tabular}

101) 0.18

Traj. \#6

\begin{tabular}{l|l}
\hline 368 & 0.64 \\
\hline
\end{tabular}

123) 0.23

Traj. \#7

\begin{tabular}{l|l}
\hline 333 & 0.56
\end{tabular}

$100 \mid 0.18$

Traj. \#8

\begin{tabular}{l|l}
\hline 48 & 0.59 \\
\hline
\end{tabular}

\begin{tabular}{l|l}
99 & 0.18
\end{tabular}

Traj. \#9

\begin{tabular}{l|l}
\hline 617 & 1.16 \\
\hline 222 & 0.42
\end{tabular}

\begin{tabular}{l|l|l|}
222 & 0.42
\end{tabular} 
Spawning reach: Lostine $\mathbf{R}$.

Lake Cr - East Lostine R (RM 124.6 - 131.7 from Snake R)

Relative Productivity
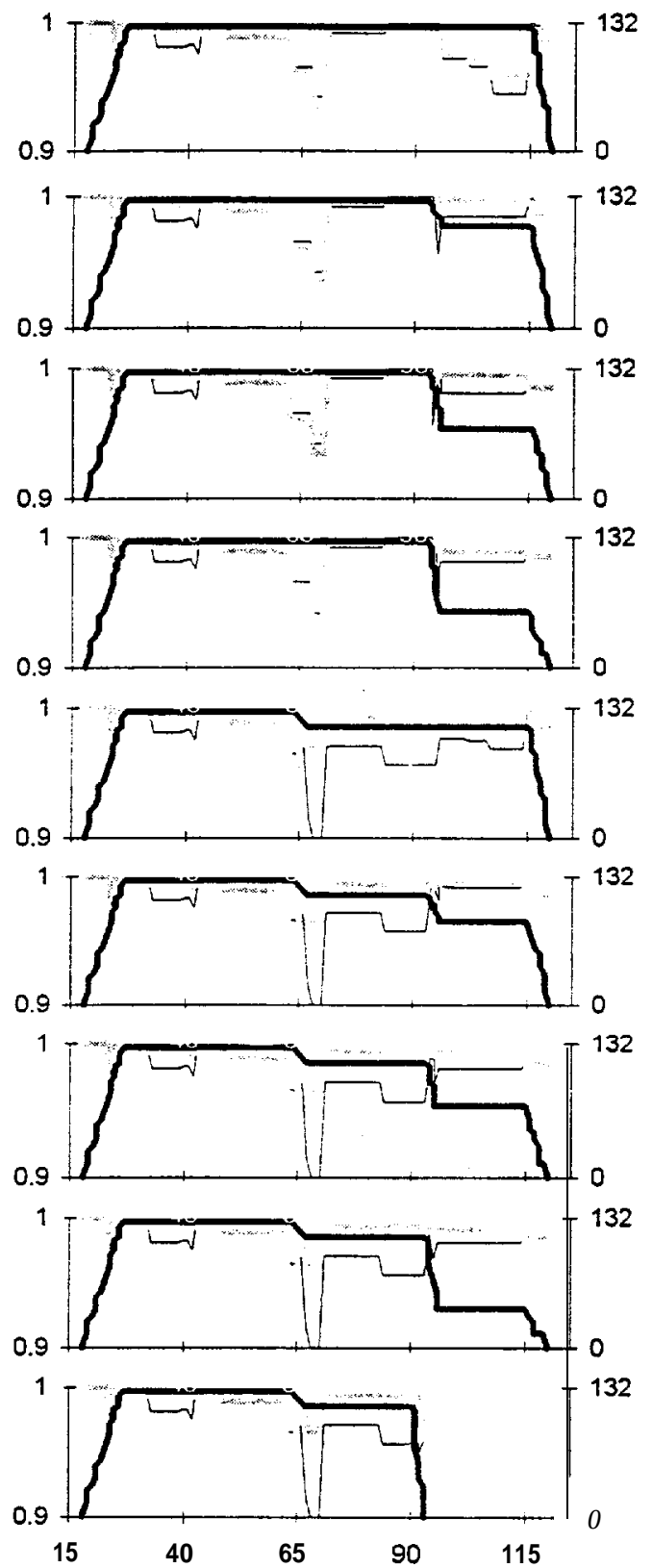

15
Relative Key Habitat Quantity
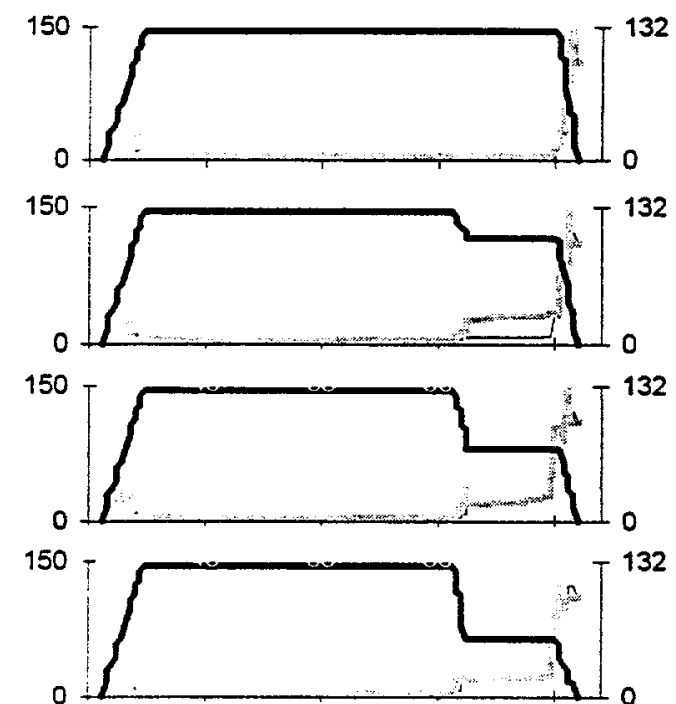

150
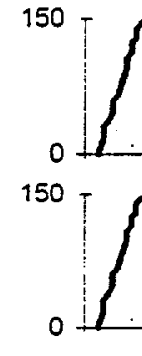

$150 T$
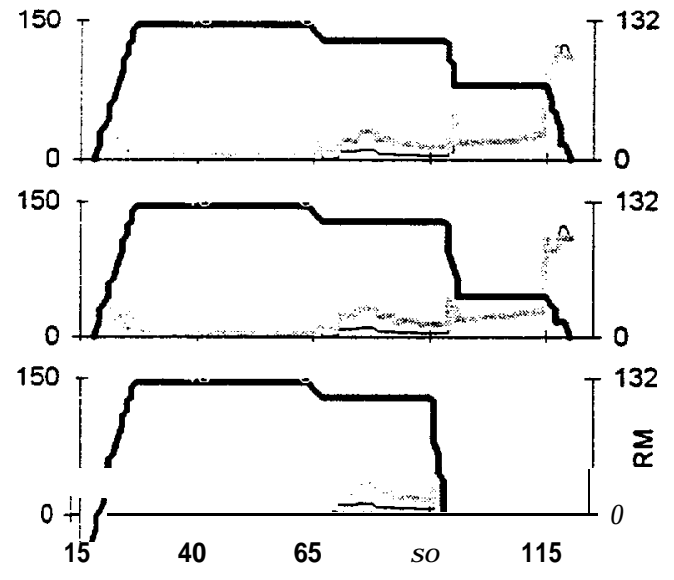

Statistical week
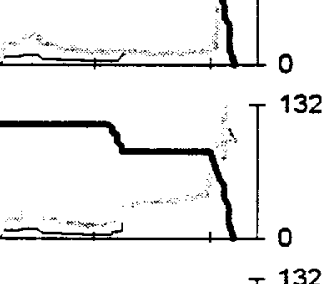

132

115

\section{Prespawn -}

Spawning

Incuba tion

Colonization

Sum. rearing

Win. rearing

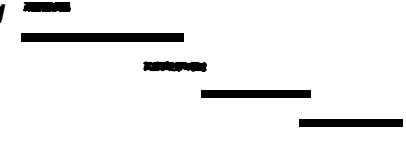

Prespawn -

Spawning

Incubation

Colonization

Sum. rearing

Win. rearing

\begin{tabular}{c|c|}
\hline $\begin{array}{c}\text { Template } \\
\text { Patient }\end{array}$ \\
\hline \multicolumn{2}{c|}{ Cum. Cum. } \\
Prod.| & Cap. \\
\multicolumn{2}{|c|}{ Traj. \#1 } \\
\hline 186 & 0.18 \\
148 & 0.14 \\
\hline
\end{tabular}

Traj. \#2

\begin{tabular}{l|l|}
\hline 332 & 0.36 \\
\hline
\end{tabular}

232 0.24

Traj. \#3

$311 \mid 0.34$

\begin{tabular}{l|l|}
219 & 0.24
\end{tabular}

Traj. \#4

\begin{tabular}{l|l|}
\hline 281 & 0.30 \\
\hline
\end{tabular}

216 0.23

\begin{tabular}{|r|c|}
\multicolumn{2}{|c|}{ Traj. \#5 } \\
\hline 286 & 0.37 \\
61 & 0.07
\end{tabular}

\begin{tabular}{c|c}
\multicolumn{3}{c}{ Traj . \#7 } \\
\hline 341 & 0.46
\end{tabular}

\begin{tabular}{r|r}
341 & 0.46 \\
73 & 0.08
\end{tabular}

Traj. \#8

\begin{tabular}{l|l}
322 & 0.44
\end{tabular}

$73 \quad 0.08$

Traj. \#9

\begin{tabular}{l|l}
575 & 0.84 \\
\hline
\end{tabular}

169 0.20
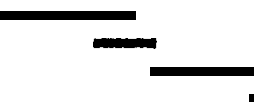
Spawning reach: Minam R.

Mouth - Cougar Cr (RM 89.5 - 99.4 from Snake R)

\section{Relative Productivity}
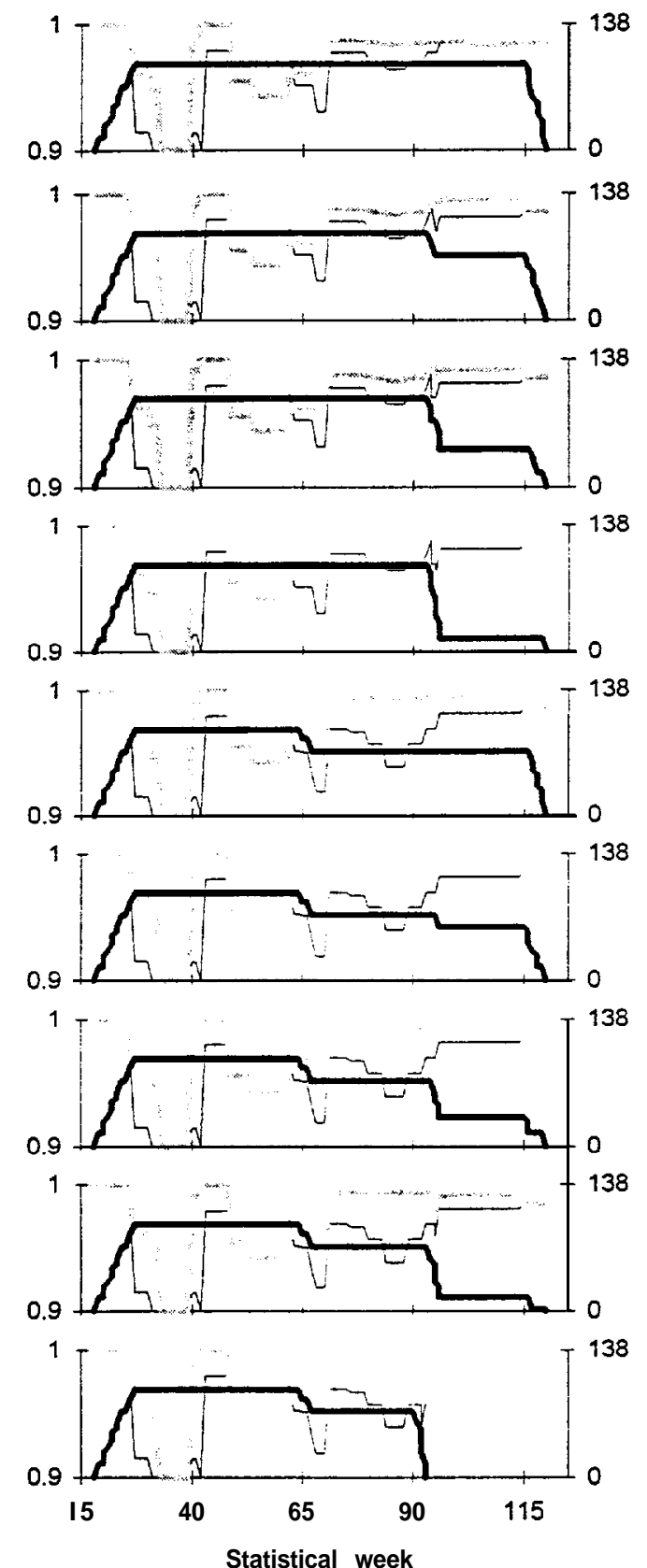

Prespawn -

Spawning

incubation
Colonization

Sum. rearing

Win. rearing
Relative Key Habitat Quantity
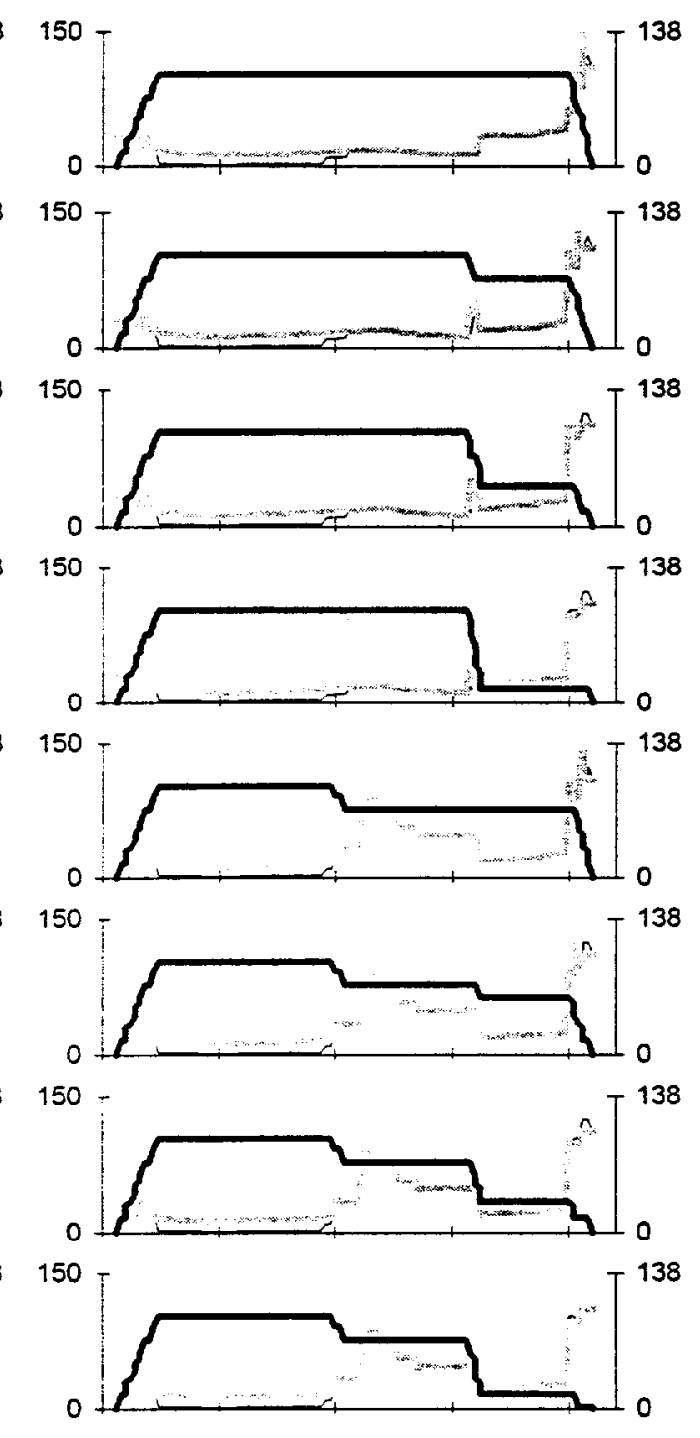

$150-$
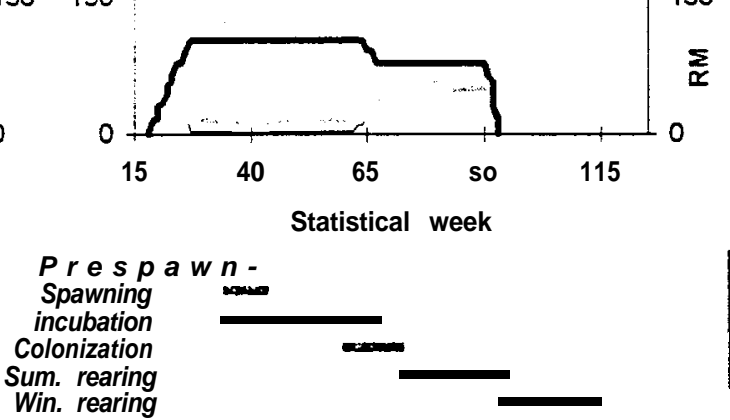

\begin{tabular}{|c|c|}
\hline \multicolumn{2}{|c|}{$\begin{array}{c}\text { Template } \\
\text { Patient }\end{array}$} \\
\hline Cum. & Cum. \\
Prod. & Cap.
\end{tabular}

Traj. \#1

$31 \quad 0.12$

10.00

Traj. \#2

$37 \mid 0.15$

10.00

Traj. \#3

\begin{tabular}{r|r|}
\hline 35 & 0.14 \\
1 & 0.00
\end{tabular}

Traj. \#4

350.14

1) 0.00

Traj. \#5

\begin{tabular}{l|l}
42 & 0.17 \\
\hline & 0.00
\end{tabular}

10.00

Traj. \#6

$38 \mid 0.16$

10.00

Traj. \#7

400.16

10.00

Traj. \#8

\begin{tabular}{r|r}
39 & 0.16 \\
1 & 0.00
\end{tabular}

Traj. \#9

\begin{tabular}{r|r}
71 & 0.29 \\
2 & 0.00
\end{tabular}

21 0.00

Trajectory

Template

Patient 
Spawning reach: Minam $\mathbf{R}$.

Cougar Cr - Little Minam R (RM 99.4 - 108.4 from Snake R)

Relative Productivity
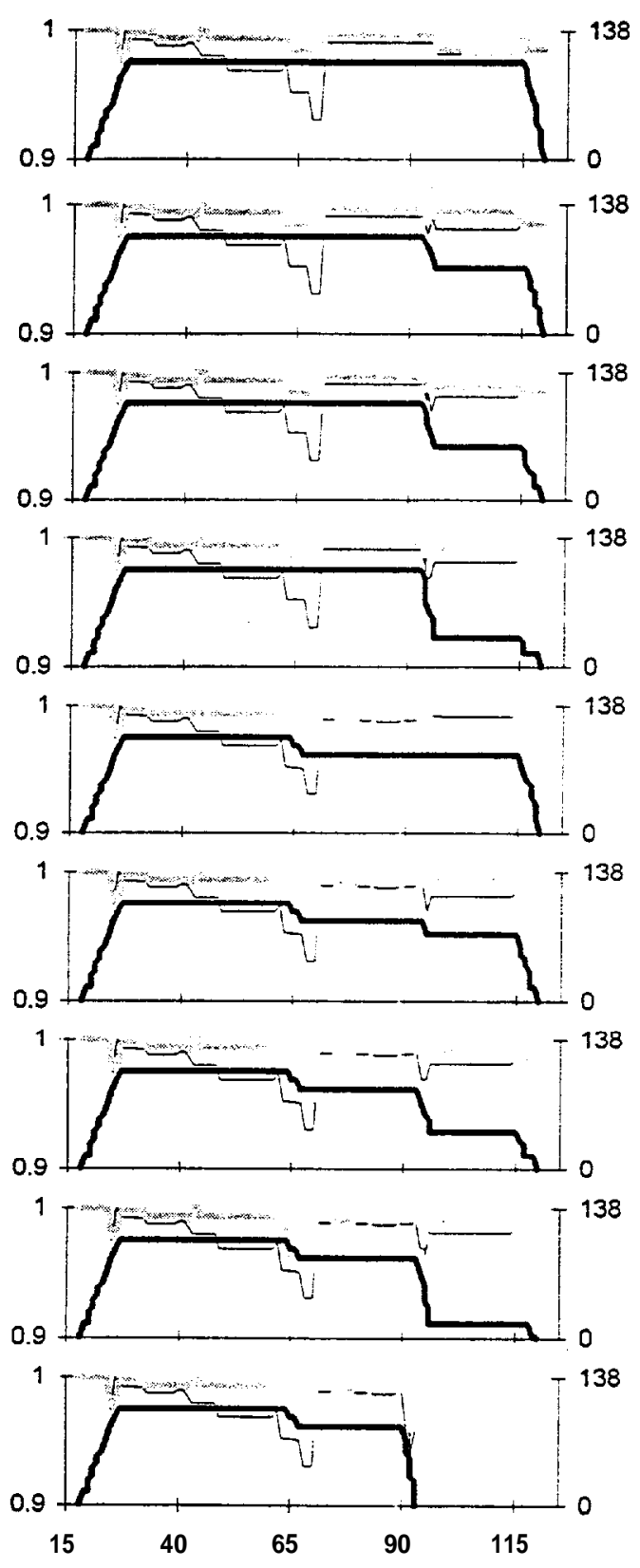

Statistical week
Relative Key Habitat Quantity
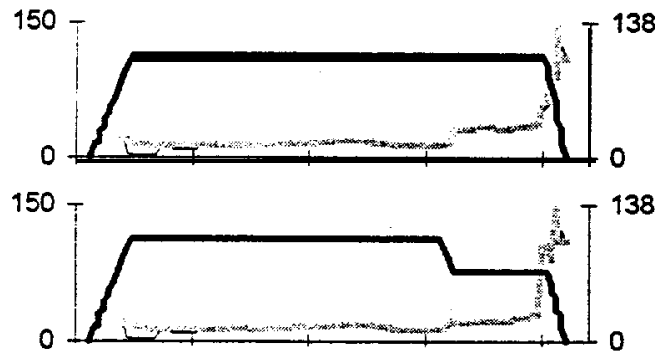

150 -

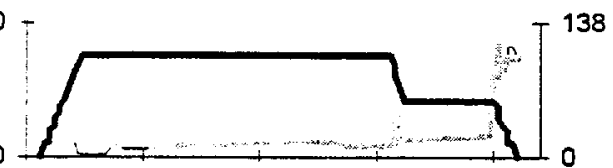

150

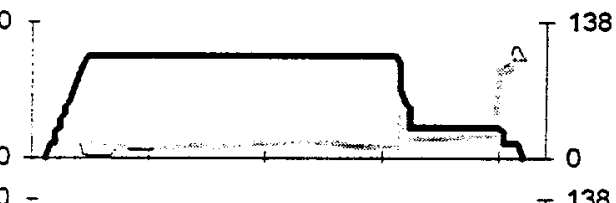

$150-$

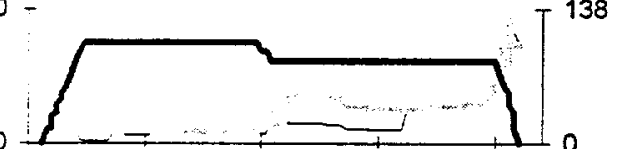

150
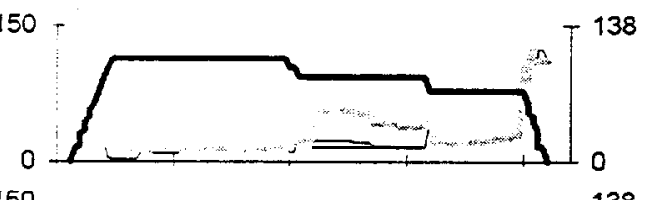

150

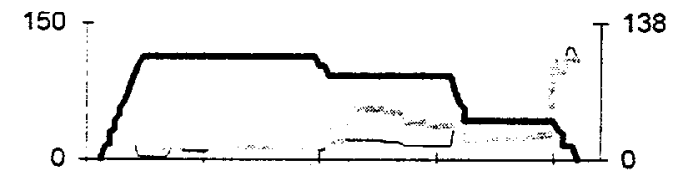

$150-$
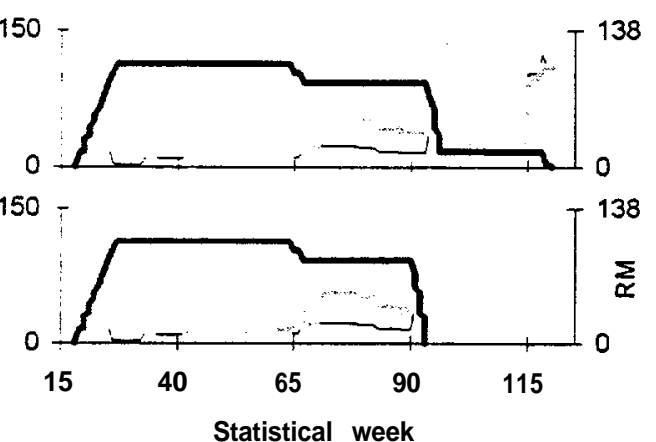

$$
\text { Prespawn - }
$$

Spawning

Incubation Colonization

Sum. rearing

Win. rearing
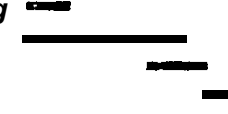

Template

Patient

Cum. Cum.

Prod. Cap.

Traj. \#1

320 $0.7 €$

1220.11

Traj. \#2

400 0.93

1220.11

Traj. \#3

\begin{tabular}{l|l}
363 & 0.85
\end{tabular}

121 0.11

Traj. \#4

\begin{tabular}{l|l}
380 & 0.89
\end{tabular}

120 0.11

Traj . \#5

\begin{tabular}{l|l}
317 & 0.95
\end{tabular}

141 0.13

Traj. \#6

\begin{tabular}{l|l}
\hline 318 & 0.91
\end{tabular}

$114 \quad 0.11$

Traj . \#7

3010.88

1120.10

Traj. \#8

\begin{tabular}{l|l}
\hline 299 & 0.87
\end{tabular}

111 0.10

Traj. \#9

\begin{tabular}{l|l}
538 & 1.73 \\
235 & 0.22
\end{tabular}

Win. rearing

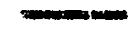

Whe rearing 
Spawning reach: Minam $\mathbf{R}$.

Little Minam R - splash dam site (1 m above Garwood Cr; RM 108.4 - 119.7 from Snake R)

Relative Productivity
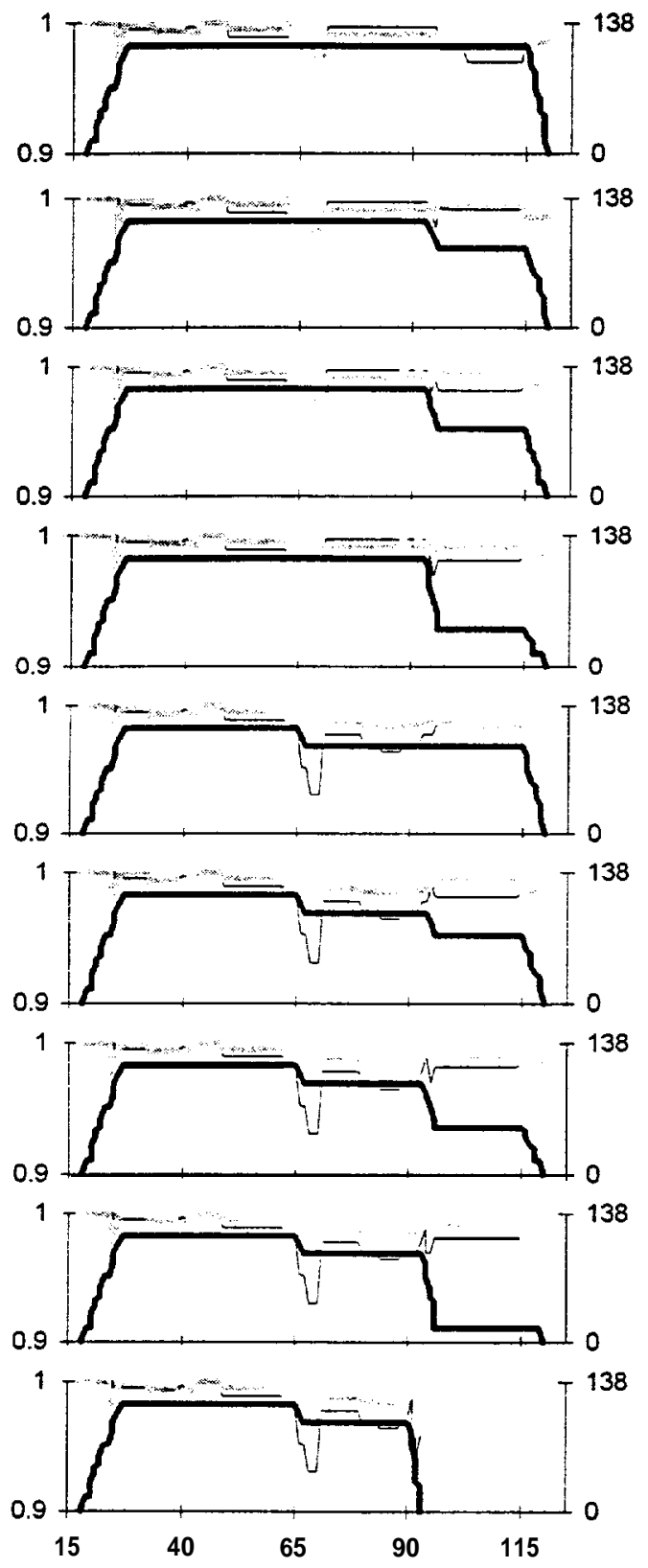

Statistical week

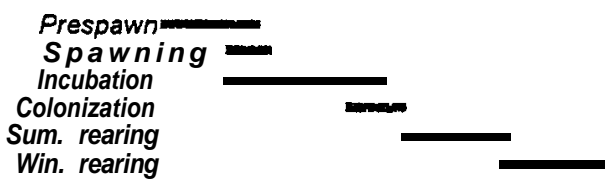

Relative Key Habitat Quantity
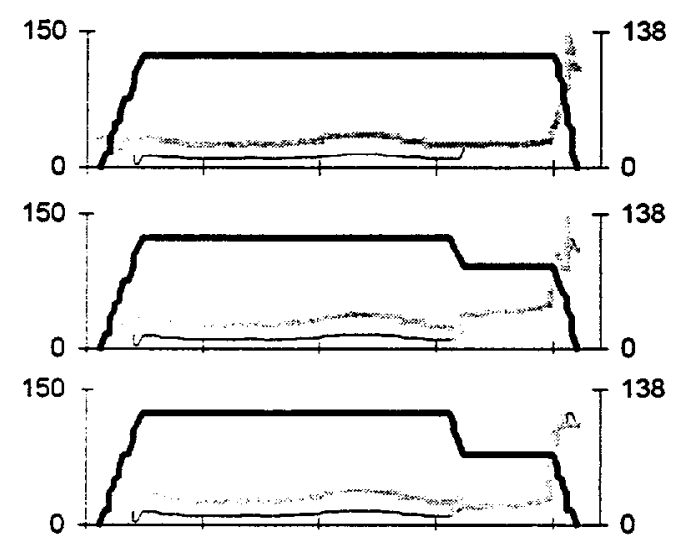

150

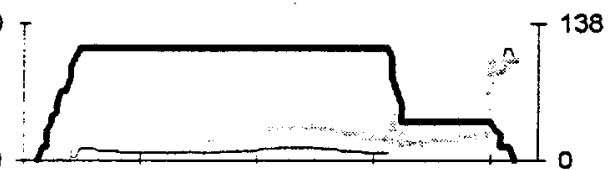

150 .

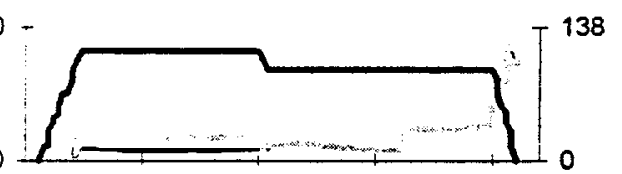

150

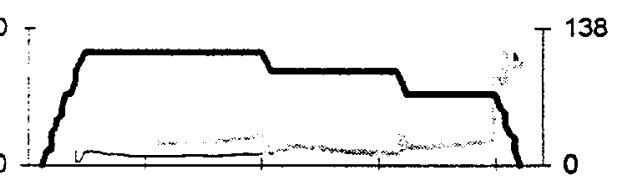

$150-$
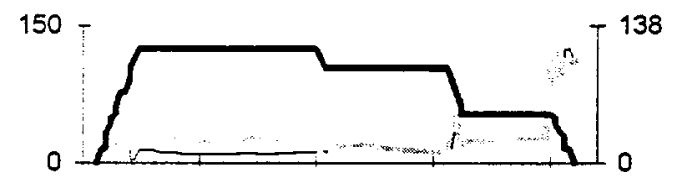

150 -
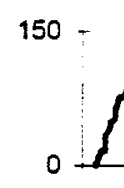

$T^{138}$

$150-$

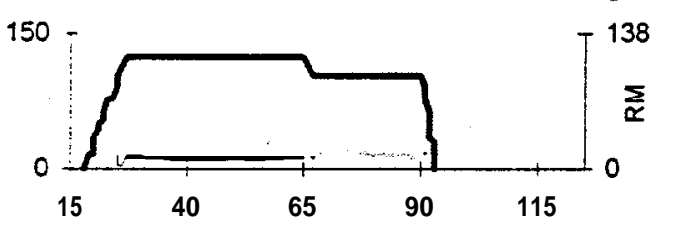

Statistical week

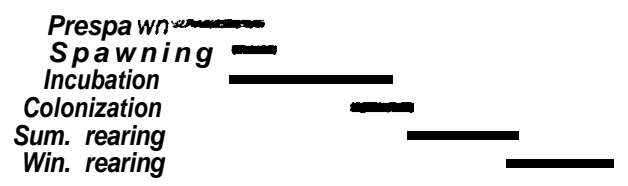

\begin{tabular}{|c|c|}
\hline \multicolumn{2}{|c|}{ Template } \\
Patient \\
\hline Cum. & Cum. \\
Prod. & Cap.
\end{tabular}

Traj. \#1

\begin{tabular}{l|l}
314 & 1.16 \\
\hline
\end{tabular}

278 0.49

Traj. \#2

\begin{tabular}{l|l}
\hline 395 & 1.47 \\
\hline
\end{tabular}

\begin{tabular}{l|l}
377 & 0.68
\end{tabular}

Traj . \#3

\begin{tabular}{|l|l|}
\hline 395 & 1.40 \\
\hline
\end{tabular}

$312 \quad 0.55$

Traj. \#4

\begin{tabular}{l|l}
374 & 1.35 \\
\hline
\end{tabular}

307 0.55

Traj. \#5

\begin{tabular}{l|l|}
272 & 0.86 \\
\hline
\end{tabular}

$153 \quad 0.32$

Traj. \#6

\begin{tabular}{|l|l|}
\hline 324 & 1.00 \\
\hline
\end{tabular}

$144 \quad 0.30$

Traj . \#7

$294 \quad 0.93$

$142 \quad 0.30$

Traj. \#8

\begin{tabular}{l|l}
\hline 08 & 0.96 \\
\hline
\end{tabular}

140 0.29

Traj. \#9

\begin{tabular}{l|l}
562 & 2.04 \\
\hline
\end{tabular}

312 0.69 
Spawning reach: Minam $\mathbf{R}$.

Splash dam site - falls (RM 119.7 - 138.0 from Snake R)

Relative Productivity
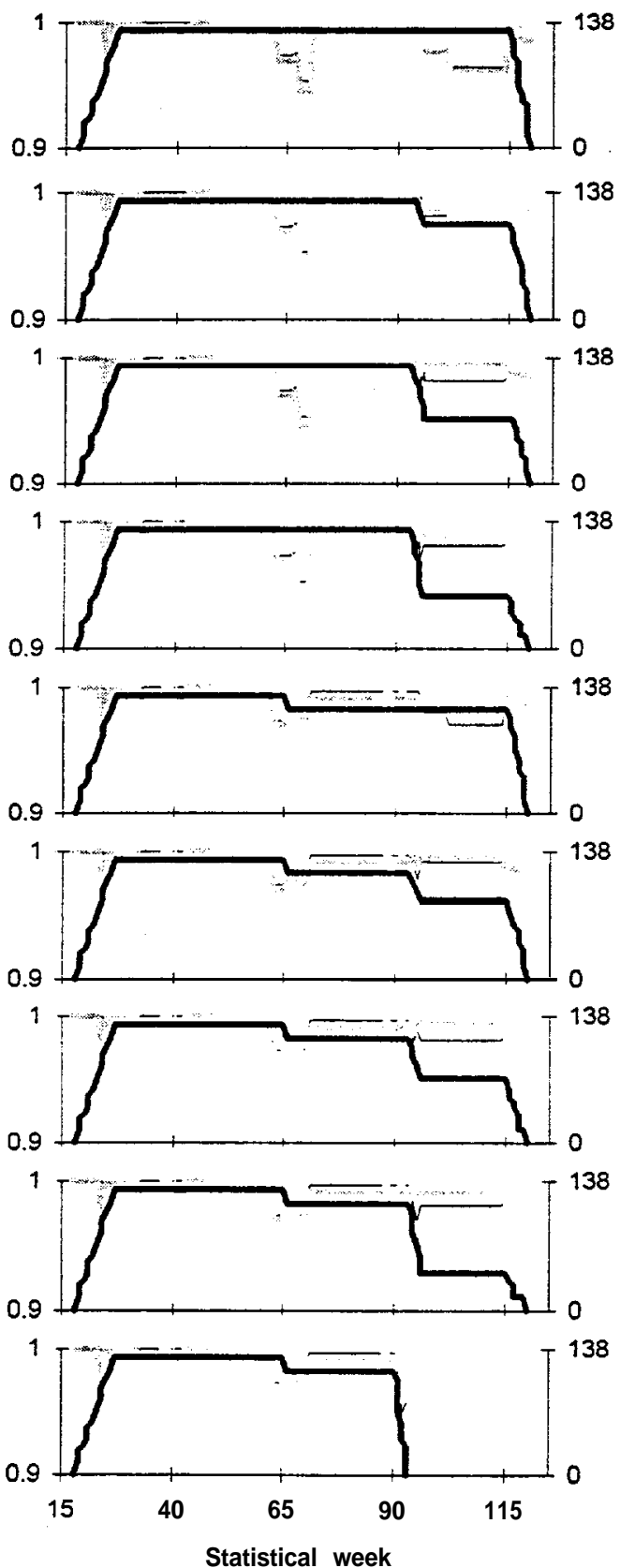

Prespawn -

Spawning

Incubation

Colonization

Sum. rearing

Win. rearing
Relative Key Habitat Quantity
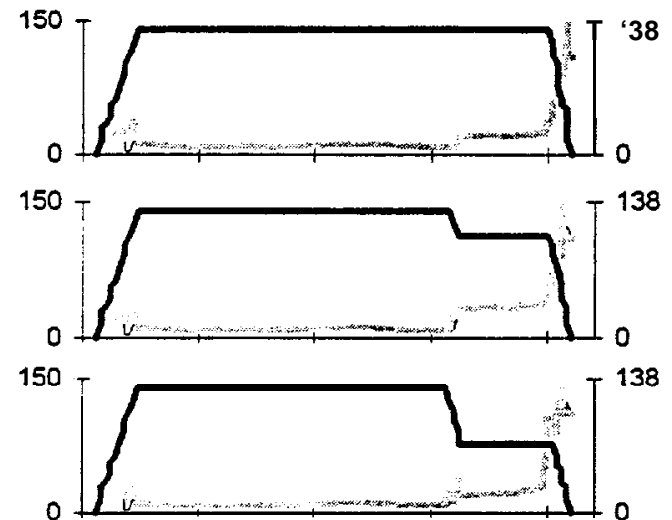

150

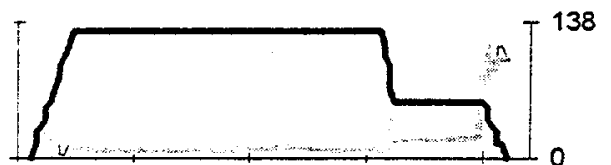

$150-$

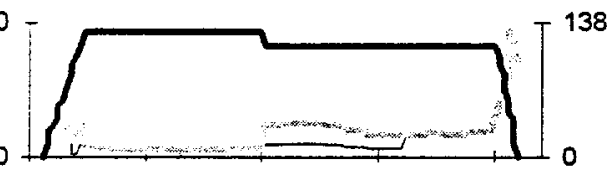

150
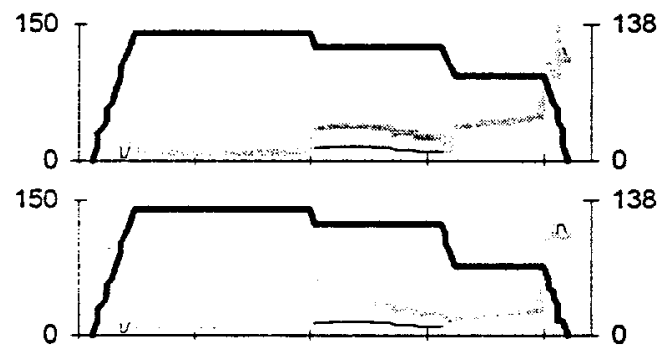

150
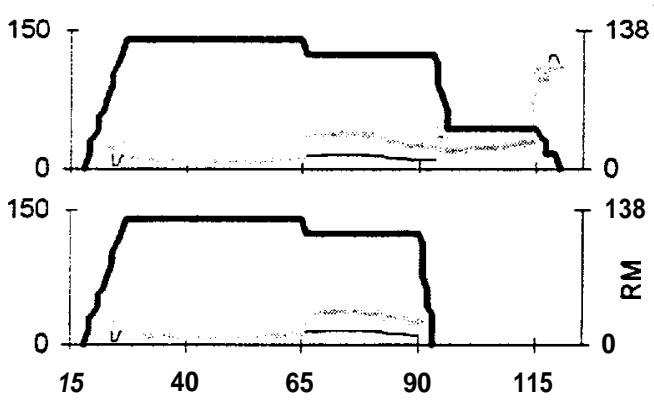

Statistical week

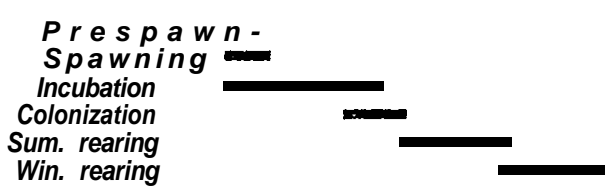

\section{Template \\ Patient \\ Cum. Cum. \\ Prod. Cap.}

Traj. \#1

2110.40

268 0.39

Traj. \#2

279 0053

32ग7 00448

Traj. \#3

$352 \quad 0.66$

$331 \quad 0.48$

Traj. \#4

318 0.60

\begin{tabular}{l|l}
327 & 0.47
\end{tabular}

Traj. \#5

\begin{tabular}{l|l|}
\hline 293 & 0.70 \\
315 & 0.48
\end{tabular}

315 0.48

Trai. \#6

3690.89

426 0.66

Traj. \#7

\begin{tabular}{|l|l|}
\hline 369 & 0.86 \\
\hline 353 & 0.53
\end{tabular}

353 0.53

Traj. \#8

\begin{tabular}{l|l}
\hline 349 & 0.83 \\
\hline
\end{tabular}

\begin{tabular}{l|l}
346 & 0.53
\end{tabular}

Traj. \#9

\begin{tabular}{|l|l|}
\hline 627 & 1.65 \\
\hline
\end{tabular}

$713 \quad 1.20$ 
Spawning reach: Prairie Cr.

Mouth - OK Gulch confluence (RM 126.8 - 131.8 from Snake R)

Relative Productivity
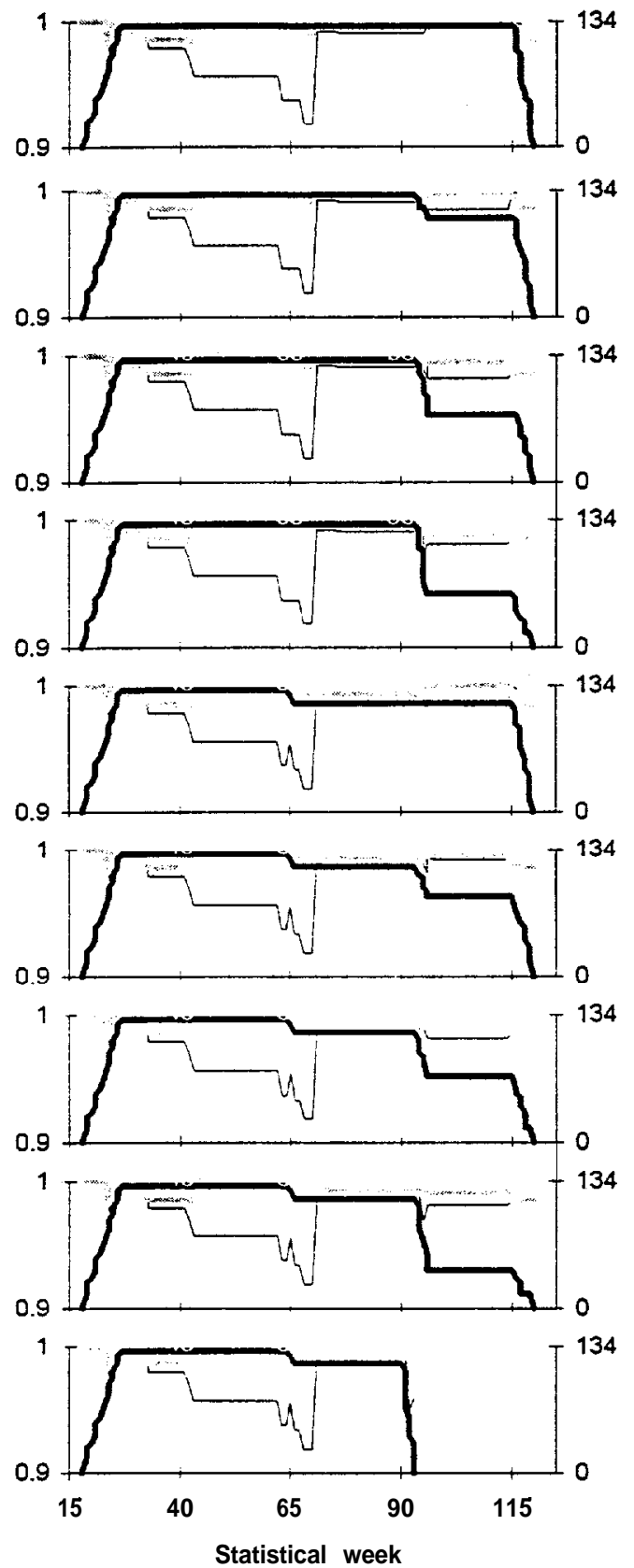

Prespawn -

spawning

Incubation

Colonization

Sum. rearing

Win. rearing
Relative Key Habitat Quantity
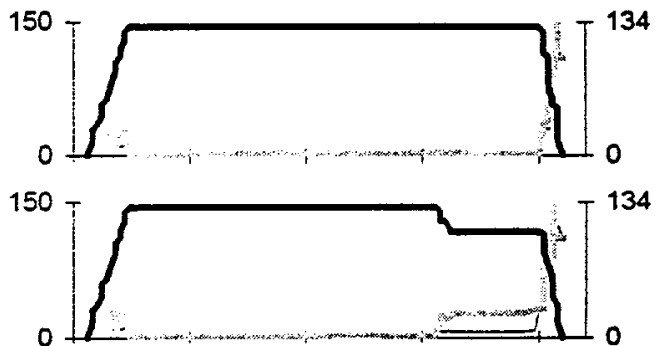

150
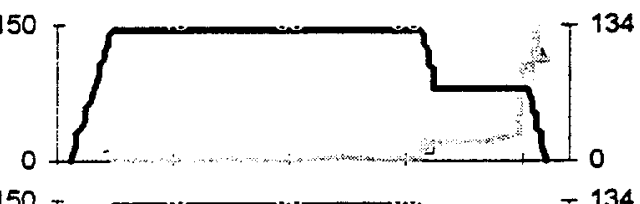

150
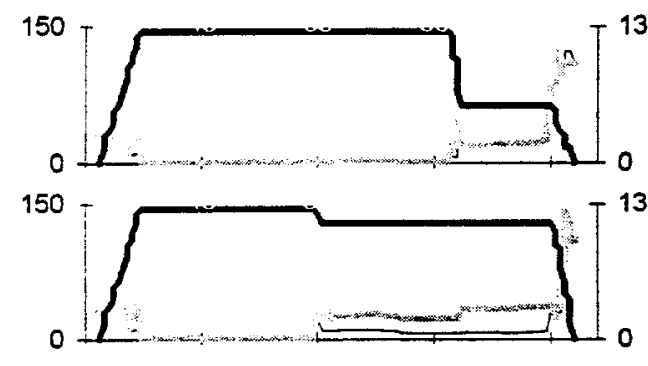

150

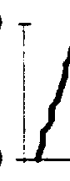

150
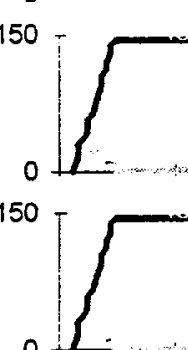

150 T
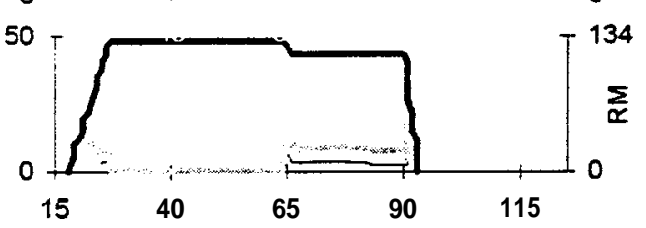

Statistical week

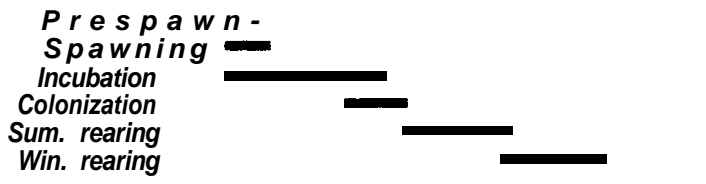

\begin{tabular}{|r|c|}
\hline \multicolumn{2}{|c|}{$\begin{array}{c}\text { Template } \\
\text { Patient }\end{array}$} \\
\hline $\begin{array}{r}\text { Cum. } \\
\text { Prod. }\end{array}$ & Cum. \\
Cap. \\
Traj. \#1 \\
\hline 508 & 0.11 \\
105 & 0.09 \\
\hline
\end{tabular}

Traj. \#2

\begin{tabular}{l|l}
499 & 0.14 \\
\hline
\end{tabular}

80 0.07

Traj. \#3

\begin{tabular}{|l|l|}
\hline 467 & 0.13 \\
\hline
\end{tabular}

73) 0.07

Traj. \#4

\begin{tabular}{l|l|}
\hline 422 & 0.12 \\
\hline & 0.07
\end{tabular}

73) 0.07

Traj. \#5

\begin{tabular}{l|l|}
476 & 0.17 \\
\hline & 0.08
\end{tabular}

810.08

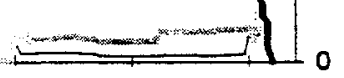

0

Traj. \#6

\begin{tabular}{l|l|}
422 & 0.15 \\
\hline & 0.09
\end{tabular}

$\begin{array}{ll}90 & 0.09\end{array}$

Traj. \#7

\begin{tabular}{l|l}
421 & 0.15 \\
\hline & 0.08
\end{tabular}

$\begin{array}{ll}74 & 0.08\end{array}$

Traj. \#8

\begin{tabular}{l|l|}
\hline 398 & 0.14 \\
\hline
\end{tabular}

720.08

Traj. \#9

\begin{tabular}{l|l|}
713 & 0.26 \\
\hline & 0.16
\end{tabular}

152 0.16 
Spawning reach: Prairie Cr.

OK Gulch fork (RM 131.8 - 133.8 from Snake R)

Relative Productivity
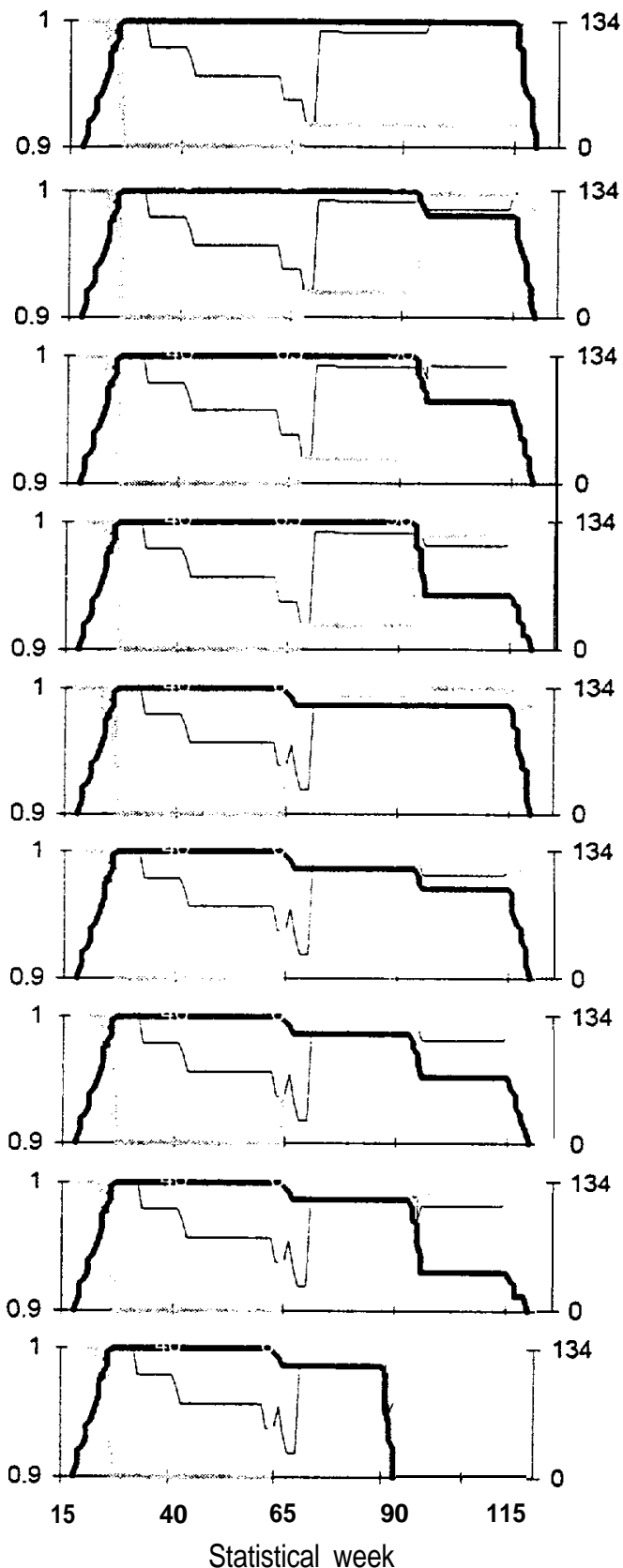

Prespawn -

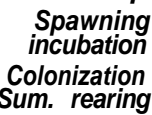

Sum. rearing

Win. rearing

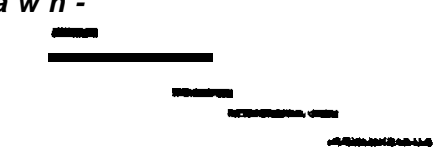

Relative Key Habitat Quantity
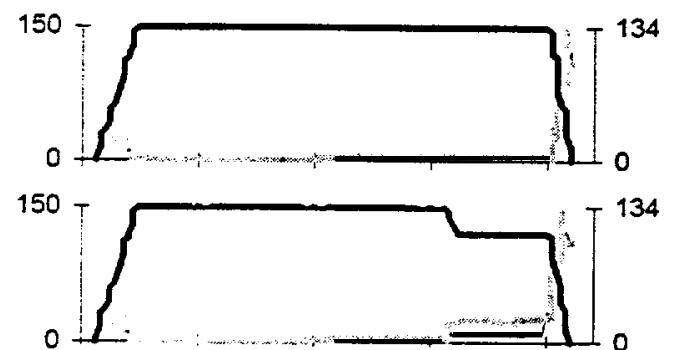

150 -
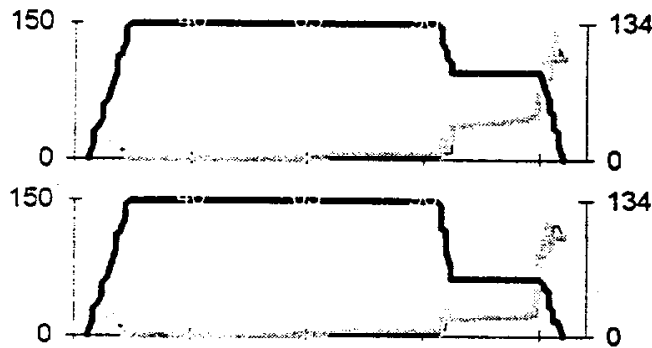

$150-$
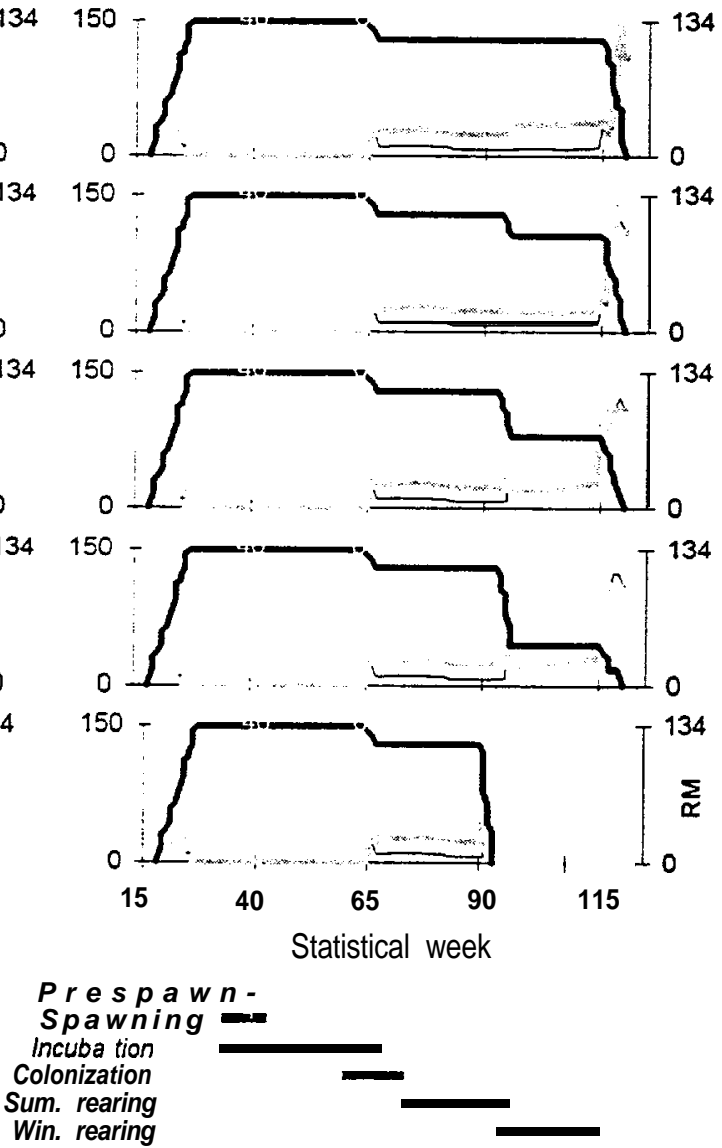

$\begin{array}{llll}40 & 65 & 90 & 115\end{array}$

Statistical week

\section{Template \\ Patient}

Cum. Cum.

Prod. Cap.

Traj. \#1

0.00

106 0.01

Traj. \#2

0.0 .00

$80 \quad 0.01$

Traj. \#3

\begin{tabular}{r|r|}
0 & 0.00 \\
89 & 0.01
\end{tabular}

$89 \quad 0.01$

Traj. \#4

\begin{tabular}{r|r|}
0 & 0.00 \\
73 & 0.01
\end{tabular}

Traj. \#5

\begin{tabular}{l|l|}
\hline 0 & 0.00 \\
\hline & 0.01
\end{tabular}

81 0.01

Traj. \#6

\begin{tabular}{r|l}
0 & 0.00 \\
75 & 0.01
\end{tabular}

Traj. \#7

\begin{tabular}{l|l|}
\hline 0 & 0.00 \\
\hline &
\end{tabular}

$74 \quad 0.01$

Traj. \#8

\begin{tabular}{l|l|}
\hline 0 & 0.00 \\
73 & 0.01
\end{tabular}

73) 0.01

Traj. \#9

\begin{tabular}{l|r|r}
$\sum_{\alpha}$ & $T$ & 0.00 \\
153 & 0.02
\end{tabular}

Trajectory 1

Template

Patient 
Spawning reach: Sheep Cr.

Mouth - Chicken Cr (RM 164.2 - 165.7 from Snake R)

Relative Productivity
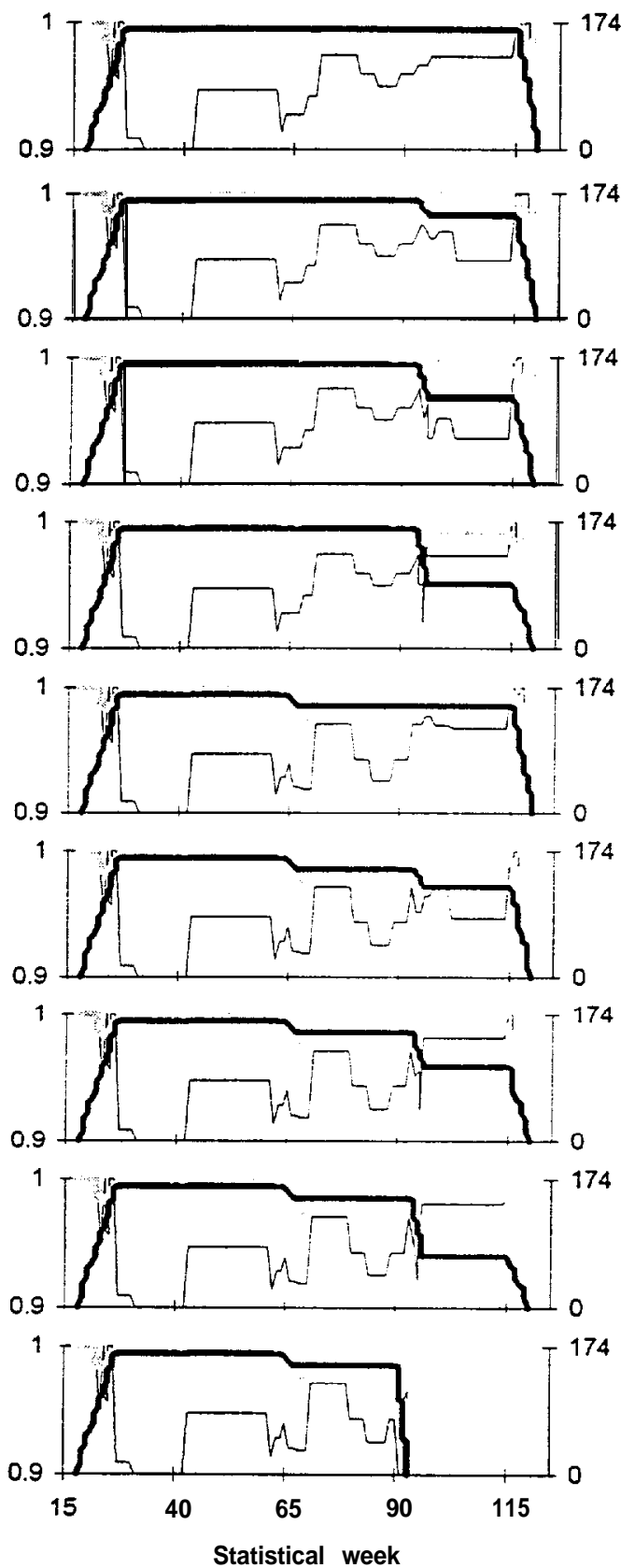

Prespawn -

Spawning

Incubation

Colonization

Sum. rearing

Win. rearing

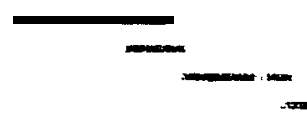

Relative Key Habitat Quantity
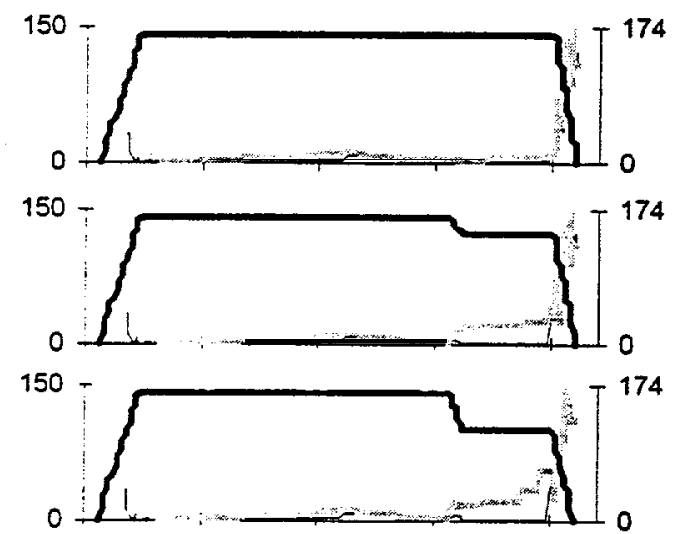

$150-$

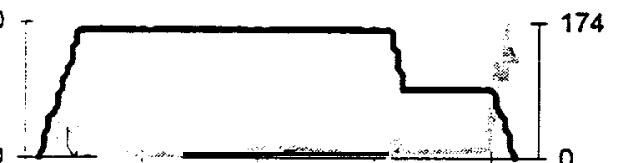

150 -

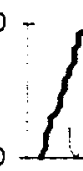

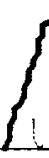
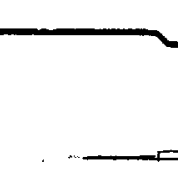

150 -

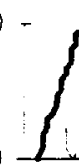

02
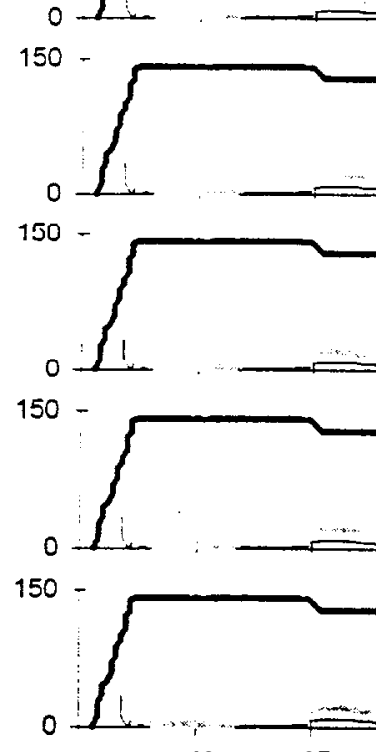

15

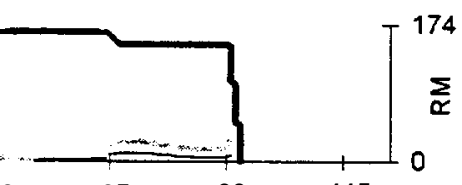

Prespawn -

Spawning

Incubation Colonization

Sum. rearing

Win. rearing \begin{tabular}{|r|c|}
\hline \multicolumn{2}{|c|}{ Template } \\
Patient \\
\hline Cum. & Cum. \\
Prod. & Cap. \\
\multicolumn{2}{|c|}{ Traj. \#l } \\
\hline 485 & 0.39 \\
1 & 0.00 \\
\hline
\end{tabular}

Traj. \#2

443 0.44

10.00

Traj. \#3

5170.51

0.00

Traj. \#4

\begin{tabular}{l|l}
466 & 0.43
\end{tabular}

10.00

Traj. \#5

\begin{tabular}{l|l}
380 & 0.47 \\
\hline
\end{tabular}

0) 0.00

Traj. \#6

\begin{tabular}{l|l}
378 & 0.48 \\
\hline & 0.00
\end{tabular}

Traj. \#7

\begin{tabular}{l|l}
374 & 0.47
\end{tabular}

10.00

Traj. \#8

$390 \quad 0.50$

10.00

Traj. \#9

$671 \quad 0.92$

Statistical week 
Spawning reach: Sheep Cr.

Chicken $\mathrm{Cr}$ - headwaters (RM 165.7 - 173.7 from Snake R)

Relative Productivity
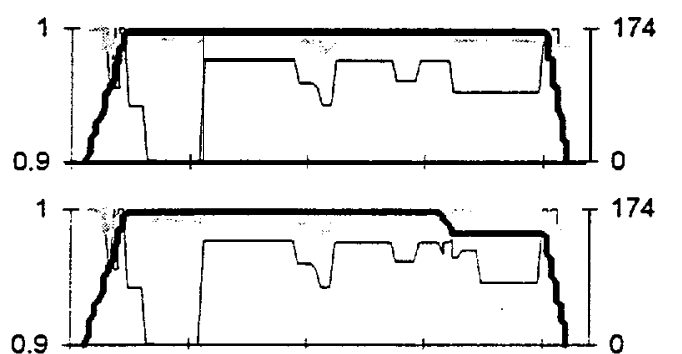

0.9
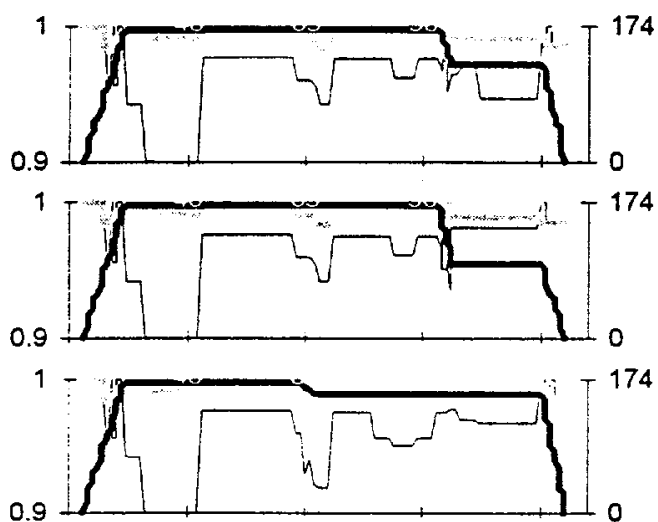

1
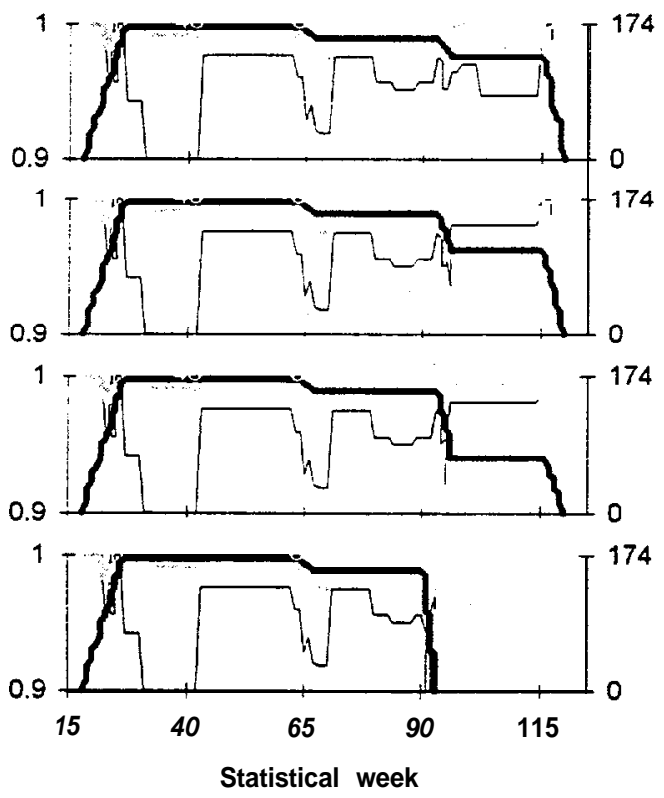

Prespaw $n-$
Spawning

Incubation

Colonization

Colonization
Sum. rearing

Win. rearing

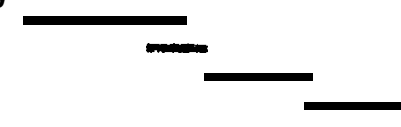

Relative Key Habitat Quantity

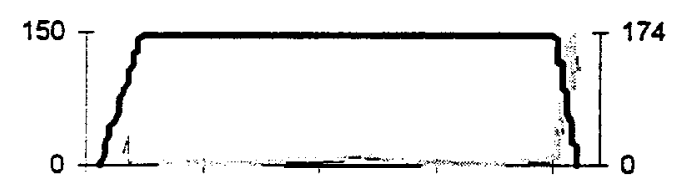

$150_{T}$

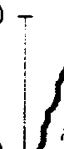

0
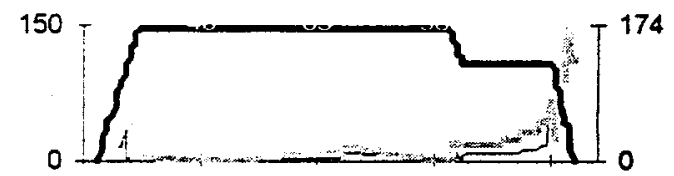

150

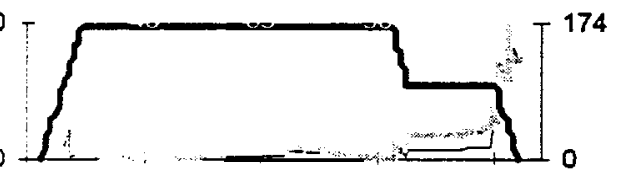

150 -
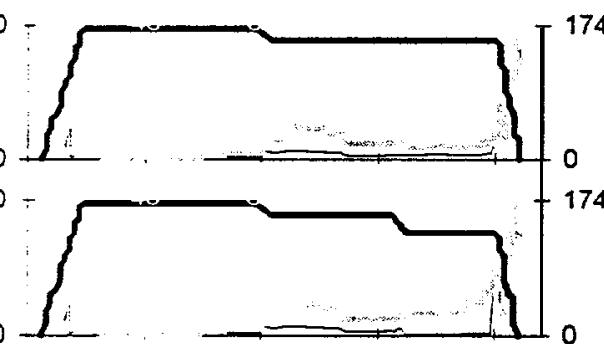

174

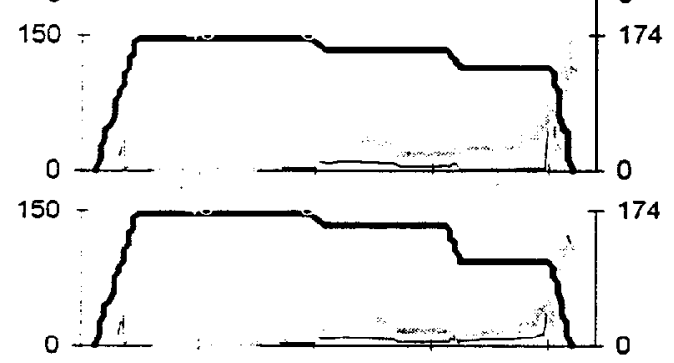

150 -
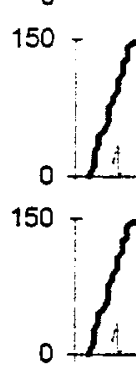

15

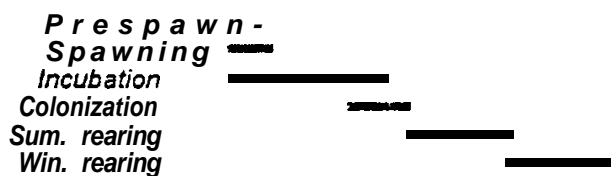

\begin{tabular}{|c|c|}
\hline \multicolumn{2}{||c|}{$\begin{array}{c}\text { Template } \\
\text { Patient }\end{array}$} \\
\hline \begin{tabular}{|c|c} 
Cum. \\
Prod.
\end{tabular} & $\begin{array}{c}\text { Cum. } \\
\text { Cap. }\end{array}$ \\
\hline
\end{tabular}

Traj. \#1

.

Traj. \#2

\begin{tabular}{r|r|}
\hline 426 & $0.3 \varepsilon$ \\
8 & 0.01
\end{tabular}

Traj. \#3

\begin{tabular}{l|l}
455 & $0.4 \mathrm{C}$
\end{tabular}

8) 0.01

Traj. \#4

\begin{tabular}{l|l}
\hline 428 & $0.3 \varepsilon$ \\
\hline
\end{tabular}

13 0.01

Traj . \#5

F

Traj. \#6

358.0 .46

5) 0.00

Traj. \#7

\begin{tabular}{r|l}
\hline 397 & 0.49 \\
9 & 0.01
\end{tabular}

Traj. \#8

-

Traj. \#9

\begin{tabular}{r|l}
\hline 678 & 0.92 \\
16 & 0.01 \\
\hline
\end{tabular}

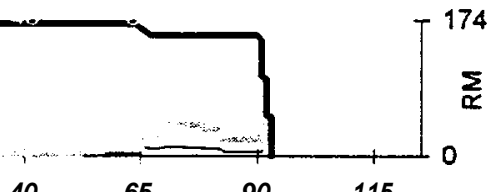

Trajectory
$-\quad$ Pemplate
Patient 
Spawning reach: Wallowa $R$.

Mouth - Minam R (RM 80.0 - 89.8 from Snake R)

Relative Productivity
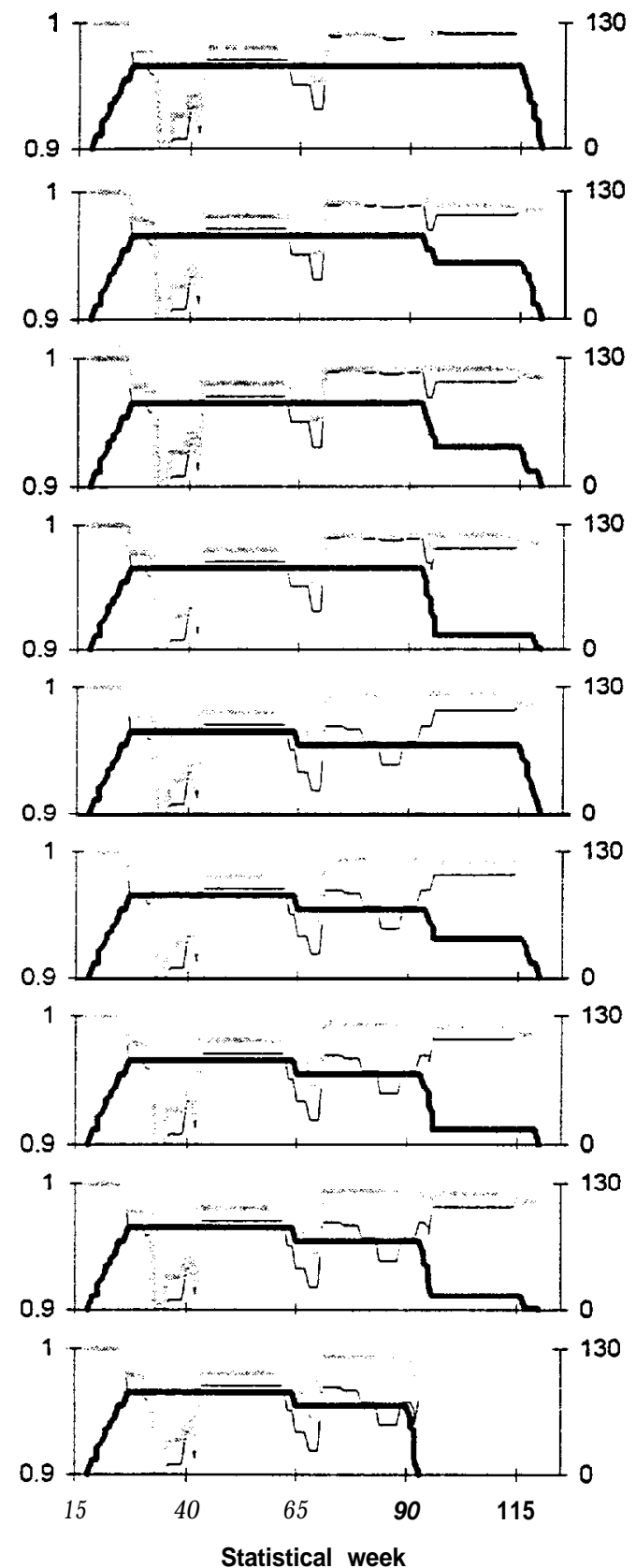

Prespawn -

Spawn
Incubation

Incubation
Colonization

Sum. rearing

Win. rearing

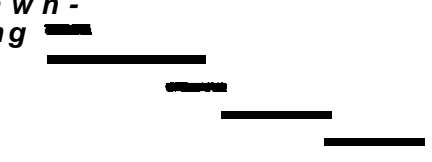

Relative Key Habitat Quantity
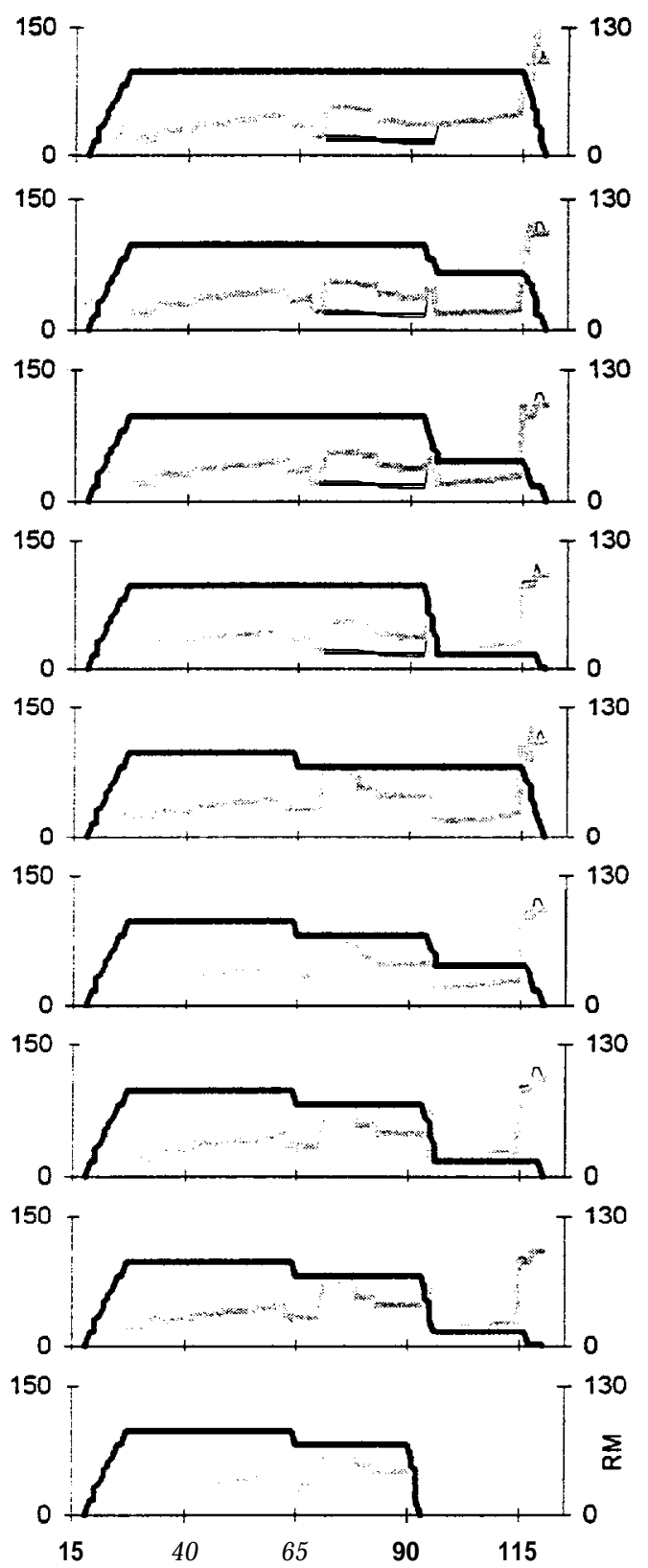

Statistical week

\begin{tabular}{|c|c|}
\hline \multicolumn{2}{|c|}{$\begin{array}{c}\text { Template } \\
\text { Patient }\end{array}$} \\
\hline $\begin{array}{c}\text { Cum. } \\
\text { Prod. }\end{array}$ & Cam. \\
\hline
\end{tabular}

Traj. \#1

$101 \quad 0.42$

47 0.21

Traj. \#2

\begin{tabular}{l|l}
\hline 91 & 0.38 \\
37 & 0.16
\end{tabular}

Traj. \#3

\begin{tabular}{l|l}
\hline 95 & 0.39 \\
37 & 0.16
\end{tabular}

$37 \quad 0.16$

Traj. \#4

\begin{tabular}{l|l}
94 & 0.39 \\
\hline &
\end{tabular}

$37 \quad 0.16$

Traj. \#5

\begin{tabular}{l|l|}
104 & 0.44 \\
\hline
\end{tabular}

16) 0.08

Traj. \#6

\begin{tabular}{l|l|}
\hline 98 & 0.41 \\
\hline
\end{tabular}

16) 0.08

Traj. \#7

\begin{tabular}{l|l|}
97 & 0.41 \\
\hline
\end{tabular}

16) 0.08

Traj. \#8

\begin{tabular}{l|l|}
\hline 96 & 0.41 \\
16 & 0.08 \\
\hline
\end{tabular}

Traj. \#9

172 0.76

37) 0.17

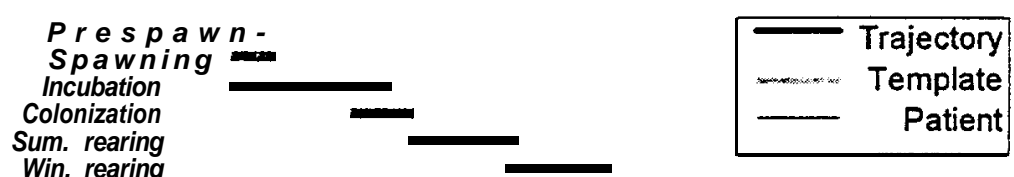


Spawning reach: Wallowa $\mathbf{R}$.

Minam R - Deer Cr (RM 89.8 - 90.8 from Snake R)

Relative Productivity
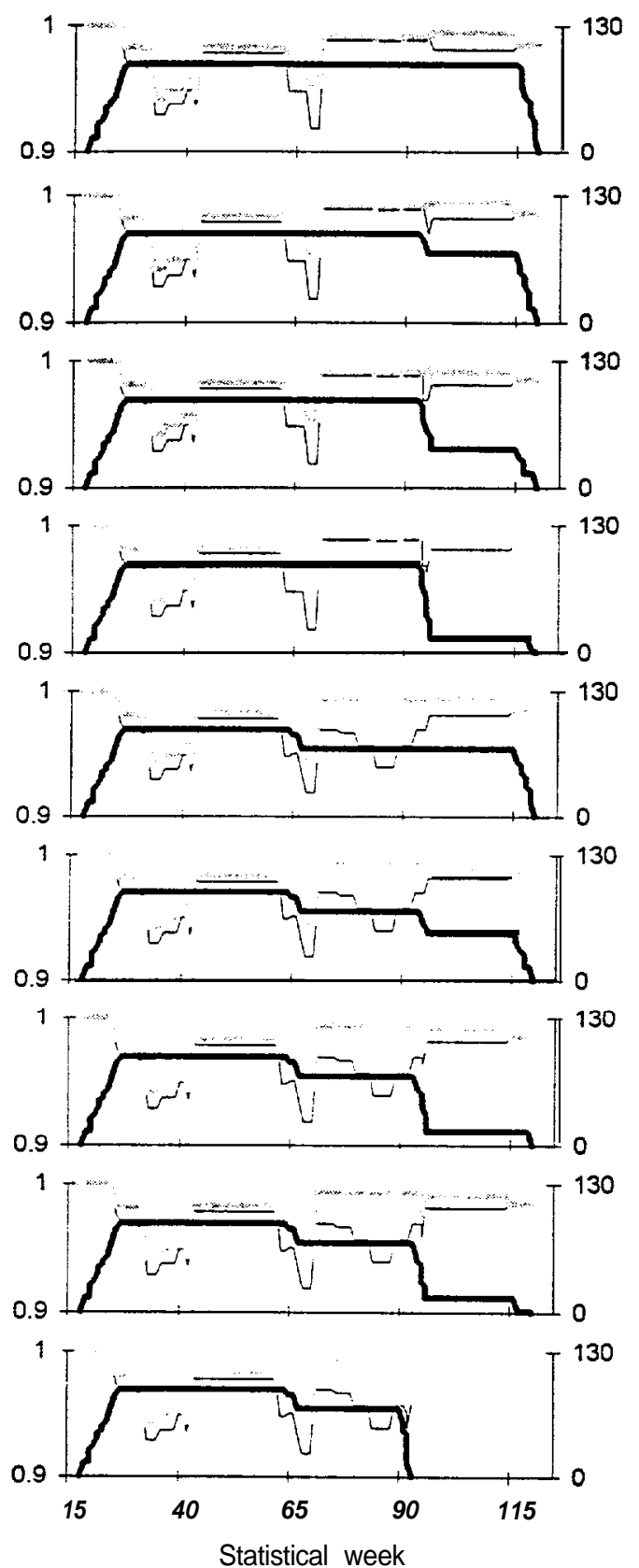

Prespa $w$ n -

Spawning

Incubation

Colonization

Sum. rearing

Win. rearing
Relative Key Habitat Quantity
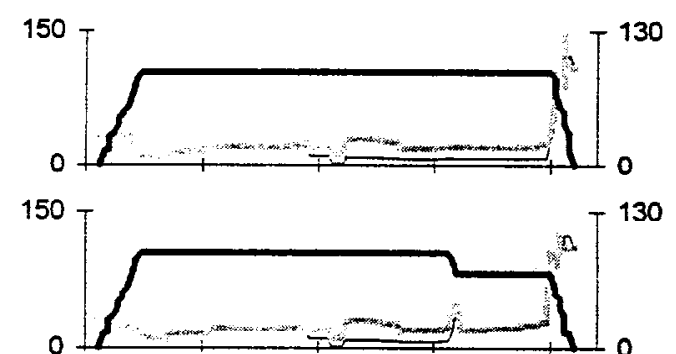

$150 \mathrm{~T}$
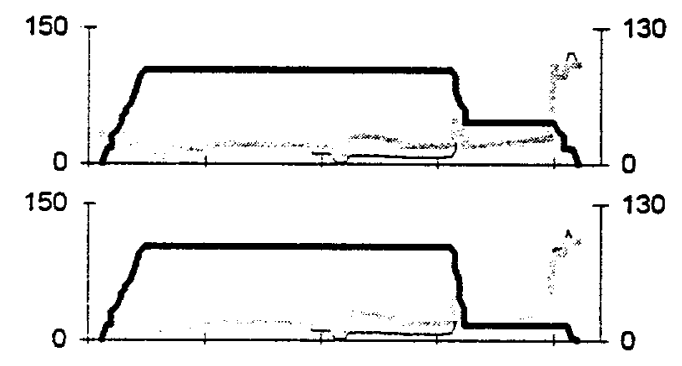

$150-$
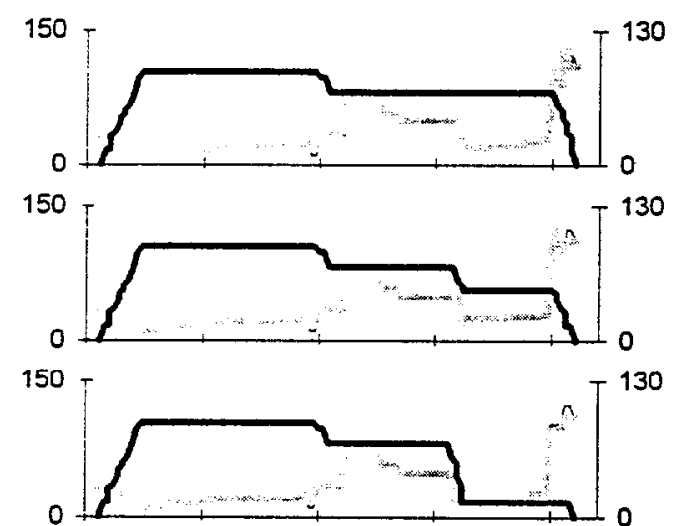

150

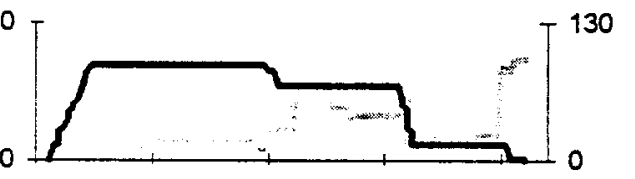

150
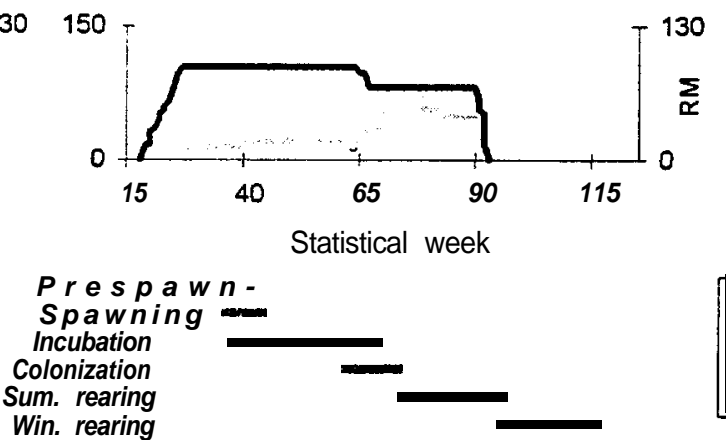

Template
Patient

\begin{tabular}{l|l}
\hline Cum. & Cum. \\
\hline
\end{tabular}

Prod. Cap.

Traj. \#1

\begin{tabular}{|l|l|}
142 & 0.36 \\
\hline & 0.13
\end{tabular}

$63 \quad 0.13$

Traj. \#2

\begin{tabular}{l|l}
143 & 0.37 \\
\hline & 0.13
\end{tabular}

62) 0.13

Traj. \#3

\begin{tabular}{l|l|}
\hline 135 & 0.35 \\
\hline
\end{tabular}

61) 0.13

Traj. \#4

$134 \quad 0.35$

$61 \quad 0.13$

Traj. \#5

\begin{tabular}{r|r|}
147 & 0.40 \\
29 & 0.08
\end{tabular}

Traj. \#6

$133 \quad 0.37$

\begin{tabular}{l|l}
29 & 0.08
\end{tabular}

Traj. \#7

\begin{tabular}{r|r|}
137 & 0.38 \\
29 & 0.08
\end{tabular}

Traj. \#8

\begin{tabular}{r|r|}
137 & 0.38 \\
29 & 0.08
\end{tabular}

Traj. \#9

\begin{tabular}{|l|l|}
\hline 245 & 0.70 \\
\hline
\end{tabular}

$66 \quad 0.18$ 
Spawning reach: Wallowa $\mathbf{R}$.

Deer Cr - Dry Cr (RM 90.8 - 98.3 from Snake R)

Relative Productivity
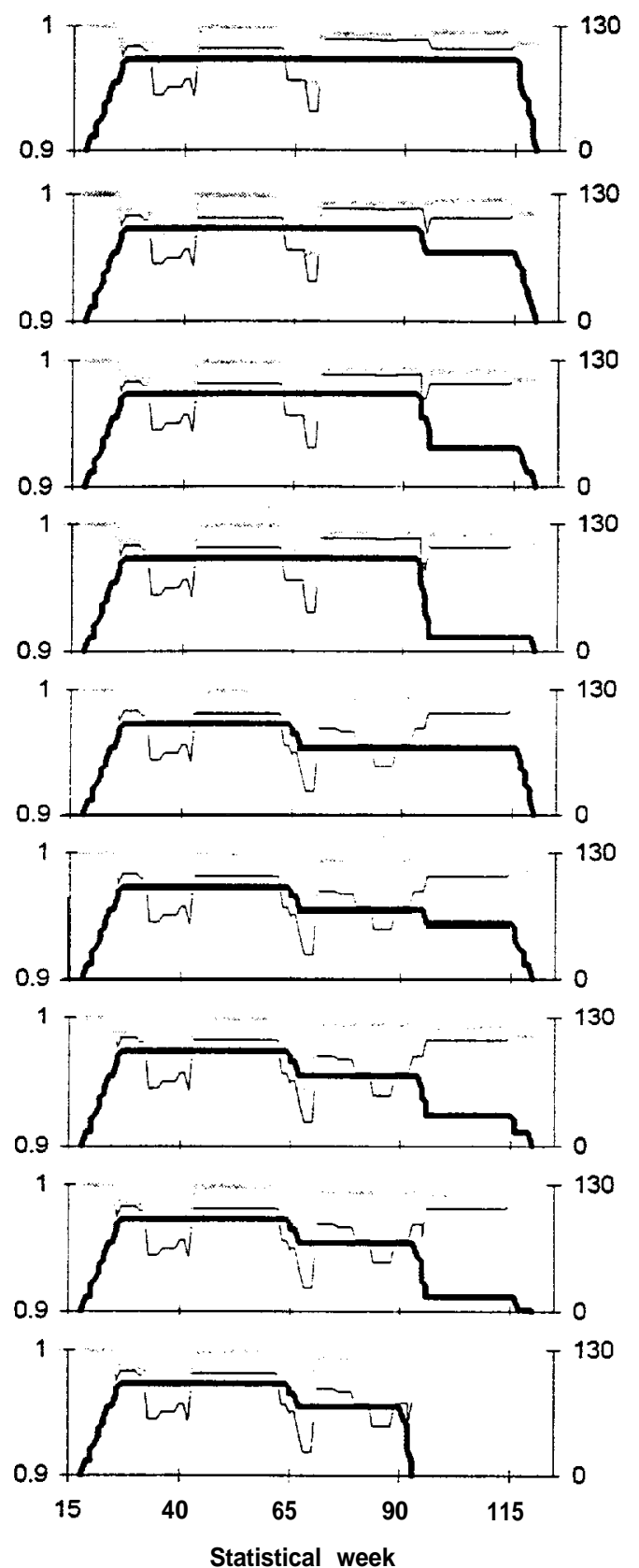

Statistical week

Prespawn -

Incubation

Colonization

Sum. rearing

Win. rearing
Relative Key Habitat Quantity
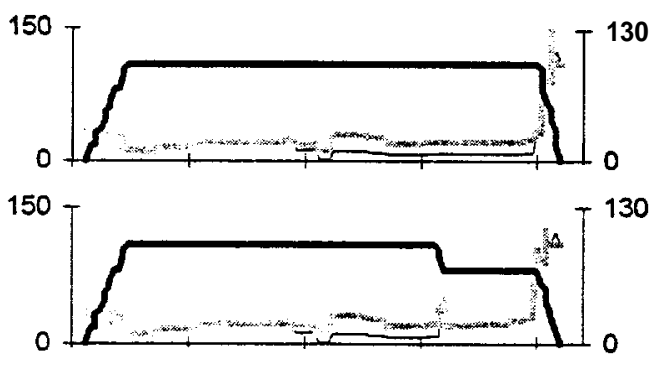

150
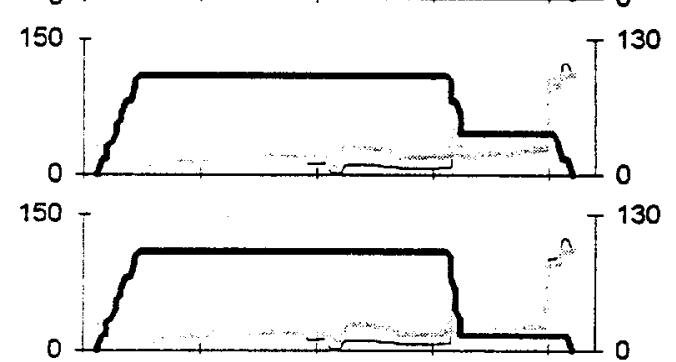

150

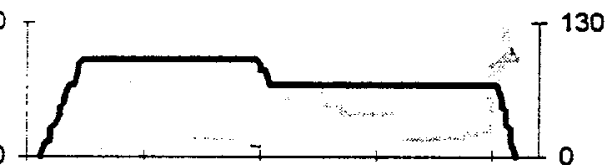

(50,

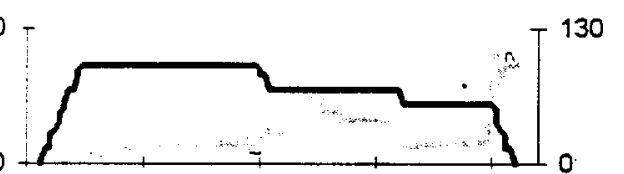

$150-$

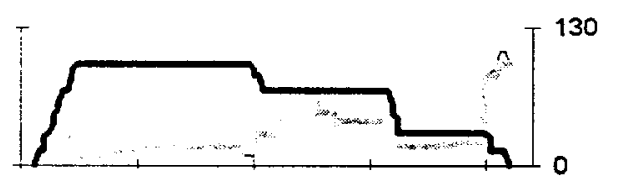

150

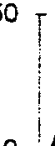

1

150

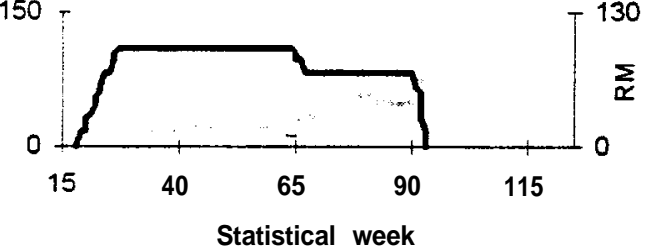

Prespaw $n$ -

Spa wn i
Incubation
Colonization
Sum. rearing

Win. rearing \begin{tabular}{|r|c|}
\hline \multicolumn{2}{|c|}{ Template } \\
Patient \\
\hline Cum. & Cum. \\
Prod. & Cap. \\
Traj. \#1 \\
\hline 270 & 0.61 \\
89 & 0.18 \\
\hline
\end{tabular}

Traj. \#2

$270 \quad 0.62$

$88 \quad 0.18$

Traj. \#3

\begin{tabular}{l|l|}
255 & 0.59 \\
\hline & 0.18
\end{tabular}

$87 \quad 0.18$

Traj. \#4

\begin{tabular}{r|r|}
254 & 0.58 \\
86 & 0.18 \\
\hline
\end{tabular}

Traj. \#5

$265 \quad 0.68$

\begin{tabular}{l|l}
39 & 0.11
\end{tabular}

Traj. \#6

$239 \quad 0.61$

39 0.11

Traj. \#7

\begin{tabular}{l|l}
\hline 249 & 0.64 \\
\hline
\end{tabular}

$39 \quad 0.11$

Traj. \#8

\begin{tabular}{|l|l|}
\hline 246 & 0.64 \\
\hline
\end{tabular}

38 0.10

Traj. \#9

\begin{tabular}{l|l|}
\hline 442 & 1.23 \\
\hline & 0.24
\end{tabular}

\begin{tabular}{l|l}
88 & 0.24
\end{tabular} 
Spawning reach: Wallowa $\mathbf{R}$.

Dry Cr - Bear Cr (RM 98.3 - 101.8 from Snake R)

Relative Productivity
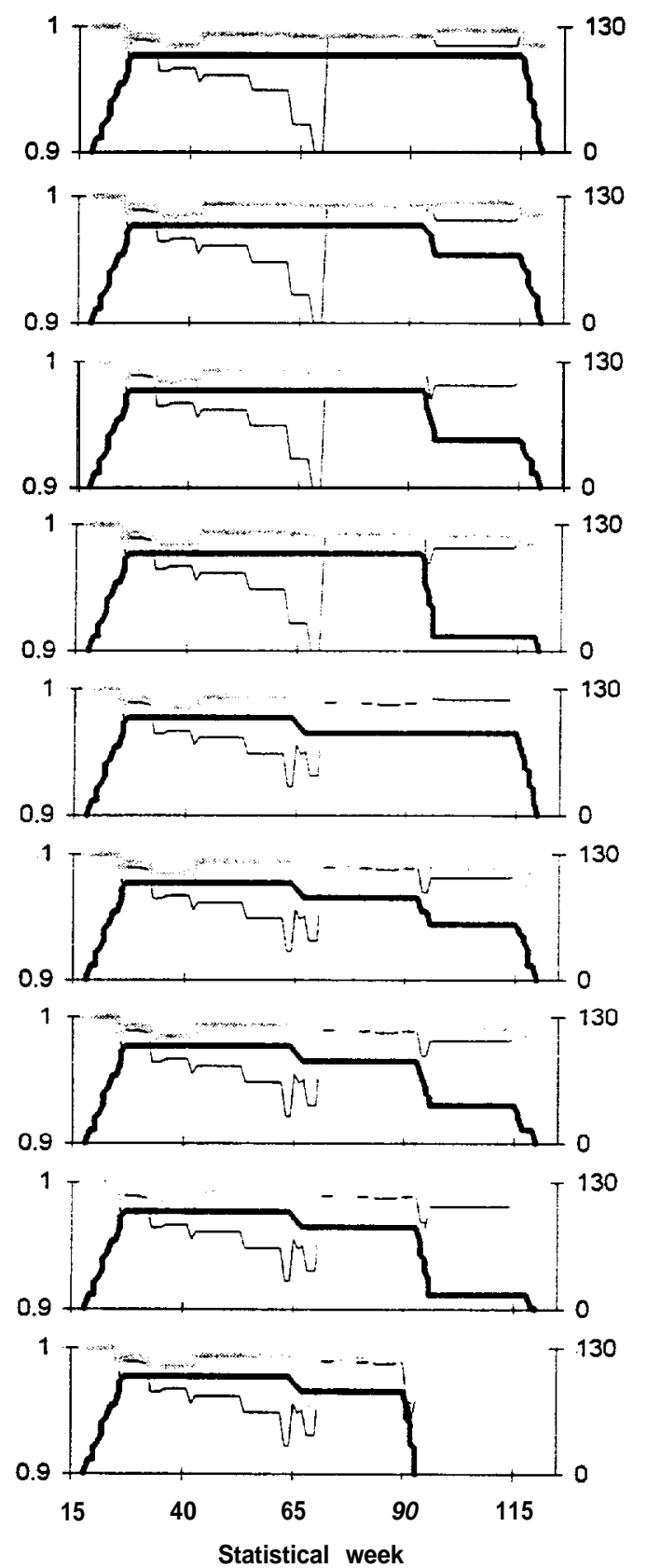

Prespawn -

Spawning
incubation Colonization Colonization
Sum. rearing Win. rearing

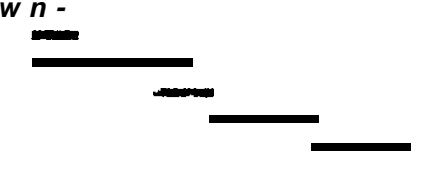

Relative Key Habitat Quantity
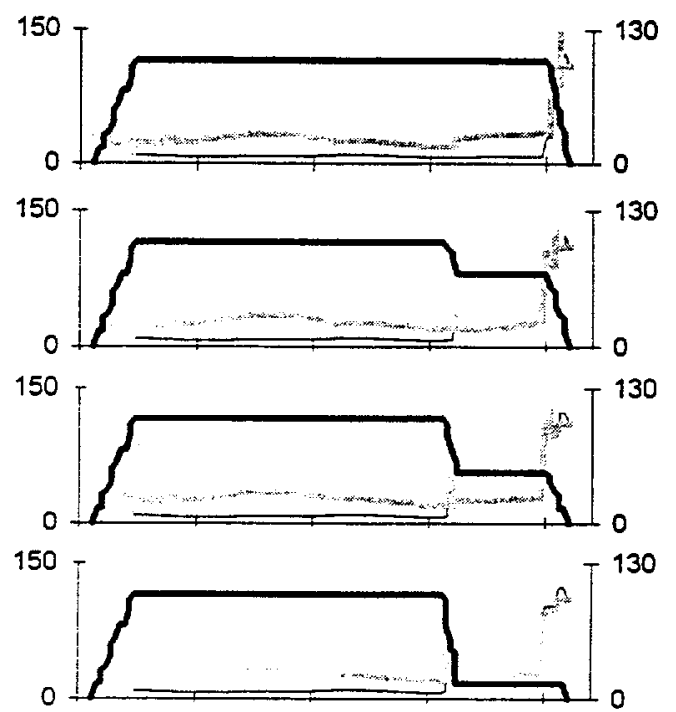

150
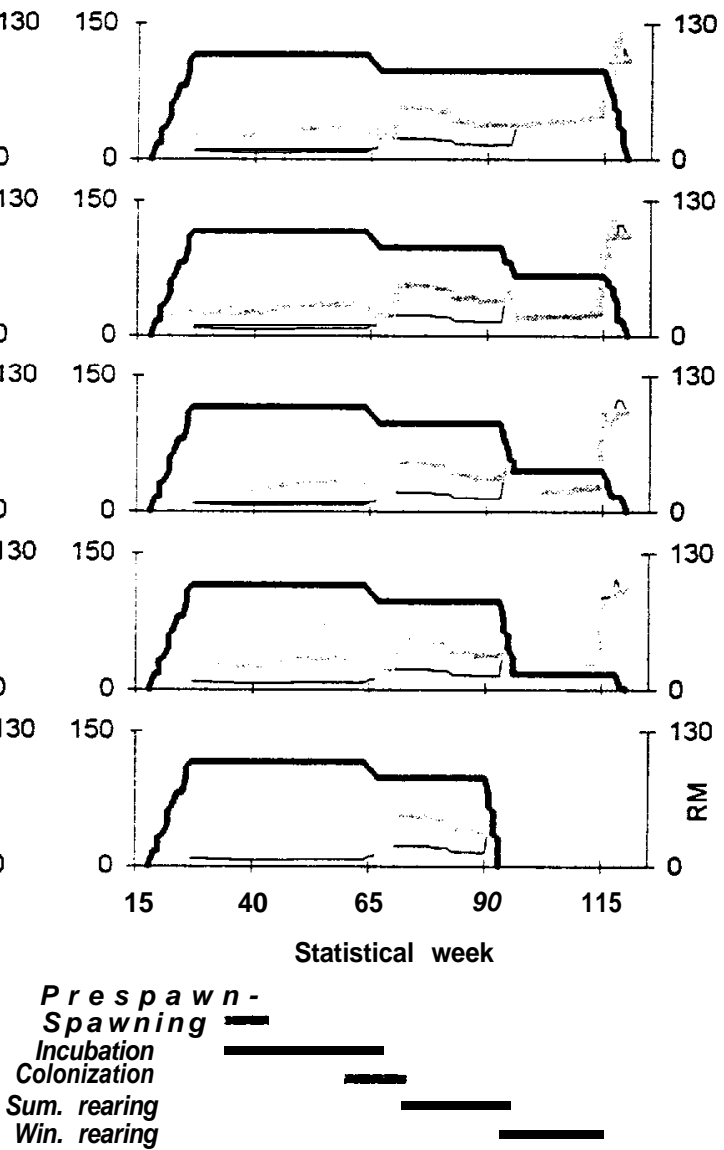

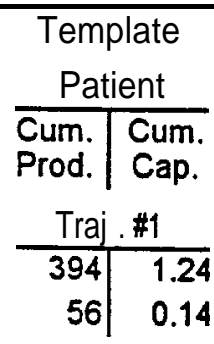

Traj. \#2

\begin{tabular}{l|l}
\hline 369 & 1.14 \\
\hline
\end{tabular}

\begin{tabular}{l|l|}
52 & 0.13 \\
\hline
\end{tabular}

Traj. \#3

Traj. \#4

\begin{tabular}{l|l|}
\hline 349 & 1.09 \\
\hline 50 & 0.12
\end{tabular}

$50 \quad 0.12$

Traj . \#5

\begin{tabular}{l|l|}
\hline 295 & 1.10 \\
\hline
\end{tabular}

73) 0.19

Traj. \#6

\begin{tabular}{l|l}
\hline 267 & 0.95 \\
\hline 58 & 0.15
\end{tabular}

58 0.15

Traj. \#7

\begin{tabular}{l|l}
\hline 280 & 1.00 \\
\hline
\end{tabular}

$58 \quad 0.15$

Traj. \#8

\begin{tabular}{l|l}
\hline 276 & 0.99 \\
\hline
\end{tabular}

\begin{tabular}{l|l}
57 & 0.15
\end{tabular}

Traj. \#9

\begin{tabular}{l|l}
\hline 499 & 2.02 \\
\hline
\end{tabular}

$121 \quad 0.32$ 
Spawning reach: Wallowa $\mathbf{R}$.

Bear Cr - Whiskey Cr (RM 101.8 - 103.9 from Snake R)

Relative Productivity
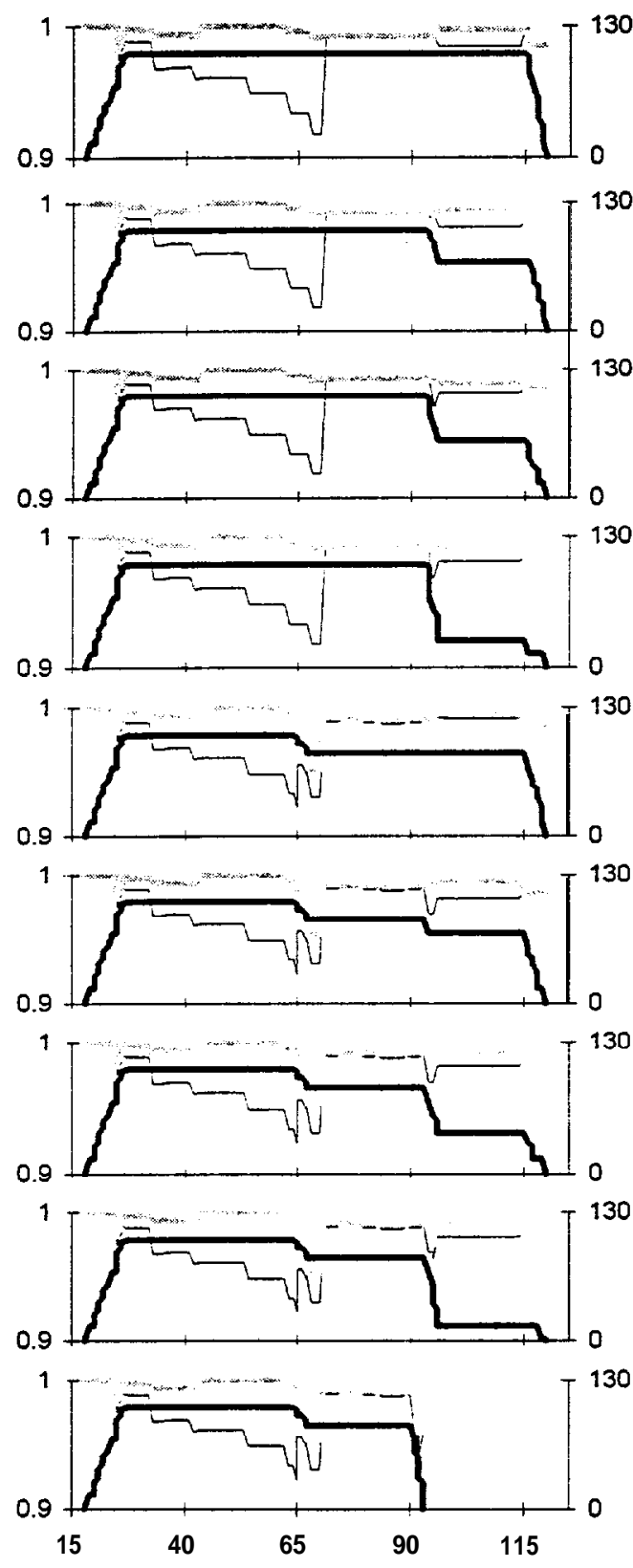

Statistical week

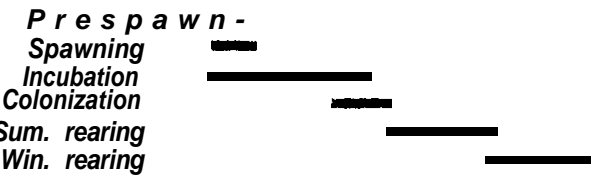

Relative Key Habitat Quantity
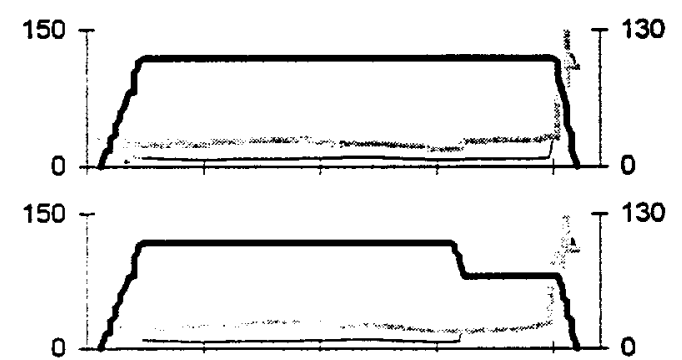

150

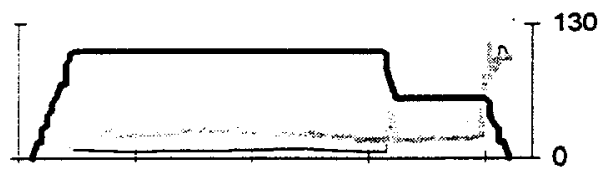

$150-$

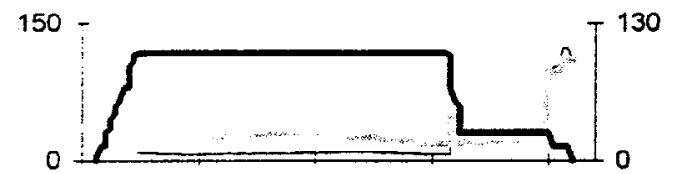

150

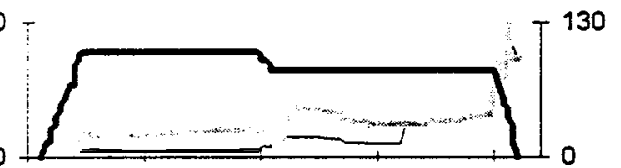

150

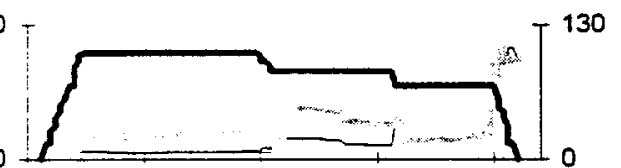

150

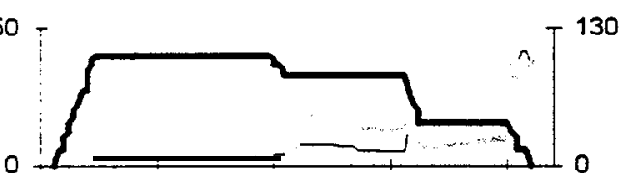

150

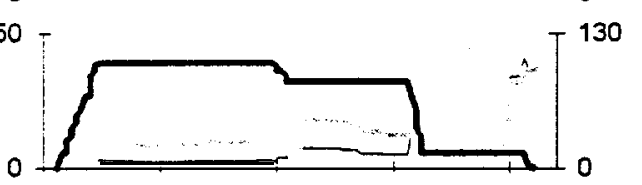

$0 \longrightarrow \longrightarrow$

150 -

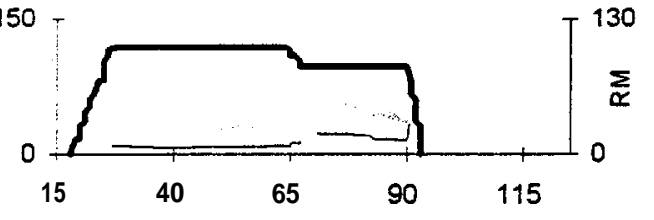

Statistical week

\begin{tabular}{|c|c|}
\hline $\begin{array}{c}\text { Template } \\
\text { Patient }\end{array}$ \\
\hline $\begin{array}{c}\text { Cum. } \\
\text { Prod. }\end{array}$ & Cum. \\
\hline
\end{tabular}

Traj. \#1

\begin{tabular}{l|l|}
\hline 510 & 1.43 \\
\hline & 07
\end{tabular}

$67 \quad 0.15$

Traj. \#2

$477 \quad 1.32$

$61 \quad 0.14$

Traj. \#3

\begin{tabular}{l|l|}
\hline 431 & 1.20 \\
\hline & 0.14
\end{tabular}

$60 \quad 0.14$

Traj. \#4

\begin{tabular}{l|l|}
\hline 451 & 1.27 \\
\hline 59 & 0.14
\end{tabular}

59 0.14

Traj. \#5

$382 \quad 1.32$

\begin{tabular}{l|l}
77 & 0.19
\end{tabular}

Traj. \#6

$384 \quad 1.25$

$61 \quad 0.15$

Traj. \#7

$363 \quad 1.20$

$61 \quad 0.15$

Traj. \#8

\begin{tabular}{l|l|}
360 & 1.18 \\
\hline
\end{tabular}

$\begin{array}{ll}60 & 0.15\end{array}$

Traj. \#9

\begin{tabular}{l|l}
648 & 2.47 \\
\hline
\end{tabular}

$127 \quad 0.32$ 
Spawning reach: Wallowa $\mathbf{R}$.

Whiskey Cr - Lostine R (RM 103.9 - 105.8 from Snake R)

\section{Relative Productivity}
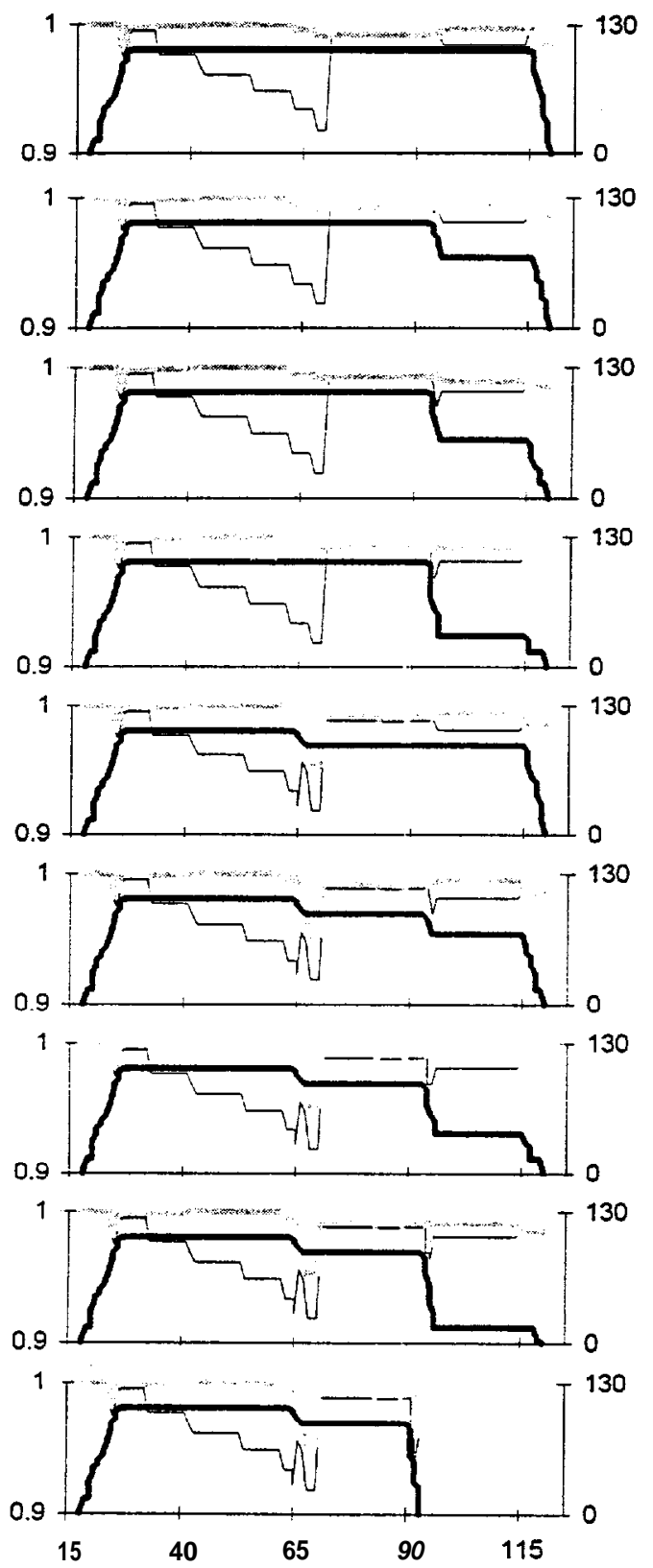

Statistical week

Prespawn-

Spawning

Incubation

Colonization

Sum. rearing

Win. rearing
Relative Key Habitat Quantity
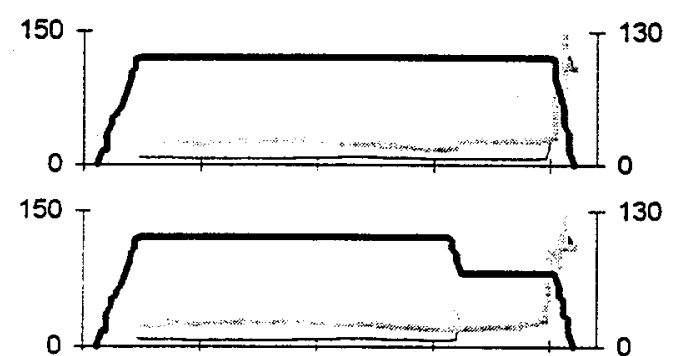

150

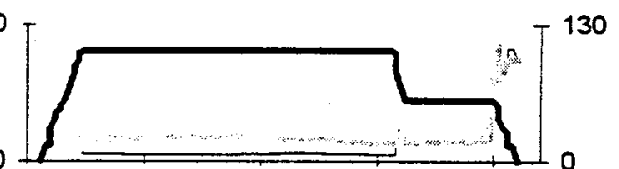

150

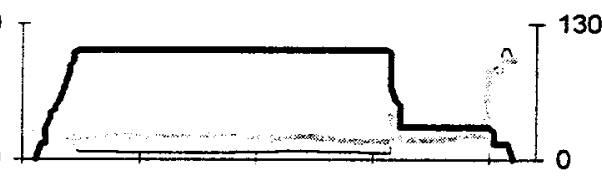

150

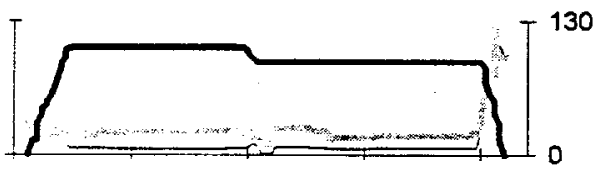

150
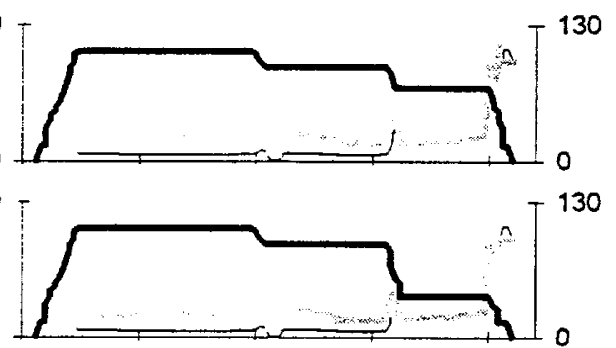

150

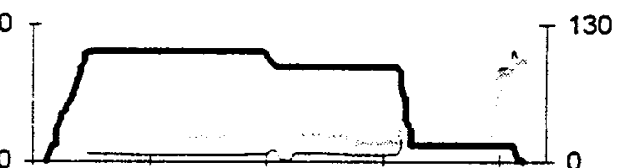

150
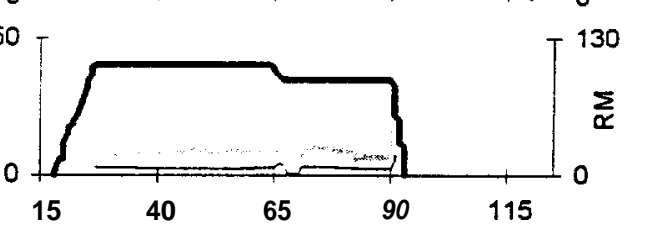

Template

Patient

Curm. Qum.

Prodil. Corap.

Traj. \#1

\begin{tabular}{l|l|}
536 & 1.41 \\
\hline
\end{tabular}

$\begin{array}{ll}74 & 0.16\end{array}$

Traj. \#2

5011.31

\begin{tabular}{l|l}
68 & 0.15
\end{tabular}

Traj. \#3

$453 \quad 1.19$

66 0.15

Traj. \#4

\begin{tabular}{l|l}
\hline 474 & 1.26 \\
\hline
\end{tabular}

66 0.15

Traj. \#5

$407 \quad 1.09$

66 0.13

Traj. \#6

\begin{tabular}{|l|l|}
\hline 409 & 1.11 \\
\hline
\end{tabular}

65 0.13

Traj. \#7

\begin{tabular}{l|l}
\hline 86 & 1.06
\end{tabular}

64 0.13

Traj. \#8

$383 \quad 1.05$

64 (0.113

Traj. \#9

\begin{tabular}{l|l}
690 & 2.18 \\
\hline
\end{tabular}

134

0.27

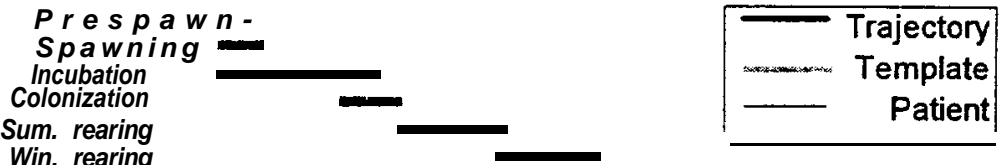


Spawning reach: Wallowa $\mathbf{R}$.

Lostine R - Parsnip Cr (RM 105.8 - 109.0 from Snake R)

\section{Relative Productivity}
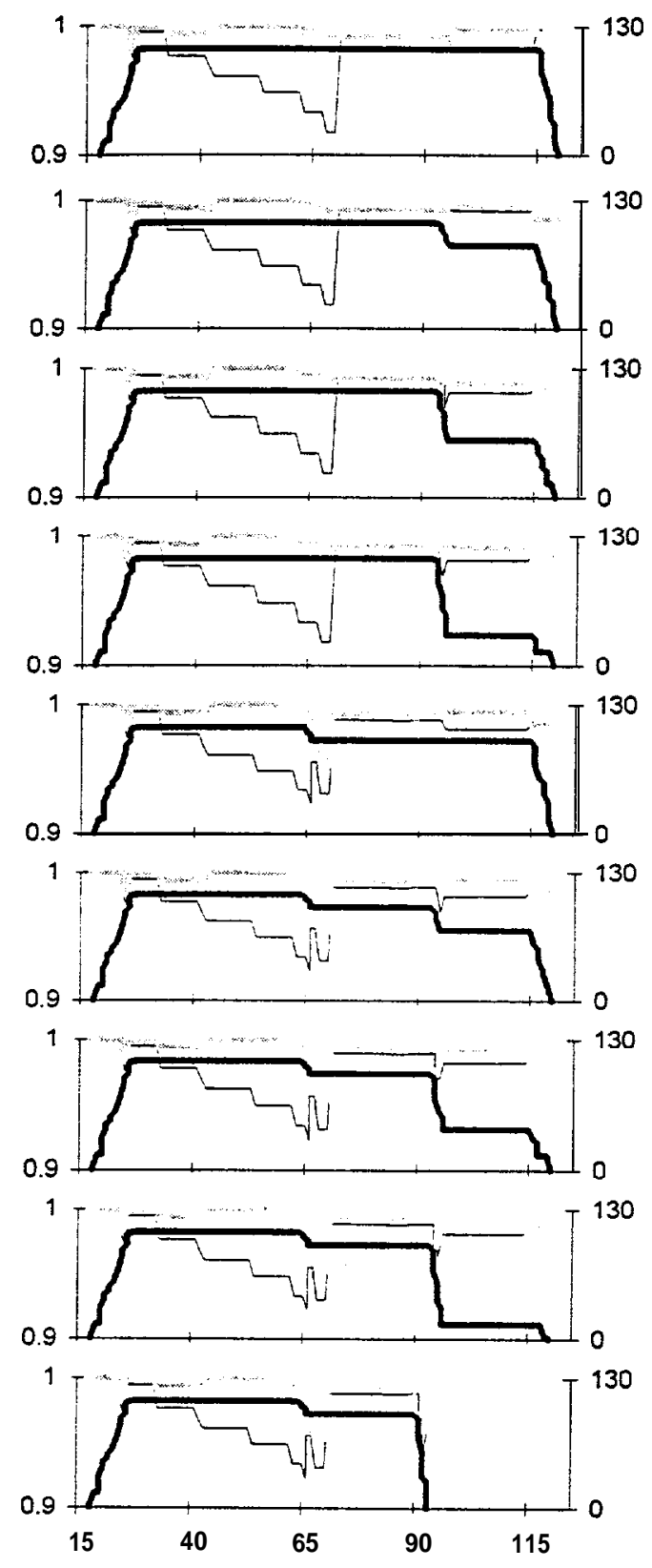

Statistical week

Prespawn-

Spawning -

Incubation

Colonization

Sum. rearing

Win. rearing
Relative Key Habitat Quantity

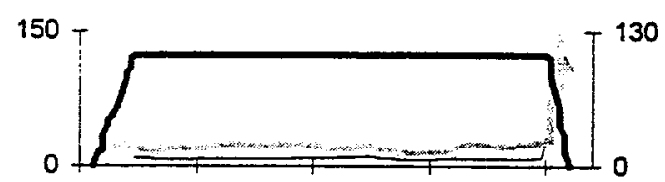

150

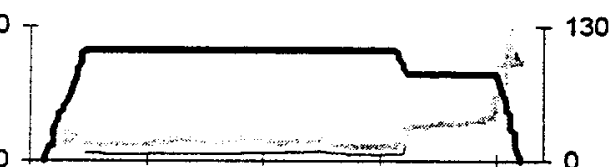

150

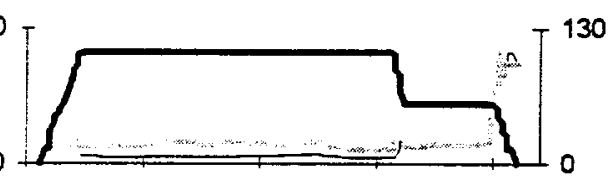

150

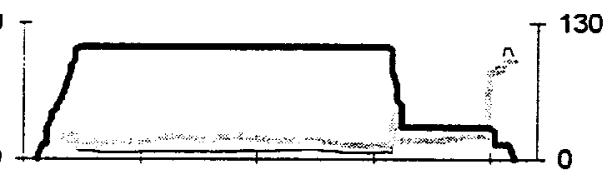

150

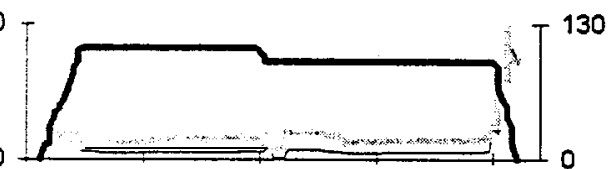

150

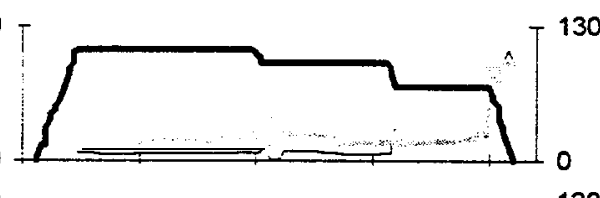

150

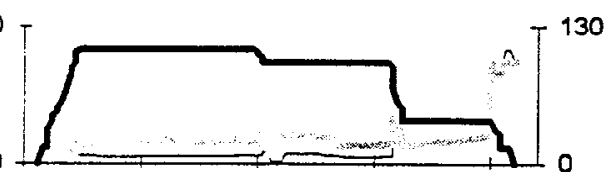

150

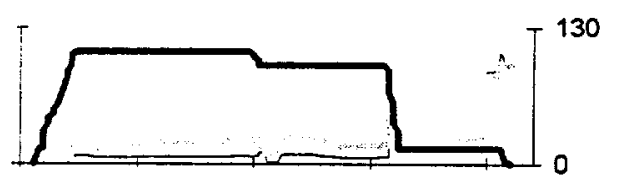

150
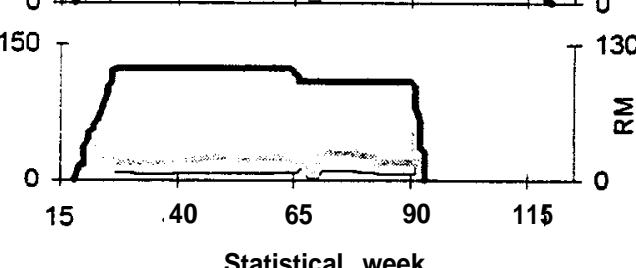

Statistical week

Prespawn Spawning Incubation Colonization sum. rearing Win. rearing

\begin{tabular}{|r|c|}
\hline \multicolumn{2}{|c|}{ Template } \\
Patient \\
\hline Cum. & Cum. \\
Prod. & Cap. \\
\multicolumn{2}{|c|}{ Traj. \#1 } \\
\hline 506 & 1.20 \\
74 & 0.16 \\
\hline
\end{tabular}

Traj. \#2

$473 \quad 1.20$

$83 \quad 0.19$

Traj. \#3

\begin{tabular}{|l|l|}
\hline 427 & 1.03 \\
\hline
\end{tabular}

67

0.15

Traj. \#4

\begin{tabular}{l|l|}
\hline 447 & 1.09 \\
\hline
\end{tabular}

67) 0.15

Traj. \#5

$402 \quad 1.02$

\begin{tabular}{l|l}
69 & 0.14
\end{tabular}

Traj. \#6

$402 \quad 1.04$

$\begin{array}{ll}68 & 0.14\end{array}$

Traj. \#7

\begin{tabular}{|l|l|}
\hline 380 & 1.00 \\
67
\end{tabular}

67 0.14

Traj. \#8

\begin{tabular}{l|l}
378 & 0.98 \\
6
\end{tabular}

67) 0.14

Traj. \#9

\begin{tabular}{l|l|}
680 & 2.03 \\
\hline
\end{tabular}

$141 \quad 0.30$ 


\section{Spawning reach: Wallowa $R$.}

Parsnip Cr - Hurricane Cr (RM 109.0 - 121.9 from Snake R)

Relative Productivity
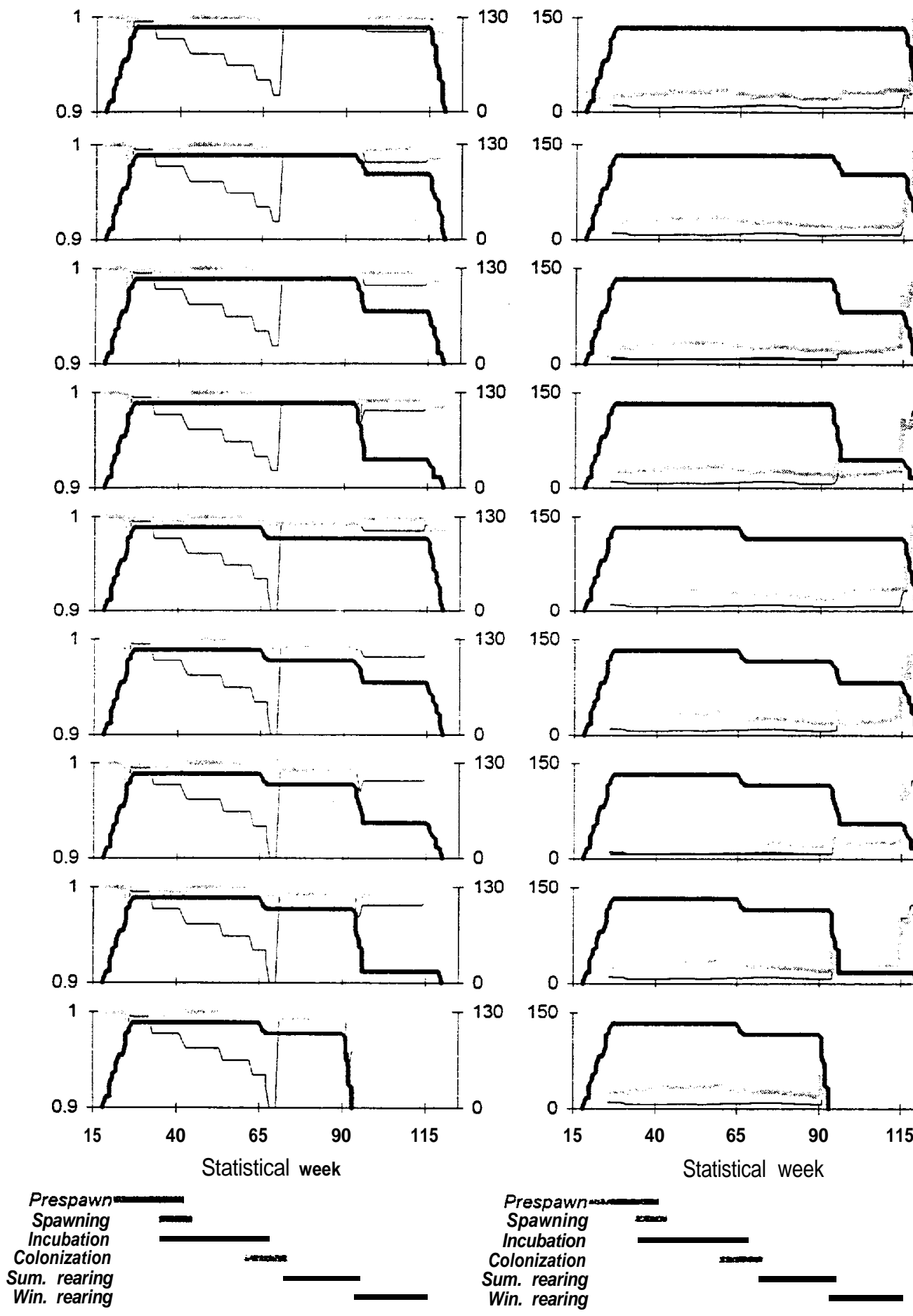

Relative Key Habitat Quantity
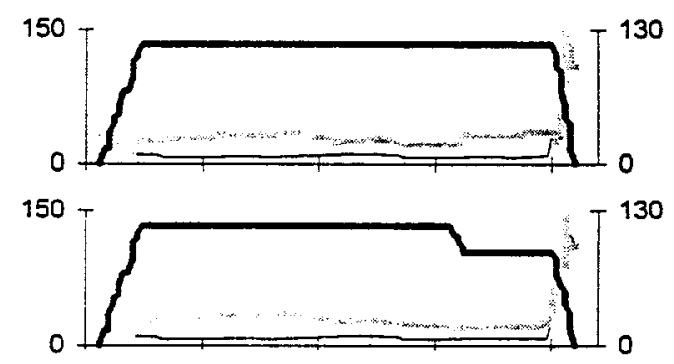

150

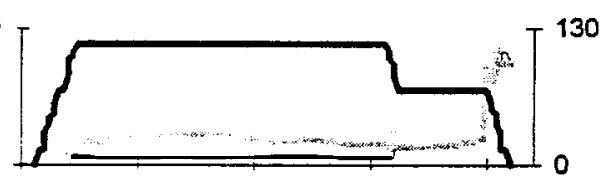

${ }^{150}$
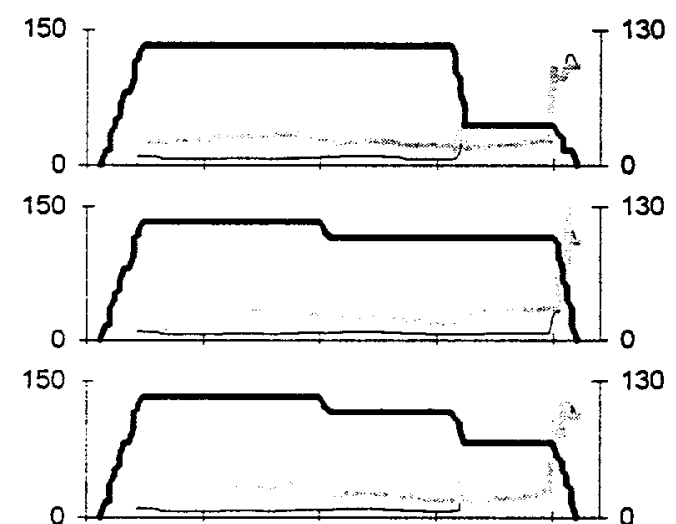

(100.

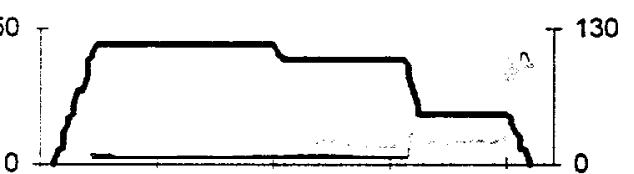

150

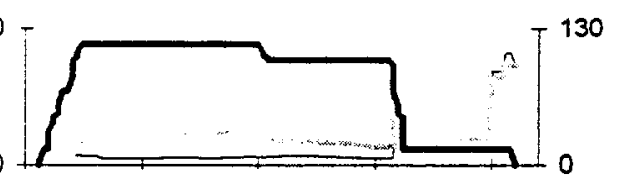

150

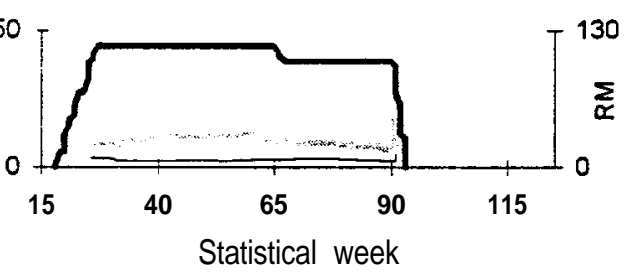

130

$\sum_{x}$

Traj. \#9

\begin{tabular}{l|l|}
\hline 805 & 2.79 \\
\hline
\end{tabular}

$129 \quad 0.30$ 
Spawning reach: Wallowa $R$.

Hurricane Cr - Lower Alderslope Ditch (RM 121.9 - 126.8 from Snake R)

Relative Productivity
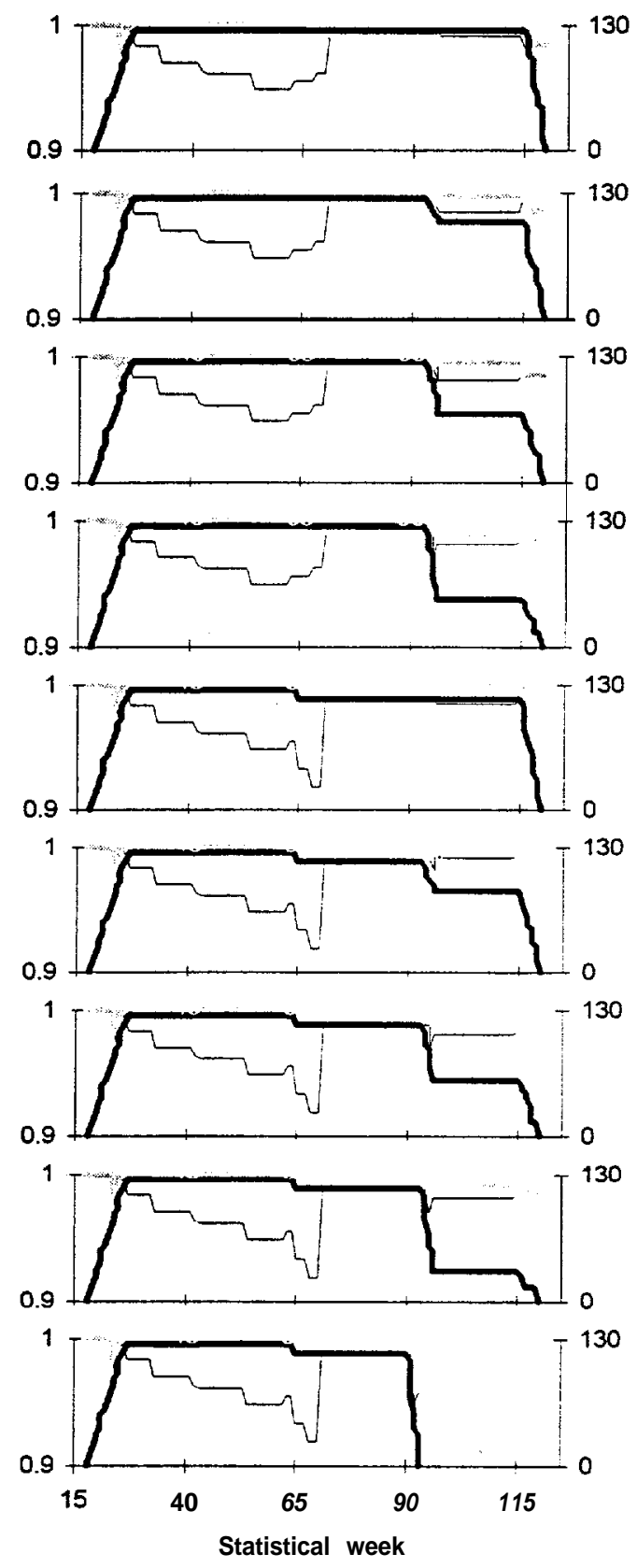

Prespawn -

spawning

Incubation
Colonization
Sum. rearing

Win. rearing

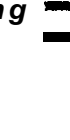

Relative Key Habitat Quantity
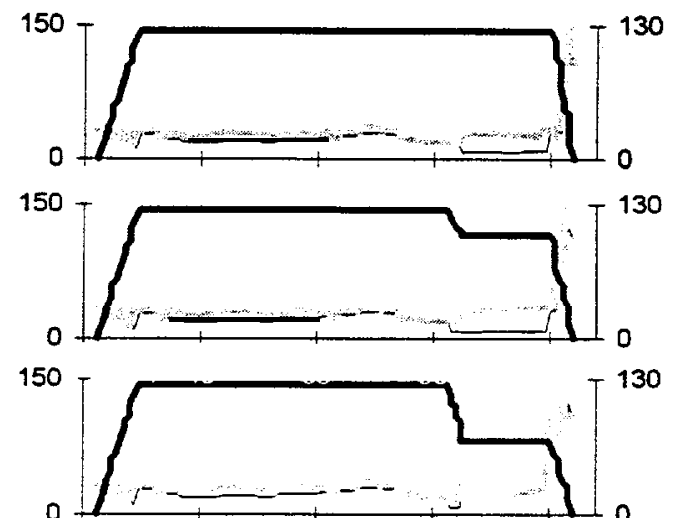

150 -

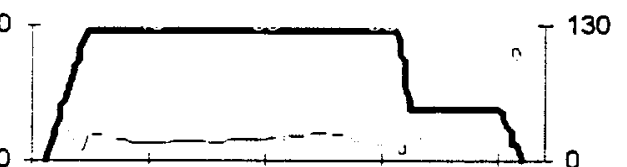

150

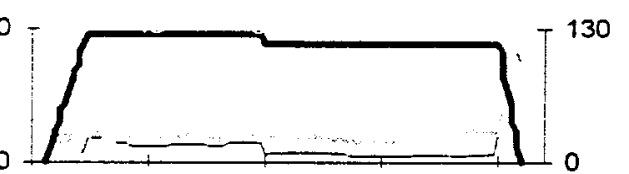

150 -

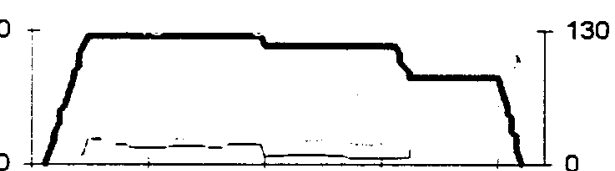

150 -

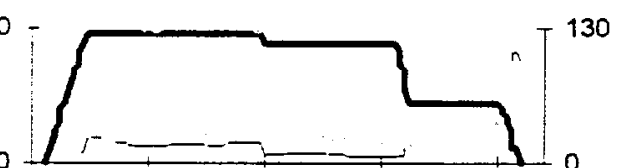

150

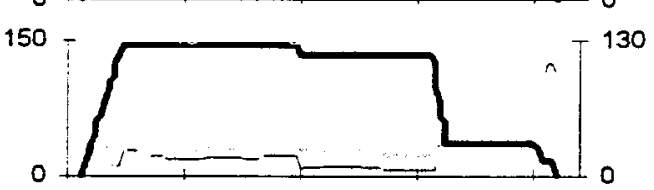

150 -

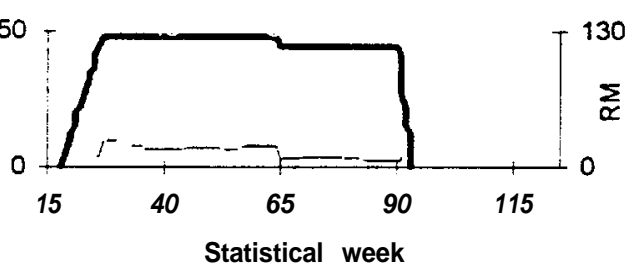

130

(1)

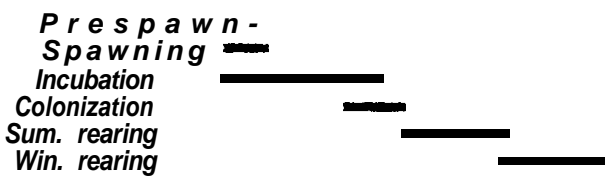

\begin{tabular}{|c|c|}
\hline \multicolumn{2}{|c|}{ Template } \\
Patient \\
\hline Cum. \\
Prod. & Cum. \\
\hline
\end{tabular}

Traj \#1

5091.51

$100 \quad 0.35$

Traj. \#2

\begin{tabular}{l|l|l|}
504 & 1.53 \\
\hline
\end{tabular}

$88 \mid 0.31$

Traj. \#3

\begin{tabular}{l|l|}
\hline 473 & 1.39 \\
\hline
\end{tabular}

$82 \quad 0.30$

Traj. \#4

427.30

$80 \quad 0.30$

Traj. \#5

532 1.62

68 0.23

Traj. \#6

472 1.48

$75 \quad 0.26$

Traj . \#7

427 1.26

$61 \quad 0.21$

Traj. \#8

446 1.34

$61 \quad 0.21$

Traj. \#9

7992.88

1270.47 
Spawning reach: Wallowa $\mathbf{R}$.

Lower Alderslope Ditch - Wallowa Lake (RM 126.8 - 130.8 from Snake R)

Relative Productivity
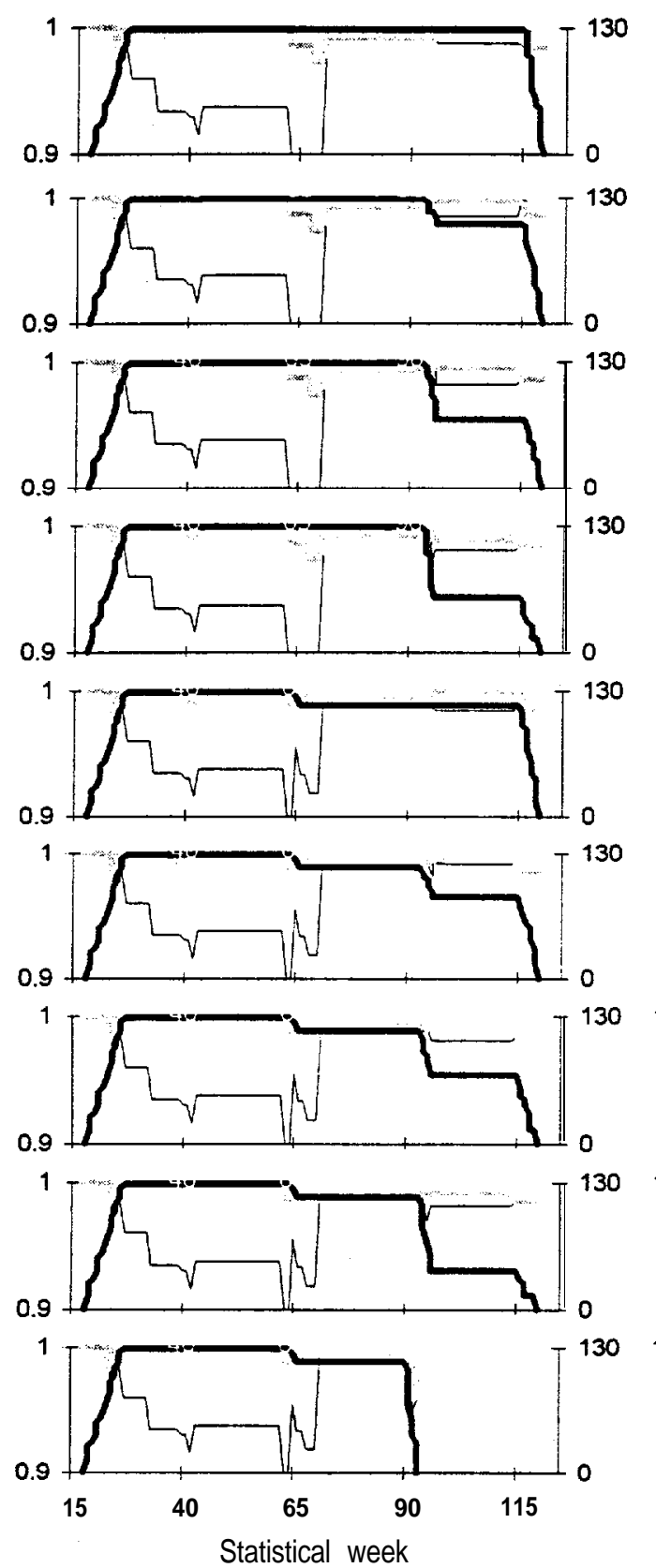

Prespawn -

Spawning

Incubation

Colonization

Sum. rearing

Win. rearing
Relative Key Habitat Quantity
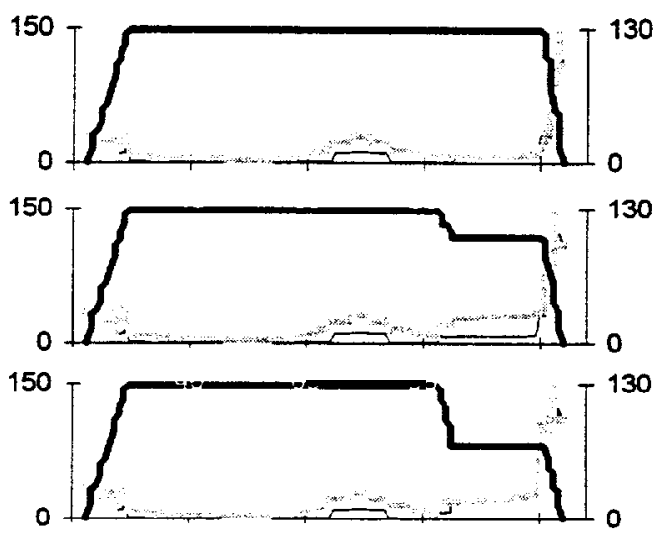

$-130$

150
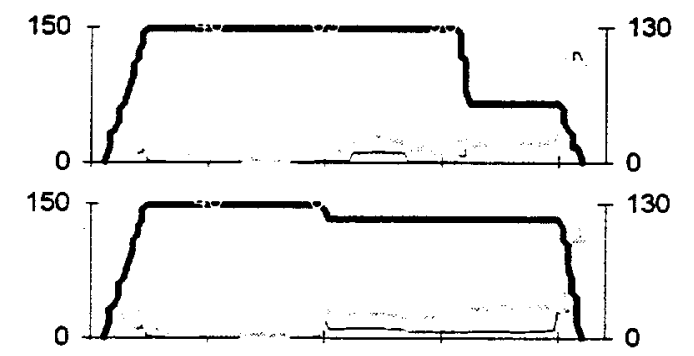

$150-$
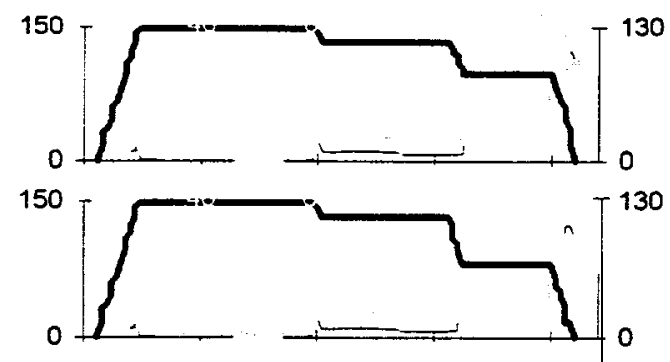

130
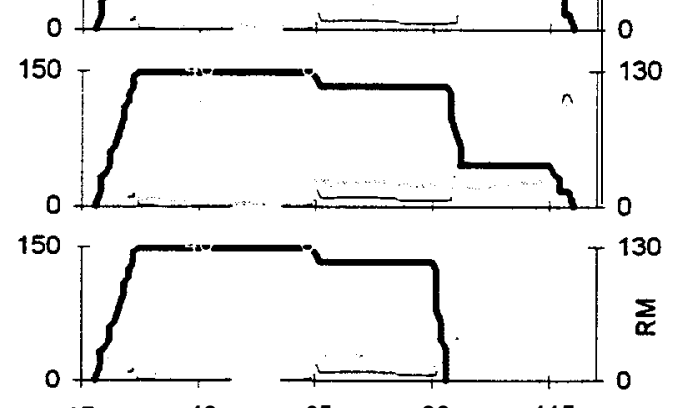

Statistical week \begin{tabular}{|r|l|}
\hline \multicolumn{2}{|c|}{ Template } \\
Patient \\
\hline $\begin{array}{r}\text { Cum. } \\
\text { Prod. }\end{array}$ & Cum. \\
Traj. \#1 \\
\hline 465 & 0.70 \\
19 & 0.00 \\
\hline
\end{tabular}

Traj. \#2

4590.81

18 0.00

Traj. \#3

$430 \quad 0.75$

$16 \quad 0.00$

Traj. \#4

388 . 0.68

16) 0.00

Traj . \#5

\begin{tabular}{|l|l|}
527 & 0.99 \\
\hline
\end{tabular}

25 0.00

Traj. \#6

\begin{tabular}{l|l}
467 & 0.89 \\
\hline
\end{tabular}

27 0.00

Traj .\#7

\begin{tabular}{l|l|}
466 & 0.86 \\
\hline
\end{tabular}

23 0.00

Traj. \#8

\begin{tabular}{l|l|}
\hline 440 & 0.83 \\
\hline
\end{tabular}

22 0.00

Traj. \#9

\begin{tabular}{|l|l|}
\hline 789 & 1.65 \\
\hline 46 & 0.01
\end{tabular}

46) 0.01 
Spawning reach: Wenaha $\mathbf{R}$.

Mouth - Crooked Cr (RM 45.1 - 51.8 from Snake R)

Relative Productivity
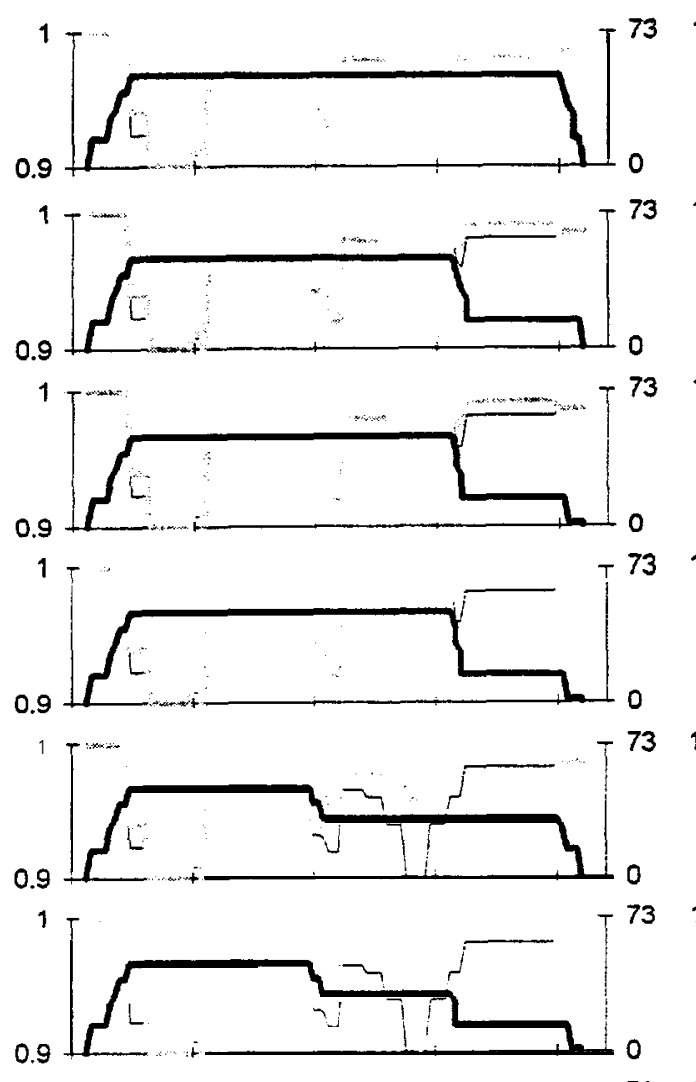

$73 \quad 150$

$73 \quad 150$
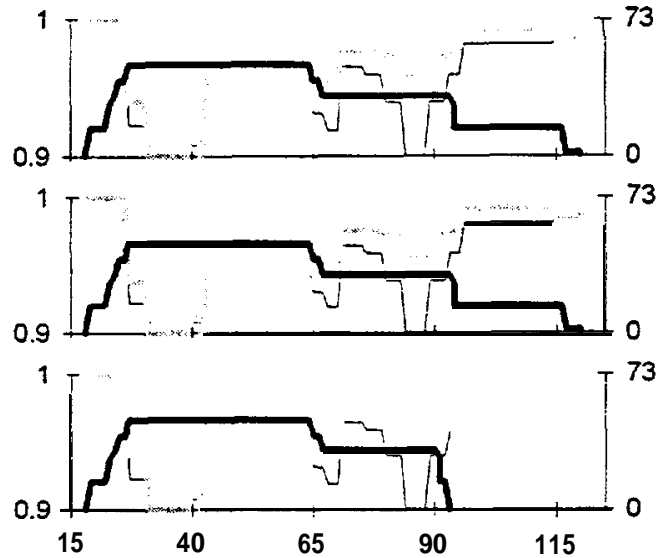

Statistical week

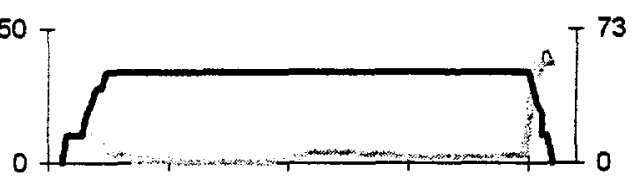

150

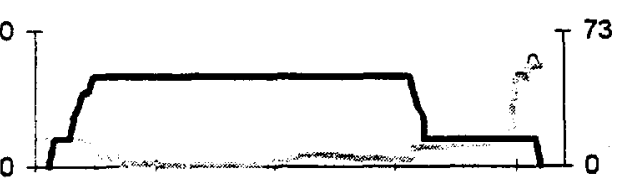

150

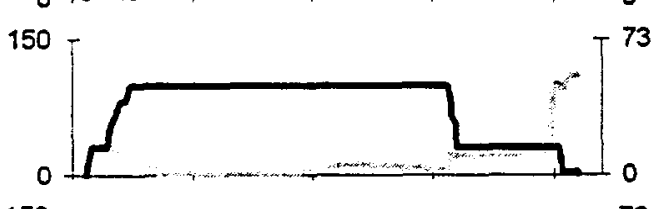

150

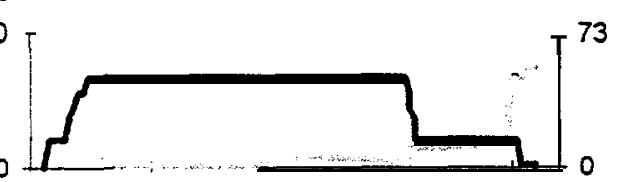

0
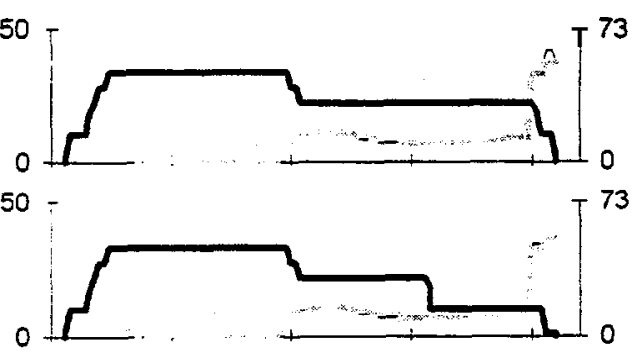

Relative Key Habitat Quantity
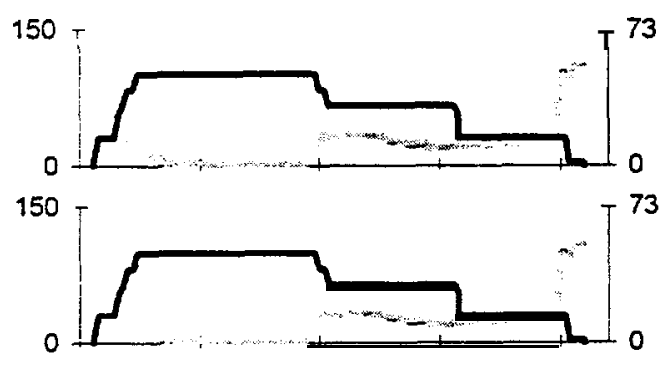

150

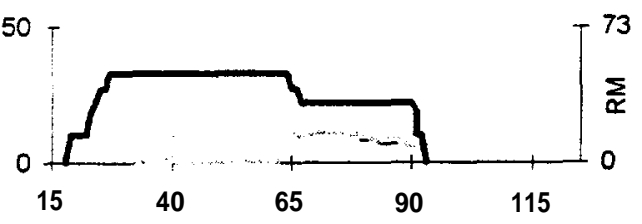

Statistical week
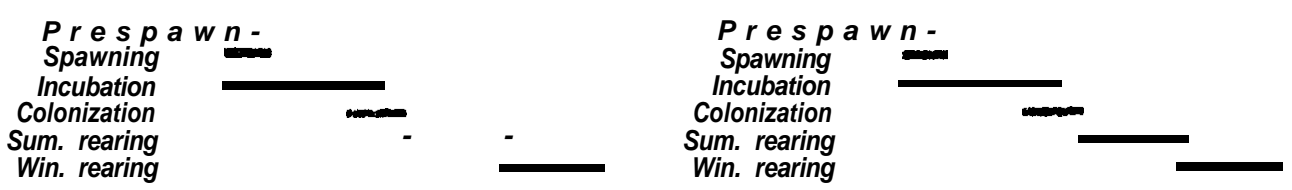

\begin{tabular}{|ll|}
\hline & Trajectory \\
Patient & Template \\
\hline
\end{tabular} 
Spawning reach: Wenaha $\mathbf{R}$.

Crooked Cr -Fairview Cr (RM 51.8 - 55.3 from Snake R)

Relative Productivity
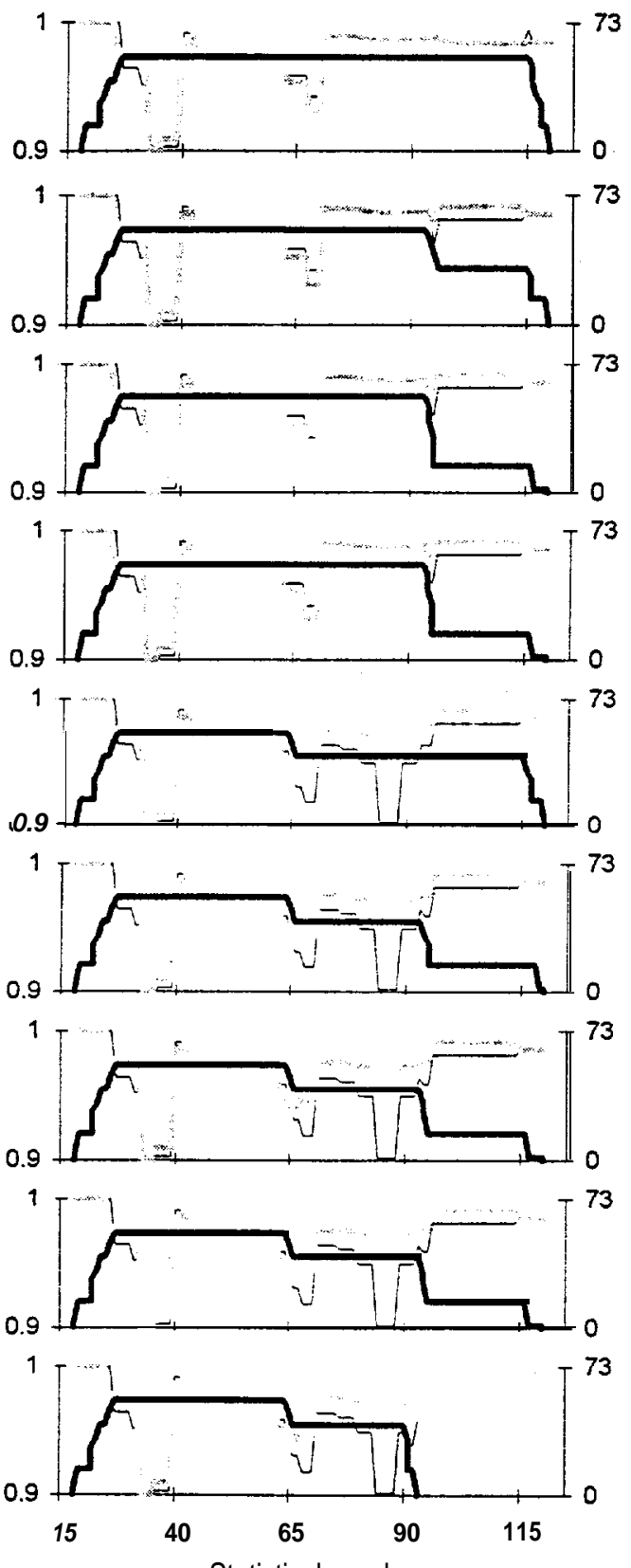

Statistical week

Prespawn -

Spawning

Incubation

Colonization

Sum. rearing

Win. rearing

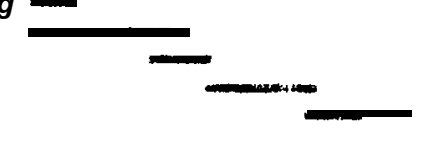

Relative Key Habitat Quantity

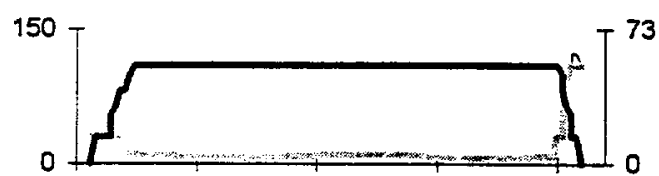

150

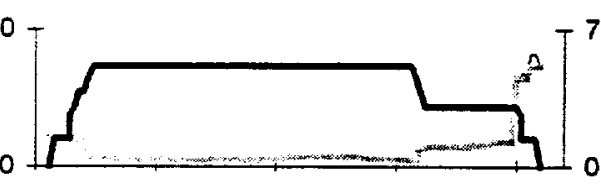

150

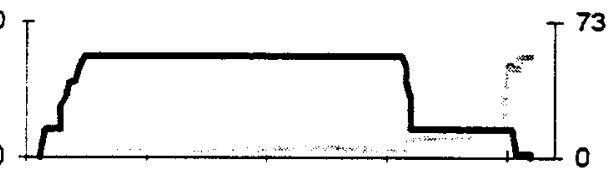

150 -

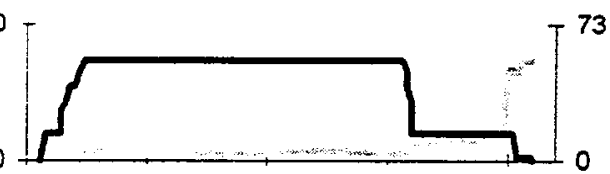

150
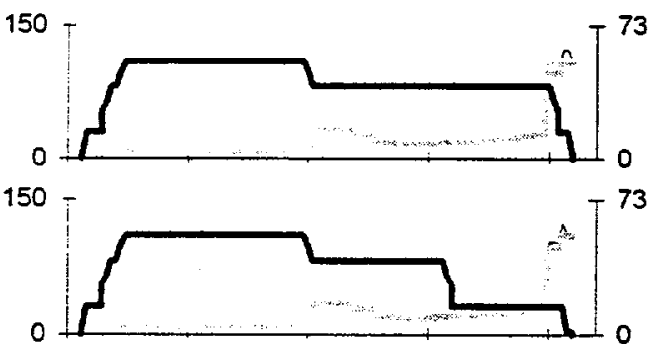

150

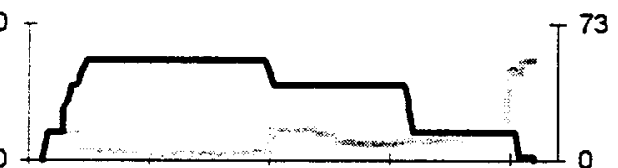

150

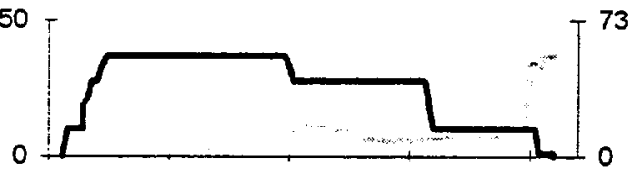

150

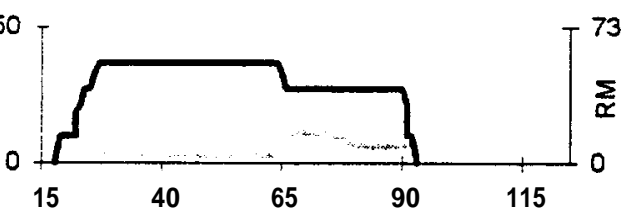

Statistical week

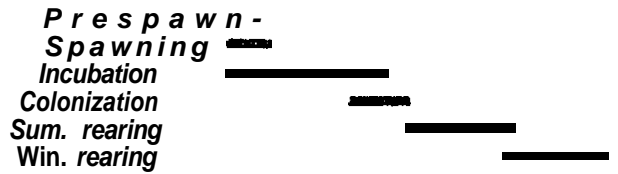

Template

Patient

Cumn. Qum.

Proded. Capp.

Traj. \#1

610.15

58

0.15

Traj. \#2

\begin{tabular}{l|l}
67 & 0.17 \\
\hline
\end{tabular}

52

0.14

Traj. \#3

\begin{tabular}{l|l}
67 & 0.17 \\
\hline & 0.13
\end{tabular}

$51 \quad 0.13$

Traj. \#4

\begin{tabular}{l|l}
67 & 0.17 \\
\hline & 0.13
\end{tabular}

51

0.13

Traj. \#5

\begin{tabular}{l|l}
44 & 0.12 \\
\hline
\end{tabular}

16 0.05

Traj. \#6

\begin{tabular}{l|l}
\hline 44 & 0.12 \\
\hline
\end{tabular}

\begin{tabular}{l|l}
16 & 0.05
\end{tabular}

Traj. \#7

\begin{tabular}{|l|l|}
\hline 44 & 0.12 \\
\hline
\end{tabular}

16) 0.05

Traj. \#8

\begin{tabular}{l|l|}
\hline 44 & 0.12 \\
\hline
\end{tabular}

16) 0.05

Traj. \#9

\begin{tabular}{l|l}
84 & 0.24
\end{tabular}

36 0.11 
Spawning reach: Wenaha $\mathbf{R}$.

Fairview $\mathrm{Cr}$ - forks (RM 55.3 - 65.9 from Snake R)

Relative Productivity
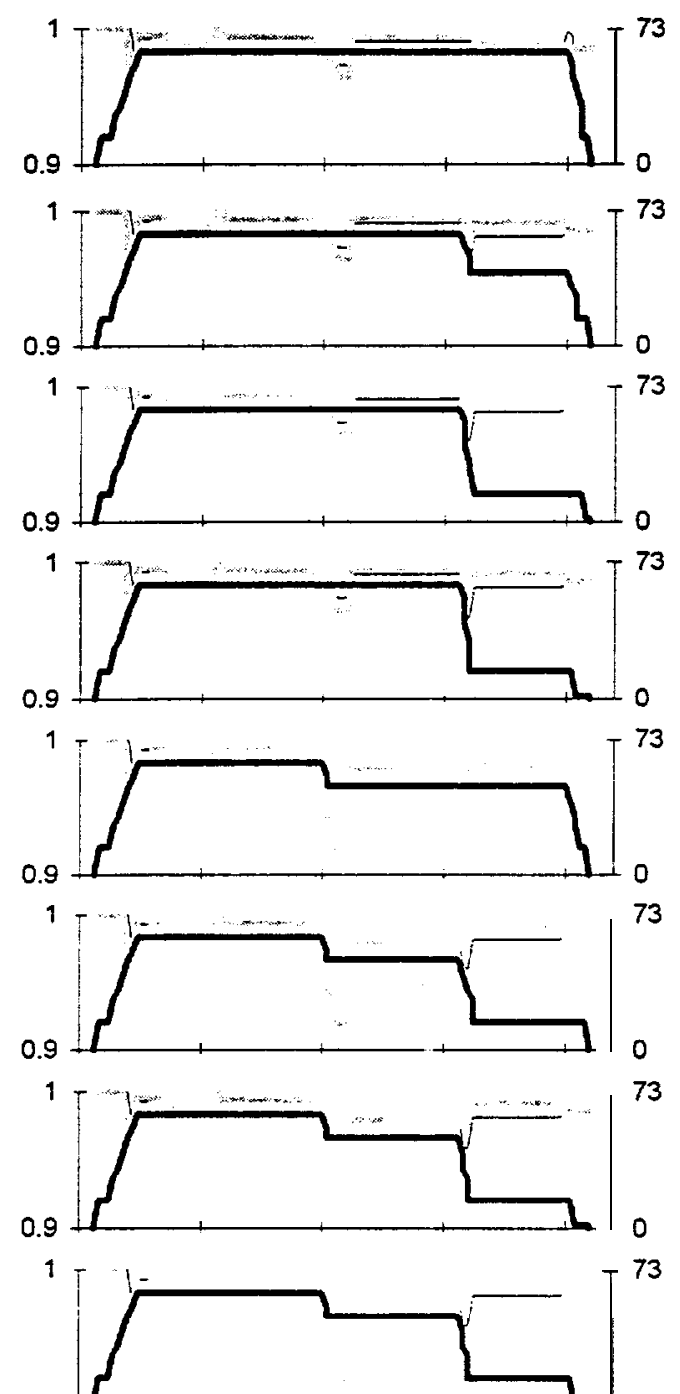

.

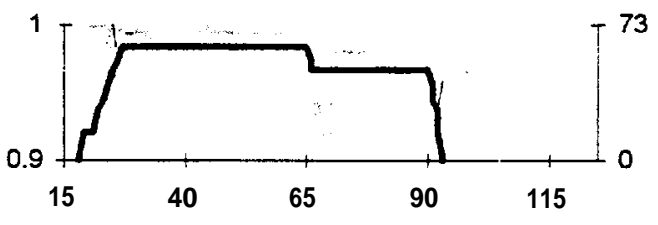

Statistical week

Prespawn -

Spawning

Incubation

Colonization

Sum. rearing

Win. rearing

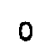

Relative Key Habitat Quantity
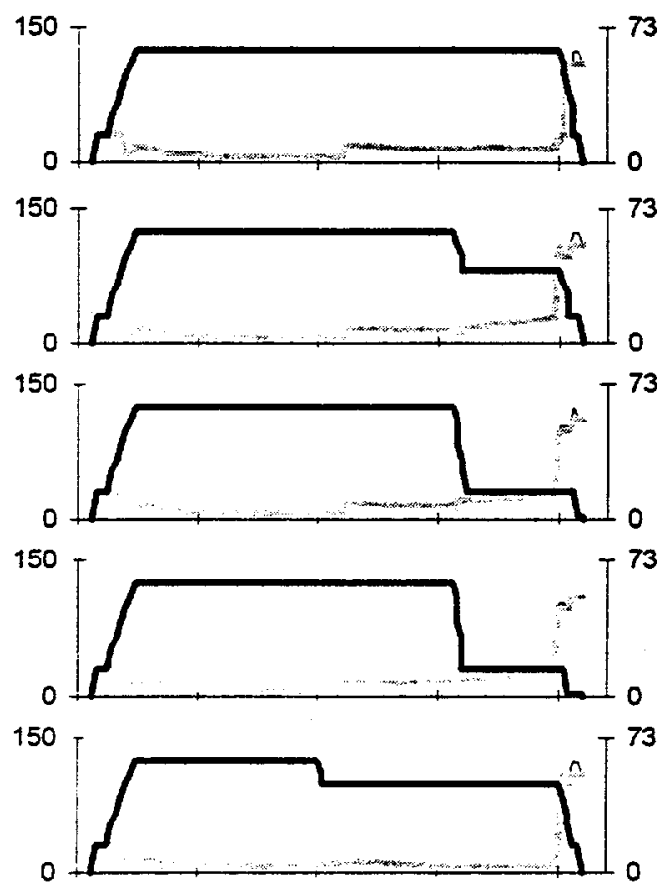

73150

$73 \quad 150$
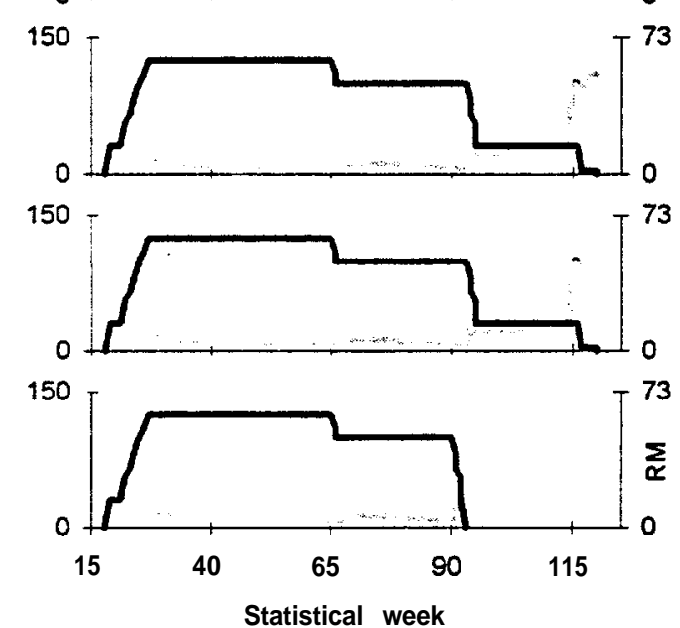

Presp a w n -

Spawning

Incubation
Colonization

Colonization

Win. rearing

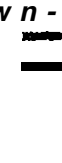

Template

Patient

Cum. Cum.

Prod. Cap.

Traj. \#1

\begin{tabular}{l|l}
\hline 294 & 0.65 \\
\hline
\end{tabular}

298 0.65

Traj. \#2

\begin{tabular}{l|l}
\hline 306 & $0.6 \mathrm{c}$ \\
\end{tabular}

$\begin{array}{ll}251 & 0.57\end{array}$

Traj. \#3

\begin{tabular}{l|l}
\hline 302 & $0.6 \mathrm{~g}$ \\
\hline
\end{tabular}

$\begin{array}{ll}244 & 0.56\end{array}$

Traj. \#4

\begin{tabular}{l|l}
\hline 302 & 0.69 \\
\hline
\end{tabular}

$\begin{array}{ll}244 & 0.56\end{array}$

Traj. \#5

\begin{tabular}{l|l}
121 & 0.27 \\
\hline
\end{tabular}

$124 \quad 0.28$

Traj. \#6

\begin{tabular}{l|l|}
\hline 146 & 0.36 \\
\hline
\end{tabular}

120) 0.29

\begin{tabular}{c|c}
\multicolumn{2}{c|}{ Traj. \#7 } \\
\hline 146 & 0.36 \\
120 & 0.29
\end{tabular}

Traj. \#8

\begin{tabular}{l|l}
146 & 0.36 \\
\hline
\end{tabular}

120 0.29

Traj. \#9

\begin{tabular}{l|l}
\hline 279 & 0.74 \\
263 & 0.69
\end{tabular} 
Spawning reach: Wenaha $\mathbf{R}$.

South Fork Wenaha R (RM 65.9 - 72.4 from Snake R)

Relative Productivity
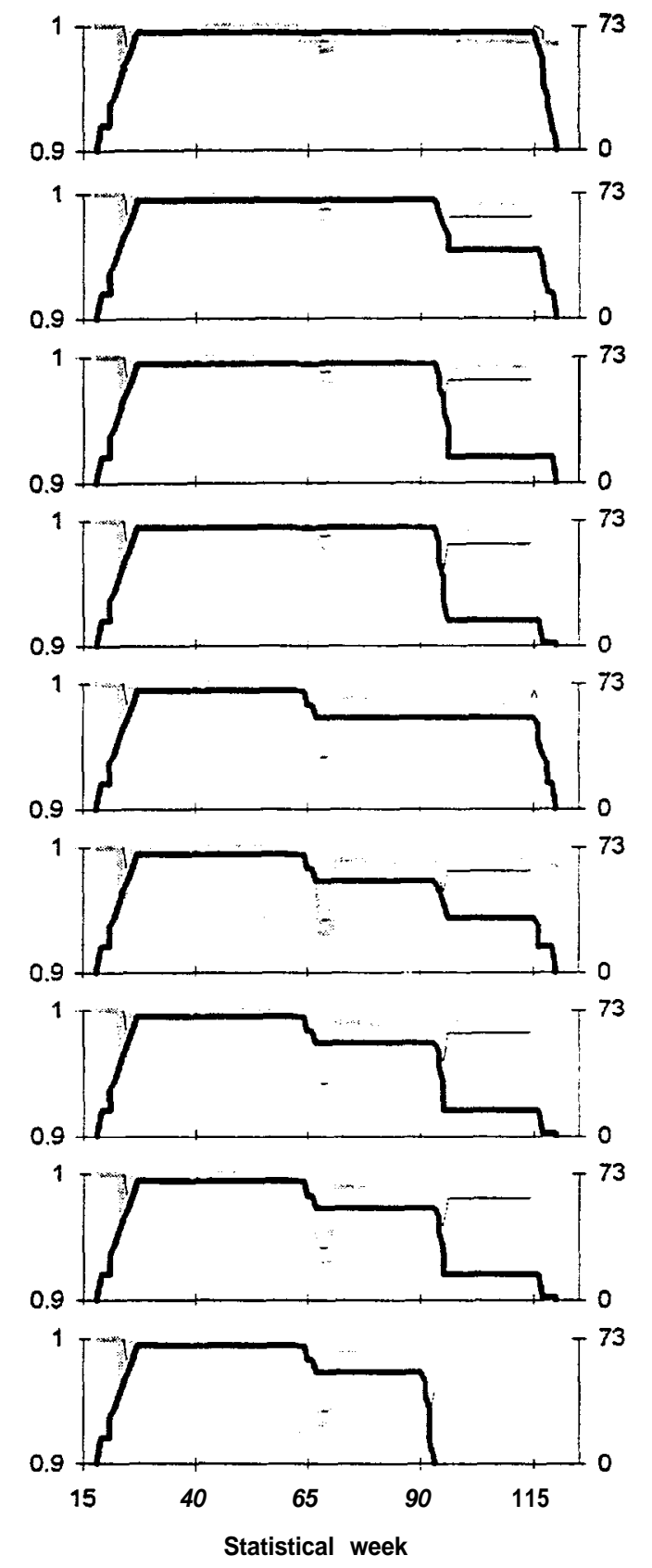

Prespawn -

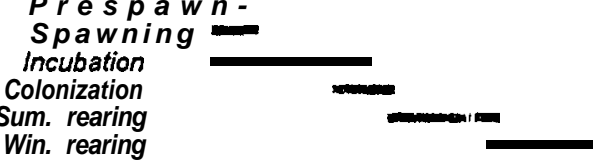

Relative Key Habitat Quantity
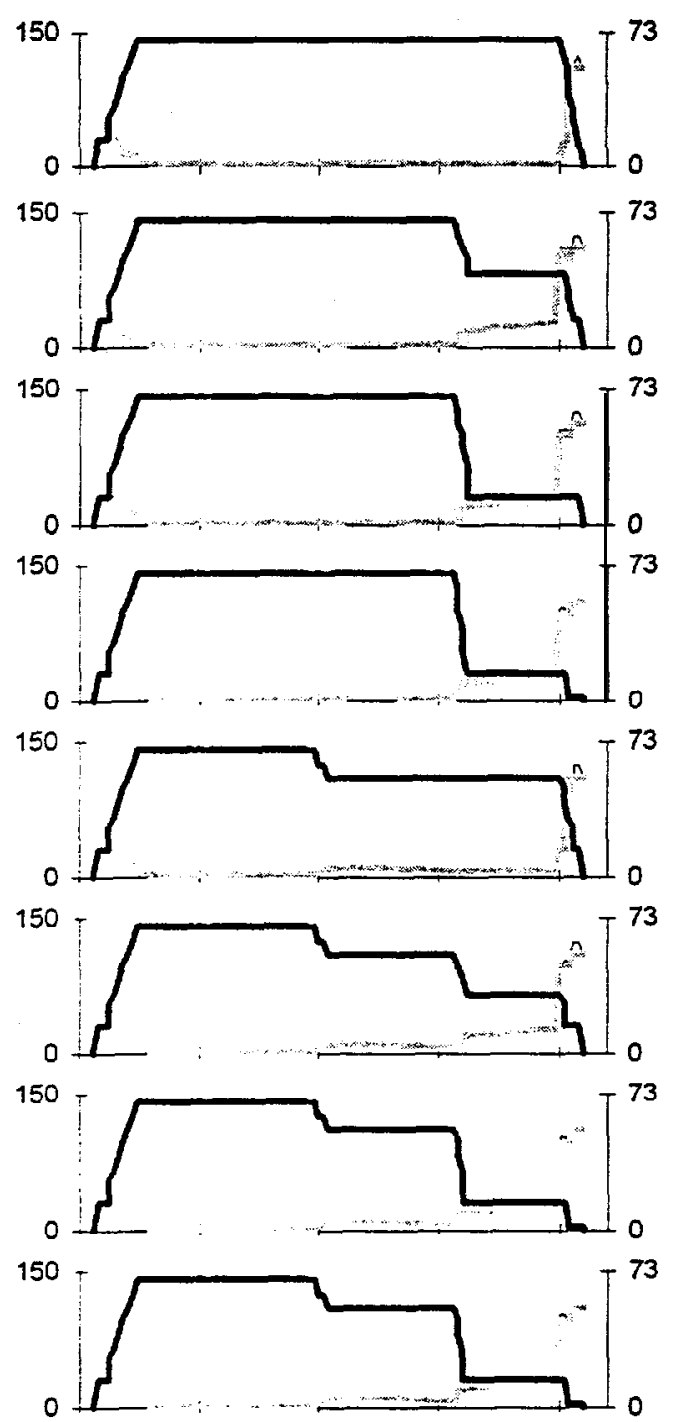

$150-$

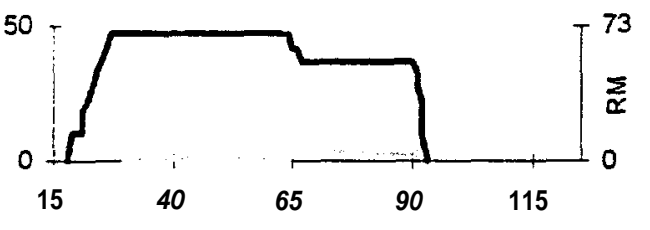

Statistical week

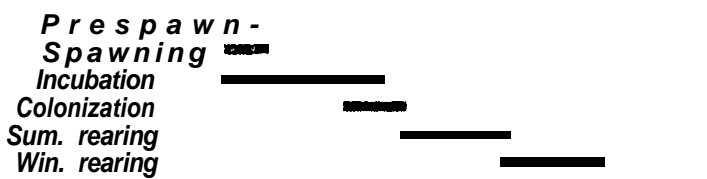

\begin{tabular}{|c|c|}
\hline \multicolumn{2}{|c|}{$\begin{array}{c}\text { Template } \\
\text { Patient }\end{array}$} \\
\hline $\begin{array}{l}\text { Cum. } \\
\text { Prod. }\end{array}$ & $\begin{array}{l}\text { Cum. } \\
\text { Cap. }\end{array}$ \\
\hline \multicolumn{2}{|c|}{ Traj. \#1 } \\
\hline 435 & 0.23 \\
\hline 486 & 0.24 \\
\hline \multicolumn{2}{|c|}{ Traj. \#2 } \\
\hline 450 & $\overline{0.25}$ \\
\hline 407 & $0.2 f$ \\
\hline \multicolumn{2}{|c|}{ Traj. \#3 } \\
\hline 445 & 0.25 \\
\hline 398 & 0.25 \\
\hline \multicolumn{2}{|c|}{ Traj. \#4 } \\
\hline 443 & $\overline{0.2 \mathrm{~s}}$ \\
\hline 393 & 0.25 \\
\hline \multicolumn{2}{|c|}{ Traj. \#5 } \\
\hline 259 & 0.23 \\
\hline 274 & 0.24 \\
\hline \multicolumn{2}{|c|}{ Traj. \#6 } \\
\hline 286 & 0.27 \\
\hline 245 & 0.22 \\
\hline \multicolumn{2}{|c|}{ Traj . \#7 } \\
\hline 286 & 0.27 \\
\hline 241 & 0.22 \\
\hline \multicolumn{2}{|c|}{ Traj. \#8 } \\
\hline \multicolumn{2}{|c|}{2861} \\
\hline $241 \mid$ & 0.22 \\
\hline Traj. & $\# 9$ \\
\hline 518 & 0.52 \\
\hline 509 & $0.4 \mathrm{~s}$ \\
\hline
\end{tabular}




\section{APPENDIX B}

Productivity and Capacity Profiles for Life History Pathways in the Imnaha River and Tributaries 
Spawning reaches analyzed in the Imnaha, listed in the order they appear in Appendix B.

Stream

Big Sheep Cr.

Big Sheep Cr.

Big Sheep Cr.

Big Sheep Cr.

Big Sheep Cr.

Big Sheep Cr.

Big Sheep Cr.

Big Sheep Cr.

Big Sheep Cr.

Imnaha R.

Imnaha $\mathrm{R}$

Imnaha R.

Imnaha R.

Imnaha $\mathrm{R}$.

Imnaha $\mathrm{R}$.

Imnaha $\mathrm{R}$

Imnaha $\mathrm{R}$.

Imnaha $\mathrm{R}$.

Imnaha R.

Imnaha $\mathrm{R}$.

Imnaha R.

Imnaha R.

Lightning Cr.

Little Sheep Cr.

Little Sheep Cr.

Little Sheep Cr.

Little Sheep Cr.

Little Sheep Cr.
Reach

Mouth - Camp Cr (RM 22.3 - 23.4 from Snake R)

Camp Cr -Little Sheep Cr (RM 23.4 - 25.4 from Snake R)

Little Sheep Cr - Squaw Cr (RM 25.4 - 37.7 from Snake R)

Squaw Cr - Man Cr (RM 37.7 - 39.3 from Snake R)

Marr Cr - Griffith Cr (RM 39.3 - 45.3 from Snake R)

Griffith Cr - Carrol Cr (RM 45.3 - 47.0 from Snake R)

Carrol Cr - Owl Cr (RM 47.0 - 53.5 from Snake R)

Owl Cr - Lick Cr (RM 53.5 - 57.4 from Snake R)

Lick Cr - South Fork (RM 57.4 - 58.9 from Snake R)

Lightning Cr - Horse Cr (RM 5.4 - 10.8 from Snake R)

Fence Cr -Big Sheep Cr (RM 16.3 - 22.3 from Snake R)

Horse Cr - Fence Cr (RM 10.8 - 16.3 from Snake R)

Big Sheep Cr - Freezeout Cr (RM 22.3 - 35.2 from Snake R)

Freezeout Cr - Grouse Cr (RM 35.2 - 40.6 from Snake R)

Grouse Cr - Summit Cr (RM 40.6 - 43.6 from Snake R)

Summit Cr - Crazyman Cr (RM 43.6 - 49.4 from Snake R)

Crazyman Cr - Gumboot Cr (RM 49.4 - 52.7 from Snake R)

Gumboot Cr - Dry Cr (RM 52.7 - 55.4 from Snake R)

Dry Cr - Skookum Cr (RM 55.4 - 61.9 from Snake R)

Blue Hole -forks (RM 66.4 - 71.4 from Snake R)

Skookum Cr - Blue Hole (RM 61.9 - 66.4 from Snake R)

South Fork Imnaha R (RM 71.4 - 76.1 from Snake R)

Mouth - forks (RM 5.4 - 13.9 from Snake R)

Mouth - Bear Gulch (RM 25.4 - 28.5 from Snake R)

Bear Gulch - Devil's Gulch (RM 28.5 - 30.9 from Snake R)

Devil's Gulch - Lightning Cr (RM 30.9 - 33.1 from Snake R)

Lightning Cr -Hayden Cr (RM 33.1 - 38.8 from Snake R)

Hayden Cr - McCully Cr (RM 38.8 - 48.8 from Snake R) 
Spawning reach: Big Sheep Cr.

Mouth - Camp Cr (RM 22.3 - 23.4 from Snake R)

\section{Relative Productivity}
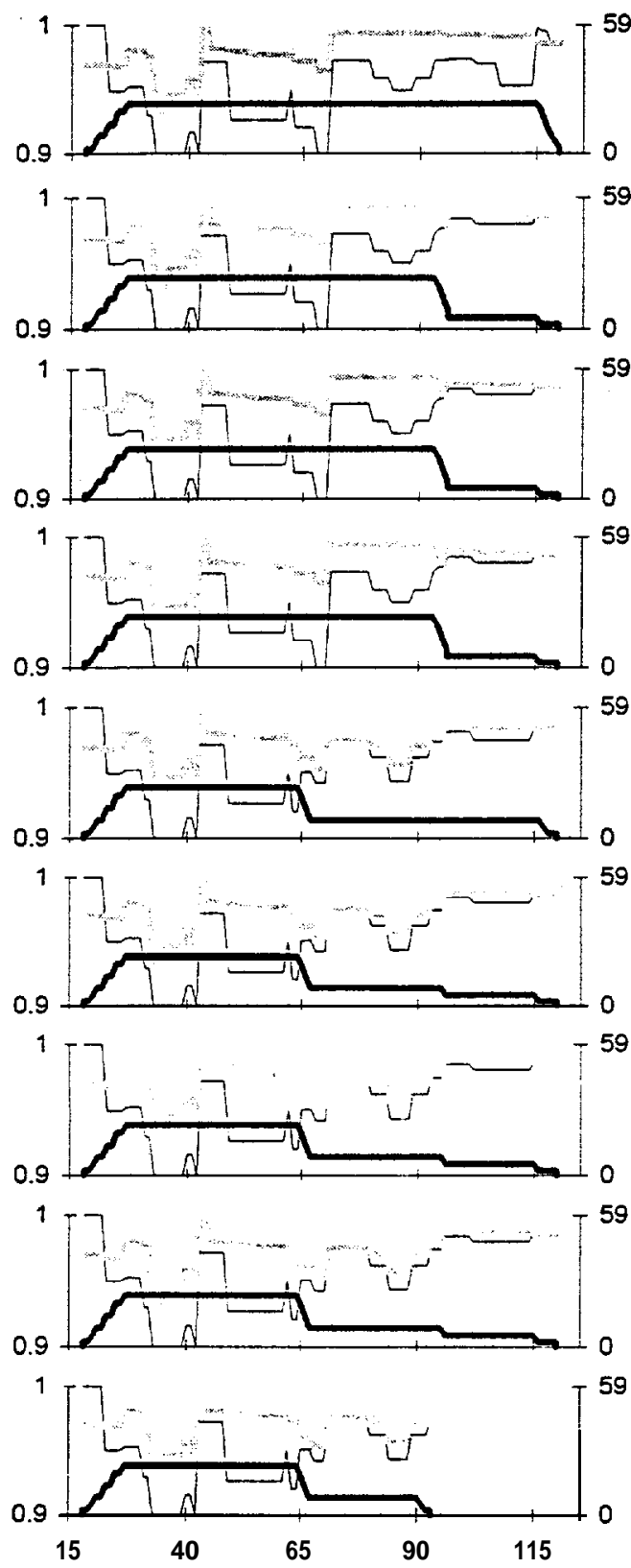

Statistical week

Prespa w n -

Spawning

Incubation Colonization

Sum. rearing

Win. rearing
Relative Key Habitat Quantity
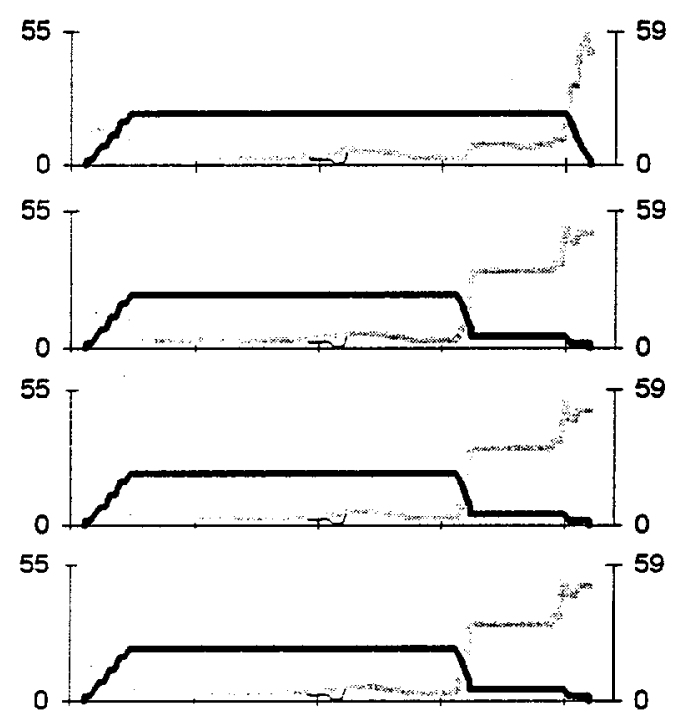

55 T
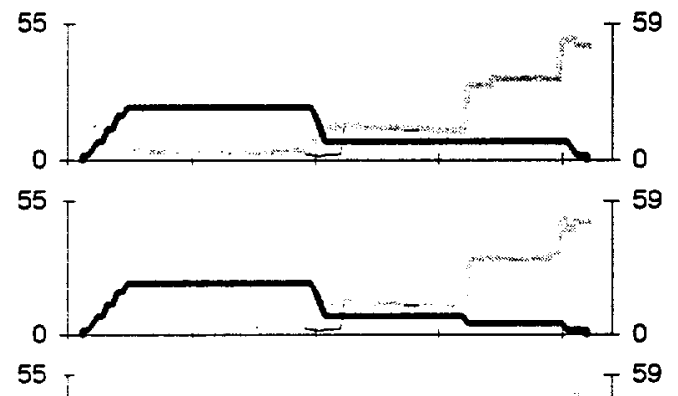

55

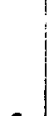

0
55
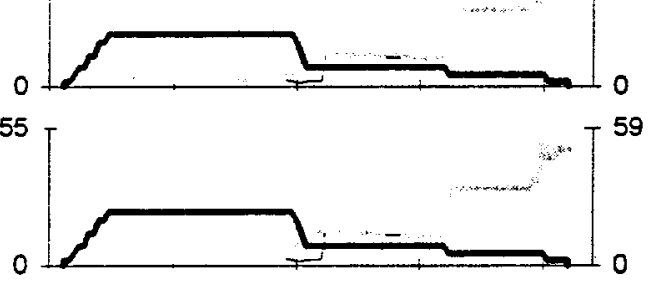

55 -

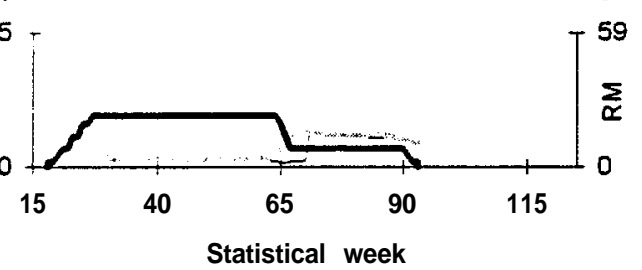

Statistical week

Prespawn -

Spawning

Incubation

Colonization

Sum. rearing

Win. rearing

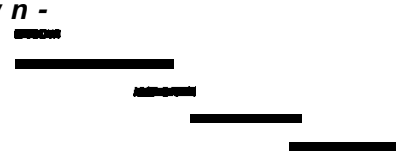

Template

Patient

Cum. Cum.

Prod. Cap.

Traj. \#1

\begin{tabular}{l|l}
\hline 106 & 0.13
\end{tabular}

2) $0.0 \mathrm{C}$

Traj. \#2

960.13

3) 0.01

Traj. \#3

96.0 .15

3) 0.01

Traj. \#4

$96 \mid 0.13$

3) 0.01

Traj. \#5

\begin{tabular}{r|l}
\hline 48 & 0.07 \\
4 & 0.01
\end{tabular}

Traj. \#6

\begin{tabular}{r|l}
\hline 52 & 0.08 \\
4 & 0.01
\end{tabular}

Traj. \#7

\begin{tabular}{r|l}
\hline 52 & 0.08 \\
4 & 0.01 \\
\hline
\end{tabular}

Traj . \#8

\begin{tabular}{l|l}
\hline 52 & 0.08 \\
\hline & 0.01
\end{tabular}

$\begin{array}{ll}4 & 0.01\end{array}$

Traj. \#9

\begin{tabular}{l|l}
\hline 104 & 0.16 \\
10 & 0.02
\end{tabular}

10) 0.02 
Spawning reach: Big Sheep Cr.

Camp Cr - Little Sheep Cr (RM 23.4 - 25.4 from Snake R)

Relative Productivity
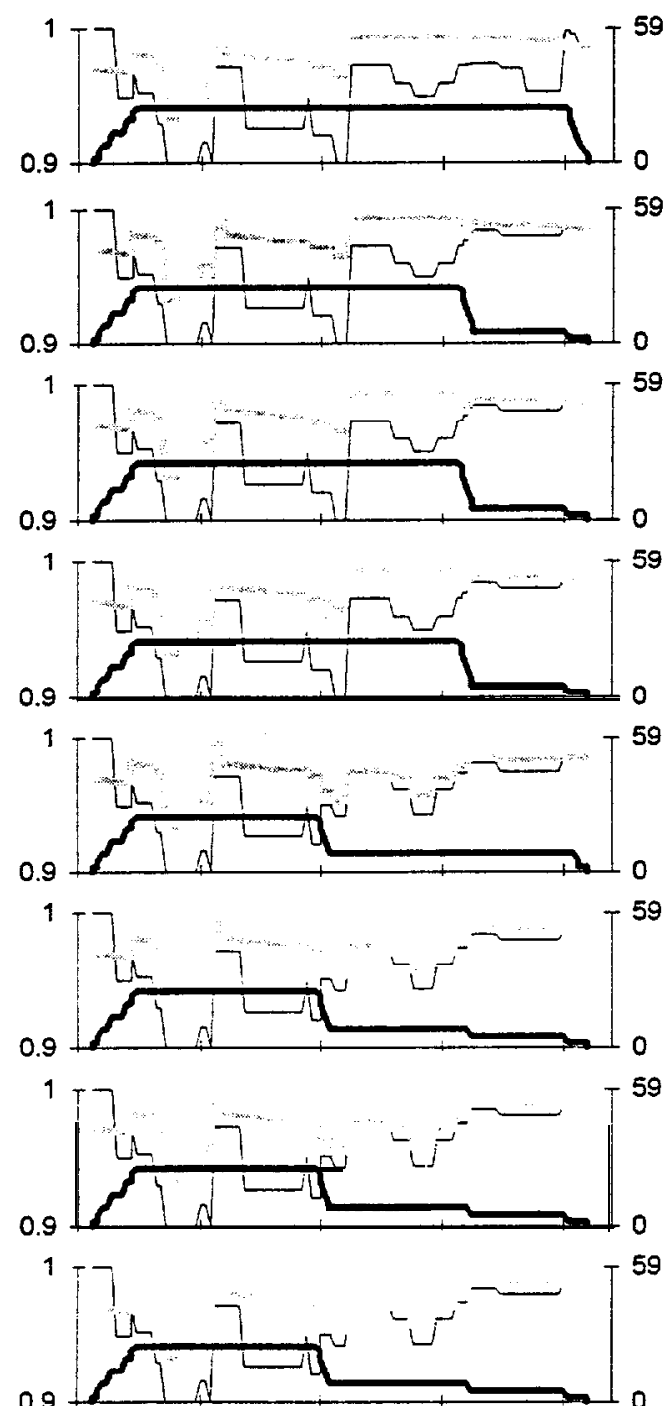

0.9

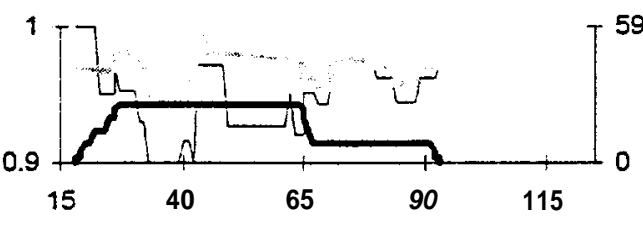

Statistical week
Relative Key Habitat Quantity
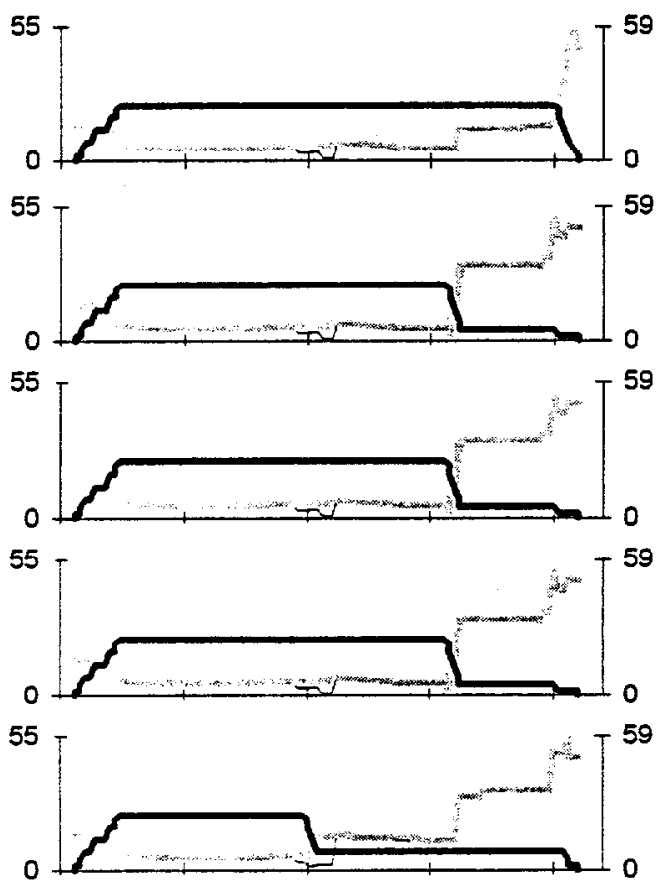

55 T

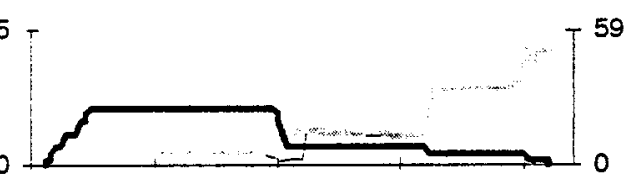

55

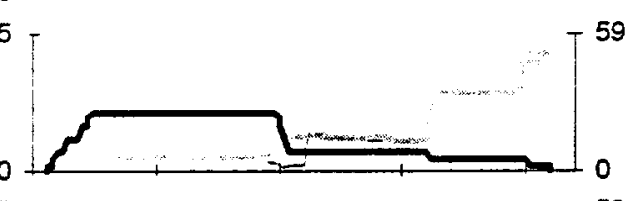

55 T

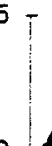

55

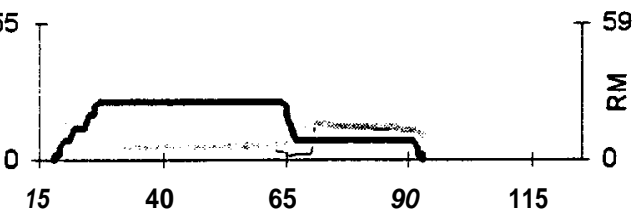

Statistical week
Prespawn -

Spawning

Incubation

Colonization

Sum. rearing

Win. rearing

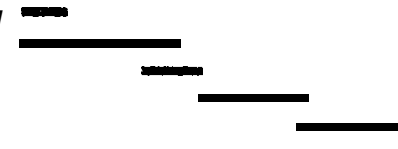

Prespawn -

Spawning

Incubation

Colonization

Sum. rearing

Win. rearing
Template

Patient

Cum. Cum.

Prod. Cap.

Traj. \#1

Traj. \#2

Traj. \#3

$9 7 \longdiv { 0 . 1 6 }$

3) 0.01

Traj. \#4

$97 \quad 0.16$

3) 0.01

Traj . \#5

490.09

4) 0.01

Traj. \#6

\begin{tabular}{r|r}
52 & 0.10 \\
4 & 0.01
\end{tabular}

Traj. \#7

\begin{tabular}{r|r}
52 & 0.10 \\
4 & 0.01
\end{tabular}

Traj. \#8

$T^{59}$

\begin{tabular}{ll|l} 
" & $:$ \\
\multicolumn{2}{|c|}{ Traj. $\# 9$} \\
\hline 104 & 0.20 \\
10 & 0.02
\end{tabular}

Trajectory
Template

Patient 
Spawning reach: Big Sheep Cr.

Little Sheep $\mathrm{Cr}$ - Squaw $\mathrm{Cr}$ (RM 25.4 - 37.7 from Snake R)

\section{Relative Productivity}
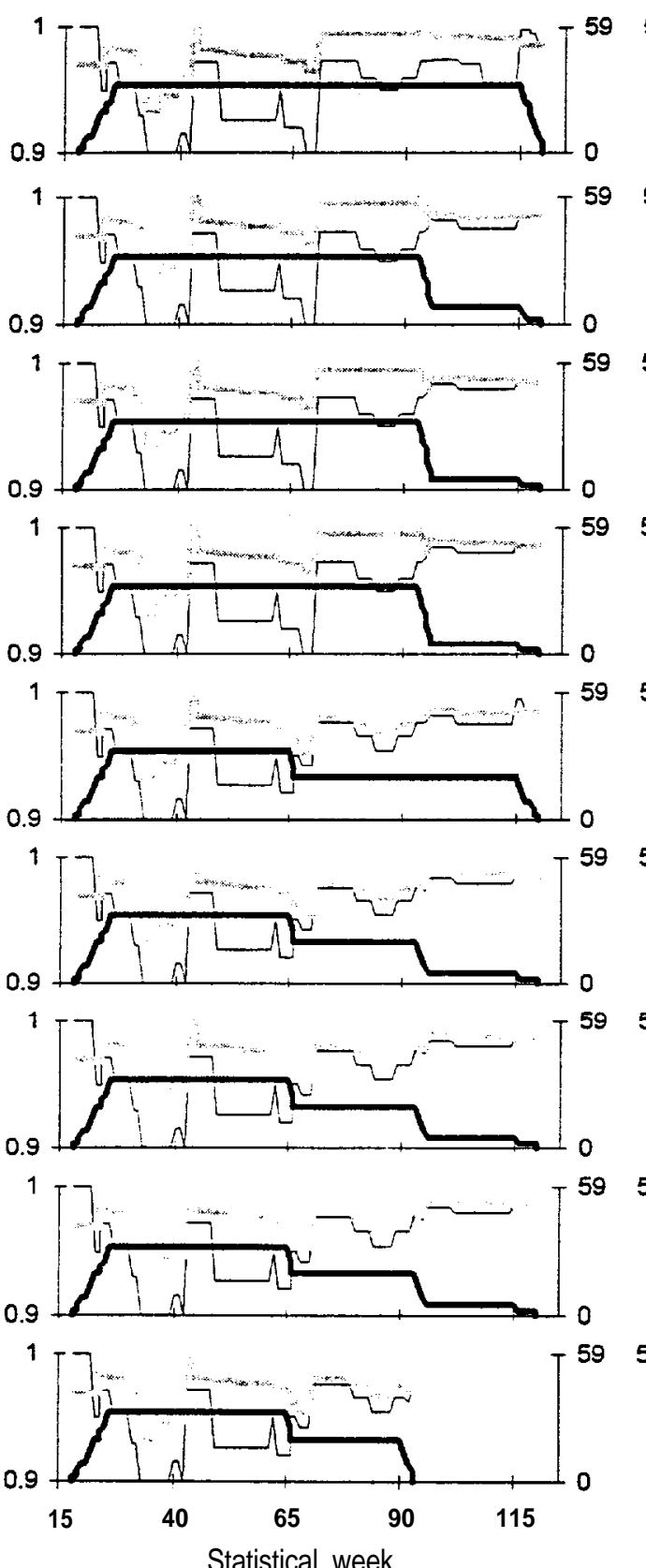

Statistical week

\section{Prespawn -}

Spawning
Incubation
Colonization

Sum. rearing

Win. rearing
Relative Key Habitat Quantity
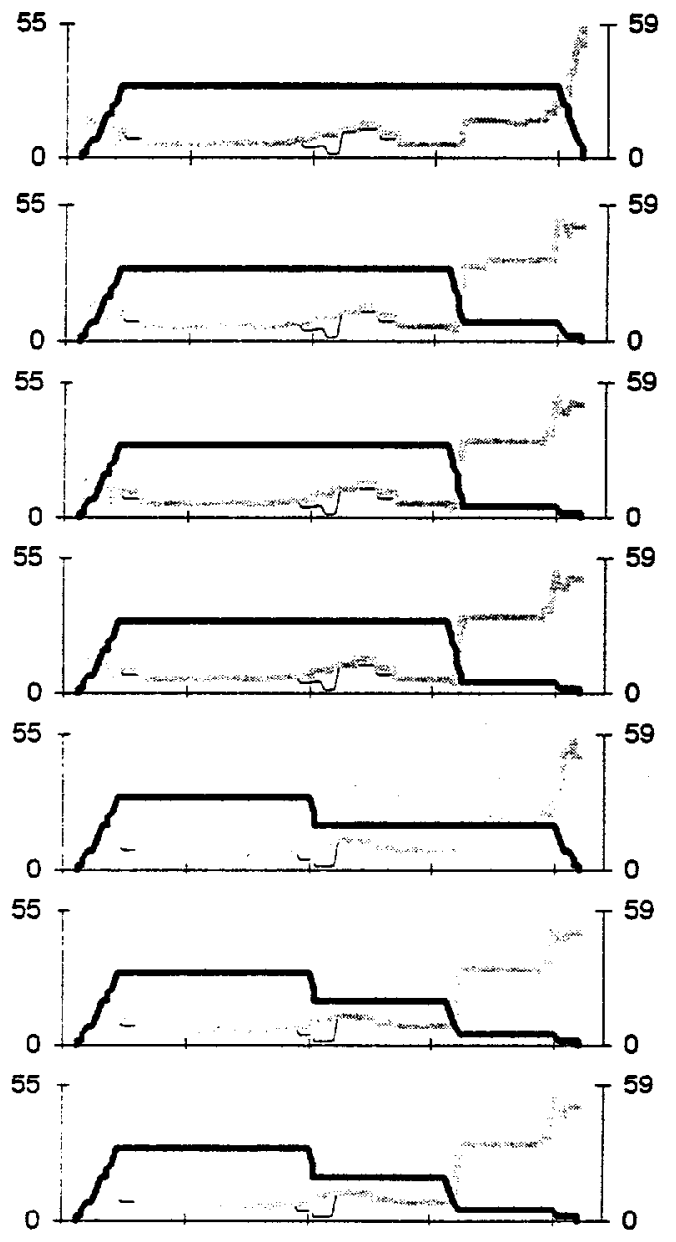

55 -
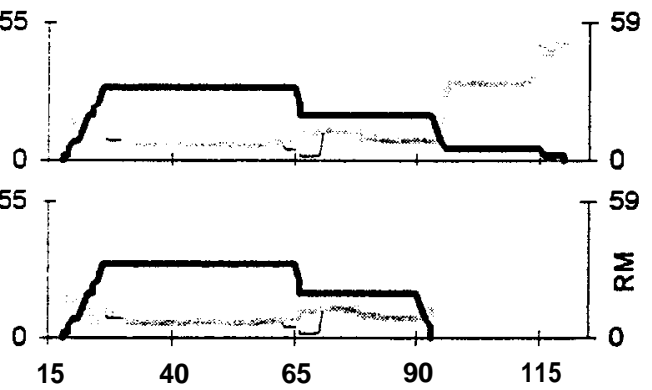

Statistical week

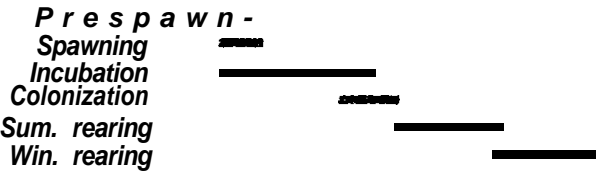

\begin{tabular}{|r|c|}
\hline \multicolumn{2}{|c|}{$\begin{array}{c}\text { Template } \\
\text { Patient }\end{array}$} \\
\hline Cum. & Cum. \\
Prod. & Cap. \\
Traj. \#1 \\
\hline 116 & 0.22 \\
3 & 0.01 \\
\hline
\end{tabular}

Traj. \#2

$99 \quad 0.19$

30.01

Traj. \#3

\begin{tabular}{|c|c|}
\hline 05 & 0.20 \\
\hline
\end{tabular}

\begin{tabular}{l|l}
4 & 0.01
\end{tabular}

Traj. \#4

$105 \quad 0.20$

\begin{tabular}{l|l|l}
4 & 0.01
\end{tabular}

Traj . \#5

\begin{tabular}{r|l}
59 & 0.12 \\
5 & 0.01
\end{tabular}

Traj. \#6

\begin{tabular}{r|l|}
\hline 62 & 0.13 \\
5 & 0.01
\end{tabular}

Traj. \#7

\begin{tabular}{r|l|}
62 & 0.13 \\
5 & 0.01
\end{tabular}

Traj . \#8

\begin{tabular}{|r|l|}
62 & 0.13 \\
5 & 0.01
\end{tabular}

Traj. \#9

\begin{tabular}{l|l}
124 & 0.26 \\
\hline & 0.02
\end{tabular}

110.02 
Spawning reach: Big Sheep Cr.

Squaw $\mathrm{Cr}$ - Man Cr (RM 37.7 - 39.3 from Snake R)

Relative Productivity
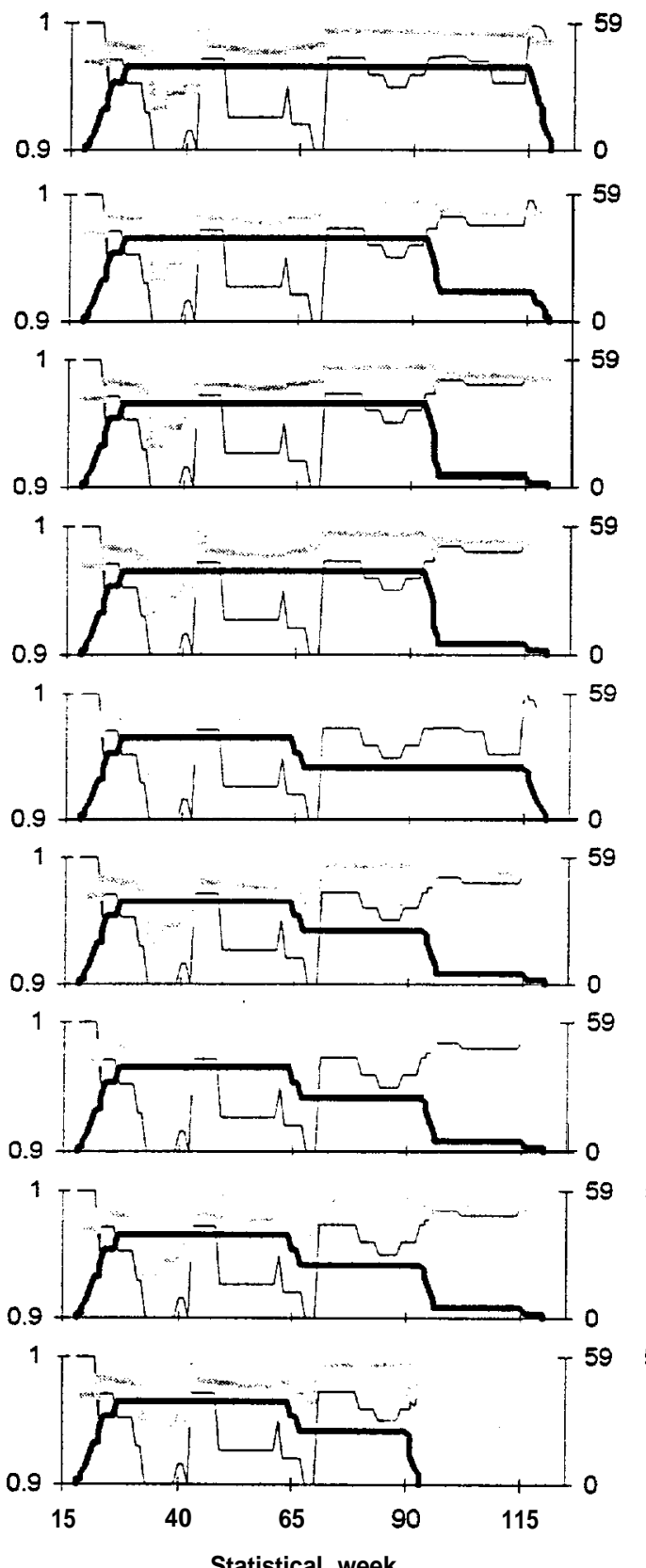

Statistical week

Prespawn -

Spawning

incubation

Colonization

Sum. rearing

Win. rearing
Relative Key Habitat Quantity
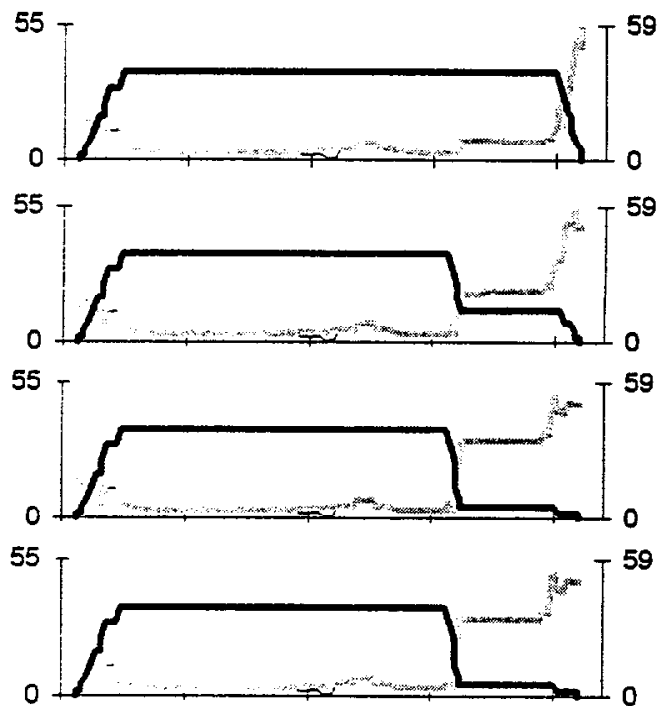

55 -

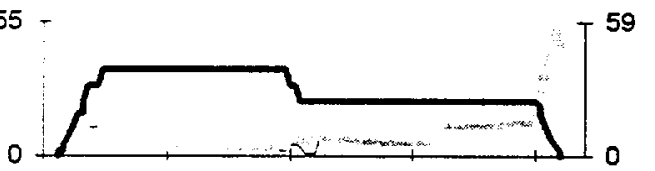

55

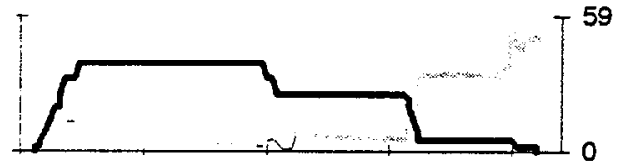

55 -
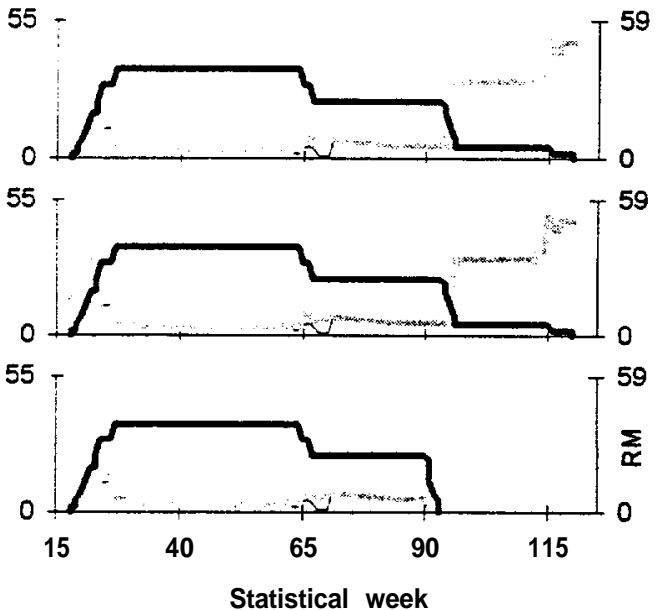

Statistical week

\section{Prespawn -}

Spawning

Incubation

Colonization

Sum. rearing

Win. rearing \begin{tabular}{|r|c|}
\hline \multicolumn{2}{|c|}{$\begin{array}{c}\text { Template } \\
\text { Patient }\end{array}$} \\
\hline Cum. & Cum. \\
Prod. & Cap. \\
Traj . \#1 \\
\hline 125 & 0.16 \\
3 & 0.00 \\
\hline
\end{tabular}

Traj. \#2

\begin{tabular}{l|l}
108 & 0.14 \\
\hline & 0.00
\end{tabular}

3) 0.00

Traj. \#3

$115 \mid 0.15$

4) 0.01

Traj. \#4

$115 \mid$\begin{tabular}{l|l}
0.15 \\
14
\end{tabular}

4) 0.01

Traj. \#5

$114 \quad 0.16$

3) 0.00

Traj. \#6

\begin{tabular}{r|r|}
104 & 0.15 \\
4 & 0.01
\end{tabular}

Traj. \#7

\begin{tabular}{l|l|}
\hline 104 & 0.15 \\
\hline
\end{tabular}

4) 0.01

Traj. \#8

$104 \quad 0.15$

$\begin{array}{lll}4 & 0.01\end{array}$

Traj. \#9

196 0.29

\begin{tabular}{l|l|}
9 & 0.01
\end{tabular} 
Spawning reach: Big Sheep Cr.

Marr Cr - Griffith Cr (RM 39.3 - 45.3 from Snake R)

Relative Productivity
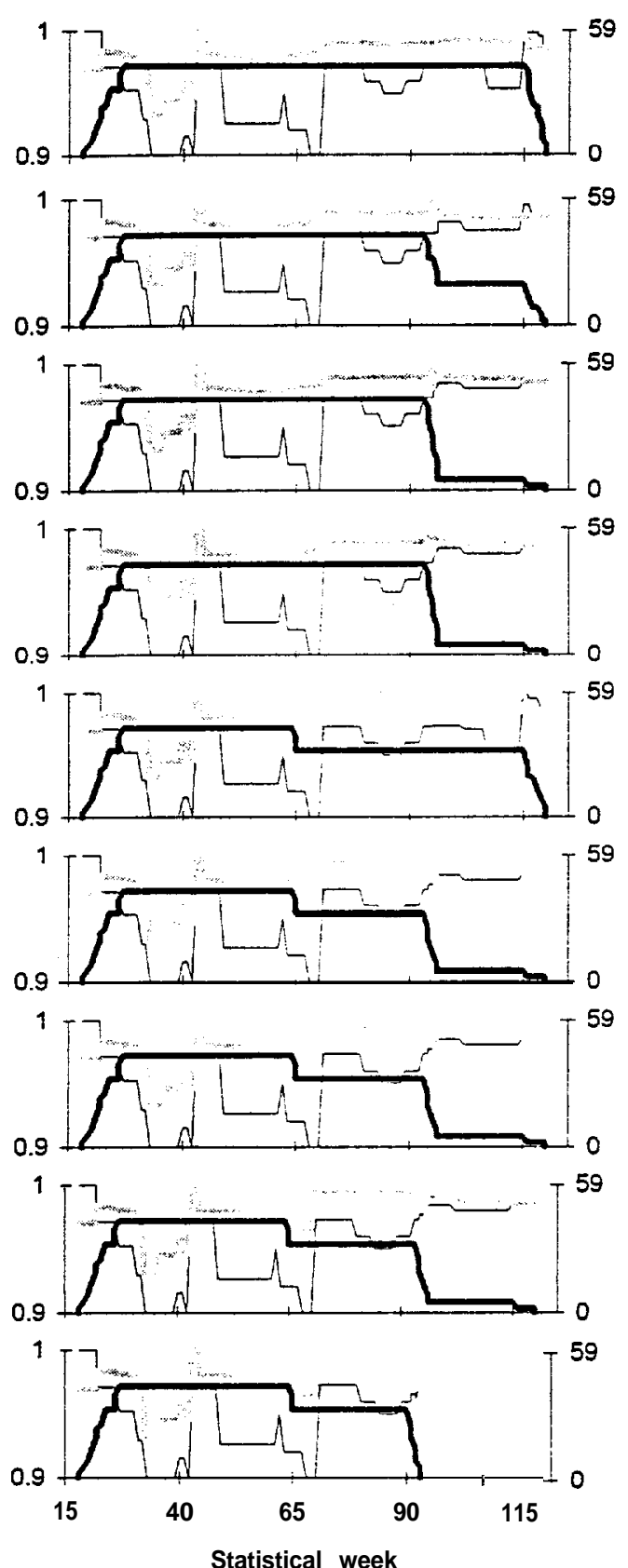

Statistical week

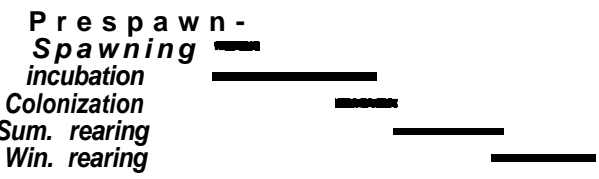

Relative Key Habitat Quantity
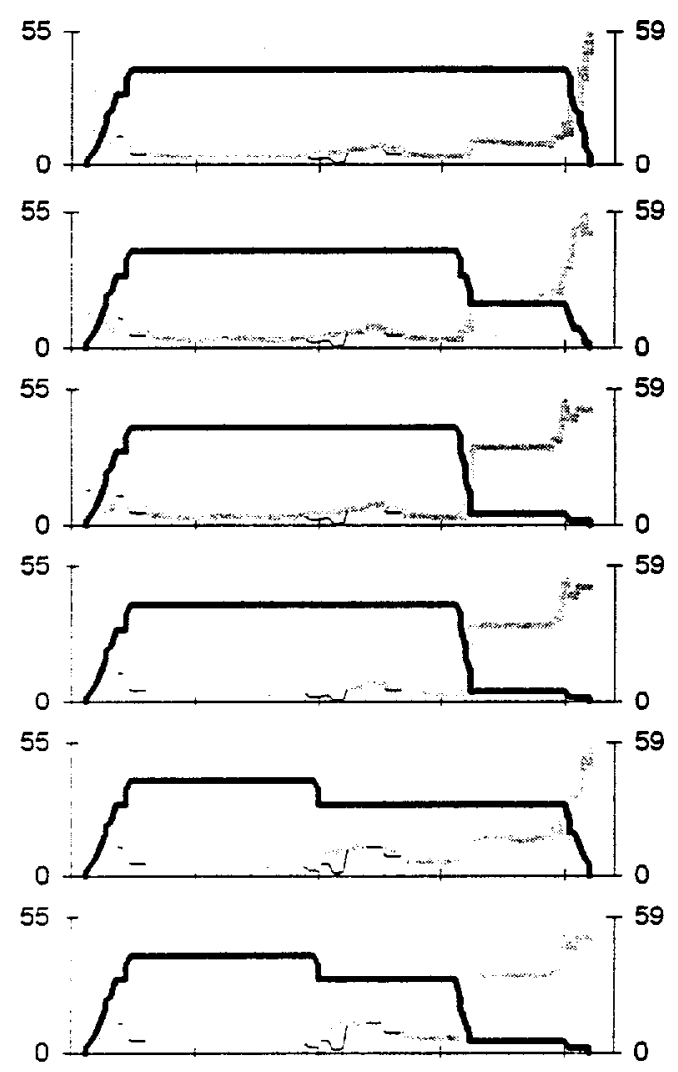

55 -

59
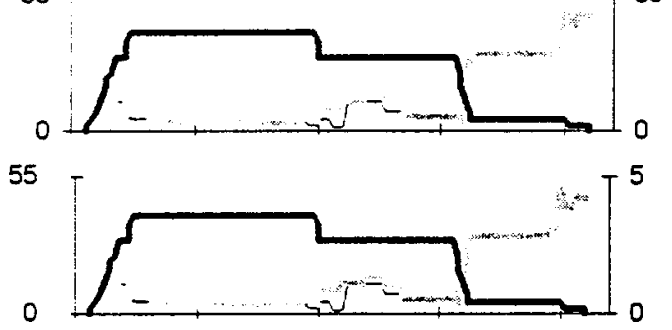

$55-$

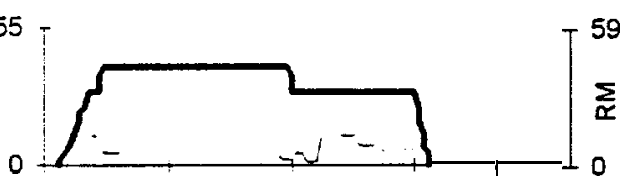

Statistical week

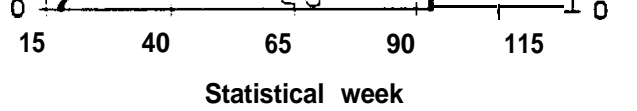

\begin{tabular}{|c|l|}
\hline \multicolumn{2}{|c|}{$\begin{array}{c}\text { Template } \\
\text { Patient }\end{array}$} \\
\hline Cum. & Cum. \\
Prod. & Cap.
\end{tabular}

Traj. \#1

$114 \mid 0.16$

30.00

Traj. \#2

101

30.01

Traj. \#3

1055.16

\begin{tabular}{l|l}
4 & 0.01
\end{tabular}

Traj. \#4

$105 \quad 0.16$

\begin{tabular}{l|l}
4 & 0.01
\end{tabular}

Traj. \#5

$122 \quad 0.19$

3) 0.00

Traj. \#6

\begin{tabular}{r|l}
110 & 0.18 \\
4 & 0.01
\end{tabular}

Traj. \#7

$110 \quad 0.18$ 4) 0.01

Traj. \#8

\begin{tabular}{r|l}
110 & 0.18 \\
4 & 0.01
\end{tabular}

Traj. \#9

\begin{tabular}{|l|l|}
\hline 206 & 0.34 \\
\hline
\end{tabular}

90.01
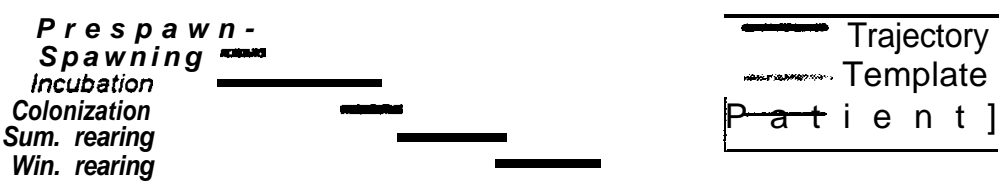
Spawning reach: Big Sheep Cr.

Griffith $\mathrm{Cr}$ - Carrol Cr (RM 45.3 - 47.0 from Snake R)

Relative Productivity
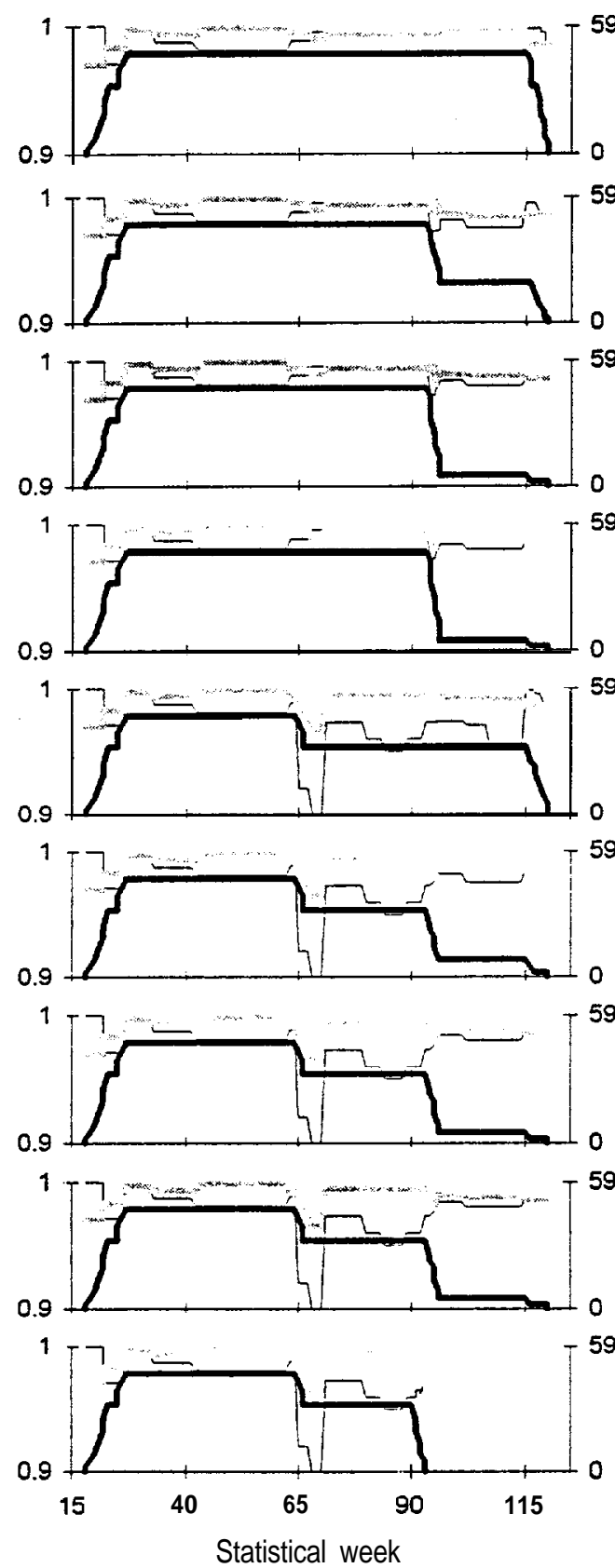

\section{Prespawn
Spawning}

Incubation

Colonization

Sum. rearing

Win. rearing
Relative Key Habitat Quantity

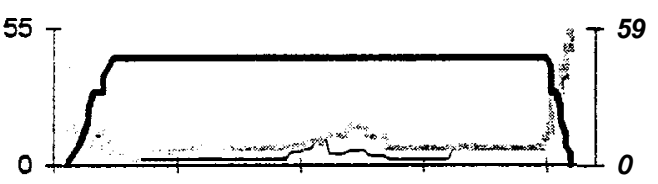

55

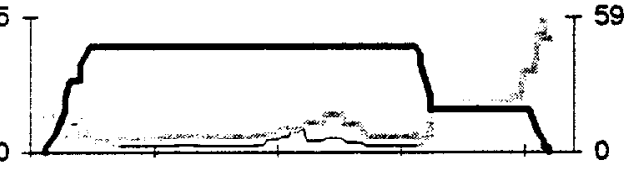

55

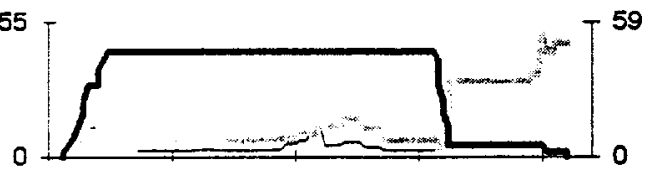

$55+$

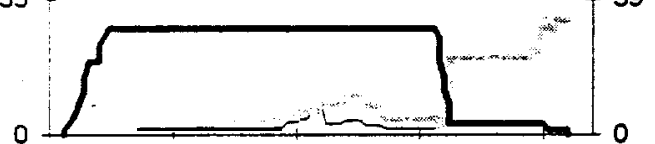

55

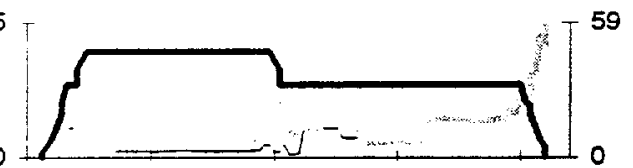

55

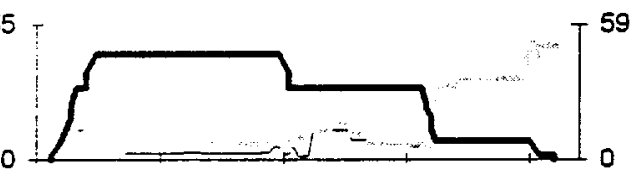

55 .

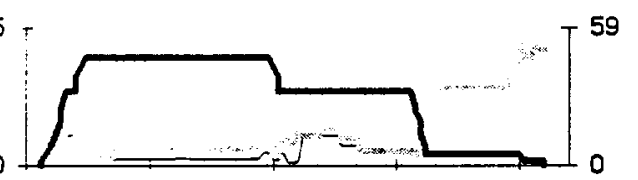

55

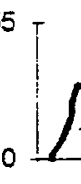

55 -
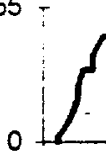

59
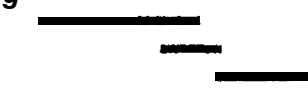

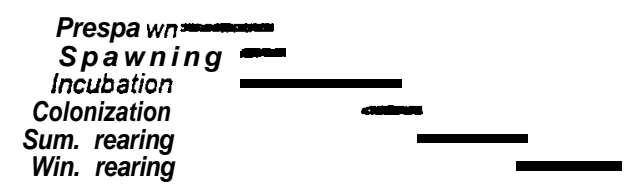

Traj. \#8

\begin{tabular}{r|r}
311 & 0.33 \\
59 & 0.06
\end{tabular}

Traj. \#9

5790.66

137 0.14 
Spawning reach: Big Sheep Cr.

Carrol Cr - Owl Cr (RM 47.0 - 53.5 from Snake R)

Relative Productivity

Relative Key Habitat Quantity
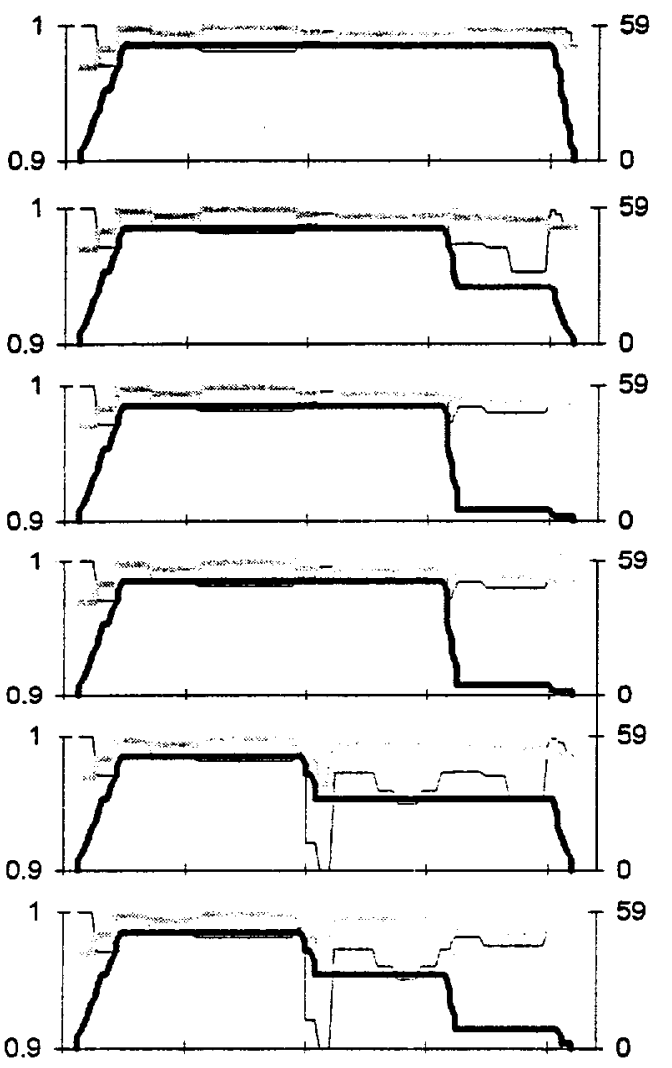

59
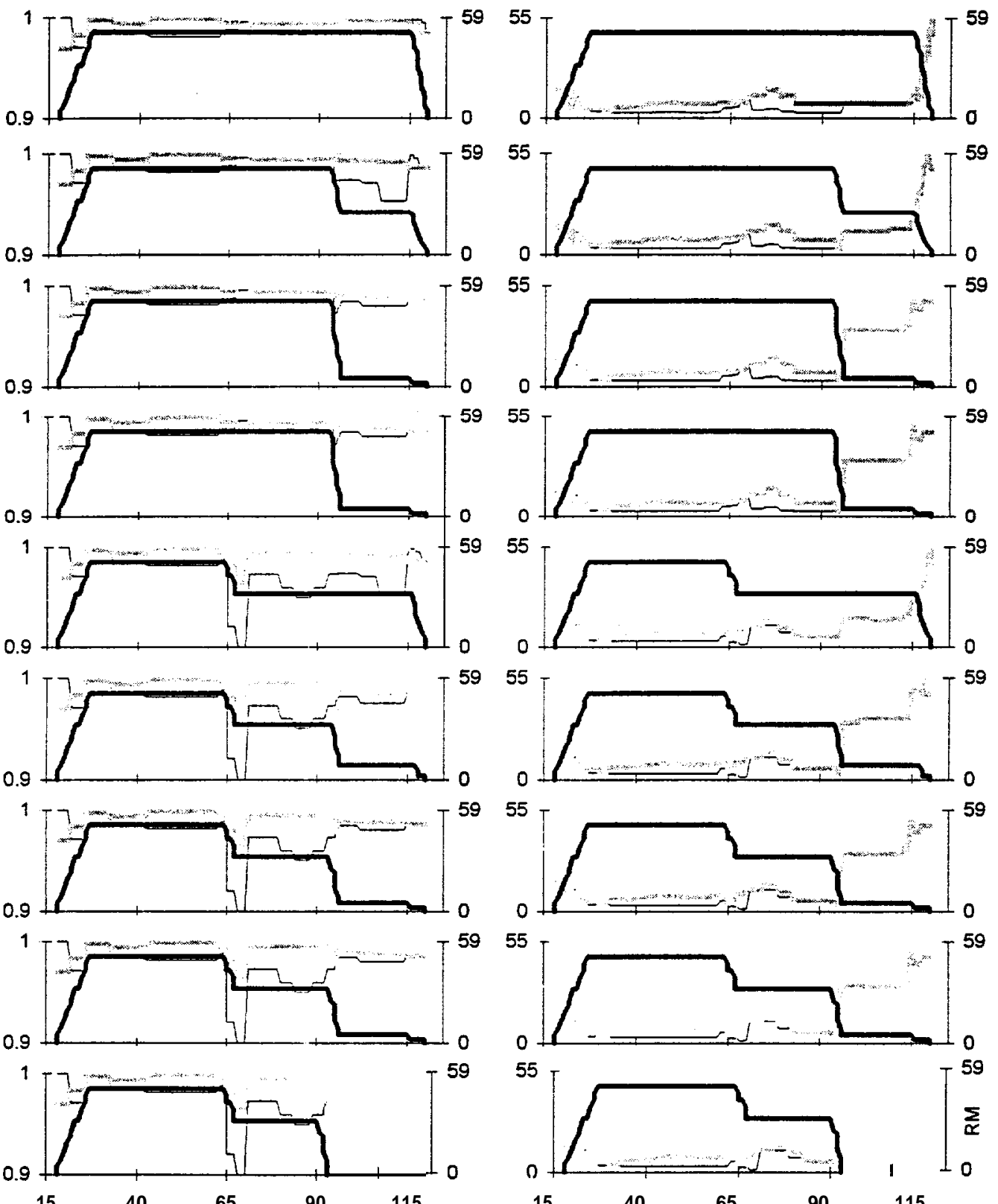

Statistical week

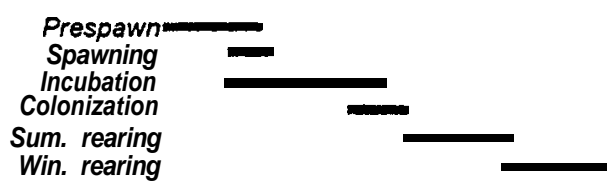

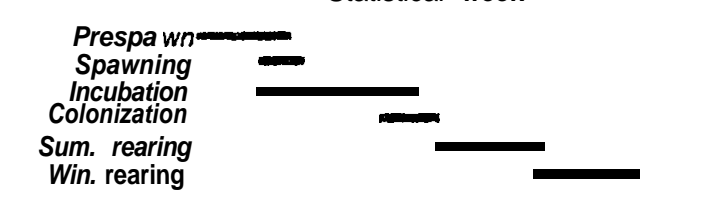

\begin{tabular}{|r|c|}
\hline \multicolumn{2}{|c|}{$\begin{array}{c}\text { Template } \\
\text { Patient }\end{array}$} \\
\hline $\begin{array}{r}\text { Cum. } \\
\text { Prod. }\end{array}$ & Cum. \\
Cap. \\
Traj. \#1 \\
\hline 416 & 0.41 \\
321 & 0.25 \\
& \\
Traj. \#2 \\
\hline 372 & 0.38 \\
159 & 0.13 \\
\end{tabular}

Traj. \#3

$344 \quad 0.37$

215 0.18

Traj. \#4

$344 \quad 0.37$

215 0.18

Traj. \#5

$350 \quad 0.36$

44 0.04

Traj. \#6

$303 \quad 0.32$

550.05

Traj. \#7

3190.34

\begin{tabular}{l|l}
59 & 0.06
\end{tabular}

Traj. \#8

\begin{tabular}{r|r}
319 & 0.34 \\
59 & 0.06
\end{tabular}

Traj. \#9

$603 \quad 0.68$

137 0.13 
Spawning reach: Big Sheep Cr.

Owl Cr - Lick Cr (RM 53.5 - 57.4 from Snake R)

Relative Productivity
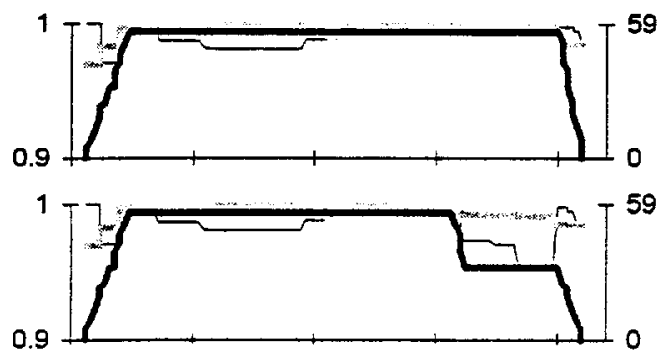

0.9
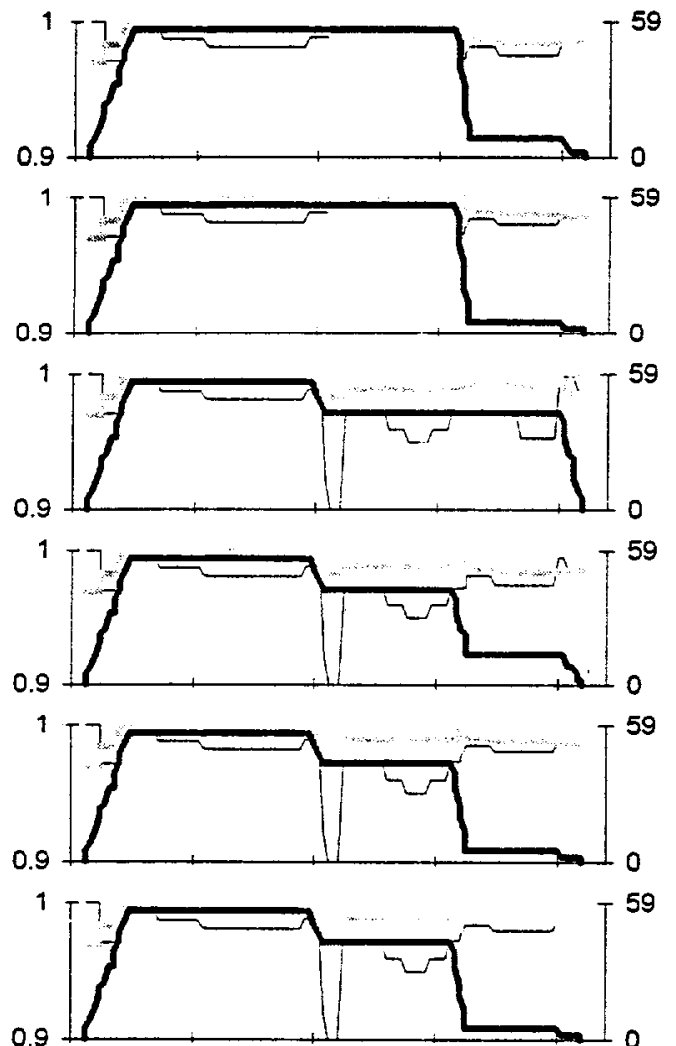

0.9

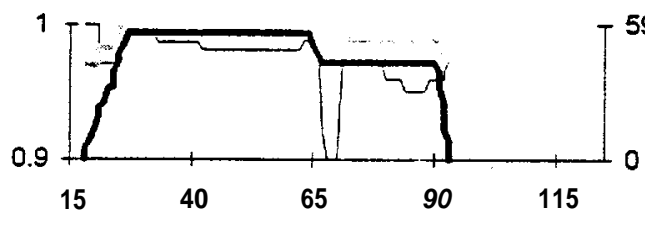

Statistical week

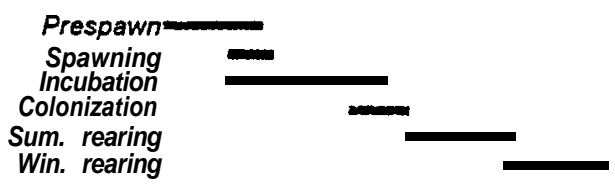

Relative Key Habitat Quantity
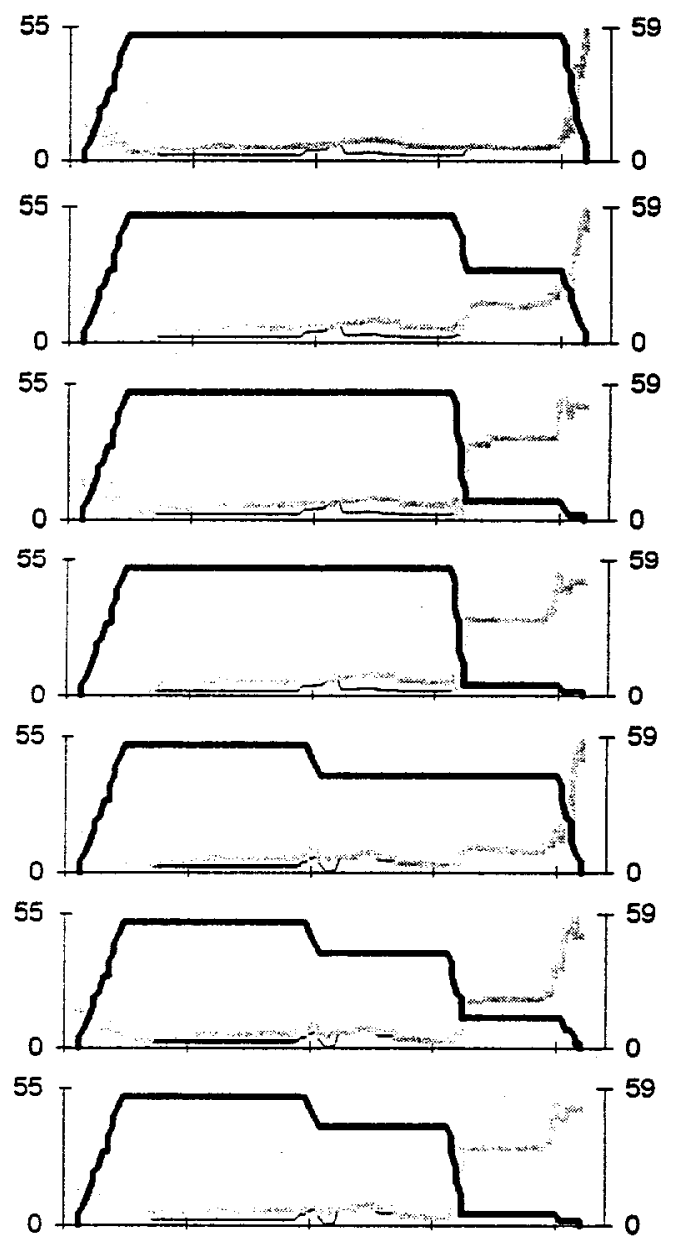

55

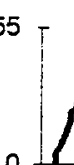

55

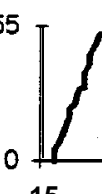

15
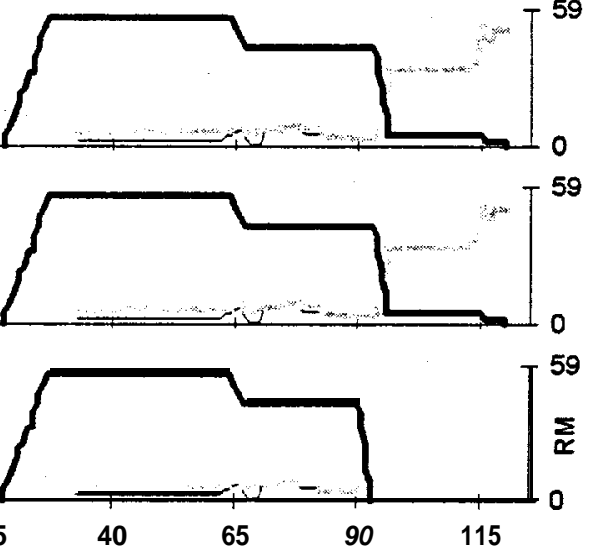

Statisticalweek

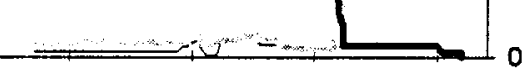

\begin{tabular}{|c|c|}
\hline \multicolumn{2}{|c|}{ Template } \\
Patient \\
\hline Cum. & Cum. \\
Prod. & Cap. \\
\hline
\end{tabular}

Traj. \#1

$477 \quad 0.35$

$330 \quad 0.19$

Traj. \#2

\begin{tabular}{l|l|}
\hline 420 & 0.33 \\
168 & 0.11
\end{tabular}

Traj. \#3

\begin{tabular}{l|l}
369 & 0.30 \\
\hline
\end{tabular}

206 0.13

Traj. \#4

$393 \quad 0.32$

$221 \quad 0.14$

Traj . \#5

$334 \quad 0.25$

$51 \quad 0.04$

Traj. \#6

\begin{tabular}{|l|l|}
\hline 294 & 0.23 \\
\hline
\end{tabular}

64) 0.04

Traj. \#7

$310 \quad 0.25$

68 0.05

Traj. \#8

\begin{tabular}{l|l|}
\hline 310 & 0.25 \\
\hline
\end{tabular}

68 0.05

Traj. \#9

\begin{tabular}{l|l}
596 & 0.51 \\
\hline
\end{tabular}

1590.11

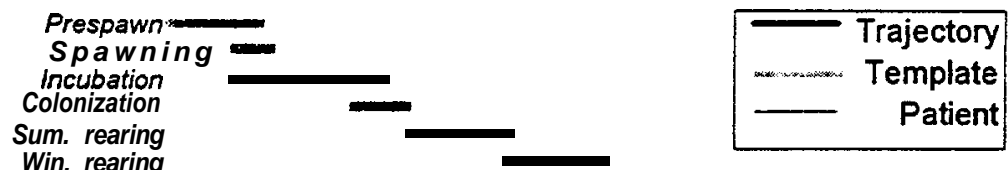


Spawning reach: Big Sheep Cr.

Lick Cr - South Fork (RM 57.4 - 58.9 from Snake R)

Relative Productivity
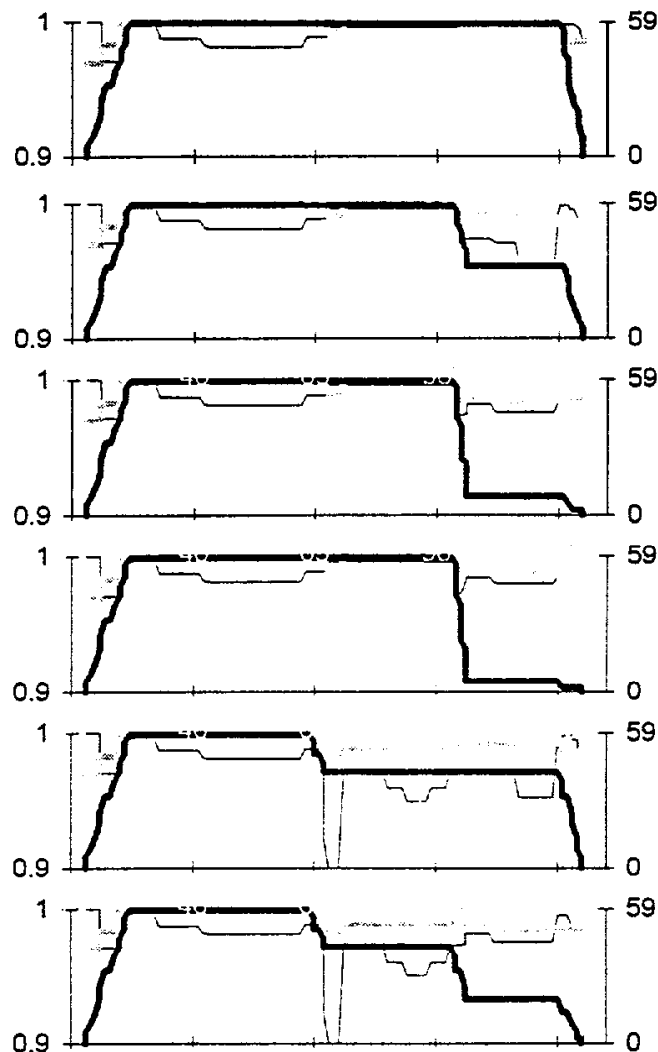

$$
1
$$

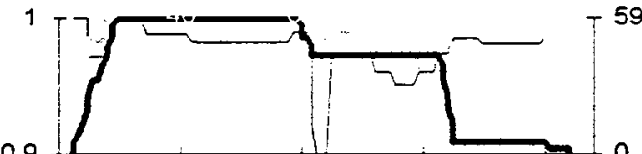

0.9

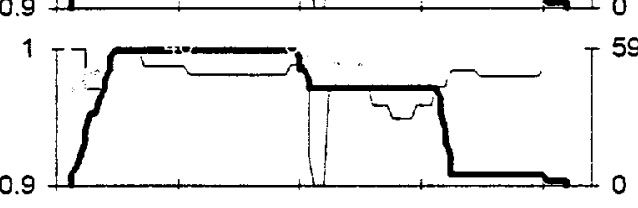

0.9

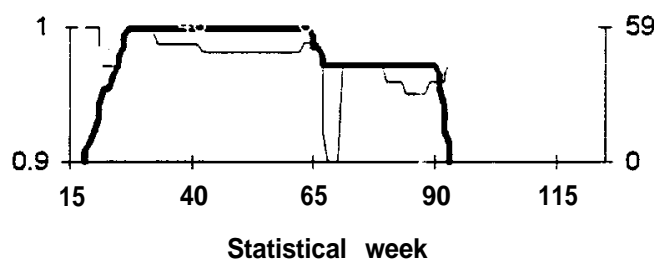

Relative Key Habitat Quantity
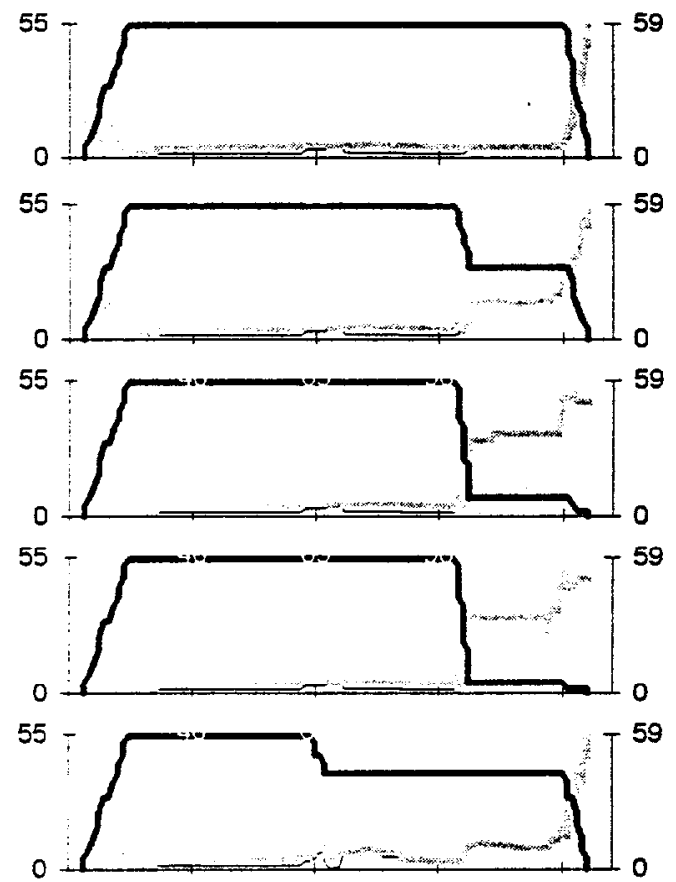

55

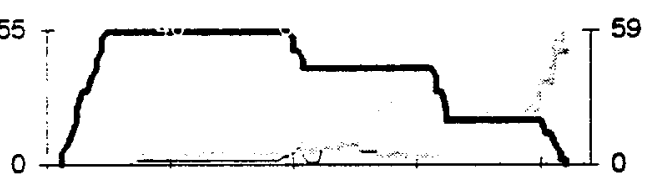

$55-$
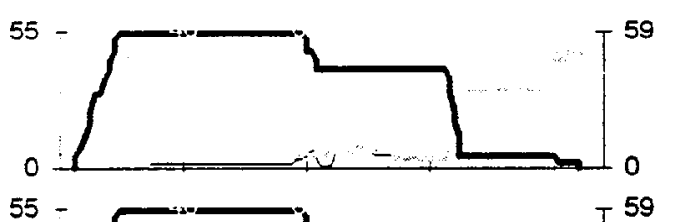

55
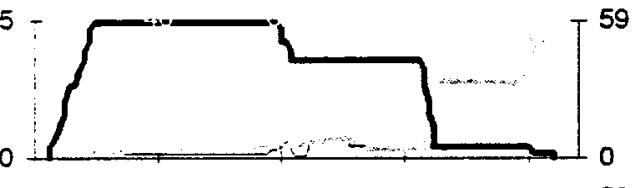

$55-$

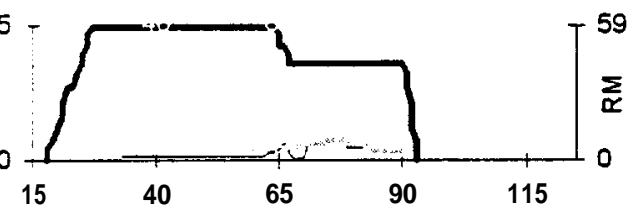

Statistical week

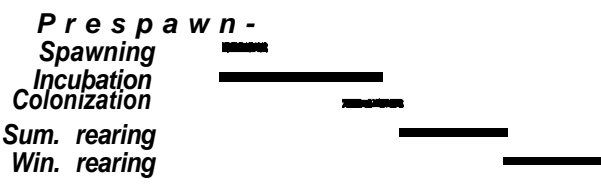

Prespawn Colonization

Win. rearing

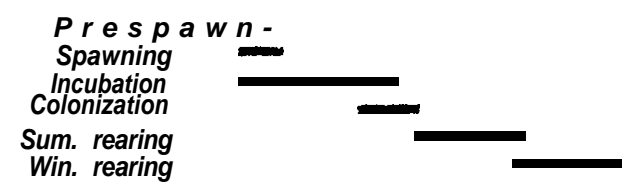

\begin{tabular}{|c|c|}
\hline \multicolumn{2}{|c|}{ Template } \\
Patient \\
\hline Cum. & Cum. \\
Prod. & Cap. \\
Traj. \#1 \\
\hline 509 & 0.26 \\
329 & 0.15 \\
\hline
\end{tabular}

\begin{tabular}{c|c}
\multicolumn{2}{c}{ Traj. \#2 } \\
\hline 448 & $0.2 E$ \\
168 & $0.0 \varepsilon$
\end{tabular}

\begin{tabular}{c|c}
\multicolumn{2}{c}{ Traj. \#3 } \\
\hline 395 & 0.23 \\
205 & $0.1 \mathrm{C}$
\end{tabular}

Traj. \#4

\begin{tabular}{l|l}
\hline 421 & 0.24 \\
\hline
\end{tabular}

221

0.11

Traj . \#5

\begin{tabular}{l|l}
\hline 357 & 0.21 \\
\hline
\end{tabular}

520.03

Traj. \#6

\begin{tabular}{l|l}
\hline 316 & $0.1 \mathrm{~s}$ \\
\hline
\end{tabular}

66 0.04

\begin{tabular}{r|r}
\multicolumn{2}{c}{ Traj. \#7 } \\
\hline 331 & 0.20 \\
69 & 0.04
\end{tabular}

Traj. \#8

\begin{tabular}{l|l}
\hline 331 & 0.20 \\
\hline
\end{tabular}

690.04

\begin{tabular}{c|c}
\multicolumn{2}{c}{ Trai. $\# 9$} \\
\hline 637 & 0.41 \\
161 & 0.10
\end{tabular}

161

0.10 
Spawning reach: Imnaha $\mathbf{R}$.

Lightning Cr - Horse Cr (RM 5.4 - 10.8 from Snake R)

Relative Productivity
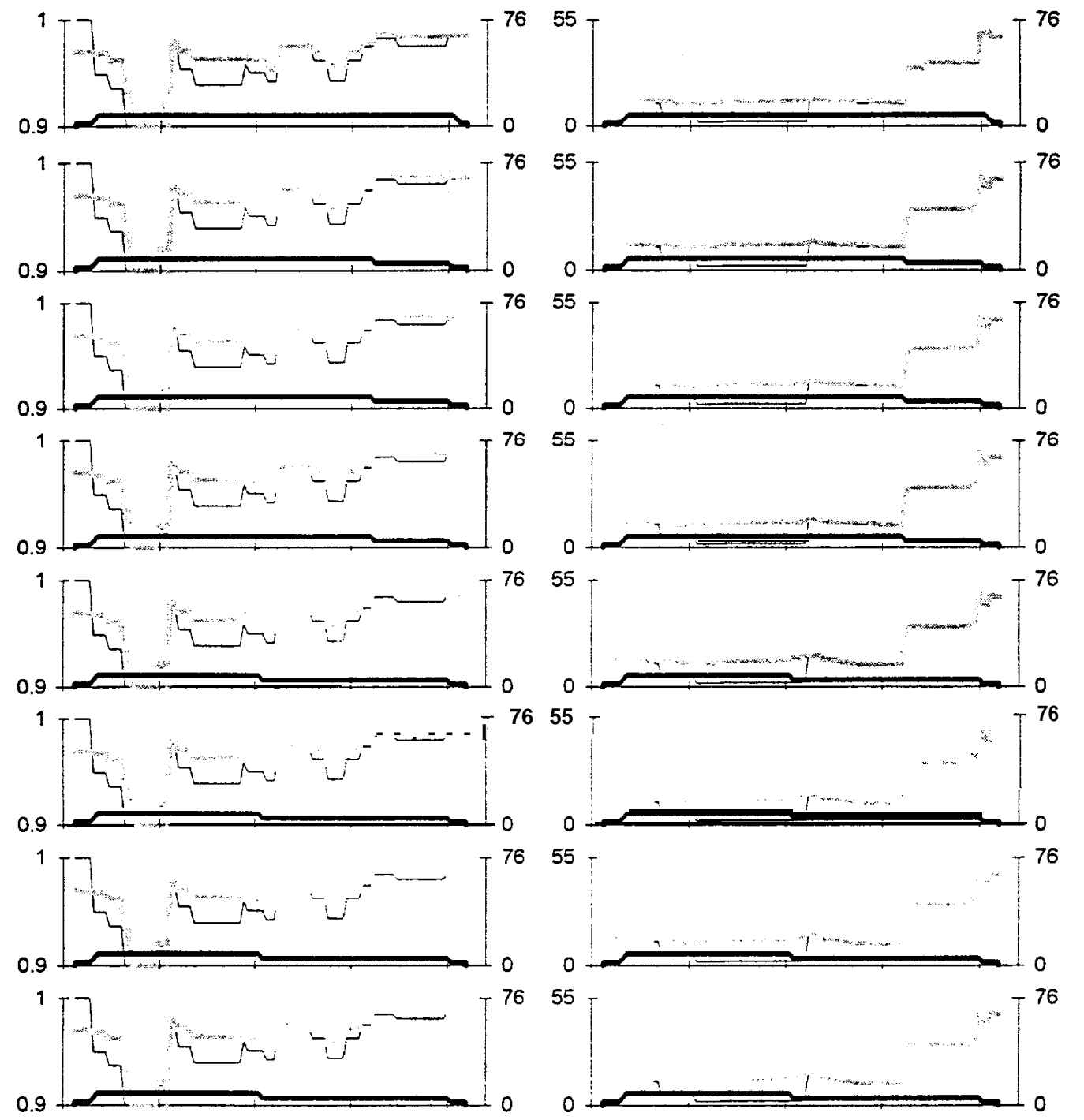
$76 \quad 55$

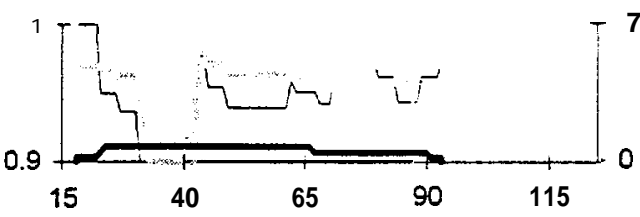
Statistical week

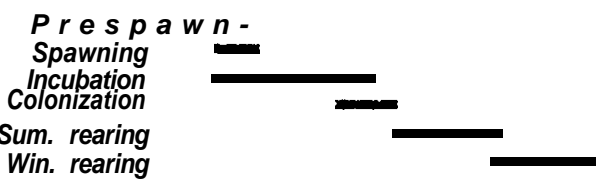

Win. rearing
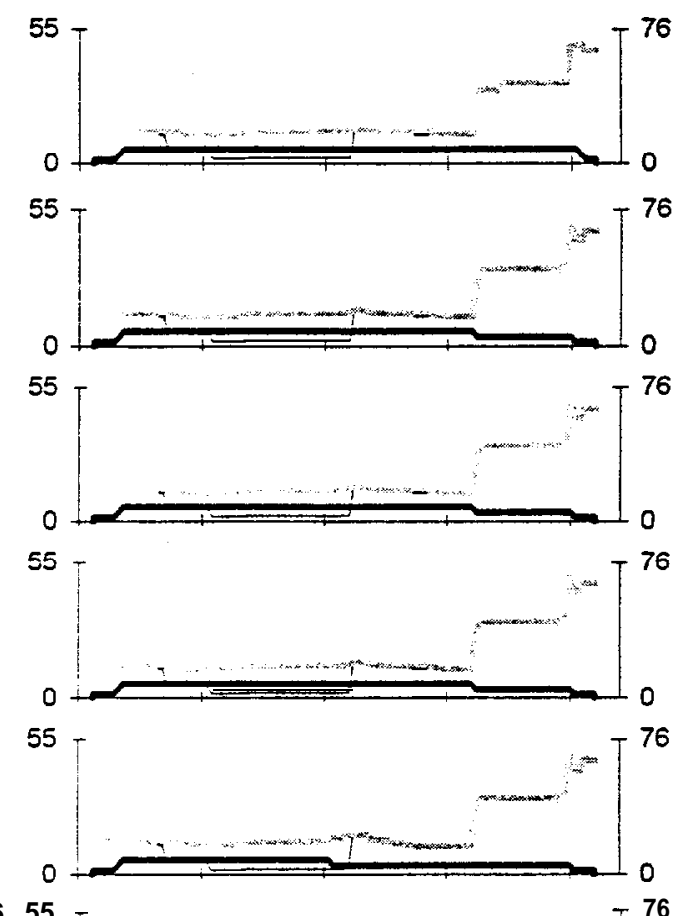

$(1, \ldots+\cdots+\infty$

$55+$

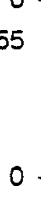

Relative Key Habitat Quantity
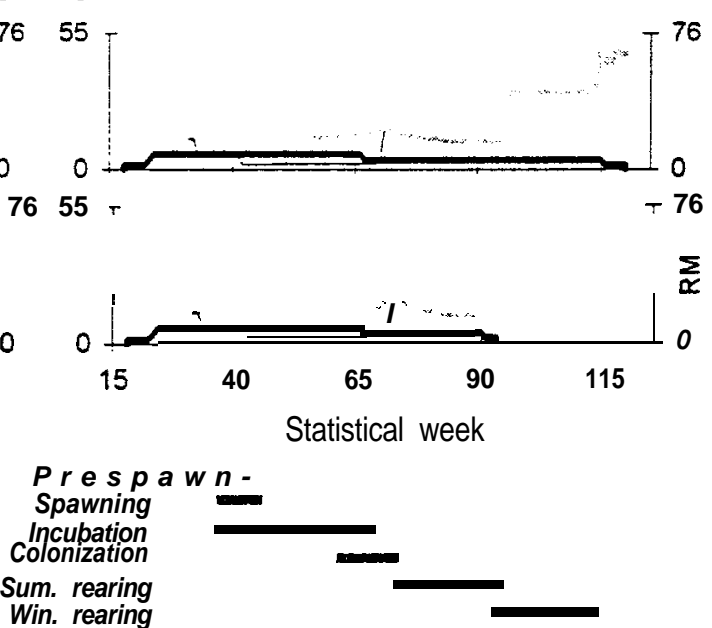

\section{Template}

Patient

Cum. Quum.

Prodi. Coap.

Traj. \#1

\begin{tabular}{l|l}
1 & 0.00 \\
0 & 0.00
\end{tabular}

Traj. \#2

\begin{tabular}{l|l}
1 & 0.00 \\
0 & 0.00
\end{tabular}

Traj. \#3

\begin{tabular}{l|l}
1 & 0.00 \\
0 & 0.00
\end{tabular}

Traj. \#4

\begin{tabular}{l|l|}
\hline 1 & 0.00 \\
0 & 0.00
\end{tabular}

Traj. \#5

\begin{tabular}{l|l}
1 & 0.00 \\
\hline & 0.00
\end{tabular}

o) 0.00

Traj. \#6

1) 0.00

0 0.00

Traj. \#7

\begin{tabular}{l|l}
1 & 0.00 \\
0 & 0.00
\end{tabular}

Traj. \#8

\begin{tabular}{l|l|}
\hline 1 & 0.00 \\
0 & 0.00
\end{tabular}

Traj. \#9

\begin{tabular}{l|l}
\hline 2 & 0.01 \\
0 & 0.00
\end{tabular} 
Spawning reach: Imnaha $\mathbf{R}$.

Horse $\mathrm{Cr}$ - Fence $\mathrm{Cr}$ (RM 10.8 - 16.3 from Snake R)

Relative Productivity
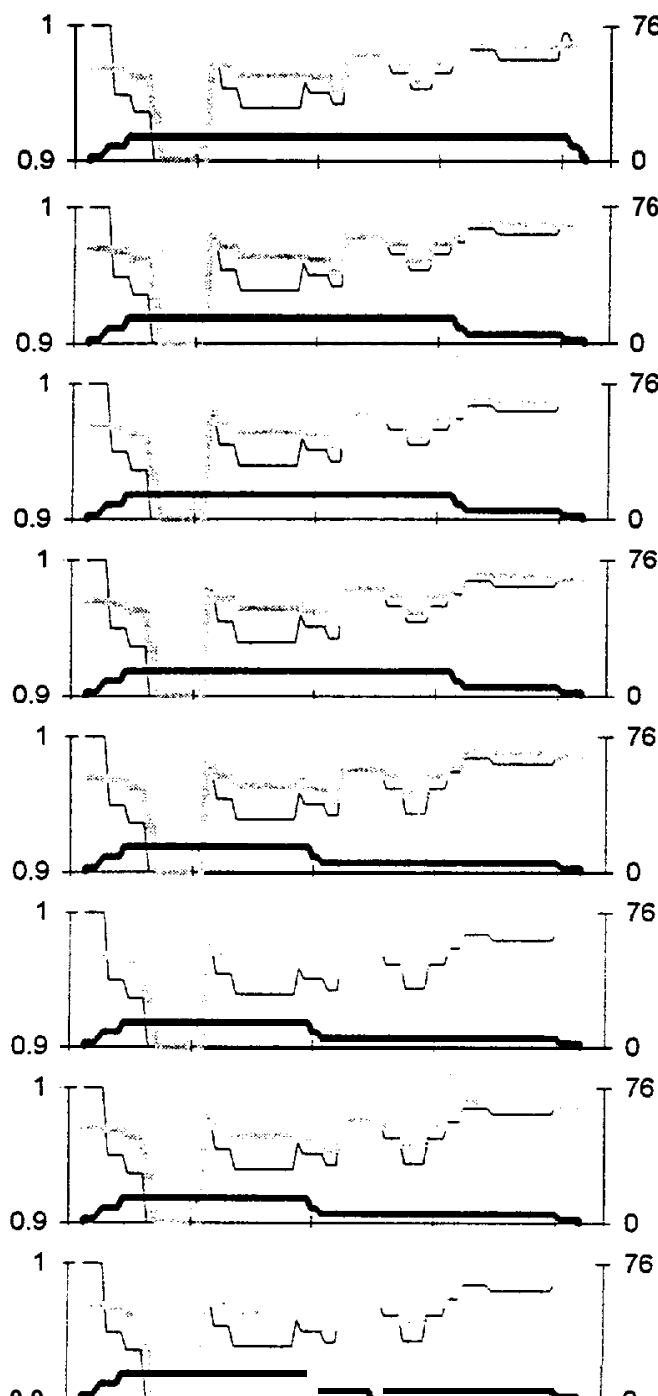

0.9

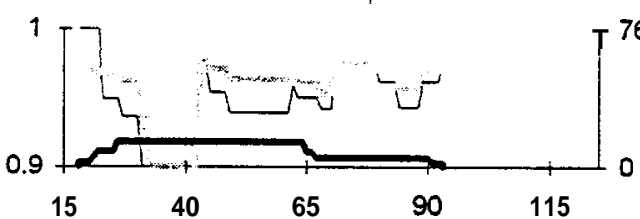

Statistical week

Prespawn -

Spawning Incubation Colonization Sum. rearing

Win. rearing
Relative Key Habitat Quantity
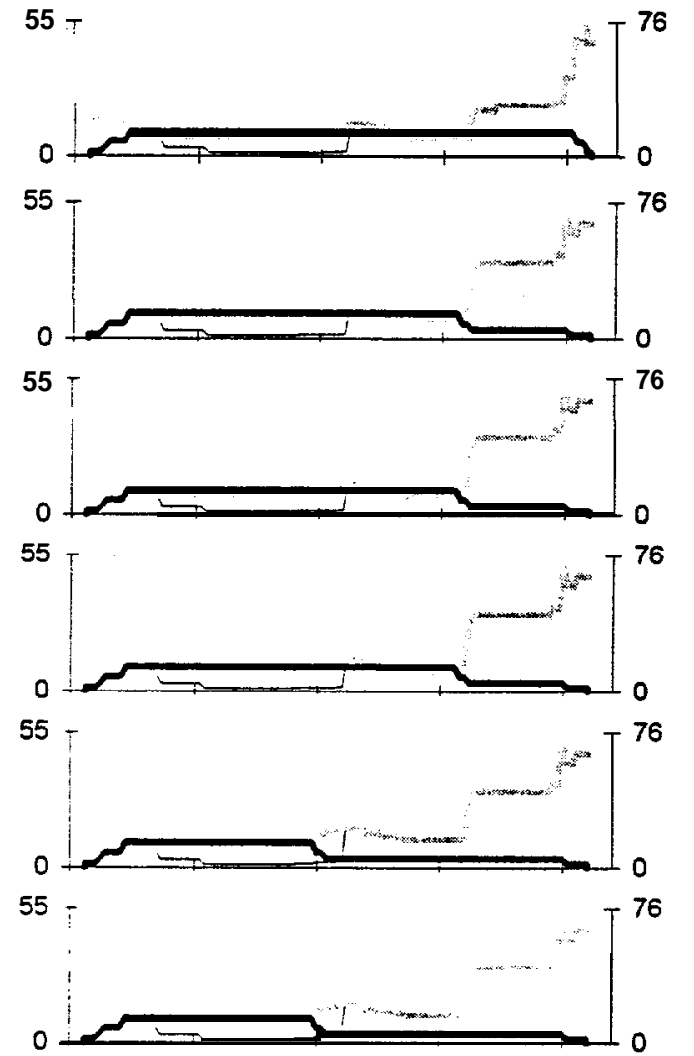

55 T
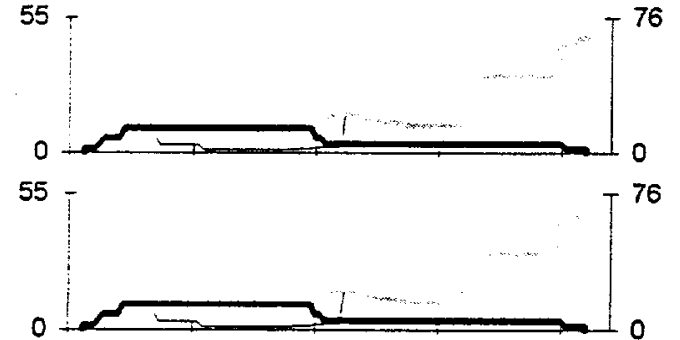

55 -

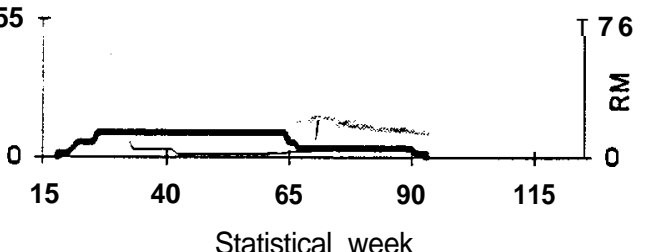

Prespawn Spawning

Incubation

Colonization

Sum. rearing

Win. rearing

Statistical week
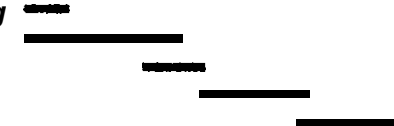

Template

Patient

Cum. Cum.

Prod. Cap.

Traj . \#1

\begin{tabular}{|l|l|}
\hline 1 & 0.00 \\
0 & 0.00
\end{tabular}

o) 0.00

Traj. \#2

10.00

Traj . \#3

10.00

0) 0.00

Traj. \#4

\begin{tabular}{l|l|}
1 & 0.00 \\
0 & 0.00
\end{tabular}

Traj. \#5

\begin{tabular}{l|l|}
\hline 1 & 0.00 \\
0 & 0.00
\end{tabular}

Traj. \#6

\begin{tabular}{l|l|}
\hline 1 & 0.00 \\
0 & 0.00 \\
\hline
\end{tabular}

Traj. \#7

\begin{tabular}{l|l}
1 & 0.00 \\
0 & 0.00
\end{tabular}

Traj. \#8

\begin{tabular}{l|l|}
\hline 1 & 0.00 \\
0 & 0.00 \\
\hline
\end{tabular}

Traj. \#9

\begin{tabular}{c|c} 
Traj. $\# 9$ \\
\hline 1.0 .00
\end{tabular}

이 0.00

Trajectory

Template

Patient 
Spawning reach: Imnaha $\mathbf{R}$.

Fence Cr - Big Sheep Cr (RM 16.3 - 22.3 from Snake R)

Relative Productivity
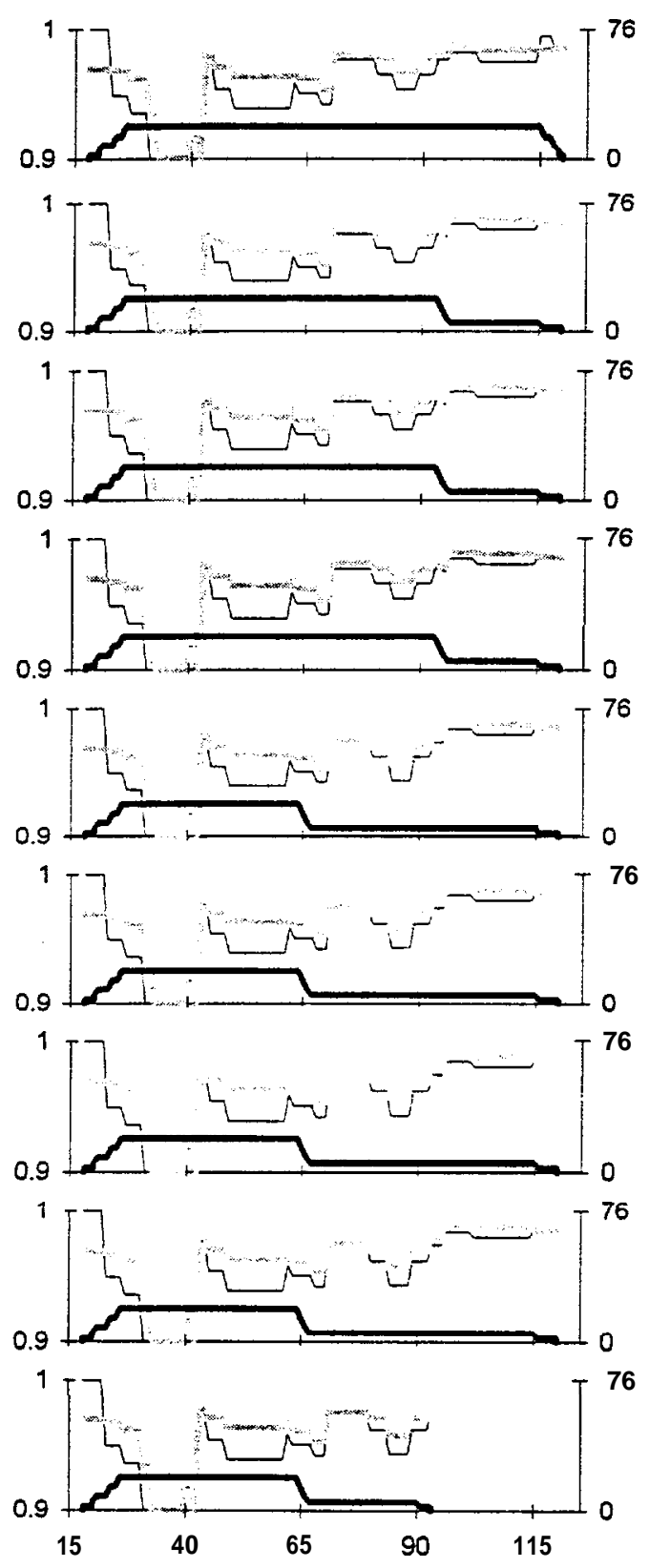

Statistical week

\section{Prespawn -}

Spawning

Incubation

Colonization

Sum. rearing

Win. rearing
Relative Key Habitat Quantity
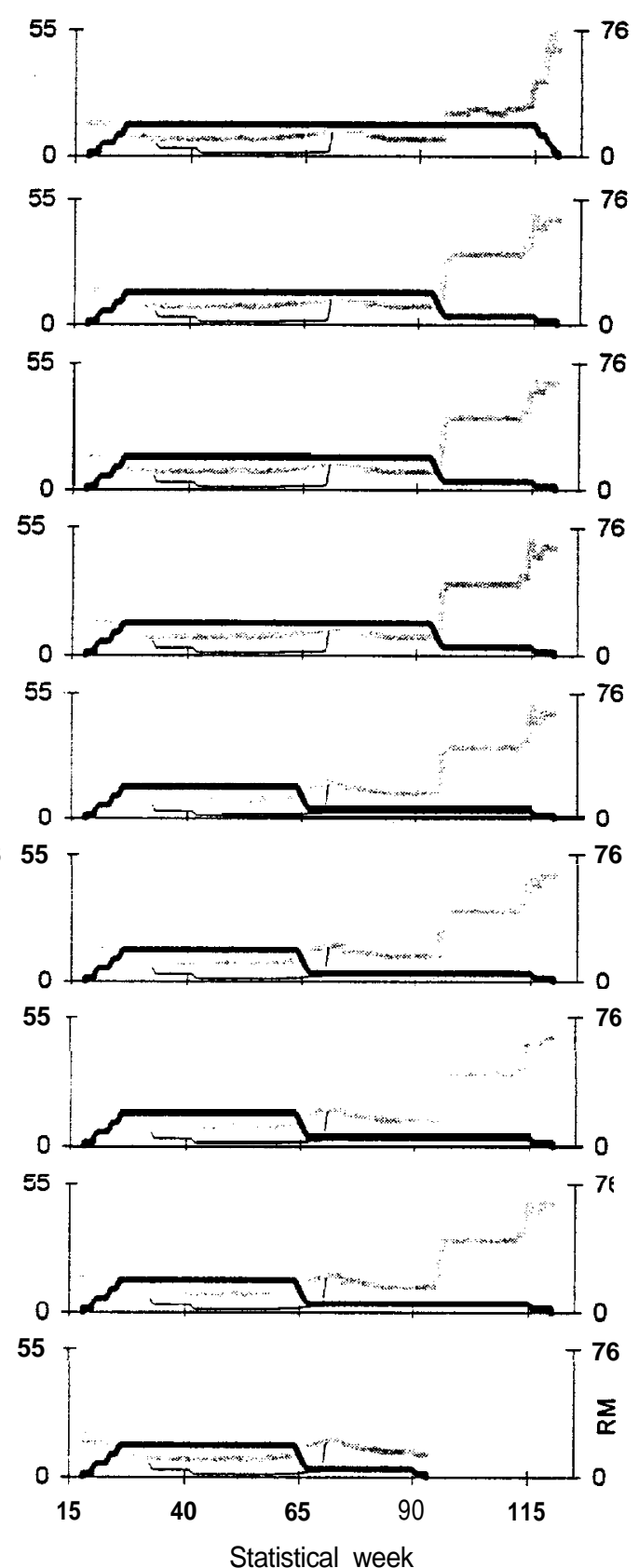

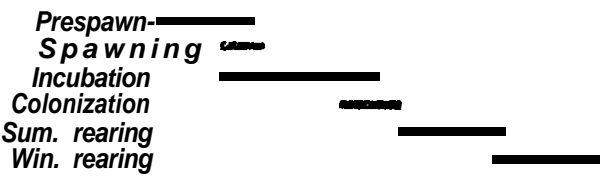

Trajectory]

Template

Patient 
Spawning reach: Imnaha $\mathbf{R}$.

Big Sheep Cr - Freezeout Cr (RM 22.3 - 35.2 from Snake R)

Relative Productivity
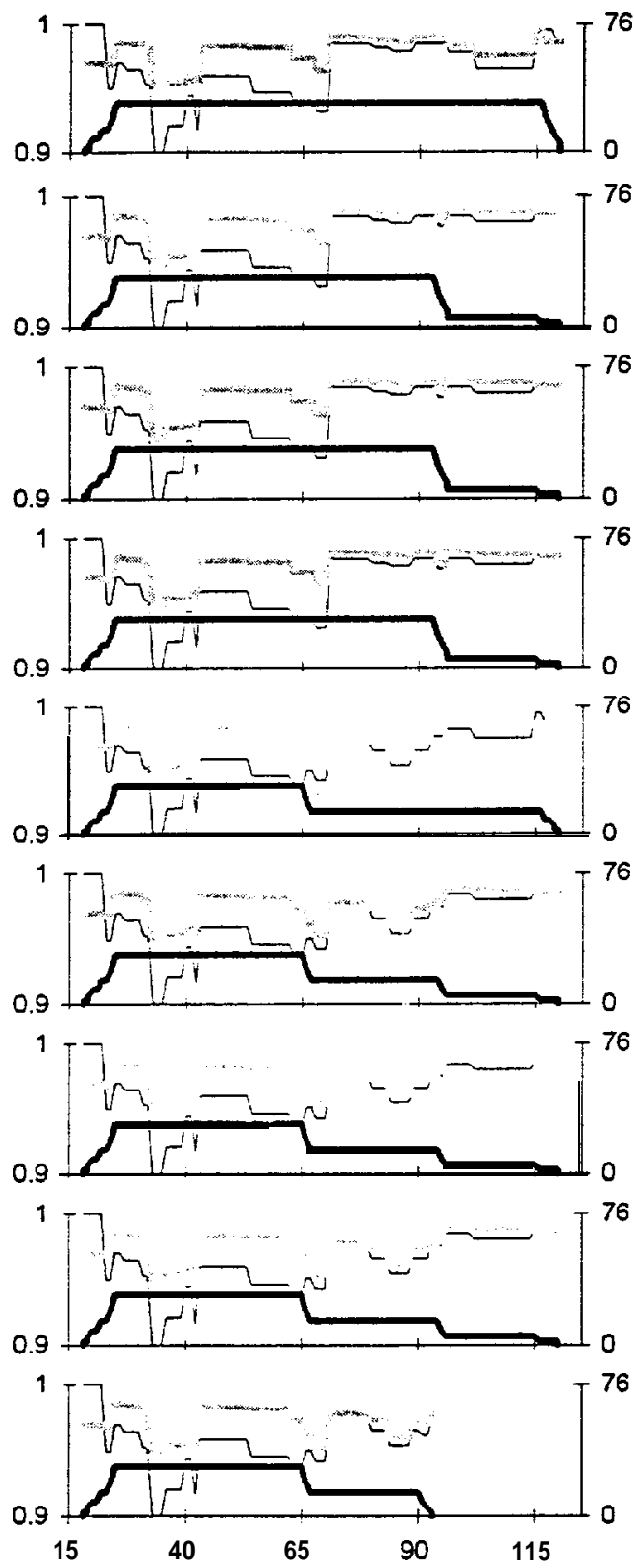

Statistical week

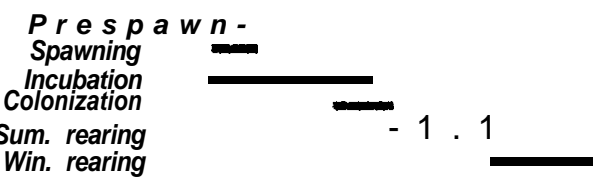

Prespawn -

Spawning

Win. rearing
Relative Key Habitat Quantity
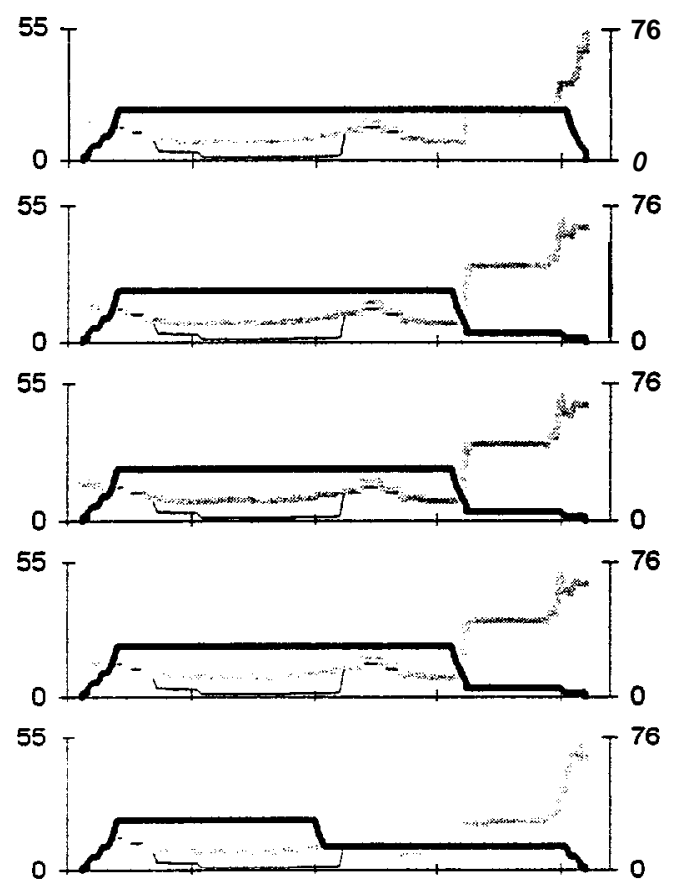

$55-$
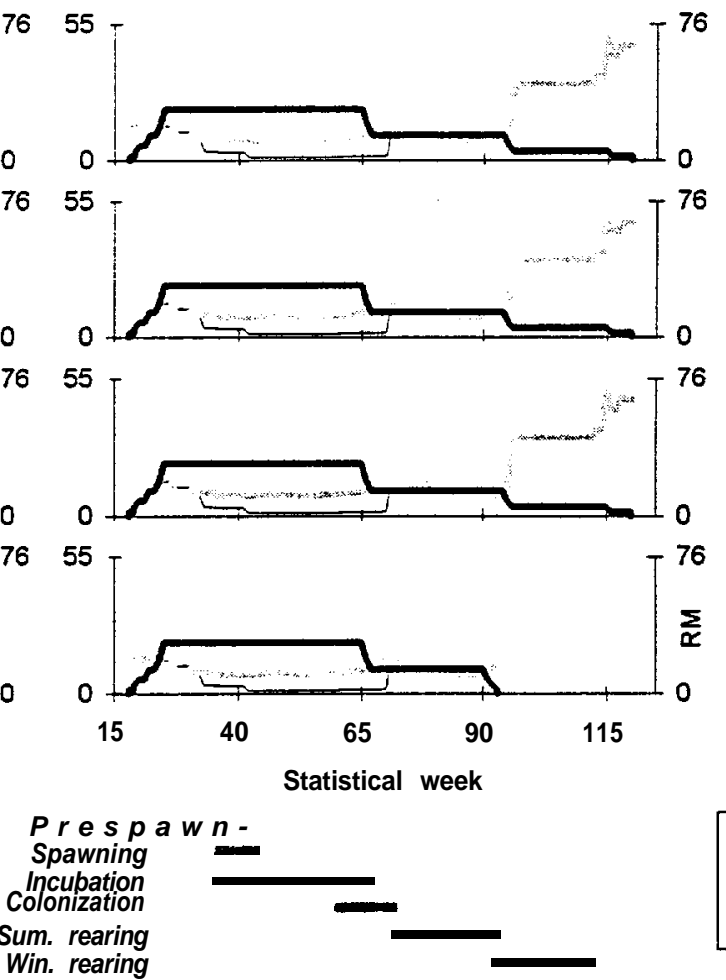

\begin{tabular}{|c|l|}
\hline $\begin{array}{c}\text { Template } \\
\text { Patient }\end{array}$ \\
\hline Cum. & Cum. \\
Prod. & Cap.
\end{tabular}

Traj. \#1

\begin{tabular}{l|l|}
\hline 85 & 0.20 \\
16 & 0.04
\end{tabular}

16) 0.04

Traj. \#2

$100 \quad 0.24$

19 0.04

Traj. \#3

$100 \quad 0.24$

$19 \quad 0.04$

Traj. \#4

\begin{tabular}{r|r|}
100 & 0.24 \\
19 & 0.04
\end{tabular}

Traj. \#5

\begin{tabular}{l|l}
\hline 61 & 0.15 \\
14 & 0.03
\end{tabular}

Traj. \#6

\begin{tabular}{l|l|}
\hline 65 & 0.16 \\
14 & 0.03
\end{tabular}

Traj. \#7

\begin{tabular}{l|l|}
\hline 65 & 0.16 \\
14 & 0.03
\end{tabular}

Traj. \#8

\begin{tabular}{l|l}
\hline 65 & 0.16 \\
\hline
\end{tabular}

$14 \quad 0.03$

Traj. \#9

\begin{tabular}{|l|l|}
\hline 129 & 0.32 \\
\hline & 0.07
\end{tabular}

33 0.07 
Spawning reach: Imnaha $\mathbf{R}$.

Freezeout $\mathrm{Cr}$ - Grouse $\mathrm{Cr}$ (RM 35.2 - 40.6 from Snake R)

Relative Productivity
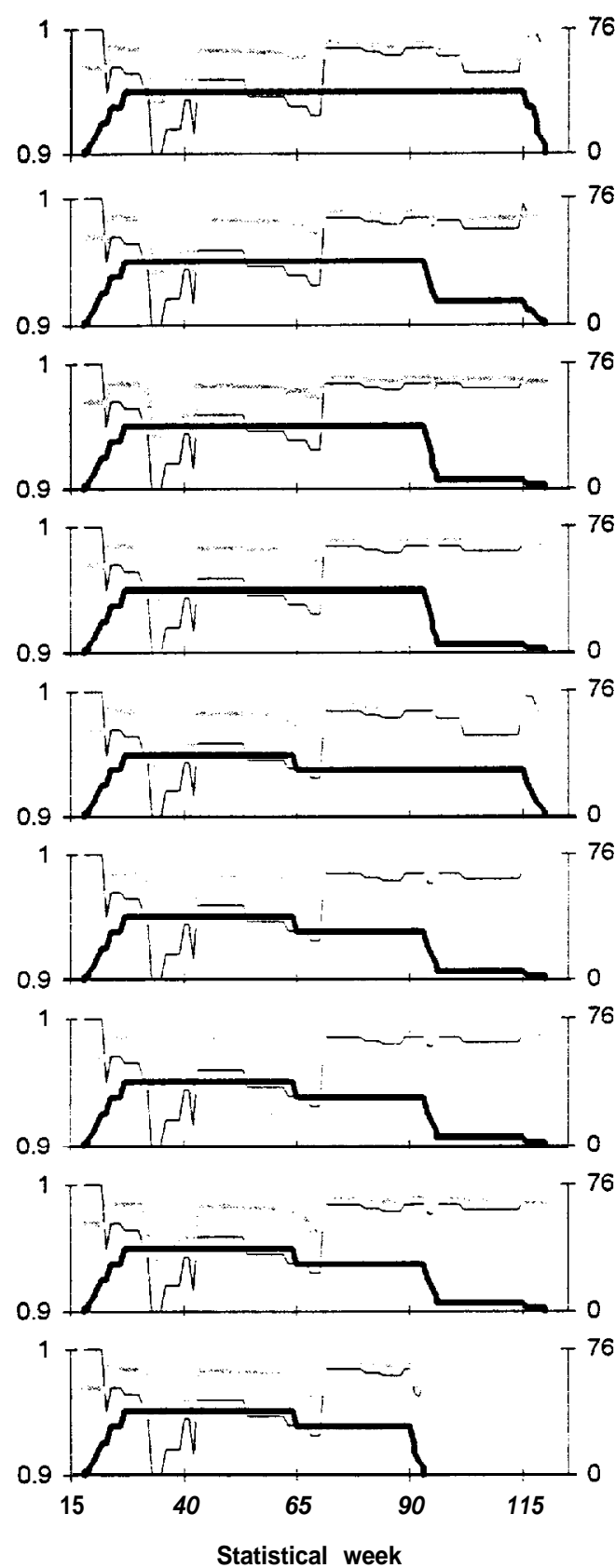

\section{Prespawn -}

Spawning

Incubation

Colonization

Sum. rearing

Win. rearing
Relative Key Habitat Quantity
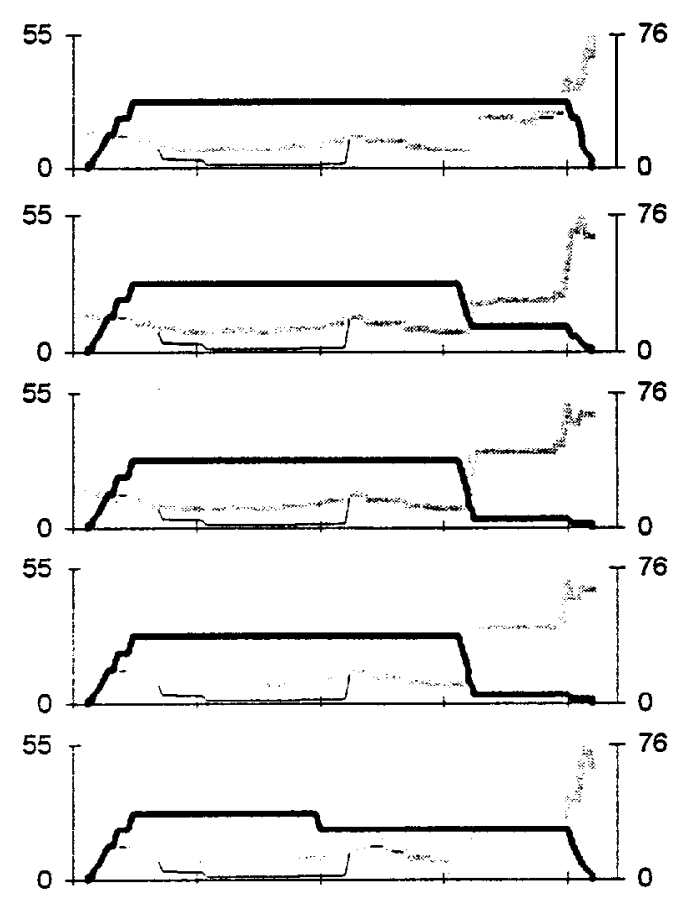

55
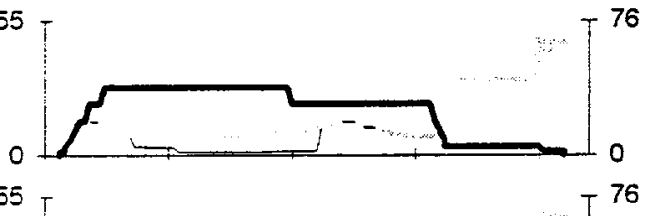

55
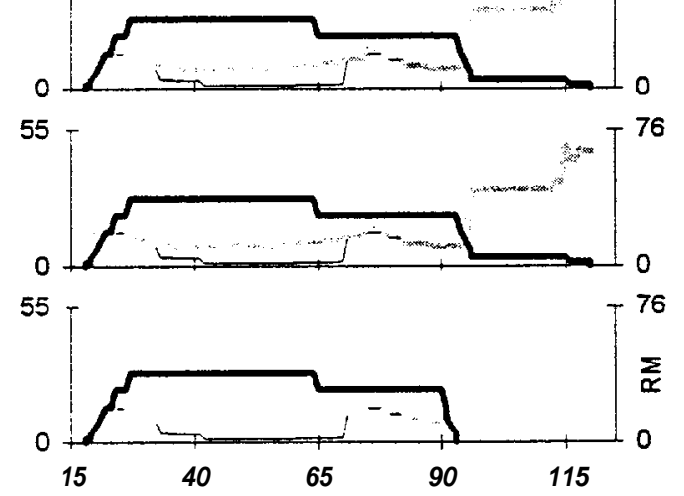

\section{Statistical week}

\begin{tabular}{|c|c|}
\hline \multicolumn{2}{|c|}{ Template } \\
Patient \\
\hline Cum. \\
Prod. & Cum. \\
\hline
\end{tabular}

Traj.\#1

92 0.21

16) 0.04

Traj. \#2

\begin{tabular}{|l|l|}
\hline 103 & 0.23 \\
\hline
\end{tabular}

18 0.04

Traj. \#3

1090.24

20) 0.04

Traj. \#4

1090.24

20) 0.04

Traj. \#5

$8 7 \longdiv { 0 . 1 9 }$

$16 \quad 0.04$

Traj. \#6

\begin{tabular}{l|l|}
103 & 0.23 \\
\hline
\end{tabular}

$19 \quad 0.04$

Traj. \#7

103 0.23

$19 \quad 0.04$

\begin{tabular}{|r|r|}
\multicolumn{2}{|c|}{ Traj. \#8 } \\
\hline 103 & 0.23 \\
19 & 0.04
\end{tabular}

Traj. \#9

\begin{tabular}{|l|l|}
198 & 0.47 \\
\hline
\end{tabular}

43) 0.09
Prespawn Spawning Incubation Colonization Sum. rearing Win. rearing
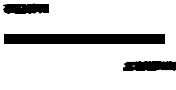

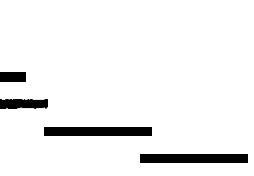


Spawning reach: Imnaha $\mathbf{R}$.

Grouse Cr - Summit Cr (RM 40.6 - 43.6 from Snake R)

Relative Productivity
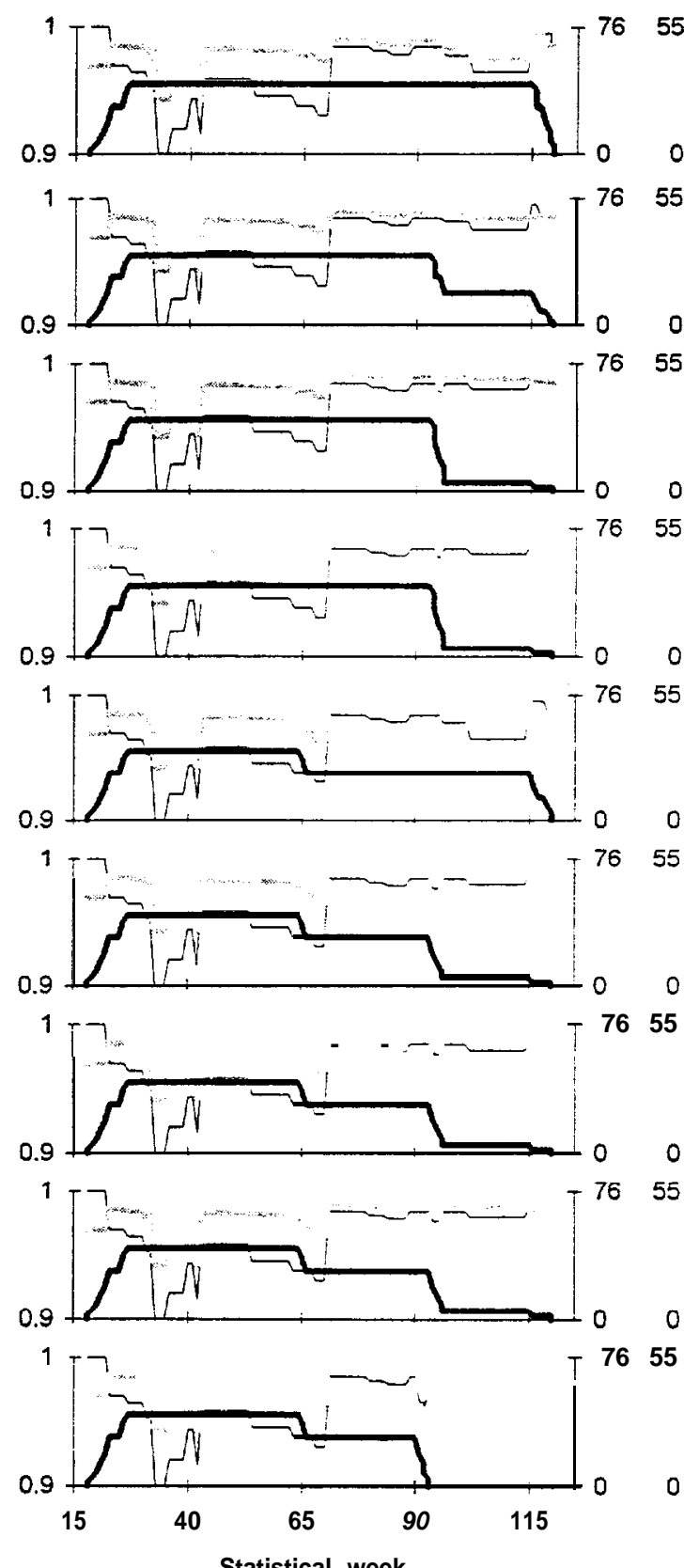

Statistical week

\section{Prespawn -}

Spawning

Incubation
Colonization

Sum. rearing

Win. rearing
Relative Key Habitat Quantity

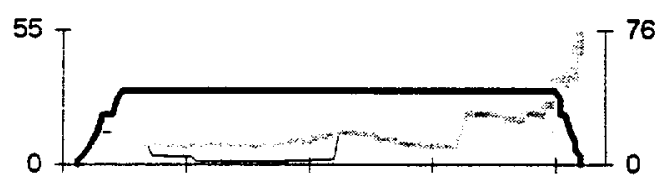

$55-$

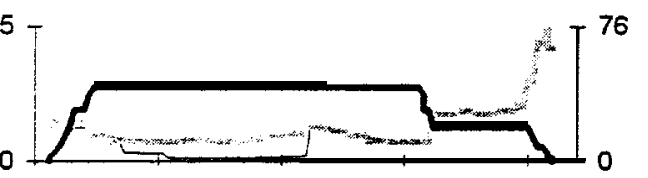

55 .

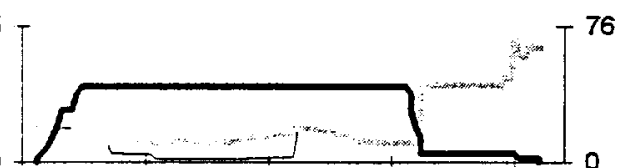

55
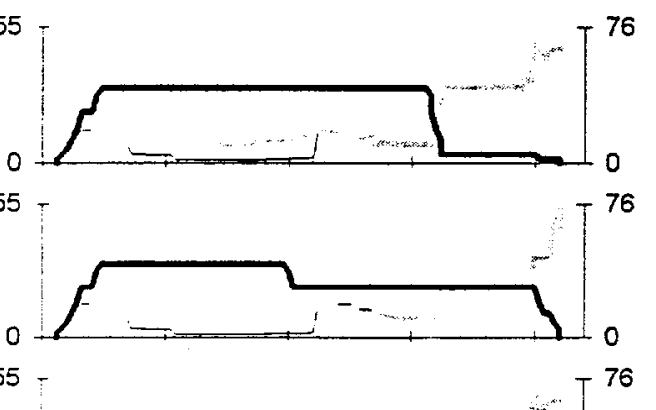

0
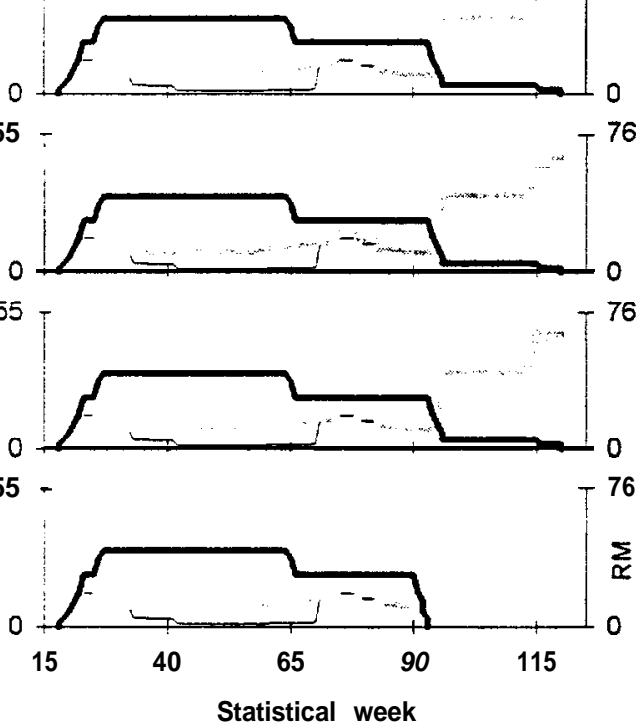

Statistical week

Prespawn -

Spawning

Incubation

Colonization

Sum. rearing

Win. rearing
Template

Patient

\begin{tabular}{l|l}
\hline Cum. & Cum. \\
\hline
\end{tabular}

Prod. Cap.

Traj .\#1

\begin{tabular}{l|l}
94 & 0.26
\end{tabular}

17) 0.04

Traj. \#2

\begin{tabular}{l|l}
\hline 105 & $0.2 ?$
\end{tabular}

190.04

Traj. \#3

\begin{tabular}{r|l}
\hline 111 & 0.24 \\
20 & 0.04
\end{tabular}

Traj. \#4

\begin{tabular}{l|l}
\hline 111 & 0.24 \\
\hline
\end{tabular}

20| 0.04

Traj. \#5

\begin{tabular}{l|l}
\hline 89 & 0.19 \\
\hline
\end{tabular}

17 0.04

Traj. \#6

$106 \quad 0.23$

20) 0.04

\begin{tabular}{r|r}
\multicolumn{2}{c}{ Traj . \#7 } \\
\hline 106 & 0.23 \\
20 & 0.04
\end{tabular}

Traj. \#8

\begin{tabular}{l|l|}
\hline 106 & 0.23 \\
\hline
\end{tabular}

20) 0.04

Traj. \#9

\begin{tabular}{l|l}
\hline 203 & 0.46
\end{tabular}

\begin{tabular}{l|l|}
44 & 0.10 \\
\hline
\end{tabular} 
Spawning reach: Imnaha $\mathbf{R}$.

Summit Cr - Crazyman Cr (RM 43.6 - 49.4 from Snake R)

Relative Productivity
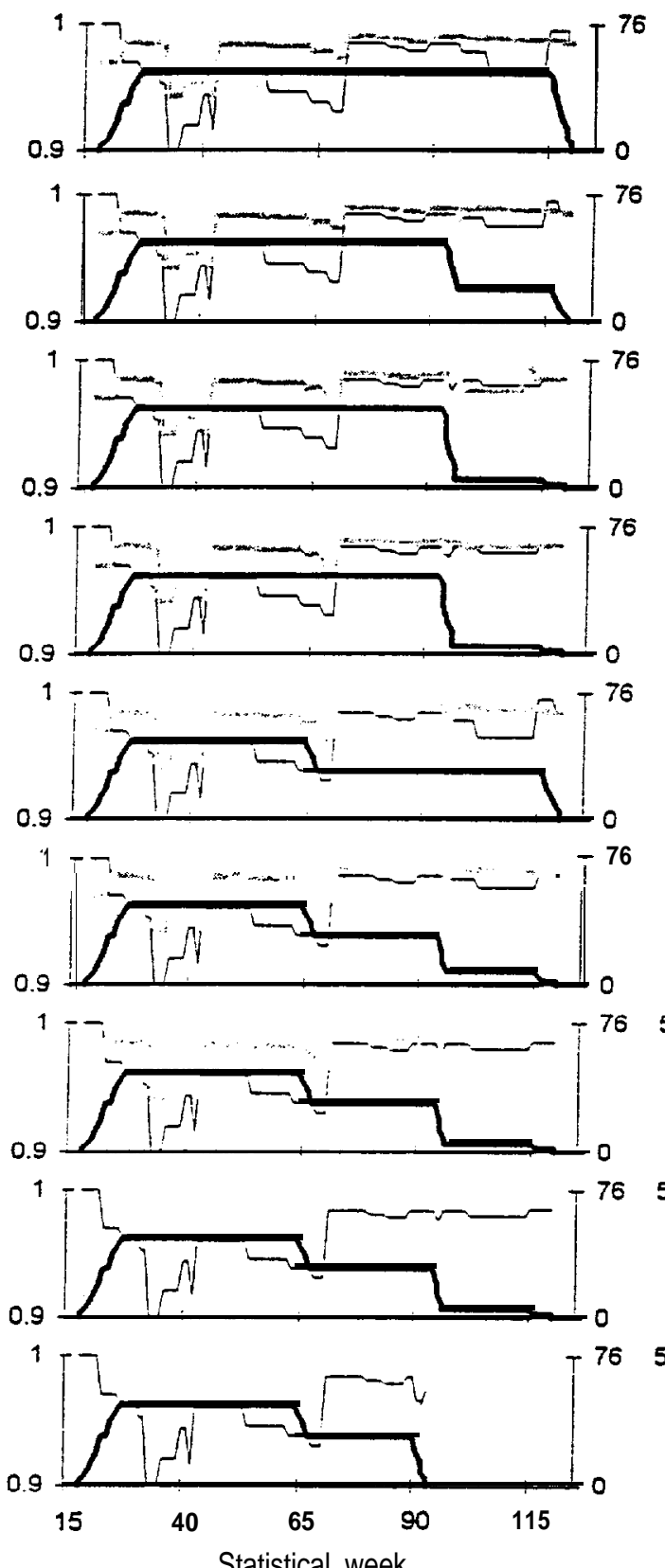

Prespawn -

Spawning

Incubation

Colonization

Sum. rearing

Win. rearing

Relative Key Habitat Quantity
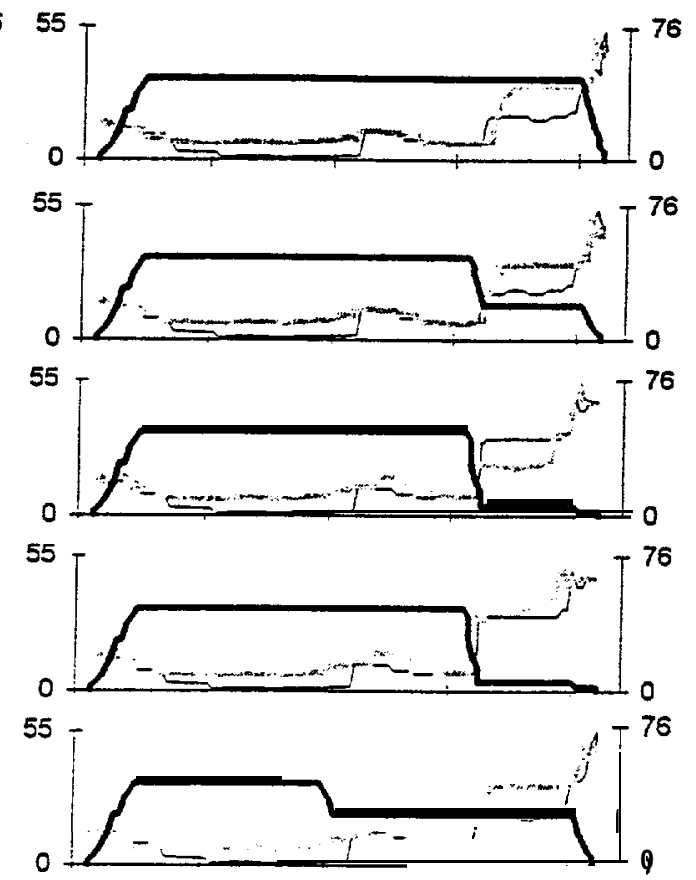

55
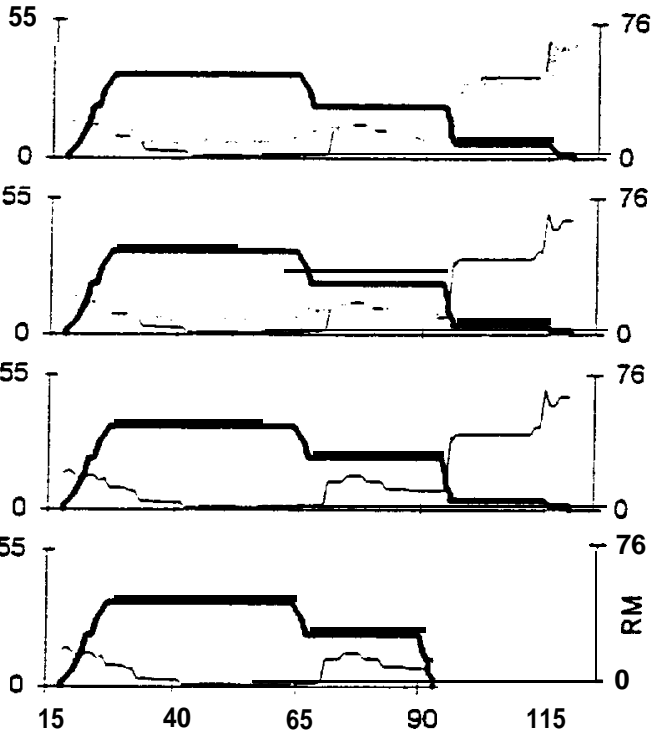

Statistical week

Prespawn -

Spawning

incubation

Colonization

Sum. rearing

Win. rearing \begin{tabular}{|c|c|}
\hline \multicolumn{2}{|c|}{$\begin{array}{c}\text { Template } \\
\text { Patient }\end{array}$} \\
\hline $\begin{array}{c}\text { Cum. } \\
\text { Prod. }\end{array}$ & Cum. \\
\hline
\end{tabular}

Traj. \#1

\begin{tabular}{l|l|}
\hline 94 & 0.19 \\
17 & 0.03
\end{tabular}

Traj. \#2

\begin{tabular}{r|r|}
105 & 0.21 \\
19 & 0.04
\end{tabular}

Traj. \#3

$111 \quad 0.2: 3$

$20 \quad-0.04$

Traj. \#4

\begin{tabular}{l|l}
\hline 111 & 0.23 \\
\hline
\end{tabular}

20) 0.04

Traj. \#5

\begin{tabular}{l|l|}
\hline 90 & 0.19 \\
\hline
\end{tabular}

17) 0.03

Traj. \#6

\begin{tabular}{l|l}
\hline 101 & 0.21 \\
\hline
\end{tabular}

19) 0.04

Traj. \#7

\begin{tabular}{ll}
\hline 107 & 0.23 \\
\hline
\end{tabular}

20) 0.04

Trai. \#8

\begin{tabular}{l|l}
\hline 107 & 0.23 \\
\hline
\end{tabular}

20) 0.04

Traj. \#9

$204 \quad 0.45$

$44 \quad 0.09$ 
Spawning reach: Imnaha $\mathbf{R}$.

Crazyman Cr - Gumboot Cr (RM 49.4 - 52.7 from Snake R)

Relative Productivity
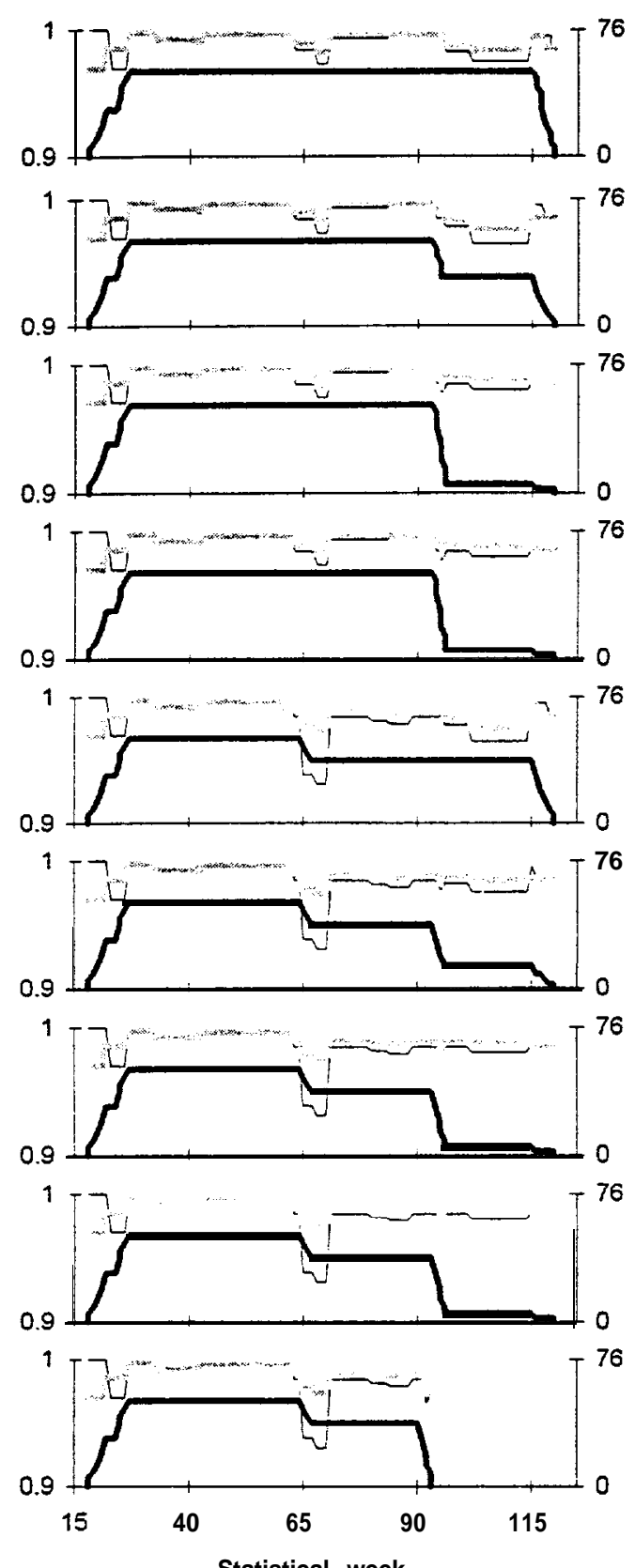

Statistical week

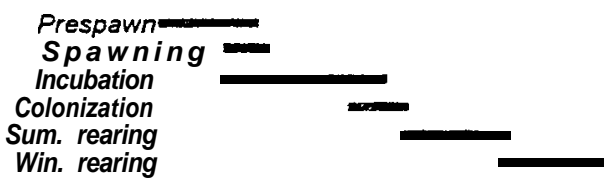

Relative Key Habitat Quantity
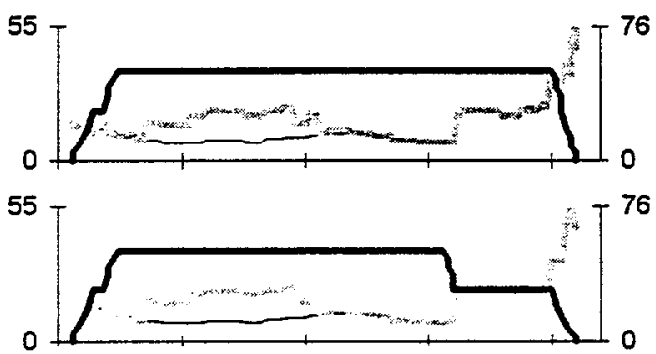

$55+T^{76}$

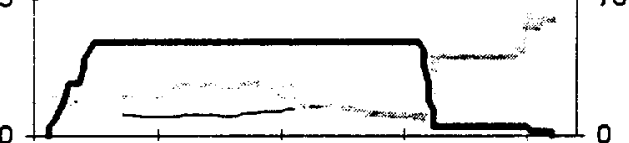

55

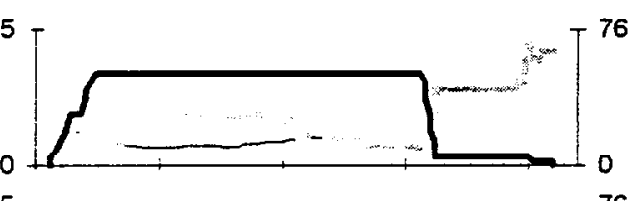

55

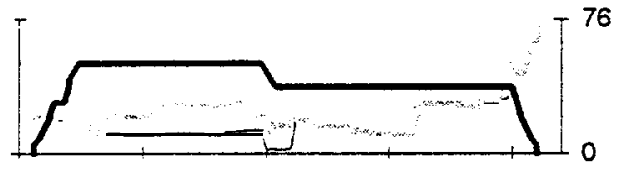

55

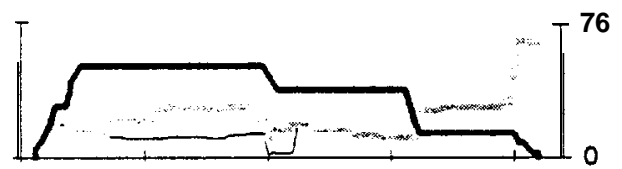

55
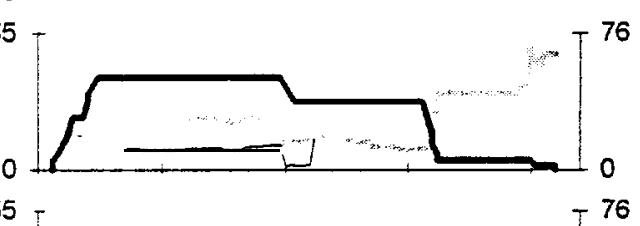

55
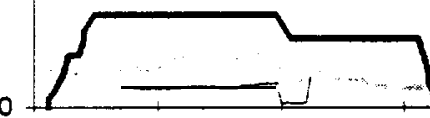

55

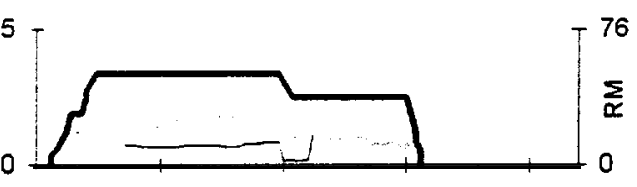

Statistical week

\begin{tabular}{l}
\hline Template \\
Patient \\
\hline Cumm. \\
Proud. \\
\hline
\end{tabular}

Traj. \#1

$323 \quad 0.51$

$2 6 8 \longdiv { 0 . 3 9 }$

Traj. \#2

$271 \quad 0.43$

$219 \quad 0.32$

Traj. \#3

$329 \quad 0.53$

$270 \quad 0.40$

Traj. \#4

$329 \quad 0.53$

$270 \quad 0.40$

Traj. \#5

\begin{tabular}{|l|l|}
216 & 0.36 \\
\hline
\end{tabular}

130 0.18

Traj. \#6

\begin{tabular}{l|l|}
\hline 242 & 0.41 \\
\hline
\end{tabular}

14890020

Traj. \#7

\begin{tabular}{l|l|}
\hline 258 & 0.44 \\
\hline
\end{tabular}

$158 \quad 0.22$

Traj. \#8

\begin{tabular}{l|l|}
258 & 0.44 \\
\hline
\end{tabular}

$158 \quad 0.22$

Traj. \#9

\begin{tabular}{|l|l|}
\hline 498 & 0.92 \\
\hline
\end{tabular}

$350 \quad 0.50$

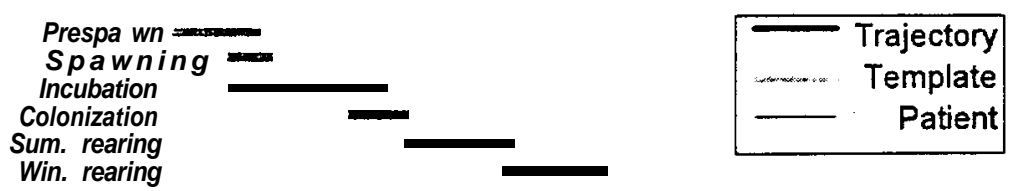


Spawning reach: Imnaha $\mathbf{R}$.

Gumboot Cr - Dry Cr (RM 52.7 - 55.4 from Snake R)

Relative Productivity
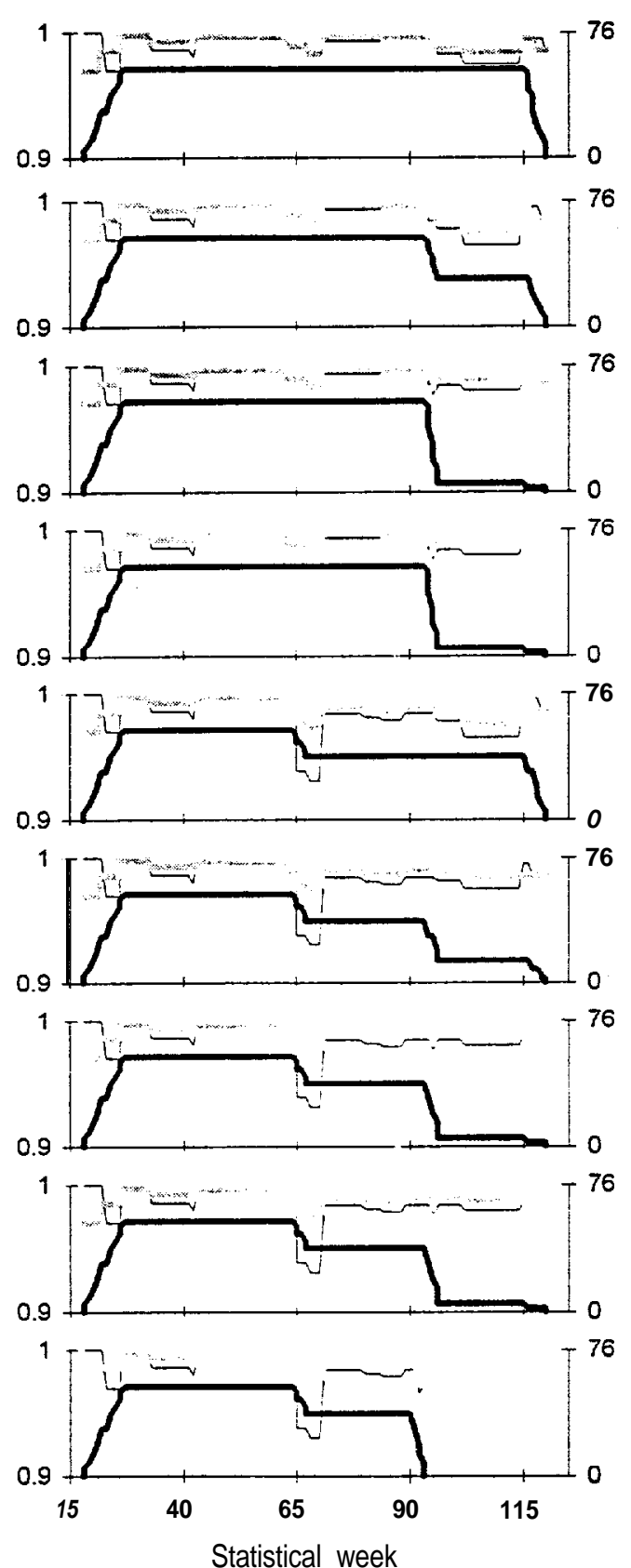

Prespa w n -

Spawning

Colonization

Colonization

Win. rearing
Relative Key Habitat Quantity
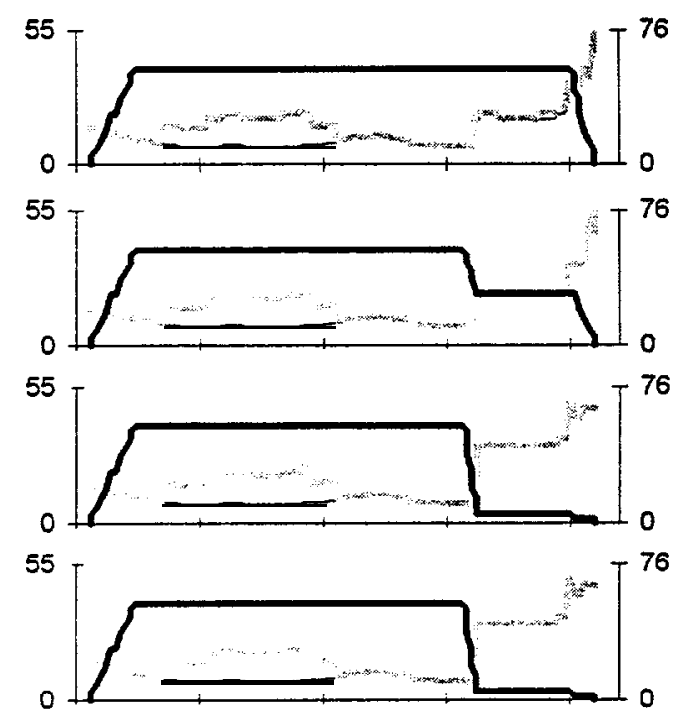

$55-$
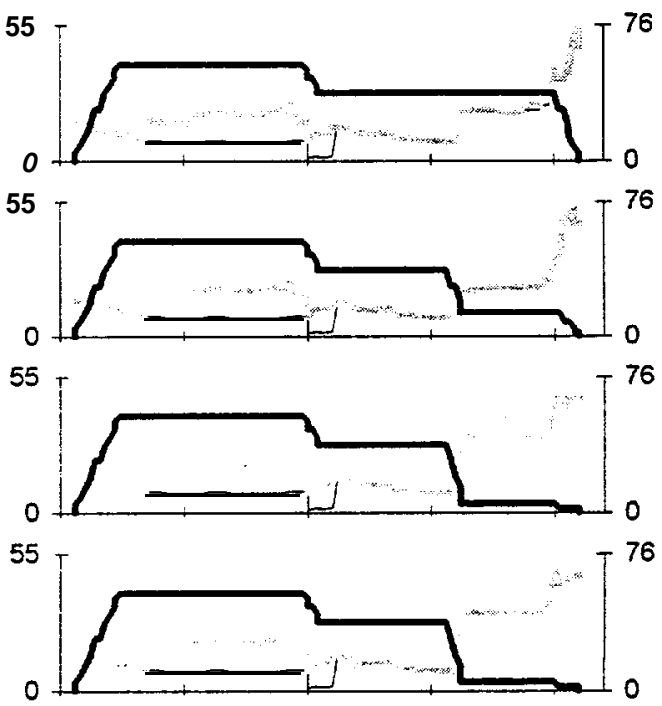

$55+$

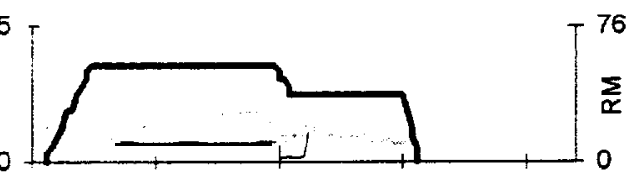

Statistical week

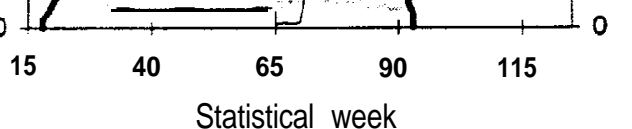

\begin{tabular}{|c|c}
\hline \multicolumn{2}{|c|}{ Template } \\
Patient \\
\hline Cum. & Cum. \\
Prod. & Cap. \\
Traj. \#1 \\
\hline 324 & $0.5 C$ \\
269 & 0.35 \\
\hline
\end{tabular}

Traj. \#2

$275 \quad 0.43$

$222 \quad 0.32$

Traj. \#3

\begin{tabular}{l|l|}
\hline 330 & 0.52 \\
\hline
\end{tabular}

$2700.4 C$

Traj. \#4

\begin{tabular}{l|l}
\hline 330 & 0.52 \\
\hline
\end{tabular}

270 0.40

Traj. \#5

\begin{tabular}{l|l|}
\hline 220 & 0.37 \\
\hline
\end{tabular}

127) 0.18

Traj. \#6

\begin{tabular}{l|l}
\hline 247 & 0.41 \\
\hline
\end{tabular}

147 $0.2 \mathrm{C}$

Traj . \#7

\begin{tabular}{l|l}
\hline 260 & 0.44 \\
\hline
\end{tabular}

\begin{tabular}{l|l}
154 & 0.21
\end{tabular}

\begin{tabular}{c|c}
\multicolumn{2}{c|}{ Traj . \#8 } \\
\hline 260 & 0.44 \\
154 & 0.21
\end{tabular}

Traj. \#9

\begin{tabular}{l|l}
\hline 503 & 0.91 \\
342 & 0.50
\end{tabular}

3420.50

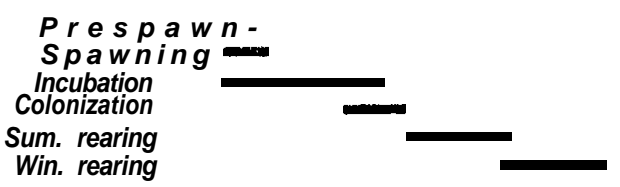


Spawning reach: Imnaha $\mathbf{R}$.

Dry Cr - Skookum Cr (RM 55.4 - 61.9 from Snake R)

Relative Productivity
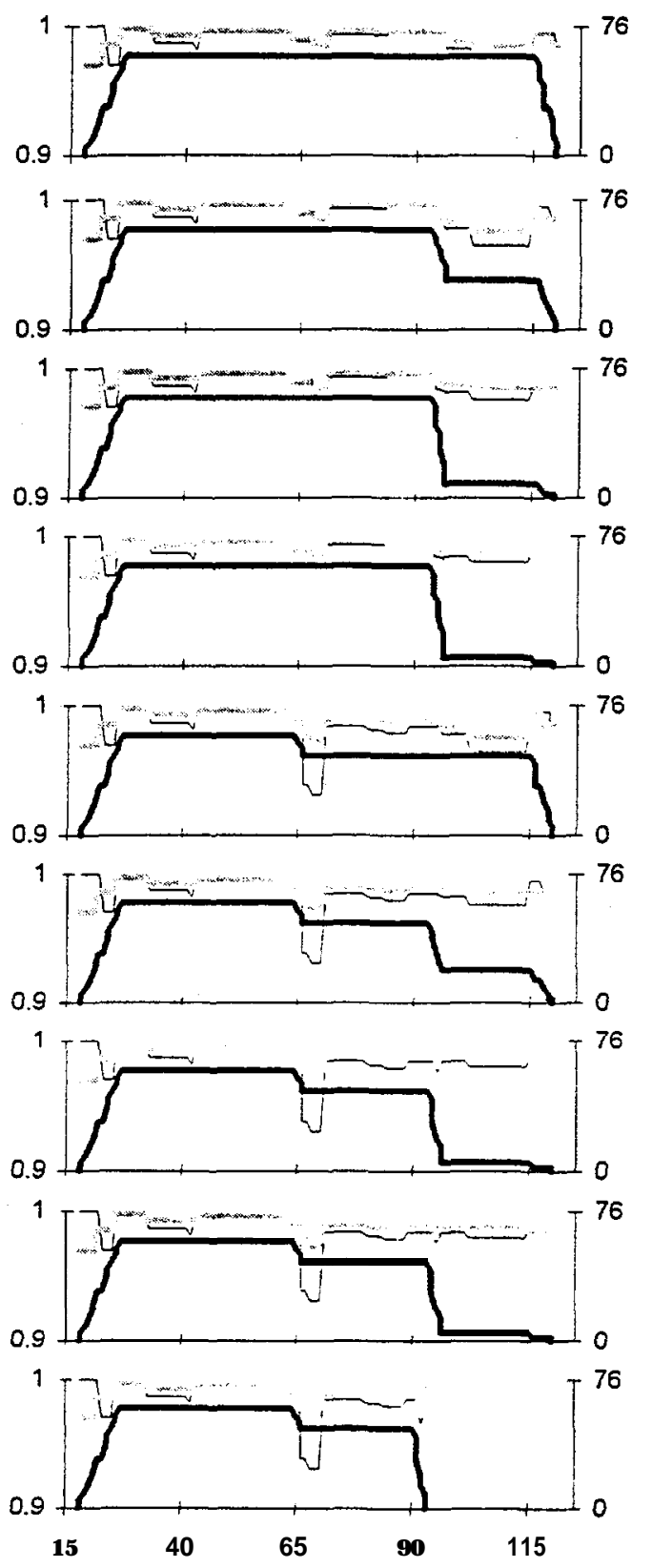

Statistical week

\section{Prespawn -}

Spawning

Incubation
Colonization

Sum. rearing

Win. rearing
Relative Key Habitat Quantity
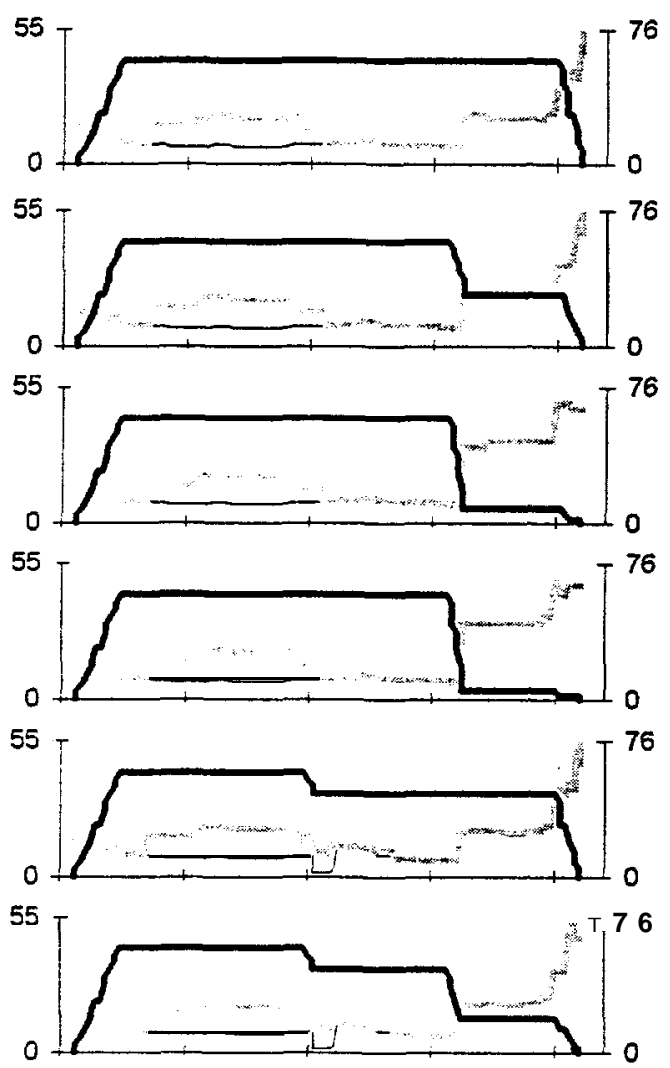

55
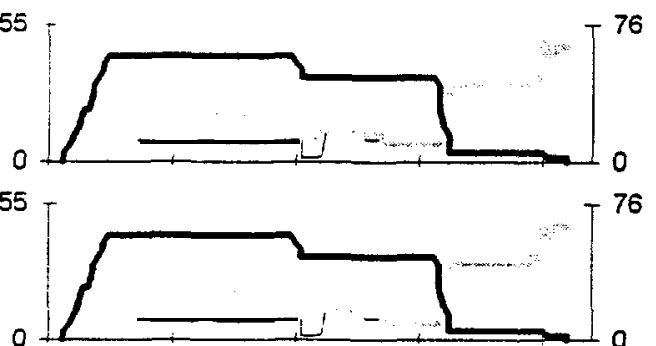

55 .

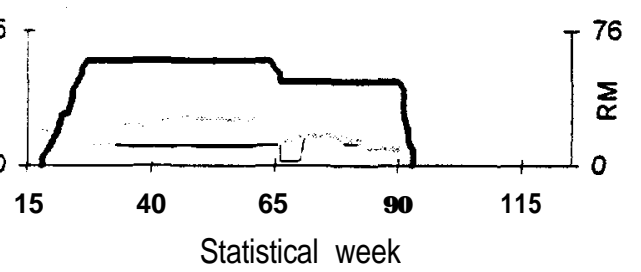

Statistical week

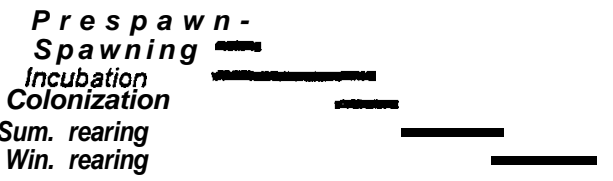

\begin{tabular}{|r|c|}
\hline \multicolumn{2}{|c|}{ Template } \\
Patient \\
\hline $\begin{array}{r}\text { Cum. } \\
\text { Prod. }\end{array}$ & Cam. \\
Traj. $\# 1$ \\
\hline 330 & 0.48 \\
288 & 0.38 \\
\hline
\end{tabular}

Traj. \#2

\begin{tabular}{l|l}
\hline 279 & 0.41 \\
\hline
\end{tabular}

2390.32

Traj. \#3

\begin{tabular}{|l|l|}
314 & 0.47 \\
\hline
\end{tabular}

270 0.37

Traj. \#4

$334 \quad 0.50$

$291 \quad 0.40$

Traj. \#5

\begin{tabular}{l|l|}
224 & 0.35 \\
\hline
\end{tabular}

143 0.18

Traj. \#6

\begin{tabular}{l|l|}
251 & 0.39 \\
\hline
\end{tabular}

167) 0.21

Traj. \#7

\begin{tabular}{l|l}
\hline 265 & 0.42 \\
\hline
\end{tabular}

173 0.22

Traj. \#8

\begin{tabular}{l|l|}
265 & 0.42 \\
\hline
\end{tabular}

173 0.22

Traj. \#9

\begin{tabular}{l|l|}
\hline 513 & 0.87 \\
\hline 384 & 0.51
\end{tabular}

\begin{tabular}{l|l}
384 & 0.51
\end{tabular} 
Spawning reach: Imnaha $\mathbf{R}$.

Skookum Cr - Blue Hole (RM 61.9 - 66.4 from Snake R)

Relative Productivity
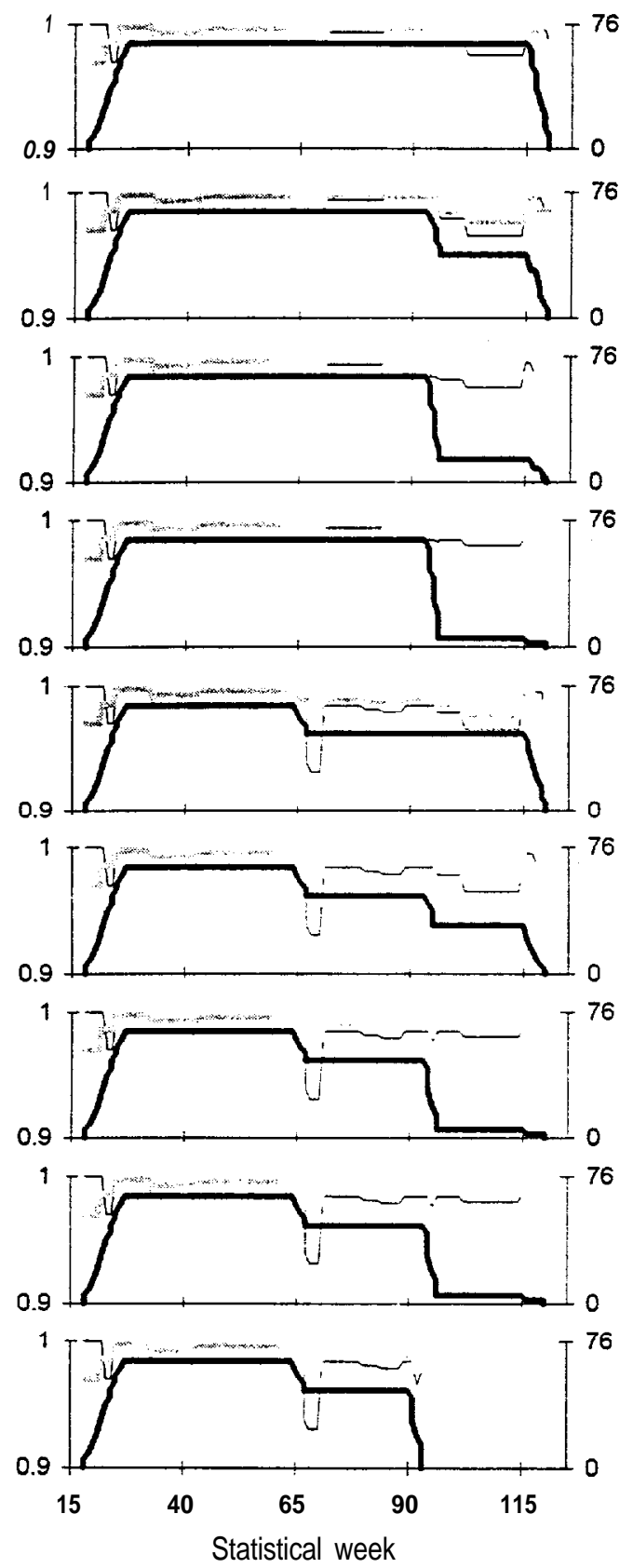

Prespa w n -

Spawning Incubation
Colonization

Sum. rearing

Win. rearing
Relative Key Habitat Quantity
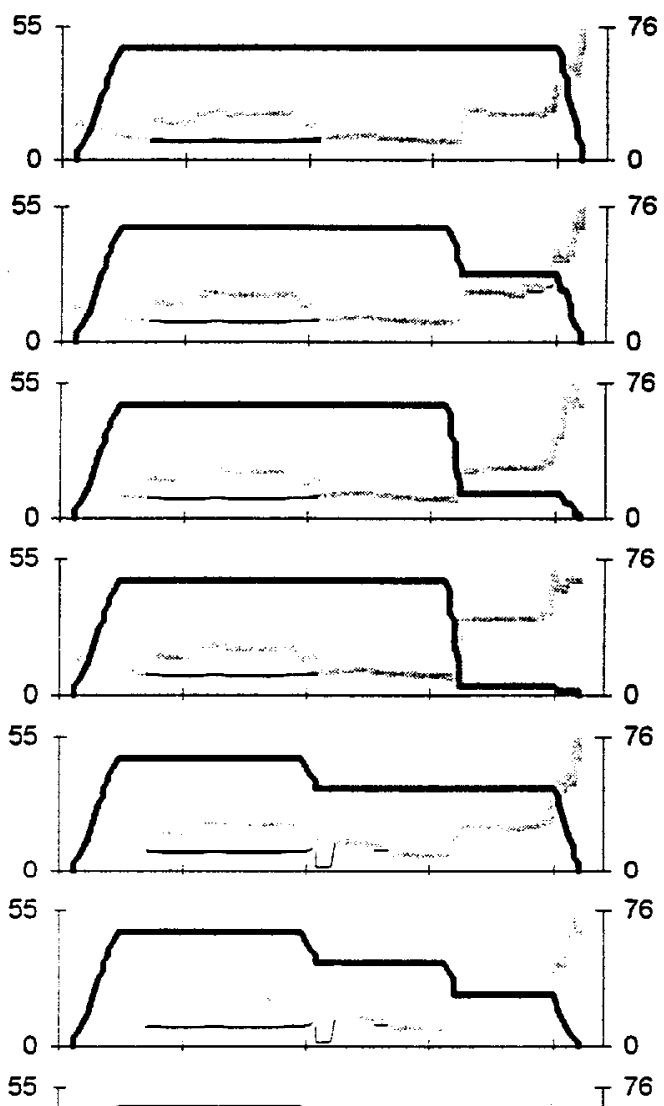

55

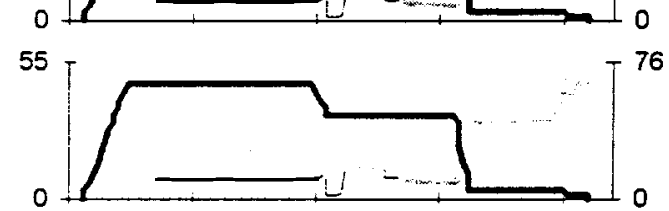

55

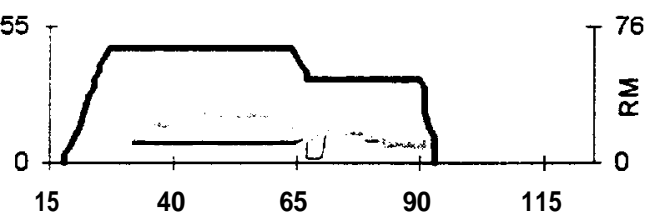

Statistical week

\begin{tabular}{|c|c|}
\hline \multicolumn{2}{|c|}{ Template } \\
Patient \\
\hline Cum. & Cum. \\
Prod. & Cap. \\
\hline
\end{tabular}

Traj. \#1

3330.47

288 0.38

Traj. \#2

2850.41

241 0.32

Traj. \#3

\begin{tabular}{l|l|}
\hline 17 & 0.45 \\
\hline
\end{tabular}

277 0.37

Traj. \#4

338 0.49

$\begin{array}{ll}292 & 0.39\end{array}$

Traj. \#5

2290.34

$\begin{array}{ll}152 & 0.18\end{array}$

Traj. \#6

\begin{tabular}{l|l}
\hline 225 & 0.34 \\
\hline
\end{tabular}

1490.18

Traj. \#7

\begin{tabular}{l|l}
271 & 0.41 \\
\hline
\end{tabular}

183 0.22

Traj. \#8

\begin{tabular}{l|l}
271 & 0.41 \\
\hline
\end{tabular}

$183 \quad 0.22$

Traj. \#9

\begin{tabular}{|l|l|}
\hline 524 & 0.86 \\
\hline
\end{tabular}

407) 0.52

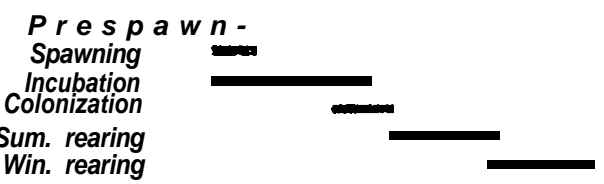

Trajectory

Template

Patient 
Spawning reach: Imnaha $\mathbf{R}$.

Blue Hole - forks (RM 66.4 - 71.4 from Snake R)

Relative Productivity
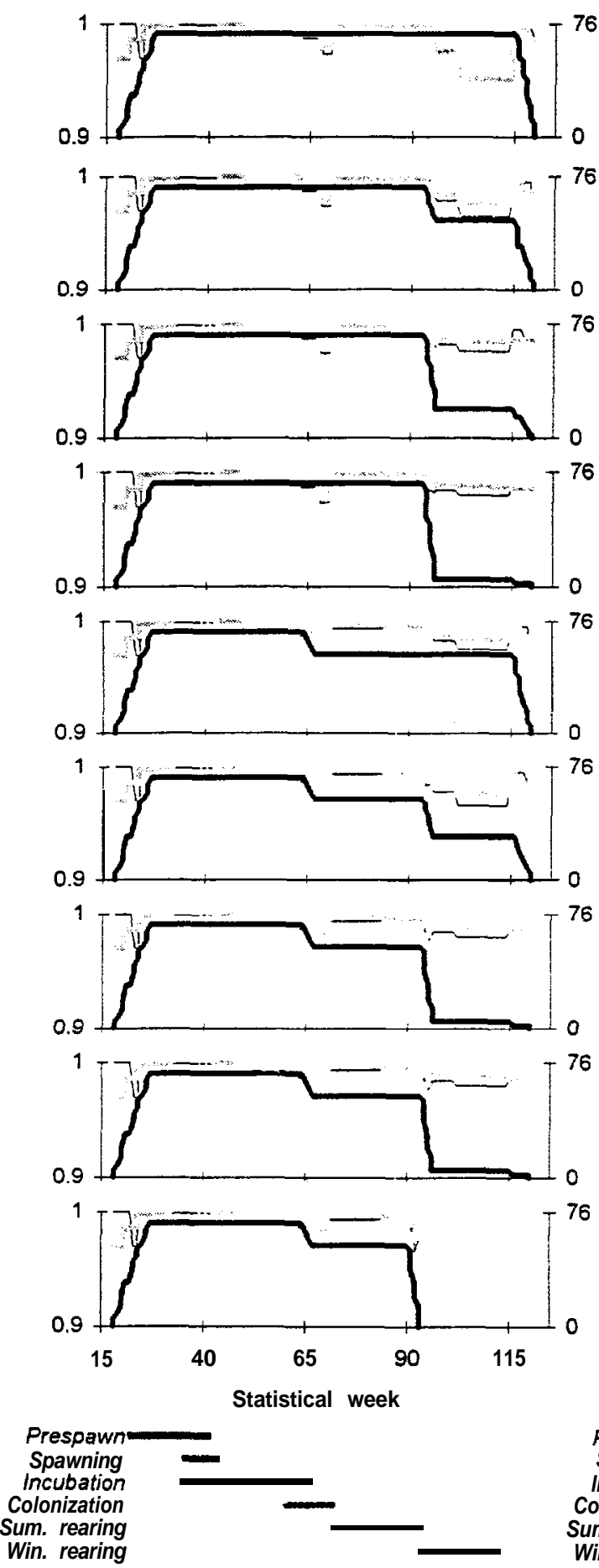

Relative Key Habitat Quantity
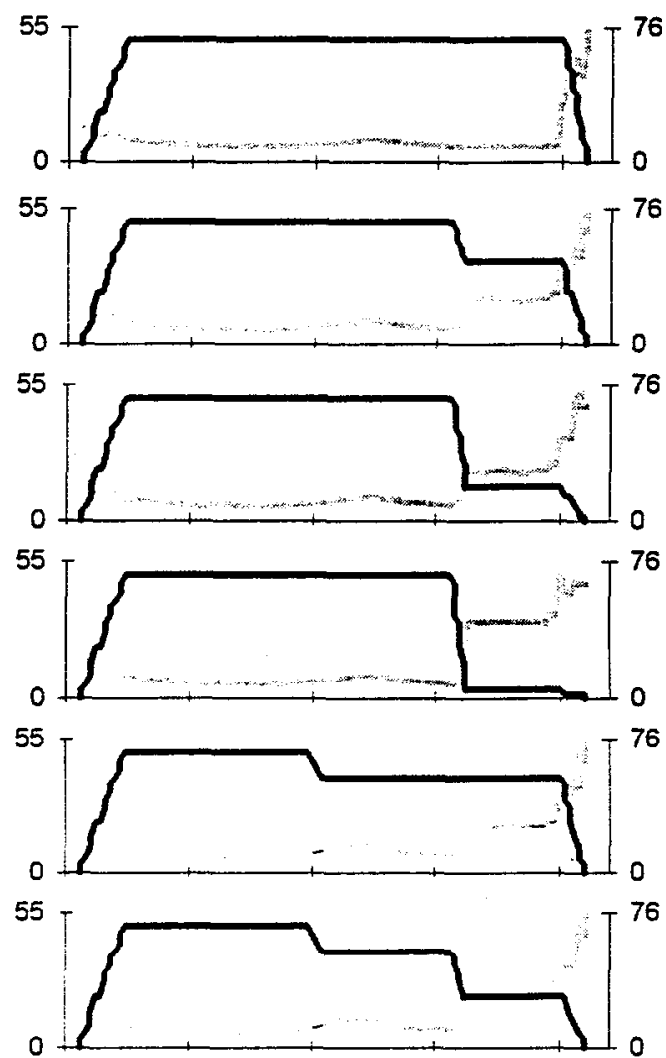

$76 \quad 55$
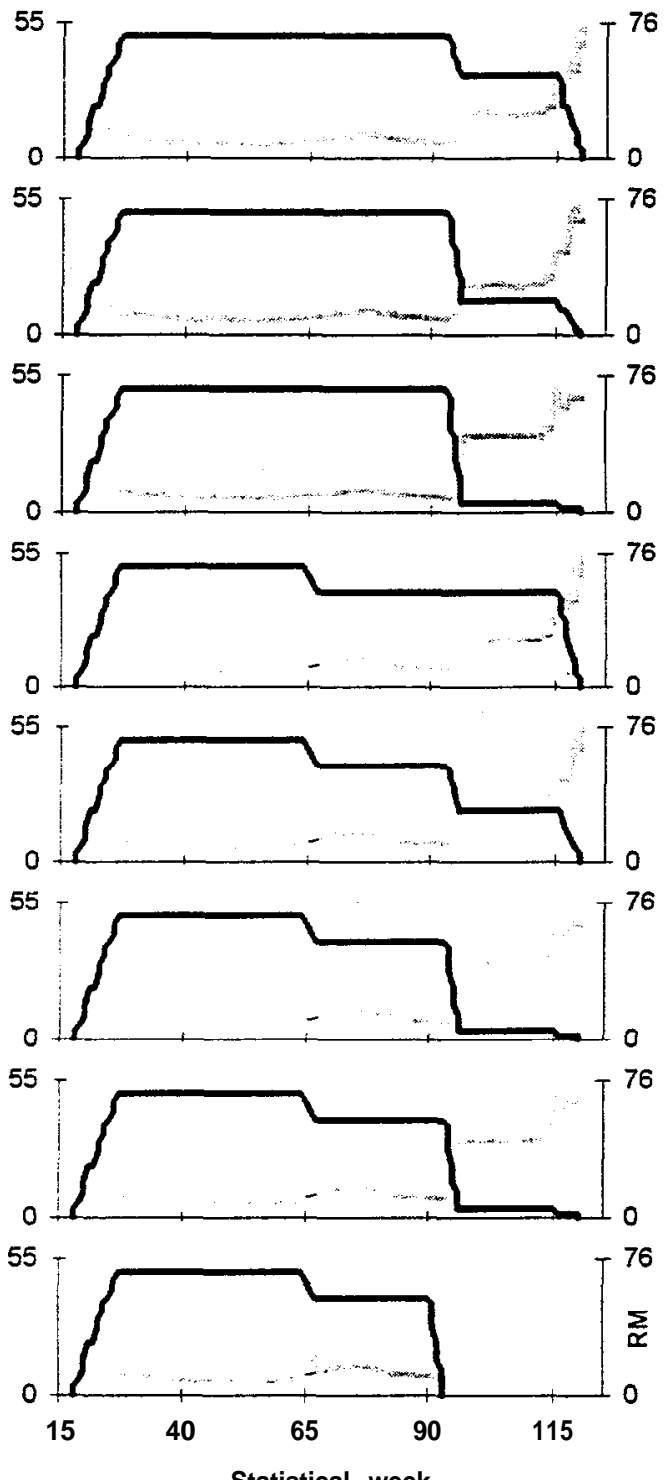

Statistical week

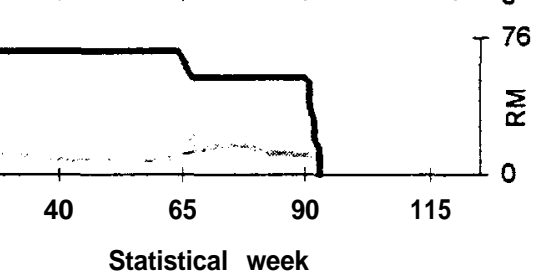

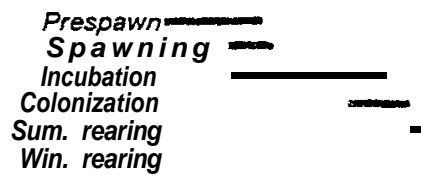

\begin{tabular}{|c|c|}
\hline \multicolumn{2}{|c|}{ Template } \\
Patient \\
\hline Cum. & Cum. \\
Prod. & Cap. \\
\hline
\end{tabular}

Traj. \#1

\begin{tabular}{l|l}
201 & 0.25 \\
\hline
\end{tabular}

236 0.25

Traj. \#2

$287 \quad 0.38$

285 0.32

Traj. \#3

$318 \quad 0.42$

\begin{tabular}{l|l}
328 & 0.37
\end{tabular}

Traj. \#4

3390.46

$343 \quad 0.39$

Traj. \#5

328.0 .46

$325 \quad 0.39$

Traj. \#6

2790.39

270 0.32

Traj. \#7

$335 \mid 0.47$

328 0.40

Traj . \#8

\begin{tabular}{|l|l|}
\hline 335 & 0.47 \\
\hline
\end{tabular}

328 0.40

Traj. \#9

\begin{tabular}{l|l}
\hline 637 & 0.98 \\
\hline
\end{tabular}

\begin{tabular}{l|l}
715 & 0.94
\end{tabular} 
Spawning reach: Lightning $\mathbf{C r}$.

Mouth - forks (RM 5.4 - 13.9 from Snake R)

Relative Productivity
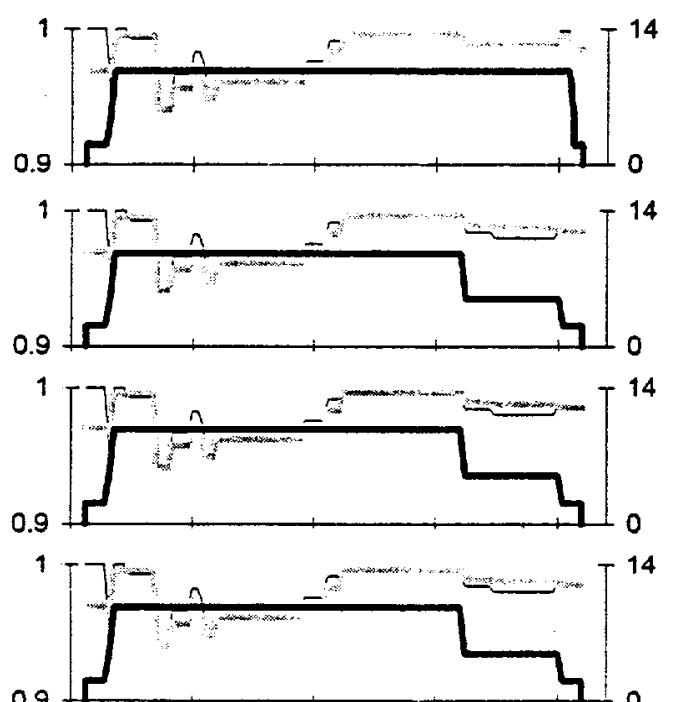

$$
0.9
$$
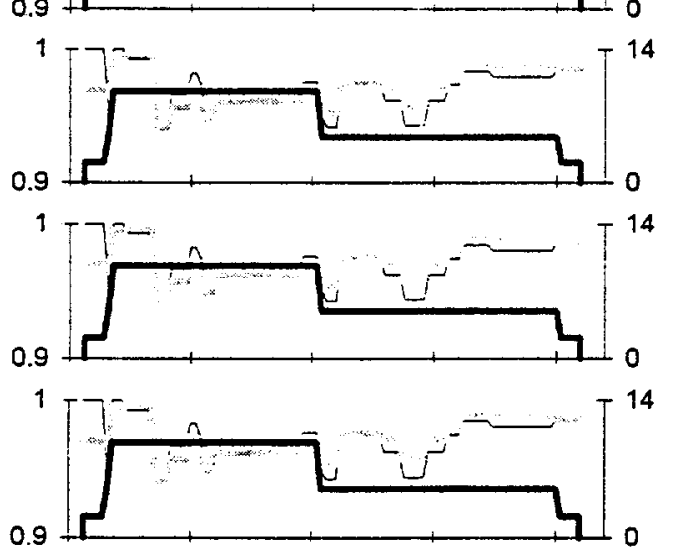

$-14 \quad 55$

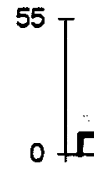

Relative Key Habitat Quantity
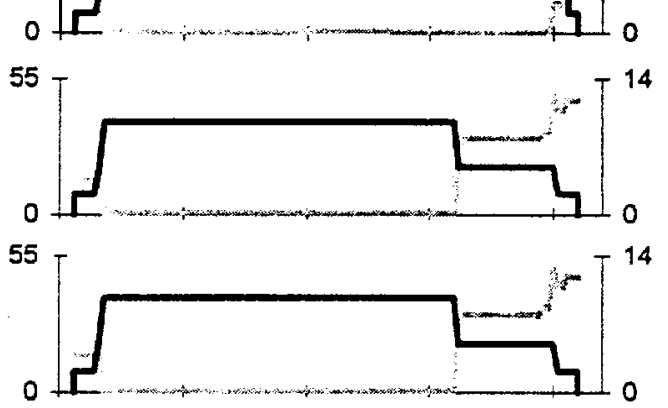

55
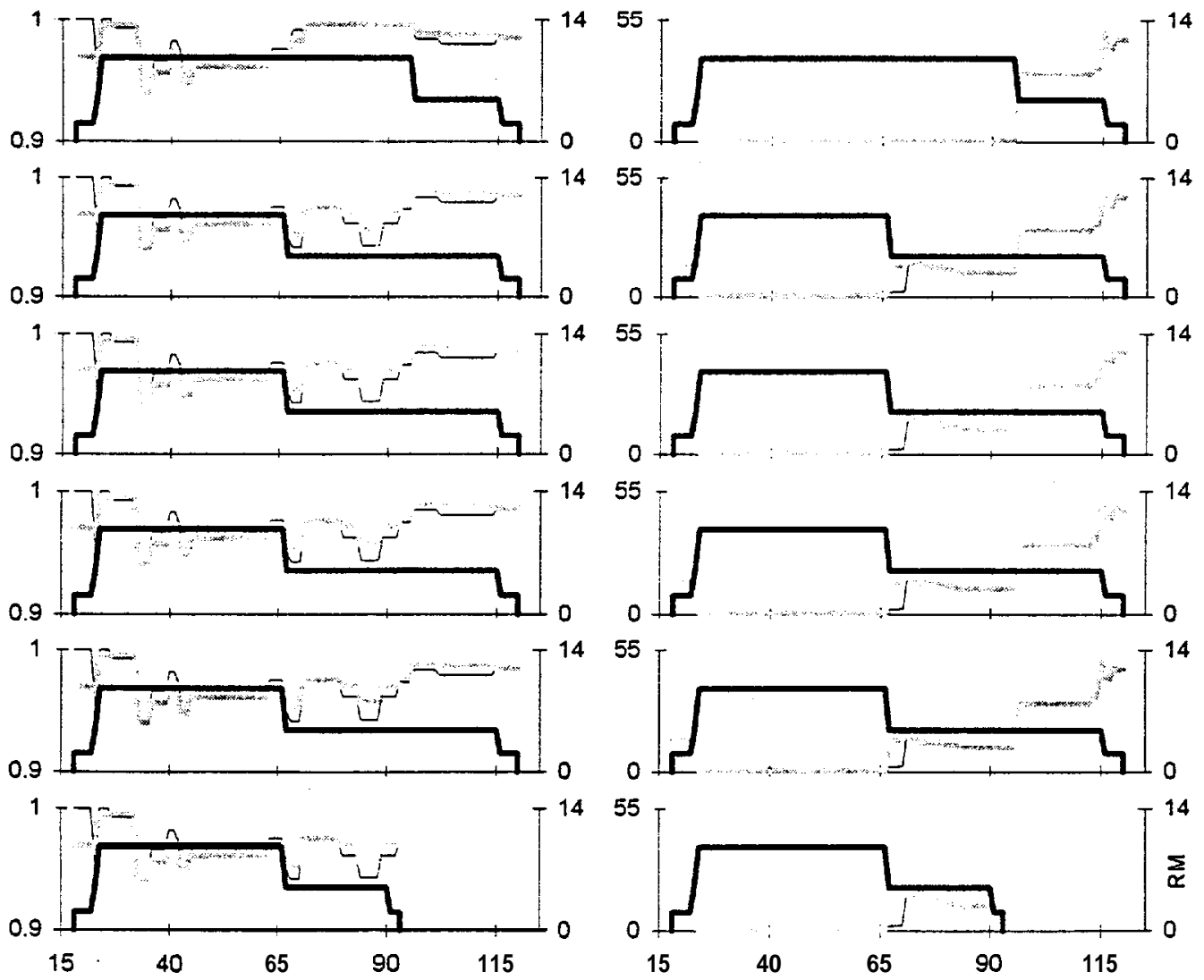

Statistical week

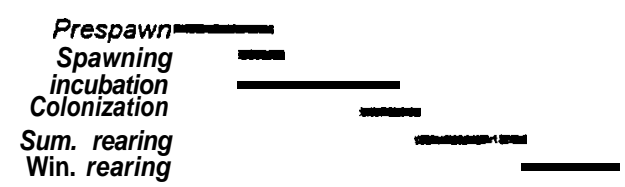

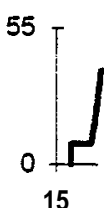

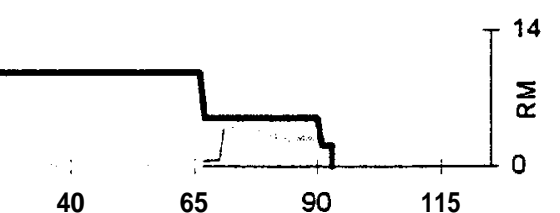

Statistical week

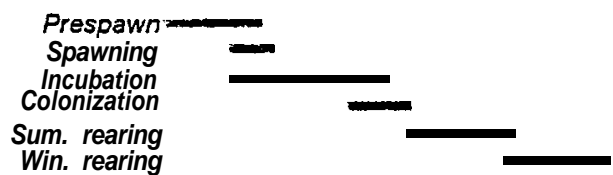

Trajectory

Template Patient 
Spawning reach: Little Sheep $\mathrm{Cr}$.

Mouth - Bear Gulch (RM 25.4 - 28.5 from Snake R)

Relative Productivity
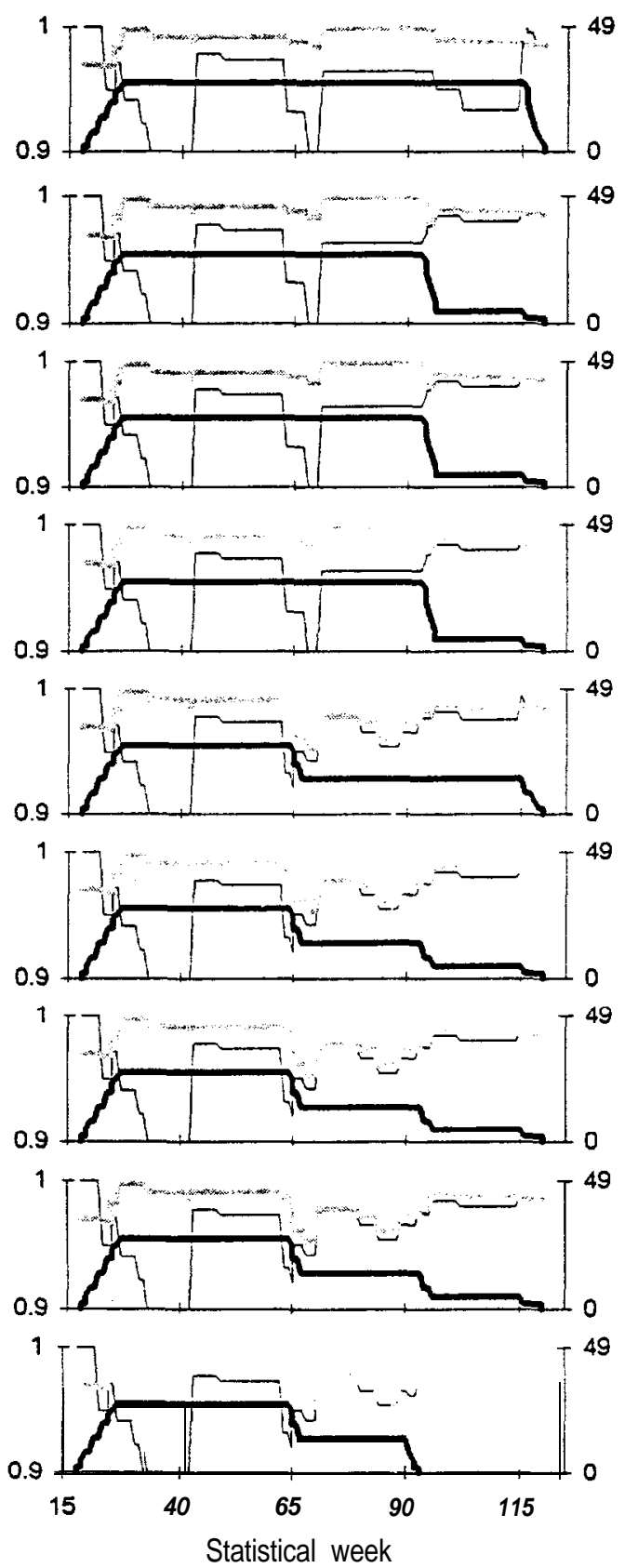

Prespawn -

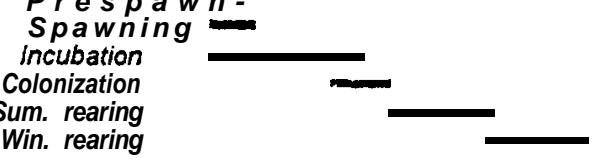

Relative Key Habitat Quantity
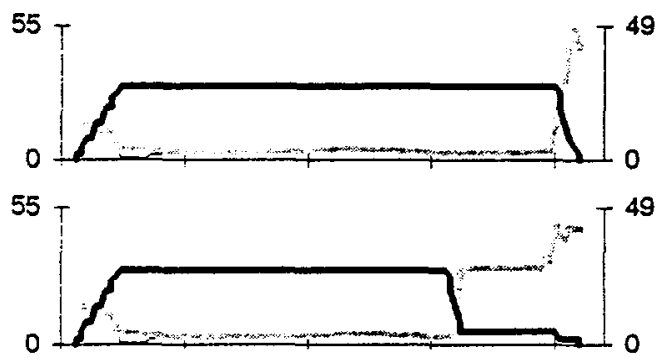

55 .

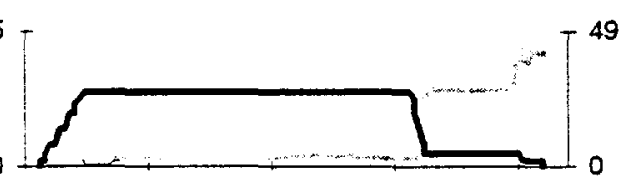

55

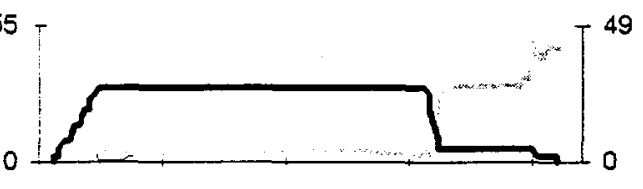

55

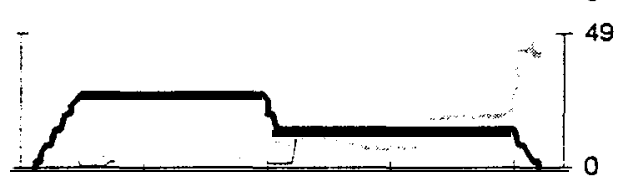

55

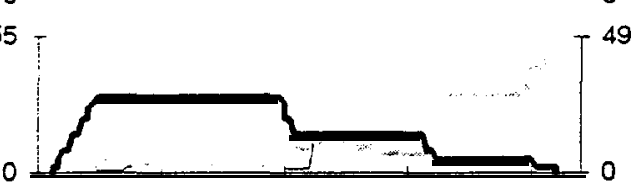

0

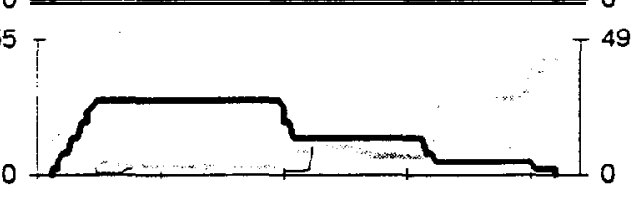

0
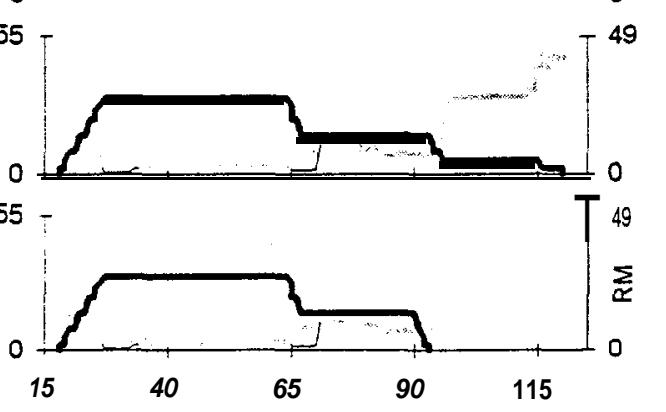

Statistical week \begin{tabular}{|c|}
\hline \multicolumn{2}{|c|}{ Template } \\
Patient \\
\hline Cum. \\
Prod. Cum. \\
\hline
\end{tabular}

Traj. \#1

$2950^{0.23}$

30.00

Traj. \#2

\begin{tabular}{r|r|}
283 & 0.27 \\
7 & 0.00 \\
\hline
\end{tabular}

Traj. \#3

\begin{tabular}{r|r|}
283 & 0.27 \\
7 & 0.00
\end{tabular}

Traj. \#4

\begin{tabular}{r|r|}
283 & 0.27 \\
7 & 0.00
\end{tabular}

Traj. \#5

\begin{tabular}{r|r|}
122 & 0.15 \\
9 & 0.00
\end{tabular}

Traj. \#6

\begin{tabular}{r|r|}
130 & 0.17 \\
\hline
\end{tabular}

$10 \quad 0.00$

Traj. \#7

\begin{tabular}{l|l}
130 & 0.17 \\
10 & 0.00
\end{tabular}

10) 0.00

Traj. \#8

1300.17

10) 0.00

Traj. \#9

\begin{tabular}{l|l}
\hline 260 & 0.34 \\
\hline
\end{tabular}

22) 000011

Prespawn -

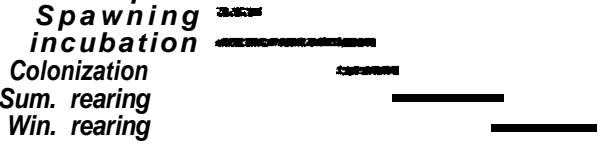


Spawning reach: Little Sheep Cr.

Bear Gulch - Devil's Gulch (RM 28.5 - 30.9 from Snake R)

\section{Relative Productivity}
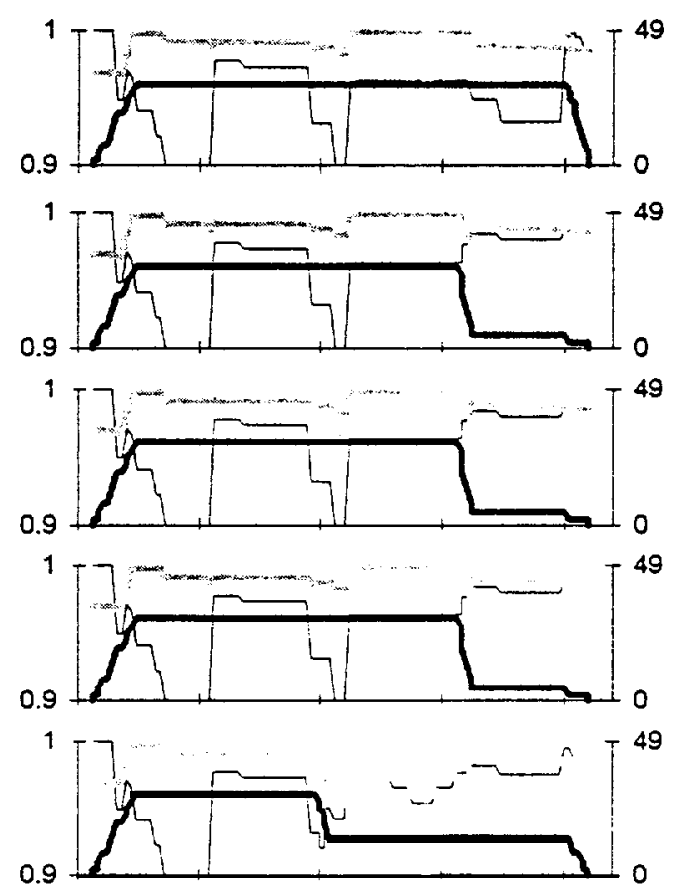

0.9
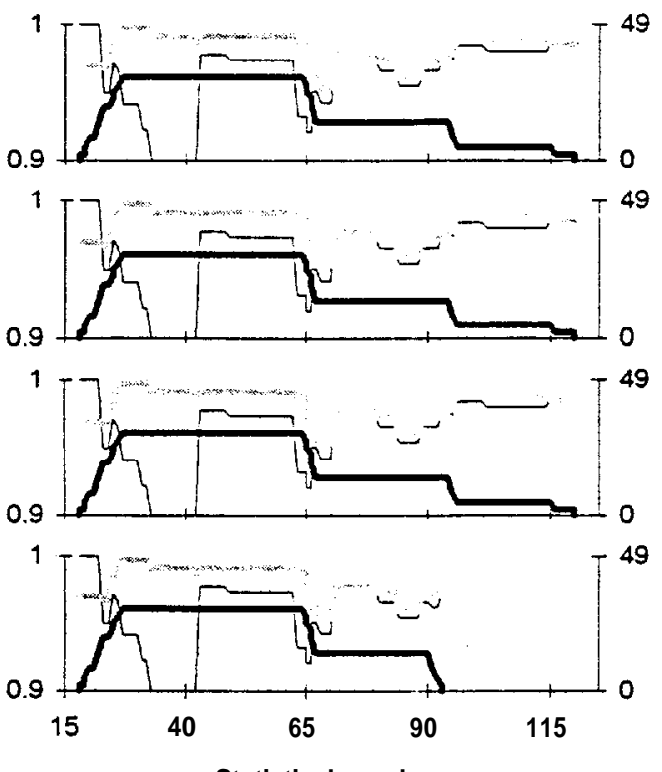

Statistical week

Prespawn -

Spawning

Incubation

Colonization

Sum. rearing

Win. rearing

\section{Relative Key Habitat Quantity}
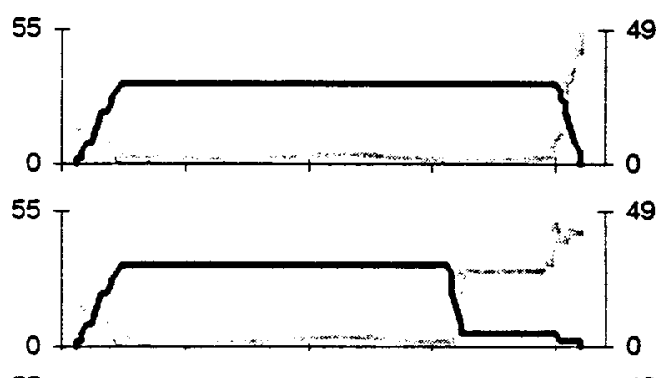

55
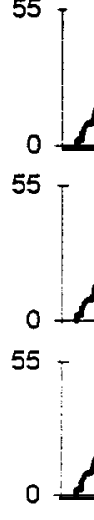

55

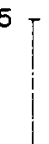

0

$55 T$

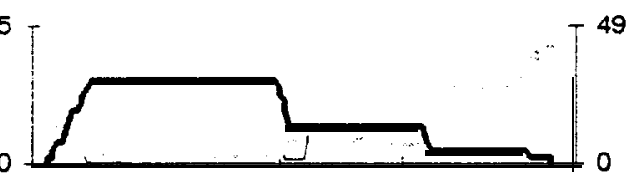

55

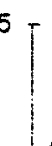

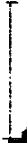

55

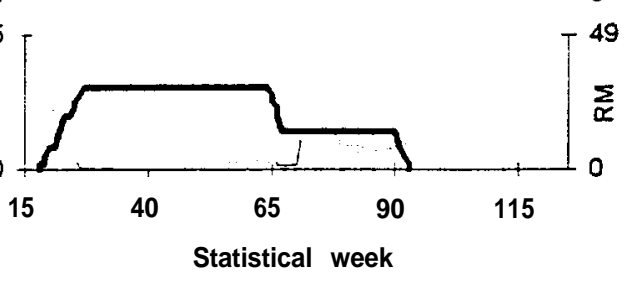

Prespawn-

Spawning Incubation

Colonization

Sum. rearing

Win. rearing

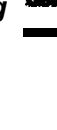

\begin{tabular}{c|c}
\hline \multicolumn{2}{|c|}{ Template } \\
Patient \\
\hline Cum. \\
Prod. & Cam. \\
\hline
\end{tabular}

Traj. \#1

\begin{tabular}{r|r|}
306 & 0.16 \\
3 & 0.00
\end{tabular}

Traj. \#2

\begin{tabular}{l|l}
292 & 0.19 \\
\hline & 0.00
\end{tabular}

$\begin{array}{ll}7 & 0.00\end{array}$

Traj. \#3

\begin{tabular}{l|l}
292 & 0.19
\end{tabular}

7) 0.00

Traj. \#4

$292 \quad 0.19$

\begin{tabular}{l|l}
7 & 0.00
\end{tabular}

Traj. \#5

128 . 0.12

9) 0.00

Traj. \#6

$136 \quad 0.13$

10) 0.00

Traj. \#7

\begin{tabular}{|l|l|}
\hline 136 & 0.13 \\
\hline
\end{tabular}

10 0.00

Traj. \#8

\begin{tabular}{|l|l|}
136 & 0.13 \\
\hline
\end{tabular}

10) 0.00

Traj. \#9

$272 \quad 0.26$

22) 0.00 
Spawning reach: Little Sheep $\mathrm{Cr}$.

Devil's Gulch - Lightning Cr (RM 30.9 - 33.1 from Snake R)

Relative Productivity
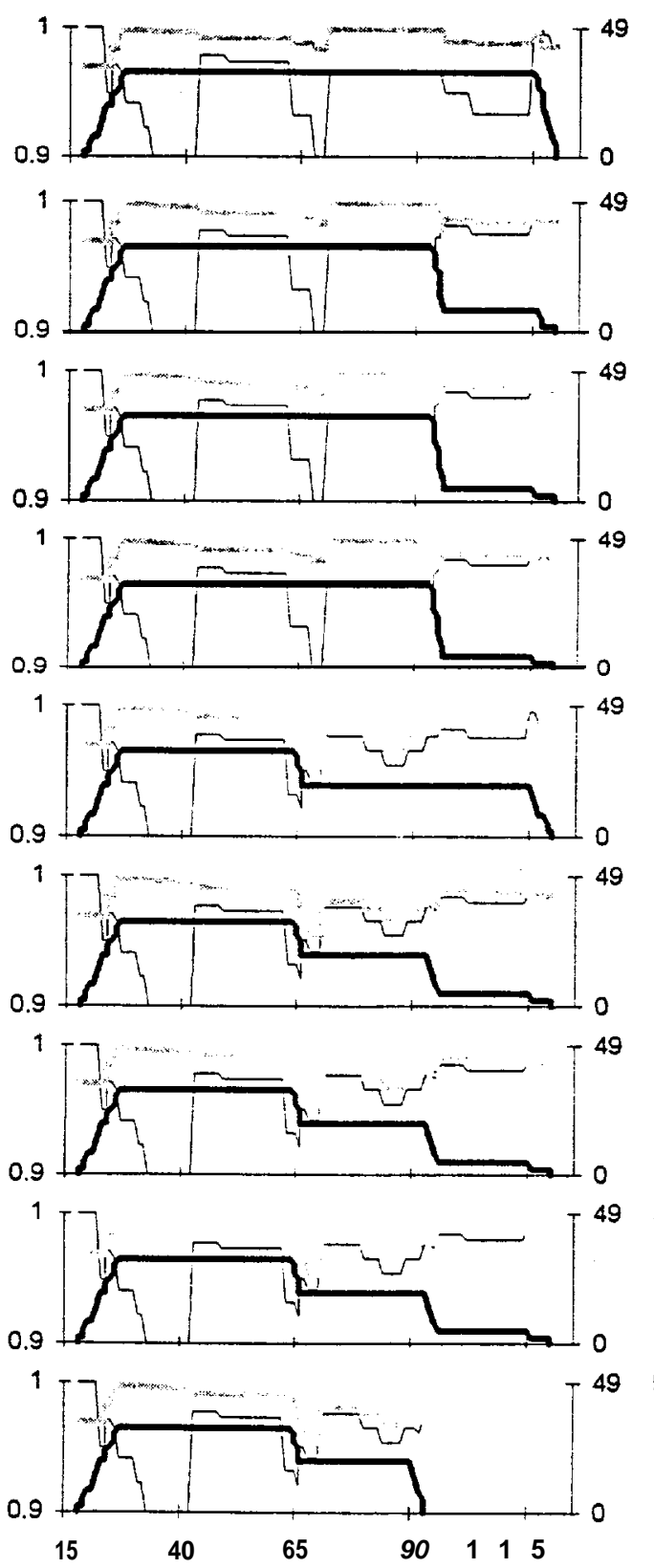

Statistical week

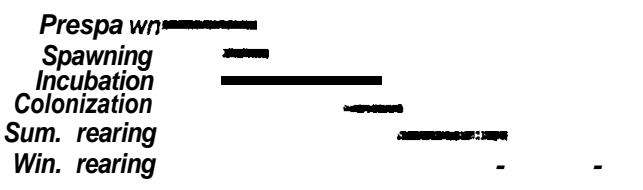

Relative Key Habitat Quantity
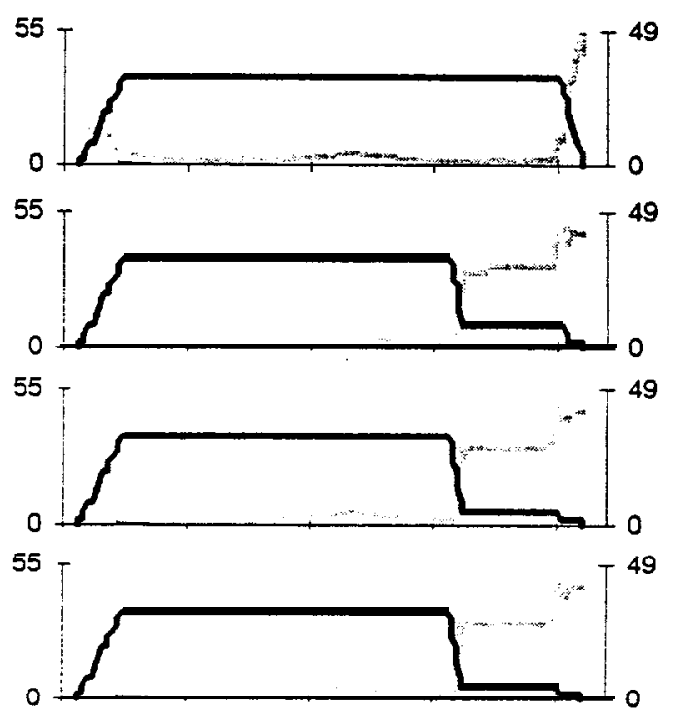

55
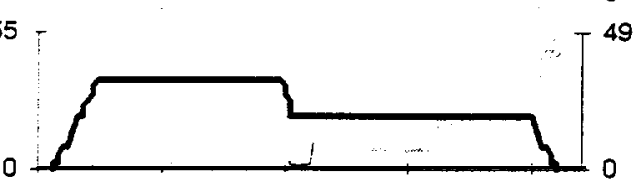

$55 T$

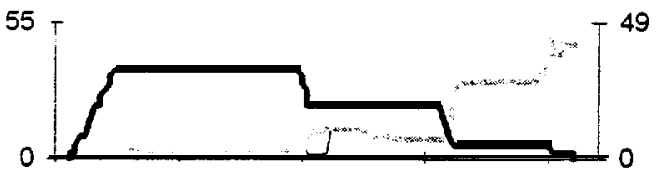

55
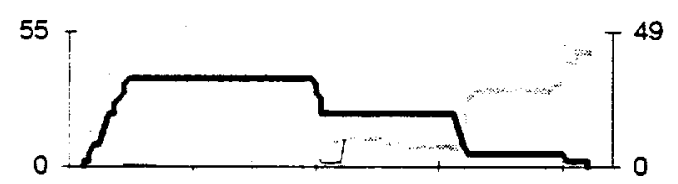

0
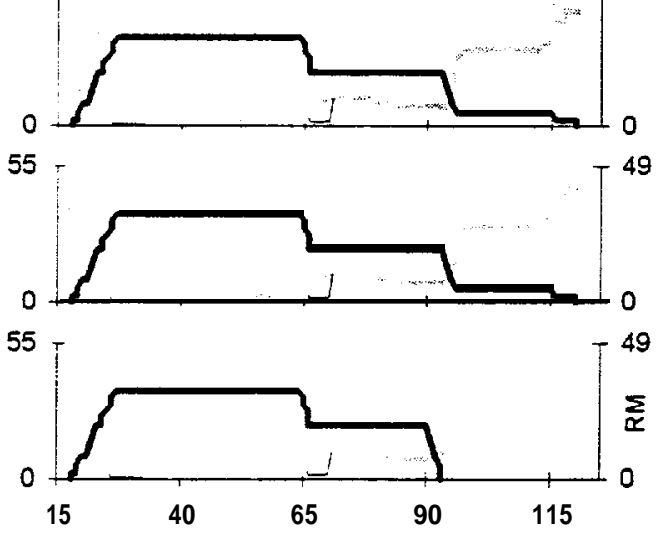

Statistical week

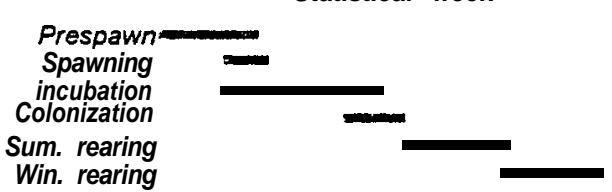

\begin{tabular}{c}
\hline Template \\
Patient \\
\hline Cumm. Qumm. \\
Prod. \\
Coatp.
\end{tabular}

Traj. \#1

\begin{tabular}{l|l|}
\hline 327 & 0.17 \\
\hline & 0.00
\end{tabular}

3) 0.00

Traj. \#2

29800119

7) 0.00

Traj. \#3

\begin{tabular}{r|r|}
313 & 0.21 \\
7 & 0.00
\end{tabular}

Traj. \#4

$313 \quad 0.21$

7) 0.00

Traj. \#5

$151 \mid 0.14$

9 0.00

Traj. \#6

1600.15

10) 0.00

Traj. \#7

$160 \quad 0.15$

10 0.00

Traj. \#8

\begin{tabular}{|l|l|}
\hline 160 & 0.15 \\
\hline
\end{tabular}

10) 0.00

Traj. \#9

\begin{tabular}{|l|l|}
\hline 318 & 0.30 \\
\hline
\end{tabular}

22 0.01 
Spawning reach: Little Sheep Cr.

Lightning $\mathrm{Cr}$ - Hayden $\mathrm{Cr}$ (RM 33.1 - 38.8 from Snake R)

Relative Productivity
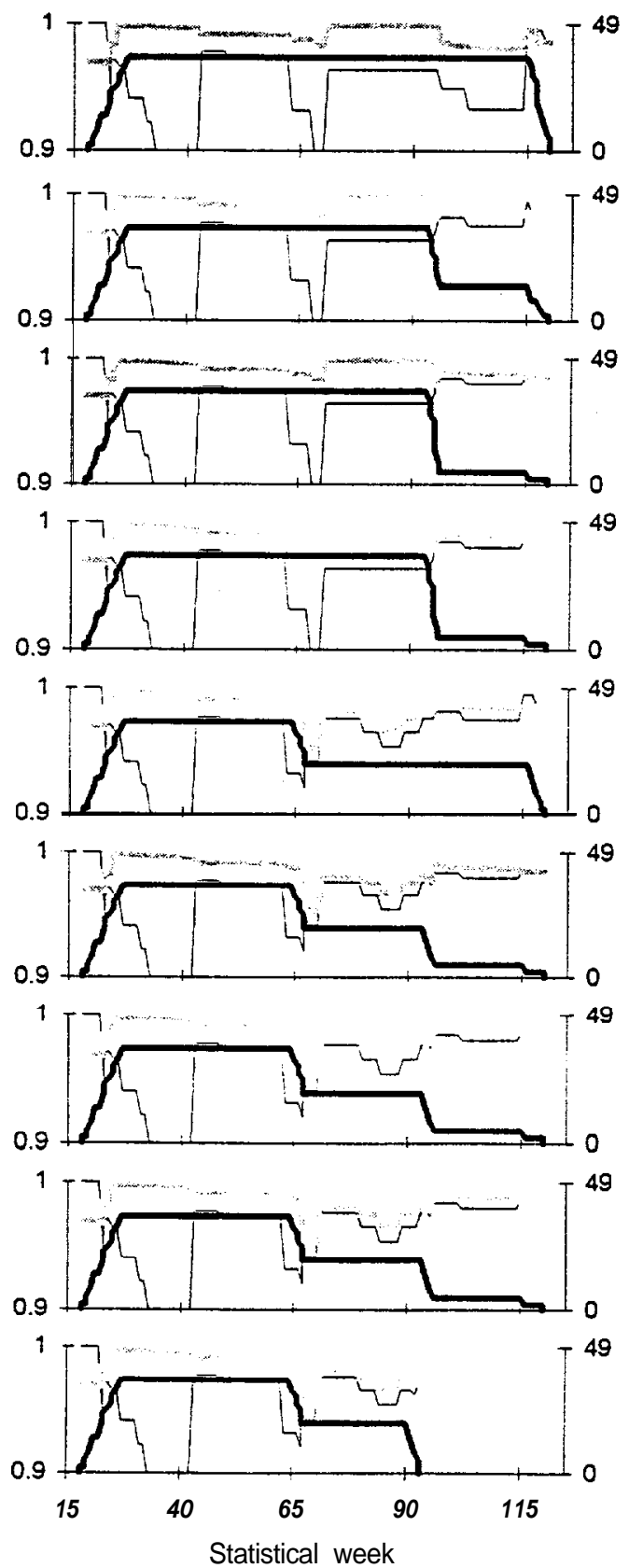

Statistical week

Prespawn. Spawning Incubation Colonization Sum. rearing Win. rearing

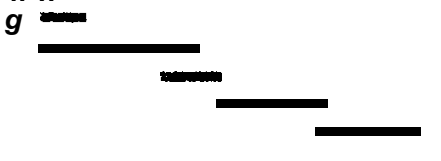

Relative Key Habitat Quantity
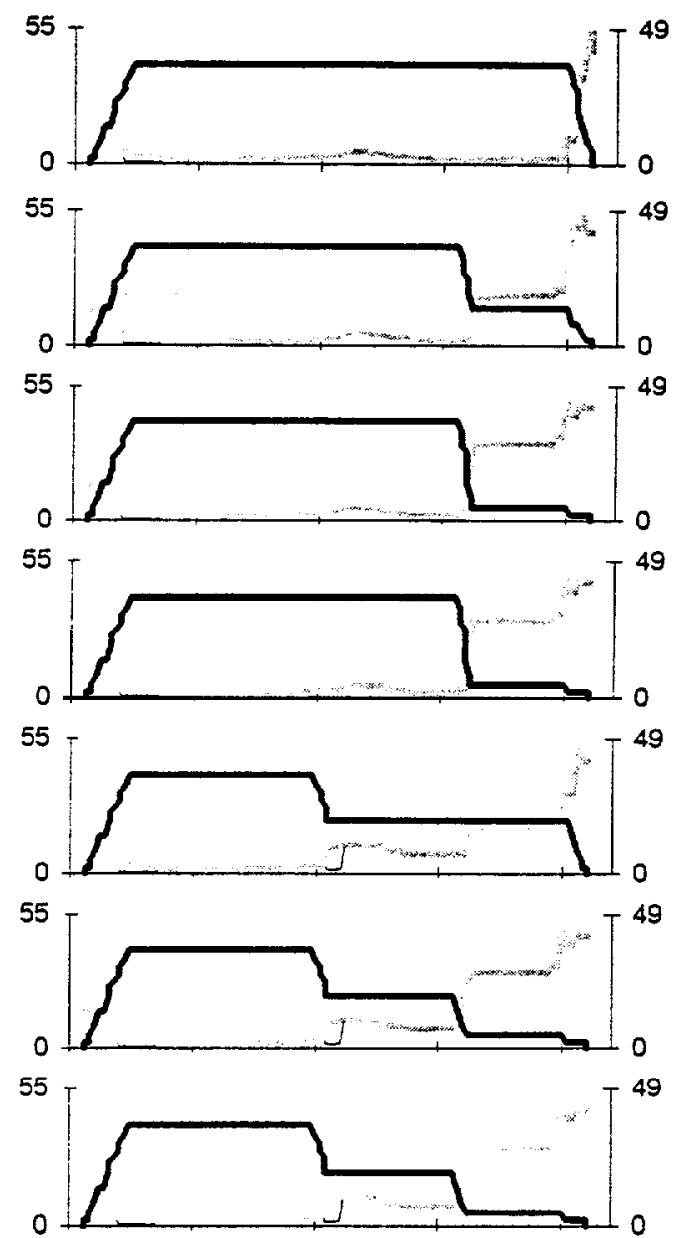

$55-$

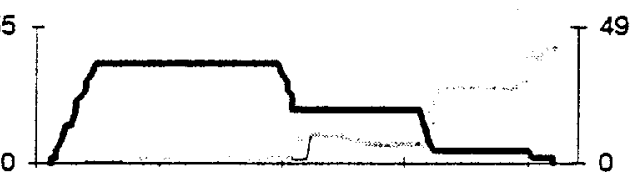

55

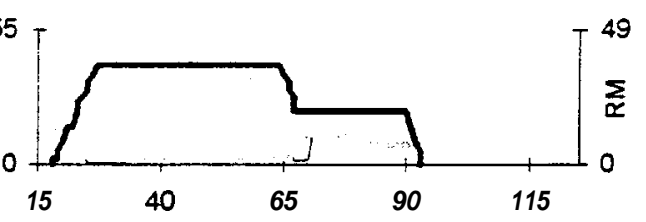

Statistical week

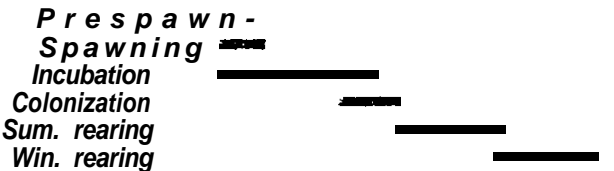

\begin{tabular}{l}
\hline \multicolumn{2}{|c|}{ Template } \\
Patient \\
\hline Cum. \\
Cum.
\end{tabular}

Prod. Cap.

Traj. \#1

\begin{tabular}{l|l|}
\hline 295 & 0.14 \\
\hline & 0.00
\end{tabular}

30.00

Traj. \#2

306 0.18

7) 0.00

Traj. \#3

$324 \quad 0.20$

$\begin{array}{ll}7 & 0.00\end{array}$

Traj. \#4

$324 \quad 0.20$

$\begin{array}{ll}7 & 0.00\end{array}$

Traj. \#5

$160 \quad 0.13$

\begin{tabular}{l|l}
9 & 0.00
\end{tabular}

Traj. \#6

\begin{tabular}{l|l}
169 & 0.14 \\
\hline
\end{tabular}

\begin{tabular}{l|l}
10 & 0.00
\end{tabular}

Traj. \#7

\begin{tabular}{l|l|}
\hline 169 & 0.14 \\
\hline
\end{tabular}

10) 0.00

Traj. \#8

\begin{tabular}{l|l|}
\hline 169 & 0.14 \\
\hline
\end{tabular}

10) 0.00

Traj. \#9

\begin{tabular}{|l|l|}
\hline 336 & 0.29 \\
\hline
\end{tabular}

22) 0.00 
Spawning reach: Little Sheep Cr.

Hayden $\mathrm{Cr}$ - McCully $\mathrm{Cr}$ (RM 38.8 - 48.8 from Snake R)

Relative Productivity
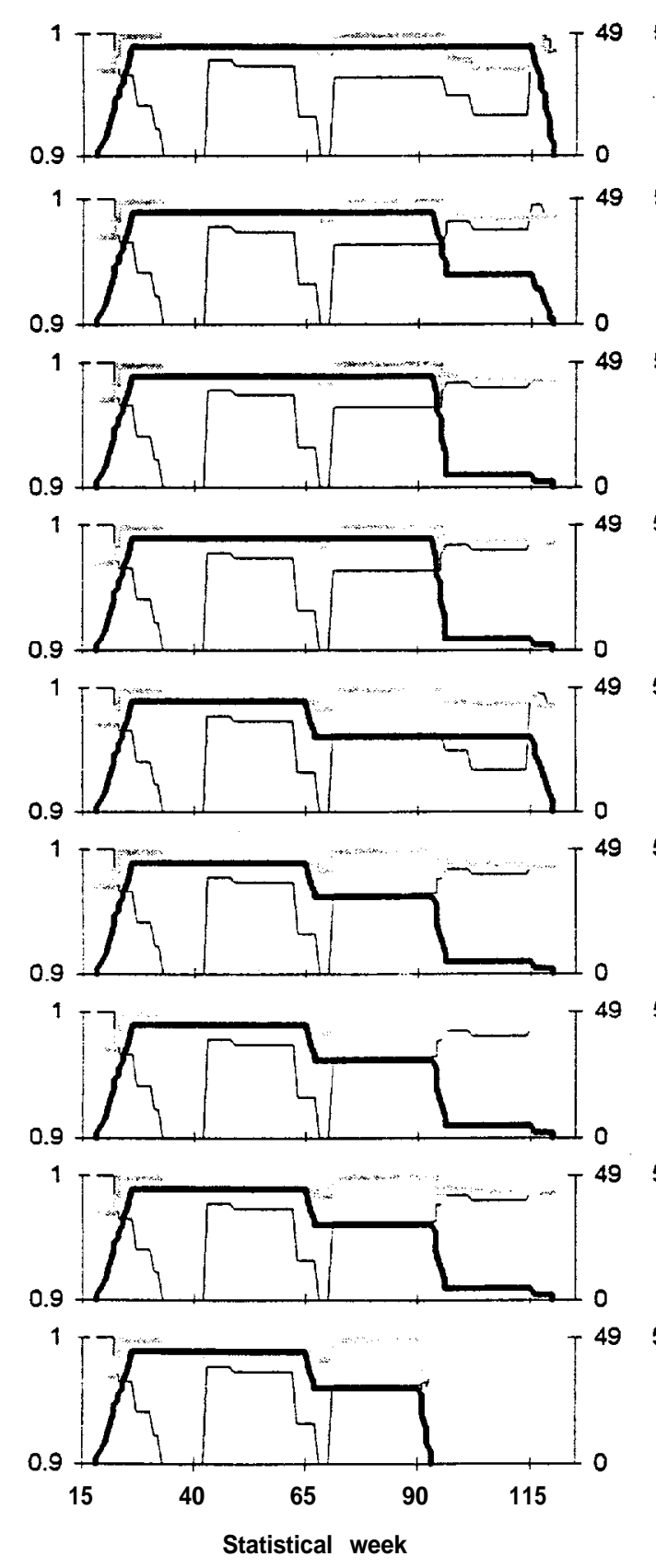

Prespawn -

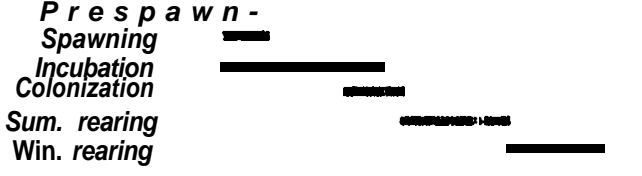

Relative Key Habitat Quantity
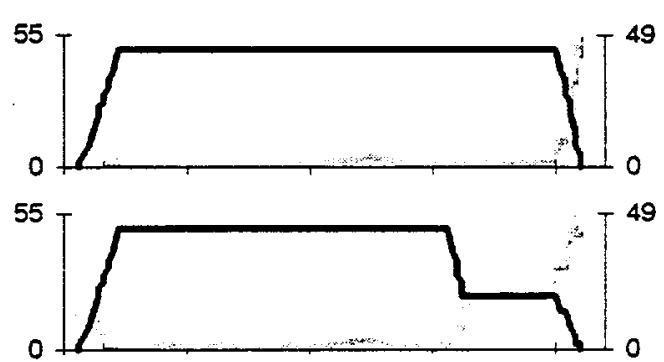

55 .

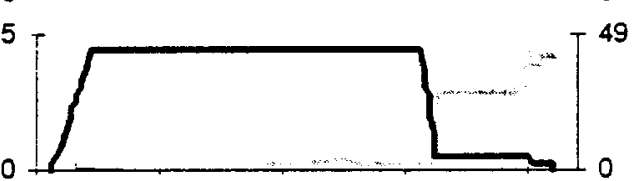

0
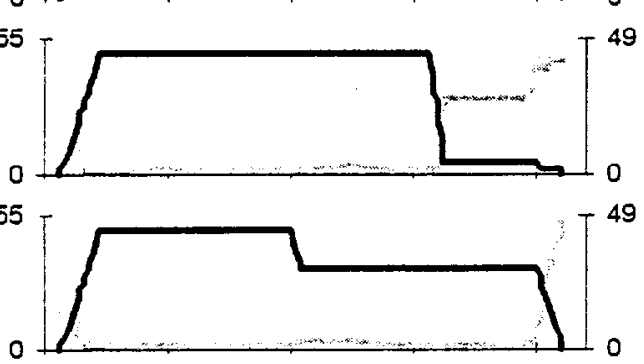

0
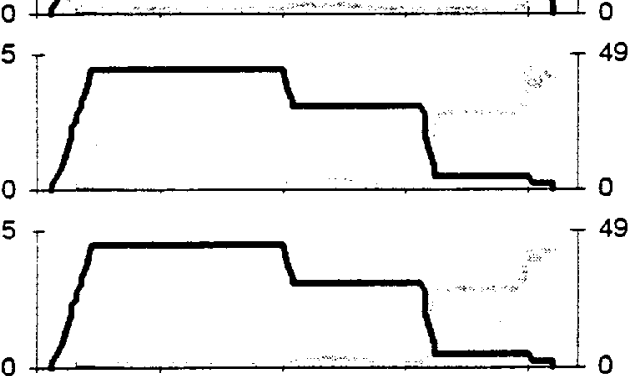

0

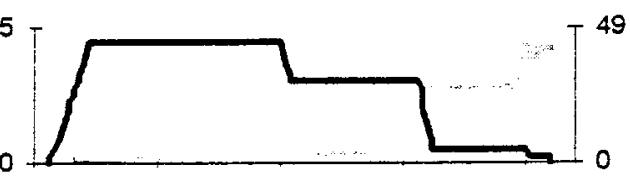

55

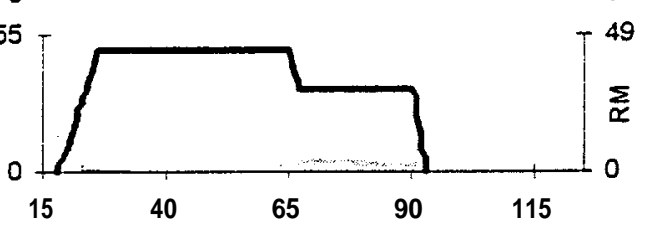

Statistical week
Template
Patient

Cum. Cum.

Prod. Cap.

Traj. \#1

\begin{tabular}{l|l}
\hline 250 & 0.11 \\
\hline & 0.00
\end{tabular}

3) 0.00

Traj. \#2

$304 \quad 0.15$

7) 0.00

Traj. \#3

$320 \quad 0.16$

\begin{tabular}{l|l}
7 & 0.00
\end{tabular}

Traj. \#4

$320 \quad 0.16$

70.00

Traj. \#5

\begin{tabular}{l|l|}
328 & 0.14 \\
\hline & 0.00
\end{tabular}

3) 0.00

Traj. \#6

$313 \quad 0.17$

70.00

Traj. \#7

3130.17

7) 0.00

Traj. \#8

313 0.17

7) 0.00

Traj. \#9

\begin{tabular}{l|l}
588 & 0.35 \\
\hline
\end{tabular}

17 0.00

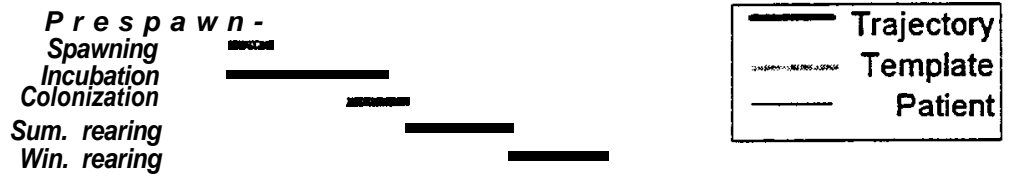




\section{APPENDIX C}

Patient Template Analysis. Landscapes showing Key Habitat, Productivity, and Environmental Attributes for each Range 
Patient Template Analysis: Landscapes showing Key Habitat, Productivity, and Environmental attributes for each stream reach range, listed in the order they appear in Appendix C.

\section{Stream Reach Range}

Grande Ronde R

Chesnimnus Cr / Joseph R / Grande Ronde R

Wenaha R / Grande Ronde R

Wallowa R / Grande Ronde R

Minam R / Wallowa R / Grande Ronde R

Bear Cr / Wallowa R / Grande Ronde R

Lostine R / Wallowa R / Grande Ronde R

Hurricane Cr / Wallowa R / Grande Ronde R

OK Gulch Cr / Prairie Cr /Wallowa R / Grande Ronde R

Lookingglass Cr / Grande Ronde R

Catherine Cr / Grande Ronde R

Sheep Cr / Grande Ronde R

Imnaha $\mathrm{R}$

Lightning Cr / Imnaha R

Big Sheep Cr / Imnaha R

Little Sheep Cr / Big Sheep Cr / Imnaha R

\section{$\underline{\text { Zoom View }}$}

Chesnimnus Cr / Joseph R

Wenaha $\mathrm{R}$

Wallowa $\mathrm{R}$

Minam R

Bear Cr

Lostine $\mathrm{R}$

Hurricane Cr

OK Gulch Cr / Prairie Cr

Lookingglass $\mathrm{Cr}$.

Catherine $\mathrm{Cr}$

Sheep Cr

Big Sheep Cr

Little Sheep Cr 


\section{Stream Reach Range}

Grande Ronde R 


\section{Patient-Template Analysis Summary}

Stream Reach Range: Grande Ronde R.

\section{Key Habitat Quantity}

The quantity of key habitat for spring chinook in the upper Grande Ronde River is greatly reduced for certain life stages compared to historic levels. Changes have been particularly severe throughout the Grande Ronde valley (- RM 95-120) for the prespawning, summer rearing, and overwintering stages. Slight to large changes have also occurred in many reaches upstream of the valley for all life stages except smolt. These losses are primarily due to land development, channel straightening and realignment, removal of large organic structure from the channel, and dewatering of the channel.

Changes in quantity of key habitat downstream of the valley have been comparatively minor.

\section{Relative Productivity (Survival)}

The capability of the mainstem Grande Ronde River of supporting spring chinook productivity (survival) is greatly reduced for most life stages compared to historic conditions. This reduction has been most severe within the Grande Ronde valley and continues for most of the distance upstream. Survival conditions have also declined markedly downstream of the valley reaches, although historic conditions there were naturally poor for some life stages in the lower 50 miles. Current survival conditions remain similar to what they were historically for the prespawning and smolt life stages over most the of the river and in the over-wintering stage in some portions of the lower river.

Survival conditions have changed in these reaches due primarily to increased water temperature, increased sediment load, loss in habitat diversity, changes in flow patterns, channel and bank destabilization, and alteration of the riparian zone. 


\section{Grande Ronde R Key Habitat Quantity}

Patient

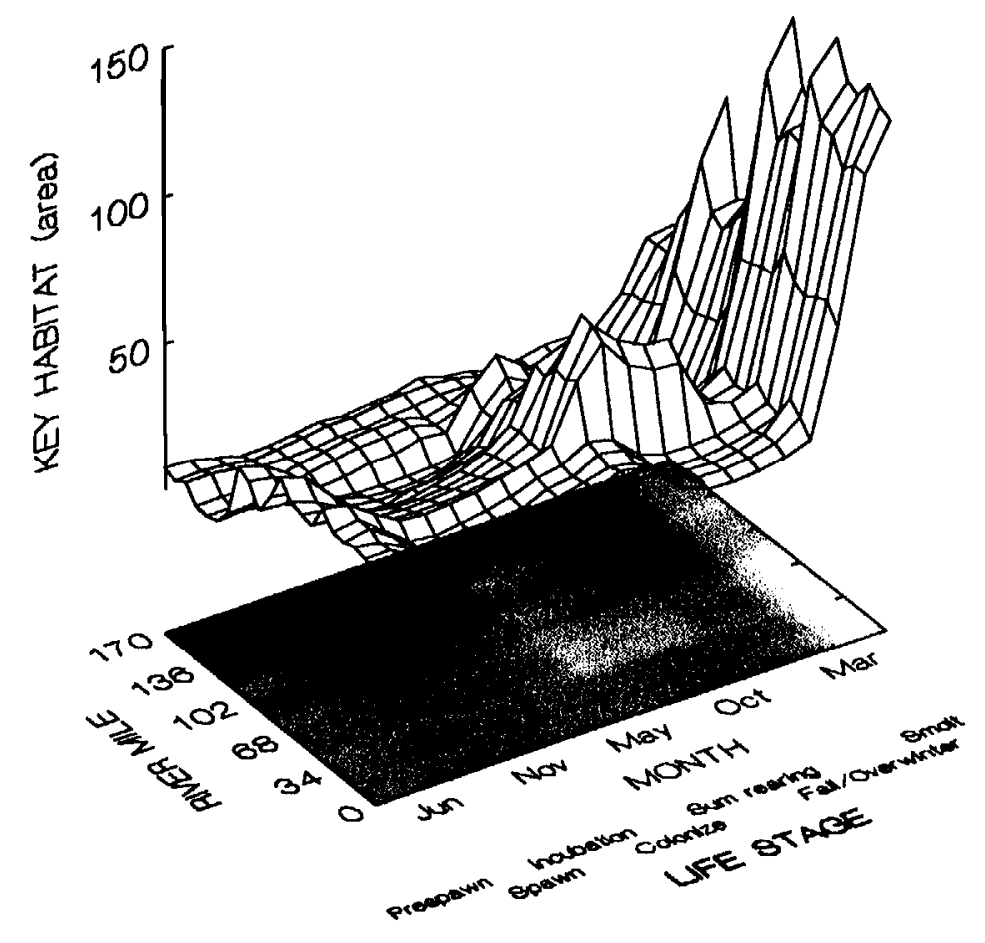

Template

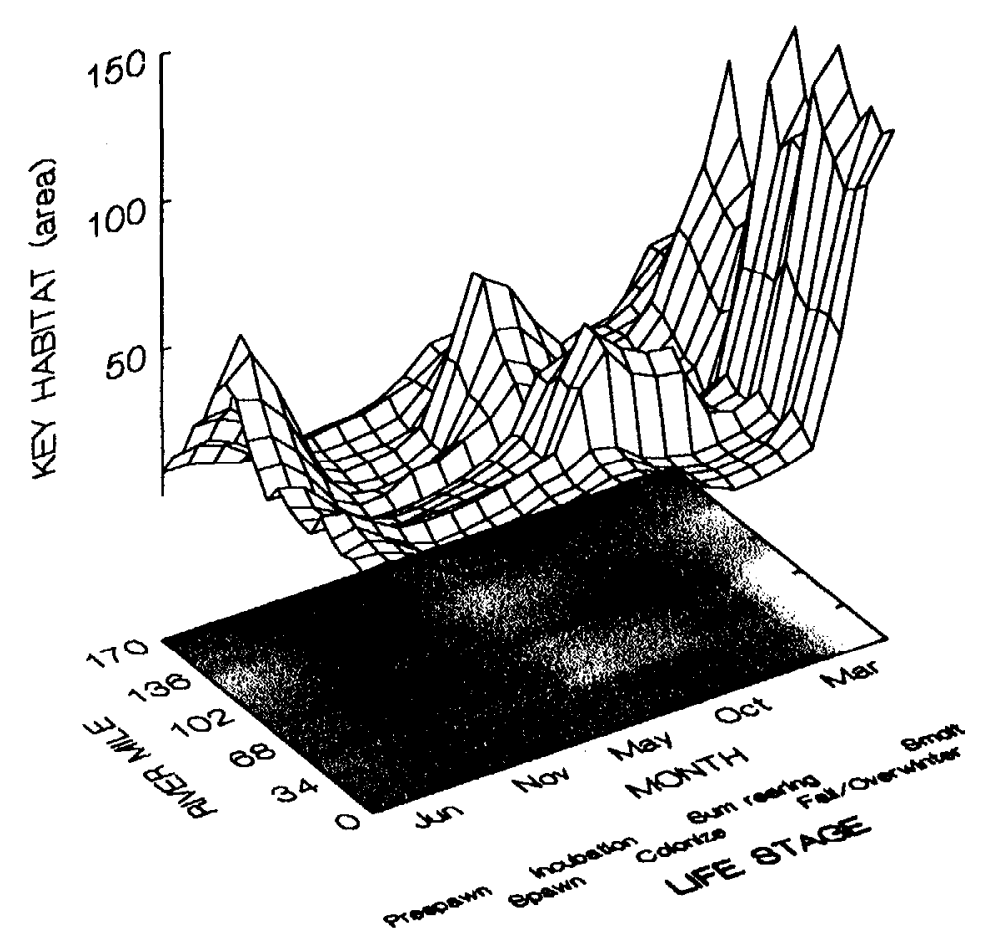




\section{Grande Ronde R Environmental Attributes}
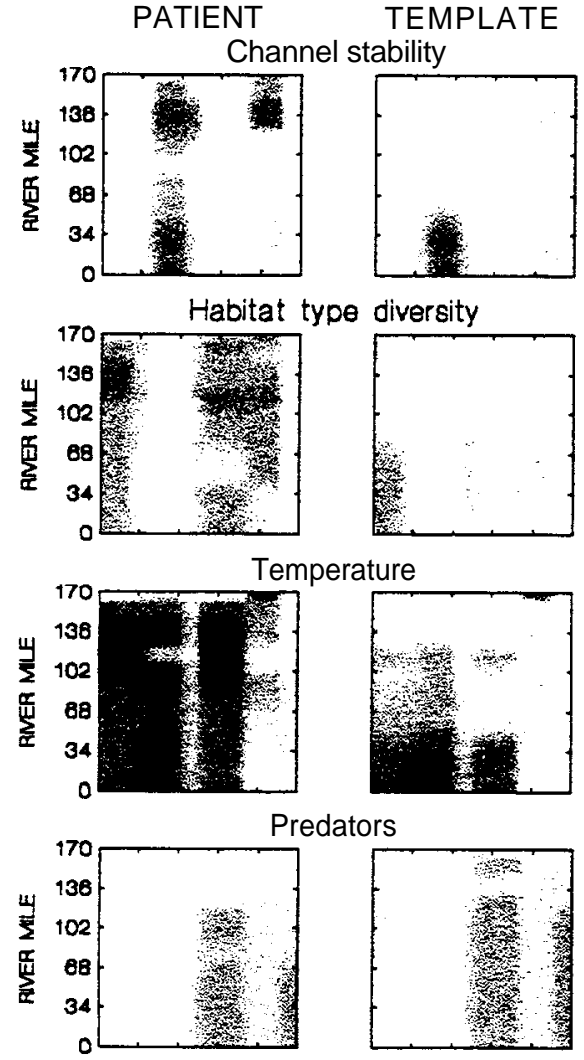

Temperature
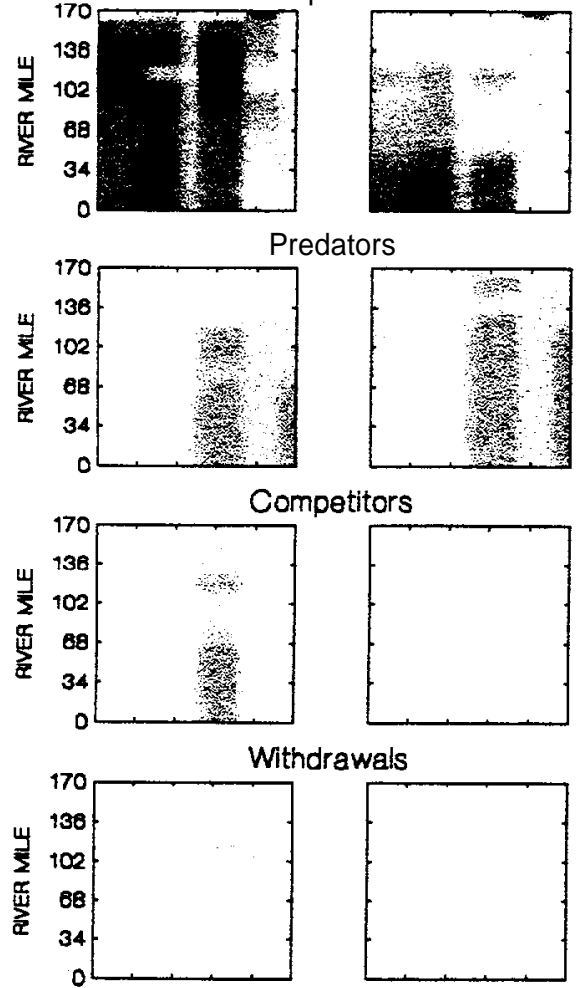

Predators

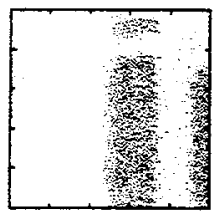

ompetitors

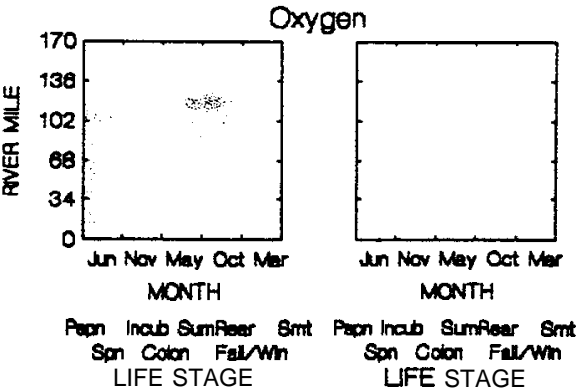

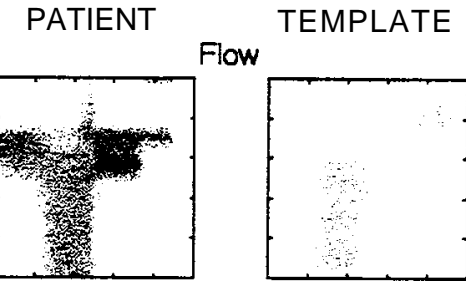

Sediment load

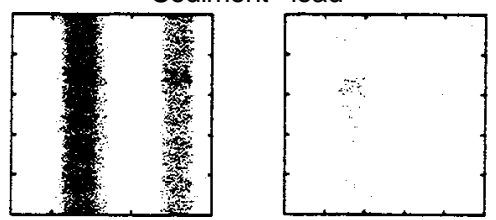

Riparian condition

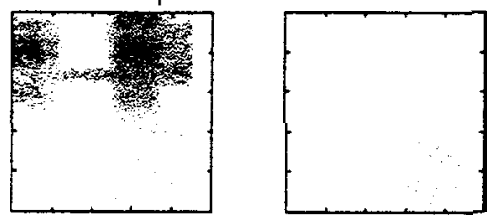

Chemicals

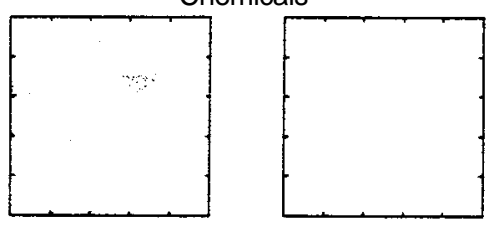

Obstructions

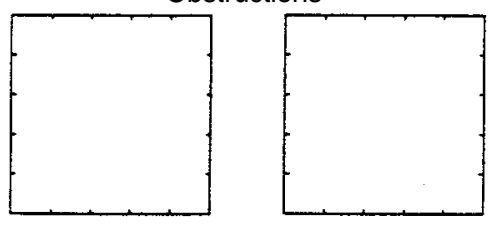

Nutrient load

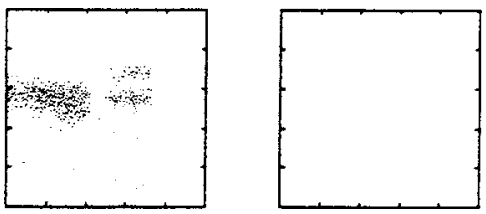

Pathogens
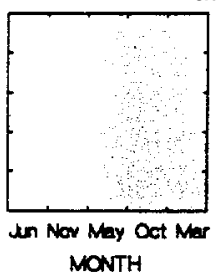

Pan that 8umfioer Sint Sin Cadon Fathwn LFE STAGE
Un Nar Nay Oet Mer MONTH

Paon hab sumfloer 8mt Son Codon Fatum 'LIFE STAGE
Level of

Effect

$\square$ No effect

$\square$ Low

Moderate

B High

Lethal 


\section{Chesnimnus Cr/Joseph Cr Key Habitat Quantity}

Patient

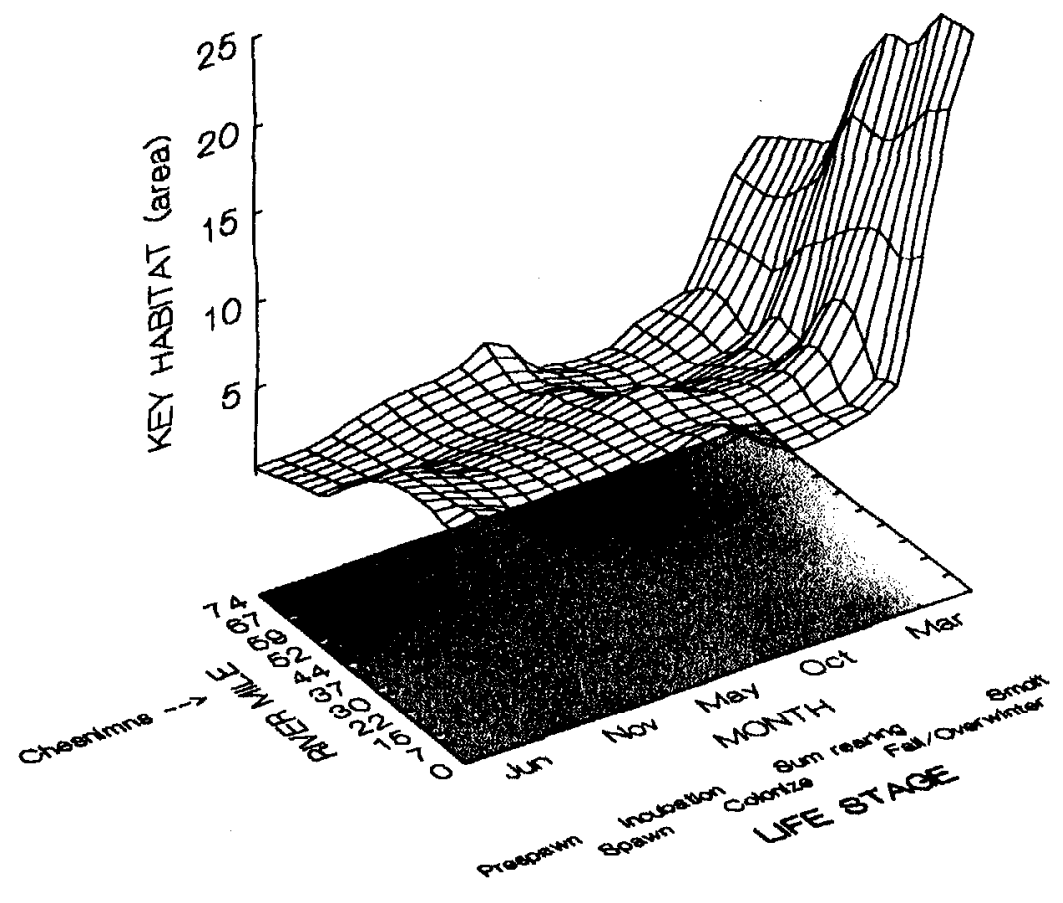

Template

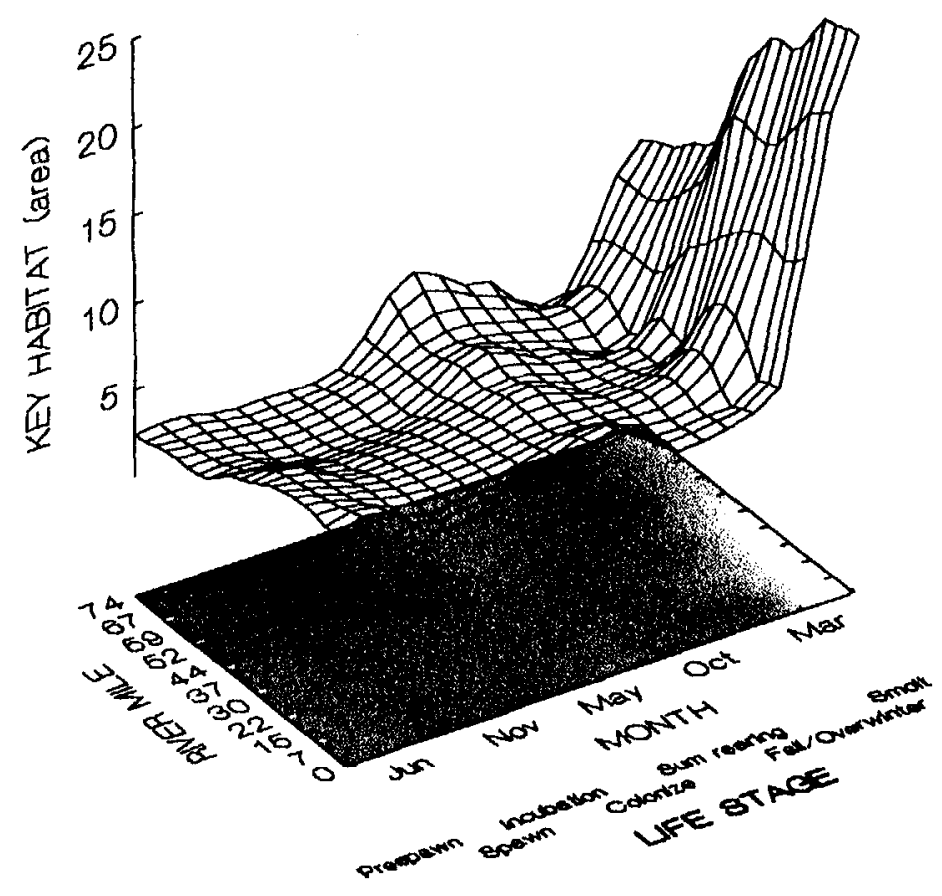




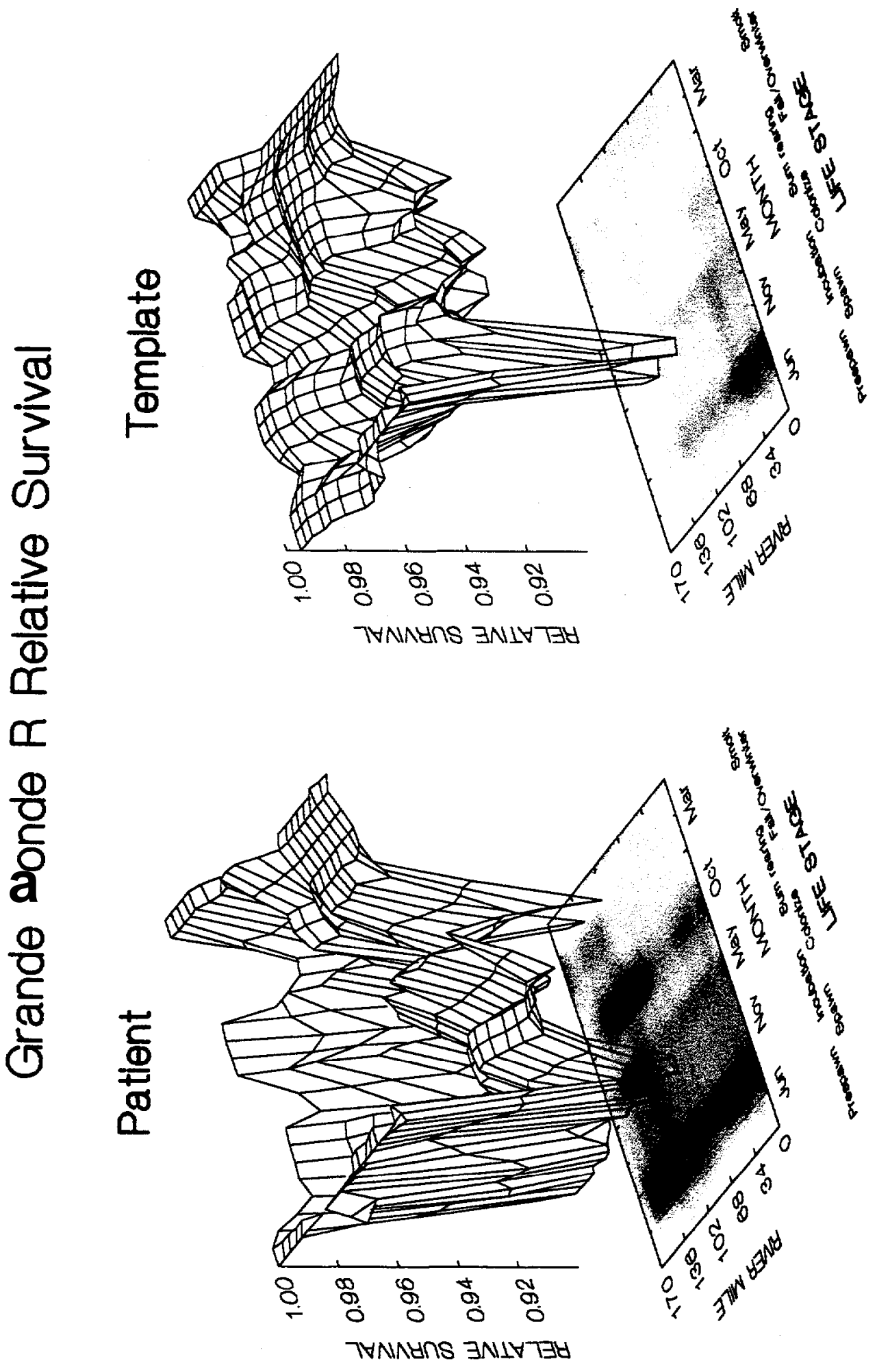




\title{
Patient-Template Analysis Summary
}

\author{
Stream Reach Range: Chesnimnus Cr./Joseph Cr./Grande Ronde R.
}

\section{Key Habitat Quantity}

Historically and continuing on to the present, the Joseph Creek subbasin has contained relatively little key habitat for spring chinook salmon.

Of the amount present, little change has occurred in the lower half of the drainage. A substantial loss has occurred in the upper reaches of the drainage, as seen in Chesnimnus Creek.

\section{Relative Productivity (Survival)}

Although this reach range's capability of supporting spring chinook productivity (survival) has been reduced substantially over the past 150 years, conditions have never been favorable for this species. In particular, survival conditions for prespawning adults, spawners, and eggs have always been poor within Chesnimnus Creek, Joseph Creek, and downstream in the Grande Ronde River. These stream sections have apparently always had water temperature too high for good survival during these life stages.

Conditions for survival have worsened for the Patient because of changes that have occurred to channel stability, flow patterns, sediment load, habitat diversity, and predation levels. 


\section{Stream Reach Range}

Chesnimnus Cr / Joseph R / Grande Ronde R 


\section{Chesnimnus Cr/Joseph Cr Environmental Attributes}
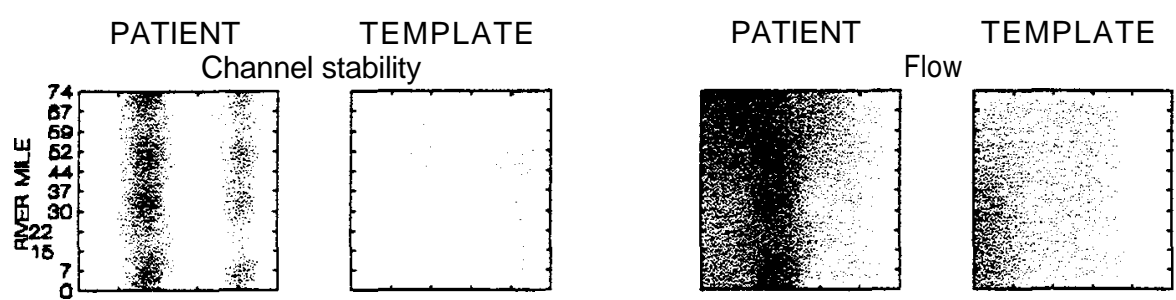

Habitat tyoe diversity
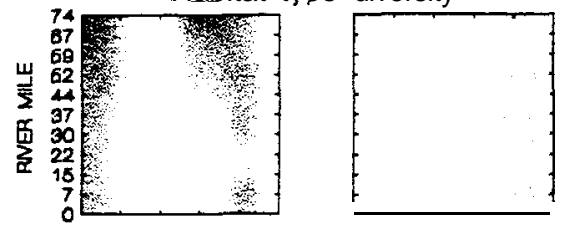

Temperature
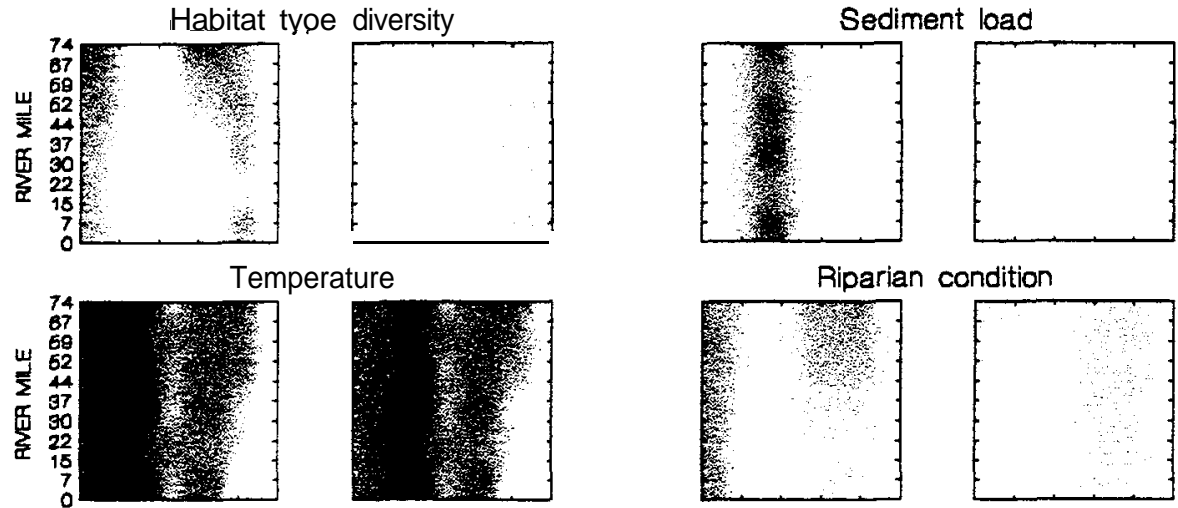

Riparian condition
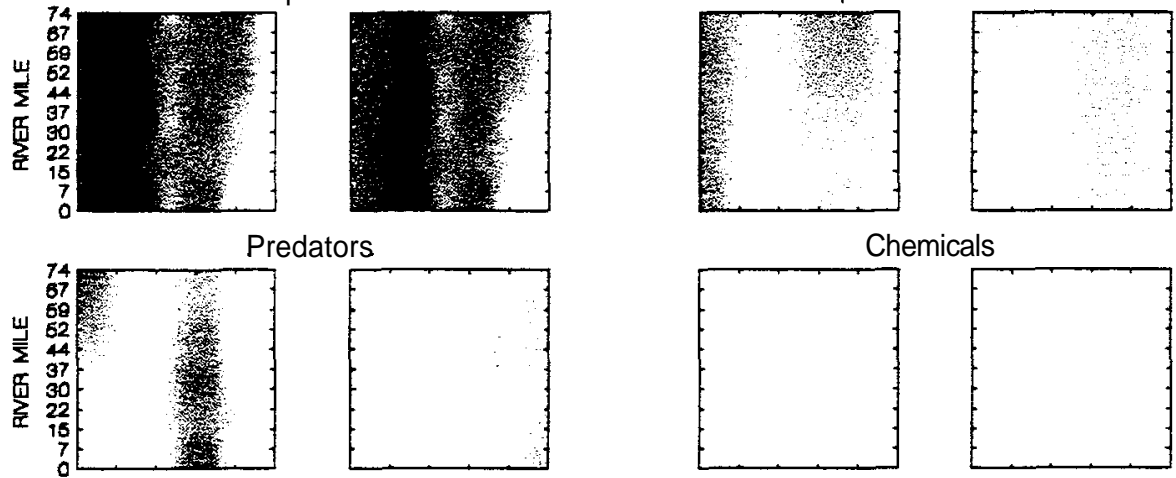

Obstructions

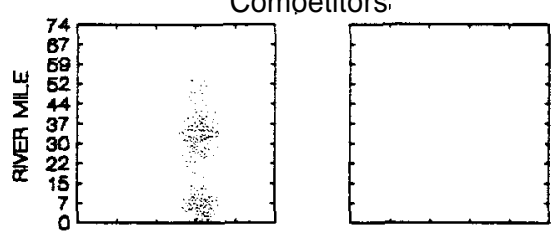

Withdrawals
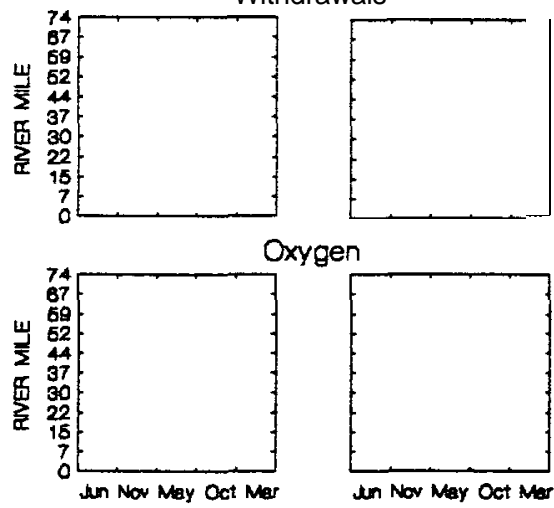

Oxygen
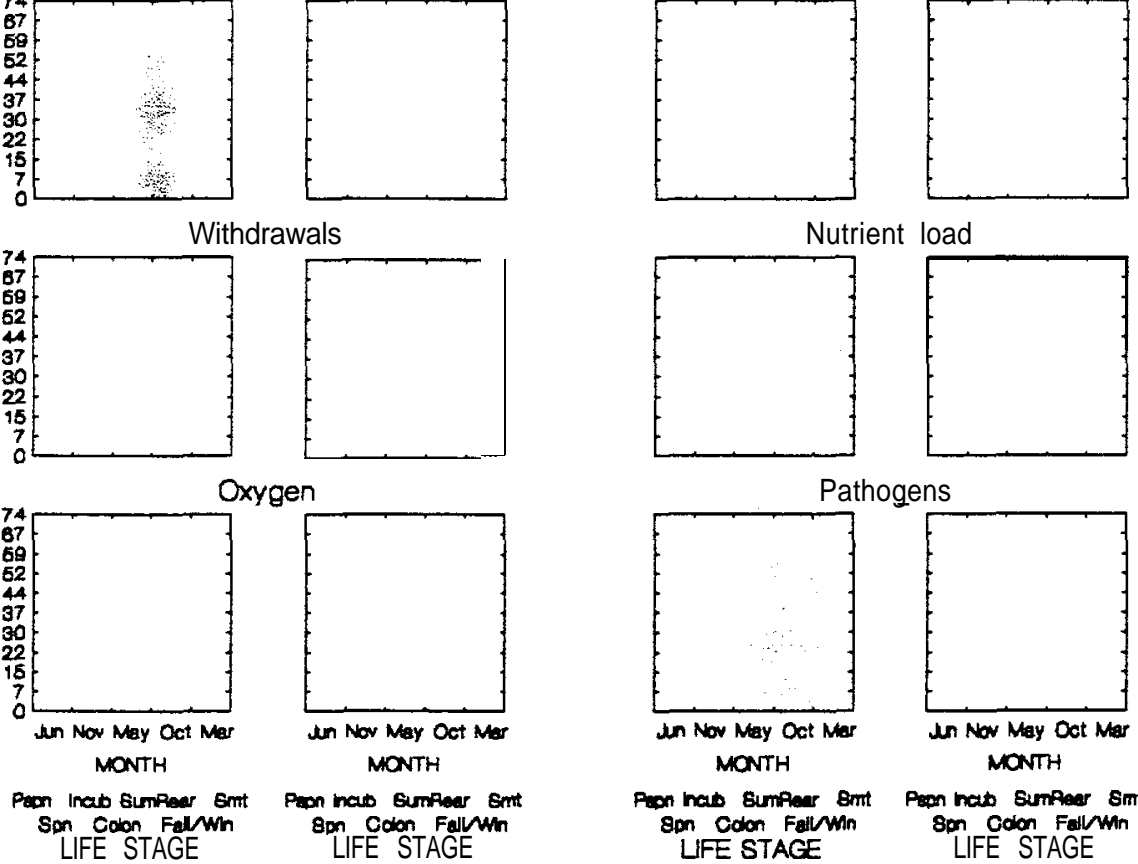

Nutrient load

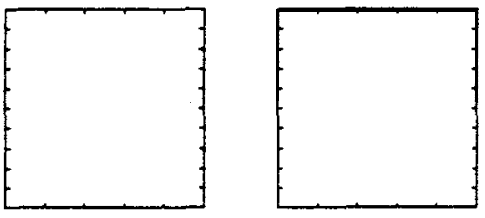

Pathogens

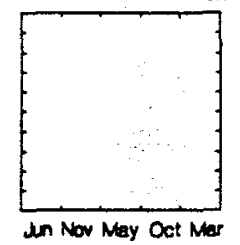

MONTH

Fron naw Sumpeer $8 \mathrm{mt}$

Son Codon Follumn

LIFE STAGE

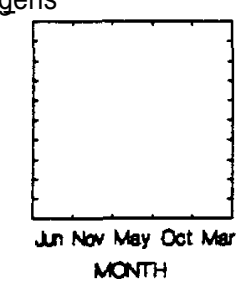

Level of

Effect

(1) $\quad$ effect

$\square$ Low

Moderate

High

- Lethal

Pron naw Sumpeer Simt Son Coion Falkmn 


\section{Chesnimnus $\mathrm{Cr} /$ Joseph Cr/Grande Ronde R Environmental Attributes}

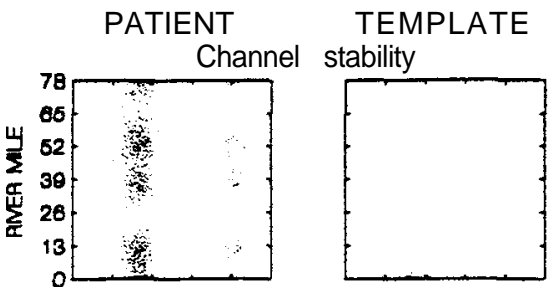

Habitat type diversity
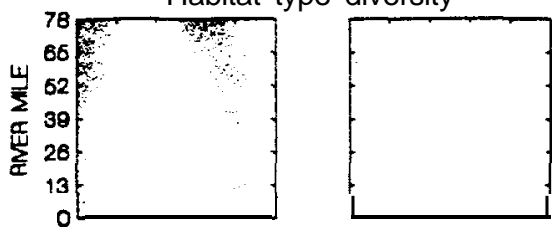

Temperature
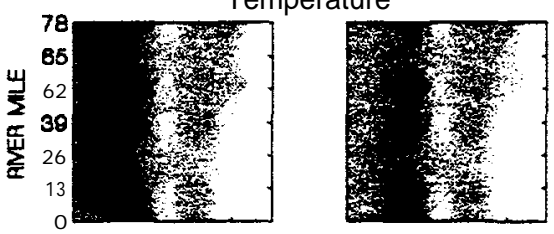

Predators
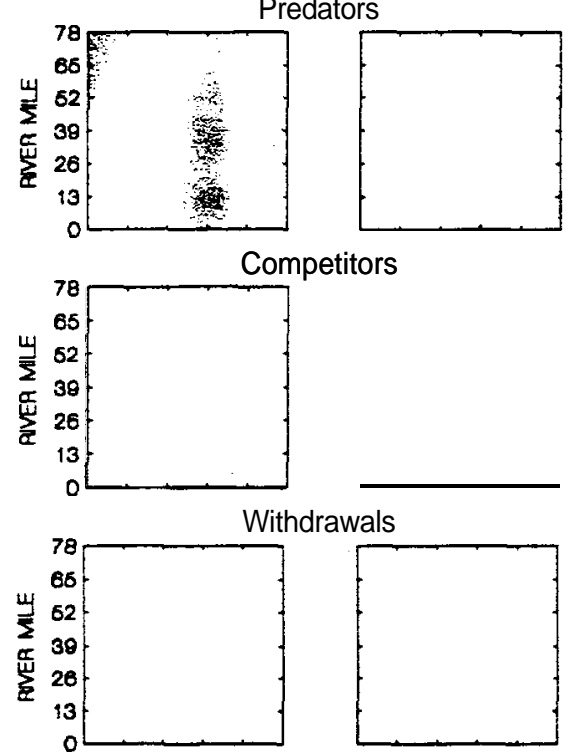

Withdrawals

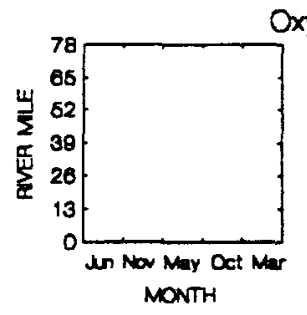

Oxygen

Puon mas bumfer 8mt Son Coon Faln

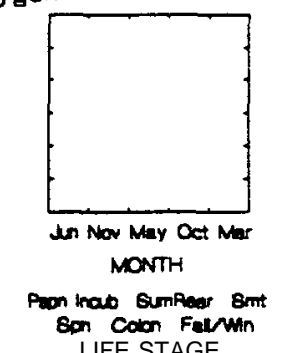

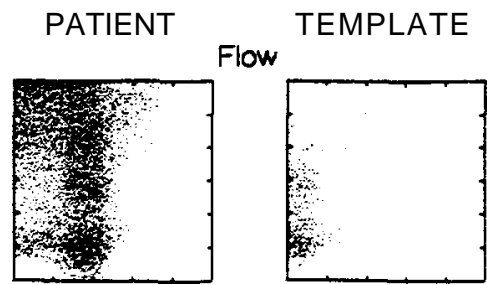

Sediment load

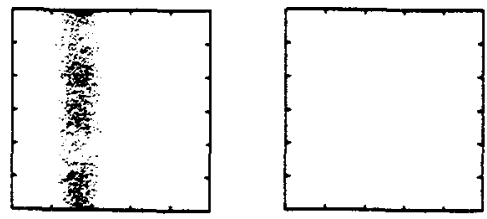

Rioarian condition

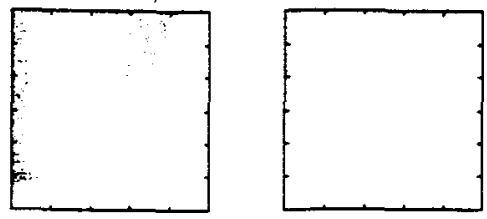

Chemicais

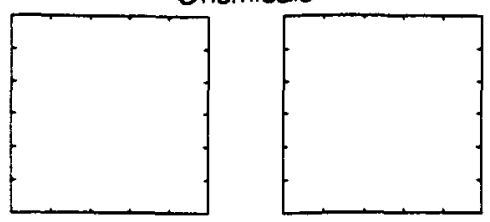

Obstructions

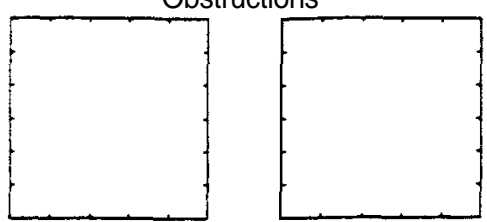

Nutrient load

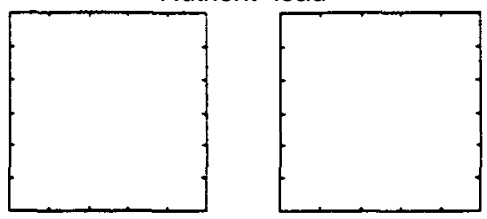

Pathogens

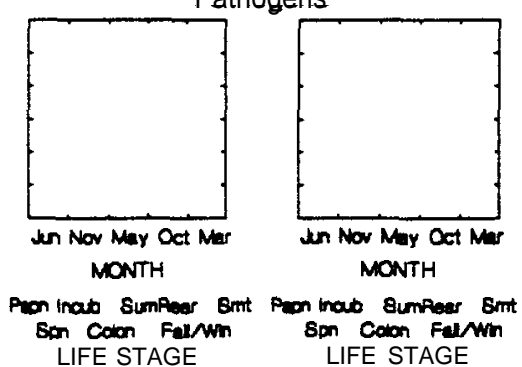

Level of Effect

No effect

cl Low

$\square$ Moderate

a High

Lethal 


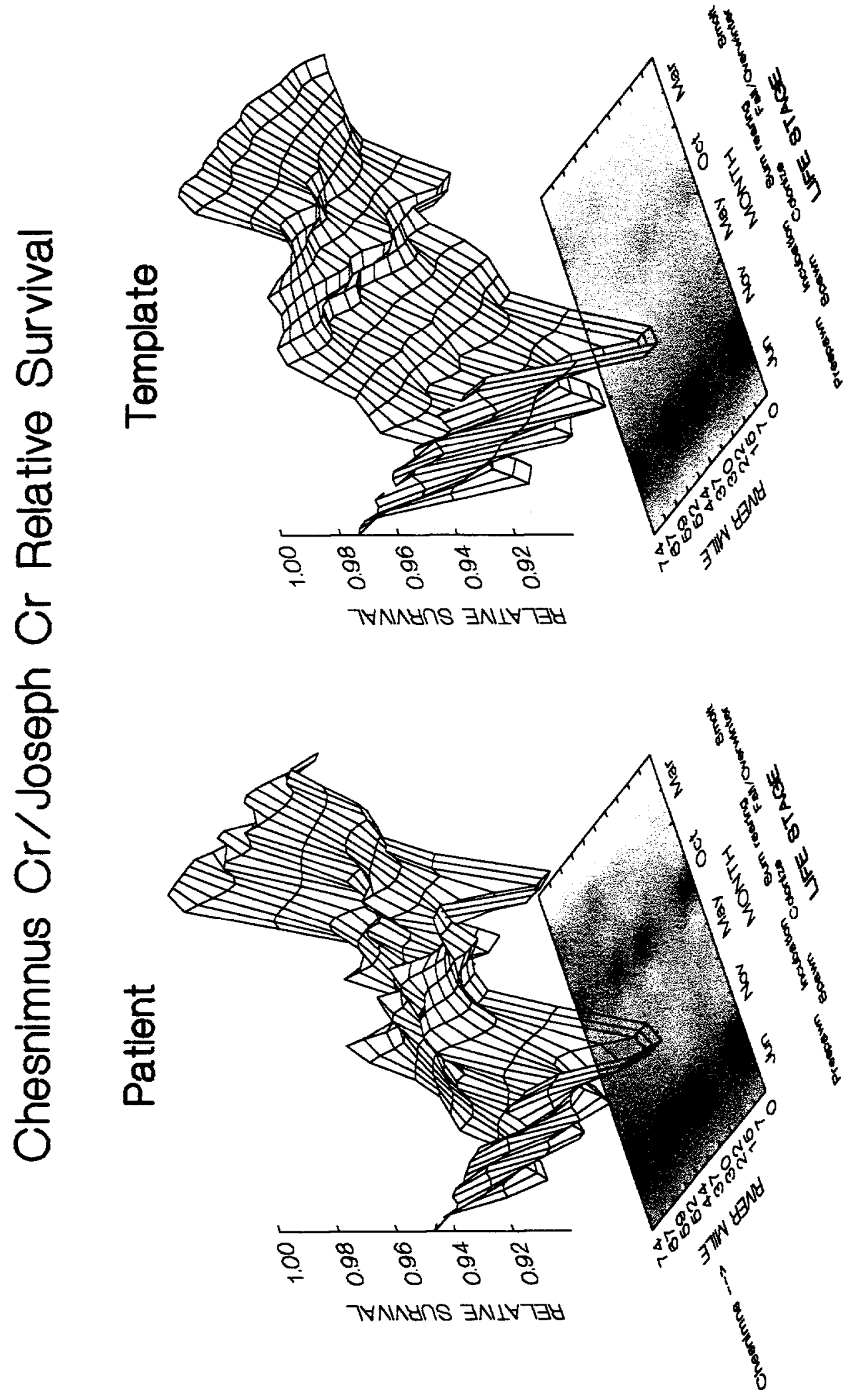


Chesnimnus Cr/Joseph Cr/Grande Ronde R Key Habitat Quantity

Patient

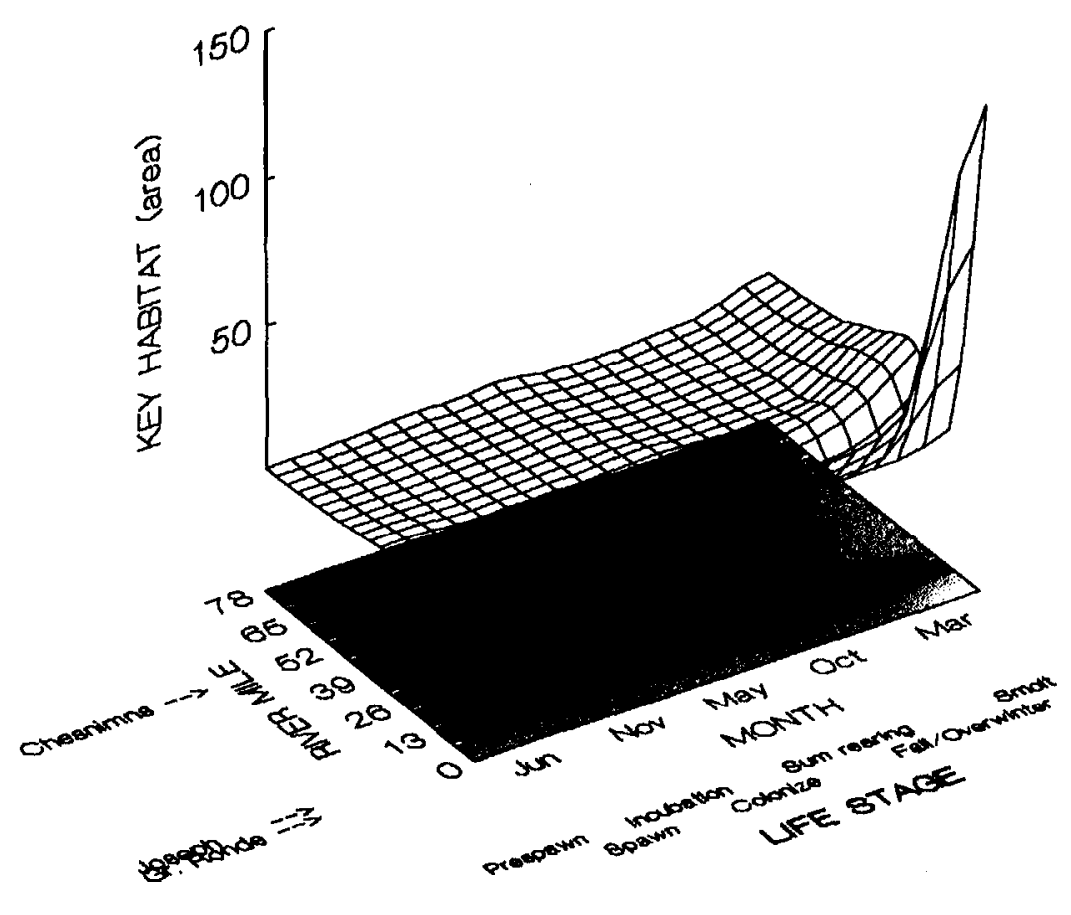

Template

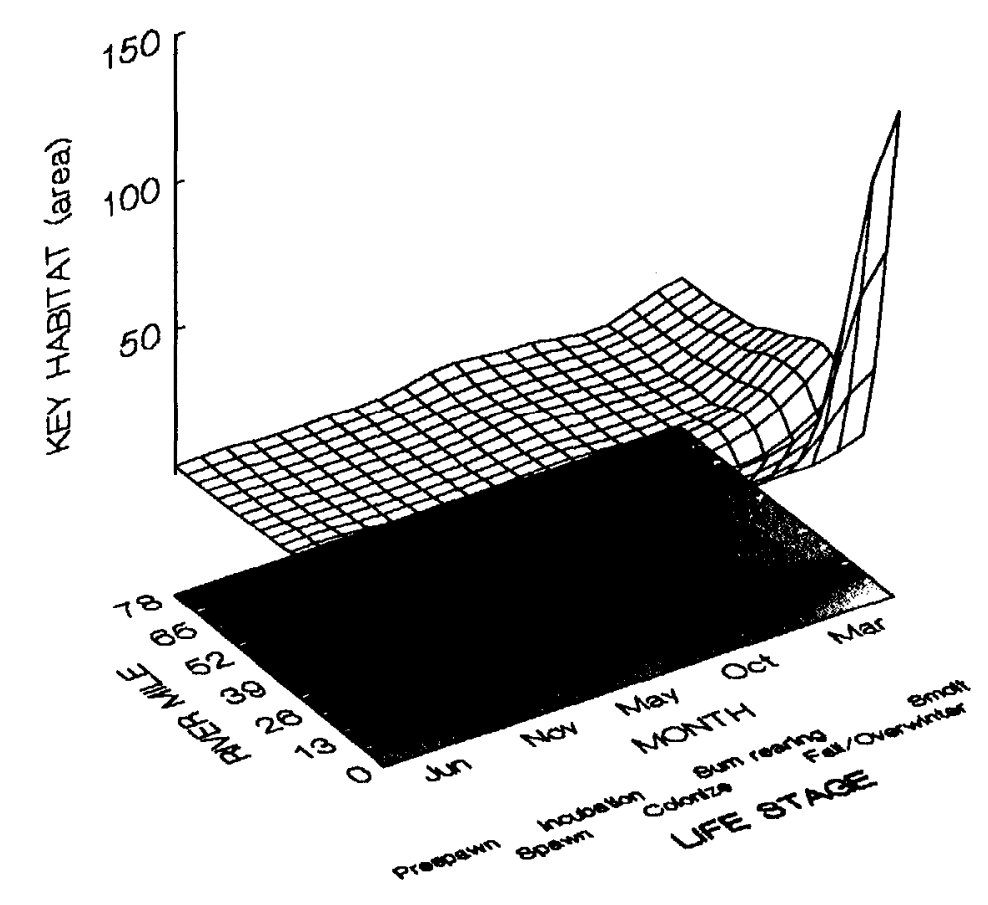




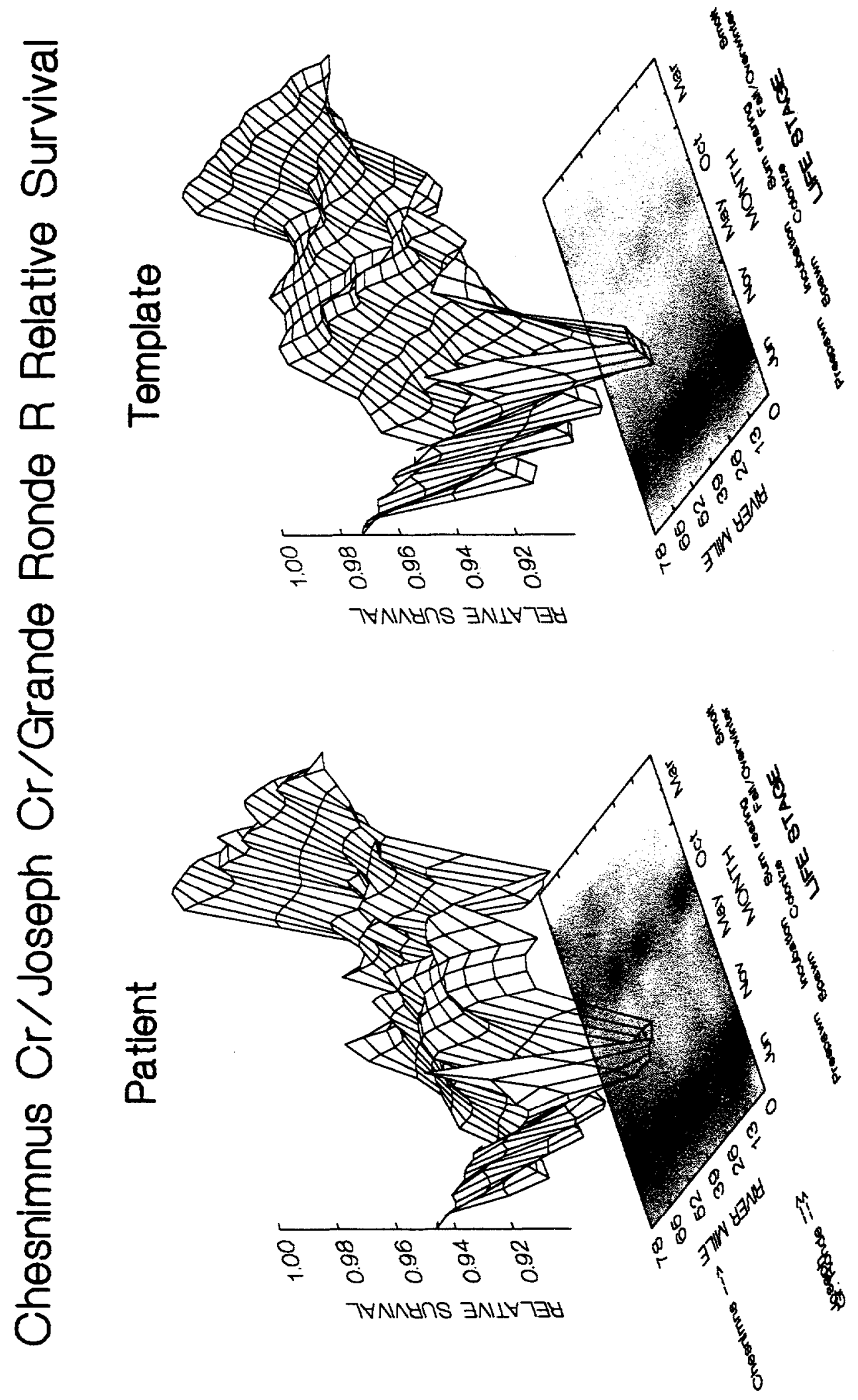




\section{Stream Reach Range}

Wenaha R / Grande Ronde R 


\section{Patient-Template Analysis Summary}

Stream Reach Range: Wenaha R./Grande Ronde R.

\section{Key Habitat Quantity}

The quantity of key habitat for spring chinook in the Wenaha River is virtually unchanged compared to historic levels. The North Fork Wenaha River is not shown in the charts but its quantity of key habitat is comparable to that shown for the South Fork.

The quantity of key habitat in the Grande Ronde River downstream of the Wenaha River is also unchanged compared to historic levels. A greater amount of key habitat occurs in the mainstem Grande Ronde River than in the Wenaha River (for a given channel length) at most life stages.

\section{Relative Productivity (Survival)}

The capability of the mainstem Wenaha River of supporting spring chinook productivity (survival) is virtually unchanged compared to historic conditions. In general, survival conditions are excellent over approximately the upper half of the Wenaha River, including its forks. The lower 14 miles of mainstem have conditions less favorable for survival, with conditions particularly poor for prespawners, spawners, and egg incubation downstream of about river mile 7 .

Survival conditions within the Grande Ronde River downstream of the Wenaha River have worsened compared to those that existed historically, although these reaches have always been unfavorable for survival during some life stages. In particular, survival conditions have always been poor in the later part of the prespawning stage and during the spawning and incubation stages. These conditions are mainly due to elevated water temperatures, sediment load, channel stability, habitat diversity, and the presence of predators. 


\section{Wenaha R Key Habitat Quantity}

\section{Patient}

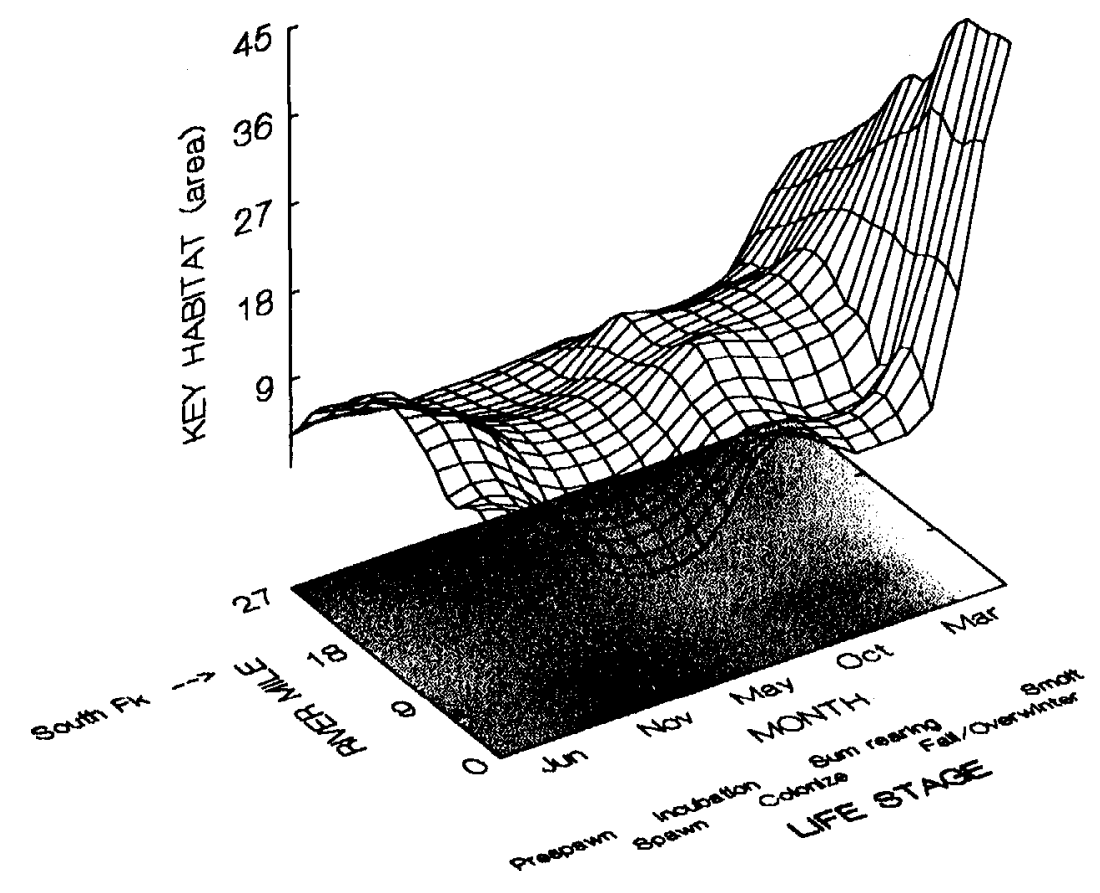

Template

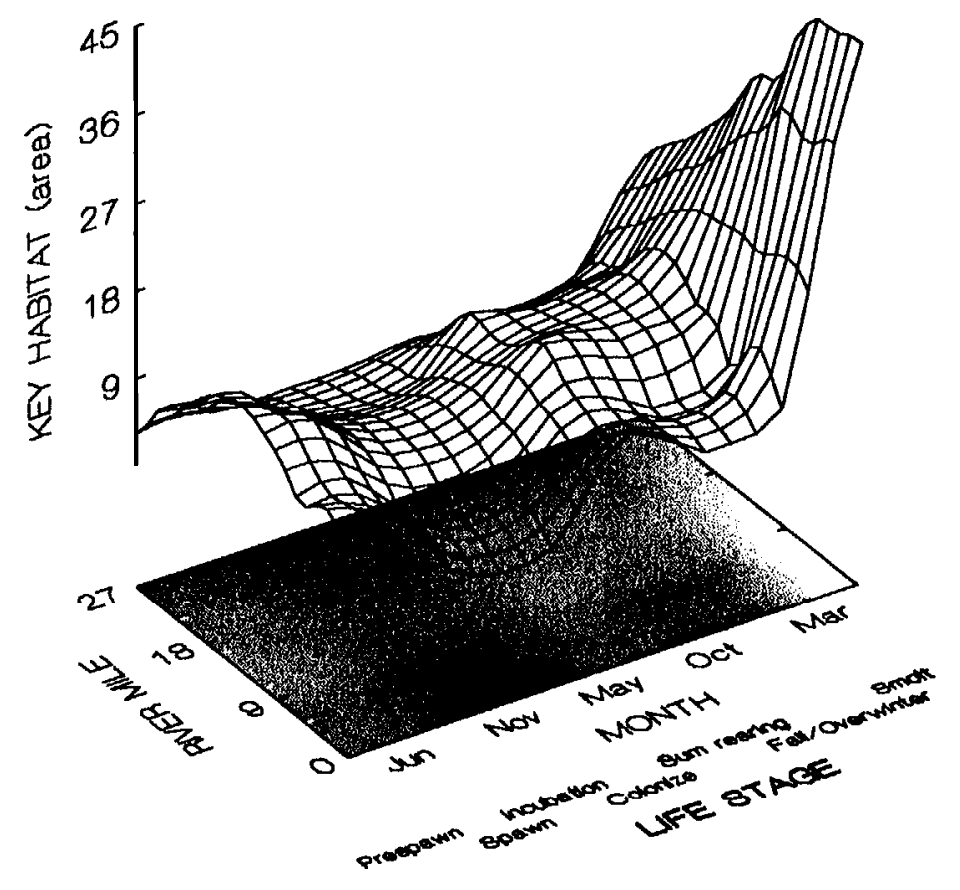


Spawning reach: Imnaha $\mathrm{R}$.

South Fork Imnaha R (RM 71.4 - 76.1 from Snake R)

Relative Productivty
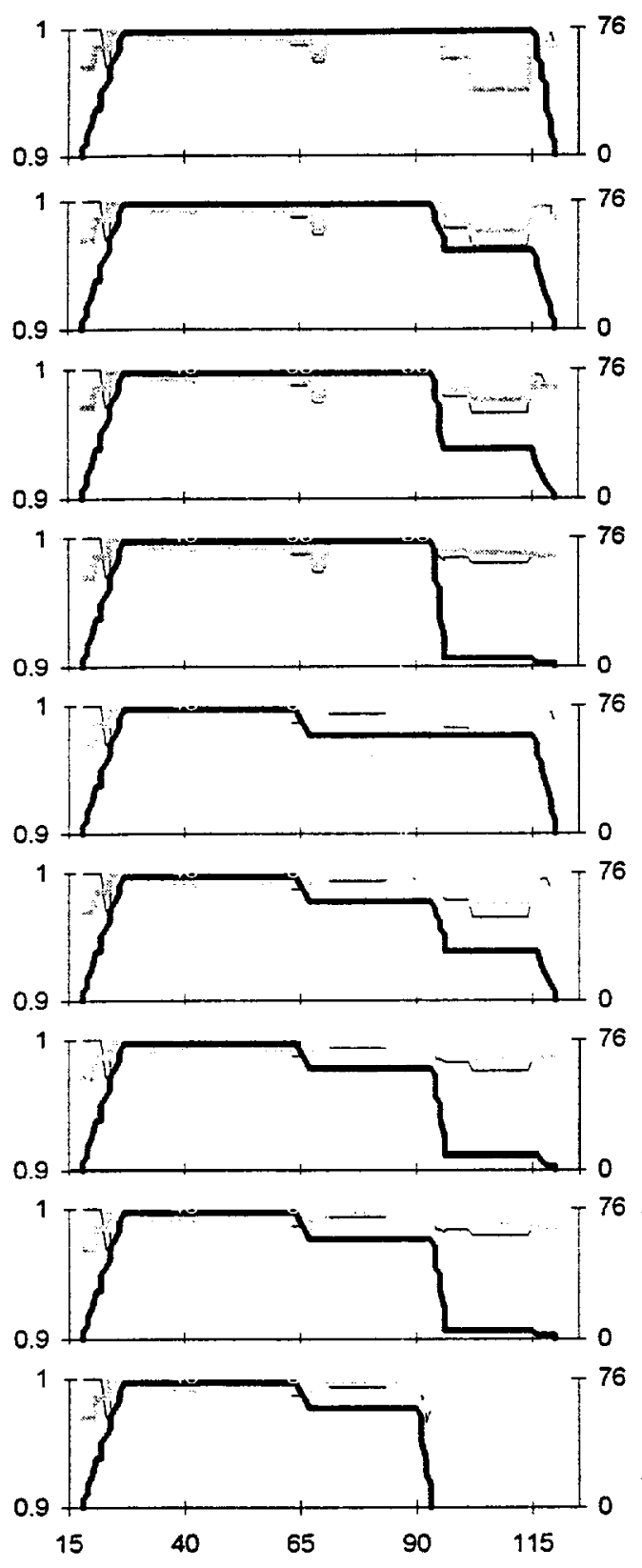

Statistical week

Prespawn -

Spawning

Incubation

Colonization

Sum. rearing

Win. rearing
Relative Key Habitat Quantity
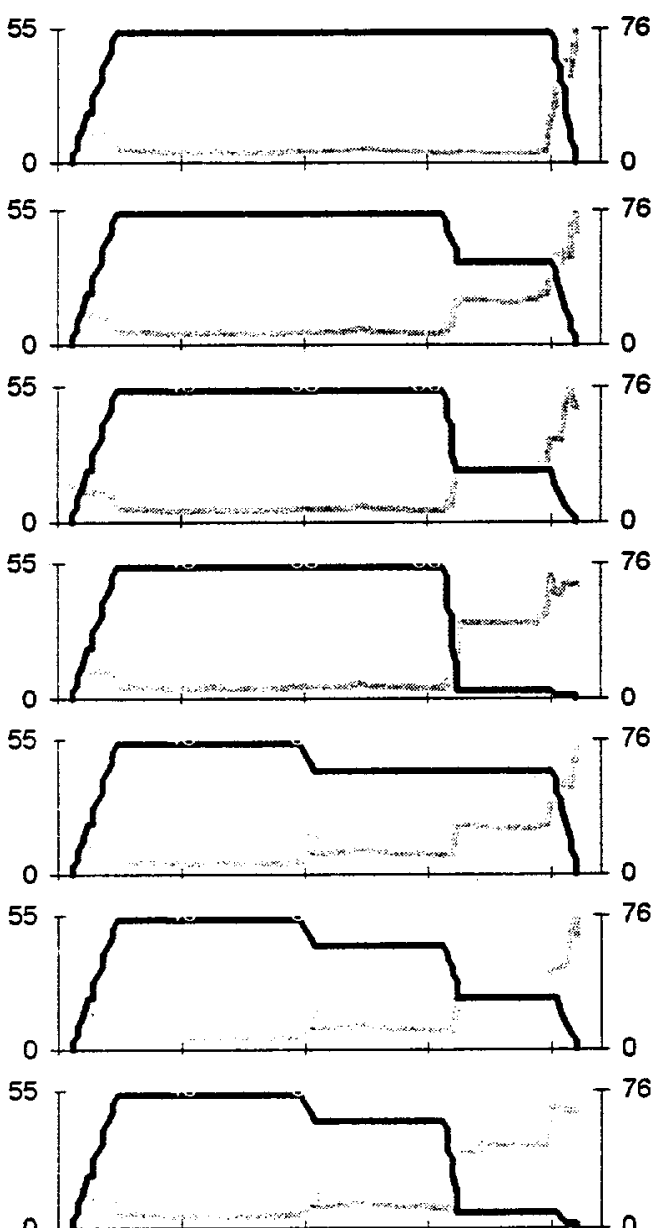

55
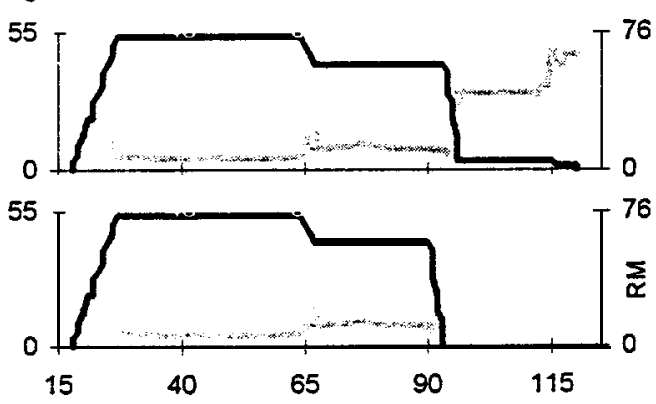

Statistical week

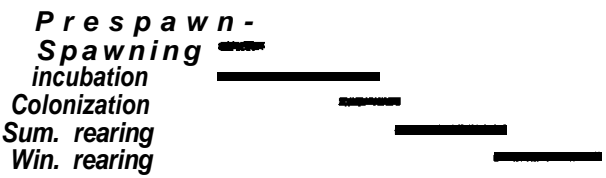

\begin{tabular}{|c|c|}
\hline \multicolumn{2}{|c|}{ Template } \\
Patient
\end{tabular}

Traj. \#1

20250.19

238 0.19

Traj. \#2

288 . 0.29

$288 \quad 0.25$

Traj. \#3

280.0 .29

$281 \quad 0.25$

Traj. \#4

$340 \quad 0.35$

$346 \quad 0.30$

Traj. \#5

\begin{tabular}{l|l}
332 & 0.38 \\
\hline
\end{tabular}

$\begin{array}{ll}327 & 0.33\end{array}$

Traj. \#6

\begin{tabular}{l|l}
\hline 280 & 0.33 \\
\hline
\end{tabular}

$\begin{array}{ll}272 & 0.27\end{array}$

Traj. \#7

\begin{tabular}{l|l}
\hline 316 & 0.37 \\
\hline
\end{tabular}

308 0.31

Traj. \#8

\begin{tabular}{l|l}
336 & 0.40
\end{tabular}

\begin{tabular}{l|l}
331 & 0.34
\end{tabular}

Traj . \#9

638 . 0.81

\begin{tabular}{l|l|}
718 & 0.78
\end{tabular} 


\section{Wenaha R Environmental Attributes}

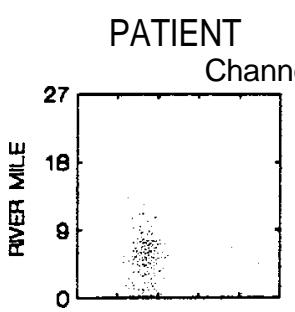

TEMPLATE

Channel stability

Habitat type diversity
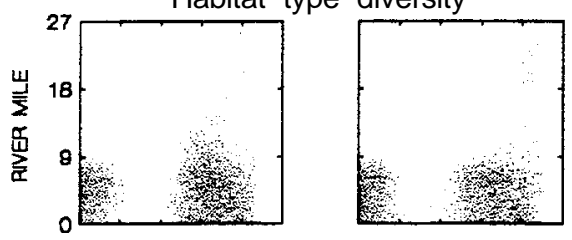

Temoerature

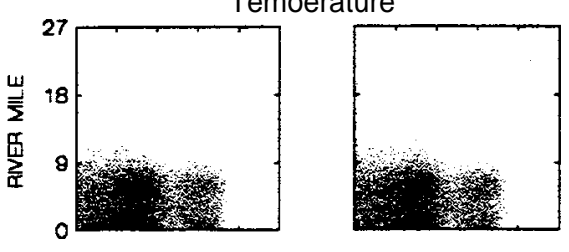

Predators

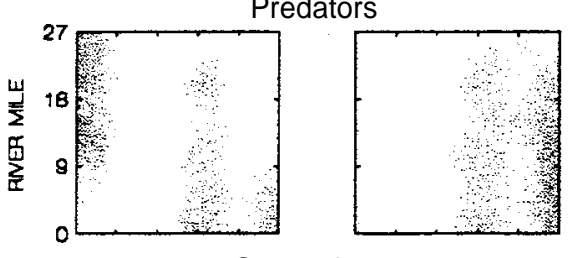

Competitors
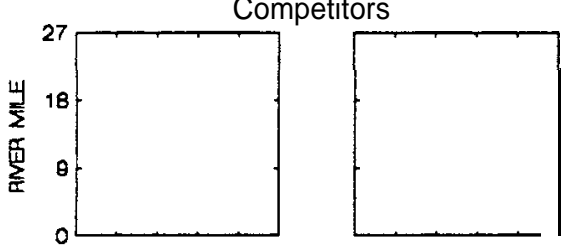

Withdrawals
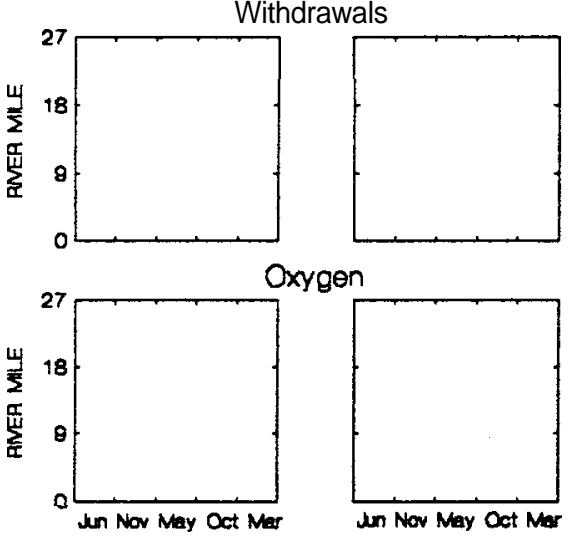

MONTH

Oxygen

Peon Irab Sumplear 8nt Pan thab 8umpiear Bnt Son Colon Falwh Gon Colon Fallwin UFE STAGE

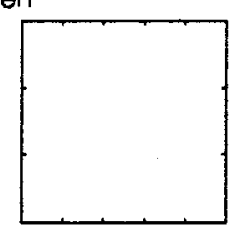

MONTH

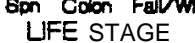

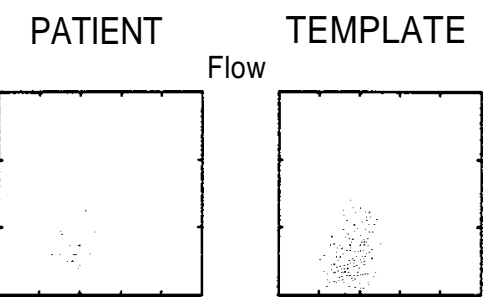

Sediment load

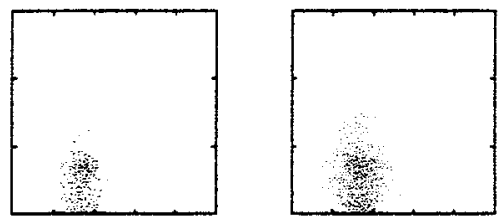

Riparian condition

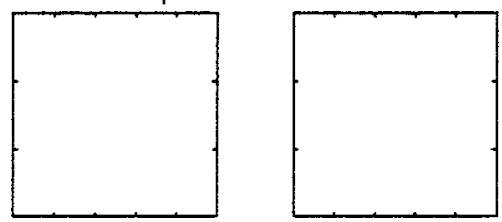

Chemicals

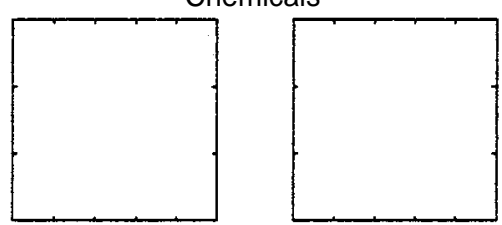

Obstructions

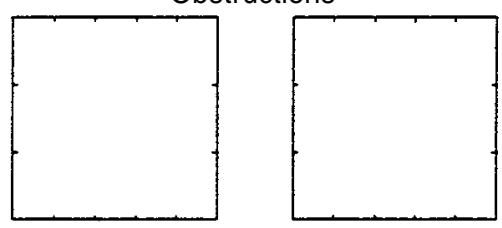

Nutrient load
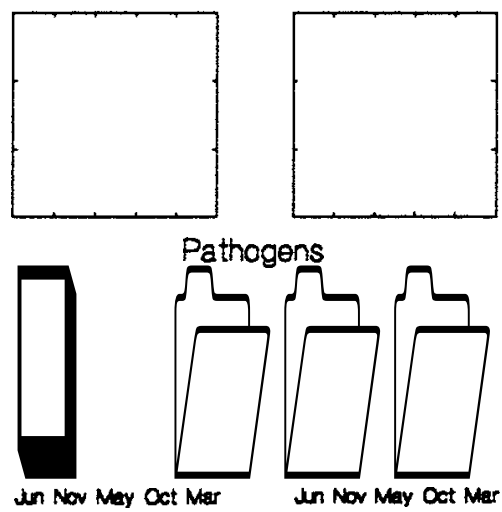

MONTH

NONTH

Feon hab Sumflas 8mi fron Irab Sumfoer 8mt Son Coion Fallum son coln Fallwin LIFE STAGE LIFE STAGE
Level of

Effect

№ effect

$\square$ Low

$\square$ Moderate

High

- Lethal 


\section{Wenaha RRelative Survival}
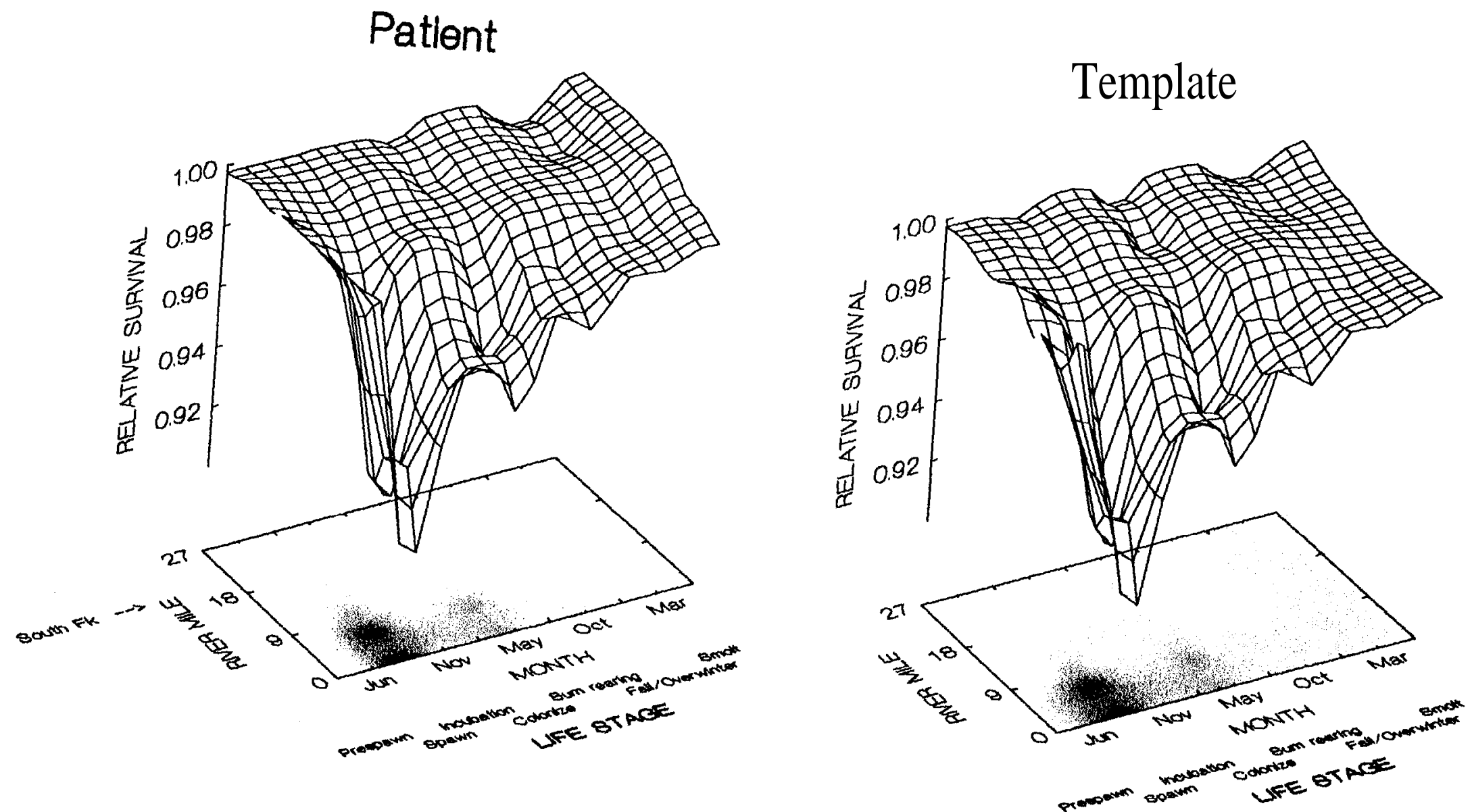


\section{Wenaha R/Grande Ronde R Key Habitat Quantity}

\section{Patient}

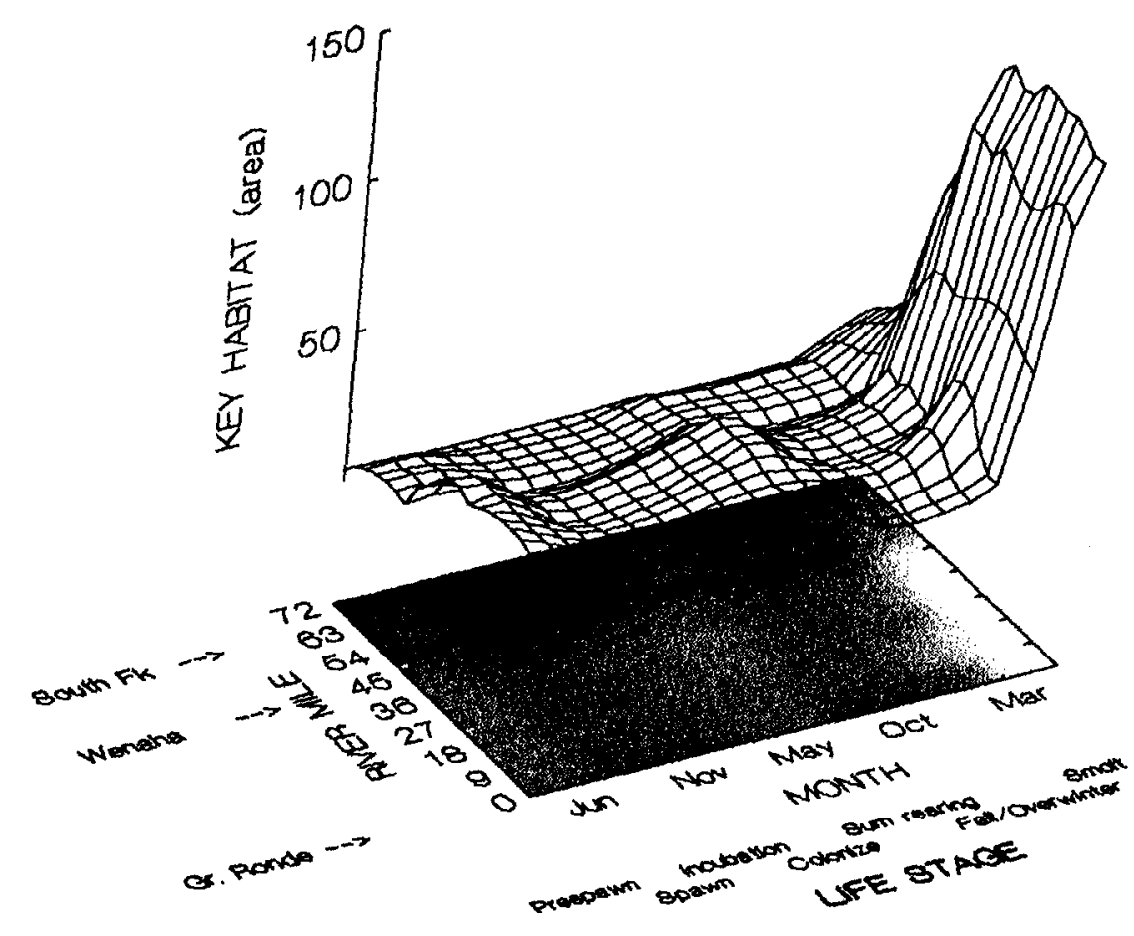

Templa te

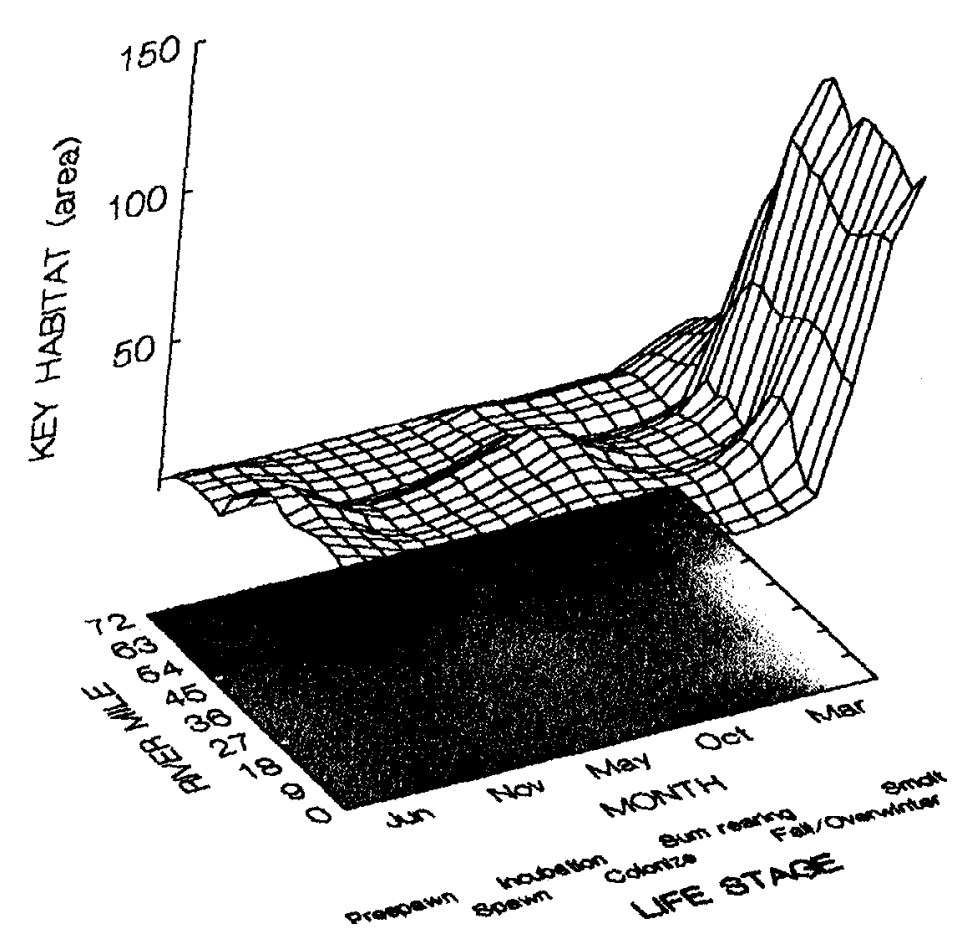




\section{Wenaha R/Grande Ronde R Environmental Attributes}

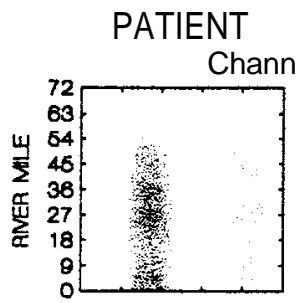

TEMPLATE

Habitat type diversity
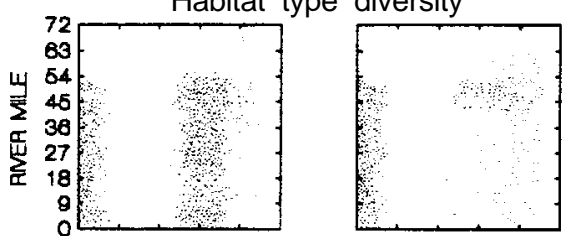

Temperature
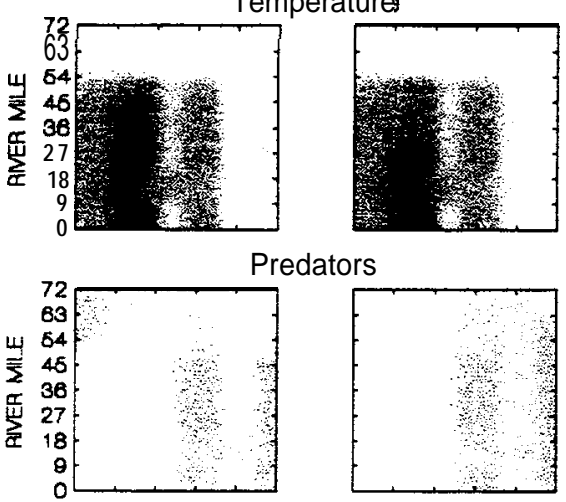

Predators
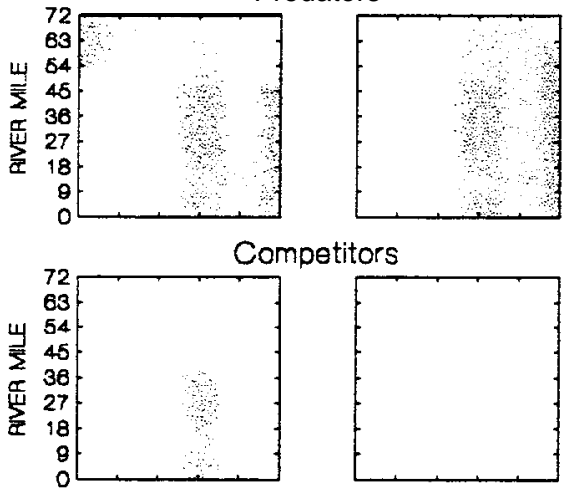

Competitors

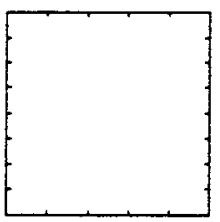

Withdrawals
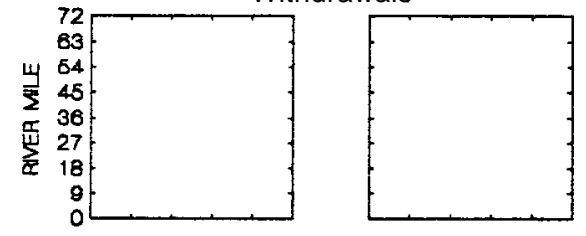

Oxygen

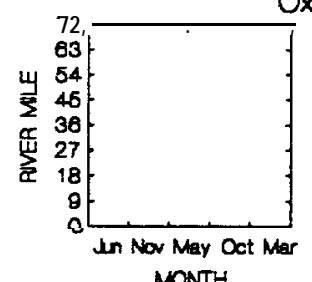

$$
\text { MONTH }
$$

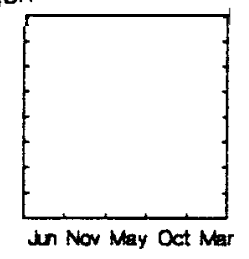

$$
\text { MONTH }
$$

Paon hat Bumfeer $S \mathrm{mt}$ Pan inat Bumpear $5 \mathrm{mt}$ Son Colon Fallwn Bon Colon Falumn

\author{
PATIENT \\ TEMPLATE
}

Flow

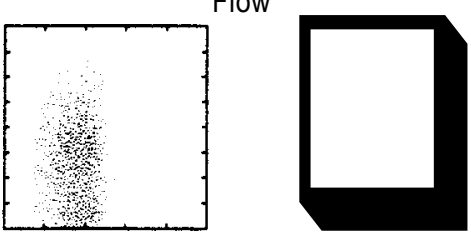

Sediment load

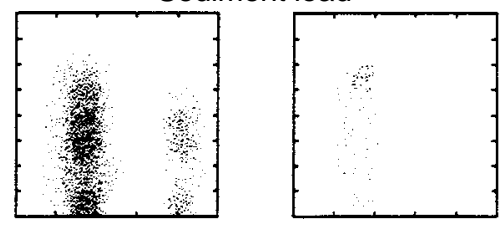

Riparian condition

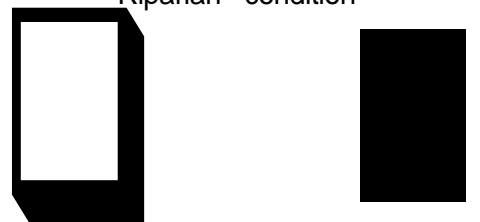

Chemicals

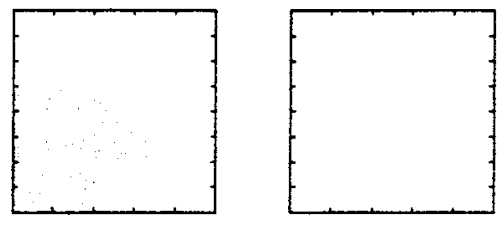

Obstructions

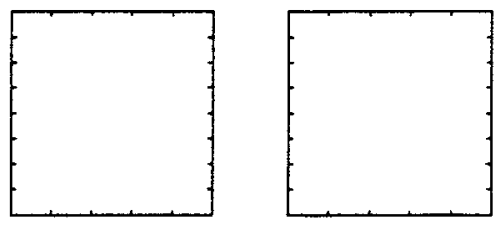

Nutrient load

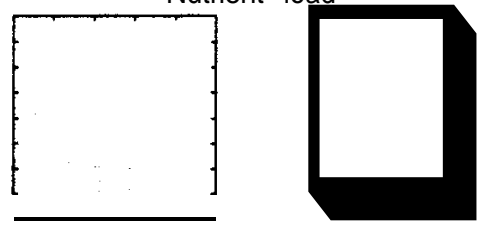

Pathoaens

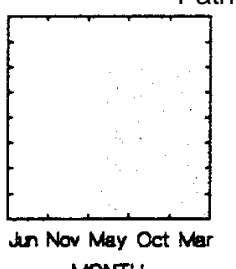
MONTH

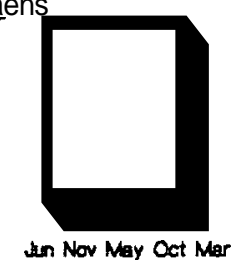

MONTH

Paon inat Bumfoer $s \mathrm{~m} \mathrm{t}$ Spn Colon Falliwn UFE STAGE
Puon Inat Sumflear Emt Bpo Colon Fallwh

LIFE STAGE
Level of

Effect

- No effect

El Low

口 Moderate

图 High

Lethal 


\section{Wenaha R/Grande Ronde R Relative Survival}
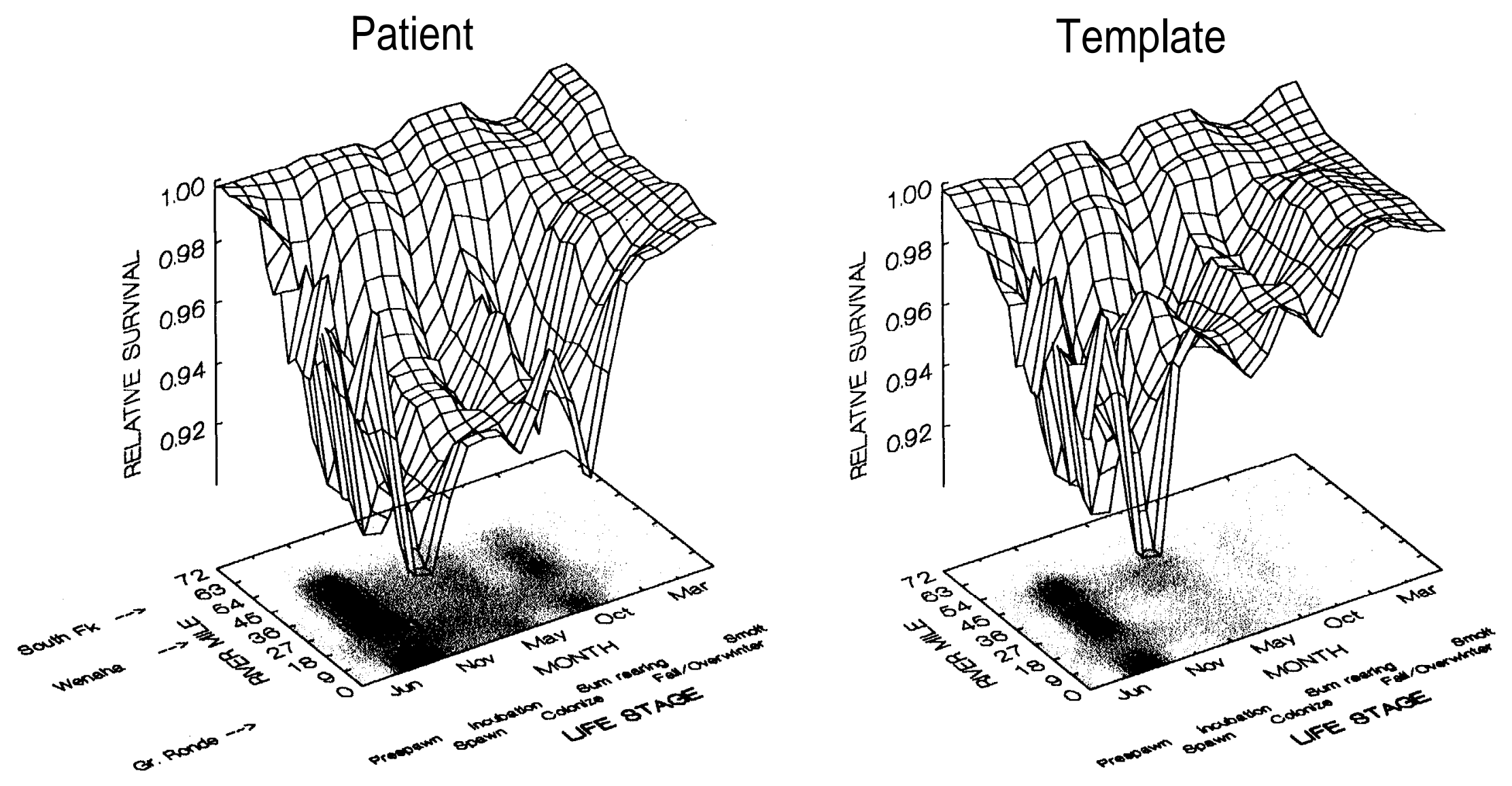
Stream Reach Range

Wallowa R / Grande Ronde R 


\section{Patient-Template Analysis Summary}

\section{Stream Reach Range: Wallowa R./Grande Ronde R.}

\section{Key Habitat Quantity}

The quantity of key habitat for most life stages of spring chinook throughout most of the mainstem Wallowa River has been substantially reduced compared to historic levels. Only habitat for the smolt stage has been largely unaffected. Habitat quantities in the lower ten miles (downstream of Minam River) are relatively unchanged for all life stages, however.

The quantity of key habitat in the Grande Ronde River downstream of the Wallowa River is nearly unchanged compared to historic levels.

\section{Relative Productivity (Survival)}

The capability of the mainstem Wallowa River of supporting spring chinook productivity (survival) is sharply reduced compared to historic conditions for several life stages. Historically, all but approximately the lower 15 miles of this river had conditions for spring chinook survival that were likely the best in the entire Grande Ronde basin. This was due to moderating effects of Wallowa Lake on environmental attributes that typically increase mortality in streams not influenced by large lakes. Currently, conditions for survival during egg incubation, fry colonization, and early summer rearing are poorest high in the system, with conditions tending to improve downstream. Survival conditions have been worsened due to alterations in most environmental attributes, most notably, sediment load, nutrient load, and habitat diversity.

Survival conditions within the Grande Ronde River downstream of the Wallowa River have worsened compared to those that existed historically, although these reaches have always been largely unfavorable for survival during some life stages. In particular, survival conditions have always been poor in the later part of the prespawning stage and during the spawning and incubation stages. These conditions are mainly due to elevated water temperatures, sediment load, channel stability, habitat diversity, and the presence of predators.

Note: Spring chinook salmon are not believed to have ever spawned in the Wallowa River upstream of Wallowa Lake. 


\section{Wallowa RKey Habitat Quantity}

\section{Patient}

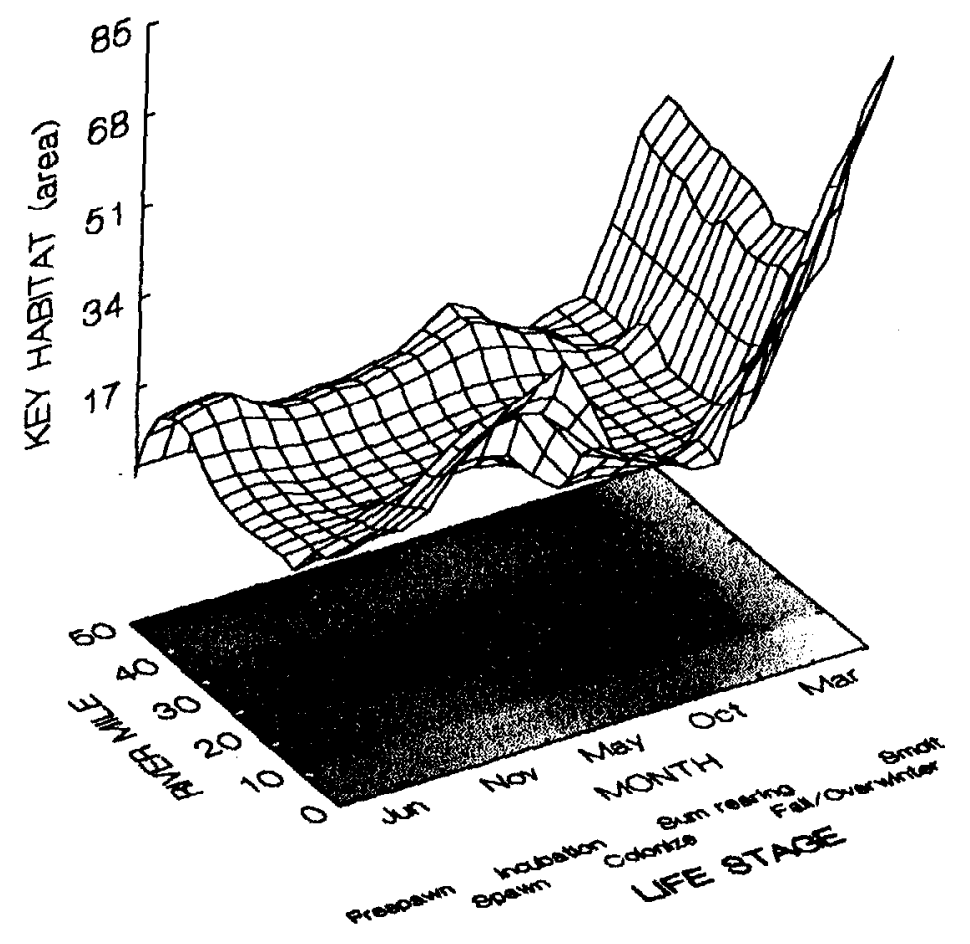

Template

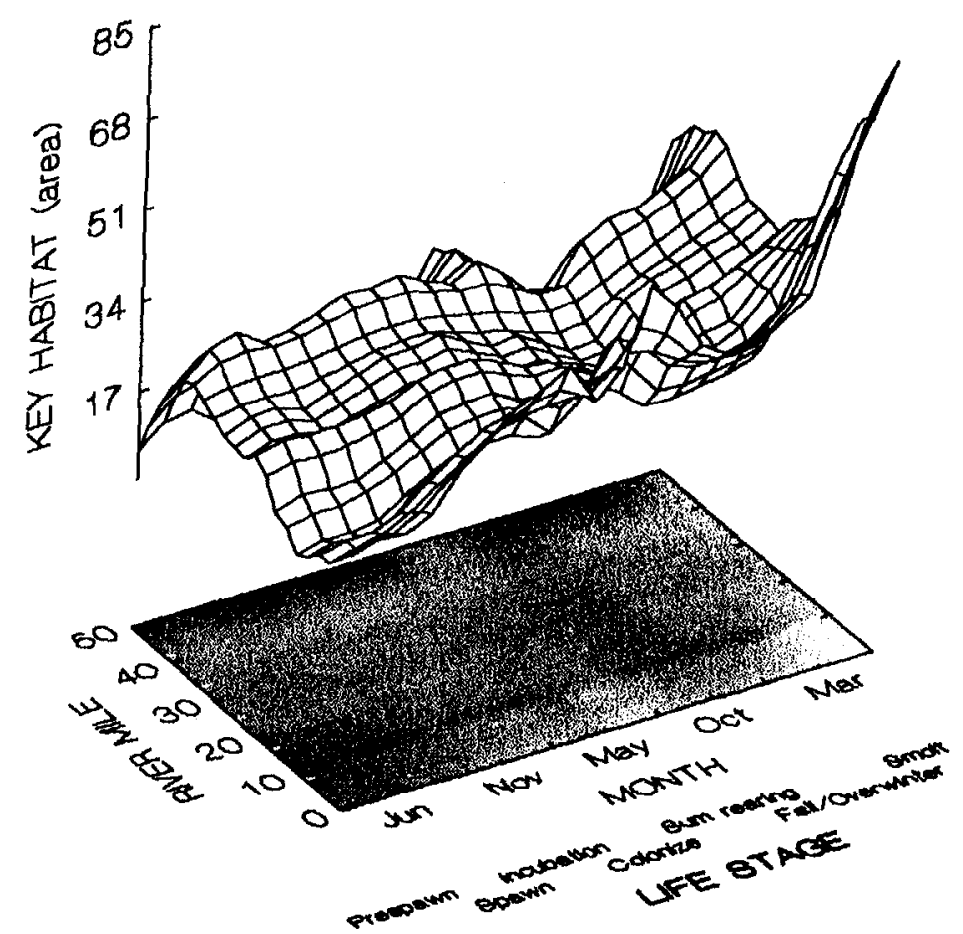




\section{Wallowa R Environmental Attributes}

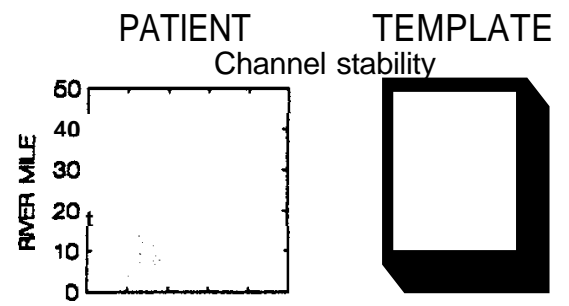

Habitat type diversity
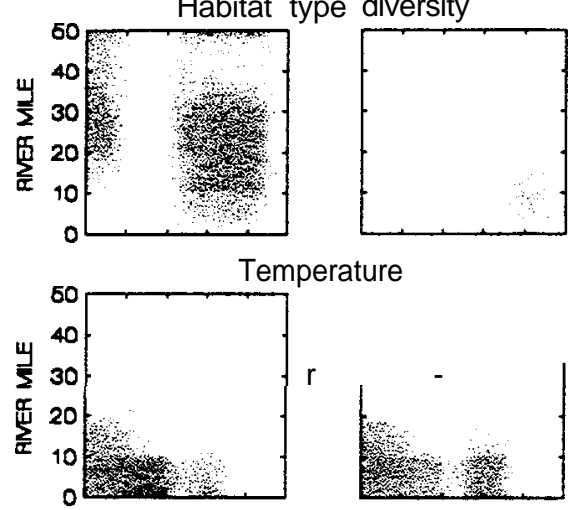

Predators
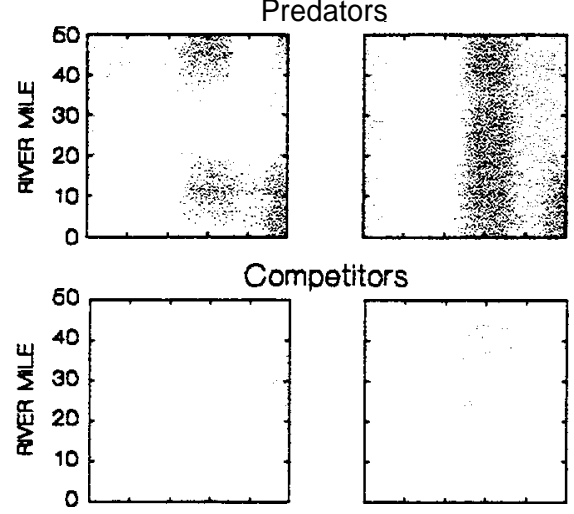

Withdrawals
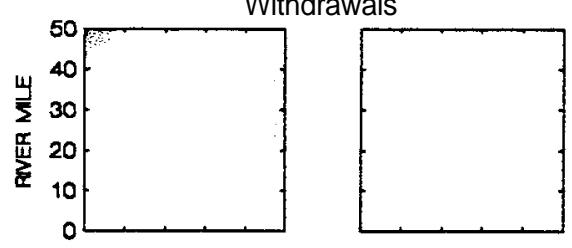

Oxygen

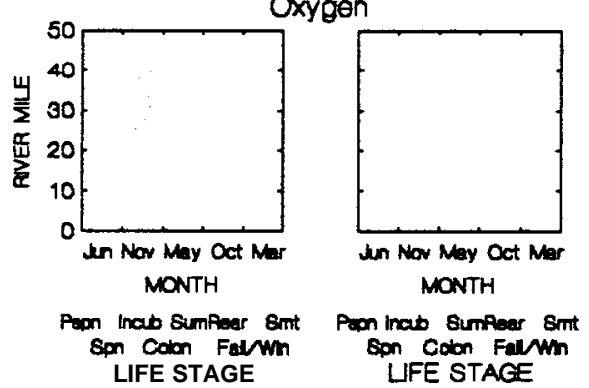

PATIENT TEMPLATE Flow

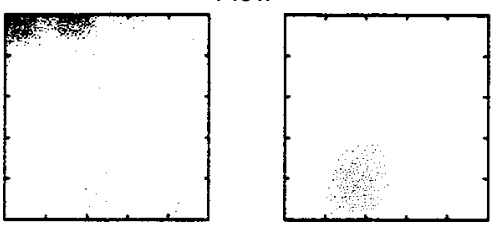

Sediment load

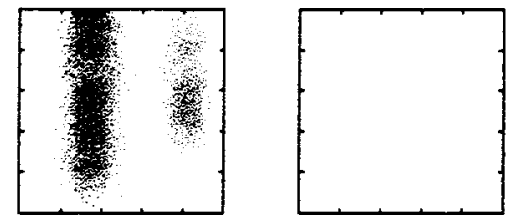

Riparian condition

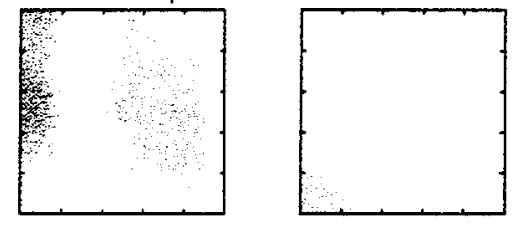

Chemicals

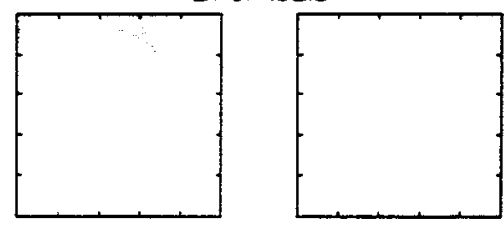

Obstructions

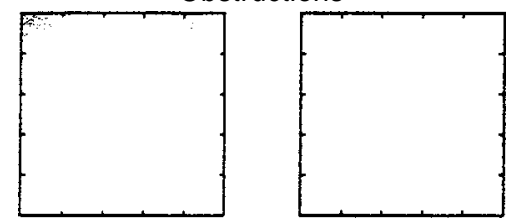

Nutrient load

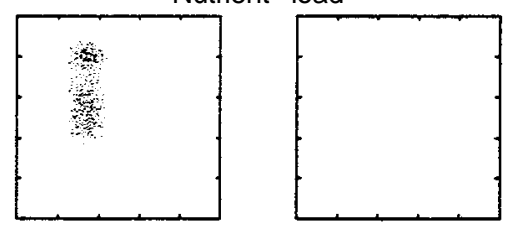

Pathogens

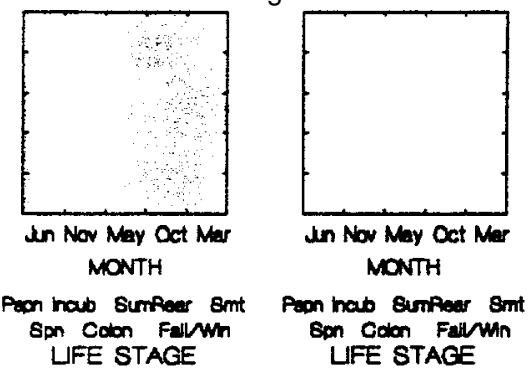

Level of

Effect

$\square$ No effect

$\square$ Low

- Moderate

a High

Lethal 


\section{Wallowa $R_{\text {Relative Survival }}$}

Pa tient

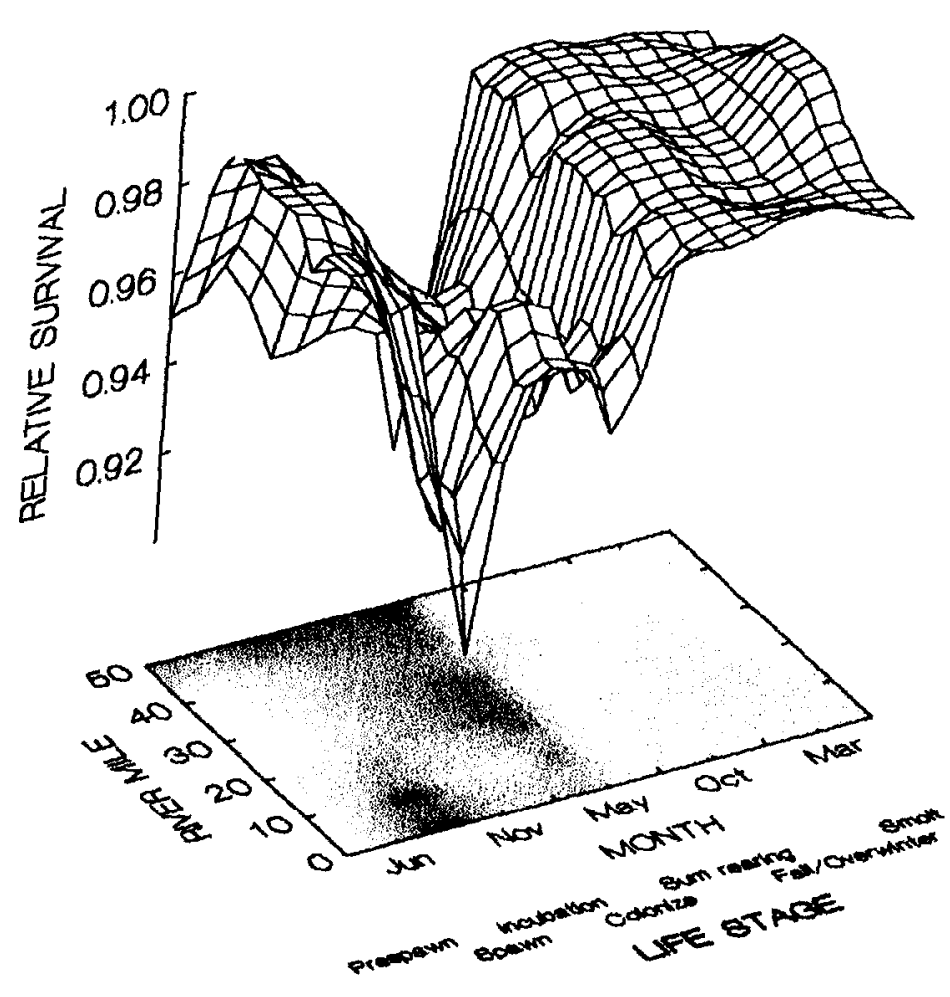

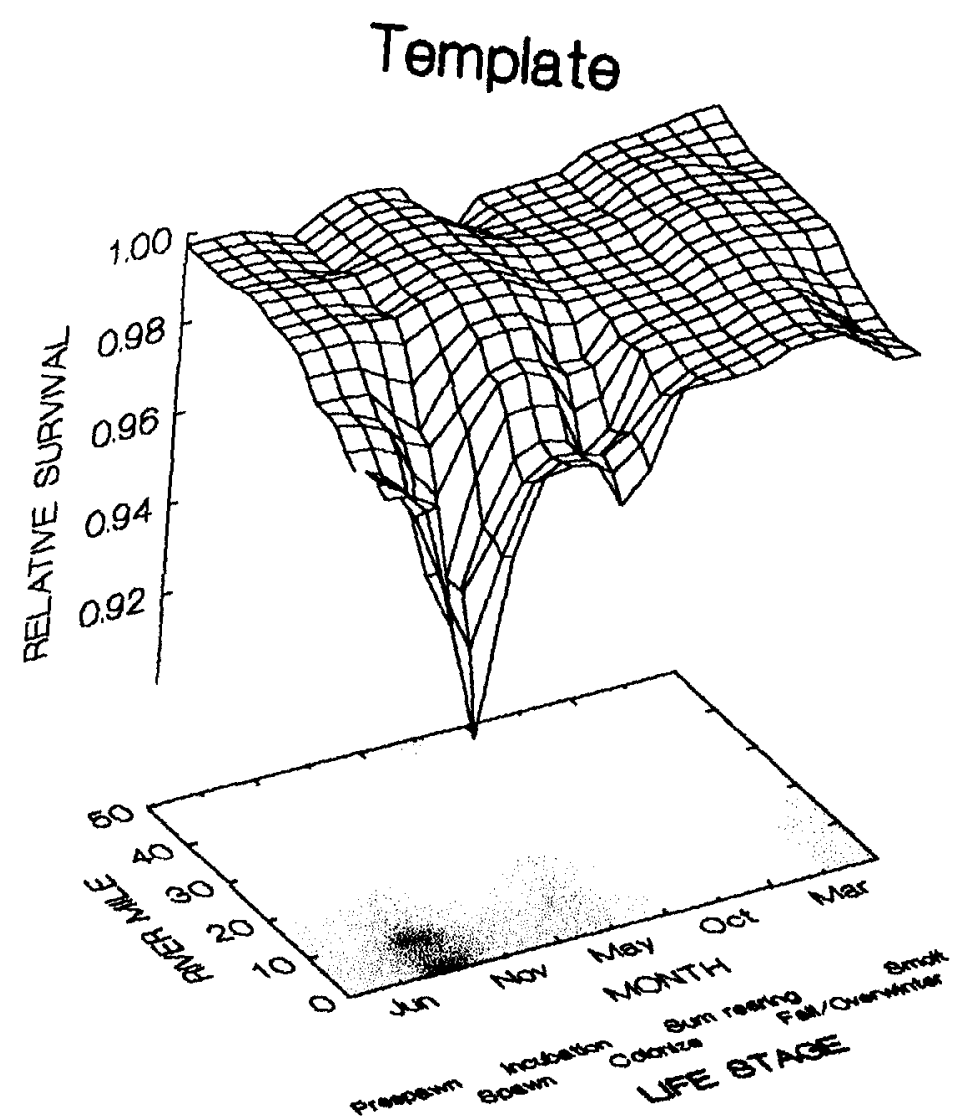




\section{Wallowa R/Grande Ronde R Key Habitat Quantity}

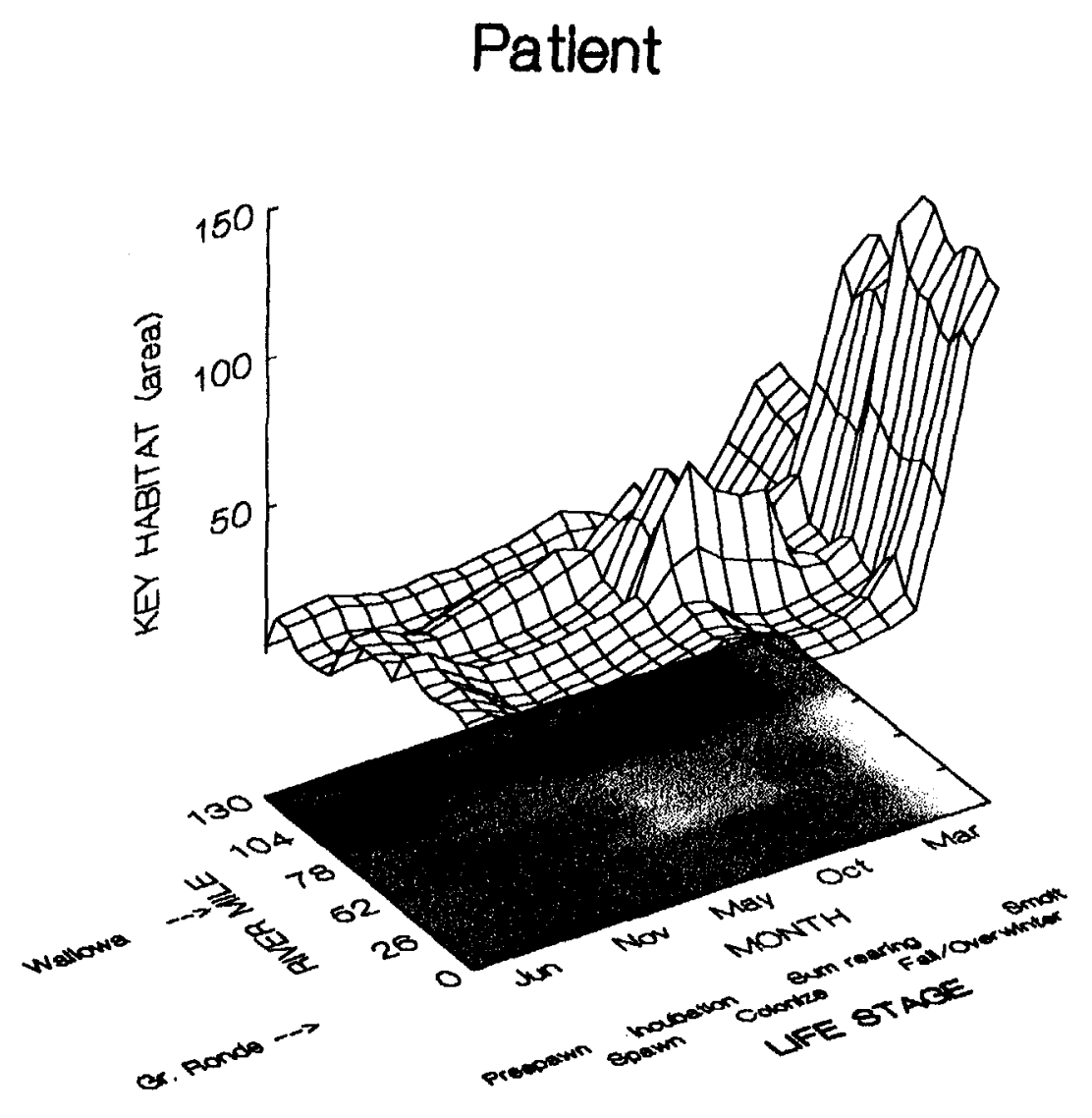

Template

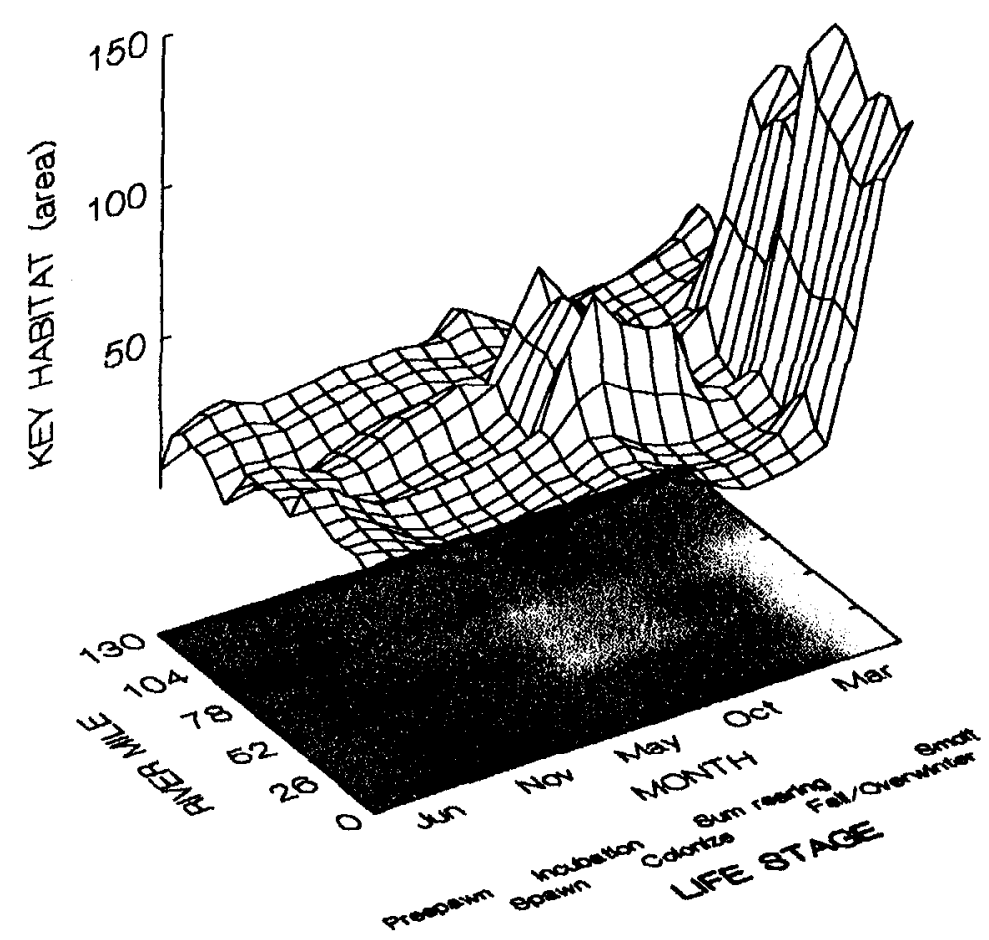




\section{Wallowa R/Grande Ronde R Environmental Attributes}

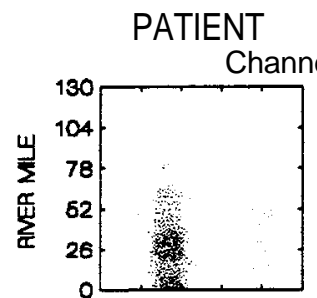

TEMPLATE

Habitat tyoe diversity
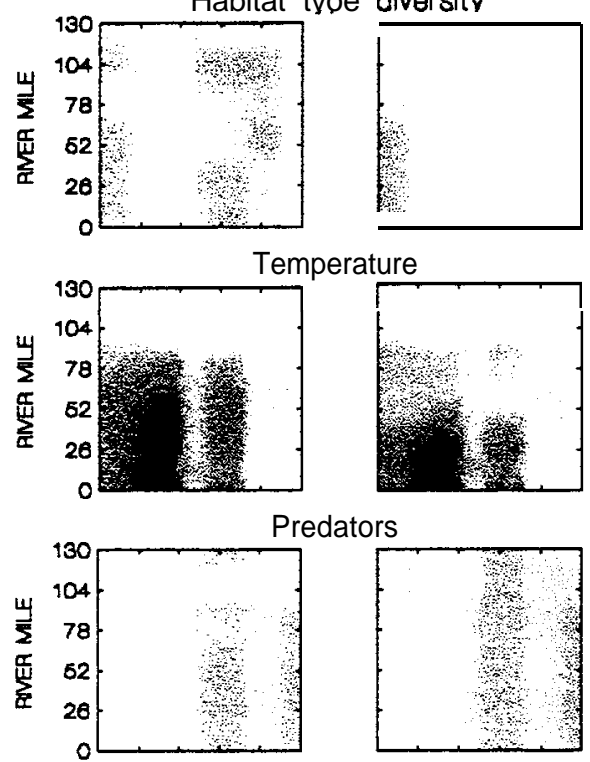

Predators

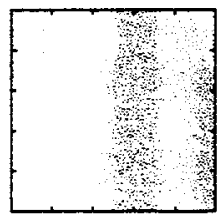

Competitors
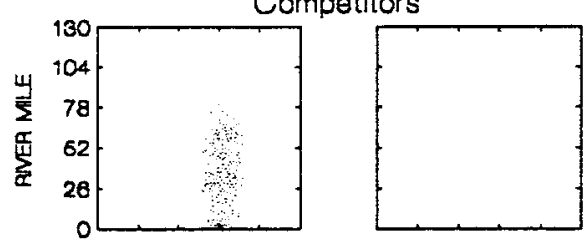

Withdrawals
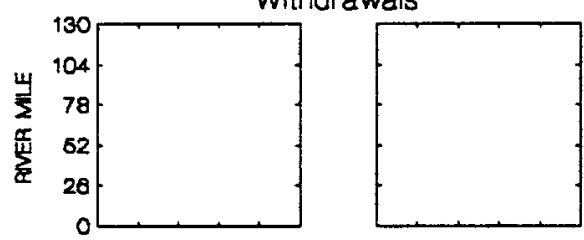

Oxygen
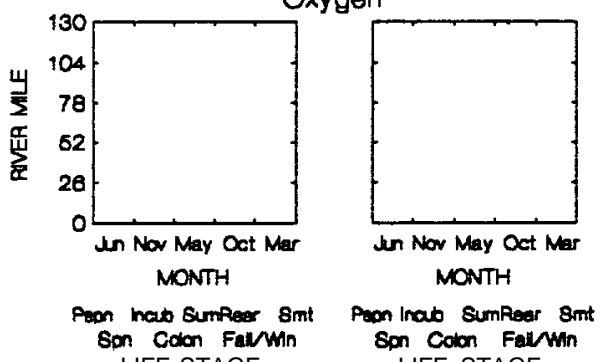

PATIENT

TEMPLATE

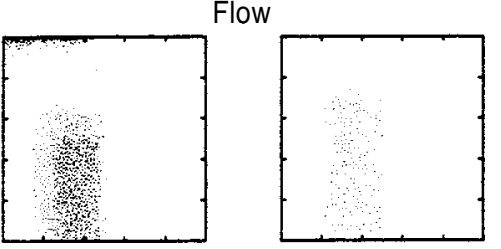

Sediment load
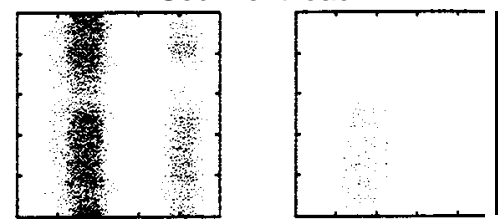

Riparian condition

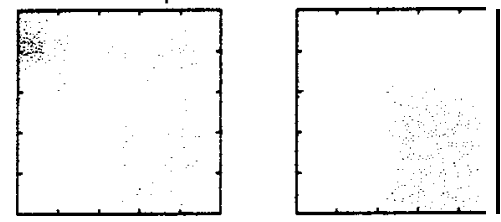

Chemicals

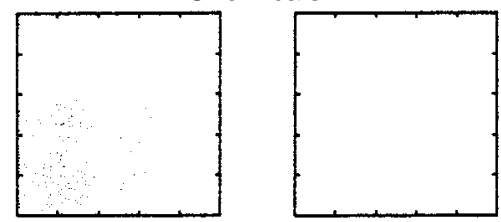

Obstructions

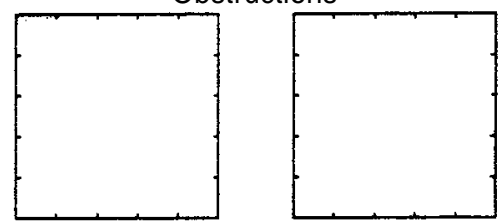

Nutrient load

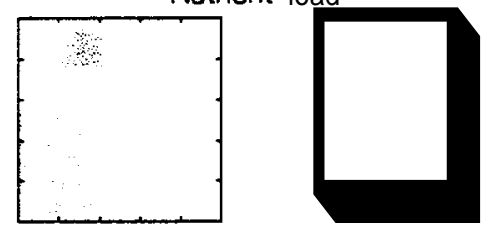

Pathogens

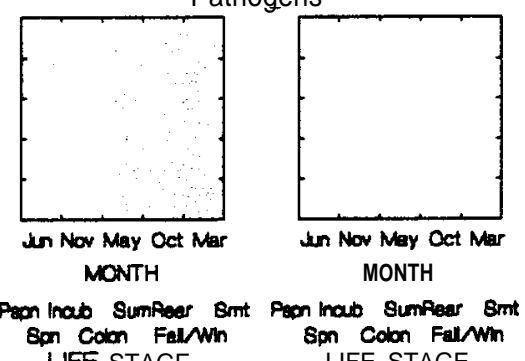

Level of

Effect

$\square$ No effect

$\square$ Low

Moderate

High

Lethal 


\section{Wallowa R/Grande Ronde R Relative Survival}
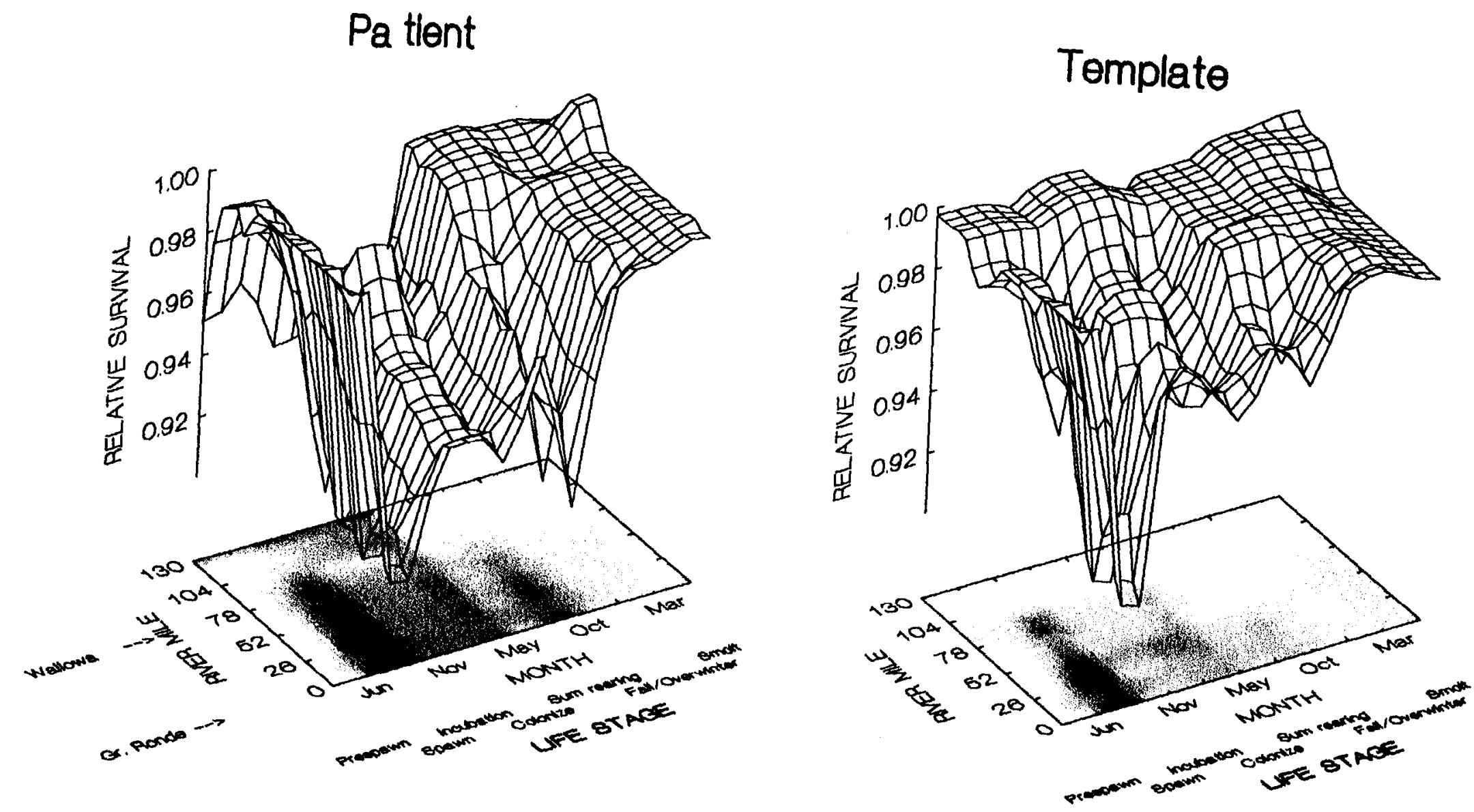
$\underline{\text { Stream Reach Range }}$

Minam R / Wallowa R / Grande Ronde R 


\section{Patient-Template Analysis Summary}

\section{Stream Reach Range: Minam R./Wallowa R./Grande Ronde R.}

\section{Key Habitat Quantity}

The extent of changes from historic levels in the quantity of key habitat for spring chinook in the Minam River differ by life stage and stream reach. The greatest reductions in key habitat have occurred in approximately the lower third of the mainstem for prespawning, spawning, and incubation life stages. Lesser, though still substantial, reductions have occurred through much of the mid portion of the mainstem for some life stages, namely fry colonization and summer rearing.

The quantities of key habitat in the Wallowa and Grande Ronde rivers downstream of the Minam River are nearly unchanged compared to historic levels.

\section{Relative Productivity (Survival)}

The capability of the mainstem Minam River of supporting spring chinook productivity (survival) is largely unchanged compared to historic conditions for all life stages. Some survival conditions in the lower ten miles of river have worsened slightly, however, due mainly to small changes in temperature regimes, habitat diversity, sediment load, channel stability, and flow regimes.

Survival conditions within the Wallowa and Grande Ronde rivers downstream of the Minam River have worsened compared to those that existed historically, although these reaches have always been moderately to largely unfavorable for survival during some life stages. In particular, conditions have always been poor in the later part of the prespawning stage and during the spawning and incubation stages in the lower Grande Ronde River. These conditions are mainly due to elevated water temperatures, sediment load, channel stability, habitat diversity, and the presence of predators. 

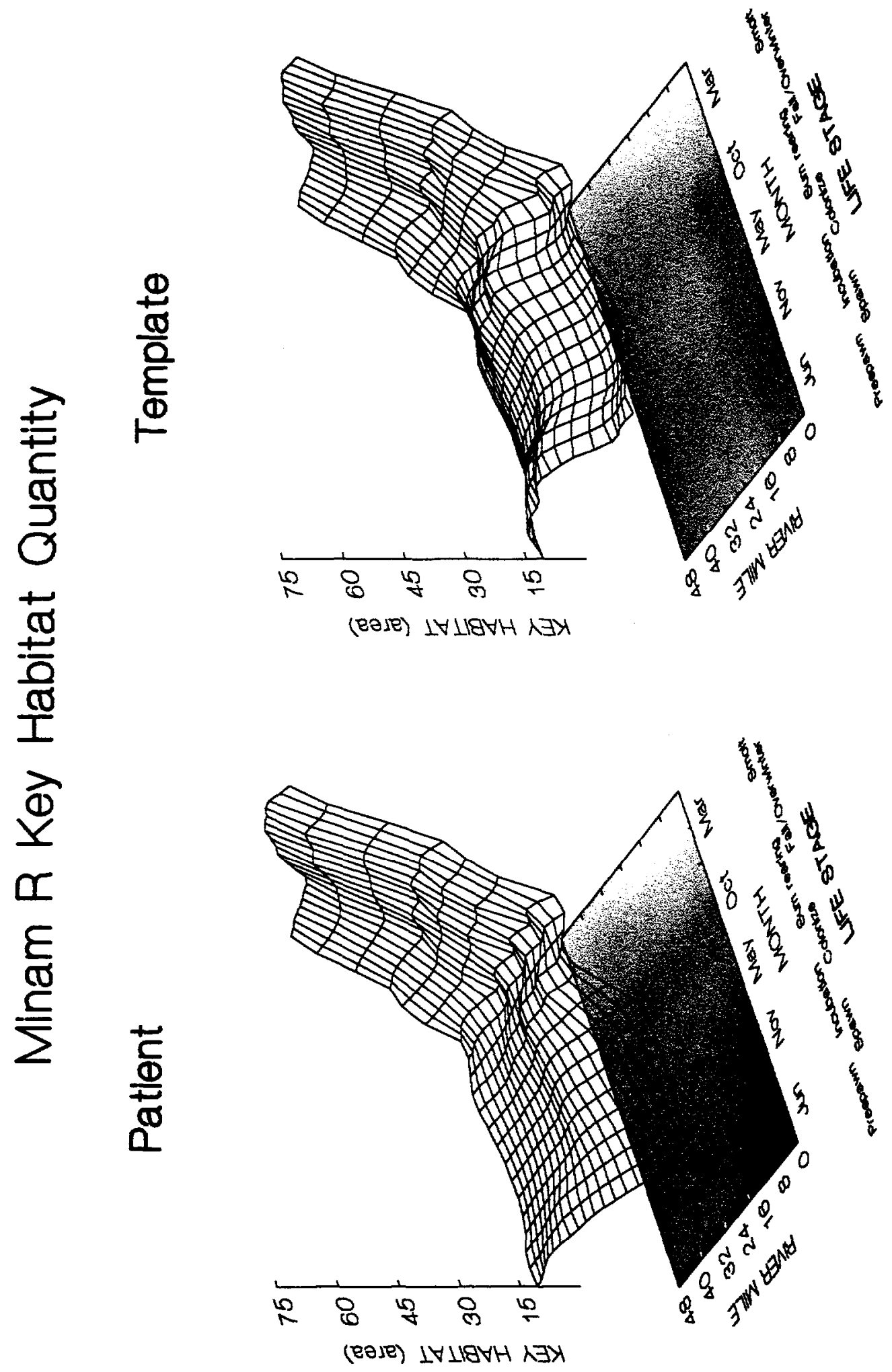


\section{Minam R Environmental Attributes}
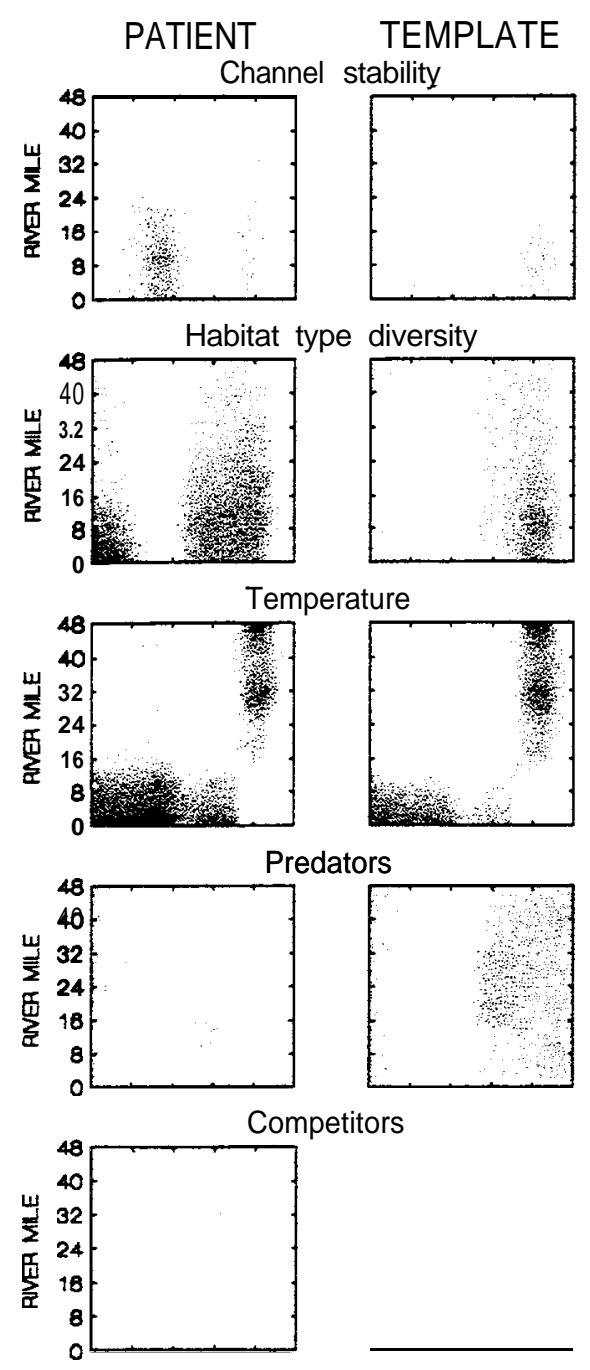

Competitors
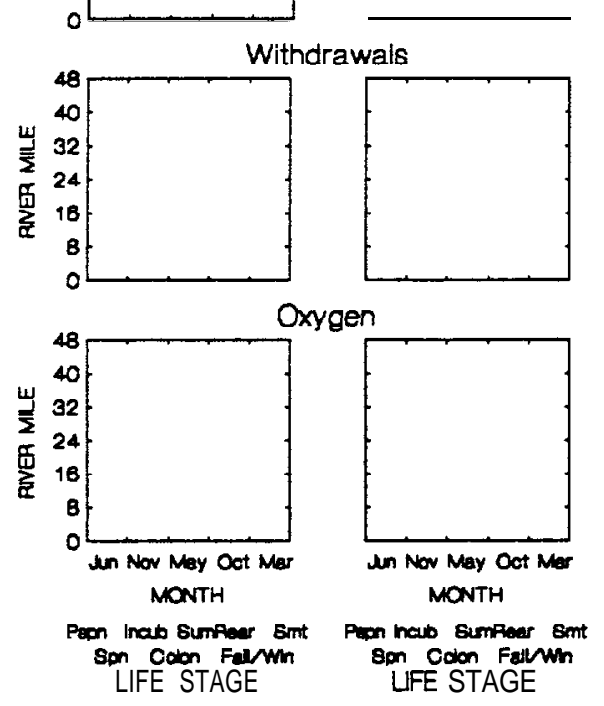

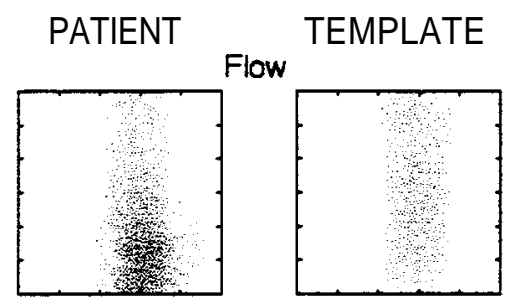

Sediment load

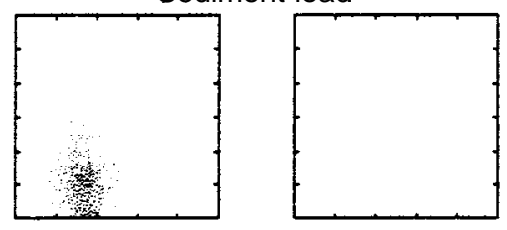

Riparian condition

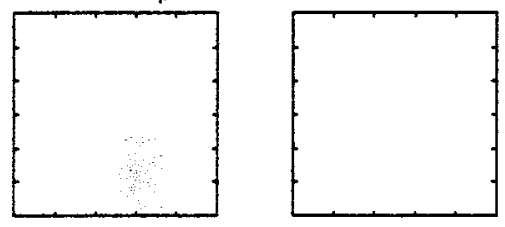

Chemicals

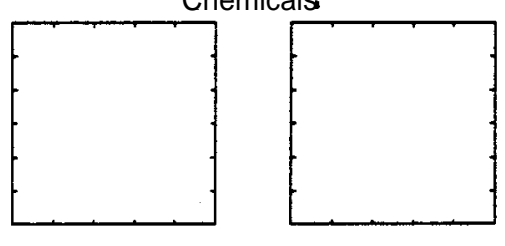

Obstructions

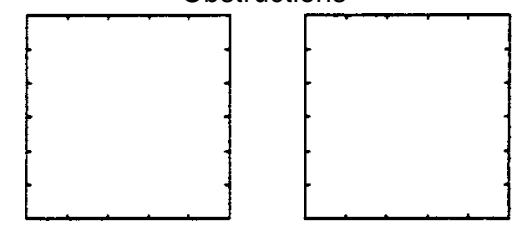

Nutrient load

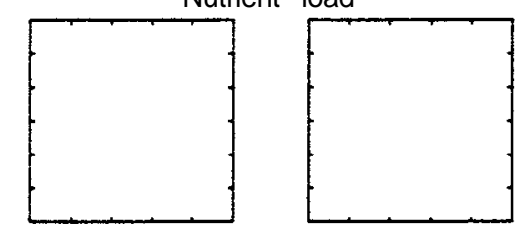

Pathoaens
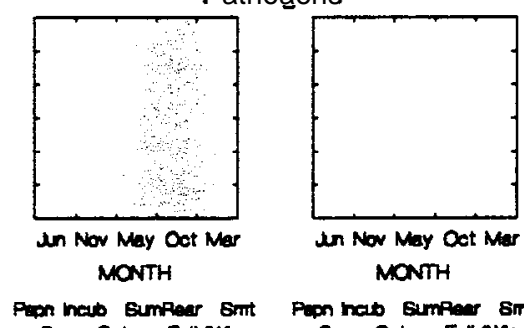

in Nor May Oet Mer MaNTH

Ent Pen hat Eumfleer $8 \mathrm{mt}$ Son Coion Fallwn Bon Coon Fallym
Level of

Effect

$\square$ No effect

$\square$ Low

울 Moderate

(]) High

Lethal 


\section{Minam R Relative Survival}
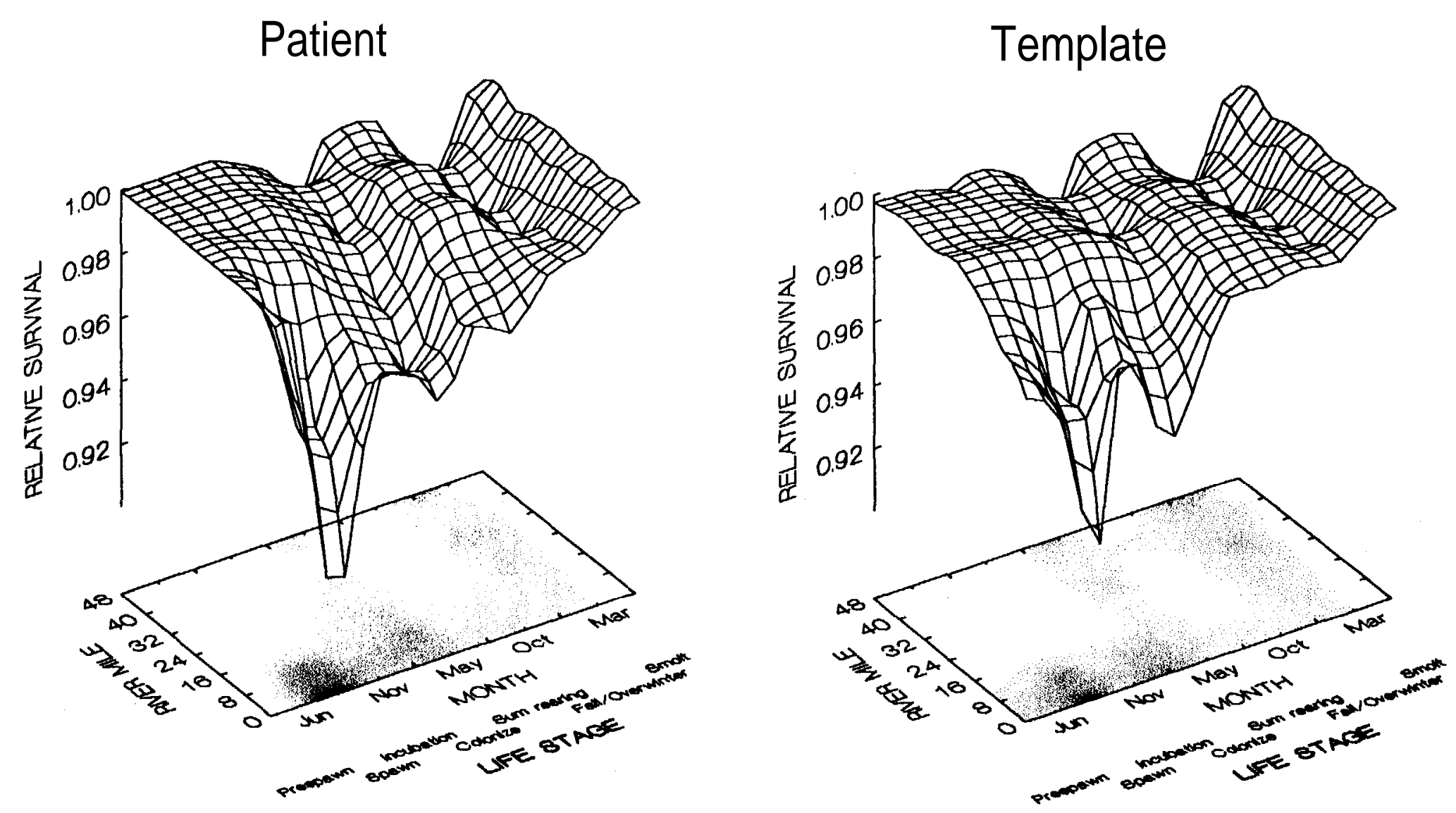


\section{Minam R/Wallowa R/Grande Ronde R Key Habitat Quantity}

\section{Patient}

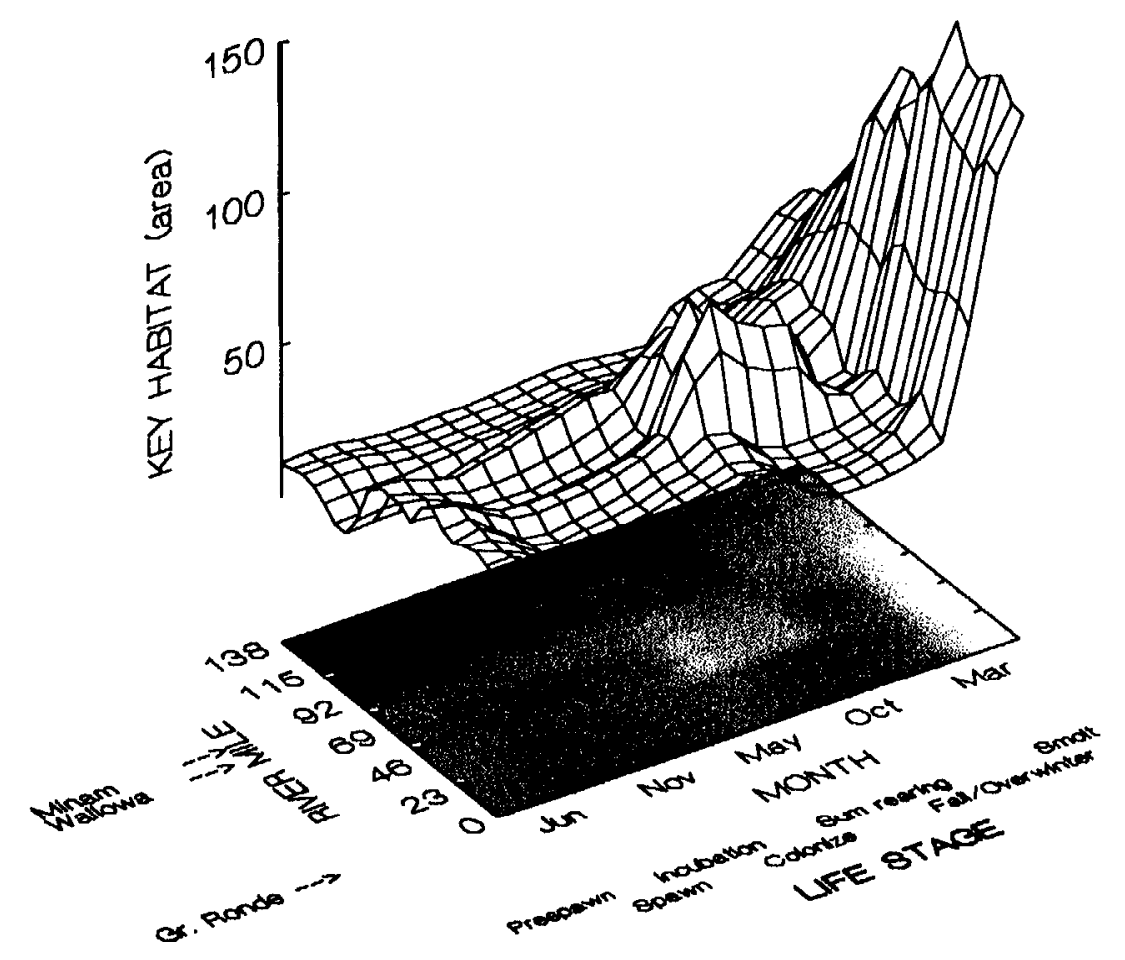

Template

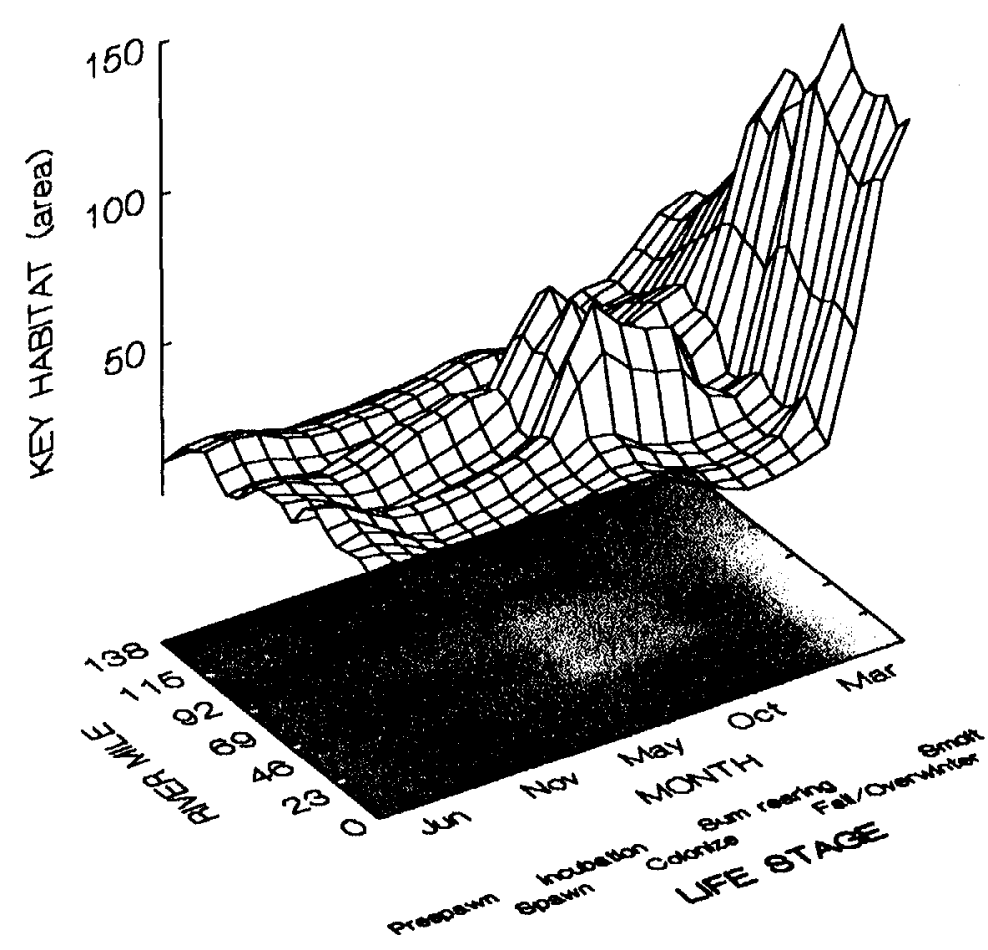




\section{Minam R/Wallowa R/Grande Ronde R Environmental Attributes}

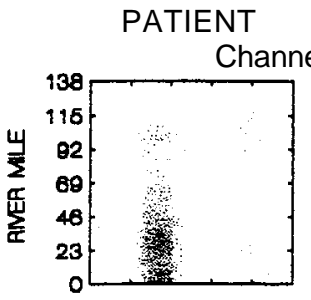

TEMPLATE

Habitat type diversity
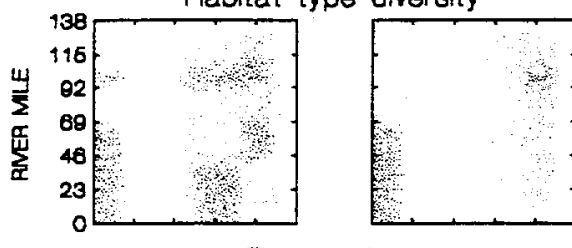

Temperature
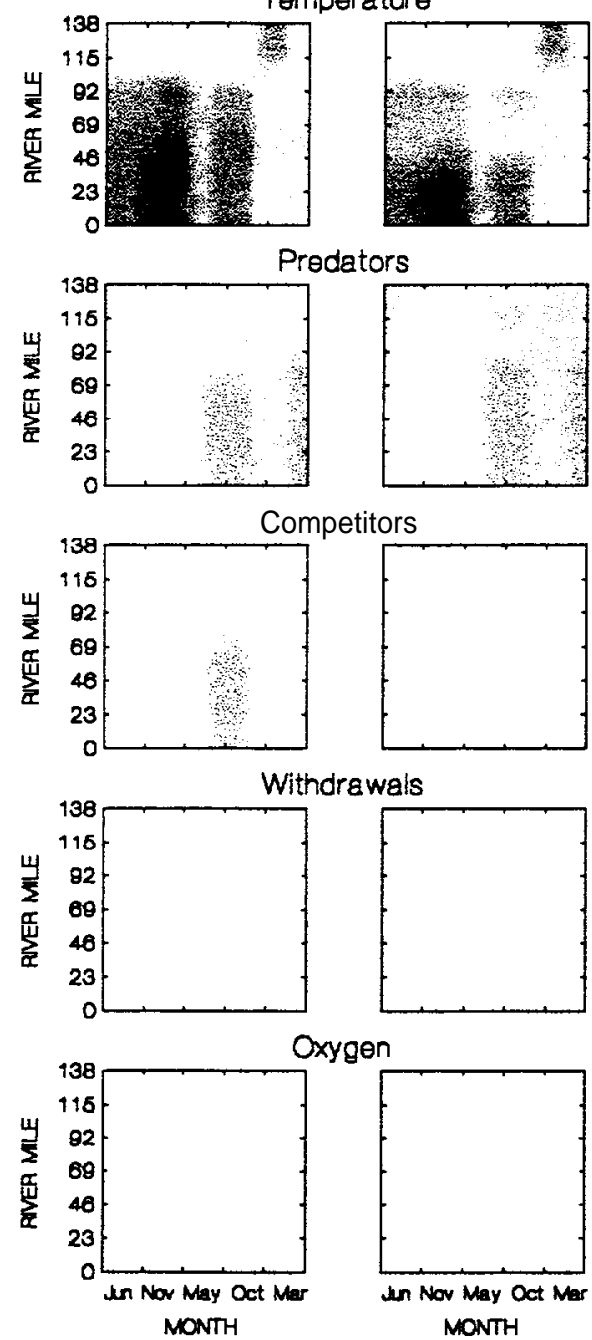

oxygen

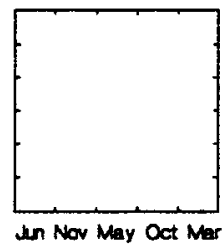

$$
\text { MONTH }
$$

Pron hab Bumfeer Sint Peon inab Sumfeer Smt son Coon Falwin Son Colon Falumn LIFE STAGE
PATIENT

FIOW TELATE

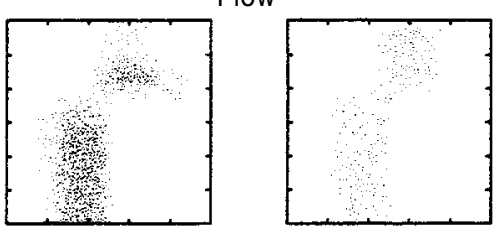

Sediment load

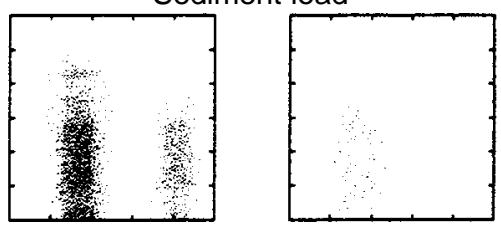

Riparian condition

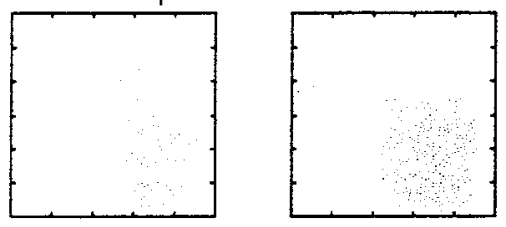

Chemicals

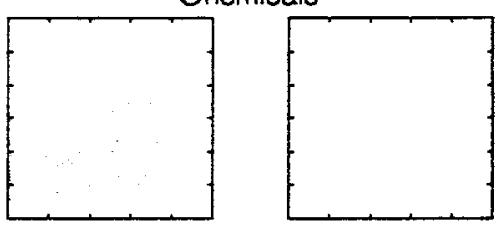

Obstructions

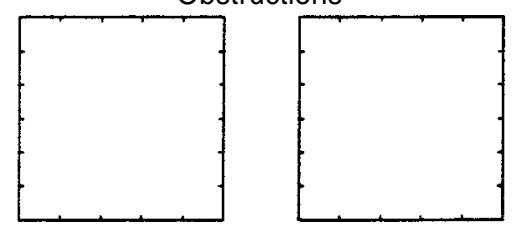

Nutrient load

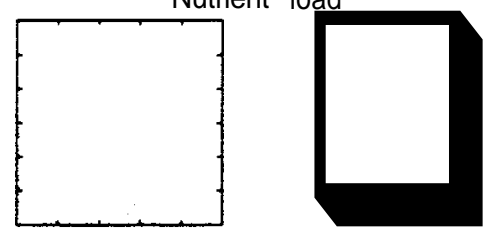

Pathogens

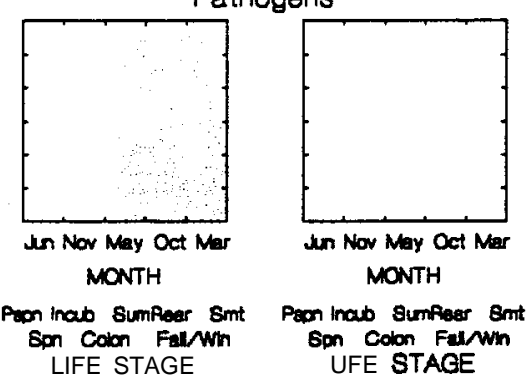

Level of

Effect

$\square$ No effect

$\square$ Low

Moderate

燿 High

Lethal 


\section{Minam R/Wallowa R/Grande Ronde R Relative Survival}
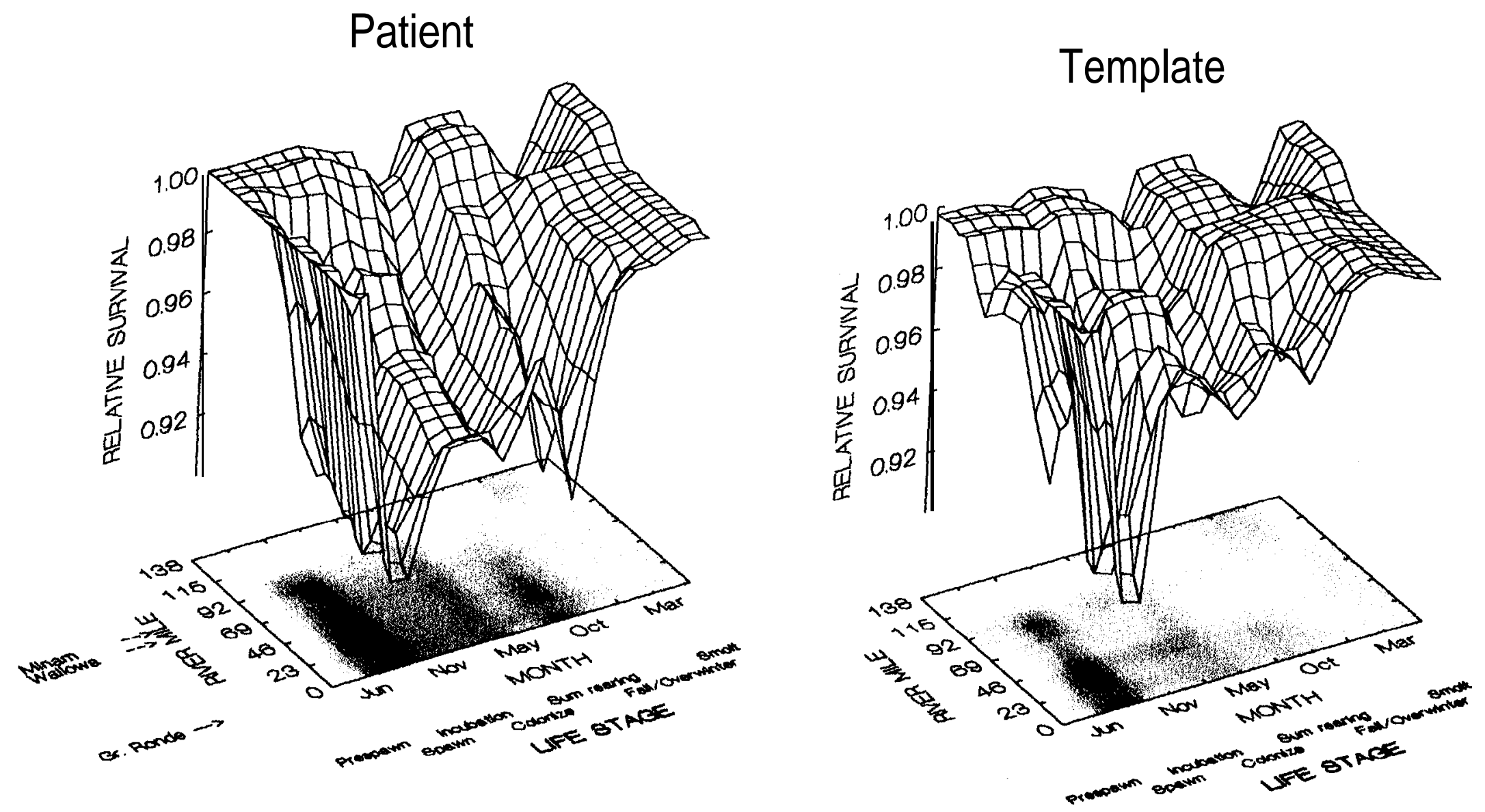


\section{Stream Reach Range}

Bear Cr / Wallowa R / Grande Ronde R 


\section{Patient-Template Analysis Summary}

Stream Reach Range: Bear Cr./Wallowa R./Grande Ronde R.

\section{Key Habitat Quantity}

The extent of changes from historic levels in the quantity of key habitat for spring chinook in Bear Creek differ by life stage and stream reach. Historically, the largest quantities of key habitat occurred in the lower six miles of stream (excluding smolt stage). Very substantial reductions have occurred in this section for all life stages except smolt. Lesser reductions have also occurred through the mid portions of the stream for some life stages. Quantity of key habitat in the upper reach is nearly unchanged.

The quantities of key habitat in some portions of the Wallowa River downstream of Bear Creek River are substantially less than historic levels, except in the lower ten miles (below Minam River) where they are relatively unchanged.

The quantity of key habitat in the Grande Ronde River downstream of the Wallowa River is nearly unchanged compared to historic levels.

\section{Relative Productivity (Survival)}

The capability of the lower six miles of Bear Creek of supporting spring chinook productivity (survival) is greatly reduced compared to historic conditions for most life stages. Survival conditions in this section are poorer due primarily to alterations in flow patterns, although other attributes (e.g., habitat diversity and temperature regimes) have also been affected. Historically, survival conditions were excellent for much of Bear Creek, and especially in the lower reaches of the stream. Changes in upper portions of the stream are comparatively minor.

Survival conditions in the Wallowa River downstream of the Bear Creek have worsened compared to those that existed historically, although the lower portions of the river have always been moderately unfavorable for survival during some life stages. Conditions have also worsened in the Grande Ronde River downstream of the Wallowa River. However, survival conditions have always been poor in the later part of the prespawning stage and during the spawning and incubation stages in the lower Grande Ronde River. These conditions are mainly due to elevated water temperatures, sediment load, channel stability, habitat diversity, and the presence of predators. 


\section{Bear Cr Key Habitat Quantity}

\section{Patient}

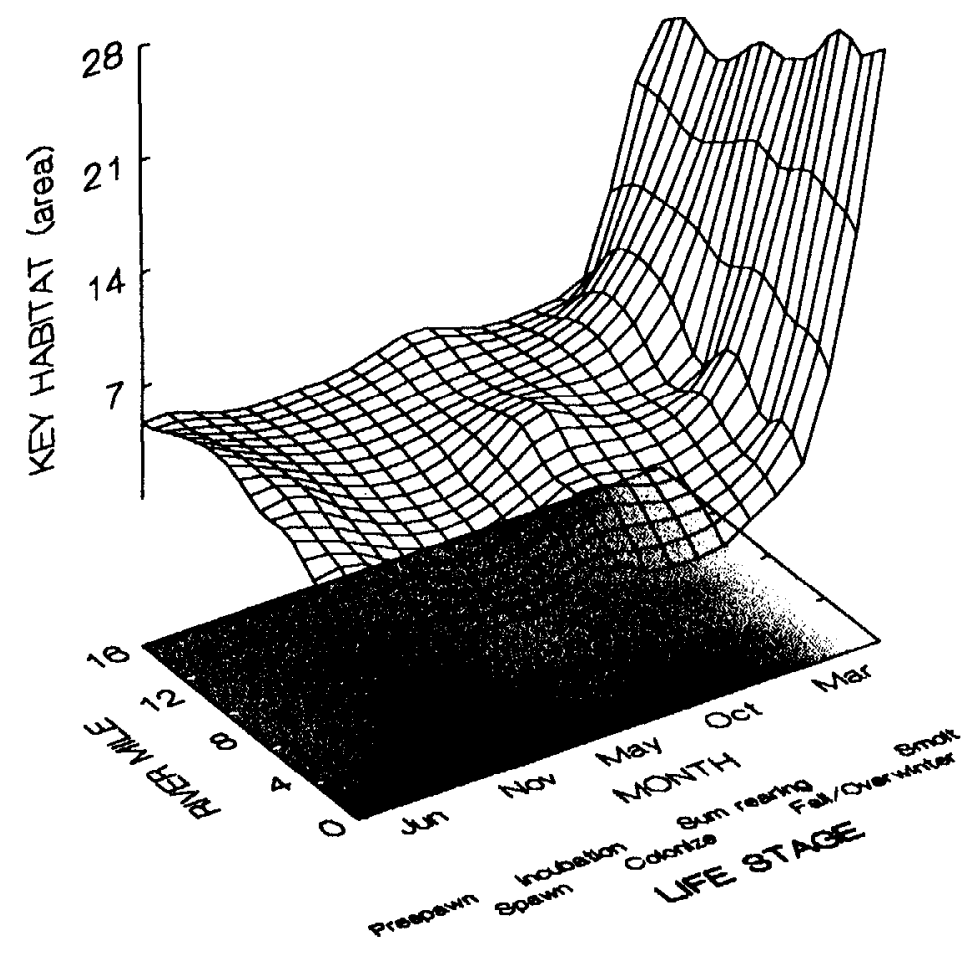

Template

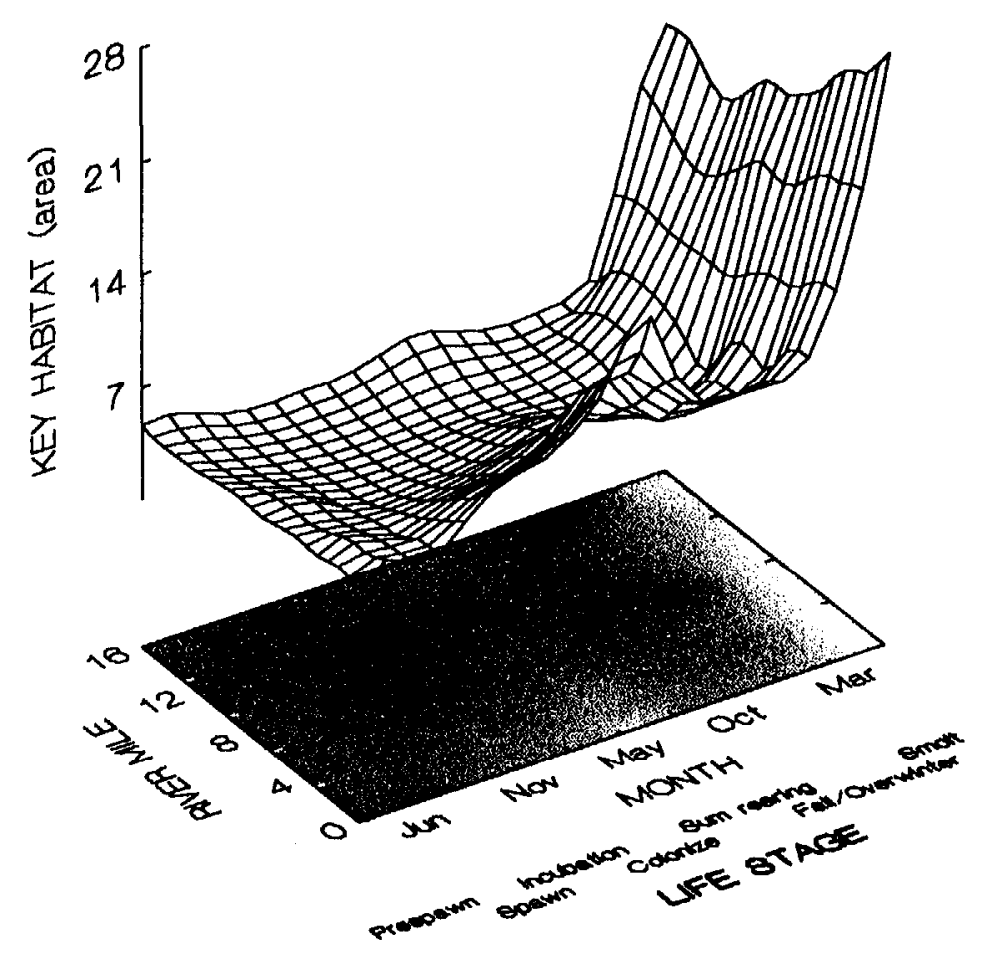




\section{Bear Cr Environmental Attributes}

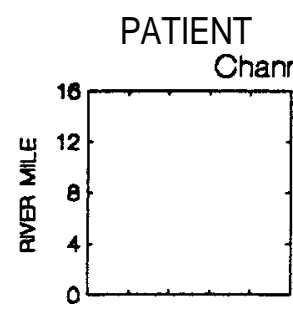

Habitat type diversity

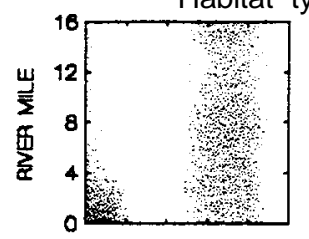

Temperature

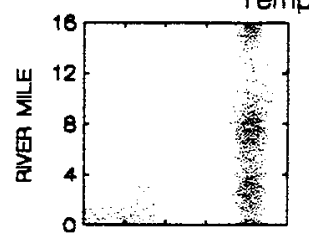

Predators
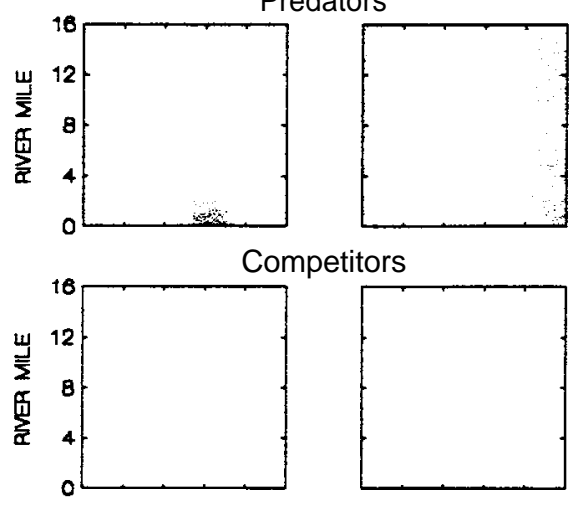

Competitors
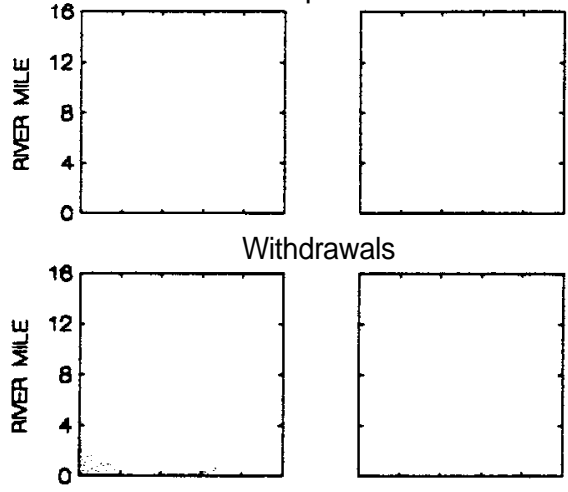

Withdrawals

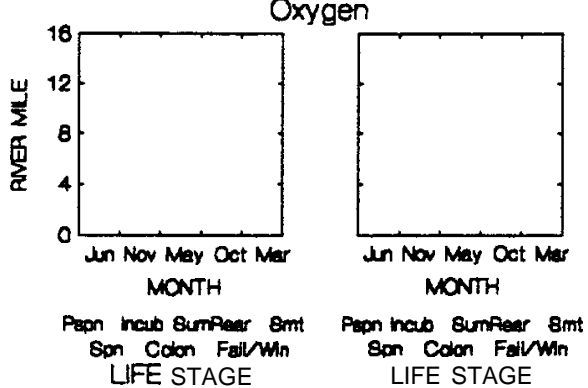

PATIENT Flow

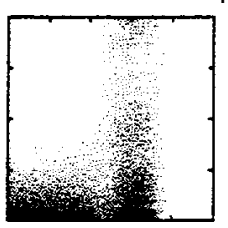

Sediment load

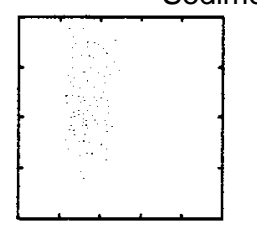

Riparian condition

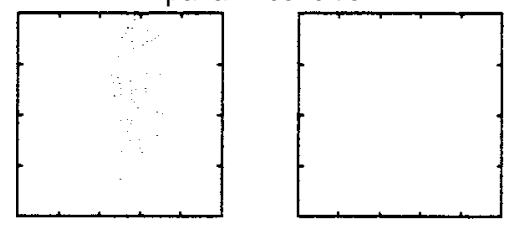

Chemicals

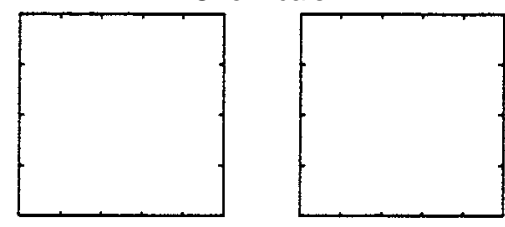

Obstructions

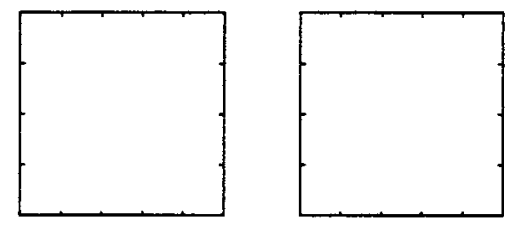

Nutrient load

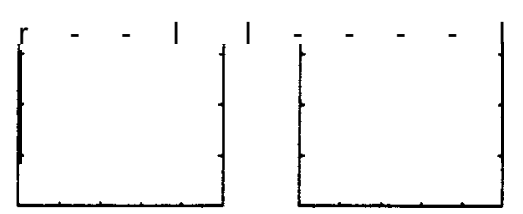

Pathogens

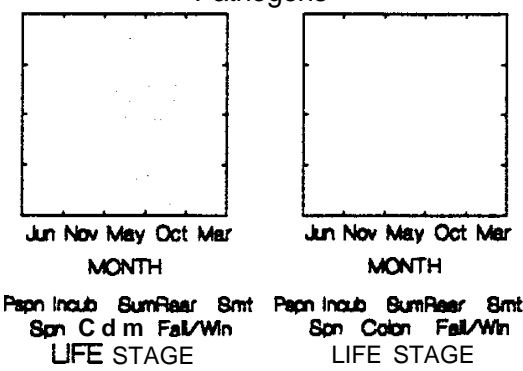

Level of

Effect

$\square$ No effect

a Low

口 Moderate

High

- Lethal 


\section{Bear $\mathrm{Cr}$ Relative Survival}
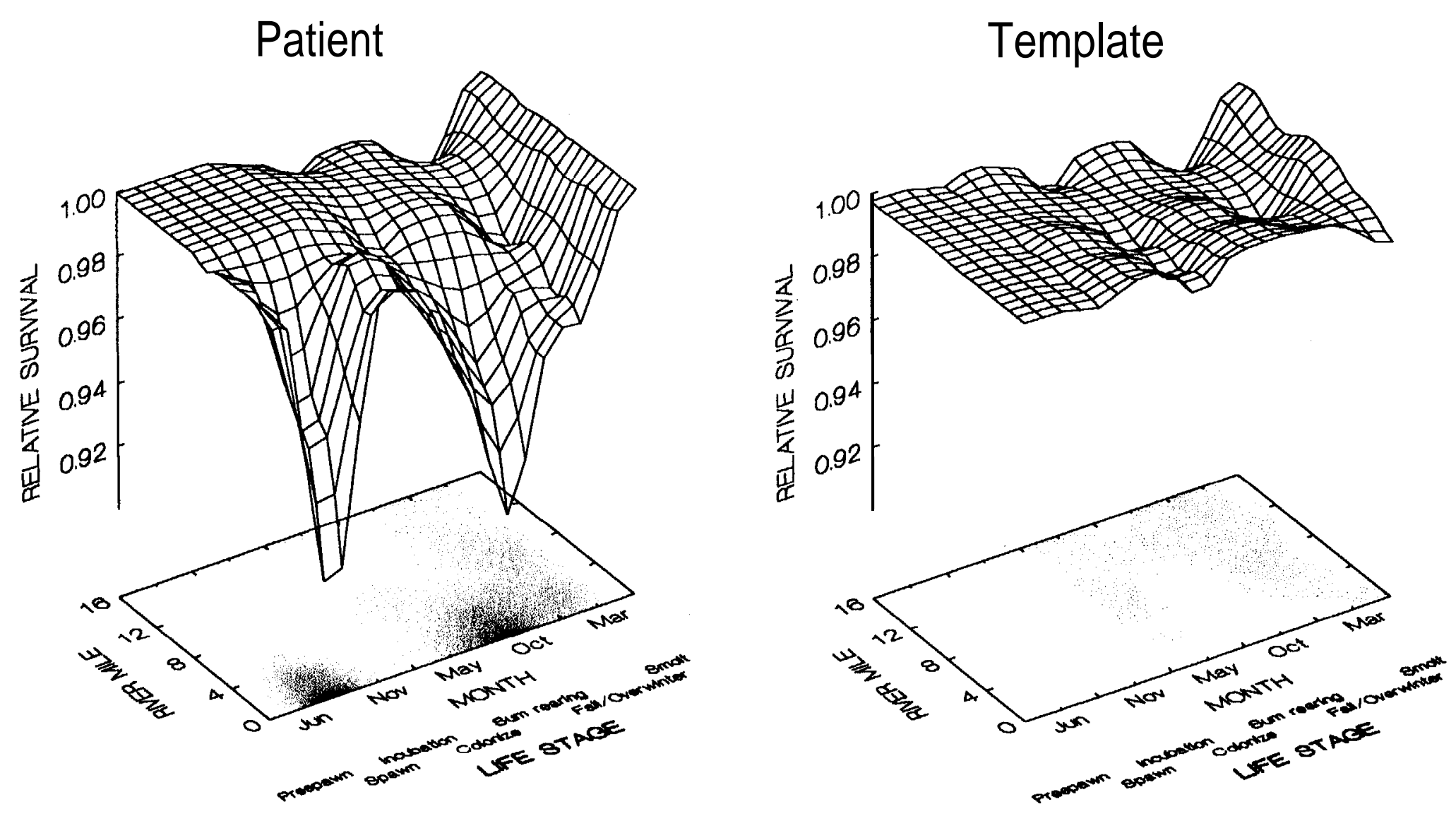


\section{Bear Cr/Wallowa R/Grande Ronde R Key Habitat Quantity}

\section{Patient}

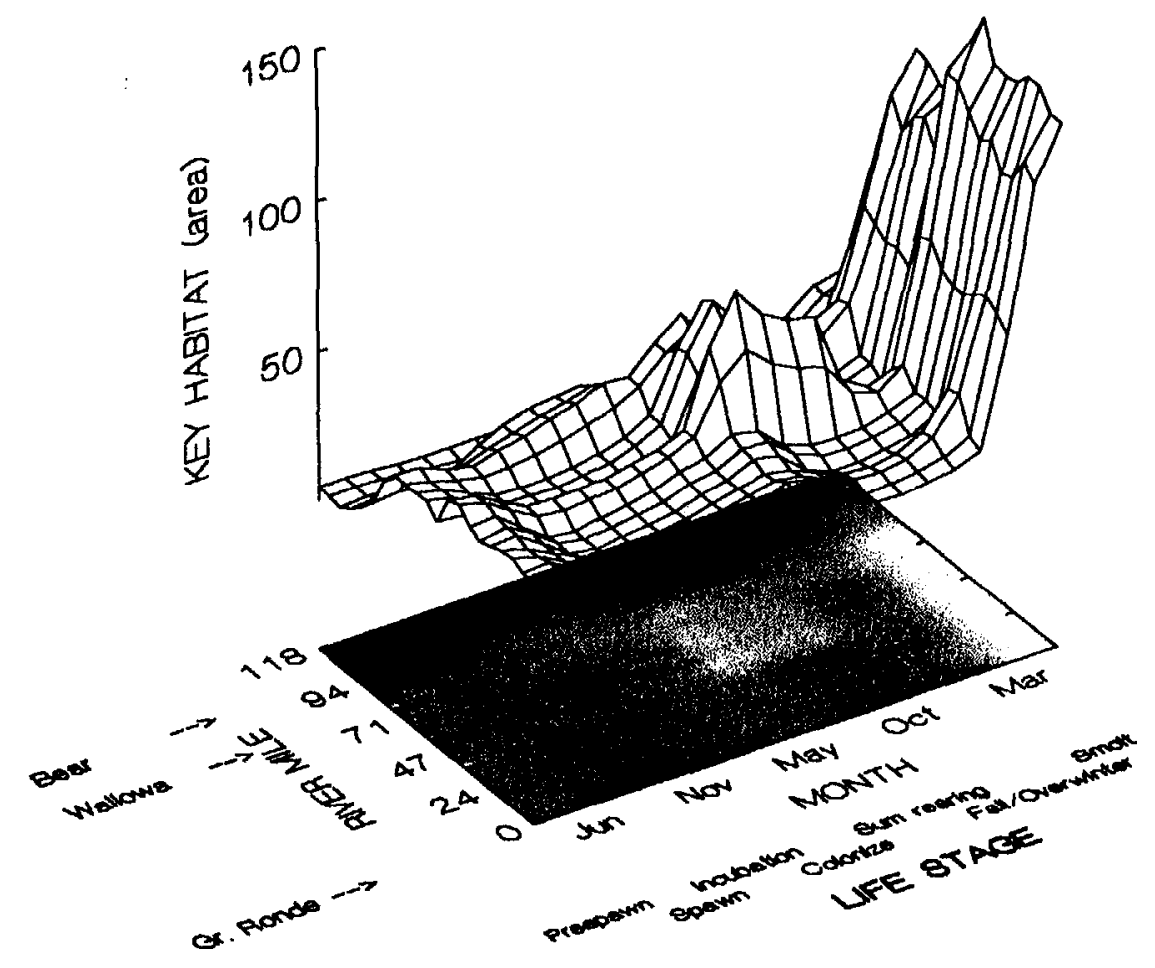

\author{
Template
}

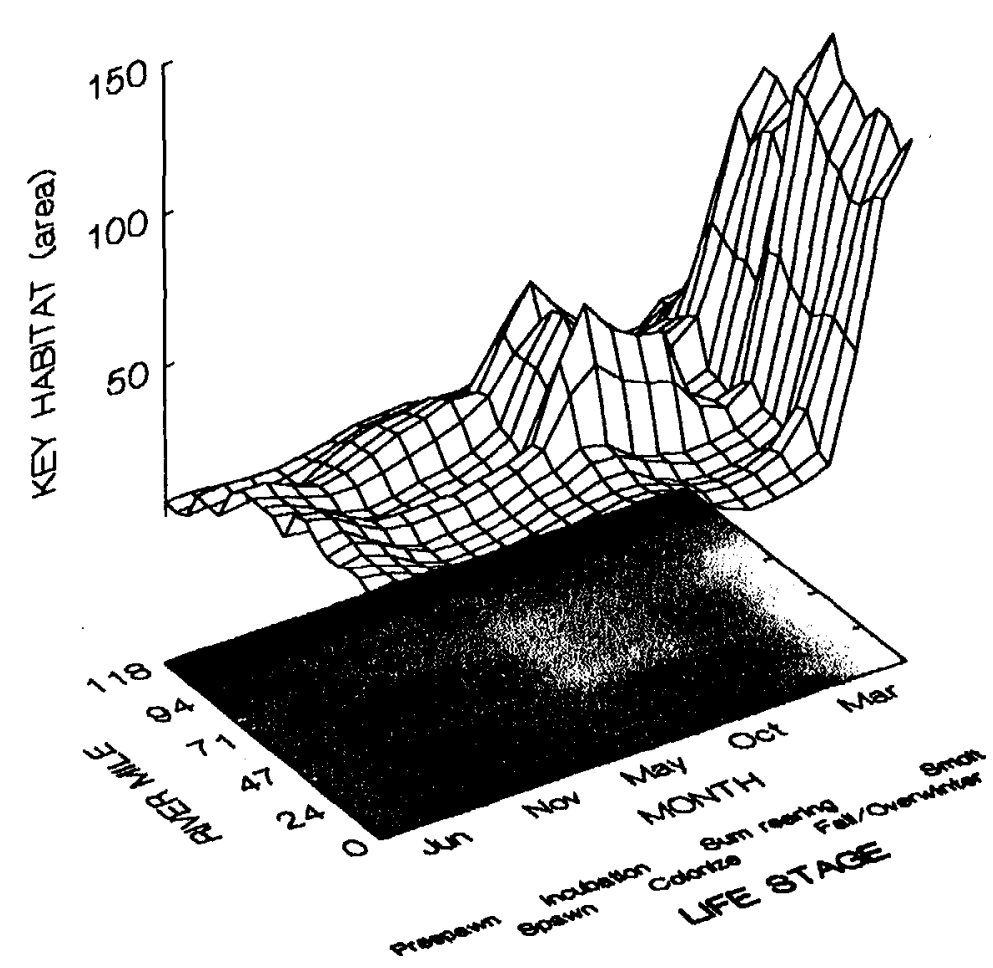




\section{Bear Cr/Wallowa R/Grande Ronde R Environmental Attributes}

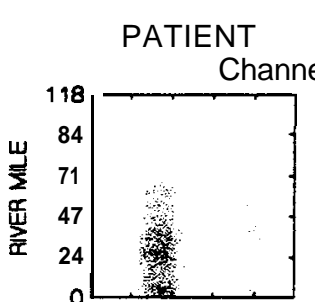

TEMPLATE

stability
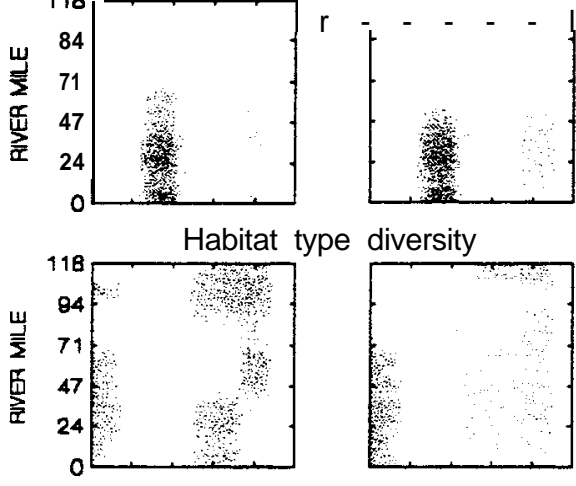

Temperature
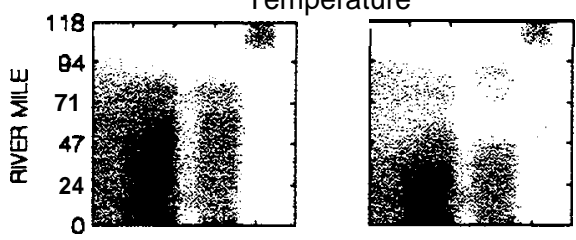

Predators
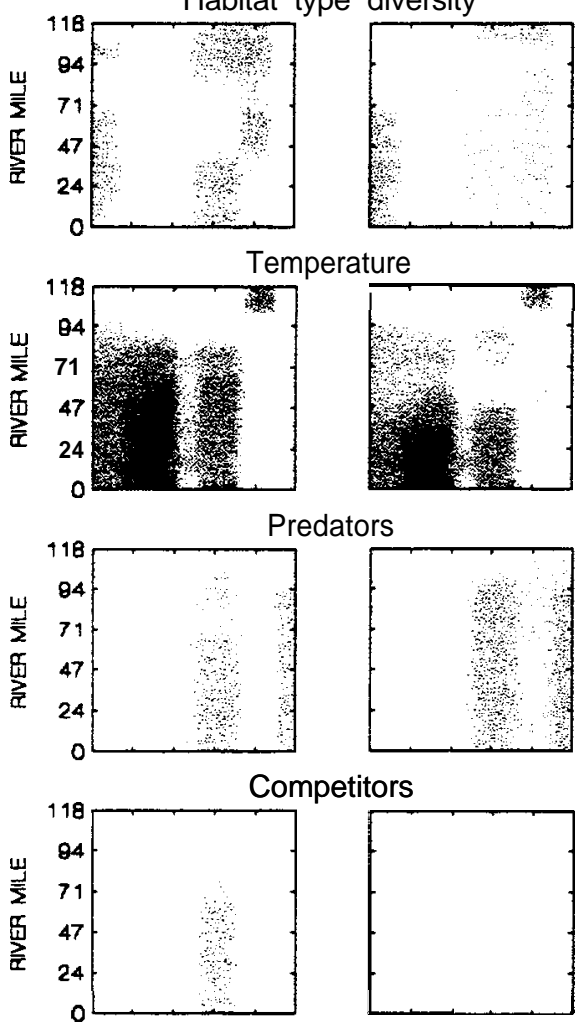

ompetitors

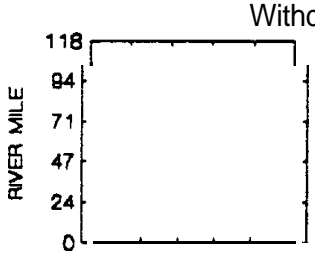

ithdrawals

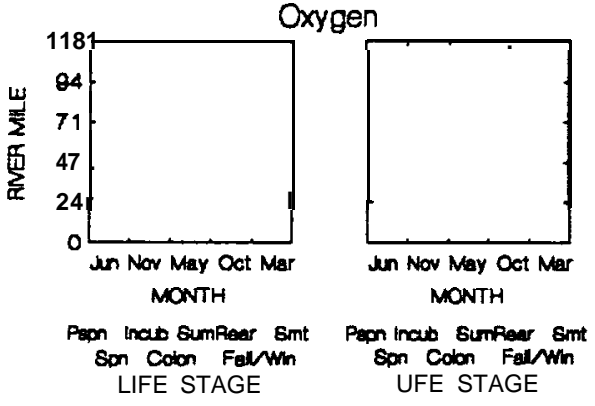

\author{
PATIENT \\ TEMPLATE
}

Flow

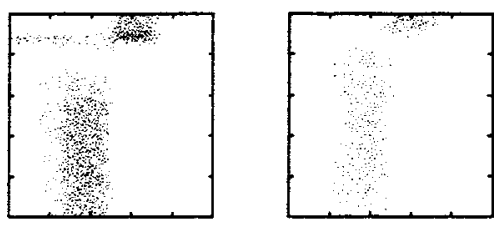

Sediment load

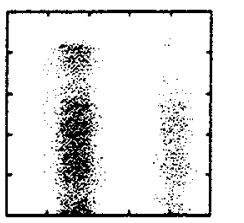

Riparian condition

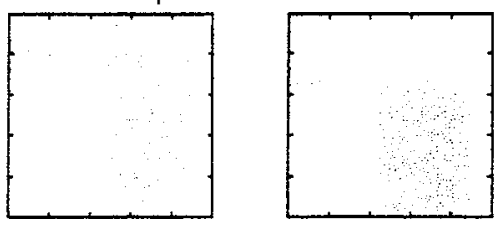

Chemicals

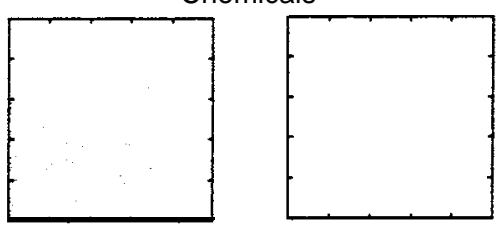

Obstructions

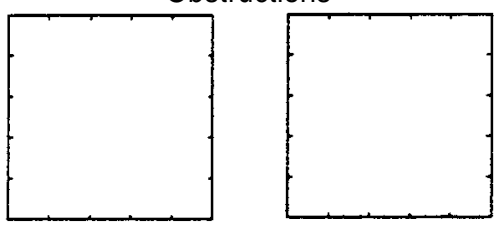

Nutrient load

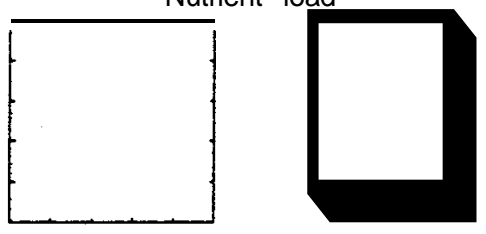

Pathogens
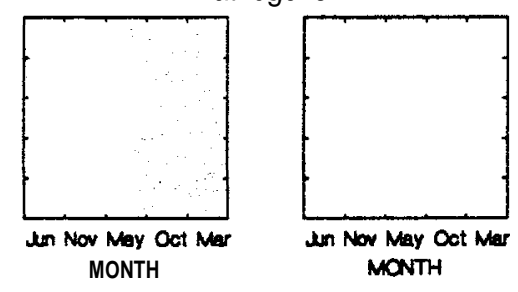

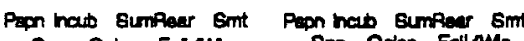

Spn Colon Fal/Wr Epn Colon Fallw

LIFE STAGE UFE STAGE
Level of Effect

$\square \quad$ No effect $\square$ Low a Moderate

High

$\square$ Lethal 


\section{Bear Cr/Wallowa R/Grande Ronde R Relative Survival}
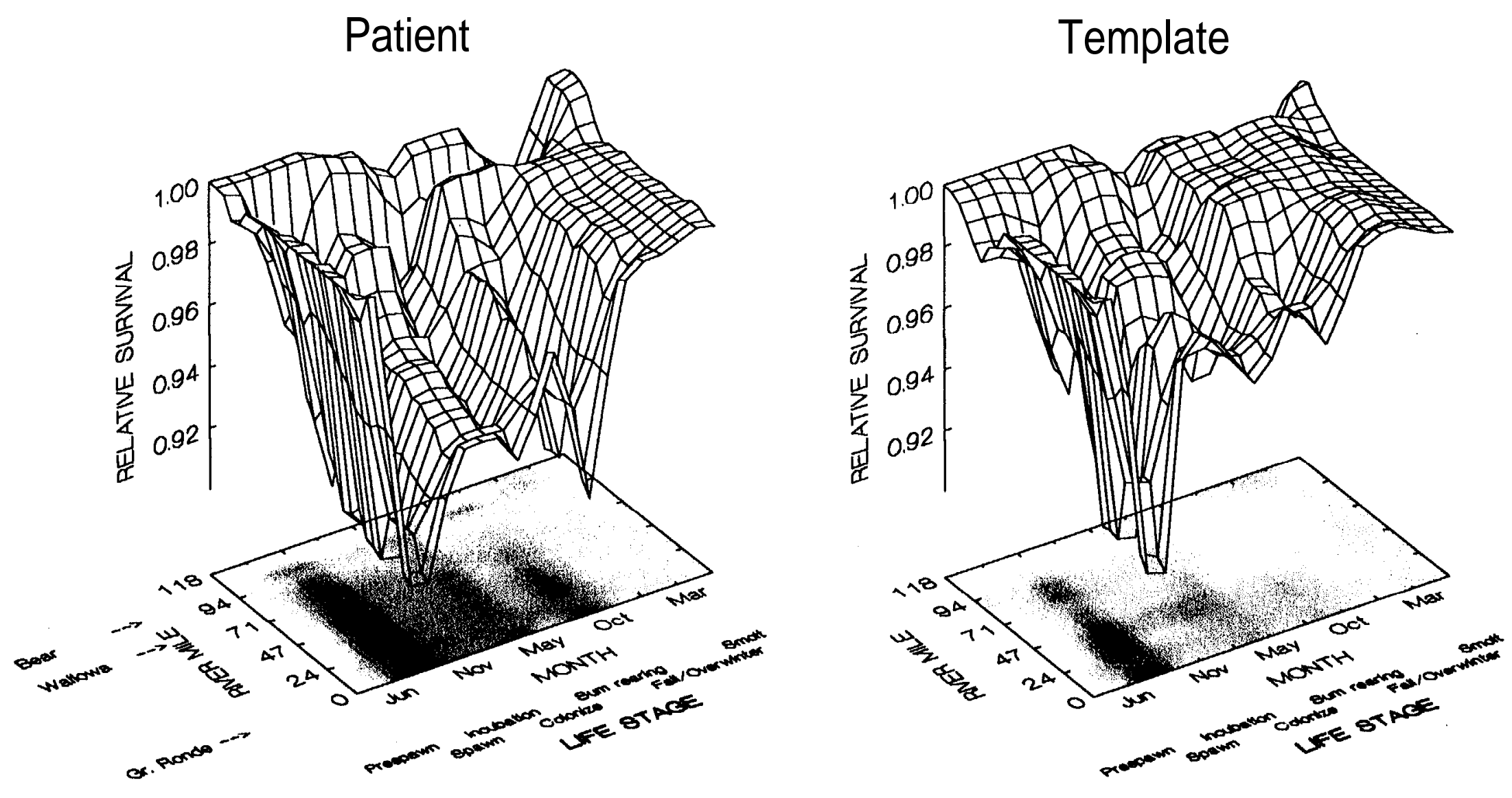


\section{Stream Reach Range}

Lostine R / Wallowa R / Grande Ronde R 


\section{Patient-Template Analysis Summary}

Stream Reach Range: Lostine R./Wallowa R./Grande Ronde R.

\section{Key Habitat Quantity}

The extent of changes from historic levels in the quantity of key habitat for spring chinook in the Lostine River differ by life stage and stream reach. Historically, the largest quantities of key habitat occurred in the lower 15 miles of river, downstream of a canyon. Substantial reductions have occurred in some parts of this section, notably for the prespawning, spawning, incubation, fry colonization, and summer rearing stages. Quantity of key habitat is virtually unchanged in the upper 10 miles of stream; this section contains much less habitat compared to quantities located downstream, however.

The quantities of key habitat in some portions of the Wallowa River downstream of the Lostine River have been reduced. Quantities in the Grande Ronde River downstream of the Wallowa River are nearly unchanged compared to historic levels.

\section{Relative Productivity (Survival)}

The capability of the lower 15 miles of mainstem Lostine River of supporting spring chinook productivity (survival) is substantially reduced compared to historic conditions for most life stages. Survival conditions in this section are poorer due primarily to higher summer temperatures, alterations in flow regimes, and loss of habitat diversity; other attributes have changed also. Historically, survival conditions were excellent in the lower ten miles; historic conditions worsened in an upstream direction with increasing stream gradient and elevation. Changes in the upper ten miles are comparatively minor.

Survival conditions in the Wallowa River downstream of the Lostine River have worsened compared to those that existed historically, although some reaches have always been moderately unfavorable for survival during some life stages. Conditions have also worsened in the Grande Ronde River downstream of the Wallowa River. However, survival conditions have always been poor in the later part of the prespawning stage and during the spawning and incubation stages in the lower Grande Ronde River. These conditions are mainly due to elevated water temperatures, sediment load, channel stability, habitat diversity, and the presence of predators. 

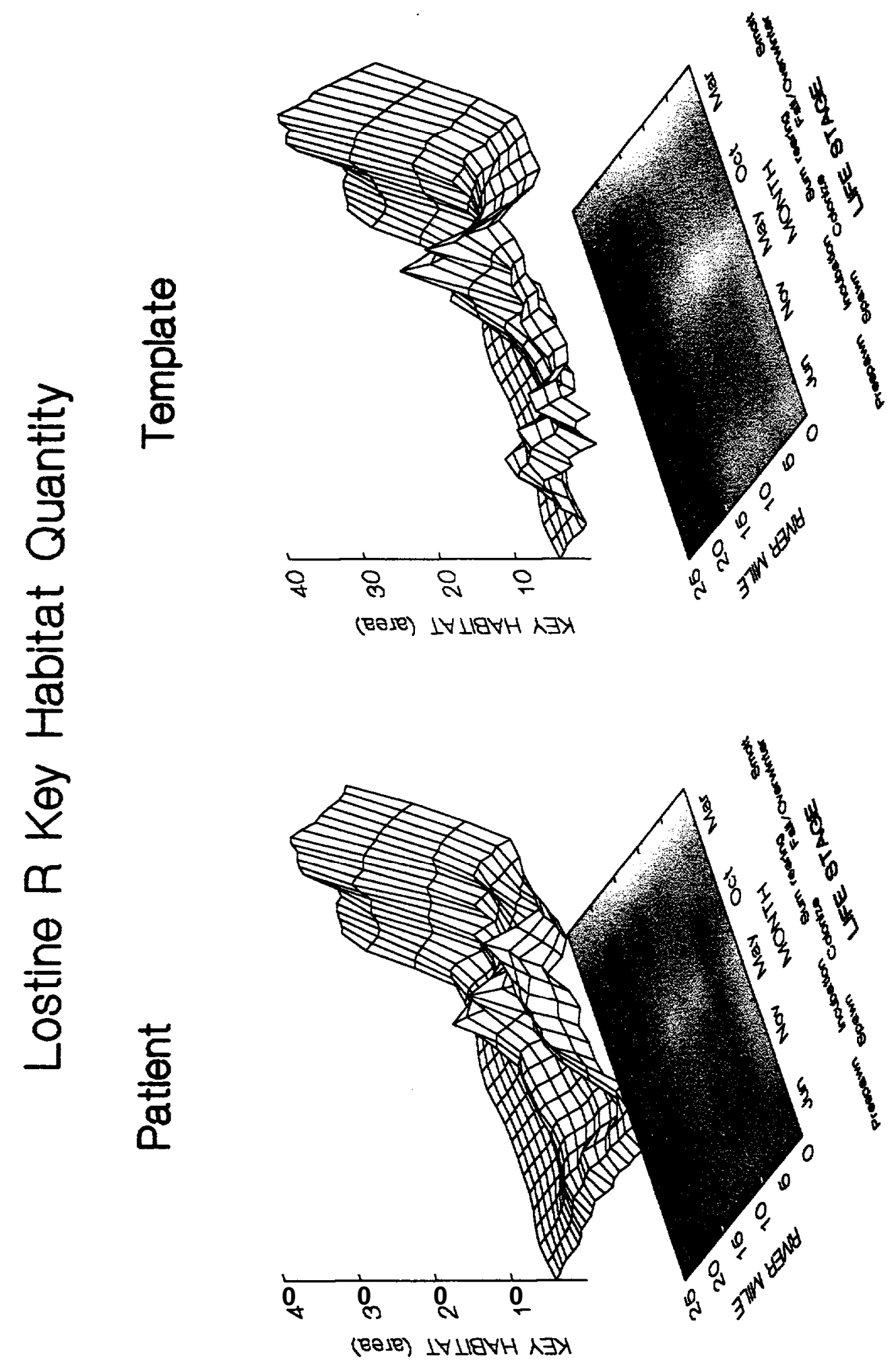


\section{Lostine R Environmental Attributes}

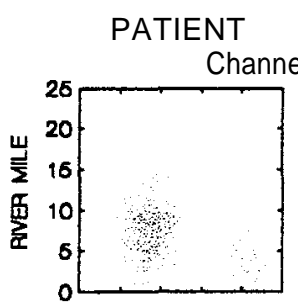

TEMPLATE

Channel stability

Habitat type diversity
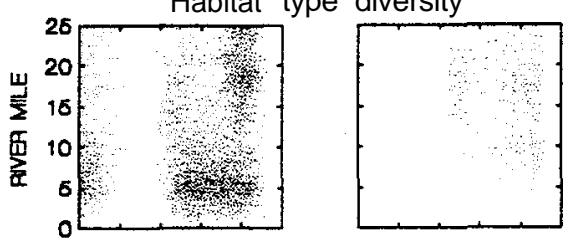

Temperature
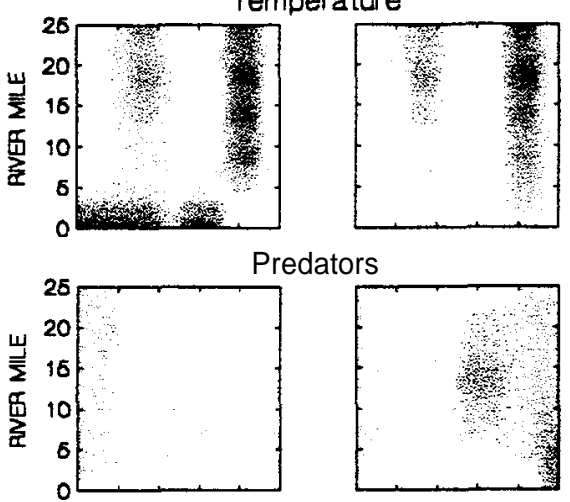

Predators

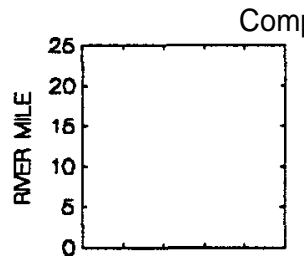

Competitors
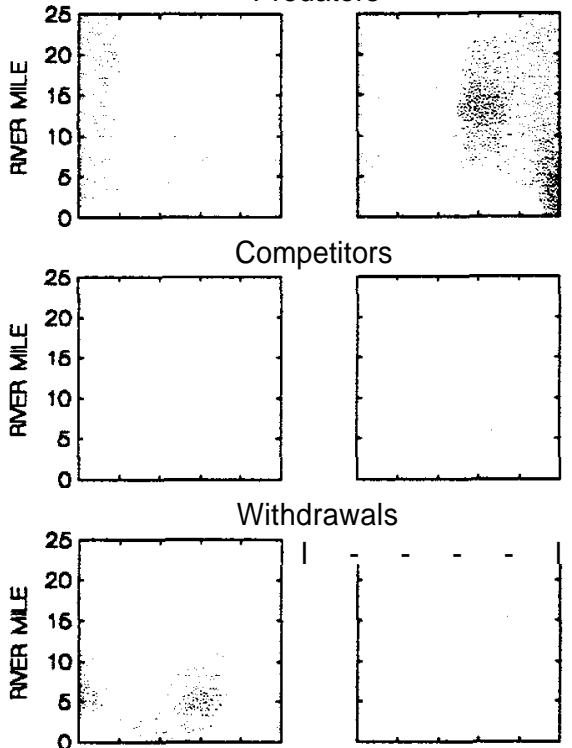

Withdrawals

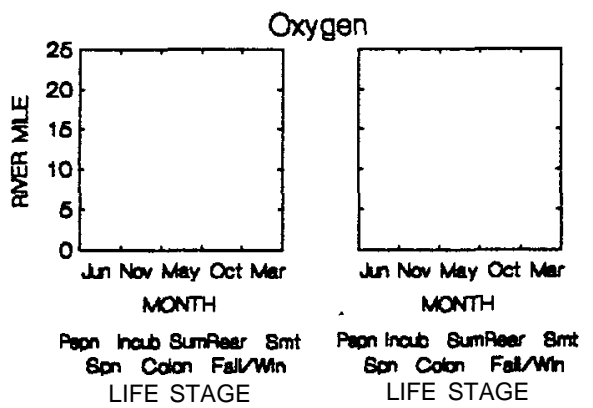

PATIENT

FIOW TEMPLATE

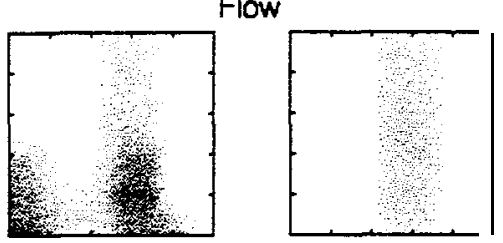

Sediment load

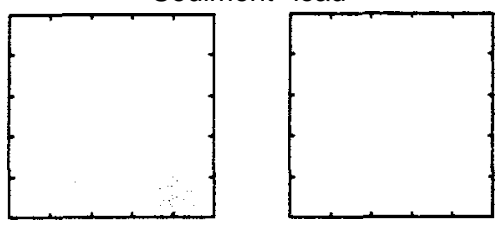

Riparian condition

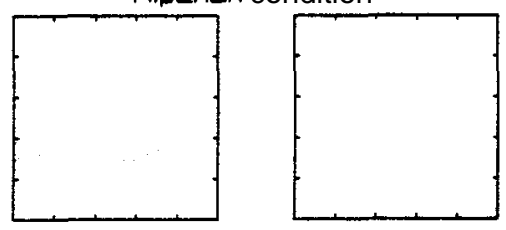

Chemicals

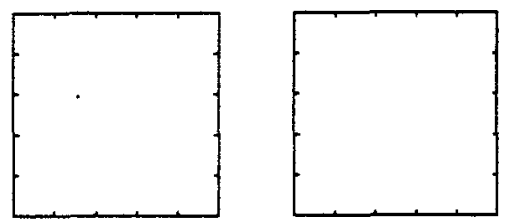

Obstructions

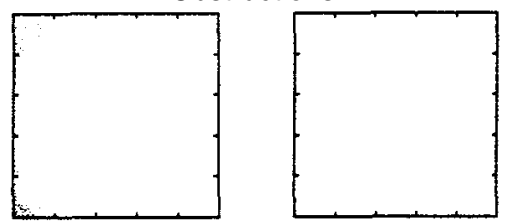

Nutrient load

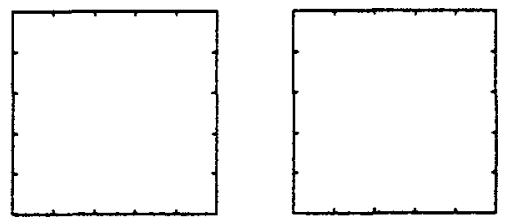

Pathogens

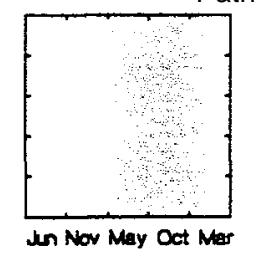

MONTH

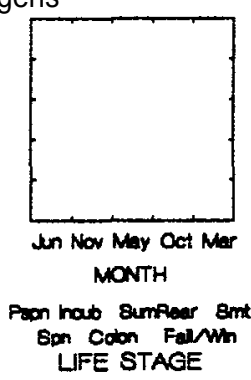

Puon hab Sumfiear 8mt Bon Colon Fallwh
LIFE STAGE
Bon Coion Falnm

LIFE STAGE
Level of Effect

$\square$ No effect

$\square$ Low

嫼 Moderate

a High

- Lethal 


\section{Lostine R Relative Survival}
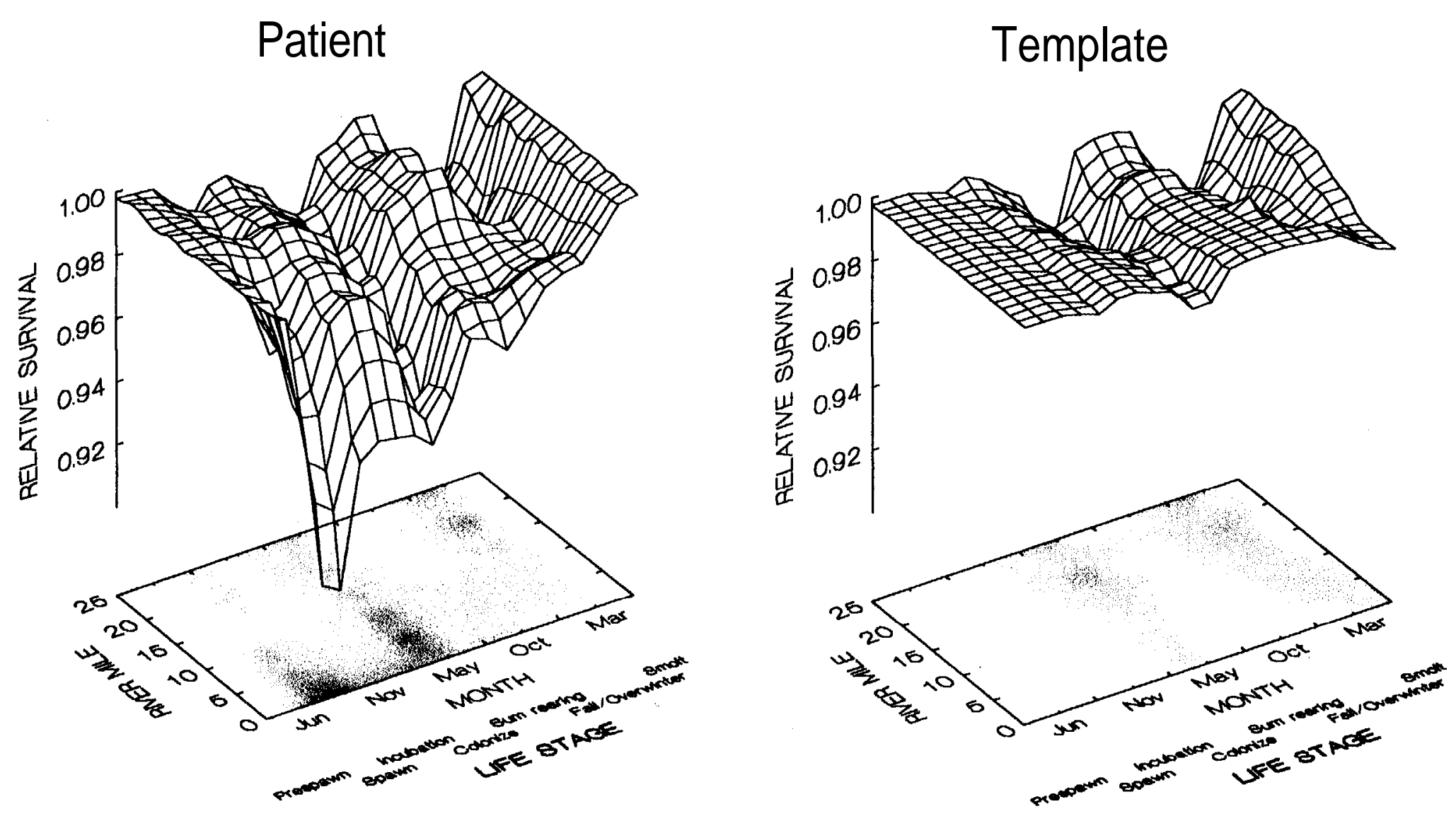


\section{Lostine R/Wallowa R/Grande Ronde R Key Habitat Quantity}

\section{Patient}

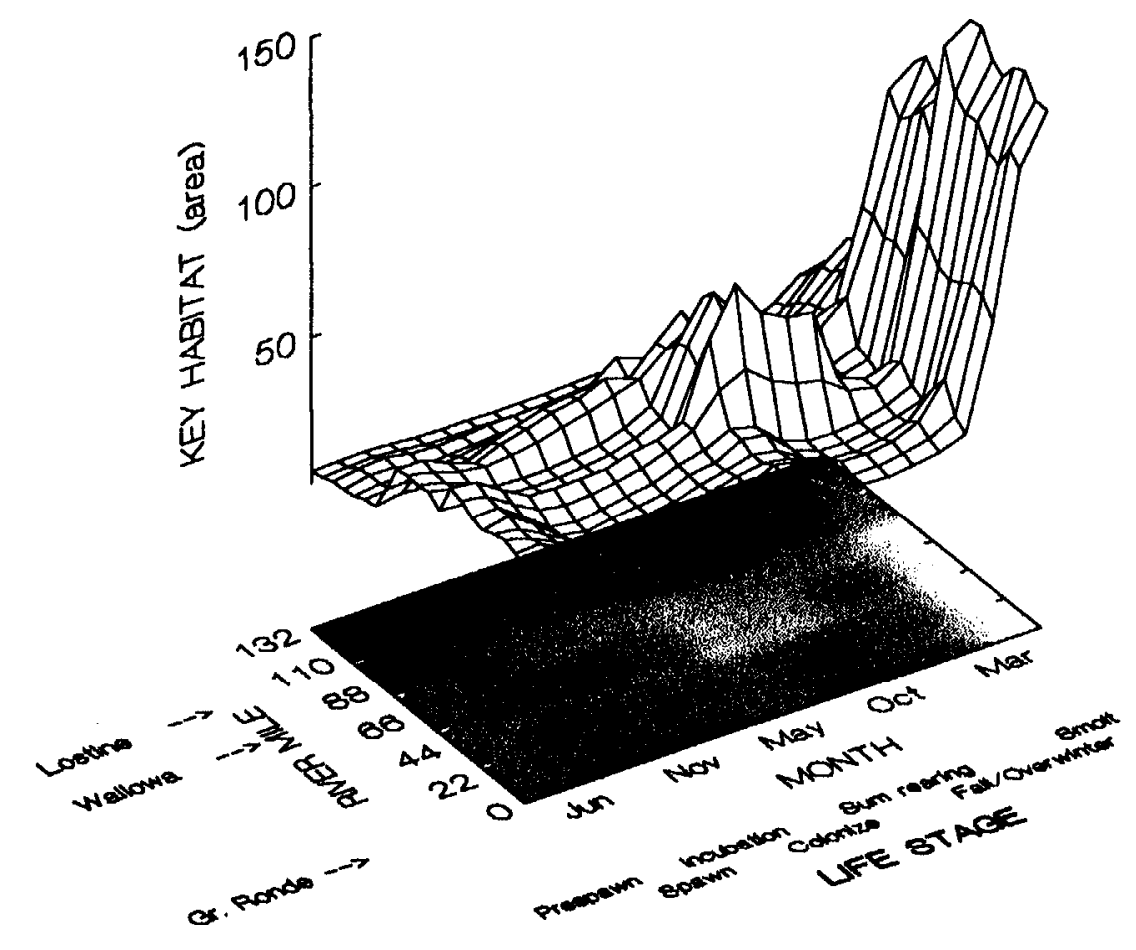

Template

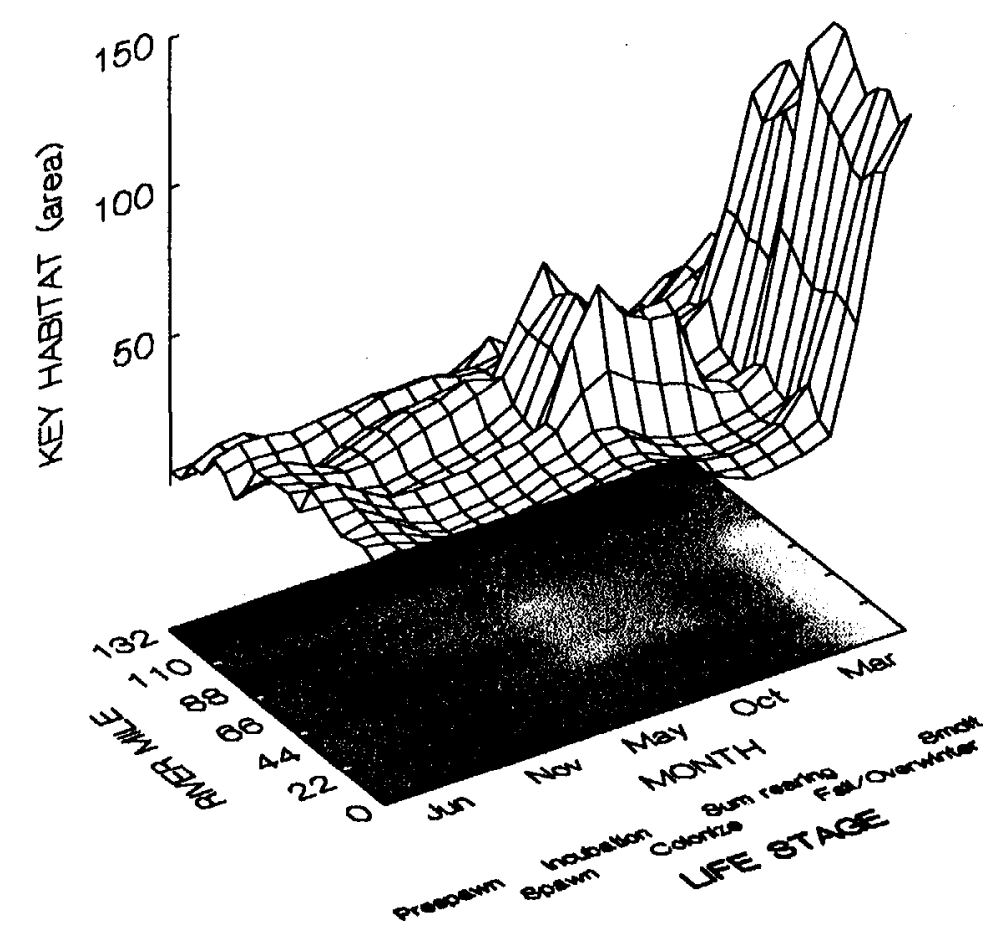




\section{Lostine R/Wallowa R/Grande Ronde R Environmental Attributes}
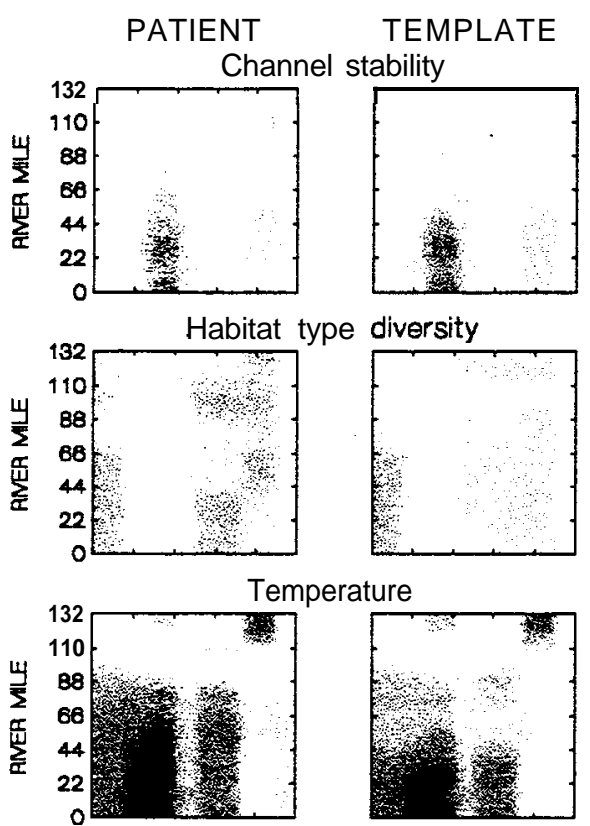

Predators
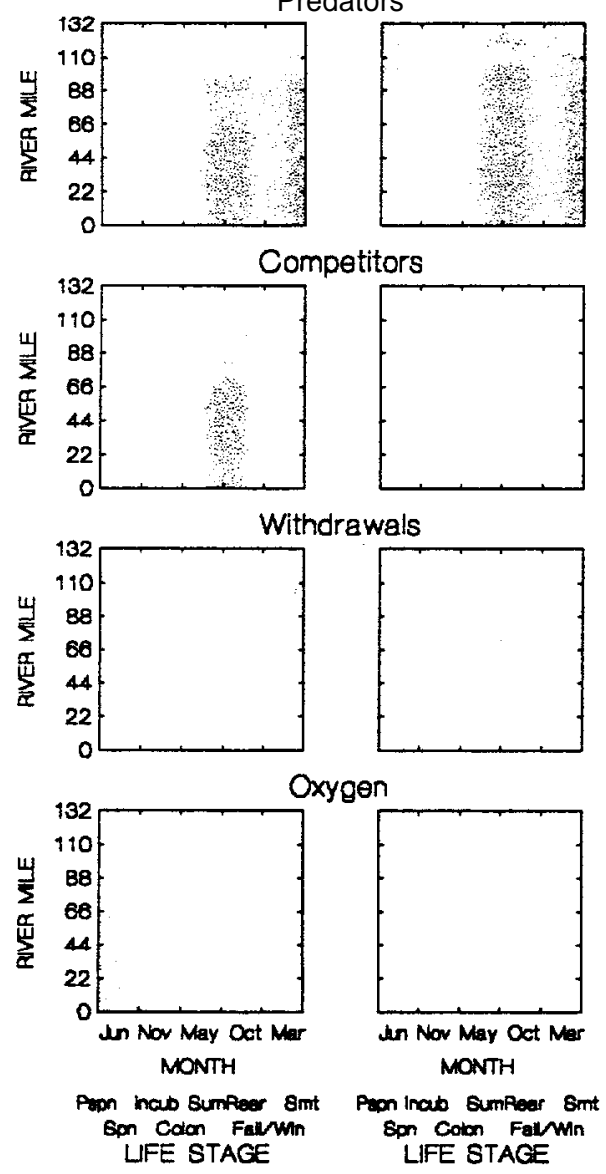

PATIENT

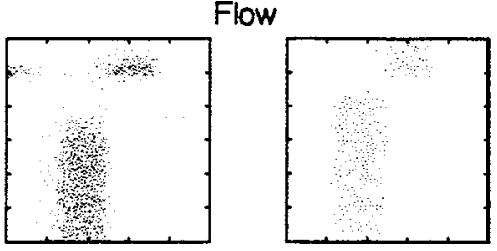

Sediment load

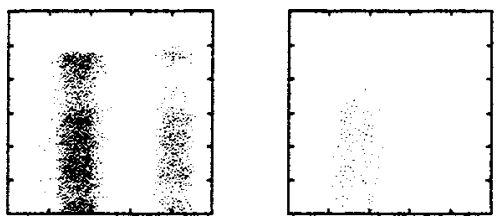

Riparian condition

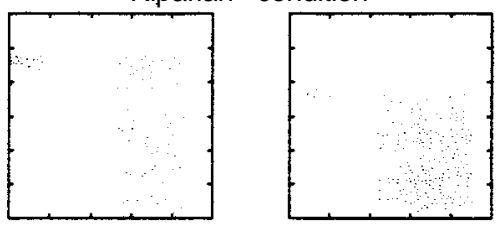

Chemicals

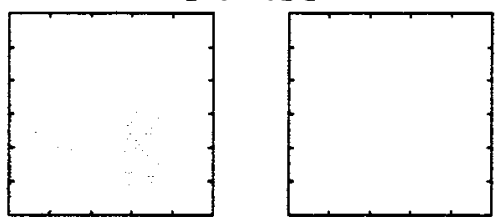

Obstructions

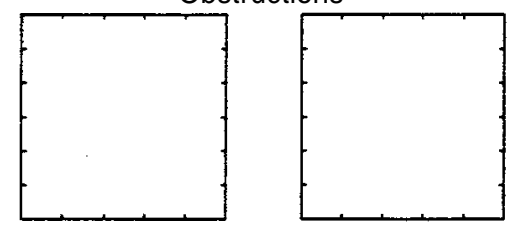

Nutrient load

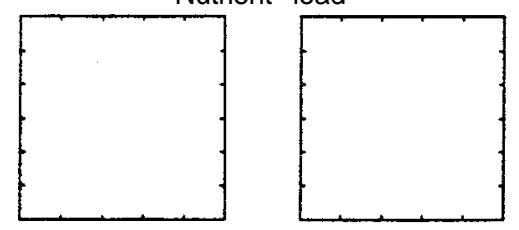

Pathogens

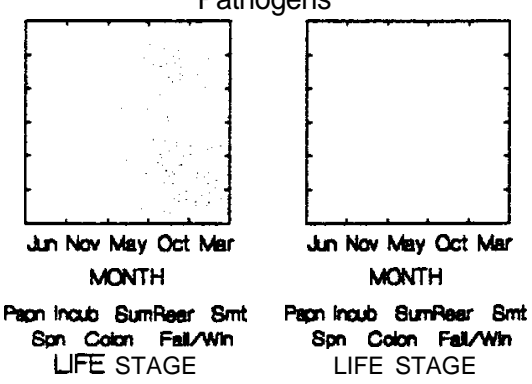

Level of

Effect

$\square$ No effect

$\square$ Low

a Moderate

䤒 High

Lethal 


\section{Lostine R/Wallowa R/Grande Ronde R Relative Survival}
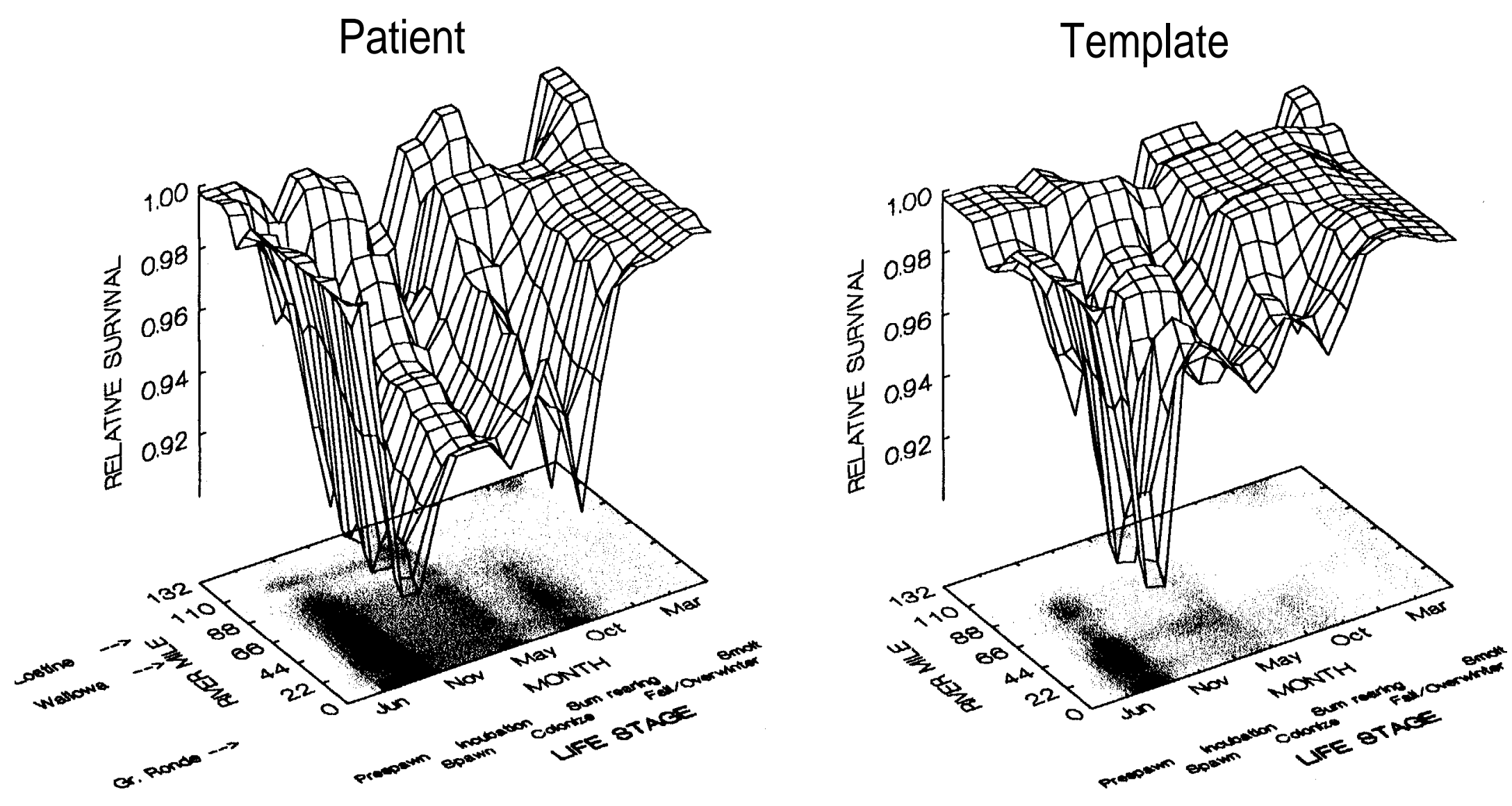


\section{Stream Reach Range}

\section{Hurricane Cr / Wallowa R / Grande Ronde R}




\section{Patient-Template Analysis Summary}

\section{Stream Reach Range: Hurricane Cr./Wallowa R./Grande Ronde R.}

\section{Key Habitat Quantity}

The quantity of key habitat for most life stages of spring chinook throughout most of Hurricane Creek (lower six miles only covered here) has been very substantially reduced compared to historic levels. The fry colonization, summer rearing, and overwintering life stages have been particularly affected.

The quantities of key habitat in most reaches of the Wallowa River downstream of Hurricane Creek River are substantially less than historic levels, except in the lower ten miles (below Minam River) where they are relatively unchanged.

The quantity of key habitat in the Grande Ronde River downstream of the Wallowa River is nearly unchanged compared to historic levels.

\section{Relative Productivity (Survival)}

The capability of Hurricane Creek of supporting spring chinook productivity (survival) is greatly reduced compared to historic conditions for every life stage except smolt. Survival conditions are worst in the upper half of the stream (upstream of Lower Alderslope Ditch). Current survival potential in this reach in mid to late summer is essentially zero due to dewatering of the channel. Survival conditions in Hurricane Creek are poor due to alterations in flow regimes, habitat diversity, sediment load, channel stability, and temperature regimes. Historically, survival conditions were excellent for the lower half of the stream, with conditions worsening with increasing gradient.

Survival conditions in the Wallowa River downstream of the Hurricane Creek have worsened compared to those that existed historically, although the lower portions of the river have always been moderately unfavorable for survival during some life stages. Conditions have also worsened in the Grande Ronde River downstream of the Wallowa River. However, survival conditions have always been poor in the later part of the prespawning stage and during the spawning and incubation stages in the lower Grande Ronde River. These conditions are mainly due to elevated water temperatures, sediment load, channel stability, habitat diversity, and the presence of predators. 


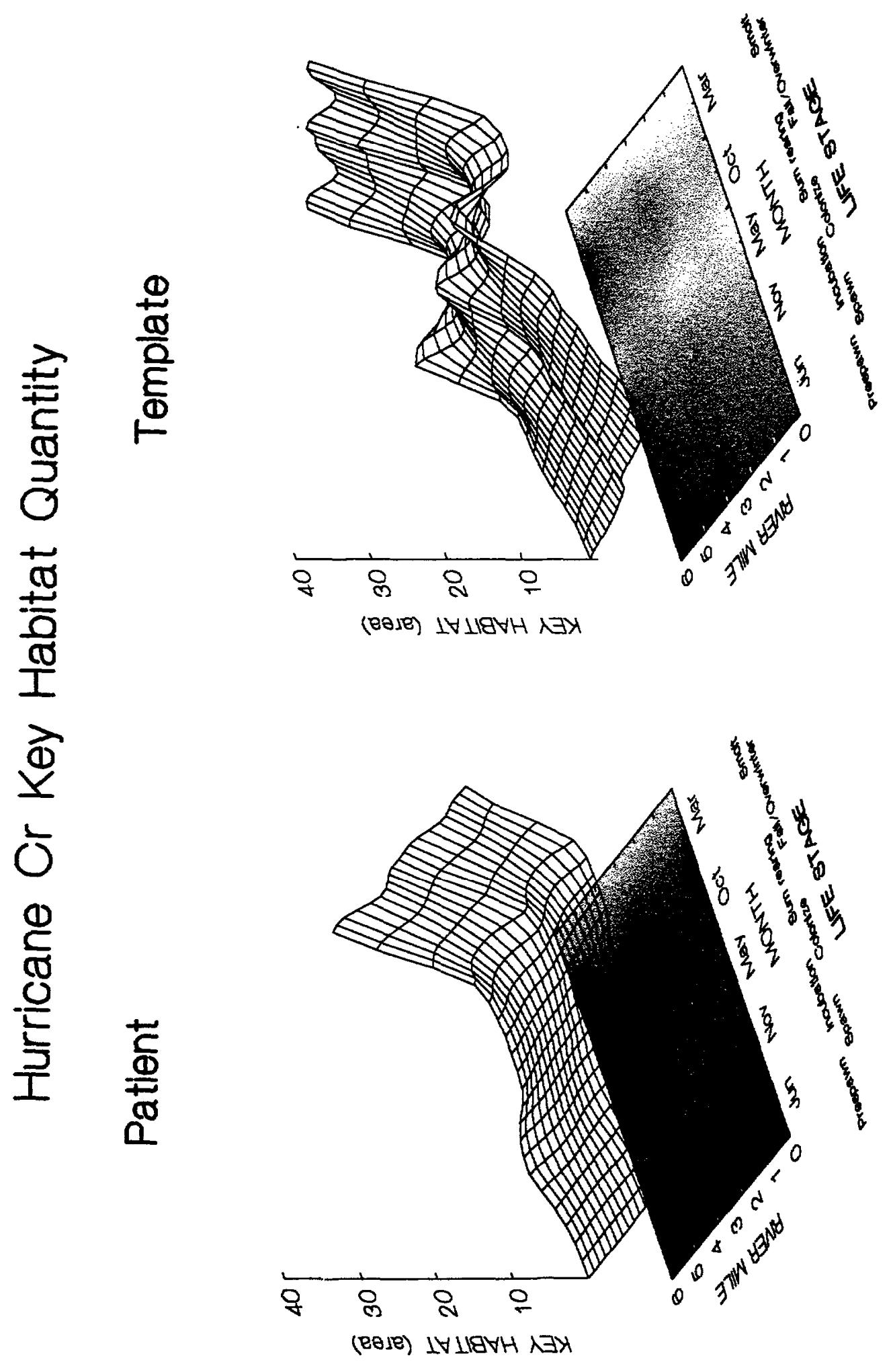


Hurricane Cr/Wallowa R/Grande Ronde R Key Habitat Quantity

Patient

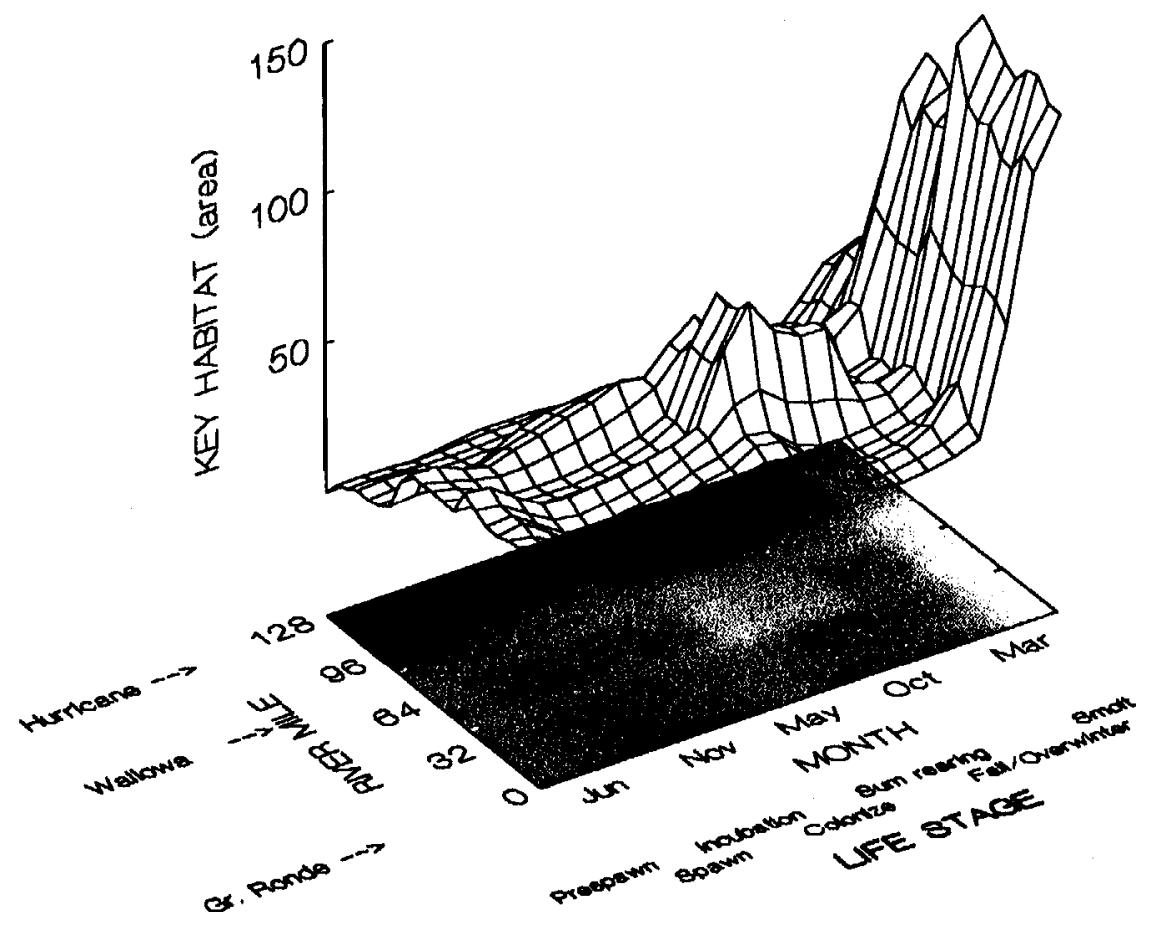

Template

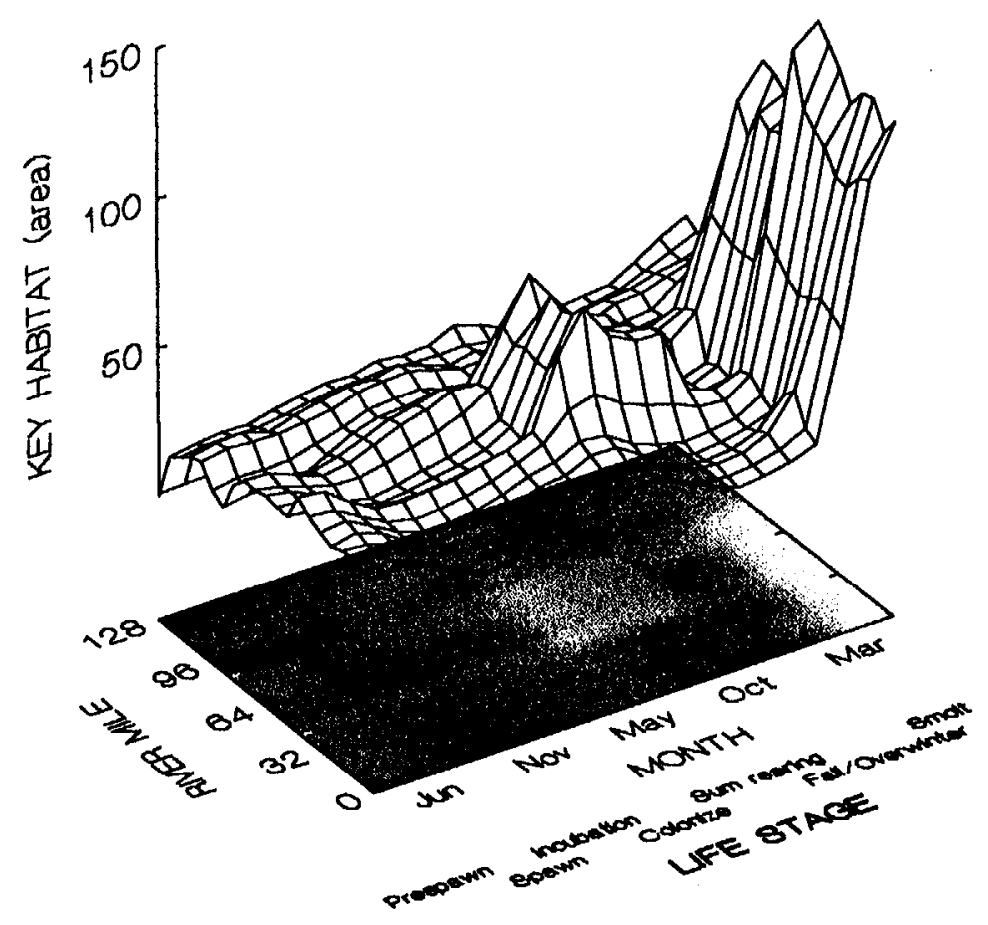




\section{Hurricane $\mathrm{Cr}$ /Wallowa R/Grande Ronde R Environmental Attributes}

PATIENT TEMPLATE

Channel stability
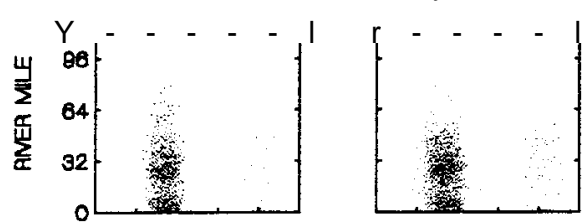

Habitat type diversity
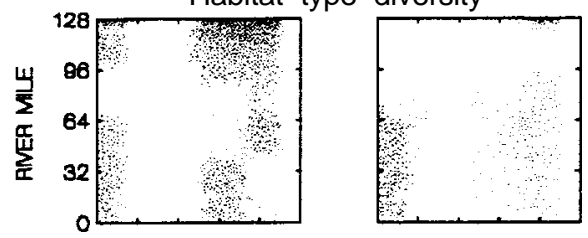

Temperature
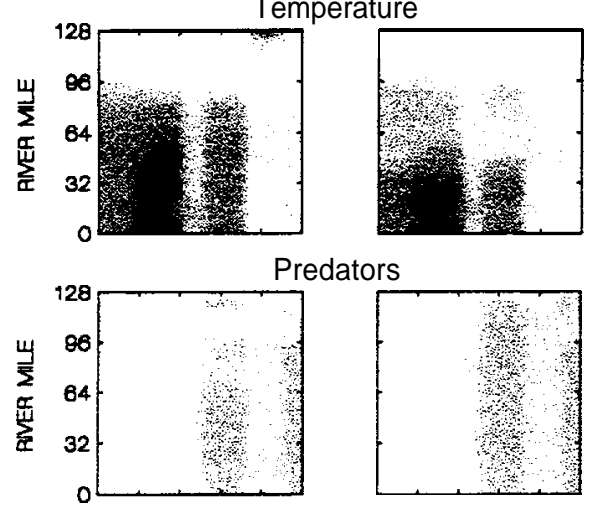

Predators
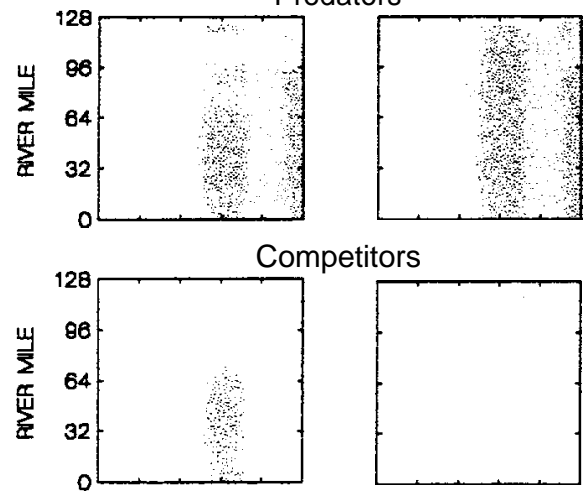

Competitors

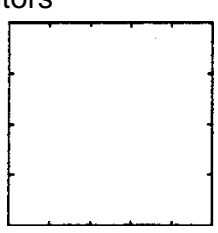

Withdrawals
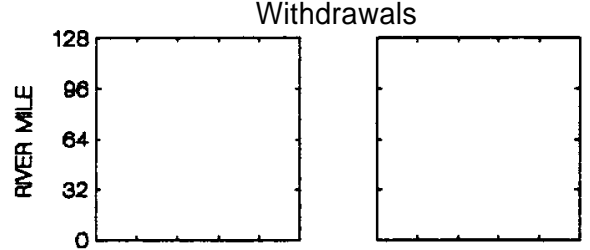

Oxygen

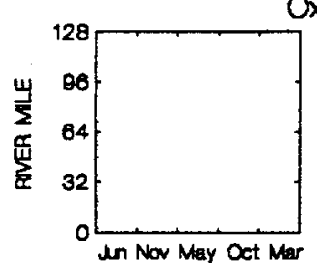
MONTH

Paon hab sumfeer smt Son Colan Fallwn LIFE STAGE

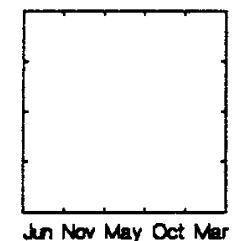

MONTH

Pan Inat Bumfacer Emt Son Coton Falnin
LIFE STAGE

\section{PATIENT \\ TEMPLATE}

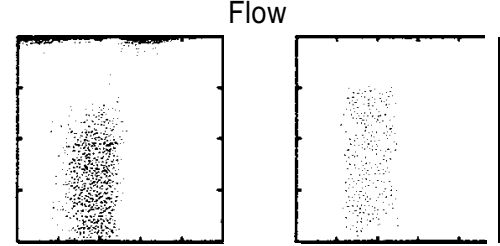

Sediment load

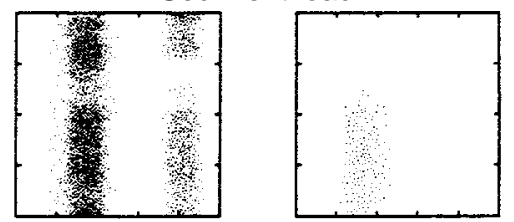

Riparian condition
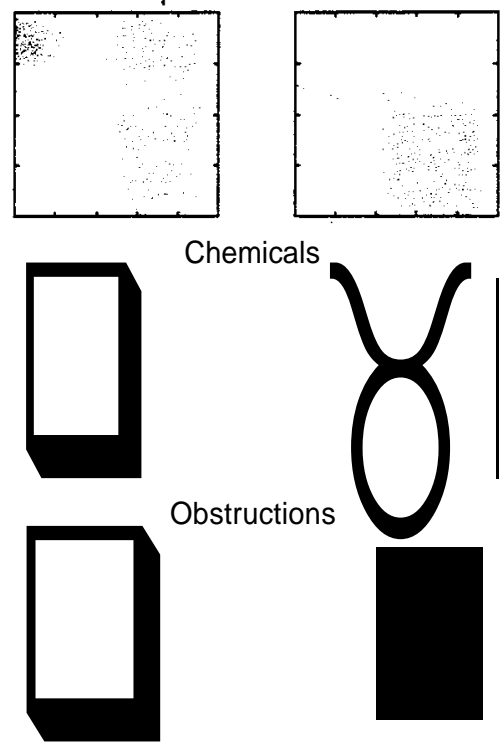

Nutrient load

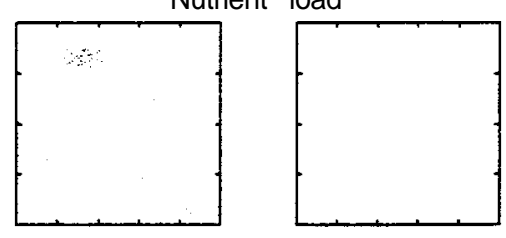

Pathogens
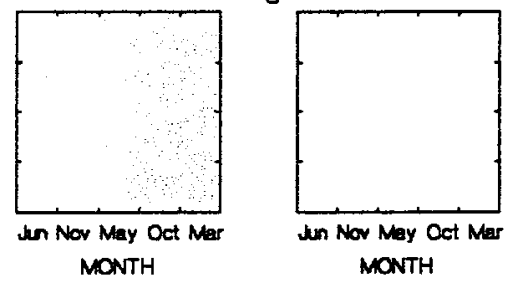

Pron inat Sumpar Simt Pan inat Sumfeer Sm Sp colon Fallwn son Coin Fallwin
LIFE STAGE
Level of

Effect

$\mathrm{Cl}$ No effect

$\square$ Low

口 Moderate

圈 High

- Lethal 


\section{Hurricane Cr/Wallowa R/Grande Ronde R Relative Survival}
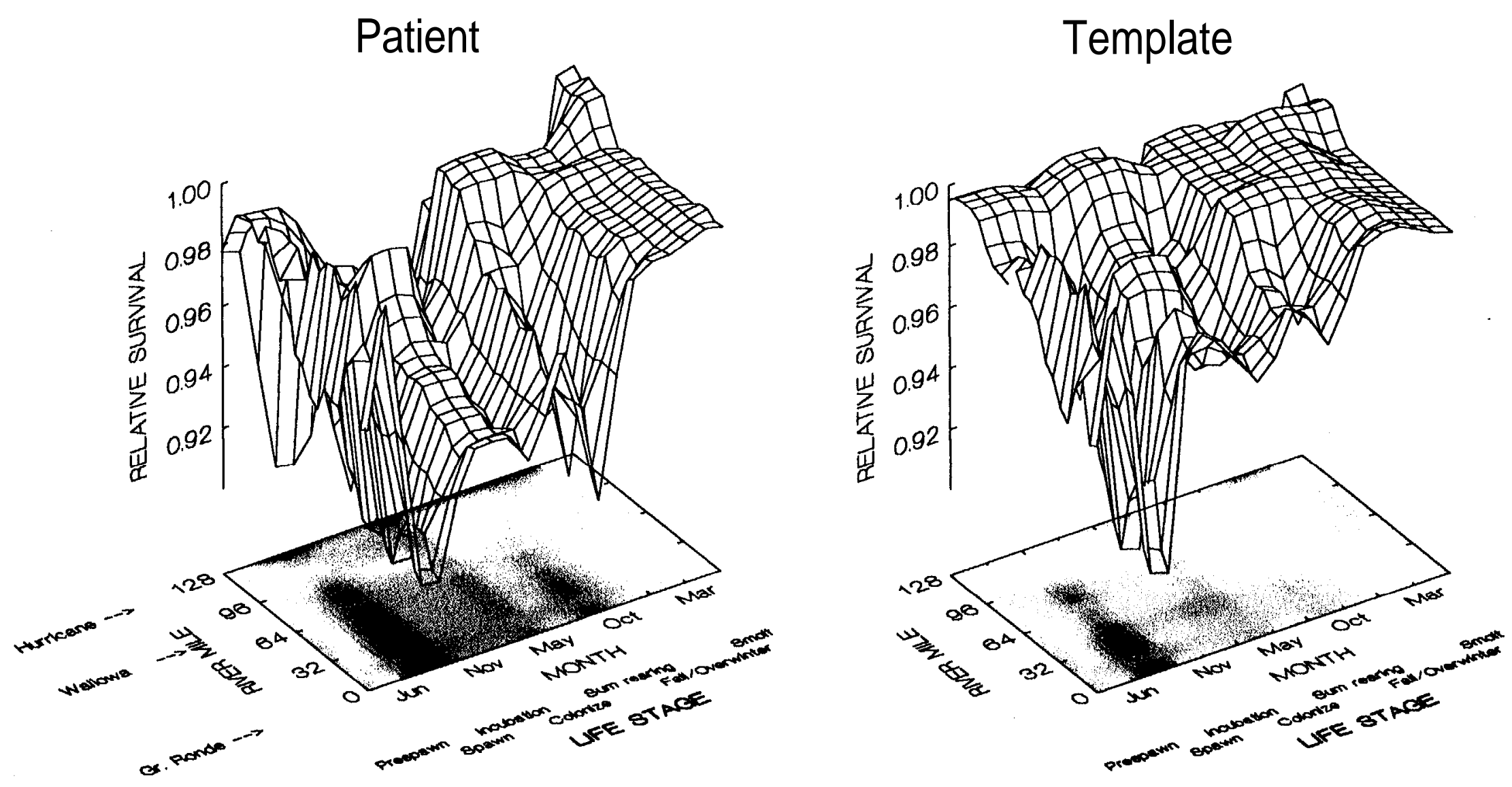


\section{Stream Reach Range}

Prairie Cr / Wallowa R / Grande Ronde R 


\section{Patient-Template Analysis Summary}

Stream Reach Range: Prairie Cr./Wallowa R./Grande Ronde R.

\section{Key Habitat Quantity}

The quantity of key habitat for all life stages of spring chinook throughout most of the Prairie Creek drainage has been greatly increased compared to historic levels. These changes are most dramatic in the upper portions of the drainage, as seen in OK Gulch (Hays and Pratt forks are not depicted in the landscapes). The reason for this increase is the addition of water into the drainage through the irrigation system. Water, withdrawn from the Wallowa River near the outlet of Wallowa Lake and from the Imnaha basin, are discharged into the Prairie subbasin, significantly enhancing flow in this system compared to historic flow levels.

The quantities of key habitat in most reaches of the Wallowa River downstream of Prairie Creek River are substantially less than historic levels, except in the lower ten miles (below Minam River) where they are relatively unchanged.

The quantity of key habitat in the Grande Ronde River downstream of the Wallowa River is nearly unchanged compared to historic levels.

\section{Relative Productivity (Survival)}

The capability of Prairie Creek of supporting spring chinook productivity (survival) compared to historic conditions differs by life stage and stream reach. In general, historic survival conditions in the lower portion of Prairie Creek were excellent for all life stages, but worsened rapidly as distance increased from the Wallowa River due to the stream's very small size. Lack of flow was the primary environmental constraint on productivity prior to the construction of the irrigation system. Survival conditions currently are good for some life stages throughout the stream, but are poor for egg incubation. The two attributes most affecting conditions currently are sediment load and habitat diversity .

Survival conditions in the Wallowa River downstream of Prairie Creek have worsened compared to those that existed historically, although the lower portions of the river have always been moderately unfavorable for survival during some life stages. Conditions have also worsened in the Grande Ronde River downstream of the Wallowa River. However, survival conditions have always been poor in the later part of the prespawning stage and during the spawning and incubation stages in the lower Grande Ronde River. These conditions are mainly due to elevated water temperatures, sediment load, channel stability, habitat diversity, and the presence of predators. 


\section{OK Gulch/Prairie Cr Key Habitat Quantity}

\section{Patient}

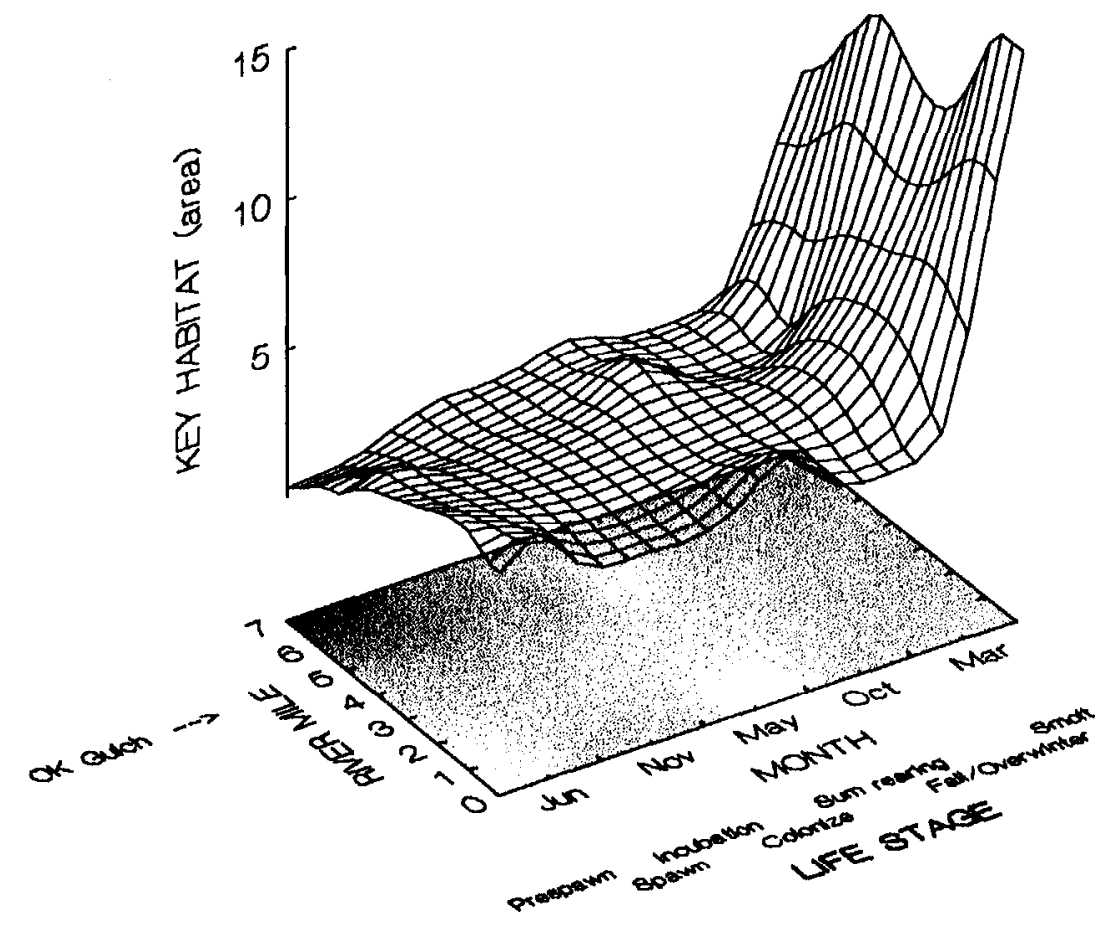

Template

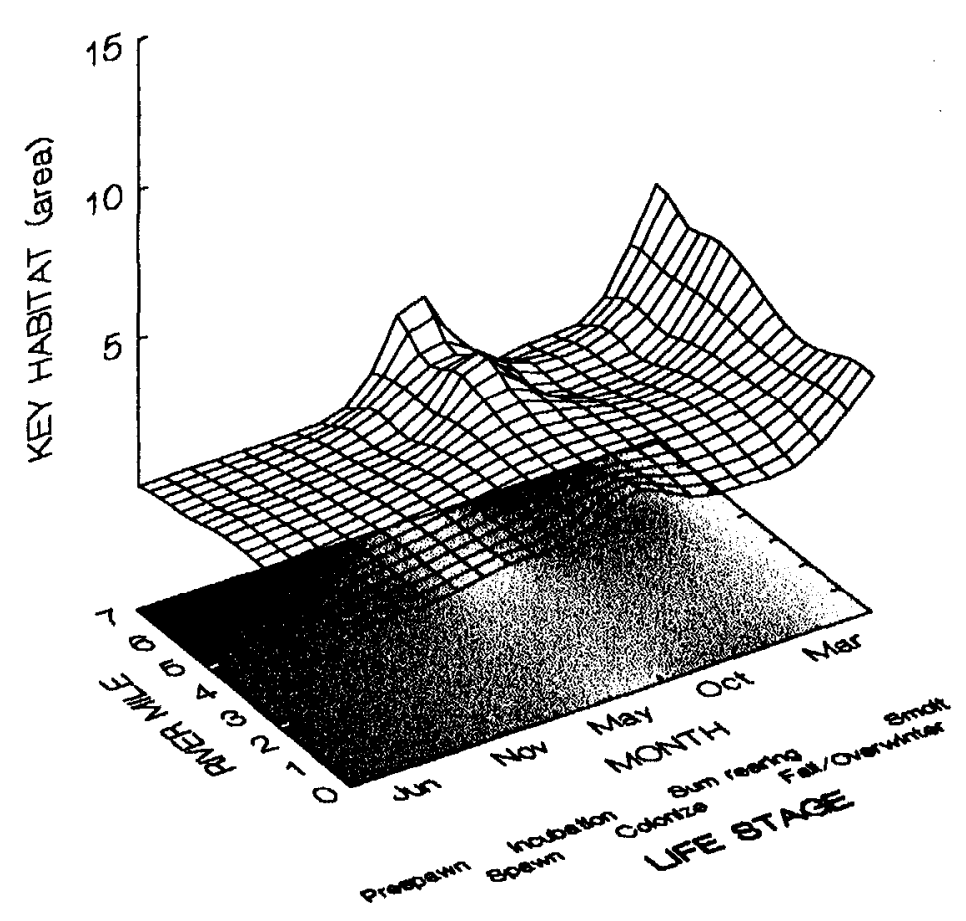




\section{OK Gulch/Prairie Cr Environmental Attributes}
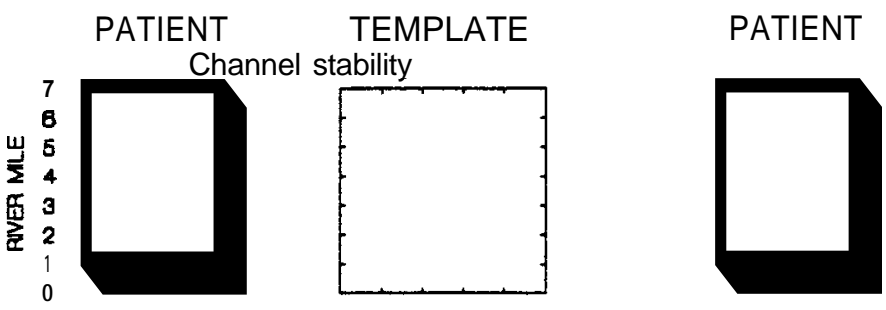

TEMPLATE

Habitat type diversity
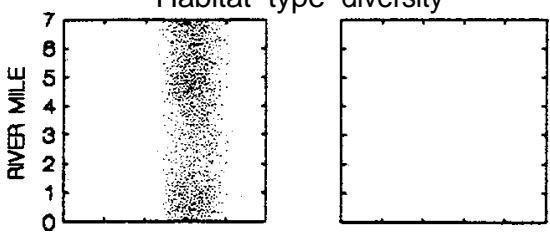

Sediment load
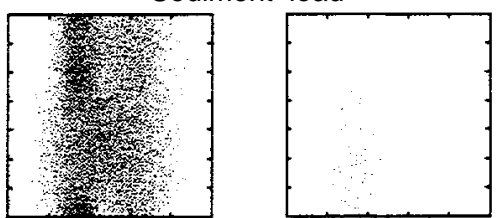

Riparian condition
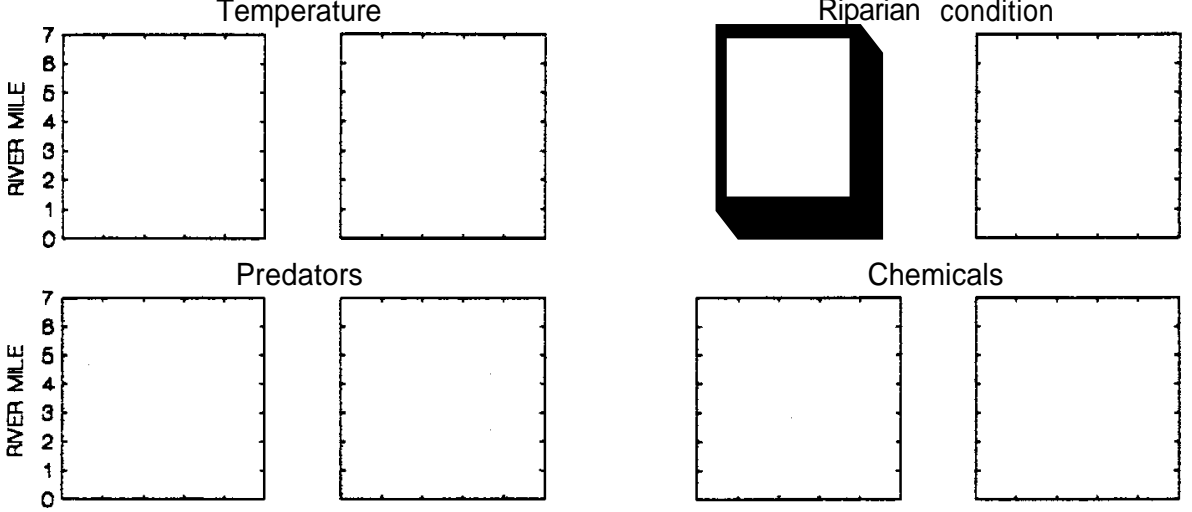

Predators

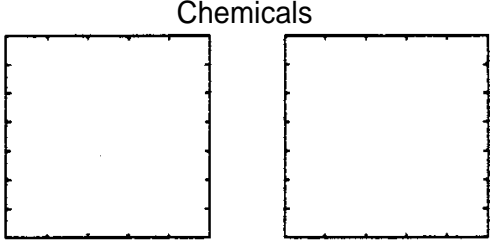

Obstructions
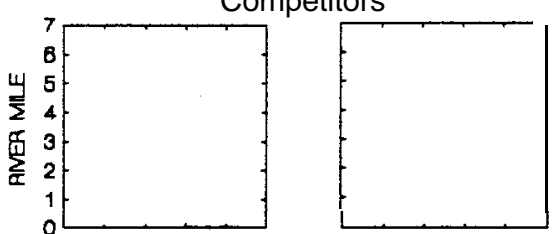

Withdrawals
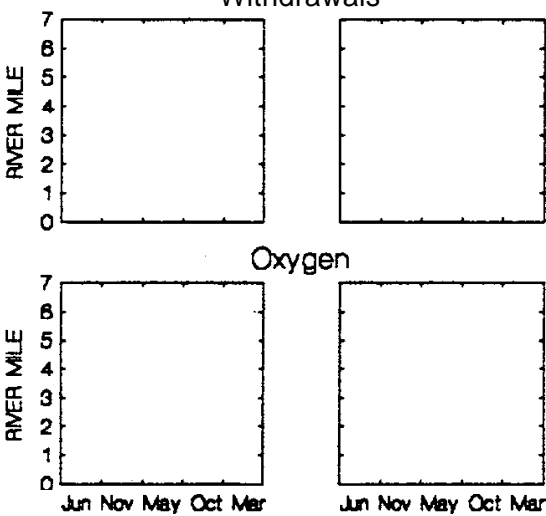

Oxygen
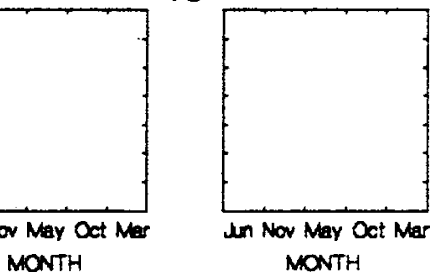
MONTH
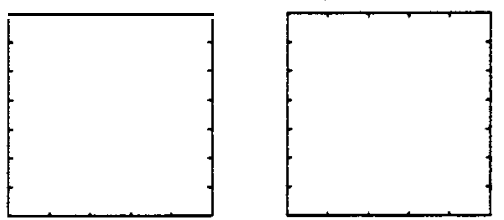

Nutrient load

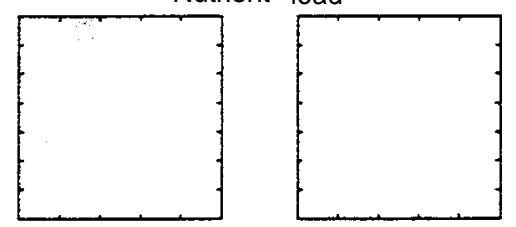

Pathogens
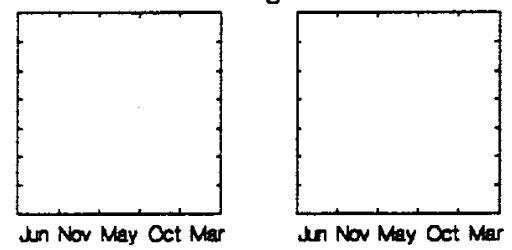

Level of

Effect

$\square$ No effect

$\square$ Low

圆 Moderate

High MONTH MONTH

Poron inab sumpear 8 int Spn Coion Fallwhin LIFE STAGE

Paon now Sumfiear $8 m$ Son Coton Fall Min LIFE STAGE Son Colon Fal Wm
UFE STAGE 


\section{Lightning $\mathrm{Cr} / \mathrm{Imnaha}$ R Relative Survival}
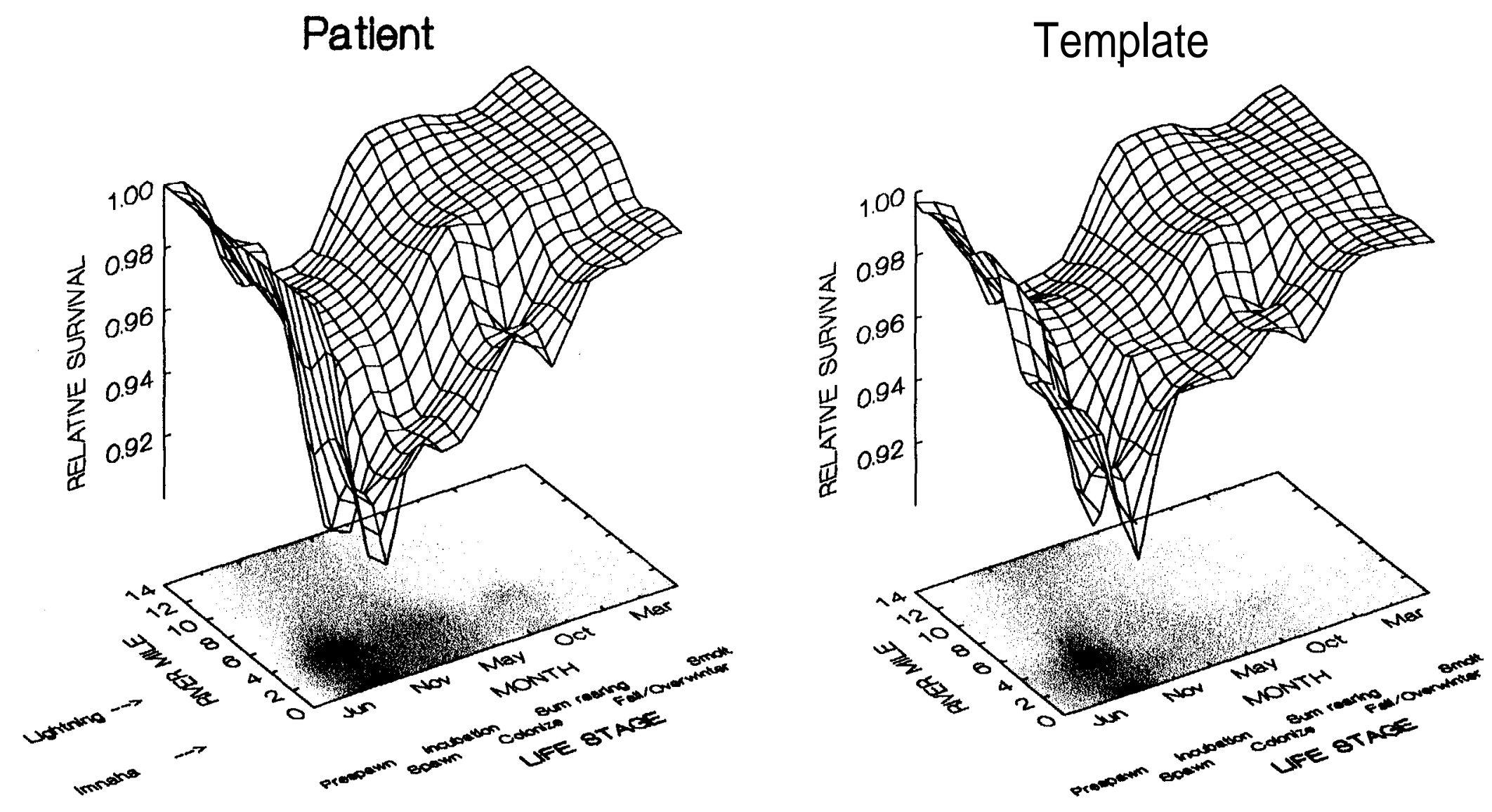

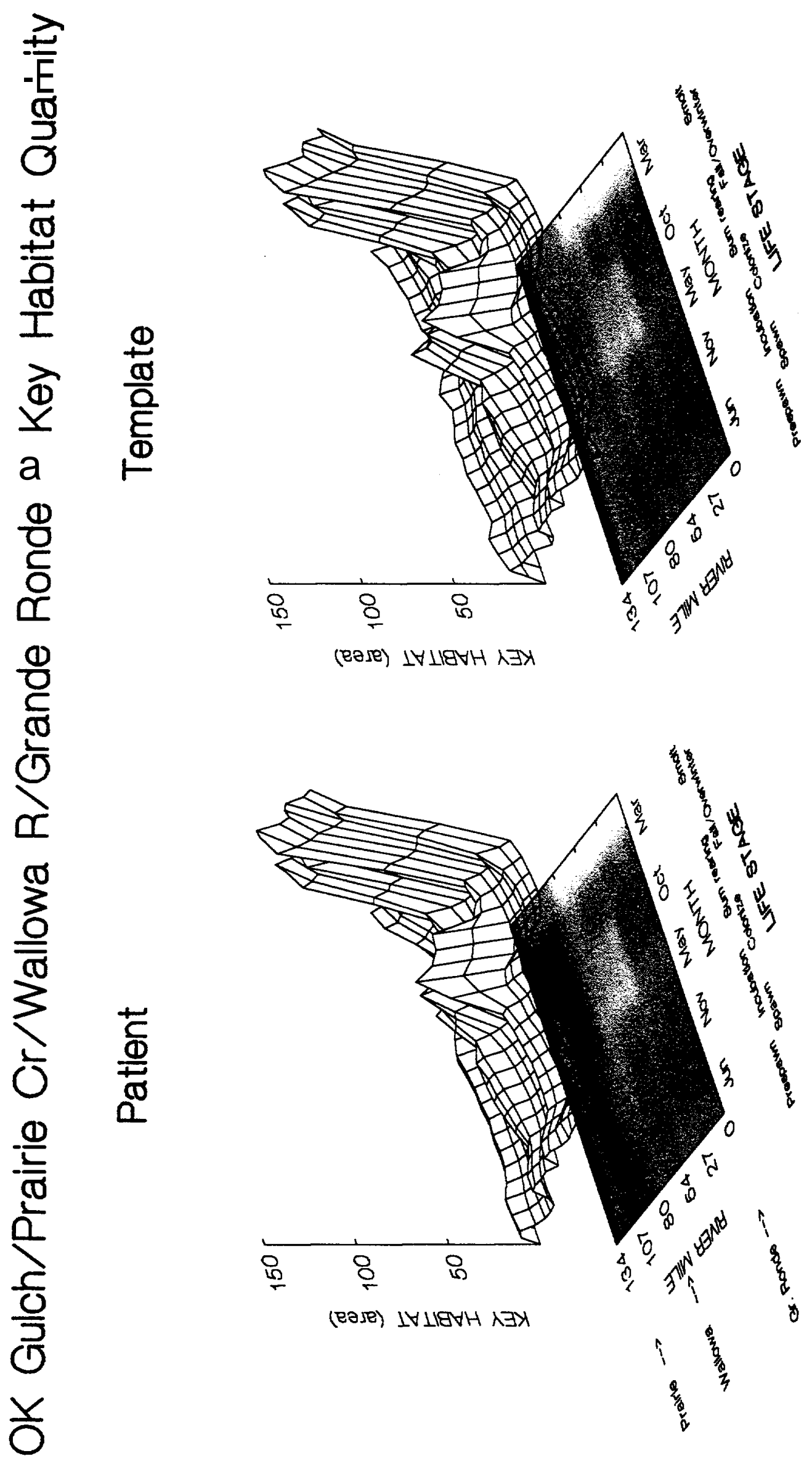


\section{Prairie $\mathrm{Cr} /$ Wallowa R/Grande Ronde R Environmental Attributes}

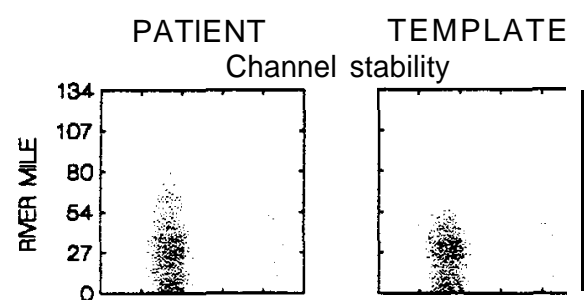

Habitat type diversity
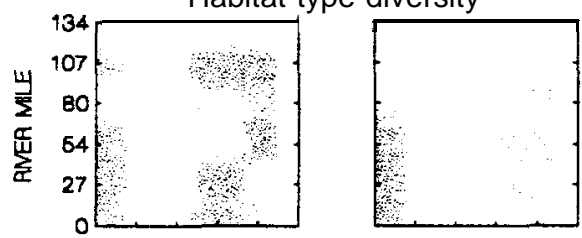

Temperature
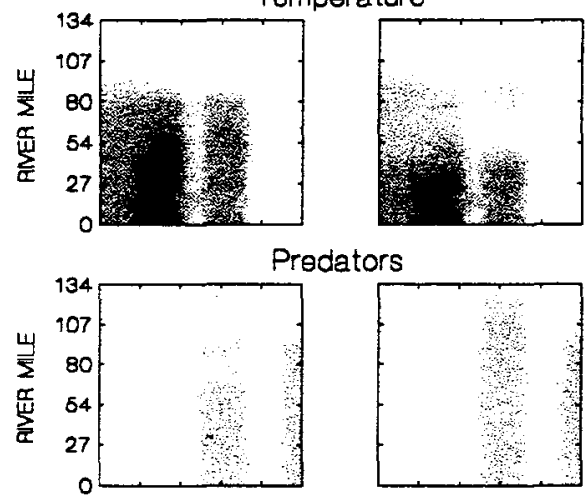

Predators

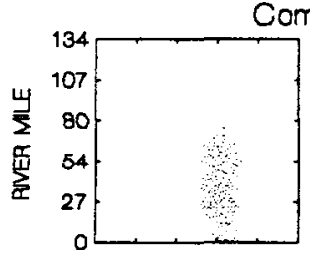

Competitors
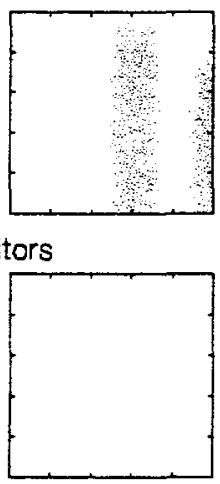

Withdrawals
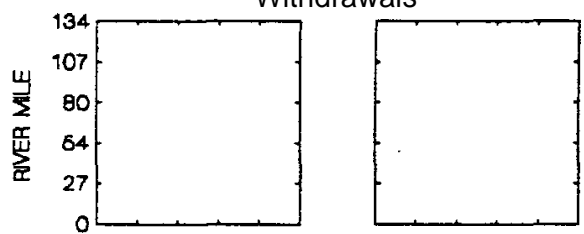

Oxygen

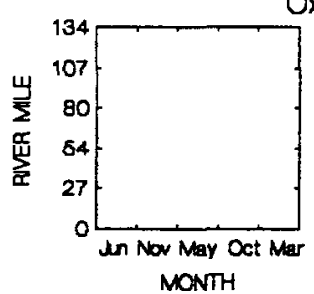

Pepn how Sumpeer 8mt Son Colon Faumn LIFE STAGE

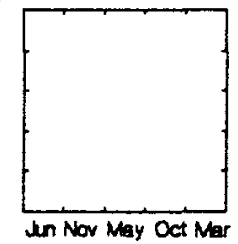
MONTH

Paon trat Sumpear Sint Son Colon Fallwn LIFE STAGE
PATIENT TEMPLATE

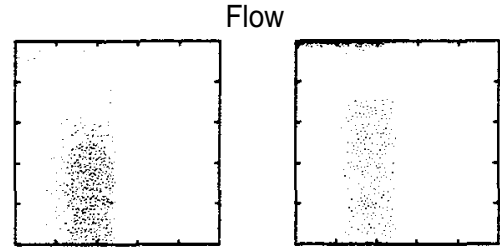

Sediment load

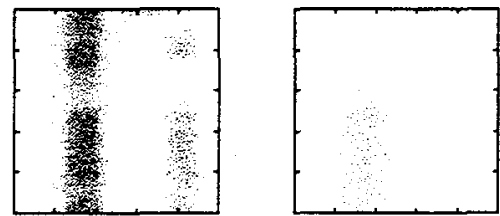

Plparian condition
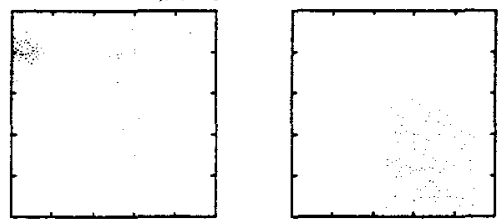

Chemicals

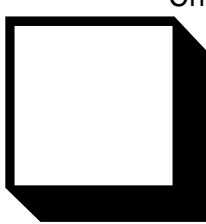

Obstructions

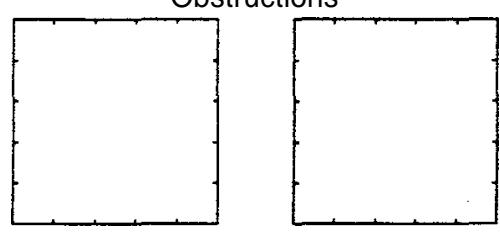

Nutrient load

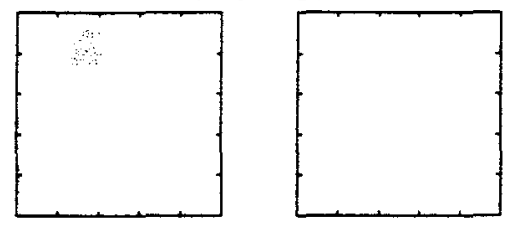

Pathogens

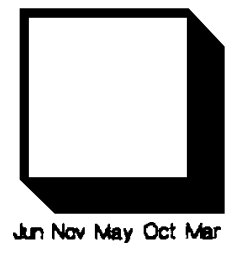

MONTH

Jun Now May Oet Mar MONTH

Peon Inat Sumfieer Sint Papn Inat Bumfear 8mt

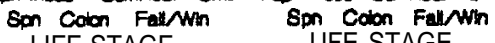
LIFE STAGE
Level of

Effect

$\square$ No effect

$\square$ Low

$\square$ Moderate

$\square$ High

, Lethal 


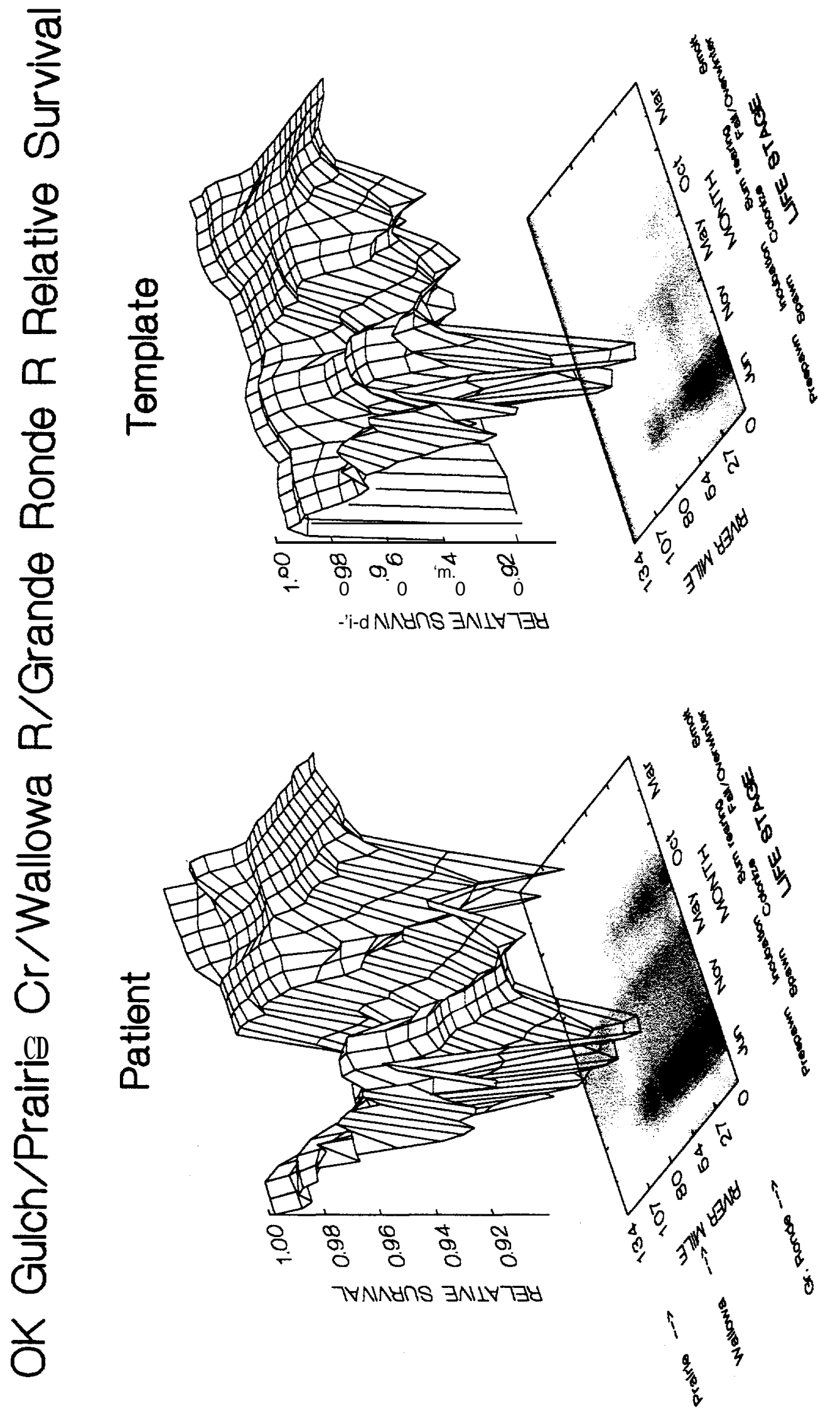




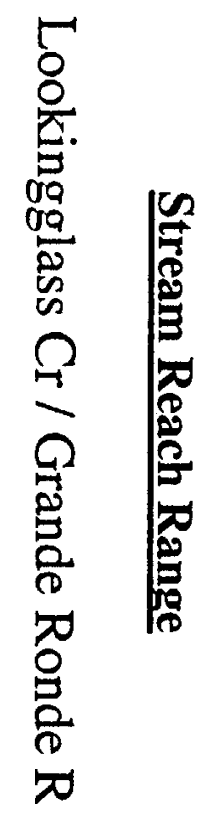




\section{Patient-Template Analysis Summary}

Stream Reach Range: Lookingglass Cr./Grande Ronde R.

\section{Key Habitat Quantity}

The quantity of key habitat for spring chinook in Lookingglass Creek is changed little compared to historic levels.

The quantity of key habitat in the Grande Ronde River downstream of Lookingglass Creek is virtually unchanged compared to historic levels.

Compared to the Grande Ronde River, Lookingglass Creek contains relatively little key habitat.

\section{Relative Productivity (Survival)}

The capability of Lookingglass Creek of supporting spring chinook productivity (survival) is largely unchanged compared to historic conditions for all life stages.

Survival conditions within the Grande Ronde River downstream of Lookingglass have worsened compared to those that existed historically, although these reaches have always been unfavorable for survival during some life stages. In particular, survival conditions have always been poor in the later part of the prespawning stage and during the spawning and incubation stages. Current survival conditions are mainly due to elevated water temperatures, sediment load, channel stability, habitat diversity, and the presence of predators. 


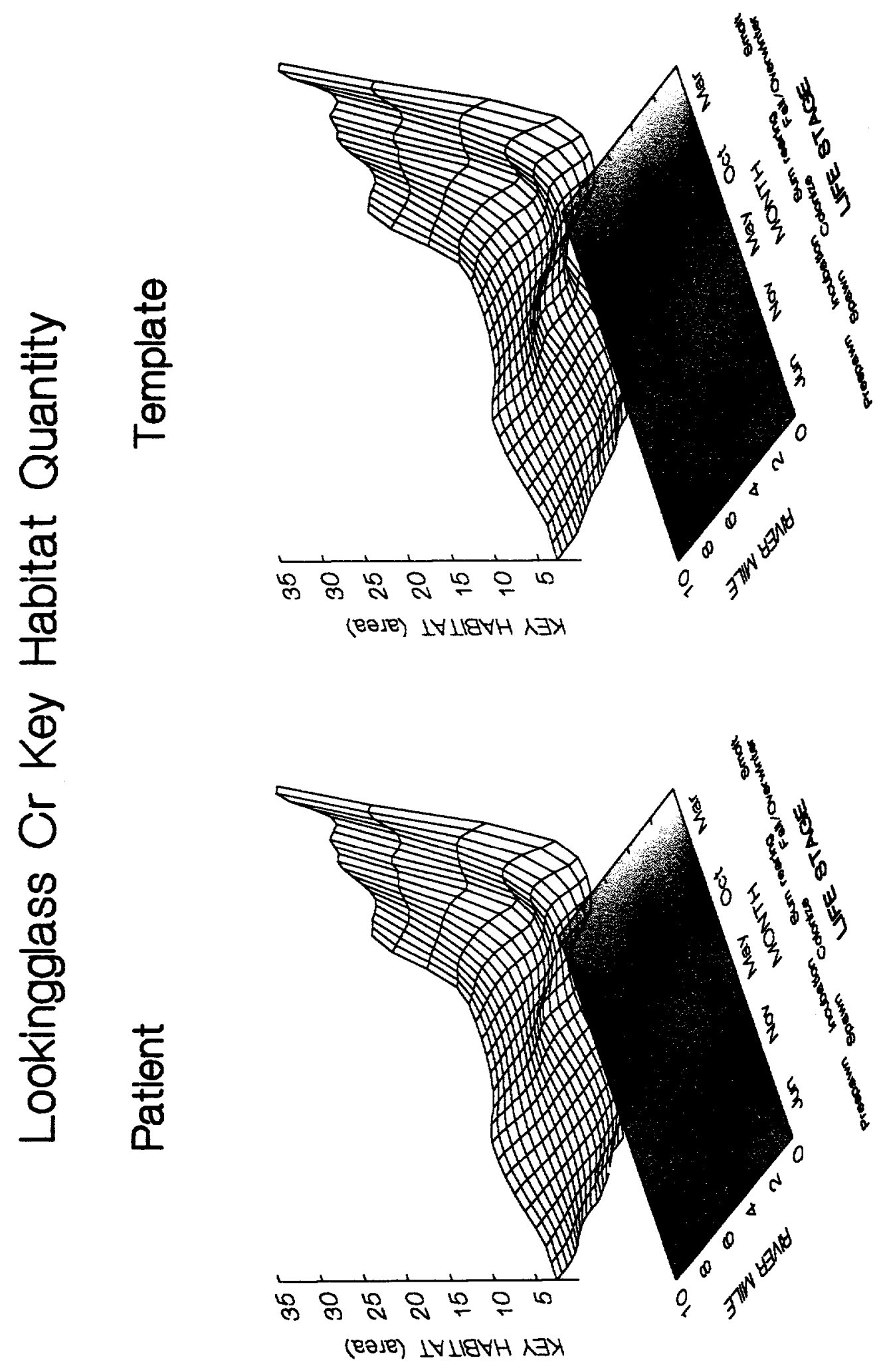




\title{
Lookingglass Cr Environmental Attributes
}

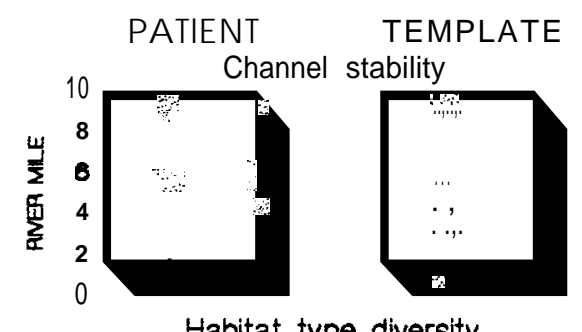

\author{
PATIENT \\ TEMPLATE
}
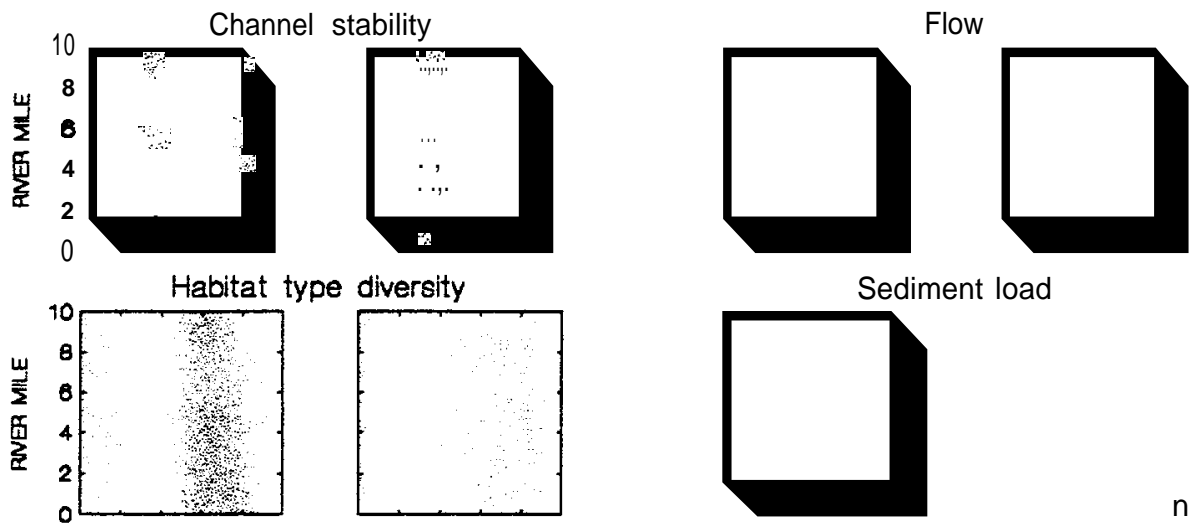

Sediment load

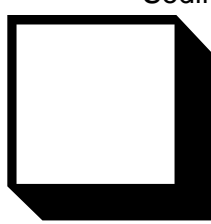

n
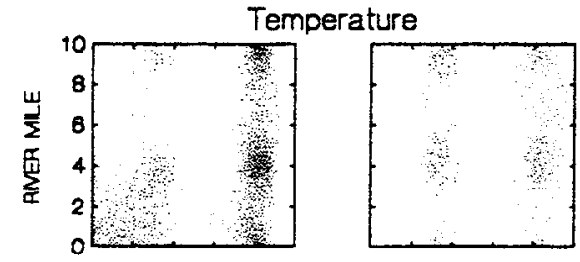

Predators

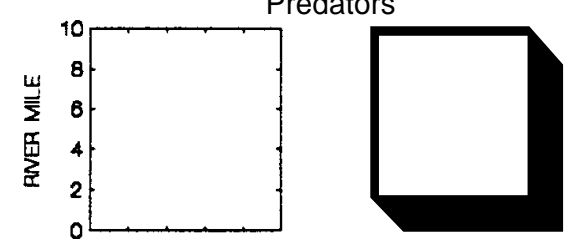

Riparian condition

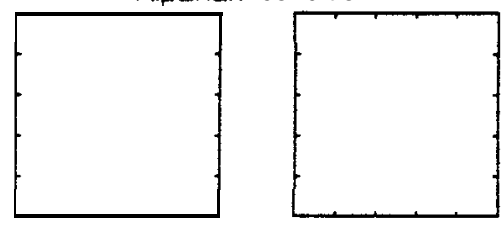

Chemicals
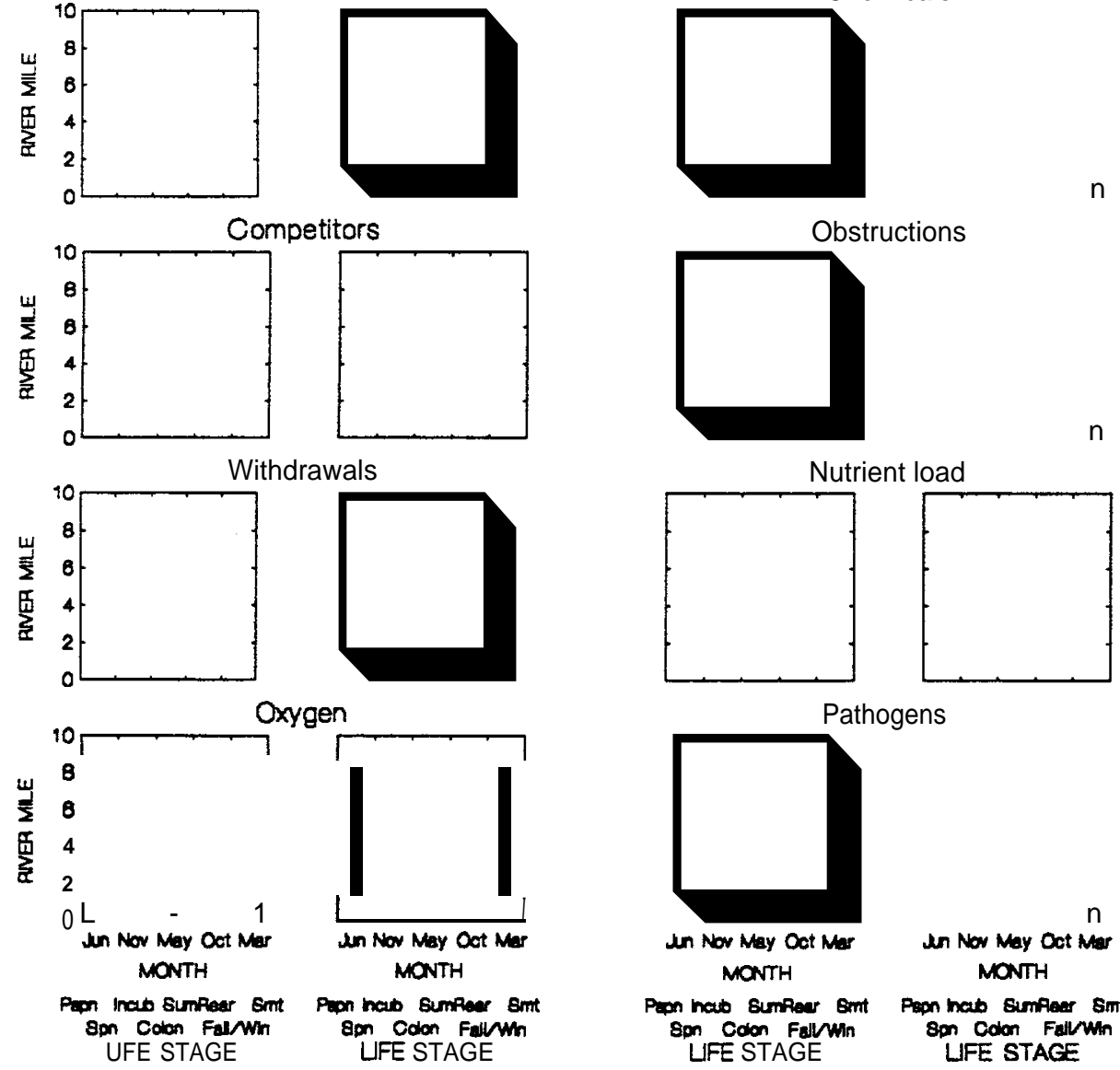

Level of

Effect

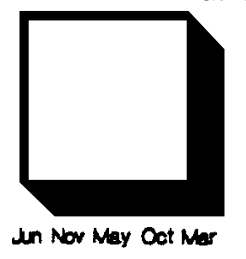

No effect

$\square$ Low

$\square$ Moderate

High

MONTH

in Now May Oct Mar MONTH

Pan now Sumfear Emt Fapn hab Eimfleer 8mt Eon Coon Fallwin
LIFE STAGE
LJFE STACE 


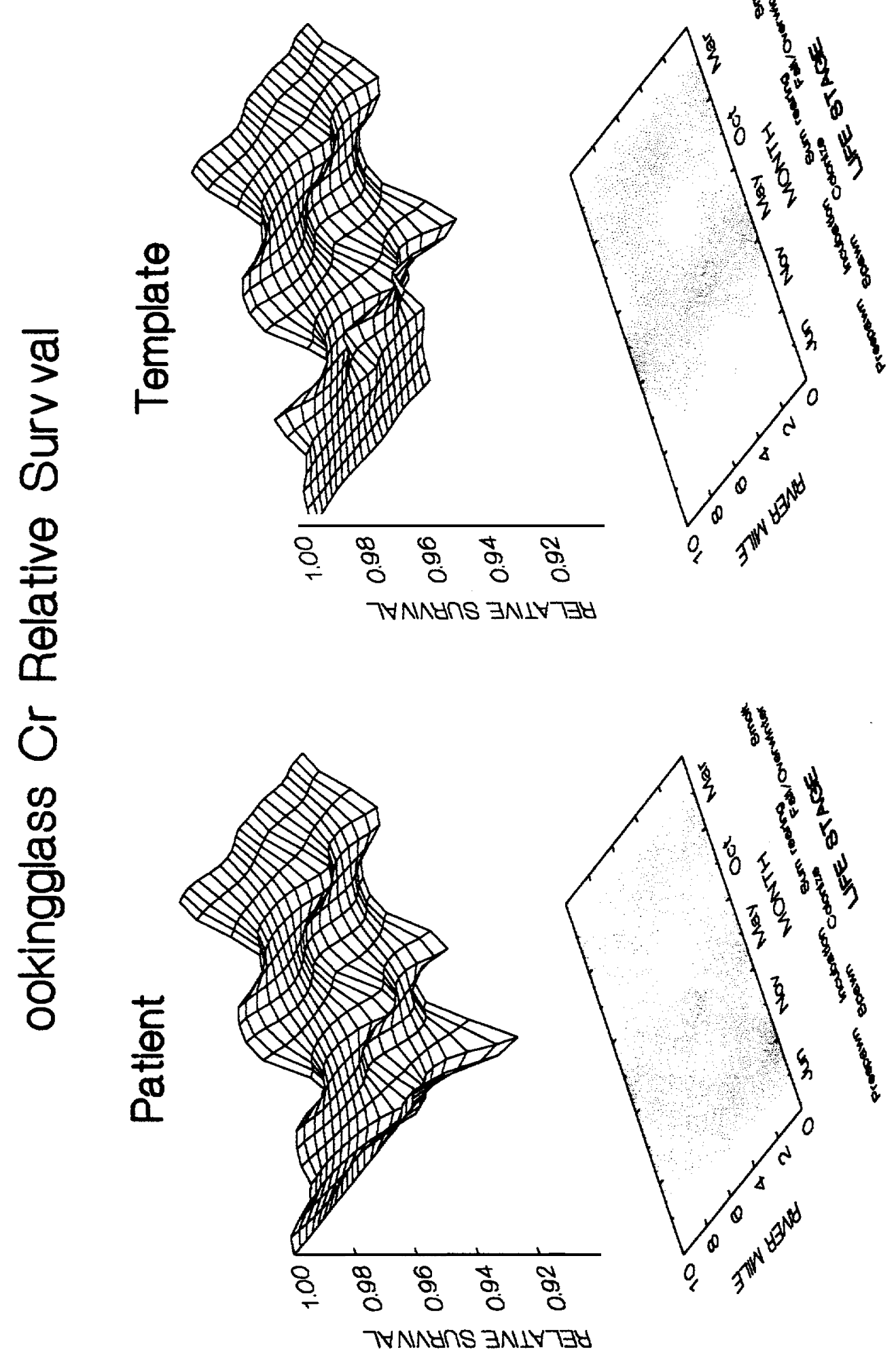




\section{Lookingglass $\mathrm{Cr} /$ Grande}

\section{Ronde $R$ Key Habitat $Q$ Template}
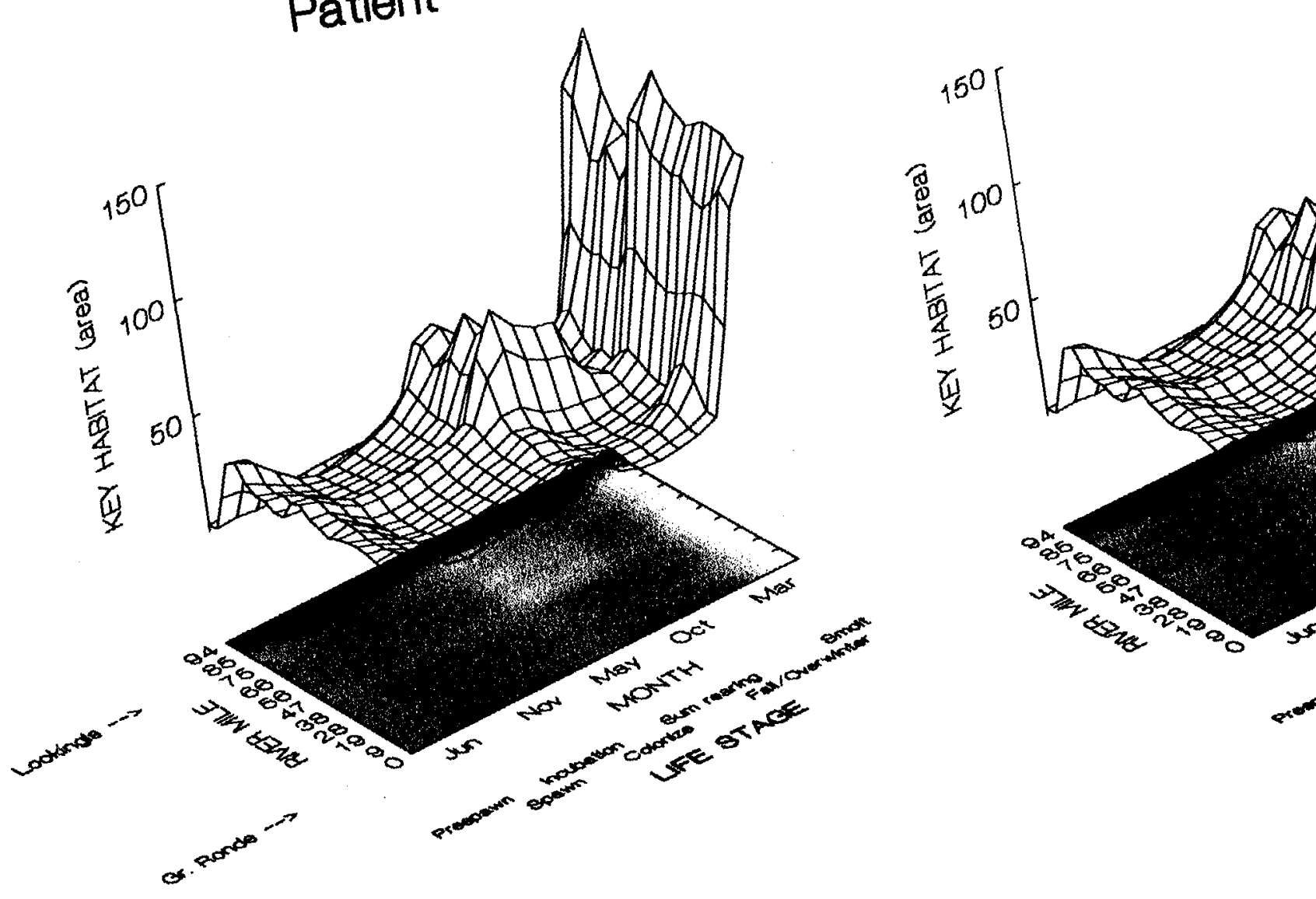


\section{Lookingglass $\mathrm{Cr}$ /Grande Ronde $\mathrm{R}$ Environmental Attributes}
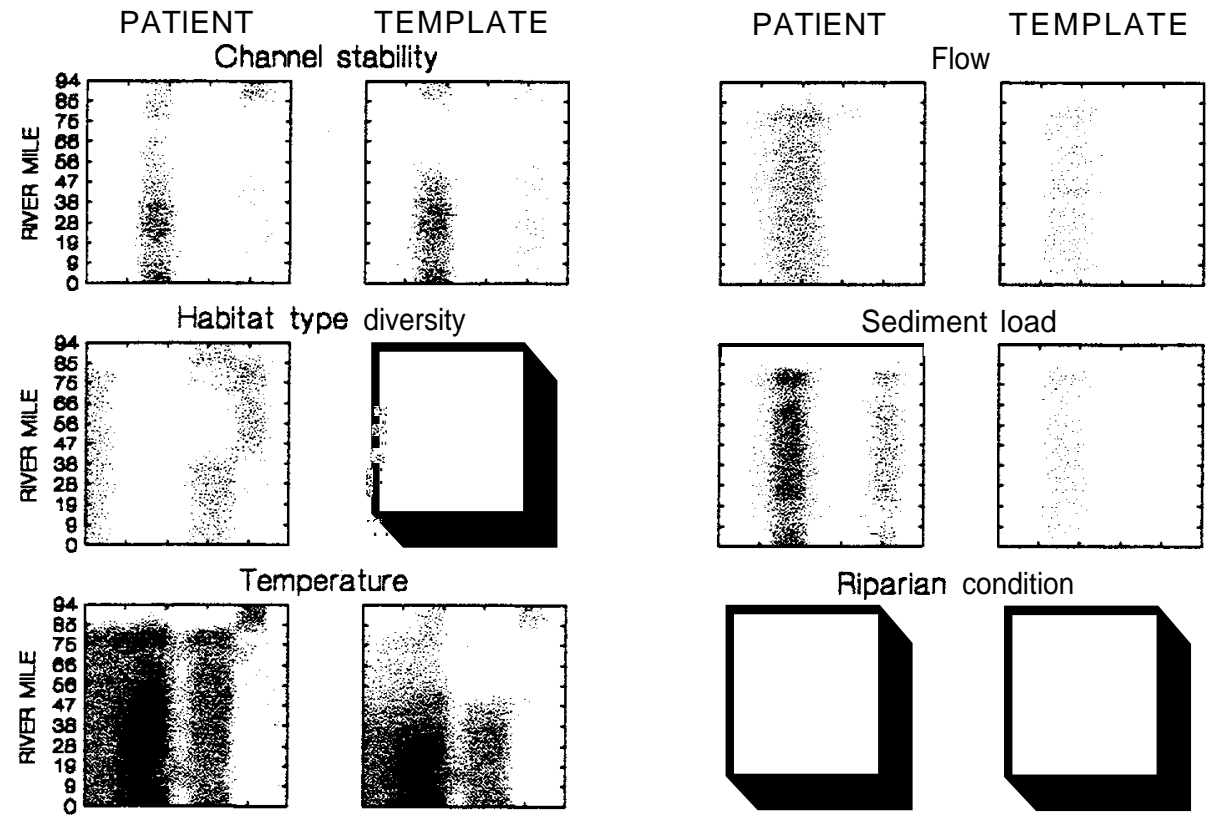

Sediment load
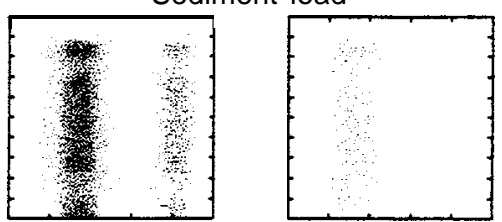

Riparian condition
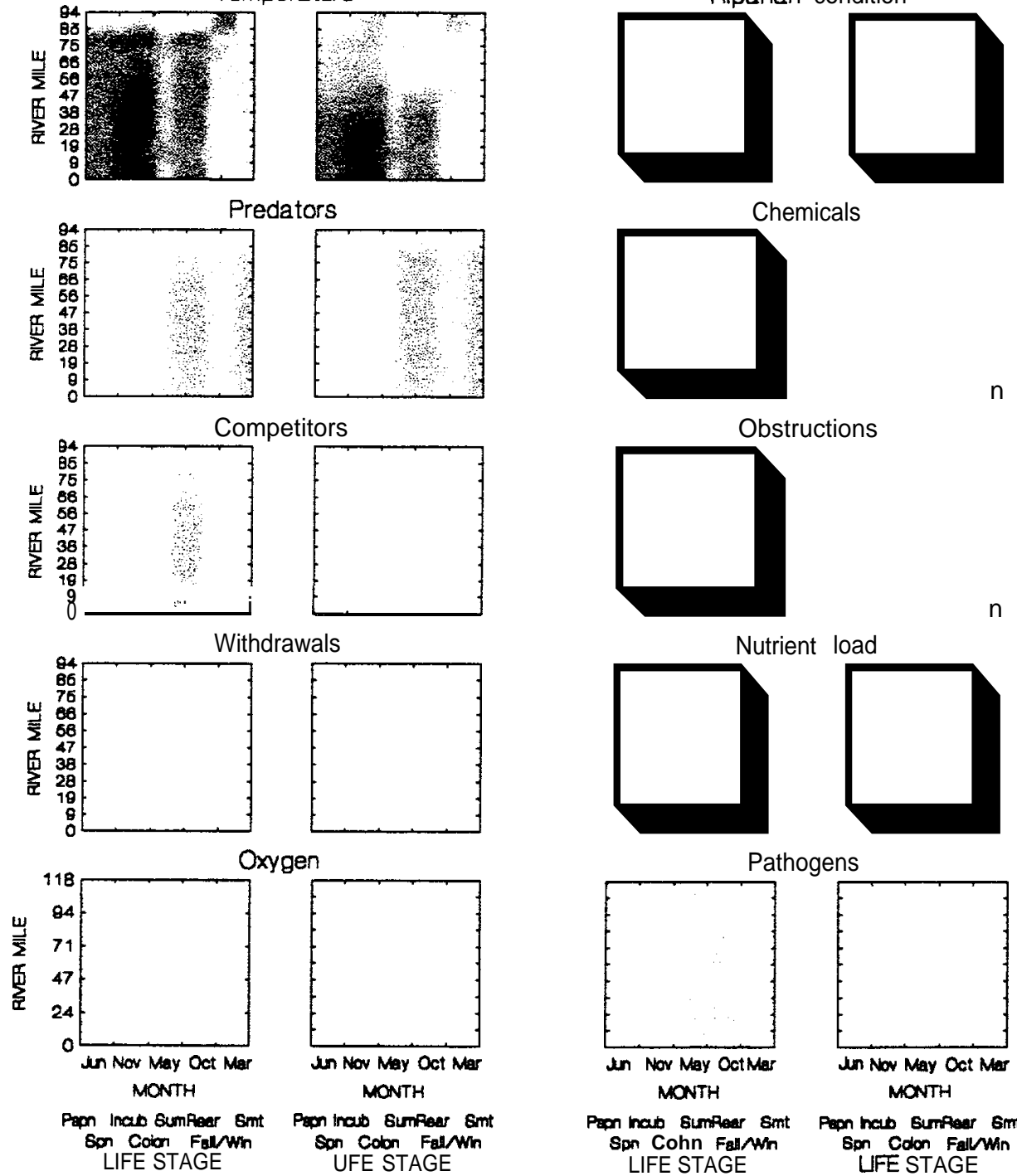

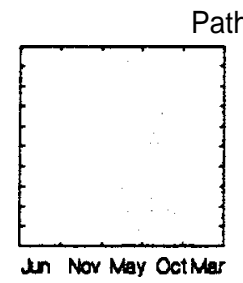

MONTH

Pam hat 8umplar 8mt Spn Cohn Fallwn LIFE STAGE

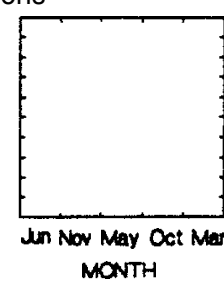

Pepn heib Sumplear $6 \mathrm{~m}$ Spn Colon Fall Wn
UFE STAGE
Level of

Effect

-! No effect

$\square$ Low

$\square$ Moderate

$\square$ High

Lethal 


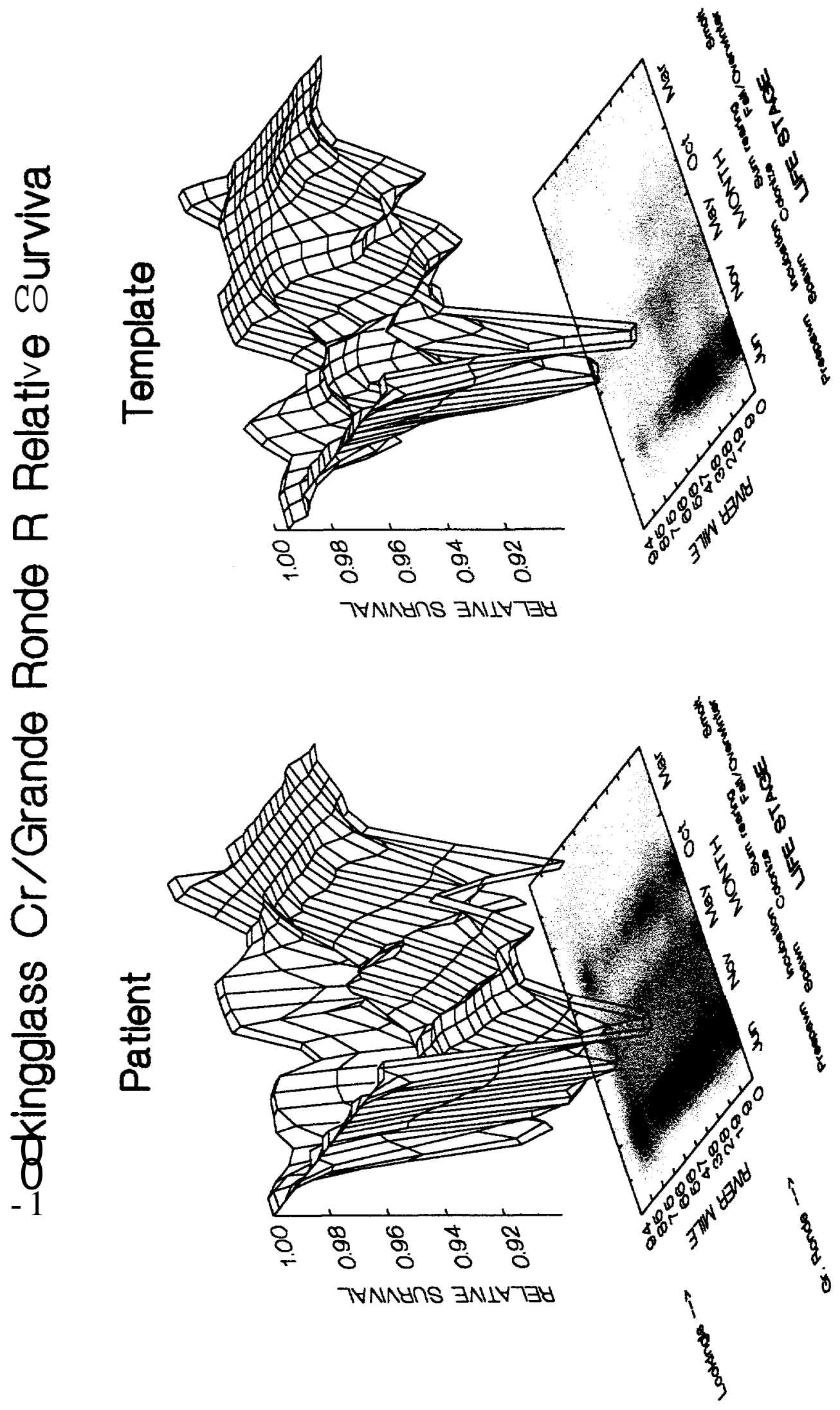




\section{Stream Reach Range}

Catherine Cr / Grande Ronde R 


\section{Patient-Template Analysis Summary}

Stream Reach Range: Catherine Cr./Grande Ronde R.

\section{Key Habitat Quantity}

The quantity of key habitat for spring chinook in Catherine Creek is greatly reduced compared to historic levels. These losses are due to land development, channel straightening and realignment, removal of large organic structure from the channel, and dewatering of the channel. Loss of key habitat has occurred for nearly every life stage, but it has been particularly severe for the prespawning, fry colonization, summer rearing, and overwintering life stages.

The quantity of key habitat within the Grande Ronde River downstream of Catherine Creek is substantially reduced compared to historic levels for a distance of approximately 30 miles. These losses have been greatest for the spawning, egg incubation, fry colonization, and overwintering life stages. Further downstream, loss in key habitat has been comparatively minor.

\section{Relative Productivity (Survival)}

The capability of Catherine Creek of supporting spring chinook productivity (survival) is sharply reduced compared to historic conditions for nearly every life stage. This reduction has been most severe in the lower 40 miles of stream, with conditions improving in the upper stream reaches. Survival conditions approach historic levels for some life stages in the Catherine Creek forks. "Survival conditions are especially low in the later phase of the prespawning stage and in the spawning, incubation, fry colonization, and summer rearing stages in the lower 30 miles of stream. Survival conditions have declined in these reaches due primarily to increased water temperature, increased sediment load, loss in habitat diversity, loss in flow, channel and bank destabilization, and alteration of the riparian corridor.

The capability of the Grande Ronde River for approximately 70 miles downstream of Catherine Creek for supporting spring chinook productivity is sharply reduced compared to historic conditions for many life stages. This loss has been most severe for a distance of approximately 40 miles downstream of Catherine Creek. Survival conditions in these reaches are especially poor in the prespawning, spawning, incubation, and summer rearing life stages. Survival conditions have declined in these reaches due primarily to increased water temperature during mid to late summer, increased sediment load, alteration of runoff patterns, and channel destabilization. 


\section{Catherine Cr Key Habitat Quantity}

Patient

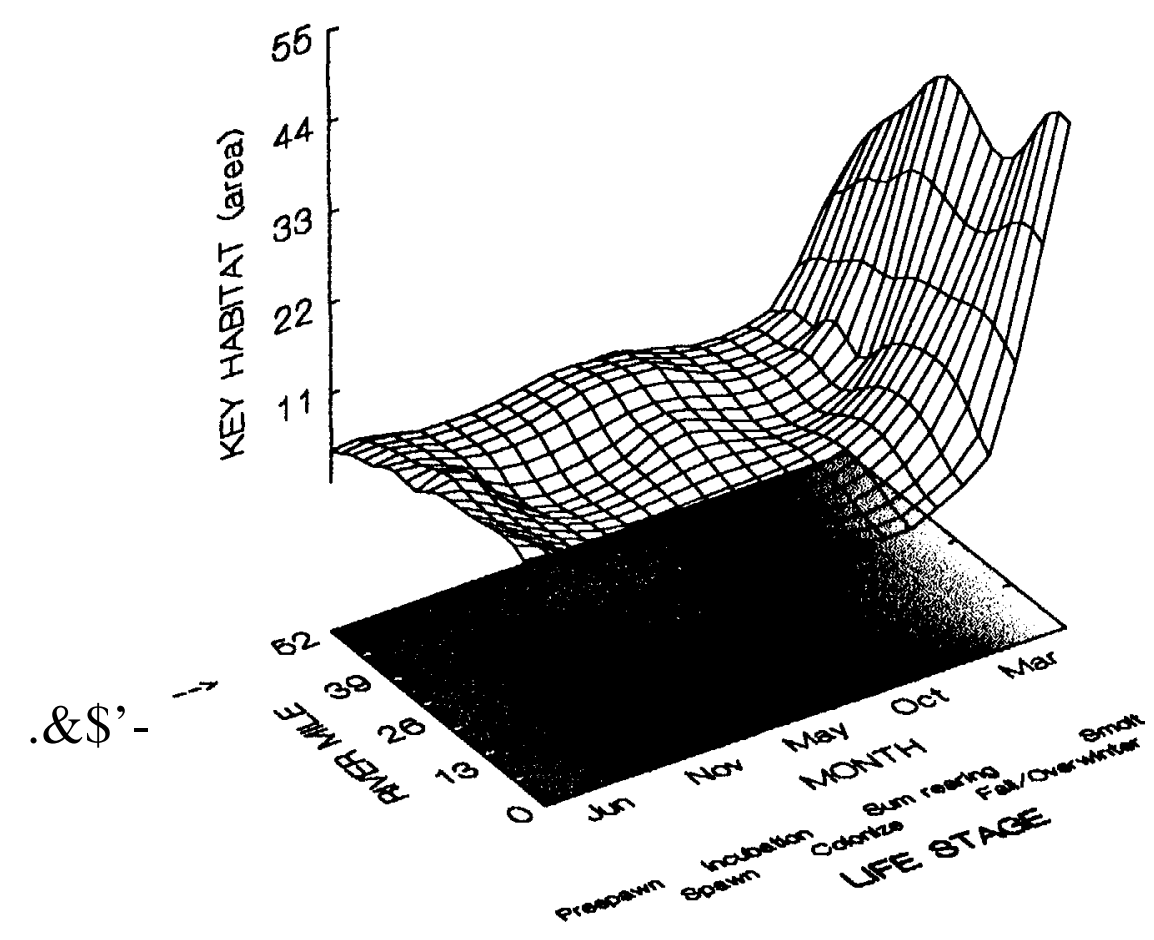

Template

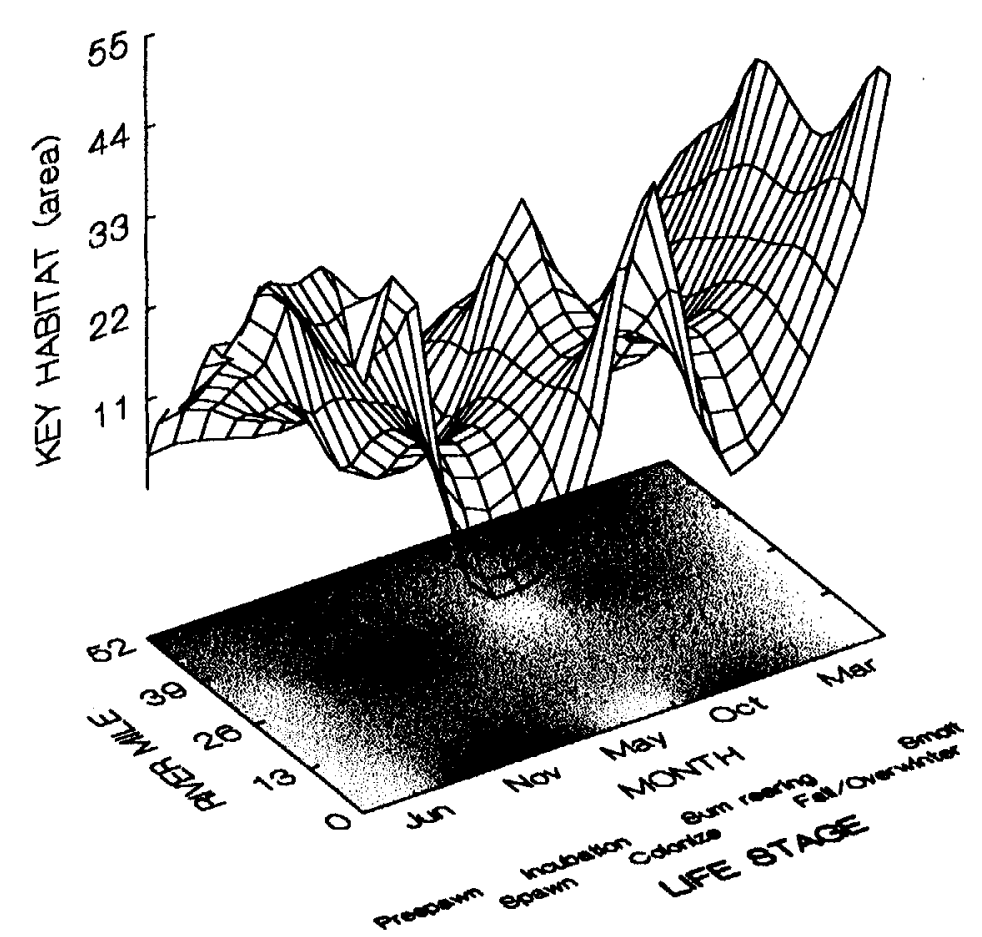




\section{Catherine Cr Environmental Attributes}
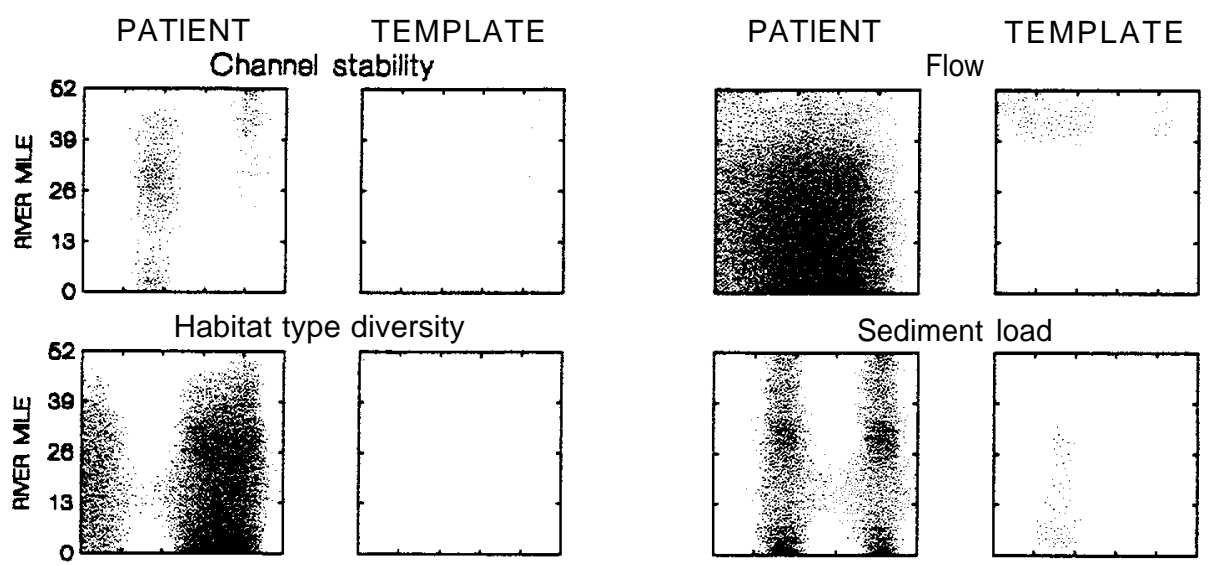

Sediment load
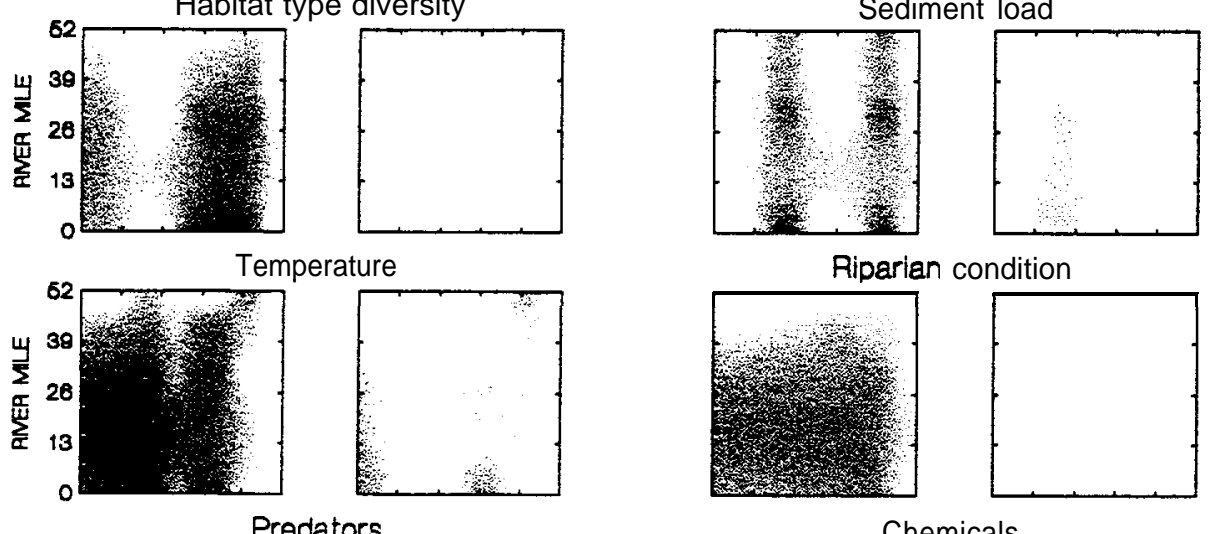

Riparian condition

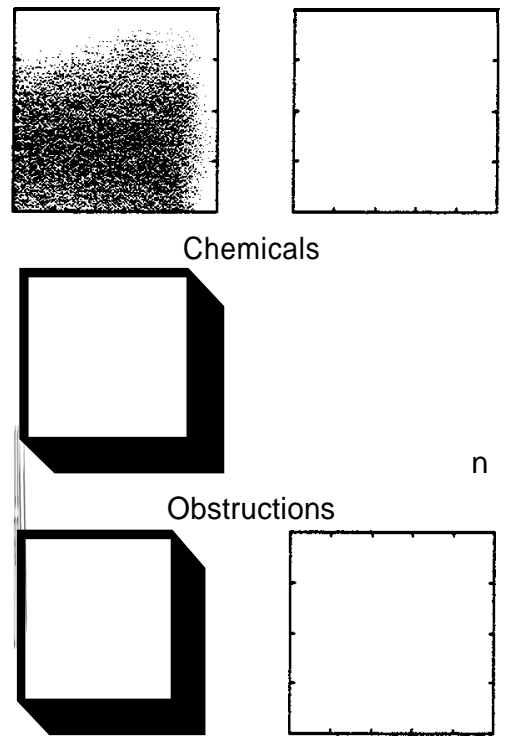

Nutrient load
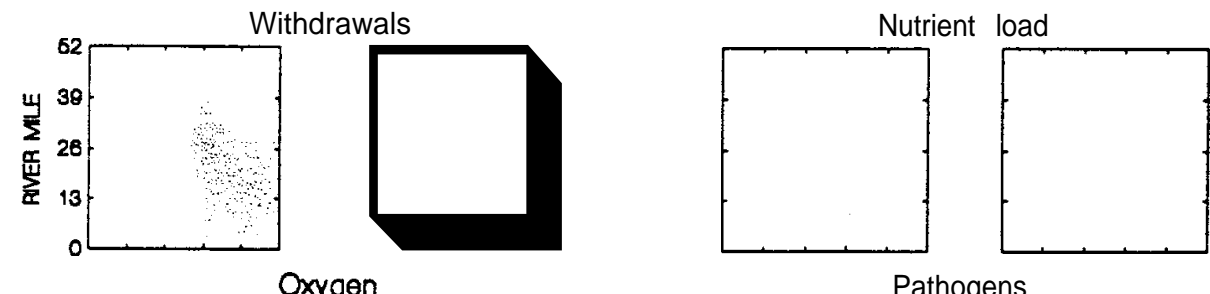

Pathogens 


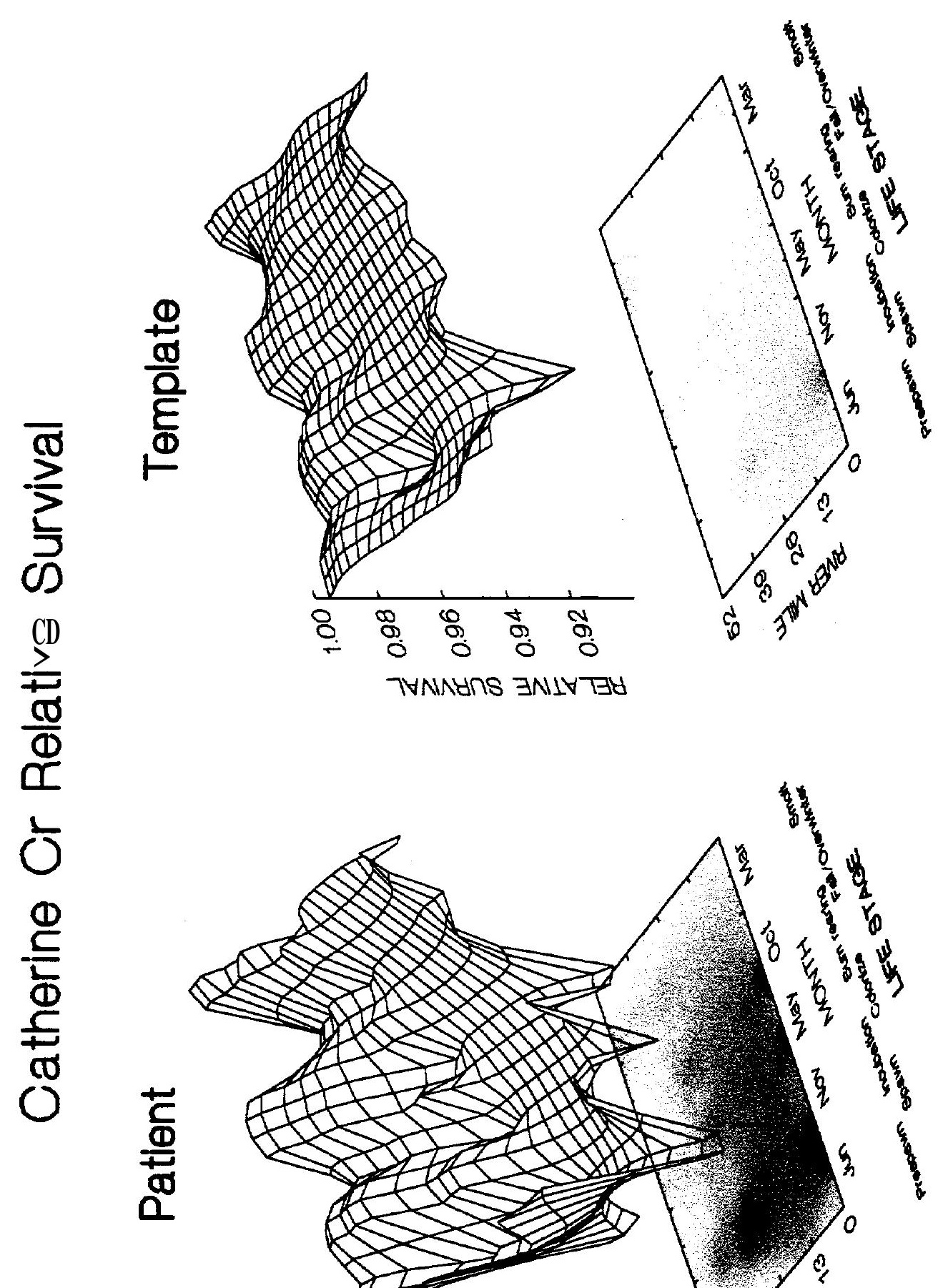




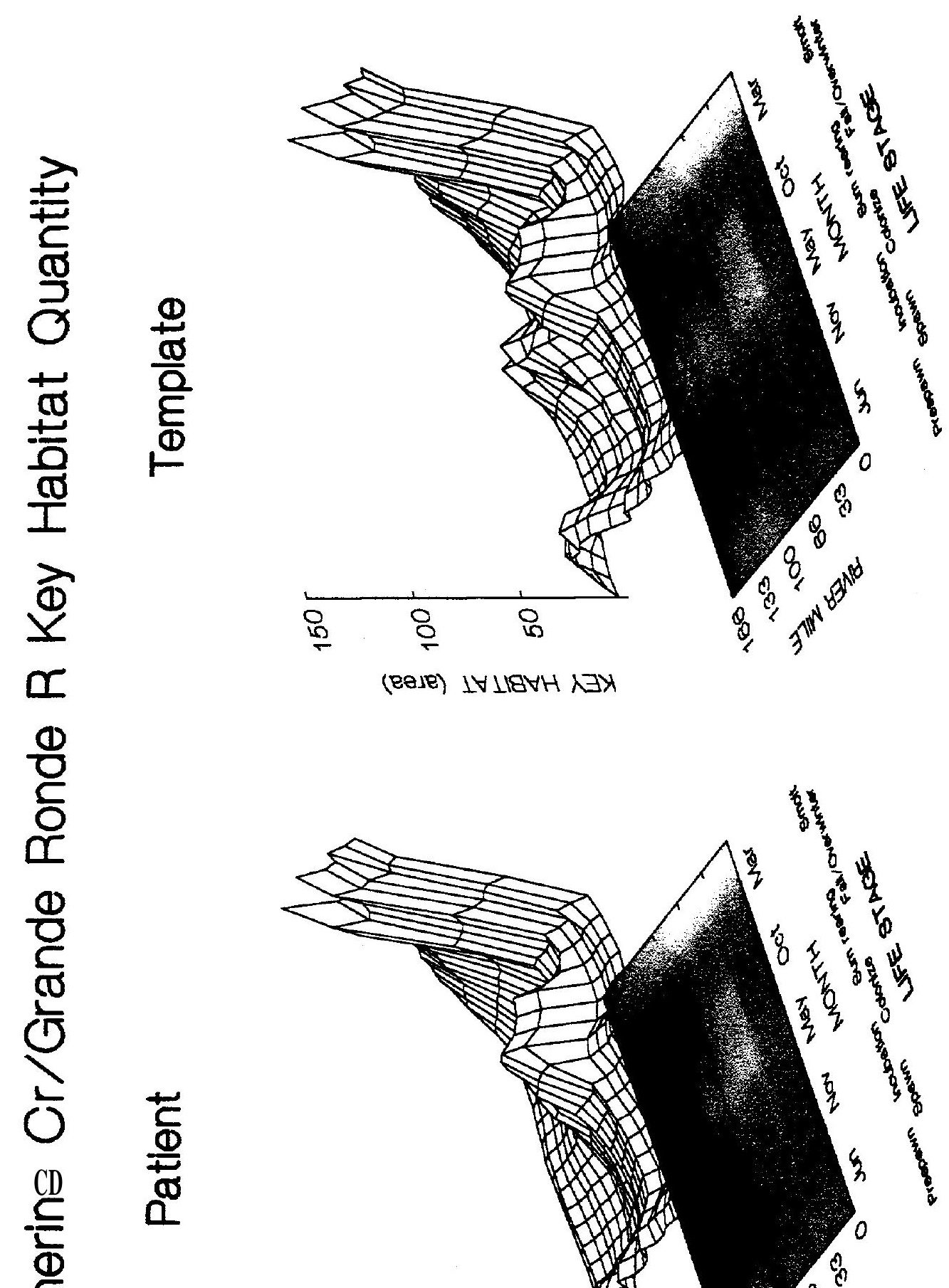




\section{Catherine CrTGrande Ronde KEnvironmental Attributes}
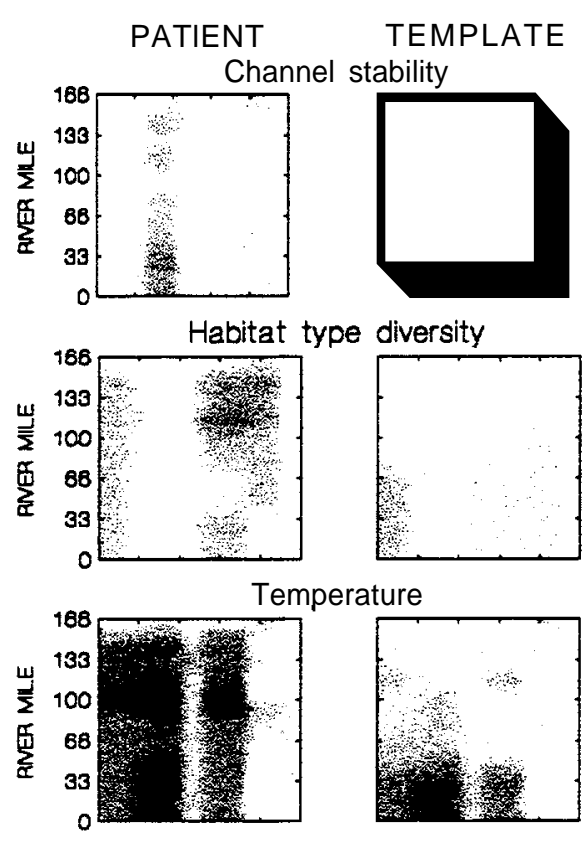

Precators
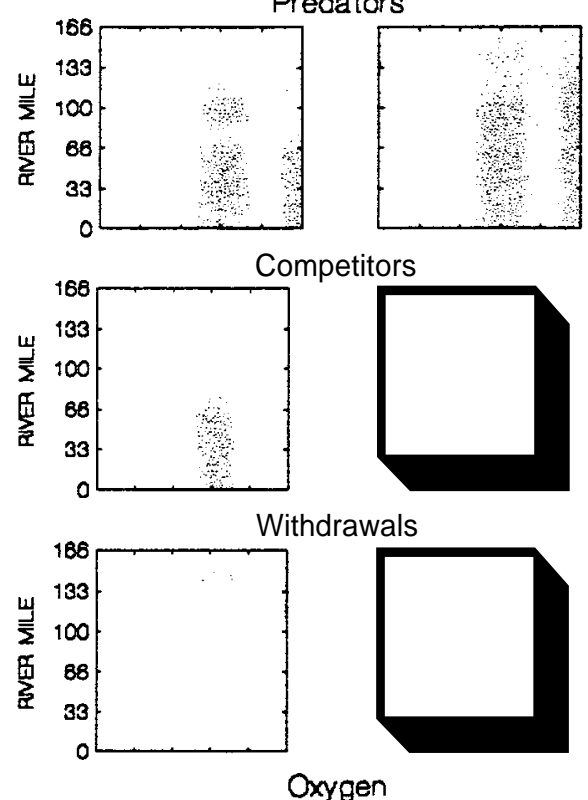

\begin{tabular}{r}
188 \\
138 \\
\hline \\
5 \\
5
\end{tabular}
PATIENT TEMPLATE Flow

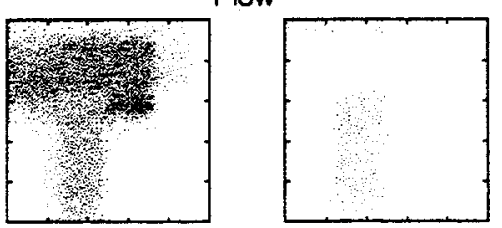

Sediment load

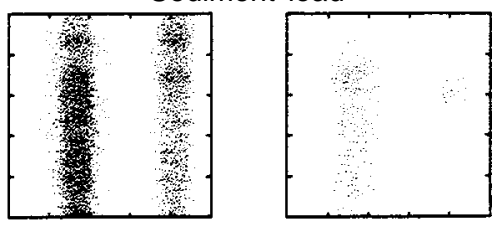

Riparian condition

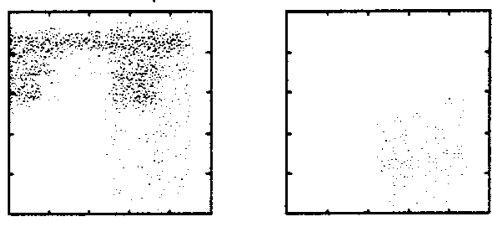

Chemicals

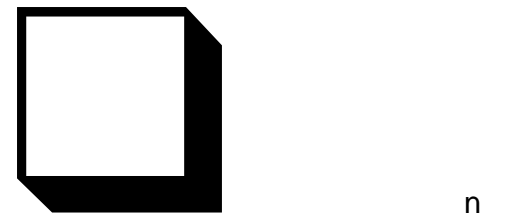

Obstructions
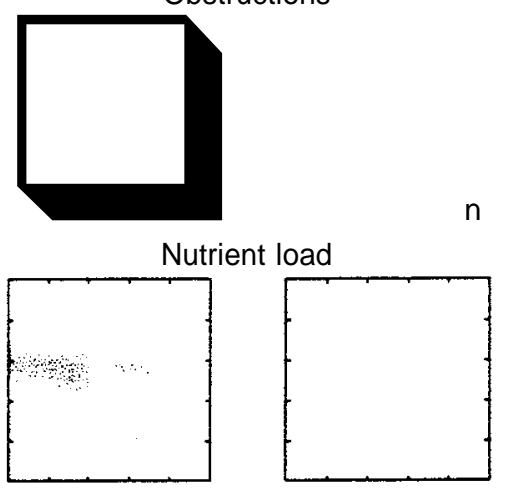

Pathagens

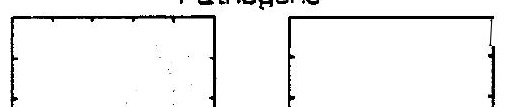

Level of

Effect

No effect 


\section{Catherine Cr/Grande Ronde R Relative Survival}
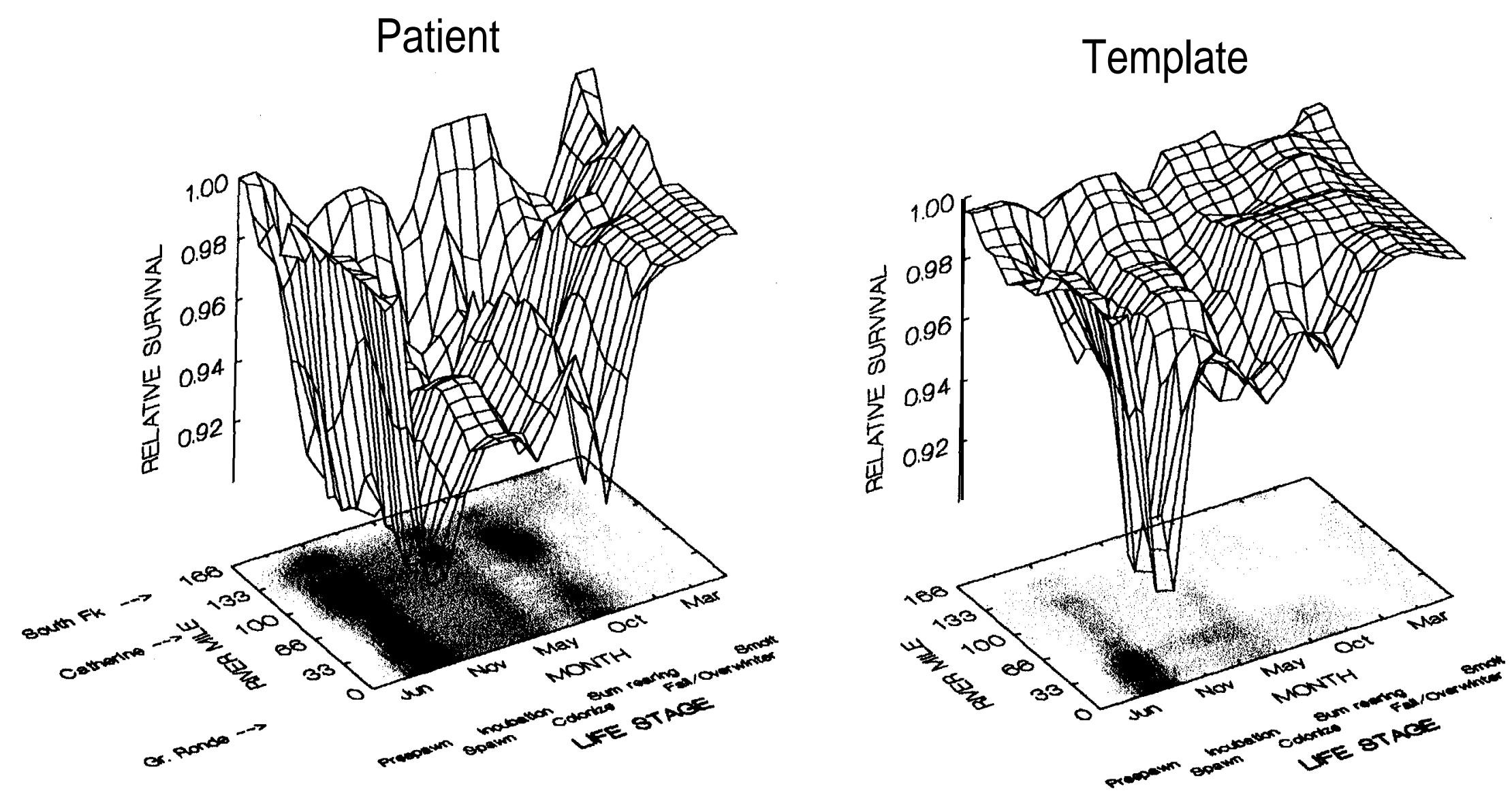
Stream Reach Range

Sheep Cr / Grande Ronde R 


\section{Patient-Template Analysis Summary}

Stream Reach Range: Sheep Cr./Grande Ronde R.

\section{Key Habitat Quantity}

The quantity of key habitat for spring chinook in Sheep Creek is substantially reduced compared to historic levels for most life stages. These losses are greatest for the prespawning, spawning, egg incubation, and overwintering life stages.

The quantity of key habitat in the Grande Ronde River downstream of Sheep Creek and extending to below the Grande Ronde valley is greatly reduced for certain life stages compared to historic levels. Changes in quantity of key habitat downstream of the valley have been comparatively minor.

\section{Relative Productivity (Survival)}

The capability of Sheep Creek of supporting spring chinook productivity (survival) is sharply reduced compared to historic conditions for nearly every life stage. Survival conditions are especially low in the later phase of the prespawning stage and in the spawning stage. Conditions are also relatively poor in the fry colonization, summer rearing, and overwintering stages. Survival conditions have declined in these reaches due primarily to increased water temperature, increased sediment load, loss in habitat diversity, changes in flow patterns, and alteration of the riparian corridor. Historic survival conditions in this stream were excellent.

The capability of the mainstem Grande Ronde River of supporting spring chinook productivity downstream of Sheep Creek is greatly reduced for most life stages compared to historic conditions. This reduction has been most severe within the Grande Ronde valley and continues for most of the distance upstream. Survival conditions have also declined markedly downstream of the valley reaches, although historic conditions there were naturally poor for some life stages in the lower 50 miles. Current survival conditions remain similar to what they were historically for the prespawning and smelt life stages over most the of the river and in the overwintering stage in some portions of the lower river. Survival conditions have changed in these reaches due primarily to increased water temperature, increased sediment load, loss in habitat diversity, changes in flow patterns, channel and bank destabilization, and alteration of the riparian zone. 


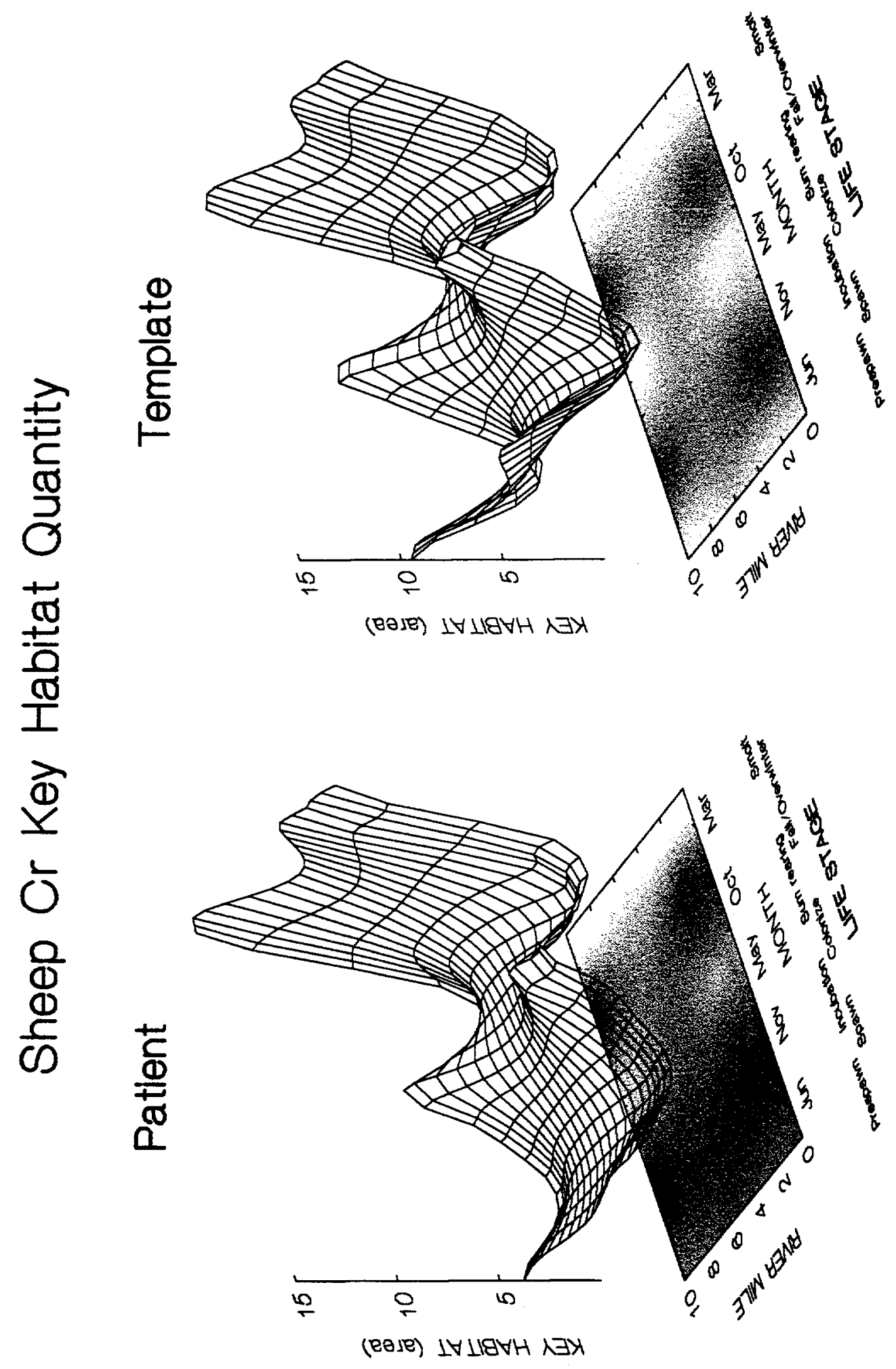




\section{Sheep Cr Environmental Attributes}

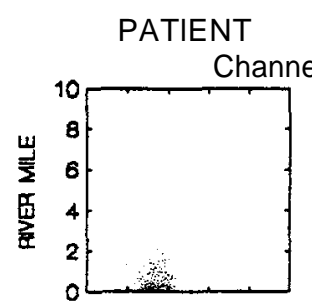

TEMPLATE

Habitat type diversity
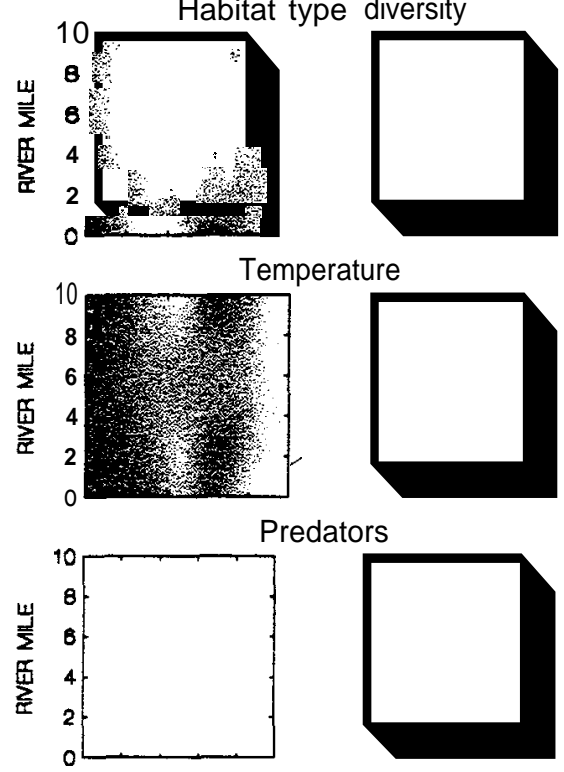

Predators

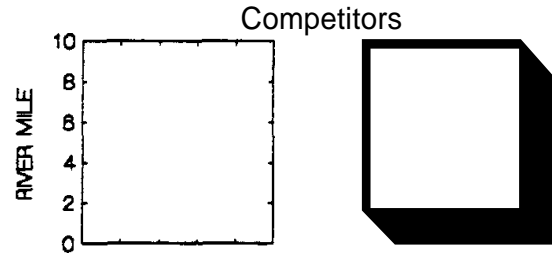

Withdrawals
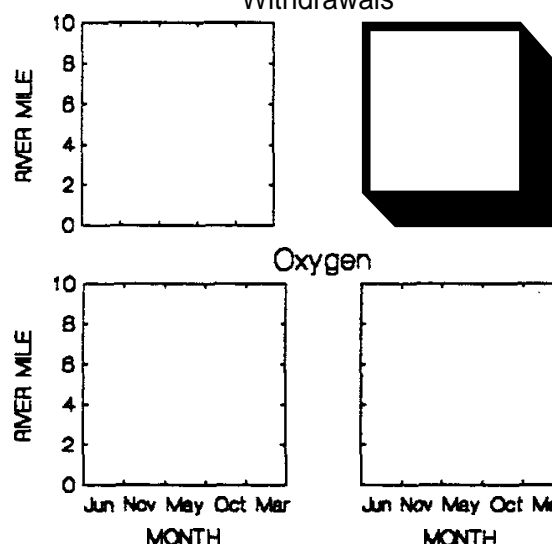

\section{Oxygen}

Fapn Inow Sumflear Bmt Son Colon FallWh LIFE STAGE

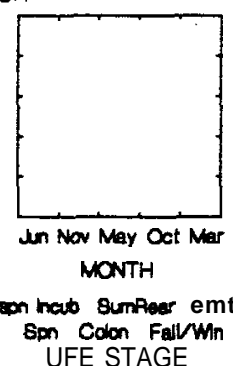

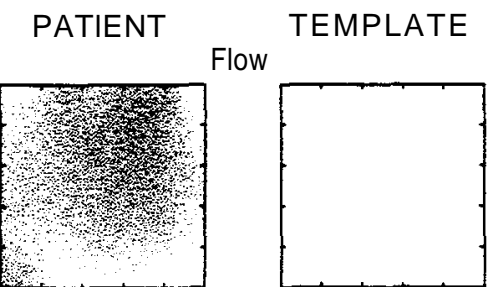

Sediment load

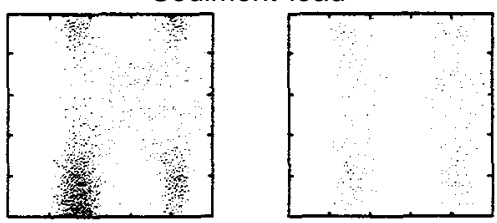

Riparian condition
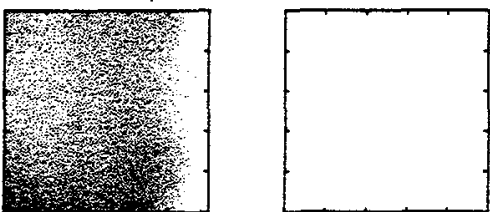

Chemicals

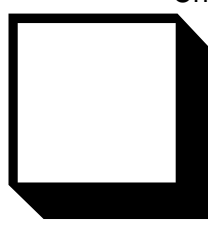

Obstructions

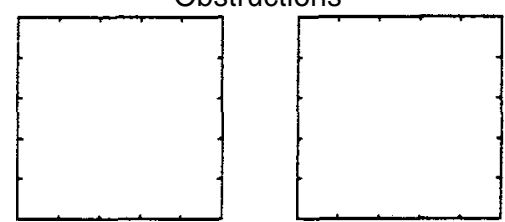

Nutrient load

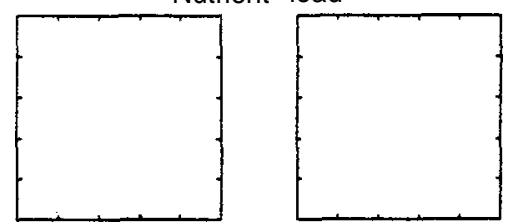

Pathogens
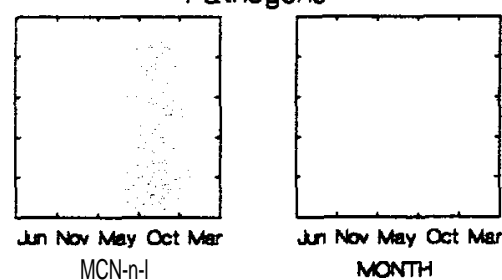

Level of

Effect

$\square$ No effect

$\square$ Low

$\square$ Moderate

$\square$ High

$$
\text { MONTH }
$$

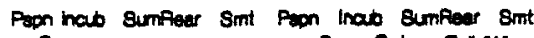
Bon Colon Follwh
LFE STAGE 


\section{Sheep Cr Relative Survival}
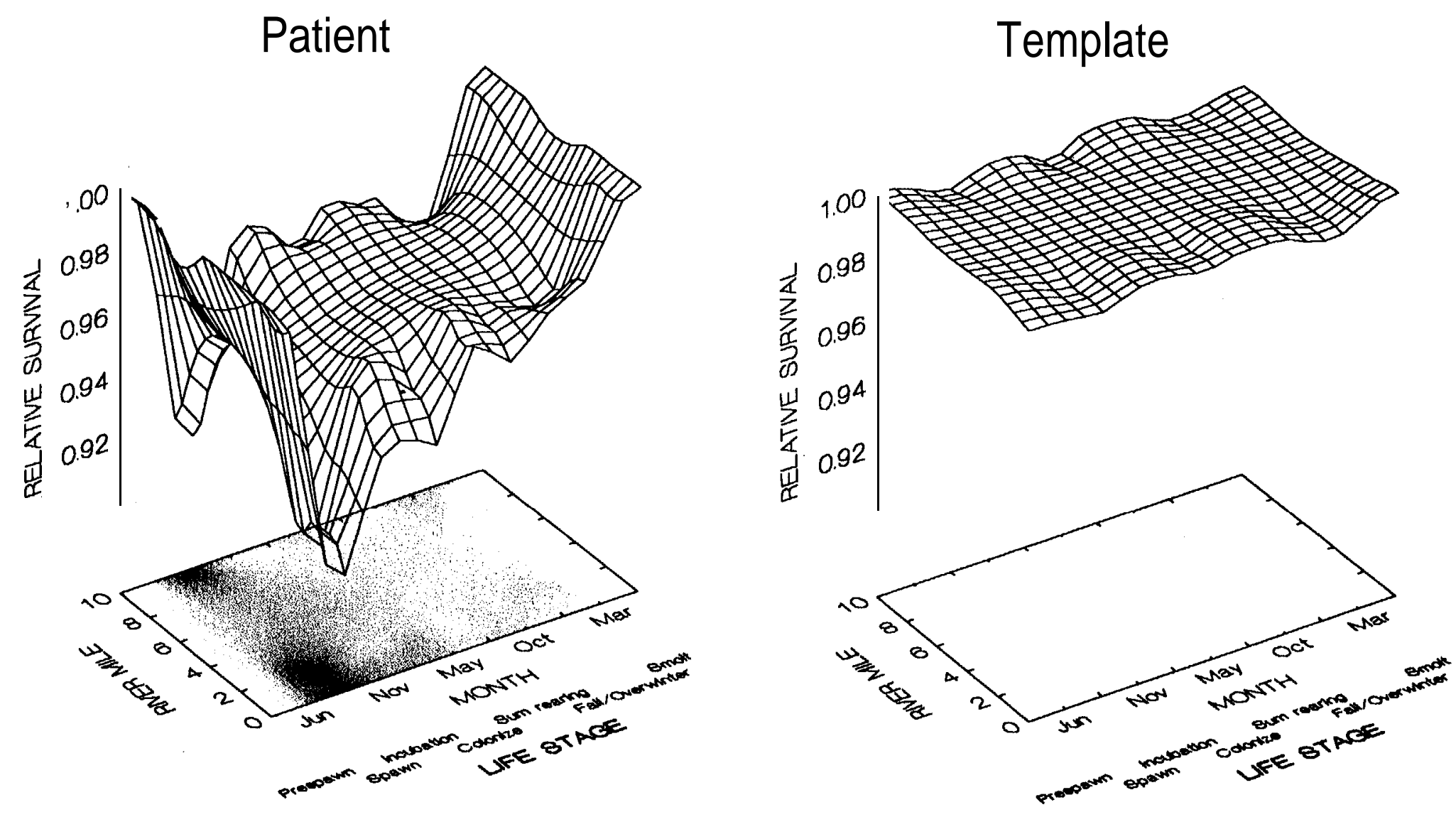


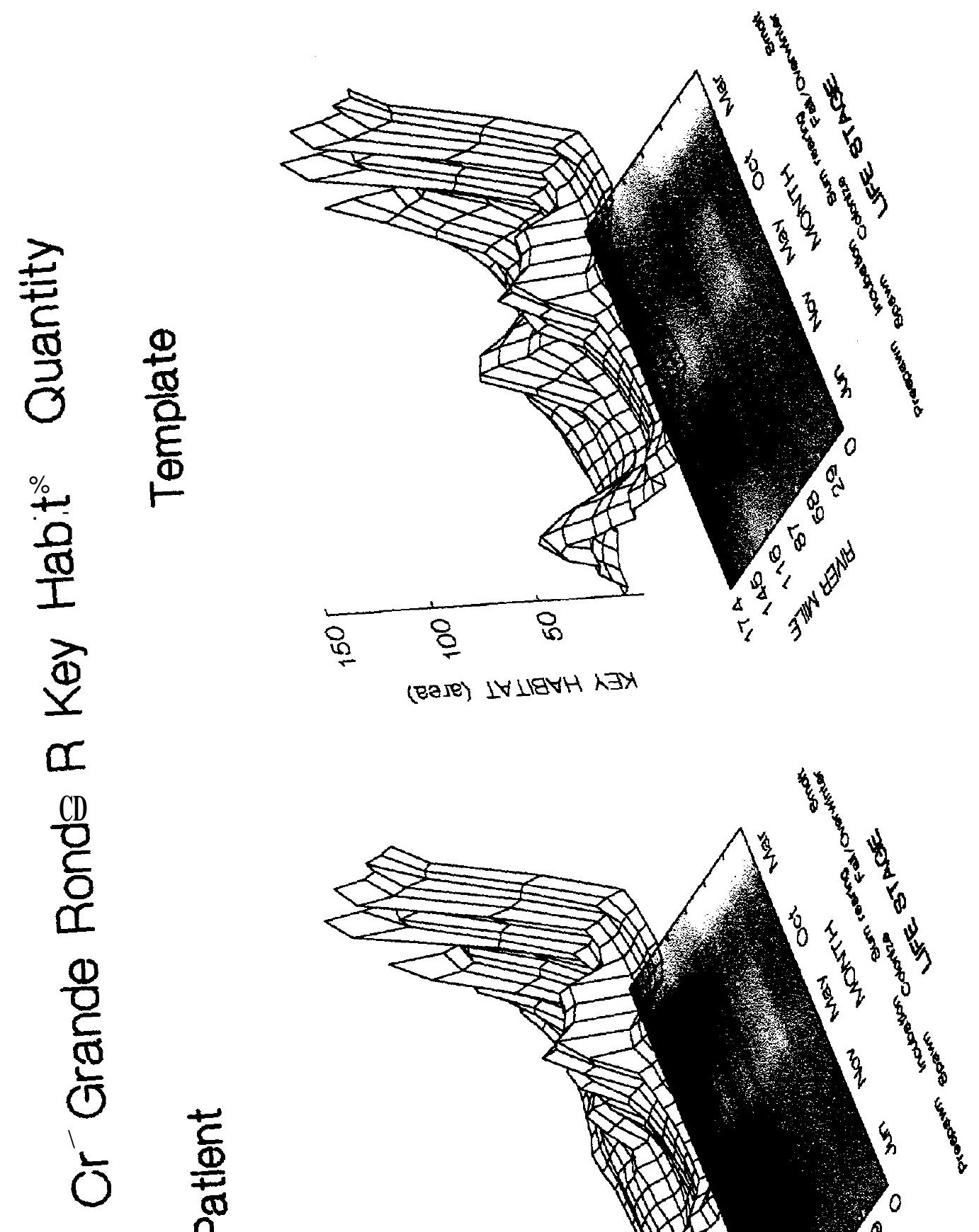




\section{Sheep Cr/Grande Ronde Fnvironmental Attributes}

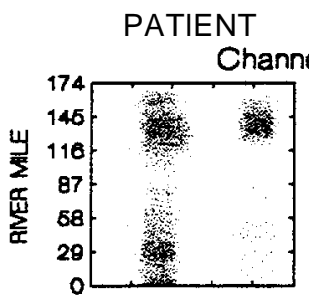

TEMPLATE

Habitat type diversity
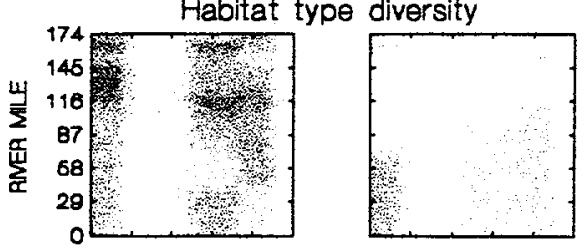

Temperature
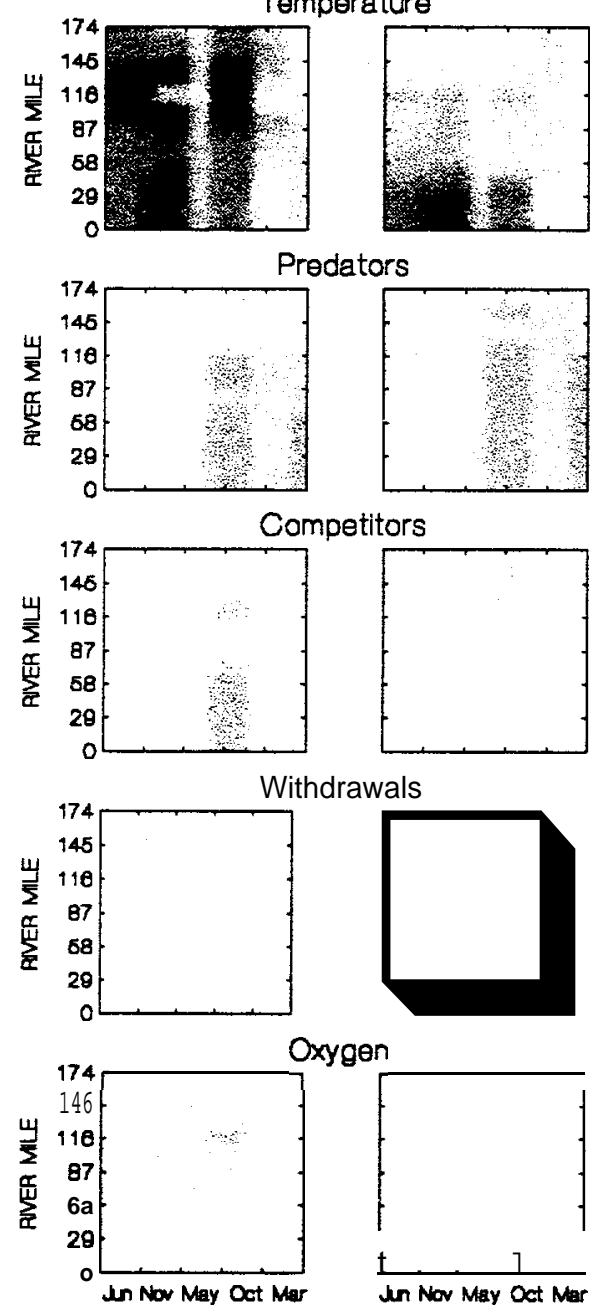

Oxygen

MONTH

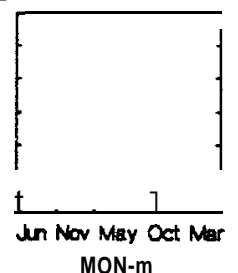

Puon nat Sumfeer $8 m$ t Son Coion Faumn

Paon incub Sumfioar $8 m$ Son Colon Fallmin

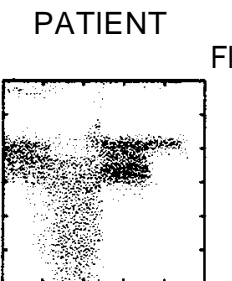

TEMPLATE Flow

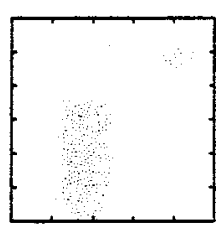

Sediment load
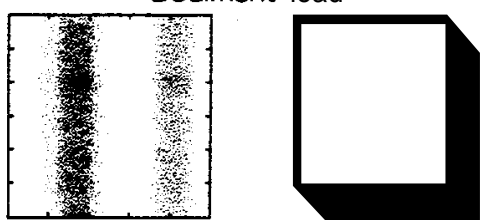

Fiparian condition

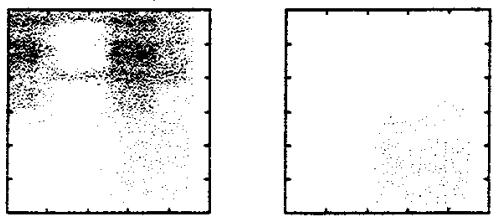

Chemicals

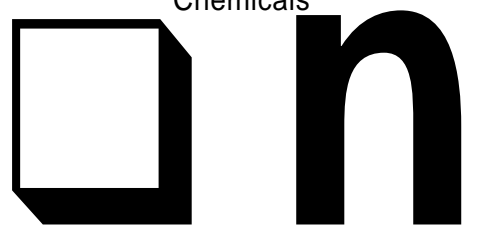

Obstructions

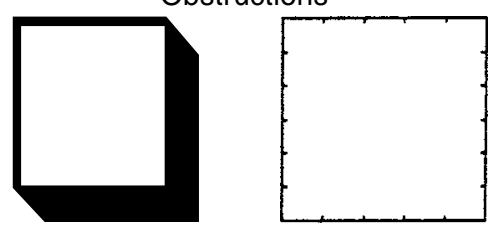

Nutrient load

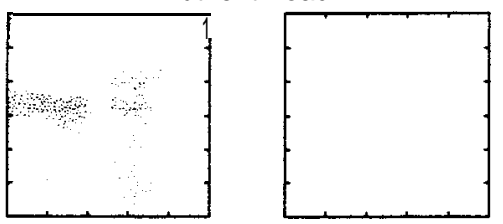

Pathogens

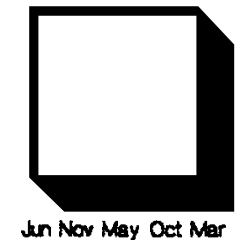
MONTH

th Nar May Oon MONTH

Puon Inab Sumpeer 8mt Pan inat 8umpear Sm Bon Colon Fallwn Sm Coin FallWm
Level of Effect

$\square$ No effect

$\square$ Low

$\square$ Moderate

$\square$ High

Lethal 


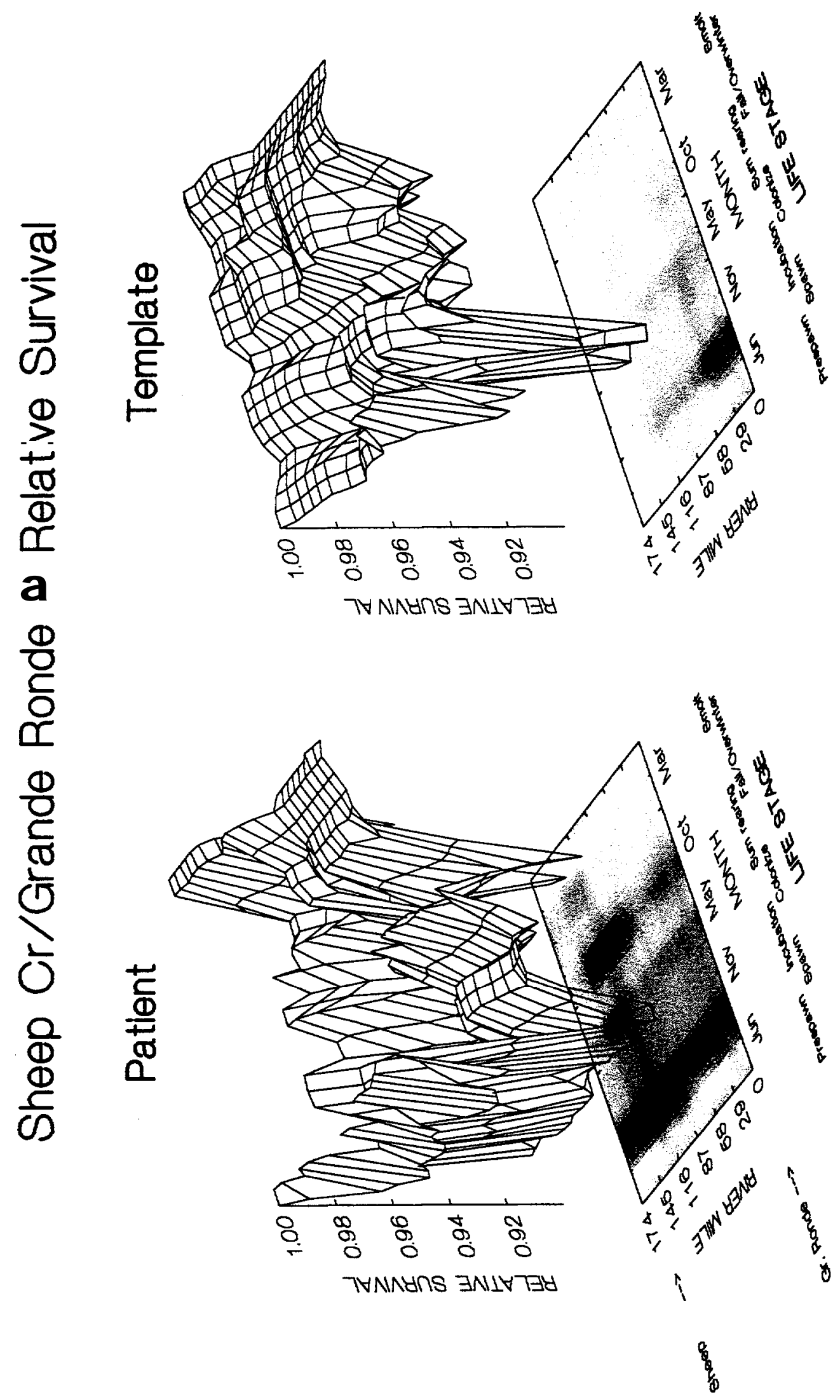


$\underline{\text { Stream Reach Range }}$

Imnaha $\mathrm{R}$ 


\section{Patient-Template Analysis Summary}

\section{Stream Reach Range: Imnaha R.}

\section{Key Habitat Quantity}

The extent of changes from historic levels in the quantity of key habitat for spring chinook in the Imnaha River differs by life stage and stream reach. The greatest reductions in key habitat have occurred through much of the mid portions and upper reaches of river (to about river mile 67) in the spawning and egg incubation stage. These losses are mainly associated with losses in gravels of a size suitable for spring chinook spawning. Causes of the loss are mainly due to removal of in-channel structure and some bank hardening.

\section{Relative Productivity (Survival)}

The capability of the mainstem Imnaha River and its South Fork of supporting spring chinook productivity (survival) is reduced for several life stages compared to historic conditions. This reduction has occurred mainly in the mid to lower reaches of the river in the prespawning, spawning, fry colonization, and summer rearing life stages. In these reaches, survival conditions have worsened due to slightly increased water temperatures, small loss in habitat diversity, and increased channel (streambed) instability. 

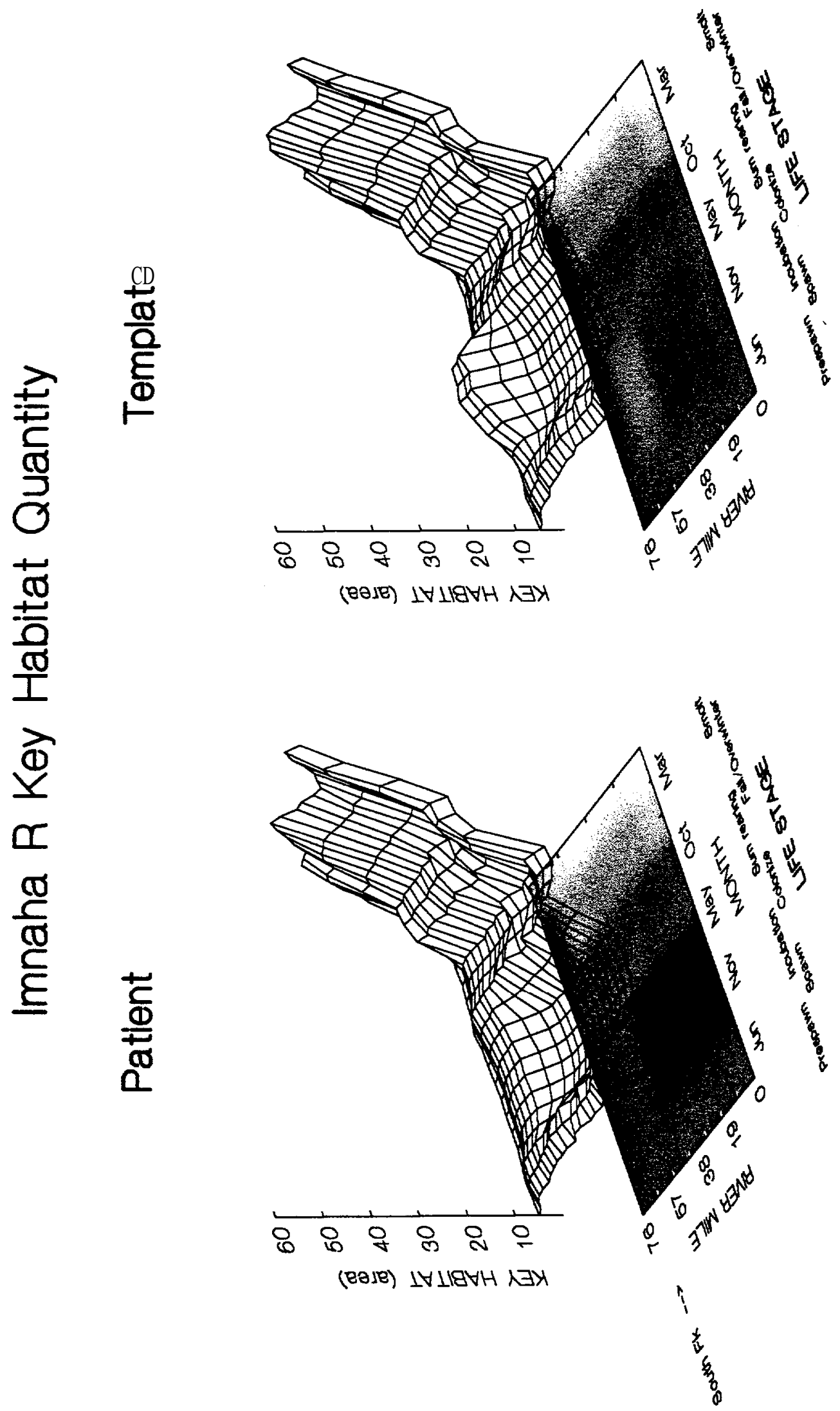


\section{Imnaha R Environmental Attributes}
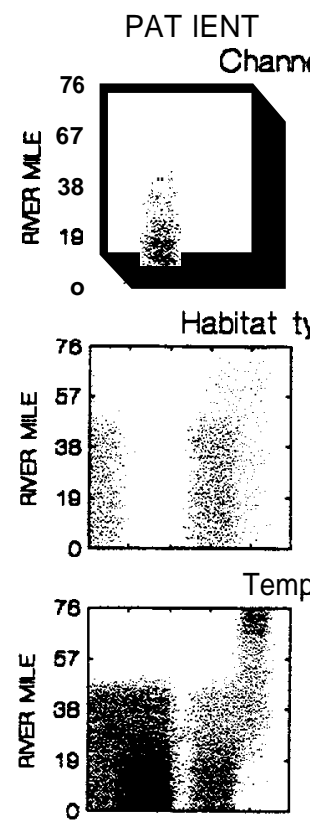

TEMPLATE

tability
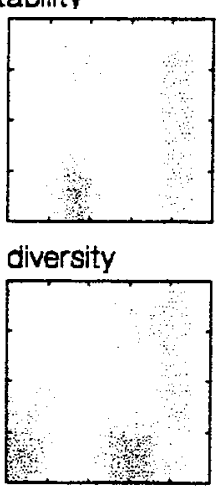

emperature

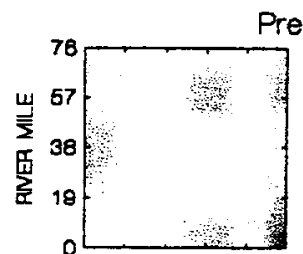

Predators
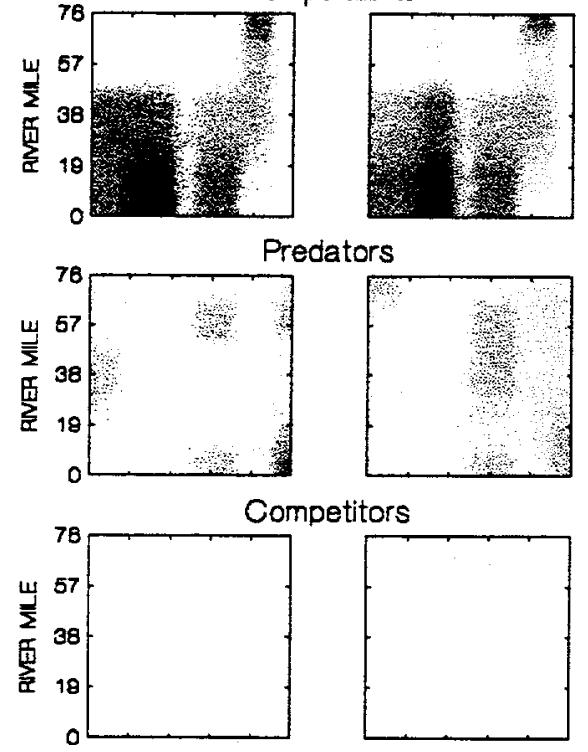

Withdrawals
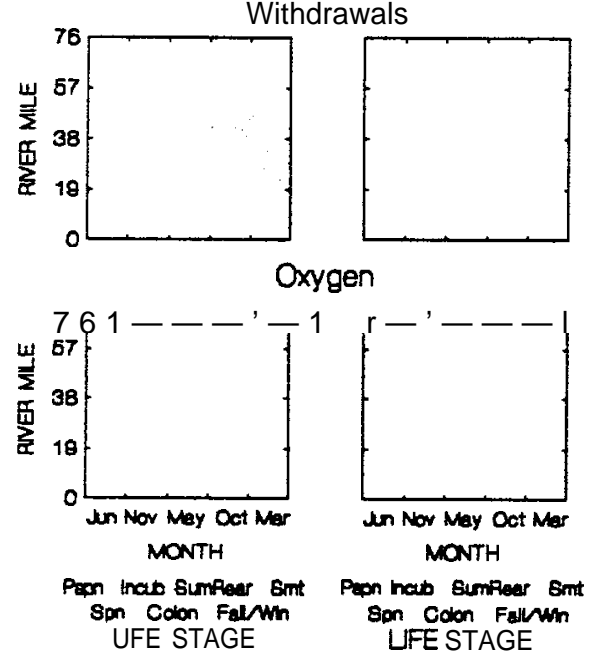

PATIENT TEMPLATE

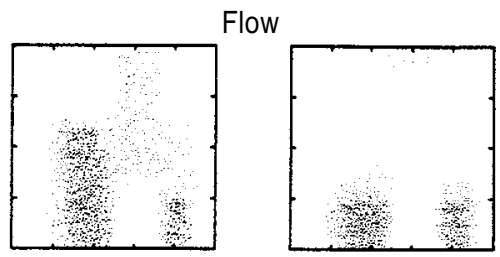

Sediment load

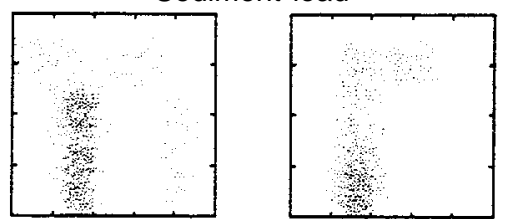

Riparian condition
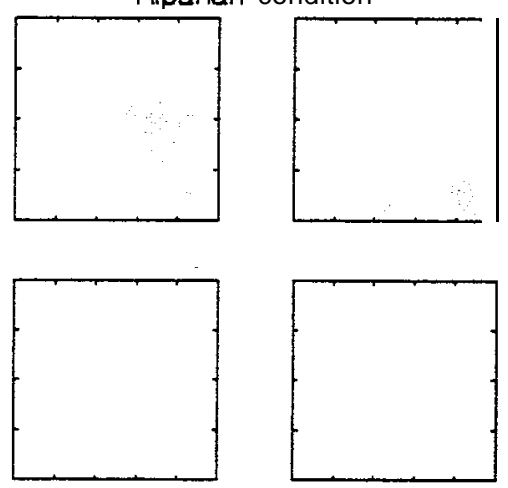

Obstructions

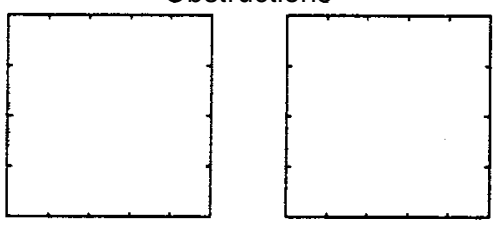

Nutrient load
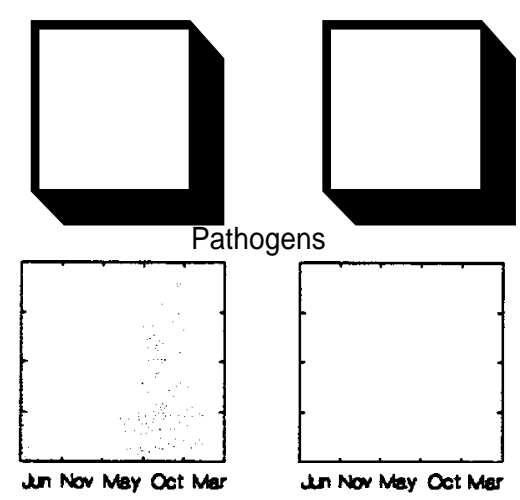

$$
\text { MONTH }
$$

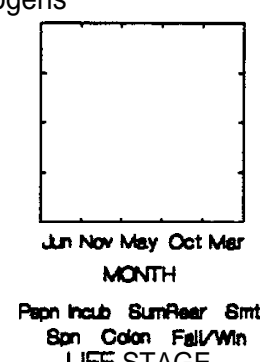

Paon naw Bumpear Emt Papn nat Sumpear Sm Epn Coion Fallwn
UFE STAGE
Level of

Effect

$\square$ No effect

$\square$ Low

Moderate

High

Lethal 


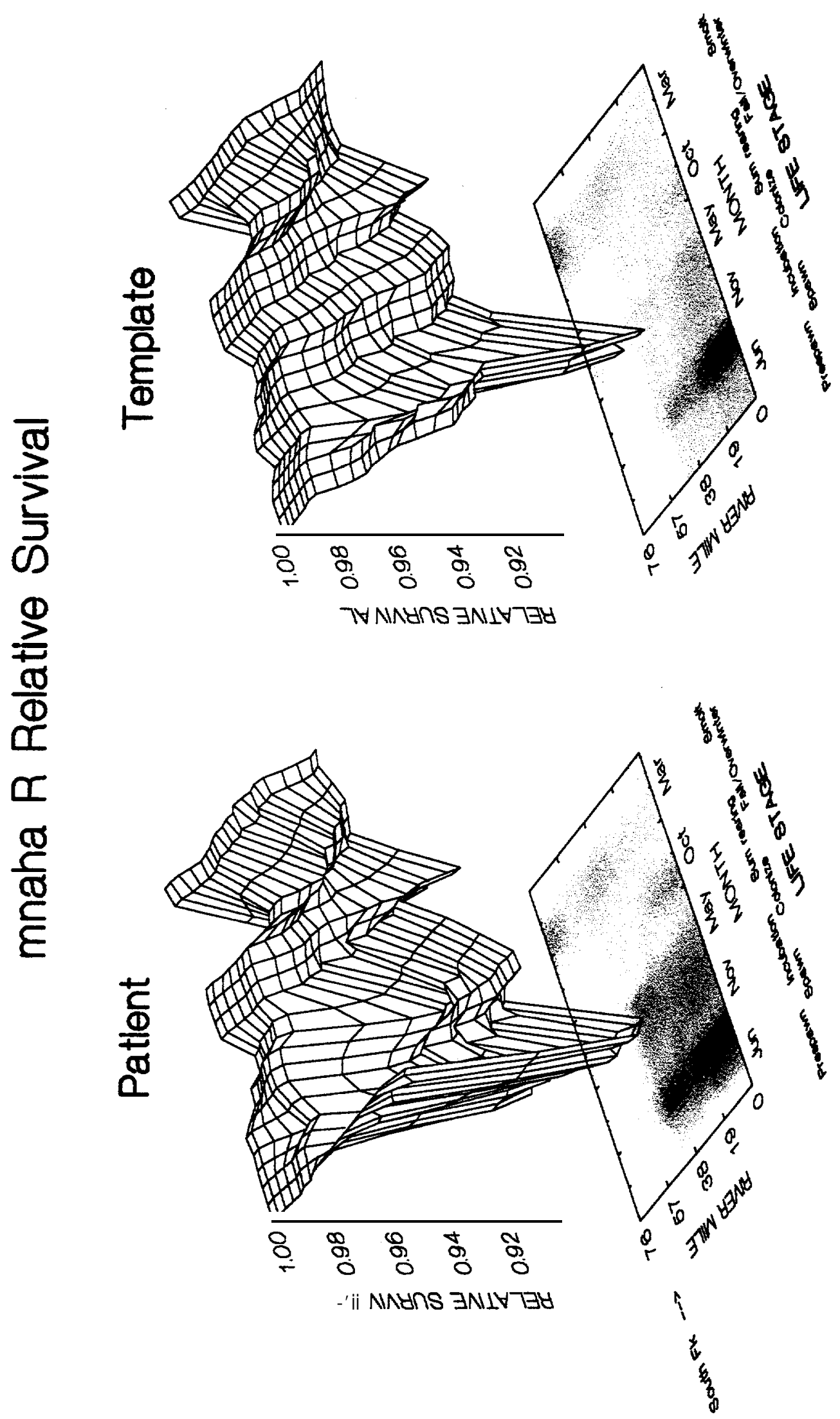


Stream Reach Range

Big Sheep Cr / Imnaha R 


\section{Patient-Template Analysis Summary}

\section{Stream Reach Range: Big Sheep Cr./Imnaha R.}

\section{Key Habitat Quantity}

The extent of changes from historic levels in the quantity of key habitat for spring chinook in Big Sheep Creek differs by life stage and stream reach. Losses have been greatest in the upper half of Big Sheep Creek in the spawning, incubation, colonization, and summer rearing stages.

The quantity of key habitat in the Imnaha River downstream of Big Sheep Creek is comparatively small, although egg incubation habitat shows relatively substantial loss compared to historic levels.

\section{Relative Productivity (Survival)}

The capability of the lower 23 miles of Big Sheep Creek of supporting spring chinook productivity (survival) is greatly reduced compared to historic conditions for most life stages. Survival conditions in this section have declined due primarily to changes in water temperature regimes, channel stability, habitat diversity, and, to a lesser extent, flow regimes and sediment load. Historically, survival conditions in the lower portion of this section were relatively poor in the prespawner, spawner, and egg incubation stages. Survival conditions in the upper ten miles of the stream, though slightly depressed compared to historic conditions, remain relatively good.

Survival conditions in the Imnaha River downstream of Big Sheep Creek have worsened compared to those that existed historically, although the lower portions of the river have always been unfavorable for survival during some life stages. Conditions are less favorable due to slightly increased water temperatures, small loss in habitat diversity, and increased channel (streambed) instability. 

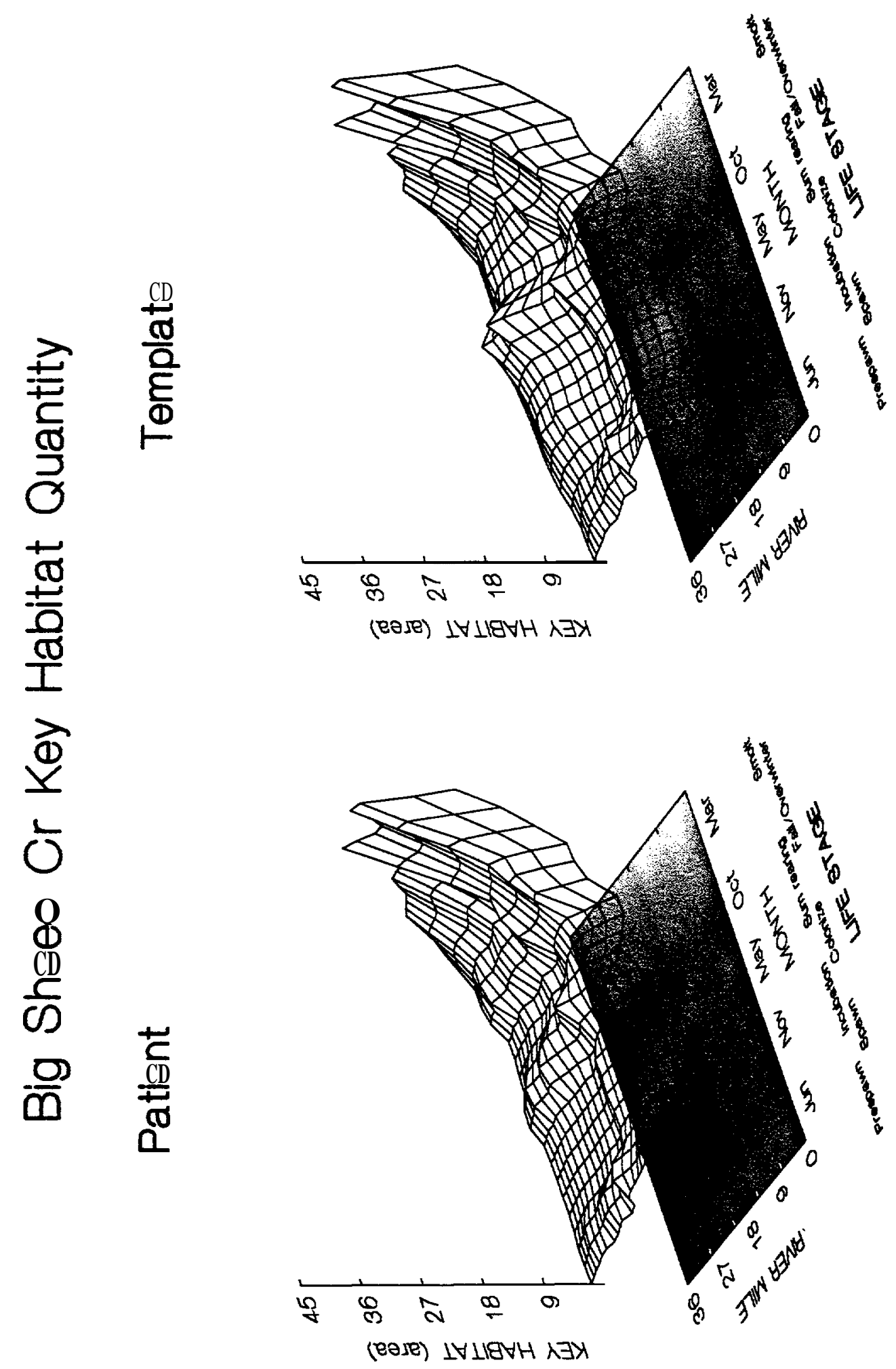


\section{Big Sheep Cr Environmental Attributes}

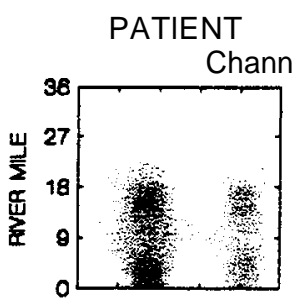

TEMPLATE

Channel stability
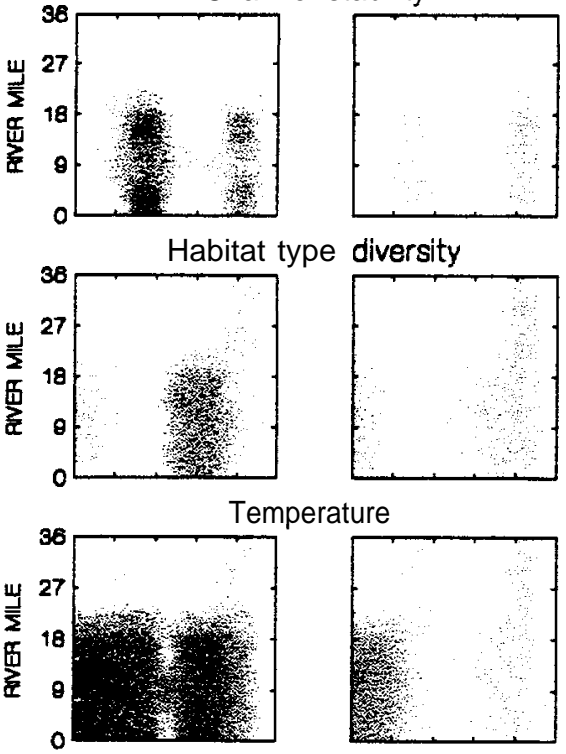

Temperature

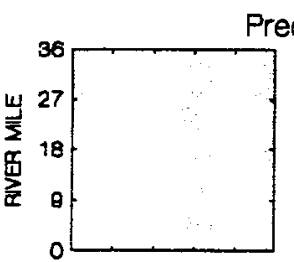

Predators

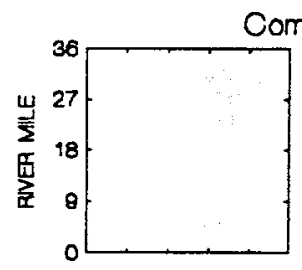

Withdrawals

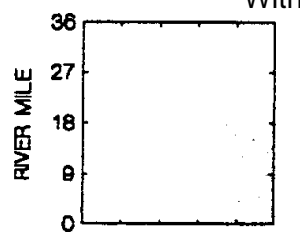

Oxygen

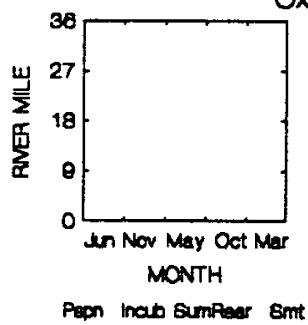

Fepn Inat Gumpear sm

Son Colon Fal
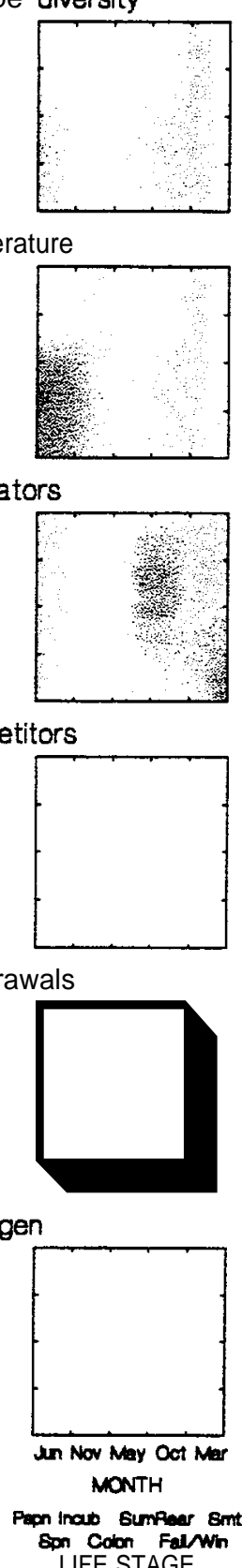

PAT IENT

TEMPLATE

Flow
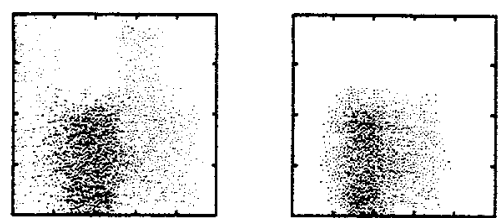

Sediment load
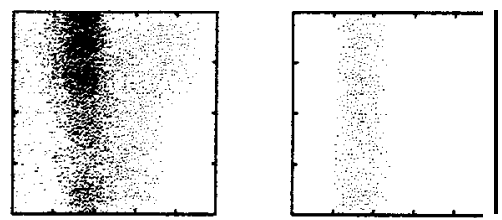

Riparian condition
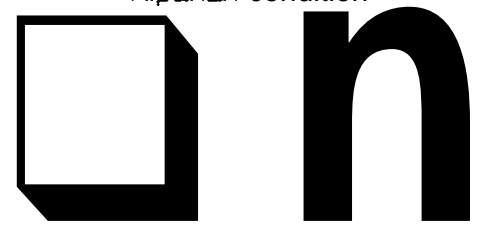

Chemicals

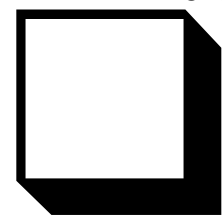

Obstructions

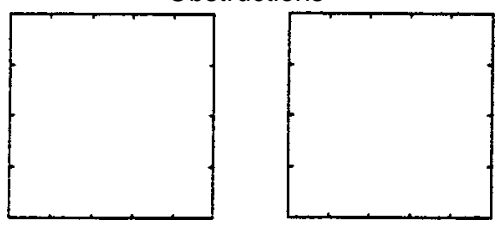

Nutrient load

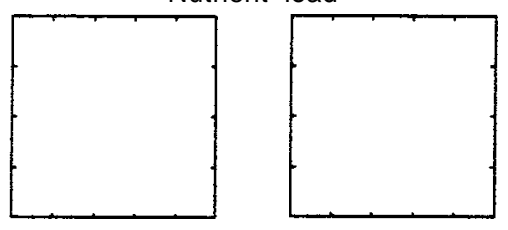

Pathogens

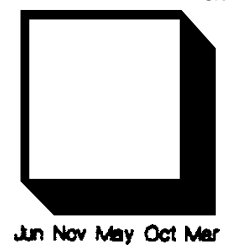
MONTH

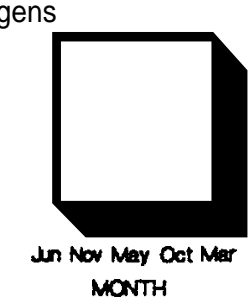

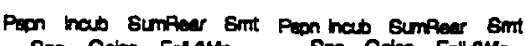
Spn Coion Falwh Bon Coion Fallwin
Level of

Effect

$\square$ No effect

Low

閣 Moderate

High

Lethal 


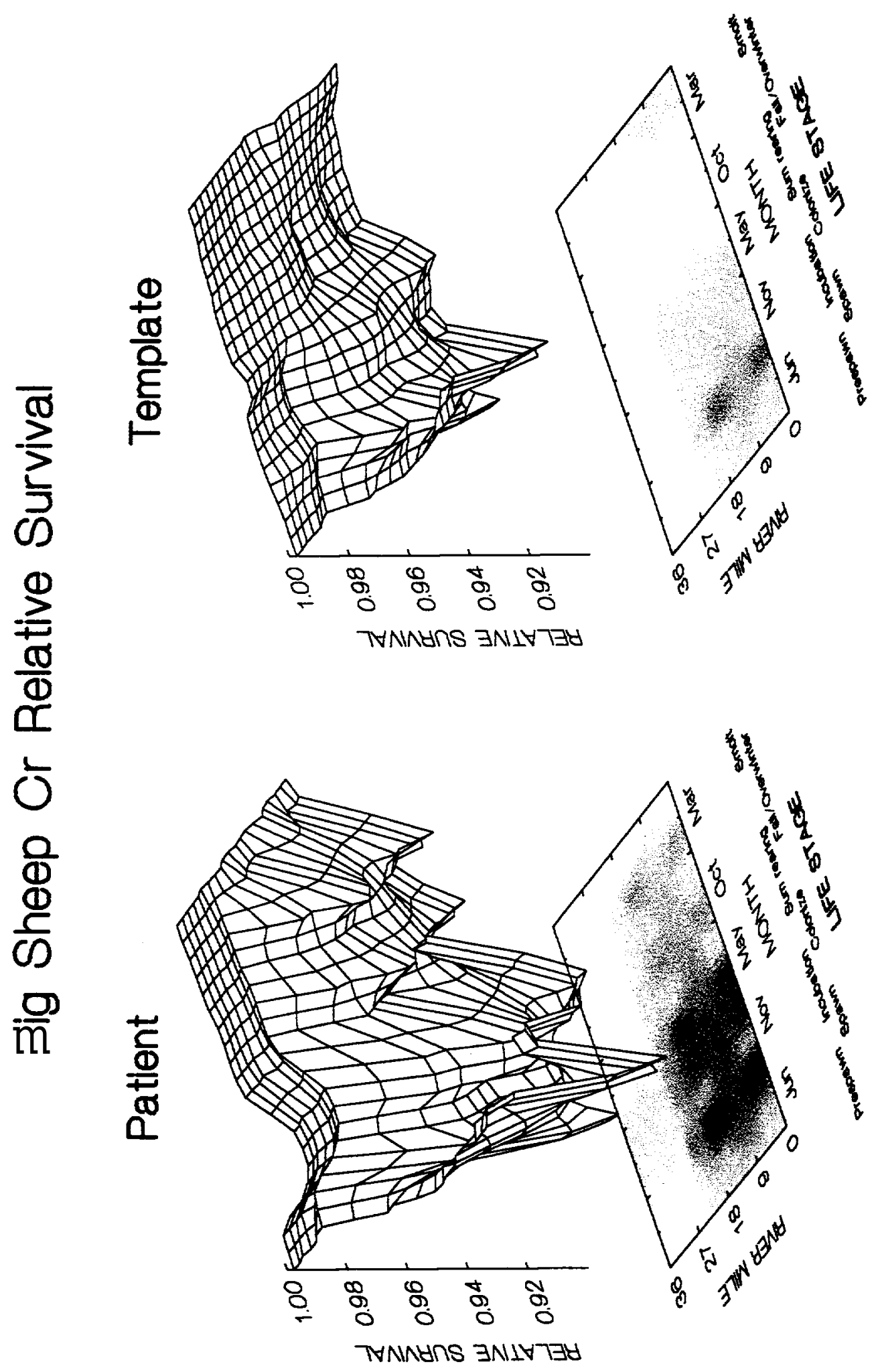




\section{Big Sheep Cr/Imnaha R Key Habitat Quantity}

Patient

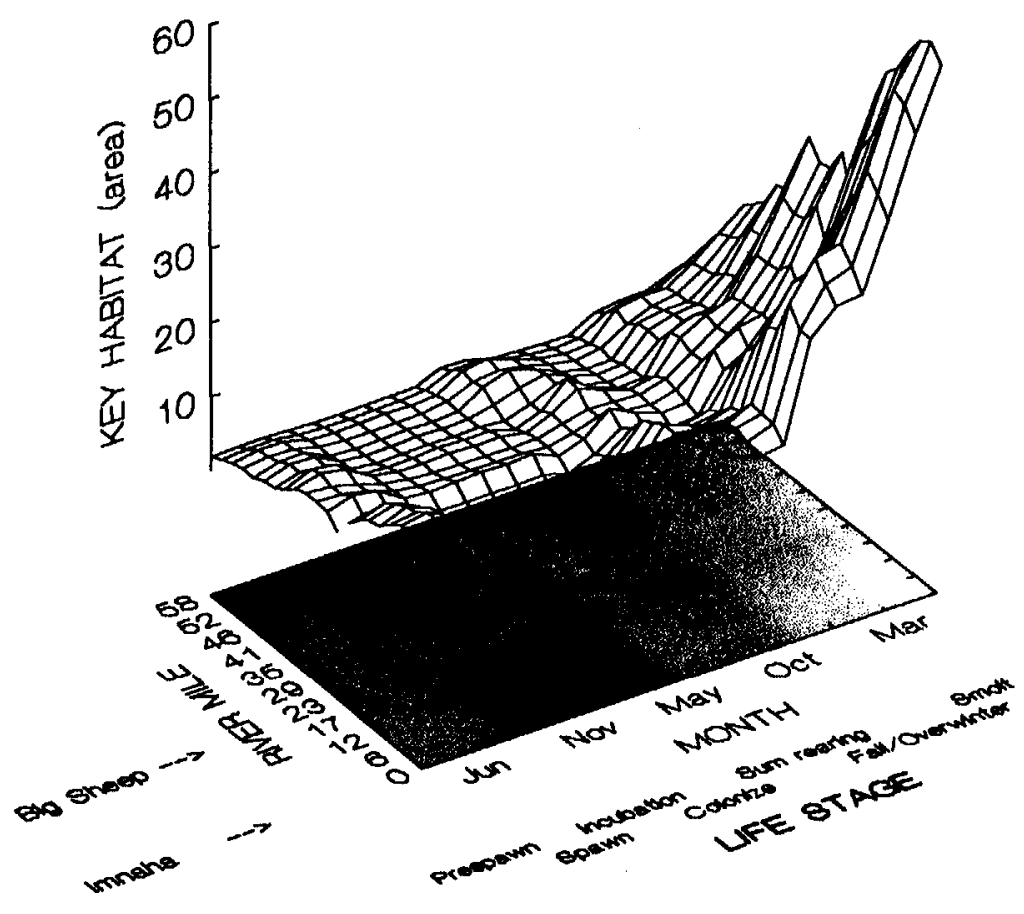

Template

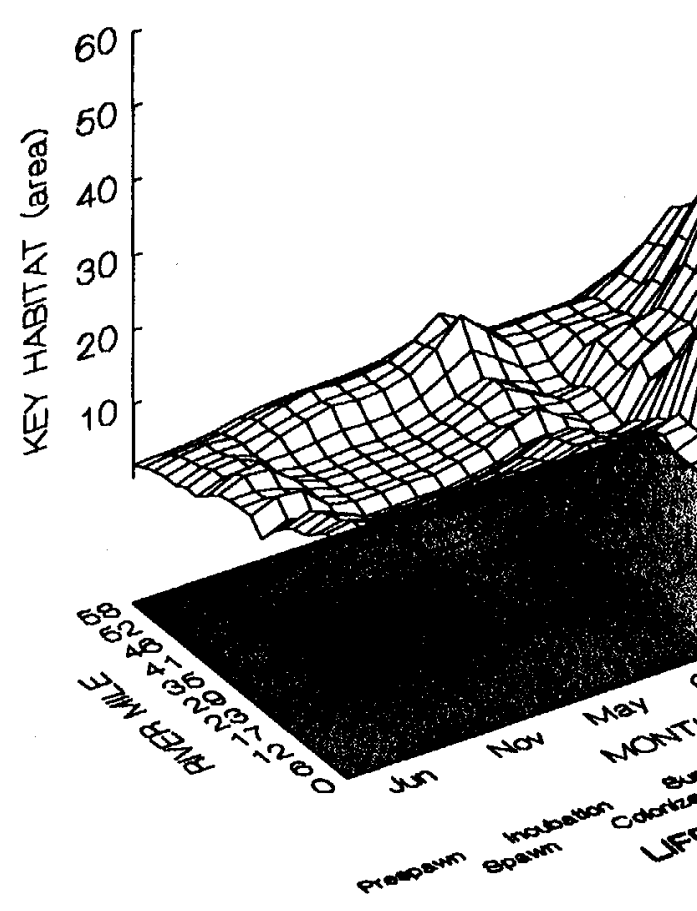




\section{Big Sheep Cr/Imnaha Fñúironmental Attributes}
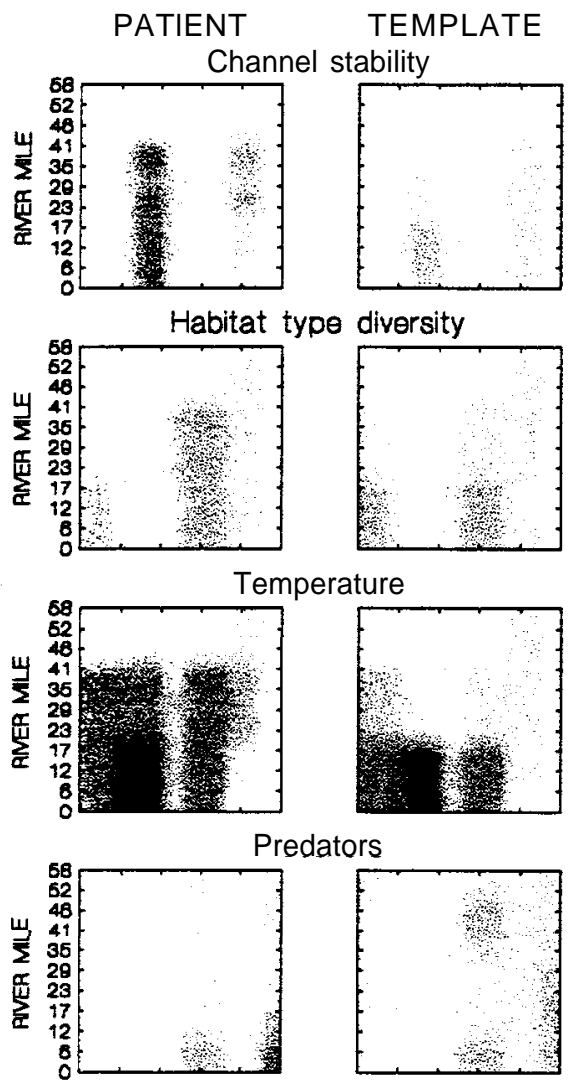

Predators
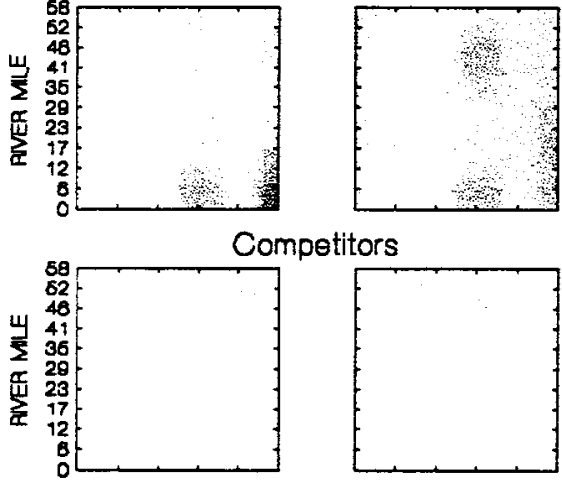

ompetitors

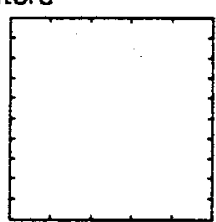

Withdrawals
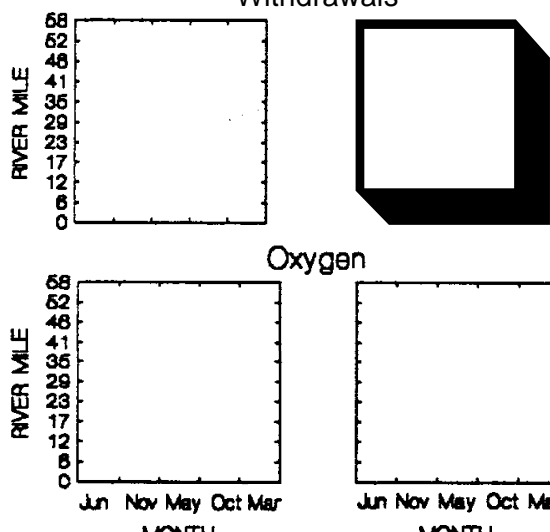

Oxygen
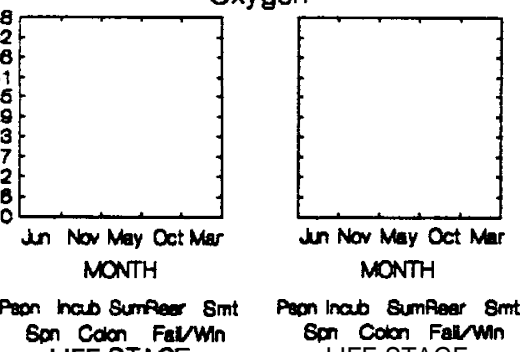

LIFE STACE

LIFE STAGE

PATIENT

Flow

TEMPLATE

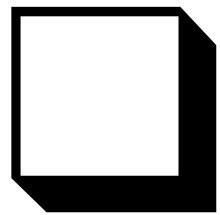

Sediment load

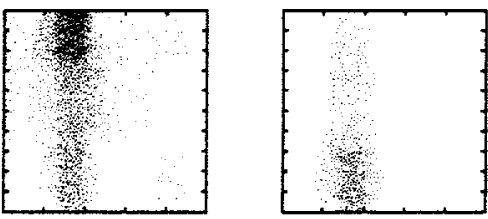

Riparian condition
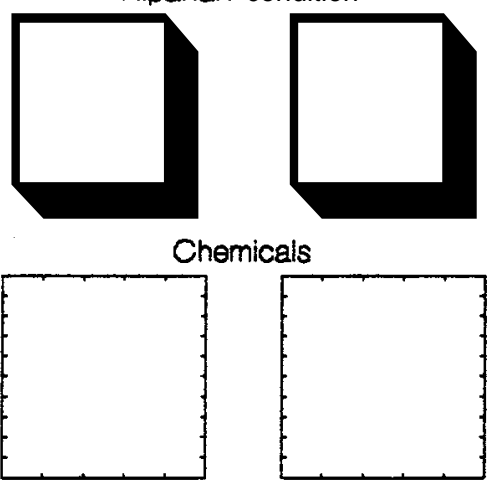

Obstructions

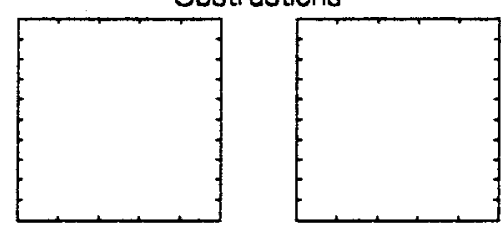

Nutrient load

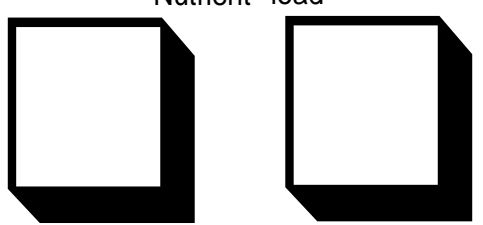

Pathogens

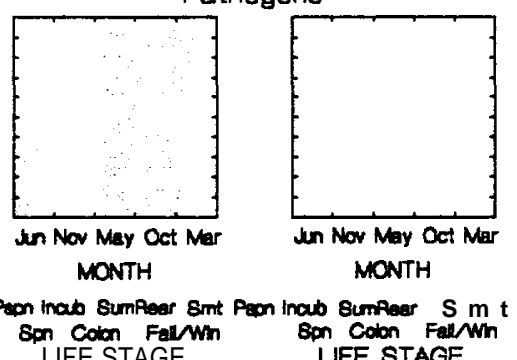

Level of

Effect

$\square$ No effect

$\square$ Low

$\square$ Moderate

$\square$ High

Lethal

Pan inat Sumfeer Bmt Pan Inab Bumpeer $S \mathrm{~m} t$

LIFE STAGE UFE STAGE 


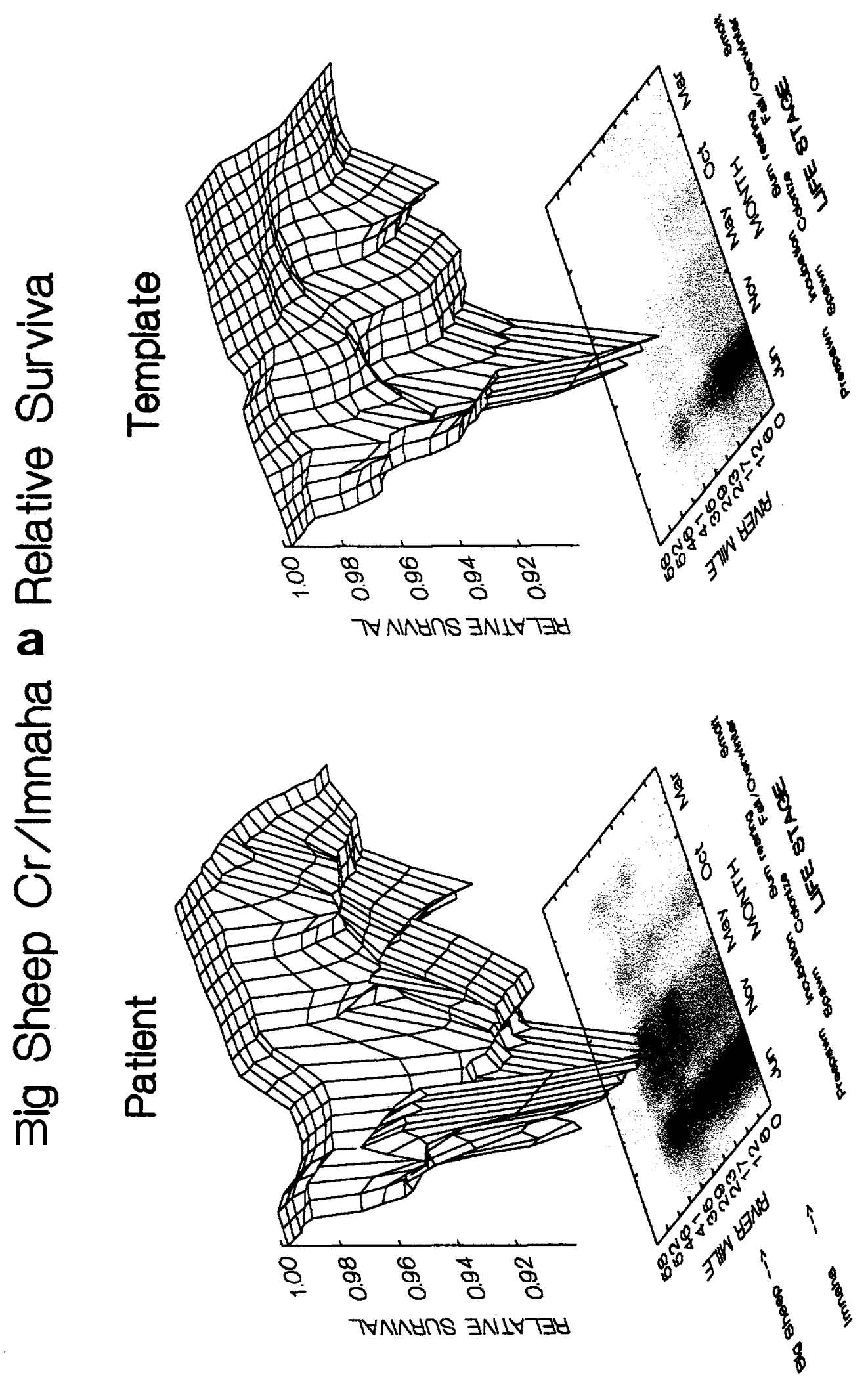




\section{Stream Reach Range}

Little Sheep Cr / Big Sheep Cr / Imnaha R 


\section{Patient-Template Analysis Summary}

Stream Reach Range: Little Sheep Cr./Big Sheep Cr./Imnaha R.

\section{Key Habitat Quantity}

The quantity of key habitat for spring chinook in Little Sheep Creek is largely unchanged compared to historic levels, except in the prespawning stage which declined. The Little Sheep Creek system has always contained relatively little spring chinook key habitat.

The quantity of key habitat in the Imnaha River downstream of Big Sheep Creek is comparatively small, although egg incubation habitat shows relatively substantial 10 ss compared to historic levels.

\section{Relative Productivity (Survival)}

The capability of Little Sheep Creek of supporting spring chinook productivity (survival) is greatly reduced compared to historic conditions for all life stages except smelt. Current survival conditions are generally poor due primarily to changes in flow regimes, channel (streambed) stability, sediment load, and habitat diversity. Historically, survival conditions in this stream were excellent for all life stages.

Survival conditions in the Imnaha River downstream of Little Sheep Creek have worsened compared to those that existed historically, although the lower portions of the river have always been unfavorable for survival during some life stages. Conditions are less favorable due to slightly increased water temperatures, small loss in habitat diversity, and increased channel (streambed) instability. 


\section{Little Sheep Cr Key Habitat Quantity}

\section{Patient}

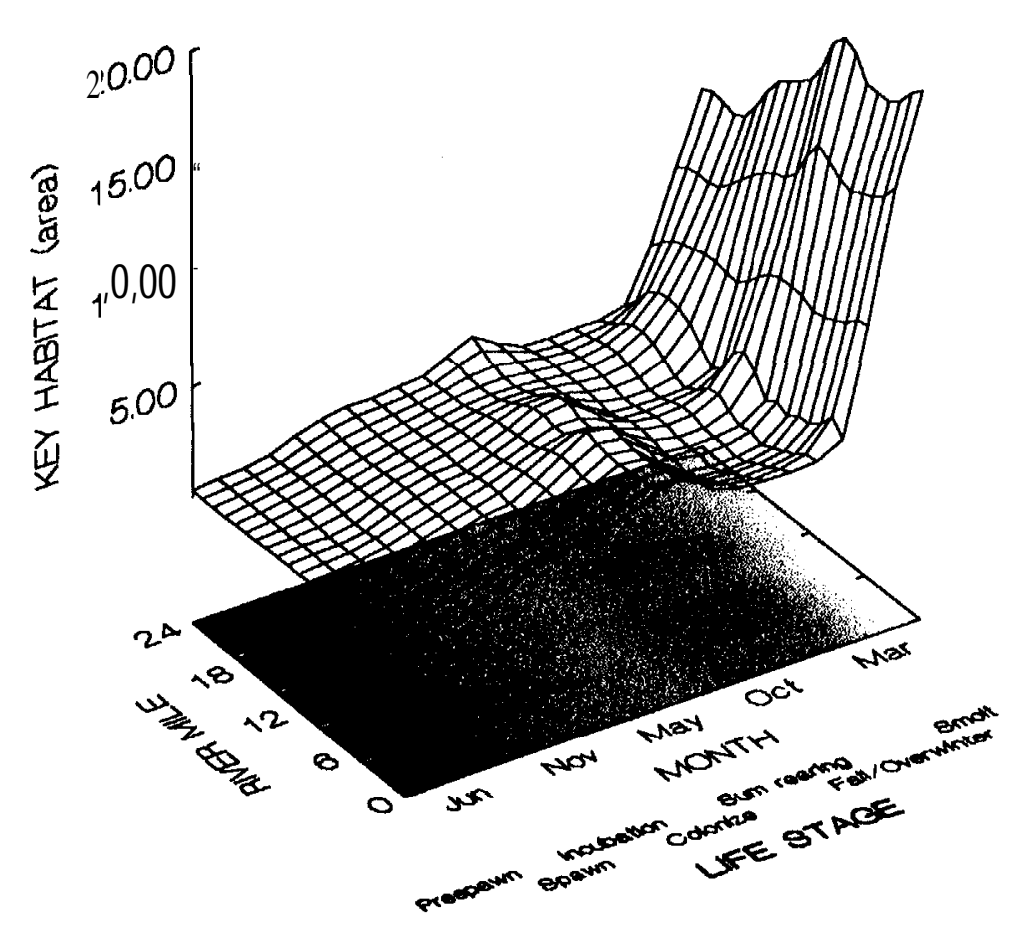

Template

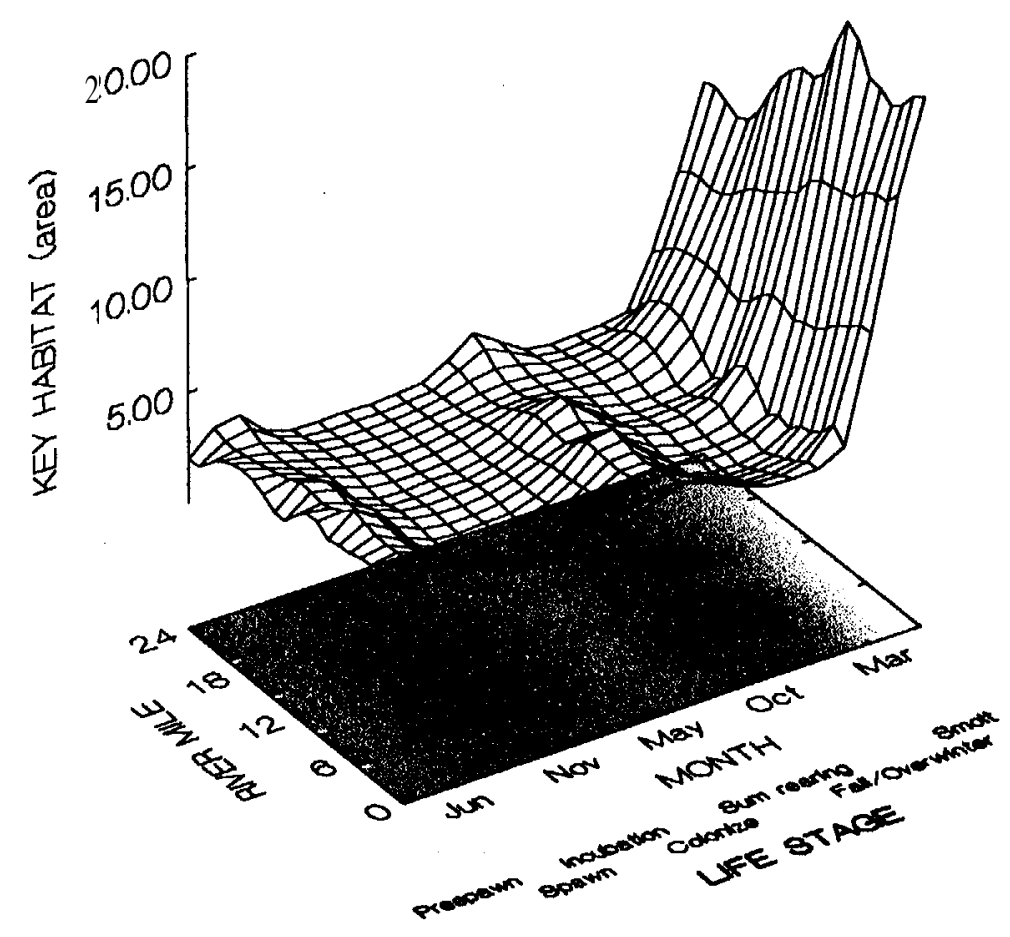




\section{Little Sheep Cr Environmental Attributes}
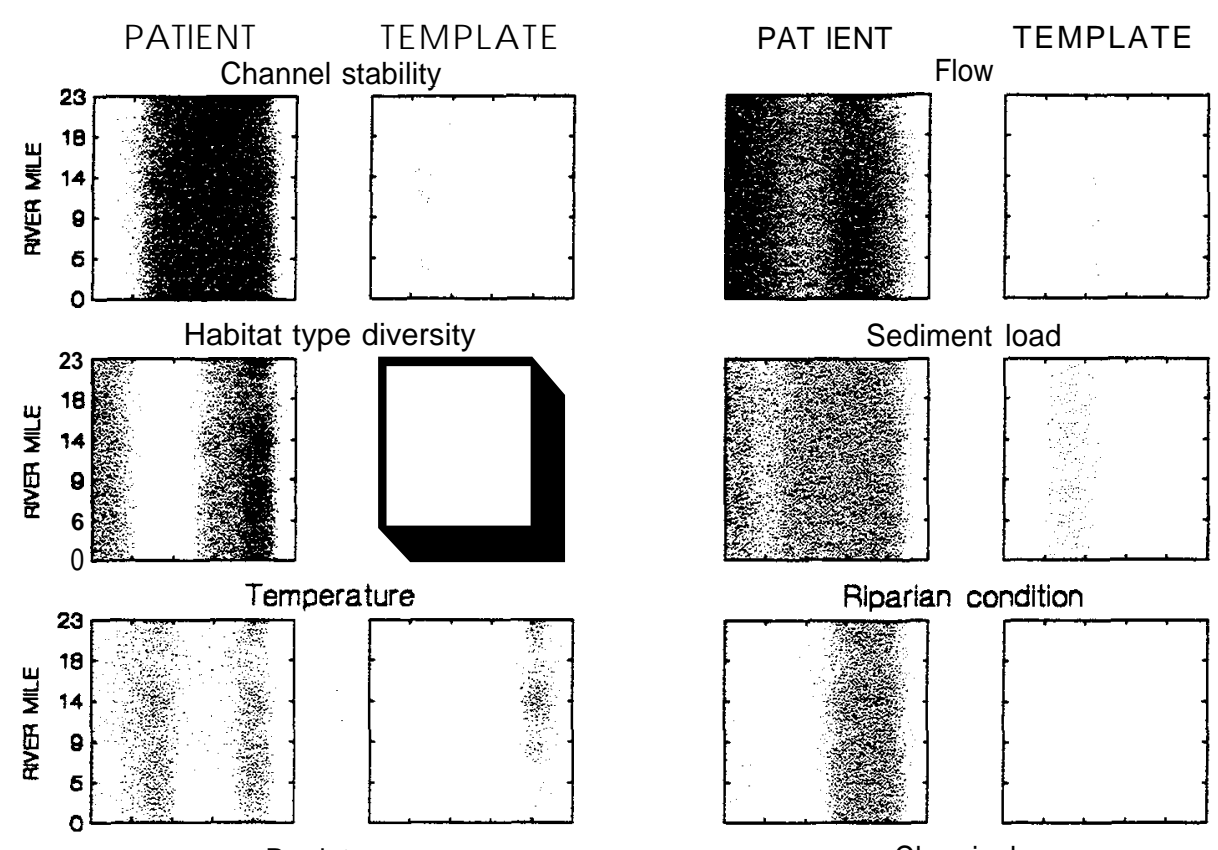

Rlparian condition
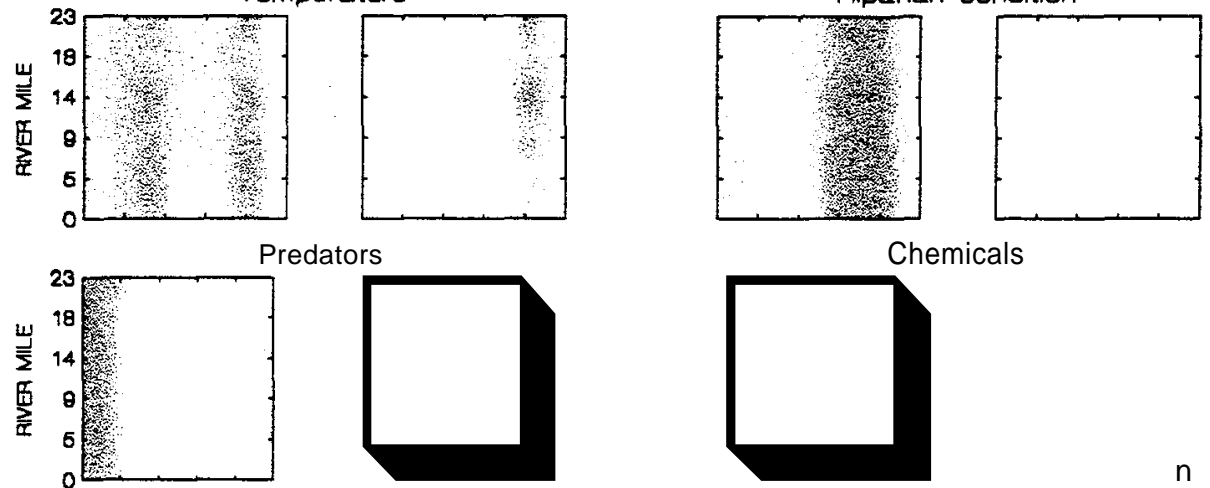

Chemicals
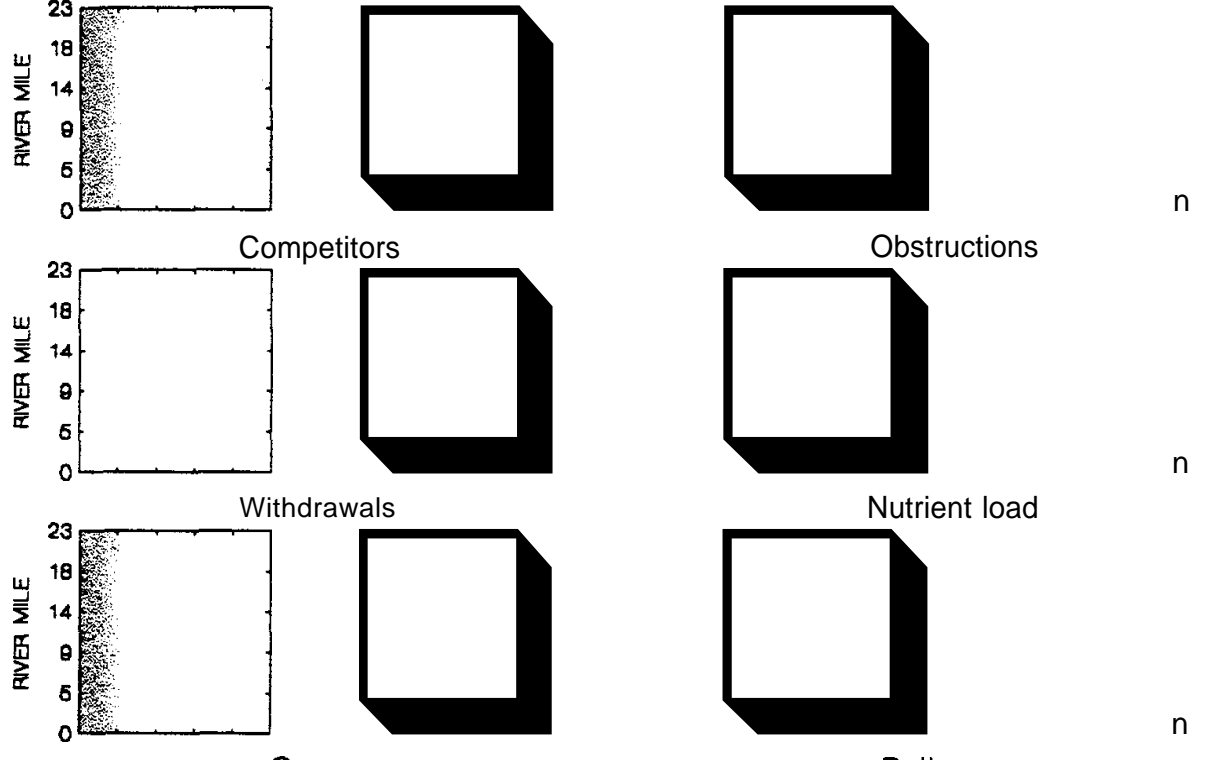

Oxygen

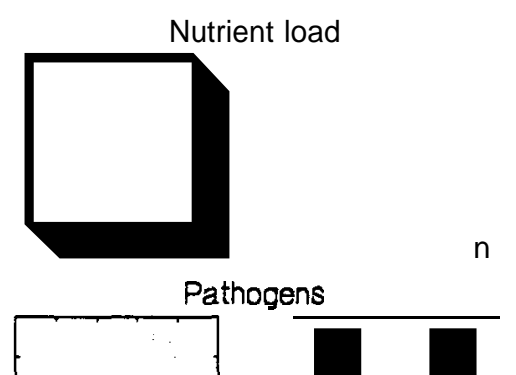




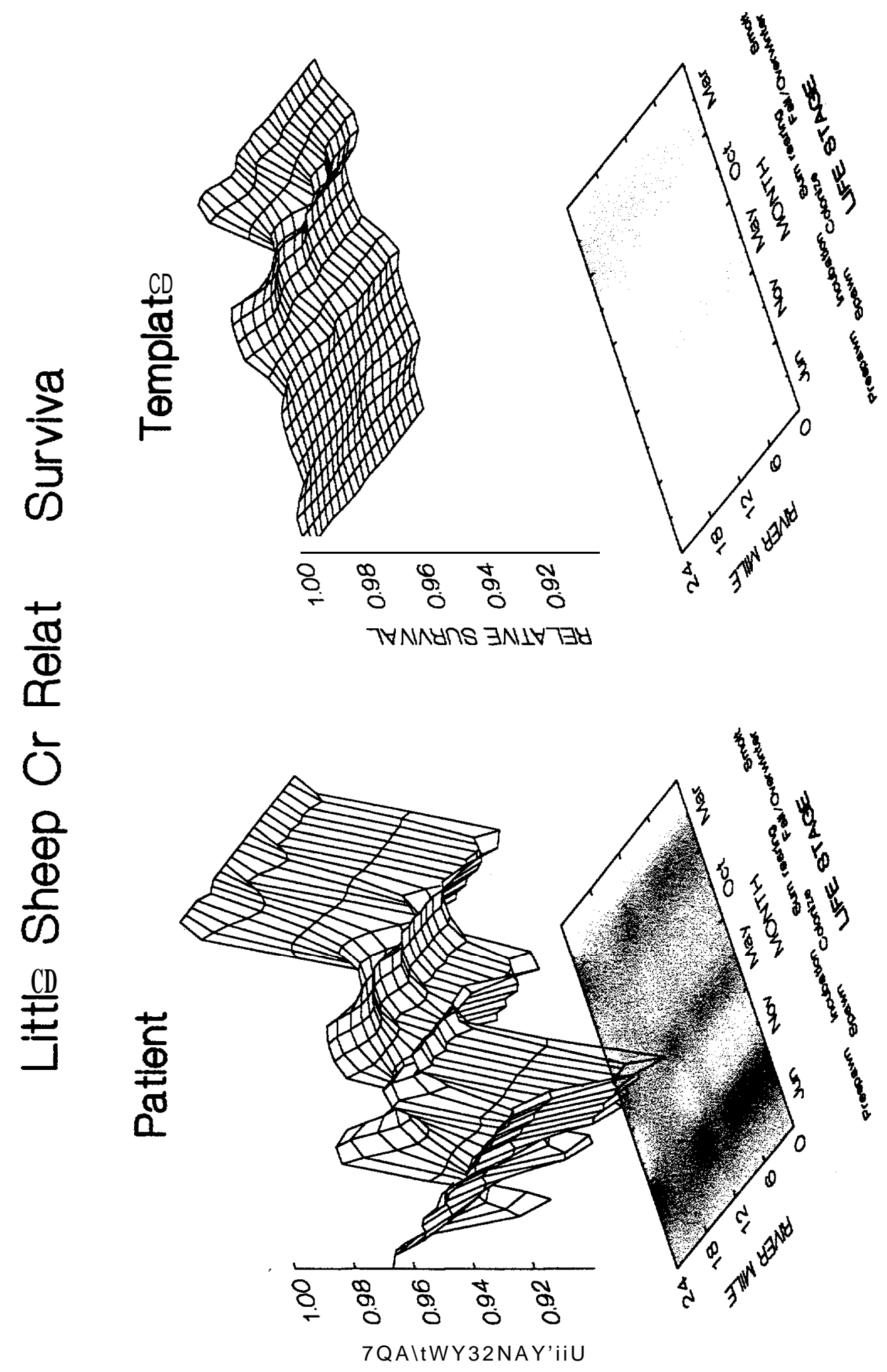



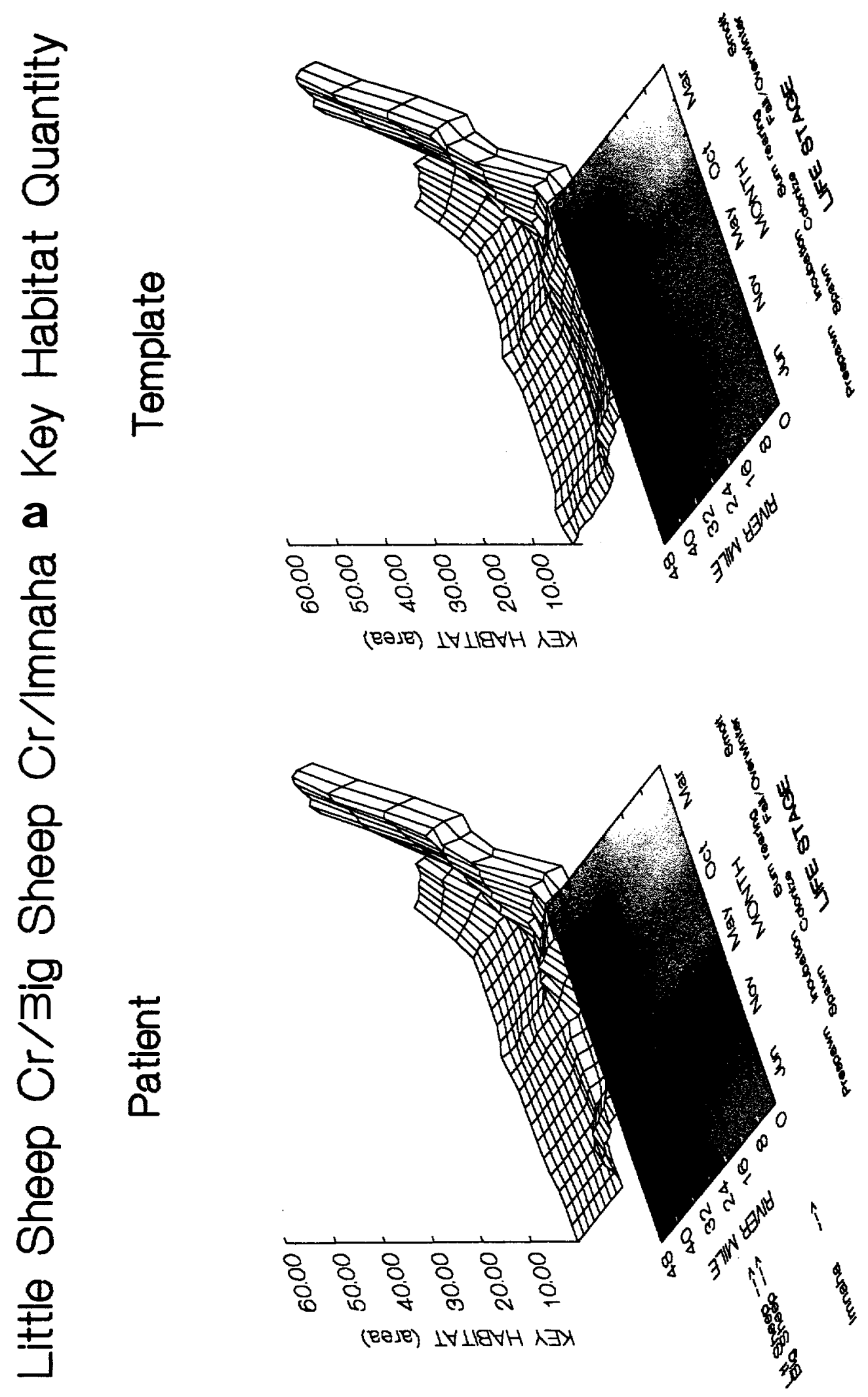


\section{Little Sheep Cr/Big Sheep Cr/Imnaha R Environmental Attributes}
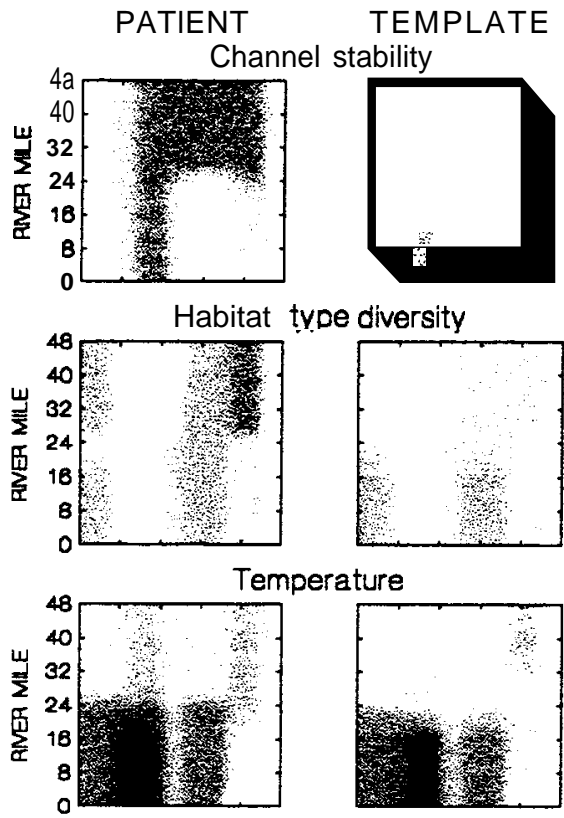

Temperature
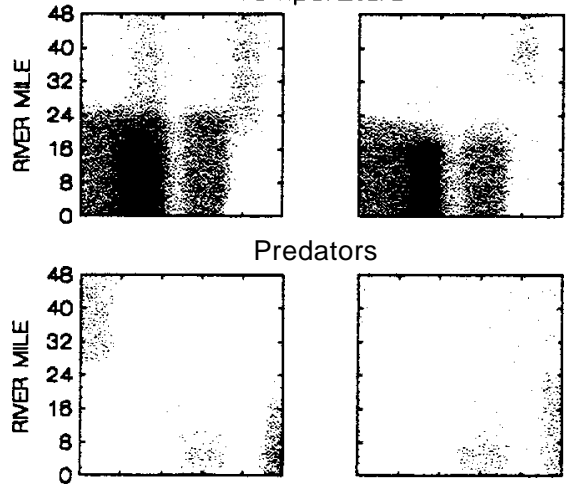

Predators
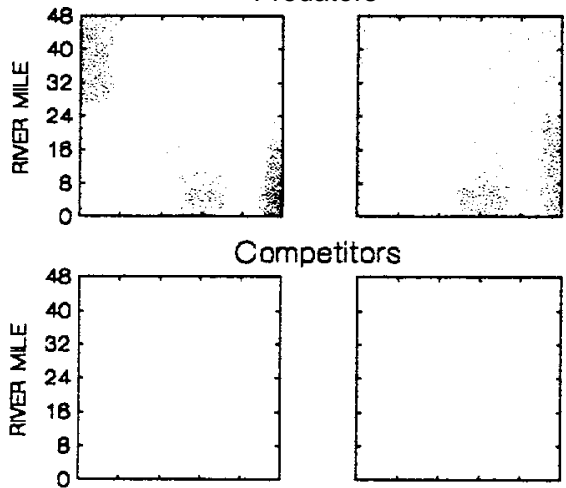

Competitors

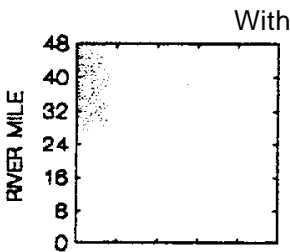

Withdrawals

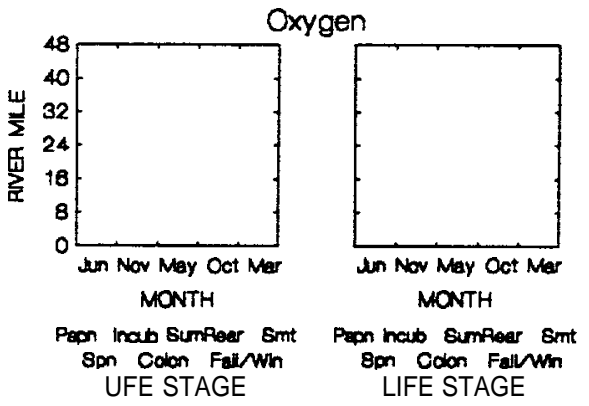

PAT IENT

TEMPLATE
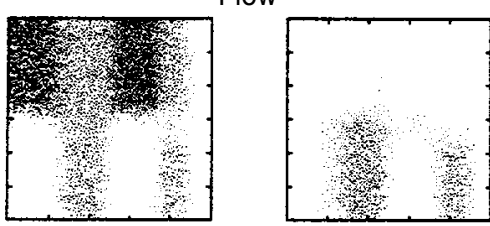

Sediment load
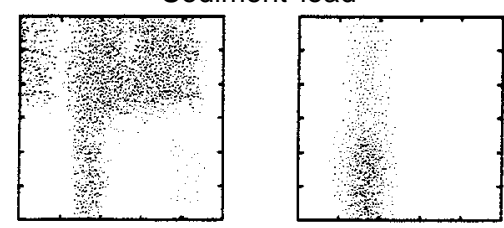

Riparian condition

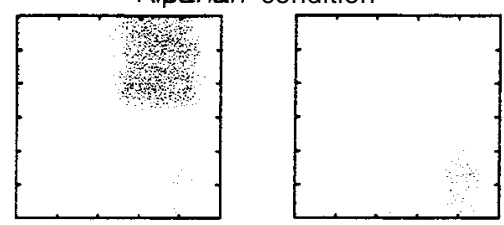

Chemicals

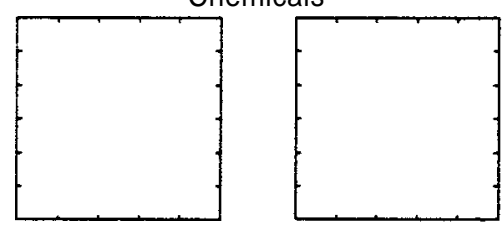

Obstructions

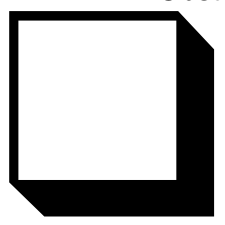

Nutrient load

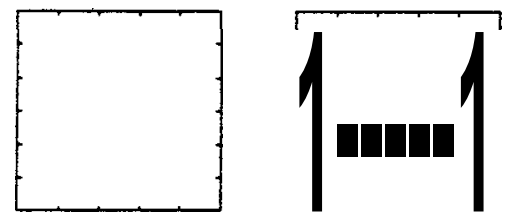

Pathogens

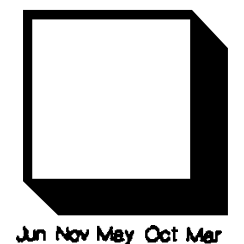

MONTH

In Now Mey Oot Mar MONTH

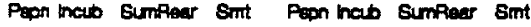
8pn Colon FallWin
LIFE STAGE
Level of

Effect

$\square$ No effect

$\square$ Low

$\square$ Moderate

High

Lethal 


\section{Little Sheep Cr/Big Sheep Cr/Imnaha R Relative Survival}
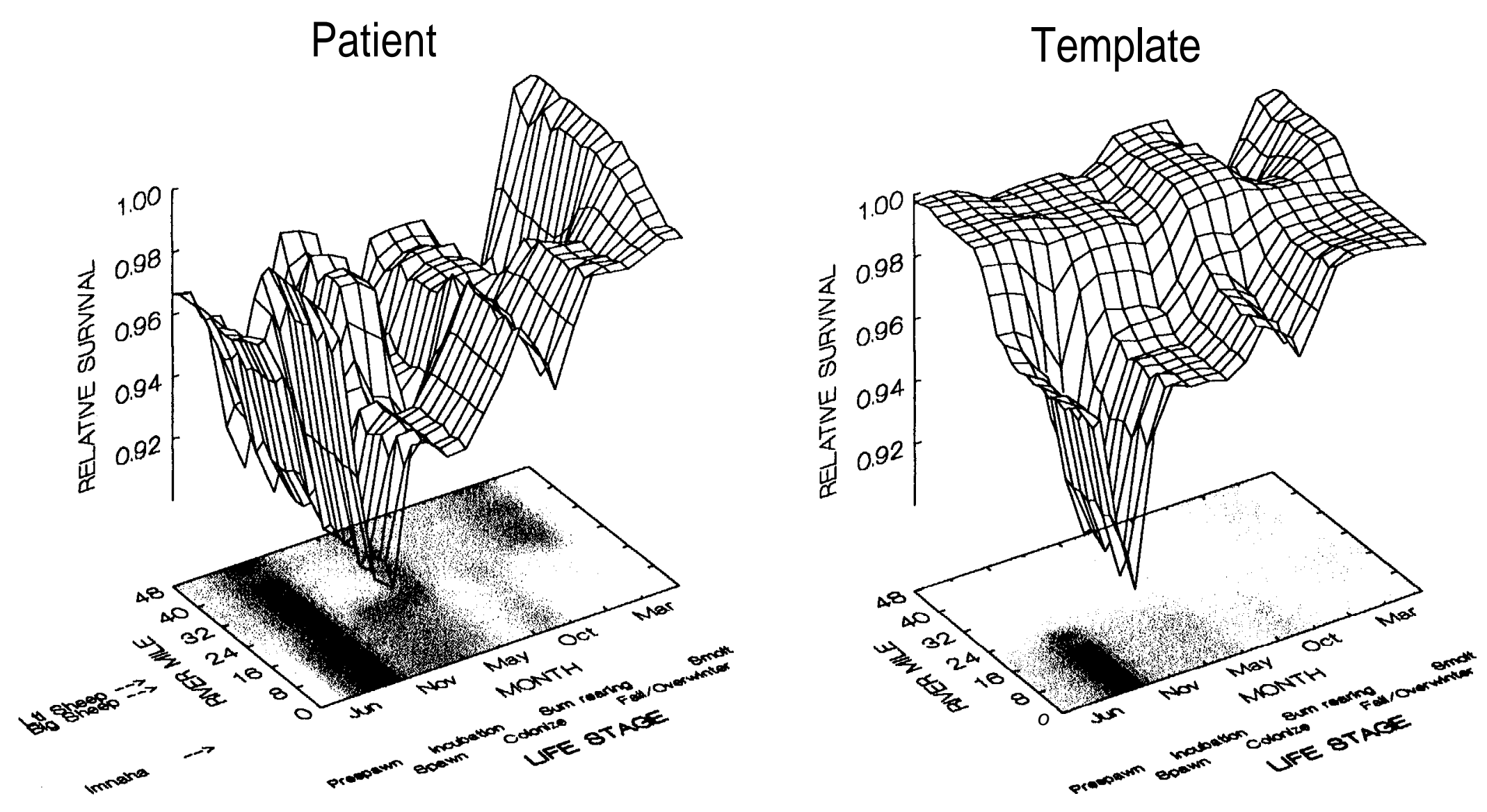
Stream Reach Range

Lightning Cr/ Imnaha R 


\title{
Patient-Template Analysis Summary
}

\author{
Stream Reach Range: Lightning Cr./Imnaha R.
}

\section{Key Habitat Quantity}

The quantity of key habitat for spring chinook in Lightning Creek is unchanged compared to historic levels. This stream has always contained little spring chinook key habitat.

The quantity of key habitat in the Imnaha River downstream of Lightning Creek is comparatively small, although egg incubation habitat shows relatively substantial loss compared to historic levels.

\section{Relative Productivity (Survival)}

The capability of Lightning Creek of supporting spring chinook productivity (survival) is essentially unchanged compared to historic conditions. In general, survival conditions are relatively poor for prespawners, spawners, and egg incubation but are good for summer rearing and overwintering.

Survival conditions in the Imnaha River downstream of Lightning have worsened slightly compared to those that existed historical y, although the lower portions of the river have always been unfavorable for survival during some life stages. Conditions are less favorable due to slightly increased water temperatures, small loss in habitat diversity, and increased channel (streambed) instability. 

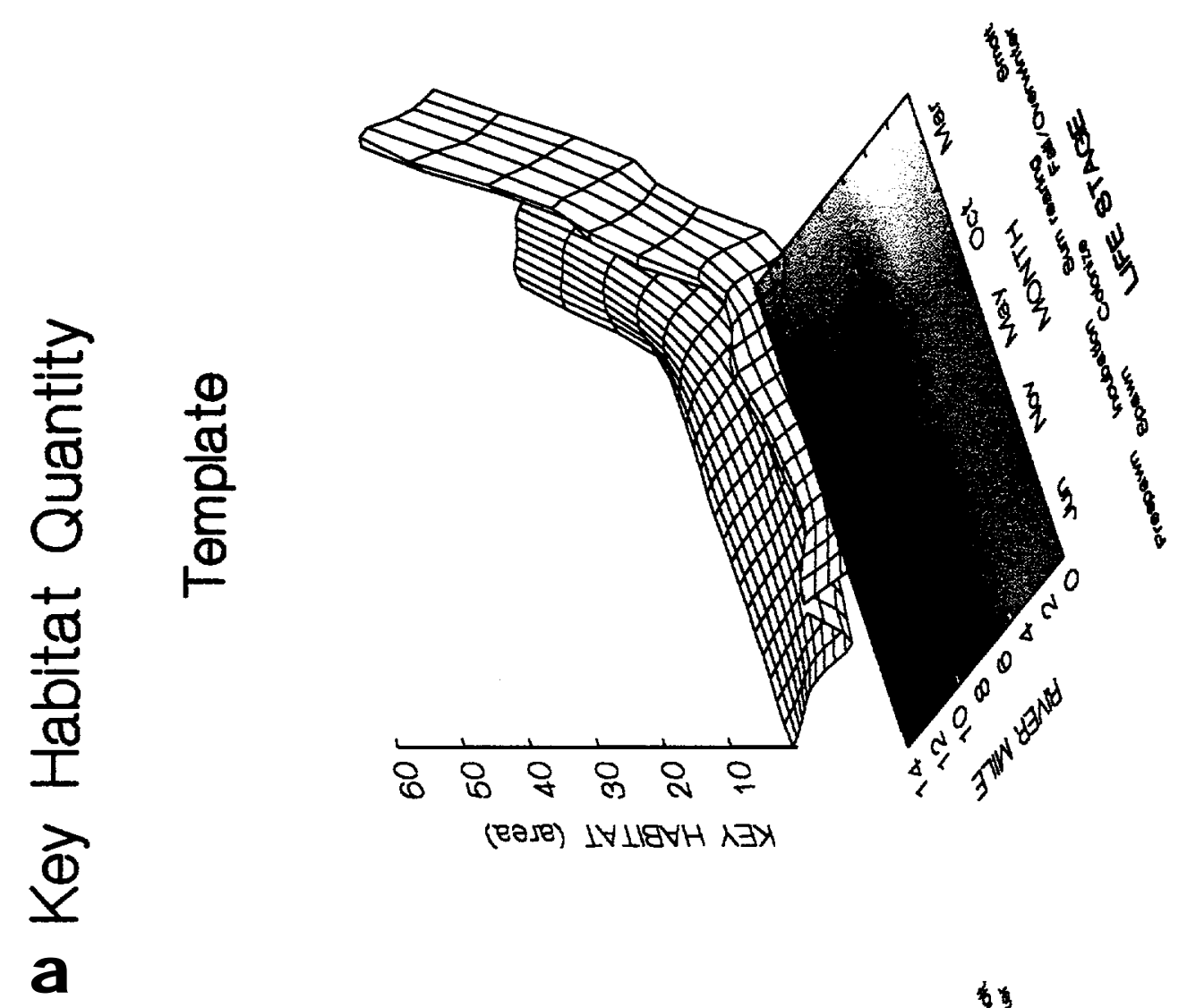

$\frac{\text { ฮ }}{\frac{\sigma}{\sigma}}$

总

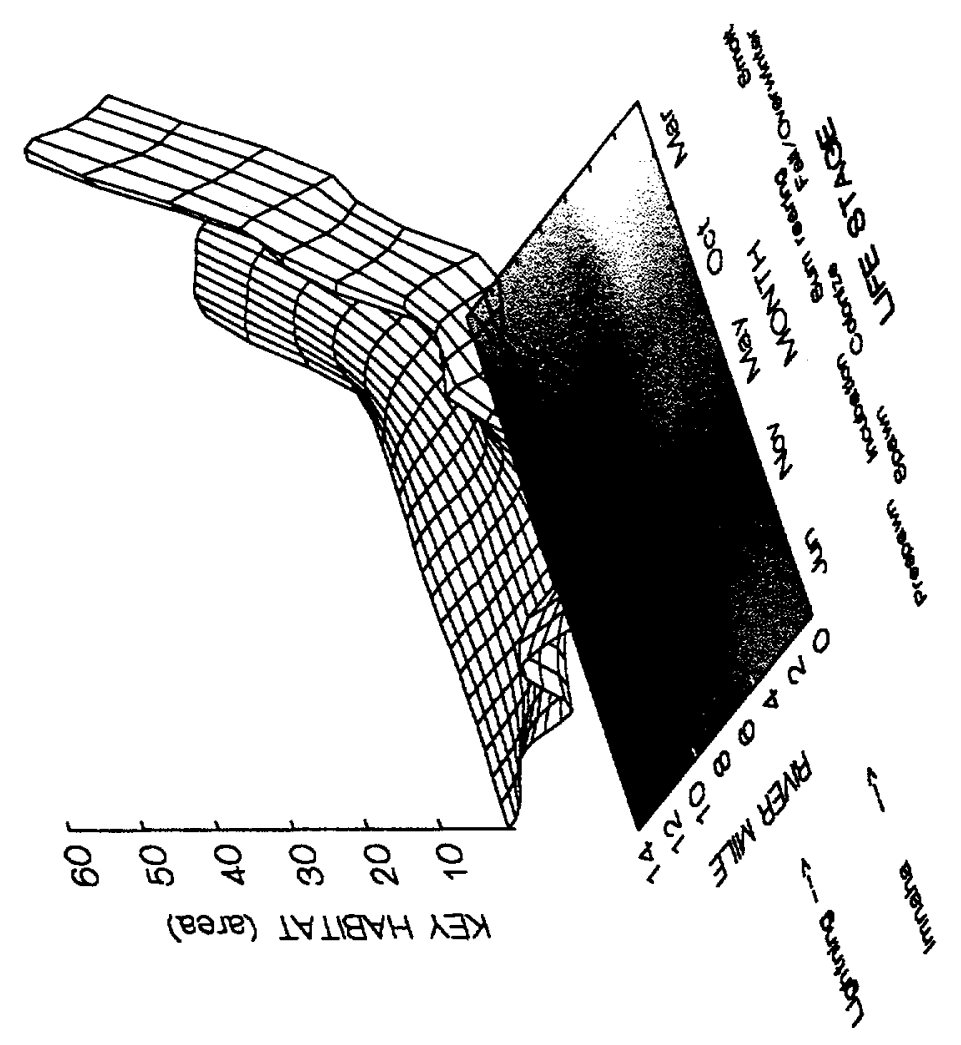




\section{Lightning $\mathrm{Cr} /$ Imnaha R Environmental Attributes}

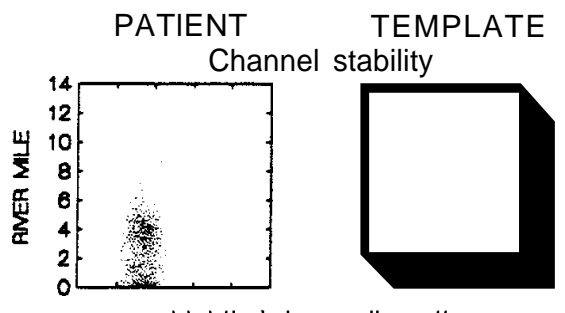

PAT IENT TEMPLATE

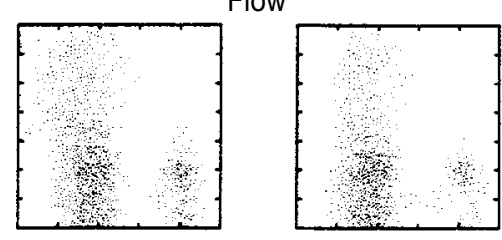

Sediment load
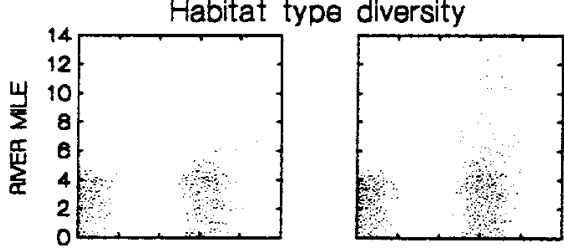

Temperature
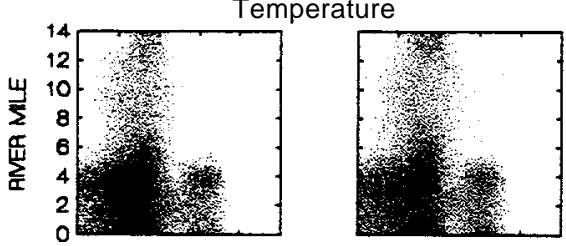

Predators
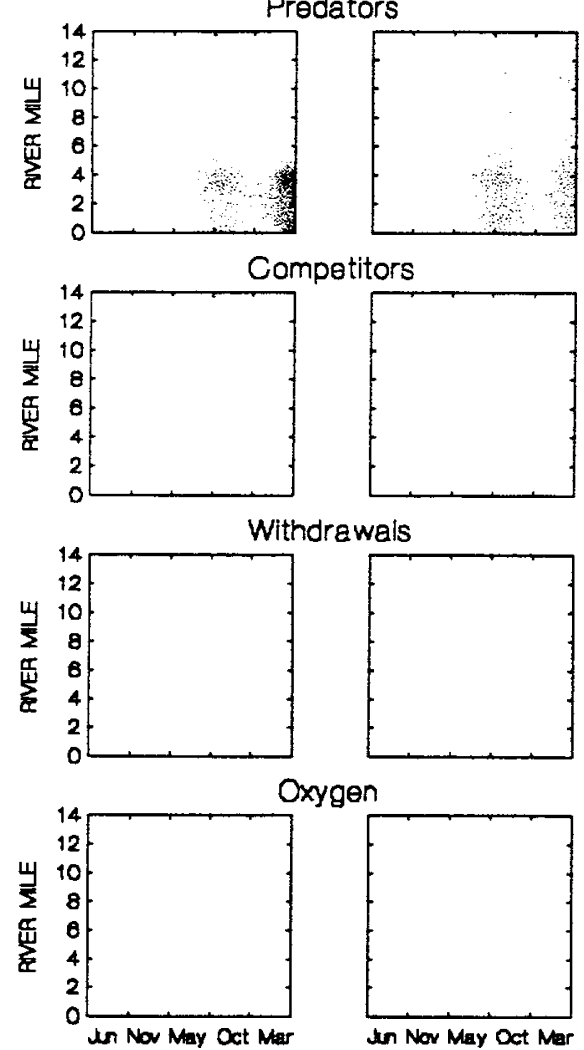

MONIH

Oxygen

Paon hous Sumper 8m Son coton Fallym

LIFE STAGE

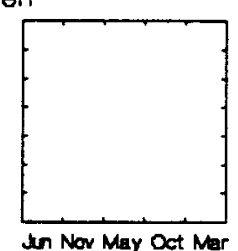
MONTH

Pam Inab Bumpear Bmt Son Colon Foumn

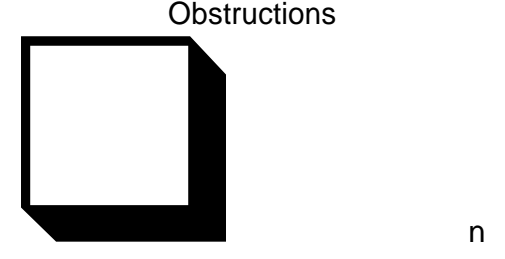

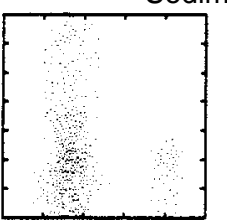

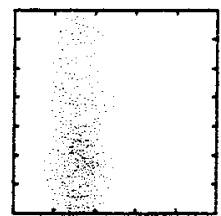

Riparian condition
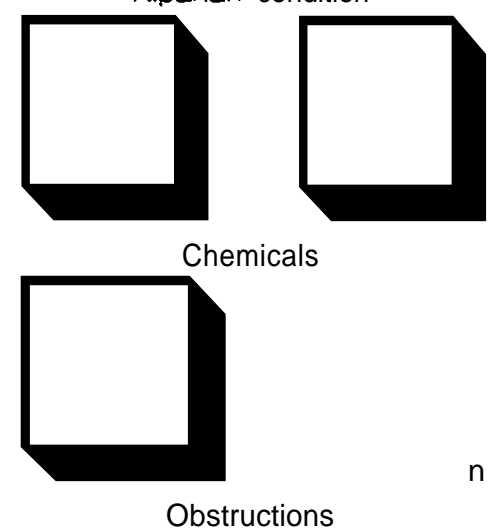

Nutrient load

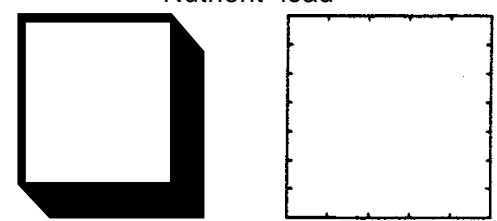

Pathogens

Level of

Effect

No effect

$\square$ Low

Moderate

- High

Lethal 

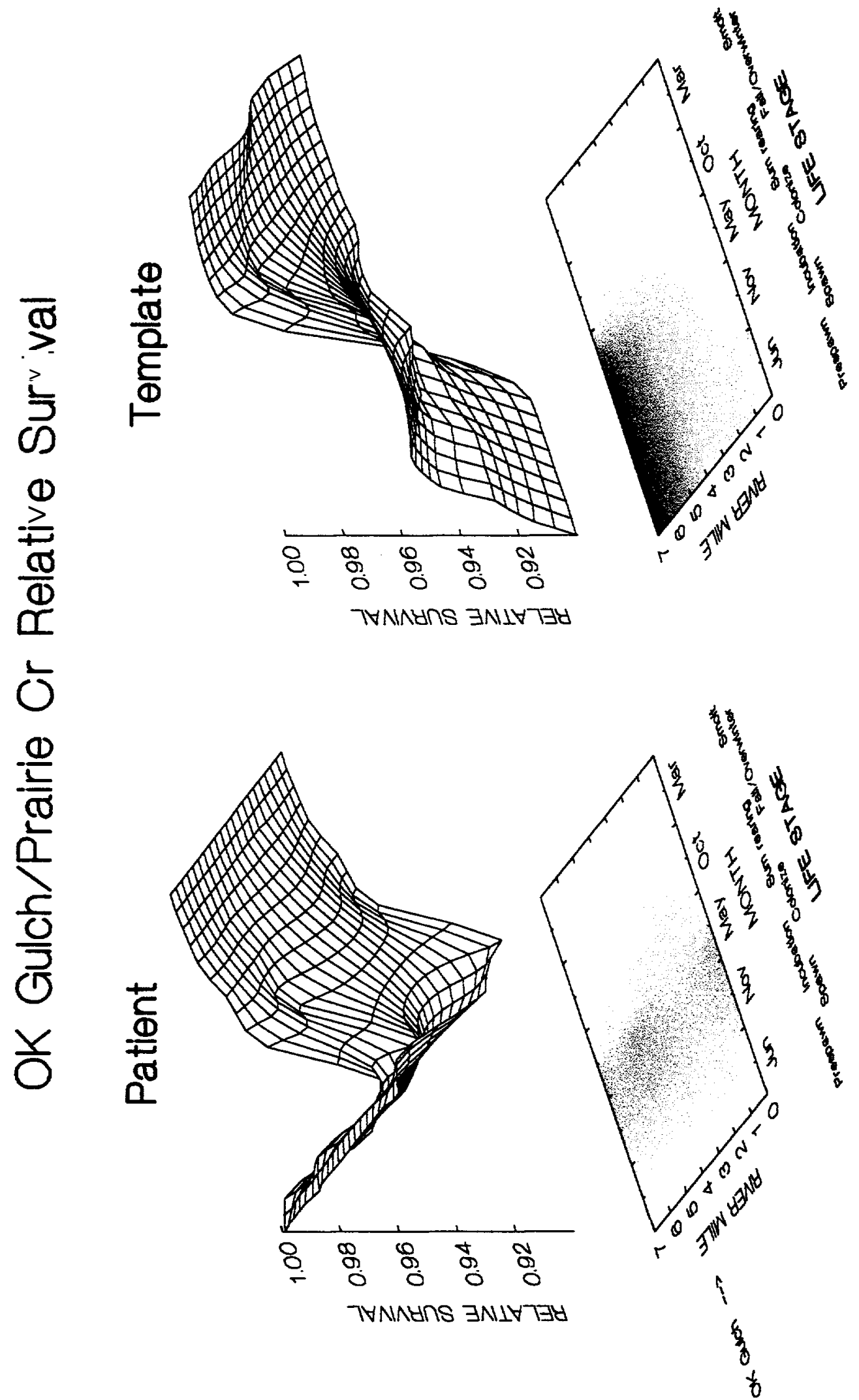


\section{APPENDIX D}

Grande Ronde Stream Reach Summaries

for Primary and Secondary Life History Pathways 


\section{Reaches analyzed in the Grande Konde, listed 'm the ordèr they appear in Appendĩ 1T.}

$\quad$ Stream
Bear Cr.
Bear Cr.
Bear Cr.
Catherine Cr.
Catherine Cr.
Catherine Cr.
Catherine Cr.
Grande Ronde R
Grande Ronde R.
Grande Ronde R.
Grande Ronde R.
Grande Ronde R
Grande Ronde R.
Grande Ronde R.
Grande Ronde R.
Grande Ronde R.
Grande Ronde R.

- Grande Ronde R.

Grande Ronde R.

Grande Ronde R.

Grande Ronde R.

Grande Ronde R.

Grande Ronde R.

Grande Ronde R.

Grande Ronde R.

Grande Ronde R.

Grande Ronde R.

Grande Ronde R.

Grande Ronde R.

Grande Ronde R.

Grande Ronde R.

Grande Ronde R.

Grande Ronde R.

Grande Ronde R.

Grande Ronde R.

Grande Ronde R.

Grande Ronde R.

Hurricane Cr.

Hurricane $\mathrm{Cr}$.

Joseph Cr.

Joseph Cr.

Joseph Cr.

Chesnimnus $\mathrm{Cr}$.

Lookingglass $\mathrm{Cr}$.

Lookingglass $\mathrm{Cr}$.
Reach

Mouth - Chamberlain Ditch (RM 101.8 -104.7 from Snake R)

Chamberlain Ditch - Little Bear Cr (RM 104.7 -109.3 from Snake R)

Little Bear Cr - headwaters (RM 109.3 -118.3 from Snake R)

Mouth - 10th Street Bridge in Union (RM 114.5 -146.0 from Snake R)

10th Street Bridge in Union - Little Catherine Cr (RM 146.0 -157.5 from Snake R)

Little Catherine Cr - forks (RM 157.5 -164.4 from Snake R)

South Fork Catherine Cr (RM 164.4 -166.8 from Snake R)

Mouth - Joseph Cr (RM 0.0- 3.7 from Snake R)

Joseph Cr - Buford Cr (RM 3.7- 26.2 from Snake R)

Buford Cr - Bear Cr (RM 26.2 -28.2 from Snake R)

Bear Cr - Menatchee Cr (RM 28.2 -34.8 from Snake R)

Menatchee Cr - Wenaha R (RM 34.8 -45.1 from Snake R)

Wenaha R - Courtney Cr (RM 45.1 -46.2 from Snake R)

Courtney Cr - Mud Cr(RM 46.2-51.4 from Snake R)

Mud Cr - Wallupa/Wildcat Cr (RM 51.4 -52.8 from Snake R)

Wallupa/Wildcat Cr - Grossman Cr (RM 52.8-6 [. 1 from Snake R)

Grossman Cr-WallowaR(RM61. 1 -80.0 from Snake R)

Wallowa R - Lookingglass Cr (RM 80.0 -83.4 from Snake R)

Lookingglass $\mathrm{Cr}$ - Gordon $\mathrm{Cr}$ (RM 83.4 -94.2 from Snake R)

Gordon Cr - Phillips Cr (RM 94.2 -96.9 from Snake R)

Phillips Cr - Clark Cr (RM 96.9 -97.3 from Snake R)

Clark Cr - Indian Cr (RM 97.3 -99.3 from Snake R)

Indian Cr - Willow Cr (RM 99.3 -103.5 from Snake R)

Willow Cr - downstream end of State Ditch (RM 103.5 -114.5 from Snake R)

Downs[ream end of State Ditch - Upstream end of State Ditch (RM 114.5 -118.9 from Snake

Upstream end of State Ditch - I-84 crossing below Devil's Slide (RM 118.9 -128.4 from Snak

I-84 crossing below Devil's Slide - Five Points Cr (RM 128.4 -133.4 from Snake R)

Five Points Cr - Rock Cr (RM 133.4 -133.8 from Snake R)

Rock Cr - Whiskey Cr (RM 133.8 -136.6 from Snake R)

Whiskey Cr - Jordan Cr (RM 136.6-139. I from Snake R)

Jordan Cr - Beaver Cr (RM 139.1 -145.8 from Snake R)

Beaver Cr - Meadow Cr (RM 145.8 -147.0 from Snake R)

Meadow Cr - Fly Cr (RM 147.0-15 [.3 from Snake R)

Fly $\mathrm{Cr}$ - head of canyon 1.3 miles downstream of Sheep Cr (RM 151.3 -159.4 from Snake R)

Head of canyon 1.3 miles downstream of Sheep Cr - LimberJim Cr (RM 159.4-164.2 from S

LimberJim Cr - Clear Cr (RM 164.2 -166.9 from Snake R)

Clear Cr - headwaters (RM 166.9- [70.1 from Snake R)

Mouth - Lower AlderSlope Ditch (RM 121.9 -124.9 from Snake R)

Lower Alderslope Ditch - Upper Alderslope Ditch Diversion (RM 124.9 -128.2 from Snake F

Mouth - Cottonwood Cr (RM 3.7- 8.2 from Snake R)

Cottonwood Cr - Swamp Cr (RM 8.2- 37.7 from Snake R)

Swamp Cr - Chesnimnus Cr (RM 37.7 -52.7 from Snake R)

Confluence with Crow Cr - South Fork (RM 52.7 -77.7 from Snake R)

Mouth - Little Lookingglass Cr (RM 83.4 -87.6 from Snake R)

Little Lookingglass $\mathrm{Cr}$ - headwaters (RM 87.6 -93.8 from Snake R)
Benefit Category

for

Primary \&

Secondary Life

Histones

$\mathrm{F}$

E

E

$\mathrm{F}$

A

D

B

B

F

E

F

F

F

F

F

F

B

A

F

F

F

F

F

F

F

D

D

F

F

F

F

A

A

A

A

A

B

B

A

E

F

F

F

B

E 


\section{Reaches analyzed in the Grande Ronde, listed in the order they appear in Appendix D.}

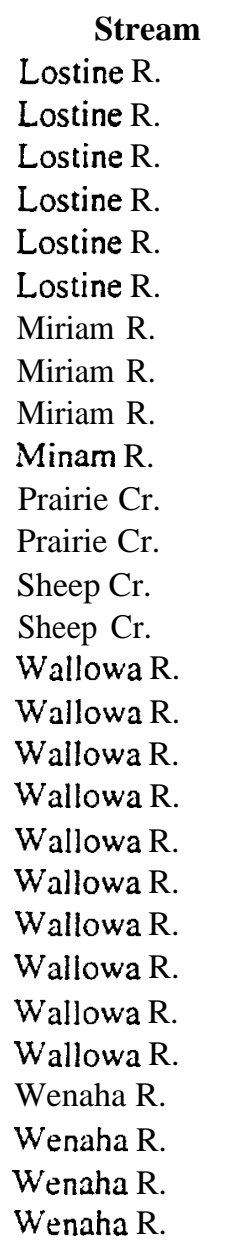

\author{
Reach \\ Mouth - ClearWater Ditch (RM 105.8 -108.3 from Snake R) \\ ClearWater Ditch - Cross Country Ditch (RN1 108.3 -110.8 from Snake R) \\ Cross Country Ditch - Sheep Ridge Dam (RM 110.8 -115.0 from Snake R) \\ Sheep Ridge Dam - Silver Cr (RM 115.0 -120.0 from Snake R) \\ Silver $\mathrm{Cr}$ - Lake Cr (RM 120.0 -124.6 from Snake R) \\ Lake Cr - East Lostine R (RM 124.6 -131.7 from Snake R) \\ Mouth - Cougar Cr (RM 89.8 -99.4 from Snake R) \\ Cougar Cr - Little Miriam R(RA499.4 -108.4 from Snake R) \\ Little Miriam R - splash dam site ( 1 m above Garwood Cr; RM 108.4 -119.7 from Snake R) \\ Splash dam site - falls (RM 119.7 -138.0 from Snake R) \\ Mouth - OK Gulch confluence (RM 126.8 -131.8 from Snake R) \\ OK Gulch fork (RM 131.8 -133.8 from Snake R) \\ Mouth - Chicken Cr (RM 164.2 -165.7 from Snake R) \\ Chicken Cr - headwaters (RM 165.7 -173.7 from Snake R) \\ Mouth - Miriam R (RM 80.0 -89.8 from Snake R) \\ Miriam R - Deer Cr (RM 89.8 -90.8 from Snake R) \\ Deer Cr - Dry Cr (RM 90.8 -98.3 from Snake R) \\ Dry Cr - Bear Cr (RM 98.3 -101.8 from Snake R) \\ Bear Cr - Whiskey Cr (RM 101.8 -103.9 from Snake R) \\ Whiskey Cr - Lostine R (RM 103.9 -105.8 from Snake R) \\ Lostine R - Parsnip Cr (RM 105.8 -109.0 from Snake R) \\ Parsnip Cr - Hurricane Cr (RM 109.0 -121.9 from Snake R) \\ Hurricane Cr - Lower Alderslope Ditch (RM 121.9 -126.8 from Snake R) \\ Lower Alderslope Ditch - Wallowa Lake (RM 126.8 -130.8 from Snake R) \\ Mouth - Crooked Cr (RM 45.1 -51.8 from Snake R) \\ Crooked Cr -Fairview Cr(RM 51.8 -55.3 from Snake R) \\ Fairview Cr - forks (RM 55.3 -65.9 from Snake R) \\ South Fork Wenaha R (RM 65.9 -72.4 from Snake R)
}

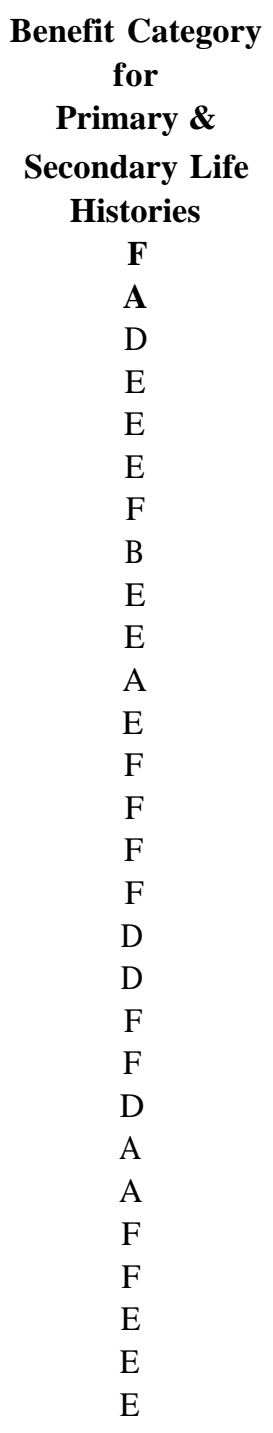


Stream Reach Summary for Primary and Secondary Spring Chinook Salmon Life Histories

Stream: Bear Cr.

Reach: $\quad$ Mouth - Chamberlain Ditch (RM $101.8-104,7$ from Snake R)

\begin{tabular}{|ll|ll|l|}
\hline Restoration benefit category: & $F$ & Chinook usage score: & 16.3 & Habitat productivity loss score: \\
\hline
\end{tabular}

\begin{tabular}{|c|c|c|c|c|c|c|c|c|c|c|c|c|c|c|}
\hline \multirow[b]{3}{*}{ Life stage } & \multirow[b]{3}{*}{ Months } & \multicolumn{10}{|c|}{ No. life history pathways by current usage abundance level } & \multicolumn{2}{|c|}{ Key habitat change } & \multirow{3}{*}{$\begin{array}{l}\text { Habital } \\
\text { productivity } \\
\text { loss score }\end{array}$} \\
\hline & & \multicolumn{5}{|c|}{ Primary } & \multicolumn{5}{|c|}{ Secondary } & \multirow{2}{*}{$\begin{array}{l}\text { Patient } \\
\text { quantity }\end{array}$} & \multirow[b]{2}{*}{$\%$ change } & \\
\hline & & Total no. & 1 & 2 & 3 & 4 & Total no. & 1 & 2 & 3 & 4 & & & \\
\hline 1. & Apr-Aug. & 2 & 2 & 0 & 0 & 0 & 14. & 14 & 0 & 0 & 0 & 1.3 & 23.9 & 1.1 \\
\hline $1,2,3$ & Aug-Oct & NA & NA & NA & NA & NA & NÄ & NA & $\mathrm{NA}$ & $\mathrm{NA}$ & $\mathrm{NA}$ & NA & $\mathrm{NA}$ & NA \\
\hline 3 & Oct-Mar & NA & NA & $\mathrm{NA}$ & $\mathrm{NA}$ & NA & $\mathrm{NA}$ & $\mathrm{NA}$ & $\mathrm{NA}$ & $\mathrm{NA}$ & $\mathrm{NA}$ & NA & $\mathrm{NA}$ & $\mathrm{NA}$ \\
\hline 3,4 & Mar-May & 0 & 0 & 0 & 0 & 0 & 8 & 8 & 0 & 0 & 0 & 2.0 & -83.8 & -4.0 \\
\hline 5 & May-Oct & 0 & 0 & 0 & 0 & 0 & 6 & 6 & 0 & 0 & 0 & 0.0 & 19.4 & -62 \\
\hline 6 & Oct-Mar & NA & NA & NA & NA & NA & NA & NA & $\mathrm{NA}$ & $\mathrm{NA}$ & $\mathrm{NA}$ & $\mathrm{NA}$ & $\mathrm{NA}$ & NA \\
\hline 7 & Mar-Jun & 2 & 2 & 0 & 0 & 0 & 0 & 0 & 0 & 0 & 0 & 13.8 & -2.4 & 0.4 \\
\hline
\end{tabular}

\begin{tabular}{|c|c|c|c|c|c|c|c|c|c|c|c|c|c|}
\hline \multirow[b]{2}{*}{ Life stage } & \multirow[b]{2}{*}{ Months } & \multicolumn{12}{|c|}{ Highest productivity impact attributes (\% change in impact from template) } \\
\hline & & Att 1 & $\%$ change & Att 2 & \% change & Att 3 & \% change & Alt 4 & $\%$ change & Att 5 & \% change & Alt 6 & $\%$ change \\
\hline 1 & Apr-Aug & FLOW & 28.7 & $\mathrm{HABD}$ & 21.5 & TEMP & 14.3 & WITH & 14.3 & & & & \\
\hline 1,3 & Aug-Öt & NA & $\mathrm{NÄ}$ & NÄ & NA & NA & NA & NA & $N A$ & $\mathrm{NA}$ & NÄ & NA & $\mathrm{NA}$ \\
\hline 3 & Ött-Mar & $\mathrm{NÄ}$ & $\mathrm{NA}$ & NÄ & NA & $\mathrm{NA}$ & NÄ & $\mathrm{NA}$ & $N A$ & $\mathrm{NA}$ & $\mathrm{NA}$ & $N A$ & $\mathrm{NA}$ \\
\hline 3,4 & Mar-May & FLOW & 62,5 & TEMP & 25.0 & WITH & 25.0 & CSTAB & 12.5 & НABD & 12.5 & PATHO & 12.5 \\
\hline 5 & May-Ott & FLOW. & 19.4 & HABD & 9.7 & PRED & 9.7 & WiTH & 9.7 & PATHO & 4.9 & & \\
\hline 6 & Ott-Mar & $\mathrm{NA}$ & NÄ & $\mathrm{NA}$ & NA & $\mathrm{NA}$ & $\mathrm{NA}$ & NA & NA & NA & $\mathrm{NA}$ & NA & $\mathrm{NA}$ \\
\hline 7 & Mar-Jun & & & & & & & & & & & & \\
\hline
\end{tabular}

Abundance /eve/ refera to the average abundance of spawners that spawn in the natal stream reaches that populate the life history pathways using the reach in this table

Level 1: $\quad 0-10$ spawners

Level 2: $\quad 10-30$ spawners

Level 30 30-60 spawners

Level $4>60$ spawners

Life stages: 1- prespawner; 2- spawner; 3- incubation; 4- fry colonization; 5- summer rearing; 6- overwintering; 7- yearling presmolt/smolt

Attributes: CHEM - chemicals

CSTAB - channel stability

COMP - competition (with other species)

FLOW - flow

HABD - habitat diversity
NUTLD - nutrient load

OBST - obstructions

OXYG - dissolved oxygen

PATHO - pathogens

PRED - predators (includes fishing-related losses)
RIPCN - riparian condition

SEDLD - sediment load

TEMP - water temperature

WITH - water withdrawals

"NA" indicates that no life history pathway meeting the criteria used in defining these pathways was present.

Blank cells under attributes indicate no additional attributes with negative effects on productivity were evident compared to those present in the template. 


\section{Stream Reach Summary for Primary and Secondary Spring Chinook SalmonLlfe Histories}

Stream: Bear Cr.

Reach: Chamberlain Ditch - Little BearCr (RM 104.7 -109.3 from Snake R)

\begin{tabular}{ll|ll|l|}
\hline Restoration benefit category: & E & Chlnook usage score: & 586,4 & Habitat productivity loss score: \\
\hline
\end{tabular}

\begin{tabular}{|c|c|c|c|c|c|c|c|c|c|c|c|c|c|c|}
\hline \multirow[b]{3}{*}{ Life stage } & \multirow[b]{3}{*}{ Montha } & \multicolumn{10}{|c|}{ No. life history pathways by current usage abundance level } & \multicolumn{2}{|c|}{ Key habitat change } & \multirow{3}{*}{$\begin{array}{c}\text { Habital } \\
\text { productivity } \\
\text { loss score }\end{array}$} \\
\hline & & \multicolumn{5}{|c|}{ Primary } & \multicolumn{5}{|c|}{ Secondary } & Patient & & \\
\hline & & Total no. & 1 & 2 & 3 & 4 & Total no. & 1 & 2 & 3 & 4 & quantity & $\%$ change & \\
\hline 1. & Apr-Aug & 7 & 7 & 0 & 0 & 0 & 49 & 49 & 0 & $\mathbf{0}$ & 0 & 5.4 & 7.8 & 0.0 \\
\hline 12,3 & Aug-Ott & 10 & 10 & 0 & 0 & 0 & 70 & 70 & 0 & 0 & 0 & 2.7 & -27.0 & $40.9 \ldots$ \\
\hline 3 & Ott-Mar & 20 & 20 & 0 & 0 & 0 & 140 & 140 & 0 & 0 & 0 & 0.7 & -63.2 & 0.0 \\
\hline 3,4 & Mar-May & 8 & 8 & 0 & 0 & 0 & 36 & 36 & 0 & 0 & 0 & 3.8 & -23.8 & -7.0 \\
\hline 5 & May-Ott & 25 & 25 & 0 & 0 & 0 & 72 & 72 & 0 & 0 & 0 & 5.1 & 8.6 & -75.6 \\
\hline 6 & Outimar & 19 & 19 & 0 & $" 0$ & 0 & 0 & 0 & 0 & 0 & 0 & 4.1 & 0.9 & -257 \\
\hline 7 & Mar-Jun & 2 & 2 & 0 & 0 & 0 & 0 & 0 & 0 & 0 & 0 & 17.9 & 5.1 & 0.3 \\
\hline
\end{tabular}

\begin{tabular}{|c|c|c|c|c|c|c|c|c|c|c|c|c|c|}
\hline \multirow[b]{2}{*}{ Life stage } & \multirow[b]{2}{*}{ Months } & \multicolumn{12}{|c|}{ Highest productivity impact attributes (\% change in impact from template) } \\
\hline & & Att 1 & $\%$ change & Att 2 & $\%$ change & Att 3 & $\%$ change & Aft 4 & T\% change & Aft 5 & $\%$ change & Att 6 & $\%$ change \\
\hline 1 & Apr-Aug & HABD & 17.8 & & & & & & & & & & \\
\hline 123 & Aug-Öct & HABD & 181 & m- & & & & & & & & & \\
\hline 131 & Optt-Mar| & CSTAB & 250 & I HALAO & 10501 & BATHÄ | & $17 p^{1}$ & RIPCN & 25.0 & & & & \\
\hline 3,4 & Mar-May & SSTAB & 25.0 & FLOW & 25.0 & HABD & & PATHO & 25.0 & RIPCN & 25.0 & & \\
\hline\|\|$\left\|||_{6}^{5}\right\|\|\|$ & in $M y_{1}-P t^{\prime}$ & FTTAB & I I I I I I I & IFLGW & 25.0. & HABD, & & PATHO & 25.0 & RIPCN & 25,0 & TEMP & 166 \\
\hline 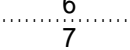 & Mar-Jun & & & PAIHO & & TE! & & & & & & & \\
\hline
\end{tabular}

Abundance /eve/ refers to the average abundance of spawners that spawn in the natal stream reaches that populate thelife history pathways using the reach in this table Level 1: $\quad 0-10$ spawners

Level 2 10-30 spawners

Level 3 30-60 spawners

Level 4: $>60$ spawnera

Life stages: 1- prespawner; 2- spawner; 3- incubation; 4- fry colonization; 5- summer rearing; 6- overwintering; 7- yearling presmolt/smolt

$\begin{array}{lll}\text { Attributes: CHEM - chemicals } & \text { NUTLD - nutrient load } & \text { RIPCN - riparian condition } \\ \text { CSTAB - channel stability } & \text { OBST - obstructions } & \text { SEDLD - sediment load } \\ \text { COMP - competition (with other species) } & \text { OXYG - dissolved oxygen } & \text { TEMP - water temperature } \\ \text { FLOW - flow } & \text { PATHO - pathogens } & \text { WITH - water withdrawals } \\ \text { HABD - habitat diversity } & \text { PRED - predators (includes fishing-related losses) }\end{array}$

"NA" indicates that no life history pathway meeting the criteria used in defining these pathwaywas present.

Blank cells under attributes indicate no additional attributes with negative effects on productivity were evident compared to those present in the template. 
Stream: Bear Cr.

Reach: Little Bear $\mathrm{Cr}$ - headwaters (RM 109.3 -118.3 from Snake R)

\begin{tabular}{lllll|l}
\hline [Restoration benefit category: & E & [Chinook usage score: & $\mathbf{5 7 5 . 2}$ & Habitat productivity loss score: \\
\hline
\end{tabular}

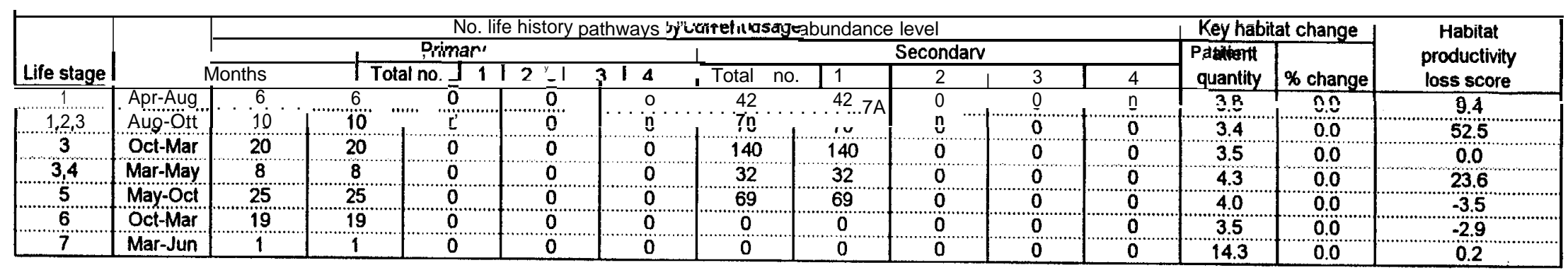

\begin{tabular}{|c|c|c|c|c|c|c|c|c|c|c|c|c|c|}
\hline \multirow[b]{2}{*}{ Life stage } & \multirow[b]{2}{*}{ Months } & \multicolumn{12}{|c|}{ Highest productivity impact attributes (\% change in impact from template) } \\
\hline & & Att 1 & $\%$ change & Att 2 & $\%$ change & Att 3 & $\%$ change & Att 4 & $\%$ change & An 5 & $\%$ changel & Aft 6 & $\%$ change \\
\hline 1 & Apr-Aug & HABD & 22.2 & & & & & & & & & & \\
\hline 123 & Aug-Ott & HABID & 8.3 & & & & & & & & & & \\
\hline 3 & Öt-Mar & PATHO & 12.5 & & & & & & & & & & \\
\hline 34 & Mar-May & PATHÖ & 25.0 & R̈IIPC̈N & 250. & 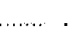 & & & & & & & \\
\hline 5 & May-Ott & PATHO & 25.0 & RIPCN & 25,0 & & & & & & & & \\
\hline 6 & Ott-Mar & PATTHO & 25.0 & 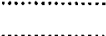 & & & & & & & & & \\
\hline 7 & Mar-Jun & & & & & & & & & & & & \\
\hline
\end{tabular}

Abundance /eve/ refers to the average abundance of spawners that spawn in the natal stream reaches that populate the life history pathwaya using the reach in this table

$$
\begin{array}{ll}
\text { Level 1: } & 0-10 \text { spawnera } \\
\text { Level 2 } & 10-30 \text { spawners } \\
\text { Level 3: } & 30-60 \text { spawnera } \\
\text { Level 4: } & \mathbf{7 6 0} \text { spawners }
\end{array}
$$

Life stages: 1- prespawner; 2- spawner; 3- incubation; 4- fry colonization; 5- summer rearing; 6- overwintering; 7-yearling presmolt/smolt

$\begin{array}{lll}\text { Attributes: } \text { CHEM - chemicals } & \text { NUTLD - nutrient load } & \text { RIPCN - riparian condition } \\ \text { CSTAB - channel stability } & \text { OBST - obstructions } & \text { SEDLD - sediment load } \\ \text { COMP - competition (with other species) } & \text { OXYG - dissolved oxygen } & \text { TEMP - water temperature } \\ \text { FLOW - flow } & \text { PATHO - pathogens } & \text { WITH - water withdrawals } \\ \text { HABD - habitat diversity } & \text { PRED - predators (includes fishing-related losses) }\end{array}$

"NA" indicates that nolife history pathway meeting the criteria used in defining these pathways was present.

Blank cells under attributes indicate no additional attributes with negative effects on productivity were evident compared to those present in the template. 
Stream Reach Summary for Primary and Secondary Spring Chinook Salmon Life Histories

Stream: Chesnimnus $\mathrm{Cr}$.

Reach: Confluence with Crow $\mathrm{Cr}$ - South Fork (RM 52.7 -77.7 from Snake R)

\begin{tabular}{ll|ll|l|}
\hline Restoration benefit category: & F & Chinook usage score: & 0.0 & Habitat productivity loss score: \\
\hline
\end{tabular}

\begin{tabular}{|c|c|c|c|c|c|c|c|c|c|c|c|c|c|c|}
\hline \multirow[b]{3}{*}{ Life stage } & \multirow[b]{3}{*}{ Months } & \multicolumn{10}{|c|}{ No. life history pathways by current usage abundance level } & \multicolumn{2}{|c|}{ Key habitat change } & \multirow{3}{*}{$\begin{array}{c}\text { Habitat } \\
\text { productivity } \\
\text { loss score }\end{array}$} \\
\hline & & \multicolumn{5}{|c|}{ Primary } & \multicolumn{5}{|c|}{ Secondary } & \multirow{2}{*}{$\begin{array}{l}\text { Patient } \\
\text { quantity }\end{array}$} & \multirow[b]{2}{*}{$\%$ change } & \\
\hline & & Total no. & 1 & 2 & 3 & 4 & Total no. & 1 & 2 & 3 & 4 & & & \\
\hline & Apr-Aug & NA & NA & $\mathrm{NA}$ & $\mathrm{NA}$ & NA & NA & $\mathrm{NA}$ & NA & NA & NA & NA & NA & NA \\
\hline 123 & Aug-ÖCl & $\mathrm{NAM}$ & $\mathrm{NA}$ & $\mathrm{NA}$ & $\mathrm{NA}$ & $\mathrm{NA}$ & $\mathrm{NA}$ & NA & NA & NA & NA & $\mathrm{NA}$ & $\mathrm{NA}$ & $\mathrm{NA}$ \\
\hline 3 & Ott-Mar & NA & NA & NA & NA & NÄ & NA & NA & NA & $\$$ & " & NA & NA & NA \\
\hline 34 & Mar-May & NA & $N A$ & $\mathrm{NA}$ & NA & $N A$ & NA & $N A$ & $\mathrm{NA}$ & & & $\cdots$ NA & NA & NA \\
\hline 5 & May-Ott & NA & NA" & NA. & $\mathrm{NA}$ & $\mathrm{NA}$ & $\mathrm{NA}$ & $\mathrm{NA}$ & $N A$ & NA & NÄ & $\mathrm{NA}$ & NA" & NÄ \\
\hline 6 & Ott-Mar & NA & $\mathrm{NA}$ & $N A$ & NA" & $\mathrm{NA}$ & NA & $\mathrm{NA}$ & NA" & NA & NA & $\mathrm{NA}$ & $\mathrm{NA}$ & $\mathrm{NA}$ \\
\hline 7 & Mar-Jun & $\mathrm{NA}$ & NA" & "̈A & NA" & NA & NA & $\mathrm{NA}$ & NA & $\mathrm{NA}$ & NA & $\mathrm{NA}$ & $\mathrm{NA}$ & $\mathrm{NA}$ \\
\hline
\end{tabular}

\begin{tabular}{|c|c|c|c|c|c|c|c|c|c|c|c|c|c|}
\hline \multirow[b]{2}{*}{ Life stage } & \multirow[b]{2}{*}{ Months } & \multicolumn{12}{|c|}{ Highest productivity impact altributes (\% change in impact from template) } \\
\hline & & Att 1 & \% change & Alt 2 & $\%$ change & Att 3 & \% change & Att 4 & $\%$ change & Att 5 & \% change & Att 6 & $\%$ change \\
\hline 1 & Apr-Aug & NA & NA & NA & NA & NA & NA & NA & NA & NA & NA & NA & NA \\
\hline $1,2,3$ & Aug-Oct & NA & $\mathrm{NA}$ & NA & $\mathrm{NA}$ & $\mathrm{NA}$ & $\mathrm{NA}$ & $N A$ & $\mathrm{NA}$ & $\mathrm{NA}$ & $\mathrm{NA}$ & $\mathrm{NA}$ & NA \\
\hline 3 & Oct-Mar & $\mathrm{NA}$ & $\mathrm{NA}$ & NA & $\mathrm{NA}$ & $\mathrm{NA}$ & $\mathrm{NA}$ & NA & $\mathrm{NA}$ & $\mathrm{NA}$ & $\mathrm{NA}$ & $\mathrm{NA}$ & NA \\
\hline 3,4 & Mar-May & $\mathrm{NA}$ & NA & NA & $N A$ & $\mathrm{NA}$ & $\mathrm{NA}$ & NA & $\mathrm{NA}$ & $\mathrm{NA}$ & $\mathrm{NA}$ & NA & NA \\
\hline 5 & May-Oct & $\mathrm{NA}$ & NA & $\mathrm{NA}$ & $\mathrm{NA}$ & $\mathrm{NA}$ & $\mathrm{NA}$ & NA & $\mathrm{NA}$ & $\mathrm{NA}$ & $\mathrm{NA}$ & $\mathrm{NA}$ & NA \\
\hline 6 & Oct-Mar & $\mathrm{NA}$ & $\mathrm{NA}$ & $\mathrm{NA}$ & $\mathrm{NA}$ & $\mathrm{NA}$ & $\mathrm{NA}$ & NA & $\mathrm{NA}$ & $\mathrm{NA}$ & $\mathrm{NA}$ & $\mathrm{NA}$ & NA \\
\hline 7 & Mar-Jun & $\mathrm{NA}$ & NA & NA & $\mathrm{NA}$ & $\mathrm{NA}$ & $\mathrm{NA}$ & NA & $\mathrm{NA}$ & $\mathrm{NA}$ & NA & $\mathrm{NA}$ & $\mathrm{NA}$ \\
\hline
\end{tabular}

Abundance/eve/ refers to the average abundance of spawners that spawn in the natal stream reaches that populate the life history pathways using the reach in this table Level 1: $\quad 0-10$ spawners

Level 2: $\quad$ 10-30 apawners

Level3: $\quad 30-60$ spawners

Level 4: $\quad>60$ spawnera

Life stages: 1- prespawner; 2- spawner; 3- incubation; 4- fry colonization; 5- summer rearing; 6- overwintering; 7- yearling presmolt/smolt

Attributes: CHEM - chemicals

CSTAB - channel stability

COMP - competition (with other species)

COW - flow

NUTLD - nutrient load

OBST - obstructions

OXYG - dissolved oxygen

PATHO - pathogens

PRED - predators (includes fishing-related losses)

RIPCN - riparian condition

SEDLD - sediment load

TEMP - water temperature

WITH - water withdrawals

"NA" indicates that no life history pathway meeting the criteria used in defining these pathways was present.

Blank cells under attributea indicate no additional attributes with negative effects on productivity were evident compared to those present in the template. 
Stream: Catherine Cr.

Reach: $\quad$ Mouth - 10th Street Bridge in Union (RM 114.5 -146.0 from Snake R)

\begin{tabular}{|ll|ll|l|}
\hline Restoration benefit category: & A & Chinook usage score: & $\mathbf{4 9 5 . 2}$ & Habitat productivity loss score: \\
\hline
\end{tabular}

\begin{tabular}{|c|c|c|c|c|c|c|c|c|c|c|c|c|c|c|}
\hline \multirow[b]{3}{*}{ Life stange } & \multirow[b]{3}{*}{ Months } & \multicolumn{10}{|c|}{ No. life history pathways by current usage abundancelevel } & \multicolumn{2}{|c|}{ Key habitat change } & \multirow{3}{*}{$\begin{array}{c}\text { Habitat } \\
\text { productivity } \\
\text { loss score }\end{array}$} \\
\hline & & \multicolumn{5}{|c|}{ Primary } & \multicolumn{5}{|c|}{ Secondary } & \multirow{2}{*}{$\begin{array}{l}\text { Patient } \\
\text { quantity }\end{array}$} & \multirow[b]{2}{*}{$\%$ change } & \\
\hline & & Total no. & 1 & 2 & 3 & 4 & Total no. & 1 & 2 & 3 & 4 & & & \\
\hline 1 & Apr-Aug & 14 & 0 & 4. & O & 10 & 10 & 0 & 4 & 0 & 6 & 11.6 & -70.3 & -37.5 \\
\hline 1.3 & Aug-Out & $\mathrm{NA}$ & $\mathrm{NA}$ & $\mathrm{NÄ}$ & "NA" & Nä & NA" & NA & NÄ & NA'A" & NA & NA & NA & NA \\
\hline 3,4 & Mar-May & 8 & 0 & 0 & 0 & 8 & 8 & 0. & 0. & 0 & 8 & 8.0. & -78.5 & -122.6 \\
\hline 5 & May out & 56 & 0 & 5 & 0 & 51 & 52 & 0 & 2 & 0 & 50 & 10 & -93.7 & -513.6 \\
\hline 6 & Oothiar & 95 & 0 & 57 & 0 & 38 & 19 & 0 & 0 & 0 & 19 & 4.3 & -61.5 & -323.3 \\
\hline 7 & Mar-Jun & 6 & 0 & 3 & 0 & 3 & 4 & 0 & 2 & 0 & 2 & 26.2 & -2.6 & 11.7 \\
\hline
\end{tabular}

\begin{tabular}{|c|c|c|c|c|c|c|c|c|c|c|c|c|c|}
\hline \multirow[b]{2}{*}{ Life stage } & \multirow[b]{2}{*}{ Months } & \multicolumn{12}{|c|}{ Highest productivity impact attributes (\% change in impact from template) } \\
\hline & & Aft 1 & $\%$ change & Att 2 & \%change & Att 3 & $\%$ change & Aft 4 & $\%$ change & Att 5 & $\%$ change & Atf 6 & $\%$ change \\
\hline 1 & Apr-Aug. & FLOW. & 45.0 & $\mathrm{HABD}$ & 450 & RiPCN & 45.0 & OBST & 22.5 & TEMP & 22.5 & & \\
\hline 1,23 & Ág-Oct & $\dot{\mathrm{NA}}$ & NÄ & "NÄ & NÄ & $\mathrm{NA}$ & $\mathrm{NÄ}$ & $\mathrm{NA}$ & $\ddot{N A}$ & NA & NA & NA & NA \\
\hline 3 & Oct-Mar & NA & NA" & NÄ & $\mathrm{NA}$ & $\mathrm{NA}$ & $\because \mathrm{NA}$ & $\mathrm{NA}$ & $\mathrm{NA}$ & $\mathrm{NA}$ & $\mathrm{NA}$ & $\mathrm{NA}$ & $\mathrm{NA}$ \\
\hline 3,4 & Mar-May & FLOẄ & 750 & HABD & 75.0 & RIPCN & 500 & NUTTED. & 25.0 & РАTHO & 25.0 & TEMP & $25.0^{\circ}$ \\
\hline 5 & May-Ott & FLOW & 75.0 & HABD & 75.0 & SEDLD & 64.4 & RIPCN & 50.0 & NÚTLD & 25.0 & РАTHO & 25.0 \\
\hline 6 & Ott-Mar & HABO & 64.0 & SEDLD & 64.0 & "FLÖW" & 42.7 & RIPCN & 42.7 & соміP & 21.3 & OBST & 21.3 \\
\hline 7 & Mar-Jun & "OBST" & 20.1 & "พ̈ітін" & $20.1^{\circ}$ & & & & & & & & \\
\hline
\end{tabular}

Abundance /eve/ refers to the average abundance of spawnera that apawn in the natal stream reaches that populate the life history pathways using the reach in this table

$$
\begin{array}{ll}
\text { Level 1: } & 0-10 \text { spawners } \\
\text { Level 2 } & 10-30 \text { spawners } \\
\text { Levei 3: } & 30-80 \text { spawners } \\
\text { Level 4: } & \mathbf{2} \mathbf{6 0} \text { spawners }
\end{array}
$$

\begin{tabular}{|c|c|c|c|}
\hline Attributes: & $\begin{array}{l}\text { CHEM - chemicals } \\
\text { CSTAB - channel stability } \\
\text { COMP - competition (with other species) } \\
\text { FLOW - fiOW } \\
\text { HABD - habitat diversity }\end{array}$ & $\begin{array}{ll}\text { NUTLD - } & \text { nutrient load } \\
\text { OBST - } & \text { obstructions } \\
\text { OXYG - } & \text { dissolved oxygen } \\
\text { PATHO - } & \text { pathogens } \\
\text { PRED - } & \text { predators (include }\end{array}$ & $\begin{array}{l}\text { RIPCN - } \\
\text { SEDLD - } \\
\text { TEMP - } \\
\text { WiTH - }\end{array}$ \\
\hline
\end{tabular}

Life stages: 1- prespawner; 2- spawner; 3- incubation; 4- fry colonization; 5- summer rearing; 6- overwintering; 7- yearling presmolt/smolt

"NA" Indicates that no life history pathway meeting the criteria used in defining these pathways was present.

Blank cells under attributea indicate no additional attributes with negative effects on productivity were evident compared to those present in the template, 


\section{Stream Reach Summary for Primary and Secondary Spring Chinook Salmon Life Histories}

Stream: Catherine $\mathrm{Cr}$

Reach: 10th Street Bridge in Union - Little Catherine Cr (RM 146.0 -157.5 from Snake R)

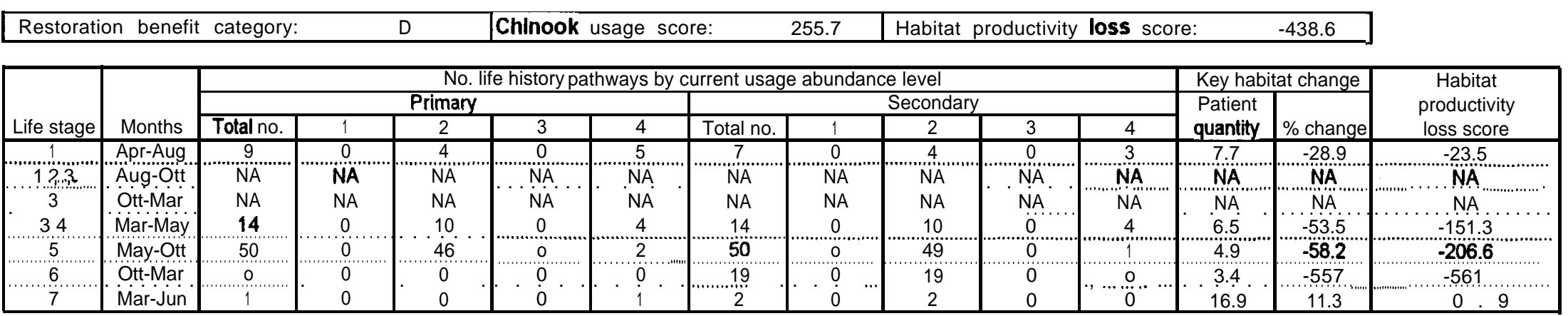

\begin{tabular}{|c|c|c|c|c|c|c|c|c|c|c|c|c|c|}
\hline \multirow[b]{2}{*}{ Life stage } & \multirow[b]{2}{*}{ Months } & \multicolumn{12}{|c|}{ Highest productivity impact attributes (\% change in impact from template) } \\
\hline & & Att 1 & $\%$ change & Aft 2 & $\%$ change & Att 3 & $\%$ change & Att 4 & \% change & Aft 5 & $\%$ change & Att 6 & $\%$ change \\
\hline 1 & Apr-Aug & TEMP & 36.1 & FLOW & 24.1 & HABD & 24.1. & OBST & 24.1 & RIPCN & 24.1 & & \\
\hline 123 & Aug-Ott & ïA & NA & NA & ïA & $\ddot{\mathrm{NA}}$ & $\dot{\mathrm{NA}}$ & $\mathrm{NA}$ & NA & NA & NA & NA & $\mathrm{NA}$ \\
\hline 3 & Ott-Mar & NA & NA & $\mathrm{NA}$ & NA & ii & NA & NA & NA & NA & NA & NA & NÄ \\
\hline 3,4 & Mar-May & TEMP & 75.0 & FLOW & 50.0 & HABD & 50.0 & RIPCN & 50.0 & WITH & 50.0 & CSTAB & 37.5 \\
\hline 5 & May-Ott & TEMP & 73.5 & FLOW & $49.0^{\circ}$ & HABD & 49,0 & RIPCN & 49.0 & WITH & 49.0 & PATHO & 245 \\
\hline 6 & Ott-Mar & HABD & 500 & RIPCN & 500 & SEDLD & 500 & CSTAB & 250 & PATHO & 250 & WiT̈H & 250 \\
\hline 7 & Mar-Jun & WITH & 16.0 & & & & & & & & & & \\
\hline
\end{tabular}

Abundance lovel refers to the average abundance of spawners that spawn in the natal stream reaches that populate the fife history pathways using the reach In thitable:

Level 1: $\quad 0$-10 spawners

Levei 2: $\quad 10-30$ spawners

Level 3: $\quad 30-80$ spawners

Levei $4>60$ spawners

Life stages: 1 - prespawner; 2- spawner; 3- incubation; 4- fry colonization; 5- summer rearing; 6- overwintering; 7- yearling presmolt/smoll

\begin{tabular}{|c|c|c|c|}
\hline Attributes: & $\begin{array}{l}\text { CHEM - chemicals } \\
\text { CSTAB - channel stability } \\
\text { COMP - competition (with other species) } \\
\text { FLOW - flow } \\
\text { HABD - habitat diversity }\end{array}$ & $\begin{array}{l}\text { NUTLD - nutrient ioad } \\
\text { OBST - obstructions } \\
\text { OXYG - dissolved oxygen } \\
\text { PATHO - pathogens } \\
\text { PRED - predators (includes fishing-related losses) }\end{array}$ & $\begin{array}{l}\text { RIPCN - } \quad \text { riparian condition } \\
\text { SEDLD - sediment load } \\
\text { TEMP - } \quad \text { water temperature } \\
\text { WITH - } \quad \text { water withdrawals }\end{array}$ \\
\hline
\end{tabular}

"NA" indicates that no fife history pathway meeting the criteria used in defining these pathways was present,

Blank cells under attributes indicate no additional attributes with negative effects on productivity were evident compared to those present in the template, 
Stream Reach Summary for Primary and Secondary Spring Chinook Salmon Life Histories

Stream: Catherine $\mathrm{Cr}$.

Reach: Little Catherine Cr - forks (RM 157.5 -164.4 from Snake R)

\begin{tabular}{|c|c|c|c|c|c|c|c|c|c|c|c|c|c|c|}
\hline \multicolumn{4}{|c|}{ [Restoration benefit category: } & $\mathrm{B}$ & \multicolumn{3}{|c|}{ Chinook usage score: } & 932.6 & \multicolumn{4}{|c|}{ Habitat productivity loss score: } & \multicolumn{2}{|l|}{-477.4} \\
\hline \multirow[b]{3}{*}{ Life stage } & \multirow[b]{3}{*}{ Months } & \multicolumn{10}{|c|}{ No. life history pathways by current usage abundance level } & \multicolumn{2}{|c|}{ Key habitat change } & Habitat \\
\hline & & \multicolumn{5}{|c|}{ Priman } & \multicolumn{5}{|c|}{ Secondarv } & \multirow{2}{*}{$\begin{array}{l}\text { Patient } \\
\text { quantity }\end{array}$} & \multirow[b]{2}{*}{ \% change } & productivity \\
\hline & & Total no. & 1 & 2 & 3 & 4 & Total no. & 1 & 2 & 3 & 4 & & & loss score \\
\hline $1 \ldots \ldots$ & Apr-Aug & 34 & $\overline{0}$ & 4 & 0 & 30 & 22 & 0 & 4. & 0 & 18 & 4.9 & -54.3 & -89.8 \\
\hline $1,2,3$ & Aug-Oct & 50 & 0 & 0 & 0 & $50^{\circ}$ & 30 & $\mathrm{n}$ & 0 & $\underline{n}$ & 30 & 63 & $=180$ & -141.5 \\
\hline 3 & Oct-Mar & 100 & 0 & 0 & 0 & 100 & 60 & 0 & 0 & 0 & 60 & 8.3 & -3.6 & -144.9 \\
\hline 34 & Mar-May & 30 & 0 & 2 & 0 & 28 & 14 & 0 & 2 & 0 & 12 & 76 & -382 & -47.2 \\
\hline 5 & May-Oct & 73 & 0 & 2 & 0 & 71 & 24 & 0 & 1 & 0 & 23 & 45 & 59.1 & -46.0 \\
\hline 6 & Oct-Mar & 19 & 0 & 0 & 0 & 19 & 0 & 0 & 0 & 0 & 0 & 3.3 & -615 & -28.5 \\
\hline 7 & Mar-Jun & 1 & 0 & 0 & 0 & 1 & 1 & 0 & 1 & 0 & 0 & 16.5 & 0.0 & 0.6 \\
\hline
\end{tabular}

\begin{tabular}{|c|c|c|c|c|c|c|c|c|c|c|c|c|c|}
\hline \multirow[b]{2}{*}{ Life stage } & \multirow[b]{2}{*}{ Months } & \multicolumn{12}{|c|}{ Highest productivity impact attributes ( $\%$ change in impact from templale) } \\
\hline & & Alt 1 & \% change & Att 2 & $\%$ change & Att 3 & $\%$ change & Att 4 & $\%$ change & Att 5 & $\%$ change & Att 6 & \% change \\
\hline 1 & Apr-Aug & SEDLD & 25.0 & TEMP & 25.0 & HABD & 20.8 & CSTAB & 12.5 & & & & \\
\hline $1,2,3$ & Aug-Oct & SEDLD & 50.0 & CSTAB & 25.0 & HABD & 25.0 & TEMP & 25.0 & & & & \\
\hline 3 & Oct-Mar & SEDLD & 500 & CSTAB & 250 & FLOWW & 25.0 & PATHO & 25.0 & TEMP & 25.0 & & \\
\hline 3,4 & Mar-May & SEDLD & 50.0 & TEMP & 50.0 & CSTAB & 25.0 & Fiow & 25.0 & PATHO & 25.0 & RIPCN & 25.0 \\
\hline 5 & May-Oct & TEMP & 50.0 & HABD & 36.0 & SEDLD & 36.0 & FLOW & 25.0 & PATHO & 25.0 & RIPCN & 25.0 \\
\hline 6 & Oct-Mar & HABD & 50.0 & SEDLD & 500 & CSTAB & 250 & PATHOO & 25.0 & TEMP & 25.0 & & \\
\hline 7 & Mar-Jun & & & & & & & & & & & & \\
\hline
\end{tabular}

Abundance /eve/ refers to the average abundance of spawners that spawn in the natal stream reaches that populate the life history pathways using the reach this table:

$$
\begin{array}{ll}
\text { Level 1: } & 0-10 \text { spawners } \\
\text { Level 2: } & 10-30 \text { spawners } \\
\text { Level 3: } & 30-60 \text { spawnera } \\
\text { Level 4: } & \mathbf{2} \mathbf{6 0} \text { spawners }
\end{array}
$$

\begin{tabular}{|c|c|c|c|}
\hline Attributes: & $\begin{array}{l}\text { CHEM - chemicals } \\
\text { CSTAB - channel stability } \\
\text { COMP - competition (with other species) } \\
\text { FLOW - flow } \\
\text { HABD - habitat diversity }\end{array}$ & 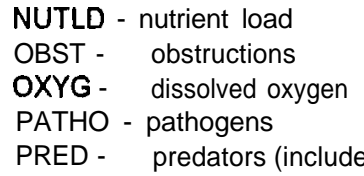 & $\begin{array}{l}\text { RIPCN - riparian condition } \\
\text { SEDLD - } \\
\text { TEMPiment load } \\
\text { TEMP - water temperature } \\
\text { WITH - water withdrawals }\end{array}$ \\
\hline
\end{tabular}

Life stages: 1- prespawner; 2- spawner; 3- incubation; 4- fry colonization; 5- summer rearing; 6- overwintering; 7- yearling presmolt/smelt

"NA" indicates that no life history pathway meeting the criteria used in defining these pathwaya was present.

Blank cells under attributea indicate no additional attributes with negative effects on productivity were evident compared to those present in the template. 
Stream Reach Summary for Primary and Secondary Spring Chinook Salmon Life Histories

Stream: Catherine $\mathrm{Cr}$

Reach: South Fork Catherine Cr (RM 164.4 -166.8 from Snake R)

\begin{tabular}{|ll|ll|l|}
\hline Restoration benefit category: & B & Chinook usage score: & $\mathbf{7 4 0 . 4}$ & Habitat productivity loss score: \\
\hline
\end{tabular}

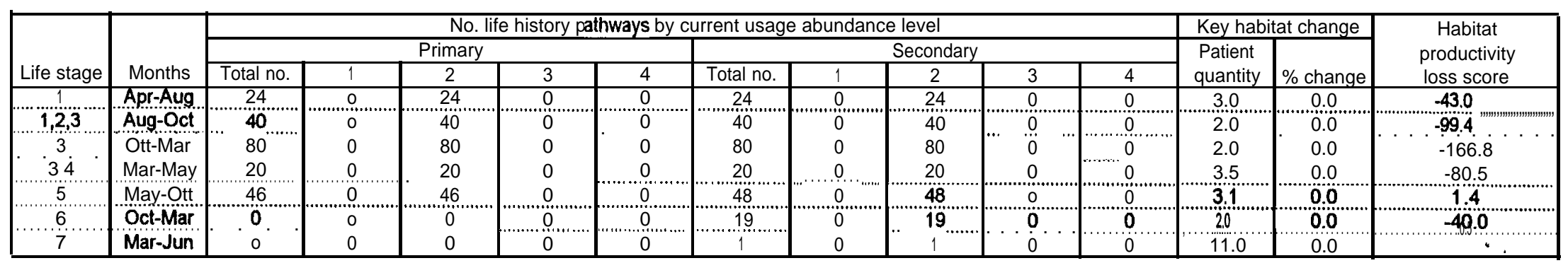

\begin{tabular}{|c|c|c|c|c|c|c|c|c|c|c|c|c|c|}
\hline \multirow[b]{2}{*}{ Life stage } & \multirow[b]{2}{*}{ Months } & \multicolumn{12}{|c|}{ Highest productivity impact attributes (\% change in impact from template) } \\
\hline & & Atf 1 & \% change & Att 2 & $\%$ change & Att 3 & $\%$ change & Att 4 & $\%$ change & Att 5 & $\%$ change & Aft 6 & \% change \\
\hline 1 & Apr-Aug & FLOW & 25.0 & SEDLD & 167 & TEMP & 8.3 & & & & & & \\
\hline 12,3 & Aug-Öct & SEDLD & 50.0 & FLOW & 25.0 & TEMMP" & 25.0 & & & & & & \\
\hline 3 & Ott-Mar & SEDLD & 50.0 & "FLOWW & 25.0 & "TËM" & 25.0 & CSTÄB & 12.5 & PÄTHÖ & 12.5 & & \\
\hline 3,4 & Mar-May & FLOW & ,25.0 & 'PAT,HO' & 25.0 & SEDED & 25.0 & CSTAB & 12.5 & "TEMPP & 12.5 & & \\
\hline 5 & May-Ott & FLOW & 25.0 & РАТНО & 25.0 & & & & & & & & \\
\hline 6 & Ott-Mar & SEDLD & 50.0 & ĊTȦÄB & 250 & FLOW & 25.0 & "HÄBD" & 25.0 & "PÄTHOO & $25.0^{\circ} " \cdots$ & & \\
\hline 7 & Mar-Jun & & & & & & & & & & & & \\
\hline
\end{tabular}

Abundance level refers to the average abundance of spawners that spawn in the natal stream reaches that populate the life history pathways using the reach In this table

$\begin{array}{ll}\text { Level 1: } & 0-10 \text { spawners } \\ \text { Level 2: } & 10-30 \text { spawners } \\ \text { Levet 3: } & 30-60 \text { spawners } \\ \text { Level 4 } & \mathbf{> 0 0} \text { spawners }\end{array}$

Life stages: 1 - prespawner; 2- spawner; 3- incubation; 4- fry colonization; 5- summer rearing; 6- overwintering; 7- yearling preamolt/smelt

$\begin{array}{lll}\text { Attributes: } \text { CHEM - chemicals } & \text { NUTLD - nutrient load } & \text { RIPCN - riparian condition } \\ \text { CSTAB - channel stability } & \text { OBST - obstructions } & \text { SEDLD - Sediment load } \\ \text { COMP - competition (with other species) } & \text { OXYG - dissolved oxygen } & \text { TEMP - water temperature } \\ \text { FLOW - flow } & \text { PATHO - pathogens } & \text { WITH - water withdrawals } \\ \text { HABD - habitat diversity } & \text { PRED - predators (includes fishing-related losses) }\end{array}$

"NA" indicates that no life history pathway meeting the criteria used in defining these pathways was present.

Blank cells under attributesindicate no additional attributes with negative effects on productivity were evident compared to those present in the template. 
Stream Reach Summary for Primary and Secondary Spring Chinook Salmon Life Histories

Stream: Grande Ronde R.

Reach: Mouth - Joseph $\mathrm{Cr}$ (RM 0,0-3.7 from Snake R)

\begin{tabular}{|ll|ll|l|}
\hline Restoration benefit category: & F & Chinook usage score: & 348.7 & Habitat productivity loss score: \\
\hline
\end{tabular}

\begin{tabular}{|c|c|c|c|c|c|c|c|c|c|c|c|c|c|c|}
\hline \multirow[b]{3}{*}{ Life stage } & \multirow[b]{3}{*}{ Months } & \multicolumn{10}{|c|}{ No. life history pathways by current usage abundance level } & \multicolumn{2}{|c|}{ Key habitat change } & \multirow{3}{*}{$\begin{array}{c}\text { Habitat } \\
\text { productivity } \\
\text { loss score }\end{array}$} \\
\hline & & \multicolumn{5}{|c|}{ Primary } & \multicolumn{5}{|c|}{ Secondary } & \multirow{2}{*}{$\begin{array}{l}\text { Patient } \\
\text { quantity }\end{array}$} & \multirow[b]{2}{*}{$\%$ change } & \\
\hline & & Total no. & 1 & 2 & 3 & 4 & Total no. & 1 & 2 & 3 & 4 & & & \\
\hline 1 & Apr-Aug & 96 & 32. & 36 & 16 & 12. & 222 & 116 & 68 & 24 & 12 & 33.8 & 0.0 & 0. \\
\hline 123 & Aug-Ott & $\mathrm{NA}$ & $\mathrm{NA}$ & NA & NA & NA & NA & NA & $\mathrm{NA}$ & $\mathrm{NA}$ & $\mathrm{NA}$ & $\mathrm{NA}$ & NA" & $\dddot{N a ̈}$ \\
\hline 3 & Ott-Mar & NA & NA & NA & $\mathrm{NA}$ & $\mathrm{NA}$ & $\mathrm{NA}$ & NA & NÄ & $\mathrm{NA}$ & NA & NA & NA & NA \\
\hline 34. & Mar-May & NA & NA & NA & NA & NA & N A " & $\mathrm{N} A$ & NA & $\mathrm{NA}$ & $\mathrm{NA}$ & NA & NA & NA \\
\hline 5 & May-Ott & ㅇ… & 0 & 0 & 0 & 0 & 3 & 0 & 2 & 1 & $\dot{0}$ & 206 & 0.0 & -54 \\
\hline 6 & Ott-Mar & NA & NA & $N A$ & NA & NA & NA & NA & NA & NA & $\mathrm{NA}$ & NA & $\mathrm{NA}$ & $\mathrm{NA}$ \\
\hline 7 & Mar-Jun & 65 & 20 & s"'”'”'”' & 12 & 8 & 203 & 110 & 60 & 22 & 11 & 109.8 & 0.0 & 31.6 \\
\hline
\end{tabular}

\begin{tabular}{|c|c|c|c|c|c|c|c|c|c|c|c|c|c|}
\hline \multirow[b]{2}{*}{ Life stage } & \multirow[b]{2}{*}{ Months } & \multicolumn{12}{|c|}{ Highest productivity impact attribute (\% change in impact from template) } \\
\hline & & Alt 1 & $\%$ change & Att 2 & \% change & Att 3 & $\%$ change & Att 4 & T\% change & Att 5 & \% change & Att 6 & $\%$ change \\
\hline 1 & Apr-Aug & CHEM & 12.5 & NUTLD & 12.5 & OXYG & 12.5 & & & & & & \\
\hline $1,2,3$ & Aug-Oct & $\mathrm{NA}$ & $\mathrm{NA}$ & NA & $N A$ & NA & $\mathrm{NA}$ & NA & NA & $\mathrm{NA}$ & $\mathrm{NA}$ & NÄ & NA \\
\hline 3 & Oct-Mar & NA & NÄ & $\mathrm{NA}$ & NA & NA & $\mathrm{NA}$ & NA & NA & NA & $\mathrm{NA}$ & $\mathrm{NA}$ & $\mathrm{NA}$ \\
\hline 3,4 & Mar-May & $\mathrm{NA}$ & $\mathrm{NA}$ & NA & $\mathrm{NA}$ & NA & $\mathrm{NA}$ & $N A$ & NA & $\mathrm{NA}$ & $\mathrm{NA}$ & $\mathrm{NA}$ & $\mathrm{NA}$ \\
\hline 5 & May-Oct & COMP & 50.0 & HABD & 25.0 & NUTLD & 25.0 & PATHO & 25.0 & & & & \\
\hline 6 & Oct-Mar & NÄ & $\mathrm{NA}$ & $\mathrm{NA}$ & $\mathrm{NA}$ & NA & $\mathrm{NA}$ & NA & $\mathrm{NA}$ & NA & $\mathrm{NA}$ & $\mathrm{NA}$ & NÄ \\
\hline 7 & Mar-jun & РАТНО & 6.5 & TEMP & 6.5 & & & & & & & & \\
\hline
\end{tabular}

Abundance/eve/refers to the average abundance of spawners that spawn in the natal stream reaches that populate the life history pathways using the reach in this table:

$$
\begin{array}{ll}
\text { Level 1: } & 0-10 \text { spawners } \\
\text { Level 2: } & 10-30 \text { spawners } \\
\text { Level 3: } & 30-60 \text { spawners } \\
\text { Level 4: } & \mathbf{>} \mathbf{6 0} \text { spawners }
\end{array}
$$

Life stages: 1 -prespawner; 2- spawner; 3- incubation; 4- fry colonization; 5- summer rearing; 6- overwintering; 7- yearling presmolt/smolt

$\begin{array}{lll}\text { Attributes: } \text { CHEM - chemicals } & \text { NUTLD - nutrient load } & \text { RIPCN - riparian condition } \\ \text { CSTAB - channel stability } & \text { OBST - obstructions } & \text { SEDLD - Sediment load } \\ \text { COMP - competition (with other species) } & \text { OXYG - dissolved oxygen } & \text { TEMP - water temperature } \\ \text { FLOW - flow } & \text { PATHO - pathogens } & \text { WITH - } \\ \text { HABD - habitat diversity } & \text { PRED - predators (includes fishing-related losses) }\end{array}$

"NA" indicates that no life history pathway meeting the criteria used in defining these pathwaywas present.

Blank cells under attributes indicate no additional attributes with negative effects on productivity were evident compared to those present in the template. 
Stream Reach Summary for Primary and Secondary Spring Chinook Salmon Life Histories

Stream: Grande Ronde R.

Reach: Joseph $\mathrm{Cr}$-Buford Cr (RM 3.7- 26.2 from Snake R)

\begin{tabular}{|ll|ll|l|}
\hline Restoration benefit category: & E & Chlnook usage score: & $\mathbf{4 2 2 . 2}$ & Habitat productivity loss score: \\
\hline
\end{tabular}

\begin{tabular}{|c|c|c|c|c|c|c|c|c|c|c|c|c|c|c|}
\hline \multirow[b]{3}{*}{ Life stage } & \multirow[b]{3}{*}{ Months } & \multicolumn{10}{|c|}{ No. life history pathways by current usage abundance level } & \multicolumn{2}{|c|}{ Key habitat change } & \multirow{3}{*}{$\begin{array}{c}\text { Habitat } \\
\text { productivity } \\
\text { loss score }\end{array}$} \\
\hline & & \multicolumn{5}{|c|}{ Primary } & \multicolumn{5}{|c|}{ Secondary } & \multirow{2}{*}{$\begin{array}{l}\text { Patient } \\
\text { quantity }\end{array}$} & \multirow[b]{2}{*}{ \% change } & \\
\hline & & Total no. & 1 & 2 & 3 & 4 & Total no. & 1 & 2 & 3 & 4 & & & \\
\hline & Apr-Aug & 74 & 26 & 25 & 17 & 6 & 168 & 90 & 46 & 26 & 6 & 33.8 & 9.8 & 0.0 \\
\hline $1,2,3$ & Aug-Ott & "Nä" & $\mathrm{NÄ}$ & $\mathrm{NA}$ & $\mathrm{NA}$ & $\mathrm{NA}$ & $\mathrm{NA}$ & $\mathrm{NA}$ & $\mathrm{NA}$ & $\mathrm{NÄ}$ & $\mathrm{NA}$ & $\mathrm{NA}$ & NA & $\mathrm{NA}$ \\
\hline 3 & Ott-Mar & NA & NA & NA & $\mathrm{NA}$ & $\mathrm{NA}$ & NA & NA & NA & NA & NA & $N A$ & "̈̈A & NA \\
\hline 34 & Mar-May & NA & NA & NA & NA & NA & NA & NA & NA & NA & NA & NA & NA & NA \\
\hline 5 & May-Ott & 0 & 0 & 0 & 0 & 0 & 3 & 0 & 2 & 1 & 0 & 20.6 & 0.0 & -3.6 \\
\hline 6 & Otț-Marar & 36 & 0 . & 18 & 18 & 0 & 185 & 93 & 37 & 55 & 0 & 23.5 & 0.2 & -44.7 \\
\hline 7 & Mar-Jun & 59 & 16 & 24 & 13 & 6 & 176 & 104 & 40 & 26 & 6 & 119.3 & 2.6 & 2.7 \\
\hline
\end{tabular}

\begin{tabular}{|c|c|c|c|c|c|c|c|c|c|c|c|c|c|}
\hline \multirow[b]{2}{*}{ Life stage } & \multirow[b]{2}{*}{ Months } & \multicolumn{12}{|c|}{ Highest productivity impact attributes (\% change in impact from template) } \\
\hline & & Att 1 & \% change & Att 2 & \% change & Att 3 & $\%$ change & Alt 4 & $\%$ change & Att 5 & \% change & Att 6 & $\%$ change \\
\hline 1 & Apr-Aug & CHEM & 19.6 & NUTLD & 19.6 & OXYG & 19.6 & & & & & & \\
\hline $1,2,3$ & Aug-Oct & $\mathrm{NA}$ & NÄ & $\mathrm{NA}$ & NA & $\mathrm{NA}$ & NA & NÄ & NÄ & NA & $\mathrm{NA}$ & NÄ & $\mathrm{NA}$ \\
\hline 3 & Oct-Mar & $N A$ & NÄ & NA & $\mathrm{NA}$ & $\mathrm{NA}$ & NA & NÄ & NA & $\mathrm{NA}$ & $\mathrm{NA}$ & $\mathrm{NA}$ & NA \\
\hline 3,4 & Mar-May & $\mathrm{NA}$ & NA & NA & $N A$ & NA & $\mathrm{NA}$ & $\mathrm{NA}$ & $\mathrm{NA}$ & $\mathrm{NA}$ & NA & $\mathrm{NA}$ & $\mathrm{NA}$ \\
\hline 5 & May-Oct & COMP & 17.3 & CHEM & 8.7 & $\mathrm{HABD}$ & 8.7 & NUTLD & 8.7 & PATHO & 8.7 & & \\
\hline 6 & Oct-Mar & SEDLD & 9.9 & PATHO & 5.3 & TËMP & 5.3 & & & & & & \\
\hline 7 & Mar-Jun & PATHO & 5.6 & TEMP & 5.6 & & & & & & & & \\
\hline
\end{tabular}

Abundance level refera to the average abundance of spawners that spawn in the natal stream reaches that populate the life history pathways using the reach in this table:

Level 1: $\quad 0-10$ spawners

Level 2 10-30 spawners

Level 3: $\quad 30-60$ spawners

Level $4>60$ spawners

Life stages: 1- prespawner; 2-spawner; 3- incubation; 4- fry colonization; 5- summer rearing; 6- overwintering; 7- yearling presmolt/smelt

Attributes: CHEM - chemicals

CSTAB - channel stability

NUTLD - nutrient load

COMP - competition (with other species)

FLOW

OBST - obstructions

OXYG - dissolved oxygen

PATHO - pathogens

RIPCN - riparian condition

HABD - habitat diversity

PRED - predatora (includes fishing-related losses)

SEDLD - sediment load

TEMP - $\quad$ water temperature

WITH - water withdrawals

"NA" indicates that no life history pathway meeting thecriteria used in defining these pathways was present.

Blank cells under attributes indicate no additional attributes with negative effects on productivity were evident compared to those present in the template. 
Stream Reach Summary for Primary and Secondary Spring Chinook Salmon Life Histories

Stream: Grande Ronde R.

Reach: Buford Cr - Bear Cr (RM 26.2 -28.2 from Snake R)

\begin{tabular}{|ll|ll|l|l|}
\hline Restoration benefit category: & F & Chinook usage score: & 141.0 & Habltat productivity loss score: \\
\hline
\end{tabular}

\begin{tabular}{|c|c|c|c|c|c|c|c|c|c|c|c|c|c|c|}
\hline \multirow[b]{3}{*}{ Life stage } & \multirow[b]{3}{*}{ Months } & \multicolumn{10}{|c|}{ No. life history pathways by current usage abundance level } & \multicolumn{2}{|c|}{ Key habitat change } & \multirow{3}{*}{$\begin{array}{c}\text { Habitat } \\
\text { productivity } \\
\text { loss score }\end{array}$} \\
\hline & & \multicolumn{5}{|c|}{ Primary } & \multicolumn{5}{|c|}{ Secondary } & \multirow{2}{*}{$\begin{array}{l}\text { Patient } \\
\text { quantity }\end{array}$} & \multirow[b]{2}{*}{ \% change } & \\
\hline & & Total no. & 1 & 2 & 3 & 4 & Total no. & 1 & 2 & 3 & 4 & & & \\
\hline 1 & Apr-Aug & 48 & 16 & 16 & 6 & 6 & 111. & 59 & 34 & 12 & 6 & 33.6 & 0.0 & 0.0 \\
\hline $1,2,3$ & Aug-Ött & NA & NA & NA & NA & NA & NA & NÄ & NA & "NÄ & NÄ & NA & NÄ & NA \\
\hline 3 & Ott-Mar & NA & NA & NA & NA & NA & NA & NA & NA & NA & NA & NA & NA & NÄ \\
\hline 3.4 & Mar-May & NA & NA & NA & NA & $" N A "$ & NA & "NA & NA & NNA & NA & NA & NA & NA \\
\hline 5 & May-Ott & 0 & 0 & 0 & 0 & 0 & 6 & 1 & 3 & 2 & 0 & 20.6 & 0.0 & -7.1 \\
\hline 6 & Ott-Mar & 2 & 0 & 1 & 1 & 0 & 7 & 4 & 1 & 2 & 0 & 20.6 & 0.0 & -5.4 \\
\hline 7 & Mar-Jun & 46 & 16 & 17 & 7 & 6 & 98 & 54 & $3 n$ & $\mathbf{R}$ & G & 1 1 3 & 0.0 & 2.3 \\
\hline
\end{tabular}

\begin{tabular}{|c|c|c|c|c|c|c|c|c|c|c|c|c|c|}
\hline \multirow[b]{2}{*}{ Life stage } & \multirow[b]{2}{*}{ Months } & \multicolumn{12}{|c|}{ 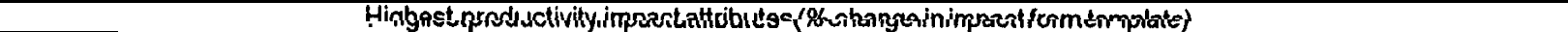 } \\
\hline & & Att 1 & $\%$ change & $\overline{\text { Att } 2}$ & $\%$ change & Att 3 & $\%$ change & An 4 & $\%$ change & Att 5 & \% change & Att 6 & $\%$ change \\
\hline 1 & Apr-Aug & CHEM & 6.1 & NUTLD & 6.1 & OXYG & 6.1 & & & & & & \\
\hline $1,2,3$ & Äug-Ött & NA & N"̈ & $\mathrm{NA}$ & Nä & $\mathrm{NA}$ & NA & N̈" & חั"̈̈ & NA & ח̈̈̈ & ח̈̈ & NÄ \\
\hline 3 & Ött-Marar & $\mathrm{NA}$ & NÄ & $\mathrm{NA}$ & "N" & Nä & NÄ & $\mathrm{NA}$ & NÄ & N" & $\mathrm{NA}$ & חัNä & NÄ \\
\hline 3,4 & Mä-May & NA & NA & NA & NA & NA & NA & NA & NA & $N A$ & $\dddot{N A}$ & NÄ & $\mathrm{NA}$ \\
\hline$\dot{5}$ & May-Ott & $\mathrm{COMP}^{\circ}$ & $2 \dot{6} . \dot{6}$ & CHEM & 13.3 & HABD & 13.3 & NIITL & 133 & " & 13.3 & & \\
\hline 6 & Ott-Mar & SEDLD & 29.7 & РАТНО & 14.9 & & & & & & & & \\
\hline 7 & Mar-Jun & PATHO & 8.0 & TEMP & 8.0 & & & & & & & & \\
\hline
\end{tabular}

Abundance level refera to the average abundance of spawners that spawn in the natal stream reaches that populate the lifehistory pathways using the reach in this table Level 1: $\quad 0-10$ spawners

Level 2 10-30 spawners

Level $3 \quad 30-60$ spawners

Level 4: $\quad>60$ spawners

Life stages.' 1- prespawner; 2- spawner; 3- incubation; 4- fry colonization; 5- summer rearing; 6- overwintering; 7- yearling presmolt/smolt

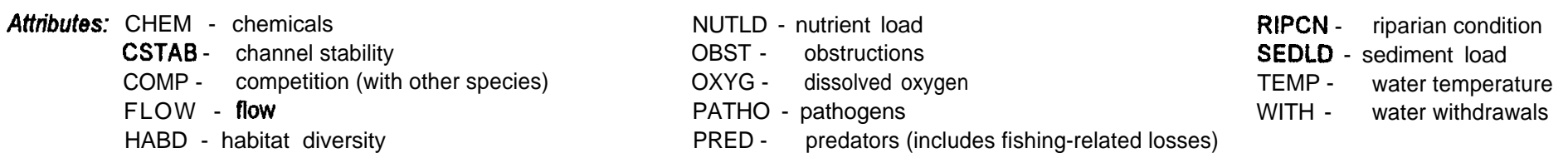

"NA" indicates that no life history pathway meeting the criteria used in defining these pathways was present.

Blank cells under attributes indicate no additional attributes withegative effects on productivity were evident compared to those present in the template. 
Stream Reach Summary for Primary and Secondary Spring Chinook Salmon Life Histories

Stream: Grande Ronde R.

Reach: Bear $\mathrm{Cr}$ - Menatchee $\mathrm{Cr}$ (RM 28.2 -34.8 from Snake R)

\begin{tabular}{|ll|ll|ll|}
\hline Restoration benefit category: & $F$ & Chinook usage score: & 245.4 & Habitat productivity loss score: & -53.0 \\
\hline
\end{tabular}

\begin{tabular}{|c|c|c|c|c|c|c|c|c|c|c|c|c|c|c|}
\hline \multirow[b]{3}{*}{ Life stage } & \multirow[b]{3}{*}{ Months } & \multicolumn{10}{|c|}{ No. life history pathways by current usage abundance level } & \multicolumn{2}{|c|}{ Key habitat change } & \multirow{3}{*}{$\begin{array}{c}\text { Habitat } \\
\text { productivity } \\
\text { loss score }\end{array}$} \\
\hline & & \multicolumn{5}{|c|}{ Primary } & \multicolumn{5}{|c|}{ Secondary } & \multirow{2}{*}{$\begin{array}{l}\text { Patient } \\
\text { quantity }\end{array}$} & \multirow[b]{2}{*}{$\%$ change } & \\
\hline & & Total no. & 1 & 2 & $\mathbf{3}$ & 4 & Total no. & 1 & 2 & 3 & 4 & & & \\
\hline 1 & Apr-Aug & 46 & 16 & 16 & 8 & 6 & 111. & 59 & 34 & 12 & 6 & 33.7 & 0.0 & 00 \\
\hline 1.2 .3 & Aug-Ott & $\mathrm{NA}$ & $\mathrm{NA"}$ & $\mathrm{NÄ}$ & NÄ & NÄ & $\ddot{N A}$ & NA & NA & $\mathrm{NÄ}$ & NA & NA & NA & NA \\
\hline 3 & Ott-Mar & NA & $N A$ & NA & NA & NA & NA & NA & $N \quad A$ & NA & $\dot{N}$ & 2 & NA & NA \\
\hline 34 & Mar-May & NA & NA & NA & $N A$ & $N A$ & NA & NA & NA & $\mathrm{NA}$ & $\mathrm{NA}$ & & NA & NA \\
\hline 5 & May-Ott & 0 & 0 & 0 & 0 & 0 & 7 & 1 & 3 & 3 & 0 & 20.6 & 0.0 & -2.8 \\
\hline 6 & Ott-Mar & 2 & 0 & 1 & 1 & 0 & 134 & 113 & 20 & 1 & 0 & 23.1 & 0.7 & -51.7 \\
\hline 7 & Mar-Jun & 46 & 16 & 17 & 7 & 6 & 98 & 54 & 30 & 8 & 6 & 106.9 & 0.0 & 1 \\
\hline
\end{tabular}

\begin{tabular}{|c|c|c|c|c|c|c|c|c|c|c|c|c|c|}
\hline \multirow[b]{2}{*}{ Life stage } & \multirow[b]{2}{*}{ Months } & \multicolumn{12}{|c|}{ Highest productivity impact attributes (\% change in impact from template) } \\
\hline & & Alt 1 & \% change & Att 2 & \% change & Att 3 & $\%$ change & Att 4 & $\%$ change & Alt 5 & $\%$ change & Att 6 & $\%$ change \\
\hline 1 & Apr-Aug & CHEM & 15.3 & NUTLD & 15.3 & OXYG & 15.3 & & & & & & \\
\hline $1,2,3$ & Aug-Oct & $\mathrm{NA}$ & NA & NÄ & NÄ & NA & $N A$ & NA & NA & NÄ & NA & NA & NA: \\
\hline 3 & Oct-Mar & $\mathrm{NA}$ & NA & NA & NA & NA & NA & NA & NA & NA & NA & NA & NA \\
\hline 3,4 & Mar-May & NA & NA & NA & NA & NA & NA & NA & NA & NA & NA & NA & NA \\
\hline 5 & May-Oct & SEDLD & 122 & PATHO & 6.1 & COMP & 5.7 & CHEM & 2.8 & HABD & 2.8 & NUTLO & 2.8 \\
\hline 6 & Oct-Mar & SEDLD & 18.1 & PATHO & 14.0 & TEMP & 14.0 & & & & & & \\
\hline 7 & Mar-Jun & PATHO & 5.1 & TEMP & 5.1 & & & & & & & & \\
\hline
\end{tabular}

Abundance level refers to the average abundance of spawners that spawn in the natal stream reaches that populate the life history pathways using the reach In this table

$$
\begin{array}{ll}
\text { Level 1: } & 0-10 \text { spawners } \\
\text { Level 2 } & 10-30 \text { spawners } \\
\text { Level 3 } & 30-60 \text { spawners } \\
\text { Level 4: } & >\mathbf{6 0} \text { spawnera }
\end{array}
$$

Life stages: 1 - prespawner; 2- spawner; 3- incubation; 4- fry colonization; 5- summer rearing; 6- overwintering; 7- yearling presmoltssmolt

Attributes: CHEM - chemicals

CSTAB - channel stability

COMP - competition (with other species)

FLOW - flow

HABD - habitat diversity
NUTLD - nutrient load

OBST - obstructions

OXYG - dissolved oxygen

PATHO - pathogens

PRED - predatora (includes fishing-related losses)
RIPCN - riparian condition

SEDLD - sediment load

TEMP - water temperature

WITH - water withdrawals

"NA" indicates that no life historv pathway meeting the criteria usad in defining these pathwavs was present.

Blank cells under attributesindicate no additional attributes with negative effects on productivity were evident compared to those present in the template. 
Stream Reach Summary for Primary and Secondary Spring Chinook Salmon Life Histories

Stream: Grande Ronde R.

Reach: Menatchee $\mathrm{Cr}$ - Wenaha R (RM $34.8-45.1$ from Snake R)

\begin{tabular}{ll|ll|ll}
\hline Restoration benefit category: & F & Chinook usage score: & 216.1 & Habitat productlvity loss score: & -35.2 \\
\hline
\end{tabular}

\begin{tabular}{|c|c|c|c|c|c|c|c|c|c|c|c|c|c|c|}
\hline \multirow[b]{3}{*}{ Life stage } & \multirow[b]{3}{*}{ Months } & \multicolumn{10}{|c|}{ No. life history pathways by current usage abundance level } & \multicolumn{2}{|c|}{ Key habitat change } & \multirow{3}{*}{$\begin{array}{c}\text { Habital } \\
\text { productivity } \\
\text { loss score }\end{array}$} \\
\hline & & \multicolumn{5}{|c|}{ Primary } & \multicolumn{5}{|c|}{ Secondary } & \multirow{2}{*}{$\begin{array}{c}\text { Patient } \\
\text { quantity }\end{array}$} & \multirow[b]{2}{*}{$\%$ change } & \\
\hline & & Total no. & 1 & 2 & 3 & 4 & Total no. & 1 & 2 & 3 & 4 & & & \\
\hline 1 & Apr-Aug & 49 & 17 & 1 & 8 & 6 & 113 & 61 & 34 & 12 & 6 & 34.2 & 0.0 & 0.0 \\
\hline $1,2,3$ & Aug-Oct & NA & NA" & NÄ & NA & $\mathrm{NA}$ & NA & NA & NA & NA & NA & $\mathrm{NA}$ & NA & NA \\
\hline 3 & Ött-Mar & NA" & $N A$ & NA & NA & NA & NA & NA & $N A$ & NA & $N A$ & $\mathrm{~N}$ & NA & $\mathrm{NA}$ \\
\hline 34 & Mar-May & NA & NA ", & NA & “' NA & NA & NA & NA " & NA & NA & NA & NA & NA & NA \\
\hline 5 & May-Ott & 2 & 0 & $\dddot{1}$ & 1 & 0 & 13 & 4 & 5 & 4 & 0 & $20.0^{\circ}$ & 0.0 & -9.2 \\
\hline 6 & Ott-Mar & 36 & 0 & 18 & 18 & o & 172 & 9 & 18 & 55 & 0 & 24.2 & 0.0 & -27.8 \\
\hline 7 & Mar-jun & $48^{\circ}$ & 16 & 17 & 7 & $\dddot{6}$ & 93 & 49 & 30 & 8 & $\dot{6}$ & $120.1^{1}$ & 0.0 & 1.7 \\
\hline
\end{tabular}

\begin{tabular}{|c|c|c|c|c|c|c|c|c|c|c|c|c|c|}
\hline \multirow[b]{2}{*}{ Life stage } & \multirow[b]{2}{*}{ Months } & \multicolumn{12}{|c|}{ Highest productivity impact attributes (\% change in impact from template) } \\
\hline & & Att 1 & $\%$ change & Att 2 & $\%$ change & Att 3 & $\%$ change & Att 4 & $\%$ change & Att 5 & $\%$ change & Att 6 & $\%$ change \\
\hline 7 & Apr-Aug & CHEM & 12.3 & NUTLD & 12.3 & OXYG & 12.3 & & & & & & \\
\hline $1,2,3$ & Aug-Oct & NA & $N A$ & $\mathrm{NA}$ & NÄ & $\mathrm{NA}$ & NÄ & $\mathrm{NA}$ & NA & $\mathrm{NA}$ & NA" & $\mathrm{NA}$ & NA \\
\hline 3 & Ott-Mar & NA & $\mathrm{NA}$ & $\mathrm{NA}$ & NA. & $\mathrm{NA}$ & $\mathrm{NA}$ & $\mathrm{NA}$ & $\mathrm{NA}$ & $\mathrm{NA}$ & $\mathrm{NA}$ & $\mathrm{NA}$ & NÄ \\
\hline ,,,,,,3,4 & Mar-May & NA & NA & NA & NA & $\mathrm{NA}$ & $\mathrm{NA}$ & NÄ & NA & $\ddot{N A}$ & NÄ & $\dot{\mathrm{NA}}$ & NA \\
\hline 5 & May-Oct & COMP & 22. & CHEM & 11.3 & HABD & $11 ?$ & NUTID & 11.3 & PATHO & 11.? & & \\
\hline 6 & Ott-Mar & SEEDLD & 6. & PATHO & 3.3 & TEMP & 3.1 & & & & & & \\
\hline 7 & Mar-Jun & PATHO & 6.1 & TEMP" & 61 & & & & & & & & \\
\hline
\end{tabular}

Abundance /eve/ refers to the average abundance of spawners that spawn in the natal stream reaches that populate the life historypathways using the reach in this table:

$$
\begin{array}{ll}
\text { Level 1: } & \text { 0-10 spawners } \\
\text { Level 2: } & 10-30 \text { spawners } \\
\text { Level 3 } & 30-80 \text { spawners } \\
\text { Level 4: } & \mathbf{2 6 0} \text { spawners }
\end{array}
$$

\begin{tabular}{|c|c|c|c|}
\hline Attributes: & $\begin{array}{l}\text { CHEM - chemicals } \\
\text { CSTAB - channel stability } \\
\text { COMP - competition (with other species) } \\
\text { FLOW - flow } \\
\text { HABD - habitat diversity }\end{array}$ & $\begin{array}{l}\text { NUTLD - } \\
\text { OBStrient load } \\
\text { OXYG - } \quad \text { obstructions } \\
\text { Oissolved oxygen } \\
\text { PATHO - } \\
\text { PRED - } \quad \text { predators (include }\end{array}$ & $\begin{array}{l}\text { RIPCN - riparian condition } \\
\text { SEDLD - } \quad \text { sediment load } \\
\text { TEMP - } \quad \text { water temperature } \\
\text { WITH - water withdrawals }\end{array}$ \\
\hline
\end{tabular}

Life stages: 1 - prespawner; 2- apawner; 3- incubation; 4- fry colonization; 5- summer rearing; 6- overwintering; 7- yearling presmolt/smolt

"NA" indicates that no tife history pathway meeting the criteria used in defining these pathways was present.

Blank cells under attributes indicate no additional attributea with negative effects on productivity were evident compared to those present in the template. 


\section{Stream Reach Summary for Primary and Secondary Spring Chinook Salmon Life Histories}

Stream: Grande Ronde R.

Reach: Wenaha R - Courtney Cr (RM 45.1 -46.2 from Snake R)

\begin{tabular}{|c|c|c|c|c|c|c|c|c|c|c|c|c|c|c|}
\hline Restoratio & n benefi & \multicolumn{2}{|c|}{ category: } & & \multicolumn{3}{|c|}{ |Chinook usage score: } & 96.0 & \multicolumn{2}{|c|}{ Habitat productivity } & \multicolumn{2}{|c|}{ loss score: } & -6.9 & \\
\hline \multirow[b]{3}{*}{ Life stage } & \multirow[b]{3}{*}{ Months } & \multicolumn{10}{|c|}{ No. life history pathways by current usage abundance level } & \multicolumn{2}{|c|}{ Key habitat change } & \multirow{3}{*}{$\begin{array}{c}\text { Habitat } \\
\text { productivity } \\
\text { loss score }\end{array}$} \\
\hline & & & & Primary & & & & & Second & & & Patient & & \\
\hline & & Total no. & 1 & 2 & 3 & 4 & Total no. & 1 & 2 & 3 & 4 & quantity & $\%$ change & \\
\hline & , Apr-A,Aug, & 44 & 15 & 16 & 7 & 6 & 102 & 57 & 29 & 10 & 6 & 39.9 & 0.0 & 0.0 \\
\hline 133. & Aug-Ott & NÄ & NA" & NÄ & "NA" & Nä & "NA" & $\mathrm{NA}$ & $\mathrm{NA}$ & NÄ & NÄ & NÄ & $\mathrm{NA}$ & NA \\
\hline 3 & Ott-Mar & NÄ & NA & NA & NA & NA & NA & NA & $\mathrm{NA}$ & NÄ & N̈̈ & $\mathrm{NA}$ & $\mathrm{NA}$ & NA \\
\hline 3,4 & Mar-May & NA & NA & NA & NA & $\mathrm{NA}$ & $\mathrm{NA}$ & NA & NA & $\ddot{N A}$ & $\mathrm{NA}$ & NA & ïA & NA \\
\hline 5 & May-Ott & 2 & 0 & 1 & 1 & 0 & 6 & 2 & 1 & 3 & 0 & 71.3 & 0.0 & 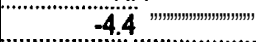 \\
\hline 6 & Ott-Mar & 2 & 0 & 1 & $i$ & 0 & 14 & 12 & $\dddot{0}$ & 2 & 0 & 28.5 & 0.0 & -3.7 \\
\hline 7 & Mar-Jun & 40 & 15 & 14 & 5 & 6 & 82 & 43 & 28 & 5 & 6 & 140.3 & 0.0 & 1.2 \\
\hline
\end{tabular}

\begin{tabular}{|c|c|c|c|c|c|c|c|c|c|c|c|c|c|}
\hline \multirow[b]{2}{*}{ Life stage } & \multirow[b]{2}{*}{ Months } & \multicolumn{12}{|c|}{ Highest productivity impact attributes (\% change in impact from template) } \\
\hline & & Att 1 & $\%$ change & Att 2 & $\%$ change & Att 3 & $\%$ change & Aft 4 & $\%$ change & Att 5 & \% change & Att 6 & \% change \\
\hline 1 & Apr-Aug & CHEM & 6.1 & NUTLD & 6.1 & $\overline{O X Y G}$ & 6,1 & TEMP & 6,1 & & & & \\
\hline $1,2,3$ & Aug-Oct & NA & NA & NA & חัก̈ & NA & $\mathrm{NA}$ & $\mathrm{NA}$ & $\mathrm{NA}$ & NÄ & $\mathrm{NA}$ & $\mathrm{NA}$ & Ä \\
\hline$\dddot{3}$ & Ott-Mar & NA & $\mathrm{NA}$ & $\mathrm{NA}$ & NA & NA & $\mathrm{NA}$ & $\mathrm{NA}$ & NÄ & NA & $\mathrm{NA}$ & NA & NA \\
\hline 34 & Mar-May & NA & Na & NA & NA & $\mathrm{NA}$ & NA & NA & NA & $\mathrm{NA}$ & $\mathrm{NÄ}$ & $\mathrm{NA}$ & $\mathrm{NA}$ \\
\hline 5 & May-Ott & SEDLD & 11.6 & COMP & 6.5 & HABD & 5.6 & PATHO & 5.8 & CHEM & 3.3 & NUTLD & 3.3 \\
\hline 6 & Ott-Mar & SEDLD & 14.8 & HABD & 7.4 & PATHO & 7.4 & & & & & & \\
\hline 7 & Mar-Jun & TEMP & 4.7 & & & & & & & & & & \\
\hline
\end{tabular}

Abundance /eve/ refers to the average abundance of spawnera that spawn in the natal stream reaches that populate the life history pathways using the reach in this table

Level 1: $\quad 0-10$ spawners

Level 2: $\quad 10-30$ spawners

Level 3 30-60 spawners

Level $4>60$ spawners

fife stages: 1- prespawner; 2- spawner; 3- incubation; 4- fry colonization; 5- summer rearing; 6- overwintering; 7-yearling presmolt/smoll

Attributes.' CHEM - chemicals

CSTAB - channel stability

COMP - competition (with other species)

FLOW - flow

HABD - habitat diversity
NUTLD - nutrient load

OBST - obstruct ions

OXYG - dissolved oxygen

PATHO - pathogena

PRED - predatora (includes fishing-related losses)
RIPCN - riparian condition

SEDLD - sediment load

TEMP - water temperature

WITH - water withdrawals

"NA" indicates that no life history pathway meeting thecriteria used in defining these pathways was present.

Blank cells under attributes indicate no additional attributes with negative effects on productivity were evident compared to those present in the template. 
Stream: Grande Ronde R.

Reach: Courtney $\mathrm{Cr}$ - Mud Cr (RM 46,2 -51.4 from Snake R)

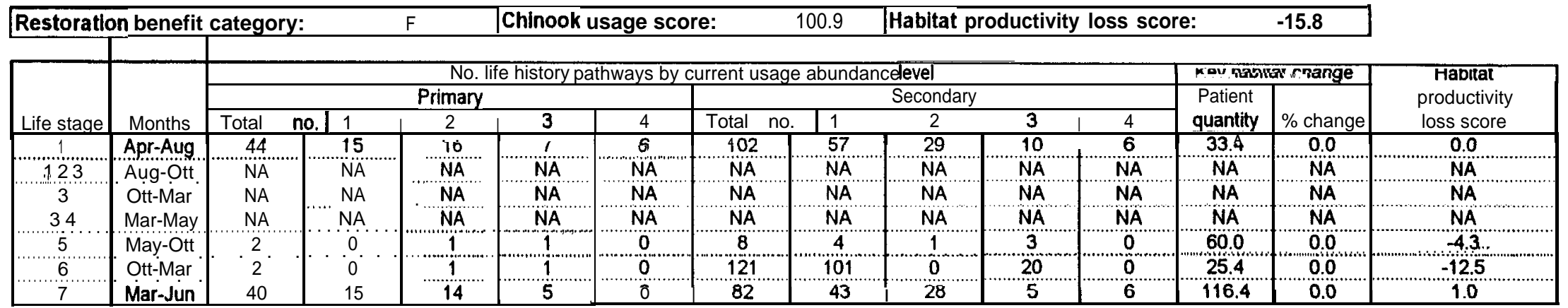

\begin{tabular}{|c|c|c|c|c|c|c|c|c|c|c|c|c|c|}
\hline \multirow[b]{2}{*}{ Life stage } & \multirow[b]{2}{*}{ Months } & \multicolumn{12}{|c|}{ Highest productivity impact attributes (\% change in impact from template) } \\
\hline & & Att 1 & \% change & Att 2 & $\%$ change & Att 3 & $\%$ change & Att 4 & $\%$ change & Att 5 & $\%$ change & Att 6 & $\%$ change \\
\hline 1 & Apr-Aug & CHEM & 5.8 & NUTLD & 5.8 & OXYG & 5.8 & TEMP & 5.8 & & & & \\
\hline $1,2,3$ & Äug-Öct & NA & NA & NA & NA & NA & NA & NA & NA & NA & NÄ & NA & NA \\
\hline 3 & Ott-Mar & NÄ & NA & NA & NA & NA & NA & $\mathrm{NA}$ & NA & NA & N"̈A & NÄ & N̈A \\
\hline 3,4 & Mar-May & $\mathrm{NÄ}$ & NA & NA & NA & NA & NA & NAA & NA & NA & NA & NA. & NA \\
\hline 5 & May-Ott & COMP & 8.9 & CHEM & 4.5 & NUTLD & 4.5 & PAT HO & “ "4.5" & TEMP & 4.5 & & \\
\hline 6 & Ott-Mar & SEDLD & 6.5 & HABD & 33 & PATHO & 33 & TEMP & 3.1 & & & & \\
\hline 7 & Mar-Jun & PATHO & 4.0 & TEMP & 4.0 & & & & & & & & \\
\hline
\end{tabular}

Abundance level refers to the average abundance of spawners that spawn in thenatal stream reaches that populate thelife history pathways using the reach in this table

$$
\begin{array}{ll}
\text { Level 1: } & 0-10 \text { spawners } \\
\text { Level 2: } & 10-30 \text { spawners } \\
\text { Level 3: } & 30-60 \text { spawners } \\
\text { Level 4: } & >60 \text { spawners }
\end{array}
$$

\begin{tabular}{|c|c|c|c|}
\hline Attributes: & $\begin{array}{l}\text { CHEM - chemicals } \\
\text { CSTAB - channel stability } \\
\text { COMP - competition (with other species) } \\
\text { FLOW - flow } \\
\text { HABD - habitat diversity }\end{array}$ & $\begin{array}{l}\text { NUTLD - nutrient load } \\
\text { OBST - obstructions } \\
\text { OXYG - dissolved oxygen } \\
\text { PATHO - pathogens } \\
\text { PRED - predators (includes fishing-related losses) }\end{array}$ & $\begin{array}{ll}\text { RIPCN - } & \text { riparian condition } \\
\text { SEDLD - } & \text { sediment load } \\
\text { TEMP - } & \text { water temperature } \\
\text { WiTH - } & \text { water withdrawals }\end{array}$ \\
\hline
\end{tabular}

Life stages: 1- prespawner; 2- spawner; 3- incubation; 4- fry colonization; 5- summer rearing; 6- overwintering; 7- yearling presmolt/smolt

"NA" indicates that no life history pathway meeting the criteria used in defining these pathways was present.

Blank cells under attributes indicate no additional attributes with negative effects on productivity were evident compared to those present in the template. 
Stream Reach Summary for Primary and Secondary Spring Chinook Salmon Life Histories

Stream: Grande Ronde R.

Reach: Mud $\mathrm{Cr}$ - WallupaWildcat $\mathrm{Cr}$ (RM 51.4 -52.8 from Snake R)

\begin{tabular}{|ll|ll|l}
\hline Restoration benefit category: & F & Chinook usage score: & 114.7 & Habltat productivity loss score: \\
\hline
\end{tabular}

\begin{tabular}{|c|c|c|c|c|c|c|c|c|c|c|c|c|c|c|}
\hline \multirow[b]{3}{*}{ Life stage } & \multirow[b]{3}{*}{ Months } & \multicolumn{10}{|c|}{ No. life history pathways by current usage abundance level } & \multicolumn{2}{|c|}{ Key habitat change } & \multirow{3}{*}{$\begin{array}{c}\text { Habitat } \\
\text { productivity } \\
\text { loss score }\end{array}$} \\
\hline & & \multicolumn{5}{|c|}{ Primary } & \multicolumn{5}{|c|}{ Secondary } & \multirow{2}{*}{$\begin{array}{l}\text { Patient } \\
\text { quantity }\end{array}$} & \multirow[b]{2}{*}{$\%$ change } & \\
\hline & & Total no. & 1 & 2 & 3 & 4 & Tolal no. & 1. & 2 & 3 & 4 & & & \\
\hline 1 & Apr-Aug & 44 & 15 & 16 & 7 & 6 & 102 & 57. & 29 & 10 & 6 & 24.2 & 0.0 & 0.0 \\
\hline $1,2,3$ & Aug-Oct & NA & NA & $\mathrm{NA}$ & $\mathrm{NA}$ & $\mathrm{NA}$ & $\mathrm{NA}$ & $\mathrm{NA}$ & NA & $\ddot{N A}$ & NA & NÄ & $\mathrm{NA}$ & $\mathrm{NA}$ \\
\hline 3 & Ocl-Mar & NÄ & NA & $\mathrm{NA}$ & $\mathrm{NA}$ & $\mathrm{NA}$ & $\mathrm{NA}$ & NA & NA & NA & NA & NA & NA & $\mathrm{NA}$ \\
\hline 3,4 & Mar-May & NA & $N A$ & $\mathrm{NA}$ & $\mathrm{NA}$ & NA & NA & $\mathrm{NA}$ & $\mathrm{NÄ}$ & $\mathrm{NA}$ & $\mathrm{NA}$ & $\mathrm{NA}$ & NA & $\mathrm{NA}$ \\
\hline 5 & May-Oct & 3 & 0 & 2 & 1 & 0 & 8 & 4 & 1 & 3 & 0 & 46.9 & 00 & -69 \\
\hline 6 & Oct-Mar & 1 & 0 & 0 & 1 & o & 18 & 15 & 0 & 3 & 0 & 18.8 & 0.0 & -3.9 \\
\hline 7 & Mar-Jun & 40 & 15 & 14 & 5 & 6 & 76 & 38 & 28 & $\dot{A}$ & 6 & 84 & 0.0 & 1.0 \\
\hline
\end{tabular}

\begin{tabular}{|c|c|c|c|c|c|c|c|c|c|c|c|c|c|}
\hline \multirow[b]{2}{*}{ Life stage } & \multirow[b]{2}{*}{ Months } & \multicolumn{12}{|c|}{ Highest productivity impact attributes (\% change in impact from template) } \\
\hline & & Atf 1 & $\%$ change & Att 2 & $\%$ change & Aft 3 & $\%$ change & Att 4 & $\%$ change & Att 5 & $\%$ change & Att 6 & $\%$ change \\
\hline 1 & Apr-Aug & CHEM & 8.5 & NUTLD & 8.5 & OXYG & 8.5 & $\overline{T E M P}$ & 8.5 & & & & \\
\hline $1,2,3$ & Aug-Oct & $\dot{N A}$ & NA & $\ddot{N A}$ & NA & NA & NÄ & $\mathrm{NA}$ & NA & NA & NA & $\mathrm{NA}$ & $\mathrm{NA}$ \\
\hline 3 & Ott-Mar & & $? !$ & NA & NÁ & NA. & NA & N.?. & NA & NA & NÄ & NÄ̈ & $\mathrm{NA}$ \\
\hline 3,4 & Mar-May & & NA & $\mathrm{NA}$ & $\mathrm{NA}$ & NA & NA & NA & NA & NA & NA & NA & $\mathrm{NA}$ \\
\hline 5 & May-Ott & COMP & 14.6. & TEṂP. & 14.6 & SEDLLP & 10.7 & Ċ HE M & 7.3 & NUTLD. & 73 & PATHO & 73 \\
\hline 6 & Ott-Mar & SEDLD & 13.2 & $\mathrm{HABD}$ & 6.6 & PATHO & 6.6 & & & & & & \\
\hline 7 & Mar-jun & PATHO & 4.4 & TËMP̈ & 4.4 & & & & & & & & \\
\hline
\end{tabular}

Abundance leve/ refers to the average abundance of spawners that spawn in the natal stream reaches that populate the life history pathways using the reach in this table:

$$
\begin{array}{ll}
\text { Level 1: } & 0-10 \text { spawners } \\
\text { Level 2 } & 10-30 \text { spawners } \\
\text { Level 3 } & 30-60 \text { spawners } \\
\text { Level 4 } & >60 \text { spawners }
\end{array}
$$

Life stages: 1 - prespawner; 2- spawner; 3- incubation; 4- fry colonization; 5- summer rearing; 6- overwintering; 7- yearling presmolt/smolt

$\begin{array}{lll}\text { Attributes: CHEM - chemicals } & \text { NUTLD - nutrient load } & \text { RIPCN - riparian condition } \\ \text { CSTAB - channel stability } & \text { OBST - obstructions } & \text { SEDLD - sediment load } \\ \text { COMP - competition (with other species) } & \text { OXYG - dissolved oxygen } & \text { TEMP - water temperature } \\ \text { FLOW - flow } & \text { PATHO - pathogens } & \text { WITH - water withdrawals } \\ \text { HABD - habitat diversity } & \text { PRED - predators (includes fishing-related losses) }\end{array}$

"NA" indicates that no life history pathway meeting the criteria used in defining these pathways was present.

Blank cells under attributes indicate no additional attributes with negative effects on productivity were evident compared to those preseintthe template. 
Stream: Grande Ronde R.

Reach: Wallupa/Wildcat $\mathrm{Cr}$ - Grossman $\mathrm{Cr}$ (RM $52.8-61.1$ from Snake R)

\begin{tabular}{ll|ll|lll}
\hline Restoration benefit category: & B & Chinook usage score: & 453.3 & Habitat productivity loss score: & -145.1 \\
\hline
\end{tabular}

\begin{tabular}{|c|c|c|c|c|c|c|c|c|c|c|c|c|c|c|}
\hline \multirow[b]{3}{*}{ Life stage } & \multirow[b]{3}{*}{ Months } & \multicolumn{10}{|c|}{ No. life history pathways by current usage abundance level } & \multicolumn{2}{|c|}{ Key habitat change } & \multirow{3}{*}{$\begin{array}{c}\text { Habitat } \\
\text { productivity } \\
\text { loss score }\end{array}$} \\
\hline & & \multicolumn{5}{|c|}{ Primary } & \multicolumn{5}{|c|}{ Secondary } & \multirow{2}{*}{$\begin{array}{l}\text { Patient } \\
\text { quantity }\end{array}$} & \multirow[b]{2}{*}{$\%$ change } & \\
\hline & & Total no. & 1 & 2 & 3 & 4 & Total no. & 1 & 2 & 3 & 4 & & & \\
\hline 1 & Apr-Aug & 44. & 15 & 16 & 7 & 6 & 102 & 57 & 29 & 10 & 6 & 22.8 & 0.0 & 0.0 \\
\hline $1,2,3$ & Aug-Oct & NÄ & NA" & NA & NA & NA & NA & NA & $\mathrm{NA}$ & NA & NA & NA & $\mathrm{NA}$ & $\mathrm{NA}$ \\
\hline 3 & Oct-Mar & NA & NA & NA & $\mathrm{NA}$ & NA & $\mathrm{NA}$ & NA & $\mathrm{NA}$ & $\mathrm{NA}$ & NA & $\mathrm{NA}$ & $\mathrm{NA}$ & $\mathrm{NA}$ \\
\hline 3,4 & Mar-May & NA & NA & $\mathrm{NA}$ & $\mathrm{NA}$ & $\mathrm{NA}$ & NA & $\mathrm{NA}$ & $\mathrm{NA}$ & $\mathrm{NA}$ & $\mathrm{NA}$ & $\mathrm{NA}$ & $\mathrm{NA}$ & $\mathrm{NA}$ \\
\hline 5 & May-OcI & 4 & 0 & 2 & 2 & 0 & 15 & 10 & 1 & 4 & 0 & 47.5 & 0.0 & -21.9 \\
\hline 6 & Oct-Mar & 19 & 0 & 19 & 0 & 0 & 181 & $160^{\circ}$ & 19 & 2 & 0 & 20.7 & 0.0 & -125.2 \\
\hline 7 & Mar-Jun & 40 & 15 & 14 & 5 & 6 & 76 & 38 & 28 & 4 & 6 & 90.5 & 0.0 & 2.1 \\
\hline
\end{tabular}

\begin{tabular}{|c|c|c|c|c|c|c|c|c|c|c|c|c|c|}
\hline \multirow[b]{2}{*}{ Life stage } & \multirow[b]{2}{*}{ Months } & \multicolumn{12}{|c|}{ Highest productivity impact attributes (\% change in impact from template) } \\
\hline & & Atf 1 & $\%$ change & Att 2 & $\%$ change & An 3 & $\%$ change & Att 4 & $\%$ change & Att 5 & $\%$ change & Att 6 & $\%$ change \\
\hline 1 & Apr-Aug & CHEM & 22,8 & NUTLD & 22.8 & OXYG & 22.8 & TEMP & 22.8 & & & & \\
\hline $1,2,3$ & Aug-Ött & NA & NA & NÄ & $\dot{\mathrm{N}} \dot{\mathrm{A}}$ & NA & $\ddot{N A}$ & NÁ & $\mathrm{NA}$ & $\mathrm{NA}$ & ח̄ä & NÄ & NA \\
\hline 3 & Ott-Mar & חั" & $\mathrm{NA}$ & $\mathrm{NA}$ & Nä & "Nä & $\mathrm{NA}$ & NÄ & $\mathrm{NA}$ & $\mathrm{NA}$ & Nä & NÄ & $\mathrm{NA}$ \\
\hline 34 & Mar-May & $\mathrm{NA}$ & $\mathrm{NA}$ & NA & $\mathrm{NA}$ & NÄ & $\mathrm{NA}$ & NA & NA & NA & $\mathrm{NA}$ & $\mathrm{NA}$ & $\ddot{N A}$ \\
\hline 5 & May-Ott & С̈OMP & 22.2 & TËMP & 22.2 & CHEM & $11.1^{\circ}$ & NUT'LD & 11.1 & PATHO & 11.1 & & \\
\hline 6 & Öt-Mar & "SEDELD" & 39.7 & "РATHO" & 25.0 & "TEMP" & 25.0 & HABD & 19.9 & & & & \\
\hline 7 & Mar-Jun & PATHO & 9.0 & TEËP̈ & 9.0 & & & & & & & & \\
\hline
\end{tabular}

Abundance /eve/ refers to the average abundance of spawners that spawn in the natal stream reaches that populate the life history pathways using the reach in this table:

$$
\begin{array}{ll}
\text { Level 1: } & \text { 0-10 spawners } \\
\text { Level 2 } & 10-30 \text { spawners } \\
\text { Level 3 } & \text { 30-60 spawners } \\
\text { Level 4: } & \mathbf{2} \mathbf{6 0} \text { spawners }
\end{array}
$$

\begin{tabular}{|c|c|c|c|}
\hline Attributes: & $\begin{array}{l}\text { CHEM - chemicals } \\
\text { CSTAB - channel stability } \\
\text { COMP - competition (with other species) } \\
\text { FLOW - flow } \\
\text { HABD - habitat diversity }\end{array}$ & $\begin{array}{l}\text { NUTLD - nutrient load } \\
\text { OBST - obstructions } \\
\text { OXYG - dissolved oxygen } \\
\text { PATHO - pathogens } \\
\text { PRED - predators (includes fishing-related losses) }\end{array}$ & $\begin{array}{l}\text { RIPCN - } \quad \text { riparian condition } \\
\text { SEDLD - sediment load } \\
\text { TEMP - } \quad \text { water temperature } \\
\text { WITH - } \quad \text { water withdrawals }\end{array}$ \\
\hline
\end{tabular}

Life stages: 1 - prespawner; 2- spawner; 3- incubation; 4- fry colonization; 5- summer rearing; 6- overwintering; 7- yearling presmolt/smolt

"NA" indicates that no tife history pathway meeting the criteria used in defining these pathways was present.

Blank cells under attributes indicate no additional attributes with negative effects on productivity were evident compared to those present in the template. 


\section{Stream Reach Summary for Primary and Secondary Spring Chinook Salmon Life Histories}

Stream: Grande Ronde R.

Reach: Grossman Cr - Wallowa R (RM 61.1 -60.0 from Snake R)

\begin{tabular}{|ll|lr|l|}
\hline Restoration benefit category: & A & Chinook usage score: & 848.6 & Habitat productivity loss score:
\end{tabular}

\begin{tabular}{|c|c|c|c|c|c|c|c|c|c|c|c|c|c|c|}
\hline \multirow[b]{3}{*}{ Life stage } & \multirow[b]{3}{*}{ Months } & \multicolumn{10}{|c|}{ No. life history pathways by current usage abundance level } & \multicolumn{2}{|c|}{ Key habitat change } & \multirow{3}{*}{$\begin{array}{c}\text { Habital } \\
\text { productivity } \\
\text { loss score }\end{array}$} \\
\hline & & \multicolumn{5}{|c|}{ Primary } & \multicolumn{5}{|c|}{ Secondary } & \multirow{2}{*}{$\begin{array}{l}\text { Patient } \\
\text { quantity }\end{array}$} & \multirow[b]{2}{*}{$\%$ change } & \\
\hline & & Total no. & 1 & 2 & 3 & 4 & Total no. & 1 & 2 & 3 & 4 & & & \\
\hline 1 & Apr-Aug & 54 & 17. & 20. & 11 & 6 & 121 & 67 & 34 & 14 & 6 & 31.5 & 00 & 00 \\
\hline $1,2,3$ & Aug-Oct & $\mathrm{NA}$ & inA' & NA & NA & $\$$ & Nin. & & NA & & N & $\mathrm{NA}$ & NJ.. & NA \\
\hline 5 & May-Ott & 6 & 0 & 3 & 3 & 0 & 166 & 28 & $21 \ldots$ & 117 & 0 & 58.1 & 0.0 & -5294 \\
\hline 6 & Outt-Mar & 36 & 19. & 0 & 19 & 0 & 408 & 225 & 108 & 57 & 16 & 22.1 & 00 & -3143 \\
\hline 7 & Mar-Jun & 40 & 16 & 13 & 5 & 6 & 71 & 32 & 29 & 4 & 6 & 107.7 & 0.0 & 2. \\
\hline
\end{tabular}

\begin{tabular}{|c|c|c|c|c|c|c|c|c|c|c|c|c|c|}
\hline \multirow[b]{2}{*}{ Life stage } & \multirow[b]{2}{*}{ Months } & \multicolumn{12}{|c|}{ Highest productivity impact attributes (\% change in impact from template) } \\
\hline & & Att 1 & $\%$ change & Att 2 & $\%$ change & Att 3 & $\%$ change & Atf 4 & $\%$ change & Att 5 & $\%$ change & Att 6 & $\%$ change \\
\hline 1 & Apr-Aug & CHEM & 22,3 & NUTLD & 22,3 & OXYG & 22.3 & TEMP & 223 & & & & \\
\hline $1,2,3$ & Äug-Öci & NA & NA & NA & NA & NA & NA & NA & NÄ & $\ddot{N A}$ & $\mathrm{NA}$ & NA & NA \\
\hline$\dddot{3}$ & Ött-Mar & NÄ & NA & $\dot{N A}$ & N̈Ä & ثंNA & $\dot{N} \ddot{A}$ & NA & NÄ & NÄ & NA & NA & $\mathrm{NA}$ \\
\hline 34 & Mar-May & COMP & 50.0 & TEMP & 50.0 & CHEM & 25,0 & FLOW & 25.0 & NUTLË & $25.0^{\circ}$ & PATHO & 25.0 \\
\hline 5 & May-Ott & COMP & 500 & TEMP & 50.0 & SEDLD & 26.9 & CHEM & 250 & NUTLLD & 25.0 & PATHO & 250 \\
\hline 6 & Ott-Mar & S்EDLD & 26.4 & PATHO & 16.6 & TEMP & 16.6 & HABD & 13.2 & & & & \\
\hline 7 & Mar-Jun & PATHO & 9.9 & TEMP & 9.9 & & & & & & & & \\
\hline
\end{tabular}

Abundance level refers to the average abundance of spawners that spawn in the natal stream reaches that populate the life history pathways using the reach in this table: $\begin{array}{ll}\text { Level 1: } & 0-10 \text { spawners } \\ \text { Level 2 } & 10-30 \text { spawners } \\ \text { Level 3: } & 30-60 \text { spawners } \\ \text { Level 4: } & \mathbf{7 6 0} \text { spawners }\end{array}$

Life stages: 1- prespawner; 2-spawner; 3- incubation; 4- fry colonization; 5- summer rearing; 6- overwintering; 7- yearling presmolt/smolt

\begin{tabular}{|c|c|c|c|c|}
\hline Attributes: & $\begin{array}{l}\text { CHEM - } \\
\text { CSTAB - } \\
\text { COMP - } \\
\text { FLOW - } \\
\text { HABD - }\end{array}$ & $\begin{array}{l}\text { chemicals } \\
\text { channel stability } \\
\text { competition (with other species) } \\
\text { flow } \\
\text { habitat diversity }\end{array}$ & $\begin{array}{l}\text { NUTLD - } \\
\text { OBST - } \quad \text { obstructiont load } \\
\text { OXYG - } \quad \text { dissolved oxygen } \\
\text { PATHO - pathogens } \\
\text { PRED - } \quad \text { predators (includes fishing-related losses) }\end{array}$ & $\begin{array}{l}\text { RIPCN - } \quad \text { riparian condition } \\
\text { SEDLD - sediment load } \\
\text { TEMP - } \quad \text { water temperature } \\
\text { WITH - } \quad \text { water withdrawals }\end{array}$ \\
\hline
\end{tabular}

"NA" indicates that no life history pathway meeting the criteria used in defining these pathways was present.

Blank cells under attributes indicate no additional attributes with negative effects on productivity were evident compared to those preseintthe template. 


\section{Stream Reach Summary for Primary and Secondary Spring Chinook Salmon Life Histories}

Stream: Grande Ronde R.

Reach: Wallowa R - Lookingglass Cr (RM $80.0-83,4$ from Snake R)

\begin{tabular}{ll|ll|ll}
\hline Restoration benefit category: & F & Chinook usage score: & 78.4 & Habitat productivity ioss score: \\
\hline
\end{tabular}

\begin{tabular}{|c|c|c|c|c|c|c|c|c|c|c|c|c|c|c|}
\hline \multirow[b]{3}{*}{ Life stag } & \multirow[b]{3}{*}{ I Months } & \multicolumn{10}{|c|}{ No. life history pathways by current usage abundance level } & \multicolumn{2}{|c|}{ Key habitat change } & \multirow{3}{*}{$\begin{array}{c}\text { Habitat } \\
\text { productivity } \\
\text { ioss score }\end{array}$} \\
\hline & & \multicolumn{5}{|c|}{ Primary } & \multicolumn{5}{|c|}{ Secondary } & \multirow{2}{*}{$\begin{array}{c}\text { Patient } \\
\text { quantity }\end{array}$} & \multirow[b]{2}{*}{$\%$ change } & \\
\hline & & Tota & \begin{tabular}{l|l} 
no. & 1 \\
\end{tabular} & 2 & 3 & 4 & Total no. & 1 & 2 & 3 & 4 & & & \\
\hline 1 & spr-maty & 23 & $n$ & 14 & & 0 & 39 & 0 & 27 & 6 & 6 & 32,3 & 0.0 & -123 \\
\hline $1,2,3$ & Aug-OCI & Nä & N"A & NA" & $\mathrm{N} A \ddot{A}$ & $N A$ & NÄ & NA & NA & NA & NA & N & "NA" & NA \\
\hline 3 & Ott-Mar & $\mathrm{NÄ}$ & $\mathrm{NA}$ & NA" & $\mathrm{NÄ}$ & NA & NA & NA & NA & NA & $\mathrm{NA}$ & N & NA & NA \\
\hline 34 & Mar-May & 0 & o & 0 & 0 & 0 & $\ddot{6}$ & 0 & $1^{*}$ & 5 & 0 & 341. & 0.0 & -137 \\
\hline 5 & May-Ott & 6 & 0 & 3 & 3 & 0 & 0 & 0 & 0 & 0 & 0 & 7.9 & -14.0 & -116 \\
\hline 6 & Ott-Mar & 0 & 0 & 0 & 0 & 0 & 24 & 0 & 23 & $\cdots$ & 1 & 110 & -29 & $=0$ \\
\hline 7 & Mar-jun & $1 \overline{7}$ & 0 & 11 & 0 & 6 & 27 & 0 & 21 & "i & 5 & 96.7 & 0.0 & 15.4 \\
\hline
\end{tabular}

\begin{tabular}{|c|c|c|c|c|c|c|c|c|c|c|c|c|c|}
\hline \multirow[b]{2}{*}{ Life stage } & \multirow[b]{2}{*}{ Months } & \multicolumn{12}{|c|}{ Highest productivity impact attributes (\% change in impact fromtemplate) } \\
\hline & & Att 1 & $\%$ change & Att 2 & $\%$ change & Att 3 & ?6 change & Att 4 & $\%$ change & Att 5 & $\%$ change & Att 6 & $\%$ change \\
\hline & Apr-Aug & TEMP & 18.8 & CHEM & 9.4 & FLOW & 9.4 & HABD & 9.4 & NUTLD & 9.4 & OXYG & 9.4 \\
\hline $1,2,3$ & Aug-Oct & NA & NA & $N A$ & NÄ & N̈̈̈ & NÄ & NÄ & Nä & $\mathrm{NÄ}$ & $\mathrm{NÄ}$ & $\mathrm{NÄ}$ & NÄ \\
\hline 3 & Ott-Mar & $\mathrm{NA}$ & NA & NA & N̈̈̈̈ & N̈Ä & NA & ח̈̈ & Nä & NÄ & $\mathrm{NA}$ & NÄ & $\mathrm{NA}$ \\
\hline ,384 & Mar-May & FLOW & 25.0 & NUTLD & $25.0^{\circ}$ & SEDLD & 25.0 & TEMP & 25.0 & CHEM"M̈ & 12.5 & CSTAB & 12.5 \\
\hline 5 & May-Oct & TEMP & “28.0” & COMP & 14.0 ' & FLOW & 14.0 & HABD & 14.0 & PATHO & 14,0 & & \\
\hline 6 & Ott-Mar & SEDLD & 12.2 & CSTAB & 6.1 & HABD & 6.1 & PATHO & 6.1 & RIPCN & 6.1 & TEMP & 6.1 \\
\hline 7 & Mar-Jun & TEMP & 6.0 & & & & & & & & & & \\
\hline
\end{tabular}

Abundance level refers to the average abundance of spawners that spawn in the natai stream reaches that populate the life history pathways using the reach in thitable:

Level 1: $\quad 0-10$ spawnera

Levei 2: $\quad 10-30$ spawners

Levei 3: $\quad 30-60$ spawners

Levei $4>60$ spawners

Life stages: 1 - prespawner; 2- spawner; 3- incubation; 4- fry colonization; 5- summer rearing; 6- overwintering; 7- yearling presmolt/smelt

Attributes: CHEM - chemicals

CSTAB - channel stability

COMP - competition (with other species)

FLOW - ffOW

HABD - habitat diversity

NUTLD - nutrient load

OBST - obstructions

OXYG - dissolved oxygen

PATHO - pathogens

PRED - predators (includes fishing-related losses)
RIPCN - riparian condition

SEDLD - sediment ioad

TEMP - $\quad$ water temperature

WiTH - water withdrawals

"NA" indicates that no life history pathway meeting the criteria used in defining these pathways was present.

Blank cells under attributes indicate no additional attributes with negative effects on productivity were evident compared to those present in titemplate. 
Stream Reach Summary for Primary and Secondary Spring Chinook Salmon Life Histories

Stream: Grande Ronde R.

Reach: Lookingglass Cr - Gordon Cr (RM 83.4 -94.2 from Snake R)

\begin{tabular}{|ll|ll|ll}
\hline Restoration benefit category: & F & Chlnook usage score: & 57.7 & Habitat productivity loss score: \\
\hline
\end{tabular}

\begin{tabular}{|c|c|c|c|c|c|c|c|c|c|c|c|c|c|c|}
\hline \multirow[b]{3}{*}{ Life stage } & \multirow[b]{3}{*}{ Months } & \multicolumn{10}{|c|}{ No. life history pathways by current usage abundance level } & \multicolumn{2}{|c|}{ Key habitat change } & \multirow{3}{*}{$\begin{array}{c}\text { Habitat } \\
\text { productivity } \\
\text { loss score }\end{array}$} \\
\hline & & \multicolumn{5}{|c|}{ Primary } & \multicolumn{5}{|c|}{ Secondary } & \multirow{2}{*}{$\begin{array}{l}\text { Patient } \\
\text { quantity }\end{array}$} & \multirow[b]{2}{*}{$\%$ change } & \\
\hline & & Total no. & 1 & 2 & 3 & 4 & \begin{tabular}{|l} 
Total no. \\
\end{tabular} & 1 & 2 & 3 & 4 & & & \\
\hline $1 \ldots$ & Apr-Aug & 17 & 0 & 11 & 0 & 6 & 31 & 0 & 25 & 0 & 6 & 12.7 & 1.8 & -45.1 \\
\hline 123 & Aug-Öet & NA" & $\mathrm{NA}$ & $\mathrm{NA}$ & $\mathrm{NA}$ & $\mathrm{NA}$ & $\mathrm{NA}$ & $\mathrm{NA}$ & NÄ & NA & NA & NA & NA & NA \\
\hline 3 & Ött-Mar & NA & NA & NA & NA & NA & NA & NA & NA & NA & NA & $\mathrm{NÄ}$ & NA & NA \\
\hline 34 & Mar-May & NA. & NA & NA & NA & NA & NA & NA & NA & NA & NA & NA & NA & NA \\
\hline 5 & May-Ott & ㅇ... & 0. & …. & 0 & 0 & 1 & 0 & i..... & $\ddot{0}$ & 0 & 6.3 & 0.0 & -0.8 \\
\hline 6 & Öt-Mar & 0 & 0 & 0 & 0 & 0 & 115 & 0 & 78 & 0 & 37 & 8.0 & $\Omega 0$ & -28.4 \\
\hline $7 \bar{c}$ & Mardilin. & .17 & $\mathrm{n}$ & {$[11$} & n & 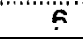 & 24 & 0 & 19 & 0 & 5 & 46.1 & 0.2 & $\ln 2$. \\
\hline
\end{tabular}

\begin{tabular}{|c|c|c|c|c|c|c|c|c|c|c|c|c|c|}
\hline \multirow[b]{2}{*}{ Life stage } & \multirow[b]{2}{*}{ Months } & \multicolumn{12}{|c|}{ Highest productivity impact attributes (\% change in impact from template) } \\
\hline & & Att 1 & \% change & Att 2 & $\%$ change & Att 3 & $\%$ change & Att 4 & \% change & Att 5 & $\%$ change & Alt 6 & $\%$ change \\
\hline 1 & Apr-Aug & FLOW & 16.8 & HABD & 16.8 & NUTLD & 16.8 & RIPCN & 16.8 & CHEM & 8.4 & OXYG & 8.4 \\
\hline $1,2,3$ & Aug-Oct & $\mathrm{NA}$ & NÄ & NA & NA & $\mathrm{NA}$ & NA & $\mathrm{NA}$ & NA & $\mathrm{NA}$ & NA & NÄ & NA \\
\hline 3 & Oct-Mar & $\mathrm{NA}$ & NA & $N A$ & NA & $\mathrm{NA}$ & $\mathrm{NA}$ & $\mathrm{NA}$ & $\mathrm{NA}$ & $\mathrm{NA}$ & NA & NA & $\mathrm{NA}$ \\
\hline 3,4 & Mar-May & NA & NA & $\mathrm{NA}$ & NA & $N A$ & $\mathrm{NA}$ & $\mathrm{NA}$ & $\mathrm{NA}$ & $\mathrm{NA}$ & NA & $\mathrm{NA}$ & NA \\
\hline 5 & May-Oct & SEDLD & 130 & FLOW & 6.5 & HABD & 6.5 & PATHO & 6.5 & TEMP & 6.5 & & \\
\hline 6 & Oct-Mar & SEDLD & 6.7 & FLOWW & 3.4 & HABD & 3.4 & PATHO & 3.4 & TEMP & 3.4 & & \\
\hline 7 & Mar-Jun & TEMP & 4.3 & & & & & & & & & & \\
\hline
\end{tabular}

Abundance /eve/ refers to the average abundance of spawners that spawn in the natal stream reaches that populate the life history pathways using the reach in this table:

$$
\begin{array}{ll}
\text { Level 1: } & 0-10 \text { spawners } \\
\text { Level 2: } & 10-30 \text { spawners } \\
\text { Level 3: } & 30-60 \text { spawners } \\
\text { Level 4: } & \mathbf{2} 60 \text { spawners }
\end{array}
$$

\begin{tabular}{|c|c|c|c|}
\hline Attributes: & $\begin{array}{l}\text { CHEM - chemicals } \\
\text { CSTAB - channel stability } \\
\text { COMP - competition (with other species) } \\
\text { FLOW - ftOW } \\
\text { HABD - habitat diversity }\end{array}$ & $\begin{array}{l}\text { NUTLD - } \\
\text { OBST - } \quad \text { obstructiont load } \\
\text { OXYG - } \quad \text { dissolved oxygen } \\
\text { PATHO - pathogens } \\
\text { PRED - } \quad \text { predators (includes fishing-related losses) }\end{array}$ & $\begin{array}{l}\text { RIPCN - } \quad \text { riparian condition } \\
\text { SEDLD - } \\
\text { TEMPiment load } \\
\text { WITH - } \quad \text { water temperature } \\
\text { Water withdrawals }\end{array}$ \\
\hline
\end{tabular}

Life stages: 1 - prespawner; 2-spawner; 3- incubation; 4- fry colonization; 5- summer rearing; 6- overwintering; 7- yearling presmolt/smolt

"NA" indicates that no life history pathway meeting the criteria used in defining these pathwaya was present.

Blank cells under attributes indicate no additional attributes with negative effects on productivity were evident compared to those present in the template. 


\section{Stream Reach Summary for Primary and Secondary Spring Chinook Salmon Life Histories}

Stream: Grande Ronde R.

Reach: Gordon $\mathbf{C r}$ - Phillips Cr (RM 94.2 -96.9 from Snake R)

\begin{tabular}{lll|ll|l|l|}
\hline Restoration benefit category: & F & Chinook usage score: & 31,0 & Habltat productivity loss score: & -14.4 \\
\hline
\end{tabular}

\begin{tabular}{|c|c|c|c|c|c|c|c|c|c|c|c|c|c|c|}
\hline \multirow[b]{3}{*}{ Life stage } & \multirow[b]{3}{*}{ Months } & \multicolumn{10}{|c|}{ No. life history pathways by current usage abundance level } & \multicolumn{2}{|c|}{ Key habitat change } & \multirow{3}{*}{$\begin{array}{c}\text { Habitat } \\
\text { productivity } \\
\text { loss score }\end{array}$} \\
\hline & & \multicolumn{5}{|c|}{ Primary } & \multicolumn{5}{|c|}{ Secondary } & \multirow{2}{*}{$\begin{array}{l}\text { Patient } \\
\text { quantity }\end{array}$} & \multirow[b]{2}{*}{$\%$ change } & \\
\hline & & Total no. & 1 & 2 & 3 & 4 & Total no, & 1 & 2 & 3 & 4 & & & \\
\hline \begin{tabular}{|l} 
\\
\end{tabular} & Apr-Aug & 17 & 0 & 11 & 0 & 6 & ? & 0 & 25 & 0 & $?$ & 18.4 & -4 & .14 .8 \\
\hline $1,2,3$ & Aug-Oct & $\dot{N} \dot{A}$ & NA & NA & ‘A & NA & $\mathrm{NA}$ & $\mathrm{NA}$ & NA & NA" & $\mathrm{NA}$ & $\mathrm{NA}$ & $\mathrm{N}$ & NÄ \\
\hline $.34_{1}$ & Mar May, & ו & וIוI, & I & NA & $N A$ & NA. & NA & $\mathrm{NA}$ & NA & NA & NA & NA & NA \\
\hline 5 & May-Ott & 0 & 0 & 0 & 0 & $\dot{0}$ & 3 & 0 & 26. & 0 & $i$ & 13.1 & ?.?? & 26 \\
\hline 6 & OctMar & 18 & 0 & 07 & 0 & 18 & 28 & 01 & 5 & & & 10.3 & -10.7 & 6.2 \\
\hline $\mathrm{Ma}$ & 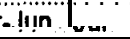 & " & & $111^{\prime}$ & & & 18 & & & & & 3.1 & $-1.1^{\circ}$ & 9.2 \\
\hline
\end{tabular}

\begin{tabular}{|c|c|c|c|c|c|c|c|c|c|c|c|c|c|}
\hline \multirow[b]{2}{*}{ Life stage } & \multirow[b]{2}{*}{ Months } & \multicolumn{12}{|c|}{ Highest productivity impact attributes (\% change in impact from template) } \\
\hline & & Att 1 & $\%$ change & Aft 2 & $\%$ change & An 3 & $\%$ change & Att 4 & $\%$ change & Att 5 & $\%$ change & Att 6 & $\%$ change \\
\hline 1 & Apr-Aug & TEMP & 6.1 & FLOW & 4.1 & HABD & 4.1 & NUTLD & 4.1 & RIPCN & 4.1 & CHEM & 2.0 \\
\hline 1.2 .3 & Aug-Öct & NA & NA & $\dot{N A}$ & NA & NA & NA & NA & & in & "NÁ" & NA & \\
\hline 3 & Ott-Mar & NA & NA & NA & NA & NA & NA. & "NA" & & "in. & "NA' & NA" & \\
\hline 3,4 & Mar-May & NA & NA & NA & NA & NA & NA & NA & NA & NA & NA & NA & $\therefore A$ \\
\hline 5 & May-Ott & FLOW & 20.6 & TEMP & 20.6 & $\mathrm{HABD}$ & 13.7 & NUTLD & 13.7 & RIPCN & 13.7 & CHEM & 6.9 \\
\hline$\dot{6}$ & Ott-Mar & $\dot{H A B D}$ & 4.4 & РАTंHO & 4.4 & SEÉLD & 4.4 & TEMP & 4.4 & & & & \\
\hline 7 & Mar-Jun & TEMP & 4.5 & & & & & & & & & & \\
\hline
\end{tabular}

Abundance level refers to the average abundance of spawners that spawn in the natal stream reaches that populate the life history pathways using the reach in this table

$$
\begin{array}{ll}
\text { Level 1: } & 0-10 \text { spawners } \\
\text { Level 2: } & 10-30 \text { spawners } \\
\text { Level 3: } & 30-60 \text { spawners } \\
\text { Level 4: } & \mathbf{7 0 0} \text { spawners }
\end{array}
$$

Life stages: 1- prespawner; 2- spawner; 3- incubation; 4- fry colonization; 5- summer rearing; 6- overwintering; 7- yearling presmolt/smolt

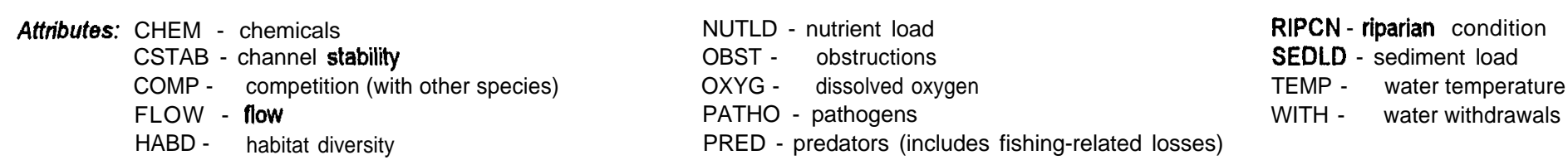

"NA" indicates that no tife history pathway meeting the criteria used in defining these pathways was present.

Blank cells under attributes indicate no additional attributes with negative effects on productivity were evident compared to those present in the template. 
Stream Reach Summary for Primary and Secondary Spring Chinook Salmon Life Histories

Stream: Grande Ronde R.

Reach: Phillips Cr - Clark Cr (RM 96.9 -97.3 from Snake R)

\begin{tabular}{|ll|ll|l|}
\hline Restoration benefit category: & F & Chlnook usage score: & 11.5 & Habitat productivity loss score: \\
\hline
\end{tabular}

\begin{tabular}{|c|c|c|c|c|c|c|c|c|c|c|c|c|c|c|}
\hline \multirow[b]{3}{*}{ Life stage } & \multirow[b]{3}{*}{ Months } & \multicolumn{10}{|c|}{ No. life history pathways by current usage abundance level } & \multicolumn{2}{|c|}{ Key habitat change } & \multirow{3}{*}{$\begin{array}{l}\text { Habitat } \\
\text { productivity } \\
\text { loss score }\end{array}$} \\
\hline & & \multicolumn{5}{|c|}{ Primary } & \multicolumn{5}{|c|}{ Secondary } & \multirow{2}{*}{$\begin{array}{l}\text { Patient } \\
\text { quantity }\end{array}$} & \multirow[b]{2}{*}{$\boldsymbol{\%}_{\text {chang }}$} & \\
\hline & & Total no. & 1 & 2 & 3 & 4 & Total no. & 1 & 2 & 3 & 4 & & & \\
\hline 1 & Apr-Aug & 17 & 0 & 11 & 0 & 6. & 31 & 0 & 25 & 0 & 6 & 13.7 & -4.0 & -11.4 \\
\hline 123 & Äug-Ott & NÄ & $\mathrm{NA}$ & NA & NA & NÄ & & NA & NÄ & $\mathrm{NAn}$ & ṆA & $\mathrm{NA}$ & NA & $\mathrm{NA}$ \\
\hline 34 & Mar-May & NA & NA & NA & $\mathrm{NA}$ & NA & $N A^{\prime}, " ; "$ & NA & $\mathrm{NA}$ & NA“““" & $N A$ & $\mathrm{~N} A$ & NA & NA \\
\hline 5 & May-Ott & 0 & . & 0. & 0 & 0 & 3 & 0 & 2 & 0 & $i$ & 131 & -0.6 & -06 \\
\hline 6 & Ott-Mar & 1 & 0 & 0 & 0 & 1 & 11 & 0 & 9 & 0 & 2 & 53 & -75 & -11 \\
\hline 7 & Mar-Jun & 16 & 0 & 11 & 0 & 5 & 17 & 0 & 14 & 0 & 3 & 36.8 & 3.0 & 3.9 \\
\hline
\end{tabular}

\begin{tabular}{|c|c|c|c|c|c|c|c|c|c|c|c|c|c|}
\hline \multirow[b]{2}{*}{ Life stage } & \multirow[b]{2}{*}{ Months } & \multicolumn{12}{|c|}{ Highest productivity impact attributes (\% change in impact from template) } \\
\hline & & Art 1 & \% change & Att 2 & $\%$ change & Att 3 & \%change & Att 4 & \% change & Att 5 & $\%$ change & Att 6 & $\%$ change \\
\hline I & Apr-Aug & TEMP & 4.7 & FLOW & 3.2 & HABD & 3.2 & NUTLD & 3.2 & RIPCN & 3.2 & CHEM & 1.6 \\
\hline $1,2,3$ & Aug-Oct & NA & NA & $\ddot{N} \mathrm{~A}$ & $\mathrm{NA}$ & NÄ & NA & NA" & NÄ & NÄ & Nä & $\mathrm{NÄ}$ & $\mathrm{NA}$ \\
\hline 3 & Ott-Mar & $N A$ & $\mathrm{NA}$ & NÄ & NA & NÄ & NÄ & NA & NÄ & NA" & NÄ & $\mathrm{NA}$ & NA \\
\hline 3,4 & Mar-May & NA & NA & NA & NA & NA & NA & NA & NA & NA & NA & NA & NA \\
\hline 5 & May-Oct & HABD & 1.6 & PATHO & 1.6 & RIPCN & 1.6 & SEDLD & 1.6 & TEMP & 1.6 & & \\
\hline 6 & Ott-Mar & HABD & 3.0 & РATHOO & 3.0 & RIPCN & 3.0 & SÉḊLD & 3.0 & TEMP & 3.0 & & \\
\hline 7 & Mar-Jun & TEMP & 2.0 & & & & & & & & & & \\
\hline
\end{tabular}

Abundance /eve/ refers to the average abundance of spawners that spawn in the natal stream reachea that populate the life history pathways using the reach in this table

$$
\begin{array}{ll}
\text { Level 1: } & 0-10 \text { spawners } \\
\text { Level 2: } & 10-30 \text { spawners } \\
\text { Level 3: } & 30-60 \text { spawners } \\
\text { Level 4 } & >\mathbf{6 0} \text { spawners }
\end{array}
$$

Life stages: 1- prespawner; 2- spawner; 3- incubation; 4- fry colonization; 5- summer rearing; 6- overwintering; 7- yearling presmolt/smolt

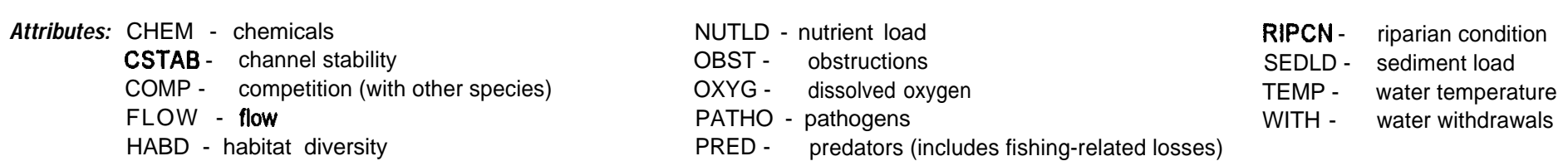

"NA" indicates that no tife history pathway meeting the criteria used in defining these pathways was present.

Blank cells under attributes indicate no additional attributes with negative effects on productivity were evident compared to those present in the template, 
Stream Reach Summary for Primary and Secondary Spring Chinook Salmon Life Histories

Stream: Grande Ronde R.

Reach: $\quad$ Clark Cr - Indian Cr (RM 97,3 -99.3 from Snake R)

\begin{tabular}{|c|c|c|c|c|c|c|c|c|c|c|c|c|c|c|}
\hline Restoration & benefit & category: & & & Chinook & usage & score: & 21,5 & Habitat & ducti & loss & & -32.0 & \\
\hline \multirow[b]{3}{*}{ Lif } & \multirow[b]{3}{*}{ Months } & \multicolumn{10}{|c|}{ No. iife history pathways by current usage abundancelevel } & \multicolumn{2}{|c|}{ Key habitat change } & \multirow{3}{*}{$\begin{array}{c}\text { Habital } \\
\text { productivity } \\
\text { loss score }\end{array}$} \\
\hline & & \multicolumn{5}{|c|}{ Primary } & \multicolumn{5}{|c|}{ Secondary } & \multirow{2}{*}{$\begin{array}{l}\text { Patient } \\
\text { quantity }\end{array}$} & \multirow[b]{2}{*}{$\%$ change } & \\
\hline & & Total no. & 1 & 2 & 3 & 4 & Total no & 1 & 2 & 3 & 4 & & & \\
\hline INA|||||||||||||||| & Aug-Oct & NÄ" & NÄ & NA" & $\mathrm{NÄ}$ & Nä & NA & NA" & $\mathrm{NA}$ & $\mathrm{NÄ}$ & NÄ & NA & NA & $\mathrm{NA}$ \\
\hline "3णा|ा। & Ott-Mar & NA & NA & NA & NA & NA & $\mathrm{NA}$ & NA & $\mathrm{NA}$ & NA & $\mathrm{NA}$ & NA & $\mathrm{NA}$ & $\mathrm{NA}$ \\
\hline 34 & Mar-May & NA & NA & NA & NA & NA & $\mathrm{NA}$ & $\mathrm{NA}$ & NA & NA & $\mathrm{NA}$ & NA & $N A$ & $\mathrm{NA}$ \\
\hline 5 & May-Ott & 0 & 0 & 0 & 0 & 0 & 4 & 0 & 3 & 0 & 1 & 131 & oo & 0.7 \\
\hline 6 & Oct-Mar & 1 & 0 & 0 & 0 & 1 & 26 & 0 & 26 & 0 & 2 & 6.8 & -6.6 & -3.8 \\
\hline 7 & Mar-Jun & 16 & 0 & 11 & 0 & 5 & 17 & 0 & 14 & 0 & 3 & 47.3 & 0.6 & 3.5 \\
\hline
\end{tabular}

\begin{tabular}{|c|c|c|c|c|c|c|c|c|c|c|c|c|c|}
\hline \multirow[b]{2}{*}{ Life stage } & \multirow[b]{2}{*}{ Months } & \multicolumn{12}{|c|}{ Highest productivity impact attributes ${ }_{\alpha}$ change in impact from template) } \\
\hline & & Aft 1 & $\%$ change & Atf 2 & $\%$ change & Atf 3 & ?6 change & Att 4 & $\%$ change & Att 5 & $\%$ change & Aft 6 & $\%$ change \\
\hline 1 & Apr-Aug & TEMP & 12.9 & FLOW & 6.6 & HABD & 6.6 & NUTLD & 8.6 & RIPCN & 8.6 & CHEM & 4.3 \\
\hline 12,3 & Aug-Ott & NA & NA & N̈̈ & NÄ & $N A^{\circ}$ & NÄ & NÄ & NA & NA & NA & $\ddot{N A}$ & NA \\
\hline 3 & Ott-Mar & NA & NA & NA & NA & ก̈̈̈ & NÄ & Nั̈̈ & $\mathrm{NA}$ & $\mathrm{NÄ}$ & $\mathrm{NA}$ & $\mathrm{NA}$ & NÄ \\
\hline 3,4 & Mar-May & NA & NA & NA & NA & NA & ח̈̈̈ & NA & NA & NA & NA & $?$ & $\mathrm{NA}$ \\
\hline 5 & May-Ott & FLOW & 3.9 & TEMP & 3.9 & HABD & 2.6 & RIPCN & 2.6 & $\mathrm{CHEM}$ & .13 & COMP & 1.3 \\
\hline 6 & Ött-Mar & TEMP & 3.1 & CSTÄB & 2.6 & "HÄB̈D̆ & 2.8 & "PÄT̈HO & 2.8 & RIPCN & 2.8 & SEDOLD & 2.8 \\
\hline 7 & Mar-Jun & TEMP & 1.8 & & & & & & & & & & \\
\hline
\end{tabular}

Abundance level refers to the average abundance of spawners that spawn in thenatal stream reaches that populate the life history pathways using the reach in this table

$$
\begin{array}{ll}
\text { Level 1: } & 0-10 \text { spawners } \\
\text { Level 2: } & 10-30 \text { spawners } \\
\text { Level 3: } & 30-60 \text { spawners } \\
\text { Level 4: } & >\mathbf{6 0} \text { spawners }
\end{array}
$$

Life stages: 1- prespawner; 2- spawner; 3- incubation; 4- fry colonization; 5- summer rearing; 6- overwintering; 7- yearling presmolt/smolt

$\begin{array}{lll}\text { Attributes: } \text { CHEM - chemicals } & \text { NUTLD - nutrient ioad } & \text { RIPCN - riparian condition } \\ \text { CSTAB - channel stability } & \text { OBST - obstructions } & \text { SEDLD - sediment ioad } \\ \text { COMP - competition (with other species) } & \text { OXYG - dissolved oxygen } & \text { TEMP - water temperature } \\ \text { FLOW - fiOW } & \text { PATHO - pathogens } & \text { WiTH - water withdrawals } \\ \text { HABD - habitat diversity } & \text { PRED - predators (includes fishing-related losses) }\end{array}$

"NA" indicates that no life history pathway meeting the criteria used in defining these pathways was present.

Blank cells under attributes indicate no additional attributes with negative effects on productivity were evident compared to those present in ttemplate. 
Stream Reach Summary for Primary and Secondary Spring Chinook Salmon Life Histories

Stream: Grande Ronde R.

Reach: Indian Cr - Willow Cr (RM 99.3 -103.5 from Snake R)

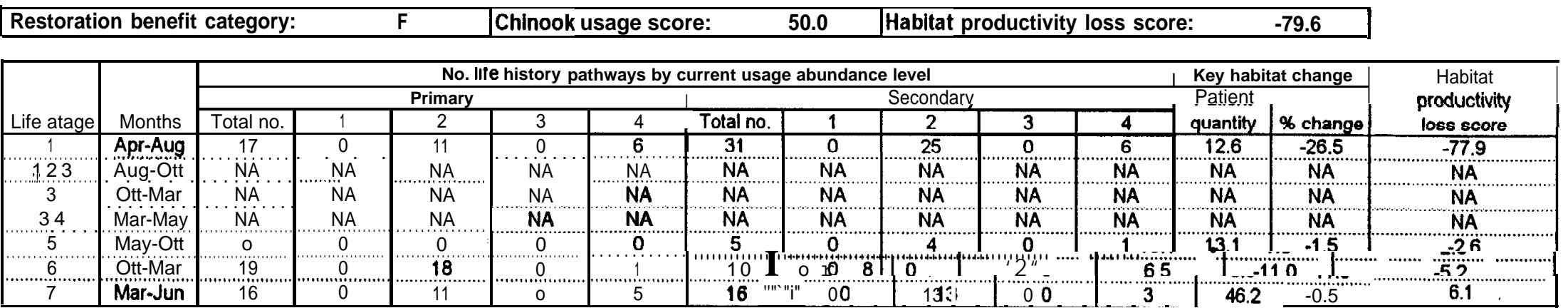

\begin{tabular}{|c|c|c|c|c|c|c|c|c|c|c|c|c|c|}
\hline \multirow[b]{2}{*}{ Life stage } & \multirow[b]{2}{*}{ Months } & \multicolumn{12}{|c|}{ Highest productivity impact attributes (\% change in impact from template) } \\
\hline & & Att 1 & $\%$ change & Att 2 & $\%$ change & Att 3 & \%change & Aft 4 & $\%$ change & Att 5 & $\%$ change & Att 6 & $\%$ change \\
\hline 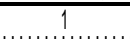 & Apr-Aug & TEMP & 322 & FLOW & 21.5 & HABD & 21.5 & NUTLD & 21.5 & RIPCN & 21.5 & CHEM & 107 \\
\hline $1,2,3$ & Äug-Öci & NA & NÄ & NA & NA & NA & NÄ & NA & NA & NÄ & NA & NA & NA \\
\hline 3 & Ott-Mar & $\mathrm{NA}$ & $\mathrm{NA}$ & NA & NA & NA & NÄ & Nä & $\mathrm{NA}$ & $\mathrm{NA}$ & $\mathrm{NA}$ & NÄ & \\
\hline 3,4 & Mar-,May & NA & NA & NA & NA & NA & NA & NA & NA & $\mathrm{NA}$ & $\mathrm{NA}$ & NA & "̈Ä \\
\hline $5^{\prime}$ & May-Ott & HABD & 41 . & PATHO & 4,1 & RIPCN & 4.1 & SEDLD & 4.1 & TEMP & 4.1 & & \\
\hline 6 & Ott-Mar & HABD & 4.4 & PATHÖ & 44 & RIPCN & 4.4 & SEELEL & 4.4 & TEMP & 4.4 & & \\
\hline 7 & Mar-Jun & TEMP & 3.3 & & & & & & & & & & \\
\hline
\end{tabular}

Abundance level refers to the average abundance of spawners that spawn in the natal stream reaches that populate the life history pathways using the reach In this table:

$$
\begin{array}{ll}
\text { Level 1: } & 0-10 \text { spawners } \\
\text { Level 2: } & 10-30 \text { spawners } \\
\text { Level 3: } & 30-60 \text { apawners } \\
\text { Level4: } & \quad \mathbf{6 0} \text { spawners }
\end{array}
$$

\begin{tabular}{|c|c|c|c|}
\hline Attributes: & $\begin{array}{l}\text { CHEM - chemicals } \\
\text { CSTAB - channel stability } \\
\text { COMP - competition (with other species) } \\
\text { FLOW - flow } \\
\text { HABD - habitat diversity }\end{array}$ & $\begin{array}{l}\text { NUTLD - nutrient load } \\
\text { OBST - obstructions } \\
\text { OXYG - dissolved oxygen } \\
\text { PATHO - pathogens } \\
\text { PRED - predators (includes fishing-related losses) }\end{array}$ & $\begin{array}{l}\text { RIPCN - } \quad \text { riparian condition } \\
\text { SEDLD - } \\
\text { TEdiment load } \\
\text { TEMP - } \quad \text { water temperature } \\
\text { WITH - } \quad \text { water withdrawals }\end{array}$ \\
\hline
\end{tabular}

Life stages: 1- prespawner; 2- spawner; 3- incubation; 4- fry colonization; 5- summer rearing; 6- overwintering; 7- yearling presmolt/smolt

"NA" indicates that no life history pathway meeting the criteria used in defining these pathways was present.

Blank cells under attributes indicate no additional attributes with negative effects on productivity were evident compared to those present in the template. 


\section{Stream Reach Summary for Primary and Secondary Spring Chinook Salmon Life Histories}

Stream: Grande Ronde R.

Reach: Willow $\mathrm{Cr}$ - downstream end of State Ditch (RM 103.5 -114,5 from Snake R)

\begin{tabular}{|ll|ll|l|}
\hline Restoration benefit category: & $F$ & Chinook usage score: & 92,3 & Habitat productivity loss score: \\
\hline
\end{tabular}

\begin{tabular}{|c|c|c|c|c|c|c|c|c|c|c|c|c|c|c|}
\hline \multirow[b]{3}{*}{ Life stage } & \multirow[b]{3}{*}{ Months } & \multicolumn{10}{|c|}{ No. life history pathways by current usage abundance level } & \multicolumn{2}{|c|}{ Key habitat change } & \multirow{3}{*}{$\begin{array}{c}\text { Habitat } \\
\text { productivity } \\
\text { loss score }\end{array}$} \\
\hline & & \multicolumn{5}{|c|}{ Primary } & \multicolumn{5}{|c|}{ Secondary } & \multirow{2}{*}{$\begin{array}{l}\text { Patient } \\
\text { quantity }\end{array}$} & \multirow[b]{2}{*}{$\%$ change } & \\
\hline & & Total no. & 1 & 2 & 3 & 4 & Total no. & 1 & 2 & 3 & 4 & & & \\
\hline 1 & Apr-Aug & 17 & …. & 11 & 0 & 6 & 31 & 0 & 25 & 0 & 6 & 43.1 & 3.6 & 51.4 \\
\hline $1,2,3$ & Aug-Öct & NA & NÄ & $\mathrm{NA}$ & NÄ & $\mathrm{NÄ}$ & $\mathrm{NA}$ & NA & $\mathrm{NA}$ & $\mathrm{NA}$ & NA & $N A$ & $N A$ & $\mathrm{NA}$ \\
\hline 3 & Ott-Mar & NA & NA & NA & NA & NA & NA & $\ddot{N A}$ & $\mathrm{NA}$ & $\mathrm{NA}$ & NA & NA & NA & NA \\
\hline 34 & Mar-May & $N \ldots A$ & $\mathrm{NA}$ & NA & NA & $\mathrm{NA}$ & NA & $\mathrm{N} \quad \mathrm{A}$ & $\mathrm{NA}$ & NA & $\mathrm{NA}$ & NA & NA & NA \\
\hline 5 & May-Ott & 1 & o & 0 & 0 & 1. & 11 & 0 & 9 & 0 & 2 & 62 & -22.7 & -18.8 \\
\hline 6 & Ott-Mar & 20 & 0 & 1 & 0 & 19 & 58 & $" 0$ & 57 & 0 & 1 & 8.5 & -7.6 & -15.3 \\
\hline 7 & Mar-Jun & 15 & 0 & 10 & 0 & 5 & 16 & 0 & 13 & 0 & $\dddot{3}$ & 49.6 & -4.0 & 12.5 \\
\hline
\end{tabular}

\begin{tabular}{|c|c|c|c|c|c|c|c|c|c|c|c|c|c|}
\hline \multirow[b]{2}{*}{ Llfe stage } & \multirow[b]{2}{*}{ Months } & \multicolumn{12}{|c|}{ Highest productivity impact attributes (\% change in impact from template) } \\
\hline & & Att 1 & $\%$ change & Att 2 & $\%$ change & Att 3 & $\%$ change & Att 4 & $\%$ change & Att 5 & $\%$ change & Att 6 & $\%$ change \\
\hline 1 & Apr-Aug & FLOW & 46.8 & CHEM & 31.2 & HABD & 31.2 & NUTLD & 31.2 & OXYG & 31.2 & RIPCN & 31.2 \\
\hline $1,2,3$ & Aug-Oct & $\mathrm{NA}$ & NA & $\mathrm{NA}$ & $\mathrm{NA}$ & NA & NA & NA & NA & $\mathrm{NA}$ & NÄ & $\mathrm{NA}$ & NA \\
\hline 3 & Oct-Mar & $\mathrm{NA}$ & $\mathrm{NA}$ & $\mathrm{NA}$ & $\mathrm{NA}$ & NA" & $\mathrm{NA}$ & $\mathrm{NA}$ & $\mathrm{NA}$ & $\mathrm{NA}$ & $\mathrm{NA}$ & $\mathrm{NA}$ & $\mathrm{NA}$ \\
\hline 3,4 & Mar-May & NA & NA" & NA & NÄ & NA & NA & NA & NA & NA & $\mathrm{NA}$ & $\mathrm{NA}$ & $\ddot{N} \ddot{n}$ \\
\hline 5 & May-Ott & $F \perp O W$ & 24.0 & HABD' & $24.0^{\circ}$ & RIPCN & 24.0"' & TEMP & 24.0 & CHEM & 12.0 & NUTLD & $12.0^{\circ}$ \\
\hline 6 & Ott-Mar & С̈HË̈̈ & 3.8 & HABDD & 28 & NÜTTLED" & 3.8 & PATTHO & 3.8 & RIPCN & 3.8 & SEDLLD & 3.8 \\
\hline 7 & Mar-Jun & TEMP & 8.9 & WİTH & 6.9 & & & & & & & & \\
\hline
\end{tabular}

Abundance lovel refers to the average abundance of spawners that spawn in the natal stream reaches that populate the life history pathways using the reach in this table:

$$
\begin{array}{ll}
\text { Level 1: } & 0-10 \text { spawners } \\
\text { Level 2: } & 10-30 \text { spawners } \\
\text { Level 3: } & 30-60 \text { spawners } \\
\text { Level 4: } & >60 \text { spawners }
\end{array}
$$

\begin{tabular}{|c|c|c|c|}
\hline Attributes: & $\begin{array}{l}\text { CHEM - chemicals } \\
\text { CSTAB - channel stability } \\
\text { COMP - competition (with other species) } \\
\text { FLOW - ffOW } \\
\text { HABD - habitat diversity }\end{array}$ & 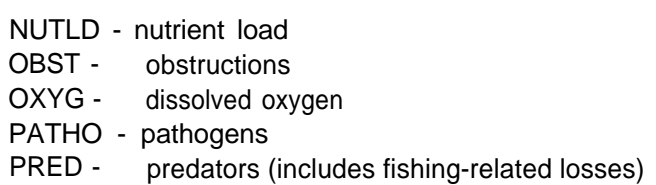 & $\begin{array}{l}\text { RIPCN - } \\
\text { SEDLD - } \\
\text { TEMP - } \\
\text { WITH - }\end{array}$ \\
\hline
\end{tabular}

Life stages: 1- prespawner; 2-spawner; 3- incubation; 4- fry colonization; 5- summer rearing; 6- overwintering; 7-yearling presmolt/smolt

"NA" indicates that no life history pathway meeting the criteria used in defining these pathwaywas present.

Blank cells under attributes indicate no additional attributes with negative effects on productivity were evident compared to those present in the template, 
Stream Reach Summary for Primary and Secondary Spring Chinook Salmon Life Histories

Stream: Grande Ronde R.

Reach: Downstream end of State Ditch - Upstream end of State Ditch (RM 114.5 -118.9 from Snake R)

\begin{tabular}{ll|ll|l|}
\hline Restoration benefit category: & D & Chinook usage score: & 62.5 & Habitat productivity loss score: \\
\hline
\end{tabular}

\begin{tabular}{|c|c|c|c|c|c|c|c|c|c|c|c|c|c|c|}
\hline \multirow[b]{3}{*}{ Life stage } & \multirow[b]{3}{*}{ Months } & \multicolumn{10}{|c|}{ No. life history pathways by current usage abundance level } & \multicolumn{2}{|c|}{ Key habitat change } & \multirow{3}{*}{$\begin{array}{c}\text { Habitat } \\
\text { productivity } \\
\text { loss score }\end{array}$} \\
\hline & & \multicolumn{5}{|c|}{ Primary } & \multicolumn{5}{|c|}{ Secondary } & \multirow{2}{*}{$\begin{array}{c}\text { Patient } \\
\text { quantity }\end{array}$} & \multirow[b]{2}{*}{$\%$ change } & \\
\hline & & Total no. & 1 & 2 & 3 & 4 & Total no & 1 & 2 & 3 & 4 & & & \\
\hline 1 & Apr-Aug & 8 & 0 & 7 & 0 & 1 & 24 & 0 & 21 & 0 & 3 & 11.6 & -318 & -29.1 \\
\hline 123 & Aug-Ott & $\ddot{N A}$ & $\mathrm{NA}$ & NA & NA & NA & NA & $\mathrm{NA}$ & $\dddot{\mathrm{NÄ}}$ & NA & $\mathrm{NA}$ & $\mathrm{NA}$ & NA & NÄ \\
\hline 3 & Ott-Mar & NA & "NA & NA & $\mathrm{NA}$ & NÄ & NA & NA & NA & NA & NA & NA & $\dddot{\mathrm{NA}}$ & NA \\
\hline 34 & Mar-May & NA & 'NA' & NA & NA & $\mathrm{NA}$ & NÄ & NA & NA & NA & NA & NA & NA & NA \\
\hline 5 & May-Ott & 0 & 0 & 0 & 0 & 0 & 11 & 0 & 10 & 0 & 1 & 8.3 & $-1 \dot{7} .1$ & -24.8 \\
\hline 6 & Ott-Mar & 0 & 0 & 0 & 0 & 0 & 58 & 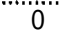 & 40 & 0 & ${ }^{118}$ & $\cdots \cdots$ & -41.8 & -153.6 \\
\hline 7 & Marolun & ? & $n$ & 7 & n & 4 & 10 & C & 8 & 0 & 2 & so.3 & $=0,4$ & $A_{\mathrm{r}}$ \\
\hline
\end{tabular}

\begin{tabular}{|c|c|c|c|c|c|c|c|c|c|c|c|c|c|}
\hline \multirow[b]{2}{*}{ Life stage } & \multirow[b]{2}{*}{ Months } & \multicolumn{12}{|c|}{ Highest productivity impact attributes (\% change in impact from template) } \\
\hline & & Aft 1 & $\%$ change & $\overline{\text { Att } 2}$ & $\%$ change & Aft 3 & $\%$ change & Aft 4 & $\%$ change & Att 5 & $\%$ change & Aft 6 & $\%$ change \\
\hline 1 & Apr-Aug & HABD & 29.6 & FLOW & 19.9 & RIPCN & 19.9 & TEMP & 19.9 & & & & \\
\hline 123 & Aug-Ott & NA & $N A \ddot{~}$ & NA & NA & NA & NA & NA & NA & NA & NA & NA & $N A^{\circ}$ \\
\hline 3 & Ott-Mar & NA & "NA & NA & NA & $\ddot{N A}$ & NA & $\mathrm{NÄ}$ & NA & NA & $\dot{\mathrm{NA}}$ & NA & NA \\
\hline 3,4 & Mar-May & $\mathrm{NA}$ & N̈A & NA & NA & NÄ & NA & NA & A & NA & NA & NA & NA \\
\hline 5 & May-Oct & FLOW & 25.7 & HABD & 25.7 & RIPCN & 25.7 & SEDLD & 25,7 & CHEM & 8,6 & PATHO & \& 6 \\
\hline 6 & Ött-Mar & FLOW & 33.6 & HABD & 336 & RIPCN & 33.6 & SEELID & 336 & СHEM & 11.2 & PATHO & 11.2 \\
\hline 7 & Mar-Jun & $\mathrm{HABD}$ & 6.5 & & & & & & & & & & \\
\hline
\end{tabular}

Abundance level refers to the average abundance of spawners that spawn in the natal stream reaches that populate the fife history pathways using the reach in this table:

$$
\begin{array}{ll}
\text { Level 1: } & 0-10 \text { spawners } \\
\text { Level 2: } & 10-30 \text { spawners } \\
\text { Level 3: } & 30-60 \text { spawners } \\
\text { Level 4: } & >60 \text { spawners }
\end{array}
$$

Life stages: 1-prespawner; 2-spawner; 3- incubation; 4- fry colonization; 5- summer rearing; 6- overwintering; 7- yearling presmoltsmolt

$\begin{array}{lll}\text { Attributes.' } \text { CHEM - chemicals } & \text { NUTLD - nutrient load } & \text { RIPCN - riparian condition } \\ \text { CSTAB - channel stability } & \text { OBST - obstructions } & \text { SEDLD - sediment load } \\ \text { COMP - competition (with other species) } & \text { OXYG - dissolved oxygen } & \text { TEMP - water temperature } \\ \text { FLOW - ftow } & \text { PATHO - pathogens } & \text { WITH - water withdrawals } \\ \text { HABD - habitat diversity } & \text { PRED - predators (includes fishing-related losses) }\end{array}$

"NA" indicates that no life history pathway meeting the criteria used in defining these pathways was present.

Blank cells under attributes indicate no additional attributes with negative effects on productivity were evident compared to those present in the template. 


\section{Stream Reach Summary for Primary and Secondary Spring Chinook Salmon Life Histories}

Stream: Grande Ronde R

Reach: Upstream end of State Ditch -1-84 crossing below Devil's Slide (RM 118.9 -128.4 from Snake R)

\begin{tabular}{|c|c|c|c|c|c|c|c|c|c|c|c|c|c|c|}
\hline \multicolumn{4}{|c|}{ Restplation benefit category: } & $\mathrm{D}$ & \multicolumn{3}{|c|}{ Chinook usage score: } & 78.9 & \multicolumn{4}{|c|}{ Habitat productivity loss score: } & -209.8 & \\
\hline \multirow[b]{3}{*}{ Life stage } & \multirow[b]{3}{*}{ Months } & \multicolumn{10}{|c|}{ No. life history pathways by current usage abundance level } & \multicolumn{2}{|c|}{ Key habitat change } & \multirow{3}{*}{$\begin{array}{c}\text { Habitat } \\
\text { productivity } \\
\text { loss score }\end{array}$} \\
\hline & & \multicolumn{5}{|c|}{ Primary } & \multicolumn{5}{|c|}{ Secondary } & \multirow{2}{*}{$\begin{array}{l}\text { Patient } \\
\text { quanlity }\end{array}$} & \multirow[b]{2}{*}{$\%$ change } & \\
\hline & & Total no. & 1 & 2 & 3 & 4 & Total no. & 1 & 2 & 3 & 4 & & & \\
\hline & Apr-Aug & 8 & 0 & 7 & 0 & 1 & 24 & 0 & 21 & 0 & 3 & 2.6 & -37.2 & -40.4 \\
\hline 110 & Aug-ÖCl & NA & NA" & $\mathrm{NA}$ & $\mathrm{NA}$ & $\mathrm{NA}$ & NA & NA & $\mathrm{NA}$ & $\mathrm{NA}$ & NA & NA & $\mathrm{NA}$ & NA \\
\hline 3 & Oct-Mar & $\mathrm{NA}$ & NA & $\mathrm{NA}$ & $\mathrm{NA}$ & $\mathrm{NA}$ & $\mathrm{NA}$ & $\mathrm{NA}$ & NA & $\mathrm{NA}$ & NÄ & NA & $\mathrm{NA}$ & NA \\
\hline 3,4 & Mar-May & $\mathrm{NA}$ & NA & $\mathrm{NA}$ & $\mathrm{NA}$ & $\mathrm{NA}$ & $\mathrm{NA}$ & $\mathrm{NA}$ & $\mathrm{NA}$ & $\mathrm{NA}$ & $N A$ & $\mathrm{NA}$ & $\mathrm{NA}$ & $\mathrm{NA}$ \\
\hline 5 & May-Öct & 0 & 0 & 0 & 0 & 0 & 14 & 0 & 13 & 0 & 1 & 15.4 & -2.8 & -15.1 \\
\hline 6 & Oct-Mar & 0 & 0 & 0 & 0 & 0 & 41 & 0 & 40 & 0 & 1 & 104 & -539 & -1542 \\
\hline & & & & 7 & 0 & 1 & 7 & 0 & 6 & 0 & 1 & 631 & 00 & -01 \\
\hline
\end{tabular}

\begin{tabular}{|c|c|c|c|c|c|c|c|c|c|c|c|c|c|}
\hline \multirow[b]{2}{*}{ Life stage } & \multirow[b]{2}{*}{ Months } & \multicolumn{12}{|c|}{ Highest productivity impact attributes ( $\%$ change in impact from template) } \\
\hline & & $\overline{\text { Att } 1}$ & $\%$ change & Att 2 & $\%$ change & Att 3 & $\%$ change & Att 4 & $\%$ change & Aft 5 & \% change & Att 6 & $\%$ change \\
\hline 1 & Apr-Aug & FLOW & 29,2 & HABD & 29.2 & TEMP & 29.2 & RIPCN & 19.5 & & & & \\
\hline $1,2,3$ & Äug-Oct & NA & NA & NA & NA & $N A^{\circ}$ & NA & NA & NA & NA & NA & $\ddot{\mathrm{NA}}$ & NÄ̈ \\
\hline 3 & Ött-Mar & NA & NA & NA & NA & NA & NA & NA & NA & NA & NA & NA & NA \\
\hline 3,4 & Mar-May & NA & NA & NA & NA & NA & NA & NA & NA & NA & NA & NA & NA \\
\hline 5 & May-Ott & FLOW & 23.2 & TEMP & '23.2 & HABD & 15.5" & OXYG & 15.5 & RIPCN & 15.5 & CHEM & 7 \\
\hline 6 & Ott-Mar & FLOW & 72.7 & HABD & 48.5 & OBST & 46,5 & RIPCN & 48.5 & SEDLD & 48.5 & WITH & 25.0 \\
\hline 7 & Mar-Jun & WITH & 7.5 & & & & & & & & & & \\
\hline
\end{tabular}

Abundancelevel refers to the average abundance of spawnera that spawn in the natal stream reaches that populate the life history pathways using the reach in this table

$$
\begin{array}{ll}
\text { Level 1: } & 0-10 \text { spawners } \\
\text { Level 2: } & 10-30 \text { spawners } \\
\text { Level 3: } & 30-60 \text { spawners } \\
\text { Level 4 } & >\mathbf{6 0} \text { spawners }
\end{array}
$$

\begin{tabular}{|c|c|c|}
\hline $\begin{array}{l}\text { Attributes: CHEM - chemicals } \\
\text { CSTAB - channel stability } \\
\text { COMP - competition (with other species) } \\
\text { FLOW - flow } \\
\text { HABD - } \quad \text { habitat diversity }\end{array}$ & $\begin{array}{ll}\text { NUTLD - } & \text { nutrient load } \\
\text { OBST - } & \text { obstructions } \\
\text { OXYG - } & \text { dissolved oxygen } \\
\text { PATHO - } & \text { pathogens } \\
\text { PRED - predators (includes }\end{array}$ & $\begin{array}{l}\text { RIPCN - } \quad \text { riparian condition } \\
\text { SEDLD - } \\
\text { TEdiment load } \\
\text { TEMP - } \quad \text { water temperature } \\
\text { WITH - } \quad \text { water withdrawals }\end{array}$ \\
\hline
\end{tabular}

Life stages.' 1- prespawner; 2- spawner; 3- incubation; 4- fry colonization; 5- summer rearing; 6- overwintering; 7-yearling presmolt/smolt

"NA" indicates that no life history pathway meeting the criteria used in defining these pathways was present.

Blank cells under attributes indicate no additional attributes with negative effects on productivity were evident compared to those present in the template. 


\section{Stream Reach Summary for Primary and Secondary Spring Chinook Salmon Life Histories}

Stream: Grande Ronde R.

Reach: $\quad 1-84$ crossing below Devil's Slide - Five Points Cr (RM 128.4 -133.4 from Snake R)

\begin{tabular}{lll|ll|ll}
\hline Restoration benefit category: & F & Chlnook usage score: & 25.6 & Habitat productivity loss score: & -62.0 \\
\hline
\end{tabular}

\begin{tabular}{|c|c|c|c|c|c|c|c|c|c|c|c|c|c|c|}
\hline \multirow[b]{3}{*}{ Life stage } & \multirow[b]{3}{*}{ Months } & \multicolumn{10}{|c|}{ No. life history pathways by current usage abundance level } & \multicolumn{2}{|c|}{ Key habitat change } & \multirow{3}{*}{$\begin{array}{c}\text { Habitat } \\
\text { productivity } \\
\text { loss score }\end{array}$} \\
\hline & & \multicolumn{5}{|c|}{ Primary } & \multicolumn{5}{|c|}{ Secondary } & \multirow{2}{*}{$\begin{array}{c}\text { Patient } \\
\text { quantity }\end{array}$} & \multirow[b]{2}{*}{$\%$ change } & \\
\hline & & Total no. & 1 & 2 & 3 & 4 & Total no. & 1 & 2 & 3 & 4 & & & \\
\hline 1 & Apr-Aug & 8 & 0 & 7 & 0 & 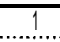 & 24 & 0 & 21 & 0 & 3 & 3.4 & -14.5 & -18.6 \\
\hline 123 & Aug-Ött & NA & NA & $N A$ & NA & NA & NQA". & NA" & NA" & $\mathrm{NA}$ & $\mathrm{NA}$ & $\mathrm{NA}$ & NA & NA \\
\hline $34 \ldots$ & Mar-May & & NA & NA & NA & NA & NA & NA & $\mathrm{N} A$ & NA & $N$ & NA & NA & NA \\
\hline 5 & May-Ott & 0 & 0 & 0 & 0 & 0 & 18 & 0 & 16 & 0 & 2 & 6.8 & -21.0 & .27 .1 \\
\hline 6 & Ott-Mar & 0 & 0 & 0 & 0 & 0 & 37 & 0 & 37 & 0 & 0 & 2.0 & -12.3 & -17.5 \\
\hline 7 & Mar-Jun & 6 & 0 & 7 & 0 & 1 & 5 & 0 & 4 & 0 & 1 & 69.3 & 0.0 & 1.1 \\
\hline
\end{tabular}

\begin{tabular}{|c|c|c|c|c|c|c|c|c|c|c|c|c|c|}
\hline \multirow[b]{2}{*}{ Life stage } & & \multicolumn{12}{|c|}{ Highest productivity impact attributes (\% change in impact from template) } \\
\hline & Months & Att 1 & $\%$ change & Att 2 & $\%$ change & Att 3 & $\%$ change & An 4 & $\%$ change & Att 5 & $\%$ change & Att 6 & \% change \\
\hline 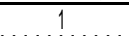 & Apr-Aug & HABD & 11.7 & RIPCN & 11.7 & TEMP & 11.7 & & & & & & \\
\hline $1,2,3$ & Aug-Ód & NA & NA & NA & $\mathrm{NA}$ & NÄ & $\mathrm{NA}$ & $\mathrm{NA}$ & NÄ & NÄ & NÄ & N̈̈ & $\mathrm{N}$ \\
\hline 3 & Öt-Mar & NÄ & NA" & NA & $\mathrm{NA}$ & NA" & $\mathrm{NA}$ & $\mathrm{NÄ}$ & $\mathrm{NA}$ & $\mathrm{NA}$ & $\mathrm{NA}$ & $\mathrm{NÄ}$ & $\mathrm{NA}$ \\
\hline 3,4 & Mar-May & NA & NA & NA & $\mathrm{NA}$ & NA & NA & NA & $\mathrm{NA}$ & $\mathrm{NÄ}$ & NA & NA & NA \\
\hline 5 & May-Ott & CSTAB & 17,5 & H A B D & 117,5 & RIPCN & 17.5 & SEDLD & 17.5 & PATHO & $8^{\circ}$ & TEMP & 8.7 \\
\hline 6 & Ott-Mar & CSTAB & 6.6 & HABD & 6.6 & RIPCN & 6.6 & SEDEDD & 6.6 & PATHO & 3.3 & TEMP & 3.3 \\
\hline 7 & Mar-Jun & & & & & & & & & & & & \\
\hline
\end{tabular}

Abundance /eve/ refers to the average abundance of spawners that spawn in the natal stream reaches that populate the life history pathways using the reach in this table

$$
\begin{array}{ll}
\text { Level 1: } & 0-10 \text { spawners } \\
\text { Level 2: } & 10-30 \text { spawners } \\
\text { Level 3: } & 30-60 \text { spawners } \\
\text { Level 4: } & \mathbf{2 0 0} \text { spawners }
\end{array}
$$

\begin{tabular}{|c|c|c|c|}
\hline Attributes: & $\begin{array}{l}\text { CHEM - chemicals } \\
\text { CSTAB - channel stability } \\
\text { COMP - competition (with other species) } \\
\text { FLOW - flow } \\
\text { HABD - habitat diversity }\end{array}$ & $\begin{array}{l}\text { NUTLD - nutrient load } \\
\text { OBST - obstructions } \\
\text { OXYG - dissolved oxygen } \\
\text { PATHO - pathogens } \\
\text { PRED - predators (includes fishing-related losses) }\end{array}$ & $\begin{array}{l}\text { RIPCN - } \quad \text { riparian condition } \\
\text { SEDLD - } \\
\text { TEMdiment load } \\
\text { WITH - } \quad \text { water temperature } \\
\text { Water withdrawals }\end{array}$ \\
\hline
\end{tabular}

Life stages.' 1- prespawner; 2- spawner; 3- incubation; 4- fry colonization; 5- summer rearing; 6- overwintering; 7- yearling presmolt/smolt

"NA" indicates that no life history pathway meeting the criteria used in defining these pathways was present.

Blank cells under attributes Indicate no additional attributes with negative effects on productivity were evident compared to those present in the template. 


\section{Stream Reach Summary for Primary and Secondary Spring Chinook Salmon Life Histories}

Stream: Grande Ronde R.

Reach: $\quad$ Five Points $\mathrm{Cr}$ - Rock Cr (RM 133.4 -133.8 from Snake R)

\begin{tabular}{|llll|l|l|l|l|l|l|l|}
\hline Restoration benefit category: & F & Chinook usage score: & 10.7 & Habitat productivity loss score: \\
\hline
\end{tabular}

\begin{tabular}{|c|c|c|c|c|c|c|c|c|c|c|c|c|c|c|}
\hline \multirow[b]{3}{*}{ Life stage } & \multirow[b]{3}{*}{ Months } & \multicolumn{10}{|c|}{ No. life history pathways by current usage abundance level } & \multicolumn{2}{|c|}{ Key habitat change } & \multirow{3}{*}{$\begin{array}{c}\text { Habitat } \\
\text { productivity } \\
\text { loss score }\end{array}$} \\
\hline & & \multirow{2}{*}{\multicolumn{5}{|c|}{$\frac{\text { Primary }}{2}$}} & \multicolumn{5}{|c|}{ Secondary } & \multirow{2}{*}{$\begin{array}{l}\text { Patient } \\
\text { quantity }\end{array}$} & \multirow[b]{2}{*}{$\%$ change } & \\
\hline & & & & 2 & 3 & 4 & Total no. & 1 & 2 & 3 & 4 & & & \\
\hline 1. & Apr-Aug & 8 & 0 & 7 & 0 & 1 & 24 & 0 & 21 & 0 & 3 & 2.3 & -8.6 & 2.1 \\
\hline $1,2,3$ & Aug-Oct & NA & NA & NÄ & NÄ & NA & NA & NÄ & NA & NÄ & NA & $N A$ & NÄ & $\mathrm{NA}$ \\
\hline 3 & Oct-Mar & $\mathrm{NA}$ & $\mathrm{NA}$ & $N A$ & NÄ & NA & $N A$ & $\mathrm{NA}$ & $\mathrm{NA}$ & NA & $\mathrm{NA}$ & NA & $\mathrm{NA}$ & NA \\
\hline 3,4 & Mar-May & $\mathrm{NA}$ & $N A$ & NA & NA & NA & NA & NA & $\mathrm{NA}$ & NA & $\mathrm{NA}$ & $\mathrm{NA}$ & $\mathrm{NA}$ & NA \\
\hline 5 & May-Oct & 0 & 0 & 0 & 0 & 0 & 18 & 0 & 16 & 0 & 2 & 6.8 & -7.8 & -10.1 \\
\hline 6 & Oct-Mar & 0 & 0 & 0 & 0 & 0 & 3 & 0 & 3 & 0 & 0 & 1.1 & -44.0 & $-5.13 .1 \mathrm{~m}$ \\
\hline 7 & Mar:jutpi & q & 0 & 7 & $a$ & 1 & j & $a$ & 2 & $\overline{\mathrm{u}}$ & 1 & 49.5 & 00 & 年 \\
\hline
\end{tabular}

\begin{tabular}{|c|c|c|c|c|c|c|c|c|c|c|c|c|c|}
\hline \multirow[b]{2}{*}{ Life stage } & \multirow[b]{2}{*}{ Months } & \multicolumn{12}{|c|}{ Highest productivity impact attributes (\% change in impact from lemplate) } \\
\hline & & Att 1 & $\%$ change & Alt 2 & \% change & Att 3 & $\%$ change & Att 4 & \% change & Att 5 & $\%$ change & Alt 6 & $\%$ change \\
\hline 1 & Apr-Aug & HABD & 6.9 & RIPCN & 6.9 & TEMP & 6.9 & & & & & & \\
\hline $1,2,3$ & Aug-Oct & NA & NA & NA & NA & NA & NA & $\mathrm{NA}$ & NA & NA & $\mathrm{NA}$ & NA & NA \\
\hline 3 & Oct-Mar & NA & NÄ & $\mathrm{NA}$ & $\mathrm{NA}$ & $\mathrm{NA}$ & NA & NA & NA & $\mathrm{NA}$ & NA & $\mathrm{NA}$ & $\mathrm{NA}$ \\
\hline 3,4 & Mar-May & NA & $\mathrm{NA}$ & NA & NA & $\mathrm{NA}$ & NA & NA & $\mathrm{NA}$ & $\mathrm{NA}$ & $\mathrm{NA}$ & $N A$ & $N A$ \\
\hline 5 & May-Oct & RIPCN & 9.8 & TEMP & 9.8 & HABD & 6.5 & PATHO & 3.3 & & & & \\
\hline 6 & Oct-Mar & CSTAB & 236 & HABD & 236 & RiPCN & 236 & SÉLIO & 236 & PATHO & 118 & TEMP & 118 \\
\hline 7 & Mar-Jun & & & & & & & & & & & & \\
\hline
\end{tabular}

Abundance level refers to the average abundance of spawners that spawn in the natal stream reaches that populate the life history pathways using the reach in this table Level 1: $\quad 0-10$ spawners

$\begin{array}{ll}\text { Level } 2 & 10-30 \text { spawners } \\ \text { Level 3: } & 30-60 \text { spawners }\end{array}$

Level 4: $\quad>60$ spawners

Life stages: 1- prespawner; 2- spawner; 3- incubation; 4- fry colonization; 5- summer rearing; 6- overwintering; 7- yearling presmott/smelt

Attributes: CHEM - chemicals

CSTAB - channel stability

COMP - competition (with other species)

FLOW - ffow

HABD - habitat diversity
NUTLD - nutrient load

OBST - obstructions

OXYG - dissolved oxygen

PATHO - pathogena

PRED - predators (includes fishing-related losses)
RIPCN - riparian condition

SEDLD - sediment load

TEMP - water temperature

WITH - water withdrawals

"NA" indicates that no life history pathway meeting the criteria used in defining these pathwaywas present.

Blank cells under attributes indicate no additional attributes with negative effects on productivity were evident compared to those present in the template. 
Stream: Grande Ronde R.

Reach: Rock Cr - Whiskey Cr (RM 133.8 -136.6 from Snake R)

\begin{tabular}{lll|ll|l}
\hline Restoration benefit category: & F & Chinook usage score: & 11.5 & Habitat productivity ioss score: \\
\hline
\end{tabular}

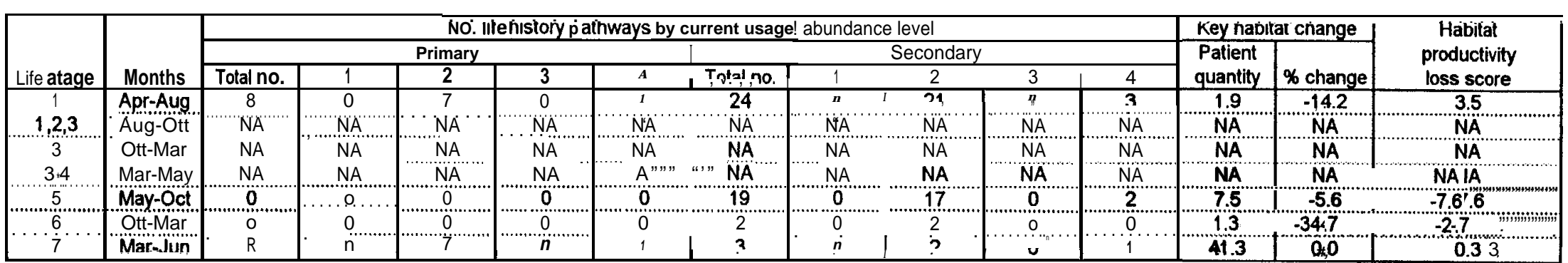

\begin{tabular}{|c|c|c|c|c|c|c|c|c|c|c|c|c|c|}
\hline \multirow[b]{2}{*}{ Life stage } & \multirow[b]{2}{*}{ Months } & \multicolumn{12}{|c|}{ Highest productivity impact attributes (\% change in impact from template) } \\
\hline & & Att 1 & \% change & Atf 2 & 96 change & Att 3 & $\%$ change & Att 4 & $\%$ change & Att 5 & $\%$ change & Atf 6 & $\%$ change \\
\hline 1 & Apr-Aug & HABD & $114 \ldots$ & RIPCN & 11.4 & TEMP & 11.4 & & & & & & \\
\hline $1,2,3$ & Aug-Oot & NA & NA & NA & NA & NA & $\mathrm{NÄ}$ & $N A$ & NA & NA & N̈Ä & $N A \ddot{~}$ & NA \\
\hline 3 & Ott-Mar & NA & NA & NA & NA" & $\mathrm{NA}$ & NÄ & NÄ & NÄ & Nä & NÄ & Nä & $\mathrm{NA}$ \\
\hline 3344, & MÁaf Way & NA & NA & NA & $N A$ & NA & NA & NA & NA & NA & NA & NA & NA \\
\hline 5 & May-Oot & CSTAB & 4.7 & HABD & 47 & RIPCN & 4.7 & SĖDL̇D & 4.7 & PATHO & 2.3 & TEMP & 2.3 \\
\hline 6 & Odtimar & CSTAB & 18.6 & HABD & 18.6 & RIPCON & 18.6 & SEDELD & 18.6 & PATHO & 9.3 & TEM MP & 9.3 \\
\hline 7 & Mar-.Jun & & & & & & & & & & & & \\
\hline
\end{tabular}

Abundance /eve/ refers to the average abundance of spawners that spawn in the natal stream reaches that populate the life history pathways using the reach in thitable:

$$
\begin{array}{ll}
\text { Levei 1: } & \text { 0-10 spawners } \\
\text { Level 2: } & 10-30 \text { spawners } \\
\text { Level 3: } & 30-60 \text { spawners } \\
\text { Level 4 } & \mathbf{2 6 0} \text { spawners }
\end{array}
$$

\begin{tabular}{|c|c|c|c|}
\hline Attributes: & $\begin{array}{l}\text { CHEM - chemicals } \\
\text { CSTAB - channel stability } \\
\text { COMP - competition (with other species) } \\
\text { FLOW - fiOW } \\
\text { HABD - habitat diversity }\end{array}$ & $\begin{array}{l}\text { NUTLD - } \text { nutrient load } \\
\text { OBST - obstructions } \\
\text { OXYG - dissolved oxygen } \\
\text { PATHO - pathogens } \\
\text { PRED - } \quad \text { predators (includes fishing-related losses) }\end{array}$ & $\begin{array}{l}\text { RIPCN - } \quad \text { riparian condition } \\
\text { SEDLD - } \\
\text { Tediment ioad } \\
\text { TEMP - } \quad \text { water temperature } \\
\text { WiTH - } \quad \text { water withdrawals }\end{array}$ \\
\hline
\end{tabular}

Life stages.' 1- prespawner; 2- spawner; 3- incubation; 4- fry colonization; 5- summer rearing; 6- overwintering; 7- yearling presmolt/smolt

"NA" indicates that no life history pathway meeting the criteria used in defining these pathways was present.

Blank cells under attributes indicate no additional attributes with negative effects on productivity were evident compared to those present in the template. 


\section{Stream Reach Summary for Primary and Secondary Spring ChinookSalmon Life Histories}

Stream: Grande Ronde R.

Reach: Whiskey Cr - Jordan Cr (RM 136.6 -139.1 from Snake R)

\begin{tabular}{|ll|ll|l|}
\hline Restoration benefit category: & F & Chinook usage score: & 43.3 & Habitat productivity loss score: \\
\hline
\end{tabular}

\begin{tabular}{|c|c|c|c|c|c|c|c|c|c|c|c|c|c|c|}
\hline \multirow[b]{3}{*}{ Life stagee } & \multirow[b]{3}{*}{ Months } & \multicolumn{10}{|c|}{ No. life history pathways by current usage abundance level } & \multicolumn{2}{|c|}{ Key habitat change } & \multirow{3}{*}{$\begin{array}{c}\text { Habitat } \\
\text { productivity } \\
\text { ioss Score }\end{array}$} \\
\hline & & \multicolumn{5}{|c|}{ Primary } & \multicolumn{5}{|c|}{ Secondary } & \multirow{2}{*}{$\begin{array}{l}\text { Patient } \\
\text { quantity }\end{array}$} & \multirow[b]{2}{*}{$\%$ change } & \\
\hline & & Total no. & 1 & 2 & 3 & 4 & Total no, & 1 & 2 & 3 & 4 & & & \\
\hline 1 & $\overline{\text { Apr-Aug }}$ & 8 & $\mathbf{0}$ & 7 & $\mathbf{0}$ & 1 & 24 & 0 & 21 & 0 & 3 & 2.1 & -24.0 & 5.8 \\
\hline 1,23 & Aug-Öt & NA & $\mathrm{NA}$ & NA & & NA & NA & $\dot{N} A$ & / & NÄ & NA & NÄ & NA & $\mathrm{NA}$ \\
\hline 3,4 & Mar-May & NA & NA & $\mathrm{NA}$ & NA & NA & NA & NA & NA & NA & NA & NA & NA & NA \\
\hline 5 & May-Oct & 0 & 0 & 0 & 0 & 0 & 21 & 0 & 19 & 0 & 2 & 83 & -127 & -19.2 \\
\hline 6 & Oct-Mar & 0 & 0 & 0 & 0 & 0 & 19 & 0 & 19 & 0 & 0 & 1.4 & -93.3 & -68.1 \\
\hline 7 & Mar-Jun & 8 & 0 & 7 & 0 & 1 & 3 & 0 & 2 & 0 & 1 & 30.3 & 0.0 & 0.9 \\
\hline
\end{tabular}

\begin{tabular}{|c|c|c|c|c|c|c|c|c|c|c|c|c|c|}
\hline \multirow[b]{2}{*}{ Life stage } & \multirow[b]{2}{*}{ Months } & \multicolumn{12}{|c|}{ Highest productivity impact attributes (\% change in impact from template) } \\
\hline & & Att 1 & \% change & Att 2 & \% change & Att 3 & \% change & Att 4 & $\%$ change & Att 5 & $\%$ change & Att 6 & $\%$ change \\
\hline 1 & Apr-Aug & HABD & 19.3 & RIPCN & 19.3 & TEMP & 19.3 & & & & & & \\
\hline $1.2,3$ & Äug-Ött & $\mathrm{NA}$ & NA & NA & $\mathrm{NA}$ & NA & NA & NA & NA & NA & NA & $\mathrm{N} \cdots$ & NA \\
\hline 3 & Ott-Mar & $\mathrm{NA}$ & NA & $\mathrm{NA}$ & NA & NA & NA & NA & NA & NA & NA & NA & NA \\
\hline $.3,4$ & Mar-May & NA & NA & NA & NA & NA & N̈A & NA & N̈A & N? & NA & & $\mathrm{NA}$ \\
\hline 5 & May-Ott & RIPCN & 15.9 & "TEMP & 15.9 & HABD & 10.8 & PATHOO & 5.3 & & & & \\
\hline 6 & Ott-Mar & CSTAB & 50.0 & HABD & 500 & RIPCN & 500 & SEDLD & 50.0 & PATHO & 250 & TEMP & 25.0 \\
\hline 7 & Mar-Jun & & & & & & & & & & & & \\
\hline
\end{tabular}

Abundancelevel refers to the average abundance of spawners that spawn in the natal stream reachea that populate the life history pathways using the reach in this table:

$$
\begin{array}{ll}
\text { Level 1: } & 0-10 \text { spawners } \\
\text { Level 2 } & 10-30 \text { spawners } \\
\text { Level 3: } & 30-60 \text { spawners } \\
\text { Level 4: } & \mathbf{>} 60 \text { spawnera }
\end{array}
$$

\begin{tabular}{|c|c|c|c|}
\hline Attributes: & $\begin{array}{l}\text { CHEM - chemicals } \\
\text { CSTAB - channel stability } \\
\text { COMP - competition (with other species) } \\
\text { FLOW - flow } \\
\text { HABD - habitat diversity }\end{array}$ & $\begin{array}{l}\text { NUTLD - } \\
\text { OBST - } \quad \text { obstructiont load } \\
\text { OXYG - } \quad \text { dissolved oxygen } \\
\text { PATHO - } \\
\text { Pathogens } \\
\text { PRED - } \quad \text { predators (includes fishing-related losses) }\end{array}$ & $\begin{array}{l}\text { RIPCN - riparian condition } \\
\text { SEDLD - sediment load } \\
\text { TEMP - water temperature } \\
\text { WITH - water withdrawals }\end{array}$ \\
\hline
\end{tabular}

Life stages: 1- prespawner; 2- spawner; 3- incubation; 4- fry colonization; 5- summer rearing; 6- overwintering; 7- yearling presmolt/smolt

"NA" indicates that no life history pathway meeting the criteria used in defining these pathwaywas present.

Blank cells under attributes indicate no additional attributes with negative effects on productivity were evident compared to those present in titemplate. 
Stream Reach Summary for Primary and Secondary Spring Chinook Salmon Life Histories

Stream: Grande Ronde R.

Reach: Jordan $\mathrm{Cr}$ - Beaver $\mathrm{Cr}$ (RM 139.1 -145.8 from Snake R)

\begin{tabular}{|c|c|c|c|c|c|c|c|c|c|c|c|c|c|c|}
\hline \multicolumn{4}{|c|}{ Restoration benefit category: } & $A$ & \multicolumn{3}{|c|}{ Chinook usage score: } & 461.2 & \multicolumn{5}{|c|}{ Habitat productivity ioss score: } & \\
\hline \multirow[b]{3}{*}{ Life stage } & \multirow[b]{3}{*}{ Months } & \multicolumn{10}{|c|}{ No. life history pathways by current usage abundancelevel } & \multicolumn{2}{|c|}{ Key habilat change } & \multirow{3}{*}{$\begin{array}{c}\text { Habitat } \\
\text { productivity } \\
\text { loss score }\end{array}$} \\
\hline & & \multicolumn{5}{|c|}{ Primary } & \multicolumn{5}{|c|}{ Secondary } & \multirow{2}{*}{$\begin{array}{l}\text { Patient } \\
\text { quantii }\end{array}$} & \multirow[b]{2}{*}{$\boldsymbol{\%}_{\text {change }}$} & \\
\hline & & Total no. & 1 & 2 & 3 & 4 & Total no. & 1 & 2 & 3 & 4 & & & \\
\hline 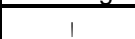 & Apr-Aug & & $?$ & & 0 & & $? ?$ & & 36 & 1 & & 2.0 & -53.8 & 218.5 \\
\hline 1,223 & Aug-Ött & & 0 & & 0 & & $30 "$ & & 30 & & & 6.2 & -42.8 & 315,8 \\
\hline 3 & Ott-Mar & 20 & 0 & 20 & 0 & 0 & 60 & 0 & 60 & 0 & $0^{\circ}$ & 8.3 & -2.2 & -513.8 \\
\hline 34 & Mar-May & 8 & 0 & 8 & 0 & 0 & 24 & 0 & 24 & 0 & 0 & 4.4 & -78.3 & -258.4 \\
\hline 5 & May-Ott & 25 & 0 & 25 & 0 & $\dot{0}$ & 88 & 0 & 86 & 0 & 2 & $10.0^{\circ}$ & -500 & -888.4 \\
\hline 6 & Ott-Mar & 19 & 0 & 19 & 0 & 0 & 36 & 0 & 18 & 0 & 18 & 1.4 & -39.9 & -840 \\
\hline 7 & Mar-Jun & $\ddot{8}$ & $\dot{0}$ & 7 & 0 & 1 & 2 & 0 & 1 & 0 & 1 & 30.3 & -4.8 & 1.0 , \\
\hline
\end{tabular}

\begin{tabular}{|c|c|c|c|c|c|c|c|c|c|c|c|c|c|}
\hline \multirow[b]{2}{*}{ Life stage } & \multirow[b]{2}{*}{ Months } & \multicolumn{12}{|c|}{ Highest productivity impact attributes (\% change in impact from template) } \\
\hline & & Att 1 & $\%$ change & An 2 & $\%$ change & Att 3 & $\%$ change & Att 4 & $\%$ change & Att 5 & $\%$ change & Att 6 & $\%$ change \\
\hline 1. & Apr-Aug & RIPCN & 75.0 & TEMP & 75,0 & HABD & 62.5 & & & & & & \\
\hline $1,2,3$ & Äug-Ối & RIPCN & 75.0 & SEDLD & 75.0 & TEMP & 75.0 & HABD & $62, \dot{5}$ & CSTAB & 50,0 & & \\
\hline 3 & Ott-Mar & SEELDD & 75.0 & TEMP & 75,0 & CSTAB & 500 & FLOW & 25,0 & HABD & 25.0 & RIPCN & 25.0 \\
\hline 3,4 & Mar-May & RIPCN & 75.0 & TEMP & 75.0 & HABD & 50.0 & CSTAB & 37.5 & SEDLD & 37.5 & FLOW. & 25.0 \\
\hline 5 & May-Ott & RIPCN & 75.0 & TEMP & 75.0 & HABD & $50.0 "$ & "“РATHO & 25.0 & CSTAB & 18.0 & SEDEL் & 18.0 \\
\hline$\ddot{6}$ & Ott-Mar & CSTAB & 21.3 & HABD & 21.3 & RIPCN & 21.3 & SEELLD & 21.3 & РАТННО & 10.7 & TंËMP & 10.7 \\
\hline 7 & Mar-Jun & & & & & & & & & & & & \\
\hline
\end{tabular}

Abundancelevel refers to the average abundance of spawners that spawn in the natal stream reaches that populate the iife history pathways using the reach in this table

$\begin{array}{ll}\text { Levei 1: } & \text { O-t O spawners } \\ \text { Levei 2: } & 10-30 \text { spawners } \\ \text { Levei 3: } & 30-60 \text { spawners }\end{array}$

Levei 4: > 60 spawners

Life stages: 1- prespawner; 2- spawner; 3- incubation 4- fry colonization; 5- summer rearing; 6- overwintering; 7- yearling presmoltsmolt

$\begin{array}{lll}\text { Attributes.' CHEM - chemicals } & \text { NUTLD - nutrient load } & \text { RIPCN - riparian condition } \\ \text { CSTAB - channel stability } & \text { OBST - obstructions } & \text { SEDLD - sediment load } \\ \text { COMP - competition (with other species) } & \text { OXYG - dissolved oxygen } & \text { TEMP - water temperature } \\ \text { FLOW - flow } & \text { PATHO - pathogens } & \text { WITH - water withdrawals } \\ \text { HABD - habitat diversity } & \text { PRED - predators (includes fishing-related losses) }\end{array}$

"NA" indicates that no life history pathway meeting the criteria used in defining these pathways was present.

Blank cells under attributes indicate no additional attributes with negative effects on productivity were evident compared to those present in the template, 
Stream Reach Summary for Primary and Secondary Spring Chinook Salmon Life Histories

Stream: Grande Ronde R.

Reach: Beaver $\mathrm{Cr}$ - Meadow $\mathrm{Cr}$ (RM 145.8 -147.0 from Snake R)

\begin{tabular}{|ll|l|l|l|}
\hline Restoration benefit category: & A & Chinook usage score: & 450.8 & Habitat productivity loss score: \\
\hline
\end{tabular}

\begin{tabular}{|c|c|c|c|c|c|c|c|c|c|c|c|c|c|c|}
\hline \multirow[b]{3}{*}{ Life stage } & \multirow[b]{3}{*}{ Months } & \multicolumn{10}{|c|}{ No. life history pathways by current usage abundance level } & \multicolumn{2}{|c|}{ Key habitat change } & \multirow{3}{*}{$\begin{array}{c}\text { Habitat } \\
\text { productivity } \\
\text { loss score }\end{array}$} \\
\hline & & \multicolumn{5}{|c|}{ Primary } & \multicolumn{5}{|c|}{ Secondary } & \multirow{2}{*}{$\begin{array}{c}\text { Patient } \\
\text { quantity }\end{array}$} & \multirow[b]{2}{*}{$\%$ change } & \\
\hline & & Total no. & 1 & 2 & 3 & 4 & Total no, & 1 & 2 & 3 & 4 & & & \\
\hline 1 & Apr-Aug & 12 & 0 & 11 & 0 & 1 & 36 & 0 & 33 & 0 & 3 & 2.1 & -53.7 & -220.4 \\
\hline 1.23 & Aug-Ott & 10 & 0 & 10 & "0” & 0 & 30 & 0 & 30 & 0 & 0 & 5.6 & -46.7 & -315.8 \\
\hline 3 & Ott-Mar & 20 & $\ddot{0}$ & 20 & 0 & 0 & 60 & 0 & 60 & 0 & 0 & 10.1 & 0.0 & -513.8 \\
\hline 3.4 & Mar-May & 8 & $\ddot{0}$ & 8 & “"” & 0 & 24 & $\dddot{0}$ & 24 & 0 & 0 & 5.6 & -76.7 & -258.4 \\
\hline 5 & May-Oct & 25 & $\dddot{0}$ & 25 & 0 & 0 & 85 & 0 & 83 & 0 & 2 & 10.2 & -53.4 & $=699.8$ \\
\hline 6 & Ott-Mar & 19 & 0 & 19 & 0 & 0 & 2 & $\ddot{0}$ & 1 & 0 & 1 & 1.7 & -91.5 & -73.6 \\
\hline 7 & Mar-Jun & 7 & 0 & 6 & 0 & 1 & 0 & 0 & 0 & $\ddot{0}$ & 0 & $41 . \dddot{3}^{\prime}$ & 0.0 & 0.8 \\
\hline
\end{tabular}

\begin{tabular}{|c|c|c|c|c|c|c|c|c|c|c|c|c|c|}
\hline \multirow[b]{2}{*}{ Life stage } & \multirow[b]{2}{*}{ Months } & \multicolumn{12}{|c|}{ Highest productivity impact attributes (\% change in impact from template) } \\
\hline & & Att 1 & $\%$ change & Art 2 & $\%$ change & Att 3 & $\%$ change & Att 4 & $\%$ change & Att 5 & $\%$ change & Att 6 & $\%$ change \\
\hline 1 & Apr-Aug & TEMP & 75.0 & HABD & 40.2 & RIPCN & 40.2 & SEDLD & 37.5 & CSTAB & 25.0 & & \\
\hline 123 & Aug-Ött & HABD & 75.0 & RIPCN & 75.0 & SEDLD & 75.0 & TEMP & 75.0 & CSTAB & 50.0 & & \\
\hline 3 & Ott-Mar & SEDLD & 75.0 & TEMP" & 750 & CSTAB" & 50.0 & FËOW" & 50,0 & HABD & 50.0 & RIPCN & 50.0 \\
\hline 3,4 & Msr-May & RIPCN & 75.0 & SÉDLD & 75.0 & TEMP" & 75.0 & CSTAB & 50.0 & FLOW & 50.0 & HABD & 50.0 \\
\hline "s & May-Ott & RIPCN & 66.7 & TEMP & 66.7 & HABD & 44.5 & PATHO & 22.2 & & & & \\
\hline$\dot{6}$ & Ott-Mar & CSTAB & 48.9 & "'HÄBD & 48.9 & RIPCN & 48.9 & SEDELD & 48.9 & PATHOO & 24.5 & TEMP & 245 \\
\hline$\dddot{7}$ & Mar-Jun & & & & & & & & & & & & \\
\hline
\end{tabular}

Abundance /eve/ refers to the average abundance of spawners that spawn in the natal stream reaches that populate the life history pathways using the reach in this table:

$$
\begin{array}{ll}
\text { Level 1: } & 0-10 \text { spawners } \\
\text { Level 2: } & 10-30 \text { spawnera } \\
\text { Level 3 } & 30-80 \text { spawners } \\
\text { Level 4: } & >\mathbf{6 0} \text { apawners }
\end{array}
$$

Life stages: 1 - prespawner; 2- spawner; 3- incubation; 4- fry colonization; 5- summer rearing; 6- overwintering; 7- yearling presmolt/smolt

$\begin{array}{lll}\text { Attributes: } \text { CHEM - chemicals } & \text { NUTLD - nutrient load } & \text { RIPCN - riparian condition } \\ \text { CSTAB - channel stability } & \text { OBST - obstructions } & \text { SEDLD - sediment load } \\ \text { COMP - competition (with other species) } & \text { OXYG - dissolved oxygen } & \text { TEMP - water temperature } \\ \text { FLOW - flow } & \text { PATHO - pathogens } & \text { WITH - water withdrawals } \\ \text { HABD - habitat diversity } & \text { PRED - predators (includes fishing-related losses) }\end{array}$

"NA" indicates that no life history pathway meeting the criteria used in defining these pathways was present.

Blank cells under attributes indicate no additional attributes with negative effects on productivity were evident compared to those present in the template. 
Stream: Grande Ronde R.

Reach: Meadow Cr - Fly Cr (RM 147.0 -151.3 from Snake R)

\begin{tabular}{|ll|ll|l}
\hline Restoration benefit category: & A & Chinook usage score: & 453.6 & Habitat productivity loss score: \\
\hline
\end{tabular}

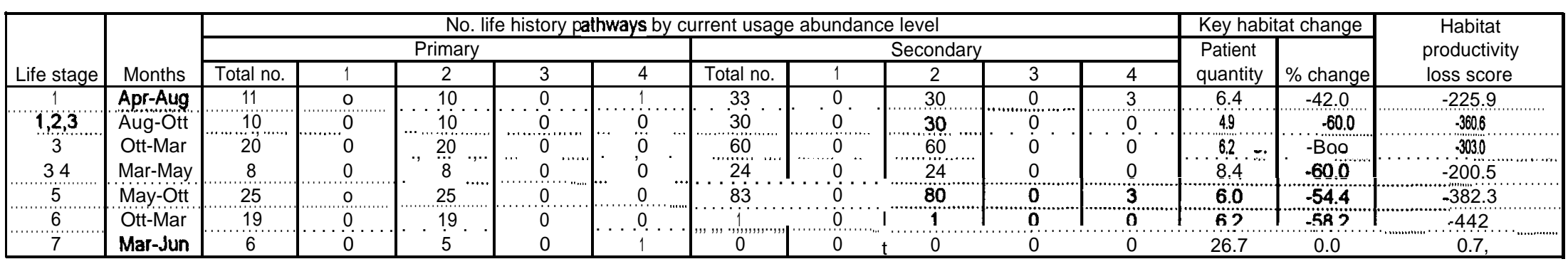

\begin{tabular}{|c|c|c|c|c|c|c|c|c|c|c|c|c|c|}
\hline \multirow[b]{2}{*}{ Life stage } & \multirow[b]{2}{*}{ Months } & \multicolumn{12}{|c|}{ Highest productivity impact attributes (\% change in impact from template) } \\
\hline & & Att 1 & $\%$ change & Atf 2 & $\%$ change & Atf 3 & $\%$ change & Att 4 & \% change & Att 5 & $\%$ change & Att 6 & $\%$ change \\
\hline 1 & Apr-Aug & TEMP & $\overline{52.5}$ & RIPCN & 35.0 & HABD & 20.9 & & & & & & \\
\hline $1,2,3$ & Aug-Öci & TËMP & 75.0 & CSTAB̈ & 50.0 & RIPCN & 50.0 & SEOQLOD & 50.0 & HABD" & 37.5 & & \\
\hline 3 & Ott-Mar & CSTAB & 50,0 & SĖDLD & 50.0 & TEMP & 50.0 & RIPCN & 25.0 & FLOÖW" & 12.5 & HABD" & 12.5 \\
\hline 3,4 & Mar-May & TEMP & 75,0 & RIPCN & 50,0 & CSTAB & 2s. 0 & $\mathrm{HABD}$ & 25.0 & PATHO & 25.0 & ȘEQLLD. & 25.0 \\
\hline 5 ." & May-Ott & TEMP & 75.0 & RIPCN & 50.0 & HABD & 25.0 & PATHO & 25.0 & CSTAB & 16.6 & SEDLD & 16.6 \\
\hline 6 & Ott-Mar & CSTAB & 48.5 & RIPCN & 46.5 & SEDLOO & 48.5 & PAT̈̈HO & 24.3 & TEMP & 24.3 & & \\
\hline 7 & Mar-Jun & & & & & & & & & & & & \\
\hline
\end{tabular}

Abundance level refera to the average abundance of spawners that spawn in thenatal stream reaches that populate the life history pathways using the reach in thistable:

Level 1: $\quad 0-10$ spawners

Level $2 \quad 10-30$ spawners

Level 3: $\quad 30-60$ spawners

Level 4: >60 spawners

Life stages: 1- prespawner; 2- spawner; 3- incubation; 4- fry colonization; 5- summer rearing; 6- overwintering; 7- yearling presmolt/smolt

\begin{tabular}{|c|c|c|c|c|}
\hline Attributes: & $\begin{array}{l}\text { CHEM - chemicals } \\
\text { CSTAB - channel stability } \\
\text { COMP - competition (with other species) } \\
\text { FLOW - flow } \\
\text { HABD - habitat diversity }\end{array}$ & $\begin{array}{l}\text { NUTLD - } \\
\text { OBST - } \quad \text { obstructiont load } \\
\text { OXYG - } \quad \text { dissolved oxygen } \\
\text { PATHO - } \\
\text { Pathogens } \\
\text { PRED - } \quad \text { predators (includes fishing-related losses) }\end{array}$ & $\begin{array}{l}\text { RIPCN - } \\
\text { SEDLD - } \\
\text { TEMP - } \\
\text { WITH - }\end{array}$ & $\begin{array}{l}\text { riparian condition } \\
\text { sediment load } \\
\text { water temperature } \\
\text { water withdrawals }\end{array}$ \\
\hline
\end{tabular}

"NA" indicates that no tifehistory pathway meeting the criteria used in defining these pathways was present.

Blank cells under attributes indicate no additional attributes with negative effects on productivity were evident compared to those present in the template. 
Stream: Grande Ronde R.

Reach: Fly $\mathrm{Cr}$ - head of canyon 1.3 miles downstream of SheepCr (RM 151.3 -159.4 from Snake R)

\begin{tabular}{|ll|ll|l}
\hline Restoration benefit category: & A & Chinook usage score: & 663.0 & Habitat productivity loss score: \\
\hline
\end{tabular}

\begin{tabular}{|c|c|c|c|c|c|c|c|c|c|c|c|c|c|c|}
\hline \multirow[b]{3}{*}{ Life stage } & \multirow[b]{3}{*}{ Months } & \multicolumn{10}{|c|}{ No. life history pathways by current usage abundance level } & \multicolumn{2}{|c|}{ Key habitat change } & \multirow{3}{*}{$\begin{array}{c}\text { Habital } \\
\text { productivity } \\
\text { loss score }\end{array}$} \\
\hline & & \multicolumn{5}{|c|}{ Primary } & \multicolumn{5}{|c|}{ Secondary } & \multirow{2}{*}{$\begin{array}{l}\text { Patient } \\
\text { quantity }\end{array}$} & \multirow[b]{2}{*}{$\%$ change } & \\
\hline & & Total no. & 1 & 2 & 3 & 4 & \begin{tabular}{|l} 
Total no. \\
\end{tabular} & 1 & 2 & 3 & 4 & & & \\
\hline 1 & Apr-Aug & 10 & 0 & 9 & 0 & 1 & 30 & 0 & 27 & 0 & 3 & 7.8 & -44.4 & -174.0 \\
\hline 1,23 & Aug-OCl & 1 & o & 101 & 0 & & & o' & 30 & "o" & 0 & "48" & 600 & $3653^{3}$ \\
\hline 3 & Ott-Mar & 1201 & 0 & 20 & 0 & & & 0 & $60^{\circ}$ & 0 & 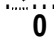 & 5,6 & -61.8 & 313,2 \\
\hline 34 & Mar-May & 13 & 0 & 13 & 0 & 0 & 39 & 0 “ & 39 & 0 & 0 & 8.4 & -60.0 & -322.0 \\
\hline "15" & May-Ott & 50 & 0 & 50 & 0 & 0 & 147 & 0 & 144 & 0 & 3 & 6.9 & .71 .0 & -540.4 \\
\hline ' & "Ött-Mar" & 38 & 1" o & $38 . .$. & $\because$ & $0 "$ & 0 & “̋ & 0 & 0 & 0 & 5.7 & -61.9 & -87.2 \\
\hline$\dot{7}$ & Mar-Jun & 5 & 0 & 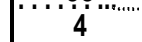 & 0 & 1 & 0 & 0 & 0 & 0 & 0 & "27.4" & 0.0 & 12 \\
\hline
\end{tabular}

\begin{tabular}{|c|c|c|c|c|c|c|c|c|c|c|c|c|c|}
\hline \multirow[b]{2}{*}{ Life stage } & \multirow[b]{2}{*}{ Months } & \multicolumn{12}{|c|}{ Highest productivity impact attributes (\% change in impact from template) } \\
\hline & & Att 1 & $\%$ change & $\overline{\text { Att } 2}$ & $\%$ change & Aft 3 & $\%$ change & Aft 4 & \% change & Att 5 & $\%$ change & Atf 6 & $\%$ change \\
\hline 1 & Apr-Aug & TEMP & 62.5 & RIPCN & 35.5 & CSTAB & 25.0 & HABD & 25.0 & SEDLD & 25.0 & & \\
\hline$j, 2,3$ & Aug-Öd & TËMP̈ & 75.0 & CSTAB & $50.0^{\circ}$ & RIPCN & 50.0 & SEDLD & 50.0 & "HÄD & $25.0^{\circ}$ & & \\
\hline 3 & Ött-Mar & "C̈TÄB" & 50.0 & RRPCN" & 50.0 & SEEDLD & 50.0 & TEMPP'." & 50.0 & FLOWW & 25.0 & HÄB̈D” & 25.0 \\
\hline 3,4 & Mar-May & TEMP & 75.0 & CSTAB & 50.0 & RIPCN & 500 & SEDLD & 50.0 & FELOWW & $250^{\circ}$ & HABD" & 25.0 \\
\hline 5 & May-Oct & TEMP & 73.3 & RIPCN & 48.8 & HABD & 24.4 & PATHO & 24.4 & & & & \\
\hline 6 & Ott-Mar & CSTAB & 50.0 & RIPCON & 50.0 & SEEDELD" & 50.0 & РÄTHO" & 25.0 & TEMP" & 25.0 & & \\
\hline 7 & Mar-Jun & & & & & & & & & & & & \\
\hline
\end{tabular}

Abundance /eve/ refers to the average abundance of spawners that spawn in the natal streamreaches that populate the life history pathways using the reach in this table

$\begin{array}{ll}\text { Level 1: } & \text { 0-10 spawners } \\ \text { Level 2: } & 10-30 \text { spawners } \\ \text { Level 3: } & 30-60 \text { spawners } \\ \text { Level 4: } & >\mathbf{6 0} \text { spawners }\end{array}$

Life stages: 1- prespawner; 2- spawner 3- incubation; 4- fry colonization; 5- summer rearing; 6- overwintering; 7- yearting presmolt/smolt

\begin{tabular}{|c|c|c|c|c|}
\hline Attributes: & $\begin{array}{l}\text { CHEM - chemicals } \\
\text { CSTAB - channel stability } \\
\text { COMP - competition (with other species) } \\
\text { FLOW - flow } \\
\text { HABD - habitat diversity }\end{array}$ & $\begin{array}{l}\text { NUTLD - } \\
\text { OBST - } \\
\text { OXYG - } \\
\text { PATHO - } \\
\text { PRED - }\end{array}$ & $\begin{array}{l}\text { nutrient load } \\
\text { obstructions } \\
\text { dissolved oxygen } \\
\text { pathogens } \\
\text { predators (includes fishing-relatedlosses) }\end{array}$ & $\begin{array}{l}\text { RIPCN - riparian condition } \\
\text { SEDLD - sediment toad } \\
\text { TEMP - water temperature } \\
\text { WITH - water withdrawals }\end{array}$ \\
\hline
\end{tabular}

"NA" indicates that no life history pathway meeting the criteria used in defining these pathways was present.

Blank cells under attributes indicate no additional attributes with negative effects on productivity were evident compared to those present in the template, 
Stream: Grande Ronde R.

Reach: Head of canyon 1.3 miles downstream of Sheep Cr - LimberJim Cr (RM 159.4 -164.2 from Snake R)

\begin{tabular}{|ll|ll|l|}
\hline Restoration benefit category: & A & Chlnook usage score: & $\mathbf{4 4 8 . 5}$ & Habltat productivity loss score: \\
\hline
\end{tabular}

\begin{tabular}{|c|c|c|c|c|c|c|c|c|c|c|c|c|c|c|}
\hline \multirow[b]{3}{*}{ Life stage } & \multirow[b]{3}{*}{ Months } & \multicolumn{10}{|c|}{ No. life history pathways by current usage abundance level } & \multicolumn{2}{|c|}{ Key habitat change } & \multirow{3}{*}{$\begin{array}{c}\text { Habital } \\
\text { productivity } \\
\text { loss score }\end{array}$} \\
\hline & & \multicolumn{5}{|c|}{\begin{tabular}{|l|l|}
\multicolumn{2}{|l|}{ Primary } \\
2 & 3
\end{tabular}} & \multicolumn{5}{|c|}{$\begin{array}{r}\text { Secondary } \\
\end{array}$} & \multirow{2}{*}{$\begin{array}{c}\text { Patient } \\
\text { quantity }\end{array}$} & \multirow[b]{2}{*}{$\%$ change } & \\
\hline & & Total no. & 1 & 2 & 3 & 4 & Total no, & 1 & 2 & 3 & 4 & & & \\
\hline 1 & Apr-Aug & 9 & 0 & 8 & 0 & 1 & 27 & 0 & 24 & 0 & 3 & 4.9 & -44.1 & -158.6 \\
\hline 12,3 & Aug-Ott & 10 & 0 & 10 & 0 & 0 & 30 & 0 & 30 & 0 & 0 & 63 & -18.0 & -367.8. \\
\hline 3 & Ott-Mar & 20 & 0 & 20 & 0 & 0 & 60 & 0 & 60 & 0 & 0 & 9.1 & -4.5 & -2865 \\
\hline 34 & Mar-May & 9 & 0 & 9 & 0 & 0 & 27 & 0 & 27 & 0 & 0 & 77 & -51.3 & -182.4 \\
\hline 5 & May-Ott. & 25 & 0 & 25 & 0 & 0 & 75 & 0 & 72 & 0 & 3 & 4.4 & -70.2 & .289 .8 \\
\hline "B" & Oct-Mar & 19 & "n & a & $\stackrel{n}{*}$ & "hin & "in" & in & $n$ & 0 & 0 & 3.6 & -619 & -529 \\
\hline 7 & Mar-Jun & 3 & 0 & 2 & 0 & 1 & 0 & 0 & 0 & 0 & 0 & 17.6 & 0.0 & 0.6 \\
\hline
\end{tabular}

\begin{tabular}{|c|c|c|c|c|c|c|c|c|c|c|c|c|c|}
\hline \multirow[b]{2}{*}{ Life stage } & \multirow[b]{2}{*}{ Months } & \multicolumn{12}{|c|}{ Highest productivity impact attributes (\% change in impact from template) } \\
\hline & & Att 1 & $\%$ change & Att 2 & $\%$ change & Att 3 & $\%$ change & An 4 & $\%$ change & Att 5 & \% change & Att 6 & $\%$ change \\
\hline 1 & Apr-Aug & RIPCN & 75.0 & TEMP & 75.0 & HABD & 62.5 & FLOW & 50.0 & OXYG & 250 & NUTLD & $15,1$. \\
\hline $1, \dot{2}, \dot{3}$ & Aug-óci & CSTÁB & 75.0 & RIPCN & 75.0 & TEMP & 75.0 & HÄB & 62.5 & FLOWW" & 50.0 & "ŠẼL̃L̆ & 500 \\
\hline 3 & Ott-Mar & CSTAB" & 75.0 & SEDLD & 50.0 & TËMP & & HABD & 37 & FLOOWW" & 25.0 & RIPCN & 25.0 \\
\hline 3,4 & Mar-,May & HABD & 75.0 & RIPCN & 75.0 & TЁМ̈ & & CSTAB & 35 & NUTLD & 25.0 & PATHO & 25.0 \\
\hline 5 & May-Ott & HABD & 75.0 & RIPCN & $750 "$ & TEMP & 75.0 & CSTAB & 27.9 & NUTLLD & 25.0 & PÄTHO & 25.0 \\
\hline 6 & Ott-Mar & CSTAB & $50.0^{\circ}$ & HABD & 50.0 & RIIPCN & 500 & PATHO & 25.0 & SEDLD & 25.0 " & TEMPO & 25.0 \\
\hline 7 & Mar-Jun & & & & & & & & & & & & \\
\hline
\end{tabular}

Abundance level refers to the average abundance of spawners that spawn in the natal stream reaches that populate the life history pathways using the reach in this table:

$$
\begin{array}{ll}
\text { Level 1: } & 0-10 \text { spawners } \\
\text { Level 2 } & 10-30 \text { spawners } \\
\text { Level 3: } & 30-60 \text { spawners } \\
\text { Level 4 } & >\mathbf{6 0} \text { spawners }
\end{array}
$$

\begin{tabular}{|c|c|c|c|c|c|}
\hline Attributes: & $\begin{array}{l}\text { CHEM - } \\
\text { CSTAB - } \\
\text { COMP - } \\
\text { FLOW - } \\
\text { HABD - }\end{array}$ & $\begin{array}{l}\text { chemicals } \\
\text { channel stability } \\
\text { competition (with other species) } \\
\text { flow } \\
\text { habitat diversity }\end{array}$ & $\begin{array}{l}\text { NUTLD - nutrient load } \\
\text { OBST - obstructions } \\
\text { OXYG - dissolved oxygen } \\
\text { PATHO - pathogens } \\
\text { PRED - predators (includes fishing-related losses) }\end{array}$ & $\begin{array}{l}\text { RIPCN - } \\
\text { SEDLD - } \\
\text { TEMP - } \\
\text { WITH - }\end{array}$ & $\begin{array}{l}\text { riparian condition } \\
\text { sediment load } \\
\text { water temperature } \\
\text { water withdrawals }\end{array}$ \\
\hline
\end{tabular}

Life stages: 1-prespawner; 2- spawner 3- incubation; 4- fry colonization; 5- summer rearing; 6- overwintering; 7- yearling presmolt/smolt

"NA" indicates that no life history pathway meeting the criteria used in defining these pathways was present.

Blank cells under attributes indicate no additional attributes with negative effects on productivity were evident compared to those present in the template. 
Stream Reach Summary for Primary and Secondary Spring Chinook Salmon Life Histories

Stream: Grande Ronde R.

Reach: LimberJim $\mathrm{Cr}$ - Clear Cr (RM 164.2 -166.9 from Snake R)

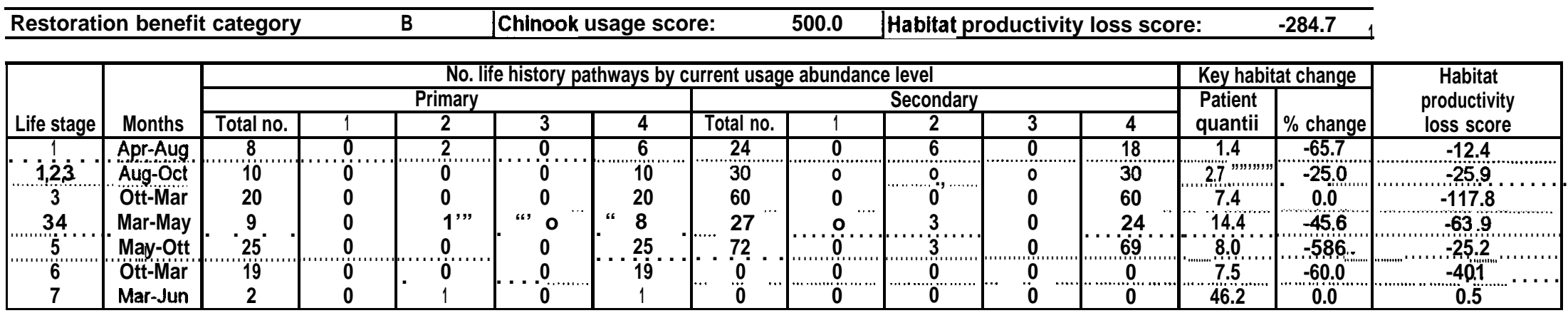

\begin{tabular}{|c|c|c|c|c|c|c|c|c|c|c|c|c|c|}
\hline \multirow[b]{2}{*}{ Life stage } & \multirow[b]{2}{*}{ Months } & \multicolumn{12}{|c|}{ Highest productivity impact attributes ("A change in impact from template) } \\
\hline & & Att 1 & $\%$ change & Att 2 & $\%$ change & Att 3 & $\%$ change & Att 4 & $\%$ change & Att 5 & $\%$ change & Att 6 & $\%$ change \\
\hline$\overline{1}$ & Apr-Aug & SEDLD & 37.5 & CSTAB & 25.0 & RIPCN & $\overline{18.9}$ & HABD & 12.5 & & & & \\
\hline $1,2,3$ & Äug-Öci & SEDLD & 50.0 & CSTAB & 25.0 & RIPCN & 25.0 & HÄBD & 12.5 & & & & \\
\hline 3 & Ott-Mar & RIPCN & 50.0 & SÉDLD & 50.0 & CSTAB & 25.0 & "РATHO" & 25.0 & & & & \\
\hline 3,4 & Mar-May & RIPCN & 75.0 & SEDLD & 39.5 & FLOW & 25.0 & HABD & 25.0 & РАTHO & 25.0 & CSTAB & $19.7^{\circ}$ \\
\hline 5 & Mav-Ott & RIPCN & 73.2 & FLOW" & 24.4 & HABD & 24.4 & PATHO & 24.4 & & & & \\
\hline 6 & Oct-Mar & CSTAB & 50.0 & FLOW & 50.0 & HÄBO & 50.0 & SEDLLD & 50.0 & PATHO & 25.0 & RIPCON & 25.0 \\
\hline 7 & Mar-Jun & & & & & & & & & & & & \\
\hline
\end{tabular}

Abundancelevel refers to the average abundance of spawners that spawn in the natal stream reaches that populate the life history pathways using the reach in this table:

$$
\begin{array}{ll}
\text { Level 1: } & 0-10 \text { spawners } \\
\text { Level 2 } & 10-30 \text { spawners } \\
\text { Level3: } & 30-60 \text { spawners } \\
\text { Level } 4 & >\mathbf{6 0} \text { spawners }
\end{array}
$$

Life stages: 1- prespawner; 2- spawner; 3- incubation; 4- fry colonization; 5- summer rearing; 8- overwintering; 7- yearling presmolt/smolt

$\begin{array}{lll}\text { Attributes: } \text { CHEM - chemicals } & \text { NUTLD - nutrient load } & \text { RIPCN - riparian condition } \\ \text { CSTAB - channel stability } & \text { OBST - obstructions } & \text { SEDLD - sediment load } \\ \text { COMP - competition (with other species) } & \text { OXYG - dissolved oxygen } & \text { TEMP - water temperature } \\ \text { FLOW - flow } & \text { PATHO - pathogens } & \text { WITH - water withdrawals } \\ \text { HABD - habitat diversity } & \text { PRED - predators (includes fishing-related losses) }\end{array}$

"NA" indicates that no life history pathway meeting the criteria usedn defining these pathways was present.

Blank cells under attributes indicate no additional attributes with negative effects on productivity were evident compared to those present in the template. 
Stream: Grande Ronde R.

Reach: $\quad$ Clear $\mathrm{Cr}$ - headwaters (RM 166.9 -170.1 from Snake R)

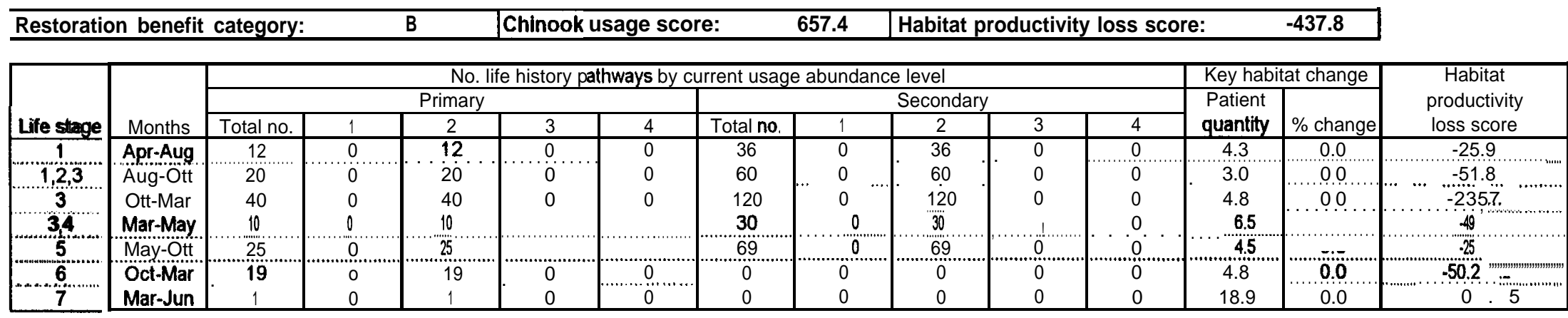

\begin{tabular}{|c|c|c|c|c|c|c|c|c|c|c|c|c|c|}
\hline \multirow[b]{2}{*}{ Life stage } & \multirow[b]{2}{*}{ Months } & \multicolumn{12}{|c|}{ Highest productivity impact attributes (\% change in impact fromtemplate) } \\
\hline & & Atf 1 & $\%$ change & Att 2 & $\%$ change & Att 3 & $\%$ change & Att 4 & $\%$ change & Att 5 & $\%$ change & Att 6 & $\%$ change \\
\hline 1 & Apr-Aug & RIPCN & 25.0 & SEDLD & 25.0 & CSTAB & 12.5 & & & & & & \\
\hline $1,2,3$ & Aug-Ott & SEDLD & 50.0 & CSTAB & 25.0 & RIPCN & 25.0 & & & & & & \\
\hline$?$ & 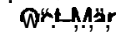 & SEDL" & 50.0 & CSTAB & 25.0 & RIPCN & 25.0 & PATHO & 12.5 & & & & \\
\hline 34 & Mar-May & RIPCN & 50.0 & CSTAB & 25.0 & PATHO & 25.0 & SEDLD & 25.0 & & & & \\
\hline 5 & May-Ott & FLOW' & 50.0 & HABD & 50.0 & RIPCN & 50.0 & SEDLD & 50.0 & CSTAB & 25,0 & РATHHO & 25.0 \\
\hline 6 & Ott-Mar & FLÖẄ & 50.0 & HABD & 50.0 & SEDLD & 50.0 & CSTAB & 25.0 & PATHO & 25.0 & RIPCN & 25.0 \\
\hline 7 & Mar-Jun & & & & & & & & & & & & \\
\hline
\end{tabular}

Abundance level refers to the average abundance of spawners that spawn in the natal stream reaches that populate the life history pathways using the reach in thitable:

$$
\begin{array}{ll}
\text { Level 1: } & 0-10 \text { apawners } \\
\text { Level 2: } & 10-30 \text { spawners } \\
\text { Level 3: } & 30-60 \text { spawners } \\
\text { Level 4: } & \mathbf{2} 60 \text { spawners }
\end{array}
$$

\begin{tabular}{|c|c|c|c|}
\hline Attributes: & $\begin{array}{l}\text { CHEM - chemicals } \\
\text { CSTAB - channel stability } \\
\text { COMP - competition (with other species) } \\
\text { FLOW - ffow } \\
\text { HABD - habitat diversity }\end{array}$ & $\begin{array}{l}\text { NUTLD - } \\
\text { OBST - } \quad \text { obstructiont ioad } \\
\text { OXYG - } \quad \text { dissolved oxygen } \\
\text { PATHO - pathogens } \\
\text { PRED - } \quad \text { predators (includes fishing-related losses) }\end{array}$ & $\begin{array}{l}\text { RIPCN - riparian condition } \\
\text { SEDLD - } \quad \text { sediment load } \\
\text { TEMP - water temperature } \\
\text { WITH - } \quad \text { water withdrawals }\end{array}$ \\
\hline
\end{tabular}

Life stages.' 1- prespawner; 2- spawner 3- incubation; 4- fry colonization; 5- summer rearing; 6- overwintering; 7- yearling presmoll/smoll

"NA" indicates that nolife history pathway meeting the criteria used in defining these pathways was present.

Blank cells under attributes indicate no additional attributes with negative effects on productivity were evident compared to those present in the template, 
Stream: Hurricane $\mathrm{Cr}$.

Reach: Mouth - Lower Alderslope Ditch (RM 121.9 -124.9 from Snake R)

\begin{tabular}{|lllll|l|}
\hline Restoration benefit category: & A & Chinook usage score: & $\mathbf{5 2 4 . 2}$ & Habitat productivity loss score: & -1973.7 \\
\hline
\end{tabular}

\begin{tabular}{|c|c|c|c|c|c|c|c|c|c|c|c|c|c|c|}
\hline \multirow[b]{3}{*}{ Life stage } & \multirow[b]{3}{*}{ Months } & \multicolumn{10}{|c|}{ No. life nistory pathways by current usage abundance level } & \multicolumn{2}{|c|}{ Key habitat change } & \multirow{3}{*}{$\begin{array}{c}\text { Habital } \\
\text { productivity } \\
\text { loss score }\end{array}$} \\
\hline & & \multicolumn{5}{|c|}{ Primary } & \multicolumn{5}{|c|}{ Secondary } & \multirow{2}{*}{$\begin{array}{l}\text { Patient } \\
\text { quantity }\end{array}$} & \multirow[b]{2}{*}{$\%$ change } & \\
\hline & & Total no. & 1 & 2 & 3 & 4 & Total no. & 1 & 2 & 3 & 4 & & & \\
\hline 1 & Apr-Aug & 6 & 6 & 0 & 0 & 0 & 42 & 42 & 0 & 0 & 0 & 0.6 & \begin{tabular}{l|l}
-94.4 \\
\end{tabular} & -165.5 \\
\hline $1,2,3$ & Aug-Oct & 10 & 10 & 0 & 0 & 0 & 70 & 70 & 0 & 0 & 0 & 2.3 & -83.6 & -660.4 \\
\hline 3 & Oct-Mar & 20 & 20 & 0 & 0 & 0 & 140 & 140 & 0 & 0 & 0 & 3.2 & -80.2 & -512.3 \\
\hline 3,4 & Mar-May & 2 & 2 & 0 & 0 & 0 & 38 & 38 & 0 & 0 & 8 & 3.6 & -83.8 & $\begin{array}{r}336.9 \\
.198 .9\end{array}$ \\
\hline 5 & May-Oct & 0 & 0 & 0 & 0 & 0 & 94 & 94 & 0 & 0 & 0 & & -80.2 & -1013 \\
\hline 6 & Oct-Mar & 0 & 0 & 0 & 0 & 0 & 19 & 19 & 0 & 0 & . & - & 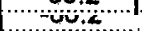 & \\
\hline 7 & Mar-Jun & 0 & 0 & 0 & 0 & 0 & 1 & 1 & 0 & 0 & 0 & 13.2 & -53.8 & 1.0 \\
\hline
\end{tabular}

\begin{tabular}{|c|c|c|c|c|c|c|c|c|c|c|c|c|c|}
\hline \multirow[b]{2}{*}{ Life stage } & \multirow[b]{2}{*}{ Months } & \multicolumn{12}{|c|}{ Highest productivity impact attributes (\% change in impact from template) } \\
\hline & & Att 1 & ?4 change & Aft 2 & $\%$ change & Att 3 & $\%$ change & Att 4 & $\%$ change & Atf 5 & $\%$ change & Att 6 & $\%$ change \\
\hline 1 & Apr-Aug & FLOW & 50.0 & HABD & 50.0 & RIPCN & 50.0 & TEMP & 50,0 & SEDLD & 37.5 & CSTAB & 12.5 \\
\hline $1,2,3$ & Aug-Oct & SEDLD & 75.0 & FLÖW & 50.0 & $\mathrm{HABD}$ & 50.0 & RIPCN & 50.0 & TEMP & 50.0 & CSTAB & 25.0 \\
\hline 3 & Ott-Mar & HABD & 75.0 & SEDLD & $75: 0$ & RIPCN & 50.0 & TЕËP̈ & 50.0 & CSTAB" & 25.0 & Filow & 25 \\
\hline 34 & Mar-May & HABD & 75.0 & SEDLD & 75.0 & FLOW & 50.0 & RIPCN & 50.0 & TEMP & 50.0 & CSTAB & $25^{\circ}$ \\
\hline 3 & May-Ott & HABD & 75.0 & FLOW & 50.0 & RIPCN & 50.0 & PATHO & 25.0 & PRED & 25.0 & & \\
\hline 6 & oct-Mar & HABD & 50.0 & SEEDLD" & 50.0 & TEM MP & 50.0 & CSTAB & 25.0 & PATHOO & 25.0 & & \\
\hline 7 & Mar-Jun & & & & & & & & & & & & \\
\hline
\end{tabular}

Abundance level refera to the average abundance of spawners that spawn in the natal stream reaches thatpopulate the iife history pathways using the reach in this table:

$\begin{array}{ll}\text { Level 1: } & 0-10 \text { spawners } \\ \text { Level 2: } & \text { 10-30 spawners } \\ \text { Level 3: } & 30-60 \text { spawners }\end{array}$

Level $4>60$ spawners

Life stages: 1- prespawner; 2- spawner; 3- incubation; 4- fry colonization; 5- summer rearing; 6- overwintering; 7- yearling presmolt/smolt

$\begin{array}{lll}\text { Attributes: } \text { CHEM - chemicals } & \text { NUTLD - nutrient load } & \text { RIPCN - riparian condition } \\ \text { CSTAB - channel stability } & \text { OBST - obstructions } & \text { SEDLD - Sediment ioad } \\ \text { COMP - competition (with other species) } & \text { OXYG - dissolved oxygen } & \text { TEMP - water temperature } \\ \text { FLOW - flow } & \text { PATHO - pathogens } & \text { WITH - water withdrawals } \\ \text { HABD - habitat diversity } & \text { PRED - predators (includes fishing-related losses) }\end{array}$

"NA" indicates that no life history pathway meeting the criteria used in defining these pathways was present.

Blank cells under attributes indicate no additional attributes with negative effects on productivity were evident compared to those present in the template. 


\section{Stream Reach Summary for Primary and Secondary Spring Chinook Salmon Life Histories}

Stream: Hurricane Cr.

Reach: Lower Alderslope Ditch - Upper Alderslope Ditch Diversion (RM 124.9 -128.2 from Snake R)

\begin{tabular}{|c|c|c|c|c|c|c|c|c|c|c|c|c|c|c|}
\hline \multicolumn{4}{|c|}{ Restoration benefit category: } & $\mathbf{F}$ & \multicolumn{3}{|c|}{ Chinook usage score: } & .0 & \multicolumn{5}{|c|}{ Habitat productivity ioss score: } & \\
\hline \multirow[b]{3}{*}{ Life stage } & \multirow[b]{3}{*}{ Months } & \multicolumn{10}{|c|}{ No. life history pathways by current usage abundance level } & \multicolumn{2}{|c|}{ Key habitat chan } & \multirow{3}{*}{\begin{tabular}{|c} 
Habitat \\
productivity \\
loss score
\end{tabular}} \\
\hline & & \multicolumn{5}{|c|}{ Primary } & \multicolumn{5}{|c|}{ Secondary } & \multirow{2}{*}{$\begin{array}{l}\text { Patient } \\
\text { quantity }\end{array}$} & \multirow{2}{*}{$\%$ change } & \\
\hline & & Total no. & 1 & 2 & 3 & 4 & \begin{tabular}{|l} 
Total no. \\
.
\end{tabular} & 1 & 2 & 3 & 4 & & & \\
\hline 1 & Apr-Aug & NA & NA & NA & NA. & NA & NA & NA & NA & NA. & NA & NA & NA & NA \\
\hline 123 & Aug-Ött & $\mathrm{NA}$ & $\mathrm{NA}$ & NÄ & $N A$ & NA & NA & NA & $\dot{N A}$ & $N A$ & NA & $N$ & NA. & NA" \\
\hline 3 & Ott-Mar & NA & NA & NA & NA & $\mathrm{NA}$ & $N A$ & $\mathrm{NA}$ & NA & NA & NA & NA & $\mathrm{NA}$ & $\mathrm{NA}$ \\
\hline 3.4 & Mar-May & NA & NA & NA & NA & NA & NA & NA. & NA & NA & NA & NA & NA & NA \\
\hline 5 & May-Ott & $\mathrm{NA}$ & NA & NA & NA & NA & NA & NA & NA & $\mathrm{NA}$ & NA & $\mathrm{NA}$ & NA & $\mathrm{NA}$ \\
\hline 6 & Ott-Mar & NA & NA & $\mathrm{NA}$ & $\mathrm{NA}$ & $\dot{N A}$ & NA & $\mathrm{NA}$ & NA & NA & NA & NA & NA & NA \\
\hline 7 & Mar-Jun & $\mathrm{NA}$ & $\mathrm{NA}$ & NA & NA & NA & NA & NA & NA & $N A$ & NA & $\mathrm{NA}$ & NA & $\mathrm{NA}$ \\
\hline
\end{tabular}

\begin{tabular}{|c|c|c|c|c|c|c|c|c|c|c|c|c|c|}
\hline \multirow[b]{2}{*}{ Life stage } & \multirow[b]{2}{*}{ Months } & \multicolumn{12}{|c|}{ Highest productivity impact attributes (\% change in impact from template) } \\
\hline & & Att 1 & $\%$ change & Att 2 & $\%$ change & Att 3 & $\%$ change & Att 4 & $\%$ change & Att 5 & $\%$ change & Att 6 & $\%$ change \\
\hline 1 & Apr-Aug & NA & NA & NA & NA & NA & NA & NA & NA & NA & NA & NA & $\mathrm{NA}$ \\
\hline $1,2,3$ & Aug-Öd & NÄ & NA & NA & NA & NA & NA & NA & inA & $\ddot{\mathrm{NA}}$ & "̈̈̈̈ & $\dddot{N A ̈}$ & NA \\
\hline 3 & Ott-Mar & $\ddot{N A}$ & NA & $\ddot{N A}$ & $\ddot{\mathrm{NA}}$ & NA & NA & NA & NA & "NÄ & NÄ & "NA" & NÄ \\
\hline 3.4 & Mar-May & NA & NA & NA & NA & NA & NA & NA & $\mathrm{NA}$ & $N A$ & $N A$ & $N A$ & $\mathrm{NA}$ \\
\hline 5 & May-Ott & $\mathrm{NA}$ & NA & NA & $\mathrm{NA}$ & NA & NA & $\mathrm{NA}$ & NA & NA & $\mathrm{NA}$ & NA & $\ddot{N} \dot{A}$ \\
\hline "6 & Ott-Mar & NA & "NÄ" & NA & NA & "NA" & NA & ...?! & NA & " & ...\% & & "in' \\
\hline 7 & "Mar-Jun & NAA & NA' & $\mathrm{NA}$ & "NÄ" & "NA" & $\mathrm{NA}$ & "NÄ & N̈̈̈ & $\mathrm{NA}$ & & & $N A$ \\
\hline
\end{tabular}

Abundance level refers to the average abundance of spawners that spawn in the natal stream reaches that populate the life history pathways using the reach in this table

Level 1: $\quad 0$-10 spawners

Level 2 10-30 spawners

Level3: $\quad 30-60$ spawners

Level4: $\quad>60$ spawners

Life stages: 1 - prespawner; 2- spawner; 3- incubation; 4- fry colonization; 5- summer rearing; 6- ovenwintering; 7- yearling presmolt/smolt

Attributes: CHEM - chemicals

CSTAB - channel stability OBST - obstructions

COMP - competition (with other species) OXYG - dissolved oxygen

PATHO - pathogens

HABD - habitat diversity PRED - predators (includes fishing-related losses)

RIPCN - riparian condition

SEDLD - sediment load

TEMP - $\quad$ water temperature

WITH - water withdrawals

"NA"indicates that no tife history pathway meeting the criteria used in defining these pathways was present.

Blank cells under attributes indicate no additional attributes with negative effects on productivity were evident compared to those present in the template. 


\section{Stream Reach Summary for Primary and Secondary Spring Chinook Salmon Life Histories}

Stream: Joseph Cr.

Reach: $\quad$ Mouth - Cottonwood $\mathrm{Cr}$ (RM 3.7- 8.2 from Snake R)

\begin{tabular}{|ll|ll|l|}
\hline Restoration benefit category: & $F$ & Chinook usage score: & 0.0 & Habitat productivity loss score: \\
\hline
\end{tabular}

\begin{tabular}{|c|c|c|c|c|c|c|c|c|c|c|c|c|c|c|}
\hline \multirow[b]{3}{*}{ Life stage } & \multirow[b]{3}{*}{ Months } & \multicolumn{10}{|c|}{ No. life history pathways by current usage abundance level } & \multicolumn{2}{|c|}{ Key habitat change } & \multirow{3}{*}{$\begin{array}{c}\text { Habitat } \\
\text { productivity } \\
\text { 10ss score }\end{array}$} \\
\hline & & \multicolumn{5}{|c|}{ Primary } & \multicolumn{5}{|c|}{ Secondary } & \multirow{2}{*}{$\begin{array}{c}\text { Patient } \\
\text { quantity }\end{array}$} & \multirow[b]{2}{*}{$\%$ change } & \\
\hline & & Tdtollal ime. & 1 & 2 & 3 & 4 & Total no. & 1 & 2 & 3 & 4 & & & \\
\hline 1 & Apr-Aug & NA & NA & $\mathrm{NA}$ & NA & NA & NA & NA & NA & NA & NA & NA & $\mathrm{NA}$ & NA \\
\hline $1,2,3$ & Aug-Odt & NA & $\mathrm{NA}$ & $\mathrm{NA}$ & $\mathrm{NA}$ & $\mathrm{NA}$ & $\mathrm{NA}$ & NA & $\mathrm{NA}$ & $\mathrm{NA}$ & $\mathrm{NA}$ & $\mathrm{NA}$ & $\mathrm{NA}$ & N̦A \\
\hline 34 & MartMby & $y$ INA & NA & NA & NA & NA & NA & NA & NA & NA & NA & NA & NA & NA \\
\hline 5 & May-Out & NA & NA & NA & NA & NA & NA & NA & NA & NA & NA & NA & NA & $\mathrm{NA}$ \\
\hline 6 & Otutivar & "NA" & NA & $\mathrm{NA}$ & $\mathrm{NA}$ & NA & NA" & $\mathrm{NA}$ & NA & NÄ & "NA" & NA & $\mathrm{NA}$ & $\mathrm{NA}$ \\
\hline 7 & Mar-Jun & NA & NA & NA & NA & NA & NA & NA & NA & $\mathrm{NA}$ & NA & NA & NA & $\mathrm{NA}$ \\
\hline
\end{tabular}

\begin{tabular}{|c|c|c|c|c|c|c|c|c|c|c|c|c|c|}
\hline \multirow[b]{2}{*}{ Life stage } & \multirow[b]{2}{*}{ Months } & \multicolumn{12}{|c|}{ Highest productivity impact attributes (\% change in impact from template) } \\
\hline & & Att 1 & $\%$ change & Att 2 & $\%$ change & Att 3 & \% change & Att 4 & \% change & Att 5 & $\%$ change & Att 6 & $\%$ change \\
\hline 1 & Apr-Aug & NA & NA & $\overline{N A}$ & $\overline{\mathrm{NA}}$ & $\overline{\mathrm{NA}}$ & $\overline{\mathrm{NA}}$ & NA & NA & NA & NA & NA & $\overline{\mathrm{NA}}$ \\
\hline $1,2,3$ & Aug-OC & NA & $\mathrm{NA}$ & $\mathrm{NA}$ & $\mathrm{NA}$ & $\mathrm{NA}$ & $\mathrm{NA}$ & $\mathrm{NA}$ & $\mathrm{NA}$ & NÄ & $\mathrm{NA}$ & $\mathrm{NA}$ & $\mathrm{NA}$ \\
\hline 3 & Ött-Mar & NÄ & Na & $\mathrm{NA}$ & $\mathrm{NA}$ & NA & "ก̈̈ & NA & NÄ & 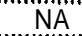 & Nä & NÄ & "NÄ \\
\hline 3,4 & Mar-May & NA & NA & NA & $\mathrm{NA}$ & NA & NÄ & NAA. & Ṇ̆ & NÄ. & NÄ & NA & NÄ \\
\hline 5 & May-Ott & NA & NA & NA & NA & NA & NA & NA & $\mathrm{NA}$ & NA & $N A$ & $\mathrm{NA}$ & $\mathrm{NA}$ \\
\hline 6 & Ott-Mar & $\mathrm{NA}$ & NA & NA & NÄ & $\mathrm{NA}$ & NA & NÄ & $\mathrm{NÄ}$ & $\mathrm{NA}$ & $\mathrm{NA}$ & $\mathrm{NÄ}$ & $\mathrm{NA}$ \\
\hline " & Mar-Jun & $\mathrm{NA}$ & $\mathrm{NA}$ & NA & $\mathrm{NA}$ & $\mathrm{NA}$ & $\mathrm{NA}$ & $\mathrm{NA}$ & NÄ & $\mathrm{NA}$ & NA & NA & $\mathrm{NA}$ \\
\hline
\end{tabular}

Abundance level refers to the average abundance of spawners that spawn in the natal stream reaches that populate the life history pathways using the reach In this table

$$
\begin{array}{ll}
\text { Level 1: } & 0-10 \text { spawners } \\
\text { Level 2: } & 10-30 \text { spawners } \\
\text { Level 3: } & 30-60 \text { spawners } \\
\text { Level } 4 & >60 \text { spawners }
\end{array}
$$

Life stages: 1-prespawner; 2-spawner; 3- incubation; 4- fry colonization; 5- summer rearing; 6- overwintering; 7- yearling presmolt/smolt

$\begin{array}{cll}\text { Attributes: } \text { CHEM - chemicals } & \text { NUTLD - nutrient load } & \text { RIPCN - riparian condition } \\ \text { CSTAB - channel stability } & \text { OBST - obstructions } & \text { SEDLD - sediment load } \\ \text { COMP - competition (with other species) } & \text { OXYG - dissolved oxygen } & \text { TEMP - water temperature } \\ \text { FLOW - flow } & \text { PATHO - pathogens } & \text { WITH - water withdrawals } \\ \text { HABD - habitat diversity } & \text { PRED - predators (includes fishing-related losses) }\end{array}$

"NA" indicates that no life history pathway meeting the criteria used in defining these pathways was present.

Blank cells under attributea indicate no additional attributes with negative effects on productivity were evident compared to those present in the template. 


\section{Stream Reach Summary for Primary and Secondary Spring Chinook Salmon Life Histories}

Stream: Joseph Cr.

Reach: $\quad$ Cottonwood $\mathrm{Cr}$ - Swamp Cr (RM 8.2- 37.7 from Snake R)

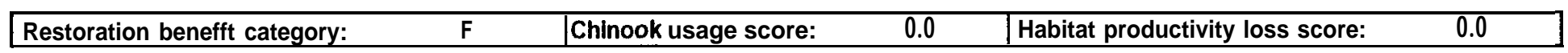

\begin{tabular}{|c|c|c|c|c|c|c|c|c|c|c|c|c|c|c|}
\hline \multirow[b]{3}{*}{ Life stage } & \multirow[b]{3}{*}{ Months } & \multicolumn{10}{|c|}{ No. life history pathways by current usage abundance level } & \multicolumn{2}{|c|}{ Key habitat change } & \multirow{3}{*}{$\begin{array}{c}\text { Habitat } \\
\text { productivity } \\
\text { loss score }\end{array}$} \\
\hline & & \multicolumn{5}{|c|}{ Primary } & \multicolumn{5}{|c|}{ Secondary } & \multirow{2}{*}{$\begin{array}{l}\text { Patient } \\
\text { quantity }\end{array}$} & \multirow[b]{2}{*}{$\%$ change } & \\
\hline & & Total no. & 1 & 2 & 3 & 4 & Total no. & 1 & 2 & 3 & 4 & & & \\
\hline 1 & Apr-Aug & NA & NA & NA & NA & NA & NA & NA & NA & NA & NA & NA & NA & NA \\
\hline $1,2,3$ & Aug-Oct & $\mathrm{NA}$ & $\mathrm{N}$ & $\mathrm{NA}$ & NÄ & "NÄ" & NA & N? & INA & NA & $\mathrm{NA}$ & $\mathrm{NA}$ & $\mathrm{NÄ}$ & \\
\hline 3 & Ott-Mar & NA & NA & NA & $\mathrm{NA}$ & NA & $N A$ & NA & NA & NA & NA & NA & NA & \\
\hline 34 & Mar-May & NA & NA & NA & NA & NA & NA & NA & $N A$ & $N A$ & NA & NA & $\mathrm{NA}$ & NA \\
\hline 5 & May-Ott & $\dot{N} \dot{A}$ & NÄ & NA & NA & $\mathrm{NA}$ & $\mathrm{NA}$ & NA & NA & NA & NA & NA & NA & NA \\
\hline " & Ött-Mar & $\mathrm{NA}$ & NA & NA & NA & NA & NA & NA & NA & NA & NA & NA & NA & NA \\
\hline 7 & Mar-jun & $\mathrm{NA}$ & $\mathrm{NA}$ & NA & $\mathrm{NA}$ & $\mathrm{NA}$ & NA & NA & NA & $\mathrm{NA}$ & $\mathrm{NA}$ & $\mathrm{NA}$ & NA & $\mathrm{NA}$ \\
\hline
\end{tabular}

\begin{tabular}{|c|c|c|c|c|c|c|c|c|c|c|c|c|c|}
\hline \multirow[b]{2}{*}{ Life stage } & \multirow[b]{2}{*}{ Months } & \multicolumn{12}{|c|}{ Highest productivity impact attributes (\% change in impact from template) } \\
\hline & & Att 1 & $\%$ change & Att 2 & $\%$ change & Att 3 & $\%$ change & Att 4 & $\%$ change & Att 5 & $\%$ change & Atf 6 & $\%$ change \\
\hline 1 & Apr-Aug & NA & NA & NA & NA & NA & NA & NA & NA & NA & NA & NA & NA \\
\hline $1,2,3$ & Aug-Oct & $\mathrm{NA}$ & $\mathrm{NA}$ & NA & $\mathrm{NA}$ & $\mathrm{NA}$ & $\mathrm{NA}$ & $\mathrm{NA}$ & $\mathrm{NA}$ & $\mathrm{NA}$ & $\mathrm{NA}$ & $\mathrm{NA}$ & $\mathrm{NA}$ \\
\hline 3 & Ott-Mar & NÄ & NÄ & $\%$ & N.A. & NÄ & NÄ & NA & NA & N & & 8 & NA \\
\hline & Mar-May & NA & NA & & $N A$ & NA & NA & NA & & N" & & & NA \\
\hline 54 & May-Ott & $\mathrm{NA}$ & NA "'," & NA & $\mathrm{NA}$ & NA & $\mathrm{NA}$ & NAA & $\mathrm{NA}_{2}$ & $\mathrm{NA}$ & $\ddot{\mathrm{NA}}$ & NÄ & NÄ \\
\hline 6 & Ott-Mar & NA & NÄ & NA & $\mathrm{NA}$ & $\dot{N} \dot{A}$ & NA & $\mathrm{NA}$ & $\mathrm{NA}$ & $\mathrm{NA}$ & $\mathrm{NA}$ & $\dot{\mathrm{NA}}$ & $\mathrm{NA}$ \\
\hline 7 & Mar-Jun & $\mathrm{NA}$ & $\mathrm{NA}$ & NÄ & $\mathrm{NA}$ & $\mathrm{NA}$ & NA & $\mathrm{NA}$ & $\mathrm{NA}$ & $\mathrm{NA}$ & $\mathrm{NA}$ & $\mathrm{NA}$ & $\ddot{\mathrm{NA}}$ \\
\hline
\end{tabular}

Abundance level refera to the average abundance of spawners that spawn in the natal stream reaches that populate the life history pathwaya using the reach in this table

Level 1: $\quad 0-10$ spawners

Level 2 10-30 spawners

Level $3 \quad 30-60$ spawners

Level 4: > 60 spawners

Life stages: 1- prespawner; 2- spawner; 3- incubation; 4- fry colonization; 5- summer rearing; 6- overwintering; 7-yearling presmolt/smolt

\section{Attributes: CHEM - chemicals}

CSTAB - channel stability

COMP - competition (with other species)

FLOW - ftOW

HABD - habitat diversity
NUTLD - nutrient load

OBST - obstructions

OXYG - dissolved oxygen

PATHO - pathogens

PRED - predators (includes fishing-reiated losses)
RIPCN - riparian condition

SEDLD - sediment ioad

TEMP - $\quad$ water temperature

WITH - water withdrawals

"NA" indicates that no life history pathway meeting the criteria used in defining these pathways wspresent.

Blank cells under attributes indicate no additional attributes with negative effects on productivity were evident compared to those present in the template. 
Stream Reach Summary for Primary and Secondary Spring Chinook Salmon Life Histories

Stream: Joseph Cr.

Reach: Swamp $\mathrm{Cr}$ - Chesnimnus $\mathrm{Cr}$ (RM 37.7 -52.7 from Snake R)

\begin{tabular}{ll|ll|l|}
\hline Restoration benefit category: & $F$ & Chlnook usage score: & 0.0 & Habitat productivity loss score: \\
\hline
\end{tabular}

\begin{tabular}{|c|c|c|c|c|c|c|c|c|c|c|c|c|c|c|}
\hline \multirow[b]{3}{*}{ Life stage } & \multirow[b]{3}{*}{ Months } & \multicolumn{10}{|c|}{ No. life history pathways by current usage abundance level } & \multicolumn{2}{|c|}{ Key habitat change } & \multirow{3}{*}{$\begin{array}{l}\text { Habitat } \\
\text { productivity } \\
\text { loss score }\end{array}$} \\
\hline & & \multicolumn{5}{|c|}{ Primary } & \multicolumn{5}{|c|}{ Secondary } & \multirow{2}{*}{$\begin{array}{l}\text { Patient } \\
\text { quantity }\end{array}$} & \multirow[b]{2}{*}{$\%$ change } & \\
\hline & & Total no. & 1 & 2 & 3 & 4 & Total no. & 1 & 2 & 3 & 4 & & & \\
\hline 1 & Apr-Aug & NA & NA & NA & NA & $N A$ & NA & NA & $N A$ & NA & NA & NA & NA & NA \\
\hline $1,2,3$ & Aug-Oct & $\mathrm{NA}$ & NA & NA & $N A$ & $\mathrm{NA}$ & NA & $\mathrm{NA}$ & NA & NA" & $\mathrm{NA}$ & NA & NA & NA" \\
\hline 3 & Oct-Mar & NA & NA & NA & $\mathrm{NA}$ & NA & $\mathrm{NA}$ & NA & NA & NA & $\mathrm{NA}$ & NA & $\mathrm{NA}$ & NA \\
\hline 3,4 & Mar-May & $\mathrm{NA}$ & NA & NA & $\mathrm{NA}$ & NA & NA & $\mathrm{NA}$ & $\mathrm{NA}$ & $\mathrm{NA}$ & $\mathrm{NA}$ & $\mathrm{NA}$ & $\mathrm{NA}$ & NA \\
\hline 5 & May-Oct & $\mathrm{NA}$ & $\mathrm{NA}$ & $\mathrm{NA}$ & NA & $\mathrm{NA}$ & $\mathrm{NA}$ & NA & $\mathrm{NA}$ & $N A$ & NÄ & $N A$ & $\mathrm{NA}$ & $\mathrm{NA}$ \\
\hline 6 & Oct-Mar & $\mathrm{NA}$ & $\mathrm{NA}$ & $\mathrm{NA}$ & NA" & $\mathrm{NA}$ & $\mathrm{NA}$ & NA" & $\mathrm{NA}$ & $\mathrm{NA}$ & $\mathrm{NA}$ & NA & NA" & NA \\
\hline 7 & Mar-Jun & $\mathrm{NA}$ & $\mathrm{NA}$ & NA & $\mathrm{NA}$ & NA & $\mathrm{NA}$ & $\mathrm{NA}$ & $N A$ & $\mathrm{NA}$ & $\mathrm{NA}$ & NA & NA & NA \\
\hline
\end{tabular}

\begin{tabular}{|c|c|c|c|c|c|c|c|c|c|c|c|c|c|}
\hline \multirow[b]{2}{*}{ Life stage } & \multirow[b]{2}{*}{ Months } & \multicolumn{12}{|c|}{ Highest productivity impact attributes (\% change in impact from template) } \\
\hline & & Aft 1 & $\%$ change & Att 2 & $\%$ change & Att 3 & $\%$ change & Att 4 & ?4 change & Att 5 & $\%$ change & Att 6 & $\%$ change \\
\hline 1 & Apr-Aug & NA & NA & NA & NA & NA & NA & NA & NA & NA & NA & NA & NA \\
\hline $1,2,3$ & Aug-Öct & NA & $\dot{N} \dot{A}$ & $\dot{N} \dot{A}$ & NA & NA & NA & NA & NA & $\mathrm{NA}$ & NA & NA & NA \\
\hline 3 & Ott-Mar & NA & $\mathrm{NA}$ & NA & NA & $\mathrm{NA}$ & NA & $\dot{\mathrm{N}} \dot{A}$ & $\mathrm{NA}$ & NA & NA & $\mathrm{NA}$ & $\mathrm{NA}$ \\
\hline 3,4 & Mar-May & NA & NA & $\mathrm{NA}$ & NA & NA & NA & NA & NA & NA & NA & NA & NA \\
\hline 5 & May-Ott & NA & $\mathrm{NA}$ & NA & NA & NA & NA & NA & NA & NA & $\mathrm{NA}$ & NA & NA \\
\hline$\dot{6}$ & Ott-Mar & ì̈ & $\mathrm{NÄ}$ & NA' & NA & NA & NA & NA & NA & NA & $\mathrm{NA}$ & NÄ & $\mathrm{NA}$ \\
\hline 7 & Mar-Jun & NA & NA & NÄ & $\mathrm{NA}$ & $\mathrm{NA}$ & $\mathrm{NA}$ & $\mathrm{NA}$ & $\mathrm{NA}$ & $\mathrm{NA}$ & NA & $\mathrm{NA}$ & NA \\
\hline
\end{tabular}

Abundance lovel refers to the average abundance of spawnera that spawn in thenatal stream reaches that populate the tife history pathwaya using the reach in this table

$\begin{array}{ll}\text { Level 1: } & 0-10 \text { spawners } \\ \text { Level 2 } & 10-30 \text { spawners } \\ \text { Level 3: } & 30-60 \text { spawners } \\ \text { Level 4: } & \mathbf{> 6 0} \text { spawners }\end{array}$

Life stages.' 1- prespawner; 2- spawner; 3- incubation; 4- fry colonization; 5- summer rearing; 6- overwintering; 7- yearling presmolt/smolt

\begin{tabular}{|c|c|c|c|}
\hline Attributes: & $\begin{array}{l}\text { CHEM - chemicals } \\
\text { CSTAB - channel stability } \\
\text { COMP - competition (with other species) } \\
\text { FLOW - ftOW } \\
\text { i-tABD - habitat diversity }\end{array}$ & $\begin{array}{l}\text { NUTLD - } \\
\text { OBST - } \quad \text { obstructions load } \\
\text { OXYG - } \quad \text { dissolved oxygen } \\
\text { PATHO - pathogens } \\
\text { PRED - } \quad \text { predators (includes fishing-related losses) }\end{array}$ & $\begin{array}{l}\text { RIPCN - } \quad \text { riparian condition } \\
\text { SEDLD - } \\
\text { Sediment load } \\
\text { TEMP - } \quad \text { water temperature } \\
\text { WITH - water withdrawals }\end{array}$ \\
\hline
\end{tabular}

"NA" indicates that no life history pathway meeting the criteria used in defining these pathways was present.

Blank cells under attributes indicate no additional attributes with negative effects on productivity were evident compared to those present in the template. 
Stream Reach Summary for Primary and Secondary Spring Chinook Salmon Life Histories

Stream: Lookingglass $\mathrm{Cr}$.

Reach: Mouth - Little Lookingglass Cr (RM 83.4 -87.6 from Snake R)

\begin{tabular}{|ll|ll|l|}
\hline Restoration benefit category: & B & Chinook usage score: & 611.0 & Habitat productivity loss score: \\
\hline
\end{tabular}

\begin{tabular}{|c|c|c|c|c|c|c|c|c|c|c|c|c|c|c|}
\hline \multirow[b]{3}{*}{ Life stage } & \multirow[b]{3}{*}{ Montha } & \multicolumn{10}{|c|}{ No. life history pathways by current usage abundance level } & \multicolumn{2}{|c|}{ Key habitat change } & \multirow{3}{*}{$\begin{array}{c}\text { Habitat } \\
\text { productivity } \\
\text { loss score }\end{array}$} \\
\hline & & \multicolumn{5}{|c|}{ Primary } & \multirow[b]{2}{*}{ Tolal no. } & & \multirow{2}{*}{\begin{tabular}{|c|} 
Secondary \\
2
\end{tabular}} & \multirow[b]{2}{*}{3} & \multirow[b]{2}{*}{4} & Patient & & \\
\hline & & Total no. & 1 & 2 & 3 & 4 & & 1 & & & & quantity & $\%$ change & \\
\hline $1 .$. & Apsing & 21. & 0 & 18 & 3 & $\overline{0}$ & 18 & 0 & 12 & 6 & 0 & 5.0 & 0.0 & -52.4 \\
\hline $1,2,3$ & Aug-Octt & $30^{\circ}$ & 0 & 30 & 0 & 0 & 20 & 0 & 20 & 0 & 0 & 2.6 & -51.0 & -191.0 \\
\hline 3,4 & Mar-May & 24,2 & 0, & 24 & 0 & 0,1 & 15 & 0 & 10 & 5 & 0 & 6.3 & -29.7 & -16.6 \\
\hline 5 & May-Öct & 72 & 0 & 69 & 3 & 0 & 25 & 0 & 25 & 0 & 0 & 4.9 & 0.0 & -25.1 \\
\hline 6 & Oct-Mar & 0 & 0 & 0 & 0 & 0 & 19 & 0 & 19 & 0 & 0 & 2.7 & 0.0 & -2.3 \\
\hline 7 & Mar-jun & $\mathrm{O}$ & 0 & 0 & 0 & 0 & 2 & 0 & 1 & 1 & 0 & 16,5 & 0.0 & 0.0 \\
\hline
\end{tabular}

\begin{tabular}{|c|c|c|c|c|c|c|c|c|c|c|c|c|c|}
\hline \multirow[b]{2}{*}{ Life stage } & \multirow[b]{2}{*}{ Months } & \multicolumn{12}{|c|}{ Highest productivity impact attributes (\% change in impact from template) } \\
\hline & & Att 1 & $\%$ change & Att 2 & $\%$ change & Att 3 & $\%$ change & Aft 4 & $\%$ change & Att 5 & $\%$ change & Att 6 & $\%$ change \\
\hline 1 & Apr-Aug & TEMP. & 42.1 & PATHO & $25 . \mathrm{n}$ & & & & & & & & \\
\hline $1,2,3$ & Aug-Oct & TEMP & 50.0 & $\mathrm{HABO}$ & 250 & 05 & 25.0 & & & & & & \\
\hline 3 & Oct-Mar & ri,nivi. & $25 n_{2}$ & PATPAT & $H O 25.0$ & & 250 & & & & & & \\
\hline 3,4 & Mar-May & FLOW & 25.0 & $\mathrm{HA}$ & DD 25250 & & & TEMP & 25.0 & & & & \\
\hline 5 & May-Oct & HABD & 25.0 & PATHO & 25.0 & TEMP & 25.0 & CSTAB & 12.6 & & & & \\
\hline 6 & Oct-Mar & CSTAB & 25.0 & HABD & 250 & OBSTT & 25.0 & РAT HO & 25.0 & TEMP & 25.0 & WITH & 25.0 \\
\hline 7 & Mar-Jun & OBST & 14.2 & РАTHO & 14.2 & Wï̈н & 14.2 & & & & & & \\
\hline
\end{tabular}

Abundance /eve/ refers to the average abundance of spawners that spawn in the natal stream reaches that populate the life history pathways using the reach in this table: Level 1: $\quad 0-10$ spawners

$\begin{array}{ll}\text { Level } 2 & 10-30 \text { spawners } \\ \text { Level 3: } & 30-60 \text { spawners }\end{array}$

$\begin{array}{ll}\text { Level 3: } & 30-60 \text { spawners } \\ \text { Level } 4 & >60 \text { spawnera }\end{array}$

Life stages: 1- prespawner; 2- spawner; 3- incubation; 4- fry colonization; 5- summer rearing; 6- overwintering; 7- yearling presmoll/smolt

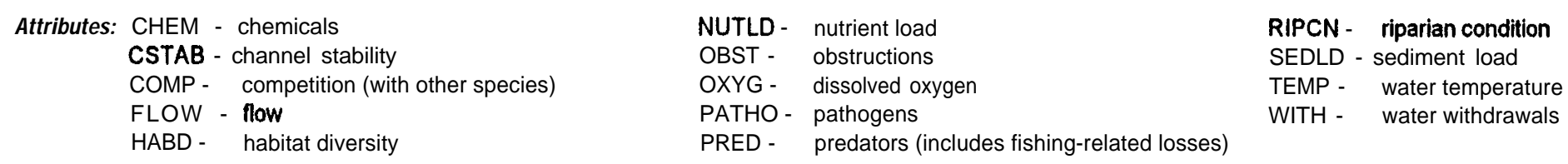

"NA" indicates that nolife history pathway meeting the criteria used in defining these pathways was present.

Blank cells under attributes indicate no additional attributes with negative effects on productivity were evident compared to those present in the template, 
Stream: Lookingglass $\mathrm{Cr}$.

Reach: Little Lookingglass $\mathbf{C r}_{\mathbf{r}}$ - headwaters (RM 87.6 -93.8 from Snake R)

\begin{tabular}{|l|l|l|l|l}
\hline Restoration benefit category: Chinook usage score: & $\mathrm{E}$
\end{tabular}

\begin{tabular}{ll|lll} 
& $\mathbf{8 2 5 . 0}$ & Habitat productivity loss score: & $\mathbf{6 5 . 5}$ \\
\hline
\end{tabular}

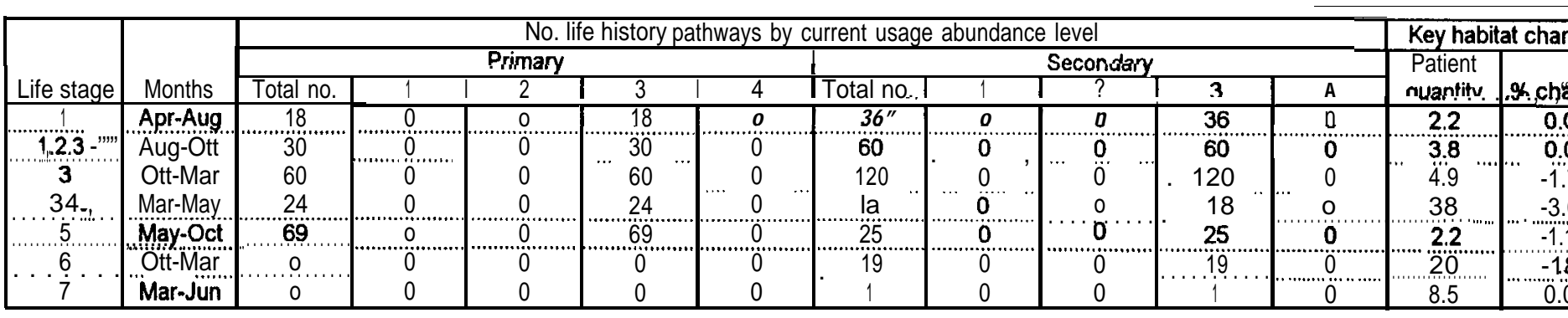

\begin{tabular}{|c|c|c|c|c|c|c|c|c|c|c|c|c|c|}
\hline \multirow[b]{2}{*}{ Life stage } & \multirow[b]{2}{*}{ Months } & \multicolumn{12}{|c|}{ Highest productivity impact attributes (\% change in impact from template) } \\
\hline & & Att 1 & $\%$ change & Att 2 & $\%$ change & At! 3 & $\%$ change & Att 4 & $\%$ change & At! 5 & $\%$ change & Att 6 & \% che \\
\hline & Apr-Aug & PATHO & 25.0 & & & & & & & & & & \\
\hline 123 & Aug-Oct & PATHO & 25.0 & & & & & & & & & & \\
\hline 3 & Ött-Märar & 'PATTHÖÖ & 250 & FLOW & 12.5 & & & & 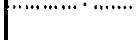 & & & & \\
\hline 3,4 & Mar-May & "HÄB̈̈ & 25.0 & PATHO & 25.0 & FLOW & 12.5 & $\cdots \cdots$ & & & $10^{\circ}$ & & \\
\hline 5 & May-Ott & HABD & 25.0 & PATHO & 25.0 & & & & & & & & \\
\hline 6 & Ott-Mar & "'"CSTAB" & $25.0^{\circ}$ & "P̈ÄTḦÖO & 25.0 & ".TËM P'" & $25.0^{\circ}$ & & & & & & \\
\hline 7 & Mar-jun & РATHO & $25.0^{\circ}$ & & & & & & & & & & \\
\hline
\end{tabular}

Abundance level refers to the average abundance of spawners that spawn in the natal stream reaches that populate the life history pathways using the reach in Level 1: $\quad 0-10$ spawners

Level 2 10-30 spawners

Level 3: $\quad 30-60$ spawners

Level $4>60$ spawners

Life stages.' 1- prespawner; 2- spawner; 3- incubation; 4- fry colonization; 5- summer rearing; 6- overwintering; 7- yearling presmolt/smolt

Attributes: CHEM - chemicals

CSTAB - channel stability

COMP - competition (with other species)

FLOW - flow

HABD - habitat diversity

\author{
NUTLD - nutrient load \\ OBST - obstructions \\ OXYG - dissolved oxygen \\ PATHO - pathogens \\ PRED - predators (includes fishing-related losses)
}

RIPCN - riparian condition SEDLD - sediment load TEMP - water temperature WITH - water withdrawals

"NA" indicates that no life history pathway meeting the criteria used in defining these pathways was present.

Blank cells under attributes indicate no additional attributes with negative effects on productivity were evident compared to those present in the template. 
Stream: Lostine R.

Reach: $\quad$ Mouth - Clear'water Ditch (RM 105.8 -108.3 from Snake R)

\begin{tabular}{|ll|ll|l|}
\hline Restoration benefit category: & F & Chinook usage score: & 15.6 & Habitat productivity loss score: \\
\hline
\end{tabular}

\begin{tabular}{|c|c|c|c|c|c|c|c|c|c|c|c|c|c|c|}
\hline \multirow[b]{3}{*}{ Life stage } & \multirow[b]{3}{*}{ Months } & \multicolumn{10}{|c|}{ No. iife history pathways by current usage abundance level } & \multicolumn{2}{|c|}{ Key habitat change } & \multirow{3}{*}{$\begin{array}{c}\text { Habitat } \\
\text { productivity } \\
\text { loss score }\end{array}$} \\
\hline & & \multicolumn{5}{|c|}{ Primary } & \multicolumn{5}{|c|}{ Secondary } & \multirow{2}{*}{$\begin{array}{c}\text { Patient } \\
\text { quantity }\end{array}$} & \multirow[b]{2}{*}{ \% change } & \\
\hline & & Total no, & 1 & 2 & 3 & 4 & Total no. & 1 & 2 & 3 & 4 & & & \\
\hline & Apr-Aug & 9 & 7 & 0 & 2 & 0 & 15 & 13 & 0 & 2 & 0 & 9.0 & -10.1 & 1.1 \\
\hline $1,2,3$ & Aug-Ôtt & $\mathrm{NA}$ & NA & NA & NA & $\dot{N} \dot{A}$ & NA & $\mathrm{NA}$ & NA & NA. & $\mathrm{NA}$ & NA" & NA & NA' \\
\hline 3 & Ott-Mar & NA & NA & NA & $\mathrm{NA}$ & NA & NA & NA & NA & $? ? ?$ & NA & NA & $\mathrm{NA}$ & $\dot{N} \dot{A}$ \\
\hline 34 & Mar-May & 2 & 2. & 0. & 0 & 0 & 6 & 6 & 0 & 0 & 0 & 18.0 & 4.1 & $-18.5^{\circ}$ \\
\hline 5 & May-Oct & 2 & 1 & 0 & 1 & 0 & 9 & 7 & 0 & 2 & 0 & 12.4 & 1.4 & -3.5 \\
\hline 6 & Oct-Mar & 1 & $\dddot{1}$ & $\dddot{0}$ & $\dddot{0}$ & 0 & 0 & 0 & 0 & 0 & 0 & 12.4 & 3.3 & 0.4 \\
\hline$\dot{7}$ & Mar-jun & 4 & 3 & 0 & 1 & 0 & 0 & 0 & 0 & 0 & $\dot{0}$ & 21.5 & 0,7 & 0 \\
\hline
\end{tabular}

\begin{tabular}{|c|c|c|c|c|c|c|c|c|c|c|c|c|c|}
\hline \multirow[b]{2}{*}{ Life stage } & \multirow[b]{2}{*}{ Months } & \multicolumn{12}{|c|}{ Highest productivity impact attributes (\% change in impact from template) } \\
\hline & & Att 1 & $\%$ change & Att 2 & $\%$ change & Att 3 & $\%$ change & Att 4 & \% change & Att 5 & $\%$ change & Att 6 & $\%$ change \\
\hline 1,23 & $\begin{array}{l}\text { Apr-Aug } \\
\text { Aug-Öof }\end{array}$ & $\begin{array}{c}\text { FLOW } \\
\text { NA }\end{array}$ & $\begin{array}{l}13.6 \\
N A\end{array}$ & $\begin{array}{c}\text { TEMP } \\
\text { NA }\end{array}$ & $\begin{array}{l}136 \\
\mathrm{NA}\end{array}$ & $\begin{array}{c}\text { OBST } \\
\text { NA }\end{array}$ & $\begin{array}{l}9.1 \\
\text { NA }\end{array}$ & $\begin{array}{c}\text { HABD } \\
\text { NA }\end{array}$ & $\begin{array}{l}4.5 \\
\text { NA }\end{array}$ & NA & NA & NA & NA \\
\hline 3 & OOtt-Mar & "NA" & $\mathrm{NAA}$ & กัลิ. & $\mathrm{NA}$ & $\mathrm{NAM}$ & $\mathrm{N} \mathrm{A}$ & $\mathrm{NÄ}$ & NA & NA & $\mathrm{NA}$ & $\mathrm{NA}$ & $\mathrm{NA}$ \\
\hline 3,4 & Mar-,May & TEMP & 24.8 & "CSṪAB & 12.4 & FLOW & 12.4 & "ẄIт" & 12.4 & & & & \\
\hline 5 & May-Ott & TEMP & 10.5 & 'FLOW' & "' 3.5 & HABD & 3.5 & РАTHO & 3.5 & "wi" WITH & 3.5 & & \\
\hline 6 & Ott-Mar & FLOW & 16.4 & CंSTÄB & 8.2 & $H A ̈ B D ”$ & 6.2 & PATHO & 8.2 & "ŠËL̄L̄". & ??? & & \\
\hline 7 & Mar-Jun & WITH & 2.1 & & & & & & & & & & \\
\hline
\end{tabular}

Abundancelevel refers to the average abundance of spawners that spawn in the natal stream reaches that populate the life history pathways using the reach in this table:

$$
\begin{array}{ll}
\text { Level 1: } & 0-10 \text { spawners } \\
\text { Level 2 } & 10-30 \text { spawners } \\
\text { Levei 3: } & 30-60 \text { spawners } \\
\text { Level 4 } & >60 \text { spawners }
\end{array}
$$

Life stages: 1- prespawner; 2- spawner; 3- incubation; 4- fry colonization; 5- summer rearing; 6- overwintering; 7- yearling presmolt/smolt

Attributes: CHEM - chemicals

CSTAB - channel stability

COMP - competition (with other species)

FLOW - ftOW

HABD - habitat diversity
NUTLD - nutrient ioad

OBST - obstructions

OXYG - dissolved oxygen

PATHO - pathogens

PRED - predators (includes fishing-related losses)
RiPCN - riparian condition

SEDLD - sediment ioad

TEMP - water temperature

WITH - water withdrawals

"NA" indicates that no life history pathway meeting the criteria used in defining these pathways was present.

Blank cells under attributes indicate no additional attributes with negative effects on productivity were evident compared to those present In thetemplate. 
Stream: Lostine R.

Reach: Clearwater Ditch - Cross Country Ditch (RM 108.3 -110.8 from Snake R)

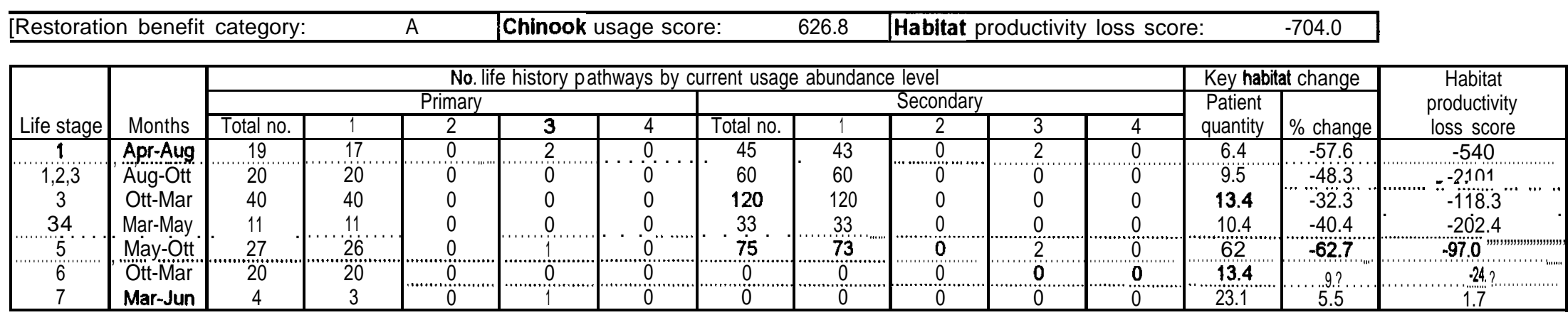

\begin{tabular}{|c|c|c|c|c|c|c|c|c|c|c|c|c|c|}
\hline \multirow[b]{2}{*}{ Life stage } & \multirow[b]{2}{*}{ Months } & \multicolumn{12}{|c|}{ Highest productivity impact attributes (\% change in impact from template) } \\
\hline & & Att 1 & $\%$ change & Att 2 & $\%$ change & Att 3 & $\%$ change & Att 4 & $\%$ change & Att 5 & $\%$ change & Att 6 & $\%$ change \\
\hline 1 & Apr-Aug & FLOW & 25.0 & TEMP & 25.0 & $\overline{H A B D}$ & 17.6 & WITH & 17.6 & & & & \\
\hline $1,2,3$ & Aug-Oct & CSTAB & 25.0 & FLOW & 25.0 & SEDLD & 25.0 & TEMP & 25.0 & WITH & 25.0 & HABD & 12.5 \\
\hline 3 & Oct-Mar & CSTAB & 25.0 & FLOW & 25.0 & HABD & 25.0 & SEDLD & 25.0 & WITH & 25.0 & РATHO & 12.5 \\
\hline 3,4 & Mar-May & HABD & 50.0 & FLOW & 25.0 & PATHO & 25.0 & TEMP & 25.0 & WITH & 25.0 & CSTAB & 11.8 \\
\hline 5 & May-Oct & $\mathrm{HABD}$ & 50.0 & FLOW & 25.0 & PATHO & 25.0 & TEMP & 25.0 & WITH & 25.0 & CSTAB & 8.9 \\
\hline 6 & Ocl-Mar & HABD & 48.4 & CSTAB & 24.2 & PATHO & 24.2 & SEDLD & 24.2 & & & & \\
\hline 7 & Mar-Jun & WITH & 8.2 & & & & & & & & & & \\
\hline
\end{tabular}

Aburrr/ante level refera to the average abundance of spawners that spawn in the natal stream reaches that populate the life history pathways using the reach in this table:

$$
\begin{array}{ll}
\text { Level 1: } & 0-10 \text { spawners } \\
\text { Level 2 } & 10-30 \text { spawners } \\
\text { Level 3 } & 30-80 \text { spawners } \\
\text { Level 4: } & >60 \text { spawners }
\end{array}
$$

Life stages: 1 - prespawner; 2- spawner; 3- incubation; 4- fry colonization; 5- summer rearing; 6- overwintering; 7- yearling presmolt/smolt

$\begin{array}{lll}\text { Attributes: } \text { CHEM - chemicals } & \text { NUTLD - nutrient load } & \text { RIPCN - riparian condition } \\ \text { CSTAB - channel stability } & \text { OBST - obstructions } & \text { SEDLD - sediment load } \\ \text { COMP - competition (with other species) } & \text { OXYG - dissolved oxygen } & \text { TEMP - water temperature } \\ \text { FLOW - flow } & \text { PATHO - pathogens } & \text { WITH - water withdrawals } \\ \text { HABD - habitat diversity } & \text { PRED - predators (includes fishing-related losses) }\end{array}$

"NA" indicates that no life history pathway meeting the criteria used in defining these pathways was present.

Blank cells under attributes indicate no additional attributes with negative effects on productivity were evident compared to those present in the template. 
Stream: Lostine $\mathrm{R}$

Reach: Cross Country Ditch - Sheep Ridge Dam (RM 110,8 -115.0 from Snake R)

\begin{tabular}{|ll|ll|l|}
\hline Restoration benefit category: & D & Chinook usage score: & 218.5 & Habitat productivity loss score: \\
\hline
\end{tabular}

\begin{tabular}{|c|c|c|c|c|c|c|c|c|c|c|c|c|c|c|}
\hline \multirow[b]{3}{*}{ Life stage } & \multirow[b]{3}{*}{ Months } & \multicolumn{10}{|c|}{ No. life history pathways by current usage abundance level } & \multicolumn{2}{|c|}{ Key habitat change } & \multirow{3}{*}{$\begin{array}{c}\text { Habitat } \\
\text { productivity } \\
\text { loss score }\end{array}$} \\
\hline & & \multicolumn{5}{|c|}{ Primary } & \multicolumn{5}{|c|}{ Secondary } & Patient & & \\
\hline & & Total no. & $T$ & 2 & $\overline{3}$ & 4 & Total no. & $T$ & 2 & $\overline{3}$ & $\overline{4}$ & quantity & $\%$ change & \\
\hline & Apr-Aug & 12 & 10 & 0 & 2 & 0 & 24 & 22 & 0 & 2 & 0 & 8.0 & -16.8 & -151.0 \\
\hline 123 & Aug-Ộtt & 10 & $10^{\circ}$ & 0 & 0 & 0 & 30 & 30 & 0 & $0^{\circ}$ & 0 & 2.0 & -664 & -1.587 \\
\hline 34 & Mar-May & 2. & 2 & 0 & 0 & 0 & 6 & “" 6 & 0 & 0 & 0 & 0.9 & -83.3 & -54.5 \\
\hline 5 & May-Ott & 3 & 2 & 0 & 1 & "ö" & 6 & 4 & 0 & 2 & 0 & 4.5 & -23.8 & -11.6 \\
\hline 6 & Oct-Mar & $\mathrm{NA}$ & $\mathrm{NÄ}$ & NäA & $\mathrm{NA}$ & $\mathrm{NA}$ & NA & $\mathrm{NA}$ & $\mathrm{NA}$ & NÄ & $\mathrm{NA}$ & $\mathrm{NA}$ & NẠ & $\mathrm{NA}$ \\
\hline$\dot{7}$ & Mar-Jun & 3 & 2 & 0 & 1 & 0 & 0 & $0^{\circ}$ & 0 & 0 & 0 & 19.8 & 0.0 & 0 \\
\hline
\end{tabular}

\begin{tabular}{|c|c|c|c|c|c|c|c|c|c|c|c|c|c|}
\hline \multirow[b]{2}{*}{ Life stage } & \multirow[b]{2}{*}{ Months } & \multicolumn{12}{|c|}{ Highest productivity impact attributes (\% change in impact from template) } \\
\hline & & Att 1 & $\%$ change & Atf 2 & $\%$ change & Att 3 & $\%$ change & Att 4 & $\%$ change & Att 5 & $\%$ change & Aft 6 & $\%$ change \\
\hline & Apr-Aug & FLOW & 56,5 & HABD & 37.7 & WITH & 37.7 & CSTAB & 25.0 & OBST & 18.8 & RIPCN & 18.8 \\
\hline 123 & Äug-Ött & FLOW & 75.0 & CSTAB & 50.0 & HABD & 50.0 & WiITH & 50.0 & ÖBST & 25.0 & RIPCN & 25.0 \\
\hline 6 & Oct-Mar & CSTAB & 50.0 & HÄBO & $\because 500$ & FLOOW & 25.0 & РATHO & 25.0 & WiTH & $25.0^{\circ}$ & & \\
\hline & Mar-May & CSTAB & 50.0 & "HÄB̈̄̈ & $500^{\circ}$ & FELÖẄ & 25.0 & "PÄTHO" & $25.0^{\circ}$ & "Ẅï̄ï" & 25.0 & & $\cdots$ \\
\hline 5 & May-Ött & F́LOW & 17.2 & WITH & $17.2^{\circ}$ & $\mathrm{HABD}$ & 8.6 & PATHO & 8.6 & RIPCN & 8.6 & & \\
\hline 8 & Ött-Msr & $\mathrm{NA}$ & $\mathrm{NA}$ & $\mathrm{NA}$ & $\mathrm{NA}$ & $\mathrm{NA}$ & $\mathrm{NA}$ & $\mathrm{NA}$ & $\mathrm{NÄ}$ & $\mathrm{NA}$ & $\mathrm{NA}$ & $\mathrm{NA}$ & $\mathrm{NÄ}$ \\
\hline 17 & Mar-Jun & 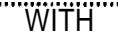 & 3.5 & & & & & & & & & & \\
\hline
\end{tabular}

Abundance level refers to the average abundance of spawners that spawn in the natal stream reaches that populate the tife history pathways using the reach in this table Level 1: $\quad 0-10$ spawners

Level 2 10-30 spawners

Level 3: $\quad 30-60$ spawners

Level 4: $>60$ spawners

Life stages: 1- prespawner; 2- spawner; 3- incubation; 4- fry colonization; 5- summer rearing; 8- overwintering; 7-yearling presmolt/smolt

Attributes: CHEM - chemicals

CSTAB - channel stability

COMP - competition (with other species)

FLOW - ftOW

HABD - habitat diversity
NUTLD - nutrient load

OBST - obstructions

OXYG - dissolved oxygen

PATHO - pathogens

PRED - predators (includes fishing-related losses)
RIPCN - riparian condition

SEDLD - sediment load

TEMP - water temperature

WITH - water withdrawals

"NA indicates that no life history pathway meeting the criteria used in defining these pathwaya was present.

Blank cells under attributes indicate no additional attributes with negative effects on productivity were evident compared to those present in the template. 
Stream Reach Summary for Primary and Secondary Spring Chinook Salmon Life Hlstorles

Stream: Lostine R.

Reach: Sheep Ridge Dam - Silver Cr (RM 115.0 -120.0 from Snake R)

\begin{tabular}{|ll|ll|l|}
\hline Restoration benefit category: & E & Chinook usage score: & 541,6 & Habitat productivity loss score: \\
\hline
\end{tabular}

\begin{tabular}{|c|c|c|c|c|c|c|c|c|c|c|c|c|c|c|}
\hline \multirow[b]{3}{*}{ Life stage } & \multirow[b]{3}{*}{ Montha } & \multicolumn{10}{|c|}{ No. life history pathways by current usage abundance level } & \multicolumn{2}{|c|}{ Key habitat change } & \multirow{3}{*}{$\begin{array}{c}\text { Habitat } \\
\text { productivity } \\
\text { loss score }\end{array}$} \\
\hline & & \multicolumn{5}{|c|}{ Primary } & \multicolumn{5}{|c|}{ Secondary } & \multirow{2}{*}{$\begin{array}{l}\text { Patient } \\
\text { quantity }\end{array}$} & \multirow[b]{2}{*}{$\%$ change } & \\
\hline & & Total no. & 1 & 2 & 3 & 4 & Total no. & 1 & 2 & 3 & 4 & & & \\
\hline 1 & Apr-Aug & 16 & 4 & 0 & 12 & 0 & 16 & 4 & 0 & 12 & 0 & 8.9 & -54.9 & 3.3 \\
\hline $1,2,3$ & Aug-OCt &. & $?$ & 0 & $\ldots ?$ & ...! & $\$$ & 0 & & & ö & $10.0^{\circ}$ & -24.1 & 25.4 \\
\hline 3 & Ött-M̈ar & & 0 & 0 & & 0 & & 0 & & & 0 & 13.3 & 0.0 & 2.7 \\
\hline 34 & Mar-May & 16 & 0 & 0 & 16 & 0 & 16 & 0 & 0 & 16 & 0 & 99 & -43.6 & 935 \\
\hline 5 & May-Ött & 50 & 2 & $0 \dddot{0}$ & 48 & 0 & 50 & 4 & 0 & 46 & $0 "$ & 18.4 & -8.9 & 40.3 \\
\hline 6 & Ott-Mar & 19 & 0 & 0 & 19 & 0 & 0 & 0 & $\dddot{0}$ & 0 & ". & 5.4 & -60\% & -13.3 \\
\hline 7 & Mar-Jun & 3 & 2 & 0 & 1 & 0 & 0 & 0 & 0 & 0 & 0 & 23.1 & 0.0 & $0 "$ \\
\hline
\end{tabular}

\begin{tabular}{|c|c|c|c|c|c|c|c|c|c|c|c|c|c|}
\hline \multirow[b]{2}{*}{ Life stage } & \multirow[b]{2}{*}{ Months } & \multicolumn{12}{|c|}{ Highest productivity impact attributes (\% change in impact from template) } \\
\hline & & Att 1 & $\%$ change & $\overline{\text { Att } 2}$ & \% change & Atf 3 & \% change & Att 4 & ?6 change & Atf 5 & $\%$ change & Att 6 & $\%$ change \\
\hline 1 & Apr-Aug & HABD & 16.2 & & & & & & & & & & \\
\hline $1,2,3$ & Aug-Öct & CSTAB & 25.0 & HABD & 12.5 & & & & & & & & \\
\hline 3 & Ött-Mär & CSTAB & 25.0 & HÄBD & $12, \dot{5}$ & "Р̈̈Т̈Н̈Ö & 12.5 & "R̈IPCAN" & $12.5^{\circ}$ & WiTH' & 12.5 & & \\
\hline 34 & Mar-May & CSTAB & 25.0 & PATHO & 25.0 & ḦABD & 12.5 & RIPCN & $12.5^{\circ}$ & WiTtH & 12.5 & & \\
\hline 5 & ,May-,Ott, & PATHO & 25.0 & TEMP & 12.9 & & & & & & & & \\
\hline 6 & Ott-Mar & PÄTHÖ & 25.0 & "T̈ËM̈P̈P" & 25,0 & & & & 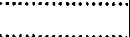 & & & & \\
\hline 7 & Mar-Jun & WITH & 11.5 & & & & & & & & & & \\
\hline
\end{tabular}

Abundancelevel refers to the average abundance of spawners that spawn in the natal stream reaches that populate the tife history pathways using the reach in this table:

$$
\begin{array}{ll}
\text { Level 1: } & 0-10 \text { spawners } \\
\text { Level 2 } & 10-30 \text { spawners } \\
\text { Level 3 } & 30-60 \text { spawners } \\
\text { Level 4: } & >\mathbf{2 0} \text { spawners }
\end{array}
$$

Life stages: 1 - prespawner; 2- spawner; 3- incubation; 4- fry colonization; 5- summer rearing; 6- overwintering; 7- yearling presmoltsmolt

Attributes: CHEM - chemicals

CSTAB - channel stability

COMP - competition (with other species)

FLOW - flow

HABD - habitat diversity
NUTLD - nutrient load

OBST - obstructions

OXYG - dissolved oxygen

PATHO - pathogens

PRED - predators (includes fishing-related losses)
RIPCN - riparian condition

SEDLD - sediment load

TEMP - water temperature

WITH - water withdrawals

"NA" indicates that no life history pathway meeting the criteria used in defining these pathways was present.

Blank cells under attributes indicate no additional attributes with negative effects on productivity were evident compared to those present in the template. 
Stream: Lostine R.

Reach: $\quad$ Silver $\mathrm{Cr}$ - Lake $\mathrm{Cr}$ (RM 120.0 -124.6 from Snake R)

\begin{tabular}{|ll|ll|l|}
\hline Restoration benefit category: & E & Chinook usage score: & 479.6 & Habitat productivity loss score: \\
\hline
\end{tabular}

\begin{tabular}{|c|c|c|c|c|c|c|c|c|c|c|c|c|c|c|}
\hline \multirow[b]{3}{*}{ Life stage } & \multirow[b]{3}{*}{ Months } & \multicolumn{10}{|c|}{ No. life history pathways by current usage abundance level } & \multicolumn{2}{|c|}{ Key habitat change } & \multirow{3}{*}{$\begin{array}{l}\text { Habitat } \\
\text { productivity } \\
\text { loss score }\end{array}$} \\
\hline & & \multicolumn{5}{|c|}{ Primary } & \multicolumn{5}{|c|}{ Secondary } & \multirow{2}{*}{$\begin{array}{l}\text { Patient } \\
\text { quantity }\end{array}$} & \multirow[b]{2}{*}{ \% change } & \\
\hline & & Total no. & 1 & 2 & 3 & 4 & Total no. & 1 & 2 & 3 & 4 & & & \\
\hline 1 & Apr-Aug & 14 & 14 & 0 & 0 & 0 & 14 & 14 & 0 & 0 & 0 & 7.0 & 0.0 & 0.0 \\
\hline $1,2,3$ & Aug-Oct & 20 & 20 & 0 & 0 & (n) & 20 & 20 & " & 0 & 0 & 5.0 & 0.0 & -50.2 \\
\hline 3 & Oct-Mar & 40 & $40^{\circ}$ & 0 & 0 & 0 & 40 & 40 & 0 & 0 & 0 & 4.8 & 0.0 & 0.0 \\
\hline 3,4 & Mar-May & 16 & 16 & 0 & 0 & 0 & 16 & 16 & 0 & 0 & 0 & 6.0 & 0.0 & 18.6 \\
\hline 5 & May-Oct & 49 & 49 & 0 & 0 & 0 & 48 & 48 & 0 & 0 & 0 & 6.2 & 0.0 & 39.5 \\
\hline 6 & Ocl-Mar & 19 & 19 & 0 & 0 & 0 & 0 & 0 & 0 & 0 & 0 & 4.8 & 0.0 & -18.1 \\
\hline 7 & Mar-Jun & 2 & 2 & 0 & 0 & 0 & 0 & 0 & 0 & 0 & 0 & 19.8 & 0.0 & 0.6 \\
\hline
\end{tabular}

\begin{tabular}{|c|c|c|c|c|c|c|c|c|c|c|c|c|c|}
\hline \multirow[b]{2}{*}{ Life stage } & \multirow[b]{2}{*}{ Months } & \multicolumn{12}{|c|}{ Highest productivity impact attributes ( $\%$ change in impact from template) } \\
\hline & & Att 1 & $\%$ change & Att 2 & $\%$ change & Atf 3 & $\%$ change & Att 4 & $\%$ change & Att 5 & $\%$ change & Att 6 & $\%$ change \\
\hline & Apr-Aug & HABD & 22.4 & OBST & 22.4 & PRED & 12.5 & & & & & & \\
\hline 123 & Äug-Ött & HABD & 25.0 & OBST & $\dddot{2} 5.0$ & PRED & 12.5 & & & & & & \\
\hline 3 & oct-Mar & PATHO & 25.0 & & & & & & & & & & \\
\hline 34 & Mar-May & PATHO & 25.0 & & & & & & & & & & \\
\hline 5 & May-Ott & 'PATHO' & 24.5 & & & & & & & & & & \\
\hline 6 & Ott-Mar & HABD & 25.0 & PATHOO & 25.0 & & & & & & & & \\
\hline 7 & Mar-Jun & & & & & & & & & & & & \\
\hline
\end{tabular}

Abundance lovel refers to the average abundance of spawners that spawn in the natal stream reaches that populate the life history pathways using the reach in this table:

$$
\begin{array}{ll}
\text { Level 1: } & 0-10 \text { apawners } \\
\text { Level 2 } & 10-30 \text { spawners } \\
\text { Level 3 } & 30-60 \text { spawners } \\
\text { Level 4: } & >60 \text { spawners }
\end{array}
$$

\begin{tabular}{|c|c|c|}
\hline $\begin{aligned} \text { Attributes: } & \text { CHEM - chemicals } \\
& \text { CSTAB - channel stability } \\
\text { COMP - competition (with other species) } & \\
& \text { FLOW - flow } \\
& \text { HABD - habitat diversity }\end{aligned}$ & $\begin{array}{l}\text { NUTLD - } \\
\text { OBST - obstructions load } \\
\text { OXYG - } \quad \text { dissolved oxygen } \\
\text { PATHO - pathogens } \\
\text { PRED - } \quad \text { predators (includes fishing-related losses) }\end{array}$ & $\begin{array}{l}\text { RIPCN - riparian condition } \\
\text { SEDLD - sediment load } \\
\text { TEMP - } \quad \text { water temperature } \\
\text { WITH - } \quad \text { water withdrawals }\end{array}$ \\
\hline
\end{tabular}

Life stages: 1 - prespawner; 2- spawner; 3- incubation; 4- fry colonization; 5- summer rearing; 6- overwintering; 7- yearling presmolt/smolt

"NA" indicates that no life history pathway meeting the criteria used in defining these pathwaya was present.

Blank cells under attributes indicate no additional attributes with negative effects on productivity were evident compared to those present in the template. 
Stream: Lostine $R$

Reach: $\quad$ Lake Cr - East Lostine R (RM 124.6 -131.7 from Snake R)

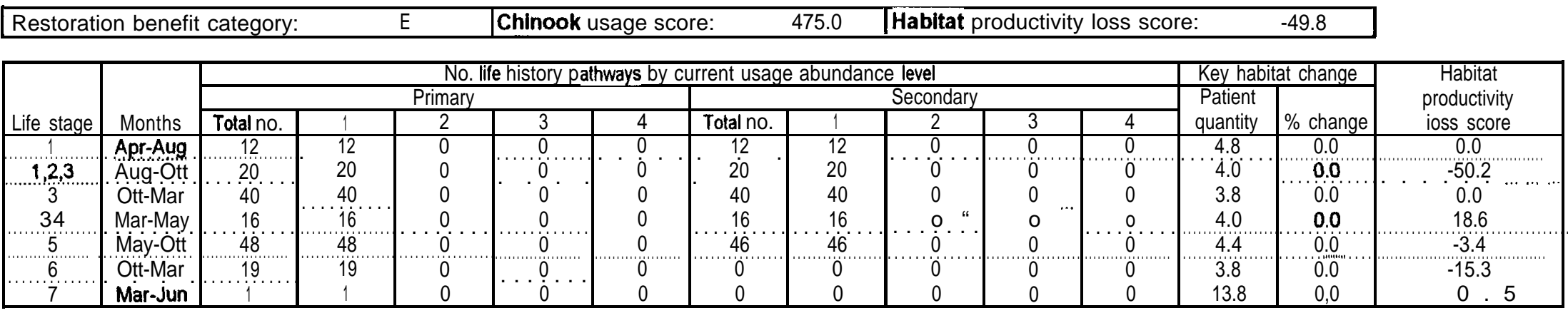

\begin{tabular}{|c|c|c|c|c|c|c|c|c|c|c|c|c|c|}
\hline \multirow[b]{2}{*}{ Life stage } & \multirow[b]{2}{*}{ Months } & \multicolumn{12}{|c|}{ Highest productivity impact attributes (\% change in impact from template) } \\
\hline & & Att 1 & $\%$ change & Att 2 & $\%$ change & Att 3 & $\%$ change & Att 4 & $\%$ change & Att 5 & $\%$ change & Att 6 & $\%$ change \\
\hline & Apr-Aug & HABD & 25.0 & OBST & 25.0 & PRED & 12.5 & & & & & & \\
\hline 1,23 & Aug-Oct & HA்க் & $25.0^{\circ}$ & ÖB̈Ṡं & 12.5 & PRED & 12.5 & & & & & & \\
\hline 3 & Ött-Mar & PÄTHÖO & 12.5 & & & & & & & & & & \\
\hline 3,4 & Mar-May & PATHHÓ & 17.2 & & & & & & & & & & \\
\hline 5 & May-Ott & HABD & 25.0 & PATHO & 25.0 & & 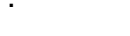 & & & & & & \\
\hline 6 & Ótt-Mar & "HABD' & 25.0 & PATHO & 25.0 & & & & & & & & \\
\hline 7 & Mar-Jun & & & & & & & & & & & & \\
\hline
\end{tabular}

Abundance lovel refers to the average abundance of spawners that spawn in the natal stream reaches that populate the life history pathways using the reach in this table Level 1: $\quad 0-10$ spawners

$\begin{array}{ll}\text { Level 2 } & 10-30 \text { spawners } \\ \text { Level 3: } & 30-60 \text { spawners } \\ \text { Level 4: } & >60 \text { apawners }\end{array}$

Lffe stages: 1- prespawner; 2- spawner; 3- incubation; 4- fry colonization; 5- summer rearing; 6- overwintering; 7 -yearling presmolt/smolt

Attributes: CHEM - chemicals

CSTAB - channel stability

COMP - competition (with other species)

FLOW - fiOW

HABD - habitat diversity
NUTLD - nutrient ioad

OBST - obstructions

OXYG - dissolved oxygen

PATHO - pathogens

PRED - predators (includes fishing-related losses)
RIPCN - riparian condition

SEDLD - sediment toad

TEMP - water temperature

WiTH - water withdrawals

"NA" indicales that no tife history pathway meeting the criteria used in defining these pathways was present.

Blank cells under attributea indicate no additional attributes with negative effects on productivity were evident compared to those present in thetemplate. 
Stream: Miriam R.

Reach: $\quad$ Mouth - Cougar Cr (RM 89.8 -99.4 from Snake R)

\begin{tabular}{|c|c|c|c|c|c|c|c|c|c|c|c|c|c|c|}
\hline \multicolumn{4}{|c|}{ Restoration benefit category: } & $\mathrm{F}$ & \multicolumn{3}{|c|}{ Chinook usage score: } & 3.8 & \multicolumn{4}{|c|}{ Habitat productivity loss score: } & 17.6 & \multirow{4}{*}{$\begin{array}{c}\text { Habitat } \\
\text { productivity } \\
\text { loss score }\end{array}$} \\
\hline \multirow[b]{3}{*}{ Life stage } & \multirow[b]{3}{*}{ Months } & \multicolumn{10}{|c|}{ No. life history pathways by current usage abundance level } & \multicolumn{2}{|c|}{ Key habitat change } & \\
\hline & & \multicolumn{5}{|c|}{ Primary } & \multicolumn{5}{|c|}{ Secondary } & \multirow{2}{*}{$\begin{array}{l}\text { Patient } \\
\text { quantity }\end{array}$} & \multirow[b]{2}{*}{$\%$ change } & \\
\hline & & Total no. & 1 & 2 & 3 & 4 & Total no. & 1 & 2 & 3 & 4 & & & \\
\hline 1 & Apr-Aug & 6 & 2 & 2 & 2 & 0 & 6 & $\overline{2}$ & 2 & 2 & 0 & 3.2 & -55.0 & 21.7 \\
\hline $1,2,3$ & Aug-Oct & Nä & $\mathrm{NA}$ & NA & $\mathrm{NA}$ & $\mathrm{NA}$ & NA & $\mathrm{NA}$ & $\mathrm{NA}$ & $\mathrm{NA}$ & NÄ & $\mathrm{NA}$ & $\mathrm{NA}$ & $\mathrm{NA}$ \\
\hline 3 & Oct-Mar & $\mathrm{NA}$ & $\mathrm{NA}$ & $\mathrm{NA}$ & $\mathrm{NA}$ & $\mathrm{NA}$ & NA & $\mathrm{NA}$ & $\mathrm{NA}$ & $\mathrm{NA}$ & NA & NA & $\mathrm{NA}$ & NA \\
\hline 3,4 & Mar-May & NA & NA & NA & NA & NA & NA & NA & $\mathrm{NA}$ & NA & $\mathrm{NA}$ & $\mathrm{NA}$ & $\mathrm{NA}$ & NA \\
\hline 5 & May-oct" & 2 & 1 & 0 & 1 & 0 & 6 & 2 & 2 & 2 & 0 & 12.8 & 0.0 & -4.0 \\
\hline 6 & Oct-Mar & NÁ & NÄ & NÄ & NA & NA & NA & NA & $\mathrm{NA}$ & $\mathrm{NA}$ & NÄ & NA & NA & NA \\
\hline 7 & Mar-Jun & 4 & 1 & 2 & 1 & 0 & 0 & 0 & 0 & 0 & 0 & 60.8 & 0.0 & 0.0 \\
\hline
\end{tabular}

\begin{tabular}{|c|c|c|c|c|c|c|c|c|c|c|c|c|c|}
\hline \multirow[b]{2}{*}{ Life stage } & \multirow[b]{2}{*}{ Months } & \multicolumn{12}{|c|}{ Highest productivity impact attributes (\% change in impact from template) } \\
\hline & & Att 1 & $\%$ change & Att 2 & $\%$ change & Att 3 & $\%$ change & Att 4 & $\%$ change & Alt 5 & $\%$ change & Att 6 & \% change \\
\hline 1 & Apr-Aug & HABD & 33.0 & & & & & & & & & & \\
\hline $1,2,3$ & Aug-Oct & $N A$ & $\mathrm{NA}$ & $\mathrm{NA}$ & $\mathrm{NA}$ & $\mathrm{NA}$ & NA & $\mathrm{NA}$ & $\mathrm{NA}$ & NA & NÄ & NÄ & NA \\
\hline 3 & Oct-Mar & $\mathrm{NA}$ & NA & $N A$ & $\mathrm{NA}$ & NA & $\mathrm{NA}$ & $\mathrm{NA}$ & $N A$ & NA & $\mathrm{NA}$ & NA & NA \\
\hline 3,4 & Mar-May & $N A$ & NA & $\mathrm{NA}$ & $N A$ & $\mathrm{NA}$ & $\mathrm{NA}$ & $\mathrm{NA}$ & $\mathrm{NA}$ & $\mathrm{NA}$ & $\mathrm{NA}$ & NA & NA \\
\hline 5 & May-Oct & FLOW & 13.2 & PATHO & 13.2 & & & & & & & & \\
\hline 6 & Oct-Mar & $\mathrm{NA}$ & NA & NA & $\mathrm{NA}$ & $\mathrm{NA}$ & NA & $\mathrm{NA}$ & NA & $\mathrm{NA}$ & $\mathrm{NA}$ & NA. & NÄ \\
\hline 7 & Mar-Jun & & & & & & & & & & & & \\
\hline
\end{tabular}

Abundance/eve/refers to the average abundance of spawners that spawn in the natal stream reaches that populate the life history pathways using the reach in this table

$$
\begin{array}{ll}
\text { Level 1: } & 0-10 \text { spawners } \\
\text { Level 2: } & 10-30 \text { spawners } \\
\text { Level 3: } & 30-60 \text { spawners } \\
\text { Level } 4 & >60 \text { spawners }
\end{array}
$$

Life stages.' 1 - prespawner; 2- apawner; 3- incubation; 4- fry colonization; 5- summer rearing; 6- overwintering; 7- yearling presmolt/smolt

Attributes: CHEM - chemicals

CSTAB - channel stability

COMP - competition (with other species)

FLOW - ftOW

HABD - habitat diversity
NUTLD - nutrient load

OBST - obstructions

OXYG - dissolved oxygen

PATHO - pathogens

PRED - predators (includes fishing-related losses)
RIPCN - riparian condition

SEDLD - sediment load

TEMP - water temperature

WITH - water withdrawals

"NA" indicates that no life history pathway meeting the criteria used in defining these pathways was present.

Blank cells under attributes indicate no additional attributes with negative effects on productivity were evident compared to those present in the template. 
Stream: Miriam R.

Reach: Cougar Cr - Little Miriam R (RM 99.4 -108.4 from Snake R)

\begin{tabular}{|ll|lllll}
\hline Restoration benefit category: & B & Chinook usage score: & 539.6 & Habitat productivity loss score: & -380.7 \\
\hline
\end{tabular}

\begin{tabular}{|c|c|c|c|c|c|c|c|c|c|c|c|c|c|c|}
\hline \multirow[b]{3}{*}{ Life stage } & \multirow[b]{3}{*}{ Months } & \multicolumn{10}{|c|}{ No. life history pathways by current usage abundance level } & \multicolumn{2}{|c|}{ Key habitat change } & \multirow{3}{*}{$\begin{array}{c}\text { Habital } \\
\text { productivity } \\
\text { loss score }\end{array}$} \\
\hline & & \multicolumn{5}{|c|}{ Primary } & \multicolumn{5}{|c|}{ Secondary } & \multirow{2}{*}{$\begin{array}{l}\text { Patient } \\
\text { quantity }\end{array}$} & \multirow[b]{2}{*}{$\%$ change } & \\
\hline & & Total no. & 1 & 2 & 3 & 4 & \begin{tabular}{|l} 
Total no. \\
.
\end{tabular} & 1 & 2 & 3 & 4 & & & \\
\hline 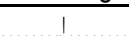 & Apr-Aug & $\$$ & 12 & 2 & 2 & 0 & 16 & 12 & 2 & $?$ & 0 & 2.8 & -81.9 & -104 \\
\hline $1,2,3$ & Aug-Ôtt & & 20 & 0 & 0 & 0 & 20 & 20 & 0 & 0 & 0 & 98 & -22.8 & -203 \\
\hline 3 & Ott-Mar & 40 & 40 & 0 & 0 & 0 & 40 & 40 & 0 & 0 & 0 & 14.4 & 6.7 & -180.7 \\
\hline 34 & Mar-May & 16 & 16 & 0 & 0 & ${ }^{\prime} 0$ & 16 & 16 & 0 & 0 & 0 & 17.5 & 7.9 & -116.6 \\
\hline 5 & May-Ött & 49 & 48 & 0 & 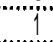 & 0 & 50 & 48 & 2 & 2 & 0 & 15.7 & 5.7 & -45.5 \\
\hline$\dddot{6}$ & Ött-M̈lar & 38 & $\cdots$ & 19 & “0” & 0 & 0 & 0 & 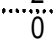 & .... & 0 & 36.2 & 7.0 & -7.3 \\
\hline 7 & Mar-Jun & 4 & 1 & 2 & 1 & 0 & “" & 0 & 0 & 0 & 0 & 60.8 & 6,2 & 0 \\
\hline
\end{tabular}

\begin{tabular}{|c|c|c|c|c|c|c|c|c|c|c|c|c|c|}
\hline \multirow[b]{2}{*}{ Life stage } & \multirow[b]{2}{*}{ Months } & \multicolumn{12}{|c|}{ 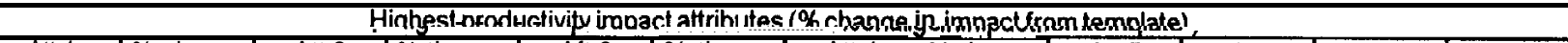 } \\
\hline & & Att 1 & $\%$ change & Att 2 & $\%$ than & Aft 3 & $\%$ than $\mathrm{e}$ & Att 4 & $\%$ than e & Alt 5 & $\%$ change & Aft 6 & \% change \\
\hline & Apr-Aug & HABD & 25.0 & TEMP & 25,0 & SEDLD & 16.7 & CSTAB & u. & & & & \\
\hline 1,23 & Aug-Oci & "SËELD & 50.0 & CSTAB & 25.0 & "TंĖM்' & 25.0 & "YABOD & 7. & & & & \\
\hline 3 & Ött-M̈ar & SEEDED & 50.0 & "C̈STÄB̈ & $25.0 \%$ & " & $25.0 \%$ & "TËMPP.". & & FLOW & 12.5 & PATHO & 12.5 \\
\hline 3,4 & Mar-May & FLOW & 25,0 & HÄBD & 25.0 & PATHO & 25.0 & RIPCN & & SEDLD & 25.0 & CSTAB & 12.5 \\
\hline 5 & May-Ott & FLOW & 24.4 & HARR & $24 \mathrm{~A}$ & PATH(7) & $24-4$ & RIPCN. & & & & & \\
\hline 6 & Ött-Mar & FLOWW & $751 \cdot 10$ & PATHOO & 250 & & & & & & & & \\
\hline 7 & Mar-Jun & & & & & & & & & & & & \\
\hline
\end{tabular}

Abundance /eve/ refers to the average abundance of spawners that apawn in the natal stream reaches that populate the life history pathways using the reach in this table:

$$
\begin{array}{ll}
\text { Level 1: } & 0-10 \text { spawners } \\
\text { Level 2: } & 10-30 \text { spawners } \\
\text { Level 3: } & 30-60 \text { spawners } \\
\text { Level 4 } & >60 \text { spawners }
\end{array}
$$

Life stages: 1-prespawner; 2-spawner; 3- incubation; 4- fry colonization; 5- summer rearing; 6-overwintering; 7- yearling presmolt/smolt

$\begin{array}{lll}\text { Attributes: } \text { CHEM - chemicals } & \text { NUTLD - nutrient load } & \text { RIPCN - riparlan condition } \\ \text { CSTAB - channel stability } & \text { OBST - obstructions } & \text { SEDLD - sediment load } \\ \text { COMP - competition (with other species) } & \text { OXYG - dissolved oxygen } & \text { TEMP - water temperature } \\ \text { FLOW - flow } & \text { PATHO - pathogens } & \text { WITH - water withdrawals } \\ \text { HABD - habitat diversity } & \text { PRED - predators (includes fishing-related losses) }\end{array}$

"NA" indicates that no life history pathway meeting the criteria used in defining these pathways was present.

Blank cells under attributes indicate no additional attributes with negative effects on productivity were evident compared to those present in the template. 
Stream: Miriam R.

Reach: Little Miriam R - splash dam site (1 m above Garwood Cr; RM 108.4 -119.7 from Snake R)

\begin{tabular}{lll|ll|l|}
\hline Restoration benefit category: & $\mathrm{E}$ & Chinook usage score: & 545.0 & Habitat productivity ioss score: \\
\hline
\end{tabular}

\begin{tabular}{|c|c|c|c|c|c|c|c|c|c|c|c|c|c|c|}
\hline \multirow[b]{3}{*}{ Lin's stage } & \multirow[b]{3}{*}{ miuriths } & \multicolumn{10}{|c|}{ No. life history pathways by current usage abundancelevel } & \multicolumn{2}{|c|}{ Key habitat change } & \multirow{3}{*}{$\begin{array}{c}\text { Habitat } \\
\text { productivity } \\
\text { loss score }\end{array}$} \\
\hline & & \multicolumn{5}{|c|}{ Primary } & \multicolumn{5}{|c|}{ Secondary } & \multirow{2}{*}{$\begin{array}{l}\text { Patient } \\
\text { quantity }\end{array}$} & \multirow[b]{2}{*}{$\%$ change } & \\
\hline & & Totalıno. & 1 & 12 & 3 & 4 & Total & no. 11 & $\dot{2}$ & 3 & 4 & & & \\
\hline 1 & Apr-Aug & 14 & $u$ & 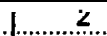 & 12 & 0 & 14 & $u$ & 2 & 12 & 0 & 13.6 & -60.0 & -5.2 \\
\hline $1,2,3$ & Aug-Oct & 20 & 0 & 0 & 20 & 0 & 20 & 0 & 0 & 20 & 0 & 107 & -60.0 & 10.2 \\
\hline 3 & Oct-Mar & 40 & 0 & 0 & 40 & $\mathbf{0}$ & 40 & 0 & 0 & 40 & 0 & 10.7 & -60.0 & -35.4 \\
\hline 3.4 & Mar-May & 16 & 0 & 0 & 16 & 0 & 16 & 0 & 0 & 16 & 0 & 13.4 & -60.0 & -14.3 \\
\hline 5 & May-Oct & 49 & 0 & 1 & 48 & 0 & 48 & 0 & 2 & 46 & 0 & 12.8 & -597 & 52.6 \\
\hline 6 & Oct-Mar & 19 & 0 & 0 & 19 & "ִo & 0 & 0 & 0 & 0 & 0 & 267 & 0.0 & -15.3 \\
\hline 7 & Mar-Jur & 2 & 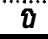 & 1 & 1 & 0 & 0 & a & 0 & a & 0 & 44.2 & 0.0 & 0.0 \\
\hline
\end{tabular}

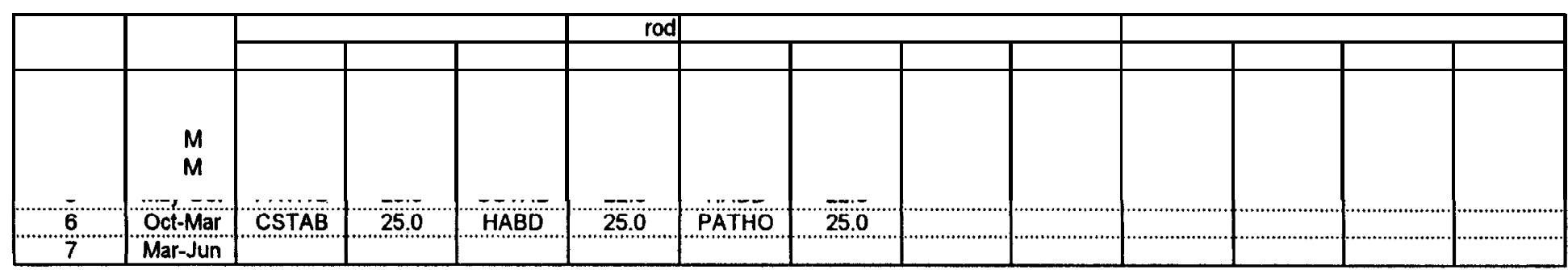

Abundance level refers to the average abundance of spawners that spawn in the natal stream reaches that populate the life history pathways using the reach in this table:

$$
\begin{array}{ll}
\text { Level 1: } & 0-10 \text { spawners } \\
\text { Level 2: } & 10-30 \text { spawners } \\
\text { Level 3: } & 30-60 \text { spawners } \\
\text { Level 4: } & >60 \text { spawners }
\end{array}
$$

Life stages.' 1- prespawner; 2- spawner; 3- incubation; 4- fry colonization; 5- summer rearing; 6- overwintering; 7- yearling presmolt/smelt

\section{Attributes: CHEM - chemicals}

CSTAB - channel stability

COMP - competition (with other species)

FLOW - ffOW

HABD - habitat diversity
NUTLD - nutrient ioad

OBST - obstructions

OXYG - dissolved oxygen

PATHO - pathogens

PRED - predators (includes fishing-related losses)
RIPCN - riparian condition

SEDLD - sediment load

TEMP - water temperature

WiTH - water withdrawals

"NA indicates that no life history pathway meeting the criteria used in defining these pathways was present.

Blank cells under attributes indicate no additional attributes with negative effects on productivity were evident compared to those present in the template. 
Stream: Miriam R.

Reach: $\quad$ Splash dam site - falls (RM 119.7 -138.0 from Snake R)

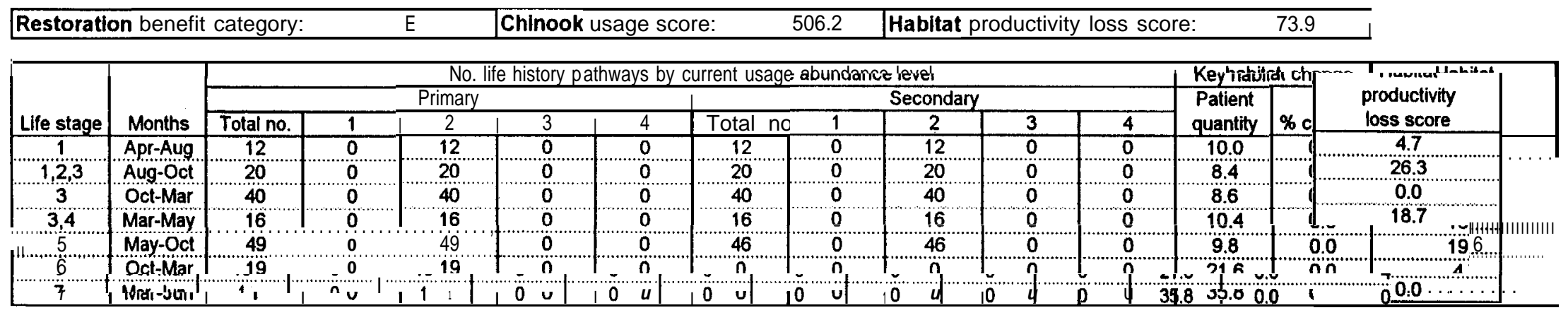

\begin{tabular}{|c|c|c|c|c|c|c|c|c|c|c|c|c|c|}
\hline \multirow[b]{2}{*}{ Life stage } & \multirow[b]{2}{*}{ Months } & \multicolumn{12}{|c|}{ Highest productivity impact attributes (\% change in impact from template) } \\
\hline & & Att 1 & $\%$ change & Alt 2 & $\%$ change & Att 3 & $\%$ change & Att 4 & $\%$ change & Att 5 & $\%$ change & Att 6 & \% change \\
\hline$\frac{1}{1}$ & Apr-Aug & & & & & & & & & & & & \\
\hline 123 & Aug-Oct & & & & & & & & & & & & \\
\hline 3 & Oct-Mar & PATHOO & 12.5 & & & & & & & & & & \\
\hline 34 & Mar-May & РATHO & 17.2 & & & & & & & & & & \\
\hline 5 & May-Oct & PATHO & 25.0 & & & & & & & & & & \\
\hline 6 & Oct-Mar & PATHO & 250 & & & & & & & & & & \\
\hline 7 & Mar-Jun & & & & & & & & & & & & 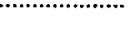 \\
\hline
\end{tabular}

Abundance/eve/refers to the average abundance of spawners that spawn in the natal stream reaches that populate the life history pathways using the reach in this table:

$$
\begin{array}{ll}
\text { Level 1: } & 0-10 \text { spawners } \\
\text { Level 2 } & 10-30 \text { spawners } \\
\text { Level 3: } & 30-60 \text { spawners } \\
\text { Levei 4 } & >\mathbf{6 0} \text { spawners }
\end{array}
$$

Life stages: 1- prespawner; 2- spawner; 3- incubation; 4- fry colonization; 5- summer rearing; 6- overwintering; 7- yearling presmolt/smolt

$\begin{array}{lll}\text { Altributes: CHEM - chemicals } & \text { NUTLD - nutrient load } & \text { RIPCN - riparian condition } \\ \text { CSTAB - channel stability } & \text { OBST - obstructions } & \text { SEDLD - Sediment toad } \\ \text { COMP - competition (with other species) } & \text { OXYG - dissolved oxygen } & \text { TEMP - water temperature } \\ \text { FLOW - flow } & \text { PATHO - pathogens } & \text { WiTH - water withdrawals } \\ \text { HABD - habitat diversity } & \text { PRED - predators (includes fishing-related losses) }\end{array}$

"NA" indicates that no life history pathway meeting the criteria used in defining these pathways was present.

Blank cells under attributes indicate no additional attributes withnegative effects on productivity were evident compared to those present in the template. 
Stream: Prairie $\mathrm{Cr}$

Reach: $\quad$ Mouth - OK Gulch confluence (RM 126.8 -131.8 from Snake R)

\begin{tabular}{ll|ll|ll}
\hline Restoration benefit category: & A & Chinook usage score: & 582.9 & Habitat productivity loss score: & -1047.5 \\
\hline
\end{tabular}

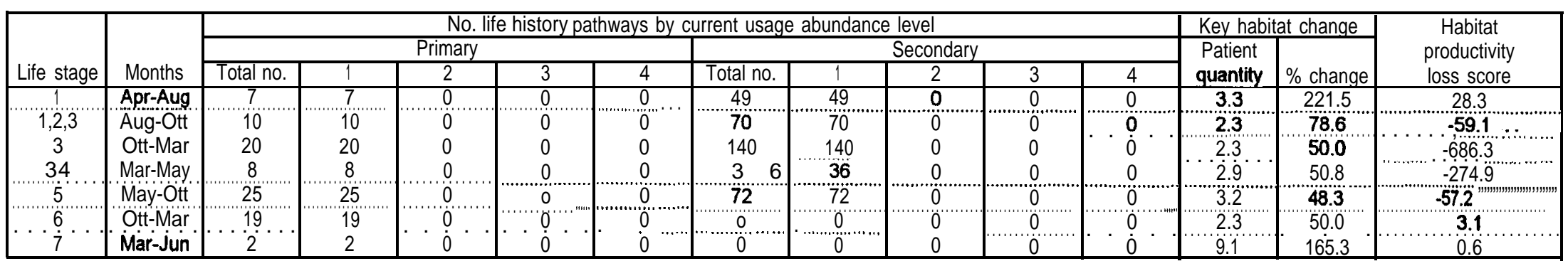

\begin{tabular}{|c|c|c|c|c|c|c|c|c|c|c|c|c|c|}
\hline \multirow[b]{2}{*}{ Life stage } & \multirow[b]{2}{*}{ Months } & \multicolumn{12}{|c|}{ Highest productivity impact attributes (\% change in impact from template) } \\
\hline & & Att 1 & $\%$ change & Att 2 & \% change & Aft 3 & $\%$ change & Att 4 & $\%$ change & Alt 5 & \% change & Att 6 & \% change \\
\hline 1 & Apr-Aug & SEDLD & 37.5 & CHEM & 25.0 & RIPCN & 25.0 & NUTLD & 20.0 & $\overline{C S T A B}$ & 12.5 & & \\
\hline $1,2,3$ & Aug-Ocl & SEDLD & 50.0 & $\mathrm{CHEM}$ & 25.0 & CSTAB & 25.0 & RIPCN & 25.0 & NUTTLD & 12.5 & & \\
\hline 3 & Oct-Mar & HABD & 500 & SEDLD & 50.0 & CHEM & 25.0 & CSTAB & 25.0 & FLOWW & 25.0 & PATHO & 25.0 \\
\hline 3,4 & Mar-May & HABD & 50.0 & SEDLD & 50.0 & CHEM & 25.0 & CSTAB & 25.0 & FLOW & 25.0 & PATHO & 25.0 \\
\hline 5 & May-Oct & HABD & 50.0 & SEDLD & 50.0 & CHEM & 25.0 & CSTAB & 25.0 & PATHO & 25.0 & RIPCN & 25.0 \\
\hline 6 & Oct-Mar & COMP & 25.0 & HABD & 250 & PATHO & 250 & SEDLD & 25.0 & & & & \\
\hline 7 & Mar-Jun & & & & & & & & & & & & \\
\hline
\end{tabular}

Abundance /eve/ refers to the average abundance of spawners that spawn in the natal stream reaches that populate the life history pathways using the reach in this table:

$$
\begin{array}{ll}
\text { Level 1: } & 0-10 \text { spawners } \\
\text { Level 2: } & 10-30 \text { spawners } \\
\text { Level 3 } & 30-60 \text { spawners } \\
\text { Level 4: } & >\mathbf{6 0} \text { spawners }
\end{array}
$$

Life stages: 1- prespawner; 2-spawner; 3- incubation; 4- fry colonization; 5- summer rearing; 6- overwintering; 7- yearling presmolt/smolt

Attributes: CHEM - chemicals

CSTAB - channel stability

COMP - competition (with other species)

FLOW - flow

HABD - habitat diversity
NUTLD - nutrient load

OBST - obstructions

OXYG - dissolved oxygen

PATHO - pathogens

PRED - predators (includes fishing-related losses)
RIPCN - riparian condition

SEDLD - sediment load

TEMP - water temperature

WITH - water withdrawals

"NA" indicates that no life history pathway meeting the criteria used in defining these pathways was present.

Blank cells under attributes Indicate no additional attributes with negative effects on productivity were evident compared to those present in the template. 
Stream: Prairie $\mathrm{Cr}$

Reach: $\quad$ OK Gulch fork (RM 131.8 -133.8 from Snake R)

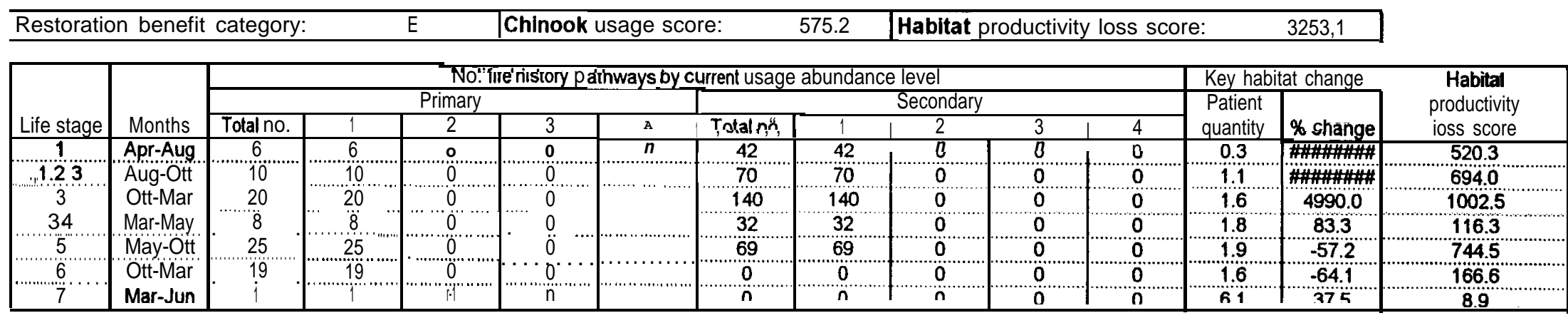

\begin{tabular}{|c|c|c|c|c|c|c|c|c|c|c|c|c|c|}
\hline \multirow[b]{2}{*}{ Life stage } & \multirow[b]{2}{*}{ Months } & \multicolumn{12}{|c|}{ Highest productivity impact attributes (\% change in impact from template) } \\
\hline & & Att 1 & \% change & Att 2 & $\%$ change & Att 3 & $\%$ change & Aft 4 & $\%$ change & Att 5 & $\%$ change & Att 6 & $\%$ change \\
\hline & Apr-Aug & HABD & 25.0 & NUTLD & 250 & PRED & 25.0 & RIPCN & 25.0 & SEDLD & 25.0 & & \\
\hline $1,2,3$ & Aug-Oct & SEDLID & 75.0 & NÜT̈T̈L̄ & 50.0 & CSTAB & 25.0 & RIPCN" & 25,0 & HABDD & 16.7 & PRED & 16.7 \\
\hline 3 & Ött-Mar & SEÉĹLD & $75.0^{\circ}$ & NUTLD & 50,0 & CSTAB & 25,0 & RIPCN & 25.0 & "Н̈AB̈D” & $12.5^{\circ}$ & "РАTHO" & 125 \\
\hline 3,4 & Mar-May & HABD & 50.0 & SEELLD & 50,0 & CHEM & 25.0 & COMP & 25,0 & C̈STAB & $25^{\circ} .0^{\circ}$ & NUTLD & 25.0 \\
\hline 5 & May-Ott & $\dot{H A B B}$ & 50,0 & SEDLLD & 50,0 & CHEM & 25.0 & "CÓMP. & 25.0 & 'CSTAB & 25.0 & PATHO & 250 \\
\hline 6 & Oct-Mar & $\mathrm{HABD}$ & 25.0 & NUTTLD & 25.0 & PिÄ HÖ & 25.0 & SEDLD & $25.0^{\circ}$ & & & & \\
\hline 7 & Mar-Jun & & & & & & & & & & & & \\
\hline
\end{tabular}

Abundancelevel refers to the average abundance of spawners that spawn in the natal stream reaches that populate the life history pathways using the reach in this table:

$$
\begin{array}{ll}
\text { Level 1: } & 0-10 \text { spawners } \\
\text { Levei 2: } & 10-30 \text { spawners } \\
\text { Level 3: } & 30-60 \text { spawners } \\
\text { Levei 4 } & >\mathbf{6 0} \text { spawners }
\end{array}
$$

Life stages: 1- prespawner; 2- spawner; 3- incubation; 4- fry colonization: 5- summer rearing; 6- overwintering; 7- yearling presmoltsmolt

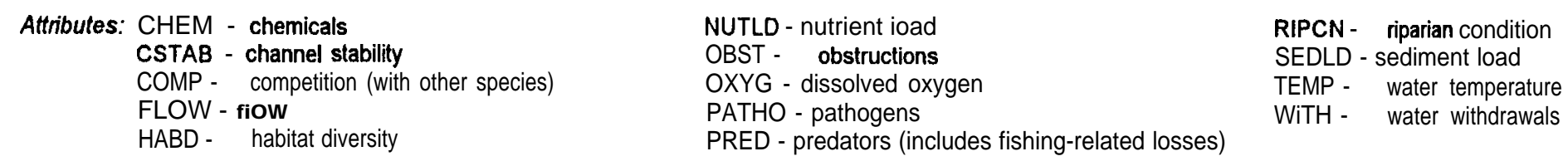

"NA" indicates that no life history pathway meeting the criteria used in defining these pathways was present.

Blank cells under attributes indicate no additional attributes with negative effects on productivity were evident compared to those present in the template. 
Stream: Sheep Cr.

Reach: $\quad$ Mouth - Chicken Cr (RM 164.2 -165.7 from Snake R)

\begin{tabular}{ll|ll|l|}
\hline Restoration benefit category: & $F$ & Chinook usage score: & 0.0 & Habitat productivity loss score: \\
\hline
\end{tabular}

\begin{tabular}{|c|c|c|c|c|c|c|c|c|c|c|c|c|c|c|}
\hline \multirow[b]{3}{*}{ Life stage } & \multirow[b]{3}{*}{ Months } & \multicolumn{10}{|c|}{ No. life history pathways by current usage abundance level } & \multicolumn{2}{|c|}{ Key habitat change } & \multirow{3}{*}{$\begin{array}{c}\text { Habitat } \\
\text { productivity } \\
\text { loss score }\end{array}$} \\
\hline & & \multicolumn{5}{|c|}{ Primary } & \multicolumn{5}{|c|}{ Secondary } & \multirow{2}{*}{$\begin{array}{l}\text { Patient } \\
\text { quantity }\end{array}$} & \multirow[b]{2}{*}{$\%$ change } & \\
\hline & & Total no. & 1 & 2 & 3 & 4 & Total no. & 1 & 2 & 3 & 4 & & & \\
\hline & Apr-Aug & $N A$ & $\mathrm{NA}$ & NA & NA & $\mathrm{NA}$ & NA & $\overline{N A}$ & NA & NA & $\overline{\mathrm{NA}}$ & NA & NA & NA \\
\hline $1,2,3$ & Aug-Ött & $\mathrm{NA}$ & $\mathrm{NA}$ & NA & $\mathrm{NA}$ & $\mathrm{NAA}$ & NÄ. & N?? & in" & NA & NÄ & NA & $\mathrm{NA}$ & NA \\
\hline 3 & Ott-Mar & NA & NA & $N A$ & NA & NA & $\mathrm{NA}$ & $\mathrm{NA}$ & N & NA & $N A^{\circ}$ & NA & $N A "$ & Nä" \\
\hline 34 & Mar-May & NA & NA & NA & NA & NA & $N A$ & NA & NA & NA & NA & NA & NA & NA \\
\hline 5 & May-Ott & $\mathrm{NA}$ & $\mathrm{NÄ}$ & $\mathrm{NA}$ & NA & NA & $\mathrm{NA}$ & $\mathrm{NÄ}$ & $\mathrm{NA}$ & NA & NÄ & $\mathrm{NA}$ & NA & NÄ \\
\hline 6 & Ött-M̄arr & $\mathrm{NA}$ & NA & N̈Ä & "NÄ" & NA" & $\mathrm{NA}$ & Nัฒ̈ & NA & $\mathrm{NA}$ & NÄ & NÄ & $\mathrm{NA}$ & $\mathrm{NA}$ \\
\hline 7 & Mar-Jun & NA & NA & NÄ & NÄ & "NÄ" & $\mathrm{NA}$ & N̈̈̈ & NA & $\mathrm{NA}$ & N̈Ä & $\mathrm{Nä}$ & $\mathrm{NA}$ & $\mathrm{NA}$ \\
\hline
\end{tabular}

\begin{tabular}{|c|c|c|c|c|c|c|c|c|c|c|c|c|c|}
\hline \multirow{2}{*}{\multicolumn{2}{|c|}{ Lifkestaged Mantths }} & \multicolumn{12}{|c|}{ Highest productivity impact attributes (\% change in impact from template) } \\
\hline & & Att 1 & $\%$ change & Aft 2 & $\%$ change & Att 3 & $\%$ change & Att 4 & $\%$ change & Att 5 & $\%$ change & Atf 6 & $\%$ change \\
\hline 1 & Apr-Aug & NA & NA & NA & $\overline{\mathrm{NA}}$ & NA & $\overline{\mathrm{NA}}$ & NA & NA & NA & $\bar{N}$ & NA & - \\
\hline $1,2,3$ & Aug-Öct & NA & NA & NA & NA & NA & NÁ & NÄ & $\mathrm{NÄ}$ & NA & N' & $\mathrm{NA}$ & \\
\hline 3 & Ötumar & NÄ & $\mathrm{N}$ & กัA & חั̈ & NA" & $\mathrm{NA}$ & NA & $\mathrm{NA}$ & $\mathrm{NA}$ & $\mathrm{NÄ}$ & $\mathrm{NÄ"}$ & $\mathrm{NA}$ \\
\hline 3,4 & Mar-May & NA & NA & $N A$ & NÄ & $\mathrm{NA}$ & $\mathrm{NÄ}$ & $\mathrm{NA}$ & NA & NA & NA & NA & $\ddot{N} A$ \\
\hline 5 & May-Ott & NA & NA & NÄ & NA & NA & $\mathrm{N} \quad \mathrm{A}$ & NA & NA & NA & $\mathrm{NA}$ & $\dot{N} A^{\circ}$ & $\mathrm{NA}$ \\
\hline 6 & OutAMar & NA & NA & "NA & NA & N̈A & N̈Ä & $N A$ & NÄ & $\mathrm{NA}$ & $\mathrm{NA}$ & $\mathrm{NA}$ & $\mathrm{NA}$ \\
\hline 7 & Mar-Jum & $\mathrm{NA}$ & NA & $\mathrm{NA}$ & NA & N̈A & ח̈̈ & NA & N̈̈̈ & $\mathrm{NA}$ & $\mathrm{Nä}$ & $\mathrm{NA}$ & $\mathrm{NA}$ \\
\hline
\end{tabular}

Abundance level refers to the average abundance of spawners that spawn in the natal stream reaches that populate the life history pathways using the reach in this table:

$$
\begin{array}{ll}
\text { Level 1: } & 0-10 \text { spawners } \\
\text { Level 2 } & 10-30 \text { spawners } \\
\text { Level 3 } & 30-60 \text { spawners } \\
\text { Level 4: } & >60 \text { spawners }
\end{array}
$$

Life stages: 1- prespawner; 2- spawner; 3- incubation; 4- fry colonization; 5- summer rearing; 6- overwintering; 7- yearling presmolt/smolt

$\begin{array}{lll}\text { Attributes: CHEM - chemicals } & \text { NUTLD - nutrient load } & \text { RIPCN - riparian condition } \\ \text { CSTAB - channel stability } & \text { OBST - obstructions } & \text { SEDLD - sediment load } \\ \text { COMP - competition (with other species) } & \text { OXYG - dissolved oxygen } & \text { TEMP - water temperature } \\ \text { FLOW - now } & \text { PATHO - pathogens } & \text { WITH - water withdrawals } \\ \text { HABD - habitat diversity } & \text { PRED - predators (includes fishing-related losses) }\end{array}$

"NA" indicates that no life history pathway meeting the criteria used in defining these pathways was present.

Blank cells under attributes indicate no additional attributes with negative effects on productivity were evident compared to those present in the template. 
Stream: Sheep Cr.

Reach: $\quad$ Chicken $\mathrm{Cr}$ - headwaters (RM 165.7 -173.7 from Snake R)

\begin{tabular}{|c|c|c|c|c|c|c|c|c|c|c|c|c|c|c|}
\hline \multicolumn{4}{|c|}{ Restoration benefit category: } & $\mathrm{F}$ & \multicolumn{3}{|c|}{ Chinook usage score: } & & \multicolumn{5}{|c|}{ Habltat productivity ioss score: } & \\
\hline \multirow[b]{3}{*}{ Life stage } & \multirow[b]{3}{*}{ Months } & \multicolumn{10}{|c|}{ No. life history pathways by current usage abundance level } & \multicolumn{2}{|c|}{ Key habitat change } & \multirow{3}{*}{$\begin{array}{c}\text { Habitat } \\
\text { productivity } \\
\text { ioss score }\end{array}$} \\
\hline & & \multicolumn{5}{|c|}{ Primary } & \multicolumn{5}{|c|}{ Secondary } & \multirow{2}{*}{$\begin{array}{l}\text { Patient } \\
\text { quantity }\end{array}$} & \multirow[b]{2}{*}{$\%$ change } & \\
\hline & & Total no. & $T$ & 2 & 3 & 4 & Total no. & 1 & 2 & 3 & 4 & & & \\
\hline $1.2 .3^{\prime \prime \prime \prime \cdots ”}$ & Aug-Ött & $\mathrm{NA}$ & "NÄ & NÄ & $\mathrm{NÄ}$ & $\mathrm{NA}$ & NA & $\mathrm{NA}$ & $\mathrm{NA}$ & NA & $\mathrm{NA}$ & $\mathrm{NA}$ & NA & $\mathrm{NA}$ \\
\hline 3 & Oct-Mar & $\mathrm{NA}$ & $\mathrm{NA}$ & $\mathrm{NA}$ & $\mathrm{NÄ}$ & $\mathrm{NA}$ & $\mathrm{NA}$ & $\mathrm{NA}$ & $\mathrm{NA}$ & "NA & NA & N̈A & $\ddot{N A}$ & Nֶ̆ \\
\hline 34 & Mar-May & NA & NA & NA & NA & NA & NA & NA & NA & NA & NA & NA & NA & NA \\
\hline 5 & May-Oct & $\mathrm{NA}$ & $\mathrm{NÄ}$ & $\mathrm{NA}$ & $\mathrm{NA}$ & NA & $\because \mathrm{NA}$ & $\because \mathrm{NÄ}$ & $\mathrm{NA}$ & $\mathrm{NA}$ & NÄ & $\mathrm{NA}$ & $\mathrm{NA}$ & $\mathrm{NA}$ \\
\hline 7 & Mar-Jun & $\mathrm{NA}$ & NÄ & $\mathrm{NA}$ & $\mathrm{NA}$ & NA & NA & NA & NA & $\mathrm{NA}$ & $\mathrm{NA}$ & $\mathrm{NA}$ & NÄ & NA \\
\hline
\end{tabular}

\begin{tabular}{|c|c|c|c|c|c|c|c|c|c|c|c|c|c|}
\hline \multirow[b]{2}{*}{ Life stage } & \multirow[b]{2}{*}{ Months } & \multicolumn{12}{|c|}{ Highest productivity impact attributes (\% change in impact from template) } \\
\hline & & Att 1 & $\%$ change & Att 2 & $\%$ change & Att 3 & $\%$ change & Att 4 & $\%$ change & Att 5 & $\%$ change & Att 6 & $\%$ change \\
\hline & Apr-Aug & $\mathrm{NA}$ & $\mathrm{NA}$ & $\overline{N A}$ & $\mathrm{NA}$ & NA & $\overline{N A}$ & NA & $\overline{\mathrm{NA}}$ & NA & $\overline{N A}$ & NA & NA \\
\hline 123 & Aug-Oct & $\mathrm{NA}$ & $\mathrm{NÄ}$ & NA & NA & $\mathrm{NA}$ & $\mathrm{NÄ}$ & Nä & NA & $\mathrm{NA}$ & $\mathrm{NA}$ & $\mathrm{NA}$ & $\because \mathrm{NA}$ \\
\hline$\dddot{3}$ & Ött-M̈ar & $\mathrm{NA}$ & $\mathrm{NA}$ & $\mathrm{NA}$ & $\mathrm{NA}$ & NÄ" & Nä & & NA & $\mathrm{NA}$ & NA" & NA: & NA \\
\hline 3,4 & Mar-May & NA & NA & NA & NA & $N A$ & $\mathrm{~N} \mathrm{NÄ}$ & N̈̈̈ & ก̈̈ & NA & NA & NÄ & $N \ddot{A}$ \\
\hline 5 & May-Öt" & NA & NA & NA & NA & NA & NA & NA & NA & NA & $\mathrm{NÄ}$ & $\mathrm{NA}$ & $\mathrm{NA}$ \\
\hline 6 & Ott-Msr & $N A$ & NA & NA & $\mathrm{NÄ}$ & $\mathrm{NÄ}$ & $\because$ & NA & NÄ & NA & NÄ & $\mathrm{NA}$ & $\mathrm{NA}$ \\
\hline 7 & Mar-jun & $\mathrm{NÄ}$ & $\mathrm{NA}$ & NÄ & N̈Ä & "Nä & $\mathrm{NA}$ & NA & $\mathrm{NA}$ & NA & NA & $\mathrm{NA}$ & $\mathrm{NA}$ \\
\hline
\end{tabular}

Abundance /eve/ refers to the average abundance of spawners that spawn in the natal stream reaches that populate the life history pathways using the reach in this table:

$$
\begin{array}{ll}
\text { Levei 1: } & 0-10 \text { spawners } \\
\text { Levei 2: } & 10-30 \text { spawners } \\
\text { Level 3: } & 30-60 \text { spawners } \\
\text { Levei 4: } & >60 \text { spawners }
\end{array}
$$

Life stages: 1- prespawner; 2- spawner; 3- incubation; 4- fry colonization; 5- summer rearing; 6- overwintering; 7- yearling presmolt/smolt

$\begin{array}{lll}\text { Attributes: } \text { CHEM - chemicals } & \text { NUTLD - nutrient load } & \text { RIPCN - riparian condition } \\ \text { CSTAB - channel stability } & \text { OBST - obstructions } & \text { SEDLD - Sediment load } \\ \text { COMP - competition (with other species) } & \text { OXYG - dissolved oxygen } & \text { TEMP - water temperature } \\ \text { FLOW - ttOW } & \text { PATHO - pathogens } & \text { WITH - water withdrawals } \\ \text { HABD - habitat diversity } & \text { PRED - predators (includes fishing-related losses) }\end{array}$

"NA" indicates that no life history pathway meeting the criteria used in defining these pathways was present.

Blank cells under attributes indicate no additional attributes with negative effects on productivity were evident compared to those present in thituriare. 
Stream: Wallowa R.

Reach: $\quad$ Mouth - Miriam R (RM 80.0 -89.8 from Snake R)

\begin{tabular}{|ll|ll|ll}
\hline Restoration benefit category & F & Chinook usage score: & 185.2 & Habitat productivity ioss score: & -51.4 \\
\hline
\end{tabular}

\begin{tabular}{|c|c|c|c|c|c|c|c|c|c|c|c|c|c|c|}
\hline \multirow[b]{3}{*}{ Life stage } & \multirow[b]{3}{*}{ Months } & \multicolumn{10}{|c|}{ No. life history pathways by current usage abundancelevel } & \multicolumn{2}{|c|}{ Key habital change } & \multirow{3}{*}{$\begin{array}{c}\text { Habitat } \\
\text { productivity } \\
\text { ioss score }\end{array}$} \\
\hline & & \multicolumn{5}{|c|}{ Primary } & \multicolumn{5}{|c|}{ Secondary } & Patient & & \\
\hline & & Total no. & 1 & 2 & 3 & 4 & Total no. & 1 & 2 & 3 & 4 & quantity & $\%$ change & \\
\hline 年 & Apr-Aug & 21 & 15 & 2 & 4 & 0 & 63 & 57 & 2 & 4 & 0 & 225 & 00 & -310 \\
\hline 123 & Äug-Ôct & $\mathrm{NA}$ & $\mathrm{NA}$ & $\mathrm{NA}$ & $\mathrm{NA}$ & NQA & $\mathrm{NA}$ & NA & $\mathrm{NA}$ & NQA" & NA & $\mathrm{NA}$ & NA & NA \\
\hline 3 & Ott-Mar & NA & NA & NA & NA & NA & NA & NA & NA & NA & NA & NA & $\mathrm{NA}$ & $\mathrm{NA}$ \\
\hline 34 & Mar-May & NA & NA & NA & NA & NA & NA & NA & $\mathrm{NA}$ & NA & NA & NA & NA & NA \\
\hline 5 & May-Ött & i & "i" & 0 & 0 & $0^{\circ}$ & 41 & 36 & 1 & 4 & 0 & 15.0 & -27.1 & -43 \\
\hline 6 & Ott-Mar & 19 & 0 & $0^{\circ}$ & 19 & 0 & 132 & 131 & $" 1$ & 0 & 0 & 41.9 & 0.0 & -16.5 \\
\hline 7 & Mar-Jun & $20^{\circ}$ & 14 & 2 & 4 & 0 & 18 & 18 & 0 & 0 & 0 & 71.5 & 0.0 & 0.4 \\
\hline
\end{tabular}

\begin{tabular}{|c|c|c|c|c|c|c|c|c|c|c|c|c|c|}
\hline \multirow[b]{2}{*}{ Life stage } & \multirow[b]{2}{*}{ Months } & & & & \multicolumn{9}{|c|}{ ghest productivity impact attributes (\% change in impact from template) } \\
\hline & & Att 1 & \% change & Att 2 & $\%$ change & Alt 3 & $\%$ change & Att 4 & $\%$ change & Att 5 & $\%$ change & Att 6 & $\%$ change \\
\hline 1 & Apr-Aug & PATHO & 11.9 & & & & & & & & & & \\
\hline $1,2,3$ & Aug-Oct & NÄ & $\mathrm{NA}$ & $\mathrm{NA}$ & NÄ & NA & NA & NÄ & NA & $\mathrm{NA}$ & $\mathrm{NÄ}$ & $\mathrm{NA}$ & NÄ \\
\hline 3 & Oct-Mar & $\mathrm{NA}$ & $\mathrm{NA}$ & $\mathrm{NA}$ & NA & NA & NA & $\mathrm{NA}$ & $\mathrm{NA}$ & $\mathrm{NÄ}$ & $\mathrm{NA}$ & $\mathrm{NÄ}$ & $\mathrm{NA}$ \\
\hline 3,4 & Mar-May & $\mathrm{NA}$ & $\mathrm{NA}$ & $\mathrm{NA}$ & $\mathrm{NA}$ & NA & $\mathrm{NA}$ & $\mathrm{NA}$ & $\mathrm{NA}$ & NA & $\mathrm{NA}$ & NA & $\mathrm{NA}$ \\
\hline 5 & May-Oct & COMP & 11.3 & HABD & 11.3 & PATHO & 11.3 & & & & & & \\
\hline 6 & Oct-Mar & FiLow & 9.4 & PATHO & 9.4 & & & & & & & & \\
\hline 7 & Mar-Jun & & & & & & & & & & & & \\
\hline
\end{tabular}

Abundance /eve/ refers to the average abundance of spawners that spawn in the natal stream reaches that populate the life history pathways using the reach in this table:

$$
\begin{array}{ll}
\text { Level 1: } & 0-10 \text { spawners } \\
\text { Level 2 } & 10-30 \text { spawners } \\
\text { Level 3 } & 30-60 \text { spawners } \\
\text { Level 4: } & >60 \text { spawners }
\end{array}
$$

Life stages.' 1- prespawner; 2- spawner; 3- incubation; 4- fry colonization; 5- summer rearing; 6- overwintering; 7- yearling presmolt/smolt

$\begin{array}{lll}\text { Attributes: CHEM - chemicals } & \text { NUTLD - nutrient load } & \text { RIPCN - riparian condition } \\ \text { CSTAB - channel stability } & \text { OBST - obstructions } & \text { SEDLD - sediment ioad } \\ \text { COMP - competition (with other species) } & \text { OXYG - dissolved oxygen } & \text { TEMP - water temperature } \\ \text { FLOW - ftOW } & \text { PATHO - pathogens } & \text { WITH - water withdrawals } \\ \text { HABD - habitat diversity } & \text { PRED - predators (includes fishing-related losses) }\end{array}$

"NA" indicates that no life history pathway meeting the criteria used in defining these pathways was present.

Blank cells under attributes indicate no additional attributes with negative effects on productivity were evident compared to those present in the template. 
Stream: Wallowa $\mathrm{R}$

Reach: Miriam R - Deer Cr (RM 89.8 -90.8 from Snake R)

\begin{tabular}{llll|lll}
\hline Restoration benefit category: & $F$ & Chinook usage score: & 54.3 & Habitat productivity loss score: & -31.4 \\
\hline
\end{tabular}

\begin{tabular}{|c|c|c|c|c|c|c|c|c|c|c|c|c|c|c|}
\hline \multirow[b]{3}{*}{ Life stage } & \multirow[b]{3}{*}{ Months } & \multicolumn{10}{|c|}{ No. life history pathway, hw current usage abundance level } & \multirow{3}{*}{\multicolumn{2}{|c|}{$\begin{array}{l}\text { Key,habita' uhamge } \\
\text { Patient } \\
\text { nuantitu I qu ghinne }\end{array}$}} & \multirow{3}{*}{$\begin{array}{l}\text { Habitat } \\
\text { productivity } \\
\text { loss score }\end{array}$} \\
\hline & & \multicolumn{5}{|c|}{ Priman } & \multirow{2}{*}{\multicolumn{5}{|c|}{$\frac{\text { Secondary }}{2}$}} & & & \\
\hline & & Total no. & 1 & 2 & 3 & 4 & & 11 & 2 & 3 & 4 & & & \\
\hline & Apr-Aug & 15 & 13 & 0 & 2, & 0 & 57 & 55 & 0 & 2 & 0 & 9.0 & -8.7 & -21.3 \\
\hline 123 & Äug-Ött & $\mathrm{NA}$ & NA & $\mathrm{NAn}$ & 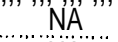 & NÄ & $\mathrm{NA}$ & $\mathrm{NA}$ & N̈Ä & NÄ & NÄ & NA & NA & NA \\
\hline 3 & Ott-Mar & $\mathrm{NA}$ & $\mathrm{NA}$ & $\mathrm{NA}$ & $\mathrm{NA}$ & $\mathrm{NÄ}$ & NA & $\mathrm{NA}$ & NA & NA & NA & NA & NA & N̈A \\
\hline 3.4 & Mar-May. & NA & $N A$ & NA & NA & NA & NA & NA & NA & NA & $\mathrm{NA}$ & NA & $\mathrm{NA}$ & NA \\
\hline 5 & May-Ott & 0 & 0 & 0 & $\cdots$ & 0 & 37 & 35 & … & 2 & 0 & 8.3 & -11.7 & -1.8 \\
\hline 6 & Oct-Mar & 0 & 0 & 0 & 0 & 0 & 9 & 9 & a' & 0 & 0 & 8.3 & -42.5 & $-8 x$ \\
\hline 7 & Mar-Jun & 15 & 13 & 0 & $\dddot{2}$ & 0 & 111 & 11 & 0 & 0 & 0 & 30.3 & -2.2 & 0 f"' \\
\hline
\end{tabular}

\begin{tabular}{|c|c|c|c|c|c|c|c|c|c|c|c|c|c|}
\hline \multirow[b]{2}{*}{ Life stage } & \multirow[b]{2}{*}{ Months } & \multicolumn{12}{|c|}{ Highest productivity impact attributes (\% change in impact from template) } \\
\hline & & Att 1 & $\%$ change & Att 2 & \% change & Att 3 & $\%$ change & Alt 4 & $\%$ change & Att 5 & $\%$ change & Att 6 & $\%$ change \\
\hline 1. & Apr-Aug & HABD & 8.7 & PATHO & 8.7 & RIPCN & 8.7 & & & & & & \\
\hline $1,2,3$ & Aug-Oad & NA & NA & NA & NA & NA & NÄ & NA. & $\mathrm{NA}$ & NA & $\mathrm{NA}$ & $\mathrm{NÄ}$ & $\mathrm{NA}$ \\
\hline 3 & Oct-Mar & NA & NÄ & $\mathrm{NA}$ & NA & $\mathrm{NA}$ & NA & NA & $\mathrm{NA}$ & NA & NA & $\mathrm{NA}$ & $\mathrm{NA}$ \\
\hline 3,4 & Mar-May & $\mathrm{NA}$ & NA & NA & NA & NA & NA & NA & $\mathrm{NA}$ & NA & NA & $\mathrm{NA}$ & NA \\
\hline 5 & May-Oct & HABD & 4.9 & PATHO & 4.9 & RIPCN & 4.9 & & & & & & \\
\hline 6 & Oct-Mar & HABD & 17.7 & PATHO & 177 & RIPCN & 17.7 & & & & & & \\
\hline 7 & Mar-Jun & & & & & & & & & & & & \\
\hline
\end{tabular}

Abundance fovel refers to the average abundance of spawners that apawn in the natal stream reaches that populate the life history pathways using the reach in this table

$$
\begin{array}{ll}
\text { Level 1: } & 0-10 \text { spawners } \\
\text { Level 2 } & 10-30 \text { spawners } \\
\text { Level 3 } & 30-60 \text { spawners } \\
\text { Level 4 } & >60 \text { spawners }
\end{array}
$$

Life stages: 1- prespawner; 2- spawner; 3- incubation; 4- fry colonization; 5- summer rearing; 6- overwintering; 7-yearling presmolt/smolt

Attributes: CHEM - chemicals

CSTAB - channel stability

COMP - competition (with other species)

FLOW - ffOW

HABD - habitat diversity
NUTLD - nutrient load

OBST - obstructions

OXYG - dissolved oxygen

PATHO - pathogens

PRED - predators (includes fishing-related losses)
RIPCN - riparian condition

SEDLD - sediment load

TEMP - water temperature

WITH - water withdrawals

"NA" Indicates that no life history pathway meeting the criteria used in defining these pathways was present.

Blank cells under attributes indicate no additional attributes with negative effects on productivity were evident compared to those present in the template, 
Stream: Wallowa $\mathrm{R}$

Reach: Deer Cr - Dry Cr (RM 90.8 -98.3 from Snake R)

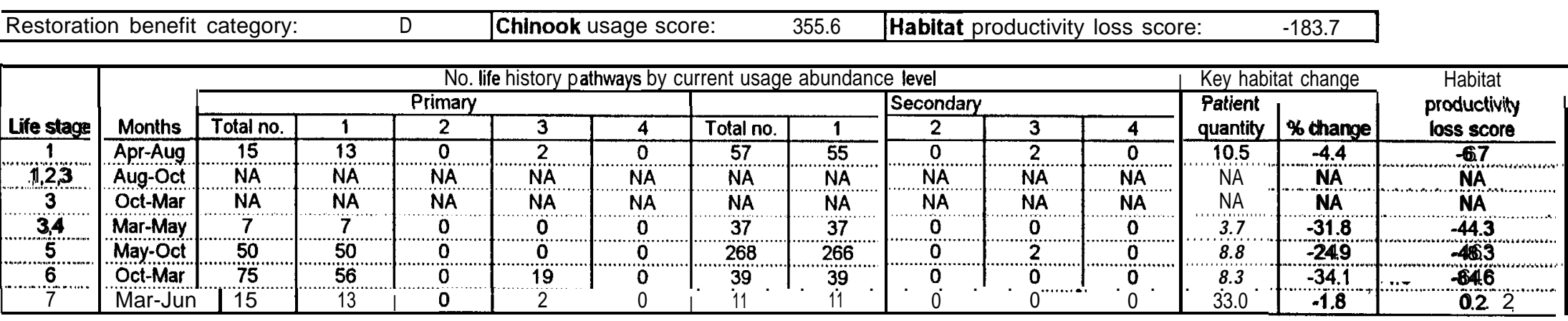

\begin{tabular}{|c|c|c|c|c|c|c|c|c|c|c|c|c|c|}
\hline \multirow[b]{2}{*}{ Life stage } & \multirow[b]{2}{*}{ Months } & \multicolumn{12}{|c|}{ Highest productivity impact attributes ( $\%$ chang in impact from template) } \\
\hline & & Atf 1 & $\%$ change & Att 2 & $\%$ change & Att 3 & $\%$ change & Att 4 & $\%$ change & Att 5 & $\%$ change & Att 6 & $\%$ change \\
\hline & Apr-Aug & HABD & 8.8 & PATHO & 8.6 & RIPCN & 8.8 & & & & & & \\
\hline $1,2,3$ & Aug-Öci & NA & NA & NA & N̈A & NัNÄ & $\dddot{\mathrm{N} A}$ & NÄ & กั & $\mathrm{NA}$ & NÄ & NÄ & ח̈̈ \\
\hline 3 & Ött-Mar & NÁ & NA & NA & $\mathrm{NÄ}$ & $\mathrm{NÄ}$ & $\mathrm{NÄ}$ & חั" & חัล & $\mathrm{NÄ}$ & $\mathrm{NA}$ & $\mathrm{NA}$ & $\mathrm{NA}$ \\
\hline $.3,4$. & Mar-May & HABD & $25.0^{\circ}$ & PATHO & 25.0 & RIPCN & 25.0 & CÖM̈P̈ & 10.4 & SEDLO & 4.6 & & \\
\hline 5 & May-Ott & HABDD & 200 & COMP & 10.0' & PATHO & 10.0 & RIPCN & 10.0 & & & & \\
\hline 6 & Ott-Mar & HABD & 14.1 & "РÄT̈Н̈Ö & 14.1 & P̈RËËD̆ & 14.1 & RIPCON & 14.1 & & & & \\
\hline 7 & Maraun & & & & & & & & & & & & \\
\hline
\end{tabular}

Abundance level refers to the average abundance of spawners that spawn in the natal stream reaches that populate the life history pathways using the reach in this table:

$$
\begin{array}{ll}
\text { Level 1: } & 0-10 \text { spawners } \\
\text { Level 2: } & 10-30 \text { spawners } \\
\text { Level 3: } & 30-60 \text { spawners } \\
\text { Level 4 } & >60 \text { spawners }
\end{array}
$$

Life stages: 1- prespawner; 2- spawner; 3- incubation; 4- fry colonization; 5- summer rearing; 6- overwintering; 7- yearling presmolt/smolt

$\begin{array}{lll}\text { Attributes: CHEM - chemicals } & \text { NUTLD - nutrient load } & \text { RIPCN - riparian condition } \\ \text { CSTAB - channel stability } & \text { OBST - obstructions } & \text { SEDLD - sediment load } \\ \text { COMP - competition (with other species) } & \text { OXYG - dissolved oxygen } & \text { TEMP - water temperature } \\ \text { FLOW - fiOW } & \text { PATHO - pathogens } & \text { WiTH - water withdrawals } \\ \text { HABD - habitat diversity } & \text { PRED - predators (includes fishing-related losses) }\end{array}$

"NA" indicates that no life history pathway meeting the criteria used in defining these pathways was present.

Blank cells under attributes indicate no additional attributes with negative effects on productivity were evident compared to those present in the template. 
Stream Reach Summary for Primary and Secondary Spring Chinook Salmon Life Histories

Stream: Wallowa $\mathrm{R}$.

Reach: $\quad$ Dry $\mathrm{Cr}$ - Bear Cr (RM 98.3 -101.8 from Snake R)

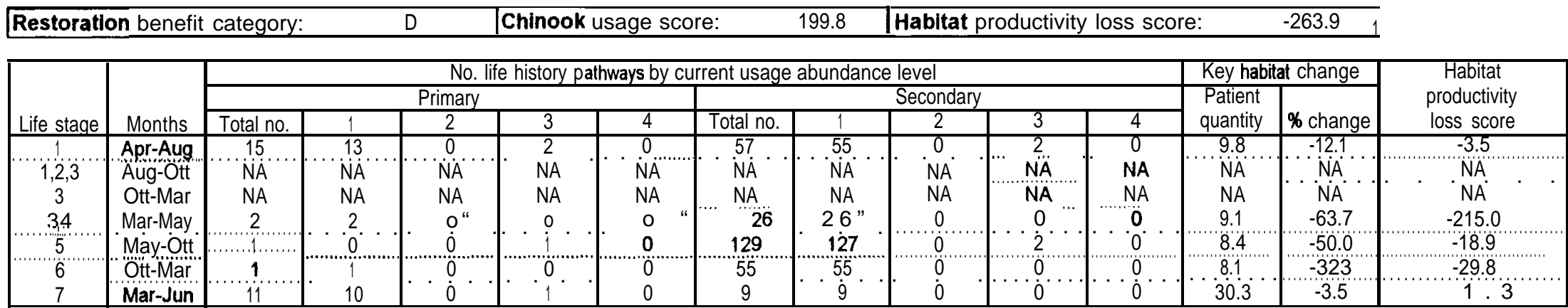

\begin{tabular}{|c|c|c|c|c|c|c|c|c|c|c|c|c|c|}
\hline \multirow[b]{2}{*}{ Life stage } & \multirow[b]{2}{*}{ Months } & \multicolumn{12}{|c|}{ Highest productivity impact attributes (\% change in impact from template) } \\
\hline & & Att 1 & $\%$ change & At! 2 & $\%$ change & Att 3 & $\%$ change & Atf 4 & $\%$ change & Art 5 & $\%$ change & Att 6 & $\%$ change \\
\hline 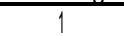 & Apr-Aug & HABD & 9.6 & RIPCN & 9.6 & PATHO & 4.8 & & & & & & \\
\hline 123 & Aug-Ôtt & NA & $\dot{N} \dot{A}$ & $\dot{\mathrm{N}} \dot{\mathrm{A}}$ & NÄ & $\dot{N} \dot{A}$ & NA & $\mathrm{NA}$ & $\mathrm{NA}$ & $\dot{\mathrm{NA}}$ & NA & $\mathrm{NA}$ & $\mathrm{NA}$ \\
\hline 3 & Ott-Mar & $\mathrm{NA}$ & NÄ & $\dot{N A}$ & $\dot{N} \dot{A}$ & NA & NA" & NA & $\mathrm{NA}$ & $\mathrm{NA}$ & $N A$ & $\mathrm{NA}$ & $\mathrm{NA}$ \\
\hline 3,4 & Mar-May & HABD & 75,0 & RIPCN & $50.0^{\circ}$ & SÉD்LD & 31.2 & COMP & 25.0 & FLOW & 25.0 & PÁTHȮ & $25.0^{\circ}$ \\
\hline 5 & , May,-Ott, & HABD & 50.0 & COMP & 25.0 & PATHO" & 25.0 & RIPCN & 25.0 & SEDLD & $17.0^{\circ}$ & & \\
\hline 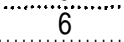 & Ott-Mar & С̈ÖM̈P“ & $25.0^{\circ}$ & "PÄT̈HÖO" & $25.0^{\circ}$ & "W̌iT̄Ḧ & $25.0^{\circ}$ & "HÄB̈D" & 20.5 & SED்LD & 20.5 & RIPCN & 10.2 \\
\hline 7 & Mar-Jun & "COMP & 5.7 & PATHHO & $5.7^{\circ}$ & $\dot{\text { WIITH }}$ & 5.7 & & & & & & \\
\hline
\end{tabular}

Abundance/eve/refers to the average abundance of spawners that spawn in the natai stream reaches that populate the life history pathways using the reach in this table:
Level 1: $\quad 0-10$ spawners
Level 2: $\quad 10-30$ spawners
Level $3 \quad 30-60$ spawners
Level $4>60$ spawners

Life stages: 1 - prespawner; 2- spawner; 3- incubation; 4- fry colonization; 5- summer rearing; 6- overwintering; 7- yearling presmolt/smolt

Attributes: CHEM - chemicals

CSTAB - channel stability

COMP - competition (with other species)

FLOW - flow

HABD - habitat diversity
NUTLD - nutrient load

OBST - obstructions

OXYG - dissolved oxygen

PATHO - pathogens

PRED - $\quad$ predators (includes fishing-related losses)
RIPCN - riparian condition

SEDLD - sediment iosd

TEMP - water temperature

WITH - water withdrawals

"NA" indicates that no life history pathway meeting the criteria used in defining these pathwayswas present.

Blank cells under attributes indicate no additional attributes with negative effects on productivity were evident compared to those present in thitemplate. 
Stream: Wallowa R.

Reach: Bear Cr - Whiskey Cr (RM 101.8 -103.9 from Snake R)

\begin{tabular}{|ll|lllll}
\hline Restoration benefit category: & $F$ & |Chinook usage score: & 30.1 & |Habitat productivity loss score: & -33.6 \\
\hline
\end{tabular}

\begin{tabular}{|c|c|c|c|c|c|c|c|c|c|c|c|c|c|c|}
\hline \multirow[b]{3}{*}{ Life stage } & \multirow[b]{3}{*}{ Months } & \multicolumn{10}{|c|}{ No. life history pathways by current usage abundance level } & \multicolumn{2}{|c|}{ Key habitat change } & \multirow{3}{*}{$\begin{array}{c}\text { Habitat } \\
\text { productivity } \\
\text { loss score }\end{array}$} \\
\hline & & \multicolumn{5}{|c|}{ Primary } & \multicolumn{5}{|c|}{ Secondary } & \multirow{2}{*}{$\begin{array}{l}\text { Patient } \\
\text { quantity }\end{array}$} & \multirow[b]{2}{*}{$\%$ change } & \\
\hline & & Total no, & 1 & 2 & 3 & 4 & Total no. & 1 & 2 & 3 & 4 & & & \\
\hline & Apr-Aug & 13 & 11. & 0 & 2 & 0 & 43 & 41 & 0 & 2 & 0 & 9.8 & -8.4 & -5.8 \\
\hline $1.2,3+\cdots$ & Aug-Ött & "NA" & $\mathrm{NA}$ & $\mathrm{NA}$ & "NA" & NA" & $\mathrm{NÄ}$ & NA & $\mathrm{NA}$ & NÄ & NA & $\mathrm{NA}$ & $\mathrm{NA}$ & $N A$ \\
\hline 3 & Ott-Mar & $\mathrm{NA}$ & $\mathrm{NA}$ & NA. & NA & $N A^{* *}$ & NA & NA & NA & NA & $\dddot{N A}$ & NA & $\mathrm{NA}$ & NA \\
\hline 34 & Mar-May & 2 & $\dddot{2}$ & 0 & 0 & 0 & 6 & $6 "$ & 0 & 0 & 0 & 9.0 & -32.3 & -22.9 \\
\hline 5 & May-Oct & 1 & 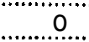 & 0 & 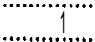 & 0 & 29 & 27 & 0 & 2 & 0 & 7.5 & -9.2 & -0.7 \\
\hline 6 & Ött-Maăr & $" 19$ & 19 & 0 & 0. & 0 & 2 & 2 & 0 & 0 & 0 & 8.2 & -12.9 & -4.6 \\
\hline 7 & Mar-Jun & 9 & 8 & 0 & 1 & $\ddot{0}$ & "̆ & 6 & 0 & o & 0 & 33.0 "',', & 0.0 & 0.5 \\
\hline
\end{tabular}

\begin{tabular}{|c|c|c|c|c|c|c|c|c|c|c|c|c|c|}
\hline \multirow[b]{2}{*}{ Life stage } & \multirow[b]{2}{*}{ Months } & \multicolumn{12}{|c|}{ Highest productivity impact attributes (\% change in impact from template) } \\
\hline & & Att 1 & $\%$ change & Att 2 & $\%$ change & Atf 3 & $\%$ change & Att 4 & $\%$ change & Att 5 & $\%$ change & Att 6 & $\%$ change \\
\hline & Apr-Aug & HABD & 6.7 & RIPCN & 6.7 & PATHO & 3.3 & TEMP & 3.3 & & & & \\
\hline 1.2." & Aug-Ött" & $\mathrm{NA}$ & $\mathrm{NÄ}$ & $\mathrm{NA}$ & "NA" & $\mathrm{NA}$ & $\mathrm{NA}$ & NA & $\mathrm{NA}$ & $\mathrm{NA}$ & $\mathrm{NA}$ & NÄ & $\mathrm{NA}$ \\
\hline 3 & Ött-Mar & $\mathrm{NA}$ & $\mathrm{NÄ̈}$ & NÄ & "ัฒ"̈ & NÄ & Nä" & $\mathrm{NA}$ & NÄ & $\mathrm{NA}$ & $\mathrm{NA}$ & No & $\mathrm{NA}$ \\
\hline$\frac{3,4}{5}$, & $\begin{array}{c}\text { Mar-May } \\
\text { May-Ott }\end{array}$ & $\begin{array}{r}\text { HABD } \\
\text { HABD }\end{array}$ & $\begin{array}{r}34.5 \\
، 7.6\end{array}$ & $\begin{array}{l}\text { RIPCN } \\
\text { PATHO }\end{array}$ & $\begin{array}{c}23.0 \\
3.8\end{array}$ & $\begin{array}{l}\text { FLOW } \\
\text { RIPCN }\end{array}$ & $\begin{array}{l}11.5 \\
3.8\end{array}$ & РÄTH்O & 11.5 & with & 11.5 & & \\
\hline 6 & OOtt-Mar & HABD & 92 & SEDED & 9,2 & PATHOOO & 4,6 & RIPCN & 4.6 & CÖMP & 3.1 & W"iт̈н" & 3.1 \\
\hline 7 & Mar-Jun & COMP & 2.8 & PATHO & 2.8 & WITH & 2.8 & & & & & & \\
\hline
\end{tabular}

Abundance /eve/ refers to the average abundance of spawners that spawn in the natal stream reaches that populate the life history pathways using the reach in this table:

$$
\begin{array}{ll}
\text { Level 1: } & 0-10 \text { spawners } \\
\text { Level 2 } & 10-30 \text { spawners } \\
\text { Level 3: } & 30-60 \text { spawners } \\
\text { Level 4 } & >60 \text { spawners }
\end{array}
$$

Life stages: 1- prespawner; 2- spawner; 3- incubation; 4- fry colonization; 5- summer rearing; 8- overwintering; 7- yearling presmolt/smolt

Attributes: CHEM - chemicals

CSTAB - channel stability

COMP - competition (with other species)

FLOW - flow

HABD - habitat diversity
NUTLD - nutrient load

OBST - obstructions

OXYG - dissolved oxygen

PATHO - pathogens

PRED - predators (includes fishing-related losses)
RIPCN - riparian condition

SEDLD - sediment load

TEMP - water temperature

WITH - water withdrawals

"NA" indicates that no tife history pathway meeting the criteria used in defining these pathways was present.

Blank cells under attributes indicate no additional attributes with negative effects on productivity were evident compared to those present in the template, 
Stream: Wallowa $\mathrm{R}$.

Reach: Whiskey $\mathrm{Cr}$ - Lostine R (RM 103.9 -105.8 from Snake R)

\begin{tabular}{|ll|ll|ll}
\hline Restoration benefit category: & $F$ & Chinook usage score: & 27.2 & Habitat productivity loss score: \\
\hline
\end{tabular}

\begin{tabular}{|c|c|c|c|c|c|c|c|c|c|c|c|c|c|c|}
\hline \multirow{3}{*}{ 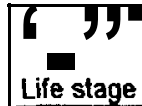 } & \multirow[b]{3}{*}{ Months } & \multicolumn{10}{|c|}{ No. life history pathways by current usage abundance level } & \multicolumn{2}{|c|}{ Key habitat change } & \multirow{3}{*}{$\begin{array}{c}\text { Habitat } \\
\text { productivity } \\
\text { loss score }\end{array}$} \\
\hline & & \multicolumn{5}{|c|}{ Primary } & \multicolumn{4}{|c|}{ Secondary } & & \multirow{2}{*}{$\begin{array}{l}\text { Patient } \\
\text { quanlity }\end{array}$} & \multirow[b]{2}{*}{ \% change } & \\
\hline & & Total no. & 1 & 2 & 3 & 4 & Total no. & 1 & 2 & 3 & 4 & & & \\
\hline 1 & Apr-Aug & 13 & 11 & 0 & 2 & 0 & 43 & 41. & $\underline{0 .}$ & $2 . .$. & 0 & 9.0 & -9.8 & -2.3 \\
\hline $1,2,3$ & Aug-Oct & $N A N A$ & $\mathrm{NA}$ & NAAA & $\mathrm{NA}$ & NA & NA & NÄ & NÄ & $\mathrm{NÄ}$ & NÄ & NA & NA & NÄ \\
\hline 3,4 & Mar-May & 2 & 2 & 0 & 0 & 0 & 6 & 6 & 0 & 0 & 0 & 8.3 & -30.7 & -22.2 \\
\hline 5 & Mà-Oct & 2 & 1 & a & 1 & 0 & 29 & 27 & 0 & 2 & 0 & 7.5 & .7 .2 & -0.6 \\
\hline 6 & Ott-Mar & 1 & 1 & 0 & 0 & 0 & 21 & 21 & 0 & 0 & 0 & 76 & .115 & -4.3 \\
\hline 7 & Mar.Jun & 8 & 7 & 0 & 1 & 0 & 6 & $\dot{6}$ & 0 & 0 & 0 & 30.3 & 0.0 & 0 \\
\hline
\end{tabular}

\begin{tabular}{|c|c|c|c|c|c|c|c|c|c|c|c|c|c|}
\hline \multirow[b]{2}{*}{ Life stage } & \multirow[b]{2}{*}{ Months } & \multicolumn{12}{|c|}{ Highest productivity impact attributes (\% change in impact from template) } \\
\hline & & Att 1 & $\%$ change & Att 2 & $\%$ change & Att 3 & $\%$ change & Att 4 & \% change & Att 5 & $\%$ change & Att 6 & \% change \\
\hline 1 & Apr-Aug & HABD & 7.8 & RIPCN & 7.8 & PATHO & 3.9 & & & & & & \\
\hline $1,2,3$ & Aug-Oct & NA & $\mathrm{NA}$ & NÄ & $\mathrm{NA}$ & NA & $N A$ & NA & NA & $N A$ & $\mathrm{NA}$ & NA & NA \\
\hline 3 & Oct-Mar & NA & $\mathrm{NA}$ & NA & NÄ & $\mathrm{NA}$ & $\mathrm{NA}$ & $\mathrm{NA}$ & NA & NÄ & $\mathrm{NA}$ & NA & NÄ \\
\hline 3,4 & Mar-May & HABD & 16.7 & SEDLD & 16.7 & NUTTLD & 11.2 & RIPCN & 11.2 & FLOWW & 5.6 & OXYG & 5.6 \\
\hline 5 & May-Oct & HABD & 6.0 & SEDLD & 6.0 & PATHO & 3.0 & RIPCN & 3.0 & & & & \\
\hline 6 & Oct-Mar & HABO & 8.1 & SEDLD & 8.1 & PATHO & 4.0 & RIPCN & 4.0 & COMP & 3.1 & WITH & 3.1 \\
\hline 7 & Mar-Jun & COMP & 2.1 & PATHO & 2.1 & WITH & 2.1 & & & & & & \\
\hline
\end{tabular}

Abundance level refers to the average abundance of spawners that spawn in the natal stream reaches that populate the life history pathways using the reach in this table $\begin{array}{ll}\text { Level 1: } & 0-10 \text { spawners } \\ \text { Level 2: } & 10-30 \text { spawners } \\ \text { Level 3: } & 30-60 \text { spawners } \\ \text { Level 4 } & >60 \text { spawners }\end{array}$

Life stages: 1- prespawner; 2- spawner; 3- incubation; 4- fry colonization; 5- summer rearing; 6- overwintering; 7- yearling presmolt/smolt

Attributes.' CHEM - chemicals

CSTAB - channel stability

COMP - competition (with other species)

FLOW - ftOW

HABD - habitat diversity
NUTLD - nutrient load

OBST - obstructions

OXYG - dissolved oxygen

PATHO - pathogens

PRED - predators (includes fishing-related losses)
RIPCN - riparian condition

SEDLD - sediment load

TEMP - water temperature

WiTH - water withdrawals

"NA" indicates that no life history pathway meeting the criteria used in defining these pathways was present.

Blank ceils under attributes indicate no additional attributes with negative effects on productivity were evident compared to those present in the template, 
Stream: Wallowa R.

Reach: $\quad$ Lostine R - Parsnip Cr (RM 105.8 -109.0 from Snake R)

\begin{tabular}{ll|ll|ll}
\hline Restoration benefit category: & D & Chinook usage score: & 240.3 & Habitat productivity loss score: \\
\hline
\end{tabular}

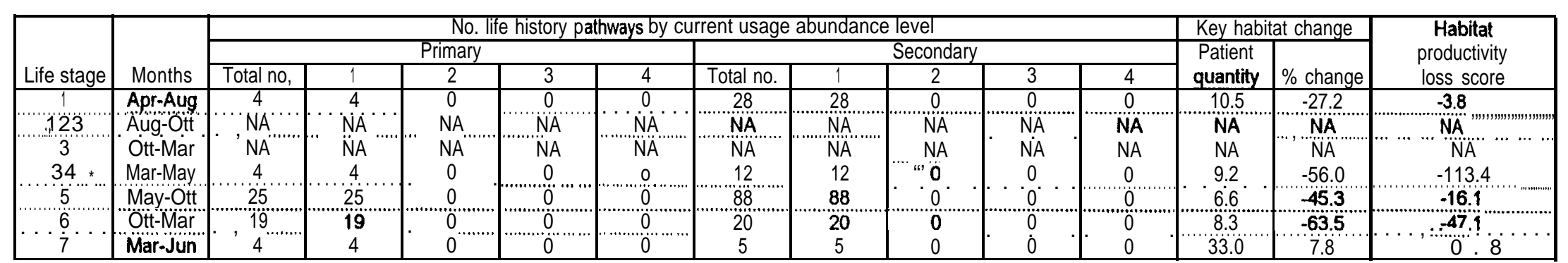

\begin{tabular}{|c|c|c|c|c|c|c|c|c|c|c|c|c|c|}
\hline \multirow[b]{2}{*}{ Life stage } & \multirow[b]{2}{*}{ Months } & \multicolumn{12}{|c|}{ Highest productivity impact attributes (\% change in impact from template) } \\
\hline & & Att 1 & \% change & Att 2 & \% change & Att 3 & $\%$ change & Aft 4 & \% change & Att 5 & \% change & Att 6 & \% change \\
\hline 1. & Apr-Aug & HABD & 24.1 & RIPCN & 24.1 & PATHO & 12.1 & & & & & & \\
\hline $1,2,3$ & Aug-Oct & $\mathrm{NA}$ & NA & NA & NA & NA & NA & NÄ & NA & NA & NÄ & NA & NA \\
\hline 3 & Oct-Mar & $\mathrm{NA}$ & NA & NA & NA & $\mathrm{NA}$ & $\mathrm{NA}$ & $\mathrm{NA}$ & $\mathrm{NA}$ & NA & NA & $\mathrm{NA}$ & NA \\
\hline 3,4 & Mar-May & HABD & 75.0 & RIPCN & 50.0 & FLOW & 25.0 & PATHO & 25.0 & WiTH & 25.0 & & \\
\hline 5 & May-Ocl & $\mathrm{HABD}$ & 440 & PATHO & 22.0 & RIPCN & 22.0 & & & & & & \\
\hline 6 & Oct-Mar & HABD & 48.8 & SEDLO & 488 & COMP & 250 & PATHO & 250 & WiTH & 25.0 & RIPCN & 24.4 \\
\hline 7 & Mar-Jun & COMP & 7.8 & PATHO & 7.8 & "WITH" & 7.8 & & & & & & \\
\hline
\end{tabular}

Abundance level refers to the average abundance of spawners that spawn in the natal stream reaches that populate the life history pathways using the reach in this table:

$$
\begin{array}{ll}
\text { Level 1: } & 0-10 \text { spawners } \\
\text { Level 2: } & 10-30 \text { spawners } \\
\text { Level 3: } & 30-60 \text { spawnera } \\
\text { Level 4: } & >\mathbf{6 0} \text { spawners }
\end{array}
$$

Life stages: 1- prespawner; 2- spawner; 3- incubation; 4- fry colonization; 5- summer rearing; 6- overwintering; 7- yearling presmolt/smoll

Attributes.' CHEM - chemicals

CSTAB - channel stability

COMP - competition (with other species)

FLOW - ftOW

HABD - habitat diversity
NUTLD - nutrient load

OBST - obstructions

OXYG - dissolved oxygen

PATHO - pathogens

PRED - predators (includes fishing-related losses)
RIPCN - riparian condition

SEDLD - sediment load

TEMP - water temperature

WITH - water withdrawals

"NA" indicates that no life history pathway meeting the criteria used in defining these pathways wea present.

Blank cells under attributes indicate no additional attributes with negative effects on productivity were evident compared to those present in the template, 
Stream: Wallowa R.

Reach: Parsnip Cr - Hurricane Cr (RM 109.0 -121.9 from Snake R)

\begin{tabular}{ll|ll|l|}
\hline Restoration benefit category: & A & Chinook usage score: & 495.3 & Habitat productivity loss score: \\
\hline
\end{tabular}

\begin{tabular}{|c|c|c|c|c|c|c|c|c|c|c|c|c|c|c|}
\hline \multirow[b]{3}{*}{ Life stage } & \multirow[b]{3}{*}{ Months } & \multicolumn{10}{|c|}{ No. life history pathways by current usage abundance level } & \multicolumn{2}{|c|}{ Key habitat change } & \multirow{3}{*}{$\begin{array}{c}\text { Habital } \\
\text { productivity } \\
\text { loss score }\end{array}$} \\
\hline & & \multicolumn{5}{|c|}{ Primary } & \multicolumn{5}{|c|}{ Secondary } & Patient & & \\
\hline & & Total no. & 1 & 2 & 3 & 4 & Total no. & 1 & 2 & 3 & 4 & quantity & $\%$ change & \\
\hline & Apr-Aug & 4 & 4 & 0 & 0 & 0 & 28 & 28 & 0 & 0 & 0 & 11.3 & -48.5 & -6.1 \\
\hline 123 & Aug-Ott & NA & NA & NA* & $\dot{N A}$ & NA & NA & $\dot{N} \dot{A}$ & $\mathrm{NA}$ & $\mathrm{NA}$ & $\mathrm{NA}$ & NA & $\mathrm{NÄ}$ & NA \\
\hline 34 & Mar-May & 2 & 2 & 0 & "o" & $0 "$ & 66 & 66 & o“ & 0 & 0 & 9.8 & -64.5 & \\
\hline 5 & May-Ott & 0 & 0 & 0 & 0 & 0 & 295 & 295 & "0”' & $0 "$ & 0 & 9.0 & -63.3 & "\%; '"',-',',',' \\
\hline 6 & Ott-Mar & 0 & 0 & 0 & 0 & 0 & 5 & 57 & $\ddot{0}$ & $\ddot{0}$ & 0 & 8.1 & -76.1 & -85.7 \\
\hline 7 & Mar-jun & 3 & 3 & $\ddot{0}$ & $" 0$ & 0 & 4 & 4 & 0 & 0 & 0 & 33.0 & -5.8 & 1.3 \\
\hline
\end{tabular}

\begin{tabular}{|c|c|c|c|c|c|c|c|c|c|c|c|c|c|}
\hline \multirow[b]{2}{*}{ Life stage } & & \multicolumn{12}{|c|}{ Highest productivity impact attributes (\% change in impact from template) } \\
\hline & Months & Att 1 & $\%$ change & Att 2 & $\%$ change & Att 3 & $\%$ change & Att 4 & $\%$ change & Att 5 & $\%$ change & Atf 6 & $\%$ change \\
\hline 1 & Apr-Aug & HABD & 39.4 & RIPCN & 39.4 & PATHO & 19.7 & & & & & & \\
\hline $1,2,3$ & Aug-Oct & $\mathrm{NA}$ & $\mathrm{NA}$ & NA & $N A$ & NA & NA & NA & NA & Nä & $\mathrm{NA}$ & Nä & NAM \\
\hline 3 & Oct-Mar & $\mathrm{NA}$ & NA & $\ddot{N A}$ & $\mathrm{NA}$ & NA & $\mathrm{NA}$ & $\dddot{N}$ & NA" & $\because \mathrm{NA}$ & $\mathrm{NA}$ & NA & $\mathrm{NA}$ \\
\hline 3,4 & Mar-May & HABD & 75.0 & RIPCN" & $50.0^{\circ}$ & SEDLD & 37.5 & FLOW & 25.0 & NUTLD & $250 \div-1$ & PÄT̈HO" & $25.0^{*}$ \\
\hline 5 & May-Oct & HABD & 50.0 & SEDLD & $36.7 "$ & PATHO & 25.0 & RIPCN & 25.0 & & & & \\
\hline 6 & Oct-Mar & HABD & 50.0 & SÉËLE & 50.0 & $\triangle O O M P$ & 25.0 & "PÄТН̈O & 25.0 & "RIPPCN" & 25.0 & ت̈iтï & 25.0 \\
\hline 7 & Mar-Jun & сомР & 158 & PATHO & 15.8 & ẄITH & 158 & & & & & & \\
\hline
\end{tabular}

Abundance /eve/ refers to the average abundance of spawners that spawn in the natal stream reaches that populate the life history pathways using the reach In this table:

$$
\begin{array}{ll}
\text { Level 1: } & 0-10 \text { spawners } \\
\text { Level 2: } & 10-30 \text { spawnera } \\
\text { Level 3: } & 30-60 \text { spawners } \\
\text { Level 4 } & >60 \text { spawners }
\end{array}
$$

Life stages.' 1- prespawner; 2- spawner; 3- incubation; 4- fry colonization; 5- summer rearing; 6- overwintering; 7- yearling presmolt/smolt

$\begin{array}{cll}\text { Attributes: CHEM - chemicals } & \text { NUTLD - nutrient load } & \text { RIPCN - riparian condition } \\ \text { CSTAB - channel stability } & \text { OBST - obstructions } & \text { SEDLD - sediment load } \\ \text { COMP - competition (with other species) } & \text { OXYG - dissolved oxygen } & \text { TEMP - water temperature } \\ \text { FLOW - flow } & \text { PATHO - pathogens } & \text { WITH - water withdrawals } \\ \text { HABD - habitat diversity } & \text { PRED - predators (includes fishing-related losses) }\end{array}$

"NA" indicates that no life history pathway meeting the criteria used in defining these pathways was present.

Blank cells under attributes indicate no additional attributes with negative effects on productivity were evident compared to those present in the template. 
Stream Reach Summary for Primary and Secondary Spring Chinook Salmon Life Histories

Stream: Wallowa R.

Reach: Hurricane Cr - Lower Alderslope Ditch (RM 121.9 -126,8 from Snake R)

\begin{tabular}{ll|ll|l|l|l|l|l|}
\hline Restoration benefit category: & A & Chinook usage score: & 595.5 & Habltat productivity ioss score: & $-1149_{4} 1$ \\
\hline
\end{tabular}

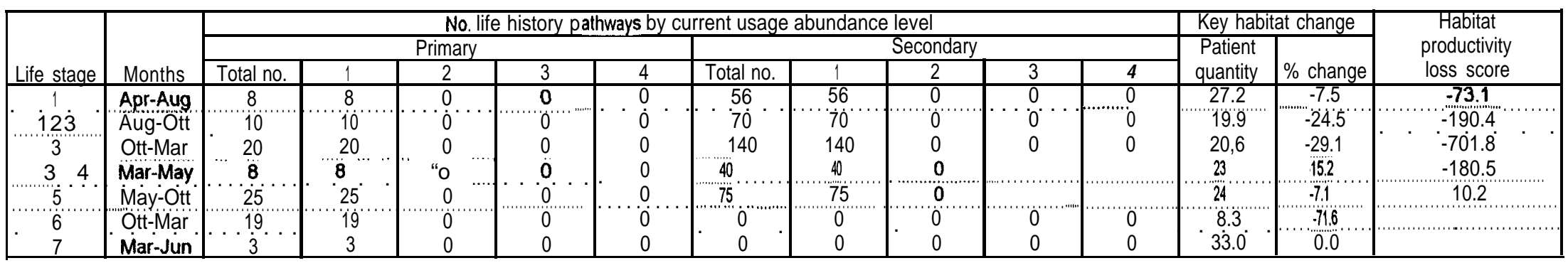

\begin{tabular}{|c|c|c|c|c|c|c|c|c|c|c|c|c|c|}
\hline \multirow[b]{2}{*}{ Life stage } & \multirow[b]{2}{*}{ Months } & \multicolumn{12}{|c|}{ Highest productivity impact attributes (\% change in impact from template) } \\
\hline & & Aft 1 & $\%$ change & Att 2 & $\%$ change & Att 3 & $\%$ change & $\overline{A t t} 4$ & $\%$ change & Att 5 & $\%$ change & Att 6 & $\%$ change \\
\hline 1 & Apr-Aug & FLOW & 40.3 & SEDLD & 37.5 & NUTLD & 25.0 & CHEM & 20.2 & HABD & 20.2 & OBST & 20.2 \\
\hline $1,2,3$ & Aug-Oct & SEDLLD & 75.0 & FLOW & 50.0 & NUTLD & 50.0 & CHEM & 25.0 & $\mathrm{HABD}$ & 25.0 & OBST & 25.0 \\
\hline 3 & Oct-Mar & SEDLD & 75.0 & NÜTLD & 50.0 & FLOW & 25.0 & HABD & 25.0 & OXYG & $25.0^{\circ}$ & PATHOO & 250 \\
\hline 3,4 & Mar-May & SEDLD & 50.0 & CHEM & 25.0 & HABD & $25.0^{\circ}$ & NUTLD & 25.0 & PATHO & 25.0 & PRED & 25.0 \\
\hline 5 & May-Oct & CHEM & 24.3 & РATHO & 24.3 & WITH & 24.3 & & & & & & \\
\hline 6 & Oct-Mar & Flow & 250 & РATHO & 250 & SEDLD & 25.0 & WiTH & 250 & & & & \\
\hline 7 & Mar-Jun & FLOWW & 14.4 & Wiтt & 14.4 & & & & & & & & \\
\hline
\end{tabular}

Abundance level refers to the average abundance of spawners that spawn in the natal stream reaches that populate the life history pathways using the reach in this table:

$$
\begin{array}{ll}
\text { Level 1: } & 0-10 \text { spawners } \\
\text { Level 2 } & 10-30 \text { spawners } \\
\text { Level 3 } & 30-60 \text { spawners } \\
\text { Level 4 } & >60 \text { spawners }
\end{array}
$$

Life stages: 1- prespawner; 2- spawner; 3-incubation; 4- fry colonization 5- summer rearing; 6- overwintering; 7- yearling presmollsmolt

Attributes: CHEM - chemicals

CSTAB - channel stability

COMP - competition (with other species)

FLOW - flow

HABD - habitat diversity
NUTLD - nutrient load

OBST - obstructions

OXYG - dissolved oxygen

PATHO - pathogens

PRED - predators (includes fishing-related losses)

\section{RIPCN - riparian condition \\ SEDLD - sediment load \\ TEMP - water temperature \\ WITH - water withdrawals}

"NA" indicates that no life history pathway meeting the criteria used in defining these pathwaya was present.

Blank cells under attributes indicate no additional attributes with negative effects on productivity were evident compared to those present in the template 
Stream: Wallowa R.

Reach: $\quad$ Lower Alderslope Ditch - Wallowa Lake (RM 126.8 -130.8 from Snake R)

\begin{tabular}{|l|l|l|l|l|}
\hline Restoration benefit category: & F & Chinook usage score: & 0.0 & Habitat productivity loss score: \\
\hline
\end{tabular}

\begin{tabular}{|c|c|c|c|c|c|c|c|c|c|c|c|c|c|c|}
\hline \multirow[b]{3}{*}{ Life stage } & \multirow[b]{3}{*}{ Months } & \multicolumn{10}{|c|}{ No. life history pathways by current usage abundance level } & \multicolumn{2}{|c|}{ Key habitat change } & \multirow{3}{*}{$\begin{array}{c}\text { Habitat } \\
\text { productivity } \\
\text { loss score }\end{array}$} \\
\hline & & \multicolumn{5}{|c|}{ Primary } & \multicolumn{5}{|c|}{ Secondary } & \multirow{2}{*}{$\begin{array}{l}\text { Patient } \\
\text { quantity }\end{array}$} & \multirow[b]{2}{*}{$\%$ change } & \\
\hline & & Total no. & 1 & 2 & 3 & 4 & \begin{tabular}{|l} 
Total no. \\
Tol
\end{tabular} & 1 & 2 & 3 & 4 & & & \\
\hline 1 & Apr-Aug & NA & NA & NA & NA & NA & NA & NA & NA & NA & NA & $\ldots$ NA ... & NA & NA \\
\hline 123 & Aug-Oct & NÄ. & Nä. & $\mathrm{NÄ}$ & N̈̈ & NÄ. & $\mathrm{NA}$ & NA & NA & $\dot{N} \dot{A}$ & NA & NA & $\mathrm{NA}$ & $\mathrm{NA}$ \\
\hline 3 & Ött-Mar & NA & NA & $\mathrm{NA}$ & 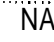 & $\mathrm{NÄ}$ & $\mathrm{NA}$ & N'A & NA & NA & $\mathrm{NA}$ & NA & NA & $\mathrm{NA}$ \\
\hline “34. & Mar-May & $\mathrm{NA}$ & NA & NA & NA & NA & NA & "'NA & NA & NA & NA & NA & NA. & NA \\
\hline 5 & May-Ott & $\dot{N A}$ & $N A$ & $\mathrm{NAM}$ & $\mathrm{NA}$ & NA & NA" & NA & NA & NA & NA & NA & NA & NÄ" \\
\hline 8 & Ott-Mar & $\mathrm{NA}$ & NA & $\mathrm{NA}$ & N̈' & $\mathrm{NA}$ & $\mathrm{NA}$ & $\mathrm{NA}$ & NA" & $\mathrm{NA}$ & $\mathrm{NA}$ & $\mathrm{NA}$ & NA & NA \\
\hline 7 & Mar-Jun & NA & NA & NA & NA & NA & NA & NA & NA & NA & NA & NA & NA & $\dot{N} \dot{A}$ \\
\hline
\end{tabular}

\begin{tabular}{|c|c|c|c|c|c|c|c|c|c|c|c|c|c|}
\hline \multirow[b]{2}{*}{ Life stage } & \multirow[b]{2}{*}{ Months } & \multicolumn{12}{|c|}{ Highest productivity impact attributes ( $\%$ change in impact from template) } \\
\hline & & Att 1 & $\%$ change & Att 2 & \% change & Att 3 & $\%$ change & Att 4 & $\%$ change & Att 5 & $\%$ change & Att 6 & $\%$ change \\
\hline- & Apr-Aug & $\mathrm{NA}$ & NA & $\mathrm{NA}$ & $\mathrm{NA}$ & $\mathrm{NA}$ & NA & NA & NA & NA & NA & NA & NA \\
\hline $1,2,3$ & Aug-Öci & NA & NA & NA & NA & $\mathrm{NÄ}$ & $\mathrm{NÄ}$ & NA & NA & NA & NA & NA & $\mathrm{NA}$ \\
\hline 3 & Ött-Mar & $\mathrm{NA}$ & NA & NA & NA. & $\mathrm{NA}$ & $\mathrm{NÄ}$ & & N̈̈̈ & NA & N̈̈̈ & NÄ & NA \\
\hline 3,4 & Mar-May & NA & NA & $\mathrm{NA}$ & $\mathrm{NA}$ & ÑA & NA & & $\mathrm{NA}$ & NÄ" & NA & NA & NA \\
\hline 5“" “"'”" & May-Ott & NA & NA & NA & $\mathrm{NA}$ & N A & NA & NA & NA & NA & $\mathrm{NA}$ & $\mathrm{NA}$ & NA \\
\hline 6 & Ott-Mar & NA & NA & NA & $N A \ddot{~}$ & NA & $\mathrm{N} \mathrm{NA}$ & $\mathrm{NÄ}$ & N̈̈" & NA & $\mathrm{NA}$ & $\mathrm{NA}$ & NA \\
\hline 7 & Mar-Jun & NA & NA & $\mathrm{NA}$ & NA & $\mathrm{NA}$ & $\mathrm{NA}$ & $\mathrm{NA}$ & $\mathrm{N} \mathrm{NA}$ & $\mathrm{NÄ}$ & $\mathrm{NA}$ & $\mathrm{NA}$ & $\mathrm{NA}$ \\
\hline
\end{tabular}

Abundance /eve/ refers to the average abundance of spawners that spawn in the natal stream reaches that populate the life history pathways using the reach in this table

$$
\begin{array}{ll}
\text { Level 1: } & 0-10 \text { spawners } \\
\text { Level 2: } & 10-30 \text { spawners } \\
\text { Level 3: } & 30-60 \text { spawners } \\
\text { Level 4: } & >60 \text { spawners }
\end{array}
$$

Life stages: 1 -prespawner; 2- spawner; 3- incubation; 4- fry colonization; 5- summer rearing; 6- overwintering; 7- yearling presmolt/smolt

$\begin{array}{lll}\text { Attributes: } \text { CHEM - chemicals } & \text { NUTLD - nutrient load } & \text { RIPCN - riparian condition } \\ \text { CSTAB - channel stability } & \text { OBST - obstructions } & \text { SEDLD - sediment load } \\ \text { COMP - competition (with other species) } & \text { OXYG - dissolved oxygen } & \text { TEMP - water temperature } \\ \text { FLOW - flow } & \text { PATHO - pathogens } & \text { WITH - water withdrawals } \\ \text { HABD - habitat diversity } & \text { PRED - predators (includes fishing-related losses) }\end{array}$

"NA" indicates that no life history pathway meeting the criteria used in defining these pathwayswas present.

Blank cells under attributes indicate no additional attributes with negative effects on productivity were evident compared to those present in the template. 
Stream Reach Summary for Primary and Secondary Spring Chinook Salmon Life Histories

Stream: Wenaha R.

Reach: $\quad$ Mouth - Crooked $\mathrm{Cr}$ (RM 45.1 -51.8 from Snake R)

\begin{tabular}{|ll|ll|l|}
\hline Restoration benefit category: & $F$ & Chinook usage score: & 31.7 & Habitat productivity loss score: \\
\hline
\end{tabular}

\begin{tabular}{|c|c|c|c|c|c|c|c|c|c|c|c|c|c|c|}
\hline \multirow[b]{3}{*}{ Life stage } & \multirow[b]{3}{*}{ Months } & \multicolumn{10}{|c|}{ No. life history pathways by current usage abundance level } & \multicolumn{2}{|c|}{ Key habitat change } & \multirow{3}{*}{$\begin{array}{c}\text { Habitat } \\
\text { productivity } \\
\text { loss score }\end{array}$} \\
\hline & & \multicolumn{5}{|c|}{ Primary } & \multicolumn{5}{|c|}{ Secondary } & \multirow{2}{*}{$\begin{array}{l}\text { Patient } \\
\text { quantity }\end{array}$} & \multirow[b]{2}{*}{$\%$ change } & \\
\hline & & Total no. & 1 & 2 & 3 & 4 & Total no. & 1 & 2 & 3 & 4 & & & \\
\hline & Apr-Aug & 4 & 1 & 2. & 1 & 0 & 9 & 2 & 5 & 2 & 0 & 12.0 & 0.0 & 52.0 \\
\hline $1,2, \hat{\beta}$ & Aug-Oct & $\mathrm{NA}$ & $\mathrm{NA}$ & $\mathrm{NÄ}$ & Nัฒ̈ & $\mathrm{NÄ}$ & $\mathrm{NA}$ & NA & NÄ & N̈Ä & “NA" & $\mathrm{NÁ}$ & NA" & NA \\
\hline 3 & Ött-Mar & NA & NA & NA & NA & NA & N̈A & NA & NA & NA & $\mathrm{NA}$ & NA & NA & $\mathrm{NA}$ \\
\hline $34 \ldots$ & Mar-May & NA & $\mathrm{NA}$ & NA & $\mathrm{NA}$ & $N A$ & $\mathrm{NA}$ & $\mathrm{NA}$ & $\mathrm{NA}$ & NA & NA & NA & NA & $\mathrm{NÄ}$ \\
\hline 5 & May-Ott & 0 & 0 & 0 & 0 & 0 & 8 & 2 & 4 & 2 & $" 0$ & $6.0^{\circ}$ & 0.0 & 0.0 \\
\hline 6 & Oct-Mar & 0 & 0 & 0 & 0 & 0 & $" i$ & 0 & 1 & 0 & $0 "$ & 6.0 & 0.0 & 0.0 \\
\hline 7 & Mar-Jun & 4 & 1 & 2 & 1 & 0 & 0 & 0 & 0 & 0 & 0 & 30.8 & 0.0 & 0.1 \\
\hline
\end{tabular}

\begin{tabular}{|c|c|c|c|c|c|c|c|c|c|c|c|c|c|}
\hline \multirow[b]{2}{*}{ Life stage } & \multirow[b]{2}{*}{ Months } & \multicolumn{12}{|c|}{ Highest productivity impact attributes (\% change in impact from template) } \\
\hline & & Att 1 & $\%$ change & Att 2 & $\%$ change & Att 3 & $\%$ change & Att 4 & $\%$ change & Att 5 & $\%$ change & Att 6 & $\%$ change \\
\hline 1 & Apr-Aug & & & & & & & & & & & & \\
\hline 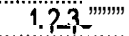 & Aug-Oct & NÄ & $\mathrm{NÄ}$ & Nั"̈ & $\mathrm{NÄ}$ & กั"̈̈ & $\mathrm{NÄ̈}$ & $\mathrm{NA}$ & $\dddot{\mathrm{NA} A ̈}$ & "NA" & NA" & NÄ & NÄ \\
\hline 3 & "Ött-Mar & ח̄"̈ & $\mathrm{NA}$ & חั" & "ก̈" & Nล̈ & N̈̈̈ & 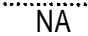 & NÄ & $\mathrm{NA}$ & $\mathrm{NA}$ & $\mathrm{NA}$ & $\mathrm{NA}$ \\
\hline 3,4 & Mar-May & NA & NA & NA & NA & $\mathrm{NA}$ & NA & NA & $\mathrm{N} 2$ & NA & N & NA & NA \\
\hline 5 & May-Ott & РÄTHO & 17.5 & & & & & & & & & & \\
\hline 6 & Oct-Mar & PATHO & 25.0 & & & 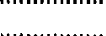 & & & & & & & \\
\hline 7 & Mar-Jun & & & & & & & & & & & & \\
\hline
\end{tabular}

Abundance level refers to the average abundance of spawners that spawn in the natal stream reaches that populate the life history pathways using the reach in this table

$$
\begin{array}{ll}
\text { Level 1: } & 0-10 \text { spawners } \\
\text { Level 2: } & 10-30 \text { spawners } \\
\text { Level 3: } & 30-60 \text { spawners } \\
\text { Level 4 } & >60 \text { spawners }
\end{array}
$$

Life stages: 1-prespawner; 2- spawner; 3- incubation; 4- fry colonization; 5- summer rearing; 6- overwintering; 7- yearling presmolt/smolt

$\begin{array}{lll}\text { Attributes: } & & \text { RHEM - chemicals } \\ \text { CSTAB - channel stability } & \text { NUTLD - nutrient load } & \text { riparian condition } \\ \text { COMP - competition (with other species) } & \text { OBST - obstructions } & \text { SEDLD - Sediment load } \\ \text { FLOW - flow } & \text { OXYG - dissolved oxygen } & \text { TEMP - water temperature } \\ \text { HABD - habitat diversity } & \text { PATHO - pathogens } & \text { WITH - water withdrawals } \\ & \text { PRED - predators (includes fishing-related losses) }\end{array}$

"NA" indicates that no life history pathway meeting the criteria used in defining these pathways was present.

Blank cells under attributes indicate no additional attributes with negative effects on productivity were evident compared to those present in the template. 
Stream: Wenaha $\mathrm{R}$

Reach: Crooked $\mathrm{Cr}$ - Fairview $\mathrm{Cr}$ (RM 51.8 -55.3 from Snake R)

\begin{tabular}{|c|c|c|c|c|c|c|c|c|c|c|c|c|c|c|}
\hline \multicolumn{3}{|c|}{ Restoration benefit category: } & \multicolumn{2}{|c|}{$\bar{E}$} & \multicolumn{3}{|c|}{ Chinook usage score: } & 555.7 & \multicolumn{5}{|c|}{ Habitat productivity ioss score: } & \multirow{4}{*}{$\begin{array}{c}\text { Habital } \\
\text { productivity } \\
\text { loss score }\end{array}$} \\
\hline \multirow[b]{3}{*}{ Life stage } & \multirow[b]{3}{*}{ Months } & \multicolumn{10}{|c|}{ No. life history pathways by current usage abundance level } & \multicolumn{2}{|c|}{ Key habitat change } & \\
\hline & & \multicolumn{5}{|c|}{ Primary } & \multicolumn{5}{|c|}{ Secondary } & \multirow{2}{*}{$\begin{array}{l}\text { Patient } \\
\text { quantity }\end{array}$} & \multirow[b]{2}{*}{$\%$ change } & \\
\hline & & Total no. & 1 & 2 & 3 & 4 & Total no. & 1 & 2 & 3 & 4 & & & \\
\hline & Apr-Aug & 9 & 6 & 2 & 1 & 0 & 19 & 12 & 5 & 2 & 0 & 9.5 & 0.0 & -364 \\
\hline 123. & Äug-Ött & 10 & 10 & 0 & 0 & $0 "$ & 20 & 20 & $\ddot{0}$ & 0 & 0 & 6.9 & 0.0 & -3.9 \\
\hline 3 & Ött-Mar & 20 & 20 & 0 & 0 & 0 & 40 & 40 & 0 & 0 & 0 & 6.7 & 00 & 0.0 \\
\hline 34 & Mar-May & 12 & 8 & 4 & 0 & 0 & 28 & 16 & 12 & 0 & 0 & 9.4 & $0.0^{\circ}$ & 37.6 \\
\hline 5 & May-Ott & $50^{\circ}$ & 25 & 25 & 0 & 0 & 116 & 46 & 68 & 2 & 0 & 8.6 & 00 & -22.4 \\
\hline 6 & Ott-Mar & 38 & la & 40 & 0 & 0 & 0 & 0 & 0 & 0 & 0 & 6.8 & 0.0 & 0.0 \\
\hline$\dot{7}$ & Mar-Jun & 4 & $1 i$ & 2 & 1 & in. & n. & n. & $n$ & 0 & 0 & 30.8 & 0.0 & 2.7 \\
\hline
\end{tabular}

\begin{tabular}{|c|c|c|c|c|c|c|c|c|c|c|c|c|c|}
\hline \multirow[b]{2}{*}{ Life stage } & \multirow[b]{2}{*}{ Months } & \multicolumn{12}{|c|}{ Highest productivity impact altributes (\% change in impact from template) } \\
\hline & & Aft 1 & $\%$ change & Att 2 & $\%$ change & Att 3 & $\%$ change & Att 4 & \% change & Att 5 & \% change & Att 6 & $\%$ change \\
\hline 1 & Apr-Aug & PRED & 24.1 & & & & & & & & & & \\
\hline $1,2,3$ & Aug-Oct & PRED & 16.7 & & & & & & & & & & \\
\hline 3 & Oct-Mar & PATHO & 125 & & & & & & & & & & \\
\hline 3,4 & Mar-May & PATHO & 19.1 & & & & & & & & & & \\
\hline 5 & May-Oct & PATHO & 24.8 & & & & & & & & & & \\
\hline 6 & Oct-Mar & PATHO & 25.0 & & & & & & & & & & \\
\hline 7 & Mar-Jun & & & & & & & & & & & & \\
\hline
\end{tabular}

Abundance lovel refers to the average abundance of spawners that spawn in the natal stream reaches that popuiate the iife history pathwaya using the reach in this table:

$$
\begin{array}{ll}
\text { Level 1: } & 0-10 \text { spawnera } \\
\text { Levei 2 } & 10-30 \text { spawners } \\
\text { Level 3: } & 30-60 \text { spawners } \\
\text { Levei 4: } & >60 \text { spawners }
\end{array}
$$

Life stages: 1- prespawner; 2- spawner; 3- incubation; 4- fry colonization; 5- summer rearing; 6- overwintering; 7- yearling presmolt/smolt

$\begin{array}{lll}\text { Attributes: CHEM - chemicals } & \text { NUTLD - nutrient ioad } & \text { RIPCN - riparian condition } \\ \text { CSTAB - channel stability } & \text { OBST - obstructions } & \text { SEDLD - sediment ioad } \\ \text { COMP - competition (with other species) } & \text { OXYG - dissolved oxygen } & \text { TEMP - water temperature } \\ \text { FLOW - fiOW } & \text { PATHO - pathogens } & \text { WITH - water withdrawals } \\ \text { HABD - habitat diversity } & \text { PRED - predators (includes fishing-related losses) }\end{array}$

"NA" indicates that no life history pathway meeting the criteria used in defining these pathways was present.

Blank cells under attributes indicate no additional attributes with negative effects on productivity were evident compared to those present in the template. 
Stream: Wenaha R.

Reach: Fairview $\mathrm{Cr}$ - forks (RM 55.3 -65.9 from Snake R)

\begin{tabular}{|ll|lll}
\hline Restoration benefit category: & E & Chinook usage score: & 405.0 & [Habitat productivity loss score:
\end{tabular}

\begin{tabular}{|c|c|c|c|c|c|c|c|c|c|c|c|c|c|c|}
\hline \multirow[b]{3}{*}{ Life stage } & \multirow[b]{3}{*}{ Months } & \multicolumn{10}{|c|}{ No. life history pathways by current usage abundance level } & \multicolumn{2}{|c|}{ Key habitat change } & \multirow{3}{*}{$\begin{array}{c}\text { Habitat } \\
\text { productivity } \\
\text { loss score }\end{array}$} \\
\hline & & \multicolumn{5}{|c|}{ Primary } & \multicolumn{5}{|c|}{ Secondary } & \multirow{2}{*}{$\begin{array}{l}\text { Patient } \\
\text { quantity }\end{array}$} & \multirow[b]{2}{*}{$\%$ change } & \\
\hline & & Total no. & 1 & 2 & 3 & 4 & Total no. & 1 & 2 & 3 & 4 & & & \\
\hline 1 & Apr-Aug & 8 & 0 & $2 \ldots$ & 6 & 0 & 17 & 0 & 5 & 12 & 0. & 16.6 & 0.0 & -5.2 \\
\hline $1,2,3$ & Aug-Oct & 10 & 0 & 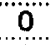 & 10 & 0 & 20 & 0 & 0 & 20 & 0 & 87 & 0.0 & -1.9 \\
\hline 3 & Oct-Mar & 20 & 0 & 0 & 20 & 0 & $40^{\circ}$ & 0 & 0 & 40 & 0 & 6.3 & 0.0 & 0.0 \\
\hline 3,4 & Mar-May & 10 & 0 & 2 & 8 & 0 & 22 & 0 & 6 & 16 & 0 & 6.7 & 0.0 & 17.5 \\
\hline 5 & May-Oci & 25 & 0 & 0 & 25 & 0 & 48 & 0 & 2 & 46 & 0 & 16.2 & 0.0 & -17.6 \\
\hline 6 & Oct-Mar & 19 & 0 & 0 & 19 & 0 & 0 & 0 & 0 & 0 & 0 & 15.8 & 0.0 & 0.0 \\
\hline 7 & Mar-Jun & 2 & 0 & 1 & 1 & 0 & 0 & 0 & 0 & 0 & 0 & 24.2 & 0.0 & 2.6 \\
\hline
\end{tabular}

\begin{tabular}{|c|c|c|c|c|c|c|c|c|c|c|c|c|c|}
\hline \multirow[b]{2}{*}{ Life stage } & \multirow[b]{2}{*}{ Months } & \multicolumn{12}{|c|}{ Highest productivity impact attributes (\% change in impact from $t_{e m} p_{a} i_{e}$ ) } \\
\hline & & Atf 1 & $\%$ change & Att 2 & $\%$ change & Att 3 & \% change & Att 4 & $\%$ change & Att 5 & \% change & Atf 6 & \% change \\
\hline & Apr-Aug & PRED & 20.5 & & & & & & & & & & \\
\hline $1,2,3$ & Aug-Ôd & PRÉD & $181^{\prime}$ & & & & $\cdots \cdots$ & & & & & & \\
\hline 3 & Ott-Mar' & PATHHO & 25.0 & & & & & & & & & & \\
\hline 3,4 & .. Mar-May & HABD & 25.0 & "РАTHÖ" & 25.0 & & & & & & & & \\
\hline 5 & May-Ott & HABD & $25.0^{\circ}$ & "PATHÖ & $25.0^{\circ}$ & & & & & & & & mim \\
\hline 6 & Oct-Mar & PATHO & 25.0 & 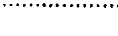 & & & & & & & & & \\
\hline 7 & Mar-Jun & & & & & & & & & & & & \\
\hline
\end{tabular}

Abundance /eve/ refers to the average abundance of spawners that spawn in the natal streamreaches that populate the life history pathways using the reach in this table

$$
\begin{array}{ll}
\text { Level 1: } & 0-10 \text { spawners } \\
\text { Level 2: } & 10-30 \text { spawners } \\
\text { Level 3 } & 30-60 \text { spawners } \\
\text { Level 4: } & >\mathbf{2 0} \text { spawners }
\end{array}
$$

Life stages: 1- prespawner; 2- spawner; 3- incubation; 4- fry colonization; 5- summer rearing; 6- overwintering; 7- yearling presmolt/smolt

$\begin{array}{lll}\text { Attributes: CHEM - chemicals } & \text { NUTLD - nutrient load } & \text { RiPCN - riparian condition } \\ \text { CSTAB - channel stability } & \text { OBST - obstructions } & \text { SEDLD - sediment load } \\ \text { COMP - competition (with other species) } & \text { OXYG - dissolved oxygen } & \text { TEMP - water temperature } \\ \text { FLOW - flow } & \text { PATHO - pathogens } & \text { WITH - water withdrawals } \\ \text { HABD - habital diversity } & \text { PRED - predators (includes fishing-related losses) }\end{array}$

"NA" indicates thst no life history pathway meeting the criteria used in defining these pathways was present.

Blank cells under attributes indicate no additional attributes with negative effects on productivity were evident compared to those present in the template. 
Stream Reach Summary for Primary and Secondary Spring Chinook Salmon Life Histories

Stream: Wenaha R.

Reach: $\quad$ South Fork Wenaha R (RM 65.9 -72.4 from Snake R)

\begin{tabular}{|c|c|c|c|c|c|c|c|c|c|c|c|c|c|c|}
\hline \multicolumn{4}{|c|}{ Restoration benefit category: } & & \multicolumn{3}{|c|}{ Chinook usage score: } & 577.0 & \multicolumn{5}{|c|}{ Habitat productivity loss score: } & \\
\hline \multirow[b]{3}{*}{ Liffestagec } & \multirow[b]{3}{*}{ Moontiths } & \multicolumn{10}{|c|}{ No. IIe nisiory pamways by current usage abundance level } & \multicolumn{2}{|c|}{ Key habitat change } & \multirow{3}{*}{$\begin{array}{c}\text { Habitat } \\
\text { productivity } \\
\text { loss score }\end{array}$} \\
\hline & & \multicolumn{5}{|c|}{ Primary } & \multicolumn{5}{|c|}{ Secondary } & \multirow{2}{*}{$\begin{array}{l}\text { Patient } \\
\text { quantity }\end{array}$} & \multirow[b]{2}{*}{$\%$ change } & \\
\hline & & Total no. & 1 & 2 & 3 & 4 & Total no. & 1 & 2 & 3 & 4 & & & \\
\hline 1 & Apr-Aug & 12. & 0 & 12 & $\mathbf{0}$ & 0 & 30. & 0 & 30 & 0 & 0 & 3.1 & 0.5 & 0.0 \\
\hline $1,2,3$ & Aug-Oct & 20 & 0 & 20 & 0 & & $50^{\circ}$ & & 5 & 0 & 0 & 2.6 & $0 . n$ & $26.2 \%$ \\
\hline 3 & Odthin & 40 & $\dot{0}$ & 40 & 0 & & 100 & & 11 & 0 & 0 & 2.8 & 0.0 & -312 \\
\hline 34 & Mar-May & 10 & 0 & 10 & 0 & 0 & 22 & 0 & 22 & ㅇ…". & 0 & 3.1 & 0.0 & 14.6 \\
\hline 5 & May-Odt & 25 & 0 & 25 & 0 & 0 & 46 & $n$ & 46 & 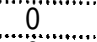 & 0 & 31 & 0.1 & $8.8^{\prime \prime \prime \prime}$ \\
\hline 6 & Odtikn & 19 & 0 & 19 & 0 & 0 & 0 & 0 & 0 & 0 & 0. & 28 & 0.0 & . \\
\hline 7 & Mar-Jun & 1 & 0 & 1 & 0 & 0 & 0 & 0 & 0 & 0 & $\ddot{0}$ & 11.2 & 0.0 & 0.5 \\
\hline
\end{tabular}

\begin{tabular}{|c|c|c|c|c|c|c|c|c|c|c|c|c|c|}
\hline \multirow[b]{2}{*}{ Life stage } & \multirow[b]{2}{*}{ Months } & \multicolumn{12}{|c|}{ Highest productivity impact attributes ( $\%$ change in impact from template) } \\
\hline & & Att 1 & $\%$ change & Att 2 & $\%$ change & Att 3 & $\%$ change & Att 4 & $\%$ change & Att 5 & $\%$ change & Att 6 & $\%$ change \\
\hline & Apr-Aug & PRED & 25.0 & TEMP & 8.3 & & & & & & & & \\
\hline $1,2,3$ & Aug-Oct & TEMP̈ & 25.0 & “P̈RËD & 16.7 & & & & & & & & \\
\hline 3 & Ött-Mär & TEMP & 23.1 & & & & & & & & & & .... \\
\hline 3,4 & MaryMay & TEMP & 12.5 & & & & & & & & & & \\
\hline 5 & May-Ott & & & & & & & & & & & & \\
\hline 6 & Ott-Mar & & & & & & & & & & & & \\
\hline 7 & Mar-Jun & CHEM & 25.0 & & & & & & & & & & .... \\
\hline
\end{tabular}

Abundance level refers to the average abundance of spawners that spawn in the natal stream reaches that populate the life history pathways using the reach in this table:

$$
\begin{array}{ll}
\text { Level 1: } & 0-10 \text { spawners } \\
\text { Level 2: } & 10-30 \text { spawners } \\
\text { Level 3: } & 30-60 \text { spawners } \\
\text { Level } 4 & >60 \text { spawners }
\end{array}
$$

Life stages: 1- prespawner; 2- spawner; 3- incubation; 4- fry colonization; 5- summer rearing; 6- overwintering; 7- yearling presmoit/smelt

Attributes: CHEM - chemicals

CSTAB - channel stability

COMP - competition (with other species)

FLOW - flow

HABD - habitat diversity
NUTLD - nutrient load

OBST - obstructions

OXYG - dissolved oxygen

PATHO - pathogens

PRED - predators (includes fishing-related losses)
RIPCN - riparian condition

SEDLD - sediment load

TEMP - water temperature

WITH - water withdrawals

"NA" indicates that no life history pathway meeting the criteria used in defining these pathways was present.

Blank cells under attributes indicate no additional attributes with negative effects on productivity were evident compared 10 those present in the template. 


\section{APPENDIX E}

Grande Ronde Stream Reach Summaries for Vacant Life History Pathways 
Reaches analyzed in the Grande Ronde, listed in the order they appear in Appendix E.

\section{Stream}

Bear Cr.

Bear Cr.

Bear Cr.

Catherine Cr.

Catherine Cr.

Catherine $\mathrm{Cr}$.

Catherine Cr.

Grande Ronde R.

Grande Ronde R.

Grande Ronde R.

Grande Ronde R.

Grande Ronde R.

Grande Ronde R.

Grande Ronde R.

Grande Ronde R.

Grande Ronde R.

Grande Ronde R.

Grande Ronde R.

Grande Ronde R.

Grande Ronde R.

Grande Ronde R

Grande Ronde R.

Grande Ronde R.

Grande Ronde R.

Grande Ronde R.

Grande Ronde R.

Grande Ronde R.

Grande Ronde R.

Grande Ronde R.

Grande Ronde R.

Grande Ronde R.

Grande Ronde R.

Grande Ronde R.

Grande Ronde R.

Grande Ronde R.

Grande Ronde R.

Grande Ronde R.

Hurricane $\mathrm{Cr}$

\section{Reach}

Mouth - Chamberlain Ditch (RM 101.8 -104.7 from Snake R)

Chamberlain Ditch - Little Bear Cr (RM 104.7 -109.3 from Snake R)

Little Bear Cr - headwaters (RM 109.3-1 18.3 from Snake R)

Mouth - 10th Street Bridge in Union (RM 114.5 -146.0 from Snake R)

10th Street Bridge in Union - Little Catherine Cr (RM 146.0 -157.5 from Snake R)

Little Catherine Cr - forks (RM 157.5 -164.4 from Snake R)

South Fork Catherine Cr (RM 164.4 -166.8 from Snake R)

Mouth - Joseph Cr (RM 0.0- 3.7 from Snake R)

Joseph Cr - Buford Cr (RM 3.7- 26.2 from Snake R)

Buford Cr - Bear Cr (RM 26.2 -28.2 from Snake R)

Bear Cr - Menatchee Cr (RM 28.2 -34.8 from Snake R)

Menatchee Cr - Wenaha R (RM 34.8 -45.1 from Snake R)

Wenaha R - Courtney Cr (RM 45.1 -46.2 from Snake R)

Courtney $\mathrm{Cr}$ - Mud Cr(RM 46.2-51.4 from Snake R)

Mud Cr - Wallupa/Wildcat Cr (RM 51.4 -52.8 from Snake R)

Wallupa/Wildcat Cr - Grossman Cr (RM 52.8 -61.1 from Snake R)

Grossman Cr - Wallowa R (RM 61.1 -80.0 from Snake R)

Wallowa R - Lookingglass Cr (RM 80.0 -83.4 from Snake R)

Lookingglass Cr - Gordon Cr (RM 83.4 -94.2 from Snake R)

Gordon Cr - Phillips Cr (RM 94.2 -96.9 from Snake R)

Phillips Cr - Clark Cr (RM 96.9 -97.3 from Snake R)

Clark Cr - Indian Cr (RM 97.3 -99.3 from Snake R)

Indian Cr - Willow Cr (RM 99.3 -103.5 from Snake R)

Willow Cr - downstream end of State Ditch (RM 103.5 -114.5 from Snake R)

Downstream end of State Ditch - Upstream end of State Ditch (RM 114.5 -118.9 from Snake

Upstream end of State Ditch - I-84 crossing below Devil's Slide (RM 118.9 -128.4 from Snak 1-84 crossing below Devil's Slide - Five Points Cr (RM 128.4 -133.4 from Snake R)

Five Points Cr - Rock Cr (RM 133.4 -133.8 from Snake R)

Rock Cr - Whiskey Cr (RM 133.8 -136.6 from Snake R)

Whiskey Cr - Jordan Cr (RM 136.6 -139.1 from Snake R)

Jordan Cr - Beaver Cr (RM 139.1 -145.8 from Snake R)

Beaver Cr - Meadow Cr (RM 145.8 -147.0 from Snake R)

Meadow Cr - Fly Cr (RM 147.0 -151.3 from Snake R)

Fly $\mathrm{Cr}$ - head of canyon 1.3 miles downstream of Sheep Cr (RM 151.3 -159.4 from Snake R)

Head of canyon 1.3 miles downstream of Sheep Cr - LimberJim Cr (RM 159.4 -164.2 from Si

LimberJim Cr - Clear Cr (RM 164.2 -166.9 from Snake R)

Clear Cr - headwaiters (RM 166.9 -170.1 from Snake R)

Mouth - I ower Alderclone Ditch (RM 1210 a 124 o from Snake P) 
Reaches analyzed in the Grande Ronde, listed in the order they appear in Appendix E.

Stream
Lostine R.
Lostine R.
Lostine R.
Lostine R.
Lostine R.
Miriam R.
Miriam R.
Miriam R.
Miriam R.
Prairie Cr.
Prairie Cr.
Sheep Cr.
Sheep Cr.
Wallowa R.
Wallowa R.
Wallowa R.
Wallowa R.
Wallowa R.
Wallowa R.
Wallowa R.
Wallowa R.
Wallowa R.
Wallowa R.
Wenaha R.
Wenaha R.
Wenaha R.
Wenaha R.

Reach

ClearWater Ditch - Cross Country Ditch (RM 108.3 -110.8 from Snake R) Cross Country Ditch - Sheep Ridge Dam (RM 110.8 -115.0 from Snake R)

Sheep Ridge Dam - Silver Cr (RM 115.0 -120.0 from Snake R)

Silver $\mathrm{Cr}$ - Lake Cr (RM 120.0 -124.6 from Snake R)

Lake Cr - East Lostine R (RM 124.6 -131.7 from Snake R)

Mouth - Cougar Cr (RM 89.8 -99.4 from Snake R)

Cougar Cr - Little Miriam R (RM 99.4 -108.4 from Snake R)

Little Miriam R - splash dam site ( I m above Garwood Cr; RM 108.4 -119.7 from Snake R)

Splash dam site - falls (RM 119.7 -138.0 from Snake R)

Mouth - OK Gulch confluence (RM 126.8 -131.8 from Snake R)

OK Gulch fork (RM 131.8 -133.8 from Snake R)

Mouth - Chicken Cr (RM 164.2 -165.7 from Snake R)

Chicken $\mathrm{Cr}$ - headwaters (RM 165.7 -173.7 from Snake R)

Mouth - Miriam R (RM 80.0 -89.8 from Snake R)

Miriam R - Deer Cr (RM 89.8 -90.8 from Snake R)

Deer $\mathrm{Cr}$ - Dry $\mathrm{Cr}(\mathrm{RM}$ 90.8 -98.3 from Snake R)

Dry Cr - Bear Cr (RM 98.3 -101.8 from Snake R)

Bear Cr - Whiskey Cr (RM 101.8 -103.9 from Snake R)

Whiskey Cr - Lostine R (RM 103.9 -105.8 from Snake R)

Lostine R - Parsnip Cr (RM 105.8 -109.0 from Snake R)

Parsnip Cr - Hurricane Cr (RM 109.0 -121.9 from Snake R)

Hurricane Cr - Lower Alderslope Ditch (RM 121.9 -126.8 from Snake R)

Lower Alderslope Ditch - Wallowa Lake (RM 126.8 -130.8 from Snake R)

Mouth - Crooked Cr (RM 45.1 -51.8 from Snake R)

Crooked Cr -Fairview Cr(RM 51.8 -55.3 from Snake R)

Fairview Cr - forks (RM 55.3 -65.9 from Snake R)

South Fork Wenaha R (RM 65.9 -72.4 from Snake R)

\section{Benefit Category}

for Vacant

Life History

Pathways

F

B

E

F

F

B

D

E 
Stream: Bear Cr.

Reach: Mouth - Chamberlain Ditch (RM 101.8 -104.7 from Snake R)

\begin{tabular}{|c|c|c|c|c|c|c|c|c|}
\hline \multicolumn{4}{|c|}{ Restoration benefit category: } & & \multicolumn{3}{|c|}{ |Chinook usage score: } & 48.3 \\
\hline \multirow[b]{3}{*}{ Life stage } & \multirow[b]{3}{*}{ Months } & \multirow{2}{*}{\multicolumn{4}{|c|}{$\begin{array}{l}\text { No. vacant life history pathways } \\
\text { by abundance level }\end{array}$}} & \multicolumn{2}{|c|}{ Key habitat change } & \multirow{3}{*}{$\begin{array}{c}\text { Habilat } \\
\text { productivity } \\
\text { loss score }\end{array}$} \\
\hline & & & & & & \multirow{2}{*}{$\begin{array}{l}\text { Patient } \\
\text { quantity }\end{array}$} & \multirow[b]{2}{*}{$\%$ change } & \\
\hline & & Total no. & 1 & 2 & 3 & & & \\
\hline 1 & Apr-Aug & 56 & 56 & 0 & 0 & 0.8 & -79.6 & -528.0 \\
\hline $1,2,3$ & Aug-Oct & 90 & 90 & 0 & 0 & 0.1 & 99.0 & -8456 \\
\hline 3 & Oct-Mar & 180 & 180 & 0 & 0 & 0.0 & -100.0 & -59.7 \\
\hline 3,4 & Mar-May & 44 & 44 & 0 & 0 & 2.8 & .78 .7 & -27.9 \\
\hline 5 & May-Öct & 94 & 94 & o" & 0 & 0.0 & -100.0 & -664.5 \\
\hline 6 & Oct-Mar & 19 & 19 & 0 & 0 & 2.4 & -75.7 & -61.1 \\
\hline 7 & Mar-Jun & 1 & 1 & 0 & 0 & 13.8 & -16.7 & 1.2 \\
\hline
\end{tabular}

\begin{tabular}{|c|c|c|c|c|c|c|c|c|c|c|c|c|c|}
\hline \multirow[b]{2}{*}{ Life stage } & \multirow[b]{2}{*}{ Months } & \multicolumn{12}{|c|}{ Highest productivity impact attributes (\% change in impact from template) } \\
\hline & & Att 1 & $\%$ change & Att 2 & $\%$ change & Att 3 & $\%$ change & Att4 & $\%$ change & Att 5 & $\%$ change & Att 6 & $\%$ change \\
\hline 1 & Apr-Aug & FLOW & 97.5 & HABD & 69.1 & TEMP & 48.7. & WITH & 47.4 & & & & \\
\hline $.1,2,3$ & Aug-Ött & FLOWW" & 100,0 & $H A B D$ & $50.0^{\circ}$ & TEMP & 50.0 & ẄITH & 41.7 & & & & \\
\hline 3 & Ott-Mar & FLOW & 100.0 & TEMP & 50.0 & WITH & 250 & CSTAB & 125 & HABD & 12.5 & PATHO & 12.5 \\
\hline 3.4 & Mar-May, & FLO' & 100 & "Н̈ABD" & 500 & PRED & 50.0 & Ẅ̈іт̈н & 500 & PATHO & ???! & TEM. & 25.0 \\
\hline 5 & May-Oct & FLOW & 1000 & $\mathrm{HABD}$ & 500 & PRED & 50.0 & ẄITḦ & 50.0 & PATHO & 25.0 & & \\
\hline 7 & $\begin{array}{l}\text { Ott-Mar } \\
\text { Mar-Jun }\end{array}$ & "TËM"P"' & $50.0^{\circ}$ & "HABD" & 25.0 & "PATHO' & 25.0 & & & & & & \\
\hline
\end{tabular}

Abundance lovel refers to estimated relative abundance of spawners that spawned in the natal stream reaches that populated the life history pathways using the reach in this table: Level 1: $\quad$ Low to average (relative to other reaches) abundance

Level 2 High (relative to other reaches) abundance

Level 3: Exceptionally high (relative to other reaches) abundance

Life stages: 1- prespawner; 2- spawner; 3- incubation; 4- fry colonization; 5- summer rearing; 6- overwintering; 7- yearling presmolt/smolt

\begin{tabular}{|c|c|c|c|}
\hline $\begin{array}{r}\text { Attributes; } \text { CHEM - } \\
\text { CSTAB - } \\
\text { COMP - } \\
\text { FLOW - } \\
\text { HABD - }\end{array}$ & $\begin{array}{l}\text { chemicals } \\
\text { channel stability } \\
\text { competition (with other species) } \\
\text { flow } \\
\text { habitat diversity }\end{array}$ & $\begin{array}{l}\text { NUTLD - nutrient load } \\
\text { OBST - obstructions } \\
\text { OXYG - dissolved oxygen } \\
\text { PATHO - pathogens } \\
\text { PRED - predators (includes fishing-related losses) }\end{array}$ & $\begin{array}{l}\text { RIPCN - riparian condition } \\
\text { SEOLD - sediment toad } \\
\text { TEMP - } \quad \text { water temperature } \\
\text { WITH - } \quad \text { water withdrawals }\end{array}$ \\
\hline
\end{tabular}

"NA" indicates that no tife history pathway meeting the criteria used in defining these pathways was present.

Blank cells under attributes indicate no additional attributes with negative effects on productivity were evident compared to those present in the template. 
Stream Reach Summary for Vacant Spring Chinook Salmon Life Histories

Stream: Bear Cr.

Reach: Chamberlain Ditch - Little Bear Cr (RM 104.7 -109.3 from Snake R)

\begin{tabular}{|ll|ll|l|}
\hline Restoration benefit category: & F & Chinook usage score: 4.0 & Habitat productivity loss score: \\
\hline
\end{tabular}

\begin{tabular}{|c|c|c|c|c|c|c|c|c|}
\hline \multirow[b]{3}{*}{ Life stage } & \multirow[b]{3}{*}{ Months } & \multirow{2}{*}{\multicolumn{4}{|c|}{$\begin{array}{l}\text { No. vacant life history pathways } \\
\text { by abundance level }\end{array}$}} & \multicolumn{2}{|c|}{ Key habitat change } & \multirow{3}{*}{$\begin{array}{c}\text { Habitat } \\
\text { productivity } \\
\text { loss score }\end{array}$} \\
\hline & & & & & & \multirow{2}{*}{$\begin{array}{l}\text { Patient } \\
\text { quantity }\end{array}$} & \multirow[b]{2}{*}{$\%$ change } & \\
\hline & & Total no. & 1 & 2 & 3 & & & \\
\hline 1 & Apr-Aug & 7 & 7 & 0 & 0 & 5.4 & 7.8 & 0.0 \\
\hline $1,2,3$ & Aug-Oct & 10 & 10 & 0 & 0 & 2.7 & -27.0 & 5.1 \\
\hline 3 & Oct-Mar & 20 & 20 & 0 & 0 & 0.7 & -83.2 & 0.0 \\
\hline 3,4 & Mar-May & 3 & 3 & 0 & 0 & 2.8 & -36.8 & -0.4 \\
\hline 5 & May-Öct & $\mathrm{NA}$ & $\mathrm{NA}$ & $\mathrm{NA}$ & $\mathrm{NA}$ & $\mathrm{NA}$ & $\mathrm{NA}$ & $\mathrm{NA}$ \\
\hline 6 & Oct-Mar & NA & NA & NA & $\mathrm{NA}$ & NA & $\mathrm{NA}$ & NA \\
\hline 7 & Mar-Jun & NA & NA & NA & NA & NA & $N A$ & NA \\
\hline
\end{tabular}

\begin{tabular}{|c|c|c|c|c|c|c|c|c|c|c|c|c|c|}
\hline \multirow[b]{2}{*}{ Life stage } & \multirow[b]{2}{*}{ Months } & \multicolumn{12}{|c|}{ Highest productivity impact attributes (\% change in impact from template) } \\
\hline & & $\overline{\text { Att } 1}$ & $\%$ change & Art 2 & $\%$ change & Att 3 & $\%$ change & Art 4 & $\%$ change & Art 5 & $\%$ change & Att 6 & $\%$ change \\
\hline 1 & Apr-Aug & HABD & 17.8 & & & & & & & & & & \\
\hline $1,2,3$ & Äug-Ött & HABD & 18.1 & & & & & & & & & & \\
\hline 3 & Oct-Mar & CSTAB & 25.0 & HABD & “250” & PATHO & 25.0 & RIPCN & 25.0 & & & & \\
\hline 34 & Mär-May & "CSTAB & 25.0 & HABD" & 250 & PATHO & 25.0 & RIPCN & $25.0^{\circ}$ & & & & \\
\hline 5 & May-Ott & $\mathrm{NA}$ & NA & NA & NAA & NAA & $\mathrm{NA}$ & $\mathrm{NA}$ & NA & $\mathrm{NA}$ & NA & $\mathrm{NA}$ & NA \\
\hline 6 & Ött-Mar & $\mathrm{NA}$ & $\ddot{N A}$ & $\mathrm{NA}$ & NÄ & $\mathrm{NA}$ & $\mathrm{NA}$ & $\mathrm{NA}$ & $\mathrm{NA}$ & $\mathrm{NA}$ & $\mathrm{NA}$ & $\mathrm{NA}$ & $\mathrm{NA}$ \\
\hline 7 & Mar-Jun & NA & $\mathrm{N} \quad \mathrm{A}$ & NA & 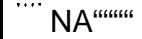 & NA & $\mathrm{NA}$ & $\mathrm{NA}$ & $\mathrm{NA}$ & $\mathrm{NA}$ & $\mathrm{NA}$ & NA & NA \\
\hline
\end{tabular}

Abundance /eve/ refers to estimated relative abundance of spawners that spawned in the natal stream reaches that populated the life history pathways using the reach in this table: Level 1: Low to average (relative to other reaches) abundance

Level 2: $\quad$ High (relative to other reaches) abundance

Level 3: Exceptionally high (relative to other reaches) abundance

Life stages: 1- prespawner; 2- spawner; 3- incubation; 4- fry colonization; 5- summer rearing; 6- overwintering; 7-yearling presmolt/smolt

$\begin{array}{clc}\text { Attributes: CHEM - chemicals } & \text { NUTLD - nutrient load } & \text { RIPCN - riparian condition } \\ \text { CSTAB - channel stability } & \text { OBST - obstructions } & \text { SEDLD - sediment load } \\ \text { COMP - competition (with other species) } & \text { OXYG - dissolved oxygen } & \text { TEMP - water temperature } \\ \text { FLOW - flow } & \text { PATHO - pathogens } & \text { WITH - water withdrawals } \\ \text { HABD - habitat diversity } & \text { PRED - predators (includes fishing-related losses) }\end{array}$

"NA" indicates that no life history pathway meeting the criteria used in defining these pathways was present.

Blank cells under attributes indicate no additional attributes with negative effects on productivity were evident compared to those present in the template. 
Stream: Bear $\mathrm{Cr}$.

Reach: Little Bear $\mathbf{C r}$ - headwaters (RM 109.3 -118.3 from Snake R)

\begin{tabular}{|ll|l|l|l|}
\hline Restoration benefit category: & F & Chinook usage score: & 4.8 & Habitat productivity loss score: \\
\hline
\end{tabular}

\begin{tabular}{|c|c|c|c|c|c|c|c|c|}
\hline \multirow[b]{3}{*}{ Life stage } & \multirow[b]{3}{*}{ Months } & \multirow{2}{*}{\multicolumn{4}{|c|}{$\begin{array}{c}\text { No. vacant life history pathways } \\
\text { by abundance level }\end{array}$}} & \multicolumn{2}{|c|}{ Key habitat change } & \multirow{3}{*}{\begin{tabular}{|c|} 
Habitat \\
productivity \\
loss score
\end{tabular}} \\
\hline & & & & & & \multirow{2}{*}{$\begin{array}{l}\text { Patient } \\
\text { quantity }\end{array}$} & \multirow[b]{2}{*}{$\%$ change } & \\
\hline & & Total no. & 1 & 2 & 3 & & & \\
\hline 1 & Apr-Aug & 6 & 6 & 0 & 0 & 38 & 0.0 & 1.2 \\
\hline $1,2,3$ & Aug-Ött & 10 & 10 & 0 & 0 & 3.4 & $0.0^{\circ}$ & 6.6 \\
\hline 3 & Ött-M̄ar & 20 & 20 & 0 & 0 & 3.5 & 0.0 & 0.0 \\
\hline 34 & Mar-May & $\ddot{2}$ & 2 & 0 & 0 & 3.9 & 0.0 & 0.9 \\
\hline 5 & May-Ôtt & $\mathrm{NÄ}$ & $\mathrm{NA}$ & ṆA" & Nä" & $\mathrm{NÄ}$ & Nä & NÄ \\
\hline 6 & Ott-Mar & NA & NA & NA & $\mathrm{NA}$ & NA & $\mathrm{NA}$ & $\mathrm{NA}$ \\
\hline 7 & Mar-Jun & $\mathrm{NA}$ & NA & $\mathrm{NA}$ & $\mathrm{NA}$ & NA & NA & NA \\
\hline
\end{tabular}

\begin{tabular}{|c|c|c|c|c|c|c|c|c|c|c|c|c|c|}
\hline \multirow[b]{2}{*}{ Life stage } & \multirow[b]{2}{*}{ Months } & \multicolumn{12}{|c|}{ Highesl pro sctivity impact attributes (\% change in impact from template) } \\
\hline & & Alt 1 & $\%$ change & Att 2 & $\%$ change & Att 3 & \% change & Att 4 & $\%$ change & Att 5 & $\%$ change & Att 6 & $\%$ change \\
\hline 1 & Apr-Aug & HABD & 22.2 & & & & & & & & & & \\
\hline $1,2,3$ & Aug-Oct & HABD & 8.3 & & & & & & & & & & \\
\hline 3 & Oct-Mar & PATHO & 12.5 & & & & & & & & & & \\
\hline 3,4 & Mar-May & PATHO & 12.5 & & & & & & & & & & \\
\hline 5 & May-Oct & NA & NA & NA & $\mathrm{NA}$ & NA & $\mathrm{NA}$ & NA & NA & $N A$ & $\mathrm{NA}$ & NA & NA \\
\hline 6 & Oct-Mar & NA & $\mathrm{NA}$ & $\mathrm{NA}$ & $\mathrm{NA}$ & NA & NA & NA & $\mathrm{NA}$ & $\mathrm{NA}$ & $\mathrm{NA}$ & NA & $\mathrm{NA}$ \\
\hline 7 & Mar-Jun & NA & NA & $\mathrm{NA}$ & $\mathrm{NA}$ & $N A$ & $\mathrm{NA}$ & $\mathrm{NA}$ & $\mathrm{NA}$ & $\mathrm{NA}$ & $\mathrm{NA}$ & $\mathrm{NA}$ & $\mathrm{NA}$ \\
\hline
\end{tabular}

Abundance lovel refers to estimated relative abundance of spawners that spawned in the natal stream reaches that populated the life history pathways using the reach in this table: Level 1: Low to average (relative to other reaches) abundance

Level 2 High (relative to other reaches) abundance

Level 3 Exceptionally high (relative to other reaches) abundance

Life stages: 1-prespawner; 2- spawner; 3- incubation; 4- fry colonization; 5- summer rearing; 6- overwintering; 7- yearlinpresmolt/smolt

Attributes: CHEM - chemicals CSTAB - channel stability

COMP - competition (with other species)

FLOW - flow

HABD - habitat diversity
NUTLD - nutrient load

OBST - obstructions

OXYG - dissolved oxygen

PATHO - pathogens

PRED - predators (includes fishing-related losses)
RIPCN - riparian condition SEDLD - sediment load

TEMP - water temperature

WITH - water withdrawals

"NA" indicates that no life history pathway meeting the criteria used in defining these pathways was present,

Blank cells under attributes indicate no additional attributes with negative effects on productivity were evident compared to those present in the template. 
Stream: Chesnimnus Cr.

Reach: Confluence with Crow $\mathrm{Cr}$ - South Fork (RM 52.7 -77.7 from Snake R)

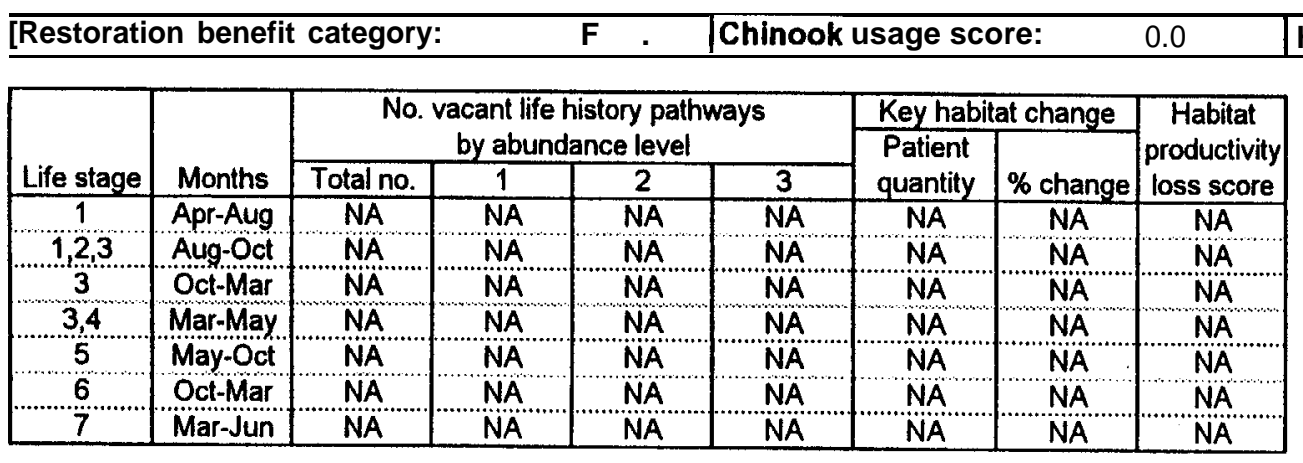

\begin{tabular}{|c|c|c|c|c|c|c|c|c|c|c|c|c|c|}
\hline \multirow[b]{2}{*}{ Life stage } & \multirow[b]{2}{*}{ Months } & & & & \multicolumn{9}{|c|}{ ighest productivity impact attributes (\% change in impact from template) } \\
\hline & & Att 1 & \% change & Att 2 & $\%$ change & Att 3 & $\%$ change & Att 4 & $\%$ change & Att 5 & $\%$ change & Att 6 & $\%$ change \\
\hline 1 & Apr-Aug & NA & NA & NA & NA & NA & NA & NA & $\mathrm{NA}$ & NA & NA & NA & NA \\
\hline $1,2,3$ & Aug-Oct & NA & $\mathrm{NA}$ & $\mathrm{NA}$ & NA & $\mathrm{NA}$ & $\mathrm{NA}$ & $\mathrm{NA}$ & $\mathrm{NA}$ & $\mathrm{NA}$ & NA & $\mathrm{NA}$ & $\mathrm{NA}$ \\
\hline 3 & Oct-Mar & $\mathrm{NA}$ & NA & NA & $\mathrm{NA}$ & NA & $\mathrm{NA}$ & NA & $\mathrm{NA}$ & $\mathrm{NA}$ & $N A$ & $\mathrm{NA}$ & NA \\
\hline 3,4 & Mar-May & $\mathrm{NA}$ & $\mathrm{NA}$ & $\mathrm{NA}$ & $\mathrm{NA}$ & $\mathrm{NA}$ & $\mathrm{NA}$ & $\mathrm{NA}$ & $\mathrm{NA}$ & $\mathrm{NA}$ & $\mathrm{NA}$ & $\mathrm{NA}$ & $\mathrm{NA}$ \\
\hline 5 & May-Oct & NA & NA & NA & NA & $\mathrm{NA}$ & NA & $N A$ & $\mathrm{NA}$ & NA & NA & $\mathrm{NA}$ & $\mathrm{NA}$ \\
\hline 6 & Oct-Mar & $\mathrm{NA}$ & $\mathrm{NA}$ & $\mathrm{NA}$ & NA & $\mathrm{NA}$ & $\mathrm{NA}$ & NA & $\mathrm{NA}$ & NA & NA & $\mathrm{NA}$ & $\mathrm{NA}$ \\
\hline 7 & Mar-Jun & $\mathrm{NA}$ & $\mathrm{NA}$ & $\mathrm{NA}$ & $\mathrm{NA}$ & NA & $\mathrm{NA}$ & NA & $\mathrm{NA}$ & NA & NA & $N A$ & $\mathrm{NA}$ \\
\hline
\end{tabular}

Abundance/eve/refers to estimated relative abundance of spawners that spawned in the natal stream reaches that populated the life history pathways using the reach in this table: Level 1: Low to average (relative to other reaches) abundance

Level 2: $\quad$ High (relative to other reaches) abundance

Level 3: Exceptionally high (relative to other reaches) abundance

Life stages: 1- prespawner; 2- spawner; 3- incubation; 4- fry colonization; 5- summer rearing; 6 - overwintering; 7- yearling presmolt/smelt

Attributes: CHEM - chemicals

CSTAB - channel stability

COMP - competition (with other species)

FLOW - flow

HABD - habitat diversity
NUTLD - nutrient load

OBST - obstructions

OXYG - dissolved oxygen

PATHO - pathogens

PRED - predators (includes fishing-related losses)
RIPCN - riparian condition

SEDLD - sediment load

TEMP - water temperature

WITH - water withdrawals

"NA" indicates that no life history pathway meeting the criteria used in defining these pathways was present.

Blank cells under attributes indicate no additional attributes with negative effects on productivity were evident compared to those present in the template. 
Stream: Catherine $\mathrm{Cr}$.

Reach: Mouth - 10th Street Bridge in Union (RM 114.5 -146.0 from Snake R)

\section{Restoration benefit category:}

A

Chinook usage score:

158.0

Habitat productivity loss score:

\begin{tabular}{|c|c|c|c|c|c|c|c|c|}
\hline \multirow[b]{3}{*}{ Life stage } & \multirow[b]{3}{*}{ Menths } & \multirow{2}{*}{\multicolumn{4}{|c|}{$\begin{array}{c}\text { No. vacant life history pathways } \\
\text { by abundance level }\end{array}$}} & \multicolumn{2}{|c|}{ Key habitat change } & \multirow{3}{*}{$\begin{array}{c}\text { Habitat } \\
\text { productivity } \\
\text { loss score }\end{array}$} \\
\hline & & & & & & \multirow{2}{*}{$\begin{array}{l}\text { Patient } \\
\text { quantity }\end{array}$} & \multirow[b]{2}{*}{$\%$ change } & \\
\hline & & Total no. & 1. & 2 & 3 & & & \\
\hline 1 & Apr-Aug & 84 & 1 & 74 & 9 & 8.6 & -77.4 & -486.3 \\
\hline $1,2,3$ & Aug-Oct & 90 & 0 & 80 & 10 & 1.0 & -75.6 & -428.4 \\
\hline 3 & Oct-Mar & $180^{\circ}$ & 0 & 160 & 20 & 0.7 & -82.8 & -820.4 \\
\hline 3,4 & Mar-May & 101 & 0 & 88 & 13 & 6.5 & -76.5 & -789.8 \\
\hline 5 & May-Oct & 352 & 1 & 311 & 40 & 1.11 & -95.7 & -18060 \\
\hline 6 & Oct-Mar & 76 & 0 & 76 & 0 & 4.3 & -72.0 & -252.4 \\
\hline 7 & Mar-Jun & 5 & 0 & 5 & 0 & 26.2 & -3.3 & 7.3 \\
\hline
\end{tabular}

\begin{tabular}{|c|c|c|c|c|c|c|c|c|c|c|c|c|c|}
\hline \multirow[b]{2}{*}{ Life stage } & \multirow[b]{2}{*}{ Months } & \multicolumn{12}{|c|}{ Highest productivity impact attributes (\% change in impact from template) } \\
\hline & & Att 1 & $\%$ change & Att 2 & $\%$ change & Att 3 & $\%$ change & Att 4 & $\%$ change & Att 5 & $\%$ change & Att 6 & $\%$ change \\
\hline 1 & Apr-Aug & FLOW & 62.5 & TEMP & 62.5 & RIPCN & 50.0 & HABD & 49.7 & CSTAB & 25.0 & SEDLD & 25.0 \\
\hline $1,2,3$ & Aug-Oct & TEMP & 1000 & FLOW & 75.0 & HABD & 75.0 & CSTAB & 500 & RIPCN & 50.0 & SEDLO & 50.0 \\
\hline 3 & Oct-Mar & TEMP & 100.0 & FLOW & 75.0 & HABD & 750 & CSTAB & 50.0 & RIPCN & 50.0 & SEDLO & 500 \\
\hline 3,4 & Mar-May & TEMP & 100.0 & FLOW & 75.0 & HABD & 75.0 & CSTAB & 50.0 & RIPCN & 50.0 & SEDLO & 50.0 \\
\hline 5 & May-Oct & HABD & 75.0 & SEDLD & 75.0 & FLOW & 74.9 & RIPCN & 500 & СOMP & 25.0 & OBST & 25.0 \\
\hline 6 & Oct-Mar & HABD & 75.0 & SEDLD & 75.0 & FLOW & 500 & RIPCN & 50.0 & COMP & 25.0 & OBST & 25.0 \\
\hline 7 & Mar-Jun & OBST & 25.0 & WITH & 25.0 & & & & & & & & \\
\hline
\end{tabular}

Abundance level refers to estimated relative abundance of spawners that spawned in the natal stream reaches that populated the life history pathways using the reach in this table: Level 1: Low to average (relative to other reaches) abundance

Level 2: $\quad$ High (relative to other reaches) abundance

Level 3: Exceptionally high (relative to other reaches) abundance

Life stages: 1- prespawner; 2- spawner; 3- incubation; 4- fry colonization; 5- summer rearing; 6- overwintering; 7- yearling presmolt/smolt

Attributes: CHEM - chemicals

CSTAB - channel stability

COMP - competition (with other species)

FLOW - flow

HABD - habitat diversity
NUTLD - nutrient load

oBST - obstructions

OXYG - dissolved oxygen

PATHO - pathogens

PRED - predators (includes fishing-related losses)
RIPCN - riparian condition

SEDLD - sediment load

TEMP - water temperature

WITH - water withdrawals

"NA" indicates that no life history pathway meeting the criteria used in defining these pathways was present.

Blank cells under attributes indicate no additional attributes with negative effects on productivity were evident compared to those present in the template. 
Stream: Catherine Cr.

Reach: 10th Street Bridge in Union - Little Catherine Cr (RM 146.0 -157.5 from Snake R)

\begin{tabular}{|c|c|c|c|c|c|c|c|c|c|c|c|c|c|}
\hline \multicolumn{4}{|c|}{ Restoration benefit category: } & $B$ & \multicolumn{3}{|c|}{ Chinook usage score: } & 78.7 & \multicolumn{4}{|c|}{ Habitat productivity loss score: } & -1986.3 \\
\hline \multirow[b]{3}{*}{ Life stage } & \multirow[b]{3}{*}{ Months } & \multirow{2}{*}{\multicolumn{4}{|c|}{$\begin{array}{l}\text { No. vacant life history pathways } \\
\text { by abundance level }\end{array}$}} & \multicolumn{2}{|c|}{ Key habitat change } & \multirow{3}{*}{$\begin{array}{l}\text { Habitat } \\
\text { productivity } \\
\text { loss score }\end{array}$} & & & & & \\
\hline & & & & & & \multirow{2}{*}{$\begin{array}{l}\text { Patient } \\
\text { quantity }\end{array}$} & \multirow[b]{2}{*}{$\%$ change } & & & & & & \\
\hline & & Total no. & 1 & 2 & 3 & & & & & & & & \\
\hline 1 & Apr-Aug & 56 & 1 & 49 & 6 & 4.8 & -59.2 & -246.4 & & & & & \\
\hline $1,2,3$ & Aug-Oct & 90 & 0 & 80 & 10 & 3.0 & -58.2 & -765.8 & & & & & \\
\hline 3 & Oct-Mar & 180 & 0 & 160 & 20 & 3.4 & -55.9 & -398.5 & & & & & \\
\hline 3,4 & Mar-May & 54 & 5 & 46 & 3 & 5.9 & -53.4 & $-275,11$ & & & & & \\
\hline 5 & May-Oct & 114 & 20 & 94 & 0 & 5.1 & $\because$ & -2428 & & & & & \\
\hline 6 & Oct-Mar & 19 & 0 & 19 & 0 & 3.4 & $\therefore$ & -5811 & & & & & \\
\hline 7 & Mar-Jun & 1 & 0 & 1 & 0 & 18.9 & 17.6 & 0.5 & & & & & \\
\hline \multirow[b]{2}{*}{ Life stage } & \multirow[b]{2}{*}{ Months } & \multicolumn{12}{|c|}{ lighest productivity impact attributes (\% change in impact from template) } \\
\hline & & $\overline{\text { Att } 1}$ & $\%$ change & Atf 2 & $\%$ change & Att 3 & $\%$ change & Att 4 & $\%$ change & Att 5 & $\%$ change & Atf 6 & $\%$ change \\
\hline 1 & Apr-Aug & TEMP & 75.0 & FLOW & 50.0 & RIPCN & 50.0 & HABD & 48.9 & OBST & 48.9 & SEDLD & 16.7 \\
\hline 123 & Aug-Ött & TEMP & 75.0 & "FLOOW" & 50.0 & "RIPCN" & 50,0 & SÉDEL'D' & $50^{\circ} 0^{\circ}$ & CSTAB & 25.0 & HABD" & 25.0 \\
\hline 3 & Ott-Mar & TEMP & 75.0 & "FLOW & “" 50.0" & RIPCN & $50 \prime 0$ & SEDLD & $50.0^{\prime}$ & "CSTAB & “ 37.5 & HABD & 25.0 \\
\hline 34 & Mar-May. & ṪĖंMṔ. & 750 & FLOW' & $50.0^{\circ}$ & HABD & 50.0 & RIPCN & $50.0^{\circ}$ & "̈̈ітї̈ & 500 & CSTAB & 37.5 \\
\hline 5 & May-Ott & TEMP & 75.0 & FLOW & 50.0 & HABD & 50,0 & RIPCN & 50.0 & WITH & 500 & РATHO & 250 \\
\hline $\begin{array}{ll}6 \\
7\end{array}$ & $\begin{array}{l}\text { Ott-Mar } \\
\text { Mar-Jun }\end{array}$ & $\begin{array}{l}\text { HABD" } \\
\text { WITH }\end{array}$ & $\begin{array}{l}50.0 \\
25.0\end{array}$ & RIPCN & 50.0 & "SEDLD" & 50,0 & CSTAB & $25.0^{\circ}$ & РATHO & 25.0 & "WITH" & 25.0 \\
\hline$I$ & & Vviाா & 20.0 & & & & & & & & & & \\
\hline
\end{tabular}

Abundance level refers to estimated relative abundance of spawners that spawned in the natal stream reaches that populated the life history pathways using the reach in this table:

Level 1: $\quad$ Low to average (relative to other reaches) abundance

Level 2 High (relative to other reaches) abundance

Level 3: Exceptionally high (relative to other reaches) abundance

Life stages: 1- prespawner; 2- spawner; 3- incubation; 4- fry colonization; 5- summer rearing; 6- overwintering; 7- yearling presmolt/smoll

Attributes: CHEM - chemicals CSTAB - channel stability

COMP - competition (with other species)

FLOW - ftOW

HABD - habitat diversity
NUTLD - nutrient load

OBST - obstructions

OXYG - dissolved oxygen

PATHO - pathogens

PRED - predators (includes fishing-related losses)
RIPCN - riparian condition

SEDLD - sediment load

TEMP - $\quad$ water temperature

WITH - water withdrawals

"NA" indicates that no life history pathway meeting the criteria used in defining these pathways was present

Blank cells under attributes indicate no additional attributes with negative effects on productivity were evident compared to those present in the template. 
Stream: Catherine Cr.

Reach: Little Catherine $\mathrm{Cr}$ - forks (RM 157.5 -164.4 from Snake R)

\begin{tabular}{|ll|ll|lll|}
\hline Restoration benefit category: & F & Chinook usage score: & 7.3 & Habitat productivity loss score: & -47.7 \\
\hline
\end{tabular}

\begin{tabular}{|c|c|c|c|c|c|c|c|c|}
\hline \multirow[b]{3}{*}{ Life stage } & \multirow[b]{3}{*}{ Months } & \multirow{2}{*}{\multicolumn{4}{|c|}{$\begin{array}{c}\text { No. vacant life history pathways } \\
\text { by abundance level }\end{array}$}} & \multicolumn{2}{|c|}{ Key habitat change } & \multirow{3}{*}{\begin{tabular}{|c|} 
Habitat \\
productivity \\
loss score
\end{tabular}} \\
\hline & & & & & & \multirow{2}{*}{$\begin{array}{l}\text { Patient } \\
\text { quantity }\end{array}$} & \multirow[b]{2}{*}{$\%$ change } & \\
\hline & & Total no. & 1 & 2 & 3 & & & \\
\hline 1 & Apr-Aug & 7 & 1 & 6 & 0 & 4.9 & -54.3 & -8.7 \\
\hline $1,2,3$ & Aug-Ott & 10 & 0 & 10 & 0 & 6.3 & -180 & -1.77 \\
\hline 3 & Ott-Mar & 20 & 0 & 20 & 0 & 8.3 & -3.6 & -18.1 \\
\hline 34 & Mar-May & 3 & 1 & 2 & 0 & 7.9 & $-30,0$ & -3.2 \\
\hline ' 5 & May-Ött & $\mathrm{NA}$ & $\mathrm{NA}$ & NÄ & NA" & NÄ & $\mathrm{NA}$ & NA \\
\hline 6 & Ott-Mar & NA & $\mathrm{NA}$ & $\mathrm{NA}$ & $\mathrm{NA}$ & NA & $\because \mathrm{NA}$ & NA \\
\hline 7 & Mar-Jun & $\mathrm{NA}$ & NA" & $\mathrm{NA}$ & NA" & $\mathrm{NA}$ & NA & NA \\
\hline
\end{tabular}

\begin{tabular}{|c|c|c|c|c|c|c|c|c|c|c|c|c|c|}
\hline \multirow[b]{2}{*}{ Life stage } & \multirow[b]{2}{*}{ Months } & \multicolumn{12}{|c|}{ Highest productivity impact attributes (\% change in impact from template) } \\
\hline & & Att 1 & $\%$ change & Att 2 & $\%$ change & Att 3 & $\%$ change & Atf 4 & $\%$ change & Att 5 & $\%$ change & Aft 6 & $\%$ change \\
\hline 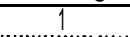 & Apr-Aug & SEDLD & 25.0 & TEMP & 25.0 & HABD & 20.8 & CSTAB & 12.5 & & & & \\
\hline $1,2,3$ & Aug-Ött & "SEEOELD" & 50.0 & CSTÄB & 25.0 & HABD & 25.0 & TEMP & 25,0 & & & & \\
\hline 3 & Ocl-Mar & SEDLD & 50.0 & CSTAB & 25.0 & FLOW & 25.0 & PATHO & 25.0 & TEMP & 25.0 & “ & " \\
\hline 34 & Mar-May & SEOLLD & 500 & CSTAB & 25.0 & FLOW & 25.0 & "PATHÖO" & 25.0 & T̈ËM̈P̈ & 25.0. & & \\
\hline 5 & May-Ott & NA & NA & $\mathrm{NA}$ & $\mathrm{NA}$ & NA & NÁ & $\mathrm{NA}$ & $\mathrm{NA}$ & $\mathrm{NA}$ & NA & $\dot{N} \dot{A}$ & $\mathrm{NA}$ \\
\hline 6 & Oct-Mar & NA & "NA" & N̈̈̈ & $\mathrm{NA}$ & NA & NA & Ñ̈̈ & Nä & $\mathrm{NA}$ & $\mathrm{NA}$ & $\mathrm{NA}$ & $\mathrm{NA}$ \\
\hline 7 & Mar-Jun & NA & NA & "NA & $\mathrm{NA}$ & $\mathrm{NA}$ & $\mathrm{NA}$ & $\mathrm{NA}$ & NA & $\mathrm{NA}$ & NA & $\mathrm{NA}$ & $\mathrm{NA}$ \\
\hline
\end{tabular}

Abundance /eve/ refers to estimated relative abundance of spawners that spawned in the natal stream reaches that populated the life history pathways using the reach in this table: Level 1: $\quad$ Low to average (relative to other reaches) abundance

Level 2: High (relative to other reaches) abundance

Level 3: Exceptionally high (relative to other reaches) abundance

Life stages: 1- prespawner; 2- spawner; 3- incubation; 4- fry colonization; 5- summer rearing; 6-overwintering; 7- yearling presmolt/smolt

Attributes: CHEM - chemicals CSTAB - channel stability

COMP - competition (with other species)

FLOW - flow

HABD - habitat diversity
NUTLD - nutrient load

OBST - obstructions

OXYG - dissolved oxygen

PATHO - pathogens

PRED - predators (includes fishing-related losses)
RIPCN - riparian condition

SEDLD - sediment load

TEMP - $\quad$ water temperature

WITH - water withdrawals

"NA" indicates that no life history pathway meeting the criteria used in defining these pathways was present,

Blank cells under attributes indicate no additional attributes with negative effects on productivity were evident compared to those present in the template. 
Stream: Catherine Cr.

Reach: South Fork Catherine $\mathrm{Cr}$ (RM 164.4 -166.8 from Snake R)

Restoration benefit category: \begin{tabular}{l|l}
$\mathrm{F}$ & Chinook usage score:
\end{tabular} \begin{tabular}{l|l}
\hline 3.8 & Habitat productivity loss score:
\end{tabular} $-42.4$

\begin{tabular}{|c|c|c|c|c|c|c|c|c|}
\hline \multirow[b]{3}{*}{ Life stage } & \multirow[b]{3}{*}{ Months } & \multirow{2}{*}{\multicolumn{4}{|c|}{$\begin{array}{l}\text { No. vacant life history pathways } \\
\text { by abundance level }\end{array}$}} & \multicolumn{2}{|c|}{ Key habitat change } & \multirow{3}{*}{$\begin{array}{c}\text { Habilatt } \\
\text { productivity } \\
\text { loss score }\end{array}$} \\
\hline & & & & & & \multirow{2}{*}{$\begin{array}{l}\text { Patient } \\
\text { quantity }\end{array}$} & \multirow[b]{2}{*}{$\%$ change } & \\
\hline & & Total no. & 1 & 2 & 3 & & & \\
\hline 1 & Apr-Aug & 6 & 6 & 0 & 0 & 3.0 & 0.0 & -5.4 \\
\hline $1,2,3$ & Aug-Oct & 10 & 10 & 0 & 0 & 2.0 & 0.0 & -124 \\
\hline 3 & Oct-Mar & 20 & 20 & 0 & 0 & 2.0 & 0.0 & -20.9 \\
\hline 3,4 & Mar-May & 2 & 2 & 0 & 0 & 3.0 & 0.0 & -37 \\
\hline 5 & May-Oct & NA & NA & NA & NA & $N A$ & NA & NA \\
\hline 6 & Oct-Mar & NA & NA & NA & NA & NA & NA & NA \\
\hline 7 & Mar-Jun & NA & NA & NA & NA & $\mathrm{NA}$ & NÄ & NA \\
\hline
\end{tabular}

\begin{tabular}{|c|c|c|c|c|c|c|c|c|c|c|c|c|c|}
\hline \multirow[b]{2}{*}{ Life stage } & \multirow[b]{2}{*}{ Months } & \multicolumn{6}{|c|}{ Highest productivity impact attribut $\epsilon$} & \multicolumn{6}{|c|}{$\%$ change in impact from template } \\
\hline & & Att 1 & $\%$ change & Att 2 & $\%$ change & Att 3 & $\%$ change & Att 4 & $\%$ change & Att 5 & $\%$ change & Atf 6 & $\%$ change \\
\hline 1 & Apr-Aug & FLOW & 25.0 & SEDLD & $\mathbf{1 6 . 7}$ & TEMP & 8.3 & & & & & & \\
\hline $1,2,3$ & Aug-Oct & SEDLD & 50.0 & FLOW & $25.0^{\circ}$ & TEMP & $250^{\circ}$ & & & & & & \\
\hline 3 & Oct-Mar & SEDLD & 50.0 & FLOW & 25.0 & TEMP & 250 & CSTAB & 12.5 & PATHO & 12.5 & & \\
\hline 3,4 & Mar-May & FLOW & $25.0^{\circ}$ & SEDLD & 25.0 & CSTAB & $12.5^{\prime}$ & PATHO & 12.5 & TEMP & 12.5 & & \\
\hline 5 & May-Oct & $\mathrm{NA}$ & NA & NA & $\mathrm{NA}$ & NA & $\mathrm{NA}$ & NA & $\mathrm{NA}$ & $\mathrm{NA}$ & $\mathrm{NA}$ & $\mathrm{NA}$ & NA \\
\hline 6 & Oct-Mar & $\mathrm{NA}$ & NÄ & $\mathrm{NA}$ & NA & NA & $\mathrm{NA}$ & $\mathrm{NA}$ & $\mathrm{NA}$ & $\mathrm{NA}$ & $\mathrm{NA}$ & $\mathrm{NA}$ & $\mathrm{NA}$ \\
\hline 7 & Mar-Jun & NA & NA & $\mathrm{NA}$ & $\mathrm{NA}$ & NA & $\mathrm{NA}$ & $\mathrm{NA}$ & NA & $\mathrm{NA}$ & $\mathrm{NA}$ & $\mathrm{NA}$ & $\mathrm{NA}$ \\
\hline
\end{tabular}

Abundance level refers to estimated relative abundance of spawners that spawned in the natal stream reaches that populated the life history pathways using the reach in this table: Level 1: Low to average (relative to other reaches) abundance

Level 2 High (relative to other reaches) abundance

Level 3 Exceptionally high (relative to other reaches) abundance

Life stages: 1- prespawner; 2-spawner; 3- incubation; 4- fry colonization; 5- summer rearing; 6- overwintering; 7- yearlingpresmolt/smolt

Attributes: CHEM - chemicals

CSTAB - channel stability

COMP - competition (with other species)

FLOW - flow

HABD - habitat diversity
NUTLD - nutrient load

OBST - obstructions

OXYG - dissolved oxygen

PATHO - pathogens

PRED - predators (includes fishing-related losses)
RIPCN - riparian condition

SEDLD - sediment load

TEMP - water temperature

WITH - water withdrawals

"NA" indicates that no life history pathway meeting the criteria used in defining these pathways was present,

Blank cells under attributes indicate no additional attributes with negative effects on productivity were evident compared to those present in the template. 
Stream Reach Summary for Vacant Spring Chinook Salmon Life Histories

Stream: Grande Ronde R.

Reach: Mouth - Joseph Cr (RM 0.0-3.7 from Snake R)

\begin{tabular}{|c|c|c|c|c|c|c|c|c|}
\hline \multicolumn{4}{|c|}{ [Restoration benefit category: } & & \multicolumn{3}{|c|}{ Chinook usage score: } & 65.4 \\
\hline \multirow[b]{3}{*}{ Life stage } & \multirow[b]{3}{*}{ Months } & \multirow{2}{*}{\multicolumn{4}{|c|}{$\begin{array}{l}\text { No. vacant life history pathways } \\
\text { by abundance level }\end{array}$}} & \multicolumn{2}{|c|}{ Key habitat change } & \multirow{3}{*}{$\begin{array}{c}\text { Habitat } \\
\text { productivity! } \\
\text { loss score }\end{array}$} \\
\hline & & & & & & \multirow{2}{*}{$\begin{array}{l}\text { Patient } \\
\text { quantity }\end{array}$} & \multirow[b]{2}{*}{$\%$ change } & \\
\hline & & Total no. & 1 & 2 & 3 & & & \\
\hline 1 & Apr-Aug & 710 & 426 & 236 & 48 & 33.8 & 0.0 & 0.0 \\
\hline $1,2,3$ & Aügo-Òt & $\mathrm{NA}$ & $\mathrm{NA}$ & NA & NA & NA & NA & NA \\
\hline 3 & Ott-Msr & $\mathrm{NA}$ & $\mathrm{NA}$ & $\mathrm{NA}$ & NA" & $\mathrm{NA}$ & $\mathrm{NA}$ & NA \\
\hline 3,4 & Mar-May & NA & NA & NA & NA & NA & $\dot{N A}$ & NA \\
\hline 5 & M̈ay-Ött & 76 & 19 & 48 & 9 & 20.6 & 0.0 & -183 \\
\hline 6 & Ott-Mar & NA & NA & N & NA & NA & $\%$ & NA \\
\hline 7 & Mar-Jun & 562 & $383^{\circ}$ & 14 & 31 & 109.8 & & 7.0 \\
\hline
\end{tabular}

\begin{tabular}{|c|c|c|c|c|c|c|c|c|c|c|c|c|c|}
\hline \multirow[b]{2}{*}{ Life stage } & \multirow[b]{2}{*}{ Months } & \multicolumn{12}{|c|}{ Highest productivity impact attributes (\% change in impact from template) } \\
\hline & & Aft 1 & $\%$ change & Atf 2 & $\%$ change & Art 3 & $\%$ change & Att 4 & $\%$ change & Art 5 & $\%$ change & Att 6 & $\%$ change \\
\hline 1 & Apr-Aug. & CHEM & 12.5 & NUTLD & $125-\cdots$ & OXYG & 12.5 & & & & & & \\
\hline$. .1,2,3$ & Aug-Ött & $\mathrm{NA}$ & NA & NA & $\dddot{N A}$ & NA & NA' & NA' & NA & $\mathrm{NÄ}$ & NÄ & NÄ & $\mathrm{NÄ}$ \\
\hline 3 & Oct-Mar & $\mathrm{NA}$ & NA & NA & NA & NA & NA & NA & NA & NA & NA & NA & NA \\
\hline $\begin{array}{l}34 \\
5\end{array}$ & Mar-May & COMP & $\begin{array}{l}\dot{N A} \\
6.6\end{array}$ & $\begin{array}{c}\mathrm{NA} \\
\mathrm{HABD}\end{array}$ & 'A & NUT'LO & $\begin{array}{c}\mathrm{NA} \\
3.3 "\end{array}$ & $\begin{array}{c}\text { NA } \\
\text { PATHO }\end{array}$ & NA & NA & $\mathrm{NA}$ & $\mathrm{NA}$ & !!??!?! \\
\hline 6 & Ott-Mar & $\begin{array}{c}\text { NA } \\
\text { PATHO }\end{array}$ & NA & $\begin{array}{l}\text { NA } \\
\text { TEMP }\end{array}$ & NA & $\mathrm{NA}$ & $\mathrm{NA}$ & $\mathrm{NA}$ & $\mathrm{NA}$ & $\mathrm{NA}^{\prime}$ & $\ddot{\mathrm{NA}}$ & $\mathrm{NA}$ & $\mathrm{NA}$ \\
\hline
\end{tabular}

Abundance/eve/ refers to estimated relative abundance of spawners that spawned in the natal stream reaches that populated the life history pathways using the reach in this table Level 1: $\quad$ Low to averaga (relative to other reaches) abundance

Level 2 High (relative to other reaches) abundance

Level 3 Exceptionally high (relative to other reaches) abundance

Life stages.' 1-prespawner; 2-spawner; 3- incubation; 4- fry colonization; 5- summer rearing; 6- overwintering; 7-yearling presmolt/smolt

Attributes: CHEM - chemicals

CSTAB - channel stability

COMP - competition (with other species)

FLOW - flow

HABD - habitat diversity
NUTLD - nutrient load

OBST - obstructions

OXYG - dissolved oxygen

PATHO - pathogens

PRED - predators (includes fishing-related losses)
RIPCN - riparian condition

SEDLD - sediment load

TEMP - water temperature

WITH - water withdrawals

"NA" indicates that no life history pathway meeting the criteria used in defining these pathways was present,

Blank cells under attributes indicate no additional attributes with negative effects on productivity were evident compared to those present in the template. 
Stream Reach Summary for Vacant Spring Chinook Salmon Life Histories

Stream: Grande Ronde R.

Reach: Joseph Cr - Buford Cr (RM 3.7- 26.2 from Snake R)

\begin{tabular}{|c|c|c|c|c|c|c|c|c|}
\hline \multicolumn{4}{|c|}{ Restoration benefit category: } & & \multicolumn{3}{|c|}{ Chinook usage score: } & 91.1 \\
\hline \multirow[b]{3}{*}{ Life staga } & \multirow[b]{3}{*}{ Months } & \multirow{2}{*}{\multicolumn{4}{|c|}{$\begin{array}{l}\text { No. vacant life history pathways } \\
\text { by abundance level }\end{array}$}} & \multicolumn{2}{|c|}{ Key habitat change } & \multirow{3}{*}{$\begin{array}{c}\text { Habitat } \\
\text { productivity } \\
\text { loss score }\end{array}$} \\
\hline & & & & & & \multirow{2}{*}{$\begin{array}{l}\text { Patient } \\
\text { quantity }\end{array}$} & \multirow[b]{2}{*}{$\%$ change } & \\
\hline & & Total no. & 1 & 2 & 3 & & & \\
\hline 1 & Apr-Aug & 571 & 342 & 190 & 39 & 33.8 & 10.1 & 00 \\
\hline 123 & Aug & NA & NA & NA & NA & NA & NA & $\mathrm{NA}$ \\
\hline 3 & Ott-Mar & $\mathrm{NA}$ & $\mathrm{NA}$ & $\mathrm{NA}$ & $\mathrm{NA}$ & $\because \mathrm{NA}$ & NA" & $\mathrm{NA}$ \\
\hline 34 & Mar-Mav & $\mathrm{NA}$ & $\mathrm{NA}$ & $\dot{N} \dot{A}$ & $\mathrm{NA}$ & NA & $\mathrm{NA}$ & $\mathrm{NA}$ \\
\hline 5 & May-Ott & 57 & 16 & 34 & 7 & 20.6 & 0.0 & -33.6 \\
\hline 6 & lar & 700 & 514 & 167 & 19 & 23.5 & 01 & -1388 \\
\hline 7 & Mar-Jun & 543 & 364 & 150 & 29 & 117.7 & 2.1 & 5.1 \\
\hline
\end{tabular}

\begin{tabular}{|c|c|c|c|c|c|c|c|c|c|c|c|c|c|}
\hline \multirow[b]{2}{*}{ Life stage } & \multirow[b]{2}{*}{ Months } & \multicolumn{12}{|c|}{ Highest productivity impact attributes (\% change in impact from template) } \\
\hline & & Atf 1 & $\%$ change & Atf 2 & $\%$ change & Att 3 & $\%$ change & Att 4 & $\%$ change & Atf 5 & $\%$ change & Att 6 & $\%$ change \\
\hline 1 & Apr-Aug & CHEM & 20.3 & NỤTLD & 203 & OXYG & 20.3 & & & & & & \\
\hline $1,2,3$ & Âug-Ött & $\mathrm{NA}$ & NA' & $N A$ & $\mathrm{NA}$ & $N A$ & NA & $\mathrm{NA}$ & N"̈̈" & $\mathrm{NA}$ & $\mathrm{NÄ}$ & $\mathrm{NÄ}$ & NÄ" \\
\hline 3 & Ött-Mar & NA & NA & NA & $\mathrm{N} A$ & $\mathrm{NA}$ & $\mathrm{NA}$ & NA & $\mathrm{NA}$ & NA & $\mathrm{NA}$ & NA & NA \\
\hline 3,4 & Mar-May & $\mathrm{NA}$ & $\mathrm{NA}$ & $\dot{N} \ddot{A}$ & $\mathrm{NA}$ & $\mathrm{NA}$ & $\mathrm{NA}$ & $\mathrm{NA}$ & NA & $\mathrm{NA}$ & $\mathrm{NA}$ & $\mathrm{NÄ}$ & NA \\
\hline 5 & May-Ott & "ŚÉḊLD" & $2 \dot{8} .1$ & COMP & 15.5 & PATHO & $14.0^{\circ}$ & CHEM & $78 \ldots$ & HABD' & 78 & NÜTLD & 7.8 \\
\hline 6 & Ött-Mar & SEDLD & 9.6 & PATHO & 7.1 & ṪËṀं & 7.1 & & & & & & \\
\hline 7 & Mar-Jun & PATHO & 4.7 & T̈EM̈P̈ & 4.7 & & & & & & & & \\
\hline
\end{tabular}

Abundance/eve/refers to estimated relative abundance of spawners that spawned in the natal stream reaches that populated the life history pathways using the reach in this table: Level 1: Low to average (relative to other reaches) abundance

Level 2: $\quad$ High (relative to other reaches) abundance

Level 3 Exceptionally high (relative to other reaches) abundance

Life stages: 1-prespawner; 2- spawner; 3- incubation; 4- fry colonization; 5- summer rearing; 6- overwintering; 7- yearling presmolt/smelt

$\begin{array}{lll}\text { Attributes: CHEM - chemicals } & \text { NUTLD - nutrient load } & \text { RIPCN - riparian condition } \\ \text { CSTAB - channel stability } & \text { OBST - obstructions } & \text { SEDLD - SEdiment load } \\ \text { COMP - competition (with other species) } & \text { OXYG - dissolved oxygen } & \text { TEMP - water temperature } \\ \text { FLOW - flow } & \text { PATHO - pathogens } & \text { WITH - water withdrawals } \\ \text { HABD - habitat diversity } & \text { PRED - predators (includes fishing-related losses) }\end{array}$

"NA" indicates that no life history pathway meeting the criteria used in defining these pathways was present.

Blank cells under attributes indicate no additional attributes with negative effects on productivity were evident compared to those present in the template. 


\section{Stream Reach Summary for Vacant Spring Chinook Salmon Life Histories}

Stream: Grande Ronde R.

Reach: Buford $\mathrm{Cr}$ - Bear Cr (RM 26.2 -28,2 from Snake R)

[Restoration benefit category:

Chinook usage score:

\begin{tabular}{l|l}
26.7 & Habitat productivity loss score.
\end{tabular}

\begin{tabular}{|c|c|c|c|c|c|c|c|c|}
\hline \multirow[b]{3}{*}{ Life stage } & \multirow[b]{3}{*}{ Months } & \multirow{2}{*}{\multicolumn{4}{|c|}{$\begin{array}{c}\text { No. vacant life history pathways } \\
\text { by abundance level }\end{array}$}} & \multicolumn{2}{|c|}{ Key habitat change } & \multirow{3}{*}{$\begin{array}{c}\text { Habitat } \\
\text { productivity } \\
\text { loss score }\end{array}$} \\
\hline & & & & & & \multirow{2}{*}{$\begin{array}{l}\text { Patient } \\
\text { quantity }\end{array}$} & \multirow[b]{2}{*}{$\%$ change } & \\
\hline & & Total no. & 1 & 2 & 3 & & & \\
\hline 1 & Apr-Aug & 355 & 213 & $\overline{118}$ & 24 & 33.8 & 0.0 & 0.0 \\
\hline 123 & Aug-Ött & NA & NA & NA & NA & NA & NA & NA \\
\hline 3 & Ott-Mar & NA & $\mathrm{NA}$ & NA & NA & $\mathrm{NA}$ & NA & NA \\
\hline 34 & Mar-May & $\mathrm{NA}$ & $\mathrm{NA}$ & NA & NA & NÄ & NA & NA \\
\hline 5 & May-Ott & 60 & 19 & 34 & 7 & 20.6 & 0.0 & -367 \\
\hline 6 & Ott-Mar & 106 & 60 & 26 & 20 & 225 & 0.0 & -268 \\
\hline 77 & Mar-Jun & 262 & 170 & 76 & 16 & 1075 & 0.0 & 3.6 \\
\hline
\end{tabular}

\begin{tabular}{|c|c|c|c|c|c|c|c|c|c|c|c|c|c|}
\hline \multirow[b]{2}{*}{ Life stage } & \multirow[b]{2}{*}{ Months } & \multicolumn{12}{|c|}{ Highest productivity impact attributes (\% change in impact from template) } \\
\hline & & Att 1 & $\%$ change & Att 2 & $\%$ change & Att 3 & $\%$ change & Att 4 & $\%$ change & An 5 & $\%$ change & Att 6 & $\%$ change \\
\hline 1 & Apr-Aug & CHEM & 6.2 & NUTLD & 6.2 & OXYG & 6.2 & & & & & & \\
\hline$i, 2,3$ & Aug-Ött & $\dot{N} \dot{A}$ & $\mathrm{NÄ}$ & NA & $\mathrm{NA}$ & $N A ̈$ & $\mathrm{NA}$ & NA & Nä" & NÄ & $\mathrm{NÄ}$ & NA" & $\mathrm{NA}$ \\
\hline 3 & Ott-Mar & NA & NA & NA & 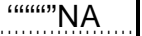 & NA & Nֵ'Ạ & $N A$ & NֵA. & NA & NA & “"NA & NA \\
\hline 3,4 & Mar-May & NA & NA. & NA & NA & NA & NA & NA & NA & NA & NA & NA & NA \\
\hline 5 & May-Ött & COMiP & $15.4^{\circ}$ & "C̈ḦËM & 7.7 & $\mathrm{HABD}$ & 7.7. & NUÛTLḶ’. & 7.7 & PATHO & 77 & & \\
\hline 6 & Ott-Mar & SEDELD & 12.9 & РAंTंHO & 6.5 & TËMP. & 3.1 & & & & & & \\
\hline 7 & Mar-Jun & PATHO & 6.9 & TEMP & 69 & & & & & & & & \\
\hline
\end{tabular}

Abundance/eve/ refers to estimated relative abundance of spawners that spawned in the natal stream reaches that populated the life history pathways using the reach in this table Level 1: Low to average (relative to other reaches) abundance

Level 2 High (relative to other reaches) abundance

Level 3 Exceptionally high (relative to other reaches) abundance

Life stages: 1-prespawner; 2- spawner; 3-incubation; 4-fry colonization; 5- summer rearing; 6-overwintering; 7-yearling presmoltsmolt

$\begin{array}{lll}\text { Attributes: } \text { CHEM - chemicals } & \text { NUTLD - nutrient load } & \text { RIPCN - riparian condition } \\ \text { CSTAB - channel stability } & \text { OBST - obstructions } & \text { SEDLD - sediment load } \\ \text { COMP - competition (with other species) } & \text { OXYG - dissolved oxygen } & \text { TEMP - water temperature } \\ \text { FLOW - flow } & \text { PATHO - pathogens } & \text { WITH - water wit hdrawals } \\ \text { HABD - habitat diversity } & \text { PRED - predators (includes fishing-related losses) }\end{array}$

"NA" indicates that no life history pathway meeting the criteria used in defining these pathways was present.

Blank cells under attributes indicate no additional attributes with negative effects on productivity were evident compared to those present in the template, 
Stream Reach Summary for Vacant Spring Chinook Salmon Life Histories

Stream: Grande Ronde R.

Reach: Bear Cr - Menatchee Cr (RM 28.2 -34.8 from Snake R)

Restoration benefit category: \begin{tabular}{l|l} 
E & Chinook usage score:
\end{tabular} \begin{tabular}{l|l} 
43.3 & Habitat productivity loss score:
\end{tabular}

\begin{tabular}{|c|c|c|c|c|c|c|c|c|}
\hline \multirow[b]{3}{*}{ Life stage } & \multirow[b]{3}{*}{ Months } & \multirow{2}{*}{\multicolumn{4}{|c|}{$\begin{array}{l}\text { No. vacant life history pathways } \\
\text { by abundance level }\end{array}$}} & \multicolumn{2}{|c|}{ Key habitat change } & \multirow{3}{*}{$\begin{array}{l}\text { Habital } \\
\text { productivity } \\
\text { loss score }\end{array}$} \\
\hline & & & & & & \multirow{2}{*}{$\begin{array}{l}\text { Patient } \\
\text { quantity }\end{array}$} & \multirow[b]{2}{*}{$\%$ change } & \\
\hline & & Total no. & 1 & 2 & 3 & & & \\
\hline 1 & Apr-Aug & 355 & 213 & 118 & 24 & 33.7 & 0.0 & 0.0 \\
\hline $1,2,3$ & Aug-Oct & $\mathrm{NA}$ & $\mathrm{NA}$ & $\mathrm{NA}$ & NA & $\mathrm{NA}$ & $\mathrm{NA}$ & $\mathrm{NA}$ \\
\hline 3 & Oct-Mar & $\mathrm{NA}$ & $\mathrm{NA}$ & $\mathrm{NA}$ & NÄ & $\mathrm{NA}$ & $\mathrm{NA}$ & NÄ \\
\hline 3,4 & Mar-May & $\mathrm{NA}$ & $\mathrm{NA}$ & NA & NA & $\mathrm{NA}$ & $\mathrm{NA}$ & NA \\
\hline 5 & May-Oct & 69 & 27 & 35 & "7 & 20.6 & 0.0 & -24.2 \\
\hline 6 & Oct-Mar & 266 & 202 & 62 & 2 & 23.0 & 0.2 & -35.4 \\
\hline 7 & Mar-Jun & 258 & 168 & 75 & 15 & 106.1 & 0.0 & 2.4 \\
\hline
\end{tabular}

\begin{tabular}{|c|c|c|c|c|c|c|c|c|c|c|c|c|c|}
\hline \multirow[b]{2}{*}{ Life stage } & \multirow[b]{2}{*}{ Months } & \multicolumn{12}{|c|}{ Highest productivity impact attributes (\% change in impact from template) } \\
\hline & & Att 1 & $\%$ change & Att 2 & $\%$ change & Att 3 & $\%$ change & Att 4 & $\%$ change & Att 5 & $\%$ change & Art 6 & $\%$ change \\
\hline 1 & Apr-Aug & CHEM & $\overline{17,4}$ & NUTLD & 17.4 & OXYG & 17.4 & & & & & & \\
\hline$, 1,2,3$ & Aug-Ött & $\dot{N A}$ & $\mathrm{NA}$ & $\mathrm{NA}$ & $\mathrm{NA}$ & NA & NÄ" & $\mathrm{NÄ}$ & NÄ & $\mathrm{NA}$ & NÄ & NA & NA \\
\hline 3 & Ott-Mar & NÄ" " & NA & NA & NA & NA & $\mathrm{NA}$ & NA" & NA & $N A$ & "'NA & NA & NA \\
\hline 34 & Mar-May & $\mathrm{NA}$ & $\mathrm{NA}$ & $\mathrm{NA}$ & $\mathrm{NA}$ & $\mathrm{NA}$ & $\mathrm{NA}$ & $\mathrm{NÄ}$ & $\mathrm{NA}$ & $\mathrm{NA}$ & $\mathrm{NA}$ & NÄ & NA" \\
\hline 5 & May-Ött & SEDLD & $11 . j$ & ĊOMP & 6.5 & ṔA்T்HO & $56^{\circ}$ & C̈HEM" & 3.3 & $\mathrm{HABD}^{\circ}$ & 3.3 & NUTTLD & 3.3 \\
\hline 6 & Ott-Mar & ŚĖḊL̇ & 6.6 & PATHO & 3.3 & TEMMP" & 31 & & & & & & \\
\hline 7 & Mar-Jun & PATHO & 4.6 & TEMP & 4.6 & & & & & & & & \\
\hline
\end{tabular}

Abundance level refers to estimated relative abundance of spawners that spawned in the natal stream reaches that populated the life history pathways using the reach in this table Level 1: Low to average (relative to other reaches) abundance

Level 2: High (relative to other reaches) abundance

Level 3 Exceptionally high (relative to other reaches) abundance

Life stages: 1- prespawner; 2- spawner; 3- incubation; 4- fry colonization; 5. summer rearing; 6- overwintering; 7- yearlingpresmolt/smolt

Attributes: CHEM - chemicals

CSTAB - channel stability

COMP - competition (with other species)

FLOW - flow

HABD - habitat diversity
NUTLD - nutrient load

OBST - obstructions

OXYG - dissolved oxygen

PATHO - pathogens

PRED - predators (includes fishing-related losses)
RIPCN - riparian condition

SEDLD - sediment load

TEMP - water temperature

WITH - water withdrawals

"NA" indicates that no life history pathway meeting the criteria used in defining these pathways was present.

Blank cells under attributes indicate no additional attributes with negative effects on productivity were evident compared to those present in the template. 
Stream: Grande Ronde R.

Reach: Menatchee $\mathrm{Cr}$ - Wenaha R (RM 34.8 -45.1 from Snake R)

\begin{tabular}{|ll|ll|l|}
\hline Restoration benefit category: & B & Chinook usage score: & 53.2 & Habitat productivity loss score: \\
\hline
\end{tabular}

\begin{tabular}{|c|c|c|c|c|c|c|c|c|}
\hline \multirow[b]{3}{*}{ Life stage } & \multirow[b]{3}{*}{ Months } & \multirow{2}{*}{\multicolumn{4}{|c|}{$\begin{array}{c}\text { No. vacant life history pathways } \\
\text { by abundance level }\end{array}$}} & \multicolumn{2}{|c|}{ Key habitat change } & \multirow{3}{*}{\begin{tabular}{|c|} 
Habitat \\
productivity \\
loss score
\end{tabular}} \\
\hline & & & & & & \multirow{2}{*}{$\begin{array}{l}\text { Patient } \\
\text { quantity }\end{array}$} & \multirow[b]{2}{*}{$\%$ change } & \\
\hline & & Total no. & 1 & 2 & 3 & & & \\
\hline song & Apr-Aug & 359 & 217 & 118 & 24 & 34.5 & 00 & $0 \Omega$ \\
\hline $1,2,3$ & Aug-Oct & NA & NA & NA & NA & $\mathrm{NA}$ & NA & $\mathrm{NA}$ \\
\hline 3 & Ott-Mar & $\mathrm{NAn}$ & NA & $\mathrm{NA}$ & N̂Ä & NÄ & NA & NA \\
\hline 34 & Mar-May & 20 & 20 & 0 & 0 & 347 & 0.0 & -422 \\
\hline 5 & May-Ött & 175 & 128 & 40 & 7 & 219 & 0.0 & -2745 \\
\hline 6 & Ott-Mar & 518 & 346 & 152 & 20 & 241 & 0.0 & -869 \\
\hline 7 & Mar-Jun & 250 & 162 & "7ั3̆" & 15 & 118.3 & 0.0 & 2.9 \\
\hline
\end{tabular}

\begin{tabular}{|c|c|c|c|c|c|c|c|c|c|c|c|c|c|}
\hline \multirow[b]{2}{*}{ Life stage } & \multirow[b]{2}{*}{ Months } & \multicolumn{12}{|c|}{ Highest productivity impact attributes (\% change in impact from template) } \\
\hline & & Atf 1 & $\%$ change & Att 2 & $\%$ change & Att 3 & $\%$ change & Att 4 & $\%$ change & Att 5 & $\%$ change & Att 6 & $\%$ change \\
\hline & Apr-Aug & CHEM & 11.6 & NUTLD & 11.6 & OXYG & 11.6 & & & & & & \\
\hline $1,2,3$ & Aug-Ött & $\mathrm{NA}$ & $\mathrm{NA}$ & $\mathrm{NÄ}$ & $\mathrm{NA}$ & $\mathrm{NA}$ & NA & NÄ & $\mathrm{NA}$ & $\mathrm{NA}$ & NA & Nä & NA \\
\hline 3 & Oct-Mar & NA & NA & NA & NA & NA & NA & NA & NA & $\mathrm{NA}$ & $\mathrm{NA}$ & NA & NA \\
\hline 3,4 & Mar-May & COÖM̈P". & 500 & .C̈ḦËM & 25.0 & FLOW & 25.0 & "HÄBD" & $250 \ldots$ & NUTíD & $25.0^{\circ}$ & РÄTḦO & 25.0 \\
\hline 5 & May-Ött & СOMP & 31.7 & $\mathrm{CHEM}$ & 15,8 & HABD & 15.8 & NUTLD & 15.8 & РАТН̈О & 15.8 & & \\
\hline 6 & Ött-Mar & SEEDLLD & 8.4 & PATHOO & 42 & TEMPP & 3.1 & & & & & & \\
\hline 7 & Mar-Jun & "PATHÖ" & 5.7 & TEMMP & 5.7 & & & & & & & & \\
\hline
\end{tabular}

Abundance leve/ refers to estimated relative abundance of spawners that spawned in the natal stream reaches that populated the life history pathways using the reach in this table:

Level 1: $\quad$ Low to average (relative to other reaches) abundance

Level 2 High (relative to other reaches) abundance

Level 3: Exceptionally high (relative to other reaches) abundance

Life stages: 1- prespawner; 2- spawner; 3- incubation; 4- fry colonization; 5- summer rearing; 6- overwintering; 7- yearting presmolt/smolt

Attributes: CHEM - chemicals

CSTAB - channel stability

COMP - competition (with other species)

FLOW - flow

HABD - habitat diversity
NUTLD - nutrient load

OBST - obstructions

OXYG - dissolved oxygen

PATHO - pathogens

PRED - predators (includes fishing-related losses)
RIPCN - riparian condition

SEDLD - sediment load

TEMP - water temperature

WITH - water withdrawals

"NA" indicates that no life history pathway meeting the criteria used in defining these pathways was present.

Blank cells under attributes indicate no additional attributes with negative effects on productivity were evident compared to those present in the template. 


\section{Stream Reach Summary for Vacant Spring Chinook Salmon Life Histories}

Stream: Grande Ronde R.

Reach: Wenaha R - Courtney Cr (RM 45.1 -46.2 from Snake R)

\begin{tabular}{|l|l|ll|l|}
\hline Restoration benefit category & F & Chinook usage score: & 20.9 & Habitat productivity loss score: \\
\hline
\end{tabular}

\begin{tabular}{|c|c|c|c|c|c|c|c|c|c|}
\hline \multirow[b]{3}{*}{ Liie stage } & \multirow[b]{3}{*}{ Months } & \multirow{2}{*}{\multicolumn{5}{|c|}{$\begin{array}{l}\text { No. vacant life history pathways } \\
\text { by abundance level }\end{array}$}} & \multicolumn{2}{|c|}{ Key habitat change } & \multirow{3}{*}{$\begin{array}{c}\text { Habitat } \\
\text { productivity } \\
\text { loss score }\end{array}$} \\
\hline & & & & & & & \multirow{2}{*}{$\begin{array}{l}\text { Patient } \\
\text { quantity }\end{array}$} & \multirow[b]{2}{*}{$\%$ change } & \\
\hline & & Total & no. & 1 & 2 & 3 & & & \\
\hline & Apr-Aug & 347 & & 209 & 114 & 24 & 398 & 0.0 & 0.0 \\
\hline $1,2,3$ & Aug-Oct & $\mathrm{NA}$ & & NA & NA & NA & $\mathrm{NA}$ & $N A$ & NA \\
\hline 3 & Oct-Mar & NÄ & & NA & NA & $\mathrm{NA}$ & $\mathrm{NA}$ & $\mathrm{NA}$ & $\mathrm{NA}$ \\
\hline 3,4 & Mar-May & NA & & $\mathrm{NA}$ & $\mathrm{NA}$ & NA & $\mathrm{NA}$ & $\mathrm{NA}$ & $\mathrm{NA}$ \\
\hline 5 & May-Oct & 82 & & 38 & 37 & 7 & 71.3 & 0.0 & -46.4 \\
\hline 6 & Oct-Mar & 103 & & 86 & 14 & 3 & 29.5 & 0.0 & -16.4 \\
\hline 7 & Mar-Jun & 217 & & 140 & 63 & 14 & 138.0 & 0.0 & 2.1 \\
\hline
\end{tabular}

\begin{tabular}{|c|c|c|c|c|c|c|c|c|c|c|c|c|c|}
\hline \multirow[b]{2}{*}{ Life stage } & \multirow[b]{2}{*}{ Months } & \multicolumn{12}{|c|}{ Highest productivity impact attributes (\% change in impact from template) } \\
\hline & & Att 1 & $\%$ change & Aft 2 & $\%$ change & Att 3 & $\%$ change & Aft 4 & $\%$ change & Aft 5 & $\%$ change & Att 6 & $\%$ change \\
\hline & Apr-Aug & CHEM & 6.0 & NUTLD & & OXYG & 6.0 & TEMP & 6.0 & & & & \\
\hline $1,2,3$, & Äug-Ött & $\mathrm{NA}$ & $\mathrm{NA}$ & NA & & $\mathrm{NA}$ & $\mathrm{NA}$ & NA & NA & NA & $\mathrm{NA}$ & NA & NÄ \\
\hline $3 " '$ & Ott-Mar & NA & NA & NA & $\mathrm{NA}$ & $\mathrm{NA}$ & NA & $" N A$ & N A & $N A$ & NA & NA & N̈A \\
\hline$., \ldots, 3,3,4, \ldots, \ldots, \ldots, \ldots$ & Mar-May & $\mathrm{NA}$ & Nï. & $\mathrm{NA}$ & $\mathrm{NA}$ & NÄ & NA & $\mathrm{NA}$ & $\ddot{N A}$ & NÄ & $\mathrm{NA}$ & $\mathrm{NA}$ & $\mathrm{NA}$ \\
\hline 5 & May-Ött & SEEDLD & 10.4 & COMPP & 8.1 & HABD & 5.2 & PATHO & & CHËEM & $40^{\circ}$ & ṄU்ṫ் & $4 ! ! ? \ldots$ \\
\hline $\begin{array}{l}6 \\
7\end{array}$ & $\begin{array}{l}\text { Ott-Mar } \\
\text { Mar-Jun }\end{array}$ & $\begin{array}{l}\text { SEDLD } \\
\text { TEMP }\end{array}$ & $\begin{array}{l}10.3 \\
4.8\end{array}$ & HABD & 5.1 & PATHO & $5.1^{\circ}$ & "TËMP" & 3.1 & & & & \\
\hline
\end{tabular}

Abundance level refers to estimated relative abundance of spawners that spawned in the natal stream reaches that populated the life history pathways using the reach in this table: Level 1: Low to average (relative to other reaches) abundance

Level 2: $\quad$ High (relative to other reaches) abundance

Level 3: Exceptionally high (relative to other reaches) abundance

Life stages: 1-prespawner; 2- spawner; 3- incubation; 4- fry colonization; 5- summer rearing; 6- overwintering; 7- yearling presmolt/smolt

\begin{tabular}{|c|c|c|c|}
\hline $\begin{array}{r}\text { Attributes: } \text { CHEM - } \\
\text { CSTAB - } \\
\text { COMP - } \\
\text { FLOW - } \\
\text { HABD - }\end{array}$ & $\begin{array}{l}\text { chemicals } \\
\text { channel stability } \\
\text { competition (with other species) } \\
\text { flow } \\
\text { habitat diversity }\end{array}$ & $\begin{array}{l}\text { NUTLD - nutrient load } \\
\text { OBST - obstructions } \\
\text { OXYG - dissolved oxygen } \\
\text { PATHO - pathogens } \\
\text { PRED - predators (includes fishing-related losses) }\end{array}$ & $\begin{array}{l}\text { RIPCN - riparian condition } \\
\text { SEDLD - } \\
\text { TEMPdiment load } \\
\text { TEMP - } \quad \text { water temperature } \\
\text { WITH - } \quad \text { water withdrawals }\end{array}$ \\
\hline
\end{tabular}

"NA" indicates that no life history pathway meeting the criteria used in defining these pathways was present.

Blank cells under attributes indicate no additional attributes with negative effects on productivity were evident compared to those present in the template. 
Stream: Grande Ronde R.

Reach: Courtney $\mathrm{Cr}$ - Mud $\mathrm{Cr}$ (RM 46.2 -51.4 from Snake R)

\begin{tabular}{ll|ll|l|}
\hline [Restoration benefit category: & F & Chinook usage score: & 20.7 & Habitat productivity loss score: \\
\hline
\end{tabular}

\begin{tabular}{|c|c|c|c|c|c|c|c|c|}
\hline \multirow[b]{3}{*}{ Life stage } & \multirow[b]{3}{*}{ Months } & \multirow{2}{*}{\multicolumn{4}{|c|}{$\begin{array}{l}\text { No. vacant life history pathways } \\
\text { by abundance level }\end{array}$}} & \multicolumn{2}{|c|}{ Key habitat change } & \multirow{3}{*}{\begin{tabular}{|c|} 
Habitat \\
productivity \\
loss score
\end{tabular}} \\
\hline & & & & & & \multirow{2}{*}{$\begin{array}{l}\text { Patient } \\
\text { quantity }\end{array}$} & \multirow[b]{2}{*}{$\%$ change } & \\
\hline & & Total no. & 1 & 2 & 3 & & & \\
\hline 1 & Apr-Aug & 347 & 209 & 114 & 24 & 33.3 & 0.0 & 0.0 \\
\hline $1,2,3$ & Aug-Oct & NA & NA & $\mathrm{NA}$ & $N A$ & $\mathrm{NA}$ & $\mathrm{NA}$ & $\mathrm{NA}$ \\
\hline 3 & Oct-Mar & $\mathrm{NA}$ & NA & NA" & $\mathrm{NA}$ & $\mathrm{NA}$ & $\mathrm{NA}$ & $\mathrm{NA}$ \\
\hline 3,4 & Mar-May & NA & $\mathrm{NA}$ & NA & $\mathrm{NA}$ & $\mathrm{NA}$ & $\mathrm{NA}$ & NA \\
\hline 5 & May-Oct & 94 & 47 & 39 & 8 & 60.0 & 0.0 & -34.8 \\
\hline 6 & Oct-Mar & 203 & 115 & 67 & 21 & 25.3 & 0.0 & -21.6 \\
\hline 7 & Mar-Jun & 214 & 137 & $63^{\circ}$ & 14 & 114.2 & 0.0 & 1.7 \\
\hline
\end{tabular}

\begin{tabular}{|c|c|c|c|c|c|c|c|c|c|c|c|c|c|}
\hline \multirow[b]{2}{*}{ Life stage } & \multirow[b]{2}{*}{ Months } & \multicolumn{12}{|c|}{ Highest productivity impact attributes (\% change in impact from template) } \\
\hline & & $\overline{\text { Att } 1}$ & $\%$ change & Atf 2 & $\%$ change & Atf 3 & $\%$ change & Att 4 & $\%$ change & Art 5 & $\%$ change & Atf 6 & $\%$ change \\
\hline 1 & Apr-Aug & CHEM & 6.1 & NUTLD & 6.1 & OXYG & 6.1 & TEMP & 6.1 & & & & \\
\hline $1,2,3$ & Aug-Ôtt & $\mathrm{NA}$ & $\mathrm{NÄ}$ & $\mathrm{NA}$ & $\mathrm{NA}$ & $\mathrm{NA}$ & NÄ & NA & NA & NÄ & $\dddot{\mathrm{N}} \mathrm{N}$ & NA.... & NĀ \\
\hline 3 & Oct-Mar & NA & NA & $\mathrm{NA}$ & NA & NA & NA & NA & NA & NA & NA & NA" & $\mathrm{NA}$ \\
\hline 34 & Mar-May & $\mathrm{NÄ}$ & $\mathrm{NA}$ & Nä & $\mathrm{NAM}$ & $\mathrm{NÄ}$ & $\mathrm{NA}$ & $\mathrm{NA}$ & $\mathrm{NA}$ & $\mathrm{NA}$ & $\because \mathrm{NA}$ & NA & NA \\
\hline 5 & fday-Ott & сÖMP & 7.1 & CHEM & 3̈.6 & NUTLD & 3.6 & PATHO & 3.6 & TEMP & 3.6 & & \\
\hline 6 & Ött-Mar & 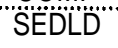 & 6.9 & $H A B B D$ & 3.4 & "PÄTHOO' & 3.4 & TËM̆P & 3.1 & & & & \\
\hline 7 & Mar-Jun & PATHO & 3.9 & TEMP & 3.9 & & & & & & & & \\
\hline
\end{tabular}

Abundance /eve/ refers to estimated relative abundance of spawners that spawned in the natal stream reaches that populated the life history pathways using the reach in this table: Levei 1: Low to average (relative to other reaches) abundance

Levei 2 High (relative to other reaches) abundance

Level 3 Exceptionally high (relative to other reaches) abundance.

Life stages: 1- prespawner; 2-spawner; 3- incubation; 4- fry colonization; 5- summer rearing; 6- overwintering; 7- yearling presmolt/smolt

Attributes: CHEM - chemicals

CSTAB - channel stability

COMP - competition (with other species)

FLOW - fiOW

HABD - habitat diversity
NUTLD - nutrient load

OBST - obstructions

OXYG - dissolved oxygen

PATHO - pathogens

PRED - predators (includes fishing-related losses)
RIPCN - riparian condition

SEDLD - sediment ioad

TEMP - water temperature

WITH - water withdrawals

"NA" indicates that no life history pathway meeting the criteria used in defining these pathways was present.

Blank cells under attributes indicate no additional attributes with negative effects on productivity were evident compared to those present in the template. 
Stream: Grande Ronde R.

Reach: $\quad$ Mud $\mathrm{Cr}$ - Wallupa/Wildcat $\mathrm{Cr}$ (RM $51.4-52.8$ from Snake $\mathrm{R}$ )

\begin{tabular}{|c|c|c|c|c|c|c|c|c|}
\hline \multicolumn{4}{|c|}{ Restoration benefit category: } & $\mathbf{F}$ & \multicolumn{3}{|c|}{ Chinook usage score: } & 25.0 \\
\hline \multirow[b]{3}{*}{ Life stage } & \multirow[b]{3}{*}{ Months } & \multirow{2}{*}{\multicolumn{4}{|c|}{$\begin{array}{l}\text { No. vacant life history pathways } \\
\text { by abundance level }\end{array}$}} & \multicolumn{2}{|c|}{ Key habitat change } & \multirow{3}{*}{$\begin{array}{c}\text { Habital } \\
\text { productivity } \\
\text { loss score }\end{array}$} \\
\hline & & & & & & \multirow{2}{*}{$\begin{array}{l}\text { Patient } \\
\text { quantity }\end{array}$} & \multirow[b]{2}{*}{$\%$ change } & \\
\hline & & Total no. & 1 & 2 & 3 & & & \\
\hline 1 & Apr-Aug & 347 & 209 & 114 & 24 & 24.1 & 0.0 & 0.0 \\
\hline $1,2,3$ & Aug-Oct & $\mathrm{NA}$ & $\mathrm{NA}$ & NA & $\mathrm{NA}$ & $\mathrm{NA}$ & $\mathrm{NA}$ & NA \\
\hline 3 & Oct-Mar & NÄ & $\mathrm{NA}$ & $\mathrm{NA}$ & $\mathrm{NA}$ & NÄ & NA & NA \\
\hline 3,4 & Mar-May & $\mathrm{NA}$ & NA & $\mathrm{NA}$ & NA & NA & $\mathrm{NA}$ & $\mathrm{NA}$ \\
\hline 5 & May-Oct & 99 & 50 & 40 & 9 & 46.9 & 0.0 & -46.9 \\
\hline 6 & Oct-Mar & 134 & 99 & 14 & 21 & 200 & 0.0 & -167 \\
\hline 7 & Mar-Jun & 205 & 132 & 60 & 13 & 89.4 & 0.0 & 1.8 \\
\hline
\end{tabular}

\begin{tabular}{|c|c|c|c|c|c|c|c|c|c|c|c|c|c|}
\hline \multirow[b]{2}{*}{ Liie stage } & \multirow[b]{2}{*}{ Months } & \multicolumn{12}{|c|}{ Highest productivity impact attributes (\% change in impact from template) } \\
\hline & & Art 1 & $\%$ change & Att 2 & $\%$ change & Att 3 & $\%$ change & Att 4 & $\%$ change & Att 5 & $\%$ change & Att 6 & $\%$ change \\
\hline & Apr-Aug & CHEM & 8.7 & NUTLD & 8.7 & OXYG & 87 & TEMP & 8.7 & & & & \\
\hline $1,2,3$ & Aug-Oct & NA & $\mathrm{NA}$ & NA & NA & $\mathrm{NA}$ & NA & NA & $\dot{N} \dot{A}$ & $\mathrm{NA}$ & $\mathrm{NAM}$ & NA & $\mathrm{NA}$ \\
\hline "' 3 ' & Ott-Mar & $\mathrm{NA}$ & $\mathrm{NA}$ & NA & NA & NA & NA & NA & NA & $\mathrm{NA}$ & NA & NA & NA \\
\hline$-.3,4$, & Mar-May & $\mathrm{NAB}$ & $\mathrm{NA}$ & $\mathrm{NA}$ & $\mathrm{NA}$ & NAA" & $\mathrm{NA}$ & $\mathrm{NA}$ & $N A$ & $\mathrm{NA}$ & $\mathrm{NA}$ & $\mathrm{NA}$ & \\
\hline 5 & May-Ott & SEDLD & 10.3 & COMP & 6.0 & TEMP & 6.0 & HABD & 5.2 & PATHOO & 5.2 & "c̈Ḣ̇̇̀̈ & \\
\hline 6 & Ott-Mar & SEDLD & 8.0 & $H A B D$ & 4.0 & PATHO & $4.0^{\circ}$ & & & & & & \\
\hline$\dot{7}$ & Mar-Jun & PATHO & 4.3 & TEMP & 4.3 & & & & & & & & \\
\hline
\end{tabular}

Abundance level refers to estimated relative abundance of spawners that spawned in the natal stream reaches that populated the life history pathways using the reach in this table:

Level 1: $\quad$ Low to average (relative to other reaches) abundance

Level 2 High (relative to other reaches) abundance

Level 3: Exceptionally high (relative to other reaches) abundance

Life stages: 1- prespawner; 2- spawner; 3- incubation; 4- fry colonization; 5- summer rearing; 6- overwintering; 7- yearling presmolt/smolf

Attributes: CHEM - chemicals

CSTAB - channel stability

COMP - competition (with other species)

FLOW - flow

HABD - habitat diversity
NUTLD - nutrient load

OBST - obstructions

OXYG - dissolved oxygen

PATHO - pathogens

PRED - predators (includes fishing-related losses)
RIPCN - riparian condition

SEDLD - sediment load

TEMP - water temperature

WITH - water withdrawals

"NA" indicates that no life history pathway meeting the criteria used in defining these pathways was present.

Blank cells under attributes indicate no additional attributes with negative effects on productivity were evident compared to those present in the template. 
Stream: Grande Ronde R.

Reach: WallupaMildcat Cr - Grossman $\mathrm{Cr}$ (RM 52.8 -61.1 from Snake R)

\begin{tabular}{|c|c|c|c|c|c|c|c|c|}
\hline \multicolumn{4}{|c|}{ [Restoration benefit category: } & $B$ & \multicolumn{3}{|c|}{ Chinook usage score: } & 88.8 \\
\hline \multirow[b]{3}{*}{ Life stage } & \multirow[b]{3}{*}{ Months } & \multirow{2}{*}{\multicolumn{4}{|c|}{$\begin{array}{c}\text { No. vacant life history pathways } \\
\text { by abundance level }\end{array}$}} & \multicolumn{2}{|c|}{ Key habitat change } & \multirow{3}{*}{$\begin{array}{c}\text { Habitat } \\
\text { productivity } \\
\text { loss score }\end{array}$} \\
\hline & & & & & & \multirow{2}{*}{$\begin{array}{l}\text { Patient } \\
\text { quantity }\end{array}$} & \multirow[b]{2}{*}{$\%$ change } & \\
\hline & & Total no. & 1 & 2 & 3 & & & \\
\hline $1+2$ & Apr-Aug & 347 & 209 & $\overline{114}$ & 24 & 22.8 & 0.0 & 00 \\
\hline 123 & Aug-Ött & NA & NA & NA & NA & NA & NA & $\mathrm{NA}$ \\
\hline 3 & Oct-Mar & NÄ & NÄ & NOÄ & NÄ & NÄ & NÄ & NÄ \\
\hline 34 & Mar-May & NA & NA & $N A$ & NA & $\mathrm{NA}$ & $\mathrm{NA}$ & $\mathrm{NA}$ \\
\hline 5 & May-Ott & 121 & 63 & 49 & 9 & 47.5 & 0.0 & -107.0 \\
\hline 6 & Ott-Mar & 459 & 339 & 99 & 21 & 207 & مِ & -219.4 \\
\hline 7 & Mar-Jun & 200 & 128 & 60 & 12 & 90.5 & 0.0 & 3.5 \\
\hline
\end{tabular}

\begin{tabular}{|c|c|c|c|c|c|c|c|c|c|c|c|c|c|}
\hline \multirow[b]{2}{*}{ Life stage } & \multirow[b]{2}{*}{ Months } & \multicolumn{12}{|c|}{ Highest productivity impact attributes (\% change in impact from template) } \\
\hline & & Att 1 & $\%$ change & Att 2 & $\%$ change & Att 3 & $\%$ change & Att 4 & $\%$ change & Aft 5 & $\%$ change & Att 6 & $\%$ change \\
\hline 1 & Apr-Aug & CHEM & 22.8 & NUTLD & 22.8 & OXYG & 22.8 & TEMP & 22.8 & & & & \\
\hline $1,2,3$ & Äug-Ött & NA & NA & NA & ìं & N̈A & NA & NA & $\mathrm{NA}$ & $\dot{N} \dot{A}$ & NA & ï̈̈ & $\mathrm{NA}$ \\
\hline 3 & Ott-Mar & $" \dddot{N} A "$ & "NA" & NA & NA & NA & NA & $\mathrm{NA}$ & NA & NA & NA & $\mathrm{NA}$ & NA \\
\hline 3,4 & Mar-Mä & "NA" & $\mathrm{NA}$ & NA & $\mathrm{NA}$ & $\mathrm{NA}$ & $\mathrm{NA}$ & $\mathrm{NA}$ & $\mathrm{NA}$ & $\mathrm{NA}$ & $\mathrm{NA}$ & $\mathrm{NA}$ & $\mathrm{NA}$ \\
\hline 5 & Mày-Ött & "ĆÖMṔp & 16.0 & TEMP & 16.0 & "CHEM & 8.0 & NUTLD & 8.0 & PATHO & 8.0 & & \\
\hline$" 6$ & Oct-Mar & "ŠËD̈ËD̈" & 30.7 & "ḦÄB̈D" & 15.3 & "PÄTHÖ & 15.3 & T̈ËMัP & 15.1 & & & & \\
\hline 7 & Mar-Jun & PATHO & 8.7 & TEMP & 8.7 & & & & & & & & \\
\hline
\end{tabular}

Abundance/eve/refers to estimated relative abundance of spawners that spawned in the natal stream reaches that populated the life history pathways using the reach in this table:

Level 1: Low to average (relative to other reaches) abundance

Level 2: High (relative to other reaches) abundance

Level 3: Exceptionally high (relative to other reaches) abundance

Life stages: 1- prespawner; 2- spawner; 3- incubation; 4- fry colonization; 5- summer rearing; 6- overwintering; 7- yearlingpresmolUsmolt

Attributes: CHEM - chemicals

CSTAB - channel stability

COMP - competition (with other species)

FLOW - ftOW

HABD - habitat diversity
NUTLD - nutrient load

OBST - obstructions

OXYG - dissolved oxygen

PATHO - pathogens

PRED - predators (includes fishing-related losses)
RIPCN - riparian condition

SEDLD - sediment load

TEMP - water temperature

WITH - water withdrawals

"NA" indicates that no life history pathway meeting the criteria used in defining these pathways was present.

Blank cells under attributes indicate no additional attributes with negative effects on productivity were evident compared to those present in the template, 
Stream Reach Summary for Vacant Spring Chinook Salmon Life Histories

Stream: Grande Ronde R.

Reach: Grossman Cr - Wallowa R (RM 61.1 -80.0 from Snake R)

\begin{tabular}{ll|ll|ll}
\hline Restoration benefit category: & A & Chinook usage score: & 221.2 & Habitat productivity loss score: & -3222.1 \\
\hline
\end{tabular}

\begin{tabular}{|c|c|c|c|c|c|c|c|c|}
\hline \multirow[b]{3}{*}{ Life stage } & \multirow[b]{3}{*}{ Months } & \multirow{2}{*}{\multicolumn{4}{|c|}{$\begin{array}{l}\text { No. vacant life history pathways } \\
\text { by abundance level }\end{array}$}} & \multicolumn{2}{|c|}{ Key habitat change } & \multirow{3}{*}{$\begin{array}{c}\text { Habitat } \\
\text { productivity } \\
\text { loss score }\end{array}$} \\
\hline & & & & & & \multirow{2}{*}{$\begin{array}{l}\text { Patient } \\
\text { quantity }\end{array}$} & \multirow[b]{2}{*}{$\%$ change } & \\
\hline & & Total no. & 1 & 2 & 3 & & & \\
\hline 1 & Apr-Aug & 496 & 303 & 154 & 39 & $?$ & 0.0 & 0.0 \\
\hline 1.23 & Aug-Ott & NA & NA & $\mathrm{NA}$ & $\mathrm{NA}$ & & NA & $\mathrm{NA}$ \\
\hline 3 & Oct-Mar & $\mathrm{NA}$ & $\mathrm{NA}$ & N̈Ä & ח̈Ä & NA & NA & $\mathrm{NA}$ \\
\hline 3,4 & Mar-May & 150 & 124 & 26 & 0 & $33.9^{\circ}$ & 0.0 & -3472 \\
\hline 5 & May-Oct & 902 & 699 & 191 & 12 & 58.3 & 0.0 & -2181.6 \\
\hline 6 & Ott-Mar & 1038 & 641 & 339 & 58 & 22.0 & 0.0 & $-697.2 "$ \\
\hline 7 & Mar-Jun & 190 & 120 & 58 & 12 & 106.2 & 0.0 & 3.9 \\
\hline
\end{tabular}

\begin{tabular}{|c|c|c|c|c|c|c|c|c|c|c|c|c|c|}
\hline \multirow[b]{2}{*}{ Life stage } & \multirow[b]{2}{*}{ Months } & \multicolumn{12}{|c|}{ Highest productivity impact attributes (\% change in impact from template) } \\
\hline & & Att 1 & $\%$ change & Att 2 & $\%$ change & Att 3 & $\%$ change & Atf 4 & \% change & Att 5 & $\%$ change & Att 6 & $\%$ change \\
\hline 1 & Apr-Aug & CHEM & 21.5 & NUTLD & 21.5 & OXYG & 21.5 & TEMP & 21.5 & & & & \\
\hline $1,2,3$ & Aug-Ott & NÄ & NÄ & N̈̈̈ & $\mathrm{NA}$ & NÄ & NÄ & NÄ & N"̈ & NA & $\mathrm{NA}$ & $\mathrm{NA}$ & $\mathrm{NA}$ \\
\hline 3 & Ott-Mar & NA & NÄ & NA & NA & NA & NA & NA & NA & NA & $\mathrm{NA}$ & NA & NA \\
\hline 3,4 & Mäar-Mày & "CÖM̄P" & 37.7 & TYËẸP̈P. & 37.7 & "FLOOW" & $25.0^{\circ}$ & РÄTHÖ & 25.0 & SEDELD & 20.2 & CHEM & 18.8 \\
\hline 5 & May-Ott & COMP & 36.2 & TEMP" & 36.2 & SEDLD & 27.2 & CHEM & 18.1 & NUTLD & 18.1 & PATHO & 18.1 \\
\hline "ख" & ött-Mar & SEDLD & 25.3 & PÄT̈HÖ & 19.0 & "TËM̈P" & $19.0 " ', " '$ & HABD & 12.6 & & & & \\
\hline 7 & Mar-Jun & PATHO & 10.2 & TEMP & 10.2 & & & & & & & & \\
\hline
\end{tabular}

Abundance lovel refers to estimated relative abundance of spawners that spawned in the natal stream reaches that populated the life history pathwavs usinathe reach in this table: Level 1: $\quad$ Low to average (relative to other reaches) abundance

Level 2: High (relative to other reaches) abundance

Level 3: Exceptionally high (relative to other reaches) abundance

Life stages.' 1- prespawner; 2- spawner; 3- incubation; 4- fry colonization; 5- summer rearing; 6- overwintering; 7- yearlincpresmolt/smolt

Attributes: CHEM - chemicals

CSTAB - channel stability

COMP - competition (with other species)

FLOW - flow

HABD - habitat diversity
NUTLD - nutrient load

OBST - obstructions

OXYG - dissolved oxygen

PATHO - pathogens

PRED - predators (includes fishing-related losses)
RIPCN - riparian condition

SEDLD - sediment load

TEMP - water temperature

WITH - water withdrawals

"NA" indicates that no life history pathway meeting the criteria used in defining these pathways was present.

Blank cells under attributes indicate no additional attributes with negative effects on productivity were evident compared to those present in the template. 
Stream: Grande Ronde R.

Reach: Wallowa R - Lookingglass Cr (RM 80.0 -83.4 from Snake R)

\begin{tabular}{|c|c|c|c|c|c|c|c|c|}
\hline \multicolumn{4}{|c|}{ [Restoration benefit category: } & $\bar{A}$ & \multicolumn{3}{|c|}{ Chinook usage score: } & 84.3 \\
\hline \multirow[b]{3}{*}{ Life stage } & \multirow[b]{3}{*}{ Months } & \multirow{2}{*}{\multicolumn{4}{|c|}{$\begin{array}{l}\text { No. vacant life history pathways } \\
\text { by abundance level }\end{array}$}} & \multicolumn{2}{|c|}{ Key habitat change } & \multirow{3}{*}{$\begin{array}{c}\text { Habitat } \\
\text { productivity } \\
\text { loss score }\end{array}$} \\
\hline & & & & & & \multirow{2}{*}{$\begin{array}{c}\text { Patient } \\
\text { quantity }\end{array}$} & \multirow[b]{2}{*}{$\%$ change } & \\
\hline & & Total no. & 1 & 2 & 3 & & & \\
\hline 1 & Apr-Aug & 228 & 152 & 68 & 8 & 26.1 & -4.1 & -516.4 \\
\hline $1,2,3$ & Aug-Oct & 80 & 70 & 10 & 0 & 7.6 & -37.5 & -487.8 \\
\hline 3 & Oct-Mar & 160 & 140 & 20 & 0 & 12.7 & -5.2 & -672.9 \\
\hline 3,4 & Mar-May & 108 & 90 & 18 & 0 & 33.3 & 0.0 & -105.7 \\
\hline 5 & May-Oct & 497 & 407 & 86 & 4 & 16.8 & -10.8 & -1353.9 \\
\hline 6 & Oct-Mar & 149 & 108 & 23 & 18 & 12.4 & -3.0 & -110.1 \\
\hline 7 & Mar-Jun & 82 & 54 & 24 & 4 & 84.0 & 0.0 & 30.1 \\
\hline
\end{tabular}

\begin{tabular}{|c|c|c|c|c|c|c|c|c|c|c|c|c|c|}
\hline \multirow[b]{2}{*}{ Life stage } & \multirow[b]{2}{*}{ Months } & \multicolumn{12}{|c|}{ Highest productivity impact attributes (\% change in impact from template) } \\
\hline & & Alt 1 & $\%$ change & Att 2 & $\%$ change & Att 3 & $\%$ change & Att 4 & $\%$ change & Att 5 & $\%$ change & Att 6 & $\%$ change \\
\hline 1 & Apr-Aug & TEMP & 50.0 & HABD & 37.5 & NUTLD & 37.5 & CHEM & 25.0 & FLOW & 25.0 & OXYG & 25.0 \\
\hline $1,2,3$ & Aug-Oct & NUTLD & 50.0 & SEDLD & 50.0 & TEMP & 50.0 & HABD & 37.5 & CHEM & 25.0 & CSTAB & 25.0 \\
\hline 3 & Oct-Mar & NUTLO & 50.0 & SEDLD & 50.0 & TEMP & 50.0 & CHEM & 25.0 & CSTAB & 25.0 & FLOW & 25.0 \\
\hline 3,4 & Mar-May & TEMP & 26.9 & FLOW & 25.0 & NÜTLLD & 250 & SEDLLD & 25.0 & PATHO & 18.7 & COMP & 13.5 \\
\hline 5 & May-Oct & TEMP & 26.0 & COMP & 13.0 & FLOW & 13.0 & $\mathrm{HABD}$ & 13.0 & PATHO & 13.0 & & \\
\hline 6 & Oct-Mar & SEDLD & 19.9 & TEMP & 10.4 & CSTAB & 10.0 & HABD & 10.0 & PATHO & 10.0 & RIPCN & 100 \\
\hline 7 & Mar-Jun & TEMP & 6.3 & & & & & & & & & & \\
\hline
\end{tabular}

Abundance level refers to estimated relative abundance of spawners that spawned in the natal stream reaches that populated the life history pathways using the reach in this table: Level 1: $\quad$ Low to average (relative to other reaches) abundance

Level 2 High (relative to other reaches) abundance

Level 3: Exceptionally high (relative to other reaches) abundance

Life stages: 1- prespawner; 2- spawner; 3- incubation; 4- fry colonization; 5- summer rearing; 6- overwintering; 7-yearlingresmolt/smolt

A/tributes: CHEM - chemicals

CSTAB - channel stability

COMP - competition (with other species)

FLOW - flow

HABD - habitat diversity
NUTLD - nutrient load

OBST - obstructions

OXYG - dissolved oxygen

PATHO - pathogens

PRED - predators (includes fishing-related losses)
RIPCN - riparian condition

SEDLD - sediment load

TEMP - $\quad$ water temperature

WITH - water withdrawals

"NA" indicates that no life history pathway meeting the criteria used in defining these pathways was present,

Blank cells under attributes indicate no additional attributes with negative effects on productivity were evident compared to those present in the template. 
Stream Reach Summary for Vacant Spring Chinook Salmon Life Histories

Stream: Grande Ronde R.

Reach: Lookingglass Cr - Gordon Cr (RM 83.4 -94.2 from Snake R)

\begin{tabular}{|llll|lll}
\hline Restoration benefit category: & A & Chinook usage score: & 105.2 & Habitat productivity loss score: & -3571.4 \\
\hline
\end{tabular}

\begin{tabular}{|c|c|c|c|c|c|c|c|c|}
\hline \multirow[b]{3}{*}{ Life stage } & \multirow[b]{3}{*}{ Months } & \multirow{2}{*}{\multicolumn{4}{|c|}{$\begin{array}{c}\text { No. vacant life history pathways } \\
\text { by abundance level }\end{array}$}} & \multicolumn{2}{|c|}{ Key habitat change } & \multirow{3}{*}{$\begin{array}{c}\text { Habitat } \\
\text { productivity } \\
\text { loss score }\end{array}$} \\
\hline & & & & & & \multirow{2}{*}{$\begin{array}{l}\text { Patient } \\
\text { quantity }\end{array}$} & \multirow[b]{2}{*}{$\%$ change } & \\
\hline & & Total no, & 1 & 2 & 3 & & & \\
\hline 1 & Apr-Aug & 216 & 106 & 102 & 8 & 12.3 & 2.1 & -575.0 \\
\hline 123 & Aug-Ott & 80 & 0 & 80 & 0 & 6.5 & 0.0 & -410.1 \\
\hline 3 & Ott-Mar & 160 & 0 & 160 & 0 & 7.9 & 0.0 & -716.1 \\
\hline 34 & Mar-May & 104 & 41 & 63 & 0 & 15.2 & 4.3 & -276.6 \\
\hline 5 & May-Ott & 386 & 150 & 232 & 4 & 9.7 & 1.4 & -1482.3 \\
\hline 6 & Ott-Mar & 303 & 189 & 113 & 1 & 8.0 & 0.0 & -133.0 \\
\hline 7 & Mar-Jun & 75 & 49 & 23 & 3 & 44.5 & 0.0 & 21.7 \\
\hline
\end{tabular}

\begin{tabular}{|c|c|c|c|c|c|c|c|c|c|c|c|c|c|}
\hline \multirow[b]{2}{*}{ Life stage } & \multirow[b]{2}{*}{ Months } & \multicolumn{12}{|c|}{ Highest productivity impact attributes (\% change in impact from template) } \\
\hline & & Att 1 & $\%$ change & Att 2 & $\%$ change & Att 3 & $\%$ change & Att 4 & $\%$ change & Att 5 & $\%$ change & Att 6 & $\%$ change \\
\hline 1 & Apr-Aug & FLOW & 50.0 & HABD & 500 & NUTLD & 500 & RIPCN & 50.0 & TEMP & 37.5 & CHEM & 25.0 \\
\hline $1,2,3$ & Aug-Oct & FLOW & 50.0 & HABD & 50.0 & NUTLD & 50.0 & RIPCN & 50.0 & SEDLD & 50.0 & TEMP & 500 \\
\hline 3 & Oct-Mar & NUTLD & 50.0 & SEDLD & 50.0 & TEMP & 500 & FLOW & 37.5 & CHEM & 25.0 & OXYG & 250 \\
\hline 3,4 & Mar-May & FLOW & 51.5 & TEMP & 51.5 & NUTLD & 34.4 & RIPCN & 34.4 & SEDLD & 21.5 & CHEM & 17.2 \\
\hline 5 & May-Oct & FLOW & 49.4 & TEMP & 49.4 & NUTLD & 32.9 & RIPCN & 32.9 & SEDLD & 17.1 & CHEM & 16.5 \\
\hline 6 & Oct-Mar & SEDLD & 12.0 & FLOW & 6.0 & HABD & 6.0 & PATHO & 6.0 & TEMP & 6.0 & & \\
\hline 7 & Mar-Jun & TEMP & 5.0 & & & & & & & & & & \\
\hline
\end{tabular}

Abundance level refers to estimated relative abundance of spawners that spawned in the natal stream reaches that populated the life history pathways using the reach in this table: Level 1: Low to average (relative to other reaches) abundance

Level 2: High (relative to other reaches) abundance

Level 3: Exceptionally high (relative to other reaches) abundance

Life stages.' 1- prespawner; 2- spawner; 3- incubation; 4- fry colonization 5- summer rearing; 6- overwintering; 7- yearling presmolt/smolt

\begin{tabular}{|c|c|c|c|}
\hline 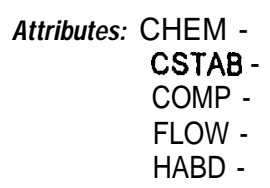 & $\begin{array}{l}\text { chemicals } \\
\text { channel stability } \\
\text { competition (with other species) } \\
\text { flow } \\
\text { habitat diversity }\end{array}$ & $\begin{array}{l}\text { NUTLD - nutrient load } \\
\text { OBST - obstructions } \\
\text { OXYG - dissolved oxygen } \\
\text { PATHO - pathogens } \\
\text { PRED - predators (includes fishing-related losses) }\end{array}$ & $\begin{array}{l}\text { RIPCN - } \quad \text { riparian condition } \\
\text { SEDLD - } \\
\text { TEdiment load } \\
\text { WEMP - } \quad \text { water temperature } \\
\text { WITH - } \quad \text { water withdrawals }\end{array}$ \\
\hline
\end{tabular}

"NA" indicates that no life history pathway meeting the criteria used in defining these pathways was present.

Blank cells under attributes indicate no additional attributes with negative effects on productivity were evident compared to those present in the template. 
Stream Reach Summary for Vacant Spring Chinook Salmon Life Histories

Stream: Grande Ronde R.

Reach: Gordon Cr - Phillips Cr (RM 94.2 -96.9 from Snake R)

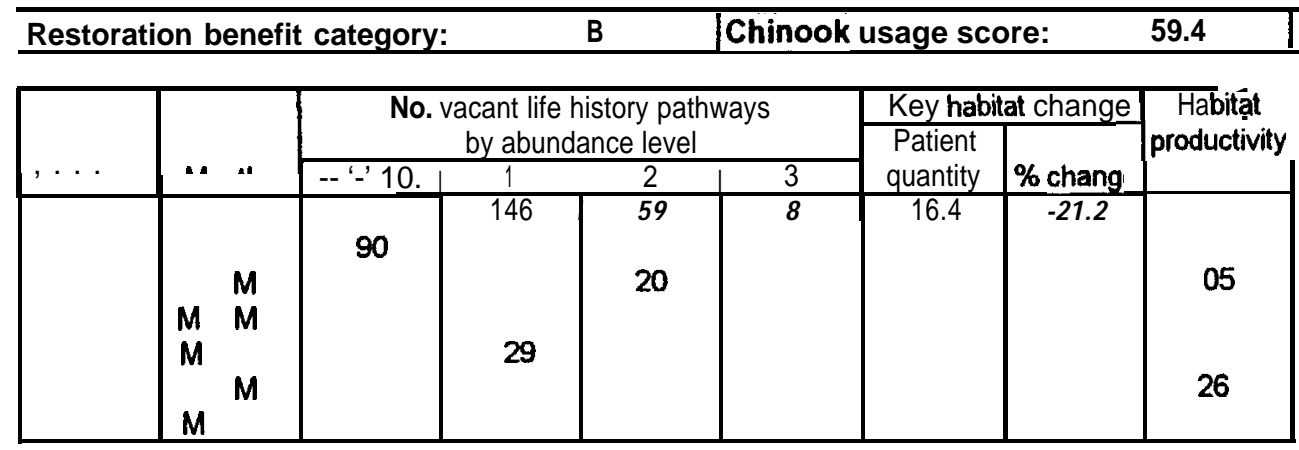

\begin{tabular}{|c|c|c|c|c|c|c|c|c|c|c|c|c|c|}
\hline \multirow[b]{2}{*}{ Life stage } & \multirow[b]{2}{*}{ Months } & \multicolumn{12}{|c|}{ Highest productivity impact attributes (\% change in impact from template) } \\
\hline & & Att 1 & $\%$ change & Att 2 & $\%$ change & Att 3 & $\%$ change & Att 4 & $\%$ change & Att 5 & $\%$ change & Att 6 & $\%$ change \\
\hline 1 & Apr-Aug & TEMP & 62,5 & NUTLD & 50.0 & FLOW & 37,5 & CHEM & 25.0 & HABD & 25.0 & OXYG & 25.0 \\
\hline $1,2,3$ & Aug-Ött & TEMP" & 75.0 & "FLOOW" & 50.0 & $\mathrm{HABD}$ & 50.0 & NüTLD & 50.0 & "RIPCN" & 50,0 & "S̈ËD̈L̈D”" & 50.0 \\
\hline '3"'”' & Ott-Mar & NUTLD & $50 ; 0$ & SEDLD & 50.0 & TEMP & 50.0 & CHEM & 25.0 & "FLOW" & 25.0 & OXYG & 25.0 \\
\hline 34 & Mar-May & FLOWW & 75.0 & TEMP & 75.0 & HABD & 50.0 & NuTiLo & 500 & RIPCN & $a \cdot$ & SEDLD & 45.7 \\
\hline 5 & May-Ott & FLOW & 52.7 & TEMP & 52.7 & $H A B D$ & 35.2 & NUTLD & 35.2 & RIPCN & & CHEM & 176 \\
\hline 6 & Ott-Mar & HABD & 9.3 & "PATHO & 9.3 & "SEDL'D" & 9.3 & "TÉM̀'P' & 9.3 & & & & \\
\hline 7 & Mar-Jun & TEMP & $5.0^{\circ}$ & & & & & & & & & & \\
\hline
\end{tabular}

Abundance level refers to estimated relative abundance of spawners that spawned in the natal stream reaches that populated the life history pathways using the reach in this table Level 1: Low to average (relative to other reaches) abundance

Level 2 High (relative to other reaches) abundance

Level 3: Exceptionally high (relative to other reaches) abundance

Life stages: 1 -prespawner; 2- spawner; 3- incubation; 4- fry colonization; 5- summer rearing; 6- overwintering; 7- yearling presmolt/smolt

Attributes.' CHEM - chemicals

CSTAB - channel stability

COMP - competition (with other species)

FLOW - ftOW

HABD - habitat diversity
NUTLD - nutrient load

OBST - obstructions

OXYG - dissolved oxygen

PATHO - pathogens

PRED - predators (includes fishing-related losses)
RIPCN - riparian condition

SEDLD - sediment load

TEMP - water temperature

WITH - $\quad$ water withdrawals

"NA" indicates that no life history pathway meeting the criteria used in defining these pathways was present.

Blank cells under attributes indicate no additional attributes with negative effects on productivity were evident compared to those present in the template. 
Stream Reach Summary for Vacant Spring Chinook Salmon Life Histories

Stream: Grande Ronde R

Reach: Phillips Cr - Clark Cr (RM 96.9 -97.3 from Snake R)

Restoration benefit category: B Chinook usage score: 53.3

Thabitat productivity loss score: $-2836.3$

\begin{tabular}{|c|c|c|c|c|c|c|c|c|}
\hline \multirow[b]{3}{*}{ Life stage } & \multirow[b]{3}{*}{ Months } & \multirow{2}{*}{\multicolumn{4}{|c|}{$\begin{array}{l}\text { No. vacant life history pathways } \\
\text { by abundance level }\end{array}$}} & \multicolumn{2}{|c|}{ Key habitat change } & \multirow{3}{*}{$\begin{array}{c}\text { Habital } \\
\text { productivity } \\
\text { loss score }\end{array}$} \\
\hline & & & & & & \multirow{2}{*}{$\begin{array}{l}\text { Patient } \\
\text { quantity }\end{array}$} & \multirow[b]{2}{*}{$\%$ change } & \\
\hline & & Total no. & 1 & 2 & 3 & & & \\
\hline 1 & Apr-Aug & 204 & 138 & 58 & 8 & 12.6 & -20.7 & -517.9 \\
\hline 12. & Aug-Oct & 90 & 80 & 10 & 0 & 5.9 & -61.1 & -548.9 \\
\hline 3 & Ött-Mar" & $180^{\circ}$ & 160 & 20 & 0 & & -59.5 & -9823 \\
\hline 34 & Mar-May & $57^{\circ}$ & 49 & 8 & 0 & 12.2 & -40.6 & -159.0 \\
\hline 5 & May-Ott & 155 & 126 & 25 & 4 & 20.2 & -3.2 & -619.5 \\
\hline 6 & Ott-Mar & 39 & 34 & 4 & 1 & 6.0 & -32.8 & -16.7 \\
\hline 7 & Mar-Jun & 55 & 35 & 17 & 3 & 35.5 & 3.7 & 7.9 \\
\hline
\end{tabular}

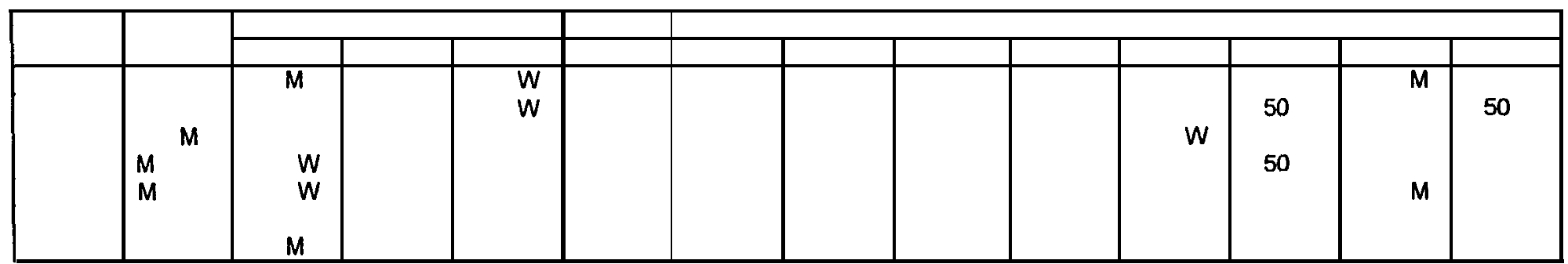

Abundance level refers to estimated relative abundance of spawners that spawned in the natal stream reaches that populated the life history pathways using the reach in this table: Level 1: Low to average (relative to other reaches) abundance

Level 2 High (relative to other reaches) abundance

Level 3: Exceptionally high (relative to other reaches) abundance

Life stages: 1- prespawner; 2- spawner; 3- incubation; 4- fry colonization; 5- summer rearing; 6- overwintering; 7- yearling presmolt/smolt

Attributes: CHEM - chemicals

CSTAB - channel stability

COMP - competition (with other species)

FLOW - flow

HABD - habitat diversity
NUTLD - nutrient load

OBST - obstructions

OXYG - dissolved oxygen

PATHO - pathogens

PRED - predators (includes fishing-related losses)
RIPCN - riparian condition

SEDLD - sediment load

TEMP - water temperature

WITH - water withdrawals

"NA" indicates that no life history pathway meeting the criteria used in defining these pathways was present.

Blank cells under attributes indicate no additional attributes with negative effects on productivity were evident compared to those present in the template. 
Stream Reach Summary for Vacant Spring Chinook Salmon Life Histories

Stream: Grande Ronde R.

Reach: Clark Cr - Indian Cr (RM 97.3 -99.3 from Snake R)

[Restoration benefit category:

Chinook usage score:

\begin{tabular}{l|l}
55.9 & Habitat productivity loss score:
\end{tabular}

$-2716.0$

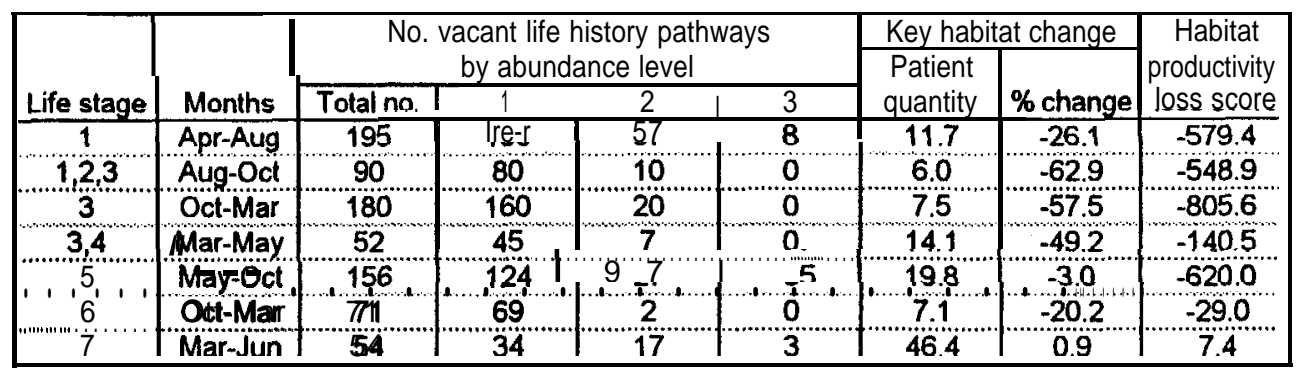

\begin{tabular}{|c|c|c|c|c|c|c|c|c|c|c|c|c|c|}
\hline \multirow[b]{2}{*}{ Life stage } & \multirow[b]{2}{*}{ Months } & \multicolumn{12}{|c|}{ Highest productivity impact attributes (\% change in impact from template) } \\
\hline & & Atf 1 & $\%$ change & Att 2 & $\%$ change & An 3 & $\%$ change & Att 4 & $\%$ change & Att 5 & $\%$ change & Att 6 & $\%$ change \\
\hline & Apr-Aug & TEMP & 62.5 & NUTLD & 50.0 & FLOW & 37.5 & CHEM & 25.0 & HABD & 25.0 & OXYG & 25.0 \\
\hline$,,, 1,, 2,3,$, & Aug-Ott & TEEMP & .75 .0 & FLOW & 50.0 & $\mathrm{HABD}$ & 50.0 & NUTLD & 50.0 & RIPCN & 50.0 & SEDLD & 50,0 \\
\hline 3 & Ott-Mar & NUTTLD & 50.0 & SEDLD & 50,0 & "TE"MP" & “50.0 & CHEM & 25.0 & FLOW & 25. & OXYG & 25. \\
\hline 3.4 & Mar-May & FLOW & 75. & "T̈ËM̈P̈" & $75.0^{\circ}$ & "HÄB̈' & 50.0 & RIPCN" & $50.0^{\circ}$ & NUTLD & 37. & "SEDLLD" & 37. \\
\hline 5 & May-Ott & FLOW & 47.5 & TE்MP & 47.5 & $\mathrm{HABD}$ & $31: 7$ & RIPCN & 31.7 & $\mathrm{CHEM}$ & 15.8 & COMP & 15.8 \\
\hline 6 & Ott-Mar & $\begin{array}{l}\text { TEMP } \\
\text { TEMP }\end{array}$ & $\begin{array}{l}10.4 \\
2.4\end{array}$ & CSTAB & 8.6 & ḢA்B் & 8.6 & "PATHÖO & 8.6 & RIPCN & 8.6 & SEDLD & 8.6 \\
\hline
\end{tabular}

Abundance level refers to estimated relative abundance of spawners that spawned in the natal stream reaches that populated the life history pathways using the reach in this table: Level 1: $\quad$ Low to average (relative to other reaches) abundance

Level 2 High (relative to other reaches) abundance

Level 3: Exceptionally high (relative to other reaches) abundance

Life stages: 1-prespawner; 2- spawner; 3- incubation; 4- fry colonization; 5- summer rearing; 6- overwintering; 7- yearlingresmolt/smolt

Attributes: CHEM - chemicals

CSTAB - channel stability

COMP - competition (with other species)

FLOW - flow

NUTLD - nutrient load

OBST - obstructions

OXYG - dissolved oxygen

RIPCN - riparian condition

HABD - habitat diversity

PATHO - pathogens

SEDLD - sediment load

TEMP - water temperature

PRED - predators (includes fishing-related losses)

"NA" indicates that no life history pathway meeting the criteria used in defining these pathways was present.

Blank cells under attributes indicate no additional attributes with negative effects on productivity were evident compared to those present in the template. 
Stream Reach Summary for Vacant Spring Chinook Salmon Life Histories

Stream: Grande Ronde R.

Reach: Indian $\mathrm{Cr}$ - Willow Cr (RM 99.3 -103.5 from Snake R)

\begin{tabular}{|ll|ll|ll}
\hline Restoration benefit category: & A & Chinook usage score: & 79.3 & Habitat productivity loss score: \\
\hline
\end{tabular}

\begin{tabular}{|c|c|c|c|c|c|c|c|c|}
\hline \multirow[b]{3}{*}{ Liie stage } & \multirow[b]{3}{*}{ Months } & \multirow{2}{*}{\multicolumn{4}{|c|}{$\begin{array}{c}\text { No. vacant life history pathways } \\
\text { by abundance level }\end{array}$}} & \multicolumn{2}{|c|}{ Key habitat change } & \multirow{3}{*}{$\begin{array}{c}\text { Habitat } \\
\text { productivity } \\
\text { loss score }\end{array}$} \\
\hline & & & & & & \multirow{2}{*}{$\begin{array}{l}\text { Patient } \\
\text { quantity }\end{array}$} & \multirow[b]{2}{*}{$\%$ change } & \\
\hline & & Total no. & 1 & 2 & 3 & & & \\
\hline 1 & Apr-Aug & 186 & 127 & 51 & $\%$ & & .37 & -688.6 \\
\hline $1,2,3$ & Aug-Ôtt & 90 & 90 & 0 & 0 & & fif. & -5489 \\
\hline 3 & Ött-Mar & 180 & $180^{\circ}$ & 0 & 0 & 70 & -63.4 & -982.3 \\
\hline 34 & Mar-May & 67 & 58 & $\ddot{g}$ & 0 & 14.7 & -59.3 & $\% ? ?$ \\
\hline 5 & May-Ott & 272 & 219 & 48 & & 2019 & & .1382 .0 \\
\hline 6 & Ött-Mar & 120 & 102 & 18 & & & " & -69.8 \\
\hline 7 & Mar-Jun & 51 & 31 & 17 & 3 & 46.2 & -0.7 & 13.2 \\
\hline
\end{tabular}

\begin{tabular}{|c|c|c|c|c|c|c|c|c|c|c|c|c|c|}
\hline \multirow[b]{2}{*}{ Life stage } & \multirow[b]{2}{*}{ Months } & \multicolumn{12}{|c|}{ Highest productivity impact attributes (\% change in impact from template) } \\
\hline & & Att 1 & $\%$ change & $\overline{\text { Att } 2}$ & $\%$ change & Att 3 & $\%$ change & Att 4 & $\%$ change & Att 5 & $\%$ change & Atf 6 & $\%$ change \\
\hline 1 & Apr-Aug & TEMP & 44.8 & FLOW & 29.8 & HABD & 29.8 & NUTLD & 29.8 & RIPCN & 29.8 & CHEM & 14.9 \\
\hline$, 1,2,3$ & Äug-Ött & TEMP & 75.0 & FLOW" & 50.0 & HABD & 50.0 & NUTTLE & 50.0 & RIPCN & 50.0 & "SEDELD" & 50.0 \\
\hline 3 & Oct-Mar & |“'NUTLD & 56.0 & SEDLD & $" 50,0$ & TEMP & 50.0 & CHEM & $25 ; 0$ & CSTAB & 25.0 & FLOW & 25.0 \\
\hline 34 & Mar-May & FLOW & 75.0 & TEMP & 75.0 & HABO & 5 & RIPCN & 50.0 & CHEM & 25.0 & COMP & 25.0 \\
\hline 5 & May-Ott & $\dot{\text { FLȮ் }}$ & ${ }^{* 6 g^{\circ}}$ & TËMP ' & 69.5 & $\mathrm{HABD}$ & 4 & RIPCN & $4 \dot{6} .3$ & "C̈HËM & 23.2 & COMP & 23.2 \\
\hline 6 & Ott-Mar & HABD & 14.1 & "РATHO & 14.1 & RIPCN' & 14.1 & SÉḊLD' & 14.1 & 'T̈ËM̈P' & 14.1 & & \\
\hline 7 & Mar-Jun & TEMP & 4.5 & & & & & & & & & & \\
\hline
\end{tabular}

Abundance level refers to estimated relative abundance of spawners that spawned in the natal stream reaches that populated the life history pathways using the reach in this table

Level 1: $\quad$ Low to average (relative to other reaches) abundance

Level 2 High (relative to other reaches) abundance

Level 3 Exceptionally high (relative to other reaches) abundance

Life stages; 1-prespawner; 2-spawner; 3- incubation; 4- fry colonization; 5- summer rearing; 6- overwintering; 7-yearling presmoltsmolt

Attributes: CHEM - chemicals

CSTAB - channel stability

COMP - competition (with other species)

FLOW - flow

HABD - habitat diversity
NUTLD - nutrient load

OBST - obstructions

OXYG - dissolved oxygen

PATHO - pathogens

PRED - predators (includes fishing-related losses)
RIPCN - riparian condition

SEDLD - sediment load

TEMP - water temperature

WITH - water withdrawals

"NA" indicates that no life history pathway meeting the criteria used in defining these pathways was present.

Blank cells under attributes indicate no additional attributes with negative effects on productivity were evident compared to those present in the template. 
Stream Reach Summary for Vacant Spring Chinook Salmon Life Histories

Stream: Grande Ronde R.

Reach: Downstream end of State Ditch - Upstream end of State Ditch (RM 114.5 -118.9 from Snake R)

\begin{tabular}{ll|ll|l|}
\hline Restoration benefit category: & A & Chinook usage score: & 105.1 & Habitat productivity loss score: \\
\hline
\end{tabular}

\begin{tabular}{|c|c|c|c|c|c|c|c|c|}
\hline \multirow[b]{3}{*}{ Life staqe } & \multirow[b]{3}{*}{ Months } & \multirow{2}{*}{\multicolumn{4}{|c|}{$\begin{array}{l}\text { No. vacant life history pathways } \\
\text { by abundance level }\end{array}$}} & \multicolumn{2}{|c|}{ Key habitat change } & \multirow{3}{*}{$\begin{array}{c}\text { Habitat } \\
\text { productivity } \\
\text { loss score }\end{array}$} \\
\hline & & & & & & \multirow{2}{*}{$\begin{array}{l}\text { Patient } \\
\text { quantity }\end{array}$} & \multirow[b]{2}{*}{$\%$ change } & \\
\hline & & Total no. & 1 & 2 & 3 & & & \\
\hline 1 & Apr-Aug & 148 & 112 & 30 & 6 & 10.5 & -51.2 & -524.4 \\
\hline $1,2,3$ & Aug-Oct & 90 & 80 & 10 & 0 & 2.5 & -75.7 & -505.4 \\
\hline 3 & Oct-Mar & $180^{\circ}$ & 160 & 20 & 0 & 0.0 & -100.0 & -1418.2 \\
\hline 3,4 & Mar-May & 87 & 60 & 19 & 8 & 1.5 & -97.3 & -651.0 \\
\hline 5 & May-Oct & 372 & 220 & 104 & 48 & 9.5 & -64.4 & -2551.3 \\
\hline 6 & Oct-Mar & 97 & 58 & 20 & 19 & 1.5 & -61.6 & -377.0 \\
\hline 7 & Mar-Jun & 29 & 24 & 3 & 2 & 36.3 & -10.5 & 8.5 \\
\hline
\end{tabular}

\begin{tabular}{|c|c|c|c|c|c|c|c|c|c|c|c|c|c|}
\hline \multirow[b]{2}{*}{ Life stage } & \multirow[b]{2}{*}{ Months } & \multicolumn{12}{|c|}{ Highest productivity impact attributes (\% change in impact from template) } \\
\hline & & Att 1 & $\%$ change & Att 2 & $\%$ change & Att 3 & $\%$ change & Att 4 & $\%$ change & Att 5 & $\%$ change & Att 6 & $\%$ change \\
\hline 1 & Apr-Aug & HABD & 75.0 & TEMP & 625 & FLOW & 50.0 & RIPCN & 28.1 & CHEM & 25.0 & NUTLD & 250 \\
\hline $1,2,3$ & Aug-Ött & RIPCN & 100.0 & 'Ḧ̈B̈B̈ & 75.00 & SEEDLD & 75.0 & "T̈ËM̈P̈P" & 62.5 & FLOW & 50.0 & C̈ं̈ंËM" & 25.0 \\
\hline 3 & Ott-Mar & RIPCN & 100.0 & SEDLD & 75.0 & CSTAB & 37.5 & FLOW & 37.5 & HABD & "'37.5 & PATHO & 12.5 \\
\hline$,, 3,4, .,$, & Mar-May & RIPCN & 87.5 & FLOW & 75.0 & $\mathrm{HABD}$ & 75.0 & CHEM & 50.0 & NUTTLD & 500 & OXYG & 50.0 \\
\hline 5 & May-Ott & FLOW & 72.9 & HABD & 72.9 & RIPCN & 72.9 & CHEM & 48.6 & NUTLD & 48.6 & OXYG & 48.6 \\
\hline $\begin{array}{l}6 \\
7\end{array}$ & $\begin{array}{l}\text { Ott-Mar } \\
\text { Mar-Jun }\end{array}$ & $\begin{array}{l}\text { FLOOW } \\
\text { HABD }\end{array}$ & $\begin{array}{l}49.4 \\
7.4\end{array}$ & HABD & .449 & RIPCN & 49.4 & S EDLD & 49.4 & CHEM & 16.5 & PATHO & 16.5 \\
\hline
\end{tabular}

Abundance /eve/ refers to estimated relative abundance of spawners that spawned in the natal stream reaches that populated the life history pathways using the reach in this table Level 1: $\quad$ Low to average (relative to other reaches) abundance

Level 2: $\quad$ High (relative to other reaches) abundance

Level 3: Exceptionally high (relative to other reaches) abundance

Life stages.' 1- prespawner; 2- spawner; 3- incubation; 4- fry colonization; 5- summer rearing; 6- overwintering; 7- yearling presmolt/smolt

$\begin{array}{cll}\text { Attributes: } \text { CHEM - chemicals } & \text { NUTLD - nutrient load } & \text { RIPCN - riparian condition } \\ \text { CSTAB - channel stability } & \text { OBST - obstructions } & \text { SEDLD - sediment load } \\ \text { COMP - competition (with other species) } & \text { OXYG - dissolved oxygen } & \text { TEMP - water temperature } \\ \text { FLOW - flow } & \text { PATHO - pathogens } & \text { WITH - water withdrawals } \\ \text { HABD - habitat diversity } & \text { PRED - predators (includes fishing-related losses) }\end{array}$

"NA" indicates that no life history pathway meeting the criteria used in defining these pathways was present.

Blank cells under attributes indicate no additional attributes with negative effects on productivity were evident compared to those present in the template. 
Stream Reach Summary for Vacant Spring Chinook Salmon Life Histories

Stream: Grande Ronde R.

Reach: Upstream end of State Ditch - i-84 crossing below Devil's Siide (RM 118.9 -128.4 from Snake R)

\begin{tabular}{|ll|ll|l}
\hline Restoration benefit category: & A & Chinook usage score: & 130.8 & Habitat productivity ioss score: \\
\hline
\end{tabular}

\begin{tabular}{|c|c|c|c|c|c|c|c|c|}
\hline \multirow[b]{3}{*}{ Life stage } & \multirow[b]{3}{*}{ Months } & \multirow{2}{*}{\multicolumn{4}{|c|}{$\begin{array}{l}\text { No. vacant life history pathways } \\
\text { by abundance level }\end{array}$}} & \multicolumn{2}{|c|}{ Key habitat change } & \multirow{3}{*}{$\begin{array}{l}\text { Habital } \\
\text { productivity } \\
\text { loss score }\end{array}$} \\
\hline & & & & & & \multirow{2}{*}{$\begin{array}{l}\text { Patient } \\
\text { quantity }\end{array}$} & \multirow[b]{2}{*}{$\%$ change } & \\
\hline & & Total no. & 1 & 2 & 3 & & & \\
\hline 1 & Apr-Aug & 139 & 104 & 29 & 6 & 2.3 & -62.5 & -593.5 \\
\hline $1,2,3$ & Aug-Oct & 90 & 80 & 10 & 0 & 1.1 & -90.8 & -546.8 \\
\hline 3 & Oct-Mar & 180 & 160 & 20 & ö & 1.7 & -81.4 & -995.7 \\
\hline 3,4 & Mar-May & 127 & 95 & 28 & 4 & 3.1 & -94.1 & -903.2 \\
\hline 5 & May-Oct & 476 & 377 & 96 & 3 & 27.2 & -25.2 & -3083.6 \\
\hline 6 & Oct-Mar & 151 & 151 & o & 0 & 10.7 & -37.5 & -401.6 \\
\hline 7 & Mar-Jun & 24 & 21 & 2 & 1 & 63.1 & 0.0 & -0.2 \\
\hline
\end{tabular}

\begin{tabular}{|c|c|c|c|c|c|c|c|c|c|c|c|c|c|}
\hline \multirow[b]{2}{*}{ Life stage } & \multirow[b]{2}{*}{ Months } & \multicolumn{12}{|c|}{ Highest productivity impact attributes (\% change in impact from template) } \\
\hline & & Art 1 & $\%$ change & Art 2 & $\%$ change & Att 3 & $\%$ change & Att 4 & $\%$ change & Att 5 & $\%$ change & Atf 6 & $\%$ change \\
\hline T & Apr-Aug & FLOW & 75.0 & TEMP & 62.5 & HA & 47.4 & SEI & 37. & RIPCN & 31.6 & CSTAB & 25.0 \\
\hline 123 & Aug-Oct & FLOWW & 75.0 & HABD & 75.0 & SEDLD & 75.0 & TE & $75 !$ & CSTAB & 50,0 & RIPCN & 50.0 \\
\hline 3 & Oct-Mar & FLOW & 75.0 & HABD & 75.0 & SEDLD & 75.0 & CSTAB & ‘50.0 & RIPCN & $50.0^{\prime \prime}$ & TEMP & 50.0 \\
\hline 3.4 & Mar-May & FLOWW & 75.0 & "HABD" & 75.0 & SEELED" & 75.0 & "TЁ̈P̈ & 75.0 & "CS̈TÄB" & 50.0 & OXYG & 50.0 \\
\hline 5 & May-Ott & F்LO்ம் & 70.4 & TÉMPP & 70.4 & HABD & 47.0 & OXYG & 47.0 & RIPCN & 47.0 & CHEM & 23.5 \\
\hline $\begin{array}{l}6 \\
7\end{array}$ & $\begin{array}{l}\text { Ott-Mar } \\
\text { Mar-Jun }\end{array}$ & $\begin{array}{l}\text { FLOWW } \\
\text { WiTH }\end{array}$ & $\begin{array}{r}51.1 \\
8.7\end{array}$ & "HABD" & 34.1 & "ÖB̈ṠT" & 34.1 & RIPCN" & 34.1 & "SEEDLD" & 34.1 & "C̈HËË" & 170 \\
\hline
\end{tabular}

Abundance level refers to estimated relative abundance of spawners that spawned in the natal stream reaches that populated the life history pathways using the reach in this table: Level 1: $\quad$ Low to average (relative to other reaches) abundance

Level 2 High (relative to other reaches) abundance

Level 3 Exceptionably high (relative to other reaches) abundance

Life stages: 1- prespawner; 2- spawner; 3- incubation; 4- fry colonization; 5- summer rearing; 6- overwintering; 7- yearling presmolt/smolt

Attributes: CHEM - chemicals

CSTAB - channel stability

COMP - competition (with other species)

FLOW - flow

HABD - habitat diversity
NUTLD - nutrient load

OBST - obstructions

OXYG - dissolved oxygen

PATHO - pathogens

PR ED - predators (includes fishing-related losses)
RIPCN - riparian condition

SEDLD - sediment load

TEMP - water temperature

WiTH - water withdrawals

"NA" indicates that no life history pathway meeting the criteria used in defining these pathways was present.

Blank cells under attributes indicate no additional attributes with negative effects on productivity were evident compared to those present in the template. 
Stream Reach Summary for Vacant Spring Chinook Salmon Life Histories

Stream: Grande Ronde R.

Reach: $\quad$ 1-84 crossing below Devil's Slide - Five Points Cr (RM 128.4 -133.4 from Snake R)

\begin{tabular}{|ll|ll|l}
\hline Restoration benefit category: & A & Chinook usage score: & 106.9 & Habitat productivity loss score: \\
\hline
\end{tabular}

\begin{tabular}{|c|c|c|c|c|c|c|c|c|}
\hline \multirow[b]{3}{*}{ Life stage } & \multirow[b]{3}{*}{ Months } & \multirow{2}{*}{\multicolumn{4}{|c|}{$\begin{array}{l}\text { No, vacant life history pathways } \\
\text { by abundance level }\end{array}$}} & \multicolumn{2}{|c|}{ Key habitat change } & \multirow{3}{*}{$\begin{array}{c}\text { Habitat } \\
\text { productivity } \\
\text { loss score }\end{array}$} \\
\hline & & & & & & \multirow{2}{*}{$\begin{array}{l}\text { Patient } \\
\text { quantity }\end{array}$} & \multirow[b]{2}{*}{$\%$ change } & \\
\hline & & Total no. & 1 & 2 & 3 & & & \\
\hline hin dicy & Apr-Aug & 130 & 56 & 38 & 36 & 3.0 & -47.8 & -548.4 \\
\hline 1.2 .3 & Aug-Ôtt & 90 & 0 & 30 & 60 & 6.5 & -46.7 & -710.4 \\
\hline 3 & Ött-Mar & 180 & 0 & 60 & 120 & 11.5 & 0.0 & -1156.0 \\
\hline 34 & Mar-Mày & 77 & 21 & 20 & 36 & 8.8 & -55.7 & -480.2 \\
\hline 5 & May-Ött & 243 & 70 & 79 & 94 & 14.7 & -28.8 & -805.9 \\
\hline 6 & Ott-Mar & 58 & 20 & 19 & $19^{\circ}$ & 1.9 & -40.3 & -89.7 \\
\hline 7 & Mar-Jun & 16 & 13 & 2 & 1 & 69.3 & 0.0 & $1.7^{\prime \prime \prime \cdots}$ \\
\hline
\end{tabular}

\begin{tabular}{|c|c|c|c|c|c|c|c|c|c|c|c|c|c|}
\hline \multirow[b]{2}{*}{ Life stage } & \multirow[b]{2}{*}{ Months } & \multicolumn{12}{|c|}{ Highest productivity impact attributes (\% change in impact from template) } \\
\hline & & $\overline{\text { Att } 1}$ & $\%$ change & At! 2 & $\%$ change & Att 3 & $\%$ change & Att 4 & $\%$ change & At! 5 & $\%$ change & Att 6 & $\%$ change \\
\hline 1 & Apr-Aug & RIPCN & 75 & TEMP & 75 & HABD & 62.5 & & & & & & \\
\hline $1,2,3$ & Aug-Oct & RIPCN & 75 & SEDLD & 75 & TEMP & 75.0 & $\begin{array}{llll}H & A & B & D^{-}\end{array}$ & & CSTAB & 50,0 & & \\
\hline 3 & Ott-Mar & SEDLD & 75.0 & TËMP “ & 75.0 & CSTAB & 50.0 & FLOW & 25.0 & HABD & 25.0 & RIPCN & 25.0 \\
\hline 3,4 & M̈ar-M̈ay & RIPCN & 38.5 & TEM"P" & 38.5 & CSTAB & 33.7 & SEDEL & 33.7 & HABD & 25.7 & FLOOW" & 225 \\
\hline 5 & May-Ott & RIPCN & 38.0 & TEMP & 38.0 & HABD & 253 & CSTAB & 15.4 & SEDLD & 15.4 & PATHO & 12.7 \\
\hline $\begin{array}{l}\dddot{6} . \\
7\end{array}$ & Ött-Mar & CSTAB & 21.6 & $\because \mathrm{HABD}$ & 21.6 & RIPCON & 21.6 & ŠÉËL̈D̆ & 21.6 & PATHO & 10.8 & TËM̈P & 10.8 \\
\hline
\end{tabular}

Abundance level refers to estimated relative abundance of spawners that spawned in the natal stream reaches that populated the life history pathways using the reach in this table: Level 1: Low to average (relative to other reaches) abundance

Level 2 High (relative to other reaches) abundance

Level 3: Exceptionally high (relative to other reaches) abundance

Life stages: 1- prespawner; 2-spawner; 3- incubation; 4- fry colonization; 5- summer rearing; 6- overwintering; 7- yearlingresmolt/smolt

Attributes: CHEM - chemicals

CSTAB - channel stability

COMP - competition (with other species)

FLOW - flow

HABD - habitat diversity
NUTLD - nutrient load

OBST - obstructions

OXYG - dissolved oxygen

PATHO - pathogens

PRED - predators (includes fishing-related losses)
RIPCN - riparian condition

SEDLD - sediment load

TEMP - water temperature

WITH - water withdrawals

"NA" indicates that no life history pathway meeting the criteria used in defining these pathways was present.

Blank cells under attributes indicate no additional attribute with negative effects on productivity were evident compared to those present in the template. 


\section{Stream Reach Summary for Vacant Spring Chinook Salmon Life Histories}

Stream: Grande Ronde R.

Reach: Five Points $\mathrm{Cr}-$ Rock $\mathrm{Cr}(\mathrm{RM} 133.4-133.8$ from Snake R)

\begin{tabular}{|ll|ll|l|}
\hline Restoration benefit category: & A & Chinook usage score: & 60.3 & Habitat productivity loss score: \\
\hline
\end{tabular}

\begin{tabular}{|c|c|c|c|c|c|c|c|c|}
\hline \multirow[b]{3}{*}{ Life stage } & \multirow[b]{3}{*}{ Months } & \multirow{2}{*}{\multicolumn{4}{|c|}{$\begin{array}{c}\text { No. vacant life history pathways } \\
\text { by abundance level }\end{array}$}} & \multicolumn{2}{|c|}{ Key habitat change } & \multirow{3}{*}{$\begin{array}{c}\text { Habitat } \\
\text { productivity y } \\
\text { loss score }\end{array}$} \\
\hline & & & & & & \multirow{2}{*}{$\begin{array}{l}\text { Patient } \\
\text { quantity }\end{array}$} & \multirow[b]{2}{*}{$\%$ change } & \\
\hline & & Total no. & 1 & 2 & 3 & & & \\
\hline 1 & Apr-Aug & 121 & 86 & 35 & 0 & 2.1 & -46.7 & -496.9 \\
\hline 123 & Aug-Ött & 90 & 60 & 30 & 0 & 5.1 & -46.7 & -710.4 \\
\hline 3 & Ött-Mar & 180 & 120 & 60 & 0 & 10.8 & 0.0 & -1156.0 \\
\hline 3.4 & Mar-M̈ay & 62 & 37 & 25 & 0 & 8.1 & -59.7 & -414.5 \\
\hline 5 & May-Ött & 126 & 68 & 58 & “o & 10.2 & -46.5 & -683.6 \\
\hline 6 & Ott-Mar & 22 & 3 & 19 & 0 & 1.7 & $-85,6$ & -72.3 \\
\hline 7 & Mar-Jun & 13 & 12 & 1 & "O & 49.5 & 0.0 & 1.0 \\
\hline
\end{tabular}

\begin{tabular}{|c|c|c|c|c|c|c|c|c|c|c|c|c|c|}
\hline \multirow[b]{2}{*}{ Life stage } & \multirow[b]{2}{*}{ Months } & \multicolumn{5}{|c|}{ Highest productivity im } & \multicolumn{7}{|c|}{ act attributes (\% change in impact from template) } \\
\hline & & Att 1 & $\%$ change & Att 2 & $\%$ change & Att 3 & $\%$ change & Att 4 & $\%$ change & Att 5 & $\%$ change & Att 6 & $\%$ change \\
\hline 1 & Apr-Aug & TEMP & 75.0 & RIPCN & 37.5 & SEDLD & 37.5 & HABD & 34.5 & CSTAB & 25.0 & & \\
\hline $1,2,3$ & Aug-Ött & HABD & 75.0 & RIPCN & 75.0 & SEDLD & 75.0 & TEMP & 75.0 & CSTAB & 50.0 & & \\
\hline 3 & Ott-Mar & SEDLD & "7s:6"، & TEMP & 75.0 & CSTAB & 50.0 & FLOW & 50.0 & HABD & 50.0” & RIPCN & 50.0 \\
\hline$,,,,,,, 3,4$. & Mar-May & RIPCN & 75.0 & TEMP & 75.0 & SEDLD & 50.6 & $\dot{H} \dot{A} \dot{B} \dot{D}$ & $50^{\circ} .0^{\circ}$ & ச்ட்Óல் & $42.9^{\circ}$ & CSTAB & 33.8 \\
\hline 5 & May-Ott & RIPCN & 58,2 & TEMP & 58.2 & HABD & 38. & PATHO & 't 9.4 & & & & \\
\hline $\begin{array}{l}6 \\
7\end{array}$ & $\begin{array}{l}\text { Oct-Mar } \\
\text { Mar-Jun }\end{array}$ & CSTAB & !?,!. & $\mathrm{HABD}$ & 45.4 & RIPCN & 45.4 & SEEDED & 45,4 & PATTHO & 22.7 & TE MP & 22 \\
\hline
\end{tabular}

Abundance lovel refers to estimated relative abundance of spawners that spawned in the natal stream reaches that populated the life history pathways using the reach in this table: Level 1: $\quad$ Low to average (relative to other reaches) abundance

Level 2 High (relative to other reaches) abundance

Level 3: Exceptionally high (relative to other reaches) abundance

Life stages: 1- prespawner; 2- spawner 3- incubation; 4- fry colonization; 5- summer rearing; 6- overwintering; 7- yearling presmolt/smolt

Attributes: CHEM - chemicals

CSTAB - channel stability

COMP - competition (with other species)

FLOW - flow

HABD - habitat diversity
NUTLD - nutrient load

OBST - obstructions

OXYG - dissolved oxygen

PATHO - pathogens

PRED - predators (includes fishing-related losses)
RIPCN - riparian condition

SEDLD - sediment load

TEMP - water temperature

WITH - water withdrawals

"NA" indicates that no life history pathway meeting the criteria used in defining these pathways was present,

Blank cells under attributes indicate no additional attributes with negative effects on productivity were evident compared to those present in the template. 
Stream: Grande Ronde R.

Reach: Rock $\mathrm{Cr}$ - Whiskey $\mathrm{Cr}$ (RM 133.8 -136.6 from Snake R)

\begin{tabular}{|ll|ll|l}
\hline Restoration benefit category: & A & Chinook usage score: & 57.4 & Habitat productivity loss score: \\
\hline
\end{tabular}

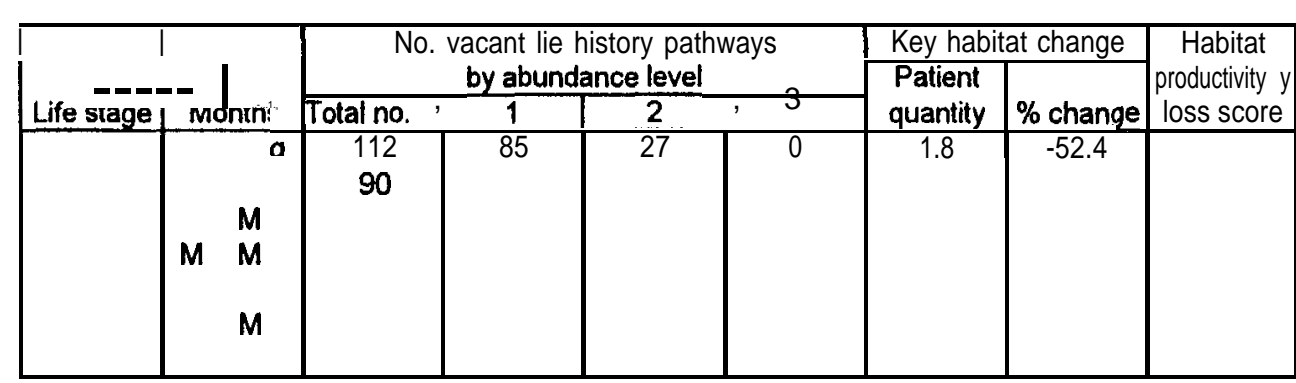

\begin{tabular}{|c|c|c|c|c|c|c|c|c|c|c|c|c|c|}
\hline \multirow[b]{2}{*}{ Life stage } & \multirow[b]{2}{*}{ Months } & \multicolumn{12}{|c|}{ Highest productivity impact attributes (\% change in impact from template) } \\
\hline & & Att 1 & $\%$ change & Att 2 & $\%$ change & Att 3 & $\%$ change & Att 4 & $\%$ change & Att 5 & $\%$ change & Att 6 & $\%$ change \\
\hline & Apr-Aug & RIPCN & 75.0 & TEMP. & 75.0 . & HABD & 62.5 & & & & & & \\
\hline $1,2,3$ & Aug-Ott & RIPCN & 75.0 & SEEDLD & 75,0 & '"TËËP̈P' & 75,0 & HABDi & 62.5 & CSTAB & 50.0 & & \\
\hline 3 & Ott-Mar & SEDLD & 75.0 & TEMP & 75.0 & CSTAB & 50.0 & FLOW & 25.0 & HABD & 25.0 & RIPCN & '25.0 \\
\hline 3,4 & Mar-May & RIPCN & 38.5 & "'TंËM̀iP" & 38.5 & HABD & 25.7 & "CSTABA' & 25.4 & SEDLD & 25.4 & FLÓW & 16.9 \\
\hline 5 & May-Ott & RIPCN & 37.9 & TEMP & 37,9 & $\mathrm{HABD}$ & “25.3”” & PATHO & 12,6 & CSTAB & 9.0 & SEDLD & 9.0 \\
\hline $\begin{array}{l}6 \\
7 \\
\end{array}$ & $\begin{array}{l}\text { Ott-Mar } \\
\text { Mar-Jun }\end{array}$ & CSTAB & 27.9 & $\mathrm{HABD}$ & 27,9 & RIPCAN & 27.9 & SEDLD & 27.9 & РАTHO & 13.9 & TEMP & 13.9 \\
\hline
\end{tabular}

Abundance/eve/refers to estimated relative abundance of spawners that spawned in the natal stream reaches that populated the life history pathways using the reach in this table: Level 1: Low to average (relative to other reaches) abundance

Level 2: $\quad$ High (relative to other reaches) abundance

Level 3 Exceptionally high (relative to other reaches) abundance

Life stages: 1- prespawner; 2- spawner; 3- incubation; 4- fry colonization; 5- summer rearing; 6- overwintering; 7- yearling presmolt/smolt

Attributes: CHEM - chemicals

CSTAB - channel stability

COMP - competition (with other species)

FLOW - flow

HABD - habitat diversity
NUTLD - nutrient load

OBST - obstructions

OXYG - dissolved oxygen

PATHO - pathogens

PRED - predators (includes fishing-related losses)
RIPCN - riparian condition

SEDLD - sediment load

TEMP - $\quad$ water temperature

WITH - $\quad$ water withdrawals

"NA" indicates that no life history pathway meeting the criteria used in defining these pathways was present.

Blank cells under attributes indicate no additional attributes with negative effects on productivity were evident compared to those present in the template. 
Stream Reach Summary for Vacant Spring Chinook Salmon Life Histories

Stream: Grande Ronde R.

Reach: Whiskey $\mathrm{Cr}$ - Jordan $\mathrm{Cr}$ (RM 136.6 -139.1 from Snake R)

\begin{tabular}{|c|c|c|c|c|c|c|c|c|c|c|c|c|c|}
\hline \multicolumn{4}{|c|}{ Restoration benefit category: } & $\bar{A}$ & \multicolumn{3}{|c|}{ Chinook usage score: } & 61.7 & \multicolumn{4}{|c|}{\begin{tabular}{|l|} 
Habitat productivity loss score: \\
\end{tabular}} & -3524.0 \\
\hline \multirow[b]{3}{*}{ Life stage } & \multirow[b]{3}{*}{ Months } & \multirow{2}{*}{\multicolumn{4}{|c|}{$\begin{array}{l}\text { No. vacant life history pathways } \\
\text { by abundance level }\end{array}$}} & \multicolumn{2}{|c|}{ Key habitat change } & \multirow{3}{*}{$\begin{array}{c}\text { Habilat } \\
\text { productivity } \\
\text { loss score }\end{array}$} & & & & & \\
\hline & & & & & & \multirow{2}{*}{$\begin{array}{l}\text { Patient } \\
\text { quantity }\end{array}$} & \multirow[b]{2}{*}{$\%$ change } & & & & & & \\
\hline & & Total no. & 1 & 2 & 3 & & & & & & & & \\
\hline 1 & Apr-Aug & 103 & 68 & 35 & 0 & 1.9 & -59.9 & -493.0 & & & & & \\
\hline $1,2,3$ & Aug-Oct & 90 & 50 & 40 & 0 & 6.2 & -467 & -710.4 & & & & & \\
\hline 3 & oct-Mar & 180 & 100 & 80 & 0 & 8.3 & 0.0 & -1156.0 & & & & & \\
\hline 34 & Mar-May & 57 & 31 & 26 & 0 & 5.0 & -65.0 & -414.6 & & & & & \\
\hline 5 & May-Oct & 119 & 68 & 51 & 0 & 9.9 & -49.3 & .682 .8 & & & & & \\
\hline 6 & Oct-Mar & 19 & 19 & 0 & 0 & 1.4 & -93.3 & -68.1 & & & & & \\
\hline 7 & Mar-Jun & 10 & $10^{\prime}$ & o & 0 & 30.3 & 0.0 & 0.9 & & & & & \\
\hline \multirow[b]{2}{*}{ Life stage } & \multirow[b]{2}{*}{ Months } & \multicolumn{12}{|c|}{ Highest productivity impact attributes (\% change in impact from template) } \\
\hline & & Att 1 & $\%$ change & Att 2 & $\%$ change & Att 3 & $\%$ change & Att 4 & $\%$ change & Att 5 & $\%$ change & Att 6 & $\%$ change \\
\hline 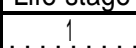 & Apr-Aug & TEMP & 75.0 & HABD & 45.6 & RIPCN & 45.6 & SEDLD & 37.5 & CSTAB & 25.0 & & \\
\hline$\ddot{1}, 2,3$ & Aug-Ött & HABD & 75.0 & RIPCN & 75.0 & SEDLD & 75.0 & TEMP & $7 \dot{5} .0$ & CSTAB & $50.0^{\circ}$ & & \\
\hline 3 & Ott-Mar & SEDLD & 75.0 & TEMP & 75.0 & CSTAB & 50.0 & FLOW' & 50.0 & HABD & 50,0 & RIPCN & 50.0 \\
\hline 34 & Mar-May. & RIPCN & 75.0 & SEDQLCD & 75.0 & TEMP & 75.0 & CSTAB & $50.0^{\circ}$ & HABD & $50.0^{\circ}$ & FLOW & 38.4 \\
\hline 4 & May-Ott & RIPCN & 61.6 & TEMP & 61,6 & HABD & 41.0 & "PATHO & 20,5 & & & & \\
\hline $\begin{array}{l}6 \\
7\end{array}$ & $\begin{array}{l}\text { Ott-Mar } \\
\text { Mar-Jun }\end{array}$ & CSTAB & $50.0^{\circ}$ & HABD & 50.0 & RIPCN & $500^{\circ}$ & SEDLD & 50,0 & PATHO & $25.0^{\circ}$ & "TEMMP" & $25.0^{\circ}$ \\
\hline
\end{tabular}

Abundance level refers to estimated relative abundance of spawners that spawned in the natal stream reaches that populated the life history pathways using the reach in this table: Level 1: Low to average (relative to other reaches) abundance

Level 2 High (relative to other reaches) abundance

Level 3: $\quad$ Exceptionally high (relative to other reaches) abundance

Life stages: 1- prespawner; 2- spawner; 3- incubation; 4- fry colonization; 5- summer rearing; 6-overwintering; 7-yearling presmoltssmolt

Attributes: CHEM - chemicals

CSTAB - channel stability

COMP - competition (with other species)

FLOW - flow

HABD - habitat diversity
NUTLD - nutrient load

OBST - obstructions

OXYG - dissolved oxygen

PATHO - pathogens

PRED - predators (includes fishing-related losses)
RIPCN - riparian condition

SEDLD - sediment load

TEMP - water temperature

WITH - water withdrawals

"NAMindicates that no life history pathway meeting the criteria used in defining these pathways was present.

Blank cells under attributes indicate no additional attributes with negative effects on productivity were evident compared to those present in the template. 
Stream Reach Summary for Vacant Spring Chinook Salmon Life Histories

Stream: Grande Ronde R.

Reach: Jordan $\mathrm{Cr}$ - Beaver Cr (RM 139.1 -145.8 from Snake R)

\begin{tabular}{ll|ll|lll}
\hline [Restoration benefit category: & D & Chinook usage score: & 32.1 & Habitat productivity loss score: & -1605.3 \\
\hline
\end{tabular}

\begin{tabular}{|c|c|c|c|c|c|c|c|c|}
\hline & & \multicolumn{4}{|c|}{ No vacant life history pathways } & Key habitat change & Habital \\
productivity
\end{tabular}

\begin{tabular}{|c|c|c|c|c|c|c|c|c|c|c|c|c|c|}
\hline \multirow[b]{2}{*}{ Life stage } & \multirow[b]{2}{*}{ Months } & \multicolumn{12}{|c|}{ Highest productivity impact attributes (\% change in impact from template) } \\
\hline & & Att 1 & $\%$ change & $\overline{\text { Att } 2}$ & $\%$ change & Att 3 & \% change & $\overline{\text { Aft } 4}$ & $\%$ change & Att 5 & $\%$ change & Att 6 & $\%$ change \\
\hline 1 & Apr-Aug & RIPCN & 75.0 & TEMP & 75.0 & HABD & 62.5 & & & & & & \\
\hline $1,2,3$ & Aug-Oct & RIPCN & 75.0 & 'S̈ĖḊL̇D̈ & 75.0 & TEMP & 75.0 & HÄB̈ & 62.5 & CSTAB' & 50.0 & & \\
\hline 3 & Oct-Mar & SEDLD & 75.0 & TEMP & 750"”' & CSTAB & 50.0 & FLOW" "'" & 25.0 & HABD & 25.0" & RIPCN & 25.0 \\
\hline 3.4 & Mar-May & CSTAB & 24.4 & SEDLD & 24.4 & TEMP & 24.4 & "F்'ÖẄ & 10.2 & HABD & 16.2 & RIPCN & 16.2 \\
\hline 5 & May-Oct & CSTAB & 12.7 & $\mathrm{HABD}$ & 127 & RIPCN & "«" $12 ; 7$ & SEDLD & 12.7 & TEMP & $\dot{9} .2$ & PÄTHO & 6.3 \\
\hline 6 & Oct-Mar & CSTAB & 6.2 & ப்ÄB் & 6.2 & RIPCN & 6.2 & SEDLD & 6.2 & PATHO & ?1 & "TËMP" & 3 \\
\hline 7 & Mar-Jun & & & & & & & & & & & & \\
\hline
\end{tabular}

Abundance level refera to estimated relative abundance of spawners that spawned in the natal stream reaches that populated the life history pathways using the reach in this table: Levei 1: Low to average (relative to other reaches) abundance

Levei 2: High (relative to other reaches) abundance

Level 3: Exceptionally high (relative to other reaches) abundance

Life stages: 1- prespawner; 2- spawner; 3- incubation; 4- fry colonization; 5- summer rearing; 6- overwintering; 7- yearling presmolt/smolt

$\begin{array}{lll}\text { Attributes: } \text { CHEM - chemicals } & \text { NUTLD - nutrient load } & \text { RIPCN - riparian condition } \\ \text { CSTAB - channel stability } & \text { OBST - obstructions } & \text { SEDLD - sediment ioad } \\ \text { COMP - competition (with other species) } & \text { OXYG - dissolved oxygen } & \text { TEMP - water temperature } \\ \text { FLOW - flow } & \text { PATHO - pathogens } & \text { WITH - water withdrawals } \\ \text { HABD - habitat diversity } & \text { PRED - predators (includes fishing-related losses) }\end{array}$

"NA" indicates that no life history pathway meeting the criteria used in defining these pathways was present.

Blank cells under attributes indicate no additional attributes with negative effects on productivity were evident compared to those present in the template. 
Stream: Grande Ronde R.

Reach: Meadow Cr - Fly Cr (RM 147.0 -151.3 from Snake R)

\begin{tabular}{|c|c|c|c|c|c|c|c|c|}
\hline \multicolumn{4}{|c|}{ Restoration benefit category: } & & \multicolumn{3}{|c|}{ Chinook usage score: } & 57.9 \\
\hline \multirow[b]{3}{*}{ Life stage } & \multirow[b]{3}{*}{ Months } & \multirow{2}{*}{\multicolumn{4}{|c|}{$\begin{array}{l}\text { No. vacant life history pathways } \\
\text { by abundance level }\end{array}$}} & \multicolumn{2}{|c|}{ Key habitat change } & \multirow{3}{*}{$\begin{array}{c}\text { Habitat } \\
\text { productivity } \\
\text { loss score }\end{array}$} \\
\hline & & & & & & \multirow{2}{*}{$\begin{array}{l}\text { Patient } \\
\text { quantity }\end{array}$} & \multirow[b]{2}{*}{$\%$ change } & \\
\hline & & Total no. & 1 & 2 & 3 & & & \\
\hline 1 & Apr-Aug & 64 & 47 & 17 & 0 & 6.5 & -38.7 & -281.0 \\
\hline $1,2,3$ & Aug-Oct & 50 & 30 & 20 & 0 & 4.9 & -60.0 & -450.8 \\
\hline 3 & Oct-Mar & 100 & 60 & 40 & 0 & 6.2 & -60.0 & -378.8 \\
\hline 3,4 & Mar-May & 60 & 48 & 12 & 0 & 8.6 & -60.0 & -369.6 \\
\hline 5 & May-Oct & 238 & 218 & 20 & ఝ & 6.1 & -58.1 & -915.4 \\
\hline 6 & Oct-Mar & 39 & 39 & 0 & 0 & 6.2 & -59.1 & -87.8 \\
\hline 7 & Mar-Jun & 5 & 5 & 0 & 0 & 28.7 & 0.0 & 1.0 \\
\hline
\end{tabular}

\begin{tabular}{|c|c|c|c|c|c|c|c|c|c|c|c|c|c|}
\hline \multirow[b]{2}{*}{ Life stage } & \multirow[b]{2}{*}{ Months } & \multicolumn{12}{|c|}{ Highest productivity impact attributes (\% change in impact from template) } \\
\hline & & Att 1 & $\%$ change & Atf 2 & $\%$ change & Att 3 & $\%$ change & Att 4 & $\%$ change & Att 5 & $\%$ change & Att 6 & $\%$ change \\
\hline 1 & Apr-Aug & TEMP & 48.4 & RIPCN & 32.3 & $\mathrm{HABD}$ & 19.1 & & & & & & \\
\hline $1,2,3$ & Aug-Ott & TEMP' & 75.0 & "CST̈ÄB & 50.0 & "RIPCN" & 50.0 & "S̈ËD̈L̈D̈" & $50.0^{\circ}$ & HÄB̈D & 37.5 & & \\
\hline 3 & Ott-Mar & CSTAB & 50.0 & SEDLD & 50.0 & TEMP & ‘50;0 & RIPCN & 25.0 & FLOW' & 12.5 & HABD & 12.5 \\
\hline 3,4 & Mar-May & TEMP. & 75.0 & RIPCN & 50.0 & CSTAB & 25.0 & HABD & 25.0 & PATHO & 25.0 & SEEDLD & 25.0 \\
\hline "'5. & May-Ott & TEMP & 74,4 & RIPCN & 49.6 & CSTAB & 26.2 & SEDLD & 26.2 & HABD & 24.8 . & PATHO & 24.8 \\
\hline $\begin{array}{l}6 \\
7\end{array}$ & $\begin{array}{l}\text { Ott-Mar } \\
\text { Mar-Jun }\end{array}$ & CSTAB & 49,2 & RIPCN & 49.2 & SEDLD & 49.2 & PATHO & 24.6 & TEMP & 246 & & \\
\hline
\end{tabular}

Abundance /eve/ refers to estimated relative abundance of spawners that spawned in the natal stream reaches that populated the life history pathways using the reach in this table:

Level 1: $\quad$ Low to average (relative to other reaches) abundance

Level 2: High (relative to other reaches) abundance

Level 3: Exceptionally high (relative to other reaches) abundance

Life stages: 1 - prespawner; 2- spawner; 3- incubation; 4- fry colonization; 5- summer rearing; 6- overwintering; 7- yearling presmolt/smolt

Attributes: CHEM - chemicals

CSTAB - channel stability

COMP - competition (with other species)

FLOW - flow

HABD - habitat diversity
NUTLD - nutrient load

OBST - obstructions

OXYG - dissolved oxygen

PATHO - pathogens

PRED - predators (includes fishing-related losses)
RIPCN - riparian condition

SEDLD - sediment load

TEMP - water temperature

WITH - water withdrawals

"NA" indicates that no life history pathway meeting the criteria used in defining these pathways was present.

Blank cells under attributes indicate no additional attributes with negative effects on productivity were evident compared to those present in the template. 


\section{Stream Reach Summary for Vacant Spring Chinook Salmon Life Histories}

Stream: Grande Ronde R.

Reach: Fly $\mathrm{Cr}$ - head of canyon $\mathbf{1 . 3}$ miles downstream of Sheep $\mathrm{Cr}$ (RM 151.3 -159.4 from Snake R)

[Restoration benefit category:

B

Chinook usage score:

\begin{tabular}{l|l}
56.2 & Habitat productivity loss score:
\end{tabular}

$-1826.5$

\begin{tabular}{|c|c|c|c|c|c|c|c|c|}
\hline \multirow[b]{3}{*}{ Life stage } & \multirow[b]{3}{*}{ Months } & \multirow{2}{*}{\multicolumn{4}{|c|}{$\begin{array}{c}\text { No. vacant life history pathways } \\
\text { by abundance level }\end{array}$}} & \multicolumn{2}{|c|}{ Key habitat change } & \multirow{3}{*}{$\begin{array}{c}\text { Habitat } \\
\text { productivity } \\
\text { loss score }\end{array}$} \\
\hline & & & & & & \multirow{2}{*}{$\begin{array}{l}\text { Patient } \\
\text { quantity }\end{array}$} & \multirow[b]{2}{*}{$\%$ change } & \\
\hline & & Total no. & 1 & 2 & 3 & & & \\
\hline 1 & Apr-Aug & 59 & 39 & 20 & 0 & 8.1 & & -216.0 \\
\hline $1,2,3$ & Aug-Ott & 50 & 20 & 30 & 0 & 4.8 & & -456.7 \\
\hline 3 & Ott-Mar & 100 & 40 & 60 & 0 & 5.6 & -61.8 & -378.8 \\
\hline 3,4 & , Mar;May, & 55 & 46 & 9 & 0 & 8.3 & -60.0 & -346.9 \\
\hline 5 & May-Ott & 140 & 140 & 0 & 0 & 7.0 & -71.1 & .385 .1 \\
\hline 6 & Ott-Mar & 19 & 19 & 0 & 0 & 5.7 & -61.9 & -43.6 \\
\hline 7 & Mar-Jun & 3 & 3 & 0 & 0 & 27.4 & 0.0 & 0.6 \\
\hline
\end{tabular}

\begin{tabular}{|c|c|c|c|c|c|c|c|c|c|c|c|c|c|}
\hline \multirow[b]{2}{*}{ Life stage } & \multirow[b]{2}{*}{ Months } & \multicolumn{12}{|c|}{ Highest productivity impact attributes (\% change in impact from template) } \\
\hline & & Atf 1 & $\%$ change & Atf 2 & $\%$ change & Att 3 & $\%$ change & Att 4 & $\%$ change & Att 5 & $\%$ change & Att 6 & $\%$ change \\
\hline 1 & Apr-Aug & TEMP & 62.5 & RIPCN & 32.3 & CSTAB & 25.0 & HABD & 25.0 & SEDLD & 250 & & \\
\hline $1,2,3$ & Aug-Ött & TEMP & $75.0^{\circ}$ & CSTAB & 50.0 & RIPCN" & 50.0 & SEDLD & 50.0 & HABD & $25.0^{\circ}$ & & \\
\hline 3 & Oct-Mar & CSTAB & 50.0 & RIPCN & 50.0 & SEDLD & 50 & TEMP & 50.0 & FLOW' & 25.0 & "HABD & 25.0 \\
\hline 3.4 & Mar-Maya & TEMP & 75.0 & CSTAB & 50.0 & RIPCN & & SEDLD" & $50.0^{\prime}$ & 1. & 25.0 & HABD & 250 \\
\hline 5 & May-Ott & TEMP & 73.3 & RIPCN & 48.8 & HABD & 24.4 & PATHO & 24,4 & & & & \\
\hline $\begin{array}{l}6 \\
7\end{array}$ & $\begin{array}{l}\text { Ott-Mar } \\
\text { Mar-Jun }\end{array}$ & CSTAB & 50.0 & RIPCN & 50.0 & SEDLD" & $50^{\circ}$ & "PATHO" & 25.0 & TEMP'... & $25.0^{\circ}$ & & \\
\hline
\end{tabular}

Abundance'level refers to estimated relative abundance of spawners that spawned in the natal stream reaches that populated the life history pathways using the reach in this table Level 1: $\quad$ Low to average (relative to other reaches) abundance

Level 2: $\quad$ High (relative to other reaches) abundance

Level 3: Exceptionally high (relative to other reaches) abundance

Life stages: 1- prespawner; 2- spawner; 3- incubation; 4- fry colonization; 5- summer rearing; 6- overwintering; 7- yearling presmolt/smolt

Attributes: CHEM - chemicals

CSTAB - channel stability

COMP - competition (with other species)

FLOW - flow

HABD - habitat diversity
NUTLD - nutrient load

OBST - obstructions

OXYG - dissolved oxygen

PATHO - pathogens

PRED - predators (includes fishing-related losses)
RIPCN - riparian condition

SEDLD - sediment load

TEMP - water temperature

WITH - water withdrawals

"NA" indicates that no life history pathway meeting the criteria used in defining these pathways was present.

Blank cells under attributes indicate no additional attributes with negative effects on productivity were evident compared to those present in the template. 
Stream Reach Summary for Vacant Spring Chinook Salmon Life Histories

Stream: Grande Ronde R.

Reach: Head of canyon 1.3 miles downstream of Sheep Cr - LimberJim Cr (RM 159.4 -164.2 from Snake R)

Restoration benefit category:

D $\quad$ Chinook usage score:

\begin{tabular}{l|l}
\hline 23.4 & Habitat productivity ioss score: \\
\hline
\end{tabular}

$-1159.8$

\begin{tabular}{|c|c|c|c|c|c|c|c|c|}
\hline \multirow[b]{3}{*}{ Life stage } & \multirow[b]{3}{*}{ Months } & \multirow{2}{*}{\multicolumn{4}{|c|}{$\begin{array}{l}\text { No. vacant life history pathways } \\
\text { by abundance level }\end{array}$}} & \multicolumn{2}{|c|}{ Key habitat change } & \multirow{3}{*}{$\begin{array}{c}\text { Habitat } \\
\text { productivity } \\
\text { loss score }\end{array}$} \\
\hline & & & & & & \multirow{2}{*}{$\begin{array}{l}\text { Patient } \\
\text { quantity }\end{array}$} & \multirow[b]{2}{*}{$\%$ change } & \\
\hline & & Total no. & 1 & 2 & 3 & & & \\
\hline 1 & Apr-Aug & 54 & 47 & 7 & 0 & 5.1 & -38.0 & -197.7 \\
\hline 1,23 & Aug-Ött & 50 & 40 & 10 & 0 & 6.3 & -18.0 & $: 459.7$ \\
\hline 3 & Ott-Mar & 100 & 80 & 20 & 0 & 9.1 & -4.5 & -358.1 \\
\hline 34 & Mar-May & 26 & $23^{\circ}$ & 3 & 0 & 8.6 & -42.6 & -139.7 \\
\hline 5 & May-Ott & 6 & 6 & $\ddot{0}$ & 0 & 3.3 & -30.5 & -4.7 \\
\hline 6 & Ott-Mar & NA & NA & NA & NA & NA & NA & NA \\
\hline 7 & Mar-Jun & 2 & 2 & 0 & 0 & 17.6 & 0.0 & 0.2 \\
\hline
\end{tabular}

\begin{tabular}{|c|c|c|c|c|c|c|c|c|c|c|c|c|c|}
\hline \multirow[b]{2}{*}{ Life stage } & \multirow[b]{2}{*}{ Months } & \multicolumn{12}{|c|}{ Highest productivity impact attributes (\% change in impact from template) } \\
\hline & & Art 1 & $\%$ change & Att 2 & $\%$ change & Art 3 & $\%$ change & Att 4 & $\%$ change & Art 5 & $\%$ change & Att 6 & $\%$ change \\
\hline 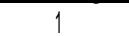 & Apr-Aug & RIPCN & 75.0 & TEMP & 75.0 & HABD & 62,5 & FLOW & 50.0 & OXYG & & NUTLD & 12.3 \\
\hline $1,2,3$ & Âug-Oci & CSTAB & 75.0 & $\dot{R} I \dot{P C} \dot{N}$ & 75.0 & TEMP & 75.0 & $\ddot{H A B D}$ & 62.5 & FLOÖW & & SEDLD & 50.0 \\
\hline 3 & Ott-Mar & CSTAB & “75;0 & SEDLD & 50.0 & TEMP & 50.0 & HABD & 37.5 & FLOW & 25.0 & RIPCN & 25.0 \\
\hline $.3,4$ & Mar-M̈ay & CSTAB & 37.0 & HÄB̈D" & 37.0 & FLOWW & 24.6 & RIPCN & 24.6 & SEẼËD̈ & 24.6 & TEMP & 24.6 \\
\hline 5 & May-Ott & CSTAB & 21.0 & HABD & 21.0 & RIPCN & 21.0 & PATHO & 10.5 & SĖDL̇D & 105 & TEMP & 10.5 \\
\hline$\dot{6}$ & Ott-Mar & $\mathrm{NA}$ & $\mathrm{NA}$ & $N A$ & $\dot{N A}$ & $\mathrm{NA}$ & iv & ..!!.. & $\mathrm{NA}$ & $\mathrm{NA}$ & $\mathrm{NA}$ & NÄ & NÄ" \\
\hline 7 & Mar-Jun & & & & & & & & & & & & \\
\hline
\end{tabular}

Abundance level refers to estimated relative abundance of spawners that spawned in the natal stream reaches that populated the life history pathways using the reach in this table: Level 1: $\quad$ Low to average (relative to other reaches) abundance

Level 2: $\quad$ High (relative to other reaches) abundance

Level 3 Exceptionally high (relative to other reaches) abundance

Life stages: 1- prespawner; 2- spawner; 3- incubation; 4- fry colonization; 5- summer rearing; 6- overwintering; 7- yearling presmolt/smolt

$\begin{array}{lll}\text { Attributes: } \text { CHEM - chemicals } & \text { NUTLD - nutrient load } & \text { RIPCN - riparian condition } \\ \text { CSTAB - channel stability } & \text { OBST - obstructions } & \text { SEDLD - sediment load } \\ \text { COMP - competition (with other species) } & \text { OXYG - dissolved oxygen } & \text { TEMP - water temperature } \\ \text { FLOW - ftOW } & \text { PATHO - pathogens } & \text { WiTH - water withdrawals } \\ \text { HABD - habitat diversity } & \text { PRED - predators (includes fishing-related losses) }\end{array}$

"NA" indicates that no life history pathway meeting the criteria used in defining these pathways was present.

Blank cells under attributes indicate no additional attributes with negative effects on productivity were evident compared to those present in the template. 
Stream: Grande Ronde R.

Reach: LimberJim Cr - Clear Cr (RM 164.2 -166.9 from Snake R)

\begin{tabular}{|c|c|c|c|c|c|c|c|c|}
\hline \multicolumn{4}{|c|}{ Restoration benefit category: } & & \multicolumn{3}{|c|}{ Chinook usage score: } & 21.0 \\
\hline \multirow[b]{3}{*}{ Life stage } & \multirow[b]{3}{*}{ Months } & \multirow{2}{*}{\multicolumn{4}{|c|}{$\begin{array}{c}\text { No. vacant life history pathways } \\
\text { by abundance level }\end{array}$}} & \multirow{2}{*}{\multicolumn{2}{|c|}{$\begin{array}{l}\text { Key habitat change } \\
\text { Patient }\end{array}$}} & \multirow{3}{*}{$\begin{array}{c}\text { Habitat } \\
\text { productivity } \\
\text { loss score }\end{array}$} \\
\hline & & & & & & & & \\
\hline & & Total no. & 1 & 2 & 3 & quantity & $\%$ change & \\
\hline \multirow{2}{*}{11} & Apr-Aug & 31 & 25 & 6 & 0 & 1.3 & -81.1 & -16.1 \\
\hline & Aug-Oct & 50 & 40 & 10 & 0 & 2.7 & -25.0 & -32.4 \\
\hline 3 & Ött-Mar & 100 & 80 & 20 & 0 & 7.4 & 0.0 & -147.3 \\
\hline 34 & Mar-Mà & 11 & 9 & 2 & 0 & 12.6 & -40.4 & -18.3 \\
\hline 5 & May-Ott & $\mathrm{NA}$ & $\mathrm{NA}$ & $\mathrm{NA}$ & $\mathrm{NA}$ & $\mathrm{NA}$ & $\mathrm{NA}$ & $\mathrm{NA}$ \\
\hline 6 & Ott-Mar & NA & "NA" & NA & $\mathrm{NA}$ & NA & NA & NA \\
\hline$\dot{7}$ & Mar-Jun & $\mathrm{NA}$ & $\mathrm{NA}$ & NA & $\mathrm{NA}$ & $\mathrm{NA}$ & NA & NA \\
\hline
\end{tabular}

\begin{tabular}{|c|c|c|c|c|c|c|c|c|c|c|c|c|c|}
\hline \multirow[b]{2}{*}{ Life stage } & \multirow[b]{2}{*}{ Months } & \multicolumn{12}{|c|}{ Highest productivity impact attributes (\% change in impact from template) } \\
\hline & & Att 1 & $\%$ change & Alt 2 & $\%$ change & Att 3 & $\%$ change & Att 4 & $\%$ change & Att 5 & $\%$ change & Att 6 & \% change \\
\hline 1 & Apr-Aug & SEDLD & 37.5 & CSTAB & 25.0 & RIPCN & 24.2 & HABD & 12.5 & & & & \\
\hline $1,2,3$ & Aug-Oct & SEDLD & 50.0 & CSTAB & 25.0 & RIPCN & 25.0 & HABD & 12.5 & & & & \\
\hline 3 & Oct-Mar & RIPCN & 50.0 & SEDLD & 50.0 & CSTAB & 25.0 & РАТНО & 25.0 & & & & \\
\hline 3,4 & Mar-May & RIPCN & $50.0^{\circ}$ & SEDLD & 44.7 & PATHO" & 25.0 & CSTAB & 22.4 & & & & \\
\hline 5 & May-Oct & NA & NA & $\mathrm{NA}$ & NA & $\mathrm{NA}$ & NA & NA & NA & NA & $\mathrm{NA}$ & $\mathrm{NA}$ & NA \\
\hline 6 & Oct-Mar & $\mathrm{NA}$ & $\mathrm{NA}$ & $\mathrm{NA}$ & $\mathrm{NA}$ & NA & NA & $\mathrm{NA}$ & $\mathrm{NA}$ & $\mathrm{NA}$ & $\mathrm{NA}$ & NA & NA \\
\hline 7 & Mar-Jun & $\mathrm{NA}$ & $\mathrm{NA}$ & $\mathrm{NA}$ & NA & $\mathrm{NA}$ & $\mathrm{NA}$ & $\mathrm{NA}$ & $\mathrm{NA}$ & NA & $\mathrm{NA}$ & $\mathrm{NA}$ & $\mathrm{NA}$ \\
\hline
\end{tabular}

Abundance /eve/ refers to estimated relative abundance of spawners that spawned in the natal stream reaches that populated the life history pathways using the reach in this table Level 1: $\quad$ Low to average (relative to other reaches) abundance

Level 2: $\quad$ High (relative to other reaches) abundance

Level 3: Exceptionally high (relative to other reaches) abundance

Life stages: 1- prespawner; 2- spawner; 3- incubation; 4- fry colonization; 5- summer rearing; 6- overwintering; 7- yearlingresmolt/smolt

Attributes: CHEM - chemicals

CSTAB - channel stability

COMP - competition (with other species)

FLOW - ftOW

HABD - habitat diversity
NUTLD - nutrient load

OBST - obstructions

OXYG - dissolved oxygen

PATHO - pathogens

PRED - predators (includes fishing-related losses)
RIPCN - riparian condition

SEDLD - sediment load

TEMP - water temperature

WITH - water withdrawals

"NA" indicates that no life history pathway meeting the criteria used in defining these pathways was present.

Blank cells under attributes indicate no additional attributes with negative effects on productivity were evident compared to those present in the template. 
Stream Reach Summary for Vacant Spring Chinook Salmon Life Histories

Stream: Grande Ronde R.

Reach: Clear $\mathrm{Cr}$ - headwaters (RM 166.9 -170.1 from Snake R)

\begin{tabular}{|c|c|c|c|c|c|c|c|c|}
\hline \multicolumn{4}{|c|}{ Restoration benefit category: } & $\bar{F}$ & \multicolumn{3}{|c|}{ Chinook usage score: } & 3.8 \\
\hline \multirow[b]{3}{*}{ Life stage } & \multirow[b]{3}{*}{ Months } & \multirow{2}{*}{\multicolumn{4}{|c|}{$\begin{array}{l}\text { No. vacant life history pathways } \\
\text { by abundance level }\end{array}$}} & \multicolumn{2}{|c|}{ Key habitat change } & \multirow{3}{*}{\begin{tabular}{|c|} 
Habitat \\
productivity \\
loss score
\end{tabular}} \\
\hline & & & & & & \multirow{2}{*}{$\begin{array}{l}\text { Patient } \\
\text { quantity }\end{array}$} & \multirow[b]{2}{*}{$\%$ change } & \\
\hline & & Total no. & 1 & 2 & 3 & & & \\
\hline 1 & Apr-Aug & 6 & 6 & 0 & 0 & 4.3 & 0.0 & -3.2 \\
\hline 123 & Aug-Ött & 10 & 10 & 0 & 0 & 3.0 & 0.0 & -6.5 \\
\hline 3 & Ött-Mar & 20 & 20 & 0 & 0 & 4.8 & 0.0 & -29.5 \\
\hline 34 & Mar-May & 2 & 2 & 0 & 0 & 5.2 & 0.0 & -2.5 \\
\hline 5 & May-Ött & NA & NA & $\dot{N} \dot{A}$ & $\mathrm{NA}$ & $\mathrm{NÄ}$ & $\mathrm{NA}$ & $\mathrm{NA}$ \\
\hline 6 & Oct-Mar & NA & NA & NA "' & NA " " & NA & NA & NA \\
\hline 7 & Mar-Jun & $\mathrm{NA}$ & $\mathrm{NA}$ & $\mathrm{NA}$ & NA & $\dot{N A}$ & $\dot{N} \dot{A}$ & $\mathrm{NA}$ \\
\hline
\end{tabular}

\begin{tabular}{|c|c|c|c|c|c|c|c|c|c|c|c|c|c|}
\hline \multirow[b]{2}{*}{ Life stage } & \multirow[b]{2}{*}{ Months } & \multicolumn{12}{|c|}{ Highest productivity impact attributes ( $\%$ change in impact from template) } \\
\hline & & Att 1 & $\%$ change & Att 2 & $\%$ change & Att 3 & \% change & Att 4 & $\%$ change & Att 5 & $\%$ change & Atf 6 & \% change \\
\hline 1 & Apr-Aug & RIPCN & 25.0 & SEDLD & 25.0 & CSTAB & 12.5 & & & & & & \\
\hline $1,2,3$ & Auy-Oet & SEDLU & Gero & CSTAB & 25.0 & RIPCN & 25.0 & & & & & & \\
\hline 3 & Oct-Mar & SEDLD & 50.0 & CSTAB & 25.0 & RIPCN & 25.0 & PATHC & 12.5 & & & & \\
\hline 3,4 & Mar-May & RIPCN & 25.0 & SEELLD & 25.0 & CSTAB & 12.5 & PATHC & 12.5 & & & & \\
\hline 5 & May-Oct & $\mathrm{NA}$ & $\mathrm{NA}$ & $\mathrm{NA}$ & NA & NA & NA & $\mathrm{NA}$ & $\mathrm{NA}$ & $\mathrm{NA}$ & NA & $\mathrm{NA}$ & $\mathrm{NA}$ \\
\hline 6 & Oct-Mar & NÄ & NÄ & $\mathrm{NA}$ & $\mathrm{NÄ}$ & NA & $\mathrm{NA}$ & $\mathrm{NA}$ & $\mathrm{NA}$ & $\mathrm{NA}$ & NA & NA & $\mathrm{NA}$ \\
\hline 7 & Mar-Jun & NA & NA & $\mathrm{NA}$ & $\mathrm{NA}$ & $\mathrm{NA}$ & NA & $\mathrm{NA}$ & $\mathrm{NA}$ & NA & $\mathrm{NA}$ & NA & $\mathrm{NA}$ \\
\hline
\end{tabular}

Abundance level refers to estimated relative abundance of spawners that spawned in the natal stream reaches that populated the life history pathways using the reach in this table Level 1: Low to average (relative to other reaches) abundance

Level 2: $\quad$ High (relative to other reaches) abundance

Level 3: $\quad$ Exceptionally high (relative to other reaches) abundance

Life stages: 1- prespawner; 2- spawner 3- incubation; 4- fry colonization; 5- summer rearing; 6- overwintering; 7- yearlingresmolt/smolt

Attributes: CHEM - chemicals

CSTAB - channel stability

COMP - competition (with other species)

FLOW - flow

HABD - habitat diversity

$\begin{array}{ll}\text { NUTLD - nutrient load } & \text { RIPCN - riparian condition } \\ \text { OBST - obstructions } & \text { SEDLD - sediment load } \\ \text { OXYG - dissolved oxygen } & \text { TEMP - water temperature } \\ \text { PATHO - pathogens } & \text { WITH - water withdrawals }\end{array}$

PRED - predators (includes fishing-related losses)

SEDLD - sediment load

WITH - $\quad$ water withdrawals
"NA $A^{N}$ indicates that no life history pathway meeting the criteria used in defining these pathways was present.

Blank cells under attributes indicate no additional attributes with negative effects on productivity were evident compared to those present in the template. 


\section{Stream Reach Summary for Vacant Spring Chinook Salmon Life Histories}

Stream: Hurricane Cr.

Reach: Mouth - Lower Alderslope Ditch (RM 121.9 -124.9 from Snake R)

\begin{tabular}{ll|ll|l}
\hline [Restoration benefit category: & F & Chinook usage score: & 6.7 & Habitat productivity loss score: \\
\hline
\end{tabular}

\begin{tabular}{|c|c|c|c|c|c|c|c|c|}
\hline \multirow[b]{3}{*}{ Life stage } & \multirow[b]{3}{*}{ Months } & \multirow{2}{*}{\multicolumn{4}{|c|}{$\begin{array}{c}\text { No. vacant life history pathways } \\
\text { by abundance level }\end{array}$}} & \multicolumn{2}{|c|}{ Key habitat change } & \multirow{3}{*}{$\begin{array}{c}\text { Habitat } \\
\text { productivity } \\
\text { loss score }\end{array}$} \\
\hline & & & & & & \multirow{2}{*}{$\begin{array}{l}\text { Patient } \\
\text { quantity }\end{array}$} & \multirow[b]{2}{*}{$\%$ change } & \\
\hline & & Total no. & 1 & 2 & 3 & & & \\
\hline 1 & Apr-Aug & 15 & 9 & 6 & 0 & 0.7 & -53.7 & \\
\hline 1,23 & Aug-Oct & 10 & 0 & 10 & 0 & 23 & -83.6 & \\
\hline 3 & Ott-Mar & 20 & 0 & 20 & 0 & 3.2 & -80.2 & -64.0 \\
\hline 34 & Mar-May & 7 & 5 & 2 & 0 & 3.6 & -815 & -615 \\
\hline 5 & May-Ott & 3 & 3 & 0 & 0 & 3.0 & -47.3 & $-3,3$ \\
\hline 6 & Ott-Mar & NA & NA & NA & NA & NA & NA & NA \\
\hline 7 & Mar-Jun & 1 & 1 & 0 & 0 & 13.2 & -14.8 & 0.3 \\
\hline
\end{tabular}

\begin{tabular}{|c|c|c|c|c|c|c|c|c|c|c|c|c|c|}
\hline \multirow[b]{2}{*}{ Life stage } & \multirow[b]{2}{*}{ Months } & \multicolumn{12}{|c|}{ Highest productivity impact attributes (\% change in impact from template) } \\
\hline & & Att 1 & $\%$ change & Att 2 & $\%$ change & Att 3 & $\%$ change & Att 4 & $\%$ change & Att 5 & $\%$ change & Att 6 & $\%$ change \\
\hline 1 & Apr-Aug & TEMP & 50,0 & SEDLD & 37.5 & FLOW & 27.0 & HABD & 27.0 & RIPCN & 27.0 & CSTAB & 125 \\
\hline $1,2,3$ & Aug-Oct & SEDLD & 75.0 & FLOW & $50.0^{\circ}$ & $\dot{H A B D}$ & 50.0 & RIPCN & 50.0 & T'EMPP & 50.0 & CSTAB & 25.0 \\
\hline 3 & Ott-Màr & HABD & 75.0 & SEDLD & 75.0 & RIPCN & 500 & TËMP' & "'” 50.0 & CSTAB & 25.0 & "FLOW & 25.0 \\
\hline 3.4 & Mar-May & $\mathrm{HABD}$ & $75.0^{\circ}$ & ŚE்DLD & 75.0 & RIPCN & 50.0 & TंÉMP & 50.0 & CSTAB & 25.0 & FLOW & 25.0 \\
\hline 5 & May-Ott & HABD & 44.3 & FLOW & 29.5 & RIPCN & 29.5 & PATHO & 14.8 & "ṔRंĖD் & 14.8 & & \\
\hline $\begin{array}{l}6 \\
7\end{array}$ & $\begin{array}{l}\text { Ott-Mar } \\
\text { Mar-Jun }\end{array}$ & $\mathrm{NA}$ & $\mathrm{NA}$ & $\mathrm{NA}$ & $\ddot{N A}$ & NA & $\mathrm{NA}$ & $\ddot{N A}$ & $\mathrm{NA}$ & $\mathrm{NA}$ & $\mathrm{NÄ}$ & $\mathrm{NA}$ & $\mathrm{NA}$ \\
\hline
\end{tabular}

Abundance level refers to estimated relative abundance of spawners that spawned in the natal stream reaches that populated the life history pathways using the reach in this table Level 1: Low to average (relative to other reaches) abundance

Level 2: $\quad$ High (relative to other reaches) abundance

Level 3: Exceptionally high (relative to other reaches) abundance

Life stages: 1-prespawner; 2- spawner; 3- incubation; 4- fry colonization; 5- summer rearing; 6- overwintering; 7-yearling presmolt/smolt

Attributes: CHEM - chemicals

CSTAB - channel stability

COMP - competition (with other species)

FLOW - flow

HABD - habitat diversity
NUTLD - nutrient load

OBST - obstructions

OXYG - dissolved oxygen

PATHO - pathogens

PRED - predators (includes fishing-related losses)
RIPCN - riparian condition SEDLD - sediment load

TEMP - water temperature

WITH - water withdrawals

"NA" indicates that no life history pathway meeting the criteria used in defining these pathwavs was present.

Blank cells under attributes indicate no additional altributes with negative effects on productivity were evident compared to those present in the template. 
Stream Reach Summary for Vacant Spring Chinook Salmon Life Histories

Stream: Hurricane Cr.

Reach: Lower Alderslope Ditch - Upper Alderslope Ditch Diversion (RM 124.9 -128,2 from Snake R)

\begin{tabular}{ll|ll|l|}
\hline Restoration benefit category: & A & Chinook usage score: & 48,0 & Habitat productivity loss score: \\
\hline
\end{tabular}

\begin{tabular}{|c|c|c|c|c|c|c|c|c|}
\hline \multirow[b]{3}{*}{ Life stage } & \multirow[b]{3}{*}{ Months } & \multirow{2}{*}{\multicolumn{4}{|c|}{$\begin{array}{c}\text { No. vacant life history pathways } \\
\text { by abundance level }\end{array}$}} & \multicolumn{2}{|c|}{ Key habitat change } & \multirow{3}{*}{$\begin{array}{c}\text { Habitat } \\
\text { productivity] } \\
\text { loss score }\end{array}$} \\
\hline & & & & & & \multirow{2}{*}{$\begin{array}{l}\text { Patient } \\
\text { quantity }\end{array}$} & \multirow[b]{2}{*}{$\%$ change } & \\
\hline & & Total no. & 1 & 2 & 3 & & & \\
\hline 1 & Apr-Aug & 54 & 54 & 0 & 0 & 0,0 & -100.0 & -515.1 \\
\hline i 2“3”” & Aüg-Ott & 90 & 80 & 0 & 0 , & 1.1 & -64.6 & $: 822,7 "$ \\
\hline 3 & Ott-Mar & 180 & 180 & 0 & 0 & 3.2 & -20.7 & -1054.4 \\
\hline 34 & Mar-May & $42^{\circ}$ & 42 & 0 & $0 "$ & 1.6 & -664 & $-199,7$ \\
\hline 5 & May-Ott & 94 & 94 & 0 & 0 & 0.0 & $-100,0$ & $-87 \ddot{3} .0^{\cdots}$ \\
\hline & Oct-Mar & 19 & 19 & 0 & 0 & " " " 0.5 & $-86,8$ & -39.9 \\
\hline 7 & Màr-Jun & 1 & 1 & 0 & $\dot{0}$ & 12,1 & -26.7 & 0.5 \\
\hline
\end{tabular}

\begin{tabular}{|c|c|c|c|c|c|c|c|c|c|c|c|c|c|}
\hline \multirow[b]{2}{*}{ Life stage } & \multirow[b]{2}{*}{ Months } & \multicolumn{12}{|c|}{ Highest productivity impact attributes (\% change in impact from template) } \\
\hline & & Att 1 & $\%$ change & Att 2 & $\%$ change & Att 3 & $\%$ change & Atf 4 & $\%$ change & Att 5 & $\%$ change & Att 6 & $\%$ change \\
\hline 1 & Apr-Aug & FLOW & 100.0 & HABD & 50,0 & & & & & & & & \\
\hline , 1,2,3 & Aug-Ott & FLOW & 100,0 & HABD & 50.0 & SEEELED & 25.0 & & & & & & \\
\hline 3 & Ọtt-Mar & FLOW & 75,0 . & SEDLD & 25.0 & HABD & 12.5 & PATHO & ‘ 12.5 & WITH & 12.5 & & \\
\hline 3,4 & Mar-May & FLOW & 50.0 & HABD & 50.0 & "CSTAB" & $25.0^{\circ}$ & PATHO & 12.5 & SEELID & 12.5 & Wัiт̈H & 12.5 \\
\hline 5 & May-Oct & SEDLD & 75,0 ' & CSTAB & 50.0 & FLOW & 50.0 & HABD & 50.0 & PATHO & 25.0 & RIPCN & $25.0^{\circ}$ \\
\hline $\begin{array}{l}6 \\
7 \\
\end{array}$ & $\begin{array}{l}\text { Oct-Mar } \\
\text { Mar-Jun }\end{array}$ & SEDLD & 75.0 & CSTAB & 50.0 & FLOW & .50 .0 & PATHO & 25.0 & RIPCN & 25.0 & & \\
\hline
\end{tabular}

Abundance/eve/ refers to estimated relative abundance of spawners that spawned in the natal stream reaches that populated the life history pathways using the reach in this table Level 1: $\quad$ Low to average (relative to other reaches) abundance

Level 2: High (relative to other reaches) abundance

Level 3: Exceptionally high (relative to other reaches) abundance

Life stages: 1 - prespawner; 2- spawner; 3- incubation; 4- fry colonization; 5- summer rearing; 6- overwintering; 7- yearling presmolt/smolt

Attributes: CHEM - chemicals

CSTAB - channel stability

COMP - competition (with other species)

FLOW - flow

HABD - habitat diversity
NUTLD - nutrient load

OBST - obstructions

OXYG - dissolved oxygen

PATHO - pathogens

PRED - predators (includes fishing-related losses)
RIPCN - riparian condition

SEDLD - sediment load

TEMP - water temperature

WITH - water withdrawals

"NA" indicates that no life history pathway meeting the criteria used in defining these pathways was present.

Blank cells under attributes indicate no additional attributes with negative effects on productivity were evident compared to those present in the template. 
Stream Reach Summary for Vacant Spring Chinook Salmon Life Histories

Stream: Joseph Cr.

Reach: Mouth - CottonwoodCr (RM 3.7- 8,2 from Snake R)

\begin{tabular}{ll|ll|l|}
\hline [Restoration benefit category: & F & Chinook usage score: & 0.0 & Habitat productivity loss score: \\
\hline
\end{tabular}

\begin{tabular}{|c|c|c|c|c|c|c|c|c|}
\hline \multirow[b]{3}{*}{ Life stage } & \multirow[b]{3}{*}{ Months } & \multirow{2}{*}{\multicolumn{4}{|c|}{$\begin{array}{l}\text { No. vacant life history pathways } \\
\text { by abundance level }\end{array}$}} & \multicolumn{2}{|c|}{ Key habitat change } & \multirow{3}{*}{$\begin{array}{c}\text { Habitat } \\
\text { productivity } \\
\text { loss score }\end{array}$} \\
\hline & & & & & & \multirow{2}{*}{$\begin{array}{l}\text { Patient } \\
\text { quantity }\end{array}$} & \multirow[b]{2}{*}{$\%$ change } & \\
\hline & & Total no. & 1 & 2 & 3 & & & \\
\hline 1 & Apr-Aug & NA & $\mathrm{NA}$ & $\mathrm{NA}$ & $\mathrm{NA}$ & NA & NA & $\overline{\mathrm{NA}}$ \\
\hline $1,2,3$ & Aug-Oct & NA & NA & NA & NA & NA & NA & NA \\
\hline 3 & Ott-Mar & $\mathrm{NA}$ & $\mathrm{NÄ}$ & $\mathrm{NA}$ & NÄ & $\mathrm{NA}$ & NÄ & $\mathrm{NA}$ \\
\hline 34 & Mar-Mà & $\mathrm{NA}$ & $\mathrm{NÄ}$ & NA & NA & NA & NA & NA \\
\hline 5 & May-Ott & $\mathrm{NA}$ & $\mathrm{NA}$ & $\mathrm{NA}$ & N̈̈ & $\mathrm{NA}$ & NÄ & NA \\
\hline 6 & Ott-Mar & NA & NA & NA & NA & NA & NA & NA \\
\hline 7 & Mar-Jun & $\mathrm{NA}$ & NA & NA & $\mathrm{NA}$ & $\mathrm{NA}$ & NA & NA \\
\hline
\end{tabular}

\begin{tabular}{|c|c|c|c|c|c|c|c|c|c|c|c|c|c|}
\hline \multirow[b]{2}{*}{ Life stage } & \multirow[b]{2}{*}{ Months } & \multicolumn{12}{|c|}{ Highest productivity impact attributes (\% change in impact from template) } \\
\hline & & Att 1 & $\%$ change & Att 2 & $\%$ change & Att 3 & $\%$ change & Att 4 & $\%$ change & Att 5 & $\%$ change & Att 6 & $\%$ change \\
\hline 1 & Apr-Aug & NA & NA & NA & NA & NA & NA & NA & $\overline{N A}$ & $\overline{N A}$ & NA & NA & $\mathrm{NA}$ \\
\hline $1,2,3$ & Âug-Ồct & $N A$ & NA & NA & NÄ & $N A \ddot{~}$ & $N{ }^{\prime \prime}$ & $N A "$ & NA & $\mathrm{NA}$ & $\mathrm{NA}$ & NA & $\mathrm{NA}$ \\
\hline 3 & Oti-M & NIA & NA & NA & NA & NA & NA & $\mathrm{NA}$ & $\mathrm{NA}$ & NA & NA " & "NA & NA \\
\hline 3,4 & Matmiala & YNA & NA & N & $\mathrm{NÄ}$ & NA & $\mathrm{NA}$ & $\mathrm{NA}$ & NA & $\mathrm{NA}$ & $\mathrm{NA}$ & $\mathrm{NA}$ & $\mathrm{NA}$ \\
\hline 5 & May $y$ O $A D$ & $t$ WNA & $\mathrm{NA}$ & NA & NA & NA & $\mathrm{NA}$ & "'"' "NA & “"'NA & NA & NA & NA & NA \\
\hline 6 & Ott-Mar & NA & Nั"̈ & NA & $\mathrm{NA}$ & N̄" & $\mathrm{NA}$ & $\dot{\mathrm{NA}}$ & $\dot{N A}$ & NA & NÄ & $\mathrm{NA}$ & NA \\
\hline 7 & Mar-Jun & $\mathrm{NNA}$ & NA & $\mathrm{NA}$ & $\mathrm{NA}$ & $" N A "$ & NA & NA & NA & NA & NA & NA & NA \\
\hline
\end{tabular}

Abundance /eve/ refers to estimated relative abundance of spawners that spawned in the natal stream reaches that populated the life history pathways using the reach in this table

Level 1: Low to average (relative to other reaches) abundance

Level 2 High (relative to other reaches) abundance

Level 3 Exceptionally high (relative to other reaches) abundance

Life stages: 1- prespawner; 2- spawner; 3- incubation: 4- fry colonization; 5- summer rearing; 6- overwintering; 7- yearling presmoltssmolt

Attributes: CHEM - chemicals

CSTAB - channel stability

COMP . competition (with other species)

FLOW - flow

HABD - habitat diversity
NUTLD - nutrient load

OBST - obstructions

OXYG - dissolved oxygen

PATHO - pathogens

PRED - predators (includes fishing-related losses)
RIPCN - riparian condition

SEDLD - sediment load

TEMP - water temperature

WITH - water withdrawals

"NA" indicates that no tife history pathway meeting the criteria used in defining these pathways was present.

Blank cells under attributes indicate no additional attributes with negative effects on productivity were evident compared to those present in the template, 
Stream Reach Summary for Vacant Spring Chinook Salmon Life Histories

Stream: Joseph Cr.

Reach: Cottonwood Cr - Swamp Cr (RM 8.2- 37.7 from Snake R)

\begin{tabular}{|ll|ll|l|}
\hline Restoration benefit category & F & Chinook usage score: & 0.0 & Habitat productivity loss score: \\
\hline
\end{tabular}

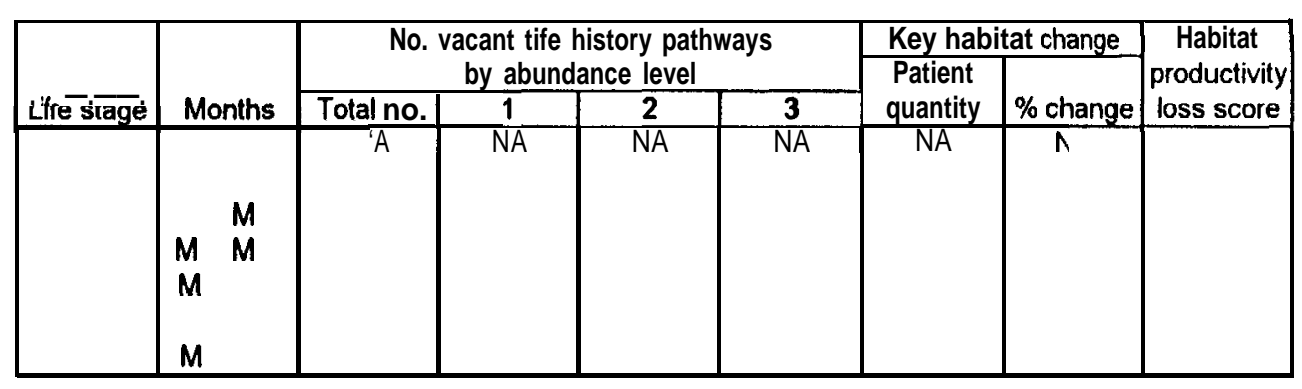

\begin{tabular}{|c|c|c|c|c|c|c|c|c|c|c|c|c|c|}
\hline \multirow[b]{2}{*}{ Life stage } & \multirow[b]{2}{*}{ Months } & \multicolumn{12}{|c|}{ Highest productivity impact attributes (?! change in impact from template) } \\
\hline & & Att 1 & $\%$ change & Att 2 & $\%$ change & Att 3 & $\%$ change & Att 4 & $\%$ change & Atf 5 & $\%$ change & Att 6 & $\%$ change \\
\hline 1 & Apr-Aug & $\mathrm{NA}$ & NA & $\mathrm{NA}$ & NA & $\mathrm{NA}$ & NA & $\overline{N A}$ & NA & $N A$ & $\mathrm{No}$ & & NA \\
\hline $1,2,3$ & Aug-Oct & $\mathrm{NA}$ & $\mathrm{NA}$ & NA' & NA & $\mathrm{NA}$ & NA & $N A$ & NA & NA" & $\mathrm{NA}$ & & $N A$ \\
\hline 3 & Ott-Mar & $\mathrm{NA}$ & $\mathrm{NA}$ & NA & $N A$ & NA & $N A$ & NA & NA & $\mathrm{NA}$ & NA & NA & NA \\
\hline 3,4 & Mar-May & $\mathrm{NA}$ & NÄ & $\mathrm{NA}$ & NÄ & $\dot{N} \dot{A}$ & $\mathrm{NA}$ & $\because \dddot{N A}$ & $\ddot{\mathrm{NA}}$ & $\mathrm{NA}$ & NA & NÄ & NA \\
\hline 5 & May-Ott" & NA & NA & $\mathrm{NA}$ & $\mathrm{NA}$ & NA & NA & NA & NA & NA & NAA & NA & NA \\
\hline 6 & Ött-Mar & NA & $\mathrm{NA}$ & NA & $\mathrm{NA}$ & $\mathrm{NA}$ & $\mathrm{NA}$ & $\mathrm{NA}$ & $\mathrm{NA}$ & $\mathrm{NA}$ & $\mathrm{NA}$ & $\mathrm{NA}$ & $\mathrm{NA}$ \\
\hline 7“ & Mar-Jun & NA & NA & NA & NA & NA & $\mathrm{NA}$ & NA & NA & NA & NA & NA & NA \\
\hline
\end{tabular}

Abundance lovel refers to estimated relative abundance of spawners that spawned in the natal stream reaches that populated the life history pathways using the reach in this table: Level 1: Low to average (relative to other reaches) abundance

Level 2 High (relative to other reaches) abundance

Level 3 Exceptionally high (relative to other reaches) abundance

Life stages: 1- prespawner; 2- spawner; 3- incubation; 4- fry colonization; 5- summer rearing; 6- overwintering; 7- yearling presmolt/smolt

Attributes: CHEM - chemicals

CSTAB - channel stability

COMP - competition (with other species)

FLOW - flow

HABD - habitat diversity

NUTLD - nutrient load

OBST - obstructions

OXYG - dissolved oxygen

PATHO - pathogens

PRED - predators (includes fishing-related losses)
RIPCN - riparian condition

SEDLD - sediment load

TEMP - water temperature

WITH - water withdrawals

"NA" indicates thst no life history pathway meeting the criteria used in defining these pathways was present.

Blank cells under attributes indicate no additional attributes with negative effects on productivity were evident compared to those present in the template. 
Stream Reach Summary for Vacant Spring Chinook Salmon Life Histories

Stream: Joseph Cr.

Reach: Swamp Cr - Chesnimnus Cr (RM $37.7-52,7$ from Snake R)

[Restoration benefit category:

\begin{tabular}{l|l}
$\mathrm{F}$ & Chinook usage score:
\end{tabular}

0.0

Habitat productivity loss score:

\begin{tabular}{|c|c|c|c|c|c|c|c|c|}
\hline \multirow[b]{3}{*}{ Life stage } & \multirow[b]{3}{*}{ Months } & \multirow{2}{*}{\multicolumn{4}{|c|}{$\begin{array}{c}\text { No. vacant lie history pathways } \\
\text { by abundance level }\end{array}$}} & \multicolumn{2}{|c|}{ Key habitat change } & \multirow{3}{*}{\begin{tabular}{|c|} 
Habitat \\
productivity \\
loss score
\end{tabular}} \\
\hline & & & & & & \multirow{2}{*}{$\begin{array}{l}\text { Patient } \\
\text { quantity }\end{array}$} & \multirow[b]{2}{*}{$\%$ change } & \\
\hline & & Total no. & 1 & 2 & 3 & & & \\
\hline 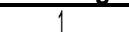 & Apr-Aug & $\overline{\mathrm{NA}}$ & $\overline{\mathrm{NA}}$ & $\overline{\mathrm{NA}}$ & $\overline{\mathrm{NA}}$ & $\mathrm{NA}$ & $\overline{\mathrm{NA}}$ & $\mathrm{NA}$ \\
\hline 123 & Aug-Oct & NA & NA & NA & $N A$ & NA & NA & NA \\
\hline 3 & Ott-Mar & $\mathrm{NA}$ & $\mathrm{NA}$ & $\mathrm{NA}$ & $\mathrm{NA}$ & NA & $\mathrm{NÄ}$ & $\mathrm{NA}$ \\
\hline 34 & Mar-May & NÄ & $\mathrm{NA}$ & $\mathrm{NA}$ & $\mathrm{NA}$ & NA & NA & NA \\
\hline 5 & May-Ott & NA & $\mathrm{NA}$ & NA & NÄ & NÄ & $\mathrm{NA}$ & NA \\
\hline 6 & Ott-Mar & NA & NA & NA & $N A$ & $\mathrm{NA}$ & $\mathrm{Nä}$ & NA \\
\hline 7 & Mar-Jun & $\mathrm{NA}$ & $\mathrm{NÄ}$ & $\mathrm{NÄ}$ & $\dot{\mathrm{NA}}$ & $\mathrm{NA}$ & $\mathrm{NA}$ & $\mathrm{NA}$ \\
\hline
\end{tabular}

\begin{tabular}{|c|c|c|c|c|c|c|c|c|c|c|c|c|c|}
\hline \multirow[b]{2}{*}{ Life stage } & \multirow[b]{2}{*}{ Months } & \multicolumn{12}{|c|}{ Highest productivity impact attributes (\% change in impact from template) } \\
\hline & & Att 1 & $\%$ change & Att 2 & $\%$ change & Att 3 & $\%$ change & Att 4 & $\%$ change & Att5 & $\%$ change & Att 6 & $\%$ change \\
\hline 1 & ,Apr -Aug & NA & NA & $\mathrm{NA}$ & NA & NA & NA & NA & NA & NA & NA & NA & NA \\
\hline $1,2,3$ & ,Aug-Ott & $\mathrm{NA}$ & $\mathrm{NA}$ & $\mathrm{NAA}$ & $\mathrm{NA}$ & $\mathrm{NA}$ & $\mathrm{NA}$ & "NA" & $\mathrm{NA}$ & $\mathrm{NÄ}$ & NÄ & $\mathrm{NA}$ & "NA" \\
\hline 3 & Oct-Mar & NA & $\mathrm{NA}$ & $\mathrm{NA}$ & NA" & NA & NA & NA & NA & "““ NA" N". & NA & NA & NA \\
\hline$\ldots ?$ ?! & Mar-May & $N$ & NA & $\mathrm{N} \quad \mathrm{A}$ & NA & NA & $\mathrm{NA}$ & NA & $!$ & NA & NA & NA & . $\$$ \\
\hline 5 & May-Ott & 'NA' & NÄ & NA & $N A$ & $\mathrm{NA}$ & NA & $N A$ & NẢ & NA & NA & $\mathrm{NA}$ & \\
\hline$\dddot{6}$ & Ött-M̈ar & NA & $\mathrm{NA}$ & $\mathrm{NA}$ & NÄ & , NA & NÄ & NA & NA & $\mathrm{NA}$ & $\mathrm{NA}$ & NA & NA \\
\hline 7 & Mar-Jun & NA & NA & NA & NA & NA & NA & NA & NA & NA & NA & NA & NA \\
\hline
\end{tabular}

Abundancelevel refers to estimated relative abundance of spawners that spawned in the natal stream reaches that populated the life history pathways using the reach in this table: Level 1: Low to average (relative to other reaches) abundance

Level 2: High (relative to other reaches) abundance

Level 3: Exceptionally high (relative to other reaches) abundance

Life stages: 1 - prespawner; 2- spawner; 3- incubation; 4- fry colonization; 5- summer rearing; 6- overwintering; 7- yearling presmolt/smolt

Attributes: CHEM - chemicals

CSTAB - channel stability

COMP - competition (with other species)

FLOW - flow

HABD - habitat diversity
NUTLD - nutrient load

OBST - obstructions

OXYG - dissolved oxygen

PATHO - pathogens

PRED - predators (includes fishing-related losses)
RIPCN - riparian condition

SEDLD - sediment load

TEMP - water temperature

WITH - water withdrawals

"NA" indicates that no life history pathway meeting the criteria used in defining these pathways was present.

Blank cells under attributes indicate no additional attributes with negative effects on productivity were evident compared to those present in the template, 
Stream Reach Summary for Vacant Spring Chinook Salmon Life Histories

Stream: Lookingglass $\mathrm{Cr}$.

Reach: $\quad$ Mouth - Little Lookingglass $\mathrm{Cr}$ (RM 83.4 -87.6 from Snake R)

Restoration benefit category:

Chinook usage score:

\begin{tabular}{l|l}
15.2 & Habitat productivity loss score:
\end{tabular}

$-207.3$

\begin{tabular}{|c|c|c|c|c|c|c|c|c|c|}
\hline \multirow[b]{3}{*}{ Life stage } & \multirow[b]{3}{*}{ Months } & \multirow{2}{*}{\multicolumn{5}{|c|}{$\begin{array}{l}\text { No. vacant life history pathways } \\
\text { by abundance level }\end{array}$}} & \multicolumn{2}{|c|}{ Key habitat change } & \multirow{3}{*}{\begin{tabular}{|c|} 
Habitat \\
productivity \\
loss score
\end{tabular}} \\
\hline & & & & & & & \multirow{2}{*}{$\begin{array}{l}\text { Patient } \\
\text { quantity }\end{array}$} & \multirow[b]{2}{*}{$\%$ change } & \\
\hline & & Total & no. & 1 & 2 & 3 & & & \\
\hline 1 & Apr-Aug & $2 A$ & & 7A & 0 & 0 & A.! & 0.0 & .43 .9 \\
\hline $1,2,3$ & Aug-Oct & 40 & & 40 & 0 & 0 & 2.6 & -51.0 & -152.8 \\
\hline 3 & Oct-Mar & 80 & & 80 & 0 & 0 & 2.7 & -60.0 & -10.5 \\
\hline 3,4 & Mar-May & 8 & & 8 & 0 & 0 & 4.5 & -42.9 & -0.1 \\
\hline 5 & May-Oct & NA & & NA & NA & NA & NA & $\mathrm{NA}$ & NA \\
\hline 6 & Oct-Mar & NA & & NA & NA & NA & $\mathrm{NA}$ & NA & NA \\
\hline 7 & Mar-Jun & NA & & NA & NA & NA & $\mathrm{NA}$ & NA & NA \\
\hline
\end{tabular}

\begin{tabular}{|c|c|c|c|c|c|c|c|c|c|c|c|c|c|}
\hline \multirow[b]{2}{*}{ Life stage } & \multirow[b]{2}{*}{ Months } & \multicolumn{12}{|c|}{ Highest productivity impact attributes (\% change in impact from template) } \\
\hline & & Att 1 & $\%$ change & Alt 2 & $\%$ change & Att 3 & $\%$ change & Alt 4 & $\%$ change & Alt 5 & $\%$ change & Att 6 & $\%$ change \\
\hline 1 & Apr-Aug & TEMP & 50.0 & PATHO & 25.0 & & & & & & & & \\
\hline $1,2,3$ & Aug.OCl & TEMP & 50.0 & HABO & 25.0 & PATHO & 250 & & & & & & \\
\hline 3 & Oct-Mar & FLOW & 25.0 & PATHO & 25.0 & & & & & & & & \\
\hline 3,4 & Mar-May & FLOW & 25.0 & PATHO & 25.0 & & & & & & & & \\
\hline 5 & May-Oct & $\mathrm{NA}$ & $\mathrm{NA}$ & $\mathrm{NA}$ & NA & $N A$ & $\mathrm{NA}$ & $\mathrm{NA}$ & NA & NA & NA & NA & $\mathrm{NA}$ \\
\hline 6 & Oct-Mar & $\mathrm{NA}$ & $\mathrm{NA}$ & $\mathrm{NA}$ & $\mathrm{NA}$ & NA & NA & $\mathrm{NA}$ & $\mathrm{NA}$ & NA & NA & $\mathrm{NA}$ & $\mathrm{NA}$ \\
\hline 7 & Mar-Jun & $\mathrm{NA}$ & $\mathrm{NA}$ & $\mathrm{NA}$ & $\mathrm{NA}$ & $\mathrm{NA}$ & $\mathrm{NA}$ & $\mathrm{NA}$ & $\mathrm{NA}$ & $\mathrm{NA}$ & $\mathrm{NA}$ & $\mathrm{NA}$ & $\mathrm{NA}$ \\
\hline
\end{tabular}

Abundance level refers to estimated relative abundance of spawners that spawned in the natal stream reaches that populated the life history pathways using the reach in this table Level 1: $\quad$ Low to average (relative to other reaches) abundance

Level 2 High (relative to other reaches) abundance

Level 3 Exceptionally high (relative to other reaches) abundance

Life stages: 1- prespawner; 2- spawner; 3- incubation; 4- fry colonization; 5- summer rearing; 6- overwintering; 7- yearling presmolt/smolt

Attributes: CHEM - chemicals

CSTAB - channel stability

NUTLD - nutrient load

COMP - competition (with other species)

FLOW - flow

OBST - obstructions

OXYG - dissolved oxygen

PATHO - pathogens

HABD - habitat diversity

PRED - predators (includes fishing-related losses)

"NA" indicates that no lie history pathway meeting the criteria used in defining these pathways was present.

Blank cells under attributes indicate no additional attributes with negative effects on productivity were evident compared to those present in the template. 
Stream: Lookingglass $\mathrm{Cr}$.

Reach: Little Lookingglass $\mathrm{Cr}_{r}$ - headwaters (RM 87.6 -93.8 from Snake R)

Restoration benefit category: Chinook usage score:

$0.0 \quad$ Habitat productivity loss score:

\begin{tabular}{|c|c|c|c|c|c|c|c|c|}
\hline \multirow[b]{3}{*}{ Life stage } & \multirow[b]{3}{*}{ Months } & \multirow{2}{*}{\multicolumn{4}{|c|}{$\begin{array}{c}\text { No. vacant life history pathways } \\
\text { by abundance level }\end{array}$}} & \multicolumn{2}{|c|}{ Key habitat change } & \multirow{3}{*}{$\begin{array}{c}\text { Habitat } \\
\text { productivity: } \\
\text { ioss score }\end{array}$} \\
\hline & & & & & & \multirow{2}{*}{$\begin{array}{l}\text { Patient } \\
\text { quantity }\end{array}$} & \multirow[b]{2}{*}{$\%$ change } & \\
\hline & & Total no. & 1 & 2 & $\overline{3}$ & & & \\
\hline 1 & Apr-Aug & NA & $\overline{\mathrm{NA}}$ & $\mathrm{NA}$ & $\overline{N A}$ & NA & NA & $\mathrm{NA}$ \\
\hline $1,2,3$ & Aug-Ott & NA & $\mathrm{N} A$ & NA & NA & NA & NA & NA \\
\hline 3 & Ott-Mar & $\mathrm{NA}$ & $\mathrm{NA}$ & $\mathrm{NA}$ & $\mathrm{NA}$ & $\mathrm{NA}$ & $\mathrm{NA}$ & $\mathrm{NA}$ \\
\hline 3,4 & Mar-Mày & $\dot{N A}$ & $\mathrm{NA}$ & NA' & NA & i & $\dot{N A}{ }_{j}$ & NA \\
\hline 5 & May-Ott & $\mathrm{NA}$ & $\mathrm{NA}$ & $\mathrm{NA}$ & $\mathrm{NA}$ & $\mathrm{NA}$ & $\mathrm{NA}$ & NA \\
\hline 6 & Ott-Mar & NA & NA & NA & NA & NA & NA & NA \\
\hline 7 & Mar-jun & $\mathrm{NA}$ & $\mathrm{NA}$ & $\mathrm{NA}$ & $\mathrm{NA}$ & $\mathrm{NA}$ & NA & $\mathrm{NA}$ \\
\hline
\end{tabular}

\begin{tabular}{|c|c|c|c|c|c|c|c|c|c|c|c|c|c|}
\hline \multirow[b]{2}{*}{ Life stage } & \multirow[b]{2}{*}{ Months } & \multicolumn{12}{|c|}{ Highest productivity impact attributes (\% change in impact from template) } \\
\hline & & Atf 1 & $\%$ change & Atf 2 & $\%$ change & Att 3 & $\%$ change & Att 4 & $\%$ change & Att 5 & $\%$ change & Atf 6 & $\%$ change \\
\hline 1 & Apr-Aug & NA & $\mathrm{NA}$ & NA & NA & NA & NA & 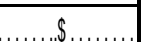 & NA & NA & NA & . & NA \\
\hline $1,2,3$ & Äug-Ött & $\mathrm{NA}$, & NA & $\mathrm{NA}$ & $\mathrm{NA}$ & $\mathrm{NA}$ & NA & & $N A$ & NA' & $\mathrm{NA}$ & & NA \\
\hline 3 & Oct-Mar & $\mathrm{NÄ}$ & $\mathrm{NA}$ & NA & NA & $\mathrm{NA}$ & NA & NA "', & $\mathrm{NA}$ & NA & $\mathrm{NA}$ & NA & NA \\
\hline$, 3,4$ & Mar-May & $\mathrm{NA}$ & $\mathrm{NA}$ & $\mathrm{NA}$ & $\mathrm{NA}$ & NA & NA" & $\mathrm{NA}$ & $\mathrm{NA}$ & NA" & NA & NA & NA \\
\hline 5 & May-Ott & NA & NA. & NA & NA & NA & $\mathrm{NA}$ & NA & NA & $\mathrm{NA}$ & $\mathrm{NA}$ & NQA & $\mathrm{NA}$ \\
\hline 6 & Ött-M̈ar & $\mathrm{NA}$ & $\dot{N A}$ & $\dot{N} \dot{A}$ & $\mathrm{NA}$ & NA & NÁ & $\mathrm{NA}$ & NÄ & NA" & NA & NA & $\mathrm{NA}$ \\
\hline 7 & Mar-Jun & NA & NA & NA““๘ & NA & NA & NA & $\mathrm{NA}$ & NA & NA & $\mathrm{NA}$ & NA & NA \\
\hline
\end{tabular}

Abundance /eve/ refers to estimated relative abundance of spawners that spawned in the natal stream reaches that populated the life history pathways using the reach in this table:

Level 1: Low to average (relative to other reaches) abundance

Level 2 High (relative to other reaches) abundance

Levei 3: Exceptionally high (relative to other reaches) abundance

Life stages: 1 - prespawner; 2- spawner; 3- incubation; 4- fry colonization; 5- summer rearing; 6- overwintering; 7- yearling presmolt/smolt

Attributes: CHEM - chemicals

CSTAB - channel stability

COMP - competition (with other species)

FLOW - flow

HABD - habitat diversity
NUTLD - nutrient load

OBST - obstructions

OXYG - dissolved oxygen

PATHO - pathogens

PRED - predators (includes fishing-relatedlosses)
RiPCN - riparian condition

SEDLD - sediment load

TEMP - $\quad$ water temperature

WiTH - water withdrawals

"NA" indicates that no life history pathway meeting the criteria used in defining these pathways was present.

Blank cells under attributes indicate no additional attributes with negative effects on productivity were evident compared to those present in the tempiate. 


\section{Stream Reach Summary for Vacant Spring Chinook Salmon Life Histories}

Stream: Lostine R.

Reach: Mouth - Clear'water Ditch(RM 105.8 -108.3 from Snake R)

\begin{tabular}{|l|l|ll|ll}
\hline Restoration benefit category: & B & Chinook usage score: & 56.6 & Habitat productivity loss score: \\
\hline
\end{tabular}

\begin{tabular}{|c|c|c|c|c|c|c|c|c|}
\hline \multirow[b]{3}{*}{ Life stage } & \multirow[b]{3}{*}{ Months } & \multirow{2}{*}{\multicolumn{4}{|c|}{$\begin{array}{l}\text { No. vacant life history pathways } \\
\text { by abundance level }\end{array}$}} & \multicolumn{2}{|c|}{ Key habitat change } & \multirow{3}{*}{$\begin{array}{c}\text { Habitat } \\
\text { roductivity } \\
\text { loss score }\end{array}$} \\
\hline & & & & & & \multirow{2}{*}{$\begin{array}{l}\text { Patient } \\
\text { quantity }\end{array}$} & \multirow[b]{2}{*}{$\%$ change } & \\
\hline & & Total no. & 1 & 2 & 3 & & & \\
\hline 1 & Apr-Aug & 75 & 55 & 15 & 5 & 6.8 & -48.9 & -467.5 \\
\hline $1,2,3$ & Aug-Oct & 90 & 70 & 20 & 0 & 13.3 & -15.3 & -845.6 \\
\hline 3 & Oct-Mar & 180 & 140 & 40 & 0 & 18.2 & 1.8 & $-794,4$. \\
\hline 3,4 & Mar-May & 64 & 44 & 15 & 5 & 18.1 & 3.8 & -211.5 \\
\hline 5 & May-Oct & 215 & 172 & 43 & 0 & 15.9 & 0.2 & -302.1 \\
\hline 6 & Oct-Mar & 38 & 38 & 0 & 0 & 12.4 & 0.8 & -26.4 “... \\
\hline 7 & Mar-Jun & "4" & 4 & 0 & 0 & 21.5 & 2.7 & 17 \\
\hline
\end{tabular}

\begin{tabular}{|c|c|c|c|c|c|c|c|c|c|c|c|c|c|}
\hline \multirow[b]{2}{*}{ Life stage } & \multirow[b]{2}{*}{ Months } & \multicolumn{12}{|c|}{ Highest productivity impact attributes (\% change in impact from template) } \\
\hline & & Art 1 & $\%$ change & Att 2 & $\%$ change & Att 3 & $\%$ change & Art 4 & $\%$ change & Att 5 & $\%$ change & Art 6 & $\%$ change \\
\hline 1 & Apr-Aug & TEMP & 62.5 & FLOW & 55,6 & OBST & 37.1 & HABD. & 18.5 . & CSTAB & 12.5 & WITH & 12.5 \\
\hline $1,2,3$ & Aug-Od & Fíow & $75.0^{\circ}$ & TEMP & 75.0 & OBST & 50.0 & Cं்ं̇ंA & 25.0 & HABD & 25.0 & "ẄIT̈H & 25.0 \\
\hline 3 & Ott-Mär' & ḦÄB̈D & 50,0 & TEMP & 50.0 & CSTAB & 25.0 & FLOW & 25,0 & PATHO & 250 & WITH & 25.0 \\
\hline 3,4 & Mar-May & TEMP & $4 \dot{6} . \dot{6}$ & HÄB̈D & $44.0^{\circ}$ & CSTAB & $23.3^{\circ}$ & F்LO் & 23,3 & 'WiT̈H' & 23.3 & "PATHO & 22.0 \\
\hline 5 & , ,May,-Q,t, & TEMP & 38.1 & FLOW & 127 & HABD & “.12.7. & РАТН̈О் & 12.7 & WITH & 12.7 & & \\
\hline 6 & $\begin{array}{l}\text { Ott-Mar } \\
\text { Mar-Jun }\end{array}$ & $\begin{array}{l}\text { FEOOWW } \\
\text { WITH }\end{array}$ & $\begin{array}{l}28.1 \\
8.1\end{array}$ & CSTAB & 14.0 & $\mathrm{HABD}$ & 14.0 & PATHÖ & 14.0 & 'ŚĖD்L்̇ & 14.0 & "พ̈іт̈ & 14.0 \\
\hline
\end{tabular}

Abundance/eve/ refers to estimated relative abundance of spawners that spawned in the natal stream reaches that populated the life history pathways using the reach in this table: Level 1: $\quad$ Low to average (relative to other reaches) abundance

Level 2: $\quad$ High (relative to other reaches) abundance

Level 3 Exceptionally high (relative to other reaches) abundance

Life stages: 1- prespawner; 2- spawner; 3. incubation; 4- fry colonization; 5- summer rearing; 6- overwintering; 7- yeartingpresmoll/smolt

Attributes: CHEM - chemicals

CSTAB - channel stability

COMP - competition (with other species)

FLOW - flow

HABD - habitat diversity

$\begin{array}{ll}\text { NUTLD - nutrient load } & \text { RIPCN - riparian condition } \\ \text { OBST - obstructions } & \text { SEDLD - sediment load } \\ \text { OXYG - dissolved oxygen } & \text { TEMP - } \text { water temperature } \\ \text { PATHO - pathogens } & \text { WITH - } \quad \text { water withdrawals }\end{array}$

PATHO - pathogens

PRED - predators (includes fishing-related losses)
TEMP - water temperature

WITH - water withdrawals

"NA $\mathrm{N}$ indicates that no life history pathway meeting the criteria used in defining these pathways was present.

Blank cells under attributes indicate no additional attributes with negative effects on productivity were evident compared to those present in the template. 
Stream Reach Summary for Vacant Spring Chinook Salmon Life Histories

Stream: Lostine R.

Reach: Clearwater Ditch - Cross Country Ditch (RM 108.3 -110.8 from Snake R)

\begin{tabular}{|c|c|c|c|c|c|c|c|c|c|c|c|c|c|}
\hline \multicolumn{4}{|c|}{ Restoration benefit category: } & $\mathbf{F}$ & \multicolumn{3}{|c|}{ Chinook usage score: } & 7.8 & \multicolumn{4}{|c|}{ Habitat productivity loss score: } & -87.8 \\
\hline \multirow[b]{3}{*}{ Life stage } & \multirow[b]{3}{*}{ Months } & \multirow{2}{*}{\multicolumn{4}{|c|}{$\begin{array}{l}\text { No. vacant life history pathways } \\
\text { by abundance level }\end{array}$}} & \multicolumn{2}{|c|}{ Key habitat change } & \multirow{3}{*}{$\begin{array}{c}\text { Habitat } \\
\text { productivity y } \\
\text { loss score }\end{array}$} & & & & & \\
\hline & & & & & & \multirow{2}{*}{$\begin{array}{l}\text { Patient } \\
\text { quantity }\end{array}$} & \multirow[b]{2}{*}{$\%$ change } & & & & & & \\
\hline & & Total no. & 1 & 2 & 3 & & & & & & & & \\
\hline 1 & Apr-Aug & 26 & 13 & 8 & 5 & 9.1 & -29.4 & -5.6 & & & & & \\
\hline $1,2.3$ & Aug-Ott & 10 & 0 & 10 & "' $\quad 0$ "” & $9: 5 “ \cdots$ & -48.3 & -26.3 & & & & & \\
\hline 3 & Ott-Mar & 20 & 0 & 20 & 0 & 13.4 & -32.3 & -14.8 & & & & & \\
\hline 34 & Mar-May & 13 & $\dot{4}$ & $\ddot{4}$ & 5 & 12.2 & -29.8 & -40.3 & & & & & \\
\hline 5 & May-Ott & " & 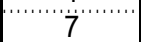 & 0 & 0 & 4.9 & -11.2 & -1.1 & & & & & \\
\hline 6 & Ott-Mar & NA & NA & NA & NA & NA & NA & NA & & & & & \\
\hline 7 & Mar-Jun & 2 & 2 & 0 & 0 & 23.1 & 1.7 & 0.3 & & & & & \\
\hline \multirow[b]{2}{*}{ Life stage } & \multirow[b]{2}{*}{ Months } & \multicolumn{12}{|c|}{ Highest productivity y impact attributes (\% change in impact from template) } \\
\hline & & Att 1 & $\%$ change & Att 2 & $\%$ change & Att 3 & $\%$ change & Att 4 & $\%$ change & An 5 & $\%$ change & Att 6 & $\%$ change \\
\hline 1 & Apr-Aug & FLOW & 25.0 & TEMP & 25.0 & HABD & 12.5 & WITH & 12.5 & & & & \\
\hline $1,2,3$ & Äug-Ött & CSTAB & 25.0 & FLOW & 25.0 & SEDLD & 25.0 & TEMP & 25.0 & ம்IT்H & 25.0 & HABD &, 12.5 \\
\hline 3 & Ott-Mar & CSTAB & 25.9 & FLOW & …ㄷ… & $\mathrm{HABD}$ & 25.0 & SEDLD & 25.0 & WITH & 25.0 & PATHO & 12.5 \\
\hline 34 & Mar-May & HABD & & WITH & $196^{\circ}$ & CSTAB & 9.8 & FLOOW & & PATHO & 9.8 & SEDED & \\
\hline 5 & May-Ott & $\because \mathrm{HABD}$ & 9.5 & CSTAB & 4.7 & PATHO & 4.7 & SEDLD & $4.7^{\prime \prime}$ & FLOW & 1.2 & TEMP & \\
\hline 6 & Ott-Mar & NA & $\mathrm{NA}$ & $\ddot{N A}$ & N̈̈" & $\mathrm{NA}$ & $\ddot{N A}$ & $\mathrm{NA}$ & $\mathrm{NA}$ & & $\mathrm{N}$ & $\mathrm{NA}$ & $\mathrm{NA}$ \\
\hline 7 & Mar-Jun & WITH & 2.5 & & & & & & & & & & \\
\hline
\end{tabular}

Abundance lovel refers to estimated relative abundance of spawners that spawned in the natal stream reaches that populated the life history pathways using the reach in this table Level 1: Low to average (relative to other reaches) abundance

Level 2 High (relative to other reaches) abundance

Level 3 Exceptionally high (relative to other reaches) abundance

Life stages.' 1 - prespawner; 2- spawner; 3- incubation; 4- fry colonization; 5- summer rearing; 6- overwintering; 7- yearling presmolt/smolt

$\begin{array}{lll}\text { Attributes: CHEM - chemicals } & \text { NUTLD - nutrient load } & \text { RIPCN - riparian condition } \\ \text { CSTAB - channel stability } & \text { OBST - obstructions } & \text { SEDLD - sediment load } \\ \text { COMP - competition (with other species) } & \text { OXYG - dissolved oxygen } & \text { TEMP - water temperature } \\ \text { FLOW - flow } & \text { PATHO - pathogens } & \text { WITH - water withdrawals } \\ \text { HABD - habitat diversity } & \text { PRED - predators (includes fishing-related losses) }\end{array}$

"NA" indicates that no life history pathway meeting the criteria used in defining these pathways was present.

Blank cells under attributes indicate no additional attributes with negative effects on productivity were evident compared to those present in the template. 
Stream Reach Summary for Vacant Spring Chinook Salmon Life Histories

Stream: Lostine R.

Reach: Cross Country Ditch - Sheep Ridge Dam (RM 110.8 -115.0 from Snake R)

\begin{tabular}{|ll|ll|l|}
\hline Restoration benefit category: & B & Chinook usage score: & 56.4 & Habitat productivity loss score: \\
\hline
\end{tabular}

\begin{tabular}{|c|c|c|c|c|c|c|c|c|}
\hline \multirow[b]{3}{*}{ Life stag } & \multirow[b]{3}{*}{ Months. } & \multirow{2}{*}{\multicolumn{4}{|c|}{$\begin{array}{c}\text { No. vacant tife history pathways } \\
\text { by abundance level }\end{array}$}} & \multicolumn{2}{|c|}{ Key habitat change } & \multirow{3}{*}{$\begin{array}{c}\text { Habitat } \\
\text { productivity\} } \\
\text { lass score }\end{array}$} \\
\hline & & & & & & \multirow{2}{*}{$\begin{array}{l}\text { Patient } \\
\text { ouantitv }\end{array}$} & \multirow[b]{2}{*}{$\%$ change } & \\
\hline & & Tolal. no & 1 & 2 & 3 & & & \\
\hline 1 & Apr-Aug & 45 & 33 & 7 & 5 & 80 & -16.8 & .1887 \\
\hline $1,2,3$ & Aug-Oct & 50 & 40 & 10 & 0 & 2.0 & -664 & -1984 \\
\hline 3 & Oct-Mar & 100 & 80 & 20 & 0 & 0.9 & -83.9 & -146.4 \\
\hline 34 & Mar-May & 64 & 56 & 3 & 5 & 11 & -83.9 & -433.8 \\
\hline 5 & May-Oct & 208 & 208 & 0 & 0 & 7.0 & -66.2 & 608.9 \\
\hline 6 & Oct-Mar & 38 & 38 & 0 & 0 & 12.9 & -2.5 & -44.2 \\
\hline 7 & Mar-Jun & 2 & 2 & 0 & $a$ & 19.8 & 0.0 & 2.5 \\
\hline
\end{tabular}

\begin{tabular}{|c|c|c|c|c|c|c|c|c|c|c|c|c|c|}
\hline \multirow[b]{2}{*}{ Life stage } & \multirow[b]{2}{*}{ Months } & \multicolumn{12}{|c|}{ Highest productivity impact attributes (\% change in impact from template) } \\
\hline & & Aft 1 & \% change & Alt 2 & $\%$ change & Att 3 & $\%$ change & Att 4 & $\%$ change & Att 5 & $\%$ change & Att 6 & $\%$ change \\
\hline 1 & Apr-Aug & FLOW & 56.5 & HABD & 37.7 & WITH & 37.7 & CSTAB & 25.0 & OBST & 18.8 & RIPCN & 18.8 \\
\hline $1,2,3$ & Aug-Oct & FLOW & 75.0 & CSTAB & 50.0 & HABD & 50.0 & WITH & 50.0 & OBST & 25.0 & RIPCN & 25.0 \\
\hline 3 & Oct-Mar & CSTAB & 50.0 & HABD & 50.0 & FLOW & 25.0 & PATHO & 25.0 & WITH & 25.0 & & \\
\hline 3,4 & Mar-May & CSTAB & 50.0 & FLOWW & 50.0 & "HABD" & 50.0 & WITH & 50.0 & PATHO & 25.0 & RIPCN & 25.0 \\
\hline 5 & May-Oct & FLOW & 50.0 & WITH & 500 & HABD & 25.0 & PATHO & 25.0 & RIPCN & 25.0 & & \\
\hline 6 & Oct-Mar & CSTAB & 25.0 & $\mathrm{HABD}$ & 25.0 & PATHO & 25.0 & WITH & 250 & & & & \\
\hline 7 & Mar-Jun & "WiTh & 25.0 & & & & & & & & & & \\
\hline
\end{tabular}

Abundance/eve/refers to estimated relative abundance of spawners that spawned in the natal stream reaches that populated the life history pathways using the reach in this table: Level 1: Low to average (relative to other reaches) abundance

Level 2: $\quad$ High (relative to other reaches) abundance

Level 3 Exceptionally high (relative to other reaches) abundance

Life stages; 1- prespawner; 2- spawner; 3- incubation; 4- fry colonization 5- summer rearing; 6- overwintering; 7- yearling presmolt/smolt

\begin{tabular}{|c|c|c|c|}
\hline $\begin{array}{l}\text { Attributes: } \\
\text { CHEM - } \\
\text { CSTAB - } \\
\text { COMP - } \\
\text { FLOW - } \\
\text { HABD - }\end{array}$ & $\begin{array}{l}\text { chemicals } \\
\text { channel stability } \\
\text { competition (with other species) } \\
\text { flow } \\
\text { habitat diversity }\end{array}$ & $\begin{array}{l}\text { NUTLD - } \\
\text { OBST - } \quad \text { obstructiont load } \\
\text { OXYG - } \quad \text { dissolved oxygen } \\
\text { PATHO - } \\
\text { Pathogens } \\
\text { PRED - } \quad \text { predators (includes fishing-related losses) }\end{array}$ & $\begin{array}{ll}\text { RIPCN - } & \text { riparian condition } \\
\text { SEDLD - } & \text { sediment load } \\
\text { TEMP - } & \text { water temperature } \\
\text { WITH - } & \text { water withdrawals }\end{array}$ \\
\hline
\end{tabular}

"NA" indicates that no life history pathway meeting the criteria used in defining these pathways was present.

Blank cells under attributes indicate no additional attributes with negative effects on productivity were evident compared to those present in the template. 
Stream Reach Summary for Vacant Spring Chinook Salmon Life Histories

Stream: Lostine R.

Reach: Sheep Ridge Dam - Silver Cr (RM 115,0 -120.0 from Snake R)

\begin{tabular}{|ll|ll|l|l|}
\hline Restoration benefit category: & E & Chinook usage score: & 44.3 & Habitat productivity loss score: & -2.5 \\
\hline
\end{tabular}

\begin{tabular}{|c|c|c|c|c|c|c|c|c|}
\hline \multirow[b]{3}{*}{ Life stage } & \multirow[b]{3}{*}{ Months } & \multirow{2}{*}{\multicolumn{4}{|c|}{$\begin{array}{c}\text { No. vacant life history pathways } \\
\text { by abundance level }\end{array}$}} & \multicolumn{2}{|c|}{ Key habitat change } & \multirow{3}{*}{$\begin{array}{c}\text { Habitat } \\
\text { productivity } \\
\text { loss score }\end{array}$} \\
\hline & & & & & & \multirow{2}{*}{$\begin{array}{l}\text { Patient } \\
\text { quantity }\end{array}$} & \multirow[b]{2}{*}{$\%$ change } & \\
\hline & & Total no. & 1 & 2 & 3 & & & \\
\hline 1 & Apr-Aug & 40 & 9 & 1 & 30 & 8.9 & -54.9 & 4.1 \\
\hline $1,2,3$ & $A$ & 50 & 0 & 0 & " 50 " & 10.0 & -24.1 & 31.7 \\
\hline 3 & Ott-Mar & 100 & 0 & 0 & 100 & 13.3 & 0.0 & 3.4 \\
\hline 34 & Mar-May & 20 & 9 & 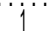 & $10^{\circ}$ & 11.2 & -31.1 & -41.7 \\
\hline 5 & May-Ott & NA & NÄ & $\mathrm{NA}$ & $\mathrm{NA}$ & NA & $\mathrm{NA}$ & $\mathrm{NA}$ \\
\hline 6 & Ott-Mar & NA & NA & NA & $\mathrm{NA}$ & $\mathrm{NA}$ & NA & NA \\
\hline 7 & Mar-Jun & $\mathrm{NA}$ & $\mathrm{NA}$ & $\mathrm{NA}$ & $\mathrm{NA}$ & $\mathrm{NA}$ & $\mathrm{NA}$ & NA \\
\hline
\end{tabular}

\begin{tabular}{|c|c|c|c|c|c|c|c|c|c|c|c|c|c|}
\hline \multirow[b]{2}{*}{ Life stage } & \multirow[b]{2}{*}{ Months } & \multicolumn{12}{|c|}{ Highest productivity impact attributes (\% change in impact from template) } \\
\hline & & Att 1 & $\%$ change & Att 2 & $\%$ change & Att 3 & $\%$ change & Att 4 & $\%$ change & Att 5 & $\%$ change & Atf 6 & $\%$ change \\
\hline 1 & Apr-Aug & HABD & 16,2 & & & & & & & & & & \\
\hline $1,2,3$ & Aug-Ött & CSṪAB & $25.0^{\circ}$ & HABD & 12.5 & & & & & & & & \\
\hline 3 & Ott-Mar & CSTAB & 25.0 & $\mathrm{HABD}$ & 12.5 & PATHO & $12: 5$ & RIPCN & 12.5 & 'WITH' & 12.5 & & \\
\hline 34 & Mar-May & CSTAB & 25.0 & HABD & 12.5 & PATHO & 12.5 & RIPCN & 12.5 & WITH & 12.5 & & \\
\hline 5 & May-Ott & $\mathrm{NA}$ & $\mathrm{NA}$ & NA & NA & $N A$ & $N A$ & NA & NA & NA & NA & NA & $\mathrm{NA}$ \\
\hline 6 & Ott-Mar & $\mathrm{NA}$ & $\mathrm{NA}$ & $\mathrm{NA}$ & NA & $\mathrm{NA}$ & & NA & $\mathrm{NA}$ & NÄ & $\mathrm{NA}$ & $\mathrm{NA}$ & $\mathrm{NA}$ \\
\hline 7 & Mar-Jun & NA & NA & NA & $\mathrm{NA}$ & NA & & $\mathrm{NA}$ & $\dot{N A}$ & $\mathrm{NÄ}$ & $\mathrm{NA}$ & $\mathrm{NA}$ & NA \\
\hline
\end{tabular}

Abundance lovel refers to estimated relative abundance of spawners that spawned in the natal stream reaches that populated the life history pathways using the reach in this table: Level 1: Low to average (relative to other reaches) abundance

Level 2: $\quad$ High (relative to other reaches) abundance

Level 3: Exceptionably high (relative to other reaches) abundance

Life stages: 1- prespawner; 2- spawner; 3- incubation; 4- fry colonization; 5- summer rearing; 6- overwintering; 7- yeariingpresmolt/smolt

$\begin{array}{cllr}\text { Attributes: CHEM - } & \text { chemicals } & \text { NUTLD - nutrient load } & \text { RiPCN - riparian condition } \\ \text { CSTAB - channel stability } & \text { OBST - obstructions } & \text { SEDLD - sediment ioad } \\ \text { COMP - } & \text { competition (with other species) } & \text { OXYG - dissolved oxygen } & \text { TEMP - water temperature } \\ \text { FLOW - flow } & \text { PATHO - pathogens } & \text { WiTH - water withdrawals } \\ \text { HABD - } & \text { habitat diversity } & \text { PREO - predators (includes fishing-related losses) }\end{array}$

"NA" indicates that no life history pathway meeting the criteria used in defining these pathways was present.

Blank cells under attributes indicate no additional attributes with negative effects on productivity were evident compared to those present in the template. 


\section{Stream Reach Summary for Vacant Spring Chinook Salmon Life Histories}

Stream: Lostine $\mathbf{R}$

Reach: Silver Cr - Lake Cr (RM 120.0 -124.6 from Snake R)

\begin{tabular}{|c|c|c|c|c|c|c|c|c|}
\hline Restorat & n benef & category & & & Chinoc & sage sc & re: & 21.8 \\
\hline & & No. & $\overline{\text { ant li }}$ & tory $p$ & ays & Key habi & t change & Habitat \\
\hline & & & $y a b u$ & ce lev & & Patient & & productivity \\
\hline Life stage & Months & Total no. & 1 & 2 & 3 & quantity & $\%$ change & loss score \\
\hline 1 & Apr-Aug & 35 & 29 & 6 & 0 & 7.0 & 0.0 & 0.0 \\
\hline $1,2,3$ & Aug-Oct & 50 & & 10 & 0 & 5.0 & 0.0 & -62.7 \\
\hline 3 & Ott-M̈ar & 100 & 80 & 20 & 0 & 48. & 0.0 & 0.0 \\
\hline 34 & Mar-May & 15 & 13 & 2 & 0 & 5.4 & 00 & 6.2 \\
\hline 5 & May-Ott & $\mathrm{NA}$ & $\mathrm{NA}$ & NÄ & NA & $\mathrm{NA}$ & NA & ‘ \\
\hline 6 & Ott-Mar & NA & NA & $\mathrm{NA}$ & NA & $\mathrm{NA}$ & NA & $\mathrm{NA}$ \\
\hline 7 & Mar-Jun & $\mathrm{NA}$ & $\mathrm{NA}$ & $\mathrm{NA}$ & $\mathrm{NA}$ & $\mathrm{NA}$ & NÄ & $\mathrm{NA}$ \\
\hline
\end{tabular}

\begin{tabular}{|c|c|c|c|c|c|c|c|c|c|c|c|c|c|}
\hline \multirow[b]{2}{*}{ Life stage } & \multirow[b]{2}{*}{ Months } & \multicolumn{12}{|c|}{ 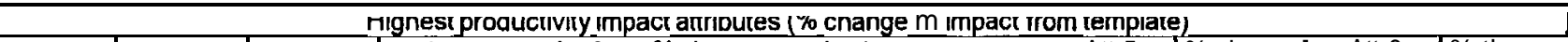 } \\
\hline & & Att 1 & $\%$ change & Att 2 & $9 \%$ than $e$ & Att 3 & $\%$ tha\%e & Att 4 & & Atti 1 南 & change] & Att 6 & $\%$ than en \\
\hline 1 & Apr-Aug & HABD & 22.4 & OBST & 22,4 & PRED & 12.5 & & & & & & \\
\hline $1,2,3$ & Aug-Ött & $H A B D$ & 25,0 & "ÖBST' & $25.0^{\circ}$ & PRED & 12.5 & & & & & & \\
\hline $3 "$ & Ott-Mar. & PATHO & 250 & & & & & & & & & & \\
\hline 3,4 & Mar-May & PATHOO & 25.0 & & & & & & & & & & \\
\hline 5 & May-Oct & NA & NA & NA & NA & NA & $\mathrm{NA}$ & $\mathrm{NA}$ & NA & $\mathrm{NA}$ & $\mathrm{NA}$ & $\mathrm{NA}$ & $\mathrm{NA}$ \\
\hline 6 & Oct-Mar & NA & NA & NÄ & $\mathrm{NA}$ & $\mathrm{NA}$ & $\mathrm{NA}$ & $\mathrm{NA}$ & NA & $\mathrm{NA}$ & $\mathrm{NA}$ & $\mathrm{NA}$ & $\mathrm{NA}$ \\
\hline 7 & Mar-Jun & $\mathrm{NA}$ & $\mathrm{NA}$ & $\mathrm{NA}$ & $\mathrm{NA}$ & $\mathrm{NA}$ & $\mathrm{NA}$ & $\mathrm{NA}$ & $\mathrm{NA}$ & $\mathrm{NA}$ & $\mathrm{NA}$ & $\mathrm{NA}$ & $\mathrm{NA}$ \\
\hline
\end{tabular}

Abundance level refers to estimated relative abundance of spawners that spawned in the natal stream reaches that populated the life history pathways using the reach in this table: Level 1: $\quad$ Low to average (relative to other reaches) abundance

Level 2 High (relative to other reaches) abundance

Level 3: Exceptionally high (relative to other reaches) abundance

Life stages: 1- prespawner; 2- spawner; 3- incubation; 4- fry colonization; 5- summer rearing; 6- overwintering; 7- yearlingpresmolt/smolt

$\begin{array}{cllr}\text { Attributes: CHEM - } & \text { chemicals } & \text { NUTLD - nutrient load } & \text { RIPCN - riparian condition } \\ \text { CSTAB - } & \text { channel stability } & \text { OBST - obstructions } & \text { SEDLD - sediment load } \\ \text { COMP - } & \text { competition (with other species) } & \text { OXYG - dissolved oxygen } & \text { TEMP - water temperature } \\ \text { FLOW - flow } & \text { PATHO - pathogens } & \text { WITH - water withdrawals } \\ \text { HABD - } & \text { habitat diversity } & \text { PRED - predators (includes fishing-related losses) }\end{array}$

"NA" indicates that no life history pathway meeting the criteria used in defining these pathways was present.

Blank cells under attributes indicate no additional attributes with negative effects on productivity were evident compared to those present in the template. 
Stream: Lostine R.

Reach: $\quad$ Lake Cr - East Lostine R (RM 124.6 -131.7 from Snake R)

\begin{tabular}{|c|c|c|c|c|c|c|c|c|}
\hline \multicolumn{4}{|c|}{ (Restoration benefit category: } & & \multicolumn{3}{|c|}{ Chinook usage score: } & 23.8 \\
\hline \multirow[b]{3}{*}{ Life stage } & \multirow[b]{3}{*}{ Months } & \multirow{2}{*}{\multicolumn{4}{|c|}{$\begin{array}{l}\text { No. vacant life history pathways } \\
\text { by abundance level }\end{array}$}} & \multicolumn{2}{|c|}{ Key habitat change } & \multirow{3}{*}{$\begin{array}{l}\text { Habitat } \\
\text { productivity } \\
\text { loss score }\end{array}$} \\
\hline & & & & & & \multirow{2}{*}{$\begin{array}{l}\text { Patient } \\
\text { quantity }\end{array}$} & \multirow[b]{2}{*}{$\%$ change } & \\
\hline & & Total no. & 1 & 2 & 3 & & & \\
\hline 1 & Apr-Aug & 30 & 30 & 0 & 0 & 4.8 & 0.0 & 0.0 \\
\hline $1,2,3$ & Aug-Oct & 50 & 50 & 0 & 0 & 4.0 & 0.0 & -62.7 \\
\hline 3 & Oct-Mar & $100^{\circ}$ & 100 & 0 & 0 & 3.8 & 0.0 & 0.0 \\
\hline 3,4 & Mar-May & 10 & 10 & 0 & 0 & 3.8 & 0.0 & 4.2 \\
\hline 5 & May-Oct & $N A$ & $\mathrm{NA}$ & NA & $\mathrm{NA}$ & $\mathrm{NA}$ & $\mathrm{NA}$ & NA \\
\hline 6 & Oct-Mar & $\mathrm{NA}$ & $\mathrm{NA}$ & $\mathrm{NA}$ & $\mathrm{NA}$ & $\mathrm{NA}$ & $\mathrm{NA}$ & NA \\
\hline 7 & Mar-Jun & NA & $\mathrm{NA}$ & $\mathrm{NA}$ & $\mathrm{NA}$ & $\mathrm{NA}$ & $\mathrm{NA}$ & NA \\
\hline
\end{tabular}

\begin{tabular}{|c|c|c|c|c|c|c|c|c|c|c|c|c|c|}
\hline \multirow[b]{2}{*}{ Life stage } & \multirow[b]{2}{*}{ Months } & \multicolumn{12}{|c|}{ Highest productivity impact attributes (\% change in impact from template) } \\
\hline & & Att 1 & $\%$ change & $\overline{\text { Att } 2}$ & $\%$ change & Att 3 & \% change & Att 4 & $\%$ change & Atf 5 & $\%$ change & Att 6 & $\%$ change \\
\hline & Apr-Aug & HABD & 25.0 & OBST & 25.0 & PRED & 12.5 & & & & & & \\
\hline $1,2,3$ & Aug-Ott & HABD & 25.0 & "ÖB̈S̈T̄" & 12.5 & " "PREËD̈ & 12.5 & & & & & & \\
\hline "3 & Ott-Mar & РАTHO & 12.5 “ & & & & & “" & & & & & \\
\hline 3,4 & Mar-May & PATHO & 12.5 & & & & & & & & & & \\
\hline 5 & May-Oct & N. & NA & NA & NA & "NA" & NA & NA & NA & NA & $\mathrm{NA}$ & $\mathrm{NA}$ & NA \\
\hline 6 & Ött-Mar & NA & NA & NA & NA & NA & $\mathrm{NA}$ & NA & $\mathrm{NÄ}$ & NA & NA & $\mathrm{NA}$ & $\mathrm{NA}$ \\
\hline 7 & Mar-Jun & NA “ “ & NA & NA & NA & NA & NA & NA & NA & $\mathrm{NA}$ & NA & NA & NA \\
\hline
\end{tabular}

Abundance level refers to estimated relative abundance of spawners that spawned in the natal stream reaches that populated the life history pathways using the reach in this table: Level 1: $\quad$ Low to average (relative to other reaches) abundance

Level 2: $\quad$ High (relative to other reaches) abundance

Level 3: Exceptionally high (relative to other reaches) abundance

Life stages: 1-prespawner; 2-spawner; 3- incubation; 4- fry colonization; 5- summer rearing; 6- overwintering; 7- yearlingpresmolt/smolt

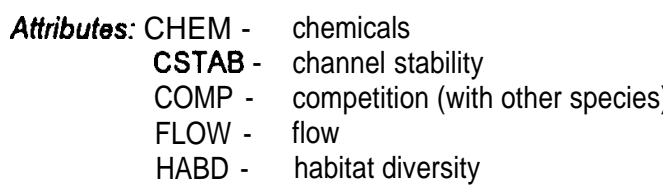

NUTLD - nutrient load

OBST - obstructions

OXYG - dissolved oxygen

PATHO - pathogens

PRED - predators (includes fishing-related losses)
RIPCN - riparian condition

SEDLD - sediment load

TEMP - water temperature

WITH - water withdrawals

"NA" indicates that no life history pathway meeting the criteria used in defining these pathways was present.

Blank cells under attributes indicate no additional attributes with negative effects on productivity were evident compared to those present in the template. 
Stream Reach Summary for Vacant Spring Chinook Salmon Life Histories

Stream: Miriam R.

Reach: Mouth - Cougar Cr (RM 89.8 -99.4 from Snake R)

\begin{tabular}{ll|ll|ll}
\hline Restoration benefit category: & B & Chinook usage score: & 88.3 & Habitat productivity loss score:
\end{tabular}

\begin{tabular}{|c|c|c|c|c|c|c|c|c|c|}
\hline \multirow[b]{3}{*}{ Life stage } & \multirow[b]{3}{*}{ Months } & \multirow{2}{*}{\multicolumn{4}{|c|}{$\begin{array}{c}\text { No. vacant life history pathways } \\
\text { by abundance level }\end{array}$}} & & \multicolumn{2}{|c|}{ Key habitat change } & \multirow{3}{*}{$\begin{array}{c}\text { Habitat } \\
\text { productivity } \\
\text { loss score }\end{array}$} \\
\hline & & & & & & & \multirow{2}{*}{$\begin{array}{l}\text { Patient } \\
\text { quantity }\end{array}$} & \multirow[b]{2}{*}{$\%$ change } & \\
\hline & & Total no. & 1 & 2 & $\overline{3}$ & & & & \\
\hline$\overline{1}$ & Apr-Aug & 69 & 58 & $\overline{9}$ & $\overline{2}$ & & 2.7 & $-77,2$ & -239.3 \\
\hline $1,2,3$ & A & 90 & 90 & " 0 & 0 & " & "' 2.2 & -83.3 & $-229,3$ \\
\hline 3 & Ott- -1 & $180^{\circ}$ & 180 & 0 & 0 & & 2.4 & -83.3 & 147.8 \\
\hline-34 & Mar-May & 72 & 50 & $188^{\circ}$ & 4 & & 13.1. & -26.0 & -140.5 \\
\hline 5 & May-Öt & 213 & 94 & 98 & $2 i$ & & $15.8^{\circ}$ & 0.0 & -286.2 \\
\hline 6 & Ott-Mar & 38 & 19 & 19 & 0 & & 36.2 & 0.0 & 0.0 \\
\hline 7 & Mar-Jun & 3 & 1 & 2 & $\dddot{0}$ & & 60.8 & 0.0 & 0.0 \\
\hline
\end{tabular}

\begin{tabular}{|c|c|c|c|c|c|c|c|c|c|c|c|c|c|}
\hline \multirow[b]{2}{*}{ Life stage } & \multirow[b]{2}{*}{ Months } & \multicolumn{12}{|c|}{ rignest productivity Impact aurioutes (\% cnange $\mathrm{m}$ impact Irom remplate) } \\
\hline & & Att 1 & $\%$ change & Att 2 & $\%$ change & Att 3 & $\%$ change & Att 4 & \% change & Att 5 & $\%$ change & Att 6 & $\%$ change \\
\hline 1 & Apr-Aug & HABD. & 43.9 & SEDLD & $25.0 \ldots$ & CSTAB. & 12.5 & teTrinta & $\frac{1}{12 . F}$ & & & & \\
\hline$, 1,2,3$ & Aug-Oct & HABD & 50.0 & SEDLD & 50.0 & CSTAB & 25.0 & TEMP & 25.0 & & & & \\
\hline & Q̈t-TMar & $\cdots H A B B$ & 50.9 & SESLL & 50.9 & - CSTAB & 25.0 & FLOW & 25.0 & PATHO & 25.0 & TEMP & 250 \\
\hline 3,4 & Mar-May & HABD & 50.0 & SEDLD & 50.0 & CSTAB & 25.0 & FLOW & 25.0 & PATHO & 25.0 & RIPCN & 250 \\
\hline 5 & May-Ott & FLOW & 24.9 & HABD & 24.9 & PATHO & 249 & RIPCN & 249 & TEMP & 24.9 & & \\
\hline$\dddot{6}$ & Ott-Mar & FL்ல் & $25.0^{\circ}$ & РATHO & 25.0 & & & & & & & & \\
\hline 7 & Mar-Jun & & & & & & & & & & & & \\
\hline
\end{tabular}

Abundance level refers to estimated relative abundance of spawners that spawned in the natal stream reaches that populated the life history pathways using the reach in this table Level 1: Low to average (relative to other reaches) abundance

Level 2 High (relative to other reaches) abundance

Level 3 Exceptionally high (relative to other reaches) abundance

Life stages: 1 - prespawner; 2- spawner; 3- incubation; 4- fry colonization; 5- summer rearing; 6-overwintering; 7-yearling presmolt/smolt

\begin{tabular}{|c|c|c|c|}
\hline $\begin{aligned} & \text { Attributes: } \text { CHEM - } \\
& \text { CSTAB - } \\
& \text { COMP - } \\
& \text { FLOW - } \\
& \text { HABD - }\end{aligned}$ & $\begin{array}{l}\text { chemicals } \\
\text { channel stability } \\
\text { competition (with other species) } \\
\text { flow } \\
\text { habitat diversity }\end{array}$ & $\begin{array}{l}\text { NUTLD - nutrient load } \\
\text { OBST - obstructions } \\
\text { OXYG - dissolved oxygen } \\
\text { PATHO - pathogens } \\
\text { PRED - predators (includes fishing-related losses) }\end{array}$ & $\begin{array}{l}\text { RIPCN - riparian condition } \\
\text { SEDLD - sediment load } \\
\text { TEMP - water temperature } \\
\text { WITH - } \quad \text { water withdrawals }\end{array}$ \\
\hline
\end{tabular}

"NA" indicates that no tife history pathway meeting the criteria used in defining these pathways was present.

Blank cells under attributes indicate no additional attributes with negative effects on productivity were evident compared to those present in the template. 


\section{Stream Reach Summary for Vacant Spring Chinook Salmon Life Histories}

Stream: Miriam R.

Reach: Cougar Cr - Liie Miriam R (RM 99.4 -108.4 from Snake R)

\begin{tabular}{|c|c|c|c|c|c|c|c|c|}
\hline \multicolumn{4}{|c|}{ [Restoration benefit category: } & $\bar{D}$ & \multicolumn{3}{|c|}{ Chinook usage score: } & 29.0 \\
\hline \multirow[b]{3}{*}{ Life stage } & \multirow[b]{3}{*}{ Months } & \multirow{2}{*}{\multicolumn{4}{|c|}{$\begin{array}{c}\text { No. vacant life history pathways } \\
\text { by abundance level }\end{array}$}} & \multicolumn{2}{|c|}{ Key habitat change } & \multirow{3}{*}{$\begin{array}{l}\text { Habitat } \\
\text { productivity } \\
\text { loss score }\end{array}$} \\
\hline & & & & & & \multirow{2}{*}{$\begin{array}{l}\text { Patient } \\
\text { quantity }\end{array}$} & \multirow[b]{2}{*}{$\%$ change } & \\
\hline & & Total no. & 1 & 2 & 3 & & & \\
\hline 1 & Apr-Aug & 40 & 24 & 14 & 2 & 2.8 & -81.9 & -13.0 \\
\hline $1,2,3$ & Aug-Oct & 50 & 40 & 10 & 0 & 9.8 & -22.8 & -25.4 \\
\hline 3 & Oct-Mar & 100 & 80 & 20 & 0 & 14.4 & 67 & -225.9 \\
\hline 3,4 & Mar-May & 15 & 8 & 6 & 1 & 17.0 & 8.1 & -48.0 \\
\hline 5 & May-Oct & 4 & 0 & 3 & 1 & 12.8 & 0.0 & -0.9 \\
\hline 6 & Oct-Mar & $\mathrm{NA}$ & $\mathrm{NA}$ & $\mathrm{NA}$ & $\mathrm{NA}$ & NA & $\mathrm{NA}$ & $\mathrm{NA}$ \\
\hline 7 & Mar-Jun & 1 & 0 & 1 & 0 & 60.8 & 4.1 & 0.0 \\
\hline
\end{tabular}

\begin{tabular}{|c|c|c|c|c|c|c|c|c|c|c|c|c|c|}
\hline \multirow[b]{2}{*}{ Life stage } & \multirow[b]{2}{*}{ Months } & \multicolumn{4}{|c|}{ Highest pro } & \multicolumn{8}{|c|}{ Ictivity impact attributes (\% change in impact from template) } \\
\hline & & Alt 1 & $\%$ change & Att 2 & $\%$ change & Att 3 & $\%$ change & Att 4 & $\%$ change & Att 5 & $\%$ change & Att 6 & $\%$ change \\
\hline 1 & Apr-Aug & HABD & 25.0 & TEMP & 25.0 & SEDLD & 16.7 & CSTAB & 8.3 & & & & \\
\hline $1,2,3$ & Aug-Oct & SEDLD & 50.0 & CSTAB & 25.0 & TEMP & 25.0 & HABD & 16.7 & & & & \\
\hline 3 & Oct-Mar & SEDLD & 500 & CSTAB & 25.0 & HABD & 25.0 & TEMP & 250 & FLOW & 12.5 & PATHO & 12.5 \\
\hline 3,4 & Mar-May & HABD & 25.0 & SEDLD & 25.0 & CSTAB & 125 & FLOW & 12.5 & PATHO & 12.5 & TEMP & 12.5 \\
\hline 5 & May-Oct & FLOW & 12.3 & HABD & 12.3 & PATHO & 123 & RIPCN & 12.3 & & & & \\
\hline 6 & Oct-Mar & $\mathrm{NA}$ & $\mathrm{NA}$ & $\mathrm{NA}$ & NÄ & NA & $\mathrm{NA}$ & $\mathrm{NA}$ & $\mathrm{NA}$ & $\mathrm{NA}$ & $\mathrm{NA}$ & $\mathrm{NA}$ & $\mathrm{NA}$ \\
\hline 7 & Mar-Jun & & & & & & & & & & & & \\
\hline
\end{tabular}

Abundancelevel refers to estimated relative abundance of spawners that spawned in the natal stream reaches that populated the life history pathways using the reach in this table: Level 1: Low to average (relative to other reaches) abundance

Level 2: $\quad$ High (relative to other reaches) abundance

Level 3: $\quad$ Exceptionally high (relative to other reaches) abundance

Life stages: 1 - prespawner; 2- spawner; 3- incubation; 4- fry colonization; 5- summer rearing; 6- overwintering; 7- yearling presmolt/smolt

Attributes: CHEM - chemicals CSTAB - channel stability

COMP - competition (with other species)

FLOW - flow

HABD - habitat diversity
NUTLD - nutrient load

OBST - obstructions

OXYG - dissolved oxygen

PATHO - pathogens

PRED - predators (includes fishing-related losses)
RIPCN - riparian condition

SEDLD - sediment load

TEMP - water temperature

WITH - water withdrawals

"NA" indicates that no life history pathway meeting the criteria used in defining these pathways was present,

Blank cells under attributes indicate no additional attributes with negative effects on productivity were evident compared to those present in the template. 


\section{Stream Reach Summary for Vacant Spring Chinook Salmon Life Histories}

Stream: Miriam R.

Reach: Little Miriam R - splash dam site (1 m aboveGarwood Cr; RM 108.4 -119.7 from Snake R)

\begin{tabular}{ll|ll|l}
\hline Restoration benefit category: & E & Chinook usage score: & 58.7 & Habitat productivity loss score: \\
\hline
\end{tabular}

\begin{tabular}{|c|c|c|c|c|c|c|c|c|}
\hline \multirow[b]{3}{*}{ Life stage } & \multirow[b]{3}{*}{ Months } & \multirow{2}{*}{\multicolumn{4}{|c|}{$\begin{array}{c}\text { No. vacant life history pathways } \\
\text { by abundance level }\end{array}$}} & \multicolumn{2}{|c|}{ Key habitat change } & \multirow{3}{*}{$\begin{array}{c}\text { Habitat } \\
\text { productivity } \\
\text { loss score }\end{array}$} \\
\hline & & & & & & \multirow{2}{*}{$\begin{array}{l}\text { Patient } \\
\text { quantity }\end{array}$} & \multirow[b]{2}{*}{$\%$ change } & \\
\hline & & Total no. & 1 & 2 & 3 & & & \\
\hline 1 & Apr-Aug & 35 & 0 & 28 & 7 & 13.6 & -60.0 & -6.5 \\
\hline 123 & Aug-Ott & 50 & 0 & 40 & " 10 & 10.7 & -60.0 & "12.7" \\
\hline 3 & Ott-Mar & 100 & 0 & 80 & 20 & 10.7 & -60.0 & -44.2 \\
\hline $3 \dot{4}$ & Mar-May & 40 & 0 & 32 & 8 & 13.4 & -60.0 & -17.9 \\
\hline 5 & May-Ott & 114 & 0 & 94 & 20 & 13.0 & -60.0 & 62,2 \\
\hline 6 & Ott-Mar & 19 & 0 & 19 & & 26.7 & 0.0 & -15.3 \\
\hline 7 & Mar-Jun & 11 & 0 & 1 & & 44.2 & 0.0 & 0.0 \\
\hline
\end{tabular}

\begin{tabular}{|c|c|c|c|c|c|c|c|c|c|c|c|c|c|}
\hline \multirow[b]{2}{*}{ Life stage } & \multirow[b]{2}{*}{ Montha } & \multicolumn{12}{|c|}{ Highest productivity impact attributes (\% change in impact from template) } \\
\hline & & Att 1 & $\%$ change & Att 2 & \% change & Att 3 & $\%$ change & Att 4 & \% change & Att 5 & $\%$ change & Atf 6 & $\%$ change \\
\hline 1 & Apr-Aug & HABD & 25.0 & CSTAB & 12.5 & & & & & & & & \\
\hline $1,2,3$ & Aug-Ott & CSTAB & 25,0 & HABD & 250 & & & & & & & & \\
\hline & Ott-Mar & CSTAB & 25.0 & HABD & 25.0 & PATHO & 25.0 & & & & & & \\
\hline 3,4 & Mar-May & Eบิด & $25 . y^{n}$ & $4 A B D$ & 25.0 & PATHO & 25.0 & & & & & & \\
\hline 5 & May-Ott & CSTAB & 25 & HABD & 25.0 & PATHÖ & 25.0 & & & & & & \\
\hline $\begin{array}{l}6 \\
7\end{array}$ & $\begin{array}{l}\text { Ott-Mar } \\
\text { Mar-Jun }\end{array}$ & CSTAB & 25. & $\dot{H A B B}$ & 25.0 & PATHHO & 25,0 & & & & & & \\
\hline
\end{tabular}

Abundancelevel refers to estimated relative abundance of spawners that spawned in the natal stream reaches that populated the life history pathways using the reach in this table Level 1: $\quad$ Low to average (relative to other reaches) abundance

Level 2 High (relative to other reaches) abundance

Level 3 Exceptionally high (relative to other reaches) abundance

Life stages: 1- prespawner; 2- spawner; 3- incubation; 4- fry colonization; 5- summer rearing; 6- overwintering; 7-yearling presmolt/smolt

Attributes: CHEM - chemicals CSTAB - channel stability

COMP - competition (with other species)

FLOW - fiOW

HABD - habitat diversity
NUTLD - nutrient load

OBST - obstructions

OXYG - dissolved oxygen

PATHO - pathogens

PRED - predators (includes fishing-reiated losses)
RIPCN - riparian condition

SEDLD - sediment ioad

TEMP - water temperature

WITH - water withdrawals

"NA" indicates that no tife history pathway meeting the criteria used in defining these pathways was present.

Blank cells under attributes indicate no additional attributes with negative effects on productivity were evident compared to those present in the template. 


\section{Stream Reach Summary for Vacant Spring Chinook Salmon Life Histories}

Stream: Minam R.

Reach: Splash dam site - falls (RM $119.7-138,0$ from Snake R)

\begin{tabular}{|c|c|c|c|c|c|c|c|c|}
\hline [Restorati & n benefit & categor & & & Chinor & Isage sc & re: & 32.2 \\
\hline & & & $\overline{\operatorname{sant} 1}$ & $\overline{\operatorname{ryp} p}$ & ys & Key hab & at change & Habitat \\
\hline & & & $y$ abe & eles & & Patient & & productivity \\
\hline Life stage & Months & Total no. & 1 & 2 & 3 & quantity & $\%$ change & loss score \\
\hline 1 & Apr-Aug & 30 & 0 & 24 & 6 & 10.0 & 0.0 & 5.9 \\
\hline $1,2,3$ & Aug-Oct & 50 & 0 & 40 & 10 & 8.4 & 0.0 & 32.8 \\
\hline 3 & Oct-Mar & 100 & 0 & 180 & 20 & 8.6 & 0.0 & 0.0 \\
\hline 3,4 & Mar-May & 15 & 0 & 12 & 3 & 9.8 & 0.0 & 6.3 \\
\hline 5 & May-Oct & $\mathrm{NA}$ & NÄ & NÄ & NA & NA & $\mathrm{NA}$ & $\mathrm{NA}$ \\
\hline 6 & Oct-Mar & $\mathrm{NA}$ & $\mathrm{NA}$ & NA & NA & $\mathrm{NA}$ & NA & $\mathrm{NA}$ \\
\hline 7 & Mar-Jun & $\mathrm{NA}$ & $\mathrm{NA}$ & $\mathrm{NA}$ & $\mathrm{NA}$ & $\mathrm{NA}$ & $\mathrm{NA}$ & $\mathrm{NA}$ \\
\hline
\end{tabular}

\begin{tabular}{|c|c|c|c|c|c|c|c|c|c|c|c|c|c|}
\hline \multirow[b]{2}{*}{ Life stage } & \multirow[b]{2}{*}{ Months } & \multicolumn{12}{|c|}{ Highest productivity impact attributes (\% change in impact from template) } \\
\hline & & Att 1 & $\%$ change & Att 2 & $\%$ change & Att 3 & $\%$ change & Att 4 & $\%$ change & Att 5 & $\%$ change & Att 6 & $\%$ change \\
\hline 1 & Apr-Aug & & & & & & & & & & & & \\
\hline $1,2,3$ & Aug-Ott & & & & & & & жан & & & & & \\
\hline 3“" “ & Ott-tiar & "'PATHO & 12.5 & & & “"”'" "' & & & & & & & \\
\hline 34 & Mar-May. & "PATHÖO & 12.5 & & & & & & & & & & \\
\hline 5 & May-Ott & $N A$ & NA & NA & NA & NA & NA & NA & NÄ & NA & $\dot{N A}$ & $\mathrm{NA}$ & $\mathrm{NA}$ \\
\hline 6 & Ött-M̈ar & NÄ & NA & "NÄ & $\dot{\mathrm{NA}}$ & NA & NÄ & NA & $\dot{N A}$ & $\mathrm{NA}$ & NA & NA & N̈̈̈ \\
\hline 7 & Mar-Jun & NA & NA & NA & NA & NA & NA & NA & NA & NA & NA & NA & NA \\
\hline
\end{tabular}

Abundance level refers to estimated relative abundance of spawners that spawned in the natal stream reaches that populated the life history pathways using the reach in this table Level 1: $\quad$ Low to average (relative to other reaches) abundance

Level 2 High (relative to other reaches) abundance

Level 3 Exceptionably high (relative to other reaches) abundance

Life stages: 1- prespawner; 2- spawner; 3- incubation; 4- fry colonization; 5- summer rearing; 6- overwintering; 7- yearling presmolt/smolt

Attributes: CHEM - chemicals

CSTAB - channel stability

COMP - competition (with other species)

FLOW - fiOW

HABD - habitat diversity
NUTLD - nutrient load

OBST - obstructions

OXYG - dissolved oxygen

PATHO - pathogens

PRED - predators (includes fishing-related losses)
RIPCN - riparian condition

SEDLD - sediment load

TEMP - $\quad$ water temperature

WiTH - water withdrawals

"NA" indicates that no life history pathway meeting the criteria used in defining these pathways was present.

Blank ceils under attributes indicate no additional attributes with negative effects on productivity were evident compared to those present in the template. 
Stream Reach Summary for Vacant Spring Chinook Salmon Life Histories

Stream: Prairie Cr.

Reach: Mouth - OK Gulch confluence (RM 126.8 -131.8 from Snake R)

\begin{tabular}{ll|l}
\hline Restoration benefit category: & $F$ & Chinook usage score \\
\hline
\end{tabular}

\begin{tabular}{l|l}
5.8 & Habitat productivity loss score
\end{tabular}

$-107.4$

\begin{tabular}{|c|c|c|c|c|c|c|c|c|}
\hline \multirow[b]{3}{*}{ Life stage } & \multirow[b]{3}{*}{ Months } & \multirow{2}{*}{\multicolumn{4}{|c|}{$\begin{array}{c}\text { No. vacant lie history pathways } \\
\text { by abundance level }\end{array}$}} & \multicolumn{2}{|c|}{ Key habitat change } & \multirow{3}{*}{$\begin{array}{c}\text { Habitat } \\
\text { productivity } \\
\text { loss score }\end{array}$} \\
\hline & & & & & & \multirow{2}{*}{$\begin{array}{l}\text { Patient } \\
\text { quantity }\end{array}$} & \multirow[b]{2}{*}{$\%$ change } & \\
\hline & & Total no. & 1 & 2 & 3 & & & \\
\hline 7 & Apr-Aug & 7 & 1 & 6 & $\overline{0}$ & 3,3 & 9.0 & 3.3 \\
\hline 1,33 & Aug-Ott & 10 & 0 & 10 & 0 & 2.3 & “ 78.6 & 7 \\
\hline 3 & Ott-Mar & 20 & 0 & 20 & 0 & 2.3 & 50.0 & -85.8 \\
\hline 34 & Mar-May & 3 & 1 & 2 & 0 & 2.5 & 50.0 & -17.5 \\
\hline 5 & May-Ott & $\mathrm{NA}$ & $\mathrm{NA}$ & $\mathrm{NA}$ & $\mathrm{NA}$ & NA & NA & $\mathrm{NA}$ \\
\hline 6 & Ott-Mar & NA & NA & NA & NA“““” & NA & NA & NA \\
\hline 7 & Mar-Jun & $\mathrm{NA}$ & $\mathrm{NA}$ & $\mathrm{NA}$ & NA' & $\mathrm{NA}$ & $\mathrm{NA}$ & $\mathrm{NA}$ \\
\hline
\end{tabular}

\begin{tabular}{|c|c|c|c|c|c|c|c|c|c|c|c|c|c|}
\hline \multirow[b]{2}{*}{ Life stage } & \multirow[b]{2}{*}{ Months } & \multicolumn{12}{|c|}{ Highest productivity impact attributes (\% change in impact from template) } \\
\hline & & $\overline{\text { Att } 1}$ & $\%$ change & $\overline{\text { Att } 2}$ & \% change & Att 3 & $\%$ change & Atf 4 & $\%$ change & Att 5 & $\%$ change & Atf 6 & $\%$ change \\
\hline 1 & Apr-Aug & SEDLD & 37.5 & CHEM & 25.0 & RIPCN & 25. & NUTLD & 20 & CSTAB & 12. & & \\
\hline $.1,2,3$ & Aug-Ott & SEDLD & 50.0 & CHEM & 25.0 & CSTAB & 25.0 & RIPCN & $25^{\circ}$ & NUTLD & 12. & & \\
\hline 3 & Ott-Mar & HÄB̈ & “ ‘50:0“ & SEDLD & 50.0 & CHEM & 25.0 & CSTAB & 25.0 & FLOW & 25.0 & PATHO & 25.0 \\
\hline 34 & Mar-May & HABD & 50.0 & SEDLD" & $50.0 \%$, & "'"'"CHEM,... & 25.0 & CSTAB & 25.0 & "FLOWW" & 250 & РАTHÖ & 25.0 \\
\hline$\dot{5}$ & May-Ott & $\mathrm{NA}$ & $\dot{\mathrm{NA}}$ & $\mathrm{NA}$ & $\mathrm{NA}$ & NA & NA & NA & NA & $\mathrm{NÄ}$ & $\dot{N A}$ & ìA & $\mathrm{NA}$ \\
\hline 6 & Ott-Mar & NA & $\mathrm{NA}$ & $\mathrm{NA}$ & $\mathrm{NA}$ & $\mathrm{NA}$ & $\mathrm{NA}$ & $\mathrm{NA}$ & $\dot{N} \dot{A}$ & $\mathrm{NA}$ & $\mathrm{NA}$ & $\mathrm{NA}$ & $\mathrm{NA}$ \\
\hline 7 & Mar-Jun & NA & NA & NA & NA & NA & NA & NA & NA & NA & NA & NA & NA \\
\hline
\end{tabular}

Abundance level refers to estimated relative abundance of spawners that spawned in the natal stream reaches that populated the life history pathways using the reach in this table Level 1: Low to average (relative to other reaches) abundance

Level 2 High (relative to other reaches) abundance

Level 3 Exceptionally high (relative to other reaches) abundance

Life stages: 1 - prespawner; 2- spawner; 3- incubation; 4- fry colonization; 5- summer rearing; 6- overwintering; 7-yearling presmolt/smolt

Attributes: CHEM - chemicals

CSTAB - channel stability

COMP - competition (with other species)

FLOW - flow

HABD - habitat diversity
NUTLD - nutrient load

OBST - obstructions

OXYG - dissolved oxygen

PATHO - pathogens

PRED - predators (includes fishing-related losses)
RIPCN - riparian condition

SEDLD - sediment load

TEMP - water temperature

WITH - water withdrawals

"NA" indicates that no life history pathway meeting the criteria used in detining these pathways was present.

Blank cells under attributea indicate no additional attributes with negative effects on productivity were evident compared to those present in the template. 
Stream Reach Summary for Vacant Spring Chinook Salmon Life Histories

Stream: Prairie Cr.

Reach: OK Gulch fork (RM 131.8 -133.8 from Snake R)

Restoration benefit category: $\mathbf{F}$

Chinook usage score: 3.8

Habitat productivity loss score:

285.4

\begin{tabular}{|c|c|c|c|c|c|c|c|c|}
\hline \multirow[b]{3}{*}{ Life stage } & \multirow[b]{3}{*}{ Months } & \multirow{2}{*}{\multicolumn{4}{|c|}{$\begin{array}{l}\text { No. vacant life history pathways } \\
\text { by abundance level }\end{array}$}} & \multicolumn{2}{|c|}{ Key habitat change } & \multirow{3}{*}{$\begin{array}{l}\text { Habitat } \\
\text { productivity } \\
\text { loss score }\end{array}$} \\
\hline & & & & & & \multirow{2}{*}{$\begin{array}{l}\text { Patient } \\
\text { quantity }\end{array}$} & \multirow[b]{2}{*}{$\%$ change } & \\
\hline & & Total no. & 1 & 2 & 3 & & & \\
\hline 1 & Apr-Aug & 6 & 6 & 0 & 0 & 0.3 & 0.0 & 65,0 \\
\hline 1,23 & Aug-Ott & 10 & 10 & 0 & 0 & 1.1 & 0.0 & 867 \\
\hline 3 & Öt-Mar & 20 & 20 & 0 & 0 & 16 & 0.0 & 125.3 \\
\hline 34 & Mar-May & 2 & 2 & 0 & 0 & 17 & 0.0 & 83 \\
\hline 5 & May-Ott & $\mathrm{NA}$ & $\mathrm{NA}$ & NA & NA & $\mathrm{NA}$ & $\mathrm{NA}$ & NA \\
\hline 6 & Ott-Mar & NA & NA & NA & NA & $\ddot{\mathrm{NA}}$ & NA & $\dot{N} \dot{A}$ \\
\hline 7 & Mar-Jun & $\mathrm{NA}$ & $\mathrm{NA}$ & NA & $\mathrm{NA}$ & $\mathrm{NA}$ & $\mathrm{NA}$ & $\mathrm{NA}$ \\
\hline
\end{tabular}

\begin{tabular}{|c|c|c|c|c|c|c|c|c|c|c|c|c|c|}
\hline \multirow[b]{2}{*}{ Life stage } & \multirow[b]{2}{*}{ Months } & \multicolumn{12}{|c|}{ Highest productivity impact attributes ( $\%$ change in impact from template) } \\
\hline & & At! 1 & $\%$ change & At! 2 & $\%$ change & At! 3 & $\%$ change & At! 4 & $\%$ change & At! 5 & $\%$ change & At! 6 & $\%$ change \\
\hline 1 & Apr-Aug & HABD & 25.0 & NUTLD & $25 \Omega$ & PRED & 25.0 & RIPCN & 25.0 & SEDLD & 25.0 & & \\
\hline$, 1,2,3$ & Äug-Ött & SEÉLLD & 75.0 & NUTLLD & 50.0 & CSTAB & 25.0 & RIPCN & 25.00 & "HÄB̈D" & 16.7 & PRED & "16.7 \\
\hline 3 & 'Ott-Mar & SEDLD & 75.0 “' & NUTLD & 50.0 & CSTAB & 25.0 & RIPCN & 25.0 & $\mathrm{HABD}$ & 125 & PATHO & 12.5 \\
\hline 34 & Mar-Mäà & SEDLD & 37.5 & CSTAB & 250 & NUTLD & 25.0 & HABD & 12.5 & PATHO & 12.5 & RIPCN & 12.5 \\
\hline 5 & "May-Ott" & $\mathrm{NA}$ & $\mathrm{NA}$ & $\mathrm{NA}$ & NA & $\mathrm{NA}$ & NA & $N A$ & NA & NA & NA & $\mathrm{NA}$ & $N A$ \\
\hline 6 & Ott-Mar & $\mathrm{NA}$ & $\mathrm{NA}$ & $\mathrm{NA}$ & $\ddot{N A}$ & $\mathrm{NA}$ & $\mathrm{NA}$ & NA & $\mathrm{NA}$ & $\mathrm{NA}$ & $\mathrm{NAA}$ & $\mathrm{NA}$ & NA \\
\hline 7 & Mar-Jun & NA & NA & NA & NA & NA & $\begin{array}{ll}\cdots & N A\end{array}$ & $N A$ & $N A^{\prime \prime}$ & $\mathrm{NA}$ & $\mathrm{NA}$ & $\mathrm{NA}$ & $\mathrm{NA}$ \\
\hline
\end{tabular}

Abundance/eve/ refers to estimated relative abundance of spawners that spawned in the natal stream reaches that populated the life history pathways using the reach in this table:

Level 1: Low to average (relative to other reaches) abundance

Level 2 High (relative to other reaches) abundance

Level 3: Exceptionally high (relative to other reaches) abundance

Life stages: 1-prespawner; 2- spawner; 3- incubation; 4- fry colonization; 5- summer rearing; 6overwintering; 7- yearling presmolt/smolt

Attributes: CHEM - chemicals CSTAB - channel stability

COMP - competition (with other species)

FLOW - flow

HABD - habitat diversity
NUTLD - nutrient load

OBST - obstructions

OXYG - dissolved oxygen

PATHO - pathogens

PRED - predators (includes fishing-related losses)
RIPCN - riparian condition

SEDLD - sediment load

TEMP - water temperature

WITH - water withdrawals

"NA" indicates that no life history pathway meeting the criteria used in defining these pathways was present.

Blank cells under attributes indicate no additional attributes with negative effects on productivity were evident compared to those present in the template. 
Stream Reach Summary for Vacant Spring Chinook Salmon Life Histories

Stream: Sheep $\mathrm{Cr}$.

Reach: $\quad$ Mouth - Chicken Cr (RM 164.2 -165.7 from Snake R)

\begin{tabular}{|c|c|c|c|c|c|c|c|c|c|c|c|c|c|}
\hline \multicolumn{4}{|c|}{ [Restoration benefit category: } & B & \multicolumn{3}{|c|}{ Chinook usage score: } & 49.0 & \multicolumn{4}{|c|}{ Habitat productivity loss score: } & -2909.9 \\
\hline \multirow[b]{3}{*}{ Life stage } & \multirow[b]{3}{*}{ Months } & \multirow{2}{*}{\multicolumn{4}{|c|}{$\begin{array}{c}\text { No. vacant life history pathways } \\
\text { by abundance level }\end{array}$}} & \multicolumn{2}{|c|}{ Key habitat change } & \multirow{3}{*}{$\begin{array}{c}\text { Habitat } \\
\text { productivity } \\
\text { loss score }\end{array}$} & & & & & \\
\hline & & & & & & \multirow{2}{*}{$\begin{array}{l}\text { Patient } \\
\text { quantity }\end{array}$} & \multirow[b]{2}{*}{$\%$ change } & & & & & & \\
\hline & & Total no. & 1 & 2 & 3 & & & & & & & & \\
\hline $\begin{array}{c}1 \\
123\end{array}$ & $\begin{array}{l}\text { Apr-Aug } \\
\text { Aug-Oct }\end{array}$ & $\begin{array}{c}63 \\
?\end{array}$ & $\begin{array}{l}63 \\
90\end{array}$ & 0 & 0 & $\begin{array}{l}1.9 \\
1.1\end{array}$ & $\begin{array}{r}-53.4 \\
-60.0\end{array}$ & $\begin{array}{l}-481.2 \\
-834 .\end{array}$ & & & & & \\
\hline 3 & Ott-Mar & 180 & $180^{\circ}$ & & & 1.9 & -60.0 & --942 & & & & & \\
\hline 34 & Mär-M̈ä̀ & $47^{\circ}$ & & 0 & 0 & 30. & -68.9 & -294.0 & & & & & \\
\hline 5 & May-Ott & 97 & 97 & 0 & 0 & 45 & -31.0 & .322 .7 & & & & & \\
\hline 6 & Ott-Mar & 19 & 19 & 0 & 0 & 2.0 & -600 & -35.1 & & & & & \\
\hline 7 & Mar-Jun & " & 2 & 0 & 0 & 10,0 & 0.0 & 0.0 & & & & & \\
\hline \multirow[b]{2}{*}{ Life stage } & \multirow[b]{2}{*}{ Months } & \multicolumn{12}{|c|}{ Highest productivity impact attributes (\% change in impact from template) } \\
\hline & & Att 1 & $\%$ change & Att 2 & $\%$ change & Att 3 & $\%$ change & Att 4 & $\%$ change & Atf 5 & $\%$ change & Att 6 & $\%$ change \\
\hline 1 & Apr-Aug & RIPCN & 75.0 & TEMP & 65.4. & FL! & 43 & HABD & 43.6 & CSTA & 37.5 & NUTLD & \\
\hline $1,2,3$ & Aug-Ött & CSTAB & 75.0 & RIPCN & 75.0 & $\mathrm{TE}$ & $75 !$ & FEOOZW & 50.0 & $\mathrm{HABC}$ & 50.0 & SEDLD & \\
\hline 3 & Ott-Mar & CSTAB & 75,0 & HABD. & 75.0 & RIPCN & 75.0 & FLOW & 50.0 & SEDLD & 50.0 & TEMP & 50.0 \\
\hline 3.4 & Mar-Mav & CSTAB & 75.0 & "HAंÁBD" & $75.0^{\circ}$ & RIPCN & 75.0 & TEMP & 75.0 & "FLOWW" & $50.0^{\circ}$ & SEELLD & 50.0 \\
\hline 5 & May-Oct & HABD & 73,2 & RIPCN & 73.2 . & TEMP & 73,2 & NÜTÜD & 24.4 & PATHHO & 24.4 & & \\
\hline $\begin{array}{l}6 \\
7\end{array}$ & $\begin{array}{l}\text { Ott-Mar } \\
\text { Mar-Jun }\end{array}$ & HABD" & $50^{\circ}$ & RIPCN & $50.0^{\circ}$ & РAं̈̈Н & 25.0 & SEDLD & $25.0^{\circ}$ & & & & \\
\hline
\end{tabular}

Abundance level refers to estimated relative abundance of spawnera that spawned in the natal stream reaches that populated the life history pathways using the reach in this table Level 1: Low to sverage (relative to other reaches) abundance

Level 2: High (relative to other reaches) abundance

Level 3: Exceptionally high (relative to other reaches) abundance

Life stages: 1 - prespawner; 2- spawner; 3- incubation; 4- fry colonization; 5- summer rearing; 6-ovenwintering; 7- yearling presmolt/smolt

Attributes: CHEM - chemicals

CSTAB - channel stability

COMP - competition (with other species)

FLOW - ftOW

HABD - habitat diversity

\section{NUTLD - nutrient load}

OBST - obstructions

OXYG - dissolved oxygen

PATHO - pathogens

PRED - predators (includes fishing-related losses)
RIPCN - riparian condition

SEDLD - sediment load

TEMP - water temperature

WITH - $\quad$ water withdrawals

"NA" indicates that no tife history pathway meeting the criteria used in defining these pathways was present.

Blank cells under attributes indicate no additional attributes with negative effects on productivity were evident compared to those present in the template. 
Stream Reach Summary for Vacant Spring Chinook Salmon Life Histories

Stream: Sheep Cr.

Reach: Chicken $\mathrm{Cr}$ - headwaters (RM 165.7 -173.7 from Snake R)

\begin{tabular}{ll|ll|l}
\hline Restoration benefit category: & B & Chinook usage score: & 60.0 & Habitat productivity loss score: \\
\hline
\end{tabular}

\begin{tabular}{|c|c|c|c|c|c|c|c|c|}
\hline \multirow[b]{3}{*}{ Life stage } & \multirow[b]{3}{*}{ Months } & \multirow{2}{*}{\multicolumn{4}{|c|}{$\begin{array}{c}\text { No. vacant life history pathways } \\
\text { by abundance level }\end{array}$}} & \multicolumn{2}{|c|}{ Key habitat change } & \multirow{3}{*}{$\begin{array}{c}\text { Habitat } \\
\text { productivity y } \\
\text { loss score }\end{array}$} \\
\hline & & & & & & \multirow{2}{*}{$\begin{array}{l}\text { Patient } \\
\text { quantity }\end{array}$} & \multirow[b]{2}{*}{$\%$ change } & \\
\hline & & Total no. & 1 & 2 & 3 & & & \\
\hline 1 & Apr-Aug & 54 & 54 & $\overline{0}$ & 0 & 1.6 & $-60,0$ & -3648 \\
\hline 1.2 .3 & Aug-Ott & 90 & 90 & 0 & '0 & 0.8 & -60.0 & -8354 \\
\hline 3 & Ott-Mar & 180 & 180 & 0 & 0 & 1.5 & -60.0 & -390.5 \\
\hline 3.4 & Mar-May & 42 & 42 & 0 & 0 & 33 & -600 & -145.8 \\
\hline 5 & May-Ott & 94 & 94 & 0 & 0 & 4.6 & -31.8 & -239.8 \\
\hline 6 & Ott-Mar & 19 & 19 & 0 & 0 & 15 & -60.0 & -74.5 \\
\hline 7 & Mar-Jun & $\dddot{11}$ & 1 & 0 & 0 & 11.0 & 0.0 & 0,5 \\
\hline
\end{tabular}

\begin{tabular}{|c|c|c|c|c|c|c|c|c|c|c|c|c|c|}
\hline \multirow[b]{2}{*}{ Life stage } & \multirow[b]{2}{*}{ Months } & \multicolumn{12}{|c|}{ Highest productivity impact attributes (\% change in impact from template) } \\
\hline & & Att 1 & $\%$ change & Att 2 & $\%$ change & Att 3 & $\%$ change & Att 4 & $\%$ change & Alt 5 & $\%$ change & Att 6 & $\%$ change \\
\hline 1 & Apr-Aug & TEMP & 75.0 & RIPCN & 43.8 & FLOW & 25.0 & HABD & 25.0 & & & & \\
\hline $1,2,3$ & Aug-Oct & TEMP & 75.0 & FLOW & 50.0 & RIPCN & 50.0 & HABD & 25.0 & SEOLO & 25.0 & & \\
\hline 3 & Oct-Mar & FLOW & 500 & RIPCN & 50.0 & SEDLD & 25.0 & TEMP & 25.0 & HABD & 12.5 & PATHO & 12.5 \\
\hline 3,4 & Mar-May & TEMP & 75.0 & FLOW & 50.0 & RIPCN & 50.0 & HABD & 25.0 & PATHO & 25.0 & SEDLD & 12.5 \\
\hline 5 & May-Oct & TEMP & 75.0 & FLOW & 50.0 & HABD & 50.0 & RIPCN & 50.0 & PATHO & 25.0 & SEDLD & 25.0 \\
\hline 6 & Oct-Mar & FLOW & 50.0 & HABD & $50.0^{\circ}$ & RIPCN & $50.0^{\circ}$ & PATHO & 25.0 & SEDLD & 25.0 & TEMP & $25.0^{\circ}$ \\
\hline 7 & Mar-Jun & & & & & & & & & & & & \\
\hline
\end{tabular}

Abundance/eve/ refers to estimated relative abundance of spawners that spawned in the natal stream reaches that populated the lie history pathways using the reach in this table: Level 1: $\quad$ Low to average (relative to other reaches) abundance

Level 2: $\quad$ High (relative to other reaches) abundance

Level 3: Exceptionally high (relative to other reaches) abundance

Life stages: 1-prespawner; 2- spawner; 3- incubation; 4- fry colonization; 5- summer rearing; 6- overwintering; 7- yearling presmoit/smelt

Attributes: CHEM - chemicals

CSTAB - channel stability

COMP - competition (with other species)

FLOW - flow

HABD - habitat diversity
NUTLD - nutrient load

OBST - obstructions

OXYG - dissolved oxygen

PATHO - pathogens

PRED - predators (includes fishing-related losses)
RIPCN - riparian condition

SEDLD - sediment load

TEMP - $\quad$ water temperature

WITH - water withdrawals

"NA" indicates that no life history pathway meeting the criteria used in defining these pathways was present.

Blank cells under attributes indicate no additional attributes with negative effects on productivity were evident compared to those present in the template. 
Stream: Wallowa R.

Reach: Mouth - Minam R (RM 80.0 -89.8 from Snake R)

Restoration benefit category: \begin{tabular}{l|l} 
B & Chinook usage score: \\
\hline
\end{tabular}

\begin{tabular}{|c|c|c|c|c|c|c|c|c|}
\hline \multirow[b]{3}{*}{ Life stage } & \multirow[b]{3}{*}{ Months } & \multirow{2}{*}{\multicolumn{4}{|c|}{$\begin{array}{l}\text { No. vacant life history pathways } \\
\text { by abundance level }\end{array}$}} & \multicolumn{2}{|c|}{ Key habitat change } & \multirow{3}{*}{$\begin{array}{c}\text { Habitat } \\
\text { productivity } \\
\text { loss score }\end{array}$} \\
\hline & & & & & & \multirow{2}{*}{$\begin{array}{l}\text { Patient } \\
\text { quantity }\end{array}$} & \multirow[b]{2}{*}{$\%$ change } & \\
\hline & & Total no. & 1 & 2 & 3 & & & \\
\hline 1 & Apr-Aug & 204 & 137 & 51 & 16 & 21.7 & 0.0 & -141.0 \\
\hline $1,2,3$ & Aug-Oct & 90 & 90 & 0 & 0 & 31.7 & 0.0 & -104.3 \\
\hline 3 & Oct-Mar & 180 & 180 & 0 & 0 & 41.6 & 0.0 & -181.4 \\
\hline 3,4 & Mar-May & 147 & 110 & 37 & 0 & 28.7 & 0.0 & -211.2 \\
\hline 5 & May-Oct & 629 & 385 & 232 & 12 & 18.5 & -36.6 & -98.8 \\
\hline 6 & Oct-Mar & 246 & 151 & 95 & 0 & 41.9 & 0.0 & -39.5 \\
\hline 7 & Mar-Jun & 40 & 23 & 13 & 4 & 71.5 & 0.0 & 0.5 \\
\hline
\end{tabular}

\begin{tabular}{|c|c|c|c|c|c|c|c|c|c|c|c|c|c|}
\hline \multirow[b]{2}{*}{ Life stage } & \multirow[b]{2}{*}{ Months } & \multicolumn{12}{|c|}{ Highest productivity impact attributes (\% change in impact from template) } \\
\hline & & Att 1 & $\%$ change & Art 2 & $\%$ change & Att 3 & $\%$ change & Aft 4 & $\%$ change & Att 5 & $\%$ change & Att 6 & $\%$ change \\
\hline 1 & Apr-Aug & PATHO & 15.8 & CSTAB & 12,5 & SEDLD & 12.5 & TEMP & 12 & & & & \\
\hline $1,2,3$ & Aug-Ött & РATHHÓ & $25.0^{\circ}$ & CSTAB & 14,6 & SEDLD & 14.6 & TEMP & 14.6 & & & & \\
\hline "3"' & Oct-Mar & CSTAB & 25.0 & HABD & 250 & PAT̈HO & 25.0 & SEDLD & 25.0 & TEMP & 25.0 & & \\
\hline 3,4 & Mar-May & HABD & 19.1 & "P̈Т̈Н̈Н̈" & 19.1 & COMP & 15.9 & CSTAB & 9.8 & SËËL̈D̆ & 9.8 & TEMP & 9.8 \\
\hline 5 & May-Öot & COMP & 15.2 & HABD & 15.2 & PAT̈HO & 15.2 & & & & & & \\
\hline $\begin{array}{l}6 \\
6 \\
7 \times\end{array}$ & $\begin{array}{l}\text { Ött-Mar } \\
\text { Mar-Jun }\end{array}$ & FFLOOWW & 13.5 & PATHOO & 13.5 & & & & & , & & & \\
\hline
\end{tabular}

Abundance level refers to estimated relative abundance of spawners that spawned in the natal stream reaches that populated the life history pathways using the reach in this table: Level 1: Low to average (relative to other reaches) abundance

Level 2 High (relative to other reaches) abundance

Level 3: Exceptionally high (relative to other reaches) abundance

Lifo stages: 1- prespawner; 2- spawner; 3- incubation; 4- fry colonization; 5- summer rearing; 6- overwintering; 7-yearling presmolt/smolt

Attributes: CHEM - chemicals

CSTAB - channel stability

COMP - competition (with other species)

FLOW - flow

HABD - habitat diversity
NUTLD - nutrient load

OBST - obstructions

OXYG - dissolved oxygen

PATHO - pathogens

PRED - predators (includes fishing-related losses)
RIPCN - riparian condition

SEDLD - sediment load

TEMP - water temperature

WITH - water withdrawals

"NA" indicates that no life history pathway meeting the criteria used in defining these pathways was present.

Blank cells under attributes indicate no additional attributes with negative effects on productivity were evident compared to those present in the template. 
Stream Reach Summary for Vacant Spring Chinook Salmon Life Histories

Stream: Wallowa R.

Reach: Miriam R - Deer Cr (RM 89.8 -90,8 from Snake R)

\begin{tabular}{ll|l|l|l|l|}
\hline Restoration benefit category & B & Chinook usage score: & 80.7 & Habitat productivity loss score: & -589.8 \\
\hline
\end{tabular}

\begin{tabular}{|c|c|c|c|c|c|c|c|c|}
\hline \multirow[b]{3}{*}{ Life stage } & \multirow[b]{3}{*}{ Months } & \multirow{2}{*}{\multicolumn{4}{|c|}{$\begin{array}{l}\text { No. vacant life history pathways } \\
\text { by abundance level }\end{array}$}} & \multicolumn{2}{|c|}{ Key habitat change } & \multirow{3}{*}{$\begin{array}{c}\text { Habitat } \\
\text { productivity } \\
\text { loss score }\end{array}$} \\
\hline & & & & & & \multirow{2}{*}{$\begin{array}{l}\text { Patient } \\
\text { quantity }\end{array}$} & \multirow[b]{2}{*}{$\%$ change } & \\
\hline & & Total no. & 1 & 2 & 3 & & & \\
\hline 1 & Apr-Aug & 171 & 115 & 42 & 14 & 8.7 & -10.3 & -101.2 \\
\hline 1,3 & Aug-Od & 90 & 90 & 0 & 0 & 159 & 0.0 & -105.2 \\
\hline 3 & Oct-Mar & 180 & $180^{\circ}$ & 0 & 0 & 20.6 & -1.5 & -77.5 \\
\hline 3,4 & Mar-May & 82 & 53 & 29 & 0 & 7.8 & -49.8 & -191.8 \\
\hline 5 & May-Oct & 272 & 125 & 137 & 10 & 8.2 & -52.1 & -56.6 \\
\hline 6 & Oct-Mar & 62 & 25 & 19 & 18 & 8.3 & -42.9 & -57.8 \\
\hline 7 & Mar-Jun & 24 & 14 & 6 & 4 & 30.3 & -3.4 & 0.2 \\
\hline
\end{tabular}

\begin{tabular}{|c|c|c|c|c|c|c|c|c|c|c|c|c|c|}
\hline \multirow[b]{2}{*}{ Life stage } & \multirow[b]{2}{*}{ Months } & \multicolumn{12}{|c|}{ Highest productivity im act attributes (\% change in impact from template) } \\
\hline & & Att 1 & $\%$ change & Att 2 & $\%$ change & Att 3 & \% change & Att 4 & $\%$ change & Att 5 & $\%$ change & Att 6 & \% change \\
\hline 1 & Apr-Aug & HABD & 25.0 & RIPCN & 25.0 & PATHO & 12.5 & & & & & & \\
\hline $1,2,3$ & Aug-Ott & SËDELD & 75.0 & "HAB́Bं' & 25.0 & RIPCN & 25.0 & PATHOO & 12.5 & & & & \\
\hline & Ort_Mar & sfnI $n$ & $75 n$ & $\mathrm{HABD}$ & 12.5 & РАT̈HÖ & $12 ; 5$ & RIPCN & "12.5"' & & & & \\
\hline & & & & SEËLLD & 34,2 & CÖMP & 25.0 & 'PATHÖO & $25.0 "$ & RIPCN & 25.0 . & & \\
\hline & & & & COMP & 23.4 & PATHO & 23.4 & RIPCN & $\Rightarrow<3$ & & & & \\
\hline & & & & PATHO & 17.7 & RIPCN & 177 & & & & & & \\
\hline
\end{tabular}

Abundance level refers to estimated relative abundance of spawners that spawned in the natal stream reaches that populated the life history pathways using the reach in this table:

Level 1: Low to average (relative to other reaches) abundance

Level 2: High (relative to other reaches) abundance

Level 3 Exceptionally high (relative to other reaches) abundance

Life stages: 1- prespawner; 2- spawner; 3- incubation; 4- fry colonization; 5- summer rearing; 6- overwintering; 7-yearlingpresmolt/smolt

Attributes.' CHEM - chemicals

CSTAB - channel stability

COMP - competition (with other species)

FLOW - flow

HABD - habitat diversity
NUTLD - nutrient load

OBST - obstructions

OXYG - dissolved oxygen

PATHO - pathogens

PRED - predators (includes fishing-related losses)
RIPCN - riparian condition

SEDLD - sediment load

TEMP - water temperature

WITH - water withdrawals

"NA" indicates that no life history pathway meeting the criteria used in defining these pathways was present.

Blank cells under attributes indicate no additional attributes with negative effects on productivity were evident compared to those present in the template. 
Stream Reach Summary for Vacant Spring Chinook Salmon Life Histories

Stream: Wallowa $\mathbf{R}$

Reach: Deer Cr - Dry Cr (RM 90.8 -98.3 from Snake R)

\begin{tabular}{ll|ll|ll}
\hline Restoration benefit category: & B & Chinook usage score: & $\mathbf{8 1 . 3}$ & Habitat productivity loss score: \\
\hline
\end{tabular}

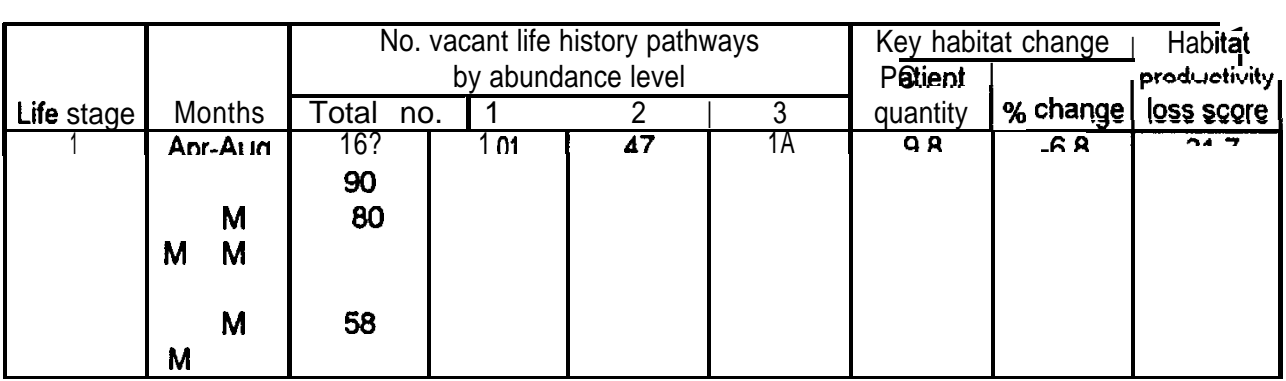

\begin{tabular}{|c|c|c|c|c|c|c|c|c|c|c|c|c|c|}
\hline \multirow[b]{2}{*}{ Life stage } & \multirow[b]{2}{*}{ Months } & \multicolumn{4}{|c|}{ Highest pror } & \multicolumn{8}{|c|}{ sctivity impact attributes (\% change in impact from template) } \\
\hline & & Att 1 & $\%$ change & $\overline{\text { Att } 2}$ & $\%$ change & Att 3 & $\%$ change & Atf 4 & $\%$ change & Atf 5 & $\%$ change & $\overline{\text { Atf } 6}$ & $\%$ change \\
\hline 1 & Apr-Aug & SEDLD & 37.5 & HABD & $\overrightarrow{15.1}$ & PATHO & 15.1 & RIPCN & 151 & & & & \\
\hline $1,2,3$ & Aug-OCt & SEDLD & 75.0 & HABD & 25.0 & PATHO & $25.0^{\circ}$ & RIPCN & 25.0 & & & & \\
\hline 3 & Oct-Mar & SEDLD & 75.0 & HABD & 25.0 & PATHO & 25.0 & RIPCN & 25.0 & & & & 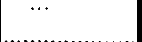 \\
\hline 3,4 & Mar-May & HABD & 19.1 & PATHO & 16.4 & RIPCN & 16.4 & COOMP" & 95 & "SEĒLEZ̄" & 46 & & \\
\hline 5 & May-Oct & $\mathrm{HABD}$ & 18.0 & COMP & 9.0 & PATHO & 9.0 & RIPCN & 90 & & & & \\
\hline 6 & Oct-Mar & HABD & 10.7 & PATHO & 10.7 & "ṔREÉD̈" & 10.7 & RIPCN & 10.7 & & & & \\
\hline 7 & Mar-Jun & & & & & & & & & & & & \\
\hline
\end{tabular}

Abundance level refers to estimated relative abundance of spawners that spawned in the natal stream reaches that populated the life history pathways using the reach in this table Level 1: Low to average (relative to other reaches) abundance

Level 2: High (relative to other reaches) abundance

Level 3: Exceptionally high (relative to other reaches) abundance

Life stages.' 1- prespawner; 2- spawner; 3- incubation; 4- fry colonization; 5- summer rearing; 6- overwintering; 7- yearling presmolt/smolt

Attributes: CHEM - chemicals

CSTAB - channel stability

COMP - competition (with other species)

FLOW - ftOW

HABD - habitat diversity

NUTLD - nutrient load

OBST - obstructions

OXYG - dissolved oxygen

PATHO - pathogens

PRED - predators (includes fishing-related losses)
RIPCN - riparian condition

SEDLD - sediment load

TEMP - water temperature

WITH - water withdrawals

"NA" indicates that no life history pathway meeting the criteria used in defining these pathwaya was present.

Blank cells under attributes indicate no additional attributes with negative effects on productivity were evident compared to those present in the template. 
Stream Reach Summary for Vacant Spring Chinook Salmon Life Histories

Stream: Wallowa R.

Reach: Dry Cr - Bear Cr (RM 98.3 -101.8 from Snake R)

\begin{tabular}{|c|c|c|c|c|c|c|c|c|}
\hline \multicolumn{4}{|c|}{ /Restoration benefit category: } & B & \multicolumn{3}{|c|}{ Chinook usage score: } & 83.9 \\
\hline \multirow[b]{3}{*}{ Life stage } & \multirow[b]{3}{*}{ Months } & \multirow{2}{*}{\multicolumn{4}{|c|}{$\begin{array}{l}\text { No. vacant tife history pathways } \\
\text { by abundance level }\end{array}$}} & \multirow{3}{*}{\multicolumn{2}{|c|}{$\begin{array}{l}\text { Key habitat change } \\
\text { Patient } \\
\text { quantity } \% \text { change }\end{array}$}} & \multirow{3}{*}{$\begin{array}{l}\text { Habitat } \\
\text { productivity } \\
\text { loss score }\end{array}$} \\
\hline & & & & & & & & \\
\hline & & Tolal no. & 1 & 2 & 3 & & & \\
\hline 1 & Apr-Aug & 153 & 63 & 76 & 14 & 9.4 & .31 .8 & -23.4 \\
\hline $1,2,3$ & Aug-Oct & 90 & 20 & 70 & 0 & 7.7 & -70.6 & -170.4 \\
\hline 3 & Oct-Mar & 180 & 40 & 140 & 0 & 8.0 & -75.0 & -693.5 \\
\hline 3,4 & Mar-May & 89 & 20 & 54 & 15 & 8.8 & -57.8 & -560.9 \\
\hline 5 & May-Oct & 277 & 47 & 109 & 121 & 8.5 & -31.3 & -22.5 \\
\hline 6 & Oct-Mar & 57 & 19 & 19 & 19 & 8.1 & -31.2 & -29.3 \\
\hline 7 & Marsiun & 18 & 11 & 4 & 3 & 30.2 & -3.4 & 1.1 \\
\hline
\end{tabular}

\begin{tabular}{|c|c|c|c|c|c|c|c|c|c|c|c|c|c|}
\hline \multirow[b]{2}{*}{ Life stage } & \multirow[b]{2}{*}{ Months } & \multicolumn{12}{|c|}{ Highest productivity impact attributes (\% change in impact from template) } \\
\hline & & An 1 & $\%$ change & Att 2 & $\%$ change & Att 3 & $\%$ change & Att 4 & $\%$ change & Att 5 & $\%$ change & Att 6 & $\%$ change \\
\hline 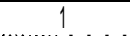 & Apr-Aug & RIPCN & 50.0 . & HABD & 375 & PATHO & 12.5 & & & & & & \\
\hline$\dddot{1}, 2, \dot{3}$ & Aug-Oct & SEDLD & $75.0^{\circ}$ & NUT்L் & $50.0^{\circ}$ & RIPCON & 50.0 & "HÄB̈" & $\dddot{3} 7 . \ddot{5}$ & OXYYG & 25.0 & PÄTHOÖ & 12.5 \\
\hline 3 & Oct-Mar & SEDLD & “".75;0 & NUTTLD. & 50.0 & HABD & 37,5 & OXYG & 25.0 & RIPCN & 25.0 & FLOW" & 125 \\
\hline 3,4, & Mar-May. & HABD & 75.0 & SEDLD & 75.0 & NUTLD & 50,0 & RIPCN & 50.0 & FLOW & 250 & OQXYG & 250 \\
\hline 5 & May-Ott & HABD & 27.2 & SEDLD & 143 & COMP. & 13.6 & PATHO & 13.6 & RIPCN & 136 & & \\
\hline 6 & Ott-Mar & "HABD" & 20.8 & SEDLD & 20.8 & PATHHO & 10.4 & RIPCN & 104 & & & & \\
\hline 7 & Mar-Jun & COMP & 5.6 & PATHO & 5.6 & WITH & 5.6 & & & & & & \\
\hline
\end{tabular}

Abundance level refers to estimated relative abundance of spawners that spawned in the natal stream reaches that populated the life history pathways using the reach in this table: Level 1: Low to average (relative to other reaches) abundance

Level 2: High (relative to other reaches) abundance

Level 3: Exceptionally high (relative to other reaches) abundance

Life stages: 1 - prespawner; 2-spawner; 3- incubation; 4- fry colonization; 5- summer rearing; 6- overwintering; 7- yearlingpresmolt/smolt

Attributes: CHEM - chemicals

CSTAB - channel stability

COMP - competition (with other species)

FLOW - flow

HABD - habitat diversity
NUTLD - nutrient load

OBST - obstructions

OXYG - dissolved oxygen

PATHO - pathogens

PRED - predators (includes fishing-related losses)
RIPCN - riparian condition

SEDLD - sediment load

TEMP - water temperature

WITH - water withdrawals

"NA" indicates that no life history pathway meeting the criteria used in defining these pathways was present.

Blank cells under attributes indicate no additional attributes with negative effects on productivity were evident compared to those present in the template, 


\section{Stream Reach Summary for Vacant Spring Chinook Salmon Life Histories}

Stream: Wallowa R.

Reach: Bear Cr - Whiskey Cr (RM 101.8 -103.9 from Snake R)

\begin{tabular}{ll|ll|l}
\hline Restoration benefit category: & B & Chinook usage score: & $\mathbf{8 1 . 2}$ & Habitat productivity loss score: \\
\hline
\end{tabular}

\begin{tabular}{|c|c|c|c|c|c|c|c|c|}
\hline \multirow[b]{3}{*}{ Life stage } & \multirow[b]{3}{*}{ Months } & \multirow{2}{*}{\multicolumn{4}{|c|}{$\begin{array}{c}\text { No. vacant life history pathways } \\
\text { by abundance level }\end{array}$}} & \multicolumn{2}{|c|}{ Key habitat change } & \multirow{3}{*}{$\begin{array}{c}\text { Habitat } \\
\text { productivit } \\
\text { loss score }\end{array}$} \\
\hline & & & & & & \multirow{2}{*}{$\begin{array}{l}\text { Patient } \\
\text { quantity }\end{array}$} & \multirow[b]{2}{*}{$\%$ change } & \\
\hline & & Total $\mathrm{n}$ & 1 & 2 & 3 & & & \\
\hline$i$ & Apr-Aug & 133 & 40 & 79 & 14 & 9.3 & -31.4 & -58.3 \\
\hline $1,2,3$ & Aug-Oct & 90 & 0 & 90 & 0 & 7.7 & -70.6 & -228.3 \\
\hline 3 & Oct-Mar & 180 & 0 & 180 & 0 & 8.3 & -72.2 & -789.5 \\
\hline 3,4 & Mar-May & 79 & 6 & 53 & 20 & 9.1 & -48.6 & -372.3 \\
\hline 5 & May-Oct & 247 & 24 & 106 & 117 & 8.5 & -28.5 & -18.3 \\
\hline 6 & Oct-Mar & 58 & 20 & 19 & 19 & 8.3 & -29.6 & -29.5 \\
\hline 7 & Mar-aun & 14 & 9 & 3 & 2 & 33.0 & 0.0 & 0.7 \\
\hline
\end{tabular}

\begin{tabular}{|c|c|c|c|c|c|c|c|c|c|c|c|c|c|}
\hline \multirow[b]{2}{*}{ Life stage } & \multirow[b]{2}{*}{ Months } & \multicolumn{12}{|c|}{ Highest productivity impact attributes $\%$ change in impact fro template) } \\
\hline & & Att 1 & $\%$ change & Atf 2 & $\%$ change & Att 3 & $\%$ change & Att 4 & $\%$ change & Att 5 & $\%$ change & Att 6 & $\%$ change \\
\hline 年 & Apr-Aug & SEDLD & 37.5 & NỤTLD & 25.0 & TEMP & 25.0 & HABD & 23.0 & RIPCN & 23.0 & OXYG & 12.5 \\
\hline $1,2,3$ & Äug-Ött & SEDLLD & 75.0 & HAB̈D" & 50.0 & NUTTLD & 50,0 & RIPCN & $50.0^{\circ}$ & OXYG & 25.0 & "PATHOOO" & 25.0 \\
\hline 3 & Oct-Mar & HABD & 75.0 & SEDLD & 750 & NUTLD & 50.0 & RIPCN & $50.0 "$ & FLOW" & 25.0 & OXYG & 25.0 ““ \\
\hline $\begin{array}{c}3,4 \\
5\end{array}$ & $\begin{array}{l}\text { Mar-May } \\
\text { May-Ott }\end{array}$ & HABD & $\begin{array}{l}71.6 \\
22.9\end{array}$ & $\begin{array}{l}\text { SEDLD } \\
\text { PATHO }\end{array}$ & 11.5 & $\begin{array}{l}\text { RIPCN } \\
\text { RIPCN }\end{array}$ & $\begin{array}{l}47.8 \\
11.5\end{array}$ & 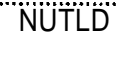 & 36.6 & "FLÖẄ & 23.9 & PATHO & 23.9 \\
\hline 6 & Ott-Mar & HABD & 20.5 & SEEDELD" & 205 & "СÖM"P" & 10.4 & "РÄT̈HÖ & 10.4 & 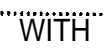 & 10.4 & RIPCN & 10.3 \\
\hline 7 & Mar-Jun & COMP & 4.5 & "PÄT̈HÖ' & 4.5 & WITH & 4.5 & & & & & & \\
\hline
\end{tabular}

Abundance/eve/ refers to estimated relative abundance of spawners that spawned in the natal stream reaches that populated the life history pathways using the reach in this table Level 1: $\quad$ Low to average (relative to other reaches) abundance

Level 2: $\quad$ High (relative to other reaches) abundance

Level 3: Exceptionally high (relative to other reaches) abundance

Life stages: 1- prespawner; 2- spawner; 3- incubation; 4- fry colonization; 5- summer rearing; 6- overwintering; 7-yearlingresmolt/smolt

\begin{tabular}{|c|c|c|c|c|}
\hline $\begin{array}{r}\text { Attributes: } \\
\text { CHEM - } \\
\text { CSTAB - } \\
\text { COMP - } \\
\text { FLOW - } \\
\text { HABD - }\end{array}$ & $\begin{array}{l}\text { chemicals } \\
\text { channel stability } \\
\text { competition (with other species) } \\
\text { flow } \\
\text { habitat diversity }\end{array}$ & $\begin{array}{l}\text { NUTLD - } \\
\text { OBST - } \\
\text { OXYG - } \\
\text { PATHO - } \\
\text { PRED - }\end{array}$ & $\begin{array}{l}\text { nutrient load } \\
\text { obstructions } \\
\text { dissolved oxygen } \\
\text { pathogens } \\
\text { predators (includes fishina-related losses) }\end{array}$ & $\begin{array}{l}\text { RIPCN - } \quad \text { riparian condition } \\
\text { SEDLD - } \\
\text { sediment load } \\
\text { TEMP - } \quad \text { water temperature } \\
\text { WITH - } \quad \text { water withdrawals }\end{array}$ \\
\hline
\end{tabular}

"NA ${ }^{N}$ indicates that no life history pathway meeting the criteria used in defining these pathways was present.

Blank cells under attributes indicate no additional attributes with negative effects on productivity were evident compared to those present in the template. 
Stream Reach Summary for Vacant Spring Chinook Salmon Life Histories

Stream: Wallowa $\mathbf{R}$.

Reach: Whiskey Cr - Lostine R (RM 103.9 -105,8 from Snake R)

\begin{tabular}{ll|ll|l}
\hline Restoration benefit category: & B & Chinook usage score: & 76.6 & Habitat productivity loss score: \\
\hline
\end{tabular}

\begin{tabular}{|c|c|c|c|c|c|c|c|c|}
\hline \multirow[b]{3}{*}{ Life stage } & \multirow[b]{3}{*}{ Months } & \multirow{2}{*}{\multicolumn{4}{|c|}{$\begin{array}{l}\text { No. vacant life history pathways } \\
\text { by abundance level }\end{array}$}} & \multicolumn{2}{|c|}{ Key habitat change } & \multirow{3}{*}{$\begin{array}{c}\text { Habitat } \\
\text { productivity] } \\
\text { loss score }\end{array}$} \\
\hline & & & & & & \multirow{2}{*}{$\begin{array}{l}\text { Patient } \\
\text { quantity }\end{array}$} & \multirow[b]{2}{*}{$\%$ change } & \\
\hline & & Total no. & 1 & 2 & 3 & & & \\
\hline 1 & Apr-Aug & 124 & 40 & 70 & $\overline{14}$ & 8.7 & -33.3 & $\begin{array}{l}-17.6 \\
\end{array}$ \\
\hline $1,2.3$ & Aug-Ott & 90 & 0 & 90 & 0 & 7.7 & -69.8 & -2084 \\
\hline 3 & Ott-Mar & 180 & 0 & 180 & 0 & 7.6 & -72.7 & -789.5 \\
\hline 34 & Mar-May & 64 & 6 & 48 & 10 & 8.3 & -571 & -3513 \\
\hline 5 & May-Ott & 130 & 24 & 103 & 3 & 8.1 & -46̣5 & -15.9 \\
\hline 6 & Ott-Mar & 21 & 2 & 19 & 0 & 7.6 & -66.7 & -23.8 \\
\hline 7 & Mar-Jun & 11 & 8 & 2 & 1 & 30,3 & 0.0 & 0.5 \\
\hline
\end{tabular}

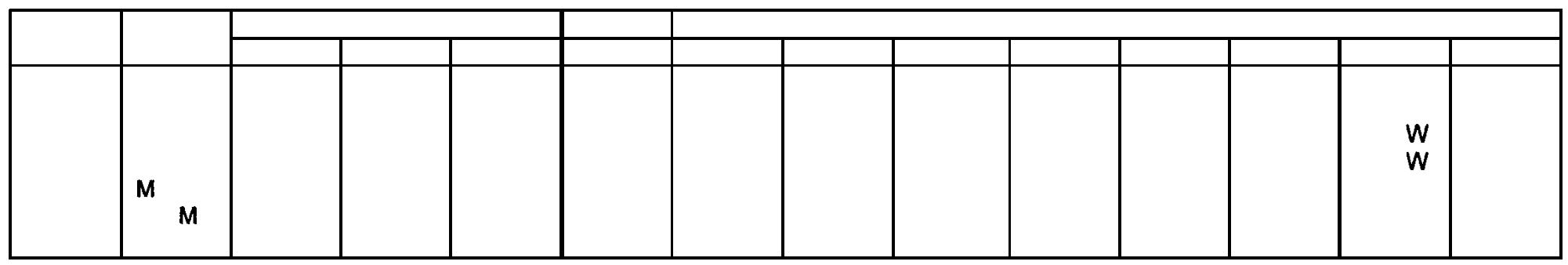

Abundance level refers to estimated relative abundance of spawners that spawned in the natal stream reaches that populated the life history pathways using the reach in this table: Level 1: Low to average (relative to other reaches) abundance

Level 2 High (relative to other reaches) abundance

Level 3: Exceptionally high (relative to other reaches) abundance

Life stages: 1- prespawner; 2- spawner; 3- incubation; 4- fry colonization; 5- summer rearing; 6- overwintering; 7-yearlingpresmolt/smolt

Attributes: CHEM - chemicals

CSTAB - channel stability

COMP - competition (with other species)

FLOW - ftOW

HABD - habitat diversity

$\begin{array}{ll}\text { NUTLD - nutrient load } & \text { RIPCN - riparian condition } \\ \text { OBST - obstructions } & \text { SEDLD - sediment load } \\ \text { OXYG - dissolved oxygen } & \text { TEMP - water temperature } \\ \text { PATHO - pathogens } & \text { WITH - } \quad \text { water withdrawals } \\ \text { PRED - }\end{array}$

"NA" indicates that no life history pathway meeting the criteria used in defining these pathways was present.

Blank cells under attributes indicate no additional attributes with negative effects on productivity were evident compared to those present in the template 
Stream Reach Summary for Vacant Spring Chinook Salmon Life Histories

Stream: Wallowa $\mathbf{R}$.

Reach: $\quad$ Lostine R - Parsnip Cr (RM 105.8 -109.0 from Snake R)

\begin{tabular}{|c|c|c|c|c|c|c|c|c|}
\hline \multicolumn{4}{|c|}{ Restoration benefit category: } & & \multicolumn{3}{|c|}{ Chinook usage score: } & \multirow{3}{*}{\begin{tabular}{|l}
75.6 \\
Halbitiat \\
productivity
\end{tabular}} \\
\hline \multirow[b]{3}{*}{ Life stage } & \multirow[b]{3}{*}{ Months } & \multirow{2}{*}{\multicolumn{4}{|c|}{$\begin{array}{l}\text { No. vacant life history pathways } \\
\text { by abundance level }\end{array}$}} & \multicolumn{2}{|c|}{ Key habitat change } & \\
\hline & & & & & & \multirow{2}{*}{$\begin{array}{l}\text { Patient } \\
\text { quantity }\end{array}$} & \multirow[b]{2}{*}{$\%$ change } & \\
\hline & & Total no. & 1 & 2 & 3 & & & loss score \\
\hline 1 & Apr-Aug & 85 & 30 & 46 & 9 & 9.2 & -44.7 & -18.7 \\
\hline $1,2,3$ & Aug-Oct & 90 & 20 & 70 & 0 & 7.7 & -61.2 & -1560 \\
\hline 3 & Oct-Mar & 180 & 40 & 140 & 0 & 8.3 & -64.9 & $-789,5$ \\
\hline 3,4 & Mar-May & 51 & 4 & 42 & 5 & 9.1 & -55.9 & -320.8 \\
\hline 5 & May-Oct & 132 & 12 & 117 & 3 & 8.7 & -46.0 & $-1-88.9$ \\
\hline 6 & Oct-Mar & 21 & 2 & 19 & 0 & 8.3 & -59.6 & -23.8 \\
\hline 7 & Mar-Jun & 6 & 4 & 1 & 1 & 33.0 & 6.7 & 0.5 \\
\hline
\end{tabular}

\begin{tabular}{|c|c|c|c|c|c|c|c|c|c|c|c|c|c|}
\hline \multirow[b]{2}{*}{ Life stage } & \multirow[b]{2}{*}{ Months } & \multicolumn{12}{|c|}{ Highest productivity impact attributes (\% change in impact from template) } \\
\hline & & $\overline{\text { Atf } 1}$ & $\%$ change & $\overline{\text { Aft } 2}$ & \% change & Aft 3 & $\%$ change & Art 4 & $\%$ change & Att 5 & $\%$ change & Atf 6 & $\%$ change! \\
\hline 1 & Apr-Aug & HABD & 408 & RIPCN & 40.8 & SEDLD & 37.5 & NUTLD & 25.0 & PATHO & 204 & OXYG & 12.5 \\
\hline $1,2,3$ & Aug-Oct & SEDLD & 75,0 & $\dot{H A B D}$ & 50.0 & NÜTLD̈ & 50.0 & RIPCN & 50.0 & OXYG & 25.0 & PATHO & 25.0 \\
\hline 3 & "Ött-Mar & $H A B D$ & 75.0 & SEDLD & 75.0 & NUTLD & 50.0 & RIPCN & $50.0 "$ & FLOW & 25.0 & OXYG & 25.0 \\
\hline 34 & Mar-May & SEDLD & 75.0 & HABD & 67.8 & "NUTLD & 50.0 & RIPCN & 45.2 & OXYG & 25.0 & PATHO & 25.0 \\
\hline 5 & May-Ott & HABD & 44.4 & PATHO & $22 . \dot{2}$ & RIPCN & 22.2 & & & & & & \\
\hline 6 & Ott-Mar & HABD & 45.4 & SEDLLD & 45.4 & COMP & 25.0 & PATHO & 25.0 & WIITH & 25.0 & RIPCN & ,22.7 \\
\hline 7 & Mar-Jun & COMP & 6.7 & PATHO & 6.7 & WITH & 6.7 & & & & & & \\
\hline
\end{tabular}

Abundance/eve/ refers to estimated relative abundance of spawners that spawned in the natal stream reaches that populated the life history pathways using the reach in this table:

Level 1: Low to average (relative to other reaches) abundance

Level 2 High (relative to other reaches) abundance

Level 3 Exceptionally high (relative to other reaches) abundance

Life stages: 1- prespawner; 2-spawner; 3- incubation; 4- fry colonization; 5- summer rearing; 6- overwintering; 7- yearlingpresmolt/smolt

\begin{tabular}{|c|c|c|c|}
\hline $\begin{array}{r}\text { Attributes: } \text { CHEM - } \\
\text { CSTAB - } \\
\text { COMP - } \\
\text { FLOW - } \\
\text { HABD - }\end{array}$ & $\begin{array}{l}\text { chemicals } \\
\text { channel stability } \\
\text { competition (with other species) } \\
\text { flow } \\
\text { habitat diversity }\end{array}$ & $\begin{array}{l}\text { NUTLD - nutrient load } \\
\text { OBST - obstructions } \\
\text { OXYG - dissolved oxygen } \\
\text { PATHO - pathogens } \\
\text { PRED - predators (includes fishing-related losses) }\end{array}$ & $\begin{array}{l}\text { RIPCN - } \quad \text { riparian condition } \\
\text { SEDLD - sediment load } \\
\text { TEMP - } \quad \text { water temperature } \\
\text { WITH - } \quad \text { water withdrawals }\end{array}$ \\
\hline
\end{tabular}

"NA" indicates that no life history pathway meeting the criteria used in defining these pathways was present,

Blank cells under attributes indicate no additional attributes with negative effects on productivity were evident compared to those present in the template. 
Stream: Wallowa R.

Reach: Parsnip $\mathrm{Cr}$ - Hurricane $\mathrm{Cr}$ (RM 109.0 -121.9 from Snake R)

\begin{tabular}{|ll|ll|lll}
\hline Restoration benefit category: & B & Chinook usage score: & 140.6 & Habitat productivity loss score: & -1686.0 \\
\hline
\end{tabular}

\begin{tabular}{|c|c|c|c|c|c|c|c|c|}
\hline \multirow[b]{3}{*}{ Life stage } & \multirow[b]{3}{*}{ Months } & \multirow{2}{*}{\multicolumn{4}{|c|}{$\begin{array}{l}\text { No. vacant life history pathways } \\
\text { by abundance level }\end{array}$}} & \multicolumn{2}{|c|}{ Key habitat change } & \multirow{3}{*}{\begin{tabular}{|c|} 
Habitat \\
productivity \\
loss score
\end{tabular}} \\
\hline & & & & & & \multirow{2}{*}{$\begin{array}{l}\text { Patient } \\
\text { quantity }\end{array}$} & \multirow[b]{2}{*}{$\%$ change } & \\
\hline & & Total no. & 1 & 2 & 3 & & & \\
\hline 1 & Apr-Aug & 76 & 18 & 4 & 54 & 9.7 & -58.7 & $\begin{array}{l}-18.4 \\
\end{array}$ \\
\hline 123 & Aug-Ött & 90 & 0 & 0 & '90',', & 7.5 & -74.8 & $-1560, " '$, \\
\hline 3 & Ött-Mar & 180 & 0 & 0 & 180 & 8.1 & -76.2 & -789.5 \\
\hline 34 & Mar-May & 114 & $49^{\prime}$ & 18 & "' $\quad 47$ & 9 & -53.2 & -616.4 \\
\hline 5 & May-Ott & 388 & 234 & $60^{\circ}$ & 94 & 9.1 & -47.0 & -45.9 \\
\hline 6" & Ott-Mar & 57 & 38 & 0 & “" "19" & "'8.1 & -53.9 & -60.6 \\
\hline 7 & "Marr-Jün & 5 & 4 & 0 & 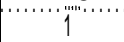 & 33.0 & -5.0 & 0.8 \\
\hline
\end{tabular}

\begin{tabular}{|c|c|c|c|c|c|c|c|c|c|c|c|c|c|}
\hline \multirow[b]{2}{*}{ Life stage } & \multirow[b]{2}{*}{ Months } & \multicolumn{12}{|c|}{ Highest productivity impact attributes (\% change in impact from template) } \\
\hline & & Att 1 & $\%$ change & Art 2 & $\%$ change & Att 3 & $\%$ change & Att 4 & $\%$ change & Att 5 & $\%$ change & Att 6 & $\%$ change \\
\hline 1 & Apr-Aug & HABD & 41.3 & RIPCN & $\overline{41.3}$ & PATHO & 18.5 & & & & & & \\
\hline $1,2,3$ & Äug-Ött & SEDLD & 75.0 & NUTLOD & $50.0^{\circ}$ & HÄB̈D̆ & 37.5 & RIIPCN & 37.5 & ÖXYG & $25.0^{\circ}$ & PATHO & 12.5 \\
\hline 3 & Ott-Mar & SEDLD & 75.0 & NUTLD & 50,0 & HABD & 37.5 & OXYG & 25.0 & RIPCN & 25.0 & FLOW & 12.5 \\
\hline 3,4 & Mar-May & Ḧ̈̈̈̈̄̈ & 75.0 & RIPCN" & 50.0 & "ŠẼËL̈D" & 32.0 & FLoW & 25.0 & "PATH'Н"'" & $25.0^{\circ}$ & WITH & 25.0 \\
\hline 5 & May-Ott & HAB'D & 36,9 & S̈ËL̈LD̈ & 25.3 & PATHO & 18.5 & RIPCN & 18.5 & & & & \\
\hline 6 & Ott-Mar & HABD & 35.4 & 'SÉDL'D' & 35.4 & PATHO & 17.7 & RIPCN & 17.7 & & & & \\
\hline 7 & Mar-Jun & CÖMP & 13.7 & РАТНO & 13.7 & WITH & 13.7 & & & & & & \\
\hline
\end{tabular}

Abundance/eve/ refers to estimated relative abundance of spawners that spawned in the natal stream reaches that populated the lie history pathways using the reach in this table: Level 1: $\quad$ Low to average (relative to other reaches) abundance

Level 2 High (relative to other reaches) abundance

Level 3 Exceptionally high (relative to other reaches) abundance

Life stages: 1-prespawner; 2- spawner; 3- incubation; 4- fry colonization; 5- summer rearing; 6- overwintering; 7-yearling presmoltsmolt

$\begin{array}{clll}\text { Attributes: } \text { CHEM - } & \text { chemicals } & \text { NUTLD - nutrient load } & \text { RIPCN - riparian condition } \\ \text { CSTAB - channel stability } & \text { OBST - obstructions } & \text { SEDLD - sediment load } \\ \text { COMP - competition (with other species) } & \text { OXYG - dissolved oxygen } & \text { TEMP - water temperature } \\ \text { FLOW - flow } & \text { PATHO - pathogens } & \text { WITH - water withdrawals } \\ \text { HABD - } & \text { habitat diversity } & \text { PRED - predators (includes fishing-related losses) }\end{array}$

"NA" indicates that no life history pathway meeting the criteria used in defining these pathways was present.

Blank cells under attributes indicate no additional attributes with negative effects on productivity were evident compared to those present in the template. 
Stream Reach Summary for Vacant Spring Chinook Salmon Life Histories

Stream: Wallowa R.

Reach: Hurricane Cr - Lower Alderslope Ditch (RM 121.9 -126.8 from Snake R)

\begin{tabular}{ll|llll}
\hline Restoration benefit category: & F & Chinook usage score: & 7.1 & /Habitat productivity loss score: & -161.3 \\
\hline
\end{tabular}

\begin{tabular}{|c|c|c|c|c|c|c|c|c|}
\hline \multirow[b]{3}{*}{ Life stage } & \multirow[b]{3}{*}{ Months } & \multirow{2}{*}{\multicolumn{4}{|c|}{$\begin{array}{l}\text { No. vacant life history pathways } \\
\text { by abundance level }\end{array}$}} & \multicolumn{2}{|c|}{ Key habitat change } & \multirow{3}{*}{$\begin{array}{c}\text { Habital } \\
\text { productivity } \\
\text { loss score }\end{array}$} \\
\hline & & & & & & \multirow{2}{*}{$\begin{array}{l}\text { Patient } \\
\text { quantity }\end{array}$} & \multirow[b]{2}{*}{$\%$ change } & \\
\hline & & Total no. & 1 & 2 & 3 & & & \\
\hline 1 & Apr-Aug & 17 & 9 & 8 & 0 & 28.7 & -7.1 & -13.3 \\
\hline $1,2,3$ & Aug-Oct & 10 & 0 & 10 & 0 & 19.9 & -24.5 & -23.8 \\
\hline 3 & Oct-Mar & 20 & 0 & 20 & 0 & 20.6 & -29.1 & -87.7 \\
\hline 3,4 & Mar-May & 9 & 5 & 4 & 0 & 22.7 & -18.9 & -36.2 \\
\hline 5 & May-Oct & 3 & 3 & 0 & 0 & 18.8 & 0.0 & 0.2 \\
\hline 6 & Ocl-Mar & $\mathrm{NA}$ & NA & $\mathrm{NA}$ & $\mathrm{NA}$ & $\mathrm{NA}$ & $\mathrm{NA}$ & $\mathrm{NA}$ \\
\hline 7 & Mar-Jun & 1 & 1 & 0 & 0 & 33.0 & 0.0 & -0.5 \\
\hline
\end{tabular}

\begin{tabular}{|c|c|c|c|c|c|c|c|c|c|c|c|c|c|}
\hline \multirow[b]{2}{*}{ Life stage } & \multirow[b]{2}{*}{ Months } & \multicolumn{12}{|c|}{ Highest productivity impact attributes (\% change in impact from template) } \\
\hline & & Aft 1 & $\%$ change & An 2 & $\%$ change & Att 3 & $\%$ change & Atf 4 & $\%$ change & Att 5 & $\%$ change & Atf 6 & $\%$ change \\
\hline 1 & Apr-Aug & SEDLD & 37.5 & FLOW & 27.0 & NUTLD & 25.0 & CHEM & 13.5 & HABD & 13.5 & OBST & 13.5 \\
\hline $1,2,3$ & Äug-Ött & ṠE்D்ட் & $750^{\circ}$ & "FLOOW" & 50,0 & NUTLD & $500^{\circ}$ & CHEM & $25.0^{\circ}$ & "HÄBD' & 25.0 & OBST & 25.0 \\
\hline 3 & Ott-Mar & SEDLD & 75.0 & NUTLD & 50.0 & FLOW & 25.0 & HABD & 25.0 & OXYG & 25.0 & PATHO & 25.0 \\
\hline 34 & Mar-May & SEDLD & 750 & NUTILO & 50.0 & FEOOOW & 25,0 & "HABD". & 25,0 & OXYG & 25.0 & PATTHO & 25.0 \\
\hline 5 & May-Ọtt & CHEM & 15.8 & PATHO & 15.8 & WITH & 15.8 & & & & & & \\
\hline 6 & Ott-Mar & $\mathrm{NA}$ & $\mathrm{NA}$ & NA & $\mathrm{NA}$ & $\mathrm{NA}$ & $\mathrm{NA}$ & $\mathrm{NA}$ & $\mathrm{NA}$ & $\mathrm{NA}$ & $\mathrm{NA}$ & $\mathrm{NA}$ & $\mathrm{NA}$ \\
\hline 7 & Mar-Jun & FLOW & 9.2 & WITH & 9.2 & "' & & & & & & & \\
\hline
\end{tabular}

Abundance level refers to estimated relative abundance of spawners that spawned in the natal stream reaches that populated the tife history pathways using the reach in this table. Level 1: Low to average (relative to other reaches) abundance

Level 2: $\quad$ High (relative to other reaches) abundance

Level 3: Exceptionally high (relative to other reaches) abundance

Life stages: 1-prespawner; 2- spawner; 3- incubation; 4- fry colonization; 5- summer rearing 6- overwintering; 7-yearlinçresmoltssmolt
Attributes.' CHEM - chemicals
CSTAB - channel stability
NUTLD - nutrient load
OBST - obstructions
RIPCN - riparian condition
COMP - competition (with other species)
FLOW - flow
OXYG - dissolved oxygen
SEDLD - sediment load
HABD - habitat diversity
PATHO - pathogens
TEMP - water temperature
PRED - predators (includes fishing-related losses)

"NA" indicates that no life history pathway meeting the criteria used in defining these pathways was present.

Blank cells under attributes indicate no additional attributes with negative effects on productivity were evident compared to those present in the template. 
Stream Reach Summary for Vacant Spring Chinook Salmon Life Histories

Stream: Wallowa R.

Reach: Lower Alderslope Ditch -Wallowa Lake (RM 126.8 -130.8 from Snake R)

[Restoration benefit category: B $\quad$ Chinook usage score: 49.9

Habitat productivity loss score: $-2251.5$

\begin{tabular}{|c|c|c|c|c|c|c|c|c|}
\hline \multirow[b]{3}{*}{ Liffestagec } & \multirow[b]{3}{*}{ Moontths } & \multirow{2}{*}{\multicolumn{4}{|c|}{$\begin{array}{l}\text { No. vacant life history pathways } \\
\text { by abundance level }\end{array}$}} & \multicolumn{2}{|c|}{ Key habitat change } & \multirow{3}{*}{$\begin{array}{c}\text { Habitat } \\
\text { productivity } \\
\text { loss score }\end{array}$} \\
\hline & & & & & & \multirow{2}{*}{$\begin{array}{l}\text { Patient } \\
\text { quantity }\end{array}$} & \multirow[b]{2}{*}{$\%$ change } & \\
\hline & & Total no. & 1 & 2 & 3 & & & \\
\hline 1 & Apr-Aug & 54 & 48 & 6 & 0 & 1.2 & -86.9 & -202.3 \\
\hline $1,2,3$ & Aug-ót & 90 & 80 & 10 & 0 & 0.1 & -96.9 & -555.0 \\
\hline 3 & Ott-Mar & 180 & 160 & 20 & 0 & 1.2 & -61.3 & -1107.4 \\
\hline 34 & Mar-May & 42 & 40 & 2 & 0 & 1.6 & -823 & -353.3 \\
\hline 5 & May-Oct & 94 & 94 & 0 & 0 & 6.1 & -74.1 & -15.2 \\
\hline 6 & Ott-Mar & 19 & 19 & 0 & 0 & 78 & -31 & -17.1 \\
\hline 7 & Mar-Jum & 1 & 1 & 0 & 0 & 330 & 0.0 & -13 \\
\hline
\end{tabular}

\begin{tabular}{|c|c|}
\hline Liffestage & Motontths \\
\hline 1 & Apr-Aug \\
\hline $1,2,3$ & Aug-Ö \\
\hline 3 & Ott=Mar \\
\hline 3,4 & $M a r=M a y$ \\
\hline 5 & May-Ot \\
\hline 6 & Otaikt \\
\hline 7 & Mar-Jum \\
\hline
\end{tabular}

\begin{tabular}{|c|c|c|c|c|}
\hline \multicolumn{3}{|c|}{ Highest productivity im } \\
\hline Att 1 & $\%$ change & Att 2 & $\%$ change & Att 3 \\
\hline FLOW & 75.0 & HABD & 50.0 & OBST \\
FLOW & 75.0 & SEDLD & 75.0 & HABD \\
FLOW & 75.0 & SEDLD & 75.0 & HABD \\
HABD & 50.0 & FLOW & 37.5 & SEDLD \\
HABD & 50.0 & CHEM & 25.0 & FLOW \\
\hdashline HABD & 50.0 & OBST & 25.0 & PATHO \\
\hdashline PRED & 25.0 & & & \\
\hline
\end{tabular}

\begin{tabular}{|c|c|c|c|c|c|c|}
\hline \multicolumn{7}{|c|}{ ct attributes (\% change in impact from template) } \\
\hline$\%$ change & Att 4 & $\%$ change & Alt 5 & $\%$ change & Att 6 & $\%$ change \\
\hline 50.0 & RIPCN & 50.0 & WITH & 50.0 & CHEM & 25.0 \\
\hline 50.0 & OBST & 50.0 & RIPCN & 50.0 & CHEM & 25.0 \\
\hline 37.5 & CHEM & 25.0 & RIPCN & 25.0 & PATHO & 12.5 \\
\hline 37.5 & CHEM & 25.0 & PATHO & 25.0 & RIPCN & 25.0 \\
\hline 250 & OBST & 25.0 & PATHO & 25.0 & RIPCN & 25.0 \\
\hline 25.0 & SEDLD & 25.0 & & & & \\
\hline
\end{tabular}

Abundance level refers to estimated relative abundance of spawners that spaw din the natal stream reaches that populated the life history pathways using the reachin this table Level 1: Low to average (relative to other reaches) abundance

Level 2: High (relative to other reaches) abundance

Level 3: Exceptionally high (relative to other reaches) abundance

Life stages: 1. prespawner; 2- spawner; 3- incubation; 4- fry colonization; 5- summer rearing; 6- overwintering; 7- yearlinpresmolt/smolt

Attributes: CHEM - chemicals

CSTAB - channel stability

COMP - competition (with other species)

FLOW - flow

HABD - habitat diversity
NUTLD - nutrient load

OBST - obstructions

OXYG - dissolved oxygen

PATHO - pathogens

PRED - predators (includes fishing-related losses)
RIPCN - riparian condition

SEDLD - sediment load

TEMP - water temperature

WITH - water withdrawals

"NA" indicates that no life history pathway meeting the criteria used in defining these pathways was present.

Blank cells under attributes indicate no additional attributes with negative effects on productivity were evident compared to those present in the template. 
Stream: Wenaha $\mathbf{R}$

Reach: $\quad$ Mouth - Crooked Cr (RM 45.1 -51.8 from Snake R)

\begin{tabular}{|ll|ll|l|}
\hline Restoration benefit category: & F & Chinook usage score: & 3.8 & Habitat productivity loss score: \\
\hline
\end{tabular}

\begin{tabular}{|c|c|c|c|c|c|c|c|c|}
\hline \multirow[b]{3}{*}{ Life atage } & \multirow[b]{3}{*}{ Months } & \multirow{2}{*}{\multicolumn{4}{|c|}{$\begin{array}{l}\text { No. vacant life history pathways } \\
\text { by abundance level }\end{array}$}} & \multicolumn{2}{|c|}{ Key habitat change } & \multirow{3}{*}{$\begin{array}{c}\text { Habitat } \\
\text { productivity } \\
\text { loss score }\end{array}$} \\
\hline & & & & & & \multirow{2}{*}{$\begin{array}{l}\text { Patient } \\
\text { quantity }\end{array}$} & \multirow[b]{2}{*}{$\%$ change } & \\
\hline & & Total no. & 1 & 2 & 3 & & & \\
\hline 1 & Apr-Aug & 8 & 4 & 4 & 0 & 12.0 & $.00 \ldots$ & 320 \\
\hline 1,23 & Aug-Oct & NA & NA & NA & NA & $\mathrm{NA}$ & NA & NÄ \\
\hline 3 & Oct-Mar & $\mathrm{NA}$ & $\mathrm{NÄ}$ & ${ }^{\mathrm{NA}}$ & NÄ & $\mathrm{NA}$ & NA & NA \\
\hline 3,4 & Mar-May & 20 & 4 & 16 & 0 & 8,2 & 0.0 & 0.0 \\
\hline 5 & May-Oct & 91 & 0 & 91 & 0 & 9,4 & 0.0 & 0.0 \\
\hline 6 & Ott-Mar & 19 & 0 & 19 & 0 & & 0.0 & 00 \\
\hline 7 & Mar-Jun & $\dddot{1}$ & 0 & 1 & 0 & 30.8 & $0.0^{\circ}$ & 0.0 \\
\hline
\end{tabular}

\begin{tabular}{|c|c|c|c|c|c|c|c|c|c|c|c|c|c|}
\hline \multirow[b]{2}{*}{ Life stage } & \multirow[b]{2}{*}{ Months } & \multicolumn{12}{|c|}{ Highest productivity impact attributes (\% change in impact fi $/ \mathrm{m}$ lemplate) } \\
\hline & & $\overline{\text { Atf } 1}$ & $\%$ change & Att 2 & $\%$ change & Att 3 & $\%$ change & Att 4 & $\%$ change & Att 5 & $\%$ change & Att 6 & $\%$ change \\
\hline 1 & Apr-Aug & & & & & & & & & & & & \\
\hline $1,2,3 \cdots$ & Aug-Ott & $\mathrm{NA}$ & Nä & $\mathrm{NA}$ & $\mathrm{NA}$ & $\mathrm{NAM}$ & "Na" & NA. & NA & NA & NÄ & NA & $\mathrm{NA}$ \\
\hline 3 & Ott-Mar & NA & $\mathrm{NA}$ & NA & NA & $\mathrm{N} A$ & NA"“"“ & NA & "NA & $\mathrm{NA}$ & $\mathrm{NA}$ & $\mathrm{NA}$ & $\mathrm{NA}$ \\
\hline 3,4 & Mar-May & PATHO & $3.1^{\prime \prime \prime \prime \prime \prime \prime ! ” ' ~}$ & & & & & & & & & & \\
\hline 5 & May-Ott & PATHO & 3.1 & & & & & & & & & & \\
\hline 6 & Oct-Mar & PATHO & 3.1 & & & & & & & & & & \\
\hline 7 & Mar-Jun & & & & & & & & & & & & \\
\hline
\end{tabular}

Abundance /eve/ refers to estimated relative abundance of spawners that spawned in the natal stream reaches that populated the life history pathways using the reach in this table Level 1: $\quad$ Low to average (relative to other reaches) abundance

Level 2: $\quad$ High (relative to other reaches) abundance

Level 3 Exceptionally high (relative to other reaches) abundance

Life stages: 1- prespawner; 2- spawner; 3- incubation; 4- fry colonization; 5- summer rearing; 6- overwintering; 7- yearling presmolt/smelt

Attributes: CHEM - chemicals

CSTAB - channel stability

COMP - competition (with other species)

FLOW - now

HABD - habitat diversity
NUTLD - nutrient load

OBST - obstructions

OXYG - dissolved oxygen

PATHO - pathogens

PRED - predators (includes fishing-related losses)
RIPCN - riparian condition

SEDLD - sediment load

TEMP - $\quad$ water temperature

WITH - water withdrawals

"NA" indicates that no tife history pathway meeting the criteria used in definina these oathwavs was present.

Blank cells under attributes indicate no additional attributes with negative effects on productivity were evident compared to those present in the template. 
Stream Reach Summary for Vacant Spring Chinook Salmon Life Histories

Stream: Wenaha $\mathbf{R}$.

Reach: Crooked $\mathbf{C r}$ - Fairview $\mathrm{Cr}$ (RM 51.8 -55,3 from Snake R)

\begin{tabular}{ll|ll|l}
\hline Restoration benefit category: & $F$ & Chinook usage score: & 16.4 & Habitat productivity loss score: \\
\hline
\end{tabular}

\begin{tabular}{|c|c|c|c|c|c|c|c|c|}
\hline \multirow[b]{3}{*}{ Life stage } & \multirow[b]{3}{*}{ Months } & \multirow{2}{*}{\multicolumn{4}{|c|}{$\begin{array}{l}\text { No. vacant life history pathways } \\
\text { by abundance level }\end{array}$}} & \multicolumn{2}{|c|}{ Key habitat change } & \multirow{3}{*}{$\begin{array}{c}\text { Habitat } \\
\text { productivity } \\
\text { loss score }\end{array}$} \\
\hline & & & & & & \multirow{2}{*}{$\begin{array}{l}\text { Patient } \\
\text { quantity }\end{array}$} & \multirow[b]{2}{*}{$\%$ change } & \\
\hline & & Total no. & 1 & 2 & 3 & & & \\
\hline 1 & Apr-Aug & 28 & 24 & 4 & 0 & 9.4 & 0.0 & -39.1 \\
\hline 123 & Aug-Ott & 40 & 40 & 0 & 0 . & 69 & 00 & -5.2 \\
\hline 3 & Ott-Mar & 80 & 80 & 0 & 0 & 6.7 & 0.0 & 0 \\
\hline 34 & Mar-May & 12 & 8 & 4 & $?$ & 8.8 & 0.0 & 7.3 \\
\hline 5 & May-Ott & $\mathrm{NA}$ & $\mathrm{NA}$ & NA & $\mathrm{NA}$ & $\mathrm{NA}$ & $\mathrm{NA}$ & $N A$ \\
\hline 6. & Ott-Mar. & NA & NA & NA & NA & NA & NA & NA \\
\hline$\ddot{7}$ & Mar-Jun & $\mathrm{N} \mathrm{A}$ & $\mathrm{NA}$ & $\mathrm{NA}$ & NÄ" & $\mathrm{NÄ}$ & $\mathrm{N} A$ & $\mathrm{NA}$ \\
\hline
\end{tabular}

\begin{tabular}{|c|c|c|c|c|c|c|c|c|c|c|c|c|c|}
\hline \multirow[b]{2}{*}{ Life stage } & \multirow[b]{2}{*}{ Months } & \multicolumn{12}{|c|}{ Highest productivify impact attribules (\% change in impacf from template) } \\
\hline & & Alt 1 & $\%$ change & Att 2 & $\%$ change & Att 3 & \% change & Att 4 & $\%$ change & Att 5 & $\%$ change & Att 6 & $\%$ change \\
\hline 1 & Apr-Aug & PRED & & & & & & & & & & & \\
\hline 123 & Alug-Oci & PRED & & & & & & & & & & & \\
\hline 3 & Ott-Mar & PATHO & 12.5 & & & & & & & & & & \\
\hline 34 & Mar-May & "PATHO' & 12.5 & & & & & & & & & & \\
\hline 5 & May-Qtt, & $\dot{N A ̈}$ & $\dot{N A}$ & NA & $\mathrm{NA}$ & NA & NA & $\mathrm{NA}$ & $\dot{N A}$ & $\mathrm{NA}$ & $\mathrm{NA}$ & $\mathrm{NA}$ & $\mathrm{NA}$ \\
\hline 6 & Ott-Mar & $\mathrm{NA}$ & $\mathrm{NA}$ & $\mathrm{NA}$ & $\mathrm{NA}$ & $\mathrm{NA}$ & $\mathrm{NA}$ & $\mathrm{NA}$ & $\mathrm{NA}$ & $\mathrm{NA}$ & $\mathrm{NA}$ & NA & NA \\
\hline 7 & Mar-Jun & NA & NA & NA & NA & NA & NA & NA & NA & NA & NA & NA & NA \\
\hline
\end{tabular}

Abundance level refers to estimated relative abundance of spawners that spawned in the natal stream reaches that populated the life history pathways using the reach in this table: Level 1: Low to average (relative to other reaches) abundance

Level 2 High (relative to other reaches) abundance

Level 3: Exceptionally high (relative to other reaches) abundance

Life stages: 1 - prespawner; 2- spawner; 3- incubation; 4- fry colonization; 5- summer rearing; 6- overwintering; 7- yearling presmolt/smolt

Attributes: CHEM - chemicals CSTAB - channel stability

NUTLD - nutrient load

COMP - competition (with other species)

FLOW - ftOW

HABD - habitat diversity

\begin{tabular}{|c|c|}
\hline 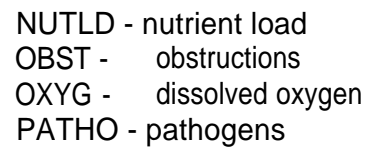 & $\begin{array}{l}\text { RIPCN - } \quad \text { riparian condition } \\
\text { SEDLD - } \\
\text { TEMPdiment load } \\
\text { TEMP - } \quad \text { water temperature } \\
\text { WITH - } \quad \text { water withdrawals }\end{array}$ \\
\hline
\end{tabular}

"NA" indicates that no lie history pathway meeting the criteria used in defining these pathways was present.

Blank cells under attributea indicate no additional attributes with negative effects on productivity were evident compared to those present in the template. 
Stream Reach Summary for Vacant Spring Chinook Salmon Life Histories

Stream: Wenaha R.

Reach: Faiview Cr - forks (RM 55.3 -65,9 from Snake R)

\begin{tabular}{ll|ll|lll}
\hline Restoration benefit category: & F & Chinook usage score: & 23.4 & Habitat productivity loss score: & -2.7 \\
\hline
\end{tabular}

\begin{tabular}{|c|c|c|c|c|c|c|c|c|}
\hline \multirow[b]{3}{*}{ Liie stage } & \multirow[b]{3}{*}{ Months } & \multirow{2}{*}{\multicolumn{4}{|c|}{$\begin{array}{c}\text { No. vacant life history pathways } \\
\text { by abundance level }\end{array}$}} & \multicolumn{2}{|c|}{ Key habitat change } & \multirow{3}{*}{\begin{tabular}{|c|} 
Habitat \\
productivity \\
10ss score
\end{tabular}} \\
\hline & & & & & & \multirow{2}{*}{$\begin{array}{l}\text { Patient } \\
\text { quantity }\end{array}$} & \multirow[b]{2}{*}{$\%$ change } & \\
\hline & & Total no. & 1 & 2 & 3 & & & \\
\hline $1,2,3$ & $\begin{array}{l}\text { Apr-Aug } \\
\text { Aug-Oct }\end{array}$ & 24 & 0 & 24 & 0 & $\begin{array}{l}16.0 \\
8.7\end{array}$ & 0.0 & $-5,1$ \\
\hline 3 & Oct-Mar & 80 & & & & 6.3 & 0.0 & \\
\hline 34 & Mar-May & 12 & $\dot{0}$ & 12 & 0 & 6.6 & 0.0 & 5.1 \\
\hline 5 & May-Ott & $\mathrm{NA}$ & $\mathrm{NA}$ & $\mathrm{NA}$ & $\mathrm{NA}$ & $\dot{\mathrm{NA}}$ & $\mathrm{NA}$ & NA' \\
\hline 6 & Ott-Mar & NA & NA & NA & NA & NA & NA & NA \\
\hline 7 & Mar-Jun & $\mathrm{NA}$ & $\mathrm{NA}$ & $\mathrm{NA}$ & $\mathrm{NA}$ & $\mathrm{NA}$ & $\mathrm{NA}$ & $\mathrm{NA}$ \\
\hline
\end{tabular}

\begin{tabular}{|c|c|c|c|c|c|c|c|c|c|c|c|c|c|}
\hline \multirow[b]{2}{*}{ Life strige } & \multirow[b]{2}{*}{ Months } & \multicolumn{12}{|c|}{ Highest productivity impact attributes (\% change in impact from templal :) } \\
\hline & & At! 1 & $\%$ change & At! 2 & $\%$ change & At! 3 & $\%$ change & At! 4 & $\%$ change & At! 5 & $\%$ change & Att 6 & $\%$ change \\
\hline 1 & Apr-Aug & PRED & 18.8 & & & & & & & & & & \\
\hline $1,2,3$ & Aug-Öct & PRED & 18.1 & & & & & & & & & & \\
\hline 3 & Oct-Mar & PATHOO & 25.0 & & & & & & & & & & \\
\hline 3,4 & Mar-May & PATHOO & 25.0 & & & & & & & & & & \\
\hline 5 & May-Oct & NA & $N A$ & $\mathrm{NA}$ & $\mathrm{NA}$ & NA & $\mathrm{NA}$ & $N A$ & NA & $\mathrm{NA}$ & $\mathrm{NA}$ & NÄ & "NA" \\
\hline 6 & Oct-Mar & NÄ & "NÄ & $\mathrm{NA}$ & NÄ & $\mathrm{NA}$ & "NA" & NÄ & $\mathrm{NA}$ & "NÄ & $\mathrm{NA"}$ & $\mathrm{NÄ}$ & $\mathrm{NA}$ \\
\hline 7 & Mar-Jun & $\mathrm{NA}$ & $\mathrm{NA}$ & $\mathrm{NA}$ & $\mathrm{NA}$ & $\mathrm{NA}$ & $\mathrm{NA}$ & $\mathrm{NA}$ & $\mathrm{NA}$ & NA & NA & NA & NA \\
\hline
\end{tabular}

Abundance/eve/refers to estimated relative abundance of spawners that spawned in the natal stream reaches that populated the life history pathways using the reach in this table: Level 1: Low to average (relative to other reaches) abundance

Level 2 High (relative to other reaches) abundance

Level 3 Exceptionally high (relative to other reaches) abundance

Life stages: 1- prespawner; 2- spawner; 3- incubation; 4- fry colonization; 5- summer rearing; 6- overwintering; 7-yearling presmolt/smolt

$\begin{array}{lll}\text { Attributes: } \text { CHEM - chemicals } & \text { NUTLD - nutrient load } & \text { RIPCN - riparian condition } \\ \text { CSTAB - channel stability } & \text { OBST - obstructions } & \text { SEDLD - sediment load } \\ \text { COMP - competition (with other species) } & \text { OXYG - dissolved oxygen } & \text { TEMP - water temperature } \\ \text { FLOW - ftOw } & \text { PATHO - pathogens } & \text { WITH - water withdrawals } \\ \text { HABD - habitat diversity } & \text { PRED - predators (includes fishing-related losses) }\end{array}$

"NA" indicates that no tife history pathway meeting the criteria used in defining these pathways was present.

Blank cells under attributes indicate no additional attributes with negative effects on productivity were evident compared to those present in the template. 


\section{Stream Reach Summary for Vacant Spring Chinook Salmon Life Histories}

Stream: Wenaha $\mathbf{R}$

Reach: South Fork Wenaha R (RM 65.9 -72.4 from Snake R)

\begin{tabular}{|ll|ll|lll}
\hline Restoration benefit category: & $F$ & Chinook usage score: & 0.0 & Habitat productivity loss score: & 0.0 \\
\hline
\end{tabular}

\begin{tabular}{|c|c|c|c|c|c|c|c|c|}
\hline \multirow[b]{3}{*}{ Life stsge } & \multirow[b]{3}{*}{ Months } & \multirow{2}{*}{\multicolumn{4}{|c|}{$\begin{array}{l}\text { No. vacant life history pathways } \\
\text { by abundance level }\end{array}$}} & \multicolumn{2}{|c|}{ Key habitat change } & \multirow{3}{*}{$\begin{array}{c}\text { Habitat } \\
\text { productivity } \\
\text { loss score }\end{array}$} \\
\hline & & & & & & \multirow{2}{*}{$\begin{array}{l}\text { Patient } \\
\text { uantit }\end{array}$} & \multirow[b]{2}{*}{$\%$ than e } & \\
\hline & & Total no. & 1 & 2 & 3 & & & \\
\hline 1 & Apr-Aug & NA & $\overline{N A}$ & $\overline{\mathrm{NA}}$ & $\overline{\mathrm{NA}}$ & & NA ${ }^{9}$ & NA \\
\hline $1, \ldots$ & Aug-Ott & NA" & NA & $\mathrm{NA}$ & $\mathrm{NÄ}$ & NA & NA & NA \\
\hline 3 & Ott-Mar & $\mathrm{NAO}$ & $\mathrm{NA}$ & $\mathrm{NA}$ & $\mathrm{NA}$ & $\mathrm{NA}$ & $\mathrm{NA}$ & $\mathrm{NA}$ \\
\hline 3,4 & Mar-May & $\mathrm{NA}$ & $\mathrm{NA}$ & $\mathrm{NA}$ & $\mathrm{NA}$ & NA & NA & $\mathrm{NA}$ \\
\hline 5 & Mäay-Ött & NA* & $\mathrm{NA}$ & $\mathrm{N}_{\mathrm{NA}}$ & $\mathrm{NA}$ & $\mathrm{NA}$ & $\mathrm{NA}$ & $\mathrm{NA}$ \\
\hline 6 & Ott-Mar & NA & $\mathrm{NA}$ & NA & $\mathrm{NA}$ & NA & $\mathrm{NA}$ & NA \\
\hline 7 & Mar-Jun & $\mathrm{NA}$ & $N A$ & $\mathrm{NA}$ & $\mathrm{NA}$ & $\mathrm{Nä}$ & $\mathrm{NA}$ & NA \\
\hline
\end{tabular}

\begin{tabular}{|c|c|c|c|c|c|c|c|c|c|c|c|c|c|}
\hline \multirow[b]{2}{*}{ Life stage } & \multirow[b]{2}{*}{ Months } & \multicolumn{12}{|c|}{ Highest productivity impact attributes (\% change in impact from template) } \\
\hline & & Att 1 & $\%$ change & Att 2 & $\%$ change & Att 3 & $\%$ change & Att 4 & $\%$ change & Att 5 & $\%$ change & Att 6 & $\%$ change \\
\hline 1 & Apr-Aug & $\mathrm{NA}$ & NA & NA & NA & NA & NA & NA & $\overline{N A}$ & $\overline{\mathrm{NA}}$ & $\mathrm{NA}$ & NA & NA \\
\hline $1,2,3$ & Äug-Ött & $\dot{\mathrm{NA}}$ & $\mathrm{NÄ̈}$ & $\mathrm{NA}$ & "NÄ & $\mathrm{NA}$ & N̈Ä & NA & $\mathrm{NA}$ & NA & $\mathrm{NA}$ & $\mathrm{NA}$ & NA \\
\hline 3 & Oct-Mar & $\mathrm{NA}$ & "NA & NA & "NA & NA & NA & $\mathrm{NA}$ & NA & NA & NA & "wa NA & NA \\
\hline 34 & Mar-May & $\mathrm{NA}$ & NA & $\mathrm{NÄ}$ & ÑÄ & "NA" & NA & NÄ & $\mathrm{NA}$ & $\mathrm{NA}$ & NA" & $\mathrm{NA}$ & $\mathrm{NA}$ \\
\hline 5 & May-Ott & $N A$ & NA & NÁ & NA "' & NA & NA & $\mathrm{NA}$ & $\dddot{\mathrm{NA}}$ & NA & $\mathrm{NA}$ & NA & '“'”,'”'NA'-"', \\
\hline 6 & Oct-Mar & NÁ & $\mathrm{NÄ}$ & NÄ" & $\mathrm{NA}$ & $\mathrm{NA}$ & NA & NA & $\mathrm{NA}$ & $\mathrm{NA}$ & $\mathrm{NÄ}$ & $\mathrm{NA}$ & $\mathrm{NA}$ \\
\hline 7 & Mar-Jun & NA & NA & $\mathbf{N} \mathbf{A}$ & NA & NA & $N A$ & NA & NA & NA & NA & NA & NA \\
\hline
\end{tabular}

Abundance level refers to estimated relative abundance of spawners that spawned in the natal stream reaches that populated the life history pathways using the reach in this table: Level 1: Low to average (relative to other reaches) abundance

Level 2 High (relative to other reaches) abundance

Level 3 Exceptionally high (relative to other reaches) abundance

Life stages: 1- prespawner; 2- spawner; 3- incubation; 4- fry colonization; 5- summer rearing; 6- overwintering; 7- yearling presmolt/smelt

Attributes: CHEM - chemicals

CSTAB - channel stability

COMP - competition (with other species)

FLOW - ftOW

HABD - habitat diversity
NUTLD - nutrient load

OBST - obstructions

OXYG - dissolved oxygen

PATHO - pathogens

PRED - predators (includes fishing-related losses)
RIPCN - riparian condition

SEDLD - sediment load

TEMP - water temperature

WITH - $\quad$ water withdrawals

"NA" indicates that no life history pathway meeting the criteria used in defining these pathways was present.

Blank cells undar attributes indicate no additional attributes with negative effects on productivity were evident compared to those present in the template. 


\section{APPENDIX F}

Imnaha Stream Reach Summaries

for Primary and Secondary Life History Pathways 
Reaches analyzed in the Imnaha, listed in the order they appear in Appendix F.

\section{Benefit Category}

for

Primary \&

Secondary Life

Histories

stream

Big Sheep Cr.

Big Sheep Cr.

Big Sheep Cr.

Big Sheep Cr.

Big Sheep Cr.

Big Sheep Cr.

Big Sheep Cr.

Big Sheep Cr.

Big Sheep Cr.

Imnaha $\mathrm{R}$.

Imnaha $R$

Imnaha $\mathrm{R}$.

Imnaha $\mathrm{R}$

Imnaha $R$.

Imnaha $R$.

Imnaha $\mathrm{R}$.

Imnaha $R$

Imnaha $R$

Imnaha $\mathrm{R}$.

Imnaha $\mathrm{R}$

Imnaha $\mathrm{R}$.

Imnaha R.

Imnaha $\mathrm{R}$.

Imnaha $\mathrm{R}$.

Lightning $\mathrm{Cr}$.

Little Sheep Cr.

Little Sheep Cr.

Little Sheep Cr.

Little Sheep Cr.

Little Sheep Cr.
Reach

Mouth - Camp Cr (RM 22.3 -23.4 from Snake R)

Camp Cr - Little Sheep Cr (RM 23.4 -25.4 from Snake R)

Little Sheep Cr - Squaw Cr (RM 25.4 -37.7 from Snake R)

Squaw Cr - Marr Cr (RM 37.7 -39.3 from Snake R)

Marr Cr - Griffith Cr (RM 39.3 -45.3 from Snake R)

Griffith Cr - Carrel Cr(RN445.3 -47.0 from Snake R)

Carrel Cr - Owl Cr (RM 47.0 -53.5 from Snake R)

Owl Cr - Lick Cr (RM 53.5 -57.4 from Snake R)

Lick Cr - South Fork (RM 57.4 -58.9 from Snake R)

Mouth - Cow Cr (RM 0.0- 4.3 from Snake R)

Cow Cr - Lightning Cr (RM 4.3 -5.4 from Snake R)

Lightning Cr - Horse Cr (RM 5.4- 10.8 from Snake R)

Fence Cr - Big Sheep Cr (RM 16.3 -22.3 from Snake R)

Horse Cr - Fence Cr (RM 10.8 -16.3 from Snake R)

Big Sheep Cr - Freezeout Cr (RM 22.3 -35.2 from Snake R)

Freezeout Cr - Grouse Cr (RM 35.2 -40.6 from Snake R)

Grouse Cr - Summit Cr (RM 40.6 -43.6 from Snake R)

Summit Cr - Crazyman Cr (RM 43.6 -49.4 from Snake R)

Crazyman Cr - GumbootCr(RA449.4 -52.7 from Snake R)

Gumboot Cr - Dry Cr (RM 52.7 -55.4 from Snake R)

Dry Cr - Skookum Cr (RM 55.4 -61.9 from Snake R)

Blue Hole - forks (RM 66.4 -71.4 from Snake R)

Skookum Cr - Blue Hole (RM 61.9 -66.4 from Snake R)

South Fork Imnaha R (RM 71.4 -76.1 from Snake R)

Mouth - forks (RM 5.4- 13.9 from Snake R)

Mouth - Bear Gulch (RM 25.4 -28.5 from Snake R)

Bear Gulch - Devil's Gulch (RM 28.5 -30.9 from Snake R)

Devil's Gulch - Lightning Cr (R.M 30.9 -33.1 from Snake R)

Lightning Cr - Hayden Cr (RM 33.1 -38.8 from Snake R)

Hayden Cr - McCully Cr (RM 38.8 -48.8 from Snake R)
$\mathrm{F}$

$\mathrm{F}$

$\mathrm{D}$

$\mathrm{D}$

B

$\mathrm{F}$ 
Stream Reach Summary for Primary and Secondary Spring Chinook Salmon Life Histories

Stream: Big Sheep Cr.

Reach: Mouth - Camp Cr (RM 22.3 -23.4 from Snake R)

\begin{tabular}{|c|c|c|c|c|c|c|c|c|c|c|c|c|c|c|}
\hline \multicolumn{4}{|c|}{ [Restoration benefit category: } & & \multicolumn{3}{|c|}{ Chinook usage score: } & 13.1 & \multicolumn{5}{|c|}{ Habitat productivity loss score: } & \\
\hline \multirow[b]{3}{*}{ Life stage } & \multirow[b]{3}{*}{ Months } & \multicolumn{10}{|c|}{ No. tife history pathways by current usage abundance level } & \multicolumn{2}{|c|}{ Key habital change } & \multirow{3}{*}{$\begin{array}{c}\text { Habitat } \\
\text { productivity } \\
\text { loss score }\end{array}$} \\
\hline & & \multicolumn{5}{|c|}{ Primary } & \multicolumn{5}{|c|}{ Secondary } & \multirow{2}{*}{$\begin{array}{l}\text { Patient } \\
\text { quantity }\end{array}$} & \multirow[b]{2}{*}{$\%$ change } & \\
\hline & & Total no. & 1 & 2 & 3 & 4 & Total no. & 1 & 2 & 3 & 4 & & & \\
\hline 1 & Apr-Aug & 5 & 5 & $\overline{0}$ & 0 & 0 & 12 & 12 & 0 & 0 & 0 & 6.0 & 0.0 & -3.9 \\
\hline $1,2,3$ & Âug-Ôtt & $\mathrm{NA}$ & $\mathrm{NA"}$ & $\mathrm{NA}$ & $\mathrm{NA}$ & NA & $\mathrm{N}^{-}$ & NAA' & & Y? $\cdots$ & $\ldots 2$ & NA" & $\mathrm{NA}$ & NA"' \\
\hline 3 & Ott-M̈ar & $\mathrm{NA}$ & NA & NA & NA & NA & $\mathrm{NA}$ & $\mathrm{NA}$ & & NA & & $\mathrm{NA}$ & $\mathrm{NA}$ & $\mathrm{NA}$ \\
\hline 34 & M̈ar-M̈lay & NA & NA & NA & NA & NA & NA & NA & NA & NA & NA & NA & N & $\mathrm{NA}$ \\
\hline 5 & May-Ött & 0 & 0 & 0 & 0 & $0^{\circ}$ & 4 & 4 & 0 & 0 & o" & 2.9 & 0.0 & 24 \\
\hline 6 & Ott-Mar & 0 & 0 & 0 & 0 & 0 & 4 & 4 & 0 & 0 & 0 & 71 & 0.0 & -3.0 \\
\hline 7 & Mar. ilun & 5 & 5 & i & $n$ & 0 & $\ddot{A}$ & A & 0 & 0 & 0 & 15.3 & 0.0 & $4 \cdot 2$ \\
\hline
\end{tabular}

\begin{tabular}{|c|c|c|c|c|c|c|c|c|c|c|c|c|c|}
\hline \multirow[b]{2}{*}{ Life stage } & \multirow[b]{2}{*}{ Months } & \multicolumn{12}{|c|}{ Highest productivity impact attributes (\% change in impact from template) } \\
\hline & & Att 1 & $\%$ change & Att 2 & $\%$ change & Att 3 & $\%$ change & Att 4 & $\%$ change & Att 5 & $\%$ change & Att 6 & $\%$ change \\
\hline 1 & Apr-Aug & FLOW & 4.7 & RIPCN & 4.7 & SEDLD & 4.7 & TEMP & 4.7 & & & & \\
\hline $1,2,3$ & Aug-Ött & NA & NÄ & $\cdots$ & NA & $\mathrm{NA}$ & $\mathrm{NA}$ & $\dot{N} \dot{A}$ & $\ddot{N A}$ & $\mathrm{NA}$ & $\mathrm{NÄ}$ & $\dot{\mathrm{NA}}$ & $\mathrm{NA}$ \\
\hline 3 & Ött-M̈ar & NA & $\mathrm{NA}$ & NA" & $\mathrm{NA}$ & $\mathrm{NA}$ & NA" & NÄ & ii" & Ni & NA" & $\mathrm{NA}$ & NA \\
\hline 34 & M̈ar-M̈ay & NA & NA & NA & $\ddot{N A}$ & NA & NA & NA & N & N & $\mathrm{NA}$ & NA & \\
\hline 5 & Ṁay-Ött & CSTÁB & 7.0 & FLOW & 7.0 & "PÄTHO & 7.0 & "TËMP" & 7.0 & 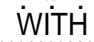 & 7.0 & & \\
\hline 6 & Ott-Mar & CSTAB & 9.9 & FLLÖẄ & 9.9 & РATHOO & 9.9 & TEMP & 9.9 & "WiTH & 9.9 & & \\
\hline 7 & Mar-Jun & 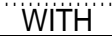 & 9.2 & & & & & & & & & & \\
\hline
\end{tabular}

Abundance lovel refera to the average abundance of spawners that spawn in the natal stream reaches that populate the tife history pathways using the reach In this table:

$$
\begin{array}{ll}
\text { Level 1: } & 0-10 \text { spawners } \\
\text { Level 2: } & 10-30 \text { spawners } \\
\text { Level 3: } & 30-60 \text { spawners } \\
\text { Level 4: } & >60 \text { spawners }
\end{array}
$$

Life stages: 1- prespawner; 2- spawner; 3- incubation; 4- fry colonization; 5- summer rearing; 6- overwintering; 7- yearling presmolt/smolt

Attributes: CHEM - chemicals

CSTAB - channel stability

COMP - competition (with other species)

FLOW - flow

HABD - habitat diversity
NUTLD - nutrient load

OBST - obstructions

OXYG - dissolved oxygen

PATHO - pathogens

PRED - predators (includes fishing-related losses)
RIPCN - riparian condition

SEDLD - sediment load

TEMP - water temperature

WITH - water withdrawala

"NA" indicates that no tife history pathway meeting the criteria usedn defining these pathwaya was present.

Blank cells under attributes indicate no additional attributes with negative effects on productivity were evident compared to those present in the template. 


\section{Stream Reach Summary for Primary and Secondary Spring Chinook Salmon Life Histories}

Stream: Big Sheep Cr.

Reach: Camp Cr - Little Sheep Cr (RM 23.4 -25.4 from Snake R)

\begin{tabular}{|ll|lllll}
\hline Restoration benefit category: & F & Chinook usage score: & 47.3 & [Habitat productivity ioss score: & -67.3 \\
\hline
\end{tabular}

\begin{tabular}{|c|c|c|c|c|c|c|c|c|c|c|c|c|c|c|}
\hline \multirow[b]{3}{*}{ Life stage } & \multirow[b]{3}{*}{ Months } & \multicolumn{10}{|c|}{ No. iife history pathways by current usage abundance level } & \multicolumn{2}{|c|}{ Key habitat change } & \multirow{3}{*}{$\begin{array}{c}\text { Habitat } \\
\text { productivity } \\
\text { ioss score }\end{array}$} \\
\hline & & \multicolumn{5}{|c|}{ Primary } & \multicolumn{5}{|c|}{ Secondary } & \multirow{2}{*}{$\begin{array}{l}\text { Patient } \\
\text { quantity }\end{array}$} & \multirow[b]{2}{*}{ \% change } & \\
\hline & & Total no. & 1 & 2 & 3 & 4 & Total no. & 1 & 2 & 3 & 4 & & & \\
\hline 1 & Apr-Aug & 5 & 5 & 0 & 0 & 0 & 12 & 12 & 0 & 0 & 0 & 6.6 & -53 & -155 \\
\hline $1,2,3$ & Aug-Ott & $\mathrm{NA}$ & $\mathrm{NA}$ & $\mathrm{NA}$ & $\mathrm{NA}$ & $\mathrm{NA}$ & NA" & NA & $\mathrm{NA}$ & $\mathrm{NA}$ & NA & $\mathrm{NA}$ & NA & NA \\
\hline 3 & Ott-Mar & NA & NA & NA & NA & NA & $\mathrm{NA}$ & NA & NA & NA & NA & NA & NA & NA \\
\hline 34 & Mar-May & NA & NA & $\dot{N}{ }^{\prime}$ & NA & NA & NA & "NA & NA & N̈̈̈̈ & $\mathrm{NA}$ & $\mathrm{NA}$ & $\mathrm{NA}$ & NA \\
\hline 5 & May-Ott & 0. & 0 & 0 & 0 & 0 & 6 & 6 & 0 & $\ldots 0$. & 0 & 5.1 & 0.0 & $\ldots \ldots$ \\
\hline 6 & "Ött-M̄ar & 0 & 00 & 0 & 0 & $\dddot{0}$ & 21 & 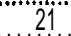 & $" 0$ & 0 & $0 "$ & 13.1 & 0.0 & -53.9 \\
\hline 7 & Mar-Jun & 5 & 5 & 0 & 0 & $\dddot{0}$ & "4 4 & 4 & 0 & 0 & 0 & 21.6 & 0.0 & 5.8 \\
\hline
\end{tabular}

\begin{tabular}{|c|c|c|c|c|c|c|c|c|c|c|c|c|c|}
\hline \multirow[b]{2}{*}{ Life atage } & \multirow[b]{2}{*}{ Months } & \multicolumn{12}{|c|}{ Highest productivity impact attributea (\% change in impact from template) } \\
\hline & & Atf 1 & $\%$ change & Aft 2 & $\%$ change & Att 3 & $\%$ change & Aft 4 & $\%$ change & Att 5 & \% change & Atf 6 & $\%$ change \\
\hline 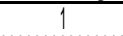 & Apr-Aug & FLOW & 18.7 & RIPCN & 18.7 & SEDLD & 18.7 & TEMP & 16.7 & & & & \\
\hline 123 & Äug-Óci & NÄ & $\dot{N} \dot{A}$ & $\dot{\mathrm{NA}}$ & NA & $\mathrm{NA}$ & NA & $\mathrm{NA}$ & $\dot{N A}$ & NA' & NÄ & $\mathrm{NÄ}$ & $\dddot{N} \mathrm{NA}^{\circ}$ \\
\hline 3 & Ott-Mar & "NÄ" & $\mathrm{NA}$ & $\mathrm{NA}$ & $\mathrm{NA}$ & $\mathrm{NÄ}$ & $\mathrm{NA}$ & $\mathrm{NÄ}$ & $\mathrm{NA}$ & NA & NA" & $\mathrm{NA}$ & NA \\
\hline 34, & Mar-May & NA & Nì & NA & NA & NA & $\dot{N} \dot{A}$ & NA & NA & $\mathrm{NÄ}$ & $\mathrm{NÄ}$ & $\mathrm{N}$ & N̈̈̈ \\
\hline 5 & May-Öt & TEMP & 14.5 & COMP & 7.2 & $\mathrm{HABD}$ & 7.2 & "PिÄT̈ḦÖ & $7.2^{\circ}$ & SEDLD & 7.2 & & \\
\hline$\dddot{6}$ & Ött-Mar & "W̄iTïi" & 25.0 & CSTAB & 23.1 & "FLOWW" & 23.1 & "PिӒТัḦО" & 23.1 & "TËM"P"' & 23.1 & & \\
\hline 7 & Mar-Jun & WITH & 12.7 & & & & & & & & & & \\
\hline
\end{tabular}

Abundance level refers to the average abundance of apawners that spawn in the natal stream reaches that populate the life history pathways using tha reach in this table:

$$
\begin{array}{ll}
\text { Level 1: } & 0-10 \text { spawners } \\
\text { Level 2 } & 10-30 \text { spawners } \\
\text { Level 3: } & 30-60 \text { spawners } \\
\text { Level 4 } & >\mathbf{7 0} \text { spawners }
\end{array}
$$

Life stages: 1- prespawner; 2- spawner; 3- incubation; 4- fry colonization; 5- summer rearing; 6- overwintering; 7- yearling presmolt/smolt

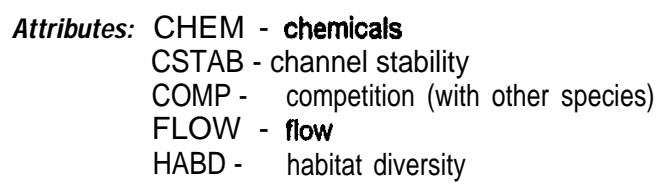

NUTLD - nutrient load

OBST - obstructions

OXYG - dissolved oxygen

PATHO - pathogens

PRED - predators (includes fishing-related losses)
RIPCN - riparian condition SEDLD - sediment load

TEMP - water temperature

WiTH - water withdrawals

"NA" indicates that no life history pathway meeting the criteria used in defining these pathways was present.

Blank cells under attributes indicate no additional attributes with negative effects on productivity were evident compared to those present in the template. 
Stream Reach Summary for Primary and Secondary Spring Chinook Salmon Life Histories

Stream: Big Sheep Cr.

Reach: Little Sheep $\mathrm{Cr}$ - Squaw $\mathrm{Cr}$ (RM 25.4 -37.7 from Snake R)

\begin{tabular}{|c|c|c|c|c|c|c|c|c|c|c|c|c|c|c|}
\hline \multicolumn{4}{|c|}{ Restoration benefit category: } & & \multicolumn{3}{|c|}{ Chinook usage score: } & 86.1 & \multicolumn{4}{|c|}{ Habitat productivity loss score: } & -114.3 & \multirow{4}{*}{$\begin{array}{c}\text { Habitat } \\
\text { productivity } \\
\text { loss score }\end{array}$} \\
\hline \multirow[b]{3}{*}{ Life stage } & \multirow[b]{3}{*}{ Months } & \multicolumn{10}{|c|}{ No. life history pathways by current usage abundance level } & \multicolumn{2}{|c|}{ Key habital change } & \\
\hline & & \multicolumn{5}{|c|}{ Primary } & \multicolumn{5}{|c|}{ Secondary } & \multirow{2}{*}{$\begin{array}{l}\text { Patient } \\
\text { quantity }\end{array}$} & \multirow[b]{2}{*}{$\%$ change } & \\
\hline & & Total no. & 1 & 2 & 3 & 4 & Total no. & 1 & 2 & 3 & 4 & & & \\
\hline & Apr-Aug & 11 & 11 & 0 & 0 & 0 & 26 & 26 & 0 & 0 & 0 & 11.8 & -13.7 & -41.7 \\
\hline $1,2,3$ & Aug-Ött & NA" & $\mathrm{NÄ}$ & $\mathrm{NA}$ & $\mathrm{NA}$ & $\mathrm{NA}$ & $\mathrm{NA}$ & $\mathrm{NA}$ & $N A$ & $N A$ & $\mathrm{NA}$ & $N A$ & $\mathrm{NA}$ & NA \\
\hline 3 & Ott-Mar & NA & NA & NA & NA & & NA & $N$ & $A$ & $\mathrm{NA}$ & NA & NA & $\mathrm{NA}$ & $\mathrm{NA}$ \\
\hline 34 & Mar-May & NA & NA & $\mathrm{NA}$ & $\mathrm{NA}$ & & NA & $\mathrm{NA}$ & $\sqrt{A}$ & NA & NA & NA & $\ddot{N A}$ & $\mathrm{NA}$ \\
\hline 5 & May-Ott & 0 & 0 & 0 & 0 & $\ddot{0}$ & 10 & 10 & 0 & $0^{\circ}$ & 0 & 57 & 0.0 & -119 \\
\hline 6 & Ött-Mar & 0 & 0 & $0 "$ & 0 & 0 & 37 & 37 & 0 & 0 & 0 & 15.6 & 0.0 & -663 \\
\hline 7 & Mar-jun & 6 & 6 & 0 & 0 & $\dot{0}$ & 4 & $4^{\circ}$ & 0 & 0 & 0 & 27.2 & 0.0 & 5.6 \\
\hline
\end{tabular}

\begin{tabular}{|c|c|c|c|c|c|c|c|c|c|c|c|c|c|}
\hline \multirow[b]{2}{*}{ Life stage } & \multirow[b]{2}{*}{ Months } & \multicolumn{12}{|c|}{ Highest productivity impact attributes (\% change in impact from template) } \\
\hline & & $\overline{\text { Aft } 1}$ & $\%$ change & Atf 2 & $\%$ change & Att 3 & $\%$ change & Att 4 & $\%$ change & Att 5 & $\%$ change & Att 6 & \% change \\
\hline 1 & Apr-Aug & FLOW & 23.1 & RIPCN & 23.1 & SEDLD & 23.1 & TEMP & 23,1 & & & & \\
\hline $1,2,3$ & Äug-Oct & NA & $\dot{N} \dot{A}$ & NA & NA & $\mathrm{NA}$ & NA & NA & $\mathrm{NA}$ & $\mathrm{NA}$ & $\mathrm{NÄ}$ & $N \dddot{A}$ & N̈A \\
\hline 3 & Ött-Mar & NÄ & $\mathrm{NA}$ & $\mathrm{NA}$ & $\mathrm{NA}$ & $\mathrm{NA}$ & NÄ & $\mathrm{NÄ}$ & $\mathrm{NA}$ & NÄ & $\mathrm{NA}$ & "NA" & NA \\
\hline 3,4 & Mar-May & $\dot{N} \dot{A}$ & NA & NA & NA & NA & NA & NA & NA & $N A$ & $\mathrm{NA}$ & $\mathrm{NÄ}$ & NA \\
\hline 5 & May-Ott & CSTAB & 11.0 & FLÖW & 11.0 & "PATHO' & $11.0^{\prime}$ & TÉMMP & 11.0 & WITHH" & 11.0 & & \\
\hline 6 & Ott-Mar & "ĆṠ̇̇̇̈' & 16.1 & "F́L̈ÖẄ & 16.1 & "PATHO & 16.1 & "TEMË'" & 161 & "พ̈IT̈H"' & 161 & & \\
\hline 7 & Mar-Jun & 'WITH' & "11.1" & & & & & & & & & & \\
\hline
\end{tabular}

Abundance lovel refers to the average abundance of spawners that spawn in the natal stream reaches that populate the life history pathways using the reach in this table $\begin{array}{ll}\text { Level 1: } & 0-10 \text { spawners } \\ \text { Level 2 } & 10-30 \text { spawners } \\ \text { Level 3: } & 30-60 \text { spawners }\end{array}$

Level 4: $\quad>60$ spawners

Life stages: 1-prespawner; 2-spawner; 3- incubation; 4- fry colonization; 5- summer rearing; 6- overwintering; 7- yearling presmolt/smolt

\begin{tabular}{|c|c|c|c|}
\hline $\begin{aligned} \text { Attributes.' } & \text { CHEM - chemicals } \\
& \text { CSTAB - channel stability } \\
& \text { COMP - competition (with other species) } \\
& \text { FLOW - ftOw } \\
& \text { HABD - habitat diversity }\end{aligned}$ & $\begin{array}{l}\text { NUTLD - } \text { nutrient ioad } \\
\text { OBST - obstructions } \\
\text { O)(YG - dissolved oxygen } \\
\text { PATHO - pathogens } \\
\text { PRED - predators (includes fishing-related losses) }\end{array}$ & $\begin{array}{l}\text { RIPCN - } \\
\text { SEDLD - } \\
\text { TEMP - } \\
\text { WITH - }\end{array}$ & $\begin{array}{l}\text { riparian condition } \\
\text { sediment load } \\
\text { water temperature } \\
\text { water withdrawals }\end{array}$ \\
\hline
\end{tabular}

"NA" indicates that no life history pathway meeting the criteria used in defining these pathways was present.

Blank cells under attribute indicate no additional attributes with negative effects on productivity were evident compared to those present in the template. 


\section{Stream Reach Summary for Primary and Secondary Spring Chinook Salmon Life Histories}

Stream: Big Sheep Cr.

Reach: Squaw $\mathrm{Cr}$ - Marr Cr (RM 37.7 -39.3 from Snake R)

\begin{tabular}{|ll|ll|lll}
\hline Restoratlon benefit category: & F & Chinook usage score: & 27.7 & Habitat productivity loss score: & -24.4 \\
\hline
\end{tabular}

\begin{tabular}{|c|c|c|c|c|c|c|c|c|c|c|c|c|c|c|}
\hline \multirow[b]{3}{*}{ Life stage } & \multirow[b]{3}{*}{ Months } & \multicolumn{10}{|c|}{ No. life history pathways by current usage abundance level } & \multicolumn{2}{|c|}{ Key habitat change } & \multirow{3}{*}{$\begin{array}{c}\text { Habitat } \\
\text { productivity } \\
10 \text { ss score }\end{array}$} \\
\hline & & \multicolumn{5}{|c|}{ Primary } & \multicolumn{5}{|c|}{ Secondary } & \multirow{2}{*}{$\begin{array}{l}\text { Pattènt } \\
\text { quantity }\end{array}$} & \multirow[b]{2}{*}{$\%$ change } & \\
\hline & & Total no. & 1 & 2 & 3 & 4 & Total no. & 1 & 2 & 3 & $\overline{4}$ & & & \\
\hline 1 & Apr-Aug & 5 & 5 & 0 & 0 & 0 & 12 & 12 & $n$ & 0 & 0 & 6.3 & -8.7 & -14.4 \\
\hline $1,2,3$ & Aug-Oct & NÄ & NA & $\mathrm{NA}$ & $\mathrm{NA}$ & NA & $1 \ldots$ NA & Nä & NAN & "NA" & $\mathrm{NA}$ & $\mathrm{NA}$ & $\mathrm{NA}$ & $\mathrm{NA}$ \\
\hline 3 & Oct-Mar & NA & NA & NA & NA & $\mathrm{NA}$ & NA & $\mathrm{NA}$ & NA & NA & NA & $N A$ & NA & NÄ \\
\hline 34 & Mar-May & NA & NA & NA & NA & $\mathrm{NA}$ & NA & $\mathrm{NA}$ & NA & NA & $\mathrm{NA}$ & $\dot{N A}$ & NA & NA \\
\hline 5 & May-Oct & 0 & 0 & 0. & 0 & 0 & 10 & 10 & 0 & 0 & 0 & 3.0 & 0.0 & -8.9 \\
\hline 6 & Oct-Mar & 0 & 0 & 0 & 0 & 0 & 1 & $i$ & 0 & 0 & 0 & 7.5 & 00 & -19 \\
\hline 7 & Mar-atth & 5 & 5 & 0 & "ח & $n$ & 1 & 1 & 0 & . & n & 13.2 & no & 0.8 \\
\hline
\end{tabular}

\begin{tabular}{|c|c|c|c|c|c|c|c|c|c|c|c|c|c|}
\hline \multirow[b]{2}{*}{ Life stage } & \multirow[b]{2}{*}{ Months } & \multicolumn{12}{|c|}{ Highest productivity impact attributes (\% change in impact from template) } \\
\hline & & $\overline{\text { Atf } 1}$ & $\%$ change & $\overline{\text { Att } 2}$ & $\%$ change & Att 3 & $\%$ change & Aft 4 & $\%$ change & Att 5 & $\%$ change & Att 6 & $\%$ change \\
\hline 1 & Apr-Aug & FLOW & 17.4 & RIPCN & 174 & SEDLD & 17.4 & TEMP & $\overline{17.4}$ & & & & \\
\hline $1,2,3$ & Äug-Oct & $\mathrm{NA}$ & "NÄ̈ & $\mathrm{N}$ & $\dddot{\mathrm{N}} \mathrm{O}$ & "NÄ & NA & NA & NÄ & $\dot{\mathrm{NA}}$ & $\dot{N} A$ & NÄ" & \\
\hline 3 & Ött-Mar & NA & $\dot{\mathrm{N}} \dot{\mathrm{A}}$ & NA & NA & NA & "NÄ & NÄ & NA & NÄ & $\mathrm{NÄ}$ & $\mathrm{NÄ̈}$ & $\dddot{\mathrm{NA}}$ \\
\hline 3,4 & Mar-May & NA & NA & $\mathrm{NA}$ & N̈̈̈ & $\mathrm{NA}$ & $\mathrm{NÄ}$ & $\mathrm{NÄ}$ & NA & $\mathrm{NA}^{\circ}$ & NA & NA & $\dot{N A}$ \\
\hline $5^{\prime \prime \prime \prime}$ & May-Oct & "TEMP & 20.8 & COMP & 10.4 & $\mathrm{HABD}$ & 10.4 & PATHO & 10.4 & SEDLD & 10.4 & & \\
\hline 6 & Ött-Mar & CSTAB & 25.0 & "FLÖW" & 25.0 & PÄTHÖ & $25.0^{\circ}$ & ṪEMPं & 25.0 & "Wiтн & 25.0 & & \\
\hline 7 & Mar-Jun & WITH & 11.6 & & & & & & & & & & \\
\hline
\end{tabular}

Abundance/eve/refers to the average abundance of spawners that spawn in the natal stream reaches that populate the life history pathways using the reach in this table Level 1: $0-10$ spawners

Level 2 10-30 spawners

Level 3: $\quad 30-60$ spawners

Level $4>60$ spawners

fife stages: 1- prespawner; 2- spawner; 3- incubation; 4- fry colonization; 5- summer rearing; 6-overwintering; 7-yearling presmolt/smolt

Attributes: CHEM - chemicals

CSTAB - channel stability

COMP - competition (with other species)

FLOW - flow

HABD - habitat diversify
NUTLD - nutrient load

OBST - obstructions

OXYG - dissolved oxygen

PATHO - pathogens

PRED - predators (includes fishing-related losses)
RIPCN - riparian condition

SEDLD - sediment load

TEMP - water temperature

WITH - water withdrawals

"NA" indicates that no tife history pathway meeting the criteria used in defining these pathways was present.

Blank cells under attributes indicate no additional attributes with negative effects on productivity were evident compared to those present in the template. 


\section{Stream Reach Summary for Primary and Secondary Spring Chinook Salmon Life Histories}

Stream: Big Sheep Cr.

Reach: $\quad$ Marr $\mathrm{Cr}$ - Griffith $\mathrm{Cr}$ (RM $39.3-45.3$ from Snake R)

\begin{tabular}{|ll|ll|l|}
\hline Restoration benefit category: & D & Chinook usage score: & 145.9 & Habitat productivity loss score: \\
\hline
\end{tabular}

\begin{tabular}{|c|c|c|c|c|c|c|c|c|c|c|c|c|c|c|}
\hline \multirow[b]{3}{*}{ Life stage } & \multirow[b]{3}{*}{ Months } & \multicolumn{10}{|c|}{ No. life history pathways by current usage abundance level } & \multicolumn{2}{|c|}{ Key habitat change } & \multirow{3}{*}{$\begin{array}{c}\text { Habitat } \\
\text { productivity } \\
\text { toss score }\end{array}$} \\
\hline & & \multicolumn{5}{|c|}{ Primary } & \multicolumn{5}{|c|}{ Secondary } & \multirow{2}{*}{$\begin{array}{l}\text { Patient } \\
\text { quantity }\end{array}$} & \multirow[b]{2}{*}{$\%$ change } & \\
\hline & & Total no. & 1 & 2 & $\overline{3}$ & 4 & Total no. & $T$ & 2 & 3 & 4 & & & \\
\hline & Apr-Aug & 5 & 5. & 0 & 0 & 0 & 12 & 12 & 0 & $\overline{0}$ & 0 & 7.2 & -7.2 & -119 \\
\hline 123 & Aug-Ött & NA & $\mathrm{NA}$ & $\mathrm{NA}$ & $\mathrm{NA}$ & NA & 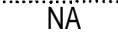 & NÄ & $\mathrm{N} A$ & "ัNÄ" & 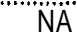 & 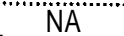 & $\mathrm{NÄ}$ & $\mathrm{NA}$ \\
\hline 3 & Ott-Mar & $\dot{\mathrm{NA}}$ & $\ddot{N} A$ & NA & NA & NA & NA & NA & NA & NA & NA & $\ddot{N} \ddot{N}$ & N̈A & N̈̈̈ \\
\hline 34 & Mar-May & 4 & 4 & 0 & “" '0 " & 0 & 16 & 16 & 0 & 0 & 0 & 1.4 & -418 & -680 \\
\hline 5 & May,-O,tt, & 25 & 25 & 0 & 0 & 0 & 102 & 102 & 0 & 0 & 0 & 4.7 & -5.7 & -177.6 \\
\hline 6 & Ott-Mar & 19 & 19 & 0 & 0 & 0 & 19 & 19 & 0 & 0 & 0 & 92 & -16 & -66.8 \\
\hline 7 & Mar-Jun & 5 & 5 & 0 & 0 & 0 & 1 & 1 & 0 & 0 & 0 & 152 & -3.4 & 08 \\
\hline
\end{tabular}

\begin{tabular}{|c|c|c|c|c|c|c|c|c|c|c|c|c|c|}
\hline \multirow[b]{2}{*}{ Life stage } & \multirow[b]{2}{*}{ Months } & \multicolumn{12}{|c|}{ Highest productivity impact attributes (\% change in impact from template) } \\
\hline & & Att 1 & $\%$ change & Att 2 & $\%$ change & At! 3 & $\%$ change & Aft 4 & $\%$ change & An 5 & $\%$ change & Att 6 & \% change \\
\hline 1 & Apr-Aug & FLOW & 14.4 & RIPCN & 14.4 & SEDLD & 14.4 & TEMP & 14.4 & & & & \\
\hline $1, \dot{2} \dot{3}$ & Aug-Öct & "NA' & "NÄ & NA & NA & & "NÄ & NA & $\dot{N A}$ & NÄ & NA & & NÄ \\
\hline 3 & Ött-Mar & $\mathrm{NA}$ & $\mathrm{NA}$ & NA" & NA & & NA & $\mathrm{NA}$ & $\mathrm{NA}$ & $\mathrm{NA}$ & NA & N̈A & NÄ' \\
\hline 3,4 & Mar-May & TEMP & 28.0 & CSTAB & 14.0 & HABD & 14.0. & PATHO & 14.0 & SEDLD & 14.0 & FLOW & 7.0 \\
\hline 5 & May-Ott & TEMP & 28.0 & $\mathrm{HABD}$ & 14.0 & PATHO & 14.0 & ŚEDLD & 14.0 & CSTAB & 103 & "F்ட்'ல்" & 103 \\
\hline 6 & Ott-Mar & CSTAB & 15.8 & FLow & 15.8 & ?ATHO & 15.8 & TEMP & 15. & WITH & 15.8 & & \\
\hline 7 & Mar-Jun & WITH & 11.0 & & & & & & & & & & \\
\hline
\end{tabular}

Abundance/eve/ refers to the average abundance of spawners that spawn in the natal stream reaches that populate the life history pathways using the reach in this table

$$
\begin{array}{ll}
\text { Level 1: } & 0-10 \text { spawners } \\
\text { Level 2: } & 10-30 \text { spawners } \\
\text { Level 3: } & 30-60 \text { spawners } \\
\text { Level 4: } & >60 \text { spawners }
\end{array}
$$

Life stages: 1- prespawner; 2- spawner; 3- incubation; 4- fry colonization; 5- summer rearing; 6- overwintering; 7- yearling presmolt/smolt

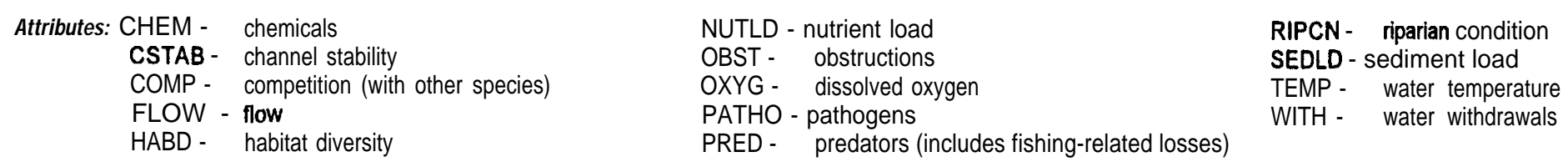

"NA" indicates that no life history pathway meeting the criteria used in defining these pathways was present.

Blank cells under attributes indicate no additional attributes with negative effects on productivity were evident compared to those present in the template. 


\section{Stream Reach Summary for Primary and Secondary Spring Chinook Salmon Life Histories}

Stream: Big Sheep $\mathrm{Cr}$.

Reach: Griffith $\mathrm{Cr}$ - Carrot $\mathrm{Cr}$ (RM $45.3-47,0$ from Snake R)

\section{\begin{tabular}{|ll|ll|ll|}
\hline Restoration benefit category: & $D$ & Chinook usage score: & 352.1 & Habitat productivity loss score: & -109.7 \\
\hline
\end{tabular}}

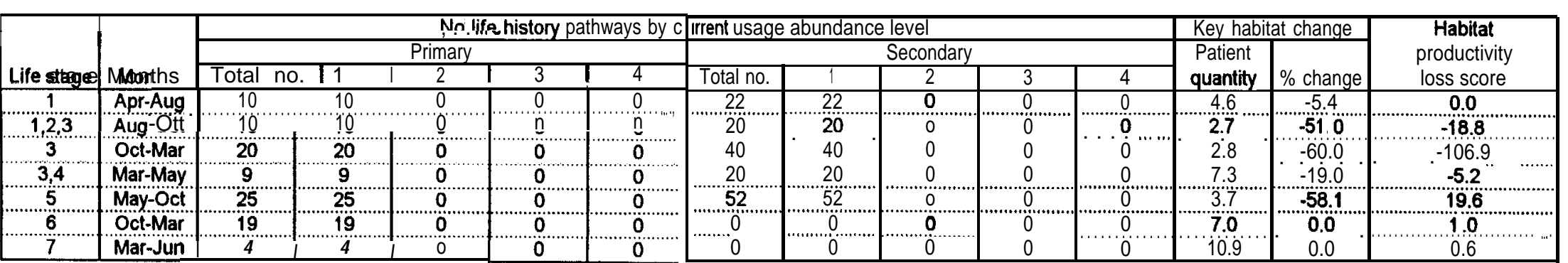

\begin{tabular}{|c|c|c|c|c|c|c|c|c|c|c|c|c|c|}
\hline \multirow[b]{2}{*}{ Life stage } & \multirow[b]{2}{*}{ Months } & \multicolumn{12}{|c|}{ Highest productivity impact attributes (\% change in impact from template) } \\
\hline & & $\overline{\text { Att } 1}$ & $\%$ change & Att 2 & $\%$ change & Att 3 & $\%$ change & Atf 4 & \% change & Att 5 & $\%$ change & Att 6 & \% change \\
\hline & Apr-Aug & SEDLD & 37.5 & FLOW & 19.2 & & & & & & & & \\
\hline $1,2,3$ & Aug-Öd & SEDLD & 50.0 & FEOOOW & 25.0 & & & & & & & & \\
\hline $3^{3}$ & OOtt-Mar & SEDLD & 50.0 & PATHOO & 25 & & & & & & & & \\
\hline 3,34 & Mar-May & SEDLD & 50,0 & FLOW & $25^{\circ}$ & PATHO & 25.0 & & & & & & \\
\hline 5 & May-Ött & FLOW & 23.7 & РАTHO & 23.7 & SEDLD & 23.7 & & "- & & & & \\
\hline 6 & Oct-Mar & COOMP & 25.0 & PATHOO & 250 & SEDLO & 250 & ........ & & & & & \\
\hline 7 & Mar-Jun & & & & & & & & & & & & \\
\hline
\end{tabular}

Abundance level refers to the average abundance of apawners that spawn in the natal stream reaches that populate the life history pathways using the reach In this table:

$$
\begin{array}{ll}
\text { Level 1: } & 0-10 \text { spawners } \\
\text { Level 2: } & 10-30 \text { apawners } \\
\text { Level 3: } & 30-60 \text { spawners } \\
\text { Level 4 } & >60 \text { spawners }
\end{array}
$$

Life stages: 1- prespawner; 2- spawner; 3- incubation; 4- fry colonization; 5- summer rearing; 6- overvvintering; 7- yearling presmoltsmolt

$\begin{array}{lll}\text { Attributes: CHEM - chemicals } & \text { NUTLD - nutrient load } & \text { RIPCN - riparian condition } \\ \text { CSTAB - channel stability } & \text { OBST - obstructions } & \text { SEDLD - sediment load } \\ \text { COMP - competition (with other species) } & \text { OXYG - dissolved oxygen } & \text { TEMP - water temperature } \\ \text { FLOW - flow } & \text { PATHO - pathogens } & \text { WITH - water withdrawals } \\ \text { HABD - habitat diversity } & \text { PRED - predators (includes fishing-related losses) }\end{array}$

"NA" indicates that no life history pathway meeting the criteria used in defining these pathways was present.

Blank cells under attributes indicate no additional attributes with negative effects on productivity were evident compared to those present in the template. 
Stream Reach Summary for Primary and Secondary Spring Chinook Salmon Life Histories

Stream: Big Sheep Cr.

Reach: Carrot $\mathrm{Cr}$ - Owt $\mathrm{Cr}$ (RM 47.0 -53.5 from Snake R)

\begin{tabular}{ll|llll|}
\hline Restoration benefit category: & D & Chinook usage score: & 353.3 & /Habitat productivity loss score: & -109.8 \\
\hline
\end{tabular}

\begin{tabular}{|c|c|c|c|c|c|c|c|c|c|c|c|c|c|c|}
\hline \multirow[b]{3}{*}{ Life stage } & \multirow[b]{3}{*}{ Months } & \multicolumn{10}{|c|}{ NO. Ire nistory pamways by current usage abundance level } & \multicolumn{2}{|c|}{ Key habitat change } & \multirow{3}{*}{$\begin{array}{c}\text { Habitat } \\
\text { productivity } \\
\text { loss score }\end{array}$} \\
\hline & & \multicolumn{5}{|c|}{ Prima-u } & \multicolumn{5}{|c|}{ Secondary } & Patient & & \\
\hline & & Total no. & 1 & 2 & 3 & 4 & Total no. & 1 & 2 & 3 & 4 & quantity & $\%$ change & \\
\hline & Apr-Aug & 9 & 9 & 0 & 0 & 0 & 20 & 20 & 0 & 0 & 0 & 4.4 & -13.4 & 0.0 \\
\hline $1,2,3$ & Aug-Ött & 10 & 10 & 0 & 0 & 0 & 20 & 20 & $\ddot{0}$ & 0 & 0 & 3.2 & .51 .0 & -18.8 \\
\hline 3 & Ott-Mar & 20 & 20 & 0 & . & 0 & 40 & 40 & 0 & 0 & 0. & 3.3 & -600 & -106.9 \\
\hline 34 & Mar-May & 9 & 9 & 0 & 0 & 0 & 20 & $m$ & 0 & $\underline{0}$ & 0 & 7.5 & -19.11 & -5.52 \\
\hline 5 & May-Oct & 25 & 25 & 0 & 0 & 0 & $50^{\circ}$ & 50 & 0 & 0 & 0 & 3.9 & $-61.0^{\circ}$ & 19.6 \\
\hline 6 & Oct-Mar & 19 & 19 & 0 & 0 & 0 & 0 & 0 & 0 & 0 & 0 & 8.3 & 0.0 & 1.0 \\
\hline 7 & Mar-Jun & 3 & 3 & 0 & 0 & 0 & 0 & 0 & 0 & 0 & 0 & 12.7 & 0.0 & 0.5 \\
\hline
\end{tabular}

\begin{tabular}{|c|c|c|c|c|c|c|c|c|c|c|c|c|c|}
\hline \multirow[b]{2}{*}{ Life stage } & \multirow[b]{2}{*}{ Months } & \multicolumn{12}{|c|}{ Highest productivity impact attributes ( $\%$ change in impact from template) } \\
\hline & & Att 1 & $\%$ change & Att 2 & $\%$ change & Att 3 & $\%$ change & Att 4 & $\%$ change & Att 5 & $\%$ change & Att 6 & $\%$ change \\
\hline & Apr-Aug & FLOW & 23.2 & SEDLD & 23.2 & & & & & & & & \\
\hline 123 & Aug-Ött & SEEEELE" & $50.0 " \cdots \cdots$ & FLOOWW" & 250 & & & & & & & & \\
\hline 3 & Ott-Mar & SEEOEZD" & $50.0^{\circ}$ & PATHOO & 125 & & & & …………, & & & & \\
\hline 3,4 & Mar-May & FEOOWW & $250^{\circ}$ & PATHOO & $25.0^{\circ}$ & 'SEEELLD & $\underline{200} 0$ & & …" & & & & \\
\hline 5 & May-Ott & FLOW & 25.0 & PÄTHO & 25.0 & SEDLD & 25.0 & COMP & 16.3 & & & & \\
\hline 6 & Ott-Mar & COMP & $25.0^{\circ}$ & PATHO & 250 & SEDLD & 25.0 & & & & & & \\
\hline 7 & 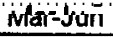 & & & & & & & & & & & & \\
\hline
\end{tabular}

Abundance level refers to the average abundance of spawners that spawn in the natal stream reaches that populate the life history pathways using the reach in this table:

$$
\begin{array}{ll}
\text { Level 1: } & 0-10 \text { spawners } \\
\text { Level 2: } & 10-30 \text { spawners } \\
\text { Level 3: } & 30-60 \text { spawners } \\
\text { Level } 4 & >60 \text { spawners }
\end{array}
$$

Life stages: 1- prespawner; 2-spawner; 3- incubation; 4- fry colonization; 5- summer rearing; 6-overwintering; 7-yearling presmolt/smolt
Attributes: CHEM - chemicals
CSTAB - channel stability
COMP - competition (with other species)
FLOW - fiOW
HABD - habitat diversity

NUTLD - nutrient load

OBST - obstructions

OXYG - dissolved oxygen

PATHO - pathogens

PRED - predators (includes fishing-related losses)

\section{RIPCN - riparian condition \\ SEDLD - sediment load \\ TEMP - water temperature \\ WITH - water withdrawals}

"NA" indicates that no life history pathway meeting the criteria used in defining these pathways was present.

Blank cells under attributes indicate no additional attributes with negative effects on productivity were evident compared to those present in the template. 
Stream Reach Summary for Primary and Secondary Spring Chinook Salmon Life Historles

Stream: Big Sheep Cr.

Reach: Owl Cr - Lick Cr (RM 53.5 -57.4 from Snake R)

\begin{tabular}{ll|ll|ll|}
\hline Restoration benefit category: & B & Chinook usage score: & 426.9 & Habitat productivity ioss score: & -231.4 \\
\hline
\end{tabular}

\begin{tabular}{|c|c|c|c|c|c|c|c|c|c|c|c|c|c|c|}
\hline \multirow[b]{3}{*}{ Life stage } & \multirow[b]{3}{*}{ Months } & \multicolumn{10}{|c|}{ No. life history pathways by current usage abundance level } & \multicolumn{2}{|c|}{ Key habitat change } & \multirow{3}{*}{$\begin{array}{c}\text { Habitat } \\
\text { productivity } \\
\text { loss score }\end{array}$} \\
\hline & & \multicolumn{5}{|c|}{ Primary } & \multicolumn{5}{|c|}{ Secondary } & \multirow{2}{*}{$\begin{array}{l}\text { Patient } \\
\text { quantity }\end{array}$} & \multirow[b]{2}{*}{ \% change } & \\
\hline & & Total no. & 1 & 2 & 3 & 4 & \begin{tabular}{|l} 
Total no. \\
\end{tabular} & 1 & 2 & 3 & 4 & & & \\
\hline 1 & Apr-Aug & $8 \ldots$ & 6 & 0 & 0 & $\overline{0}$ & 28 & 28 & 0 & 0 & 0 & 2.7 & -14.0 & 00 \\
\hline $1,2,3$ & Aug-Oct & $100^{\circ}$ & to & $\dddot{0}$ & 0 & 0 & 40 & 40 & 0 . & "i & 0 & 2.3 & -52.4 & -314 \\
\hline 3,4 & Mar-May & 9 " & 9 & 0 & $0 “$ & " 0 & $22^{\prime}$ & 22 & '0 & 0 & 0 & 5.2 & -220 & -77 \\
\hline 5 & May-Ot & 25 & 25 & $\dot{0}$ & 0 & 0 & $48^{\circ}$ & 48 & 0 & 0 & 0 & 26 & -62.0 & -15.5 \\
\hline 6 & Ott twat & 19 & 19 & 0 & 0 & 0 & 0 & 0 & 0 & 0 & 0 & 5.8 & 0.0 & 10 \\
\hline 7 & Mar-Jun & 2 & 2 & 0 & 0 & 0 & 0 & 0 & 0 & 0 & 0 & $9.1^{\circ}$ & 0.0 & 0 \\
\hline
\end{tabular}

\begin{tabular}{|c|c|c|c|c|c|c|c|c|c|c|c|c|c|}
\hline \multirow[b]{2}{*}{ Liftestagec } & \multirow[b]{2}{*}{ Mounthes } & \multicolumn{12}{|c|}{ Highest productivity impact attributes (\% change in impact from lemplate) } \\
\hline & & Att 1 & \% change & Att 2 & \% change & Att 3 & \% change & Att 4 & \% change & Att 5 & $\%$ change & Att 6 & \% change \\
\hline 1 & $\overline{\text { Apr-Aug }}$ & SEDLD & 37.5 & FLOW & 21.8 & & & & & & & & \\
\hline 123 & Aug=Ö́t & SEDLD & 50.0 & FLOW & 25.0 & & & & & & & & \\
\hline 3 & Oth What & SEDLD & 50.0 & PATHO & 25.0 & & & & & & & & \\
\hline 3,4 & Mar-May & SEDLD & 50.0 & FLOW & 25.0 & PATHO & 25.0 & & & & & & \\
\hline 5 & May-out & FLOW & 24.6 & PATHO & 24.6 & SEDLD & 24.6 & & & & & & \\
\hline 6 & Ode- Whar & COMP & 25.0 & PATHO & 25.0 & SEDLD & 25.0 & & & & & & \\
\hline 7 & Mar = lium & & & & & & & & & & & & \\
\hline
\end{tabular}

Abundance /eve/ refers to the average abundance of spawners thst spawn in the natal stream reaches that populate the life history pathways using the reach in this table:

Level 1: $\quad 0-10$ spawners

Levei 2: $\quad 10-30$ spawners

Levei 3: $\quad 30-60$ spawners

Level 4: $\quad>60$ spawners

Life stages.' 1- prespawner; 2- spawner; 3- incubation; 4- fry colonization; 5- summer rearing; 6- overwintering; 7- yearling presmolt/smolt

$\begin{array}{lll}\text { Attributes: } \text { CHEM - chemicals } & \text { NUTLD - nutrient ioad } & \text { RIPCN - riparian condition } \\ \text { CSTAB - channel stability } & \text { OBST - obstruct ions } & \text { SEDLD - Sediment ioad } \\ \text { COMP - competition (with other species) } & \text { OXYG - dissolved oxygen } & \text { TEMP - water temperature } \\ \text { FLOW - fiOW } & \text { PATHO - pathogens } & \text { WiTH - water withdraws } \\ \text { HABD - habitat diversity } & \text { PRED - predators (includes fishing-related losses) }\end{array}$

"NA" indicates that no life history pathway meeting the criteria used in defining these pathways was present.

Blank cells under attributes indicate no additional attributes with negative effects on productivity were evident compared to those present in thetemplate 


\section{Stream Reach Summary for Primary and Secondary Spring Chinook Salmon Life Histories}

Stream: Big Sheep Cr.

Reach: $\quad$ Lick Cr - South Fork (RM 57.4 -58.9 from Snake R)

\begin{tabular}{|c|c|c|c|c|c|}
\hline Restoration benefit category: & $\mathrm{B}$ & Thlnook usage score: & 497.7 & Habitat productivity loss score: & -308.0 \\
\hline
\end{tabular}

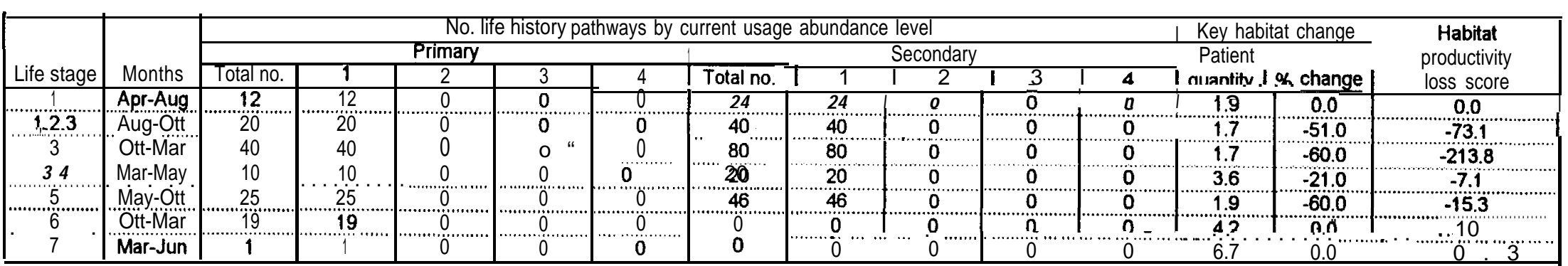

\begin{tabular}{|c|c|c|c|c|c|c|c|c|c|c|c|c|c|}
\hline \multirow[b]{2}{*}{ Life stage } & \multirow[b]{2}{*}{ Months } & \multicolumn{12}{|c|}{ Highest productivity impact attributes (\% change in impact from template) } \\
\hline & & Aft 1 & $\%$ change & Alt 2 & $\%$ change & Alt 3 & \% change & Att 4 & \% change & Att 5 & $\%$ change & Att 6 & $\%$ change \\
\hline 1 & Apr-Aug & FLOW & 25.0 & SEDLD & 25.0 & & & & & & & & \\
\hline $1,2,3$ & Aug-Od & SEDLD & 50.0 & FLOW & 25.0 & & & & & & & & \\
\hline 3 & Oct-Mar & SEOLD & 50.0 & PATHO & 12.5 & & & & & & & & ............... \\
\hline 3,4 & Mar-May & FLOW & 25.0 & PATHO & 25.0 & SEDID & 25.0 & & & & & & \\
\hline 5 & May-Oct & COMP & 25.0 & FLOW & 25.0 & PATHO & 25.0 & SEDLD & 25.0 & & & & \\
\hline 6 & Ocl-Mar & COMP & 25.0 & РATHO & 25.0 & SEDLD & 250 & & & & & & \\
\hline 7 & Mar-Jun & & & & & & & & & & & & \\
\hline
\end{tabular}

Abundance /eve/ refers to the average abundance of spawners that spawn in the natal stream reaches that populate the life history pathways using the reach in this table:

$$
\begin{array}{ll}
\text { Level 1: } & 0-10 \text { spawners } \\
\text { Level 2: } & 10-30 \text { spawners } \\
\text { Level 3: } & 30-60 \text { spawners } \\
\text { Level 4 } & >60 \text { spawners }
\end{array}
$$

Life stages.' 1- prespawner; 2- spawner; 3- incubation; 4- fry colonization; 5- summer rearing; 6- overwintering; 7- yearling presmolt/smolt

$\begin{array}{lll}\text { Attributes: CHEM - chemicals } & \text { NUTLD - nutrient load } & \text { RIPCN - riparian condition } \\ \text { CSTAB - channel stability } & \text { OBST - obstructions } & \text { SEDLD - Sediment load } \\ \text { COMP - competition (with other species) } & \text { OXYG - dissolved oxygen } & \text { TEMP - water temperature } \\ \text { FLOW - ftOW } & \text { PATHO - pathogens } & \text { WITH - water withdrawals } \\ \text { HABD - habitat diversity } & \text { PRED - predators (includes fishing-related losses) }\end{array}$

"NA" indicates that no life history pathway meeting the criteria used in defining these pathways was present.

Blank cells under attributea indicate no additional attributes with negative effects on productivity were evident compared to those present in the template. 


\section{Stream Reach Summary for Primary and Secondary Spring Chinook Salmon Life Historles}

Stream: Imnaha R

Reach: Mouth - Cow $\mathrm{Cr}(\mathrm{RM}$ 0.0- 4,3 from Snake R)

\begin{tabular}{ll|ll|lll}
\hline Restoration benefit category: & F & Chinook usage score: & 132.1 & Habltat productivity loss score: & 133.8 \\
\hline
\end{tabular}

\begin{tabular}{|c|c|c|c|c|c|c|c|c|c|c|c|c|c|c|}
\hline \multirow[b]{3}{*}{ Life stage } & \multirow[b]{3}{*}{ Months } & \multicolumn{10}{|c|}{ No. life history pathways by current usage abundance level } & \multicolumn{2}{|c|}{ Key habitat uirainge } & \multirow{3}{*}{$\begin{array}{l}\text { Habittat } \\
\text { prodi!rtivity } \\
\text { loss score }\end{array}$} \\
\hline & & \multicolumn{5}{|c|}{ Primary } & \multicolumn{5}{|c|}{ Secondar! } & Eationt & & \\
\hline & & Total no. & 1 & 2 & 3 & 4 & Total no. & 1 & 2 & 3 & 4 & quaintury & \% chañge & \\
\hline 1 & Apr-Aug & 35 & 25 & 6 & 4 & 0 & 91 & 63 & 16 & 12 & 0 & 14.6 & 0.0 & 136.8 \\
\hline $1,2,3$ & Aug-Oct & NA & NA & NA & NÄ & NÄ & NA & NÄ & NA & NÄ & NÄ & $\mathrm{NA}$ & $\mathrm{NA}$ & NA \\
\hline 3,4 & Mar-May & $\mathrm{NA}$ & $\mathrm{NA}$ & NA & NA & $\mathrm{NA}$ & NA & NA & NÄ & NA & $\mathrm{NA}$ & $\mathrm{NA}$ & NA & $\mathrm{NA}$ \\
\hline 5 & May-Oct & 0 & 0 & 0 & 0 & 0 & 11 & 6 & 3 & 2 & 0 & 9.8 & 0.0 & -3.0 \\
\hline 6 & Oct-Mar & NA & NÄ & $\mathrm{NA}$ & $\mathrm{NA}$ & NA & $\mathrm{NA}$ & NA & NA & NA & NÄ & $\mathrm{NA}$ & $\mathrm{NA}$ & NÄ \\
\hline 7 & Mar-run & 32 & 21 & 7 & 4 & 0 & 132 & 93 & 211 & 18 & 0 & 45.9 & 0.0 & 0 \\
\hline
\end{tabular}

\begin{tabular}{|c|c|c|c|c|c|c|c|c|c|c|c|c|c|}
\hline \multirow[b]{2}{*}{ Life stage } & \multirow[b]{2}{*}{ Months } & \multicolumn{12}{|c|}{ Highest productivity impact attributes (\% change in impact from template) } \\
\hline & & Att 1 & $\%$ change & Att 2 & $\%$ change & Att 3 & $\%$ change & Att 4 & $\%$ change & Att 5 & $\%$ change & Att 6 & $\%$ change \\
\hline & Apr-Aug & PATHO & 8.6 & & & & & & & & & & \\
\hline 123 & Aug-OCi & NA & NA & NA & $\mathrm{NÄ}$ & $\mathrm{NA}$ & N̈̈̈ & $\mathrm{Nä"}$ & $\mathrm{NA}$ & NA & NÄ & NÄ & $N A \ddot{A}$ \\
\hline 3 & Ött-Mar & N̈A & $\mathrm{NA}$ & $\mathrm{N}^{\mathrm{NA}}$ & "Nä & $\mathrm{NA}$ & N̈̈̈ & $\mathrm{NA}$ & $\mathrm{NA}$ & $\mathrm{NÄ}$ & $\mathrm{NÄ}$ & $\mathrm{NA}$ & $\mathrm{NA}$ \\
\hline & Mar-May & NA & $\mathrm{NA}$ & & NA' & $\mathrm{NA}$ & & NA & & NA & $\mathrm{NA}$ & $\mathrm{NÄ}$ & N̈Ä" \\
\hline 34 & May-Ott' & PATHO & 13.3 & ** & & & & & $A$ & & & & \\
\hline 6 & Ott-Mar & $\dot{N} \dot{A}$ & NA & $\mathrm{NA}$ & NA" & $\mathrm{NA}$ & $\mathrm{NA}$ & NA & $\mathrm{NA}$ & NA & $\mathrm{NA}$ & $\mathrm{NA}$ & $\mathrm{NÄ}$ \\
\hline$"$ & Mar-Jun & "PRËËD” & 7,4 & & & & & & & & & & \\
\hline
\end{tabular}

Abundance /eve/ refers to the average abundance of spawners that spawn in the natal stream reaches that populate the life history pathways using the reach in this table:

$$
\begin{array}{ll}
\text { Level 1: } & 0-10 \text { spawners } \\
\text { Level 2: } & 10-30 \text { spawners } \\
\text { Level 3: } & 30-60 \text { spawners } \\
\text { Level } 4 & >60 \text { spawners }
\end{array}
$$

Life stages: 1- prespawner; 2- spawner; 3- incubation; 4- fry colonization; 5- summer rearing; 6- overwintering; 7- yearling presmolt/smelt

$\begin{array}{lll}\text { Attributes: } \text { CHEM - chemicals } & \text { NUTLD - nutrient load } & \text { RIPCN - riparian condition } \\ \text { CSTAB - channel stability } & \text { OBST - obstructions } & \text { SEDLD - sediment load } \\ \text { COMP - competition (with other species) } & \text { OXYG - dissolved oxygen } & \text { TEMP - water temperature } \\ \text { FLOW - flow } & \text { PATHO - pathogens } & \text { WITH - water withdrawals } \\ \text { HABD - habitat diversity } & \text { PRED - predators (includes fishing-related losses) }\end{array}$

"NA" indicates that no life history pathway meeting the criteria used in defining these pathways was present.

Blank cells under attributes indicate no additional attributes with negative effects on productivity were evident compared to those present in the template. 


\section{Stream Reach Summary for Primary and Secondary Spring Chinook Salmon Life Histories}

Stream: Imnaha R.

Reach: Cow $\mathrm{Cr}$ - Lightning $\mathrm{Cr}$ (RM 4.3- 5.4 from Snake R)

\begin{tabular}{|llll|ll|}
\hline Restoration benefit category: & $F$ & Chinook usage score: & $\mathbf{1 7 3 . 0}$ & Habitat productivity ioss score: & 10.8 \\
\hline
\end{tabular}

\begin{tabular}{|c|c|c|c|c|c|c|c|c|c|c|c|c|c|c|}
\hline \multirow[b]{3}{*}{ Life stage } & \multirow[b]{3}{*}{ Months } & \multicolumn{10}{|c|}{ No. iife history pathways by current usage abundance level } & \multicolumn{2}{|c|}{ Key habitat change } & \multirow{3}{*}{$\begin{array}{c}\text { Habitat } \\
\text { productivity } \\
\text { loss score }\end{array}$} \\
\hline & & \multicolumn{5}{|c|}{ Primary } & \multicolumn{5}{|c|}{ Secondary } & Patient & & \\
\hline & & Total no. & 1 & 2 & 3 & 4 & Total no. & 1 & 2 & 3 & 4 & quanlity & $\%$ change & \\
\hline & Apr-Aug & 16 & 11 & 3 & 2 & 0 & 44 & 30 & 8 & 6 & 0 & 15.7 & 0.0 & 76.9 \\
\hline $123:=$ & Aug-Ött & NÄ" & $\mathrm{NA}$ & $\mathrm{NA}$ & $\mathrm{NA}$ & $\mathrm{NA}$ & $\mathrm{NA}$ & NA & $\mathrm{NA}$ & $\mathrm{NÄ}$ & $\mathrm{NA}$ & $\mathrm{NA}$ & $\mathrm{NA}$ & $\mathrm{NA}$ \\
\hline 34 & Mar-May & 0 & o. & $0 "$ & 0 & 0 & 4 & 4 & 10 & “ 0 ““ & 0 & 2.5 & -83.3 & -4.6 \\
\hline 5 & May-Ọtt & 0 & 0 & 0 & 0 & 0 & 24 & 21 & 2 & 1 & 0 & 12.6 & 0.0 & -14.6 \\
\hline 6 & Ott-Mar & 18 & 18 & 0 & 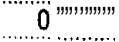 & 0 & 220 & 146 & 37 & 37 & 0 & 31.2 & 0.0 & -46.9 \\
\hline 7 & Mar-Jun & 10 & 11 & 3 & 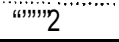 & 0 & 39 & 28 & 6 & 5 & $0^{\circ}$ & 52.4 & 0.0 & $0.0^{\prime}$ \\
\hline
\end{tabular}

\begin{tabular}{|c|c|c|c|c|c|c|c|c|c|c|c|c|c|}
\hline \multirow[b]{2}{*}{ Life stage } & \multirow[b]{2}{*}{ Months } & \multicolumn{12}{|c|}{ Highest productivity impact attributes (\% change in impact from $t_{e m} p_{a} i_{e}$ ) } \\
\hline & & Att 1 & $\%$ change & Att 2 & $\%$ change & Att 3 & $\%$ change & Att 4 & $\%$ change & Att 5 & $\%$ change & Att 6 & $\%$ change \\
\hline 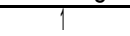 & Apr-Aug & PATHO & 110 & & & & & & & & & & \\
\hline $12,3,-$ & Aug-Ött & $\mathrm{NA}$ & $\mathrm{NÄ}$ & $\cdots, \ldots$ & NÄ & $\mathrm{NA}$ & $\mathrm{NA}$ & N̈Ä & $\mathrm{NA}$ & $\mathrm{NÄ}$ & $\mathrm{NÄ}$ & NÄ & $\mathrm{NA}$ \\
\hline $3 \ldots$ & Ott-Mar & NA & NA & NA & $\mathrm{NA}$ & NA & $\mathrm{NA}$ & Nin̈ & NA" & "̃̈̆ & חin" & No....... & $\mathrm{NA}$ \\
\hline $\begin{array}{c}3,4 \\
5\end{array}$ & $\begin{array}{l}\text { Mar-May } \\
\text { May-Ott }\end{array}$ & $\begin{array}{l}\text { PATHO } \\
\text { PATHO }\end{array}$ & $\begin{array}{l}25.0 \\
22.0\end{array}$ & "' & $\cdots$ & & & & & & & & \\
\hline 6 & Ott-Mar & PATHO & 70 & SEEDELE & 7.0 & PREED & 6.6 & & & ". & & & " \\
\hline$\dddot{7}$ & Mar-Jun & "P̈REËD" & 8.7 & & & & & & & & & & \\
\hline
\end{tabular}

Abundance/eve/refers to the average abundance of spawners that spawn in the natal stream reaches that populate the life history pathways using the reach in this table

$$
\begin{array}{ll}
\text { Level 1: } & 0-10 \text { spawners } \\
\text { Level 2: } & 10-30 \text { spawners } \\
\text { Level 3: } & 30-60 \text { spawners } \\
\text { Level 4: } & >60 \text { spawners }
\end{array}
$$

\begin{tabular}{|c|c|c|c|c|c|}
\hline $\begin{array}{r}\text { Attributes: } \\
\text { CHEM - } \\
\text { CSTAB - } \\
\text { COMP - } \\
\text { FLOW - } \\
\text { HABD - }\end{array}$ & $\begin{array}{l}\text { chemicals } \\
\text { channel stability } \\
\text { competition (with other species) } \\
\text { flow } \\
\text { habitat diversity }\end{array}$ & $\begin{array}{l}\text { NUTLD - } \\
\text { OBST - } \\
\text { OXYG - } \\
\text { PATHO - } \\
\text { PRED - }\end{array}$ & $\begin{array}{l}\text { nutrient load } \\
\text { obstructions } \\
\text { dissolved oxygen } \\
\text { pathogens } \\
\text { predators (includes fishing-reiated losses) }\end{array}$ & $\begin{array}{l}\text { RIPCN - } \\
\text { SEDLD - } \\
\text { TEMP - } \\
\text { WiTH - }\end{array}$ & $\begin{array}{l}\text { riparian condition } \\
\text { sediment load } \\
\text { water temperature } \\
\text { water withdrawals }\end{array}$ \\
\hline
\end{tabular}

Life stages.' 1- prespawner; 2- spawner; 3- incubation; 4- fry colonization; 5- summer rearing; 6- overwintering; 7-yearling presmolt/smolt

"NA" indicates that no life history pathway meeting the criteria used in defining these pathways was present,

Bıanik cells uniaer atributes Indicate no additional attributes with negative effects on productivity were evident compared to those present in the template. 


\section{Stream Reach Summary for Primary and Secondary Spring Chinook Salmon Life Histories}

Stream: Imnaha R.

Reach: Lightning $\mathrm{Cr}$ - Horse $\mathrm{Cr}$ (RM 5.4- 10.8 from Snake R)

\begin{tabular}{|ll|ll|llll}
\hline Restoration benefit category: & F & Chinook usage score: & 134.9 & Habitat productivity loss score: & 153.8 \\
\hline
\end{tabular}

\begin{tabular}{|c|c|c|c|c|c|c|c|c|c|c|c|c|c|c|}
\hline \multirow[b]{3}{*}{ Life stage } & \multirow[b]{3}{*}{ Months. } & \multicolumn{10}{|c|}{ No. life history pathways by current usage abundance level } & \multicolumn{2}{|c|}{ Key habitat change } & \multirow{3}{*}{$\begin{array}{c}\text { Habitat } \\
\text { productivity } \\
\text { loss score }\end{array}$} \\
\hline & & \multicolumn{5}{|c|}{ Primary } & \multicolumn{5}{|c|}{ Secondary } & \multirow{2}{*}{$\begin{array}{l}\text { Patient } \\
\text { quantii }\end{array}$} & \multirow[b]{2}{*}{$\%$ change } & \\
\hline & & Total no. & 1 & 2 & 3 & 4 & Total no. & 1 & 2 & 3 & 4 & & & \\
\hline 1 & Apr-Aug & 15 & 10 & 3 & 2 & 0 & 43 & 29 & 8 & 6 & 0 & 144 & 0.0 & 1708 \\
\hline 123 & Äug-Ött & $\mathrm{NA}$ & $\ddot{\mathrm{NA}} \dot{ }$ & $\mathrm{NA}$ & $\mathrm{NA}$ & $\dot{N A}$ & $\ddot{\mathrm{NA}}$ & $\ddot{\mathrm{N}} \ddot{\mathrm{A}}$ & $\dot{N A}$ & NA & $\mathrm{NA}$ & NÄ & $\mathrm{NA}$ & NA \\
\hline 3 & Ott-Mar & NA & NA & NA & NA & NA & NA & NA & NA & & NA & NA" & $\left(\begin{array}{lll}\cdots \\
\%\end{array}\right.$ & NA \\
\hline 34 & Mar-May. & $N A$ & $N A$ & NA & NA & $\mathrm{NA}$ & $\mathrm{NA}$ & $\mathrm{NA}$ & $" N A$ & & NA & $\mathrm{NA}$ & $. .70 \ldots . .$. & NA \\
\hline 5 & May-Ott & 0 & 0 & 0 & 0 & 0 & 4 & 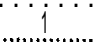 & 2 & 1 & 0 & 12.0 & 0.0 & -0.3 \\
\hline $6 "$ & Oct-Mar & 19 & "1." & 18 & 0 & 0 & 66 & 62 & $?$ & $?$ & 0 & 32.0 & 0.0 & -166 \\
\hline$\dot{7}$ & Mar-Jun & 16 & $\ddot{g}$ & 4 & 3 & 0 & 31 & 24 & 4 & 3 & 0 & 48.4 & 0.0 & 0 \\
\hline
\end{tabular}

\begin{tabular}{|c|c|c|c|c|c|c|c|c|c|c|c|c|c|}
\hline \multirow[b]{2}{*}{ Life stage } & \multirow[b]{2}{*}{ Months } & \multicolumn{12}{|c|}{ Highest productivity impact attributes (\% change in impact from template) } \\
\hline & & Aft 1 & $\%$ change & Att 2 & $\%$ change & Att 3 & $\%$ change & Att 4 & $\%$ change & Att 5 & $\%$ change & Att 6 & \% change \\
\hline $\begin{array}{c}1 \\
1,2,3\end{array}$ & $\begin{array}{l}\text { Apr-Aug } \\
\text { Äug-Oct }\end{array}$ & $\begin{array}{c}\text { PATHO } \\
\text { NA }\end{array}$ & $\begin{array}{c}23.2 \\
\text { NA }\end{array}$ & NA & NA & NA & NA & NA & NA & NA & NA & NA & NA \\
\hline 3 & ött-Mär & NA & NÄ & N A & Nล̈ & Nั"̈ & $\mathrm{NÄ}$ & $\mathrm{NA}$ & חั" & "Nä" - & NÄ & NÄ & $\mathrm{NA}$ \\
\hline $\begin{array}{l}3,4,, \cdot, \cdot, \\
5\end{array}$ & $\begin{array}{l}\text { Mar-May } \\
\text { May-Ött }\end{array}$ & $\begin{array}{c}\text { NA } \\
\text { PATHOO }\end{array}$ & $\begin{array}{l}\mathrm{NA} \\
7.5\end{array}$ & NA & NA & NA & NA & NA & NA & $N \ddot{A}$ & $N A$ & $\mathrm{NA}$ & $\because \mathrm{NA}$ \\
\hline 6 & Ött-Mar & PATHOO & 68 & SEDLD & 68 & PRED & 6.6 & & & 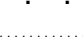 & & " & \\
\hline 7 & Mar-Jun & PRED & 9.8 & & & & & & & & & & \\
\hline
\end{tabular}

Abundance level refers to the average abundance of spawners that spawn in the natal stream reaches that populate the life history pathways using the reach in this table:

$\begin{array}{ll}\text { Levei 1: } & 0-10 \text { spawners } \\ \text { Level 2 } & 10-30 \text { spawners } \\ \text { Levei 3 } & 30-60 \text { spawners }\end{array}$

Levei 4: $\quad>60$ spawners

Life stages: 1- prespawner; 2- spawner; 3- incubation; 4- fry colonization; 5- summer rearing; 6- overwintering; 7- yearling presmolt/smolt

$\begin{array}{lll}\text { Attributes: } \text { CHEM - chemicals } & \text { NUTLD - nutrient ioad } & \text { RIPCN - riparian condition } \\ \text { CSTAB - channel stability } & \text { OBST - obstructions } & \text { SEDLD - sediment ioad } \\ \text { COMP - competition (with other species) } & \text { OXYG - dissolved oxygen } & \text { TEMP - water temperature } \\ \text { FLOW - ftOW } & \text { PATHO - pathogens } & \text { WiTH - water withdrawals } \\ \text { HABD - habitat diversity } & \text { PRED - predators (includes fishing-related losses) }\end{array}$

"NA" indicates that no life history pathway meeting the criteria used in defining these pathways was present.

Blank ceils under attributes indicate no additional attributes with negative effects on productivity were evident compared to those present in the template. 


\section{Stream Reach Summary for Primary and Secondary Spring Chinook Salmon Life Historles}

Stream: Imnaha R.

Reach: Fence $\mathrm{Cr}$ - Big Sheepcr (RM 16.3 -22.3 from Snake R)

\begin{tabular}{|ll|ll|ll|}
\hline Restoration benefit category: & $F$ & Chinook usage score: & 132.0 & Habitat productivity loss score: & 144.0 \\
\hline
\end{tabular}

\begin{tabular}{|c|c|c|c|c|c|c|c|c|c|c|c|c|c|c|}
\hline \multirow[b]{3}{*}{ Life stage } & \multirow[b]{3}{*}{ Months } & \multicolumn{10}{|c|}{ No. life history pathways by current usage abundance level } & \multicolumn{2}{|c|}{ Key habitat change } & \multirow{3}{*}{$\begin{array}{c}\text { Habitat } \\
\text { productivity } \\
\text { loss score }\end{array}$} \\
\hline & & \multicolumn{5}{|c|}{ Primary } & \multicolumn{5}{|c|}{ Secondary } & \multirow{2}{*}{$\begin{array}{l}\text { Patient } \\
\text { quantity }\end{array}$} & \multirow[b]{2}{*}{$\%$ change } & \\
\hline & & Total no. & 1 & 2 & 3 & 4 & Total no. & 1 & 2 & 3 & 4 & & & \\
\hline 1 & Apr-Aug & 15 & 10 & 3 & 2. & 0. & 43 & 29 & 8 & 6 & 0 & 12.0 & 0.0 & 150.3 \\
\hline $1,2,3$ & Aug-Ott & $\mathrm{NA}$ & $\mathrm{NA}$ & NÄ & Nä. & $\mathrm{NA}$ & $\mathrm{NA}$ & $\mathrm{NÄ}$ & 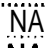 & $\mathrm{NA}$ & $\mathrm{NA}$ & $\mathrm{NA}$ & NA & NA \\
\hline 34 & Mar-May & “NA & $\mathrm{NA}$ & $\mathrm{NA}$ & NA & NA & $N A$ & $N A$ & NA & $N A$ & " NA NA & NA & NA & \\
\hline 5 & May-Oct & 1 & "ị" & 0 & 0 & 0 & 13 & 7 & 4 & 2 & 0 & 7.5 & 0.0 & : ye"' \\
\hline 6. & Ott-Mar & 2 & 0 & $1^{\cdots}$ & "ij" & 0 & 82 & 62 & 19 & 1 & 0 & 19.8 & 0.0 & -19.9 \\
\hline 7 & Mar-Jun & 12 & 9 & 2 & "i" & 0 & 23 & 16 & 4 & 3 & 0 & 36.1 & 0.0 & 16.6 \\
\hline
\end{tabular}

\begin{tabular}{|c|c|c|c|c|c|c|c|c|c|c|c|c|c|}
\hline \multirow[b]{2}{*}{ Life slage } & \multirow[b]{2}{*}{ Months } & \multicolumn{12}{|c|}{ Highest productivity impact attributes (\% change in impact from template) } \\
\hline & & Att 1 & \% change & Aft 2 & $\%$ change & Alt 3 & \% change & Alt 4 & $\%$ change & Att 5 & $\%$ change & Att 6 & \% change \\
\hline 1 & Apr-Aug & PATHO & 20.4 & & & & & & & & & & to change \\
\hline $1,2,3$ & Aug-Oct & $N A$ & $\mathrm{NA}$ & NÄ & NA & NÄ & $\mathrm{NA}$ & NA & NA & NA & NÄ & NA & $\mathrm{NA}$ \\
\hline 3 & Oct-Mar & $\mathrm{NA}$ & $\mathrm{NA}$ & $\mathrm{NA}$ & $\mathrm{NA}$ & NA & $\mathrm{NA}$ & $N A$ & $\mathrm{NA}$ & $\mathrm{NA}$ & $\mathrm{NA}$ & NA & $\mathrm{NA}$ \\
\hline 3,4 & Mar-May & $\mathrm{NA}$ & $\mathrm{NA}$ & $\mathrm{NA}$ & $\mathrm{NA}$ & $\mathrm{NA}$ & $\mathrm{NA}$ & $N A$ & NA & $\mathrm{NA}$ & $\mathrm{NA}$ & NA & $\mathrm{NA}$ \\
\hline 5 & May-Oct & PATHO & 8.7 & & & & & & & & & & \\
\hline 6 & Oct-Mar & PATHO & 8.2 & RIPCN" & 8.2 & SEDLLD & 8.2 & & & & & & \\
\hline 7 & Mar-Jun & & & & & & & & & & & & \\
\hline
\end{tabular}

Abundance lovel refers to the average abundance of spawners that spawn in the natal stream reaches that populate the life history pathways using the reach in this table:

$$
\begin{array}{ll}
\text { Level 1: } & 0-10 \text { spawners } \\
\text { Level 2: } & 10-30 \text { apawners } \\
\text { Level 3: } & 30-60 \text { spawners } \\
\text { Level 4 } & >60 \text { spawners }
\end{array}
$$

Life stages: 1-prespawner; 2- spawner; 3- incubation; 4- fry colonization; 5- summer rearing; 6- overwintering; 7- yearling presmolt/smelt

$\begin{array}{lll}\text { Attributes: CHEM - chemicals } & \text { NUTLD - nutrient load } & \text { RIPCN - riparian condition } \\ \text { CSTAB - channel stability } & \text { OBST - obstructions } & \text { SEDLD - Sediment load } \\ \text { COMP - competition (with other species) } & \text { OXYG - dissolved oxygen } & \text { TEMP - water temperature } \\ \text { FLOW - ftOW } & \text { PAT HO - pathogens } & \text { WITH - water withdrawals } \\ \text { HABD - habitat diversity } & \text { PRED - predators (includes fishing-related losses) }\end{array}$

"NA" indicates that no life history pathway meeting the criteria used in defining these pathways was present.

Blank cells under attributes indicate no additional attributes with negative effects on productivity were evident compared to those present in the template. 
Stream Reach Summary for Primary and Secondary Spring Chinook Salmon Life Histories

Stream: Imnaha $\mathbf{R}$

Reach: Horse Cr - Fence Cr (RM 10.8 -16.3 from Snake R)

\begin{tabular}{|ll|ll|l|}
\hline Restoration benefit category: & F & Chinook usage score: & 133.4 & Habitat productivity loss score: \\
\hline
\end{tabular}

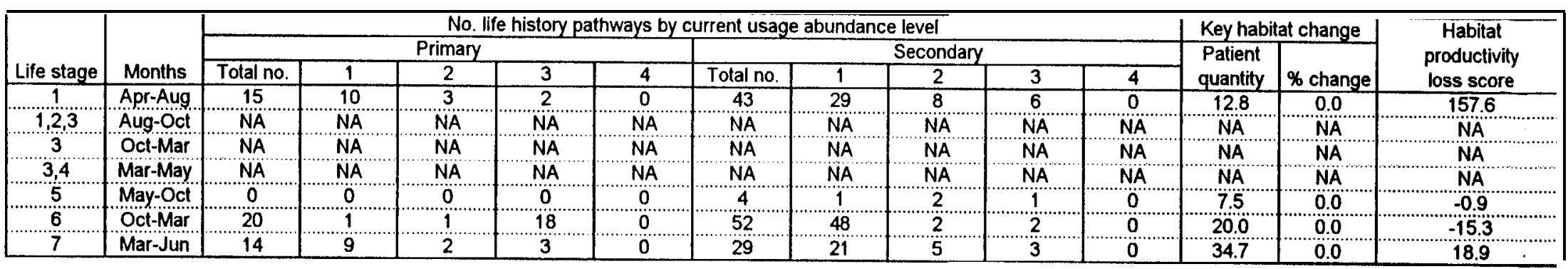

\begin{tabular}{|c|c|c|c|c|c|c|c|c|c|c|c|c|c|}
\hline \multirow[b]{2}{*}{ Life stage } & \multirow[b]{2}{*}{ Months } & \multicolumn{12}{|c|}{ Highest productivity impact attributes (\% change in impact from template) } \\
\hline & & Att 1 & \% change & Att 2 & \% change & Att 3 & $\%$ change & Att 4 & $\%$ change & Att 5 & $\%$ change & Att 6 & $\%$ change \\
\hline 100 & Apr-Aug & PATHO & 21.4 & & & & & & & & & & \\
\hline 1,23 & Äug-Öci & NA & NA & NA & $\because \mathrm{N}$ & NÄ & NQA & NA & $N A$ & NA & $\mathrm{NÄ}$ & $\mathrm{NÄ}$ & $N A ̈$ \\
\hline 3 & Ött-Mar & NA & Na & NA & NA & NA & NÄ & NÄ" & & N̈̈̈ & $\dot{N} \dot{n} A \dot{a}$ & ï̈̈̈ & NA \\
\hline 3,4 & Mar-May & NA & NA & NÁ & NA & $\mathrm{NA}$ & NA & NA & $\mathrm{NA}$ & NA & NA & NA' & NA" \\
\hline 5 & May-Ott & PATHO & 8.0 & & & & & & & & & & \\
\hline 6 & Ott-Mar & $\mathrm{HABD}$ & 7.9 & "PÄT̈HÖ" & 7.96 & SEEDLLD" & 7.9 & & . & & & & \\
\hline 7 & Mar-Jun & & & & & & & & & & & & \\
\hline
\end{tabular}

Abundance /eve/ refers to the average abundance of spawners that spawn in the natal stream reaches that populate the life history pathways using the reach in this table

$$
\begin{array}{ll}
\text { Level 1: } & 0-10 \text { spawners } \\
\text { Level 2: } & 10-30 \text { spawners } \\
\text { Level 3: } & 30-60 \text { spawners } \\
\text { Level 4: } & >60 \text { spawners }
\end{array}
$$

Life stages.' 1- prespawner; 2- spawner; 3- incubation; 4- fry colonization; 5- summer rearing; 6- overwintering; 7- yearling presmott/smelt

Attributes: CHEM - chemicals

CSTAB - channel stability

COMP - competition (with other species)

FLOW - ffOW

HABD - habitat diversity
NUTLD - nutrient load

OXYG - dissolved oxygen

PATHO - pathogens

PRED - predators (includes fishing-related losses)
RIPCN - riparian condition

SEDLD - sediment ioad

TEMP - water temperature

WITH - water withdrawals

"NA" indicates that no life history pathway meeting the criteria used in defining these pathways was present,

Blank cells under attributes indicate no additional attributes with negative effects on productivity were evident compared to those present in the template, 


\section{Stream Reach Summary for Primary and Secondary Spring Chinook Salmon Life Histories}

Stream: Imnaha R.

Reach: $\quad$ Big Sheep Cr - Freezeout Cr (RM 22.3 -35.2 from Snake R)

\begin{tabular}{|c|c|c|c|c|c|c|c|c|c|c|c|c|c|c|}
\hline \multicolumn{4}{|c|}{ Restoration benefit category: } & 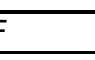 & \multicolumn{3}{|c|}{ Chinook usage score: } & 224.5 & \multicolumn{5}{|c|}{ Habitat productivity loss score: } & \multirow{4}{*}{$\begin{array}{c}\text { Habitat } \\
\text { productivity } \\
\text { loss score }\end{array}$} \\
\hline \multirow[b]{3}{*}{ Life stage } & \multirow[b]{3}{*}{ Months } & \multicolumn{10}{|c|}{ No. life history pathways by current usage abundance level } & \multicolumn{2}{|c|}{ Key habitat change } & \\
\hline & & \multicolumn{5}{|c|}{ Primary } & \multicolumn{5}{|c|}{ Secondary } & \multirow{2}{*}{$\begin{array}{l}\text { Patient } \\
\text { quantity }\end{array}$} & \multirow[b]{2}{*}{$\%$ change } & \\
\hline & & Total no. & 1 & 2 & 3 & 4 & Total no. & 1 & 2 & 3 & 4 & & & \\
\hline 123 & Aug-Öct & $\mathrm{NA}$ & NÄ & $\dot{N A}$ & NÁ & NA" & NA & NA & $\mathrm{NA}$ & NA & $\mathrm{NA}$ & $\mathrm{NA"}$ & NA & NA \\
\hline 3 & Ött-M̈ar & NÄ & NA & NA & NA & NA & NA & NA & NA & NA & NA & NA & NA & $\ddot{N A}$ \\
\hline 34 & Mar-May & NA & NA & $\mathrm{NA}$ & NA & NA & NA & NA & NA & NA & NA & $\ddot{N A}$ & NA & NA \\
\hline$\cdots$ & May-Ött & 3 & 1 & $i$ & 1 & 0 & 18 & $\ddot{g}$ & 5 & 4 & 0 & 7.5 & $0.0^{\circ}$ & -42 \\
\hline$\dot{7}$ & Mar-jun & 7 & 4 & 2 & 1 & 0 & 21 & 12 & 5 & 4 & 0 & 34.0 & 0.0 & $0.0^{\circ}$ \\
\hline
\end{tabular}

\begin{tabular}{|c|c|c|c|c|c|c|c|c|c|c|c|c|c|}
\hline \multirow[b]{2}{*}{ Life stage } & \multirow[b]{2}{*}{ Months } & \multicolumn{12}{|c|}{ Highest productivity impact attributes (\% change in impact from template) } \\
\hline & & Att 1 & \% change & Att 2 & \% change & Att 3 & $\%$ change & Alt 4 & \% change & Att 5 & \% change & Att 6 & \% change \\
\hline$\overline{1}$ & Apr-Aug & HABD & 24.2 & OBST & 24.2 & PATHO & 24.2 & PRED & 24.2 & TEMP & $\overline{24.2}$ & & \\
\hline $1,2,3$ & Aug-Oat & NA & $\mathrm{NA}$ & NÄ & NA & $\mathrm{NA}$ & NÄ & NA & NA & NA & NA & NÄ & NA \\
\hline 3 & Oct-Mar & NA & $\mathrm{NA}$ & $\mathrm{NA}$ & NA & $\mathrm{NA}$ & NÄ & $\mathrm{NA}$ & $\mathrm{NA}$ & $\mathrm{NA}$ & $N A$ & $\mathrm{NA}$ & NAA \\
\hline 3,4 & Mar-May & $\mathrm{NA}$ & $\mathrm{NA}$ & NA & NA & $\mathrm{NA}$ & NA & $\mathrm{NA}$ & NA & $\mathrm{NA}$ & NA & $\mathrm{NA}$ & NA \\
\hline 5 & May-Oct & HABD & 22.5 & COMP & 11.2 & FLOW & 11.2 & PATHO & 11.2 & RIPCN & 11.2 & WITH & 11.2 \\
\hline 6 & Oct-Mar & COMP & 14.7 & FLOWW & 14.7 & PATHO & 14.7 & RIPCN & 14.7 & SEOLLD & 14.7 & WiTïi & 14.7 \\
\hline 7 & Mar-Jun & WITH & 12.2 & & & & & & & & & & \\
\hline
\end{tabular}

Abundancelevel refers to the average abundance of spawners that spawn in the natal stream reaches that populate the life history pathways using the reach in this table:

$$
\begin{array}{ll}
\text { Level 1: } & 0-10 \text { spawners } \\
\text { Level 2: } & 10-30 \text { spawners } \\
\text { Level 3: } & 30-60 \text { spawners } \\
\text { Level 4 } & >60 \text { spawners }
\end{array}
$$

\begin{tabular}{|c|c|c|c|}
\hline $\begin{array}{l}\text { Attributes: } \text { CHEM - chemicals } \\
\text { CSTAB - channel stability } \\
\text { COMP - competition (with other species) } \\
\text { FLOW - fiOW } \\
\text { HABD - habitat diversity }\end{array}$ & $\begin{array}{l}\text { NUTLD - } \\
\text { OBST - } \quad \text { obstructiont load } \\
\text { OXYG - } \text { dissolved oxygen } \\
\text { PATHO - pathogens } \\
\text { PRED - } \quad \text { predators (includes fishing-related losses) }\end{array}$ & $\begin{array}{l}\text { RIPCN - } \\
\text { SEDLD - } \\
\text { TEMP - } \\
\text { WITH - }\end{array}$ & $\begin{array}{l}\text { riparian condition } \\
\text { sediment load } \\
\text { water temperature } \\
\text { water withdrawals }\end{array}$ \\
\hline
\end{tabular}

Life stages: 1- prespawner; 2- spawner; 3- incubation; 4- fry colonization; 5- summer rearing; 6- overwintering; 7- yearling presmolt/smolt

"NA" indicates that no life history pathway meeting the criteria used in defining these pathways was present.

Blank cells under attributes indicate no additional attributes with negative effects on productivity were evident compared to those present in the template, 


\section{Stream Reach Summary for Primary and Secondary Spring Chinook Salmon Life Histories}

Stream: Imnaha $\mathbf{R}$.

Reach: Freezeout $\mathrm{Cr}$ - Grouse $\mathrm{Cr}$ (RM 35.2 -40.6 from Snake R)

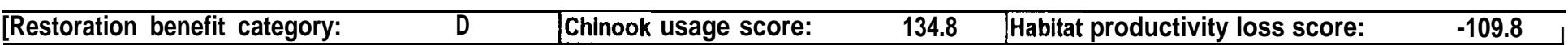

\begin{tabular}{|c|c|c|c|c|c|c|c|c|c|c|c|c|c|c|}
\hline \multirow[b]{3}{*}{ Life stage } & \multirow[b]{3}{*}{ Months } & \multicolumn{10}{|c|}{ No. iife history pathways by current usage abundancelevel } & \multicolumn{2}{|c|}{ Key habitat change } & \multirow{3}{*}{$\begin{array}{c}\text { Habitat } \\
\text { productivity } \\
\text { ioss score }\end{array}$} \\
\hline & & \multicolumn{5}{|c|}{ Primary } & \multicolumn{5}{|c|}{ Secondary } & \multirow{2}{*}{$\begin{array}{l}\text { Patient } \\
\text { quantity }\end{array}$} & \multirow[b]{2}{*}{ \% chanqe } & \\
\hline & & Total no. & 1 & 2 & 3 & 4 & Total no. & 1 & 2 & 3 & 4 & & & \\
\hline $1 . .$. & Apr-Aug & 10 & 5 & 3 & 2. & 0 & 31. & 17 & 8 & 6 & 0 & 11.5 & -03 & -34.5 \\
\hline $1,2,3$ & Äug-Ött & NA & NA & 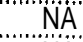 & 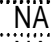 & 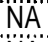 & NA & NA & NA & NÄ & NA & NÄ & NA & NA \\
\hline 34 & Mar-May & 0 & 0 & 0 & 0 & 0 & 20 & 16 & $\dddot{4}$ & 0 & 0 & 1.8 & -35.1 & -35.3 \\
\hline 5 & May-OQtt & 3 & 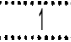 & 11 & 11 & 0 & 129 & $100^{\circ}$ & 25 & 4 & 0 & 9.5 & -1.4 & $-30 "$ \\
\hline 6 & Oct-Mar & 0 & 0 & 0 & 0 & 0 & 39 & 20 & 1 & 18 & 0 & 20.3 & -0.7 & -9.6 \\
\hline 7 & Mar-Jun & 7 & 4 & 2 & "i & 0 & 7 & “"'4"”' & "i" & 2 & 0 & 33.4 & -3.8 & 0.0 \\
\hline
\end{tabular}

\begin{tabular}{|c|c|c|c|c|c|c|c|c|c|c|c|c|c|}
\hline \multirow[b]{2}{*}{ Liffestagee } & \multirow[b]{2}{*}{ Madontths } & \multicolumn{12}{|c|}{ Highest productivity impact attributes (\% change in impact from template) } \\
\hline & & Aft 1 & $\%$ change & Att 2 & $\%$ change & Att 3 & $\%$ change & Att 4 & \% change & Att 5 & $\%$ change & Att 6 & 96 change \\
\hline 1 & Apr-Aug & $\mathrm{HABD}$ & 16.0 & OBST & 16.0 & PATHO & 16.0 & PRED & 16.0 & TEMP & 16.0 & & \\
\hline $1,2,3$ & Aug-Öt & NA & $\mathrm{NA}$ & $\mathrm{NA}$ & $\mathrm{NA}$ & $\mathrm{NA}$ & $\mathrm{NA}$ & $\mathrm{NA}$ & $\mathrm{NÄ}$ & NA" & $\mathrm{NA}$ & $\mathrm{NÄ}$ & NA \\
\hline 3 & Otthar & NA & NA & $\dot{N A}$ & NA & $\mathrm{NA}$ & NA & $\mathrm{NA}$ & & NÄ & $\mathrm{NABA}$ & NÄ & ? \\
\hline 3,4 & Mar-May & HABD & 20.6 & COMP & 10.3 & CSTAB & 10.3 & FLOW & 1 & "PATHO' & 10.3 & RIPCN & \\
\hline 5 & May-Ott & HABD & 20.2 “'” & COMP & 10.1 & FLOW & $" 10.1$ & PATHO & $10.1^{\cdots}$ & RIPCN & 10.1 & WiTH & 10.1 \\
\hline 6 & Odteivar & COMP & 7.6 & "FL'ÖW" & 76 & "PÄTHÖ" & 7.6 & RIPCN & 7.6 & SEDLD & 76 & "Wiт̈н" & 7.6 \\
\hline 7 & Mar-Jun & WHTḦ & 11.2 & & & & & & & & & & \\
\hline
\end{tabular}

Abundancelevel refers to the average abundance of spawners that spawn in the natal stream reaches that populate the life history pathways using the reach in this table:

$$
\begin{array}{ll}
\text { Level 1: } & 0-10 \text { spawners } \\
\text { Level 2 } & 10-30 \text { spawners } \\
\text { Level 3: } & 30-60 \text { spawners } \\
\text { Levei 4: } & >60 \text { spawners }
\end{array}
$$

Life stages: 1- prespawner; 2- spawner; 3- incubation; 4- fry colonization; 5- summer rearing; 6- overwintering; 7- yearling presmolt/smolt

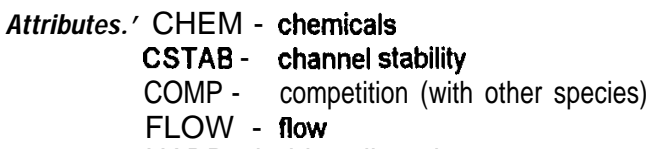

"NA" indicates that no life history pathway meeting the criteria used in defining these pathways was present.

Blank cells under attributes indicate no additional attributes with negative effects on productivity were evident compared to those present in the template, 
Stream Reach Summary for Primary and Secondary Spring Chinook Salmon Life Histories

Stream: Imnaha R.

Reach: Grouse $\mathrm{Cr}$ - Summit $\mathrm{Cr}$ (RM 40.6 -43.6 from Snake R)

\begin{tabular}{|ll|ll|lll}
\hline Restoratlon benefit category: & F & Chinook usage score: & 57.3 & Habitat productivity loss score: & -57.0 \\
\hline
\end{tabular}

\begin{tabular}{|c|c|c|c|c|c|c|c|c|c|c|c|c|c|c|}
\hline \multirow[b]{3}{*}{ Life stage } & \multirow[b]{3}{*}{ Months } & \multicolumn{10}{|c|}{ No. life history pathways by current usage abundance level } & \multicolumn{2}{|c|}{ Key habitat change } & \multirow{3}{*}{$\begin{array}{c}\text { Habitat } \\
\text { productivity } \\
\text { loss score }\end{array}$} \\
\hline & & \multicolumn{5}{|c|}{ Primary } & \multicolumn{5}{|c|}{ Secondary } & Patient & & \\
\hline & & Total no. & 1 & 2 & 3 & 4 & Total no. & 1 & 2 & 3 & 4 & quantity & $\%$ change & \\
\hline & Apr-Aug & 10 & 5 & 3 & 2 & 0 & 31 & 17 & 8 & 6 & 0 & 12.8 & 0.0 & -45.2 \\
\hline $1,2,3$ & Äug-Ött & $\mathrm{NA}$ & $\ddot{\mathrm{NA}}$ & NA & NA & NA & NA" & N̈A" & N̈̈̈ & "NA & NA" & $\mathrm{NA}$ & NA & \\
\hline 34 & Mar-May & 0 & 0 & 0 & 0 & 0 & “ 5 & 4 & $i$ & 0 & 0 & 1.9 & -34.4 & -83 \\
\hline 5 & May-Ott & 3 & "'i.". & 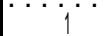 & $\cdots$ & 0 & $20^{\circ}$ & $10 "$ & 6 & 4 & 0 & 7.5 & 0.0 & -3.0 \\
\hline 6 & Ott-Mar & 0 & 0 & 0 & "0" & ơ" & $\cdots$ & 0 & $0^{\circ}$ & 1 & 0 & 18.8 & 0.0 & -0.5 \\
\hline $7^{\circ}$ & Mar-Jun & 7 & 4 & 2 & 1 & 0 & 5 & $\dddot{3}$ & 1 & 1 & 0 & 36.2 & 0.0 & 0 \\
\hline
\end{tabular}

\begin{tabular}{|c|c|c|c|c|c|c|c|c|c|c|c|c|c|}
\hline \multirow[b]{2}{*}{ Life stage } & \multirow[b]{2}{*}{ Months } & \multicolumn{12}{|c|}{ Highest productivity impact attributes (\% change in impact from template) } \\
\hline & & Att 1 & $\%$ change & Att 2 & $\%$ change & Att 3 & $\%$ change & Aft 4 & \% change & Att 5 & $\%$ change & Att 6 & $\%$ change \\
\hline 1 & Apr-Aug & HABD & 172 & OBST & 17.2 & PATHO & 17.2 & PRED & 17.2 & TEMP & 17.2 & & \\
\hline 1,23 & Äug-Öci & NA & $\mathrm{NA}$ & NA & $\mathrm{NA}$ & $\mathrm{NA}$ & $\mathrm{NA}$ & NA & $\mathrm{NÄ}$ & $N A "$ & $\dddot{\mathrm{N}} \mathrm{A}$ & $\mathrm{NA}$ & NÄ" \\
\hline 3 & Ött-Mar & $\dot{\mathrm{NA}}$ & $\mathrm{NA}$ & NA & $\mathrm{NÄ}$ & NÄ & $\dddot{N}$ & Nä̈ & ח̈̈ & $N$ & $\dddot{N} \dddot{N}$ & NA" & NÄ \\
\hline 3,4 & Mar-May & COMPP & 10.3 & HABBD & 10.3 & PATHO & 10,3 & RIPCN & 10,3 & "Ẅ̈̈т̈' & $10.3^{\circ}$ & & \\
\hline $5, "$ & May-Ött & HABD & 13.3 & COMP & "' 6.7' "' & FLOW & $6.7^{\prime}$ & PATHO & 6.7 & RIPCN & 6.7 & SĖDiL & 6.7 \\
\hline 6 & Ott-Mar & COMP & 25.0 & F̈LÖẄ & $25.0^{\circ}$ & "PATHÖ & 25.0 & RIPICN & $25.0^{\circ}$ & SEDLLD & 25.0 & WiTH & $25.0^{\circ}$ \\
\hline 7 & Mar-Jun & Wiтt & 7.5 & & & & & & & & & & \\
\hline
\end{tabular}

Abundance level refers to the average abundance of spawners that spawn in the natal stream reaches that populate the iife history pathways using the reach in this table:

$$
\begin{array}{ll}
\text { Levei 1: } & 0-10 \text { spawners } \\
\text { Level 2: } & 10-30 \text { spawners } \\
\text { Level } 3: & 30-60 \text { spawners } \\
\text { Level } 4 & >60 \text { spawners }
\end{array}
$$

\begin{tabular}{|c|c|c|c|}
\hline $\begin{aligned} & \text { Attributes: } \text { CHEM - chemicals } \\
& \text { CSTAB - channel stability } \\
& \text { COMP - competition (with other species) } \\
& \text { FLOW - ftOW }\end{aligned}$ & $\begin{array}{l}\text { NUTLD - } \text { nutrient load } \\
\text { OBST - obstructions } \\
\text { OXYG - dissolved oxygen } \\
\text { PATHO - pathogens }\end{array}$ & $\begin{array}{l}\text { RIPCN - } \\
\text { SEDLD - } \\
\text { TEMP - } \\
\text { WiTH - }\end{array}$ & $\begin{array}{l}\text { riparian condition } \\
\text { sediment load } \\
\text { water temperature } \\
\text { water withdrawals }\end{array}$ \\
\hline
\end{tabular}

Life stages: 1- prespawner; 2- spawner; 3- incubation; 4- fry colonization; 5- summer rearing; 6- overwintering; 7- yearling presmolt/smolt

"NA" indicates that no tife history pathway meeting the criteria used in defining these pathways was present.

Blank cells under attributes indicate no additional attributes with negative effects on productivity were evident compared to those present in the template. 
Stream Reach Summary for Primary and Secondary Spring Chinook Salmon Life Histories

Stream: Imnaha R.

Reach: Summit $\mathrm{Cr}$-Crazyman $\mathrm{Cr}$ (RM 43.6 -49.4 from Snake R)

\begin{tabular}{ll|ll|lll}
\hline Restoration benefit category: & D & Chinook usage score: & 163.6 & Habltat productivity loss score: & -143.5 \\
\hline
\end{tabular}

\begin{tabular}{|c|c|c|c|c|c|c|c|c|c|c|c|c|c|c|}
\hline \multirow[b]{3}{*}{ Life stage } & \multirow[b]{3}{*}{ Months } & \multicolumn{10}{|c|}{ No. life history pathways by current usage abundance level } & \multirow{2}{*}{\multicolumn{2}{|c|}{$\begin{array}{l}\text { Key habitat change } \\
\text { Patient }\end{array}$}} & \multirow{3}{*}{$\begin{array}{c}\text { Habitat } \\
\text { productivity } \\
\text { loss score }\end{array}$} \\
\hline & & \multicolumn{5}{|c|}{ Primary } & \multicolumn{5}{|c|}{ Secondary } & & & \\
\hline & & Total no. & 1 & 2 & 3 & A & T,asalıno. & 1 & 2 & 3 & 4 & quanțtity & $\%$ change & \\
\hline & Apr-Aug & 10 & 5 & 3. & 2. & $\pi$ & $2 J_{1}$ & 17 & 8 & 6 & 0 & 1A-8 & -3.6 & -42.6 \\
\hline $1,2,3$ & Aug-Oct & $\mathrm{NA}$ & NA & NA & NA & $\mathrm{NÄ}$ & $\mathrm{NA}$ & NA & NA & NÁ & $\mathrm{NA}$ & $\mathrm{NA}$ & NA & NA \\
\hline 3 & Ött-Mar & NA & NA & NA & NA & NA & NAA & NA & NA & NA & NA & $\mathrm{NA}$ & NA & NA \\
\hline 34 & Mar-May & 0 & 0 & 0 & $0 .$. & 0 & 33 & 4 & 17 & 12 & 0 & 1.7 & -24.0 & -404 \\
\hline 5 & May-Ott & 3 & 1 & 1 & 1 & 0 & 197 & 10 & 94 & 93 & 0 & 8.6 & -11 & $-3 \cap 7$ \\
\hline 6 & Ott-Mar & 0 & 0 & 0 & 0. & 0 & 75 & 37 & 19 & 19 & 0 & 18.3 & 0.0 & -29.7 \\
\hline 7 & Mar-Jun & 7 & 4 & 2 & 1 & 0 & 5 & 3 & 1 & 1 & 0 & 29.7 & 0.0 & 0.0 \\
\hline
\end{tabular}

\begin{tabular}{|c|c|c|c|c|c|c|c|c|c|c|c|c|c|}
\hline \multirow[b]{2}{*}{ Life stage } & \multirow[b]{2}{*}{ Months } & \multicolumn{12}{|c|}{ Highest productivity impact attribute (\% change in impact from template) } \\
\hline & & Att 1 & $\%$ change & Att 2 & $\%$ change & Att 3 & $\%$ change & Att 4 & $\%$ change & Att 5 & \% change & Att 6 & \% change \\
\hline 1 & Apr-Aug & HABD & 16.2 & OBST & 16.2 & PATHO & 16.2 & PRED & 16.2 & TEMP & 16.2 & & \\
\hline $1,2,3$ & Aug-Oct & $\mathrm{NA}$ & $\mathrm{NA}$ & $\mathrm{NA}$ & $\mathrm{NA}$ & $\mathrm{NA}$ & $\mathrm{NA}$ & $\mathrm{NA}$ & NÄ & NÄ & NÄ & NÄ & $\mathrm{NA}$ \\
\hline 3 & Oct-Mar & $\mathrm{NA}$ & $\mathrm{NA}$ & $\mathrm{NA}$ & $\mathrm{NA}$ & $\mathrm{NA}$ & $\mathrm{NA}$ & $\mathrm{NA}$ & $N A$ & NA & NA & NA & NA \\
\hline 3,4 & Mar-May & HABD & 13.3 & CSTAB & 8.7 & FLOW & 8.7 & COMP & 6.6 & PATHO & 6.6 & RIPCN & 6.6 \\
\hline 5 & May-Oct & HABD & 13.5 & COMP & 6.7 & FLOW & 6.7 & PATHO & 6.7 & RIPCN & 6.7 & WITH & 6.7 \\
\hline 6 & Oct-Mar & COMP & 11.3 & FLOW & 11.3 & PATHO & 11.3 & RIPCN & 11.3 & SEDLD & 11.3 & WiTH" & 11.3 \\
\hline 7 & Mar-Jun & "шітн & 9.7 & & & & & & & & & & \\
\hline
\end{tabular}

Abundancelevel refers to the average abundance of spawners that spawn in the natal stream reaches that populate the life history pathways using the reach in this table

$$
\begin{array}{ll}
\text { Level 1: } & \text { 0-10 spawners } \\
\text { Level 2 } & \text { 10-30 spawners } \\
\text { Level 3 } & 30-60 \text { spawners } \\
\text { Level } 4 & >60 \text { spawners }
\end{array}
$$

Life stages: 1 - prespawner; 2- spawner; 3- incubation; 4- fry colonization; 5- summer rearing; 6- overwintering; 7- yearling presmolt/smolt

$\begin{array}{lll}\text { Attributes: CHEM - chemicals } & \text { NUTLD - nutrient load } & \text { RIPCN - riparian condition } \\ \text { CSTAB - channel stability } & \text { OBST - obstructions } & \text { SEDLD - Sediment load } \\ \text { COMP - competition (with other species) } & \text { OXYG - dissolved oxygen } & \text { TEMP - water temperature } \\ \text { FLOW - flow } & \text { PATHO - pathogens } & \text { WITH - water withdrawals } \\ \text { HABD - habitat diversity } & \text { PRED - predators (includes fishing-related losses) }\end{array}$

"NA" indicates that no life history pathway meeting the criteria used in defining these pathways was present.

Blank cells under attributes indicate no additional attributes with negative effects on productivity were evident compared to those present in the template. 


\section{Stream Reach Summary for Primary and Secondary Spring Chinook Salmon Life Histories}

Stream: Imnaha R.

Reach: Crazyman Cr - Gumboot Cr (RM $49.4-52,7$ from Snake R)

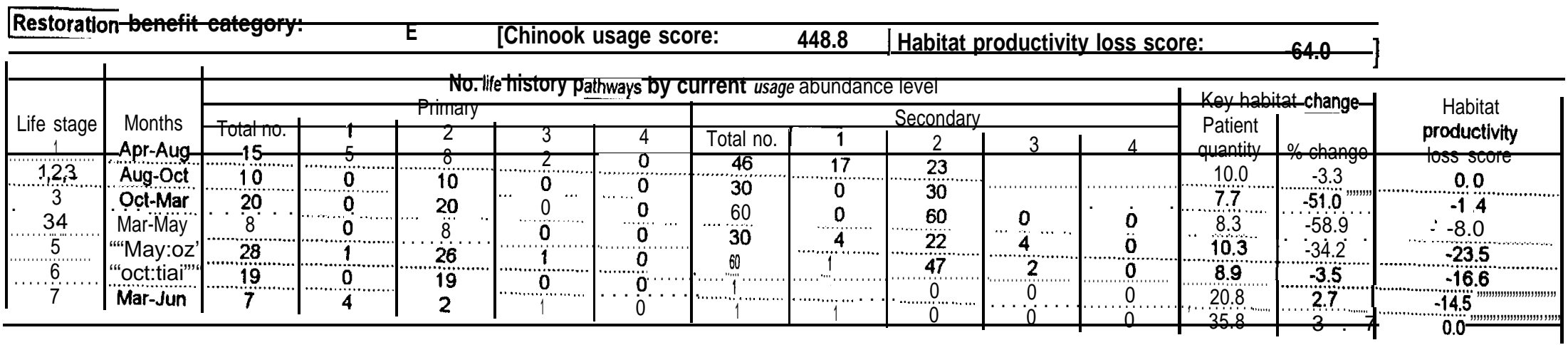

\begin{tabular}{|c|c|c|c|c|c|c|c|c|c|c|c|c|c|}
\hline Life stage & Months & \multicolumn{12}{|c|}{ Highest productivity impact attributes. (\%, channeinimpartfom template) } \\
\hline 1 & Apr-Aug & HABD & $\frac{\text { \% change }}{129}$ & Att 2 & \% change & Att 3 & \% change & Att 4 & \% change & Att 5 & \% change & Att 6 & \% change \\
\hline $1,2,3$ & Aug-Oct & HABD & 25.0 & $\begin{array}{l}\text { OBST } \\
\text { PRED }\end{array}$ & 12.9 & PATHO & 12.9 & SEDLD & 12.9 & & & & \\
\hline 3 & Oct-Mar & COMP & 25.0 & $\begin{array}{l}\text { PRED } \\
\text { PATHO }\end{array}$ & 25.0 & SEDLD & 25.0 & OBST & 12.5 & PATHO & 12.5 & & \\
\hline 3,4 & Mar-May & COMP & 25.0 & FLOW & 25.0 & PATHO & & & & & & & \\
\hline 5 & May-Oct & FLOW & 25.0 & PATHO & 250 & TEMP & 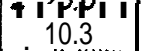 & I & $\ldots \ldots . .$. & & & & \\
\hline 6 & Oct-Mar & PATHO & 25.0 & TEMP & 24.4 & & & & $\begin{array}{lll}\cdots & \cdots & \cdots\end{array}$ & $\ldots \ldots . . . . . . . . .$. & & & \\
\hline 7 & Mar-Jun & PATHO & 11.1 & & & & & & & & & & \\
\hline
\end{tabular}

Abundance level refers to the average abundance of spawners that spawn in the natal stream reaches that populate the life history pathways using the reach in this table

$$
\begin{array}{ll}
\text { Level 1: } & 0-10 \text { spawners } \\
\text { Level 2: } & 10-30 \text { spawners } \\
\text { Level 3: } & \mathbf{3 0 - 6 0} \text { spawners } \\
\text { Level 4: } & >60 \text { spawners }
\end{array}
$$

Life stages: 1- prespawner; 2-spawner; 3- incubation; 4- fry colonization; 5- summer rearing; 6-overwintering; 7- yearling presmolt/smolt

Attributes: CHEM - chemicals

CSTAB - channel stability

COMP - competition (with other species)

FLOW - flow

HABD - habitat diversity

NUTLD - nutrient load

OBST - obstructions

OXYG - dissolved oxygen

PATHO pathogens

PRED - predators (includes fishing-related losses)
RIPCN - riparian condition

SEDLD - sediment load

TEMP - water temperature

WITH - water withdrawals

"NA" indicates that no life history pathway meeting the criteria used in defining these pathways was present

Blank cells under attributes indicate no additional attributes with negative effects on productivity were evident compared to those present in the template. 
Stream Reach Summary for Primary and Secondary Spring Chinook Salmon Life Historles

Stream: Imnaha R.

Reach: Gumboot $\mathrm{Cr}$ - Dry Cr (RM 52.7 -55.4 from Snake R)

Restoration benefit category: \begin{tabular}{l|l} 
B & Chlnook usage score:
\end{tabular}

\begin{tabular}{l|l}
774.0 & Habitat productivity loss score:
\end{tabular}

$-122.1$

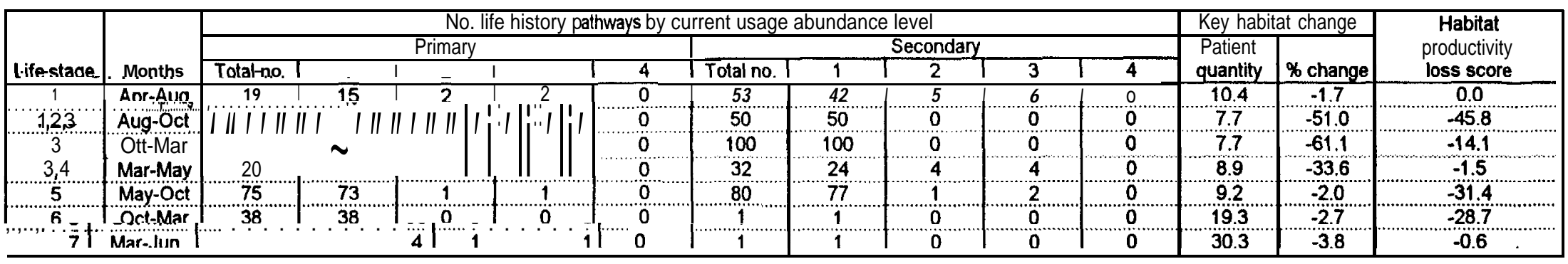

\begin{tabular}{|c|c|c|c|c|c|c|c|c|c|c|c|c|c|}
\hline \multirow[b]{2}{*}{ Life stage } & \multirow[b]{2}{*}{ Months } & \multicolumn{12}{|c|}{ Highest productivity impact attributes (\% change in impact from template) } \\
\hline & & Att 1 & $\%$ change & Att 2 & $\%$ change & Att 3 & $\%$ change & Att 4 & $\%$ change & Att 5 & $\%$ change & Atf 6 & $\%$ change \\
\hline 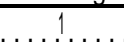 & Apr-Aug & HABD & 20.8 & SEDLL & 20.8 & PRED & 6.3 & & & & & & \\
\hline 1,23 & Aug-Ocl & HABD' & 18.7 & SEDLD & 16.7 & PRED & 8,3 & & & & & & \\
\hline 3 & Ött-M̈ar & PATHO & 12.5 & & & & & & & & & & \\
\hline 3,4 & Mar-May & FLOW & 250 & 'PATHO & 25.0 & & & & & & & & \\
\hline 5. & May-Oct & FLOWW & 23.4 & PẠTHŌO & 23.4 & & & & 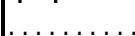 & & & & \\
\hline $6^{\circ}$ & Ött-Mar & "PATHÖO & 24.6 & TEMP & $24 . \dot{6}$ & & & & & & & & \\
\hline$" 7$ & Mar-jun & "ṔŔÉÉD" & 11.5 & & & & & & & & & & \\
\hline
\end{tabular}

Abundance level refers to the average abundance of spawners that spawn in the natal stream reaches that populate the life history pathways using the reach in this table Level 1: $\quad 0-10$ spawners

Level $2 \quad 10-30$ spawners

Level 3: $\quad 30-60$ spawners

Level 4: $>60$ spawners

Life stages: 1- prespawner; 2- spawner; 3- incubation; 4- fry colonization; 5- summer rearing; 6- overwintering; 7- yearling presmolt/smolt

$\begin{array}{lll}\text { Attributes: CHEM - chemicals } & \text { NUTLD - nutrient load } & \text { RIPCN - riparian condition } \\ \text { CSTAB - channel stability } & \text { OBST - obstructions } & \text { SEDLD - Sediment ioad } \\ \text { COMP - competition (with other species) } & \text { OXYG - dissolved oxygen } & \text { TEMP - water temperature } \\ \text { FLOW - flow } & \text { PATHO - pathogens } & \text { WITH - water withdrawals } \\ \text { HABD - habitat diversity } & \text { PRED - predators (includes fishing-related losses) }\end{array}$

"NA" indicates that no life history pathway meeting the criteria used in defining these pathwaywas present.

Blank cells under attributes indicate no additional attributes with negative effects on productivity were evident compared to those present in the template. 


\section{Stream Reach Summary for Primary and Secondary Spring Chinook Salmon Life Histories}

Stream: Imnaha R.

Reach: Dry Cr - Skookum Cr (RM 55.4 -61.9 from Snake R)

\begin{tabular}{ll|ll|lll}
\hline Restoration benefit category: & E & Chinook usage score: & 842.3 & Habitat productivity ioss score: & -92.5 \\
\hline
\end{tabular}

\begin{tabular}{|c|c|c|c|c|c|c|c|c|c|c|c|c|c|c|}
\hline \multirow[b]{3}{*}{ Life stage } & \multirow[b]{3}{*}{ Months } & \multicolumn{10}{|c|}{ No, life history pathways by current usage abundance level } & \multicolumn{2}{|c|}{ Key habitat change } & \multirow{3}{*}{$\begin{array}{c}\text { Habitat } \\
\text { productivity } \\
\text { loss score }\end{array}$} \\
\hline & & \multicolumn{5}{|c|}{ Primary } & \multicolumn{5}{|c|}{ Secondary } & \multirow{2}{*}{$\begin{array}{l}\text { Patient } \\
\text { quantii }\end{array}$} & \multirow[b]{2}{*}{$\%$ change } & \\
\hline & & Total no. & $T$ & 2 & 3 & 4 & Total no. & $T$ & 2 & $\overline{3}$ & 4 & & & \\
\hline & Apr-Aug & 17 & 3 & 12 & 2 & 0 & 48 & 12 & 30 & 6 & 0 & 9.4 & 0.0 & 0.0 \\
\hline 1.2 .3 & Äüg-Ött & $20 "$ & 0 & 20 & "O" & 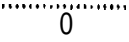 & $50^{\circ}$ & 0 & 50 & 0 & 0 & 7.7 & -52.8 & -41.2 \\
\hline 3 & Ott-Mar & 40 & 0 & $\dddot{40}$ & 0 & 0 & 100 & 0 & 100 & 0 & 0 & 7.7 & -60.0 & $\because 4$ \\
\hline 34. & Mar-May & 21 & 5. & 16 & 'o & 0 & 34 & $14^{\circ}$ & 16 & 4 & 0 & 8.3 & -265 & \\
\hline 5 & ,May-Oيtt & 74 & 25 & 48 & 1 & 0 & 100 & 75 & 23 & 2 & 0 & 8.6 & -1.5 & -366 \\
\hline 6 & Ott-Mar & 38 & 19 & 19 & "ִo." & $\dddot{0}$ & $0 "$ & 0 & 0 & 0 & 0 & 193 & 00. & -285 \\
\hline$\dot{7}$ & Mar-jun & 4 & 2 & 1 & $i$ & $\dddot{0}$ & 1 & 1 & 0 & $\dot{0}$ & 0 & 30.3 & 0.0 & -0.6 \\
\hline
\end{tabular}

\begin{tabular}{|c|c|c|c|c|c|c|c|c|c|c|c|c|c|}
\hline \multirow[b]{2}{*}{ Life stage } & & \multicolumn{12}{|c|}{ Highest productivity impact attributes $(\%$ change in impact from template) } \\
\hline & Months & Att 1 & $\%$ change & Att 2 & $\%$ change & Att 3 & $\%$ change & $\overline{\text { Att } 4}$ & $\%$ change & Att 5 & $\%$ change & Att 6 & $\%$ change \\
\hline & Apr-Aug & HABD & 19.7 & SEDLD & 19.7 & & & & & & & & \\
\hline $1,2,3$ & Aug-Ocl" & "HÄB̈D̆" & $25.0^{\circ}$ & "P̌R̈ËD" & 25.0 & "S̈ËËLË & $25.0^{\circ}$ & & & & & & \\
\hline 3 & Oct-Mar & '"'PATHO- & $25.0^{\circ}$ & & & & & & & & & & \\
\hline 34 & Mar-May & FLOW & 25.0 & РÄTḦO & 25.0 & & & & & & & & \\
\hline 5 & May-Ott & FLÓW & $25.0^{\circ}$ & "P̈ATHÖ & 25.0 & TEMP & 15,4 & & & & {$\left[\begin{array}{lll}* & .\end{array}\right.$} & & \\
\hline$\ddot{6}$ & Ött-M̈ar & PATHO & $250^{\circ}$ & "P̈RËË" & 25.0 & TEMP & 25.0 & & & & & & \\
\hline 7 & Mar-Jun & PRËD̈ & 15.2 & & & & & & & & & & \\
\hline
\end{tabular}

Abundancelevel refera to the average abundance of spawners that spawn in the natal stream reaches that populate the life history pathwaya using the reach in this table

$\begin{array}{ll}\text { Level 1: } & 0-10 \text { spawners } \\ \text { Level 2: } & 10-30 \text { spawners } \\ \text { Level 3: } & 30-60 \text { spawners } \\ \text { Level 4 } & >60 \text { apawners }\end{array}$

Life stages: 1- prespawner; 2- spawner; 3- incubation; 4- fry colonization; 5- summer rearing; 6- overwintering; 7- yearling presmoft/smelt

Aftributes: CHEM - chemicals

CSTAB - channel stability

COMP - competition (with other species)

FLOW - flow

HABD - habitat diversity
NUTLD - nutrient load

OBST - obstructions

OXYG - dissolved oxygen

PATHO - pathogens

PRED - predators (includes fishing-related losses)
RIPCN - riparian condition

SEDLD - sediment load

TEMP - water temperature

WITH - water withdrawals

"NA" indicates that no fife history pathway meeting the criteria used in defining these pathwayswas present.

Blank cells under attributes indicate no additional attributes with negative effects on productivity were evident compared to those present in the template, 


\section{Stream Reach Summary for Primary and Secondary Spring Chinook Salmon Life Histories}

\section{Stream: Imnaha R}

Reach: Blue Hole - forks (RM 66.4 -71.4 from Snake R)

\begin{tabular}{|ll|l|lll}
\hline Restoration benefit category: & E & Chinook usage score: & 495.0 & Habitat productivity loss score: & 45.6 \\
\hline
\end{tabular}

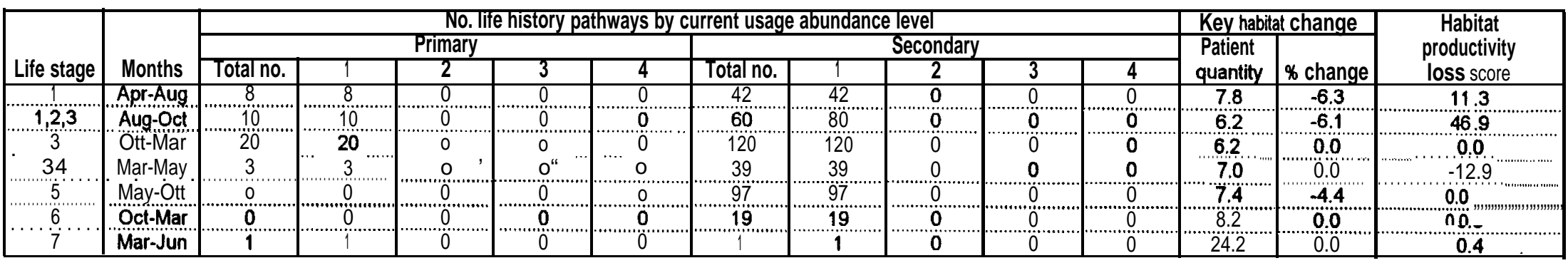

\begin{tabular}{|c|c|c|c|c|c|c|c|c|c|c|c|c|c|}
\hline \multirow[b]{2}{*}{ Life stage } & \multirow[b]{2}{*}{ Months } & \multicolumn{12}{|c|}{ Highest productivity impact attributes (\% change in impact from template) } \\
\hline & & Att 1 & \% change & Att 2 & \% change & Att 3 & $\%$ change & Att 4 & \% change & Att 5 & \% change & Att 6 & $\%$ change \\
\hline 1 & Apr-Aug & & & & & & & & & & & & \\
\hline $1,2,3$ & Aug-Oct & & & & & & & & & & & & \\
\hline 3 & Oct-Mar & РАTHO & 25.0 & & & & & & & & & & ․․․․…… \\
\hline 3,4 & Mar-May & PATHO & 25.0 & & & & & & & & & & \\
\hline 5 & May-Oct & PATHO & 24.5 & & & & & & & & & & \\
\hline 6 & Oct-Mar & PATHO & 25.0 & & & & & & & & & & \\
\hline 7 & Mar-jun & & & & & & & & & & & & \\
\hline
\end{tabular}

Abundance /eve/ refers to the average abundance of spawners that spawn in the natal stream reaches that populate the life history pathways using the reach in this table

$$
\begin{array}{ll}
\text { Level 1: } & 0-10 \text { spawners } \\
\text { Level 2: } & 10-30 \text { spawners } \\
\text { Level 3: } & 30-60 \text { spawners } \\
\text { Level 4 } & >60 \text { spawners }
\end{array}
$$

Life stages.' 1 -prespawner; 2- spawner; 3- incubation; 4- fry colonization; 5- summer rearing; 6- overwintering; 7- yearling presmolt/smolt

$\begin{array}{lll}\text { Attributes: CHEM - chemicals } & \text { NUTLD - nutrient load } & \text { RIPCN - riparian condition } \\ \text { CSTAB - channel stability } & \text { OBST - obstructions } & \text { SEDLD - Sediment load } \\ \text { COMP - competition (with other species) } & \text { OXYG - dissolved oxygen } & \text { TEMP - water temperature } \\ \text { FLOW - flow } & \text { PATHO - pathogens } & \text { WITH - water withdrawals } \\ \text { HABD - habitat diversity } & \text { PRED - predators (includes fishing-related losses) }\end{array}$

"NA" indicates that no life history pathway meeting the criteria used in defining these pathways was present.

Blank cells under attributes indicate no additional attributes with negative effects on productivity were evident compared to those present in the template, 


\section{Stream Reach Summary for Primary and Secondary Spring Chinook Salmon Life Histories}

Stream: Imnaha R.

Reach: Skookum Cr - Blue Hole (RM 61.9 -66.4 from Snake R)

Restoration benefit category: $E$

Chinook usage score:

\begin{tabular}{l|l}
\hline 753.4 & Habitat productivity loss score:
\end{tabular} $-84.8$

\begin{tabular}{|c|c|c|c|c|c|c|c|c|c|c|c|c|c|c|}
\hline \multirow[b]{3}{*}{ Life stage } & \multirow[b]{3}{*}{ Months } & \multicolumn{10}{|c|}{ No. life history pathways by current usage abundance level } & \multicolumn{2}{|c|}{ Key habitat change } & \multirow{3}{*}{$\begin{array}{c}\text { Habitat } \\
\text { productivity } \\
\text { loss score }\end{array}$} \\
\hline & & \multicolumn{5}{|c|}{ Primary } & \multicolumn{5}{|c|}{ Secondary } & \multirow{2}{*}{$\begin{array}{c}\text { Patient } \\
\text { quanlity }\end{array}$} & \multirow[b]{2}{*}{$\%$ change } & \\
\hline & & Total no. & 1 & 2 & 3 & 4 & Total no. & 1 & 2 & 3 & 4 & & & \\
\hline 12 & Apr-Aug & 15 & 3 & 0 & 12. & 0 & 46 & 12 & 0 & 36 & 0 & 9.2 & 0.0 & 0.0 \\
\hline $1,2,3$ & Aug-Ött & 20 & 0 & 0 & 20 & 0 & 60 & 0 & 0 & 60 & 0 & 7.7 & -51.0 & $.49 .7 "$ \\
\hline 3 & Ott-Mar & 40 & 0 & 0 & 40 & 0 & 120 & 0 & 0 & 120 & 0 & 7.7 & -60.0 & 0.3 \\
\hline $34, " ' \cdots$ & Mar-May & 18 & 2 & 0 & 16 & 0 & 29 & 5 & 0 & 24 & 0 & 8.7 & -31.9 & 0.1 \\
\hline 5 & May-Ott & 48 & 0 & 0 & 48 & 0 & 52 & 6 & 0 & 46 & 0 & 8.7 & 0.0 & -20.8 \\
\hline 6 & Ọtt-Mar & 19 & 0 & $\dddot{00}$ & 19 & 0 & 0 & 0 & 0 & 0 & 0 & 19.3 & 0.0 & .143 \\
\hline 7 & Mar-Jun & 2 & $1 "$ & 0 & "'i" & i & 1 & $1^{\circ}$ & n" & $n$ & in & $3 n 3$ & nก & $\Omega$ \\
\hline
\end{tabular}

\begin{tabular}{|c|c|c|c|c|c|c|c|c|c|c|c|c|c|}
\hline \multirow[b]{2}{*}{ Life stage } & \multirow[b]{2}{*}{ Months } & \multicolumn{12}{|c|}{ Highest productivity impact attributes (\% change in impact from template) } \\
\hline & & Att 1 & $\%$ change & Att 2 & \% change & Att 3 & $\%$ change & Att 4 & \% change & Att 5 & \% change & Att 6 & \% change \\
\hline 1 & Apr-Aug & HABD & 21.5 & SEDLD & 21.5 & PRED & 8.3 & & & & & & \\
\hline $1,2,3$ & Aug-Oct & HABD & 16.7 & SEOLLD & 16.7 & PRED & 8.3 & & & & & & \\
\hline 3 & Oct-Mar & РАТНО & 12.5 & & & & & & & & & & \\
\hline 3,4 & Mar-May & FLOW & 25.0 & PATHO & 25.0 & & & & & & & & \\
\hline 5 & May-Oct & FLOW & 24.1 & PATHO & 24.1 & & & & & & & & \\
\hline 6 & Oct-Mar & PATHO & 250 & TEMP & 25.0 & & & & & & & & \\
\hline 7 & Mar-Jun & PRED & 14.9 & & & & & & & & & & \\
\hline
\end{tabular}

Abundance /eve/ refers to the average abundance of spawners that spawn in the natal stream reaches that populate the life history pathways using the reach in this table

Level 1: $\quad 0-10$ spawners

Level 2: $\quad 10-30$ spawners

Level 3: $\quad 30-60$ spawners

Level 4: $>60$ spawners

Life stages: 1-prespawner; 2- spawner; 3- incubation; 4- fry colonization; 5- summer rearing; 6- overwintering; 7- yearling presmolt/smolt

Attributes: CHEM - chemicals

CSTAB - channel stability

COMP - competition (with other species)

FLOW - ftOW

HABD - habitat diversity
NUTLD - nutrient load

OBST - obstructions

OXYG - dissolved oxygen

PATHO - pathogens

PRED - predators (includes frshing-related losses)
RIPCN - riparian condition

SEDLD - sediment load

TEMP - water temperature

WITH - water withdrawals

"NA" indicates that no life history pathway meeting the criteria used in defining these pathways was present.

Blank cells under attributes indicate no additional attributes with negative effects on productivity were evident compared to those present in the template. 


\section{Stream Reach Summary for Primary and Secondary Spring Chinook Salmon Life Histories}

Stream: Imnaha R.

Reach: South Fork Imnaha R (RM 71.4 -76.1 from Snake R)

\begin{tabular}{ll|ll|ll|}
\hline Restoration benefit category: & $\mathrm{E}$ & Chinook usage score: & 613.2 & Habltat productivity loss score: & 52.9 \\
\hline
\end{tabular}

\begin{tabular}{|c|c|c|c|c|c|c|c|c|c|c|c|c|c|c|}
\hline \multirow[b]{3}{*}{ Life stage } & \multirow[b]{3}{*}{ Montha } & \multicolumn{10}{|c|}{ No. life history pathways by current usage abundance level } & \multicolumn{2}{|c|}{ Key habitat change } & \multirow{3}{*}{$\begin{array}{c}\text { Habitat } \\
\text { productivity } \\
\text { loss score }\end{array}$} \\
\hline & & \multicolumn{5}{|c|}{ Primary } & \multicolumn{5}{|c|}{ Secondary } & Patient & & \\
\hline & & Total no. & 1 & 2 & 3 & 4 & Total no. & 1 & 2 & 3 & 4 & quantity & $\%$ change & \\
\hline 1 & Apr-Aug & 12 & 12 & 0 & 0 & 0 & 36 & 36 & 0 & 0 & 0 & 5.0 & -56 & 117 \\
\hline 123 & Aug-Ött & 20 & 20 & 0 & 0 & 0 & 60 & $60^{\circ}$ & 0 & 0 & 0 & 4.6 & 0.0 & 536 \\
\hline 3 & Ott-Mar & $40^{\circ}$ & 40 & 0 & 0 & 0 & $t 20$ & 120 & 0 & 0 & 0 & 4.6 & $0.0^{\circ}$ & 00 \\
\hline 34 & Mar-May & 10 & 10 & 0 & 0 & 0 & 30 & 30 & 0 & 0 & 0 & 5.0 & 0.0 & -12.5 \\
\hline 5 & May-Ott & 25 & 25 & 0 & 0 & 0 & 69 & 69 & 0 & 0 & 0 & 5,0 & -2.6 & 0.0 \\
\hline 6 & Ott-Mar & 19 & 19 & ". & $\dddot{0}$ & $" 0$ & "ö & 0 & "0 & 0 & "ö & 4.6 & 0.0 & 00 \\
\hline 7 & Mar-Jun & $i$ & 1 & 0 & 0 & 0 & 0 & 0 & $\dot{0}$ & 0 & 0 & 18.2 & 0.0 & 0.0 \\
\hline
\end{tabular}

\begin{tabular}{|c|c|c|c|c|c|c|c|c|c|c|c|c|c|}
\hline \multirow[b]{2}{*}{ Life stage } & \multirow[b]{2}{*}{ Months } & \multicolumn{12}{|c|}{ Highest productivity impact attributes (\% change in impact from template) } \\
\hline & & $\overline{A t t 1}$ & $\%$ change & $\overline{\text { Aft } 2}$ & $\%$ change & Att 3 & $\%$ change & Att 4 & $\%$ change & Att 5 & $\%$ change & Alt 6 & \% change \\
\hline 1 & Apr-Aug & & & & & & & & & & & & \\
\hline $1,2,3$ & Aug-Oct & & & & & & & & & & & & \\
\hline 3 & Oct-Mar & PATHO & 12.5 & & & & & & & & & & \\
\hline 3,4 & Mar-May & PATHO & 16.3 & & & & & & & & & & \\
\hline 5 & May-Oct & PATHO & 25.0 & & & & & & & & & & \\
\hline 6 & Oct-Mar & PATHO & 25.0 & & & & & & & & & & \\
\hline$\dddot{7}$ & Mar-Jun & & & & & & & & & & & & \\
\hline
\end{tabular}

Abundance lovel refers to the average abundance of spawners that spawn in the natal stream reaches that populate the life history pathways using the reach in this table

$$
\begin{array}{ll}
\text { Level 1: } & 0-10 \text { spawners } \\
\text { Level 2 } & 10-30 \text { spawners } \\
\text { Level 3: } & 30-60 \text { spawners } \\
\text { Level 4 } & >60 \text { spawners }
\end{array}
$$

Life stages: 1- prespawner; 2- spawner; 3- incubation; 4- fry colonization; 5- summer rearing; 6- overwintering; 7- yearling preamott/smelt

Attributes: CHEM - chemicals

CSTAB - channel stability

COMP - competition (with other species)

FLOW - flow

HABD - habitat diversity
NUTLD - nutrient load

OBST - obstructions

OXYG - dissolved oxygen

PATHO - pathogens

PRED - predators (includes fishing-related losses)
RIPCN - riparian condition

SEDLD - sediment load

TEMP - water temperature

WITH - water withdrawal

"NA" indicates that no life history pathway meeting the criteria used in defining these pathways was present.

Blank cells under attributes indicate no additional attribute with negative effects on productivity were evident compared to those present in the template. 
Stream Reach Summary for Primary and Secondary Spring Chinook Salmon Life Histories

Stream: Lightning $\mathrm{Cr}$

Reach: $\quad$ Mouth - forks (RM 5.4- 13.9 from Snake R)

\begin{tabular}{|llll|lll}
\hline Restoration benefit category: & $\mathrm{F}$ & [Chinook usage score: & 108.7 & Habltat productivity ioss score: & 14.5 \\
\hline
\end{tabular}

\begin{tabular}{|c|c|c|c|c|c|c|c|c|c|c|c|c|c|c|}
\hline \multirow[b]{3}{*}{ Life stage } & \multirow[b]{3}{*}{ Months } & \multicolumn{10}{|c|}{ No. life history pathways by current usage abundance level } & \multicolumn{2}{|c|}{ Key habitat change } & \multirow{3}{*}{$\begin{array}{c}\text { Habitat } \\
\text { productivity } \\
\text { loss score }\end{array}$} \\
\hline & & \multicolumn{5}{|c|}{ Primary } & \multicolumn{5}{|c|}{ Secondary } & \multirow{2}{*}{$\begin{array}{l}\text { Patient } \\
\text { quantity }\end{array}$} & \multirow[b]{2}{*}{$\%$ change } & \\
\hline & & Total no. & 1 & 2 & 3 & 4 & Total no. & 1 & 2 & 3 & 4 & & & \\
\hline 1 & $\overline{\text { Apr-Aug }}$ & 9 & 9 & 0 & 0 & 0 & 9 & & 0 & & 0 & 0.6 & 0.0 & \\
\hline $1,2,3$ & Aug-Oct & $10^{\circ}$ & $10^{\circ}$ & $\dot{0}$ & 0 & 0 & 10 & 9 & $\dot{0}$ & & 0 & 0.45 & 0 & \\
\hline 3,4 & Mar-May & 8 & 8 & "“" " "Q & "o"'” & 0 & 4 & 4 & 0 ", & 0 & 0 & $0.6 " ' ”$ & 6.3 & 3.2 \\
\hline 5 & May-Oct & 25 & 25 & 0 & $0 "$ & 0 & 0 & 0 & 0 & $\dot{0}$ & 0 & 0.5 & 0.0 & 0.0 \\
\hline 6 & Oct-Mar & 19 & 19 & 0 & 0 & 0 & $\ddot{0}$ & 0 & 0 & 0 & 0 & 0.5 & 16 & 1.6 \\
\hline 7 & Mar-Jun & 3 & $\dot{3}$ & 0 & $\dot{0}$ & 0 & 0 & 0 & 0 & 0 & 0 & 13.2 & 4.8 & 0.4 \\
\hline
\end{tabular}

\begin{tabular}{|c|c|c|c|c|c|c|c|c|c|c|c|c|c|}
\hline \multirow[b]{2}{*}{ Lifiesthgec } & \multirow[b]{2}{*}{ Matorthe } & \multicolumn{12}{|c|}{ Highest productivity impact attributes (\% change in impact from template) } \\
\hline & & Att 1 & $\%$ change & $\overline{\text { Att } 2}$ & \% change & Att 3 & $\%$ change & $\overline{\text { Att } 4}$ & $\%$ change & Att 5 & $\%$ change & Att 6 & $\%$ change \\
\hline 1 & Apr-Aug & FLOW & 15.2 & & & & & & & & & & \\
\hline 12,3 & Âug-Oct & FLOW & 5.5 & & & & $\cdot$ & & & & & & \\
\hline 3 & Ottivinar & "PATHO & 8.2 & & & & & & & & & & \\
\hline 3,44 & Mar-May & COMP & 7.8 & PATHO & 7.8 & & & & & & & & \\
\hline 5 & May-Odt & COMP & 7.8 & PATHO & 7.8 & & & & & & & & \\
\hline 6 & "Othidar & PATHO & 7.8 & & & & & & & & & & \\
\hline 7 & Mar-jun & & & & & & & & & & & & \\
\hline
\end{tabular}

Abundance /eve/ refers to the average abundance of spawners that spawn in the natal stream reaches that populate the life history pathways using the reach in this table:
Level 1: $\quad 0-10$ spawnera
Level 2 10-30 spawners
Level 3: $\quad 30-60$ spawners
Level $4>60$ spawnera

Life stages: 1 - prespawner; 2- spawner; 3- incubation; 4- fry colonization; 5- summer rearing; 6- overwintering; 7- yearling presmolt/smolt

\begin{tabular}{|c|c|c|}
\hline $\begin{array}{l}\text { Attributes: } \mathrm{CHEM} \mathrm{-} \mathrm{chemicals} \\
\text { CSTAB - channel stability } \\
\text { COMP - competition (with other species) } \\
\text { FLOW - flow } \\
\text { HABD - habitat diversity }\end{array}$ & $\begin{array}{l}\text { NUTLD - } \\
\text { OBST - } \quad \text { obstrient ioad } \\
\text { OXYG - } \quad \text { dissolved oxygen } \\
\text { OXYG } \\
\text { PATHO - pathogens } \\
\text { PRED - } \quad \text { predators (includes fishing-related losses) }\end{array}$ & $\begin{array}{l}\text { RIPCN - riparian condition } \\
\text { SEDLD - } \\
\text { Tediment load } \\
\text { TEMP - } \quad \text { water temperature } \\
\text { WiTH - } \quad \text { water withdrawals }\end{array}$ \\
\hline
\end{tabular}

"NA" indicates that no life history pathway meeting the criteria used in defining these pathways was present.

Blank cells under attributes indicate no additional attributes with negative effects on productivity were evident compared to those present in thetemplate. 
Stream Reach Summary for Primary and Secondary Spring Chinook Salmon Life Histories

Stream: Little Sheep Cr.

Reach: Mouth - Bear Gulch (RM 25.4 -28.5 from Snake R)

\begin{tabular}{|ll|ll||lll|}
\hline Restoration benefit category: & $F$ & Chinook usage score: & 0.0 & Habitat productivity ioss score: & 0.0 \\
\hline
\end{tabular}

\begin{tabular}{|c|c|c|c|c|c|c|c|c|c|c|c|c|c|c|}
\hline \multirow[b]{3}{*}{ Life stage } & \multirow[b]{3}{*}{ Months } & \multicolumn{10}{|c|}{ No. life history pathwsys by current usage abundance level } & \multicolumn{2}{|c|}{ Key habitat change } & \multirow{3}{*}{$\begin{array}{c}\text { Habitat } \\
\text { productivity } \\
\text { 10ss score }\end{array}$} \\
\hline & & \multicolumn{5}{|c|}{ Primary } & \multicolumn{5}{|c|}{ Secondary } & Patient & & \\
\hline & & Total no. & 1 & 2 & 3 & 4 & Total no. & 1 & 2 & 3 & 4 & quantity & $\%$ change & \\
\hline & Apr-Aug & $\overline{N A}$ & $\mathrm{NA}$ & NA & NA & $\overline{N A}$ & $\overline{N A}$ & $\overline{N A}$ & NA & $\overline{N A}$ & $\overline{N A}$ & NA & NA & NA \\
\hline $1,2,3$ & Aug-Ött & $\mathrm{NA}$ & "NA" & $\mathrm{NA}$ & Nä & NA & $N A{ }^{\prime}$ & $\dot{N A}$ & $\mathrm{NA}$ & $\mathrm{NA}$ & $\mathrm{NA}$ & NA & $N A$ & $N A$ \\
\hline 34 & Mar-May & NA & NA & $\mathrm{NA}^{\prime}$ & NA & $N A$ & NA & NA & NA & NA & NA & NA & NA & NA \\
\hline 5 & May-Ott & $\mathrm{NA}$ & $\mathrm{NA}$ & NA & NÄ & $\mathrm{NA}$ & $\mathrm{NÄ}$ & $\mathrm{NA}$ & $\mathrm{NA}$ & $\mathrm{NA}$ & NA & $\dot{N} \dot{A}$ & NA & NA \\
\hline 6 & Öt-Mar & $\mathrm{NA}$ & $\mathrm{NA}$ & $\mathrm{NA}$ & $\mathrm{NA}$ & $\mathrm{NA}$ & $\mathrm{NA}$ & $\mathrm{NA}$ & $\mathrm{NA}$ & $\mathrm{NA}$ & $\mathrm{NA}$ & $\mathrm{NA}$ & "NÄ" & $\mathrm{NÄ}$ \\
\hline 7 & Mar-Jun & $\mathrm{NA}$ & NA & NA & $\dot{N} A^{\circ}$ & $\dot{N A}$ & NA & $\mathrm{NA}$ & $\mathrm{NA}$ & $\mathrm{NA}$ & $\mathrm{NA}$ & $\mathrm{NA}$ & NA & $\mathrm{NA}$ \\
\hline
\end{tabular}

\begin{tabular}{|c|c|c|c|c|c|c|c|c|c|c|c|c|c|}
\hline \multirow[b]{2}{*}{ Life stage } & \multirow[b]{2}{*}{ Months } & \multicolumn{12}{|c|}{ Highest productivity impact attributes (\% change in impact from template) } \\
\hline & & Aft 1 & $\%$ change & Att 2 & \% change & Att 3 & $\%$ change & Att 4 & $\%$ change & Att 5 & $\%$ change & Att 6 & $\%$ change \\
\hline & Apr-Aug & NA & NA & NA & NA & NA & NA & NA & NA & NA & NA & NA & NA \\
\hline$i, 2,3$ & Aug-Oct & NÄ & NÄ & "NÄ & $\dot{N A}$ & NA & NA & ì̈ & $\mathrm{NÄ}$ & NA & $\ddot{\mathrm{NA}}$ & $\mathrm{NA}$ & $\mathrm{NA}$ \\
\hline 3 & Ött-Mar & NÄ & N̈A & $\mathrm{NA}$ & $\mathrm{NA}$ & ïA & $\mathrm{NA}$ & $\mathrm{NA}$ & $\mathrm{NA}$ & $\mathrm{NA}$ & Y? & $\mathrm{NA}$ & $\mathrm{NÄ}$ \\
\hline 3,4 & Mar-May & $\dot{N A}$ & NA & NA & NA & NA & NA & NA & NA & NA & NÄ & $\mathrm{NA}$ & $N A$ \\
\hline 5 “ & May-Ott & NA & NA & NA & NA & $\mathrm{N} A$ & NA & "NA & $\mathrm{N} A$ & NA & NA & NA & NA \\
\hline 6 & Ótt-Mar & $\mathrm{NA}$ & NA & NA & $\mathrm{NA}$ & NA & $\mathrm{NA}$ & NA & $\mathrm{NA}$ & NA & $\mathrm{NA}$ & $\mathrm{NA}$ & $\mathrm{NA}$ \\
\hline 7 & Mar-Jun & $\mathrm{NÄ}$ & $\mathrm{NA}$ & NAA" & $\mathrm{NA}$ & $\mathrm{NA}$ & $\mathrm{NA}$ & $\mathrm{NA}$ & $\mathrm{NA}$ & $\mathrm{NA}$ & $\mathrm{NA}$ & $\mathrm{NA}$ & $\mathrm{NA}$ \\
\hline
\end{tabular}

Abundance/eve/refers to the average abundance of spawners that spawn in the natal stream reaches that populate the life history pathways using the reach in this table

$$
\begin{array}{ll}
\text { Level 1: } & 0-10 \text { spawners } \\
\text { Level 2 } & 10-30 \text { spawners } \\
\text { Level 3 } & 30-60 \text { spawners } \\
\text { Level } 4 & >60 \text { spawners }
\end{array}
$$

Life stages: 1- prespawner; 2- spawner; 3- incubation; 4- fry colonization; 5- summer rearing; 6- overwintering; 7- yearling presmolt/smolt

\section{Attributes: CHEM - chemicals}

CSTAB - channel stability

COMP - competition (with other species)

FLOW - fiow

HABD - habitat diversity
NUTLD - nutrient load

OBST - obstructions

OXYG - dissoived oxygen

PATHO - pathogens

PRED - predators (includes fishing-related losses)
RIPCN - riparian condition

SEDLD - sediment ioad

TEMP - water temperature

WiTH - water withdrawsis

"NA" indicates that no fife history pathway meeting the criteria used in defining these pathways was present.

Blank ceils under attributes indicate no additional attributes with negative effects on productivity were evident compared to those present in the template. 
Stream Reach Summary for Primary and Secondary Spring Chinook Salmon Life Histories

Stream: Little Sheep Cr.

Reach: Bear Gulch - Devil's Gulch (RM 28.5 -30.9 from Snake R)

\begin{tabular}{|c|c|c|c|c|c|c|c|c|c|c|c|c|c|c|}
\hline \multicolumn{4}{|c|}{ Restoration benefit category: } & $\mathbf{F}$ & \multicolumn{3}{|c|}{ Chinook usage score: } & .0 & \multicolumn{5}{|c|}{ [Habitat productivity loss score: } & \\
\hline \multirow[b]{3}{*}{ Life stage } & \multirow[b]{3}{*}{ Months } & \multicolumn{10}{|c|}{ No. life history pathways by current usage abundance level } & \multicolumn{2}{|c|}{ Key habitat change } & \multirow{3}{*}{$\begin{array}{c}\text { Habitat } \\
\text { productivity } \\
\text { loss soore }\end{array}$} \\
\hline & & \multicolumn{5}{|c|}{ Primary } & \multicolumn{5}{|c|}{ Secondary } & \multirow{2}{*}{$\begin{array}{l}\text { Patient } \\
\text { quantity }\end{array}$} & \multirow[b]{2}{*}{$\%$ change } & \\
\hline & & Total no. & 1 & 2 & 3 & 4 & Total no. & $T$ & 2 & 3 & 4 & & & \\
\hline 1 & Apr-Aug & NA & NA & NA. & NA & $\overline{N A}$ & NA & NA. & $\overline{N A}$ & $\mathrm{NA}$ & NA & NA & NA & NA \\
\hline 10.3 & Äug-Ött & $\mathrm{NA}$ & NA & $\hat{N A}$ & $\mathrm{NA}$ & $\mathrm{NA}$ & $\mathrm{NA}$ & $\mathrm{NA}$ & NA & NA & $\mathrm{NA}$ & NA & $\mathrm{NA}$ & NA \\
\hline 3 & Ott-Mar & NA & $N A$ & NA & NA & $\mathrm{NA}$ & NA & NA & NA & NA & NA & NA & $\dot{\mathrm{NA}}$ & $\dot{N A}$ \\
\hline 34 & Mar-May & NA & $\mathrm{N} A$ & $\mathrm{NA}$ & NA & $N A$ & $\mathrm{NA}$ & NA & NA & NA & NA & NA. & NA & NA \\
\hline 5 & May-Ott & $\mathrm{NA}$ & $\mathrm{NA}$ & $\mathrm{NA}$ & NA & NA & NA & NA & NÄ & NA & $\mathrm{NA}$ & $\dot{N A}$ & NA" & NA \\
\hline 7 & Mar-Jun & NA & NA & NA. & $\mathrm{NA}$ & NA: & NA. & NA & $\mathrm{NA}$ & NA & $\mathrm{NA}$ & NA & $\mathrm{NA}$ & $\mathrm{NA}$ \\
\hline
\end{tabular}

\begin{tabular}{|c|c|c|c|c|c|c|c|c|c|c|c|c|c|}
\hline \multirow[b]{2}{*}{ Life stage } & \multirow[b]{2}{*}{ Montha } & \multicolumn{12}{|c|}{ Highest productivity impact attributes (\% change in impact from template) } \\
\hline & & Att 1 & $\%$ change & Att 2 & $\%$ change & Att 3 & $\%$ change & Att 4 & $\%$ change & Att 5 & $\%$ change & Atf 6 & \% change \\
\hline 1 & Apr-Aug & $\mathrm{NA}$ & NA & NA & $\mathrm{NA}$ & NA & NA & $\overline{\mathrm{NA}}$ & $\mathrm{NA}$ & NA & NA & NA & NA \\
\hline 12,3 & Äug-Oct & NA & $\dot{N} \dot{A}$ & $\mathrm{NA}$ & NA & $N A$ & NA & NA & NA & NA & NA & NA & NA \\
\hline 3 & Ött-M̆är & NA" & NÄ" & NÄ" & $\mathrm{NA}$ & NÄ & $\mathrm{NA}$ & $\mathrm{NA}$ & NA & NA & $N A$ & $N A$ & NA \\
\hline 3,4 & Mar-May & NA & NA & NA & NA & NA & & NA & NA & NA & Na & Na & NA. \\
\hline 5 & May-Ott & "NÄ" & NA & $\mathrm{NA}$ & NÄ' & NA & NA & $N A$ & $N A$ & $\mathrm{NA}$ & & & $\%$ \\
\hline 6 & Ott-Mar & $N A$ & NA & NA & NA & NA & NA & NA & NA & $\mathrm{NA}$ & & & \\
\hline 7 & Mar.jun & 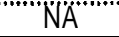 & "NÄ" & $\mathrm{NA}$ & $\mathrm{NA}$ & $\mathrm{NA}$ & NA & $\mathrm{NA}$ & $\mathrm{NA}$ & $\mathrm{NA}$ & $\mathrm{NA}$ & $\mathrm{NA}$ & $\mathrm{NA}$ \\
\hline
\end{tabular}

Abundance /eve/ refers to the average abundance of spawners that spawn in the natal stream reaches that populate the life history pathways using the reach in this table $\begin{array}{ll}\text { Level 1: } & 0-10 \text { spawners } \\ \text { Level 2: } & 10-30 \text { spawners } \\ \text { Level 3: } & 30-60 \text { spawners } \\ \text { Level 4: } & \mathbf{>} 60 \text { spawners }\end{array}$

Life stages: 1 - prespawner; 2- spawner; 3- incubation; 4- fry colonization; 5- summer rearing; 6- overwintering; 7-yearling presmolt/smolt

Attributes: CHEM - chemicals

CSTAB - channel stability

COMP - competition (with other species)

FLOW - ffow

HABD - habitat diversity
NUTLD - nutrient load

OBST - obstructions

OXYG - dissolved oxygen

PATHO - pathogens

PRED - predators (includes fishing-related losses)
RIPCN - riparian condition

SEDLD - sediment load

TEMP - water temperature

WITH - water withdrawals

"NA" indicates that no life history pathway meeting the criteria used in defining these pathways was present.

Blank cells under attributes indicate no additional attributes with negative effects on productivity were evident compared to those present in the template. 
Stream Reach Summary for Primary and Secondary Spring Chinook Salmon Life Histories

Stream: Little Sheep Cr.

Reach: Devil's Gulch - Lightning $\mathrm{Cr}$ (RM 30.9 -33.1 from Snake R)

\begin{tabular}{|ll|ll|lll}
\hline Restoration benefit category: & $F$ & Chinook usage score: & 0.0 & Habitat productivity loss score: & 0.0 \\
\hline
\end{tabular}

\begin{tabular}{|c|c|c|c|c|c|c|c|c|c|c|c|c|c|c|}
\hline \multirow[b]{3}{*}{ Life stage } & \multirow[b]{3}{*}{ Months } & \multicolumn{10}{|c|}{ No. life history pathways by current usage abundance level } & \multicolumn{2}{|c|}{ Key habitat change } & \multirow{3}{*}{$\begin{array}{c}\text { Habitat } \\
\text { productivity } \\
\text { loss score }\end{array}$} \\
\hline & & \multicolumn{5}{|c|}{ Primary } & \multicolumn{5}{|c|}{ Secondary } & \multirow{2}{*}{$\begin{array}{l}\text { Patient } \\
\text { quantity }\end{array}$} & \multirow[b]{2}{*}{$\%$ chanae } & \\
\hline & & Total no. & 1 & 2 & 3 & 4 & Total no. & 1 & 2 & 3 & 4 & & & \\
\hline 1 & Apr-Aug & $\mathrm{NA}$ & NA & $\mathrm{NA}$ & $\mathrm{NA}$ & $\mathrm{NA}$ & NA & $\overline{N A}$ & $\overline{\mathrm{NA}}$ & $\mathrm{NA}$ & $\mathrm{NA}$ & NA & NA & NA \\
\hline 123 & Âug-Ött & $\mathrm{NA}$ & $\mathrm{NA}$ & NÄ & $\mathrm{NA}$ & NA & $\mathrm{NA}$ & NA & $N A$ & "NA" & $\mathrm{NA}$ & NÁ' & "NA" & "NA" \\
\hline 3 & Ott-Mar & NA & NA & NA & NA & NA & NA & NA & NA & NA & NA & NA & NA & NA \\
\hline 34 & Mar-May & $\mathrm{NA}$ & NA & NA & NA & NA & NA & NA & NA & NA & NA & $\mathrm{N}$ & NA & NA \\
\hline 5 & May-Ott & "NÄ & NA & NA & $\mathrm{NA}$ & NA & $\mathrm{NA}$ & NA & NA & NA & NA & NA & NA & NA \\
\hline 6 & ontiolar. & NA & NA & NA. & NA & $\mathrm{NA}$ & $\mathrm{NA}$ & NA: & $\mathrm{NA}$ & $\mathrm{NA}$ & $\mathrm{NA}$ & $\mathrm{NA}$ & $\mathrm{NA}$ & $\mathrm{NA}$ \\
\hline 7 & Marillin $\lfloor\ldots . . .1$ & & NA,$\cdots$ & $\ldots$ & NA & NA & NA & NA & $\mathrm{N}$ & $\mathrm{N} A$ & & NA & & $\mathrm{NA}$ \\
\hline
\end{tabular}

\begin{tabular}{|c|c|c|c|c|c|c|c|c|c|c|c|c|c|}
\hline \multirow[b]{2}{*}{ Life stage } & \multirow[b]{2}{*}{ Months } & \multicolumn{12}{|c|}{ Highest productivity impact attributes ( $\%$ change in impact from template) } \\
\hline & & Att 1 & $\%$ change & Att 2 & $\%$ change & Att 3 & $\%$ change & Att 4 & $\%$ change & Att 5 & $\%$ change & Att 6 & $\%$ change \\
\hline 1 & Apr-Aug & NA & NA & NA & NA & NA & NA & NA & NA & NA & NA & NA & NA \\
\hline 1,23 & Aug-Oct & NA & NÄ Kं & NA & NA & NA & NA & NA & NA & NÄ & $\%$ & NA & \\
\hline 3 & OOtt-Mar" & $\mathrm{NÄ}$ & $\mathrm{NA}$ & NA & $\mathrm{NA}$ & $\mathrm{NA}$ & NA & NÄ & "NA' & NA & & NAA & \\
\hline 3,4 & Mar-May & NA & NA & $\mathrm{NA}$ & NA & NA & NA & NA & $N A$ & NA & NA & NA & NA \\
\hline 5 & ,May=-Qtt, & NA & NA & NA & $\mathrm{NA}$ & NA & $\mathrm{NA}$ & $\mathrm{NA}$ & NA & NA & NA & NA & NA \\
\hline 6 & Ott-Mar & $\mathrm{NA}$ & $\mathrm{NA}$ & $\mathrm{NA}$ & $\mathrm{NA}$ & $\mathrm{NA}$ & NA & NA & NA & NA & NÁ & NA & "NA" \\
\hline 7 & Mar-Jun & $\mathrm{NA}$ & $\mathrm{NA}$ & $\mathrm{NA}$ & NA & NA & NA & NA & NÄ & $\mathrm{NA}$ & $\mathrm{NA}$ & $\mathrm{NA}$ & NA \\
\hline
\end{tabular}

Abundance lovel refers to the average abundance of spawners that spawn in the natal stream reaches that populate the life history pathways using the reach in this table

$$
\begin{array}{ll}
\text { Level 1: } & 0-10 \text { spawners } \\
\text { Level 2: } & 10-30 \text { spawners } \\
\text { Level 3: } & 30-60 \text { spawners } \\
\text { Level 4 } & >60 \text { spawners }
\end{array}
$$

Life stages: 1 - prespawner; 2- spawner; 3- incubation; 4- fry colonization; 5- summer rearing; 6- overwintering; 7- yearling presmolt/smolt

Attributes: CHEM - chemicals

CSTAB - channel stability

COMP - competition (with other species)

FLOW - flow

HABD - habital diversity
NUTLD - nutrient load

OBST - obstructions

OXYG - dissolved oxygen

PATHO - pathogens

PRED - predators (includes fishing-related losses)
RIPCN - riparian condition

SEDLD - sediment load

TEMP - water temperature

WITH - water withdraws

"NA" indicates that no life history pathway meeting the criteria used in defining these pathways was present.

Blank cells under attributes indicate no additional attributes with negative effects on productivity were evident compared to those present in the template, 
Stream Reach Summary for Primary and Secondary Spring Chinook Salmon Life Histories

Stream: Little Sheep Cr.

Reach: Lightning $\mathrm{Cr}$ - Hayden $\mathrm{Cr}(\mathrm{RM} 33.1$-38.8 from Snake R)

\begin{tabular}{|c|c|c|c|c|c|c|c|c|c|c|c|c|c|c|}
\hline \multicolumn{4}{|c|}{ Restoration benefit category: } & & \multicolumn{3}{|c|}{ Chinook usage score: } & $\overline{0.0}$ & \multicolumn{5}{|c|}{ Habitat productivity loss score: } & \multirow{4}{*}{$\begin{array}{l}\text { Habitat } \\
\text { productivity } \\
\text { 10ss score }\end{array}$} \\
\hline \multirow[b]{3}{*}{ Life stage } & \multirow[b]{3}{*}{ Months } & \multicolumn{10}{|c|}{ No, life history pathways by current usage abundance level } & \multicolumn{2}{|c|}{ Key habitat change } & \\
\hline & & \multicolumn{5}{|c|}{ Primary } & \multicolumn{5}{|c|}{ Secondary } & \multirow{2}{*}{$\begin{array}{l}\text { Patient } \\
\text { quantity }\end{array}$} & \multirow[b]{2}{*}{$\%$ change } & \\
\hline & & Total no. & 1 & 2 & 3 & 4 & Total no. & 1 & 2 & 3 & 4 & & & \\
\hline 1 & Apr-Aug & NA & $\overline{N A}$ & $\overline{\mathrm{NA}}$ & $\overline{\mathrm{NA}}$ & NA & $\overline{\mathrm{NA}}$ & $\overline{\mathrm{NA}}$ & $\overline{N A}$ & $\mathrm{NA}$ & $\mathrm{NA}$ & $\overline{N A}$ & NA & $\overline{\mathrm{NA}}$ \\
\hline 123 & Aug-Oct & $\mathrm{NA}$ & $\mathrm{NA}$ & NA & NA & NA & NA & NA & NA & NA & NA & NA & $\mathrm{NA}$ & $\mathrm{NA}$ \\
\hline 3 & Ott-M̈ar & NA & NA & NA & NA & NA & NA & NA & NA & NA & NA & NA & NA & NA \\
\hline 34 & Mar-May & NA & NA & NA & NA & NA & NA & NA & NA & NA & NA & NA & NA & NA \\
\hline 5 & May-Ött & ìA & NA & NA & NA & NA & NA & NA & NA & $\dot{N A}$ & "NÄ & NÄ & NA & $\mathrm{NÄ}$ \\
\hline 6 & Ött-Mar & $\dot{N A}$ & $\mathrm{NA}$ & $\mathrm{NA}$ & NÄ & $\mathrm{NA}$ & $\mathrm{NA}$ & NA & $\mathrm{NA}$ & NA" & $\mathrm{NA}$ & $\mathrm{NA}$ & $\mathrm{NA}$ & $\mathrm{NÄ}$ \\
\hline 7 & Mar-Jun & $\ddot{\mathrm{NA}}$ & NA & NA & NA & NA & NA & NA & NA & NA & NA & NA & NA & NA \\
\hline
\end{tabular}

\begin{tabular}{|c|c|c|c|c|c|c|c|c|c|c|c|c|c|}
\hline \multirow[b]{2}{*}{ Life stage } & \multirow[b]{2}{*}{ Months } & \multicolumn{12}{|c|}{ Highest productivity impact attributes (\% change in impact from template) } \\
\hline & & Att 1 & $\%$ change & Att 2 & $\%$ change & Att 3 & $\%$ change & Att 4 & $\%$ change & Att 5 & $\%$ change & Att 6 & $\%$ change \\
\hline & Apr-Aug & NA & $\overline{N A}$ & NA & $\overline{N A}$ & NA & $\overline{N A}$ & $\overline{\mathrm{NA}}$ & NA & NA & $\overline{\mathrm{NA}}$ & NA & $\overline{N A}$ \\
\hline 1,23 & Äug-Oci & $\mathrm{NA}$ & NÄ & NA & NA & NA & $\mathrm{NÄ}$ & NÄ & $\dot{N A}$ & NA & NA & "NA & NA \\
\hline 3 & ott-Mar & $\mathrm{NA}$ & NÄ & $\mathrm{NA}$ & $\mathrm{NA}$ & NA & NA & NA & NA & $\mathrm{NA}$ & NÄ & $\mathrm{NA}$ & $\mathrm{NA}$ \\
\hline 3,4 & Mar-May, & NA & NA & NA & NA & NA & NA & NA & NA & NA & NA & NA & NA \\
\hline 5 & May-Ott & $\mathrm{NA}$ & NA & NA & NA & NA & NA & "NA & A"”' & NA & NA & NA & NA \\
\hline 6 & Ott-Mar & $\dot{N A} \dot{A}^{\circ}$ & NA & NA & NA & NA & NA & NA & NA & NA & $\mathrm{NA}$ & $\mathrm{NA}$ & $\mathrm{NA}$ \\
\hline$\dot{7}$ & Mar-jun & $\mathrm{NA}$ & $\mathrm{NA}$ & NA" & $\mathrm{NA}$ & $\mathrm{NA}$ & $\mathrm{NA}$ & $\mathrm{NA}$ & NA & $\mathrm{NA}$ & NA & NA & NA \\
\hline
\end{tabular}

Abundance level refers to the average abundance of spawners that spawn in the natal stream reaches that populate the life history pathways using the reach in this table

$$
\begin{array}{ll}
\text { Level 1: } & 0-10 \text { spawners } \\
\text { Level 2 } & 10-30 \text { spawners } \\
\text { Level 3: } & 30-60 \text { spawners } \\
\text { Level 4: } & >\mathbf{6 0} \text { spawners }
\end{array}
$$

Life stages: 1-prespawner; 2- spawner; 3- incubation; 4- fry colonization; 5- summer rearing; 6-overwintering; 7- yearling presmolt/smolt

Attributes: CHEM - chemicals

CSTAB - channel stability

COMP - competition (with other species)

FLOW - flow

HABD - habitat diversity
NUTLD - nutrient load

OBST - obstructions

OXYG - dissolved oxygen

PATHO - pathogens

PRED - predators (includes fishing-related losses)
RIPCN - riparian condition

SEDLD - sediment load

TEMP - water temperature

WITH - $\quad$ water withdrawals

"NA" indicates that no tife history pathway meeting the criteria used in defining these pathways was present

Blank cells under attributes indicate no additional attributes with negative effects on productivity were evident compared to those present in the template. 
Stream Reach Summary for Primary and Secondary Spring Chinook Salmon Life Histories

Stream: Little Sheep Cr.

Reach: Hayden $\mathrm{Cr}-\mathrm{McCully} \mathrm{Cr}$ (RM $38.8-48.8$ from Snake $\mathrm{R}$ )

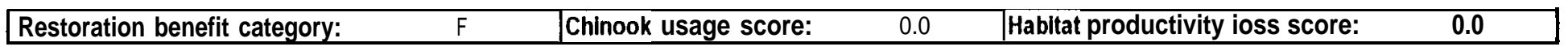

\begin{tabular}{|c|c|c|c|c|c|c|c|c|c|c|c|c|c|c|}
\hline \multirow[b]{3}{*}{ Life stage } & \multirow[b]{3}{*}{ Months } & \multicolumn{10}{|c|}{ No. life history pathways by current usage abundance level } & \multicolumn{2}{|c|}{ Key habitat change } & \multirow{3}{*}{$\begin{array}{c}\text { Habital } \\
\text { productivity } \\
\text { loss score }\end{array}$} \\
\hline & & \multicolumn{5}{|c|}{ Primary } & \multicolumn{5}{|c|}{ Secondary } & \multirow{2}{*}{$\begin{array}{l}\text { Patient } \\
\text { quantity }\end{array}$} & \multirow[b]{2}{*}{$\%$ change } & \\
\hline & & Total no. & $\overline{1}$ & 2 & 3 & 4 & Total no. & 1 & 2 & 3 & 4 & & & \\
\hline & Apr-Aug & NA & $\mathrm{NA}$ & $\mathrm{NA}$ & $\mathrm{NA}$ & $\mathrm{NA}$ & $\mathrm{NA}$ & $\overline{N A}$ & $\mathrm{NA}$ & NA & NA & $\mathrm{NA}$ & $\mathrm{NA}$ & $\mathrm{NA}$ \\
\hline $1,2,3$ & Aug-Ött & $\mathrm{NA}$ & $\mathrm{NÄ}$ & NÄ & $\dot{N} \dot{A}$ & NA & NA & NA & NA & NÄ & $\mathrm{NA}$ & $\mathrm{NA}$ & $\mathrm{NA}$ & $\mathrm{NA}$ \\
\hline 3 & Ott-Mar & NA & NA & NA & NA & NA & NA & NA & NA & NA & NA & NA & NA & NA \\
\hline 34. & Mar-May & NA & NA & $\mathbf{N} \mathbf{A}$ & NA & NA & NA?? & $N A$ & NA & $N A$ & NJ...... & $\mathrm{NJ}$ & NA & NA \\
\hline 5 & "Mày-Ött" & NA & $\mathrm{NA}$ & NÄ & $\mathrm{NA}$ & Ni & NAA & $\mathrm{NA}$ & & NA & & & & $?$ \\
\hline 8 & Öt-Mar & $\mathrm{NA}$ & $\mathrm{NA}$ & $\mathrm{NA}$ & $N A$ & NA & NA & NA & & NA & Na & NA & & \\
\hline 7 & Mar-Jun & NA & NA & NA & NA & NA & NA & $\dot{N A}$ & NA & NA & NA & NA & $\mathrm{NA}$ & $\dot{N}$ \\
\hline
\end{tabular}

\begin{tabular}{|c|c|c|c|c|c|c|c|c|c|c|c|c|c|}
\hline \multirow[b]{2}{*}{ Life stage } & \multirow[b]{2}{*}{ Months } & \multicolumn{12}{|c|}{ Highest productivity impact attributes (\% change in impact from template) } \\
\hline & & Atf 1 & $\%$ change & Att 2 & \% change & Att 3 & $\%$ change & Att 4 & \% change & Att 5 & \% change & Att 6 & \% change \\
\hline - & Apr-Aug & NA & NA & NA & NA & $\mathrm{NA}$ & NA & $\mathrm{NA}$ & $\mathrm{NA}$ & $\mathrm{NA}$ & $\mathrm{NA}$ & NA & $\mathrm{NA}$ \\
\hline 123 & Aug-oci & $\mathrm{NA}$ & NA & NA & NA & NA & NA & $\dot{N} \dot{A}$ & NA & NA & NA & $\dot{\mathrm{NA}}$ & $\dot{N} \dot{A}$ \\
\hline 3 & OOtt-Mar & NA & NA & NA & NA & NA & $\dot{\mathrm{NA}}$ & NA & $\dot{N A}$ & $\ddot{N A ̈}$ & $\mathrm{NÄ}$ & $\mathrm{NÄ}$ & $\mathrm{NA}$ \\
\hline 3,4 & Mar-Mav & NA & NA & NA & NA & NA & NA & NA & NA & NA & NA & & NA \\
\hline 5 & May-Oct & $\mathrm{NA}$ & "NA & $\mathrm{NA}$ & $\mathrm{NA}$ & $N A$ & NA & $\mathrm{NA}$ & NA & $\mathrm{NA}$ & NA. & & $N A$ \\
\hline$\ddot{6}$ & ött-Mar & NA & NA & & $N \dot{A}$ & NA & & NA & NA & & & & NA \\
\hline$\dot{7}$ & Mar-Jun & $\mathrm{NA}$ & $\mathrm{NA}$ & & NA" & $\mathrm{NA}$ & & NÄ & NÄ & & & & NA \\
\hline
\end{tabular}

Abundance level refers to the average abundance of spawnera that spawn in the natal stream reaches that populate the life history pathways using the reach in this table:

$$
\begin{array}{ll}
\text { Level 1: } & 0-10 \text { spawnera } \\
\text { Level 2 } & 10-30 \text { spawners } \\
\text { Level 3: } & 30-60 \text { spawners } \\
\text { Level 4 } & >60 \text { spawners }
\end{array}
$$

Life stages: 1- prespawner; 2- spawner; 3- incubation; 4- fry colonization; 5- summer rearing; 6- overwintering; 7- yearling presmolt/smolt

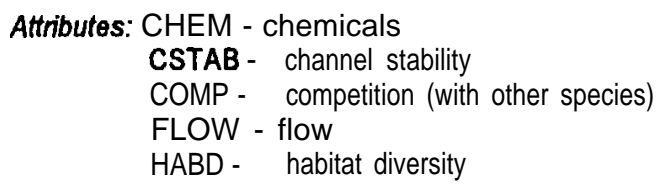

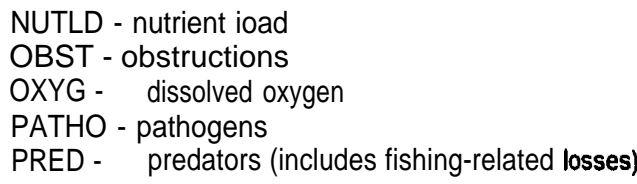

\begin{abstract}
RIPCN - riparian condition
SEDLD - sediment load

TEMP - water temperature

WITH - water withdrawals
\end{abstract}

"NA" indicates that no tife history pathway meeting the criteria used in defining these pathways was present.

Blank cells under attributes indicate no additional attributes with negative effects on productivity were evident compared to those present in the template. 


\section{APPENDIX G}

Imnaha Stream Reach Summaries for Vacant Life History Pathways 
Benefit Category

for Vacant

stream

Reach

Big Sheep Cr.

Mouth - Camp Cr (RM 22.3 -23.4 from Snake R)

Life History

Pathways

Camp Cr - Little Sheep Cr (RM 23.4 -25.4 from Snake R)

D

Big Sheep Cr.

Little Sheep Cr - Squaw Cr (RM 25.4 -37.7 from Snake R)

$\mathrm{D}$

Big Sheep Cr.

Squaw Cr - Marr Cr (RM 37.7 -39.3 from Snake R)

Marr Cr - Griffith Cr (RM 39.3 -45.3 from Snake R)

Griffith Cr - Carrel Cr (RM 45.3 -47.0 from Snake R)

Carrol Cr - Owl Cr (RM 47.0 -53.5 from Snake R)

Owl Cr - Lick Cr (RM 53.5 -57.4 from Snake R)

Lick Cr - South Fork (RM 57.4 -58.9 from Snake R)

Mouth - Cow Cr (RM 0.0- 4.3 from Snake R)

Cow Cr - Lightning Cr (RM 4.3- 5.4 from Snake R)

Lightning Cr - Horse Cr (RM 5.4- 10.S from Snake R)

Fence Cr - Big Sheep Cr (RM 16.3 -22.3 from Snake R)

Horse Cr - Fence Cr (RM 10.8 -16.3 from Snake R)

Big Sheep Cr - Freezeout Cr (RM 22.3 -35.2 from Snake R)

Freezeout Cr - Grouse Cr (RM 35.2 -40.6 from Snake R)

Grouse Cr - Summit Cr(RN440.6 -43.6 from Snake R)

Summit Cr - Crazyman Cr (RM 43.6 -49.4 from Snake R)

Crazyman Cr - Gumboot Cr (RM 49.4 -52.7 from Snake R)

Gumboot Cr - Dry Cr (RM 52.7 -55.4 from Snake R)

Dry Cr - Skookum Cr (RM 55.4 -61.9 from Snake R)

Blue Hole - forks (RM 66.4 -71.4 from Snake R)

Skookum Cr - Blue Hole (RM 61.9 -66.4 from Snake R)

South Fork Imnaha R (RM 71.4 -76.1 from Snake R)

Mouth - forks (RM 5.4- 13.9 from Snake R)

Mouth - Bear Gulch (RM 25.4 -28.5 from Snake R)

Bear Gulch - Devil's Gulch (RM 28.5 -30.9 from Snake R)

Devil's Gulch - Lightning Cr (RM 30.9 -33.1 from Snake R)

Lightning Cr - Hayden Cr (RM 33.1 -38.8 from Snake R)

Hayden Cr - McCully Cr (RM 38.8 -48.8 from Snake R) 


\section{Stream Reach Summary for Vacant Spring Chinook Salmon Life Histories}

Stream: Big Sheep Cr.

Reach: Mouth - Camp Cr (RM 22.3 -23.4 from Snake R)

\begin{tabular}{ll|ll|l}
\hline Restoration benefit category: & D & Chinook usage score: & 19.9 & Habitat productivity loss score:
\end{tabular}

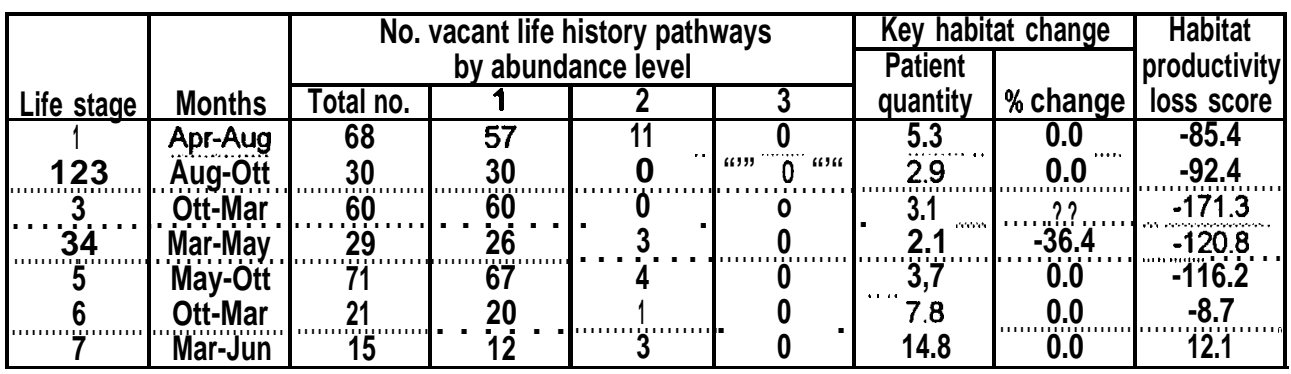

\begin{tabular}{|c|c|c|c|c|c|c|c|c|c|c|c|c|}
\hline \multirow[b]{2}{*}{ Life stage } & \multirow[b]{2}{*}{ Months } & \multicolumn{11}{|c|}{ Highest productivity impact attributes (\% change in impact from template) } \\
\hline & & Att 1 & $\%$ change & Att 2 & $\%$ change & Att 3 & $\%$ change & Att 4 & $\%$ change & Art 5 & $\%$ change & Att 6 \\
\hline 1 & Apr-Aug & FLOW & 17.7 & TEMP & 17.7 & RIPCN & 17.2 & SEDLD & 17.2 & HABD & 8.8 & \\
\hline $1,2,3$ & Aüg-Ott & CSTAB & $35 . \dot{4}$ & TEMP & $3 \dot{5} .4$ & FLOW & $1 \dot{7} .7$ & ṠĖḊL̇D & $\dot{17 . \dot{7}}$ & 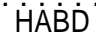 & 8.8 & RIPCN \\
\hline 3 & Ott-Mar & CSTAB & 35.4 & TEMP & $3 s .4$ & HABD & 17.7 & SEDLD & 17.7 & COMP & 8.8 & FLOW \\
\hline 34 & Mar-May & 'TEMMP' & 28.1 & CSTAB & 20.2 & HABD & 20.2 & "SEDLD' & 20.2 & COMMP & 14.0 & "РATHO \\
\hline 5 & May-Ott & TEMP & 27.3 & CSTAB & "17.3 & FLOW & 17.3 & PATHO & 17.3 & WIITH & 17.3 & COMP \\
\hline 6 & Ott-Mar & CSTAB & 4.2 & FLOW & 4.2 & PAT HO & 4.2 & TEMP & 4.2 & "WiTH & 4.2 & \\
\hline 7 & Mar-Jun & WITH & 15,9 & & & & & & & & & \\
\hline
\end{tabular}

Abundancelovel refera to estimated relative abundance of spawners that spawned in the natal stream reaches that populated the life history pathways us Level 1: Low to average (relative to other reaches) abundance

Level 2 High (relative to other reaches) abundance

Level 3: $\quad$ Exceptionally high (relative to other reaches) abundance

Life stages: 1- prespawner; 2- spawner; 3- incubation; 4- fry colonization; 5- summer rearing; 6- overwintering; 7- yearling presmolt/smolt

$\begin{array}{lll}\text { Attributes: CHEM - chemicals } & \text { NUTLD - nutrient load } & \text { RIPCN - riparian conc } \\ \text { CSTAB - channel stability } & \text { OBST - obstructions } & \text { SEDLD - sediment loa } \\ \text { COMP - competition (with other species) } & \text { OXYG - dissolved oxygen } & \text { TEMP - water tempe } \\ \text { FLOW - flow } & \text { PATHO - pathogens } & \text { WITH - water withdra } \\ \text { HABD - habitat diversity } & \text { PRED - predators (includes fishing-related losses) }\end{array}$

"NA" indicates that no life history pathway meeting the criteria used in defining these pathways was present.

Blank cells under attributes indicate no additional attributes with negative effects on productivity were evident compared to those present in the template. 
Stream: Big Sheep Cr.

Reach: Camp Cr - Little Sheep Cr (RM 23.4 -25,4 from Snake R)

\begin{tabular}{|ll|ll|l}
\hline Restoration benefit category: & D & Chinook usage score: & 37.9 & Habitat productivity loss score: \\
\hline
\end{tabular}

\begin{tabular}{|c|c|c|c|c|c|c|c|c|}
\hline \multirow[b]{3}{*}{ Life stage } & \multirow[b]{3}{*}{ Months } & \multirow{2}{*}{\multicolumn{4}{|c|}{$\begin{array}{c}\text { No. vacant life history pathways } \\
\text { by abundance level }\end{array}$}} & \multicolumn{2}{|c|}{ Key habitat change } & \multirow{3}{*}{$\begin{array}{c}\text { Habitat } \\
\text { productivity } \\
\text { loss score }\end{array}$} \\
\hline & & & & & & \multirow{2}{*}{$\begin{array}{l}\text { Patient } \\
\text { quantity }\end{array}$} & \multirow[b]{2}{*}{$\%$ change } & \\
\hline & & Total no. & 1 & 2 & 3 & & & \\
\hline 1 & Apr-Aug & 65 & 54 & 11 & 0 & 6.1 & -4.8 & -111.7 \\
\hline $1,2,3$ & Aug-Oct & 30 & 30 & 0 & 0 & 5.1 & 0.0 & -130.5 \\
\hline 3 & Ött-Mar & $60^{\circ}$ & 60 & ”o & $\dddot{0}$ & 5.3 & 0 & -2420 \\
\hline 34 & Mar-May & 40 & 37 & 3 & 0 & 2.6 & -57.4 & -232.8 \\
\hline 5 & May-Ott & 136 & 132 & 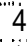 & 0 & 5.6 & -2.2 & -379.5 \\
\hline 6 & Ott-Mar & 40 & 39 & $\dddot{1}$ & 0 & 131 & $0.0 \%$ & -107.0 \\
\hline '7' & Mar-Jun & 14 & 111 & 3 & 0 & 20.7 & 0,0 & 12.5 \\
\hline
\end{tabular}

\begin{tabular}{|c|c|c|c|c|c|c|c|c|c|c|c|c|}
\hline \multirow[b]{2}{*}{ Life stage } & \multirow[b]{2}{*}{ Months } & \multicolumn{11}{|c|}{ Highest productivity impact attributes (\% change in impact from template) } \\
\hline & & An 1 & $\%$ change & Att 2 & $\%$ change & Att 3 & $\%$ change & Att 4 & $\%$ change & Att 5 & $\%$ change & Att 6 \\
\hline 1 & Apr-Aug . & TEMP & 37.5 & CSTAB & 25.0 . & FLOW & 22.5 & RIPCN & 22.5 & SEDLD & 22.5 & HABD \\
\hline $1,2,3$ & Aug-Oct & $\dot{C} \mathbf{S T A B}$ & 50.0 & TEMP & 50.0 & F́L̇W் & $25.0^{\circ}$ & RIPCN & 25.0 & SEEDELD̄ & $25.0^{\circ}$ & HAABD \\
\hline 3 & Ott-Mar & CSTAB & 50.0 & HABD & 50.0 & TEMP & 50.0 & COMP & “' 25.0 & FLOW. & 2.50 & PATHO. \\
\hline 3,4 & 'Mar-May' & "CST'AB'. & '50.0' & 'HA'B'D' & 50.0 & "TEḾ' & 50.0 & COMP & 25.0 & "FlÖẄW' & 25.0 & PATHO \\
\hline 5 & Mày-Ottt & TEM̆P & $47.0^{\prime}$ & CÖMP & 23.5 & HABD & 23.5 & "PATHO & 235 & SEDLD & 23.5 & \\
\hline 6 & Ott-Mar & 'Ẅiт̈H' & 25.0 & "CSTAB & 24.1 & "FLOWW & 24.1 & PATHO & 24,1 & TEMMP' & 24.1 & \\
\hline 7 & Mar-Jun & WITH & 17.6 & & & & & & & & & \\
\hline
\end{tabular}

Abundance level refers to estimated relative abundance of spawners that spawned in the natal stream reaches that populated the life history pathways us Level 1: $\quad$ Low to average (relative to other reaches) abundance

Level 2: $\quad$ High (relative to other reaches) abundance

Level 3: Exceptionally high (relative to other reaches) abundance

Life stages: 1- prespawner; 2- spawner; 3- incubation; 4- fry colonization; 5- summer rearing; 6- overwintering; 7-yearling presmolt/smolt

Attributes: CHEM - chemicals

CSTAB - channel stability

COMP - competition (with other species)

FLOW - flow

HABD - habitat diversity
NUTLD - nutrient load

OBST - obstructions

OXYG - dissolved oxygen

PATHO - pathogens

PRED - predators (includes fishing-related losses)
RIPCN - riparian cond

SEDLD - sediment loa

TEMP - water temper

WITH - water withdre

"NA" indicates that no life history pathway meeting the criteria used in defining these pathways was present.

Blank cells under attributes indicate no additional attributes with negative effects on productivity were evident compared to those present in the template, 
Stream Reach Summary for Vacant Spring Chinook Salmon Life Histories

Stream: Big Sheep $\mathrm{Cr}$.

Reach: Little Sheep Cr - Squaw Cr (RM 25.4 -37.7 from Snake R)

\begin{tabular}{|ll|ll|lll}
\hline Restoration benefit category: & B & Chinook usage score: & 62.1 & Habitat productivity loss score: & -1585.7 \\
\hline
\end{tabular}

\begin{tabular}{|c|c|c|c|c|c|c|c|c|}
\hline \multirow[b]{3}{*}{ Life slage } & \multirow[b]{3}{*}{ Months } & \multirow{2}{*}{\multicolumn{4}{|c|}{$\begin{array}{l}\text { No. vacant life history pathways } \\
\text { by abundance level }\end{array}$}} & \multicolumn{2}{|c|}{ Key habital change } & \multirow{3}{*}{$\begin{array}{c}\text { Habital } \\
\text { productivity } \\
\text { loss score }\end{array}$} \\
\hline & & & & & & \multirow{2}{*}{$\begin{array}{l}\text { Patient } \\
\text { quantity }\end{array}$} & \multirow[b]{2}{*}{$\%$ change } & \\
\hline & & Total no. & 1 & 2 & $\overline{3}$ & & & \\
\hline 1 & Apr-Aug & 90 & 55 & 35 & $\overline{0}$ & 10.4 & -14.9 & -172.0 \\
\hline $1,2,3$ & Aug-Oct & 50 & 0 & 50 & 0 & 5.7 & 0.0 & -217.5 \\
\hline 3 & Oct-Mar & 100 & 0 & 100 & $\dddot{0}$ & 6.2 & 0.0 & -403.3 \\
\hline 3,4 & Mar-May & 70 & 45 & 25 & 0 & 3.2 & -38.8 & -267.2 \\
\hline 5 & May-Oct & 259 & 211 & 48 & 0 & 8.0 & -4.0 & -417.0 \\
\hline 6 & Oct-Mar & 76 & 57 & 19 & 0 & 15.6 & 0.0 & -113.7 \\
\hline 7 & Mar-Jun & 7 & 6 & 1 & $\dddot{0}$ & 27.2 & 0.0 & 5.0 \\
\hline
\end{tabular}

\begin{tabular}{|c|c|c|c|c|c|c|c|c|c|c|c|c|c|}
\hline \multirow[b]{2}{*}{ Life stage } & \multirow[b]{2}{*}{ Months } & \multicolumn{12}{|c|}{ Highest productivity impact attributes (\% change in impact from template) } \\
\hline & & Att 1 & $\%$ change & Att 2 & $\%$ change & Att 3 & $\%$ change & Alt 4 & $\%$ change & Alt 5 & $\%$ change & Alt 6 & $\%$ change \\
\hline 1 & Apr-Aug & FLOW & 25.0 & TEMP & 25.0 & RIPCN & 23.8 & SEDLD & 23.8 & HABD & 12.5 & & \\
\hline $1,2,3$ & Aug-Oct & $\mathrm{CSTAB}$ & 50.0 & TEMP & 500 & FLOW & 25.0 & SEDLD & 25.0 & HABD & 12.5 & RIPCN & 12.5 \\
\hline 3 & Oct-Mar & CSTAB & 50.0 & TEMP & 50.0 & HABD & 25.0 & SEDLD & 25.0 & COMP & 12.5 & FLOW & 125 \\
\hline 3,4 & Mar-May & TEMP & 24.8 & CSTAB & 20.1 & HABD & 20.1 & SEDLL & 20.1 & COMP & 12.4 & PATHO & 12.4 \\
\hline 5 & May-Oct & TEMP & 25.0 & CSTAB & 14.5 & FLOW & 14.5 & PATHO & 14.5 & WITH & 14.5 & COMP & 12.5 \\
\hline 6 & Oct-Mar & CSTAB & 13.5 & FLOOW & 13.5 & PATHO & 13.5 & TEMP & 13.5 & WITH & 13.5 & & \\
\hline 7 & Mar-Jun & WITH & 14.0 & & & & & & & & & & \\
\hline
\end{tabular}

Abundance level refers to estimated relative abundance of spawners that spawned in the natal stream reaches that populated the life history pathways using the reach in this table Level 1: Low to average (relative to other reaches) abundance

Level 2 High (relative to other reaches) abundance

Level 3: Exceptionally high (relative to other reaches) abundance

Life stages.' 1 - prespawner; 2- spawner; 3- incubation; 4- fry colonization; 5- summer rearing; 6-overwintering; 7- yearling presmolt/smolt

Attributes: CHEM - chemicals

CSTAB - channel stability

COMP - competition (with other species)

FLOW - flow

HABD - habitat diversity
NUTLD - nutrient load

OBST - obstructions

OXYG - dissolved oxygen

PATHO - pathogens

PRED - predators (includes fishing-related losses)
RIPCN - riparian condition

SEDLD - sediment load

TEMP - water temperature

WITH - water withdrawals

"NA" indicates that no life history pathway meeting the criteria used in defining these pathways was present.

Blank ceils under attributes indicate no additional attributes with negative effects on productivity were evident compared to those present in the template. 
Stream Reach Summary for Vacant Spring Chinook Salmon Life Histories

Stream: Big Sheep Cr.

Reach: Squaw Cr - Marr Cr (RM $37.7-39,3$ from Snake R)

\begin{tabular}{|c|c|c|c|c|c|c|c|c|c|c|c|c|c|}
\hline \multicolumn{4}{|c|}{ Restoration benefit category: } & D & \multicolumn{3}{|c|}{ Chinook usage score: } & 35.7 & \multicolumn{4}{|c|}{ Habitat productivity loss score: } & -1398.4 \\
\hline \multirow[b]{3}{*}{ Life stage } & \multirow[b]{3}{*}{ Months } & \multirow{2}{*}{\multicolumn{4}{|c|}{$\begin{array}{l}\text { No. vacant life history pathways } \\
\text { by abundance level }\end{array}$}} & \multicolumn{2}{|c|}{ Key habitat change } & \multirow{3}{*}{$\begin{array}{c}\text { Habitat } \\
\text { productivity } \\
\text { loss score }\end{array}$} & & & & & \\
\hline & & & & & & \multirow{2}{*}{$\begin{array}{l}\text { Patient } \\
\text { quantity }\end{array}$} & \multirow[b]{2}{*}{$\%$ change } & & & & & & \\
\hline & & Total no. & 1 & 2 & 3 & & & & & & & & \\
\hline 1 & Apr-Aug & 50 & 50 & 0 & 0 & 4.5 & , & -132.9 & & & & & \\
\hline 1,2 & Aug-Od & 60 & gp & & 0 & 3.0 & & -261.0 & & & & & \\
\hline 3 & Oct-Mar & $120^{\circ}$ & 120 & & 0 & 3.2 & & -483.9 & & & & & \\
\hline 34 & Mar-May & 39 & 39 & 0 & 0 & 1.9 & "47.!5" & 246.8 & & & & & \\
\hline 5 & May-Ott & 75 & 75 & 0 & 0 & 4.1 & -5.7 & -221.4 & & & & & \\
\hline 6 & Ott-Mar & 19 & 19 & 0 & 0 & 8.0 & $0.0 “$ & -52.8 & & & & & \\
\hline 7 & Mar-Jun & 2 & 2 & 0 & $\ddot{0}$ & 13.2 & 0.0 & 0.4 & & & & & \\
\hline \multirow[b]{2}{*}{ Life stage } & \multirow[b]{2}{*}{ Months } & \multicolumn{12}{|c|}{ Highest productivity impact attributes (\% change in impact from template) } \\
\hline & & Att 1 & $\%$ change & Atf 2 & $\%$ change & An 3 & $\%$ change & Att 4 & $\%$ change & An 5 & $\%$ change & Att 6 & $\%$ change \\
\hline 1 & Apr-Aug, & TEMP & 37.5 & CSTAB & 25.0 & FLOW & 21.3 & RIPCN & 21.3 & SEDLD & 21.3 & HABD & 12.5 \\
\hline $1,2,3$ & Äug-Ött & CSTAB & 50.0 & TÉMP & 50,0 & F́LÓW் & 25.0 & RIPCN & $25.0^{\circ}$ & SEDLD & 25.0 & $\mathrm{HABD}$ & 12.5 \\
\hline 3 & Ott-Mar & CSTAB & 50.0 & HABD & 50.0 & TEMP & 50.0 & COMP & $25.0^{\circ}$ & FLOW & 250 & PATHO & 25.0 \\
\hline 3,4 & Mar-May & CSTAB & 50.0 & TEMP & 50.0 & HABD' & 43.9 & COMP & 25.0 & "РATHO & 250 & SEDLD & 25.0 \\
\hline 5 & May-Ott & TEMP & 48.8 & COMP & 24.4 & "HA60"“" & 24.4 & РАTHO & 24.4 & SEEDLD" & 244 & & \\
\hline $\begin{array}{l}6 \\
7\end{array}$ & $\begin{array}{l}\text { Ott-Mar } \\
\text { Mar-Jun }\end{array}$ & $\begin{array}{l}\text { CSTAB } \\
\text { WITH }\end{array}$ & $\begin{array}{l}25.0 \\
18.7\end{array}$ & FLOOWW" & 25.0 & PATHO & 25.0 & "TEMP" & 25,0 & "WITH" & 25.0 & & \\
\hline
\end{tabular}

Abundance level refers to estimated relative abundance of spawners that spawned in the natal stream reaches that populated the tife history pathways using the reach in this table: Level 1: Low to average (relative to other reaches) abundance

Level 2: $\quad$ High (relative to other reaches) abundance

Level 3: Exceptionally high (relative to other reaches) abundance

Life stages: 1- prespawner; 2- spawner; 3- incubation; 4- fry colonization; 5- summer rearing; 6- overwintering; 7-yearling presmolt/smolt

Attributes: CHEM - chemicals

CSTAB - channel stability

COMP - competition (with other species)

FLOW - flow

HABD - habitat diversity
NUTLD - nutrient load

OBST - obstructions

OXYG - dissolved oxygen

PATHO - pathogens

PRED - predators (includes fishing-related losses)
RIPCN - riparian condition

SEDLD - sediment load

TEMP - water temperature

WITH - water withdrawals

"NA" indicates that no life history pathway meeting the criteria used in defining these pathways was present.

Blank cells under attributes indicate no additional attributes with negative effects on productivity were evident compared to those present in the template. 


\section{Stream Reach Summary for Vacant Spring Chinook Salmon Life Histories}

Stream: Big Sheep Cr.

Reach: Marr Cr - Griffith $\mathrm{Cr}$ (RM $39.3-45.3$ from Snake R)

\begin{tabular}{|ll|l|l|}
\hline Restoration benefit category: & B & Chinook usage score: 46,1 & Habitat productivity loss score: \\
\hline
\end{tabular}

\begin{tabular}{|c|c|c|c|c|c|c|c|c|}
\hline \multirow[b]{3}{*}{ Life stage } & \multirow[b]{3}{*}{ Months } & \multirow{2}{*}{\multicolumn{4}{|c|}{$\begin{array}{c}\text { No. vacant life history pathways } \\
\text { by abundance level }\end{array}$}} & \multicolumn{2}{|c|}{ Key habitat change } & \multirow{3}{*}{$\begin{array}{c}\text { Habitat } \\
\text { productivity } \\
\text { loss score }\end{array}$} \\
\hline & & & & & & \multirow{2}{*}{$\begin{array}{l}\text { Patient } \\
\text { quantity }\end{array}$} & \multirow[b]{2}{*}{$\%$ change } & \\
\hline & & Total no. & 1 & $\overline{2}$ & 3 & & & \\
\hline 1 & Apr-Aug & $\overline{44}$ & 44 & $\overline{0}$ & 0 & 4.9 & -20.4 & -130.4 \\
\hline 123 & Aug-Oct & 60 & 60 & 0 & 0 & 3.5 & -50 & -2610 \\
\hline 3 & Oct-Mar & 120 & 120 & $\dddot{0}$ & 0 & 3.7 & -2.4 & -483.9 \\
\hline 34 & Mar-May & 44 & 44 & 0 & 0 & 2.0 & -51.8 & -268.2 \\
\hline 5 & May-Oct & 111 & 111 & $\ddot{0}$ & $\dddot{0}$ & 4.9 & $-10.0^{\circ}$ & -251.6 \\
\hline 6 & Oct-Mar & 19 & 19 & 0 & 0 & 9.2 & -2.6 & -52.8 \\
\hline 7 & Mar-Jun & 1 & 1 & 0 & 0 & 15.2 & -7.7 & 0.3 \\
\hline
\end{tabular}

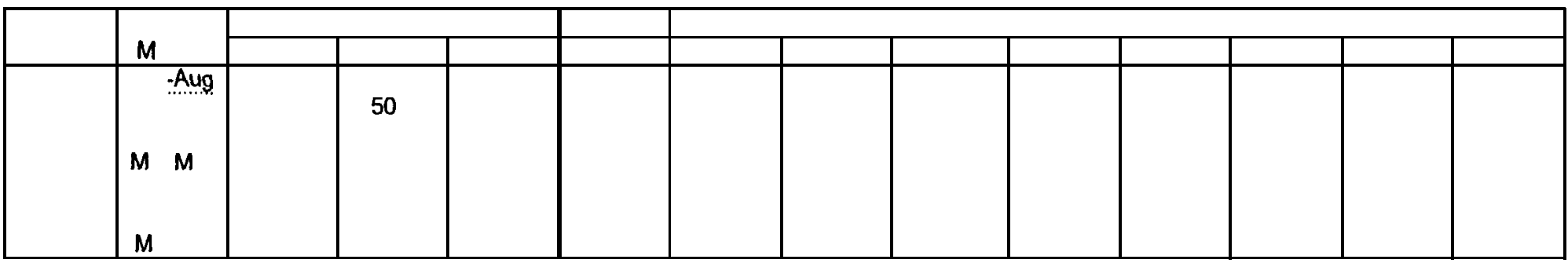

Abundance/eve/refers to estimated relative abundance of spawners that spawned in the natal stream reaches that populated the life history pathways using the reach in this table: Level 1: $\quad$ Low to average (relative to other reaches) abundance

Level 2: $\quad$ High (relative to other reaches) abundance

Level 3 Exceptionally high (relative to other reaches) abundance

Life stages: 1. prespawner; 2-spawner; 3- incubation; 4- fry colonization; 5- summer rearing; 6- overwintering; 7-yearlingresmolt/smolt

Attributes: CHEM - chemicals

CSTAB - channel stability

COMP - competition (with other species)

FLOW - flow

NUTLD - nutrient load

OBST - obstructions

OXYG - dissolved oxygen

REN - riparian condition

HABD - habitat diversity

PATHO - pathogens

SEDLO - sediment load

TEMP - water temperature

"NA" indicates that no life history pathway meeting the criteria used in defining these pathways was present.

Blank cells under attributes indicate no additional attributes with negative effects on productivity were evident compared to those present in the template. 
Stream Reach Summary for Vacant Spring Chinook Salmon Life Histories

Stream: Big Sheep Cr.

Reach: Griffith $\mathrm{Cr}$ - Carrel Cr (RM 45.3 -47.0 from Snake R)

\begin{tabular}{ll|ll|ll} 
Restoration benefit category: & F & Chinook usage score: & 12.2 & Habitat productivity loss score: & -131.9 \\
\hline
\end{tabular}

\begin{tabular}{|c|c|c|c|c|c|c|c|c|}
\hline \multirow[b]{3}{*}{ Life stage } & \multirow[b]{3}{*}{ Months } & \multirow{2}{*}{\multicolumn{4}{|c|}{$\begin{array}{c}\text { No. vacant life history pathways } \\
\text { by abundance level }\end{array}$}} & \multicolumn{2}{|c|}{ Key habitat change } & \multirow{3}{*}{$\begin{array}{c}\text { Habitat } \\
\text { productivity } \\
\text { loss score }\end{array}$} \\
\hline & & & & & & \multirow{2}{*}{$\begin{array}{l}\text { Patient } \\
\text { quantiiy }\end{array}$} & \multirow[b]{2}{*}{$\%$ change } & \\
\hline & & Total no, & 1 & 2 & 3 & & & \\
\hline 1 & Apr-Aug & 23 & 23 & 0 & 0 & $4.1 \ldots$ & -6.6 & 0.0 \\
\hline 1,23 & Aug-Ott & 30 & 30 & 0 & 0 & 2.7 & -51.0 & -18.8 \\
\hline 3 & Ott-Mar & 60 & 60 & 0 & “" & 2.8 & -60.0 & -106.9 \\
\hline 34 & Mar-May & 11 & 11 & 0 & 0 & 5.5 & -280 & -6.1 \\
\hline 5 & May-Ött & $\mathrm{NA}$ & NA & $\mathrm{NA}$ & $\mathrm{NA}$ & NA & NA & NÄ \\
\hline 6 & Ott-Mar & NA & NA & NA & NA & NA & NA & NA \\
\hline 7 & Mar-Jun & $\mathrm{NA}$ & NA & $\mathrm{NA}$ & NA & NA & NÄ & $\mathrm{NA}$ \\
\hline
\end{tabular}

\begin{tabular}{|c|c|c|c|c|c|c|c|c|c|c|c|c|c|}
\hline \multirow[b]{2}{*}{ Life stage } & \multirow[b]{2}{*}{ Months } & \multicolumn{12}{|c|}{ Highest productivity impact attributes (\% change in impact from template) } \\
\hline & & Att 1 & $\%$ change & An 2 & $\%$ change & Art 3 & \% change & Att 4 & $\%$ change & Att 5 & $\%$ change & Att 6 & $\%$ change \\
\hline & Apr-Aug & SEDLD & 37.5 & FLOW & 72.0 & & & & & & & & \\
\hline $1,2,3$ & Aug-Ôtt & SEDLD" & 50.0 & "Flöw" & 25.0 & & & & & & & & \\
\hline 3 & Ott-Mar & SEDLD & 50.0 & PATHO & 250 & & & & & & & & \\
\hline 3,4 & Mar-May & SEDLD &, 50.0 & PATHO & 213 & & & & & & & & \\
\hline 5 & May-Ott & NA & NA & $\mathrm{N} A$ & NA & NA & $N A$ & "NÁ & NA & NA & NA & $\dot{N} \dot{A}$ & $\mathrm{NA}$ \\
\hline 6 & Öt-Mar & NA & $\mathrm{NA}$ & $\mathrm{NA}$ & NA & $\mathrm{NA}$ & $\mathrm{NA}$ & $\mathrm{NA}$ & $\mathrm{NA}$ & $\mathrm{NA}$ & $N A$ & NA & NA \\
\hline 7 & Mar-Jun & NA & NA & NA & NA & NA & NA & NA & NA & NA & NA & NA & NA \\
\hline
\end{tabular}

Abundance/eve/refers to estimated relative abundance of spawners that spawned in the natal stream reaches that populated the life history pathways using the reach in this table: Level 1: Low to average (relative to other reaches) abundance

Level 2 High (relative to other reaches) abundance

Level 3: Exceptionally high (relative to other reaches) abundance

Life stages: 1- prespawner; 2- spawner; 3- incubation: 4- fry colonization; 5- summer rearing; 6- overwintering; 7-yearling presmolt/smolt

Attributes: CHEM - chemicals

CSTAB - channel stability

COMP - competition (with other species)

FLOW - flow

HABD - habitat diversity
NUTLD - nutrient load

OBST - obstructions

OXYG - dissolved oxygen

PATHO - pathogens

PRED - predators (includes fishing-related losses)
RIPCN - riparian condition

SEDLD - sediment load

TEMP - water temperature

WITH - water withdrawals

"NA" indicates that no tife history pathway meeting the criteria used in defining these pathways was present.

Blank cells under attributes indicate no additional attributes with negative effects on productivity were evident compared to those present in the template. 
Stream Reach Summary for Vacant Spring Chinook Salmon Life Histories

Stream: Big Sheep Cr.

Reach: Carrel $\mathrm{Cr}-\mathrm{Owl} \mathrm{Cr}(\mathrm{RM} 47.0-53.5$ from Snake R)

\begin{tabular}{|ll|ll|lll}
\hline Restoration benefit category: & F & Chinook usage score: & 14.5 & Habitat productivity loss score: & -130.1 \\
\hline
\end{tabular}

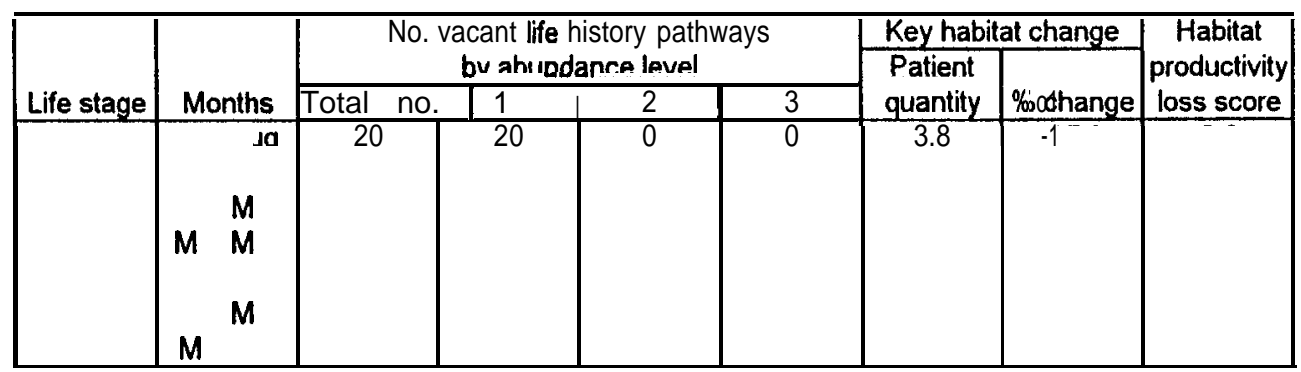

\begin{tabular}{|c|c|c|c|c|c|c|c|c|c|c|c|c|c|}
\hline \multirow[b]{2}{*}{ Life stage } & \multirow[b]{2}{*}{ Months } & \multicolumn{12}{|c|}{ Highest productivity ime ıct attributes (\% change in impact from template) } \\
\hline & & Att 1 & $\%$ change & Atf 2 & $\%$ change & An 3 & $\%$ change & Atf 4 & $\%$ change & Att 5 & $\%$ change & Att 6 & $\%$ change \\
\hline 1 & Apr-Aug & FLOW & 24,6 & SEDLD & 24.6 & & & & & & & & \\
\hline$,, 1,2,3$ & Aug-Oct & SEDLD & 500 & FL'OW & 25.0 & & & & & & & & \\
\hline 3 & Ött-Mar & SEDLD & 50.0 & PATHO & $" 12.5$ & & & & & & & & \\
\hline 34 & Mar-May & SEDLD & 22.7 & PAT̈HO & 47.4 & & & & & & & & \\
\hline 5 & May-Ott & $\mathrm{NA}$ & NA & NA & NA & NA & NA & NA & NA & NA & NA & NA & NA \\
\hline 6 & Ott-Mar & NA & NA & NA & NA & NA & NA & NA & NA & NA & NA & NA & NA \\
\hline 7 & Mar-Jun & NA & NA & NA & NA & NA & NA & NA & NA & NA & NA & NA & NA \\
\hline
\end{tabular}

Abundance level refers to estimated relative abundance of spawners that spawned in the natal stream reaches that populated the life history pathways using the reach in this table Level 1: $\quad$ Low to average (relative to other reaches) abundance

Level 2: High (relative to other reaches) abundance

Level 3 Exceptionally high (relative to other reaches) abundance

Life stages.' 1- prespawner; 2- spawner; 3- incubation; 4- fry colonization; 5- summer rearing; 6- overwintering; 7-yearlingpresmolt/smolt

\begin{tabular}{|c|c|c|c|}
\hline $\begin{array}{r}\text { Attributes: } \\
\text { CHEM - } \\
\text { CSTAB - } \\
\text { COMP - } \\
\text { FLOW - } \\
\text { HABD - }\end{array}$ & $\begin{array}{l}\text { chemicals } \\
\text { channel stability } \\
\text { competition (with other species) } \\
\text { flow } \\
\text { habitat diversity }\end{array}$ & $\begin{array}{l}\text { NUTLD - } \text { nutrient load } \\
\text { OBST - obstructions } \\
\text { OXYG - dissolved oxygen } \\
\text { PATHO - pathogens } \\
\text { PRED - predators (includes fishing-related losses) }\end{array}$ & $\begin{array}{l}\text { RIPCN - } \quad \text { riparian condition } \\
\text { SEDLD - sediment load } \\
\text { TEMP - } \quad \text { water temperature } \\
\text { WITH - } \quad \text { water withdrawals }\end{array}$ \\
\hline
\end{tabular}

"NA" indicates that no tife history pathway meeting the criteria used in defining these pathways was present.

Blank cells under attributes indicate no additional attributes with negative effects on productivity were evident compared to those present in the template 
Stream Reach Summary for Vacant Spring Chinook Salmon Life Histories

Stream: Big Sheep Cr.

Reach: Owl Cr - Lick Cr (RM 53.5 -57.4 from Snake R)

\begin{tabular}{|ll|ll|lll|}
\hline Restoration benefit category: & F & Chinook usage score: & 3.9 & Habitat productivity loss score & -43.7 \\
\hline
\end{tabular}

\begin{tabular}{|c|c|c|c|c|c|c|c|c|}
\hline \multirow[b]{3}{*}{ Life stage } & \multirow[b]{3}{*}{ Months } & \multirow{2}{*}{\multicolumn{4}{|c|}{$\begin{array}{l}\text { No. vacant life history pathways } \\
\text { by abundance level }\end{array}$}} & \multicolumn{2}{|c|}{ Key habitat change } & \multirow{3}{*}{$\begin{array}{c}\text { Habitat } \\
\text { productivity } \\
\text { loss score }\end{array}$} \\
\hline & & & & & & \multirow{2}{*}{$\begin{array}{l}\text { Patient } \\
\text { quantity }\end{array}$} & \multirow[b]{2}{*}{$\%$ change } & \\
\hline & & Total no. & 1 & 2 & 3 & & & \\
\hline$T$ & Apr-Aug & 7 & 7 & 0 & 0 & 2.7 & -14.3 & 0.0 \\
\hline $1,2,3$ & Aug-Oct & 10 & 10 & 0 & 0 & 2.3 & -52.4 & -6.3 \\
\hline 3 & Oct-Mar & 20 & 20 & 0 & 0 & 2.3 & -60.0 & .35 .6 \\
\hline 3,4 & Mar-May & 3 & 3 & 0 & 0 & 4.3 & -30.0 & -1.8 \\
\hline 5 & May-Oct & NA & NÄ & NÄ & NA & $\mathrm{NA}$ & $\mathrm{NA}$ & $\mathrm{NA}$ \\
\hline 6 & Oct-Mar & $\mathrm{NA}$ & NA & NA & NA & $\mathrm{NA}$ & NA & NA \\
\hline 7 & Mar-Jun & $\mathrm{NA}$ & NA & $\mathrm{NA}$ & "NA & NÄ & $\mathrm{NA}$ & $\mathrm{NA}$ \\
\hline
\end{tabular}

\begin{tabular}{|c|c|c|c|c|c|c|c|c|c|c|c|c|c|}
\hline \multirow[b]{2}{*}{ Life stage } & \multirow[b]{2}{*}{ Months } & \multicolumn{12}{|c|}{ Highest productivity impact attributes (\% change in impact from template) } \\
\hline & & Att 1 & $\%$ change & Att 2 & \% change & Att 3 & $\%$ change & Att 4 & $\%$ change & Art 5 & $\%$ change & Atf 6 & $\%$ change \\
\hline & Apr-Aug & SEDLD & ??? & FLOW & 22.3 & & & & & & & & \\
\hline 123 & Aug-Ött & SEDLD & 50.0 & FLOW & 25.0 & & & A & & & & & \\
\hline 3 & Oct-Mar & SEDLD & 50.0 & РАTHO & 25.0 & & & & . & & & & \\
\hline 34 & Mar-May & SEDLD & 50.0 & PATHO & 25.0 & ïia & $\dot{\mathrm{N}} \mathrm{A}^{\prime}$ & AN & 'NA & $N A$ & $N A$ & AiA & $\Delta A$ \\
\hline 5 & May-Ott & $N A$ & NA & NA & NA & Niñ & NA & Nin & NA & Nin" & Nin" & Nin & Nin \\
\hline $\begin{array}{l}6 \\
7\end{array}$ & Ott-Mar & $\begin{array}{l}\text { NA } \\
\text { NA }\end{array}$ & $\begin{array}{l}\text { NA } \\
\text { NA }\end{array}$ & NA & NA & NA & $\begin{array}{l}\text { INA } \\
\text { NA }\end{array}$ & NA & NA & NA & NA & NA & NA \\
\hline & & & & & & & & & & & & & \\
\hline
\end{tabular}

Abundance/eve/ refers to estimated relative abundance of spawners that spawned in the natal stream reaches that populated the life history pathways using the reach in this table: Level 1: Low to average (relative to other reaches) abundance

Level 2 High (relative to other reaches) abundance

Level 3: Exceptionally high (relative to other reaches) abundance

Life stages: 1- prespawner; 2- spawner; 3- incubation; 4- fry colonization; 5- summer rearing; 6- overwintering; 7- yearling presmolt/smolt

Attributes: CHEM - chemicals

CSTAB - channel stability

COMP - competition (with other species)

FLOW - ftOW

HABD - habitat diversity
NUTLD - nutrient load

OBST - obstructions

OXYG - dissolved oxygen

PATHO - pathogens

PRED - predators (includes fishing-related losses)
RIPCN - riparian condition

SEDLD - sediment load

TEMP - water temperature

WITH - water withdrawals

"NA" indicates that no life history pathway meeting the criteria used in defining these pathways was present.

Blank cells under attributes indicate no additional attributes with negative effects on productivity were evident compared to those present in the template. 
Stream: Big Sheep Cr.

Reach: Lick Cr - South Fork (RM 57.4 -58.9 from Snake R)

\begin{tabular}{|c|c|c|c|c|c|c|c|c|}
\hline \multicolumn{4}{|c|}{ Restoration benefit category: } & & \multicolumn{3}{|c|}{ [Chinook usage score: } & 3.8 \\
\hline \multirow[b]{3}{*}{ Life stage } & \multirow[b]{3}{*}{ Months } & \multirow{2}{*}{\multicolumn{4}{|c|}{$\begin{array}{l}\text { No. vacant life history pathways } \\
\text { by abundance level }\end{array}$}} & \multicolumn{2}{|c|}{ Key habitat change } & \multirow{3}{*}{$\begin{array}{c}\text { Habilat } \\
\text { productivity } \\
\text { loss score }\end{array}$} \\
\hline & & & & & & \multirow{2}{*}{$\begin{array}{l}\text { Patient } \\
\text { quantity }\end{array}$} & \multirow[b]{2}{*}{$\%$ change } & \\
\hline & & Total no. & 1 & 2 & 3 & & & \\
\hline 1 & Apr-Aug & 6 & 6 & 0 & $\overline{0}$ & 1.9 & 0.0 & 0.0 \\
\hline $1,2,3$ & Aug-Oct & 10 & 10 & 0 & 0 & 1.7 & -51.0 & -12.2 \\
\hline 3 & Oct-Mar & 20 & 20 & 0 & 0 & 1.7 & -60.0 & -35.6 \\
\hline 3,4 & Mar-May & 2 & 2 & 0 & 0 & 3.2 & -30.0 & -1.2 \\
\hline 5 & May-Oct & $\mathrm{NA}$ & NA & $\mathrm{NA}$ & $\mathrm{NA}$ & $\mathrm{NA}$ & NA & $\mathrm{NA}$ \\
\hline 6 & Oct-Mar & $\mathrm{NA}$ & $\mathrm{NA}$ & $\mathrm{NA}$ & NA & NA & NA & $\mathrm{NA}$ \\
\hline 7 & Mar-Jun & $\mathrm{NA}$ & NÄ & $\mathrm{NA}$ & NA & $\mathrm{NA}$ & NA & NA \\
\hline
\end{tabular}

\begin{tabular}{|c|c|c|c|c|c|c|c|c|c|c|c|c|c|}
\hline \multirow[b]{2}{*}{ Life stage } & \multirow[b]{2}{*}{ Months } & \multicolumn{9}{|c|}{ Highest productivity impact attributes ( $\%$ change in impact from templat } & \\
\hline & & Att 1 & $\%$ change & Att 2 & $\%$ change & Att 3 & $\%$ change & Att 4 & $\%$ change & Att 5 & $\%$ change & Att 6 & $\%$ change \\
\hline 1 & Apr-Aug & FLOW & 25.0 & SEDLD & 25.0 & & & & & & & & \\
\hline $1,2,3$ & Aug-Oct & SEDLD & 50.0 & FLOW & 25.0 & & & & & & & & \\
\hline 3 & Oct-Mar & SEDLD & 50.0 & PATHO & 12.5 & & & & & & II & & (1.11, I \\
\hline 3,4 & Mar-May & SEDLD & 25.0 & PATHO & 12.5 & & & & & & & & \\
\hline 5 & May-Oct & $\mathrm{NA}$ & $\mathrm{NA}$ & NA & NA & NA & NA & NA & NA & NA & NA & NA & NA \\
\hline 6 & Oct-Mar & $\mathrm{NA}$ & $\mathrm{NA}$ & $\mathrm{NA}$ & $\mathrm{NA}$ & $\mathrm{NA}$ & $\mathrm{NA}$ & $\mathrm{NA}$ & $\mathrm{NA}$ & NA & NA & NA & NA \\
\hline 7 & Mar-Jun & NA & $\mathrm{NA}$ & $\mathrm{NA}$ & $\mathrm{NA}$ & $\mathrm{NA}$ & $\mathrm{NA}$ & $\mathrm{NA}$ & $\mathrm{NA}$ & NA & $\mathrm{NA}$ & NA & NA \\
\hline
\end{tabular}

Abundance /eve/ refers to estimated relative abundance of spawners that spawned in the natal stream reaches that populated th ! life history pathways using the reach in this table

Level 1: $\quad$ Low to average (relative to other reaches) abundance

Level 2 High (relative to other reaches) abundance

Level 3 Exceptionally high (relative to other reaches) abundance

Life stages: 1- prespawner; 2-spawner; 3- incubation; 4- fry colonization; 5- summer rearing; 6- overwintering; 7-yearlingpresmolt/smolt

Attributes: CHEM - chemicals

CSTAB - channel stability

COMP - competition (with other species)

FLOW - flow

HABD - habitat diversity
NUTLD - nutrient load

OBST - obstructions

OXYG - dissolved oxygen

PATHO - pathogens

PRED - predators (includes fishing-related losses)
RIPCN - riparian condition

SEDLD - sediment load

TEMP - water temperature

WITH - water withdrawals

"NA" indicates that no life history pathway meeting the criteria used in defining these pathways was present

Blank cells under attributes indicate no additional attributes with negative effects on productivity were evident compared to those present in the template. 
Stream Reach Summary for Vacant Spring Chinook Salmon Life Histories

Stream: Imnaha R.

Reach: Mouth - Cow Cr (RM 0.0- 4.3 from Snake R)

\begin{tabular}{|c|c|c|c|c|c|c|c|c|}
\hline \multicolumn{4}{|c|}{ Restoration benefit category: } & & \multicolumn{3}{|c|}{ Chinook usage score: } & 39.0 \\
\hline \multirow[b]{3}{*}{ Life stage } & \multirow[b]{3}{*}{ Months } & \multirow{2}{*}{\multicolumn{4}{|c|}{$\begin{array}{c}\text { No. vacant life history pathways } \\
\text { by abundance level }\end{array}$}} & \multicolumn{2}{|c|}{ Key habitat change } & \multirow{3}{*}{$\begin{array}{l}\text { Habitat } \\
\text { productivity } \\
\text { loss score }\end{array}$} \\
\hline & & & & & & \multirow{2}{*}{$\begin{array}{l}\text { Patient } \\
\text { quantity }\end{array}$} & \multirow[b]{2}{*}{$\%$ change } & \\
\hline & & Total no. & 1 & 2 & 3 & & & \\
\hline 1 & Apr-Aug & 168 & 136 & 3 & 0 & 14.6 & 0.0 & 400.0 \\
\hline $1,2,3$ & & $\mathrm{~N}$ & NA & NA & $\mathrm{N}$ & $\mathrm{N}$ & NA & NA' “ \\
\hline 3 & Ött-Mar & $\mathrm{NA}$ & $\mathrm{NA}$ & $\mathrm{NA}$ & $\mathrm{N}$ & $\mathrm{NA}$ & $\mathrm{NA}$ & $\mathrm{NA}$ \\
\hline 34 & Mar-May & $\dot{\mathrm{N}}$ & NA & $\mathrm{NA}$ & NA & $\mathrm{N}$ & NA & NÄ \\
\hline 5 & May-Oct & 4 & 35 & 7 & 0 & 9. & 0,0 & -12.4 \\
\hline 6 & Ott- & $\mathrm{N}$ & NA & NA & NA & $\mathrm{NA}$ & NA & NA \\
\hline 7 & Mar-Jun & 215 & 170 & 45 & 0 & 45.8 & $0.0^{\circ}$ & 0.0 \\
\hline
\end{tabular}

\begin{tabular}{|c|c|c|c|c|c|c|c|c|c|c|c|c|c|}
\hline \multirow[b]{2}{*}{ Life stage } & \multirow[b]{2}{*}{ Months } & \multicolumn{12}{|c|}{ Highest productivity impact attributes (\% change in impact from template) } \\
\hline & & Aft 1 & $\%$ change & Att 2 & $\%$ change & Att 3 & $\%$ change & Att 4 & $\%$ change & Atf 5 & $\%$ change & Atf 6 & $\%$ change \\
\hline 1 & Apr-Aug & PATHO & 18.9 & & & & & & & & & & \\
\hline$\dot{1}, \dot{2}, \dot{3}$ & Aug-Oct & $\mathrm{NA}$ & NA & & $\dddot{\mathrm{N}} \mathrm{M}$ & $\mathrm{NA}$ & $\dot{N} \dot{A}$ & NA & "NA" & NA & NA & $\mathrm{NA}$ & $\mathrm{NA}$ \\
\hline 3 & Ott-Mär & $" \mathrm{NA}$ & $\mathrm{NA}$ & $\mathrm{NA}$ & NA & NA & NA & & NA & $\mathrm{NA}$ & .““”NA & NA & NA \\
\hline $\begin{array}{c}3,4 \\
5\end{array}$ & Mar-May & PAA & $\frac{N A}{192}$ & NA & $\mathrm{NA}$ & NA & NA & $\ddot{N A}$ & NA & $N A$ & NA & NA & N \\
\hline 6 & Ott-Mar & NA & NA & "NA" & $\mathrm{NA}$ & NÄ & $\mathrm{NÄ}$ & $\mathrm{NA}$ & $\mathrm{NA}$ & NA & NÖ̈ & Nä & $\mathrm{NA}$ \\
\hline 7 & Mar-Jun & PRED & 19.6 & & & & & & & & & & \\
\hline
\end{tabular}

Abundance level refers to estimated relative abundance of spawners that spawned in the natal stream reaches that populated the life history pathways using the reach in this table Level 1: Low to average (relative to other reaches) abundance

Level 2: High (relative to other reaches) abundance

Level 3: Exceptionally high (relative to other reaches) abundance

Life stages: 1-prespawner; 2- spawner; 3- incubation; 4- fry colonization; 5- summer rearing; 6- overwintering; 7- yearlingresmolt/smolt

$\begin{array}{lll}\text { Attributes: CHEM - chemicals } & \text { NUTLD - nutrient load } & \text { RIPCN - riparian condition } \\ \text { CSTAB - channel stability } & \text { OBST - obstructions } & \text { SEDLD - sediment load } \\ \text { COMP - competition (with other species) } & \text { OXYG - dissolved oxygen } & \text { TEMP - water temperature } \\ \text { FLOW - flow } & \text { PATHO - pathogens } & \text { WITH - water withdrawals } \\ \text { HABD - habitat diversity } & \text { PRED - predators (includes fishing-related losses) }\end{array}$

"NA" indicates that no life history pathway meeting the criteria used in defining these pathways was present.

Blank cells under attributes indicate no additional attributes with negative effects on productivity were evident compared to those present in the template 
Stream Reach Summary for Vacant Spring Chinook Salmon Life Histories

Stream: Imnaha R.

Reach: Cow $\mathrm{Cr}$ - Lightning $\mathrm{Cr}$ (RM 4.3- 5.4 from Snake R)

\begin{tabular}{ll|ll|ll}
\hline Restoration benefit category: & E & Chinook usage score: & 54.6 & Habitat productivity loss score: & \#\#\# \\
\hline
\end{tabular}

\begin{tabular}{|c|c|c|c|c|c|c|c|c|}
\hline \multirow[b]{3}{*}{ Life stage } & \multirow[b]{3}{*}{ Morithis } & \multirow{2}{*}{\multicolumn{4}{|c|}{$\begin{array}{c}\text { No. vacant life history pathways } \\
\text { by abundance level }\end{array}$}} & \multicolumn{2}{|c|}{ Key habitat change } & \multirow{3}{*}{$\begin{array}{l}\text { Habitat } \\
\text { productivity } \\
\text { loss score }\end{array}$} \\
\hline & & & & & & \multirow{2}{*}{$\begin{array}{l}\text { Patient } \\
\text { quamity }\end{array}$} & \multirow[b]{2}{*}{ Mícnäge } & \\
\hline & & iolápitro. & & $z$ & 3 & & & \\
\hline- & Apr-Aug & 81 & $6^{\prime} 5$ & 16 & 0 & 15.7 & 0.0 & 223.9 \\
\hline 123 & Aug-Ott & NA. & NA & NA & NA & NA & $\mathrm{NA}$ & NA \\
\hline 3 & Ott-Mar & NA & N̈Ä & N̈Ä & NA & NA" & NัN" & N̈Ä \\
\hline 3,4 & Mar-M̀à & $\ddot{4}$ & ? & is? & 1 & 2 & -83 & .46 \\
\hline 5 & May-Ott & 40 & 36 & 4 & 0 & 12 & 0.0 & -21.4 \\
\hline 6 & Ott-Mar & 375 & 300 & 75 & 0 & 31.1 & 0.9 & -203.2 \\
\hline 7 & Mar-Jun & 60 & 48 & 12 & 0 & 52.0 & 0.7 & 0.0 \\
\hline
\end{tabular}

\begin{tabular}{|c|c|c|c|c|c|c|c|c|c|c|c|c|c|}
\hline \multirow[b]{2}{*}{ Life stage } & \multirow[b]{2}{*}{ Months } & \multicolumn{12}{|c|}{ Highest productivity impact attributes (\% change in impact from template) } \\
\hline & & Att 1 & $\%$ change & $\overline{\text { Att } 2}$ & $\%$ change & An 3 & $\%$ change & Att 4 & $\%$ change & Att 5 & $\%$ change & Att 6 & $\%$ change \\
\hline 1 & Apr-Aug & PATHO & 226 & & & & & & & & & & \\
\hline $1,2,3$ & Äug-Ött & $\mathrm{NA}$ & NA & $\mathrm{NA}$ & NA & $\mathrm{NA}$ & $\mathrm{NA}$ & $\mathrm{NA}$ & $\mathrm{NA}$ & $\mathrm{NA}$ & $\mathrm{NA}$ & $\mathrm{NA}$ & $\mathrm{NA}$ \\
\hline 3 “ & Ott-Mar & $\mathrm{NA}$ & NA & NA & NA & $\mathrm{NA}$ & NA & NA & $N A$ & NA & $\mathrm{NA}$ & NA & NA \\
\hline$>\quad, 3,4$. & Mar-May & PATHO & 25.6 & & & & & & & & & & \\
\hline 5 & May-Ott & РӒTHÓ & $21 . \dot{1}$ & & & & & & & & & & \\
\hline 6 & Ott-Mar & PATHO & 20.1 & SEDLD & 20.1 & PRED & 18.4 & & & & & & \\
\hline 7 & Mar-Jun & PRED & 19.2 & & & & & & & & & & \\
\hline
\end{tabular}

Abundance lovel refers to estimated relative abundance of spawners that spawned in the natal stream reaches that populated the life history pathways using the reach in this table Level 1: $\quad$ Low to average (relative to other reaches) abundance

Level 2 High (relative to other reaches) abundance

Level 3: Exceptionally high (relative to other reaches) abundance

Life stages: 1- prespawner; 2- spawner; 3- incubation; 4- fry colonization; 5- summer rearing; 6- overwintering; 7- yearlingpresmolt/smolt

Attributes: CHEM - chemicals

CSTAB - channel stability

COMP - competition (with other species)

FLOW - flow

HABD - habitat diversity
$N$ UTLD - nutrient load

OBST - obstructions

OXYG - dissolved oxygen

PATHO - pathogens

PRED - predators (includes fishing-related losses)
RIPCN - riparian condition

SEDLD - sediment load

TEMP - water temperature

WITH - water withdrawals

"NA" indicates that no life history pathway meeting the criteria used in defining these pathways was present.

Blank cells under attributes indicate no additional attributes with negative effects on productivity were evident compared to those present in the template. 
Stream Reach Summary for Vacant Spring ChinookSalmon Life Histories

Stream: Imnaha R.

Reach: Lightning $\mathrm{Cr}$ - Horse $\mathrm{Cr}$ (RM 5.4- 10.8 from Snake R)

\begin{tabular}{|llll|ll}
\hline Restoration benefit category: & F & [Chinook usage score: & 32.0 & Habitat productivity loss score:
\end{tabular}

\begin{tabular}{|c|c|c|c|c|c|c|c|c|}
\hline \multirow[b]{3}{*}{ Life stage } & \multirow[b]{3}{*}{ Months } & \multirow{2}{*}{\multicolumn{4}{|c|}{$\begin{array}{l}\text { No. vacant life history pathways } \\
\text { by abundance level }\end{array}$}} & \multicolumn{2}{|c|}{ Key habitat change } & \multirow{3}{*}{$\begin{array}{c}\text { Habitat } \\
\text { productivity } \\
\text { loss score }\end{array}$} \\
\hline & & & & & & \multirow{2}{*}{$\begin{array}{l}\text { Patient } \\
\text { quantity }\end{array}$} & \multirow[b]{2}{*}{$\%$ change } & \\
\hline & & Total no. & 1 & 2 & 3 & & & \\
\hline 1 & Apr-Aug & 111 & 86 & 25 & 0 & 14.4 & 0.0 & 320.5 \\
\hline $1,2,3$ & Aug-Oct & NA & $\mathrm{NA}$ & NA & NA & $\mathrm{NA}$ & NA & NA \\
\hline 3 & Oct-Mar & NA & $\mathrm{NA}$ & NÄ & NÄ & NÄ & NÄ & $\mathrm{NÄ}$ \\
\hline 3,4 & Mar-May & 9 & 9 & 0 & 0 & 2.2 & -83.3 & -10.3 \\
\hline 5 & May-Oct & 62 & 57 & 5 & 0 & 12.6 & -1.5 & -40.2 \\
\hline 6 & Oct-Mar & 92 & 70 & 22 & 0 & 31.8 & 0.2 & -44.9 \\
\hline 7 & Mar-Jun & 52 & 42 & 10 & 0 & 48.4 & 0.3 & 0.0 \\
\hline
\end{tabular}

\begin{tabular}{|c|c|c|c|c|c|c|c|c|c|c|c|c|c|}
\hline \multirow[b]{2}{*}{ Life stage } & \multirow[b]{2}{*}{ Months } & \multicolumn{12}{|c|}{ Highest productivity impact attributes (\% change in impact from template) } \\
\hline & & Att 1 & $\%$ change & Att 2 & $\%$ change & Att 3 & $\%$ change & Att 4 & $\%$ change & Att 5 & $\%$ change & Att 6 & $\%$ change \\
\hline 1 & Apr-Aug & PATHO & 24.1 & & & & & & & & & & \\
\hline $1,2,3$ & Aug-Ott & $\mathrm{NÄ}$ & $\mathrm{NA}$ & $\mathrm{NÄ}$ & $\mathrm{NA}$ & $\mathrm{NA}$ & NA & $\mathrm{NÄ}$ & $\mathrm{NÄ}$ & NÄ & NÄ & "NA"' & $\mathrm{NA}$ \\
\hline 3 & Ott-Mar & NA & NA & NA & NA & NA & NA & NA & NA & NA & NA & NA & NA \\
\hline $3,4, \ldots$ & Mar-May & PATHOO & 25.0 & CSTABB' & 12.5 & & & & & & & & \\
\hline 5 & May-Oct & PATHO & 22.4 & & & & & & & & & & \\
\hline 6 & Ott-M̈ar & "̈ÄT̈ḦÖO & 17.4 & SEDLD & 17.4 & PRED & 16.9 & & & & & & \\
\hline 7 & Mar-Jun & PRED & 19.4 & & & & & & & & & & \\
\hline
\end{tabular}

Abundance/eve/refers to estimated relative abundance of spawners that spawned in the natal stream reaches that populated the life history pathways using the reach in this table Level 1: $\quad$ Low to average (relative to other reaches) abundance

Level 2: High (relative to other reaches) abundance

Level 3 Exceptionally high (relative to other reaches) abundance

Life stages: 1-prespawner; 2- spawner; 3- incubation; 4- fry colonization; 5- summer rearing; 6- overwintering; 7-yearlincpresmolt/smolt

Attributes: CHEM - chemicals CSTAB - channel stability

COMP - competition (with other species)

FLOW - flow

HABD - habitat diversity
NUTLD - nutrient load

OBST - obstructions

OXYG - dissolved oxygen

PATHO - pathogens

PRED - predators (includes fishing-related losses)
RIPCN - riparian condition SEDLD - sediment ioad

TEMP - $\quad$ water temperature

WITH - water withdrawals

"NA" indicates that no life history pathway meeting the criteria used in defining these pathways was present.

Blank cells under attributes indicate no additional attributes with negative effects on productivity were evident compared to those present in the template. 
Stream Reach Summary for Vacant Spring Chinook Salmon Life Histories

Stream: Imnaha R.

Reach: Fence $\mathrm{Cr}_{\mathrm{r}}$ - Big Sheep $\mathrm{Cr}(\mathrm{RM} 16.3-22.3$ from Snake $\mathrm{R})$

\begin{tabular}{|l|l|ll|l}
\hline Restoration benefit category: & E & Chinook usage score: & 83.6 & Habitat productivity loss score: \\
\hline
\end{tabular}

\begin{tabular}{|c|c|c|c|c|c|c|c|c|}
\hline \multirow[b]{3}{*}{ Life stage } & \multirow[b]{3}{*}{ Months } & \multirow{2}{*}{\multicolumn{4}{|c|}{$\begin{array}{c}\text { No. vacant life history pathways } \\
\text { by abundance level }\end{array}$}} & \multicolumn{2}{|c|}{ Key habitat change } & \multirow{3}{*}{$\begin{array}{c}\text { Habitat } \\
\text { productivity } \\
\text { loss score }\end{array}$} \\
\hline & & & & & & \multirow{2}{*}{$\begin{array}{l}\text { Patient } \\
\text { quantity }\end{array}$} & \multirow[b]{2}{*}{$\%$ change } & \\
\hline & & Total no. & 1 & 2 & 3 & & & \\
\hline 1 & Apr-Aug & 130 & 109 & 21. & 0 & 111 & 0.0 & -5.34 \\
\hline 1,23 & Aug-Oct & 30 & 30 & 0 & 0 & 31 & -58.3 & -30 \\
\hline 3 & Ott-Mar & 60 & 60 & 0 & 0 & 1.3 & -83.3 & -124.3 \\
\hline 34 & Mar-May & 62 & 45 & 17 & 0 & 1.7 & -833 & -71.0 \\
\hline 5 & May-Ott & 265 & 191 & 74 & 0 & 9.0 & -0.2 & -202.0 \\
\hline 6 & Ott-Mar & 151 & 114 & 37 & 0 & 19.9 & -3.9 & -95.6 \\
\hline 7 & Mar-Jun & 34 & 28 & 6 & 0 & 34.0 & $-15^{\prime \prime \prime \prime}$ & 25.9 \\
\hline
\end{tabular}

\begin{tabular}{|c|c|c|c|c|c|c|c|c|c|c|c|c|c|}
\hline \multirow[b]{2}{*}{ Life stage } & \multirow[b]{2}{*}{ Months } & \multicolumn{12}{|c|}{ Highest productivity impact attributes (\% change in impact from template) } \\
\hline & & Att 1 & $\%$ change & Att 2 & $\%$ chan & Att 3 & $\%$ change & $\overline{A t !} 4$ & $\%$ change & Att 5 & $\%$ change & Aft 6 & \% change \\
\hline & Apr-Aug & PATHO & 22.9 & TEMP & 8.3 & CSTAB & 8.3 & & & & & & \\
\hline 123 & Auq-Ött & CSTAB & $25.0^{\circ}$ & TEMP & 83 & PATHO & 8.3 & & & & & & \\
\hline 3“"” & Oct-Mar & CSTAB & 25.0 & PATHO & 12.5 & & & & & & & & \\
\hline 34 & Mar-May & $\begin{array}{ll}\text { CSTAR } \\
\text { CST }\end{array}$ & 250 & PATHO & 250 & & & & & & & & \\
\hline 5 & Mav-Ott & PATHO & 24.2 & & & & & & & & & & \\
\hline 6 & $\mathrm{OCt}-\mathrm{Mar}$ & PATHO & 19.9 & RIPCN & 19.9 & SEDLD & 19.9 & & & & & & \\
\hline 7 & Mar-Jun & & & & & & & & & & & & \\
\hline
\end{tabular}

Abundancelevel refers to estimated relative abundance of spawners that spawned in the natal stream reaches that populated the life history pathways using the reach in this table Level 1: $\quad$ Low to average (relative to other reaches) abundance

Level 2: $\quad$ High (relative to other reaches) abundance

Level 3: Exceptionally high (relative to other reaches) abundance

Life stages: 1-prespawner; 2- spawner; 3- incubation; 4- fry colonization; 5- summer rearing; 6- overwintering; 7- yearlingpresmolt/smolt

$\begin{array}{lll}\text { Attributes: } & \text { NHEM - chemicals } & \text { RIPCN - riparian condition } \\ \text { CSTAB - channel stability } & \text { OBST - nutrient load } & \text { SEstructions } \\ \text { COMP - competition (with other species) } & \text { OXYG - dissolved oxygen } & \text { TEMP - wadiment load } \\ \text { FLOW - tt Ow } & \text { PATHO - pathogens temperature } \\ \text { HABD - habitat diversity } & \text { PRED - predators (includes fishing-related losses) } & \text { WITH - water withdrawals }\end{array}$

"NA" indicates that no life history pathway meeting the criteria used in defining these pathways was present.

Blank cells under attributes indicate no additional attributes with negative effects on productivity were evident compared to those present in the template. 


\section{Stream Reach Summary for Vacant Spring Chinook Salmon Life Histories}

Stream: Imnaha R.

Reach: Horse $\mathbf{C r}$ - Fence $\mathbf{C r}$ (RM 10.8 -16.3 from Snake R)

\begin{tabular}{ll|ll|ll|}
\hline [Restoration benefit category: & $\mathrm{E}$ & Chinook usage score: & 43.7 & Habitat productivity loss score:
\end{tabular}

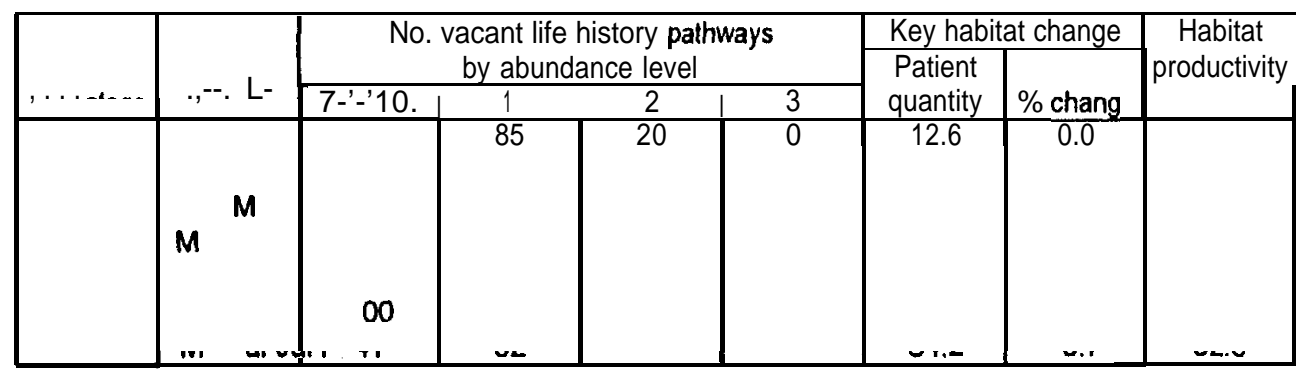

\begin{tabular}{|c|c|c|c|c|c|c|c|c|c|c|c|c|c|}
\hline \multirow[b]{2}{*}{ Life stage } & \multirow[b]{2}{*}{ Months } & \multicolumn{12}{|c|}{ Highest productivity impact attributes (\% change in impact from template) } \\
\hline & & Att 1 & $\%$ change & Att 2 & $\%$ change & Art 3 & $\%$ change & Att 4 & $\%$ change & Att 5 & $\%$ change & Att 6 & $\%$ change \\
\hline 1 & Apr-Aug & PATHO & 23.9 & & & & & & & & & & \\
\hline $1,2,3$ & Aug.Oct & NA & NA & NA & NA & NA & NA & NA & NA & NA & NA & NA & NA \\
\hline $3 “$ & Ött-Mar & NA & NA & NA & NA & NA & NA & NA & NA & “ NA & NA & NA & NA \\
\hline 3.4 & Mar-May & CSTAB & $25.0^{\circ}$ & PATHO & 250 & RIPCN & 250 & & & & & & \\
\hline 5 & May-Oct & PATHO & 23.5 & HABD & 22.3 & SEDLD & 22.3 & & & & & & \\
\hline 6 & Oct-Mar & HABD & 24.4 & PATHO & 24.4 & SEDLD & 24.4 & & & & & & \\
\hline 7 & Mar-Jun & & & & & & & & & & & & \\
\hline
\end{tabular}

Abundancelevel refers to estimated relative abundance of spawners that spawned in the natal stream reaches that populated the life history pathways using the reach in this table Level 1: Low to average (relative to other reaches) abundance

Level 2 High (relative to other reaches) abundance

Level 3: Exceptionally high (relative to other reaches) abundance

Life stages: 1- prespawner; 2. spawner; 3- incubation; 4- fry colonization; 5- summer rearing; 6- overwintering; 7- yearling presmolt/smolt

\section{Attributes; CHEM - chemicals}

CSTAB - channel stability

COMP - competition (with other species)

FLOW - flow

HABD - habitat diversity

\section{NUTLD - nutrient load}

OBST - obstructions

OXYG - dissolved oxygen

PATHO - pathogens

PRED - predators (includes fishing-related losses)
RIPCN - riparian condition

SEDLD - sediment load

TEMP - water temperature

WITH - water withdrawals

"NA" indicates that no life history pathway meeting the criteria used in defining these pathways was present.

Blank cells under attributes indicate no additional attributes with negative effects on productivity were evident compared to those present in the template. 
Stream Reach Summary for Vacant Spring Chinook Salmon Life Histories

Stream: Imnaha $\mathbf{R}$.

Reach: Big Sheep Cr - Freezeout Cr (RM 22,3 -35.2 from Snake R)

\begin{tabular}{|c|c|c|c|c|c|c|c|c|}
\hline Restorati & n benefit & category: & & $E$ & Chinook & usage sc & ore: & 70.3 \\
\hline & & No. $x$ & nnt I & istory pat & ays & Key hab & at change & Habitat \\
\hline & & & & ance leve & & Patient & & productivity \\
\hline Life stage & Mointns & Tota'nor: & 4 & 2 & 3 & quantity & $\%$ change & loss score \\
\hline 1 & Apr-Aug & 85 & $\sqrt{51}$ & 34 & 0 & 12.4 & -12.2 & -129.1 \\
\hline $1,2,3$ & Aug-Oct & 1190 & ipi & 100 & Ia & $\$ 2$ & -59.4 & -133.6 \\
\hline 3 & Oct-Mar & $80^{\circ}$ & 0 & 80 & 0 & 1.4 & -83.3 & -237.5 \\
\hline 3,4 & Mar-May & 52 & 30 & 22 & 0 & 1.7 & -77.1 & -167.1 \\
\hline 5 & May-Oct & 193 & 144 & 49 & 0 & 9.9 & -6.3 & -96.6 \\
\hline 6 & Oct-Mar & 76 & 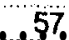 & 1919 & 0 & 20.3 & -7.9 & -860 \\
\hline 7 & Mar-Juń & 11 & & $z^{\prime}$ & 0 & 20,0 & -0.6 & 233 \\
\hline
\end{tabular}

\begin{tabular}{|c|c|c|c|c|c|c|c|c|c|c|c|c|c|}
\hline \multirow[b]{2}{*}{ Life stage } & \multirow[b]{2}{*}{ Months } & \multicolumn{12}{|c|}{ Highest productivity impact attributes (\% change in impact from template) } \\
\hline & & Att 1 & $\%$ change & Att 2 & $\%$ change & Att 3 & $\%$ change & Att 4 & $\%$ change & Att 5 & $\%$ change & Att 6 & $\%$ change \\
\hline 1 & Apr-Aug & CSTAB & 25.0 & FLOW & 250 & HABD & 24.9 & OBST & 24,9 & PATHO & 24.9 & PRED & 249 \\
\hline$\ddot{1} \ddot{2}, \dot{3}$ & Aug-Ocd & CSTAB & 50,0 & FLÖW & 50.0 & HABD & $25 . \dot{0}$ & PÄT̈HÖ & 25.0 & PRÉĖ & 25.0 & SEDLD & 25.0 \\
\hline "3 & Ott-Mar & CSTAB & 500 & FLoW & 50.0 & COMP & 25.0 & HABD & 25.0 & PATHO & 25.0 & RIPCN & 25.0 \\
\hline 3,4 & Mar-May & CSTAB & 50.0 & FLOW & 50.0 & Ḧ̈̈̈̈ं & 50.0 & CंÖMP & 25.0 & PATHO & 25.0 & RIPCN & 25.0 \\
\hline 5 & May-Ott & $\mathrm{HABD}$ & 49.8 & COMP & 24.9 & FLOW & 24.9 & PATेНÖO & $24.9^{\circ}$ & RIPCN & 249 & WITH & 24.9 \\
\hline 6 & Ott-Mar & "CÖM̄P" & $25.0^{\circ}$ & FLOOWW & 25.0 & PӒТНӦ & 25.0 & RIPCN & 25.0 & SEEDELD" & 250 & Ẅ|TัT̈H & 25.0 \\
\hline
\end{tabular}

Abundance level refers to estimated relative abundance of spawners that spawned in the natal stream reaches that populated the life history pathways using the reach in this table Level 1: Low to average (relative to other reaches) abundance

Level 2 High (relative to other reaches) abundance

Level 3: Exceptionally high (relative to other reaches) abundance

Life stages: 1- prespawner; 2-spawner; 3-incubation; 4- fry colonization; 5- summer rearing; 6- overwintering; 7-yearling presmolt/smolt

Attributes: CHEM - chemicals

CSTAB - channel stability

COMP - competition (with other species)

FLOW - flow

HABD - habitat diversity

$\begin{array}{ll}\text { NUTLD } & \text { nutrient load } \\ \text { OBST - } & \text { obstructions } \\ \text { OXYG - } & \text { dissolved oxygen } \\ \text { PATHO } & \text { - pathogens } \\ \text { PRED - } & \text { predators (includes fishing-related losses) }\end{array}$

\begin{abstract}
RPCN - riparian condition
SEDLD - sediment load

TEMP - water temperature

WITH - water withdrawals
\end{abstract}

"NA" indicates that no life history pathwsy meeting the criteria used in defining these pathways was present.

Blank cells under attributes indicate no additional attributes with negative effects on productivity were evident compared to those present in the template. 
Stream Reach Summary for Vacant Spring Chinook Salmon Life Histories

Stream: Imnaha R.

Reach: Freezeout Cr - Grouse Cr (RM 35.2 -40.6 from Snake R)

\begin{tabular}{llll|ll}
\hline Restoration benefit category: & E & [Chinook usage score: & 50.1 & Habitat productivity loss score:
\end{tabular}

\begin{tabular}{|c|c|c|c|c|c|c|c|c|}
\hline \multirow[b]{3}{*}{ Life stage } & \multirow[b]{3}{*}{ Months } & \multirow{2}{*}{\multicolumn{4}{|c|}{$\begin{array}{c}\text { No. vacant life history pathways } \\
\text { by abundance level }\end{array}$}} & \multicolumn{2}{|c|}{ Key habitat change } & \multirow{3}{*}{$\begin{array}{c}\text { Habitat } \\
\text { productivity } \\
\text { loss score }\end{array}$} \\
\hline & & & & & & \multirow{2}{*}{$\begin{array}{l}\text { Patient } \\
\text { quantity }\end{array}$} & \multirow[b]{2}{*}{$\%$ change } & \\
\hline & & Total no. & 1 & 2 & 3 & & & \\
\hline 1 & Apr-Aug & 45 & 44 & 1 & 0 & 10.3 & -4.9 & -86.2 \\
\hline 123 & Aug-Ott & 50 & 50 & 0 & 0 & 3.2 & -59.4 & -167.1 \\
\hline 3 & Ott-Mar & 100 & 100 & 0 & 0 & 1.4 & -83.7 & -296.8 \\
\hline 34 & Mar-May & 44 & 44 & 0 & 0 & 1.7 & $-83 . \dot{1}$ & -1794 \\
\hline 5 & May-Ott & 148 & 147 & 1 & 0 & 96 & -3.7 & -83.3 \\
\hline 6 & Ott-Mar & 38 & 38 & 0 & 0 & 203 & -2.6 & -33.2 \\
\hline 7 & Mar-Jun & 4 & 4 & 0 & 0 & 33.0 & -6.2 & 0.5 \\
\hline
\end{tabular}

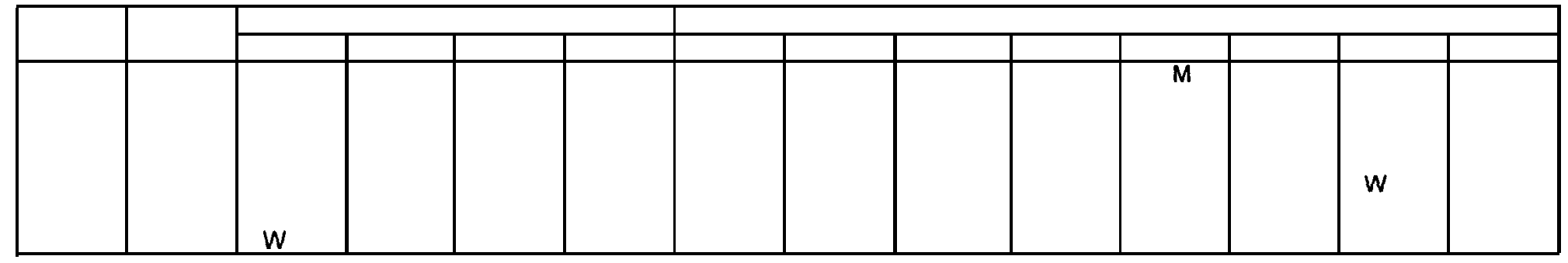

Abundance level refers to estimated relative abundance of spawners that spawned in the natal stream reaches that populated the life history pathways using the reach in this table Level 1: $\quad$ Low to average (relative to other reaches) abundance

Level 2: $\quad$ High (relative to other reaches) abundance

Level 3: Exceptionally high (relative to other reaches) abundance

Life stages: 1- prespawner; 2- spawner; 3-incubation; 4- fry colonization; 5- summer rearing; 6-overwintering; 7- yearling presmoll/smolt

Attributes: CHEM - chemicals

CSTAB - channel stability

COMP - competition (with other species)

FLOW - flow

HABD - habitat diversity
NUTLD - nutrient load

OBST - obstructions

OXYG - dissolved oxygen

PATHO - pathogens

PRED - predators (includes fishing-related losses)
RIPCN - riparian condition

SEDLD - sediment load

TEMP - water temperature

WITH - water withdrawals

"NA" indicates that no life history pathway meeting the criteria used in defining these pathways was present.

Blank cells under attributes indicate no additional attributes with negative effects on productivity were evident compared to those present in the template. 
Stream Reach Summary for Vacant Spring Chinook Salmon Life Histories

Stream: Imnaha $\mathbf{R}$.

Reach: Grouse $\mathrm{Cr}$ - Summit $\mathrm{Cr}$ (RM 40.6 -43.6 from Snake R)

\begin{tabular}{|c|c|c|c|c|c|c|c|c|c|c|c|c|c|}
\hline \multicolumn{4}{|c|}{ Restoration benefit category: } & $\mathbf{F}$ & \multicolumn{3}{|c|}{ [Chinook usage score: } & 31.6 & \multicolumn{4}{|c|}{ Habitat productivity loss score: } & \#\#\#\#\# \\
\hline \multirow[b]{3}{*}{ Life stage } & \multirow[b]{3}{*}{ Months } & \multirow{2}{*}{\multicolumn{4}{|c|}{$\begin{array}{l}\text { No. vacant life history pathways } \\
\text { by abundance level }\end{array}$}} & \multicolumn{2}{|c|}{ Key habitat change } & \multirow{3}{*}{$\begin{array}{c}\text { Habitat } \\
\text { productivit }\end{array}$} & & & & & \\
\hline & & & & & & \multirow{2}{*}{$\begin{array}{l}\text { Patient } \\
\text { quantity }\end{array}$} & & & & & & & \\
\hline & & Total no. & 1 & 2 & 3 & & & & & & & & \\
\hline 1 & Apr-Aug & 40 & 39 & 1 & 0 & 10.5 & - & & & & & & \\
\hline $1,2,3$ & Aug-Oct & 50 & 50 & 0 & 0 & 3.2 & -58.3 & -1671 & & & & & \\
\hline 3 & Oct-Mar & 100 & 100 & 0 & 0 & 1.4 & -83.3 & -296.8 & & & & & \\
\hline 3,4 & Mar-May & 33 & 33 & 0 & 0 & 1.8 & -80.8 & -132.0 & & & & & \\
\hline 5 & May-Oct & 75 & 74 & 1 & 0 & 9.9 & 0.0 & -41.3 & & & & & \\
\hline 6 & Oct-Mar & 19 & 19 & 0 & 0 & 20.5 & 0.0 & -16.6 & & & & & \\
\hline 7 & Mar-Jun & 2 & 2 & 0 & 0 & 35.8 & 2.4 & 0.2 & & & & & \\
\hline \multirow[b]{2}{*}{ Life stage } & \multirow[b]{2}{*}{ Months } & \multicolumn{12}{|c|}{ Highest productivity impact attributes (\% change in impact from template) } \\
\hline & & Alt 1 & $\%$ change & Aft 2 & $\%$ change & Att 3 & $\%$ change & Att 4 & $\%$ change & Att 5 & $\%$ change & Att 6 & $\%$ change \\
\hline 1 & Apr-Aug & CSTAB & 25.0 & FLOW & 25.0 & HABD & 23.0 & OBST & 23.0 & PATHO & 23.0 & PRED & 23.0 \\
\hline $1,2,3$ & Aug-Oct & CSTAB & 50.0 & FLOW & 50.0 & HABD & 25.0 & PATHO & 25.0 & PRED & 25.0 & SEDLD & 25.0 \\
\hline 3 & Oct-Mar & CSTAB & 50.0 & FLOW & 50.0 & COMP & 25.0 & HABD & 25.0 & PATHO & 25.0 & RIPCN & 25.0 \\
\hline 3,4 & Mar-May & CSTAB & 50.0 & FLOW & 50.0 & HABD & 50.0 & COMP & 25.0 & PATHO & 250 & RIPCN & 25.0 \\
\hline 5 & May-Oct & HABD & 48.8 & COMP & 24.4 & FLOW & 24.4 & PATHO & 24.4 & RIPCN & 24.4 & WITH & 24.4 \\
\hline 6 & Oct-Mar & COMP & 25.0 & FLOW & 25.0 & PATHO & 25.0 & RIPCN & 25.0 & SEDLD & 25.0 & "wiтн" & 25.0 \\
\hline 7 & Mar-Jun & WITH & 16.4 & & & & & & & & & & \\
\hline
\end{tabular}

Abundance level refers to estimated relative abundance of spawners that spawned in the natal stream reaches that populated the life history pathways using the reach in this table Level 1: $\quad$ Low to average (relative to other reaches) abundance

Level 2 High (relative to other reaches) abundance

Level 3 Exceptionally high (relative to other reaches) abundance

Life stages: 1 - prespawner; 2- spawner; 3- incubation; 4- fry colonization; 5- summer rearing; 6overwintering; 7-yearling presmolt/smolt

Attributes: CHEM - chemicals

CSTAB - channel stability

COMP - competition (with other species)

FLOW - flow

HABD - habitat diversity
NUTLD - nutrient load

OBST - obstructions

OXYG - dissolved oxygen

PATHO - pathogens

PRED - predators (includes fishing-related losses)
RIPCN - riparian condition

SEDLD - sediment load

TEMP - water temperature

WITH - water withdrawals

"NA" indicates that no life history pathway meeting the criteria used in defining these pathways was present.

Blank cells under attributes indicate no additional attributes with negative effects on productivity were evident compared to those present in the template. 
Stream Reach Summary for Vacant Spring Chinook Salmon Life Histories

Stream: Imnaha R.

Reach: Summit Cr - Crazyman Cr (RM 43.6 -49.4 from Snake R)

\begin{tabular}{|ll|ll|ll|}
\hline Restoration benefit category: & F & Chinook usage score: & 38.1 & Habitat productivity loss score: \\
\hline
\end{tabular}

\begin{tabular}{|c|c|c|c|c|c|c|c|c|}
\hline \multirow[b]{3}{*}{ Life stage } & \multirow[b]{3}{*}{ Months } & \multirow{2}{*}{\multicolumn{4}{|c|}{$\begin{array}{c}\text { No. vacant life history pathways } \\
\text { by abundance level }\end{array}$}} & \multirow{2}{*}{\multicolumn{2}{|c|}{ Key habit it thange }} & \multirow{3}{*}{$\begin{array}{c}\text { Habiltat } \\
\text { productivity } \\
\text { dessssomre }\end{array}$} \\
\hline & & & & & & Patient & & \\
\hline & & Total no. & 1 & $\overline{2}$ & 3 & quantity & $\%$ thange & \\
\hline 1 & Apr-Aug & 35 & 34 & $\frac{7}{1}$ & 0 & 8.7 & -6.8 & -61.7 \\
\hline $1,2,3$ & Aug-Ott & 50 & 50 & 0 & 0 & 2.9 & -46.7 & -133.6 \\
\hline 3 & Ott-Mar & 100 & 100 & 0 & " & 1.2 & -647 & -237.5 \\
\hline 34 & Mar-May & 31 & $31^{\circ}$ & 0 & 0 & 1.5 & -78.4 & -116.5 \\
\hline 5 & May-Ott & 73 & 72 & ‘ & 0 & 8.7 & -8.7 & -41.0 \\
\hline 6 & Ott-Mar & 19 & 19 & 0 & 0 & 18.3 & -339 & -36.4 \\
\hline 7 & Mar-Jun & 1 & 1 & 0 & 0 & 29.7 & -4.0 & 0.8 \\
\hline
\end{tabular}

\begin{tabular}{|c|c|c|c|c|c|c|c|c|c|c|c|c|c|}
\hline \multirow[b]{2}{*}{ Life stage } & \multirow[b]{2}{*}{ Months } & \multicolumn{12}{|c|}{ Highest productivity impact attributes (\% change in impact from template) } \\
\hline & & Atf 1 & $\%$ change & Att 2 & $\%$ change & Art 3 & $\%$ change & Att 4 & $\%$ change & Att 5 & $\%$ change & Att 6 & $\%$ change \\
\hline 年 & Apr-Aug & HABD & 24.1 & oBST & 24.1 & PATHO & 24.1 & PRED & 24.1 & TEM & 24.1 & CSTAB & 16.7 \\
\hline $1,2,3$ & Äug-Ött & CSTAB & $50.0^{\circ}$ & FLOW & $50.0^{\circ}$ & SEÉËL̄ & $25.0^{\circ}$ & PATHO & $167^{\circ}$ & TEMP & 16.7 & HABD & 16.7 \\
\hline 3 & Oct-Mar & CSTAB & 50.0 & FLOW & 50.0 & SEDLD & $25^{\circ} .0$ & COMP & 12.5 & HABD & 12.5 & PATHO & 12.5 \\
\hline 3,4 & Mar-May & HABD & 5,000 & COMP & 25.0 & CSTAB & 25.0 & FLOW & 25.0 & РATHO & 250 & RIPCN & 25.0 \\
\hline $5, ", \cdots$ & May-Ott & $\mathrm{HABD}$ & 48.9 & COMP & 24.4 & F́'ÓW' & 24.4 & PATHO & 24.4 & RIPCN & 24.4 & WIṪH் & 24.4 \\
\hline 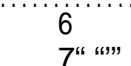 & Ott-Mar & COMP & $\begin{array}{l}25.0 \\
25.0\end{array}$ & F்ட்ல் & 25.0 & 'PATHO' & 25.0 & RIPCN & 25.0 & SEEDÉD & 25.0 & WITH & 25.0 \\
\hline
\end{tabular}

Abundance level refers to estimated relative abundance of spawners that spawned in the natal stream reaches that populated the life history pathways using the reach in this table Level 1: Low to average (relative to other reaches) abundance

Level 2: $\quad$ High (relative to other reaches) abundance

Level 3: Exceptionally high (relative to other reaches) abundance

Life stages.' 1-prespawner; 2-spawner; 3- incubation; 4- fry colonization; 5- summer rearing; 6- overwintering; 7- yearlingpresmolt/smolt

Attributes: CHEM. chemicals

CSTAB - channel stability

COMP - competition (with other species)

FLOW - flow

HABD - habitat diversity
NUTLD - nutrient load

OBST - obstructions

OXYG - dissolved oxygen

PATHO - pathogens

PRED - predators (includes fishing-related losses)
RIPCN - riparian condition

SEDLD - sediment load

TEMP - water temperature

WITH - water withdrawals

"NA" indicates that no life history pathway meeting the criteria used in defining these pathways was present

Blank cells under attributes indicate no additional attributes with negative effects on productivity were evident compared to those present in the template. 
Stream Reach Summary for Vacant Spring Chinook Salmon Life Histories

Stream: Imnaha R.

Reach: Crazyman $\mathrm{Cr}$ - Gumboot $\mathrm{Cr}$ (RM 49.4 -52.7 from Snake R)

\begin{tabular}{|ll|ll|lll|}
\hline Restoration benefit category: & F & Chinook usage score: & 11.5 & Habitat productivity loss score: & -10.2 \\
\hline
\end{tabular}

\begin{tabular}{|c|c|c|c|c|c|c|c|c|}
\hline \multirow[b]{3}{*}{ Life stage } & \multirow[b]{3}{*}{ Months } & \multirow{2}{*}{\multicolumn{4}{|c|}{$\begin{array}{l}\text { No. vacant life history pathways } \\
\text { by abundance level }\end{array}$}} & \multicolumn{2}{|c|}{ Key habitat change } & \multirow{3}{*}{$\begin{array}{c}\text { Habitat } \\
\text { productivity } \\
\text { loss score }\end{array}$} \\
\hline & & & & & & \multirow{2}{*}{$\begin{array}{l}\text { Patient } \\
\text { quantity }\end{array}$} & \multirow[b]{2}{*}{$\%$ change } & \\
\hline & & Total no. & 1 & 2 & 3 & & & \\
\hline & Apr-Aug & 20 & 19 & 1 & 0 & 9.4 & -4.5 & 0.0 \\
\hline $1,2,3$ & Aug-Ott & 30. & 30 & 0 & 0 & 7.7 & -51.0 & -1.1 \\
\hline 3 & Ott-Mar & 60 & "艹ö & 0 & 0 & 8.3 & -58.9 & -60 \\
\hline 34 & Mar-Mày & 6 & 6 & 0 & $\dot{0}$ & 9.8 & -38.1 & -31 \\
\hline 5 & May-Ott & 2 & 11 & 1 & 0 & 7.5 & 0.0 & -0.1 \\
\hline 6 & Ott-Mar & NA & NA & NA & NA & NA & NA & NA \\
\hline 7 & Mar-Jun & $\mathrm{NA}$ & NA & NA & NA & NA & NA & NA \\
\hline
\end{tabular}

\begin{tabular}{|c|c|c|c|c|c|c|c|c|c|c|c|c|c|}
\hline \multirow[b]{2}{*}{ Life stage } & \multirow[b]{2}{*}{ Months } & \multicolumn{12}{|c|}{ Highest productivity impact attributes (\% change in impact from template) } \\
\hline & & Att 1 & $\%$ change & Att 2 & $\%$ change & An 3 & $\%$ change & Att 4 & $\%$ change & Att 5 & $\%$ change & Att 6 & $\%$ change \\
\hline 1 & Apr-Aug & HABD & 17,7 & OBST & 17.7 & PATHO & 17.7 & SEDLD & 17.7 & & & & \\
\hline$, 1,2,3$ & Aug-Ött & "HABD & 25,0 & PRRED & 25.0 & SEDLD & 25.0 & OBST & 12.5 & PATHO & 12.5 & & \\
\hline 3 & Oct-Mar & COMP & 25.0 & PATHO & 25.0 & & & & & & & & \\
\hline 3,4 & Mar-May & COMP & 25,0 & PATHO & 250 & & & & & & & & \\
\hline 5 & May-Ott & FLLOW & 4.8 & PATHO & 4.8 & & & & & & & & \\
\hline 6 & Ött-Mar & $\mathrm{NA}$ & "NÄ & NÄ & NÄ & NA & $\mathrm{NA}$ & $\mathrm{NA}$ & NA & NA & NA" & $\mathrm{NÄ}$ & NA \\
\hline 7 ، & Mar-Jun & NA & NA & NA & NA & NA & NA" & NA & NA & NA & NA & NA & NA \\
\hline
\end{tabular}

Abundance level refers to estimated relative abundance of spawners that spawned in the natal stream reaches that populated the life history pathways using the reach in this table Level 1: Low to average (relative to other reaches) abundance

Level 2: $\quad$ High (relative to other reaches) abundance

Level 3: Exceptionally high (relative to other reaches) abundance

Life stages: 1- prespawner; 2- spawner; 3- incubation; 4- fry colonization; 5- summer rearing; 6- overwintering; 7- yearling presmolt/smolt

$\begin{array}{clc}\text { Attributes: } & \text { NHEM - chemicals } & \text { RIPCN - riparian condition } \\ \text { CSTAB - channel stability } & \text { OBST - nutrient load } & \text { obstructions } \\ \text { COMP - competition (with other species) } & \text { OXYG - dissolved oxygen } & \text { TEMP - sediment load } \\ \text { FLOW - flow } & \text { PATHO - pathogens temperature } \\ \text { HABD - habitat diversity } & \text { PRED - predators (includes fishing-related losses) } & \text { WITH - water withdrawals }\end{array}$

"NA" indicates that no life history pathway meeting the criteria used in defining these pathways was present.

Blank cells under attributes indicate no additional attributes with negative effects on productivity were evident compared to those present in the template, 
Stream Reach Summary for Vacant Spring Chinook Salmon Life Histories

Stream: ImnahaR.

Reach: Gumboot Cr - Dry Cr (RM 52.7 -55.4 from Snake R)

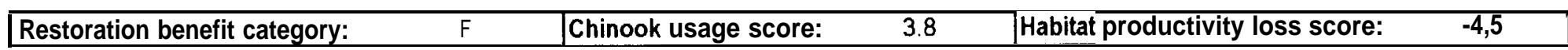

\begin{tabular}{|c|c|c|c|c|c|c|c|c|}
\hline \multirow[b]{3}{*}{ Life stage } & \multirow[b]{3}{*}{ Months } & \multirow{2}{*}{\multicolumn{4}{|c|}{$\begin{array}{c}\text { No. vacant life history pathways } \\
\text { by abundance level }\end{array}$}} & \multicolumn{2}{|c|}{ Key habitat change } & \multirow{3}{*}{$\begin{array}{c}\text { Habitat } \\
\text { productivity } \\
\text { loss score }\end{array}$} \\
\hline & & & & & & \multirow{2}{*}{$\begin{array}{l}\text { Patient } \\
\text { quantity }\end{array}$} & \multirow[b]{2}{*}{$\%$ change } & \\
\hline & & Total no. & 1 & 2 & 3 & & & \\
\hline 1 & Apr-Aug & 2 & 1 & 1 & 0 & 11.3 & -4.3 & 0.0 \\
\hline $1,2,3$ & Aug-Oct & NA & $\mathrm{NA}$ & NA & $\mathrm{NA}$ & NA & NA & $\mathrm{NA}$ \\
\hline 3 & Oct-Mar & $\mathrm{NA}$ & NÄ & $N A$ & $\mathrm{NA}$ & $\mathrm{NA}$ & $\mathrm{NA}$ & $\mathrm{NA}$ \\
\hline 3,4 & Mar-May & 4 & 0 & 4 & 0 & 9.4 & -17.3 & 0.0 \\
\hline 5 & May.Oct & 21 & 1 & 20 & 0 & 9.5 & -2.5 & -4.4 \\
\hline 6 & Oct-Mar & $\mathrm{NA}$ & NA & NA & NA & $\mathrm{NA}$ & NA & $\mathrm{NA}$ \\
\hline 7 & Mar-Jun & $\mathrm{NA}$ & NÄ & NÄ & NÄ & NÄ & NA & $\mathrm{NA}$ \\
\hline
\end{tabular}

\begin{tabular}{|c|c|c|c|c|c|c|c|c|c|c|c|c|c|}
\hline \multirow[b]{2}{*}{ Life stage } & \multirow[b]{2}{*}{ Months } & \multicolumn{12}{|c|}{ Highest productivity impact attributes (\% change in impact from template) } \\
\hline & & Att 1 & $\%$ change & Att12 & $\%$ change & Att 3 & $\%$ change & Att 4 & $\%$ change & Att 5 & $\%$ change & Att 6 & $\%$ change \\
\hline 1 & Apr-Aug & HABD & 17.2 & SEDLD & 17.2 & & & & & & & & \\
\hline $1,2,3$ & Aug-Oct & NA & NA & $\mathrm{NA}$ & $\mathrm{NA}$ & $\mathrm{NA}$ & $\mathrm{NA}$ & NA & NA & NA & NÄ & NA & NA \\
\hline 3 & Oct-Mar & $\mathrm{NA}$ & $\mathrm{NA}$ & NA & $\mathrm{NA}$ & $\mathrm{NA}$ & NA & $\mathrm{NA}$ & $\mathrm{NA}$ & $\mathrm{NA}$ & $\mathrm{NA}$ & $\mathrm{NA}$ & $\mathrm{NA}$ \\
\hline 3,4 & Mar-May & FLOW & 25.0 & PATHO & 250 & & & & & & & & \\
\hline 5 & May-Oct & FLOW & 24.0 & PATHO & 24.0 & & & & & & & & \\
\hline 6 & Oct-Mar & NA & $\mathrm{NA}$ & $\mathrm{NA}$ & $\mathrm{NA}$ & NA & NA & $\mathrm{NA}$ & NA & $\mathrm{NA}$ & $\mathrm{NA}$ & $\mathrm{NA}$ & $\mathrm{NA}$ \\
\hline 7 & Mar-Jun & NA & $\mathrm{NA}$ & $\mathrm{NA}$ & $\mathrm{NA}$ & $\mathrm{NA}$ & NA & $\mathrm{NA}$ & NA & NA & $\mathrm{NA}$ & $\mathrm{NA}$ & $\mathrm{NA}$ \\
\hline
\end{tabular}

Abundance lovel refers to estimated relative abundance of spawners that spawned in the natal stream reaches that populated the life history pathways using the reach in this table: Level 1: Low to average (relative to other reaches) abundance

Level 2: $\quad$ High (relative to other reaches) abundance

Level 3: Exceptionally high (relative to other reaches) abundance

Life stages: 1- prespawner; 2- spawner; 3- incubation; 4- fry colonization; 5- summer rearing; 6- overwintering; 7- yearling presmolt/smolt

$\begin{array}{lll}\text { Attributes: CHEM - chemicals } & \text { NUTLD - nutrient load } & \text { RIPCN - riparian condition } \\ \text { CSTAB - channel stability } & \text { OBST - obstructions } & \text { SEDLD - sediment load } \\ \text { COMP - competition (with other species) } & \text { OXYG - dissolved oxygen } & \text { TEMP - water temperature } \\ \text { FLOW - flow } & \text { PATHO - pathogens } & \text { WITH - water withdrawals } \\ \text { HABD - habitat diversity } & \text { PRED - predators (includes fishing-related losses) }\end{array}$

"NA" indicates that no lie history pathway meeting the criteria used in defining these pathways was present.

Blank cells under attributes indicate no additional attributes with negative effects on productivity were evident compared to those present in the template. 
Stream Reach Summary for Vacant Spring Chinook Salmon Life Histories

Stream: Imnaha R.

Reach: Dry Cr - Skookum Cr (RM 55.4 -61.9 from Snake R)

\begin{tabular}{ll|ll|lll}
\hline [Restoration benefit category: & F & Chinook usage score: & 2.7 & Habitat productivity loss score: & -4.3 \\
\hline
\end{tabular}

\begin{tabular}{|c|c|c|c|c|c|c|c|c|}
\hline \multirow[b]{3}{*}{ Life stage } & \multirow[b]{3}{*}{ Months } & \multirow{2}{*}{\multicolumn{4}{|c|}{$\begin{array}{c}\text { No. vacant life history pathways } \\
\text { by abundance level }\end{array}$}} & \multicolumn{2}{|c|}{ Key habitat change } & \multirow{3}{*}{$\begin{array}{c}\text { Habitat } \\
\text { productivity } \\
\text { loss score }\end{array}$} \\
\hline & & & & & & \multirow{2}{*}{$\begin{array}{l}\text { Patient } \\
\text { quantity }\end{array}$} & \multirow[b]{2}{*}{$\%$ change } & \\
\hline & & Total no. & 1 & 2 & 3 & & & \\
\hline 1 & Apr-Aug & 2 & 1 & 1 & $\overline{0}$ & 10.5 & 0.0 & 0.0 \\
\hline $1,2,3$ & Aug-Oct & $N A$ & NA & NA & NA & NA & NA & NA \\
\hline 3 & Ott-Mar & $\mathrm{NA}$ & $\mathrm{NA}$ & NA & $\mathrm{NA}$ & NA & $\mathrm{NA}$ & $\mathrm{NA}$ \\
\hline 34 & Mar-May & 5 & 4 & 1 & 0 “ & 8.4 & -171 & 0.1 \\
\hline 5 & May-Ott & 20 & 20 & 0 & 0 & 8.9 & -1.4 & -4.4 \\
\hline 6 & Ott-Mar & NA & NA & NA & NA & NA & NA & NA \\
\hline 7 & Mar-Jun & $\mathrm{NA}$ & $\mathrm{NA}$ & $\mathrm{NA}$ & $\mathrm{NA}$ & $\mathrm{NA}$ & $\mathrm{NA}$ & $\mathrm{NA}$ \\
\hline
\end{tabular}

\begin{tabular}{|c|c|c|c|c|c|c|c|c|c|c|c|c|c|}
\hline \multirow[b]{2}{*}{ Life stage } & \multirow[b]{2}{*}{ Months } & \multicolumn{12}{|c|}{ Highest productivity impact attributes (\% change in impact from template) } \\
\hline & & Att 1 & $\%$ change & Att 2 & $\%$ change & Att 3 & $\%$ change & Att 4 & $\%$ change & Att 5 & $\%$ change & Atf 6 & $\%$ change \\
\hline 1 & Apr-Aug & HABD & 192 & SEDLD & 19.2 & & & & & & & & \\
\hline $1,2,3$ & Aug-Ött & NA & & NA & NA & 'NA' & NA' & NA" & NA' & NA' & 'NA" & NA' & NA" \\
\hline 3 “ & "Ott-Mai & NA & NA & $\mathrm{NA}$ & NA & NA & NA & $\mathrm{NA}$ & NA“““ & NA & NA & NA. & NA \\
\hline 3,4 & Mar-May & "FLOOW" & 25.0 & PATHO & $25.0^{\circ}$ & & & & & & & & \\
\hline 5 & May-Ott & FLOW & 25.0 & PATHO & 25.0 & & & & & & & & \\
\hline$\dot{6}$ & Ott-Mar & $\mathrm{NA}$ & NÄ & $\ddot{N A}$ & $\mathrm{NA}$ & $\ddot{\mathrm{NA}}$ & $\ddot{N A}$ & $\ddot{\mathrm{NA}}$ & NÄ & $\ddot{\mathrm{NA}}$ & $\mathrm{NA}$ & $\dot{N A} \dot{A}^{\circ}$ & iNA \\
\hline 7 & Mar-Jun & NA & $\mathrm{NA}$ & NA & NA & "NA & NA & NA & NA & NA & NA & NA & NA \\
\hline
\end{tabular}

Abundance/eve/ refers to estimated relative abundance of spawners that spawned in the natal stream reaches that populated the life history pathways using the reach in this table:

Level 1: $\quad$ Low to average (relative to other reaches) abundance

Level 2: High (relative to other reaches) abundance

Level 3 Exceptionally high (relative to other reaches) abundance

Life stages: 1-prespawner; 2- spawner; 3- incubation; 4- fry colonization; 5- summer rearing; 6- overwintering; 7-yearting presmolt/smolt

Attributes: CHEM - chemicals

CSTAB - channel stability

COMP - competition (with other species)

FLOW - ftOW

HABD - habitat diversity

\section{NUTLD - nutrient load}

OBST - obstructions

OXYG - dissolved oxygen

PATHO - pathogens

PRED - predators (includes fishing-related losses)
RIPCN - riparian condition

SEDLD - sediment load

TEMP - water temperature

WITH - water withdrawals

"NA" indicates that no life history pathway meeting the criteria used in defining these pathways was present.

Blank cells under attributes indicate no additional attributes with negative effects on productivity were evident compared to those present in the template. 
Stream Reach Summary for Vacant Spring Chinook Salmon Life Histories

Stream: Imnaha R.

Reach: Blue Hole - forks (RM 66.4 -71.4 from Snake R)

\begin{tabular}{|c|c|c|c|c|c|c|c|c|}
\hline \multicolumn{4}{|c|}{ /Restoration benefit category: } & & \multicolumn{3}{|c|}{ Chinook usage score: } & 5.9 \\
\hline \multirow[b]{3}{*}{ Life stage } & \multirow[b]{3}{*}{ Months } & \multirow{2}{*}{\multicolumn{4}{|c|}{$\begin{array}{l}\text { No. vacant life history pathways } \\
\text { by abundance level }\end{array}$}} & \multicolumn{2}{|c|}{ Key habitat change } & \multirow{3}{*}{$\begin{array}{c}\text { Habitat } \\
\text { productivity } \\
\text { loss score }\end{array}$} \\
\hline & & & & & & \multirow{2}{*}{$\begin{array}{l}\text { Patient } \\
\text { quantity }\end{array}$} & \multirow[b]{2}{*}{$\%$ change } & \\
\hline & & Total no. & 1 & 2 & 3 & & & \\
\hline 1 & Apr-Aug & 7 & 1 & 6 & 0 & 7.7 & -6.5 & 1.6 \\
\hline $1,2,3$ & Aug-Oct & 10 & 0 & 10 & 0 & 6.2 & -6.1 & 67 \\
\hline 3 & Oct-Mar & 20 & 0 & 20 & 0 & 6.2 & 0.0 & 0.0 \\
\hline 3,4 & Mar-May & 3 & 1 & 2 & 0 & 6.6 & 0.0 & -0.7 \\
\hline 5 & May-Ocl & $\mathrm{NA}$ & NA & NA & $\mathrm{NA}$ & NA & NA & $\mathrm{NA}$ \\
\hline 6 & Oct-Mar & $\mathrm{NA}$ & NA & NA & NA & NA & NA & NA \\
\hline 7 & Mar-Jun & $\mathrm{NA}$ & $\mathrm{NA}$ & $\mathrm{NA}$ & $\mathrm{NA}$ & $\mathrm{NA}$ & $\mathrm{NA}$ & $\mathrm{NA}$ \\
\hline
\end{tabular}

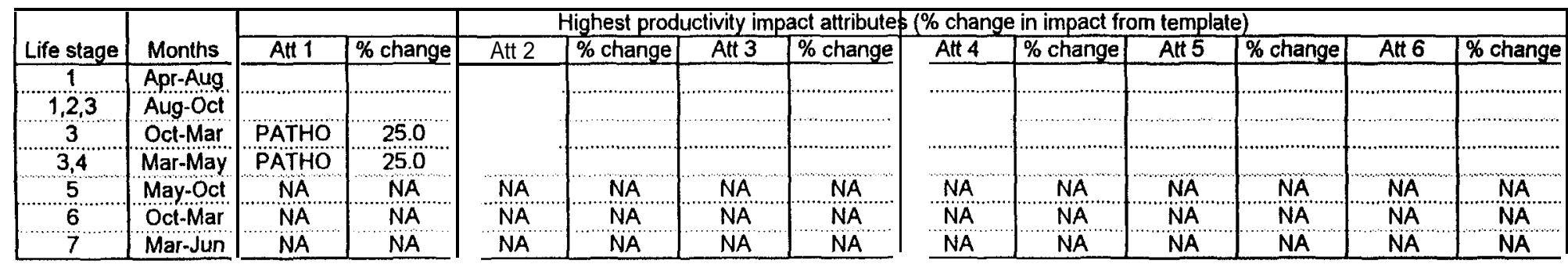

Abundance/eve/ refers to estimated relative abundance of spawners that spawned in the natal stream reaches that populated the life history pathways using the reach in this Level 1: Low to average (relative to other reaches) abundance

Level 2: $\quad$ High (relative to other reaches) abundance

Level 3: Exceptionally high (relative to other reaches) abundance

Life stages: 1-prespawner; 2-spawner; 3- incubation; 4- fry colonization; 5- summer rearing; 6- overwintering; 7-yearling presmolt/smolt

\begin{tabular}{|c|c|c|}
\hline $\begin{aligned} & \text { Attributes: } \text { CHEM - chemicals } \\
& \text { CSTAB - channel stability } \\
& \text { COMP - competition (with other species) } \\
& \text { FLOW - flow } \\
& \text { HABD - habitat diversity }\end{aligned}$ & $\begin{array}{l}\text { NUTLD - nutrient load } \\
\text { OBST - obstructions } \\
\text { OXYG - dissolved oxygen } \\
\text { PATHO - pathogens } \\
\text { PRED - predators (includes fishing-related losses) }\end{array}$ & $\begin{array}{l}\text { RIPCN - riparian condition } \\
\text { SEDLD - sediment load } \\
\text { TEMP - water temperature } \\
\text { WITH - } \quad \text { water withdrawals }\end{array}$ \\
\hline
\end{tabular}

"NA" indicates that no life history pathway meeting the criteria used in defining these pathways was present.

Blank cells under attributes indicate no additional attributes with negative effects on productivity were evident compared to those present in the template. 
Stream Reach Summary for Vacant Spring Chinook Salmon Life Histories

Stream: Imnaha R.

Reach: Skookum Cr - Blue Hole (RM 61.9 -66.4 from Snake R)

\begin{tabular}{|ll|ll|l|}
\hline Restoration benefit category: & $F$ & Chinook usage score: & 0.4 & Habitat productivity loss score: \\
\hline
\end{tabular}

\begin{tabular}{|c|c|c|c|c|c|c|c|c|}
\hline \multirow[b]{3}{*}{ Life stage } & \multirow[b]{3}{*}{ Months } & \multirow{2}{*}{\multicolumn{4}{|c|}{$\begin{array}{c}\text { No. vacant life history pathways } \\
\text { by abundance level }\end{array}$}} & \multicolumn{2}{|c|}{ Key habitat change } & \multirow{3}{*}{\begin{tabular}{|c|} 
Habitat \\
productivity \\
loss score
\end{tabular}} \\
\hline & & & & & & \multirow{2}{*}{$\begin{array}{l}\text { Patient } \\
\text { quantity }\end{array}$} & \multirow[b]{2}{*}{$\%$ change } & \\
\hline & & Total no. & 1 & 2 & 3 & & & \\
\hline 1 & Apr-Aug & 2 & 1 & 1 & 0 & 10.5 & 0.0 & 0.0 \\
\hline $1,2,3$ & Aug-Oct & $N A$ & NA & $\dot{N A}$ & NA & $\mathrm{NA}$ & $\mathrm{NA}$ & NA \\
\hline 3 & Ött-M̄ar & NA & NA & $N A$ & NA & NA & $\mathrm{NA}$ & NA \\
\hline 34 & Mar-May & $\ddot{2}$ & 1 & 1 & 0 & 8.6 & -42.9 & 0.0 \\
\hline 5 & May-Ott & "NA & $\mathrm{NA}$ & NÄ & NA & $\mathrm{NA}$ & $\mathrm{NA}$ & $\mathrm{NA}$ \\
\hline 6 & Ott-Mar & NA & $N A$ & $\mathrm{NA}$ & $\mathrm{NA}$ & $\mathrm{NA}$ & $N A$ & $\mathrm{NA}$ \\
\hline 7 & Mar-Jun & $\mathrm{NA}$ & NA & $N A$ & $\mathrm{NÄ}$ & $\mathrm{NA}$ & $\dot{\mathrm{NA}}$ & NA \\
\hline
\end{tabular}

\begin{tabular}{|c|c|c|c|c|c|c|c|c|c|c|c|c|c|}
\hline \multirow[b]{2}{*}{ Life stage } & \multirow[b]{2}{*}{ Months } & \multicolumn{12}{|c|}{ Highest productivity impact attributes (\% change in impact from template) } \\
\hline & & Att 1 & $\%$ change & Att 2 & $\%$ change & Att 3 & $\%$ change & Att 4 & $\%$ change & An 5 & $\%$ change & Att 6 & $\%$ change \\
\hline 1 & Apr-Aug & HABD & 12.0 & SEDLD & 12.0 & & & & & & & & \\
\hline $1,2,3$ & $\begin{array}{l}\text { Aug-Oct } \\
\text { Ott-Mar }\end{array}$ & NA & ' $\sqrt{A}$ & $\begin{array}{l}\text { NA } \\
\text { N A }\end{array}$ & NA & $\begin{array}{l}\text { NA } \\
N A \\
N A \cdots \cdots\end{array}$ & ${ }_{N A}^{N A}$ & $\begin{array}{l}\mathrm{NA} \\
\mathrm{NA}\end{array}$ & $\begin{array}{l}\text { NA } \\
\text { NA }\end{array}$ & $\begin{array}{l}\text { NA } \\
\text { NA }\end{array}$ & $\begin{array}{l}\text { NA } \\
\text { NA } \cdots \cdot \cdots\end{array}$ & $\begin{array}{l}\text { NA } \\
\text { NA }\end{array}$ & $\begin{array}{l}\text { NA } \\
\text { NA }\end{array}$ \\
\hline $\begin{array}{l}34 \\
5 \\
5 \\
6 \\
7\end{array}$ & $\begin{array}{l}\text { Mar-May } \\
\text { May-Ott } \\
\text { Ött-Mar } \\
\text { Mar-Jun }\end{array}$ & $\begin{array}{l}\text { PATHO } \\
\text { NA . } \\
\text { NA } \\
\text { NA }\end{array}$ & $\begin{array}{c}125 \\
\mathrm{NA} \\
\mathrm{NA} \\
\mathrm{NA}\end{array}$ & $\begin{array}{c}\text { NA } \\
\cdots \\
\text { NA } \\
\text { NA } \\
\text { NA }\end{array}$ & $\begin{array}{l}\text { NA } \\
\text { NA } \\
\text { NA }\end{array}$ & $\begin{array}{l}\text { NA } \\
\text { NA } \\
\text { NA }\end{array}$ & {$\left[\begin{array}{rr}N A \\
\cdots & N A \\
\cdots & N A\end{array}\right.$} & $\begin{array}{l}\mathrm{NA} \\
\mathbf{N A} \\
\mathbf{N A}\end{array}$ & $\begin{array}{c}N A \\
N A \\
\text { "N A" }\end{array}$ & $\begin{array}{l}\text { NA } \\
\dot{N} \dot{A} \\
\tilde{N A}\end{array}$ & $\begin{array}{l}\text { NÄ } \\
\text { NA" } \\
\text { NA }\end{array}$ & $\begin{array}{l}\text { NA } \\
\text { NA"' } \\
\text { NA }\end{array}$ & $\begin{array}{l}\text { NA } \\
\dot{N} \dot{A} \\
N A\end{array}$ \\
\hline
\end{tabular}

Abundance level refers to estimated relative abundance of spawners that spawned in the natal stream reaches that populated the life history pathways using there ch in this table Level 1: $\quad$ Low to average (relative to other reaches) abundance

Level 2: $\quad$ High (relative to other reaches) abundance

Level 3: Exceptionally high (relative to other reaches) abundance

Life stages.' 1 - prespawner; 2- spawner; 3- incubation; 4- fry colonization; 5- summer rearing; 6- overwintering; 7- yearling presmolt/smolt

$\begin{array}{ll}\text { Attributes.' } & \text { NHEM - chemicals } \\ \text { CSTAB - channel stability } & \text { OBTLD - nutrient load } \\ \text { COMP - competition (with other species) } & \text { OXYG - dissolved oxygen } \\ \text { FLOW - ftOW } & \text { PATHO - pathogens } \\ \text { HABD - habitat diversity } & \text { PRED - predators (includes fishing-related losses) }\end{array}$

RIPCN - riparian condition

SEDLD - sediment load

TEMP - water temperature

WITH - water withdrawala

"NA" indicates that no tife history pathway meeting the criteria used in defining these pathways was present.

Blank cells under attributes indicate no additional attributes with negative effects on productivity were evident compared to those present in the template, 
Stream Reach Summary for Vacant Spring Chinook Salmon Life Histories

Stream: Imnaha R.

Reach: South Fork Imnaha R (RM 71.4 -76.1 from Snake R)

\begin{tabular}{|c|c|c|c|c|c|c|c|c|}
\hline \multicolumn{4}{|c|}{ Restoration benefit category: } & & \multicolumn{3}{|c|}{ [Chinook usage score: } & \multirow{3}{*}{$\begin{array}{l}3.8 \\
\text { Habiltant } \\
\text { productivitty }\end{array}$} \\
\hline \multirow[b]{3}{*}{ Life stage } & \multirow[b]{3}{*}{ Months } & \multirow{2}{*}{\multicolumn{4}{|c|}{$\begin{array}{l}\text { No. vacant life history pathways } \\
\text { by abundance level }\end{array}$}} & \multicolumn{2}{|c|}{ Key habitat change } & \\
\hline & & & & & & \multirow{2}{*}{$\begin{array}{l}\text { Patient } \\
\text { quantity }\end{array}$} & \multirow[b]{2}{*}{$\%$ change } & \\
\hline & & Total no. & 1 & 2 & 3 & & & loss score \\
\hline 1 & Apr-Aug & 6 & 6 & $\overline{0}$ & 0 & 5.0 & -5.6 & 1.5 \\
\hline $1,2,3$ & Aug-Oct & 10 & 10 & 0 & 0 & 4.6 & 0.0 & 6.7 \\
\hline 3 & Oct-Mar & 20 & 20 & 0 & 0 & 4.6 & 0.0 & 0.0 \\
\hline 3,4 & Mar-May & 2 & 2 & 0 & 0 & 5.0 & 0.0 & -0.5 \\
\hline 5 & May-Oct & NA & NA & NA & NA & NA & NA & NA \\
\hline 6 & Oct-Mar & NA & NA & $\mathrm{NA}$ & $\mathrm{NA}$ & NÄ & $\mathrm{NA}$ & NA \\
\hline 7 & Mar-Jun & $\mathrm{NA}$ & $\mathrm{NA}$ & $\mathrm{NA}$ & $\mathrm{NA}$ & NA & $N A$ & $\mathrm{NA}$ \\
\hline
\end{tabular}

\begin{tabular}{|c|c|c|c|c|c|c|c|c|c|c|c|c|c|c|}
\hline \multirow[b]{2}{*}{ Life stage } & \multirow[b]{2}{*}{ Months } & \multicolumn{13}{|c|}{ Highest productivity impact attributes (\% change in impact from template) } \\
\hline & & Att 1 & $\%$ change & Att 2 & & change & Att 3 & $\%$ change & Att 4 & $\%$ change & Att 5 & $\%$ change & Att 6 & $\%$ change \\
\hline 1 & Apr-Aug & & & & & & & & & & & & & \\
\hline $\begin{array}{l}1,2,3 \\
3 \% \text { “ }\end{array}$ & $\begin{array}{l}\text { Aug-Ôtt } \\
\text { Oct-Mar }\end{array}$ & 'PATHO & 12.5 & & & & & & & & & & & \\
\hline 3,4 & Mar-May & "PATHÖ & 12.5 & & & & & & & & & & & \\
\hline 5 & May-Ott & NA & NA & NA & & NA & NA & "' "NA" & NA & NA & NA & NA & NA & $\mathrm{NA}$ \\
\hline 6 & Ött-Mar & $\mathrm{NA}$ & $\mathrm{NA}$ & NÄ & & $\dot{\mathrm{NA}}$ & $\mathrm{NA}$ & NA & NA & NA & NA & $\mathrm{NA}$ & NA & $\mathrm{NA}$ \\
\hline 7 & Mar-Jun & NA & NA & NA & & $\mathrm{N} \quad \mathrm{A}$ & NA & " NA & NA & $\mathrm{N} A$ & NA & $\mathrm{NA}$ & $\mathrm{NA}$ & $\mathrm{NA}$ \\
\hline
\end{tabular}

Abundance lovel refers to estimated relative abundance of spawners that spawned in the natal stream reaches that populated the life history pathways using the reach in this table:

Level 1: Low to average (relative to other reaches) abundance

Level 2: High (relative to other reaches) abundance

Level 3 Exceptionally high (relative to other reaches) abundance

Life stages: 1 - prespawner; 2- spawner; 3- incubation; 4- fry colonization; 5- summer rearing; 6overwintering; 7- yearling presmolt/smolt

Attributes: CHEM - chemicals

NUTLD - nutrient load

OBST - obstructions

RIPCN - riparian condition

CSTAB - channel stability

OXYG - dissolved oxygen

PATHO - pathogens

FLOW - flow

PRED - predators (includes fishing-related losses)

cediment load

TEMP - water temperature

HABD - habitat diversity

"NA" indicates that no life history pathway meeting the criteria used in defining these pathways was present.

Blank cells under attributes indicate no additional attributes with negative effects on productivity were evident compared to those present in the template. 
Stream Reach Summary for Vacant Spring Chinook Salmon Life Histories

Stream: Lightning $\mathrm{Cr}$.

Reach: Mouth - forks (RM 5.4- 13.9 from Snake R)

\begin{tabular}{ll|ll|lll}
\hline Restoration benefit category & $F$ & Chinook usage score: & 9.0 & Habitat productivity loss score: & 11.9 \\
\hline
\end{tabular}

\begin{tabular}{|c|c|c|c|c|c|c|c|c|}
\hline \multirow[b]{3}{*}{ Life stage } & \multirow[b]{3}{*}{ Months } & \multirow{2}{*}{\multicolumn{4}{|c|}{$\begin{array}{l}\text { No. vacant life history pathways } \\
\text { by abundance level }\end{array}$}} & \multicolumn{2}{|c|}{ Key habitat change } & \multirow{3}{*}{$\begin{array}{c}\text { Habitat } \\
\text { productivity } \\
\text { loss score }\end{array}$} \\
\hline & & & & & & \multirow{2}{*}{$\begin{array}{l}\text { Patient } \\
\text { quantity }\end{array}$} & \multirow[b]{2}{*}{$\%$ change } & \\
\hline & & Total no. & 1 & 2 & 3 & & & \\
\hline 1 & Apr-Aug & 9 & 9 & 0 & 0 & 0.6 & 0.0 & 0.1 \\
\hline 123 & Aug-Oct & 10 & 10 & & 0 & 0.4 & 0.0 & \\
\hline 3 & Ött-Mar & 20 & 20 & & 0 & 0.5 & 4.9 & \\
\hline 34 & Mar-May & 8 & 8 & 0 & 0 & 0.6 . & 9.1 & 4.7 \\
\hline 5 & May-Ott & 25 & 25 & 0 & 0 & 0.5 & 0.0 & 0.0 \\
\hline 6 & Ott-Mar & NA & $\mathbf{N A}$ & NA" & $\mathrm{NA}$ & "NA & NA & NA \\
\hline 7 & Mar-Jun & NA & NA & NA & NA & $\mathrm{NA}$ & NA & NA \\
\hline
\end{tabular}

\begin{tabular}{|c|c|c|c|c|c|c|c|c|c|c|c|c|c|}
\hline \multirow[b]{2}{*}{ Life stage } & \multirow[b]{2}{*}{ Months } & \multicolumn{12}{|c|}{ Highest productivity impact attributes (\% change in impact from template) } \\
\hline & & Atf 1 & $\%$ change & Att 2 & $\%$ change & Att 3 & $\%$ change & Att 4 & $\%$ change & Att 5 & $\%$ change & Atf 6 & $\%$ change \\
\hline 1 & Apr-Aug & FLOW & 23.1 & & & & & & & & & & \\
\hline $1,2,3$ & Aug-Ôtt & F்ட்ல் & 8.3 & & $\ldots .$. & 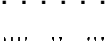 & $\because$. & . & $\ldots .$. & & ..... & & \\
\hline 3 ' & Ott-Mar & PATHO & "12.5 & & & . & & & & & & & \\
\hline 3,4 & Mar-May & COMP. & ,25.0 & PATHO & 25.0 & & & & & & & & \\
\hline 5 & May-Ott & ĊOMP & 25.0 & PATHO & 25.0 & & & & & & & & \\
\hline 6 & Ott-Mar & NA & NA & NA & NA & NA & NA & $\because N A$ & $\mathrm{NA}$ & $\mathrm{NA}$ & $\mathrm{NA}$ & $\mathrm{NA}$ & $\mathrm{NA}$ \\
\hline 7 & Mar-Jun & NA & NA & NA & NA & NA & NA & NÄ & NA & NA & NA & NA & NA \\
\hline
\end{tabular}

Abundance level refers to estimated relative abundance of spawners that spawned in the natal stream reaches that populated the life history pathways using the reach in this table Level 1: Low to average (relative to other reaches) abundance

Level 2: High (relative to other reaches) abundance

Level 3: Exceptionally high (relative to other reaches) abundance

Life stages: 1 - prespawner; 2- spawner; 3- incubation; 4- fry colonization; 5- summer rearing; 6- overwintering; 7- yearlingresmolt/smolt

\begin{tabular}{|c|c|c|c|c|c|}
\hline $\begin{aligned} & \text { Attributes: } \text { CHEM - } \\
& \text { CSTAB - } \\
& \text { COMP - } \\
& \text { FLOW - } \\
& \text { HABD - }\end{aligned}$ & $\begin{array}{l}\text { chemicals } \\
\text { channel stability } \\
\text { competition (with other species) } \\
\text { flow } \\
\text { habitat diversity }\end{array}$ & $\begin{array}{l}\text { NUTLD - } \\
\text { OBST - } \\
\text { OXYG - } \\
\text { PATHO - } \\
\text { PRED - }\end{array}$ & $\begin{array}{l}\text { nutrient load } \\
\text { obstructions } \\
\text { dissolved oxygen } \\
\text { pathogens } \\
\text { predators (includes fishing-related losses) }\end{array}$ & $\begin{array}{l}\text { RIPCN - } \\
\text { SEDLD - } \\
\text { TEMP - } \\
\text { WITH - }\end{array}$ & $\begin{array}{l}\text { riparian condition } \\
\text { sediment load } \\
\text { water temperature } \\
\text { water withdrawals }\end{array}$ \\
\hline
\end{tabular}

"NA" indicates that no life history pathway meeting the criteria used in defining these pathways was present.

Blank cells under attributes indicate no additional attributes with negative effects on productivity were evident compared to those present in the template. 
Stream Reach Summary for Vacant Spring Chinook Salmon Life Histories

Stream: Little Sheep Cr.

Reach: Mouth - Bear Gulch (RM 25.4 -28.5 from Snake R)

\section{Restoration benefit category:}

Chinook usage score:

15.3 $\mid$ Habitat productivity loss score:

$-620.2$

\begin{tabular}{|c|c|c|c|c|c|c|c|c|}
\hline \multirow[b]{3}{*}{ Life slage } & \multirow[b]{3}{*}{ Months } & \multirow{2}{*}{\multicolumn{4}{|c|}{$\begin{array}{l}\text { No. vacant life history pathways } \\
\text { by abundance level }\end{array}$}} & \multicolumn{2}{|c|}{ Key habitat change } & \multirow{3}{*}{$\begin{array}{c}\text { Habitat } \\
\text { productivity } \\
\text { loss score }\end{array}$} \\
\hline & & & & & & \multirow{2}{*}{$\begin{array}{l}\text { Patient } \\
\text { quantity }\end{array}$} & \multirow[b]{2}{*}{$\%$ change } & \\
\hline & & Total no. & 1 & 2 & 3 & & & \\
\hline 1 & Apr-Aug & 37 & 31 & 6 & 0 & 0.6 & $-66,1$ & -131.1 \\
\hline $1,2,3$ & Aug-Oct & 30 & 30 & 0 & 0 & 2,3 & $-20.3^{\prime \prime}$ & -194.0 \\
\hline 3 & Oct-Mar & 60 & 60 & 0 & 0 & 3.1 & 0.0 & $-72.5^{\circ}$ \\
\hline 3,4 & Mar-May & 24 & 24 & 0 & 0 & 3.8 & 0.0 & -104.6 \\
\hline 5 & May-Oct & 56 & 52 & 4 & $\dot{0}$ & 3.5 & -3.4 & -107.1 \\
\hline 6 & Oct-Mar & 19 & 19 & 0 & $0^{\prime, \prime \prime \prime}$ & & 0.0 & -11.8 \\
\hline 7 & Mar-Jun & 6 & 4 & 2 & 0 & 13.2 & 0.0 & 0.9 \\
\hline
\end{tabular}

\begin{tabular}{|c|c|c|c|c|c|c|c|c|c|c|c|c|c|}
\hline \multirow[b]{2}{*}{ Life stage } & \multirow[b]{2}{*}{ Months } & \multicolumn{12}{|c|}{ Highest productivity impact attributes (\% change in impact from template) } \\
\hline & & $\overline{\text { Att } 1}$ & $\%$ change & Att 2 & $\%$ change & Att 3 & $\%$ change & Att 4 & $\%$ change & Att 5 & $\%$ change & $\overline{\text { Atf } 6}$ & $\%$ change \\
\hline 1 & Apr-Aug & FLOW & 60.2 & HABD & 40.2 & SEDLD & 40.2 & WITH & 40.2 & OBST. & 20.1 & PRED & 17.7 \\
\hline $1,2,3$ & Äug-Ött & F்L̈Öய் & 53.1 & CSTAB & 35.4 & HABD & 35,4 & WITH & 35,4 & ÓB́ST் & 17.7 & PRED" & 17.7 \\
\hline 3 & Ott-Mar & CSTAB & 44.2 & FLOW & 35.4 & SEDLD & 26,5 & TEMP & 17.7 & PATHO & $8.8 "$ & RIPCN & 8.8 \\
\hline 3 & Mar-May & CSTAB & 47,3 & FLOOW & $42.1^{\circ}$ & SÉDLDD & 28.4 & RIPCN & 28.1 & $\mathrm{HABD}$ & $14.0^{\circ}$ & NUTLDD & 14.0 \\
\hline 5 & May-Ott & CSTAB & 43.5 & FLOW & 42.0 & HABD & 29.0 & RIPCN & 29.0 & SEDLD & 29.0 & PATHO & 14.5 \\
\hline 6 & Ott-Mar & CSTAB & 9.3 & FLOW & 6.2 & HABD & 6.2 & RIPCN & 6.2 & SÉḊL் & 6.2 & PATHO & $\dot{3} . \dot{1}$ \\
\hline 7 & Mar-Jun & & & & & & & & & & & & \\
\hline
\end{tabular}

Abundance lovel refers to estimated relative abundance of spawners that spawned in the natal stream reaches that populated the life history pathways using the reach in this table

Level 1: Low to average (relative to other reaches) abundance

Level 2: $\quad$ High (relative to other reaches) abundance

Level 3: Exceptionally high (relative to other reaches) abundance

Life stages: 1- prespawner; 2- spawner; 3- incubation; 4- fry colonization; 5- summer rearing; 6- overwintering; 7-yearling presmoltsmolt

Attributes: CHEM - chemicals

CSTAB - channel stability

COMP - competition (with other species)

FLOW - flow

HABD - habitat diversity
NUTLD - nutrient load

OBST - obstructions

OXYG - dissolved oxygen

PATHO - pathogens

PRED - predators (includes fishing-related losses)
RIPCN - riparian condition

SEDLD - sediment load

TEMP - water temperature

WITH - water withdrawals

"NA" indicates that no life history pathway meeting the criteria used in defining these pathways was present.

Blank cells under attributes indicate no additional attributes with negative effects on productivity were evident compared to those present in the template. 
Stream Reach Summary for Vacant Spring Chinook Salmon Life Histories

Stream: Little Sheep Cr.

Reach: Bear Gulch - Devil's Gulch (RM 28.5 -30.9 from Snake R)

\begin{tabular}{ll|l|l|l}
\hline Restoration benefit category: & D & Chinook usage score: & 28.6 & Habitat productivity loss score:
\end{tabular}

\begin{tabular}{|c|c|c|c|c|c|c|c|c|}
\hline \multirow[b]{3}{*}{ Life stage } & \multirow[b]{3}{*}{ Months } & \multirow{2}{*}{\multicolumn{4}{|c|}{$\begin{array}{c}\text { No. vacant life history pathways } \\
\text { by abundance level }\end{array}$}} & \multicolumn{2}{|c|}{ Key habitat change } & \multirow{3}{*}{\begin{tabular}{|c|} 
Habitat \\
productivity \\
loss score
\end{tabular}} \\
\hline & & & & & & \multirow{2}{*}{$\begin{array}{l}\text { Patient } \\
\text { quantity }\end{array}$} & \multirow[b]{2}{*}{$\%$ change } & \\
\hline & & Total no. & 1 & 2 & 3 & & & \\
\hline 1 & Apr-Aug & 34 & 28 & 6 & 0 & 0.5 & -75.8 & -151.8 \\
\hline 123 & Aug-Ott & 30 & 30 & 0 & 0 & 1.1 & -31.2 & -274.0 \\
\hline 3 & Ott-Mar & 60 & 60 & 0 & 0 & 1.9 & 0.5 & -102.4 \\
\hline 34 & Mar-May & 32 & 23 & 9 & 0 & 3.3 & -6.7 & -179.4 \\
\hline 5 & May-Ott & 121 & 51 & 70 & 0 & 2.4 & -5.6 & -278.2 \\
\hline $6 “$ & Oct-Mar & 8 & 19 & 19 & $" 0 "$ & 1.9 & 4 & -137.9 \\
\hline 7 & Mar-Jun & 5 & 3 & 2 & 0 & 10.5 & -3.8 & 0.9 \\
\hline
\end{tabular}

\begin{tabular}{|c|c|c|c|c|c|c|c|c|c|c|c|c|c|}
\hline \multirow[b]{2}{*}{ Life stage } & \multirow[b]{2}{*}{ Months } & \multicolumn{12}{|c|}{ Highest productivity impact attributes (\% change in impact from template) } \\
\hline & & Att 1 & $\%$ change & Att 2 & $\%$ change & Att 3 & $\%$ change & $\overline{\text { Att } 4}$ & $\%$ change & Att 5 & $\%$ change & Att 6 & $\%$ change \\
\hline 1 & Apr-Aug & FLOW & 65.8 & HABD & 43.9 & SEDLD & 43.9 & WITH & 43.9 & CSTAB & 25.0 & TEMP & 25.0 \\
\hline $1,2.3$ & Auq-Ött & FL̈ÖW & 75.0 & CSTAB & 50.0 & HABD & 50.0 & SEDLD & 50.0 & "WiT̈H' & 50. & OBST" & 25.0 \\
\hline 3 & Oct-Mar & CSTAB & 75.0 & FLOW & 50.0 & SEDLD & 50.0 & PATHO & $25.0^{\circ}$ & RIPCN & 25.0 & TEMP & 25.0 \\
\hline 3,4 & Mar-May & CSTAB & 75.0 & F́löw் & $500^{\circ}$ & SĖDL̇D & $50.0^{\circ}$ & RIPCN & 33.3 & PATHO & 25.0 & "TËMP" & $25.0^{\circ}$ \\
\hline 5 & May-Ott & CSTAB & 50.7 & FLOW & 50.7 & RIPCN & 33.0 & SEDLD & 33.8 & HABD & 16.9 & NU்ṪLD & 16.9 \\
\hline$\dot{6}$ & Ött-Mar & CSTAB & 54.1 & ḞLÖẄ & 36.1 & HAंBं' & 36.1 & RIPCN & 36.1 & SEDLD & 36.1 & PATHO & $18.0^{\circ}$ \\
\hline 7 & Mar-Jun & & & & & & & & & & & & \\
\hline
\end{tabular}

Abundance level refers to estimated relative abundance of spawners that spawned in the natal stream reaches that populated the life history pathways using the reach in this table: Level 1: Low to average (relative to other reaches) abundance

Level 2: High (relative to other reaches) abundance

Level 3: Exceptionally high (relative to other reaches) abundance

Life stages: 1- prespawner; 2- spawner 3- incubation; 4- fry colonization; 5- summer rearing; 6- overwintering; 7- yearling presmolt/smolt

\section{Attributes: CHEM - chemicals}

CSTAB - channel stability

COMP - competition (with other species)

FLOW - ftOW

HABD - habitat diversity
NUTLD - nutrient load

OBST - obstructions

OXYG - dissolved oxygen

PATHO - pathogens

PRED - predators (includes fishing-related losses)
RIPCN - riparian condition

SEDLD - sediment load

TEMP - water temperature

WITH - $\quad$ water withdrawals

"NA" indicates that no life history pathway meeting the criteria used in defining these pathways was present.

Blank cells under attributes indicate no additional attributes with negative effects on productivity were evident compared to those present in the template. 
Stream Reach Summary for Vacant Spring Chinook Salmon Life Histories

Stream: Little Sheep Cr.

Reach: Devil's Gulch - Lightning Cr (RM 30.9 -33.1 from Snake R)

\begin{tabular}{ll|ll|l|}
\hline Restoration benefit category: & D & Chinook usage score: & 25.8 & Habitat productivity loss score:
\end{tabular}

$-1134.7]$

\begin{tabular}{|c|c|c|c|c|c|c|c|c|}
\hline \multirow[b]{3}{*}{ Life stage } & \multirow[b]{3}{*}{ Months } & \multirow{2}{*}{\multicolumn{4}{|c|}{$\begin{array}{l}\text { No. vacant life history pathways } \\
\text { by abundance level }\end{array}$}} & \multicolumn{2}{|c|}{ Key habitat change } & \multirow{3}{*}{$\begin{array}{c}\text { Habital } \\
\text { productivity } \\
\text { loss score }\end{array}$} \\
\hline & & & & & & \multirow{2}{*}{$\begin{array}{l}\text { Patient } \\
\text { quantity }\end{array}$} & \multirow[b]{2}{*}{$\%$ change } & \\
\hline & & Total no. & 1 & 2 & 3 & & & \\
\hline 1 & Apr-Aug & 36 & 30 & 6 & 0 & 0.5 & -79.5 & -181.9 \\
\hline $1,2,3$ & Aug-Oct & 40 & 40 & 0 & 0 & 1.4 & 27.0 & -386.9 \\
\hline 3 & Oct-Mar & $80^{\circ}$ & 80 & 0 & 0 & 2.1 & 5.6 & -136.5 \\
\hline 3.4 & Mar-May & 26 & 23 & 3 & 0 & 3.6 & 16.5 & -162.5 \\
\hline 5 & May-Oct & 52 & 50 & 2 & 0 & 2.8 & -5.6 & -171.9 \\
\hline 6 & Oct-Mar & 19 & 19 & 0 & 0 & 2.1 & 5.9 & -95.5 \\
\hline 7 & Mar-Jun & 3 & 2 & 1 & $\ddot{o}$ & 11.9 & 15.4 & 0.5 \\
\hline
\end{tabular}

\begin{tabular}{|c|c|c|c|c|c|c|c|c|c|c|c|c|c|}
\hline \multirow[b]{2}{*}{ Lifs stage } & \multirow[b]{2}{*}{ Months } & \multicolumn{12}{|c|}{ Highest productivity impact attributes (\% change in impact from template) } \\
\hline & & Atf 1 & $\%$ change & Att 2 & $\%$ change & Att 3 & \% change & $\overline{\text { Att } 4}$ & $\%$ change & Atf 5 & \% change & Atf 6 & $\%$ change \\
\hline 1 & Apr-Aug & FLOW & 75.0 & HABD & 50.0 & WITH & 50.0 & PRED & 45.4 & SEDLD & 45.4 & OBST & 25.0 \\
\hline $1,2,3$ & Aug-Ott & FLOW & $75.0^{\circ}$ & CŚTÁB & 50.0 & HABD & 50.0 & WITH & 50,0 & "P̈R̈ËD̆ & 37.5 & ÖB̈STं & $25.0^{\circ}$ \\
\hline 3 & Ott-Mar & CSTAB & 62.5 & Fiow & 50.0 & SEDLD & 37.5 & TEMP & 25.0 & PATHO" & 12.5 & RIPCN & 12.5 \\
\hline 3.4 & Mar-May & CSTAB & 75.0 & $\dot{F} \dot{L} \dot{O} \dot{W}$ & & 'RIPCN' & $50^{\prime} 0$ & SÉDLL & $50^{\circ}$ & HÄBÖ & 250 & NUTLD. & 25.0 \\
\hline 5 & May-Ott & CSTAB & $" 75.0$ & FLOW & & RIPCN & 50,0 & SEDLD & “50.0”"' & $\dot{H A B D}$ & $i 20$ & NUTLD & 25 \\
\hline $\begin{array}{l}6 \\
7\end{array}$ & $\begin{array}{c}\text { Ott-Mar } \\
\text { Mar-Jun }\end{array}$ & CSTAB & $75.0^{\circ}$ & FLÖẂ & $500^{\circ}$ & $H A B D$ & 50.0 & RIPCN & 50.0 & SEDLD & 50.0 & PATHO & 25 \\
\hline
\end{tabular}

Abundance level refers to estimated relative abundance of spawners that spawned in the natal stream reaches that populated the life history pathways using there_ch in this table: Level 1: Low to average (relative to other reaches) abundance

Level 2: High (relative to other reaches) abundance

Level 3 Exceptionally high (relative to other reaches) abundance

Life stages: 1- prespawner; 2- spawner; 3- incubation; 4- fry colonization; 5- summer rearing; 6- overwintering; 7- yearlingpresmoltsmolt

Attributes.' CHEM - chemicals

CSTAB - channel stability

COMP - competition (with other species)

FLOW - flow

HABD - habitat diversity
NUTLD - nutrient load

OBST - obstructions

OXYG - dissolved oxygen

PATHO - pathogens

PRED - predators (includes fishing-related losses)
RIPCN - riparian condition

SEDLD - sediment load

TEMP - water temperature

WITH - water withdrawals

"NA" indicates that no life history pathway meeting the criteria used in defining these pathways was present.

Blank cells under attributes indicate no additional attributes with negative effects on productivity were evident compared to those present in the template. 
Stream Reach Summary for Vacant Spring Chinook Salmon Life Histories

Stream: Little Sheep Cr.

Reach: Lightning $\mathrm{Cr}$ - Hayden $\mathrm{Cr}$ (RM 33.1 -38.8 from Snake R)

Restoration benefit category: \begin{tabular}{l|l} 
B & Chinook usage score:
\end{tabular}

\begin{tabular}{l|l}
43.8 & Habitat productivity loss score:
\end{tabular}

\begin{tabular}{|c|c|c|c|c|c|c|c|c|}
\hline \multirow[b]{3}{*}{ Life stage } & \multirow[b]{3}{*}{ Months } & \multirow{2}{*}{\multicolumn{4}{|c|}{$\begin{array}{l}\text { No. vacant life history pathways } \\
\text { by abundance level }\end{array}$}} & \multicolumn{2}{|c|}{ Key habitat change } & \multirow{3}{*}{$\begin{array}{c}\text { Habitat } \\
\text { productivity } \\
\text { loss score }\end{array}$} \\
\hline & & & & & & \multirow{2}{*}{$\begin{array}{l}\text { Patient } \\
\text { quantity }\end{array}$} & \multirow[b]{2}{*}{$\%$ change } & \\
\hline & & Total no. & 1 & 2 & 3 & & & \\
\hline 1 & Apr-Aug & 42 & 36 & 6 & 0 & 0.5 & -83.5 & -245.2 \\
\hline $1,2,3$ & Aug-Oct & 60 & 60 & 0 & 0 & 1.4 & -27.0 & -580.4 \\
\hline 3 & Oct-Mar & 120 & 120 & 0 & 0 & 2.1 & 5.6 & -204.8 \\
\hline 3,4 & Mar-May & 33 & 30 & 3 & 0 & 36 & 16.0 & -210.2 \\
\hline 5 & May-Oct & 73 & 71 & 2 & 0 & 2.8 & -4.1 & -246.4 \\
\hline 6 & Oct-Mar & 19 & 19 & 0 & 0 & 2.1 & 5.9 & -82.8 \\
\hline 7 & Mar-Jun & 2 & 1 & 1 & 0 & 11.9 & 20.0 & 0.5 \\
\hline
\end{tabular}

\begin{tabular}{|c|c|c|c|c|c|c|c|c|c|c|c|c|c|}
\hline \multirow[b]{2}{*}{ Life stage } & \multirow[b]{2}{*}{ Months } & \multicolumn{12}{|c|}{ Highest productivity impact attributes (\% change in impact from template) } \\
\hline & & Att 1 & $\%$ change & Att 2 & $\%$ change & Att 3 & $\%$ change & Att 4 & $\%$ change & Att 5 & $\%$ change & Att 6 & $\%$ change \\
\hline & Apr-Aug & FLOW & 75.0 & HABD. & 50.0 & SEDLD & 50.0 & WITH & 50.0 & CSTAB & 25.0 & OBST & 25.0 \\
\hline $1,2,3$ & Aug-Oct & FLOW & 75.0 & CSTAB & 50.0 & HABD & 50.0 & SEDLD & 50.0 & WITH & 50.0 & OBST & 25.0 \\
\hline 3 & Oott-Mar & CSTAB & 75.0 & FLOW & 50.0 & SEOLD & 50.0 & PATHO & 25.0 & RIPCN & 25,0 & TEMP & 25.0 \\
\hline 34 & Mar-May & CST AB & 75.0 & FLOW & 75.0 & RIPCN & 50.0 & SEDLD & 50.0 & HABD & 25.0 & NUTLD & 25.0 \\
\hline 5 & May-Ott & CSTAB & 74.4 & FLOW & 74.4 & RIPCN & 49.6 & SEDLD & 49.6 & HABD & 24.8 & NUTLD & 24. \\
\hline $\begin{array}{l}6 \\
7\end{array}$ & $\begin{array}{l}\text { Ott-Mar } \\
\text { Mar-Jun }\end{array}$ & CSTAB & $75.0^{\prime}$ & "F́L'ÓẂ" & $50.0^{\circ}$ & "HÄB'B'" & $50.0^{\circ}$ & RIPCN" & 50.0 & SEDLD & $50.0^{\circ}$ & PATHOO" & 25. \\
\hline
\end{tabular}

Abundance level refers to estimated relative abundance of spawners that spawned in the natal stream reaches that populated the life history pathways using the reach in this table: Level 1: Low to average (relative to other reaches) abundance

Level 2: $\quad$ High (relative to other reaches) abundance

Level 3: Exceptionally high (relative to other reaches) abundance

Life stages: 1-prespawner; 2- spawner; 3- incubation; 4- fry colonization; 5- summer rearing; 6- overwintering; 7-yearlingpresmolt/smolt

Attributes: CHEM - chemicals

CSTAB - channel stability

COMP - competition (with other species)

FLOW - flow

HABD - habitat diversity

\section{NUTLD - nutrient load}

OBST - obstructions

OXYG - dissolved oxygen

PATHO - pathogens

PRED - predators (includes fishing-related losses)
RIPCN - riparian condition

SEDLD - sediment load

TEMP - water temperature

WITH - water withdrawals

"NA" indicates that no life history pathway meeting the criteria used in defining these pathways was present.

Blank cells under attributes indicate no additional attributes with negative effects on productivity were evident compared to those present in the template. 
Stream Reach Summary for Vacant Spring Chinook Salmon Life Histories

Stream: Little Sheep Cr.

Reach: Hayden $\mathrm{Cr}$ - McCully $\mathrm{Cr}$ (RM 38.8 -48.8 from Snake R)

Restoration benefit category: B $\quad$ Chinook usage score:

$51.9 \quad$ Habitat productivity loss score:

$-1517.4$

\begin{tabular}{|c|c|c|c|c|c|c|c|c|}
\hline \multirow[b]{3}{*}{ Liie stage } & \multirow[b]{3}{*}{ Months } & \multirow{2}{*}{\multicolumn{4}{|c|}{$\begin{array}{c}\text { No. vacant life history pathways } \\
\text { by abundance level }\end{array}$}} & \multicolumn{2}{|c|}{ Key habitat change } & \multirow{3}{*}{$\begin{array}{c}\text { Habitat } \\
\text { productivit) } \\
\text { loss score }\end{array}$} \\
\hline & & & & & & \multirow{2}{*}{$\begin{array}{l}\text { Patient } \\
\text { quantity }\end{array}$} & \multirow[b]{2}{*}{$\%$ change } & \\
\hline & & Total no. & 1 & 2 & 3 & & & \\
\hline 1 & Apr-Aug & 42 & 0 & 42 & 0 & 0.4 & -85.8 & -245.2 \\
\hline $1,2,3$ & Aug-Oct & 60 & 0 & $60 "$ & "0 & 12 & -270 & -5479 \\
\hline 3 & Ött-Mar & 120 & 0 & 120 & 0 & 17 & -1.8 & -204.8 \\
\hline 34 & Mar-May & $33^{\circ}$ & 0 & 33 & 0 & 2.0 & -0.5 & -210.2 \\
\hline 5 & May-Ott & 71 & 0 & 71 & 0 & 2.2 & -8.7 & -241.5 \\
\hline 6 & Ott-Mar & 19 & 0 & 19 & " 0 & $" 1.7$ & -1.9 & -68.0 \\
\hline 7 & Mar-Jun & & ö & "i & "1 & 67 & 00 & 02 \\
\hline
\end{tabular}

\begin{tabular}{|c|c|c|c|c|c|c|c|c|c|c|c|c|c|}
\hline \multirow[b]{2}{*}{ Life stage } & \multirow[b]{2}{*}{ Months } & \multicolumn{12}{|c|}{ Highest productivity impact attributes (\% change in impact from template) } \\
\hline & & At! 1 & $\%$ change & At! 2 & $\%$ change & At! 3 & $\%$ change & At! 4 & $\%$ change & At! 5 & $\%$ change & At! 6 & $\%$ change \\
\hline 1 & Apr-Aug & FLOW & 75.0 & $\mathrm{HABD}$ & 50.0 & SEDLL & 50.0 & WITH & 50.0 & OBST & 250 & PRED & 25.0 \\
\hline $1,2,3$ & Äug-Ött & "FLÖẄ & 75.0 & CSTAB & $50.0^{\circ}$ & "HABD" & 50.0 & WITH & $50 \ddot{0}$ & OBBST & 25.0 & PRED & 25.0 \\
\hline 3 & Ott-Mar & "CSTAB" & 62.5 & FLOW & 500 & SEDLD & 37.5 & TEMP & 25.0 & PATHO & 12.5 & RIPCN & 125 \\
\hline 3,4 & Mar-Mày & CSTAB & $75.0^{\circ}$ & FLOOW & $75.0^{\circ}$ & RIPCN & 50.0 & SEDLD & 50.0 & HABD" & 250 & NÜTLD & 250 \\
\hline 5 & May-Ött & CSTAB & 75.0 & FLOW & 75.0 & "HAED" & 50.0 & RIPCN & 50.0 & SEDLLD & 50.0 & NUUTL̇D & 250 \\
\hline $\begin{array}{l}6 \\
7\end{array}$ & $\begin{array}{l}\text { Ott-Mar } \\
\text { Mar-Jun }\end{array}$ & CSTAB & 75.0 & FLOW & 50.0 & HABD & . & RIPCN & 50.0 & SEDLD' & 50.0 & PATHO & 250. \\
\hline
\end{tabular}

Abundance level refers to estimated relative abundance of spawners that spawned in the natal stream reaches that populated the life history pathways using the reach in this table Level 1: Low to average (relative to other reaches) abundance

Level 2: High (relative to other reaches) abundance

Level 3: $\quad$ Exceptionally high (relative to other reaches) abundance

Life stages: 1- prespawner; 2- spawner; 3- incubation; 4- fry colonization; 5- summer rearing; 6- overwintering; 7- yearling presmolt/smolt

Attributes: CHEM - chemicals

CSTAB - channel stability

COMP - competition (with other species)

FLOW - flow

HABD - habitat diversity
NUTLD - nutrient load

OBST - obstructions

OXYG - dissolved oxygen

PATHO - pathogens

PRED - predators (includes fishing-related losses)
RIPCN - riparian condition

SEDLD - aadiment load

TEMP - water temperature

WITH - water withdrawals

"NA" indicates that no life history pathway meeting the criteria used in defining these pathways was present.

Blank cells under attributes indicate no additional attributes with negative effects on productivity were evident compared to those present in the template, 


\section{APPENDIX H}

Bibliography 


\section{Bibliography}

Agee, J.K. 1994. Fire and weather disturbances in terrestrial ecosystems of the eastern Cascades. General Technical Report PNW-GTR-320, Report. US. Department of Agriculture, U.S. Forest Service, Pacific Northwest Research Station, Portland, Oregon.

Akin, J. Jr. 1909. Diary of James Akin, Jr. Page 259 in Transactions of the Thirty-sixth Annual Reunion of the Oregon Pioneer Association Portland, June 11, 1908. Chausse-Prudhomme Co., Portland, Oregon.

Alexander, C. W., R.L. Moffatt, P. R. Boucher, and M. L. Smith, 1987. Water resources data Oregon water year 1985, Volume 1: eastern Oregon. Report. U.S. Geological Survey, Portland, Oregon.

Allen, E. 1946. Canvas Caravans. Binfords \& Mort, Publishers, Portland, Oregon.

Allyn, H. 1924. Journal of Henry Allyn, 1853. Page 372 in Transactions of the Fortyninth Annual Reunion of the Oregon Pioneer Association Portland, June 16, 1921. Chausse-Prudhomme Co., Portland, Oregon.

Barry, J. N. 1911. The first-born on the Oregon Trail. Page 164 in F.G. Young, editor. The Quarterly of the Oregon Historical Society. XII. The Ivy Press, Portland, Oregon.

Bell, F. M., L. Crandall, T.R. Newell, K.N. Phillips, F.M. Veatch, and M.T. Wilson. 1956. Compilation of records of surface waters of the United States through September 1950. Part 13: Snake River Basin. Department of the Interior, Washington, D.C.

Bells, D. A. Ethics and the credibility of applied science. Oregon State University, Corvallis, Oregon.

Bengtsson, J. 1991. Interspecific competition in metapopulations. Biological Journal of the Linnean Society 42:219-237.

Beschta, R., W. Platts, and B. Kauffman, 1991. Field review of fish habitat improvement projects in the Grande Ronde and John Day River Basins of eastern Oregon. Report. Bonneville Power Administration, Portland, Oregon.

Bevan, D., J. Harville, P. Bergman, T. Bjornn, J. Crutchfield, P. Klingeman, and J. Litchfield, 1994. Snake River Salmon Recovery Team: Final Recommendations to the National Marine Fisheries Service. Appendices to May 1994 Report. National Marine Fisheries Service, Portland, Oregon.

Bjornn, T. C. and D.W.Reiser. 1991. Habitat requirements of salmonids in streams. Pages 83-138 in W.R. Meehan, editor. Influences of forest and rangeland management on salmonid fishes and their habitats. American Fisheries Society, Bethesda, Maryland. 
Booth, M. editor. Historical reprints: Overland from Indiana to Oregon, the Dinwiddie journal. State University of Montana, Missoula.

Bryson, D., 1994. Northeast Oregon hatchery project Grande Ronde River management plan. Final report, Bonneville Power Administration, Portland, Oregon.

Buckingham, H. T. 1984. Kenneth L. Holmes, editor. Covered wagon women, diaries \& letters from the western trails 1840-1890- Volume III 1851. The Arthur H. Clark Company, Glendale, California.

Buckley, G. L. 1992, Desertification of the Camp Creek drainage in central Oregon, 1826-1905. Master's thesis. University of Oregon, Eugene, Oregon.

Bunnell, F. L., 1989. Alchemy and uncertainty: what good are models? General Technical Report PNW-GTR-232, Report. United States Department of Agriculture, Forest Service, Pacific Northwest Research Station, Portland, Oregon.

Burck, W. A., 1994. Life history of spring chinook salmon in Lookingglass Creek, Oregon. Information Report 94-1. Oregon Department of Fish and Wildlife, Portland, Oregon.

Burnett, P. H. 1904. Recollections and opinions of an old pioneer. Page 4 in The Quarterly of the Oregon Historical Society. Volume V, March 1904- December 1904. J.R. Whitney, State Printer, Salem, Oregon.

Carmichael, R.W. and R.T. Messmer, 1985. Lower Snake River compensation plan, Oregon evaluation studies. Annual Progress Report. Oregon Department of Fish and Wildlife, Portland, Oregon.

Carmichael, R. W., B. A. Miller, and R. T. Messmer, 1986. Lower Snake River compensation plan, Oregon evaluation studies. Annual Progress Report. Oregon Department of Fish and Wildlife, Portland, Oregon.

Carmichael, R. W., R.T. Messmer, and B. A. Miller, 1987. Lower Snake River compensation plan, Oregon evaluation studies. Annual progress report, Oregon Department of Fish and Wildlife, Portland, Oregon.

Carmichael, R. W., R.T. Messmer, and B. A. Miller, 1988. Lower Snake River compensation plan, Oregon evaluation studies. Annual Progress Report. Oregon Department of Fish and Wildlife, Portland, Oregon.

Case, T.J. 1991. Invasion resistance, species build-up and community collapse in metapopulation models with interspecies competition. Biological Journal of the Linnean Society 42:239-266. 
Castle, G. 1931. Belshaw journey, Oregon Trail, 1853. L. M. Scott, editor. The Oregon Historical Quarterly. XXXII. Statesman Publishing Co., Salem, Oregon.

Caswell, H. and J.E. Cohen. 1991. Disturbance, interspecific interaction and diversity in metapopulations. Biological Journal of the Linnean Society 42:193-218.

Cheng, K. M., 1. M. McCallum, R. I. McKay, and B.E. March. 1987. A comparison of survival and growth of two strains of chinook salmon (Oncorhynchus tshawytscha) and their crosses reared in confinement. Aquiculture 67:301-311.

Clarke, W. C., R. E. Withler, and J.E. Shelbourn. 1992. Genetic control of juvenile life history pattern in chinook salmon (Oncorhynchus tshawytscha). Canadian Journal of Fisheries and Aquatic Sciences 49:2300-2306.

Collie, J. S. and C .J. Walters. 1991. Adaptive management of spatially replicated groundfish populations. Canadian Journal of Fisheries and Aquatic Sciences 48(7): 1273-1284.

Columbia Basin Fish \& Wildlife Authority, 1990. Grande Ronde River subbasin salmon and steelhead production plan, Columbia basin system planning. Northwest Power Planning Council, the Agencies and Indian Tribes of the Columbia Basin Fish and Wildlife Authority, Portland, Oregon.

Conyers, E. W. 1906. Diary of E. W. Conyers, a pioneer of 1852. Page 423 in Transactions of the Thirty-Third Annual Reunion of the Oregon Pioneer Association June 15, 1905. Peaslee Bros. Company, Printers, Portland, Oregon.

Costanza, R., B. G. Norton, and B. D. Haskell, editors. 1992. Ecosystem health: new goals for environmental management. island Press, Washington, D.C.

Cranston, S. A. 1984. K.L. Holmes, editor. Covered wagon women, diaries\& letters from the western trails 1840-1890- Volume III 1851. The Arthur H. Clark Company, Glendale, California.

Crawford, P. V. 1924. Journal of a trip across the plains, 1851. F.G. Young, editor. The Quarterly of the Oregon Historical Society. Koke-Tiffany Co., Eugene, Oregon.

Crouse, D. T., L. B. Crowder, and H. Caswell. 1987. A stage-based population model for loggerhead sea turtles and implications for conservation. Ecology 68(5): 1412-1423.

Doppelt, B., M. Scurlock, C. Frissell, and J. Karr. 1993. Entering the watershed: a new approach to save America's river ecosystems . Island Press, Covelo, California.

Douglas, D. 1914. Journal kept by David Douglas during his travels in North America 1823-1827 together with a particular description of thirty-three species of American oaks and eighteen species of pines. William Wesley\& Son, London. 
Drury, C. M., editor. 1958. The diaries and letters of Henry H. Spalding and Asa Bowen Smith relating to the Nez Perce Mission 1838-1842. The Arthur H. Clark Company, Glendale, California.

Ecosystem Charter Drafting and Advisory Committee members, 1993. Draft elements of an ecosystem charter for the Great Lakes - St. Lawrence Basin. Ecosystem Charter Drafting and Advisory Committee,

Eigenmann, C. H. 1895. Results of explorations in western Canada and the northwestern United States. Bulletin of the United States Fish Commission. Vol. XIV, for 1894. Government Printing Office, Washington D.C.

Ellison, J. W. 1932. Diary of Maria Parsons Belshaw, 1853. Page 320 in L. M. Scott, editor. The Oregon Historical Quarterly. XXXIII. Statesman Publishing Co., Salem, Oregon.

Endicott, E. 1993. Land conservation through public/private partnerships. Lincoln Institute of Land Policy and Island Press, Washington, D.C.

Everett, R., 1994. Volume II, Ecosystem management: principles and applications. General Technical Report, PNW-GTR-318, U.S. Department of Agriculture, Forest Service, Pacific Northwest Research Station, Portland, Oregon.

Everett, R., P. Hessburg, M. Jensen, and B. Bormann, 1993. Eastside forest ecosystem health assessment, Volume 1: executive summary. Report. United States Department of Agriculture, Forest Service, Forest Service Research, Portland, Oregon.

Everett, R., P. Hessburg, M. Jensen, and B. Bormann, 1994. Volume 1: Executive summary. General Technical Report, PNW-GTR-317, U.S. Department of Agriculture, Forest Service, Pacific Northwest Research Station, Portland, Oregon.

Evermann, B.W. and S.E. Meek, 1898. A report on salmon investigations in the Columbia River Basin and elsewhere on the Pacific coast in 1896. United States Bureau of Fisheries Bulletin, 1897 (1898), vol. XVII, p. 15-84. Dec. 352; F12-241, United States Bureau of Fisheries, Washington, D.C.

Forest Ecosystem Management Assessment Team, 1993. Forest ecosystem management: an ecological, economic, and social assessment. Report. U.S. Forest Service, U.S. Fish and Wildlife Service, National Park Service, National Oceanic and Atmospheric Administration, National Marine Fisheries Service, U.S. Bureau of Land Management, Environmental Protection Agency, Washington, D.C.

Fremont, B. Capt. Report of the exploring expedition to the Rocky Mountains in the year 1842, and to Oregon and North California in the years 1843-44. Blair and Rives, Washington, DC. 
Friday, J. and S.J. Miller, 1984. Statistical summaries of streamflow data in Oregon, Volume 1: eastern Oregon. Report. U.S. Geological Survey, Portland, Oregon.

Frissell, C. A. and T. Hirai. 1988. Life history patterns, habitat change, and productivity of fall chinook stocks of southwest Oregon. Pages 1-9 in Proceedings of the Northeast Pacific Chinook and Coho Workshop. North Pacific International Chapter of the American Fisheries-Society.

Gaumer, T. F., 1968. Closing report: behavior of juvenile anadromous salmonids in the Imnaha River, September 1964- June 1967. Report. Pacific Northwest Power Company, Portland, Oregon.

Gilbert, C. H. and B.W. Evermann. 1895. A report upon investigations in the Columbia River Basin, with descriptions of four new species of fishes. Bulletin of the United States Fish Commission. Vol. XIV, for 1894. Government Printing Office, Washington D.C.

Gildemeister, J., 1992. Bull trout, walking grouse and buffalo bones. Oregon Department of Fish and Wildlife, LaGrande, Oregon.

Gilpin, M. 1991. The genetic effective size of a metapopuiation. Biological Journal of the Linnean Society 42:165-175.

Glenn, J.G. 1852. Diary of John G. Glenn.

Grande Ronde Model Watershed Board. 1994. Operations - action plan. Report 5, Working draft. Grande Ronde Model Watershed Board, LaGrande, Oregon.

Grande Ronde Model Watershed Board. 1994. Operations - action plan. Report 6b, Draft Report. Grande Ronde Model Watershed Board, LaGrande, Oregon.

Gullixson, R., 1978. Ground water investigation of the upper Grande Ronde River Basin Union County, Oregon. Report. Army Corps of Engineers, Walla Walla District, Walla Walla, Washington.

Hanski, 1. 1991. Single-species metapopulation dynamics: concepts, models and observations. Biological Journal of the Linnean Society 42:17-38.

Hanski, 1. and M. Gilpin. 1991. Metapopulation dynamics: brief history and conceptual domain. Biological Journal of the Linnean Society 42:3-16.

Hansson, L. 1991. Dispersal and connectivity in metapopulations. Biological Journal of the Linnean Society 42:89-103.

Harden, A. B. 1847. Diary of Absolom B. Harden.

Harrison, S. 1991. Local extinction in a metapopulation context: an empirical evaluation. Biological Journal of the Linnean Society 42:73-88. 
Harvey, A. E., M. Geist, G. 1. McDonald, M.F. Jurgensen, P. H. Cochran, D. Zabowski, and R.T. Meurisse, 1994. Biotic and abiotic processes in eastside ecosystems: the effects of management on soil properties, processes, and productivity. General Technical Report PNW-GTR-323, U.S. Department of Agriculture, Forest Service, Pacific Northwest Research Station, Portland, Oregon.

Hasselstrom, L. M. editor. 1984. Journal of a mountain man James Clyman. Mountain Press Publishing Company, Missoula.

Hastings, L. B. 1926. Diary of Loren B. Hastings, a pioneer of 1847. Page 12 in Transactions of the Fifty-first Annual Reunion of the Oregon Pioneer Association Portland, June 21, 1923. F.W.Baltes and Company, Portland, Oregon.

Healey, M. C. Life history of chinook salmon (Oncorhynchus tshawytscha). Pages 313393 in Pacific salmon life histories.

Healey, M.C. 1983. Coastwide distribution and ocean migration patterns of streamand ocean-type chinook salmon, Oncorhynchus tshawytscha. Canadian FieldNaturalist 97(4): 427-433.

Hiatt, 1. 1893. Thirty-one years in Baker County: a history of the county from 1861 to 1893. Abbot \& Foster, Baker City, Oregon.

Hines, C. 1986. K.L. Holmes, editor. Covered wagon women, diaries \& letters from the western trails 1840-1890- Volume VI 1853-1854. The Arthur H. Clark Company, Glendale, California.

Homer, N. and T.C. Bjornn, 1981. Status of upper Columbia and Snake River coho salmon in relation to the Endangered Species Act. Report. U.S. Fish and Wildlife Service, Moscow, Idaho.

Hubbard, L. E., R.L.Moffatt, T. A. Herrett, R.L. Kraus, and G. P. Ruppert, 1990. Water resources data Oregon water year 1989, Volume 1: eastern Oregon. Report. U.S. Geological Survey, Portland, Oregon.

Hulbert, A. B. and D. P. Hulbert. The Oregon crusade: across land and seato Oregon. Overland to the Pacific vol. 5. Stuart Commission of Colorado College, Denver Public Library.

Hunt, W. P. 1973. The overland diary of Wilson Price Hunt. H. C. Franchere, translator and editor. Oregon Book Society.

Huntington, C. W., 1994. Stream and riparian conditions in the Grande Ronde Basin 1993. Grande Ronde Model Watershed Board, LaGrande, Oregon.

Irving, W. 189? Astoria or anecdotes of an enterprise beyond the Rocky Mountains. Belford-Clarke Co., Chicago. 
Jimenez, J., 1994. Biological assessment: upper main Grande Ronde River, section 7 major drainage. April, 1994 Report. La Grande and Walla Walla Ranger Districts, Wallow-Whitman and Umatilla National Forests.

Johnson, C. G. Jr., R.R. Clausnitzer, P.J. Mehringer, and C.D. Oliver, 1994. Biotic and abiotic processes of eastside ecosystems: the effects of management on plant and community ecology, and on stand and landscape vegetation dynamics. General Technical Report, PNW-GTR-322, U.S. Department of Agriculture, Forest Service, Pacific Northwest Research Station, Portland, Oregon.

Johnson, L. 1994. Patterns and process in ecological systems: a step in the development of a general ecological theory. Canadian Journal of Fisheries and Aquatic Sciences 51(1): 226-246.

Johnson, O. and Wm. H. Winter. 1900. Route across the Rocky Mountains with a description of Oregon and California, their geographical features, their resources, soil, climate, productions, etc., etc. Page 62 in The Quarterly of the Oregon Historical Society.

Johnson, S. L., M.F. Solazzi, and J. D. Rodgers, 1993. Development and evaluation of techniques to rehabilitate Oregon's wild salmonids. Progress Reports 1993. Oregon Department of Fish and Wildlife, Portland, Oregon.

Johnson, S. W., J.F. Thedinga, and K. V. Koski. 1992. Life history of juvenile oceantype chinook salmon (Oncorhynchus tshawytscha) in the Situk River, Alaska. Canadian Journal of Fisheries and Aquatic Sciences 49:2621-2629.

Kellogg, J. D. 1916. Memories of Jane D. Kellogg. Page 86 in Transactions of the Forty-First Annual Reunion of the Oregon Pioneer Association Portland, June 19, 1913. Chausse-Prudhomme Co., Portland, Oregon.

Knight, A. 1986. K.L. Holmes, editor. Covered wagon women, diaries \& letters from the western trails 1840-1890- Volume VI 1853-1854. The Arthur H. Clark Company, Glendale, California.

Lackey, R. T., (Adapted from a presentation given at the symposium: "Pacific Salmon and their Ecosystems: Status and Future Options," University of Washington, Seattle, January 10-12, 1994). Ecological risk assessment: protecting northwest anadromous salmonid stocks. Draft Report. Environmental Research Laboratory, U.S. EPA, Corvallis, Oregon.

Lehmkuhl, J. F., P.F. Hessburg, R.L. Everett, M.H. Huff, and R. D. Ottmar. Historical and current forest landscapes of eastern Oregon and Washington, Part 1: vegetation pattern and insect and disease hazards. US Department of Agriculture, Forest Service, Pacific Northwest Research Station in cooperation with Pacific Northwest Region. 
Li, H. W., G. A. Lamberti, T. N. Pearsons, C.K. Tait, J.L. Li, and J.C. Buckhouse. 1994. Cumulative effects of riparian disturbances on high desert trout streams of the John Day Basin, Oregon. Transactions of the North American Fisheries Society. 123(4): 627-640.

Ludwig, D., R. Hilborn, and C. Walters. 1993. Uncertainty, resource exploitation, and conservation: lessons from history. Science 260:17-36.

McDonnell, L.J. and S.F. Bates. 1993. Natural resources policy and law: trends and directions. Natural Resources Law Center and Island Press, Washington, D.C.

Masterson, M. G. 1986. One woman's west: recollections of the Oregon Trail and settling in the northwest country by Martha Gay Masterson 1838-1916. L. Barton, editor. Spencer Butte Press, Eugene, Oregon.

May, J. E., J.P. Kline, R.M. Richmond, D. P. Jr. Brigham, and R.L. Meinen, 1993. Wallowa and Grande Ronde Rivers. Final management plan/ environmental assessment. Department of the Interior, Washington, D.C.

McAllister, J. 1925. Diary of Rev. John McAllister a pioneer of 1852. Page 471 in Transactions of the Fiftieth Annual Reunion of the Oregon Pioneer Association Portland, June 15, 1922. Chausse-Prudhomme Co., Portland, Oregon.

McClung, J. S. 1862. Journal to Oregon, April 22nd 1862.

Mclntosh, B. A. 1992. Historical changes in anadromous fish habitat in the upper Grande Ronde River, Oregon, 1941-1990. Master's thesis. Oregon State University, Corvallis, Oregon.

McIntosh, B. A., J.R. Sedell, J.E. Smith, R.C. Wismar, S.E. Clarke, G.H. Reeves, and L. A. Brown, 1994. Management history of eastside ecosystems: Changes in fish habitat over 50 years, 1935 to 1992. General Technical Report, PNW-GTR-321, Report. U.S. Department of Agriculture, Forest Service, Pacific Northwest Research Station, Portland, Oregon.

McIntosh, B. A., S.E. Clarke, and J.R. Sedell, 1991. Summary report for Bureau of Fisheries stream habitat surveys: Clearwater, Salmon, Weiser, and Payette River Basins, 1934-1942. BPA Project No. 89-104, Historical data base, Pacific Northwest Research Station, USDA Forest Service, Oregon State University, Corvallis, Oregon.

McIntosh, B. A., S.E. Clarke, and J.R.Sedell, 1991. Summary report for Bureau of Fisheries stream habitat surveys: Columbia River Basin, 1934-1942. BPA Project No. 89-104, Historical data base, Report. Pacific Northwest Research Station, USDA Forest Service, Oregon State University, Corvallis, Oregon. 
Mclntosh, B. A., S.E. Clarke, and J.R. Sedell, 1991. Summary report for Bureau of Fisheries stream habitat surveys: Cowlitz River Basin, 1934-1942. BPA Project No. 89-104, Historical data base, Report. Pacific Northwest Research Station, USDA Forest Service, Oregon State University, Corvallis, Oregon.

Mclntosh, B. A., S.E. Clarke, and J.R. Sedell, 1991. Summary report for Bureau of Fisheries stream habitat surveys: Umatilla, Tucannon, Asotin, and Grande Ronde River Basins, 1934-1942. BPA Project No. 89-104, Historical data base., Report. Pacific Northwest Research Station, USDA Forest Service, Oregon State University, Corvallis, Oregon.

McIntosh, B. A., S.E. Clarke, and J.R. Sedell, 1991. Summary report for Bureau of Fisheries stream habitat surveys: Willamette River Basin, 1934-1942. BPA Project No. 89-104, Historical data base, Report. Pacific Northwest Research Station, USDA Forest Service, Oregon State University, Corvallis, Oregon.

Mclntosh, B. A., S.E. Clarke, and J.R. Sedell, 1991. Summary Report for Bureau of Fisheries stream habitat surveys: Yakima River Basin, 1934-1942. BPA Project No. 89-104, Historical data base, Report. Pacific Northwest Research Station, USDA Forest Service, Oregon State University, Corvallis, Oregon.

McMullin, R., 1994. Assessment of ongoing and proposed activities: Miriam River section 7 watershed. Final Report. Eagle Cap and La Grande Ranger Districts, Wallowa-Whitman National Forest.

McMullin, R., 1994. Assessment of ongoing and proposed activities: Wallowa River section 7 watershed. Final Report. Eagle Cap and Wallowa Valley Ranger Districts, Wallowa-Whitman National Forest.

Messmer, R. T., R.W. Carmichael, and M.W. Flesher, 1989. Lower Snake River compensation plan, Oregon evaluation studies. Annual Progress Report. Oregon Department of Fish and Wildlife, Portland, Oregon.

Messmer, R. T., R.W. Carmichael, and M.W.Flesher, 1990. Lower Snake River compensation plan, Oregon evaluation studies. Annual Progress Report. Oregon Department of Fish and Wildlife, Portland, Oregon.

Metcalfe, N.B. and J.E. Thorpe. 1990. Determinants of geographical variation in the age of seaward-migration salmon, Salmo salar. Journal of Animal Ecology 59: 135-145.

Minto, J. 1901. Reminiscences of Experiences on the Oregon Trailin 1844. F.G. Young, editor. The Quarterly of the Oregon Historical Society. II. W. H. Leeds, State Printer, Salem, Oregon.

Nachman, G. 1991. An acarine predator-prey metapopulation system inhabiting greenhouse cucumbers. Biological Journal of the Linnean Society 42:285-303. 
Naiman, R.J. 1992. Watershed management: balancing sustainability and environmental change. Springer-Verlag, New York.

National Commission on the Environment. 1993. Choosing a sustainable future. Island Press, Washington, D.C.

Nehlson, W., 1994. South Umpqua River Basin case study. Draft Report. Pacific Rivers Council, Portland, Oregon.

Nesmith, J. 1906. Emigration of 1843. F.G. Young, editor. The Quarterly of the Oregon Historical Society. VII. J. R. Whitney, State Printer, Salem, Oregon.

Nicholas, J.W. and D.G. Hankin, 1988. Chinook salmon populations in Oregon Coastal River Basins: description of life histories and assessment of recent trends in run strengths. Information Report 88-1, Oregon Department of Fish and Wildlife, Portland, Oregon.

Northcote,T.G. 1992. Migration and residency in stream salmonids--some ecological considerations and evolutionary consequences. Nordic Journal of Freshwater Resources 67:5-17.

Northrop, M. and G. Westlund, 1994. Biological assessment: middle Grande Ronde River watershed, for spring and fall Snake River chinook salmon. May, 1994. Report. Walla Walla Ranger District, Wallow-Whitman and Umatilla National Forests.

Olson, R. K., editor. 1992. Integrating sustainable agriculture, ecology, and environmental policy. Food Products Press, New York.

O'Neill, R. V., D.L.DeAngelis, J.B. Waide, and T.F.H. Allen. 1986. A hierarchical concept of ecosystems. Princeton University Press, Princeton, New Jersey.

Oregon Department of Fish and Wildlife and Confederated Tribes of the Warm Springs Reservation of Oregon, 1990. Columbia Basin system planning production plan: Salmon and steelhead. Hood River Subbasin. Northwest Power Planning Council, Confederated Tribes of the Warm Springs Reservation of Oregon, Portland, Oregon.

Palmer, J. 1906. Journal of travels over the Rocky Mountains to the mouth of the Columbia River, made during the years 1845 and 1846. R.G. Thwaites, editor. Arthur H. Clarke Company, Cleveland, Ohio.

Parker, A.J. 1878. Diary of Armeda Jane Parker.

Pearsons, T. N., H.W. Li, and G.A. Lamberti. 1992. Influence of habitat complexity on resistance to flooding and resilience of stream fish assemblages. Transactions of the American Fisheries Society 121:427-436. 
Pengra, C. E. Diary of Mrs. Bynon J. Pengra, Maiden name Charlotte Emily Stearns. Lane County Pioneer-Historical Society, Inc., Eugene, Oregon.

Perciasepe, R. 1994. EPA's watershed approach to ecosystem management. Fisheries 19(4): 4.

Perkins, S. S. 1986. K.L. Holmes, editor. Covered wagon women, diaries\& letters from the western trails 1840-1890, Volume VI 1853-1854. The Arthur H. Clark Company, Glendale, California.

Pillay, T.V. 1992. Aquiculture and the environment (sections 14.3-14.5). Halsted Press, New York.

Platz, I., 1994. Assessment of ongoing and proposed activities: Imnaha River Section 7 Watershed. Final Report. Eagle Cap, Wallowa Valley, and Pine Ranger Districts and Hells Canyon National Recreation Area, Wallowa-Whitman National Forest.

Platz, J., 1994. Assessment of ongoing and proposed activities: Joseph Creek Section 7 Watershed. Final Report. Hells Canyon National Recreation Area, Wallowa-Whitman National Forest.

Platz, J., 1994. Assessment of ongoing and proposed activities: Sheep Creek Section 7 Watershed. Final Report. Eagle Cap Ranger District, Hells Canyon National Recreation Area, Wallowa Valley Ranger District, Wallowa-Whitman National Forest.

Platz, J., 1994. Assessment of ongoing and proposed activities: Snake River Section 7 Watershed. Final Report. Hells Canyon National Recreation Area, Wallowa Valley Ranger District, Wallowa-Whitman National Forest.

Porter, E.L. 1864. Crossing the plains, a diary by Elizabeth Lee Porter 1864.

Ray, C., M. Gilpin, and A.T. Smith. 1991. The effect of nonspecific attraction on metapopulation dynamics. Biological Journal of the Linnean Society 42:123-134.

Reed, F. C. 1900. Annual reports of the Department of Fisheries of the state of Oregon to the legislative assembly, twenty-first regular session, 1901. W. P. Leeds, state printer, Salem, Oregon.

Regier, H. 1993. A perspective for fish and fishing in Wisconsin. Kemp Workshop, September 10-12, 1993, University of Wisconsin, Madison, Wisconsin.

Regier, H., 1993. Ecoscience as emerging in the Great Lakes Basin. Draft Report. National Water Research Institute, Burlington, Ontario.

Renshaw, R.H. 1851. Diary of Robert Harvey Renshaw. 
Rieman, B.E. and J.D. McIntyre, 1993. Demographic and habitat requirements for conservation of bull trout. General Technical Report INT-302. U.S. Forest Service, Intermountain Research Station, Ogden, UT.

Robbins, W.G. and D.W. Wolf, 1994. Landscape and the intermontane northwest: An environmental history. General Technical Report, PNW-GTR-319. U.S. Department of Agriculture, Forest Service, Pacific Northwest Research Station, Portland, Oregon.

Robe, R. 1928. Robert Robe's diary while crossing the plains in 1851. E.S. Meany, Managing Editor. The Washington Historical Quarterly. XIX(I). The Washington University State Historical Society, Seattle .

Root, R. 1955. Journal of travels from St. Josephs to Oregon with observations of that country, together with a description of California its agricultural interests, and a full description of its gold mines. Biobooks, Oakland 10, California.

Rose, R., 1994. Assessment of ongoing and proposed activities: Lostine River Section 7 Watershed. Final Report. Eagle Cap Ranger District, WallowaWhitman National Forest.

Sabelis, M. W., O. Diekmann, and V.A. Jansen. 1991. Metapopulation persistence despite local extinction: predator-prey patch models of the Lotka-Volterra type. Biological Journal of the Linnean Society 42:267-283.

Schluchter, M.D. and J.A. Lichatowich, 1977. Juvenile life histories of Rogue River spring chinook salmon Oncorhynchus tshawytscha (Walbaum), as determined by scale analysis. Report. Oregon Department of Fish and Wildlife, Corvallis, Oregon.

Scott, A. J. 1986. K.L. Holmes and D.C. Duniway, editor. Covered wagon women, diaries \& letters from the western trails 1840-1890- Volume V 1852. The Arthur H. Clark Company, Glendale, California.

Settle, R.W. editor. 1940. The march of the mounted riflemen, first United States military expedition to travel the full length of the Oregon Trail from Fort Leavenworth to Fort Vancouver May to October, 1849 as recorded in the journals of Major Osborne Cross and George Gibbs and the official report of Colonel Loring. The Arthur H. Clark Company, Glendale, California.

Shannon, M.A. and C. Robinson, 1993. Institutional strategies for landscape management. Report. College of Forest Resources, University of Washington, Seattle, Washington.

Shupp, B. 1994. Issues facing traditional fisheries management. Fisheries 19(4): 2425. 
Sims, W. E., 1994. Biological assessment: Catherine Creek, section 7 major drainage. Final Report, April, 1994. La Grande Ranger District, Wallow-Whitman National Forest.

Sims, W. E., 1994. Biological assessment: upper Grande Ronde River, section 7 major drainage. Final Report, April, 1994. La Grande and North Fork John Day Ranger Districts, Wallow-Whitman and Umatilla National Forests.

Sjögren, P. 1991. Extinction and isolation gradients in metapopulations: the case of the pool frog (Ranalessonae). Biological Journal of the Linnean Society 42:135147.

Skovlin, J.M. and J.W. Thomas, 1995. Interpreting long-term trends in Blue Mountain ecosystems from repeat photography. PNW-GTR-315, General technical Report . United States Department of Agriculture, Forest service, Pacific Northwest Research Station, Portland, Oregon.

Smith, E. D. 1983. K.L. Holmes, editor. Covered wagon women, diaries\& letters from the western trails 1840-1890, Volume I -1890-1849. The Arthur H. Clark Company, Glendale, California.

Smith-Gill, S.J. 1983. Developmental plasticity: developmental conversion versus phenotypic modulation. American Zoology 23:47-55.

Spinden, H. J. The Nez Perce Indians. American Anthropological Association.

State Fish and Game Protector. 1896. Third and fourth annual reports of the state fish and game protector of the state of Oregon 1895-1896. W.H. Leeds, State Printer, Salem, Oregon.

State Fish and Game Protector. 1898. Fifth and sixth annual reports of the state fish and game protector of the state of Oregon 1897-1898. W.H. Leeds, State Printer, Salem, Oregon.

Stout, L. 1852. A guide from the junction of the California and Oregon road, prepared only by guess, although is correct enough to travel.

Stout, L. 1852. Crossing the plains to Oregon in 1852.

Stuart, R. 1935. The discovery of the Oregon Trail: Robert Stuart's narratives. P.A. Rollins, editor. Charles Scribners Sons, New York.

Sutton, S. 1854. Diary of Sarah Sutton.

Symons, P.E. 1979. Estimated escapement of Atlantic salmon (Salmo salar) for maximum smelt production in rivers of different productivity. Journal of the Fisheries Research Board of Canada 36:132-140. 
Tait, C, K., J.L. Li, G.H.Lamberti, T.N. Pearsons, and H.W. Li. 1994. Relationships between riparian cover and the community structure of high desert streams. Journal of the North American Benthological Society :

Taylor, A.D. 1991. Studying metapopulation effects in predator-prey systems. Biological Journal of the Linnean Society 42:305-323.

Taylor, E.B. 1990. Environmental correlates of life-history variation in juvenile chinook salmon, Oncorhynchus tshawytscha(Walbaum). Journal of Fish Biology 37:117.

Taylor, E.B. 1990. Phenotypic correlates of life-history variation in juvenile chinook salmon, Oncorhynchus tshawytscha. Journal of Animal Ecology 59:455-468.

Thomas, J.W., M.G. Raphael, R.G. Anthony, E.D. Forsman, A.G. Gunderson, R.S. Holthausen, B.G. Marcot, G.H. Reeves, J.R. Sedell, and D.M. Solis, 1993. Viability assessments and management considerations for species associated with late-successional and old-growth forests of the Pacific Northwest. Report of the Scientific Analysis Team. United States Department of Agriculture, Forest Service Research.,

Thompson, R.N. and J.B. Haas, 1960. Environmental survey report pertaining to salmon and steelhead in certain rivers of eastern Oregon and the Willamette River and its tributaries, Part 1: Survey reports of eastern Oregon rivers. Report. Fish Commission of Oregon, Clackamas, Oregon.

Townsend, J. K. Narrative of a journey across the Rocky Mountains to the Columbia River. University of Nebraska Press, Lincoln, Nebraska.

U.S. Army Corps of Engineers Walla Walla District, 1984. Lower Snake River fish and wildlife compensation Big Canyon fish facilities. Design memorandum 13.1, U.S. Army Corps of Engineers, Walla Walla District, Walla Walla, Washington.

U.S. Army Corps of Engineers Walla Walla District, 1986. Lower Snake River fish and wildlife compensation Imnaha River satellite facilities. Design memorandum 13.2, U.S. Army Corps of Engineers, Walla Walla District, Walla Walla, Washington.

U.S. Army Corps of Engineers Walla Walla District., 1975. Special report: lower Snake River fish and wildlife compensation plan. U.S. Army Corps of Engineers, Walla Walla District, Walla Walla, Washington.

U.S. Army Corps of Engineers Walla Walla District., 1983. Lower Snake River fish and wildlife compensation Irrigon Fish Hatchery. Design memorandum 11, U.S. Army Corps of Engineers, Walla Walla District, Walla Walla, Washington. 
U.S. Army Corps of Engineers Walla Walla District., 1985. Lower Snake River fish and wildlife compensation Little Sheep Creek satellite facility. Design memorandum 13, U.S. Army Corps of Engineers, Walla Walla District, Walla Walla, Washington.

U.S. Army Corps of Engineers Walla Walla District., 1985. Lower Snake River fish and wildlife compensation Washington satellite fish facilities. Design memorandum 10, U.S. Army Corps of Engineers, Walla Walla District, Walla Walla, Washington.

U.S. Forest Service and U.S. Bureau of Land Management, 1993. Anadromous fish habitat and watershed conservation strategy (PACFISH). U.S. Department of Interior, Washington, D.C.

U.S. Forest Service, Oregon Department of Fish and Wildlife, Columbia River Intertribal Fish Commission, Confederated Tribes of the Umatilla Indian Reservation, Nez Perce Tribe, and Oregon State University, 1992. Upper Grande Ronde River anadromous fish habitat protection, restoration and monitoring plan. U.S. Forest Service, Oregon Department of Fish and Wildlife, Columbia River Intertribal Fish Commission, Confederated Tribes of the Umatilla Indian Reservation, Nez Perce Tribe, Oregon State University, Corvallis, Oregon.

U.S. Forest Service, U.S. Department of Agriculture, Bureau of Land Management, and U.S. Department of the Interior, 1994. Record of decision for amendments to Forest Service and Bureau of Land Management planning documents within the range of the northern spotted owl: Standards and guidelines for management of habitat for late-successional and old-growth forest related species within the range of the northern spotted owl. U.S. Forest Service; U.S. Deparment of Agriculture; Bureau of Land Management; U.S. Department of the Interior, Washington, D.C.

U.S. Forest Service, U.S. Department of Agriculture, Bureau of Land Management, and U.S. Department of the Interior, 1993. Draft supplemental environmental impact statement on management of habitat for late-successional and old-growth forest related species within the range of the northern spotted owl. U.S. Forest Service, U.S. Deparment of Agriculture, Bureau of Land Management, U.S. Department of the Interior, Washington, D.C.

U.S. Forest Service. Influence of forest and rangeland management on anadromous fish habitat in western North America - USDA Forest Service anadromous fish habitat program. U.S. Forest Service, Washington, D.C.

United States Commissioner of Fish and Fisheries. 1894. Report of the Commissioner of Fish and Fisheries on investigations in the Columbia River Basin in regard to the salmon fisheries. 53 rd Congress, 2nd Session. Senate Miscellaneous Document No. 200. Government Printing Office, Washington. 
United States Department of the Interior, 1950. Survey of the Columbia River and Its Tributaries. Fisheries No. 39, Special scientific Report. Fish and Wildlife Service, W a s h ington D.C.

Van Dusen, H. G. 1903. Annual reports of the Department of Fisheries of the state of Oregon to the legislative assembly, twenty-second regular session 1903. W.H. Leeds, state printer, Salem, Oregon.

Van Dusen, H. G. 1904. Annual reports of the Department of Fisheries of the state of Oregon for the years 1903 and 1904 to the twenty-third legislative assembly (regular session). W.H. Leeds, state printer, Salem, Oregon.

Van Dusen, H. G. 1907. Annual reports of the Department of Fisheries of the state of Oregon to the legislative assembly, twenty-fourth regular session 1907. W.H. Leeds, state printer, Salem, Oregon.

Van Dusen, H. G. Annual reports of the Department of Fisheries of the state of Oregon to the legislative assembly, twenty-second regular session 1903. W.H. Leeds, state printer, Salem, Oregon.

Verboom, J., K. Lankester, and J.A. Metz. 1991. Linking local and regional dynamics in stochastic metapopulation models. Biological Journal of the Linnean Society 42:39-55.

Walters, C. J. and J.S. Collie. 1989. An experimental strategy for groundfish management in the face of large uncertainty about stock size and production. Pages 13-25 in R. J. Beamish and G. A. MacFarlane, editors. Effects of ocean variability on recruitment and an evaluation of parameters used in stock assessment models. Canadian Special Publication of Fisheries and Aquatic Sciences 108.

Walters, C., L. Gunderson, and C.S. Helling. 1992. Experimental policies for water management in the Everglades. Ecological Applications 2(2): 189-202.

Walters, C., R.D. Goruk, and D. Radford. 1993. Rivers inlet sockeye salmon: an experiment in adaptive management. North American Journal of Fisheries Management 13:253-262.

Walters, C.J. 1989. Value of short-term forecasts of recruitment variation for harvest management. Canadian Journal of Fisheries and Aquatic Sciences 46(11): 1969-1976.

Walters, C.J. 1993. Dynamic models and large scale field experiments in environmental impact assessment and management. Australian Journal of Ecology 18:53-61.

Walters, C.J.and C.S. Helling. 1990. Large-scale management experiments and learning by doing. Ecology 71 (6): 2060-2068. 
Walters, C.J. and J.S. Collie. 1988. Is research on environmental factors useful to fisheries management? Canadian Journal of Fisheries and Aquatic Sciences $45(10)$ : $1848-1854$.

Walters, C.J. and R. Hilborn. 1976. Adaptive Control of Fishing Systems. Journal of the Fisheries Research Board of Canada 33(1): 145-159.

Walters, C.J. and R. Hilborn. 1978. Ecological optimization and adaptive management. Ann. Rev. Ecol. Syst. 9:157-188.

Warren, E. S. Memoirs of the west, the Spaldings.

Watson, W. C. 1876. The disappearance of the salmon and its causes. Pages 534538 in R.M.E. MacDonald, Appendix V: An analytical subject bibliography of the publications of the Bureau of Fisheries, 1871-1920. United States Bureau of Fisheries, Washington, D.C.

Watson, Wm. J. Journal of an overland journey to Oregon made in the year 1849. Ye Galleon Press, Fairfield, Washington.

Whitman, M. M. 1963. Mrs. Whitman's Diary, July to December 1836. C.M.Drury, editor. First white women over the Rockies, diaries, letters, and biographical sketches of the six women of the Oregon Mission who made the overland journey in 1836 and 1838- Vol. 1. The Arthur H. Clark Company, Glendale, California.

Wilkes, G. 1845. The history of Oregon, geographical and political. William H. Colyer, New York.

Winton, H. N. M. 1939. William T. Newby's Diary, Emigration of 1843. R.C. Clark, editor. The Oregon Historical Quarterly, XL. Statesman Publishing Co., Salem, Oregon.

Withler, R. E., W.C. Clarke, B.E. Ridden, and H. Kreiberg. 1987. Genetic variation in freshwater survival and growth of chinook salmon (Oncorhynchus tshawytscha). Aquiculture 64:85-96.

Young, F.G. editor. 1899. Sources of the History of Oregon, Volume 1, Parts 3 to 6 inclusive. University Press, Eugene, Oregon.

Zeiber, J. S. 1923. Diary of John S. Zeiber, 1851. Page 301 in Transactions of the Forty-Eighth Annual Reunion of the Oregon Pioneer Association Portland, July 1, 1920. Chausse-Prudhomme Co., Portland, Oregon. 